\title{
Development of a Recyclable Molybdenum Dearomatization Agent
}

\author{
Jeffery Todd Myers \\ Rock Hill, South Carolina
}

B. S., Winthrop University, 2012

A Dissertation presented to the Graduate Faculty of the University of Virginia in Candidacy for the Degree of

Doctor of Philosophy

Department of Chemistry

University of Virginia

May 2017
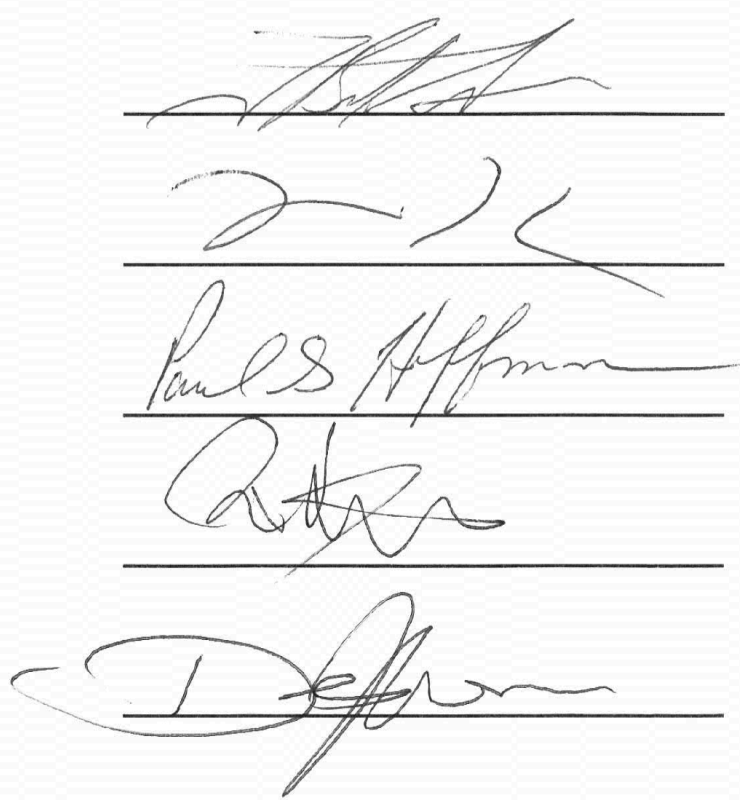


\section{Copyright Information}

Chapters 2 and 3 are compilations of two published works and have been reproduced in accordance with Section II.1 or American Chemical Society Journal Publishing Agreement. Proper citations for Chapters 2 and 3 are given. Chapter 5 is an outline for an upcoming publication.

Chapter 2: Jeffery T. Myers, Philip J. Shivokevich, Jared A. Pienkos, Michal Sabat, William H. Myers, and W. Dean Harman, Organometallics, 2015, 34 (14), pp 36483657.

Chapter 3: Jeffery T. Myers, Steven J. Dakermanji, Timothy R. Chastanet, Philip J. Shivokevich, Laura J. Strausberg, Michal Sabat, William H. Myers, and W. Dean Harman, DOI: 10.1021/acs.organomet.6b00780.

Chapter 5: Jeffery T. Myers, Jacob A. Smith, Steven J. Dakermanji, Katy B. Wilson, Philip J. Shivokevich, W. Dean Harman. 


\section{Acknowledgments/ A Reflection on the Past 10 Years}

There are a lot of people that I would like to acknowledge for their help and guidance as I have explored chemistry. This section cannot come close to conveying how indebted I feel to all of you. Whether you have helped me understand a concept, encouraged me not to give up, or simply helped me relax by enjoying a beer with me, I am truly grateful for your aid. Thank you.

When I was in high school I had no desire to go to college: I wanted to be a firefighter. In my senior year of high school I was learning how to connect SCBAs and clear rooms when I took my first class in chemistry. I started to think that this might be a fun thing to study further, but college felt so out of reach because I didn't have a financial plan. One night after work I called my grandparents and asked if there was any help they could give me. They told me that if I got into college, then they would pay for my books. This was the spark that I needed. I had to take the SATs three times to qualify for South Carolina's LIFE scholarship, but I did it, got accepted into Winthrop University, and began my journey in chemistry.

I would like to thank all of the professors in the chemistry department at Winthrop. There are two key events during my time at Winthrop that specifically altered my life. The first occurred in my general chemistry class, taught by Dr. Patrick Owens. Dr. Owens commanded respect when he entered a room. He is a man who demanded your focus and accepted nothing but your best. I would not have a career in chemistry without him. His class was comprised of two tests and a final exam. I failed his first test. I went to his office after receiving my grade and tried to explain. 
"I tried hard. I studied a lot and just didn't get it, but_-"

"No you didn't." said Dr. Owens. "You didn't study hard enough. You failed, Jeff. However, unlike other people who got a good grade and now think they don't have to study as hard, you know differently. You are going to work harder, study longer, and you are going to pass."

I did. I studied harder than I ever had before and I passed. If he hadn't said that, then I would not have even met the next person to change my life: my advisor, Dr. James Hanna. Dr. Hanna saw that I had done well in Dr. Owens' class and offered me a research opportunity. I had no lab experience and a pyromaniac mindset. Not the best combination for an organic chemistry lab. Before I got my hands on these Molotov cocktail mixers, Dr. Hanna asked me to write a literature review on "Palladium-Catalyzed Cross Coupling Reactions." I had to look up the definition of every word, but once I finished this assignment and began hands-on research, I knew chemistry was my passion. I stayed in Dr. Hanna's lab for three and a half years and loved every second of it. Without this experience, I probably would not have stuck with chemistry. I learned that although I am not the smartest person, I am a good researcher.

During my time at Winthrop, my graduating class grew as a family. We studied together, got coffee together, and complained about classes together. We stayed in this love-hate relationship because we knew how rewarding chemistry was to us. One of these late nights of studying led me to look outside the window and admire the trees outside. With the building stress of the impending organic exam, all I could think to say was, "I'd like to be a tree." Then, without skipping a beat, Paisley said, "Yeah." I was hooked. This beautiful girl that could outperform me in any chemistry class had my full attention. She 
had caught my attention in our first chemistry class. Sitting behind me, I grew jealous as she talked to the guy sitting beside her, Zayed. Admittedly, she may have been partly to blame for my failing Dr. Owens' first chemistry test. Paisley, Amber and I would study all hours of the night in Wofford Hall. We would make competitions out of studying; Who Can Write the Electron Configuration the Fastest was a game I always lost. But this, this understanding of wanting to be a tree, confirmed that she would be the one.

Senior year at Winthrop I began planning my next step: I wanted to be a nuclear engineer for the Navy. Around the time that I began interviews I decided to make my move on Paisley. After numerous rejections, she eventually agreed. I will not bore you with the details of how we accidentally wedding crashed or how we got lost in the middle of nowhere, but needless to say it was perfect. Again, I was hooked but there was a problem. Paisley wanted to go to grad school and the boomers that I would be stationed on would make a relationship with Paisley difficult. So, again I changed my direction and set my eyes on grad school. We applied to the same schools, with a few different ones, and hoped for the best. I really wanted to go to Wake Forest to work with Dr. Mark Welker on some cool Diels-Alder reactions. Of course, Paisley's impressive academic record won her a fellowship at Wake, but I got rejected. Out of the 7 or 8 schools that we applied to, the University of Virginia was the only one we both got in to. Paisley was ecstatic because she wanted to work for Dr. Don Hunt, but I had no clue who I wanted to work for.

During graduate prospective weekend at UVa I met Dr. Dean Harman. Dean's explanation of chemistry was like a child's obsession with his toys. He wanted me to understand how every little part worked. "The metal donates electron density into the $\pi^{*}$ 
orbital of the aromatic and this disruption of aromaticity allows us to activate the aromatic for chemical transformations...For example, we can take a pyrrole and through a cycloaddition create this epibatidine derivative. Have you ever heard of epibatidine?" Little did Dean know, I had just given a presentation on a total synthesis of epibatidine at Winthrop. This was the sign.

After joining Dean's lab I was asked if I wanted to work on the inorganic or the organic side. I decided the inorganic side because I wanted to broaden my horizons; this answer led me to be trained by Laura Straussberg. Laura was a great teacher. She taught me how to use a glovebox for the first time, how to interpret 2D NMR spectra, and how to keep my glovebox clean. With Laura's training, I soon became proficient at setting up small-scale reactions and the in-and-outs of the lab. I always think of Laura when I fold a sheet of paper in half to write on it. I am very grateful for Laura's patience with me. I may have been too anxious for independence and as such I was a little... annoying. Soon after I started, Laura went off to write her dissertation and Phil Shivokevich joined the Harman Lab. This changed everything.

From this point on, Jared Pienkos trained the two of us. Phil and I joined forces and began working on a single project that Dean set out for us. I still have the first sheet of paper that Dean handed us, which listed our project goals. It was titled The Road to Molybdenum Dearomatization. In essence, our project was to get "molly" off the ground. Our first of many goals was to synthesize $\mathrm{TpMo}(\mathrm{NO})(\mathrm{L})(\mathrm{Br})$ on a "usable" scale. So, Phil, Jared and I tackled this one step at a time. Every step in this procedure had some issue that we fixed together. Phil and I had weekly meetings with Dean to discuss our progress (more often our lack of progress), but these meetings always digressed into a 
discussion on general chemistry. In these meetings Dean showed us that the success of a reaction was not whether the reaction yielded the desired product, rather the success of a reaction is setting it up correctly. Did we consider our previous observations? Did we attempt a logical way to address these observations? Did we consider the error in our assumptions? These fundamental questions were instilled in us during our meetings with Dean.

A very important lesson Dean taught us was how to respect one another. I never felt afraid to ask for an explanation no matter how basic of a concept it would seem. Dean encouraged us to understand that in grad school everyone has a different background and to be sensitive towards someone's lack of knowledge. This was very important to me because I felt (and still feel) less knowledgeable than my colleagues. There were times when I was literally red in the face with embarrassment. One particular example of this was when I couldn't define the difference between diastereomers and enantiomers during group meeting. Dean did not let this lack of knowledge degrade his respect for me. What I did to earn this respect I do not know, but I am very grateful that he gave me a chance. Furthermore, I am honored that Dean has offered me a position in his lab as a post-doc. I know that there is still much for me to learn from Dean and I am excited that I will have another couple of years to do so. Thank you.

Another respectful man in the Harman Lab was Dr. Bill Myers. Bill was a postdoc for Dean that, thankfully, never left. After moving to Richmond to teach, Bill stayed on as our lab manager during the summer. Lab manager doesn't do justice to Bill's title. He was our teacher of all things (chemistry and more), counselor, spectra interpreter, but most importantly he was our friend. On my first day in lab, I walked into the conference 
room to find this gentle giant sitting at his desk. "You look like someone who has had a rough go," Bill said. I was baffled. What do you say to that?! Our time with Bill would be riddled with interesting events like this. From discussions of beautiful women in convertibles to The Terror of Tiny Town, Bill was always a fierce friend and avid teacher.

In the Harman Lab we always know that we can call on Dean whenever we need help, but more often we call on one another for help. The respect that Dean taught us to have for one another allows us to enjoy meetings and discussions unencumbered by a fear of being singled out for not knowing something. Furthermore, during our time in lab we have enjoyed a melting pot of music. From the Green Day fanatics to the Les Miserables enthusiasts, we have an unspoken respect for individuals' choices in music. A fun game Phil and I would play is Who Put that Song On, where we would identify the individual in lab that started a particular song. From this we have developed a few key identifying songs that I would like to share.

"Wake Me Up" by Avicii

What can I say about Dr. Jared Pienkos? You are a great friend first and foremost. You saved my life after I stabbed myself with an NMR tube, but were too good to wash my dishes for me. Jared had an incredible knack for scaling up the molly preps. He perfected the dimethylfuran synthesis (with MeIm as the ligand) while using me as an iodo complex monkey. He was the first person I showed Paisley's engagement ring to and, after she amazingly said yes, was the best man at our wedding. One of my favorite of many moments with Jared was when he (dressed up as a sandwich) got into a fight 
with a person (Tom Dawson maybe?) that was dressed up as a hot dog during a Halloween party. Jared did it for the sheer corniness of saying he was in a food fight.

"The Middle" by Jimmy Eat World

Dr. Ben Liebov is by far the most honest man I know. Ben would never miss an opportunity to help someone. Nor would Ben ever miss an opportunity to learn. With his Trader O's and sushi grade tuna, Ben always stuck to his principles and passions. Whether he was reminding us to wash the white caps or to keep the waste closed, Ben kept our lab in line. Two more songs that I cannot help but relate to him are "It Wasn't Me" by Shaggy and "Sugar" by Maroon 5. Whenever Shaggy came on, I knew that he was annoyed with Bri and I couldn't help but laugh. Whenever "Sugar" came on I would (and still do) curse Ben's name for ruining the music video's magic by explaining that it was not real. Lastly, I miss going to brunch at Beer Run with him and Nicole. Mimosas on Sunday mornings just aren't the same without you, Ben.

"Shake it Off" by Taylor Swift

Dr. Bri MacLeod drove me to be a better chemist. With Bri in lab I was driven to obtain the cleanest spectra and highest yields possible. Imperfections in my NMRs during group meeting would not go unnoticed by Bri's eagle eye. I believe she obtained an eagle eye enhancement during her "eye bubble" doctor's visits second year. Bri had me questioning the validity of my research quite a bit, which, although annoying at times, encouraged me to define what I found to be important with my own work. Phil will probably read that and say, "Meh." Bri was an overbearing mother at times, but was also 
a fun person to vent with. Sharing our failures with one another in hopes of making the other feel better became a small tradition between us. Bri was a strong proponent of our weekly outings, and as such our Thursday night trips to Three Notch'd (TKT!) have significantly decreased since she left, which is disheartening.

“Whiskey Lullaby” by Brad Paisley

There is not enough room to say all that I want to about Philip Shivokevich. Phil and I have spent the past 5 years figuring out everything to do with molybdenum dearomatization. Phil has a wealth of knowledge and every desire to share it with whoever wants to learn. Furthermore, Phil has been our local handyman when it comes to all things rotovap and more. Anywhere Phil goes he will be an asset. I will greatly miss our conversations about the future of the lab. Like Ben, I cannot help but relate another song to Phil, "The Gummy Bear Song" by Gummibär. We would play Señor Gummibär's song on a loop until someone would turn it off.

During our time as boxmates we would often argue about the stupidest things. For instance, whether lions sleep in the jungle or not, if our gloves are purple or blue, if high school is over or not... Phil always won those arguments, but never could win a beer bet with me. Speaking of not winning, I will be surprised if the North will defeat the South before you graduate, bet a 6-pack?

"I hate u, I love u" by Gnash

Katy "Jumbo Zinc" Wilson came into our lab shortly after Phil and I passed candidacy. At first Katy came off as quiet and shy. How wrong was that first 
impression?! What I mistook for bashfulness turned out to be Katy listening intently and learning everything she could. She has a vast knowledge of organic chemistry with tungsten. Katy has kindly put up with my continued badgering for her to try experiments, even when they don't align with Dean's desired reactions. She never misses a chance to hear about my "cool" observations or whacky thoughts. Even during my ludicrous discussion about an electrophile that can sometimes be a nucleophile, Katy listened; for that I am grateful. One of these days she is going to try Chicken Salad Chick. Like all of my suggestions, after a year of my suggesting it she will realize that I am right.

"Hooked on a Feeling” by Blue Suede

Tim Chastanet was the first person I trained as a graduate student. Tim taught me how to teach. His first project was to optimize the allyl preps in Chapter 3, so big shout out to him for that. Tim never missed an opportunity to talk about food. His gourmet tastes and my fast food tendencies often clashed, but he nonetheless was always excited to talk about his most recent culinary adventure. Tim never feared trying a new reaction. He perfected a methanol quenching of the acenaphthene reduction that would revolutionize the dimethylfuran prep (with DMAP as the ligand). Furthermore, without this "methanol workup" I doubt that the TFT prep in Chapter 5 would have become so successful. I owe you a great debt of gratitude, Tim.

"Amish Paradise" by "Weird" Al Yankovich

Steven Dakermanji optimized the $\mathrm{TpMo}(\mathrm{NO})(\mathrm{DMAP})\left(\eta^{2}\right.$-dimethylfuran) prep discussed in Chapter 3. This is the first of many advances Steven has contributed to the 
molly system. Steven's love for the lab is evident in his desire to help change tanks, do stockroom runs, and volunteer for random lab maintenance. This motivation is a rare quality and I am grateful to have him help keep the lab running. Furthermore, Steven's persistence in keeping Jared's/Ben's/my tradition alive by playing "Friday" by Rebecca Black on Fridays is much appreciated.

“Closer" by The Chainsmokers (ft. Halsey)

Alex Heyer has a drive for research that is unstoppable. Even to the point where he focuses on his own research while others are giving group meeting. He continues to push forward with a meticulous scientific approach, even if day after day he gets "black death". Furthermore, Alex encourages a community among the lab with weekly hypothetical/would you rather/personal questions on his board. For the official "Fun Friday" record, I won the whole pizza challenge, which will be added to my CV.

“Arrival” by Jóhann Jóhannsson

Jacob Smith is a titan of all things fluorine. All of the kinetic data, the newly bound fluorinated aromatics, and the benzene complex in Chapter 5 were all accomplished by Jacob. Let me repeat that, just in case someone didn't catch it. THE MOLLY BENZENE COMPLEX! Wow! When Jacob joined our lab it instantly felt like he had been there all along. One of the first challenges we tackled together was optimizing the TFT prep. This beast of an optimization was only accomplished by careful collaboration between the two of us. Sadly, we could not find a good enough reason to incorporate sand, whether white or brown, into this prep. Sorry, Dean. Once the 
optimization was completed, Jacob took off like a baby bird kicked out of the nest. Wranglin'!

Although he has not played any songs in lab I imagine Kevin's song to be something along the lines of the following: 80's song... by 80 's artist...

Dr. Kevin Welch has been a tutor to me for the past four months. I've only known him for this short period of time and have noticed a few key qualities about him. He is difficult to piss off, fun to argue with, and talentedly sarcastic. Most memorable to me was his comment in Chapter 5, "Technically naphthalene is a substituted benzene..." After reading this comment I promptly shouted “@\#\$ you!” at my laptop. Thank you for your time and advice, Kevin.

For the rest of the members in lab, I cannot pick out a specific song that I associate with you, my apologies. Ben "Bento Box" Cavannaugh is a mystery to me. His shy temperament yet happy personality are calming and intriguing. I think of Mr. Box as a Bruce Wayne of sorts. I would like to thank Hannah Nedz "Baller" for giving Katy an adversary worthy of her competitiveness. I also thank Hannah for being a whistler and enjoyer of Eric Hutchinson. Justin Wilde has an uncanny knowledge of organic chemistry. I give full credit to him for the pyridine-borane, pyridinium, and MVKpyridine complexes in Chapter 6. I have no doubt that molly dearomatization of ANHs will soon take off with Justin at the helm. Spenser Simpson has recently joined our lab. He has the spunk of a new kid, which will go far in our lab. Furthermore, he enjoys the outreach group, LEAD, and has a real talent with teaching kids science. I hope to pass on 
some of my experiences with LEAD in the hopes that he will continue with this important group.

Now to come back to the person who this dissertation is dedicated to: my wife, Dr. Paisley Myers. I don't know why you eventually said yes to my repeated attempts to ask you out, but you did, and I am so happy for that. It was in Dr. Owens' general chemistry class that our relationship began, and it was there that our relationship took its next step. You have a kind, caring, passionate heart and an incredible intelligence. Over the past five years you have made the tough times easier and the easy times joyful. You are my best friend and most cherished confidant. Thank you.

Aside from Kevin and Dean, our dog, Riley, was probably the most helpful in writing this dissertation. When I was stressed from writing at home, I knew I could come out to the living room and find him relaxed on the couch without a care in the world. The relaxing walks we'd take or belly rubs I'd give him during those times were a blessing that I treasure. Thank you.

My family has been a great supporter of my studying in Virginia. Specifically I want to thank my mom and sister. I am very thankful to have a mom who has sacrificed so much for us. Your example of perseverance and determination has driven me to do all that I have done. Jessica, I am so proud of the wonderful family you have created. I hope Paisley and I are as great of parents as you and Josh. To Dad and Carolyn, thank you for your support and guidance. I continue to live by the motto "adapt and overcome" that you taught me. To the rest of my family, I cannot thank you all personally, but I want each individual to know that I am very thankful for your continued support and encouragement. Thank you. 


\section{Abstract}

Chapter 1 includes a review on the past 30 years of electron-rich dearomatization agents. Specifically, the binding of aromatics to $\left\{\mathrm{Os}\left(\mathrm{NH}_{3}\right)_{5}\right\}^{2+},\{\operatorname{TpRe}(\mathrm{CO})(\mathrm{L})\}$, $\left\{\mathrm{TpW}(\mathrm{NO})\left(\mathrm{PMe}_{3}\right)\right\}$, and $\{\mathrm{TpMo}(\mathrm{NO})(\mathrm{MeIm})\}$ fragments is investigated. A primary focus of this review is to identify advantages and disadvantages discovered with each metal fragment. With this knowledge, ideal expectations of a more universally applicable dearomatization agent are stated, and the ensuing Chapters address instances where these expectations are met.

In Chapter 2 we demonstrate the ability of the $\{\mathrm{TpMo}(\mathrm{NO})(\mathrm{MeIm})\}$ fragment to activate naphthalene and anthracene towards organic transformations. After isolating these organically transformed complexes, the oxidative decomplexation of the new organics is achieved by stirring overnight in the presence of air. Next, we show that by using a halogen as an oxidant (iodine) we can improve the yield of the isolated organic, while also recovering a reusable metal center, $\mathrm{TpMo}(\mathrm{NO})(\mathrm{MeIm})(\mathrm{I})$.

Chapter 3 addresses a common disadvantage found in the comparison of molybdenum to its third-row congeners. Eta-2 bound aromatic complexes of $\{\mathrm{TpMo}(\mathrm{NO})(\mathrm{MeIm})\}$ are more susceptible to oxidation in the presence of electrophiles. By adjusting the ligand set, specifically replacing MeIm with DMAP, molybdenum becomes more resistant towards oxidation by electrophiles. A testament to this resistance is shown by the isolation of arenium complexes of naphthalene and anthracene bound to $\{$ TpMo(NO)(DMAP) $\}$. Furthermore, molybdenum's resistance towards electrophilic oxidation is investigated with the addition of carbon-based electrophiles. 
Chapter 4 continues the investigation of carbon-based electrophilic additions to TpMo(NO)(DMAP) $\left(\eta^{2}\right.$-naphthalene). Specifically, we explore the ability to perform MIMIRC additions on eta-2 bound naphthalene. This reaction is shown to be successful with various Michael acceptors and is used in the isolation of a fused ring system with a steroidal core.

In Chapters 5 and 6 we identify two new classes of aromatics that can bind to $\{\mathrm{TpMo}(\mathrm{NO})(\mathrm{DMAP})\}$ in an eta-2 fashion, fluorintated aromatics and pyridines. We then explore initial reactions that these complexes are capable of. 


\section{List of Abbreviations}

\begin{tabular}{|c|c|}
\hline $\mathrm{Cp}$ & Cyclopentadienyl (Cyclopentadienide Anion) \\
\hline $\mathrm{Cp}^{*}$ & Pentamethylcyclopentadienyl (Pentamethylcyclopentadienide Anion) \\
\hline DMA & N,N-dimethylacetamide \\
\hline DMAP & 4-N,N-Dimethylaminopyridine \\
\hline DME & 1,2-Dimethoxyethane \\
\hline DMF & N,N-Dimethylformamide \\
\hline EA & Elemental analysis \\
\hline ESI & Electrospray ionization \\
\hline EVK & Ethyl vinyl ketone \\
\hline HATR & Horizontal attenuated total reflectance \\
\hline IR & Infrared \\
\hline $\mathrm{KTp}$ & Potassium hydridotris(pyrazolyl)borate \\
\hline MeIm & 1-Methylimidazole \\
\hline MS & Mass spectrometry \\
\hline MTDA & Methyl trimethylsilyl dimethylketene acetal \\
\hline MVK & Methyl vinyl ketone \\
\hline NHE & Normal hydrogen electrode \\
\hline NMR & Nuclear magnetic resonance \\
\hline NOE & Nuclear Overhauser effect \\
\hline ORTEP & Oak Ridge Thermal Ellipsoid Program \\
\hline OTf & Trifluoromethanesulfonate (Triflate) anion \\
\hline $\mathrm{Pz}$ & A pyrazole group in hydridotris(pyrazolyl)borate \\
\hline TBAH & Tetrabutylammonium hexafluorophosphate \\
\hline TBDMSOTf & Tert-butyl-dimethylsilyl triflate \\
\hline
\end{tabular}


THF Tetrahydrofuran

TIPSOTf Tri-isopropylsilyl triflate

TLC Thin layer chromatography

Tp Hydridotris(pyrazolyl)borate anion 


\section{Table of Contents}

Copyright Information $\quad$ ii

Acknowledgments/ A Reflection on the Past 10 Years iii

List of Abbreviations xvii

List of Figures $\quad$ xxi

List of Schemes $\quad$ xxiii

Chapter 1: Introduction

1.1 Introduction 2

$1.2\left\{\mathrm{Os}\left(\mathrm{NH}_{3}\right)_{5}\right\}^{2+}$ Dearomatization 3

$1.3\{\operatorname{TpRe}(\mathrm{CO})(\mathrm{L})\}$ Dearomatization 6

$1.4\{\mathrm{TpMo}(\mathrm{NO})(\mathrm{MeIm})\}$ Dearomatization 15

$1.5\left\{\mathrm{TpW}(\mathrm{NO})\left(\mathrm{PMe}_{3}\right)\right\}$ Dearomatization 18

1.6 Developing the $\{$ TpMo(NO)(L)\} Dearomatization Agent 23

1.7 References 24

Chapter Two: Synthesis of 2-Substituted 1,2-Dihydronaphthalenes and 1,2Dihydroanthracenes Using a Recyclable Molybdenum Dearomatization Agent

2.1 Introduction 29

2.2 Results $\quad 30$

2.2.1 Improved Synthesis of TpMo(NO) $(\mathrm{MeIm})(L \pi)(L \pi=$ naphthalene or anthracene) 30

2.2.2 Oxidation of TpMo(NO)(MeIm) $\left(\eta^{2}\right.$-naphthalene) with Iodine 33

2.2.3 Spectroscopic Profile of TpMo(NO)(MeIm) $\left(\eta^{2}\right.$-1,2-dihydroarene) 36

2.2.4 Isolation of 1,2-Dihydroarenes Through Air Oxidation 39

2.2.5 Recyclable Oxidation of TpMo(NO) $\left(\right.$ MeIm) $\left(\eta^{2}\right.$-1,2-dihydroarenes $) \quad 41$

2.2.6 Chemical Elaboration of Isolated 1,2-Dihydroarenes 44

2.3 Discussion $\quad 44$

2.4 Conclusion $\quad 55$

2.5 Experimental

2.6 References $\quad 83$

Chapter 3: 4-(Dimethylamino)pyridine (DMAP) as an Acid-Modulated Donor Ligand for PAH Dearomatization

3.1 Introduction $\quad 86$

3.2 Results $\quad 87$

3.2.1. Synthesis of TpMo(NO) $(D M A P)(L \pi)$

3.2.2. Synthesis of TpMo(NO)(DMAP) $\left(\eta^{2}\right.$-arenium) complexes 90

3.2.3. Protonation and nucleophilic addition to 24 and 25

3.2.4. Nucleophilic additions to 27 and 28 
3.3 Discussion 101

3.4 Conclusion 113

$\begin{array}{ll}3.5 \text { Experimental } & 114\end{array}$

$\begin{array}{ll}3.6 \text { References } & 163\end{array}$

Chapter 4: Michael-Michael Ring Closures on TpMo(NO)(DMAP)( $\eta^{2}$-naphthalene)

4.1. Introduction 165

$\begin{array}{ll}4.2 \text { Results and Discussion } & 168\end{array}$

4.3 Conclusion 191

4.4 Experimental 192

4.5 References 206

Chapter 5: Binding and Activation of $\alpha, \alpha, \alpha$-Trifluorotoluene with $\{$ TpMo(NO)(DMAP)\}

5.1 Introduction

5.2 Results

5.3 Discussion

5.4 Conclusion

5.5 Experimental

5.6 References

Chapter 6: Activation of Pyridines Using \{TpMo(NO)(DMAP)\}

$\begin{array}{ll}6.1 \text { Introduction } & 238\end{array}$

6.2 Results and Discussion 240

6.3 Conclusion 257

6.4 Experimental 258

$\begin{array}{ll}6.5 \text { References } & 270\end{array}$

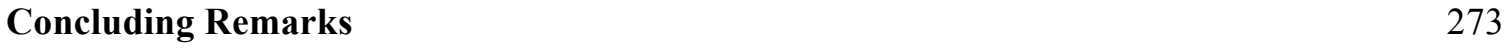

$\begin{array}{ll}\text { Appendix } & 277\end{array}$ 


\section{List of Figures}

Figure 1.1. Tandem electrophilic-nucleophilic addition to [Os]-bezene complex.

Figure 1.2. Regioselectivity obtained through greater retention of aromaticity. 5

Figure 1.3. Coordination diastereomers of $\operatorname{TpRe}(\mathrm{CO})(\mathrm{L})\left(\eta^{2}\right.$-naphthalene). $\quad 11$

Figure 2.1. NOESY correlations for $\mathbf{6 p}$ and the proposed assignment of $\mathbf{6 d}$ as its 37 coordination diastereomer, rather than a constitutional isomer resulting from a 1,4-addition. $J_{\mathrm{H} 1 \mathrm{~b}-\mathrm{H} 2}<1 \mathrm{~Hz}$.

Figure 2.2. Crystal structure of dihydronaphthalene complex 6.

Figure 2.3. Cyclic voltammogram of the reaction mixture following oxidation of 6 and air (top), CAN (middle), or iodine (bottom).

Figure 2.4. Naphthafuranones pygmaeocin $\mathrm{A}$ and arthrinin $\mathrm{A}$, and the anthrafuranone olivinolide.

Figure 2.5. Analysis using NOE (red arrows).

Figure 3.1. Solid-state structures of two dihydronaphthalene complexes 30, using MeIm (Left) ${ }^{11}$ and DMAP (Right).

Figure 3.2. NOESY correlations for 30p.

Figure 3.4. Analysis of NOE interactions.

Figure 4.1. Selected proton assignments for $\mathbf{6 1 .}$

Figure 4.2. Crystal structure of 87 .

Figure 4.3. Numbering scheme of 87 .

Figure 4.4. Analysis of possible steric interaction among different geometries of 61 .

Figure 4.6. Cis and trans orientations of H13 in 92.

Figure 4.7. Examples of steroidal cores found in the literature.

Figure 5.1. ${ }^{1} \mathrm{H}$ NMR spectra of $\mathrm{TpMo}(\mathrm{NO})(\mathrm{DMAP})\left(\eta^{2}-\mathrm{TFT}\right)(\mathbf{1 0 0})$ in 
$\mathrm{d}^{6}$-acetone at various temperatures.

Figure 5.2. Coordination diastereomers of $\mathbf{1 0 0}$ and their relative free energies as predicted from DFT calculations.

Figure 5.3. Intrafacial isomerization between 100-A and 100-B.

Figure 5.4. Pharmaceuticals containing $\mathrm{CF}_{3}$ groups.

Figure 5.5. Newly bound fluorinated aromatic complexes of $\{\mathrm{TpMo}(\mathrm{NO})(\mathrm{DMAP})\}$.

Figure 6.1. Resonance structures showing the delocalization of electron density 244 on 2-methoxypyridine via coordinating at the $\mathrm{C} 3-\mathrm{C} 4$ double bond.

Figure 6.2. Crystal structure of 124A. The triflate anion is omitted for clarity. $\quad 247$

Figure 6.3. ${ }^{1} \mathrm{H}$ NMR of $\mathbf{1 2 5}\left(\mathrm{d}^{6}\right.$-Acetone, $\left.25^{\circ} \mathrm{C}\right)$. 251

Figure 6.4. ${ }^{1} \mathrm{H}$ NMR spectra of $\mathbf{1 2 4}$ at various temperatures in $\mathrm{CD}_{3} \mathrm{CN}$. 253 
Scheme 1.1. Examples of hydrogenation, electrophilic addition, and cycloaddition 6 mediated by $\left\{\mathrm{Os}\left(\mathrm{NH}_{3}\right)_{5}\right\}^{+2}$.

Scheme 1.2. Organic products isolated as a racemic mixture from a diastereomeric 9 mixture of a stereogenic metal center.

Scheme 1.3. Diastereoselective control of electrophilic-nucleophilic additions to $\mathrm{TpRe}(\mathrm{CO})(\mathrm{L})\left(\eta^{2}\right.$-naphthalene $)$.

Scheme 1.4. Enantioenrichment of $\operatorname{TpRe}(\mathrm{CO})(\mathrm{L})\left(\eta^{2}\right.$-benzene $)$.

Scheme 1.5. Enantioselective organic modifications using $\{\mathrm{TpRe}(\mathrm{CO})(\mathrm{MeIm})\}$.

Scheme 1.6. Synthesis of $\mathrm{TpMo}(\mathrm{NO})(\mathrm{MeIm})\left(\eta^{2}-\mathrm{L} \pi\right)$.

Scheme 1.7. Diels-Alder synthesis of cycloadducts promoted by $\{\mathrm{TpMo}(\mathrm{NO})(\mathrm{MeIm})\}$.

Scheme 1.8. In situ protonation during the exchange of TpW(NO) $\left(\mathrm{PMe}_{3}\right)\left(\eta^{2}\right.$-benzene) 19 with aniline, indoline, or quinoline.

Scheme 1.9. Organic modification of $\mathrm{TpW}(\mathrm{NO})\left(\mathrm{PMe}_{3}\right)\left(\eta^{2}\right.$-indolinium $)$.

Scheme 1.10. Isolation of enantionenriched $\mathrm{TpW}(\mathrm{NO})\left(\mathrm{PMe}_{3}\right)\left(\eta^{2}\right.$-benzene $)$ complex. 21

Scheme 1.11. Synthesis of the eta-2 bound pyrrolium complex and subsequent synthesis of an indolizidine core.

Scheme 2.1. Synthesis of dihapto-coordinated naphthalene and anthracene complexes 32 of molybdenum.

Scheme 2.2. Proposed formal catalytic cycle for the dearomatization of naphthalene 34 with $\{\mathrm{TpMo}(\mathrm{NO})(\mathrm{MeIm})\}$.

Scheme 2.3. Oxidation of 4 with iodine.

Scheme 2.4. Iodolactonization of 12 and 13.

Scheme 2.5. Dearomatization of naphthalene using $\left\{\mathrm{Cr}(\mathrm{CO})_{3}\right\}$.

Scheme 2.6. Organic additions to anthracene. 
Scheme 2.7. Tandem electrophilic-nucleophilic addition reactions to 4 or $\mathbf{5}$.

Scheme 2.8. Potential pathways from the addition of an electrophile to $\mathbf{4}$ or $\mathbf{5}$.

Scheme 2.9. Exchange of 2,5-dimethylfuran with $\mathrm{CH}_{3} \mathrm{CN}$ to form $\mathrm{TpMo}(\mathrm{NO})(\mathrm{MeIm}) 52$ ( $\eta^{2}$-acetonitrile), and the resulting complex observed through $\mathrm{CV}$.

Scheme 2.10. Potential pathways after adding a nucleophile to an allylic species of 453 or 5.

Scheme 3.1. Arene protonation versus metal oxidation.

Scheme 3.2. Synthesis of $\eta^{2}$-coordinated naphthalene, anthracene, and

Scheme 3.3. Protonation and isolation of allylic naphthalene and anthracene complexes.

Scheme 3.4. Formal catalytic cycle for the generation of dihydronaphthalenes and dihydroanthracenes.

Scheme 3.5. Tandem addition of dimethoxypropane, and LiDMM, to 24 and $\mathbf{2 5} .99$

Scheme 3.6. Possible pathways after the addition of electrophile $\left(E^{+}\right)$to $\mathbf{2 4}$ or $\mathbf{2 5} . \quad 104$

Scheme 3.7. Oxidative decomposition of $\mathbf{C}$ by excess electrophile, $\mathrm{H}^{+}$in this case. 105

Scheme 3.8. Addition of dimethoxypropane and LiDMM to 24 using HOTf.

Scheme 3.9. Comparison of regiochemistry between 1,2 and 1,4-additions on $25 . \quad 109$

Scheme 3.10. Addition of dimethoxycyclohexane as an electrophile to $\mathbf{2 5}$, followed 111 by a nucleophilic addition with either $\mathrm{NaCNBH}_{3}$ (bottom) or LiDMM (top).

Scheme 3.11. Formation of the propanylidine dihydroanthracene complex 60.

Scheme 3.12. Electrophilic addition of HOTf and LiDMM to form 14 and the product of a Michael-Michael ring closure, 61.

Scheme 4.1. Tandem Michael-Michael-ring closure to yield ( \pm )-9,11-Dehydroestrone. 
Scheme 4.2. MIMIRC reactions on $\left\{\mathrm{Os}\left(\mathrm{NH}_{3}\right)_{5}\left(\eta^{2}-2 \text {-methylfuran }\right)\right\}^{2+}$ and $\mathrm{TpRe}(\mathrm{CO})(\mathrm{MeIm})\left(\eta^{2}-2-\right.$ methoxynaphthalene $)$.

Scheme 4.3. Attempted tandem electrophilic-nucleophilic addition of EVK and LiDMM to 24.

Scheme 4.4. Analysis of mixture resulting from the attempted tandem electrophilic 170 nucleophilic addition of EVK and LiDMM to 24.

Scheme 4.5. Proposed mechanism for the synthesis of $\mathbf{6 1 .}$

Scheme 4.6. Synthesis of $\mathbf{6 1}$ from 24 as well as common side-products (e.g., 85, 86, 175 and free naphthalene)

Scheme 4.7. Synthesis of 87 and 88 and their subsequent oxidations to yield 61 and 89.

Scheme 4.8. $[\mathrm{A}+\mathrm{B}] \mathrm{MIMIRC}$ additions to 24.

Scheme 4.8. Samarium diiodide-induced cyclization of naphthyl-substituted ketone (95).

Scheme 4.9. Competitive pathways encountered during the synthesis of 87 .

Scheme 5.1. Exchange of $\mathbf{2 6}$ with TFT to yield $\mathbf{1 0 0 .}$

Scheme 5.2. Reduction of $\mathbf{2 3}$ to $\mathbf{1 0 0 .}$

Scheme 5.3. Tandem addition reaction sequence with

$\mathrm{TpRe}(\mathrm{CO})(\mathrm{MeIm})\left(\eta^{2}\right.$-toluene) (101).

Scheme 5.4. Tandem electrophilic-nucleophilic additions to TpMo(NO)(DMAP) 219 $\left(\eta^{2}\right.$-TFT) (100) complex.

Scheme 5.5. Future reactivity for chemical elaboration from $\mathbf{1 0 0}$.

Scheme 6.1. Traditional synthetic methods for the reduction of pyridines.

Scheme 6.2. Coordination and reactivity of pyridines dearomatized through coordination to $\left\{\mathrm{TpW}(\mathrm{NO})\left(\mathrm{PMe}_{3}\right)\right\}$.

Scheme 6.3. Exchange of 2,5-dimethylfuran with 2-methoxy- and 
Scheme 6.4. Electrophilic addition to 121. 245

Scheme 6.5. Attempted electrophilic-nucleophilic additions to $\mathbf{1 2 3}$ and $\mathbf{1 2 4 .} 248$

Scheme 6.6. Reduction of 124 to yield $125 . \quad 248$

Scheme 6.7. Oxonium demethylation of $\mathbf{1 2 4} \quad 249$

Scheme 6.8. Attempted tandem electrophilic-nucleophilic additions to $\mathbf{1 2 3} 250$ and 124.

Scheme 6.9. Protonation of $122 . \quad 254$

Scheme 6.10. Exchange of TFT ligand for pyridine-borane and subsequent 255 reactivity. 


\section{Chapter 1}

\section{Introduction}




\subsection{Introduction}

Molecular libraries are largely made up of unsaturated, "flat" molecules. ${ }^{1}$ These planar, achiral molecules do not provide the complexity that medicinal chemists need to fully investigate the importance of molecular topology in the design and development of successful drug candidates. For this reason, functionalized alicyclic molecules are inherently more advantageous than their planar analogues because they contain potential stereocenters. Arenes represent a wealth of opportunities as synthons for functionalized alicyclic molecules because of their abundance in nature, their stability, and the diversity of substituents present. Being unsaturated, aromatic molecules offer numerous locations for addition and thus the functionalization and creation of stereocenters can, in theory, easily be achieved. Traditional methods for creating alicyclic molecules from aromatics include the Birch reduction, ${ }^{2,3}$ photocycloaddition, ${ }^{4}$ and enzymatic oxidation. ${ }^{5}$ However, perhaps the greatest potential lies in the ability to complete these transformations using transition metals.

Transition metal fragments such as $\left\{\mathrm{Cr}(\mathrm{CO})_{3}\right\}^{6-8},\left\{\mathrm{Mn}(\mathrm{CO})_{3}\right\}^{+9-11}$, and $\{\mathrm{FeCp}\}^{+12,13}$ can activate aromatics through an electron-withdrawing, $\eta^{6}$-coordination that makes the arene susceptible to nucleophilic attack. Although this method can yield alicyclic molecules, it more commonly results in the creation of substituted aromatics. A complementary strategy to this use of these electron-poor transition metals lies in the activation of aromatic molecules through $\eta^{2}$-coordination to an electron-rich metal complex. ${ }^{14,15}$ This coordination is controlled by the donation from a $\mathrm{d} \pi$ orbital of the metal into a $\pi^{*}$ orbital of the aromatic ligand. This donation results in a disruption of the aromaticity (e.g., dearomatization) making the ligand susceptible to tandem electrophilic- 
nucleophilic additions. Utilizing this dearomatization method, the stepwise, controlled synthesis of functionalized alicyclic compounds directly from aromatic molecules can be achieved. The use of electron-rich transition metals in the disruption of aromaticity has been investigated over the past three decades with the following metals: osmium (II), ${ }^{14}$ rhenium (I), ${ }^{14}$ tungsten $(0),{ }^{15}$ and molybdenum $(0) .{ }^{16,17}$

\section{$1.2\left\{\mathrm{Os}\left(\mathrm{NH}_{3}\right)_{5}\right\}^{2+}$ Dearomatization}

The ability to form a thermally stable, $\eta^{2}$-bound arene complex was first demonstrated in the coordination of $\left\{\mathrm{Os}\left(\mathrm{NH}_{3}\right)_{5}\right\}^{+2}([\mathrm{Os}])$ to benzene. ${ }^{18}$ The coordination of the metal occurs through the electron donation from a metal $\mathrm{d} \pi$ orbital into a $\pi^{*}$ orbital of the aromatic as well as a $\sigma$-donation from the aromatic to the metal (Figure 1.1). A key difference in comparison to other $\eta^{2}$-bound aromatic complexes (i.e., $\mathrm{Cu}(\mathrm{I}),{ }^{19} \mathrm{Ag}(\mathrm{I}),{ }^{19}$ $\mathrm{Pt}(0),{ }^{20} \mathrm{Ni}(0),{ }^{21}$ etc. $)$ is that the resulting [Os]-arene complex shows enhanced reactivity of the arene with electrophiles (vide infra). In contrast to the other $\eta^{2}$-bound aromatic complexes mentioned above, [Os] complexes are coordinatively saturated which prevents disfavored electrophilic attack at the metal center when attempting electrophilic additions to the aromatic ligand (Figure 1.1).

The coordination of benzene to [Os] accomplishes two key changes to the reactivity of the arene. First, the double bond bound to the metal is generally protected from further reactivity. In the case of benzene, the resulting dearomatization imparted by the metal yields an organic ligand resembling a diene. Second, the unbound portion of the ring exhibits a greater localization of electron density. The donation of electron density into the ring makes the uncoordinated organic more nucleophilic and promotes the 
addition of an electrophile. After the addition of the electrophile, the resulting positively charged allyl complex is stabilized through electron donation from the metal and, in some cases, this cationic complex can be isolated (Figure 1.1). ${ }^{22}$

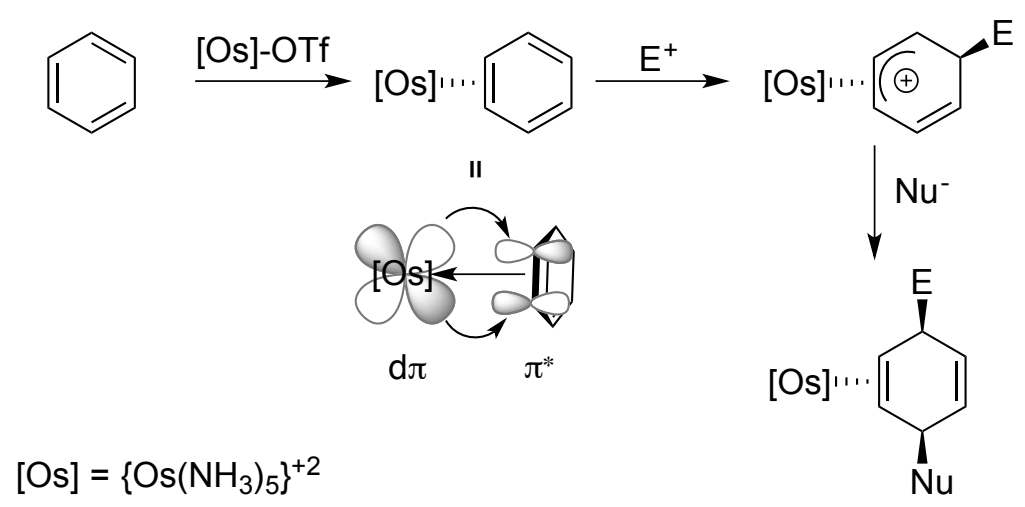

Figure 1.1. Tandem electrophilic-nucleophilic addition to [Os]-bezene complex.

These allyl complexes can then be subjected to nucleophilic attack yielding a net tandem electrophilic-nucleophilic addition product: an [Os]-( $\left.\eta^{2}-1,4-c y c l o h e x a d i e n e\right)$ complex. Upon oxidation of the metal complex, donation into the $\pi^{*}$ orbital is reduced and the functionalized organic ligand is released. Using this method while varying the electrophile (i.e., HOTf, acetals, ketals, Michael acceptors, etc.) and nucleophile (i.e., silyl ketene acetals, enolates, pyrroles, etc.), a variety of functionalized alicyclic molecules were synthesized. ${ }^{22-26}$

Further expanding upon this approach, [Os] has been shown to bind substituted benzenes, naphthalenes, ${ }^{27}$ pyrroles ${ }^{28}$ pyridines,${ }^{29}$ thiophenes,${ }^{30}$ and furans. ${ }^{31}$ The regiochemistry of the coordination is dictated by the electronic preference of the aromatic. Binding occurs where aromaticity is least disrupted or linear conjugation is best retained. ${ }^{14}$ For instance, $[\mathrm{Os}]$ binds naphthalene across the $\mathrm{C}(1)$ and $\mathrm{C}(2)$ rather than the $\mathrm{C}(2)$ and $\mathrm{C}(3)$ bond. Binding across the $\mathrm{C}(1)$ and $\mathrm{C}(2)$ bond retains the aromatic nature of 
one of the benzene rings, whereas coordination across the $\mathrm{C}(2)$ and $\mathrm{C}(3)$ bond disrupts the aromaticity of both rings (Figure 1.2). Once bound, these ligands can undergo simple organic transformations (i.e., Michael additions, Michael-Michael ring closure, DielsAlder, hydrogenation, etc.) to yield novel small molecules (Scheme 1.1). ${ }^{24,32-34}$

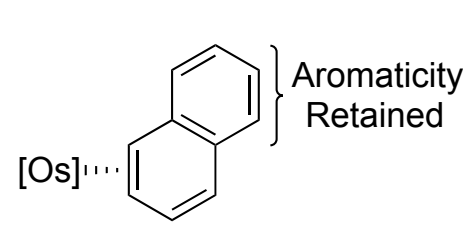

Binding through $\mathrm{C}(1)$ and $\mathrm{C}(2)$

Preferred

$$
\left.[\mathrm{Os}]=\left\{\mathrm{Os}\left(\mathrm{NH}_{3}\right)_{5}\right)\right\}^{+2}
$$

vs.

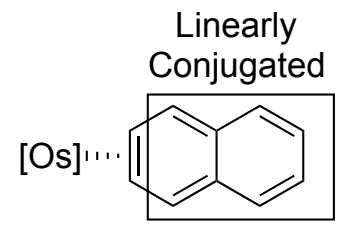

Binding through $\mathrm{C}(2)$ and $\mathrm{C}(3)$

Figure 1.2. Regioselectivity obtained through greater retention of aromaticity.

[Os], being achiral, cannot differentiate the two entiofaces of prochiral, aromatic ligands being coordinated. Thus racemic mixtures of the [Os]-aromatic complexes are isolated. Due to the large steric bulk of the metal complex, additions to the coordinated organics occur stereoselectively anti to the metal center. However, because the [Os]arene complex is racemic, the product of these additions generates enantiomers of the liberated organic products. Aromatics containing chiral substituents have been used to impact the preference in binding orientation; however, this limits the variability in products that can be synthesized using [Os]. ${ }^{35}$ Incorporating chiral amines into the osmium (II) ligand set impacted the sterics and electronics of the metal center, thus preventing coordination of aromatics. ${ }^{36,37}$ 
Scheme 1.1. Examples of hydrogenation, electrophilic addition, and cycloaddition mediated by $\left\{\mathrm{Os}\left(\mathrm{NH}_{3}\right)_{5}\right\}^{+2}$.

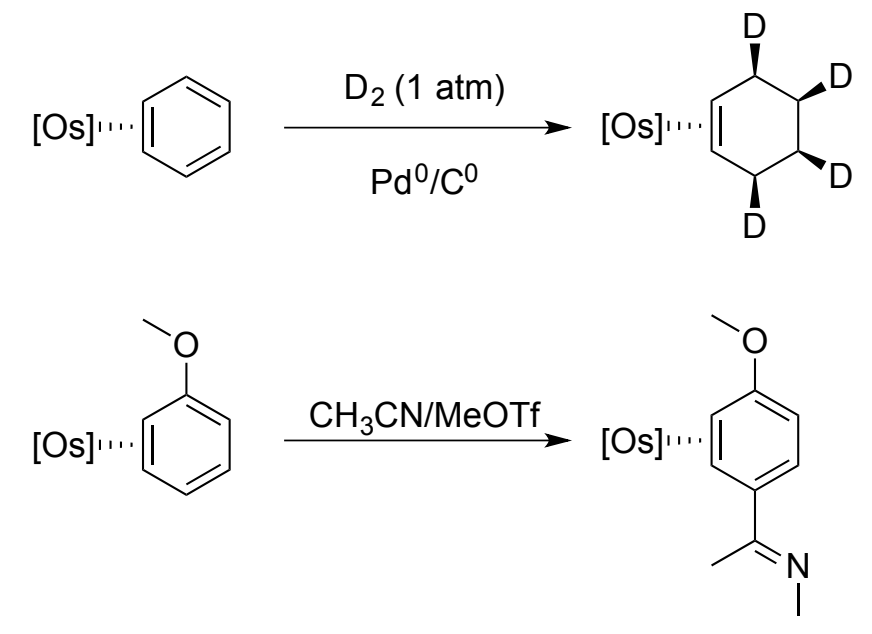

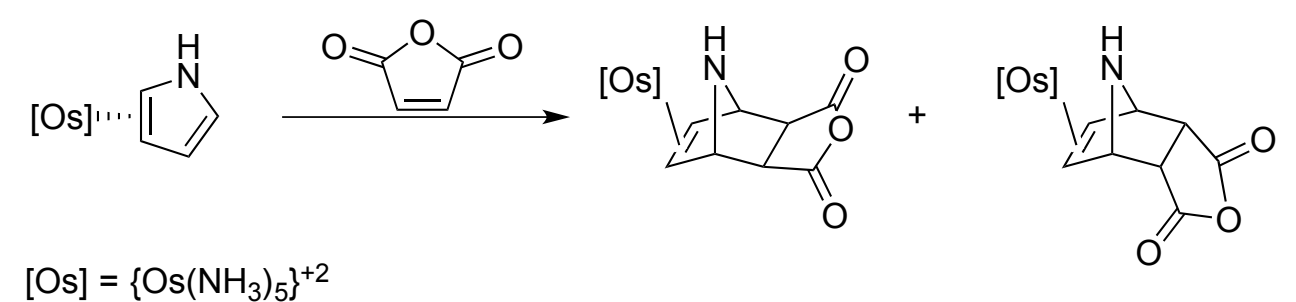

Although [Os] has proven useful for the synthesis of novel organics from aromatic molecules, its utility was limited due to the high cost and lack of chirality. With the expectation of fixing these issues, the development of dearomatization agents using other transition metals was explored.

\section{$1.3\{\operatorname{TpRe}(\mathrm{CO})(\mathrm{L})\}$ Dearomatization}

Initial attempts to form a new dearomatization agent were focused on retaining several key features that differentiate [Os] from the unstable or unreactive $\eta^{2}$-aromatic complexes reported in the literature. To maintain the high electron density needed to overcome aromatic stability, a low-valent $\mathrm{d}^{6}$ metal with primarily $\sigma$-donating ligands is needed. Also desired was a metal center that is coordinatively saturated upon 
coordination of the aromatic ligand. As seen by [Os], a coordinatively saturated metal center offers greater compatibility with electrophiles as it disfavors oxidation at the metal center. With these stringent requirements, sufficient electron donation to overcome aromatic stability can be achieved, while oxidation during organic modification is hindered.

Among the literature, reports showed rhenium (I) to have potential in forming thermally stable eta- 2 bound aromatic complexes. ${ }^{38-40}$ The significantly lower cost $(\sim 1 / 12$ the cost per gram) to use rhenium (I) in place of osmium (II) fixed one of the greatest disadvantages of [Os]. This cost difference can be seen by a comparison of the starting material used for rhenium (I) and osmium (II), perrhenic acid and osmium tetroxide respectively. Although the benefit of cost reduction was promising, the electron-donating ability of rhenium (I) needed to be investigated to determine its ability to backbond and stabilize eta-2 bound aromatics. To compare rhenium (I) and osmium (II), dinitrogenligand stretching frequencies were used to probe the electron density of potential new dearomatization agents. ${ }^{14}$ This analysis showed that the initial rhenium (I) complexes investigated (e.g., $\quad\left\{c i s-\left[\operatorname{Re}(\mathrm{en})_{2}\left(\mathrm{PPh}_{3}\right)\right]\right\}^{+1}, \quad\left\{c i s-\left[\operatorname{Re}(\mathrm{ampy})_{2}\left(\mathrm{PPh}_{3}\right)\right]\right\}^{+1}, \quad$ and $\quad\{c i s-$ $\left[\operatorname{Re}(\operatorname{ampy})(\right.$ tbpy $\left.\left.\left.)\left(\mathrm{PPh}_{3}\right)\right]\right\}^{+1}\right) \quad($ where en $=$ ethylendiamine, ampy = 2-amino-6methylpyridinate, and tbpy $=\operatorname{tris}($ bipyridine $))$ are too electron rich. $^{41}$ To reduce the electron density on rhenium (I), a single $\pi$-acidic ligand (e.g., $\mathrm{CO}$ ) was added to the metal center.

Incorporation of a $\mathrm{CO}$ into the ligand set provided enough stability so that a $\mathrm{fac}$ $\left[\operatorname{Re}(\right.$ dien $)\left(\mathrm{PPh}_{3}\right)(\mathrm{CO})\left(\eta^{2}\right.$-furan $\left.)\right](\mathrm{OTf})$ could be isolated. ${ }^{41}$ The isolation of this complex demonstrated the ability of a rhenium (I) complex to coordinate an aromatic molecule; 
however, no other eta- 2 bound complexes of this fragment could be isolated. This limitation was believed to be a consequence of the greater steric bulk of the ligand set, mainly the large $\mathrm{PPh}_{3}$ ligand; and so, a complex with similar electronics, but decreased steric hindrance was sought after. In this vein, studies had shown that a $\mathrm{Cp} * \operatorname{Re}(C O)_{2}\left(\eta^{2}-\right.$ benzene) complex $\left(\mathrm{Cp}^{*}=\right.$ pentamethylcyclopentadiene $)$ was believed to be the intermediate to a bridged binuclear complex, $\mathrm{Cp}{ }^{*}-(\mathrm{CO})_{2} \operatorname{Re}\left(\mu-1,2-\eta^{2}-3,4-\eta^{2-}\right.$ $\left.\mathrm{C}_{6} \mathrm{H}_{6}\right) \operatorname{Re}(\mathrm{CO})_{2} \mathrm{Cp}^{*}{ }^{42}$ Although similar in their electronic donations, Tp $(\mathrm{Tp}=$ hydridotris(pyrazolyl)borate) shows a greater ability than $\mathrm{Cp}^{*}$ to stabilize a 6-coordinate octahedral geometry. ${ }^{43-46}$ This enforced geometry is believed to reduce the likelihood of potential $\operatorname{TpRe}(\mathrm{L})_{2}\left(\eta^{2}\right.$-aromatic) complexes to undergo aromatic $\mathrm{C}-\mathrm{H}$ oxidative addition. Using this information, the synthesis of $\operatorname{TpRe}(\mathrm{CO})_{2}\left(\eta^{2}\right.$-aromatic) complexes was investigated. However, the $\left\{\mathrm{TpRe}(\mathrm{CO})_{2}\right\}$ fragment proved too electron poor to stabilize eta-2 binding of aromatics. ${ }^{14}$

To provide more electron density within the rhenium (I) metal center, one of the $\mathrm{CO}$ ligands of $\left\{\operatorname{TpRe}(\mathrm{CO})_{2}\right\}$ was replaced with a more $\sigma$-donating ligand (L). The donating abilities of the $\{\operatorname{TpRe}(\mathrm{CO})(\mathrm{L})\}$ fragment vary based on the differing degree of б-donation from L. Subsequent success was found with complexes of the form $\operatorname{TpRe}(\mathrm{CO})\left(\mathrm{PMe}_{3}\right)(\mathrm{L} \pi)$ (where $\mathrm{L} \pi=$ naphthalene, thiophene, and furan). Thus a new generation of dearomatization agents was born of the form $\{\operatorname{TpM}(\pi$-acid $)(L)\} \quad$ (where $M$ $=\mathrm{Re}, \mathrm{Mo}$, or $\mathrm{W} ; \pi$-acid $=\mathrm{CO}$ or $\mathrm{NO}$; and $\mathrm{L}=1$-methylimidazole $(\mathrm{MeIm})$, 1 butylimidazole (BuIm), trimethylphosphine $\left(\mathrm{PMe}_{3}\right)$, triphenylphosphine $\left(\mathrm{PPh}_{3}\right)$, trimethylphosphite $\left(\mathrm{POMe}_{3}\right)$, tert-butylnitrile $\left({ }^{\mathrm{t}} \mathrm{BuCN}\right)$, pyridine, $\mathrm{NH}_{3}$, or 4dimethylaminopyridine (DMAP)). ${ }^{47-49}$ In the case of $\{\operatorname{TpRe}(\mathrm{CO})(\mathrm{L})\}$, using MeIm as an 
ancillary ligand creates a complex able to bind the largest variety of aromatics. ${ }^{15}$ These include substituted pyrroles, benzenes, and pyridines, which can be coordinated directly during the reduction of the $\operatorname{Tp} \operatorname{Re}(\mathrm{CO})(\mathrm{Br})_{2}$ precursor in the presence of MeIm. It was found that other ligands could replace benzene in the complex $\operatorname{TpRe}(\mathrm{CO})(\mathrm{MeIm})\left(\eta^{2}\right.$ benzene) as a result of the lability of the coordinated benzene ligand. ${ }^{48}$ Through this exchange the binding of lutidine, which is sensitive to the reduction conditions (i.e., $\mathrm{Na}^{0}$ ) mentioned above, occurred to yield the $\operatorname{TpRe}(\mathrm{CO})(\mathrm{MeIm})\left(\eta^{2}\right.$-lutidine) complex. ${ }^{48}$

Complexes of the form $\operatorname{TpM}(\pi$-acid)(L)(L $\pi)$ are chiral. Assuming one could start with a single enantiomer, stereoselective organic transformations on the aromatic ligand would theoretically yield enantio-enriched products. However, coordination of prochiral, unsaturated molecules yields a diastereomeric mixture differing in either the position or enantioface that the metal binds to (Scheme 1.2).

Scheme 1.2. Organic products isolated as a racemic mixture from a diastereomeric mixture of a stereogenic metal center.

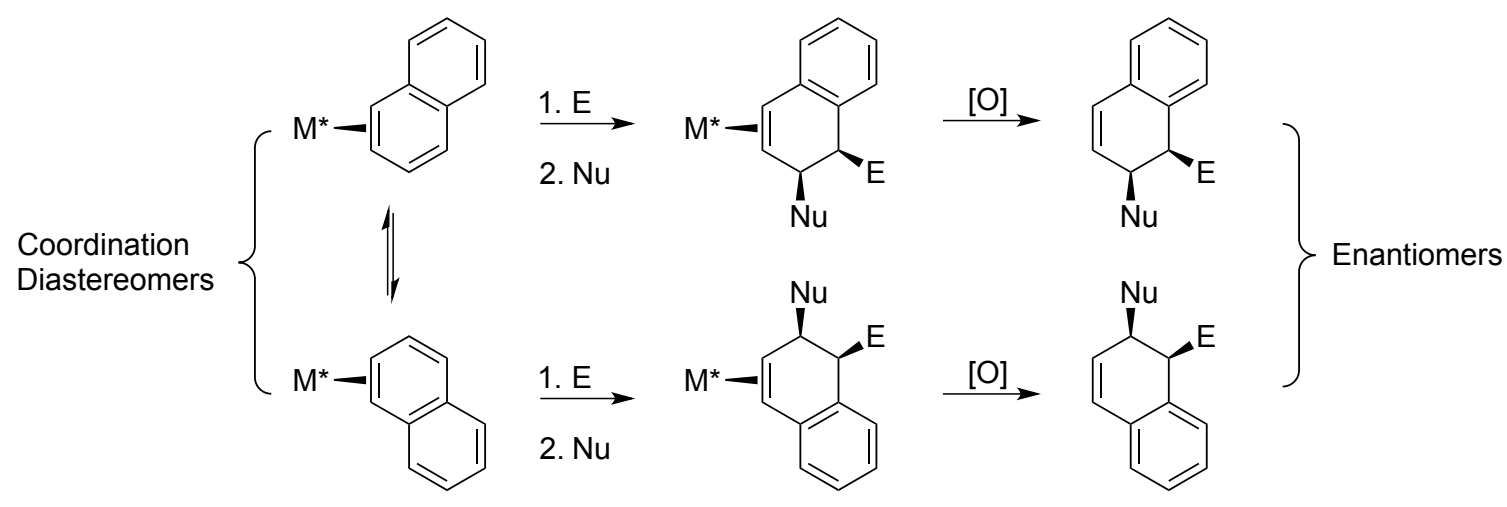


Theoretically, if these coordination diastereomers were isolated as a 1:1 ratio, then organic transformations on said mixture would yield the organic as a racemic mixture. Thus, an in-depth analysis on how to selectively obtain or react one coordination diastereomer over the other is required.

In complexes of the form $\operatorname{TpM}(\pi$-acid) $(\mathrm{L})(\mathrm{L} \pi)$ the coordinated ligand $(\mathrm{L} \pi)$ will orient itself so that the $\pi$-bond is parallel to the M-L bond. This preference is due to the double-faced $\pi$-acid (e.g., $\mathrm{CO}$ or NO) that lowers the energy of two $\mathrm{d} \pi$ orbitals, leaving the remaining filled $\mathrm{d} \pi$ orbital (the HOMO) unaffected. This metal-centered HOMO overlaps with the antibonding orbital of the ligand, favoring the ligand to orient orthogonal to the M- $\pi$-acid bond. ${ }^{50}$ In addition to this electronic impact on ligand orientation, the electronic preference to orient partial positive charges distal to the ancillary ligand (L) as well as potential $\pi$-stacking with L (i.e., where L contains a $\pi$ system) can determine the preferred orientation of the coordinated ligand ( $\mathrm{L} \pi)$. Furthermore, the prevention of steric hindrance can cause a preference for different coordination diastereomers. ${ }^{49}$ By observing the preferred coordination diastereomers formed by coordination of various ligands to $\{\mathrm{TpRe}(\mathrm{CO})(\mathrm{MeIm})\}$, the steric repulsion in each quadrant formed by the ligands cis to the L $\pi$ ligand was found to increase in the order of $\mathrm{d}<\mathrm{a}<\mathrm{b}<\mathrm{c}$ (Figure 1.3). ${ }^{51}$ Following this trend, coordination diastereomers of $\operatorname{TpRe}(\mathrm{CO})(\mathrm{MeIm})\left(\eta^{2}\right.$-aromatic) favor placing the bulk of the unbound portion of the ring in the least hindered quadrant $\mathrm{d}$. This can be seen in Figure 1.3 where coordination diastereomer A positions the naphthalene ring "up" or proximal to the ancillary ligand where $\mathrm{L}=$ MeIm or Py. For rhenium (I), replacing $\mathrm{L}$ with a bulkier ancillary ligand increases the steric hindrance in quadrant d. For instance, $\operatorname{TpRe}(\mathrm{CO})\left(\mathrm{PMe}_{3}\right)\left(\eta^{2}-\right.$ 
naphthalene) has a greater steric hindrance in quadrant $d$ thus favoring the "down" coordination diastereomer $(\mathbf{B}){ }^{52}$

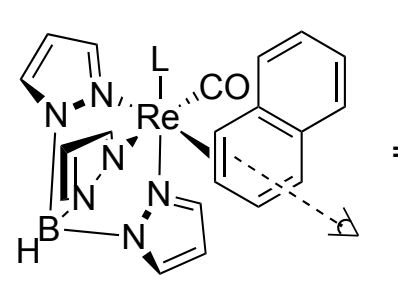

\begin{tabular}{|ll|}
\hline \multicolumn{1}{c}{$\mathrm{L}$} & \multicolumn{1}{c|}{ A/B } \\
\hline Melm & $4.5: 1$ \\
Py & $3: 1$ \\
$\mathrm{PMe}_{3}$ & $<1 / 19$ \\
\hline
\end{tabular}

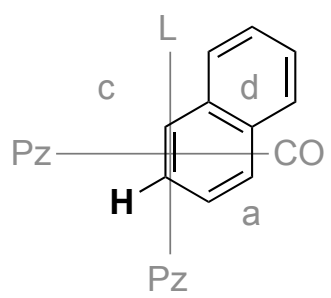

A
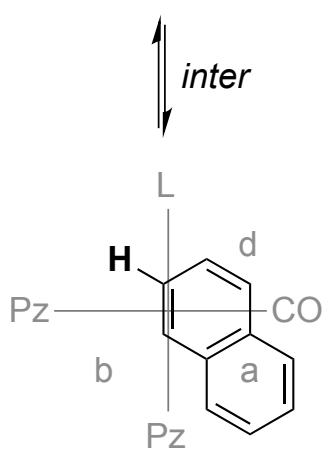

B
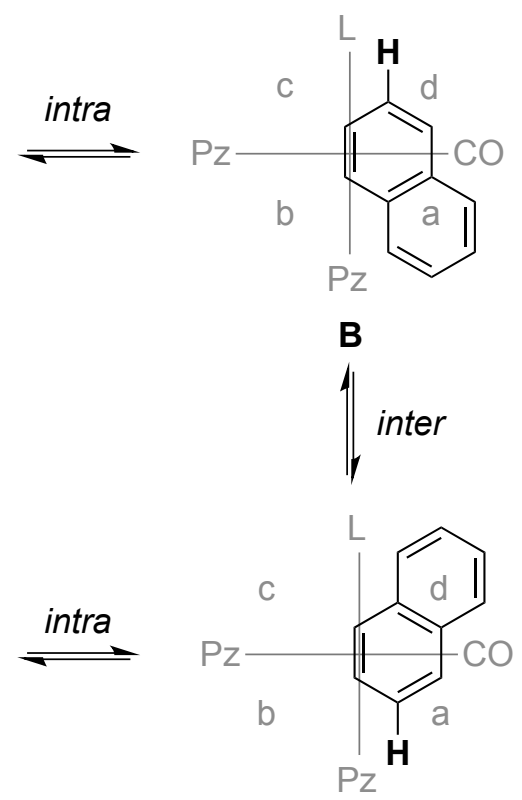

A

Figure 1.3. Coordination diastereomers of $\operatorname{Tp} \operatorname{Re}(\mathrm{CO})(\mathrm{L})\left(\eta^{2}\right.$-naphthalene).

Although the coordination diastereomers can be controlled by sterics, as described above, these diastereomers maintain isomerization equilibrium. The mechanism of this isomerization has been determined to occur through two non-dissociative pathways, interfacial (face-flip) and intrafacial (ring walk) isomerization. By studying spinsaturation experiments of $\operatorname{Tp} \operatorname{Re}(\mathrm{CO})(\mathrm{L})(\mathrm{L} \pi)$, it was found that both face-flip and ring walk mechanisms are operative and preference of either mechanism depends on the aromatic being bound as well as the ability to backbond from the metal. In the case of naphthalene, spin-saturation experiments, depicted by the bold hydrogens in Figure 1.3, support a preference for intrafacial isomerization during the conversion between $\mathbf{A}$ and B. $^{51}$ 
Scheme 1.3. Diastereoselective control of electrophilic-nucleophilic additions to $\operatorname{TpRe}(\mathrm{CO})(\mathrm{L})\left(\eta^{2}\right.$-naphthalene).

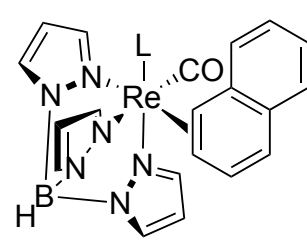

4

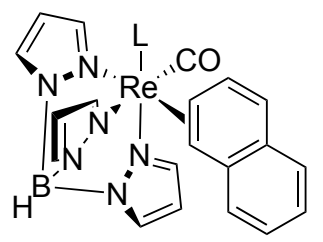

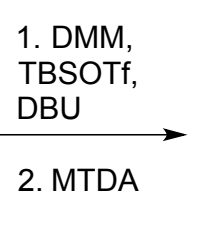

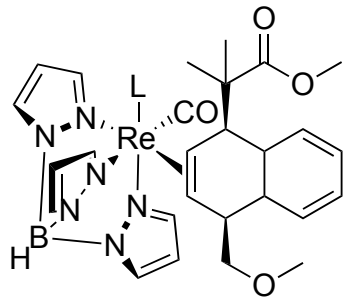

A<smiles>COC[C@H]1C=C[C@@H](C(C)(C)C(=O)OC)[C@@H]2C=CC=CC12</smiles>

Enantiomers
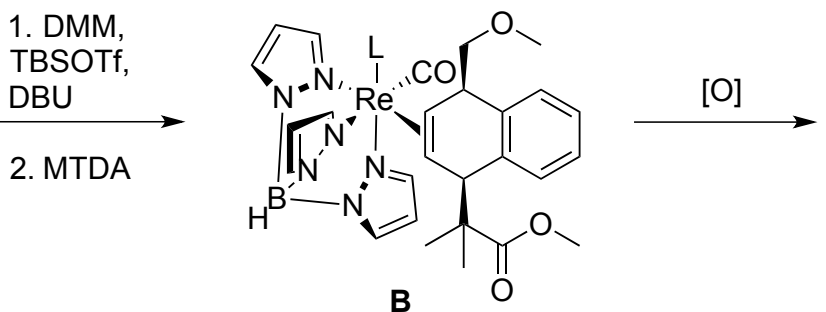<smiles>COC[C@H]1C=C[C@H](C(C)(C)C(=O)OC)c2ccccc21</smiles>

\begin{tabular}{|ll|}
\hline \multicolumn{1}{c}{$\mathrm{L}$} & \multicolumn{1}{c|}{ A/B } \\
\hline Melm & $>20: 1$ \\
Py & $5: 1$ \\
$\mathrm{PMe}_{3}$ & $>1: 20$ \\
\hline
\end{tabular}

With this steric control of coordination diastereomers, the desired enantiomer of an organic can be selectively synthesized, provided that the metal stereocenter is of a single configuration $(\mathrm{R} / \mathrm{S})$. For instance, $\operatorname{TpRe}(\mathrm{CO})(\mathrm{L})\left(\eta^{2}\right.$-naphthalene) undergoes a tandem electrophilic-nucleophilic addition to form a specific enantiomer of a 1,4dihydronaphthalene depending on the ancillary ligand (L) used (Scheme 1.3). ${ }^{52}$ In addition to the stereoselective control, $\operatorname{TpRe}(\mathrm{CO})(\mathrm{L})\left(\eta^{2}\right.$-naphthalene) has demonstrated regioselective control by isolating a 1,2- or 1,4-dihydronaphthalene based on which ancillary ligand is used. ${ }^{53}$ Although controlling the diastereomeric ratio allows for the selective synthesis of enantiomers, because complexes of the form $\operatorname{TpRe}(\mathrm{CO})(\mathrm{L})\left(\eta^{2}-\right.$ 
aromatic) are synthesized as a racemic mixture of the two enantiomers of the metal, the organic products isolated directly from these complexes will also be racemic mixtures.

Utilizing the steric profile described above, it was found that enantioenrichment of rhenium (I) could be induced using the coordination of the chiral and bulky $\alpha$ pinene. ${ }^{54}$ Stirring the $\operatorname{TpRe}(\mathrm{CO})(\mathrm{MeIm})\left(\eta^{2}\right.$-benzene) complex with $(R)$ - $\alpha$-pinene yields two diastereomers of the metal alkene complex. To minimize steric repulsion in these diastereomers, the metal coordinates to the face of the alkene opposite to the geminal methyls. A more stable, "matched" diastereomer ( $S$ hand of the metal) is isolated where the olefinic methyl is placed into quadrant $b$ with no substituents in quadrant $c$. The less stable, "mismatched" diastereomer ( $R$ hand of the metal) is suspected to form by placing the olefinic methyl in quadrant $\mathrm{c}$, creating great steric repulsion. Stirring a mixture of the matched and mismatched complexes in benzene allows for the steric repulsion in the mismatched isomer to be relieved by exchanging with benzene. The resulting $R$ $\operatorname{TpRe}(\mathrm{CO})(\mathrm{MeIm})\left(\eta^{2}\right.$-benzene) complex can then be separated from the matched $\alpha$ pinene complex through chromatography. The remaining matched $\alpha$-pinene complex can then be oxidized and reduced in the presence of benzene to isolate the $S$ $\operatorname{TpRe}(\mathrm{CO})(\mathrm{MeIm})\left(\eta^{2}\right.$-benzene) complex (Scheme 1.4), effectively resolving the two hands of the chiral metal complex. 
Scheme 1.4. Enantioenrichment of $\operatorname{TpRe}(\mathrm{CO})(\mathrm{L})\left(\eta^{2}\right.$-benzene $)$.
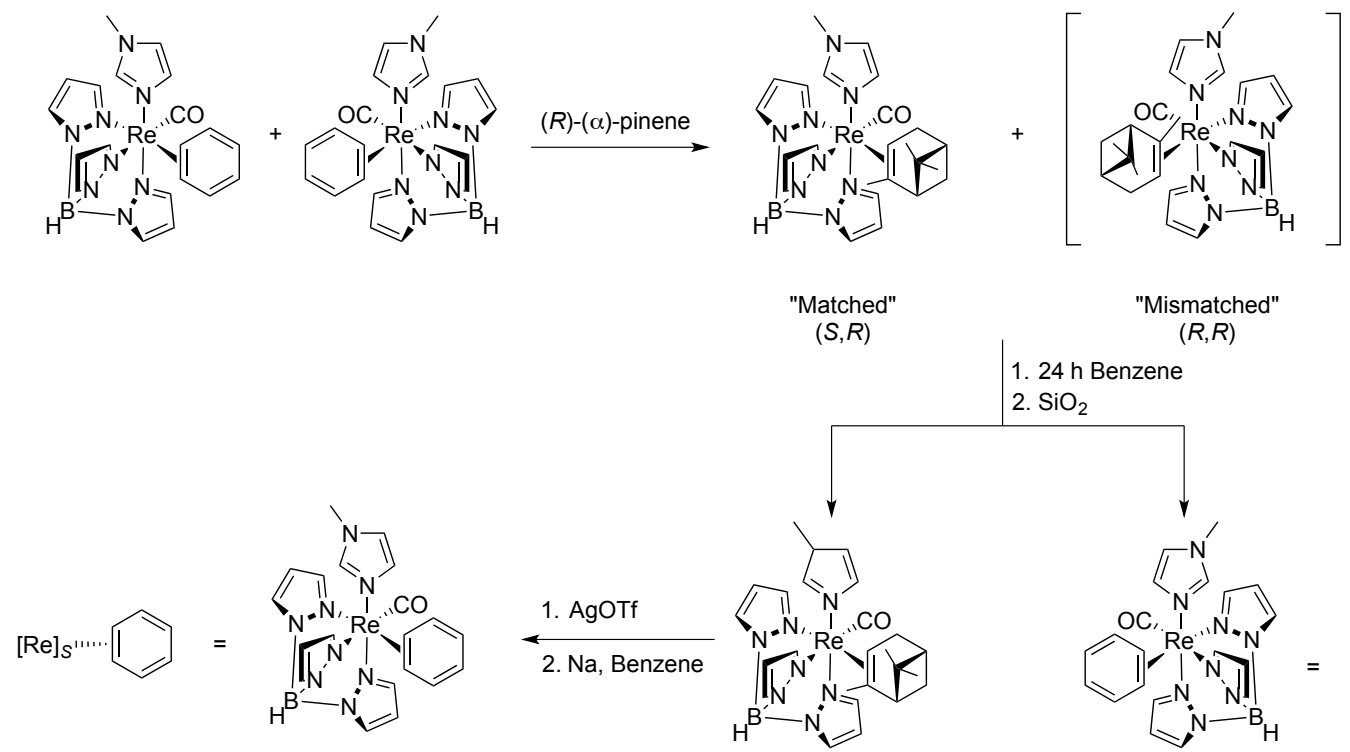

$(S)$

$(S, R)$

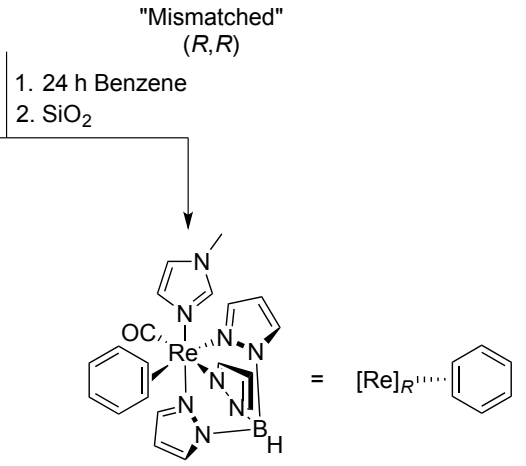

$(R)$

The benzene ligands of these enantioenriched complexes can then be exchanged for other aromatic ligands, while retaining the chiral enrichment of the metal center. The resulting enantioenriched complexes can undergo organic transformations to yield organics with high levels of enantiopurity (Scheme 1.5). ${ }^{52,55,56}$

Scheme 1.5. Enantioselective organic modifications using $\{\mathrm{TpRe}(\mathrm{CO})(\mathrm{MeIm})\}$.<smiles></smiles>

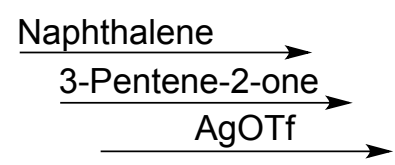<smiles>C[C@H]1CC(=O)C[C@H]2C=Cc3ccccc3[C@H]12</smiles>

$96 \%$ ee, $\mathrm{dr}>20: 1$<smiles>[TeH]c1ccccc1</smiles>

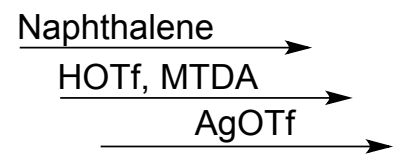<smiles>COC(=O)C(C)(C)[C@H]1C=Cc2ccccc2C1</smiles>

$86 \%$ ee, $d r>20: 1$ 
$\{\mathrm{TpRe}(\mathrm{CO})(\mathrm{L})\}$ demonstrated a wide breadth of reactivity and adjustability in the ligand set. However, in addition to the high cost associated with its stoichiometric use, another disadvantage was the inability to consistently synthesize the $\operatorname{TpRe}(\mathrm{CO})(\mathrm{MeIm})\left(\eta^{2}\right.$-benzene) on a multigram scale. Some reports even indicate that upwards of 30 days are needed for the completion on a 5 g scale. ${ }^{57}$ Over the course of 15 years, the development of $\{\operatorname{TpRe}(\mathrm{CO})(\mathrm{L})\}$ provided well-learned lessons that were subsequently applied to other transition metals.

\section{$1.4\{$ TpMo(NO)(MeIm)\} Dearomatization}

Molybdenum offers an attractive candidate for the center of these metal scaffolds as it is a nontoxic, second row metal that is commercially available at a fraction of the cost of osmium (II) or rhenium (I). Comparing the reduction potentials of $\left[\operatorname{Re}(\mathrm{CO})_{2}(\mathrm{dmpe})_{2}\right]^{+1}$ $(1.66 \mathrm{~V})$ and $\mathrm{Mo}(\mathrm{CO})_{2}(\mathrm{dmpe})_{2}(0.22 \mathrm{~V})$ suggests that molybdenum, being more electron rich, requires a stronger $\pi$-acid than $\mathrm{CO}$. Through a comparison of $\left[\mathrm{TpMo}(\mathrm{CO})_{3}\right]^{-1}$ and $\mathrm{TpMo}(\mathrm{CO})_{2}(\mathrm{NO})$ it was found that replacement of $\mathrm{CO}$ with $\mathrm{NO}^{+}$raises the reduction potential of molybdenum by approximately $1 \mathrm{~V} \cdot{ }^{17}$ This increase in reduction potential provides the correct electronics needed to stabilize an eta- 2 coordinated aromatic. The addition of $\mathrm{NO}^{+}$also balances the charge from the Tp ligand, which provides a neutral complex and allows for a greater compatibility with purification through chromatography. Thus the development of $\mathrm{TpMo}(\mathrm{NO})(\mathrm{L})(\mathrm{L} \pi)$ was pursued.

Utilizing reports by $\mathrm{McCleverty,} \mathrm{TpMo}(\mathrm{NO})(\mathrm{Br})_{2}$ was made and subsequent reductions were attempted. ${ }^{58}$ By reducing $\mathrm{TpMo}(\mathrm{NO})(\mathrm{Br})_{2}$ in the presence of the desired MeIm and an excess of $\mathrm{L} \pi$ (where $\mathrm{L} \pi=$ thiophene, furan, naphthalene), the desired 
TpMo(NO)(MeIm)(L $\pi$ ) can be isolated in fair yields ( $40 \%$ average) ${ }^{17}$ In addition to this method, a stepwise synthesis was developed wherein $\mathrm{TpMo}(\mathrm{NO})(\mathrm{MeIm})(\mathrm{Br})$ is isolated, thereby preventing potential decomposition pathways in the aforementioned one-pot synthesis. ${ }^{16}$

Scheme 1.6. Synthesis of $\mathrm{TpMo}(\mathrm{NO})(\mathrm{MeIm})\left(\eta^{2}-\mathrm{L} \pi\right)$.

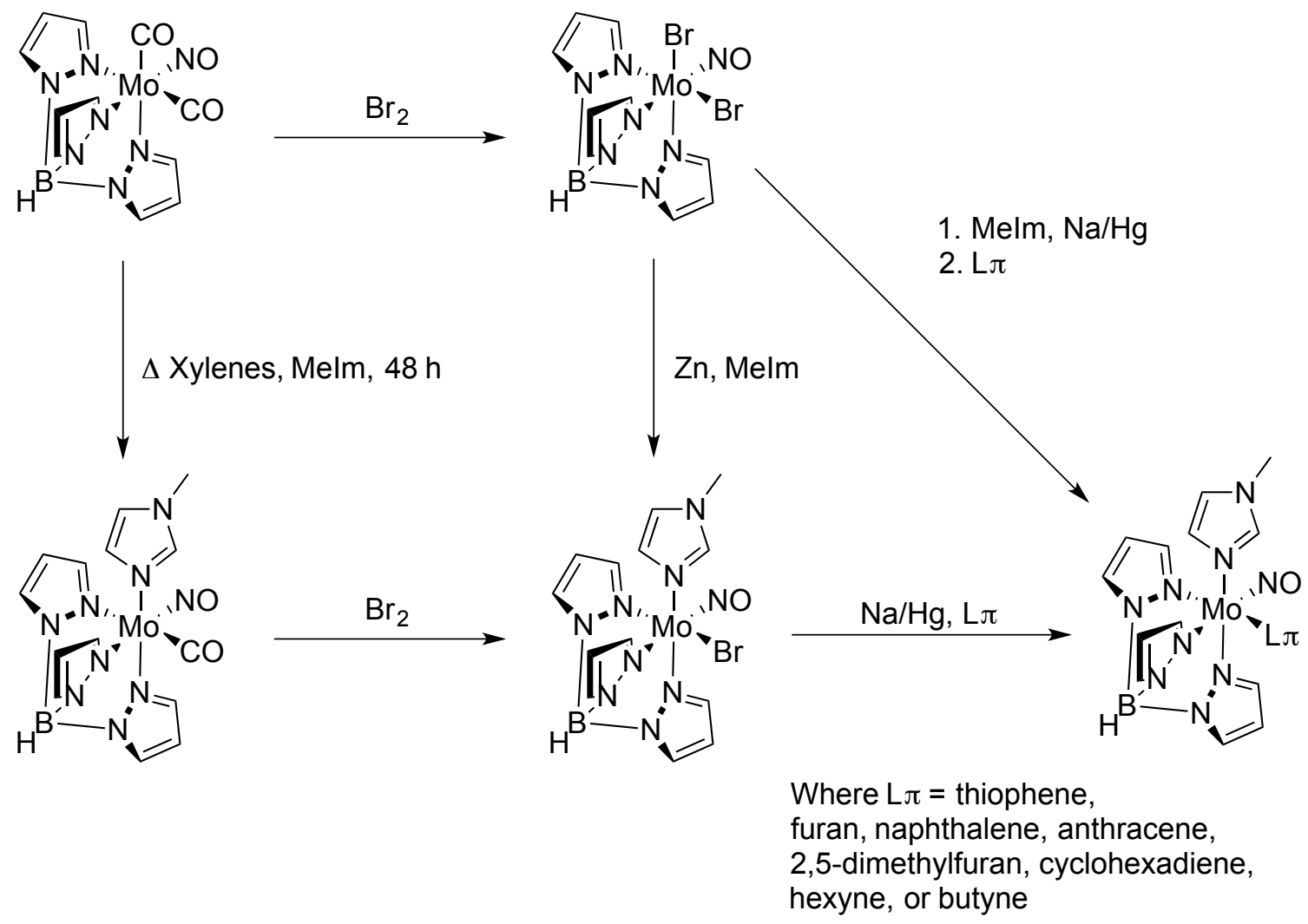

The isolation of $\mathrm{TpMo}(\mathrm{NO})(\mathrm{MeIm})(\mathrm{Br})$ is accomplished through a reduction of $\mathrm{TpMo}(\mathrm{NO})(\mathrm{Br})_{2}$ with $\mathrm{Zn}^{0}$ in the presence of MeIm. Alternatively, by refluxing a xylenes solution of $\mathrm{TpMo}(\mathrm{CO})_{2}(\mathrm{NO})$ and $\mathrm{MeIm}, \mathrm{TpMo}(\mathrm{CO})(\mathrm{NO})(\mathrm{MeIm})$ can be isolated and subsequently oxidized to yield $\mathrm{TpMo}(\mathrm{NO})(\mathrm{MeIm})(\mathrm{Br})$ (Scheme 1.6). In addition to isolating the $\mathrm{TpMo}(\mathrm{NO})(\mathrm{MeIm})(\mathrm{L} \pi)$ described above in the one-step process from $\mathrm{TpMo}(\mathrm{NO})(\mathrm{Br})_{2}$, the reduction of $\mathrm{TpMo}(\mathrm{NO})(\mathrm{MeIm})(\mathrm{Br})$ in presence of the desired 
ligand yielded $\operatorname{TpMo}(\mathrm{NO})(\mathrm{MeIm})(\mathrm{L} \pi) \quad(\mathrm{L} \pi=$ anthracene, 2,5-dimethylfuran, cyclohexadiene, hexyne, and butyne) in comparable yields (Scheme 1.6).

Organic modifications with complexes of the form $\mathrm{TpMo}(\mathrm{NO})(\mathrm{MeIm})(\mathrm{L} \pi)$ were demonstrated for complexes where $\mathrm{L} \pi=$ naphthalene or cyclohexadiene. TpMo(NO)(MeIm) $\left(\eta^{2}\right.$-naphthalene) was found to undergo a tandem electrophilicnucleophilic addition via protonation and subsequent addition of methyl trimethylsilyl dimethylketene acetal (MTDA) to yield a 1,2-dihydronaphthalene. For the complex $\mathrm{TpMo}(\mathrm{NO})(\mathrm{MeIm})\left(\eta^{2}\right.$-cyclohexadiene), the electron donation from the metal fragment is able to activate the conjugated diene towards Diels-Alder transformations, resulting in the formation of cycloadducts not directly accessible from the uncoordinated diene (Scheme 1.7). ${ }^{59}$ However, further elaboration of the organic modification of arenes using the $\{\mathrm{TpMo}(\mathrm{NO})(\mathrm{MeIm})\}$ was prevented due to a greater sensitivity of $\{\mathrm{TpMo}(\mathrm{NO})(\mathrm{MeIm})\}$ towards oxidation in comparison to its osmium (II) and rhenium (I) analogues. For instance, relative to $\operatorname{TpRe}(\mathrm{CO})(\mathrm{MeIm})\left(\eta^{2}\right.$-naphthalene $) \quad\left(E_{1 / 2}=0 \quad \mathrm{~V}\right)$, $\mathrm{TpMo}(\mathrm{NO})(\mathrm{MeIm})\left(\eta^{2}\right.$-naphthalene), with a more negative anodic wave $\left(E_{\mathrm{p}, \mathrm{a}}\right.$ of $\left.-0.26 \mathrm{~V}\right)$, is more prone to oxidation by electrophilic reagents.

Scheme 1.7. Diels-Alder synthesis of cycloadducts promoted by $\{\mathrm{TpMo}(\mathrm{NO})(\mathrm{MeIm})\}$.

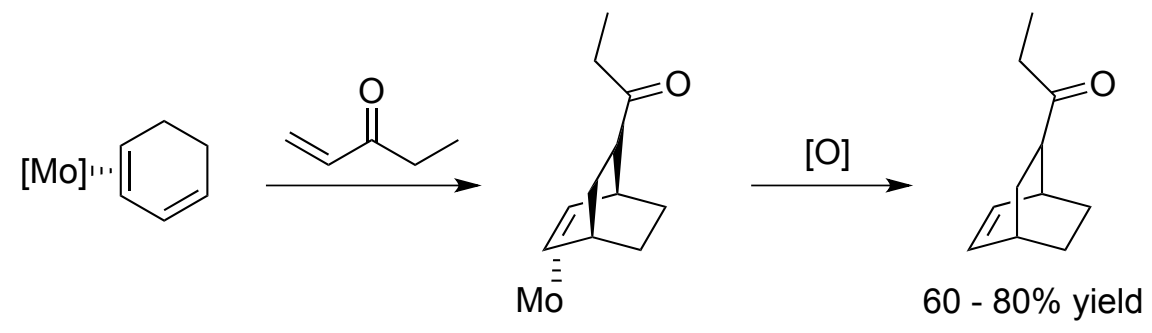


In addition to the difficulties with transforming aromatics through organic modifications, $\{\mathrm{TpMo}(\mathrm{NO})(\mathrm{MeIm})\}$ also failed to form stable complexes with benzene, pyrrole, pyridine, and their derivatives. These limitations led to a discontinuation of the pursuit of molybdenum metal complexes as dearomatization agents; however, a promising tungsten moiety proved successful.

\section{$1.5\left\{\mathrm{TpW}(\mathrm{NO})\left(\mathrm{PMe}_{3}\right)\right\}$ Dearomatization}

It was hypothesized that $\{\mathrm{TpMo}(\mathrm{NO})(\mathrm{MeIm})\}$ lacks the sufficient backbonding capabilities that the third-row transition metal fragments $\left\{\mathrm{Os}\left(\mathrm{NH}_{3}\right)_{5}\right\}^{2+}$ and $\{\mathrm{TpRe}(\mathrm{CO})(\mathrm{MeIm})\}$ provide to support eta-2 binding with benzene. For this reason, the development of $\{\mathrm{TpW}(\mathrm{NO})(\mathrm{L})\}$ was targeted as a more capable group VI congener for dearomatization. Comparing the reduction potentials of $\mathrm{TpRe}(\mathrm{CO})_{3}$ and $\mathrm{TpW}(\mathrm{NO})(\mathrm{CO})_{2}$, it is seen that the latter is approximately $300 \mathrm{mV}$ more reducing. ${ }^{60}$ Analyzing the various $\sigma$-donating capabilities of ancillary ligands used with $\{\operatorname{TpRe}(\mathrm{CO})(\mathrm{L})\}^{49}$, it was hypothesized that $\mathrm{PMe}_{3}$ would properly balance the increased reducing power of $\{\mathrm{TpW}(\mathrm{NO})(\mathrm{CO})\}$ to generate a complex capable of coordinating aromatic molecules in an eta-2 fashion. $\mathrm{TpW}(\mathrm{NO})(\mathrm{Br})_{2}$ is synthesized following a similar method used to make TpMo(NO)(Br $)_{2}$. By subjecting TpW(NO)(Br $)_{2}$ to reductive conditions in the presence of $\mathrm{PMe}_{3}$, the precursor $\mathrm{TpW}(\mathrm{NO})\left(\mathrm{PMe}_{3}\right)(\mathrm{Br})$ is made and can subsequently be reduced in the presence of benzene to isolate $\mathrm{TpW}(\mathrm{NO})\left(\mathrm{PMe}_{3}\right)\left(\eta^{2}\right.$-benzene). ${ }^{61}$

Similar to the previous dearomatization agents mentioned above, the $\left\{\mathrm{TpW}(\mathrm{NO})\left(\mathrm{PMe}_{3}\right)\right\}$ ([W]) fragment is able to bind a broad array of aromatic molecules (i.e., substituted benzenes, pyridines, furans, thiophenes, pyrroles, etc.). ${ }^{62}$ In contrast to 
small scales and long reaction times in the synthesis of $\mathrm{TpRe}(\mathrm{CO})(\mathrm{MeIm})\left(\eta^{2}\right.$-benzene), $\mathrm{TpW}(\mathrm{NO})\left(\mathrm{PMe}_{3}\right)\left(\eta^{2}\right.$-benzene) can be isolated on a multigram scale (upwards of $12 \mathrm{~g}$ ) requiring only $24 \mathrm{~h}$ for reaction completion. ${ }^{63}$ Attempts to coordinate more electron-rich aromatic molecules, such as $\mathrm{N}$-alkylated anilines, indolines, and quinolines, through a direct ligand exchange from the benzene complex result in the formation of intractable mixtures. However, purported $\eta^{2}$-coordinated complexes of these aromatics have been observed during these exchanges using cyclic voltammetry (CV). These unstable complexes can be trapped by in situ protonation during the ligand exchange reaction through the addition of weak anilinium acids (Scheme 1.8). ${ }^{64,65}$

Scheme 1.8. In situ protonation during the exchange of $\mathrm{TpW}(\mathrm{NO})\left(\mathrm{PMe}_{3}\right)\left(\eta^{2}\right.$-benzene $)$ with aniline, indoline, or quinoline.

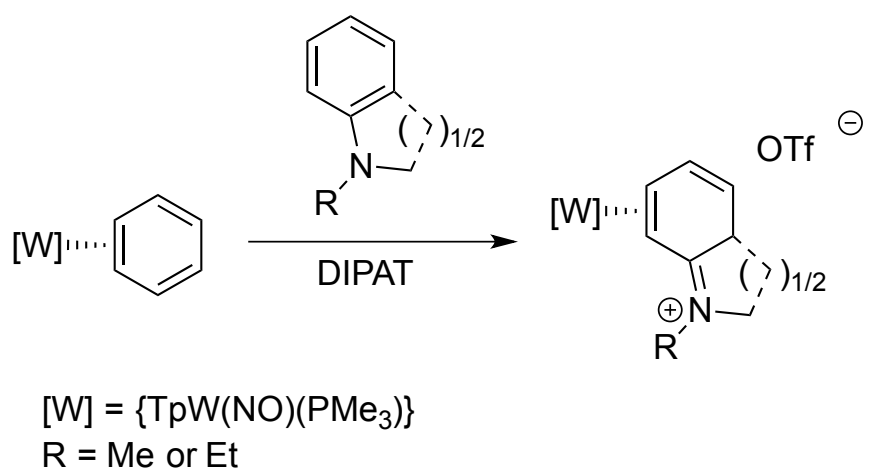

Following a similar method for organic modification, the indolinium species has shown a great utility in organic modification utilizing tandem electrophilic-nucleophilic additions. The resulting functionalized tetrahydroindolines contain upwards of four additional stereocenters controlled by the metal (Scheme 1.9). ${ }^{66}$ 
Scheme 1.9. Organic modification of $\mathrm{TpW}(\mathrm{NO})\left(\mathrm{PMe}_{3}\right)\left(\eta^{2}\right.$-indolinium $)$.

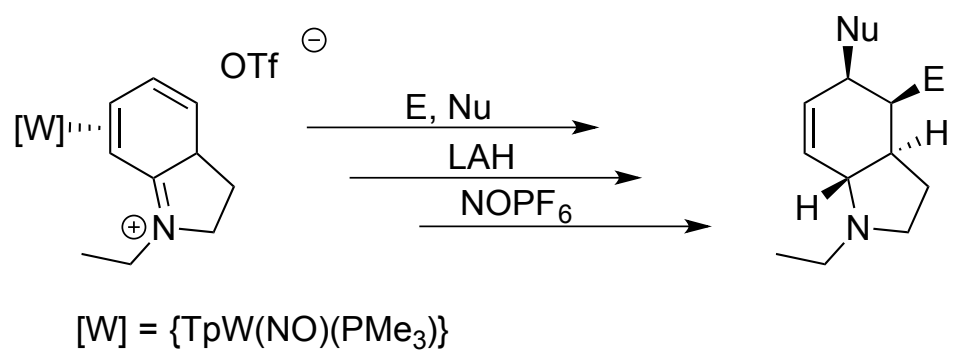

Similar to $\{\operatorname{TpRe}(\mathrm{CO})(\mathrm{MeIm})\}$, the chiral [W] fragment can be resolved to generate enantioenriched complexes of aromatic molecules. Although attempts to follow the same enrichment method as $\{\operatorname{TpRe}(\mathrm{CO})(\mathrm{MeIm})\}$ (e.g., $\alpha$-pinene exchange) showed limited effectiveness, ${ }^{67}$ the use of chiral tartrate salts proved much more successful. Protonation of TpW(NO) $\left(\mathrm{PMe}_{3}\right)\left(\eta^{2}\right.$-1,3-dimethoxybenzene) with $L$-dibenzoyltartaric acid results in the formation of thermally stable oxonium complexes. The diastereomeric salts of these complexes can be separated by the differences in their solubility. Once separated, the salts can be deprotonated and the resulting resolved dimethoxybenzene complex can undergo exchange with benzene, yielding the primary precursor for dearomatization complexes formed using [W] (Scheme 1.10). ${ }^{68}$ Current methods to utilize this enantioenriched complex for organic modifications are underway.

Although the $\left\{\mathrm{TpW}(\mathrm{NO})\left(\mathrm{PMe}_{3}\right)\right\}$ fragment has shown a great versatility in organic synthesis and scale, a few disadvantages remain. The use of $\mathrm{PMe}_{3}$ as an ancillary ligand provides a useful handle for monitoring reactions through ${ }^{31} \mathrm{P} \mathrm{NMR}$; however, the high cost of this ligand limits the practicality of the widespread use of this complex. Unlike $\{\operatorname{TpRe}(\mathrm{CO})(\mathrm{L})\}$, the ancillary ligand of $[\mathrm{W}]$ cannot be altered without sacrificing the ability to bind a broad array of aromatics. 
Scheme 1.10. Isolation of enantionenriched TpW(NO) $\left(\mathrm{PMe}_{3}\right)\left(\eta^{2}\right.$-benzene $)$ complex.

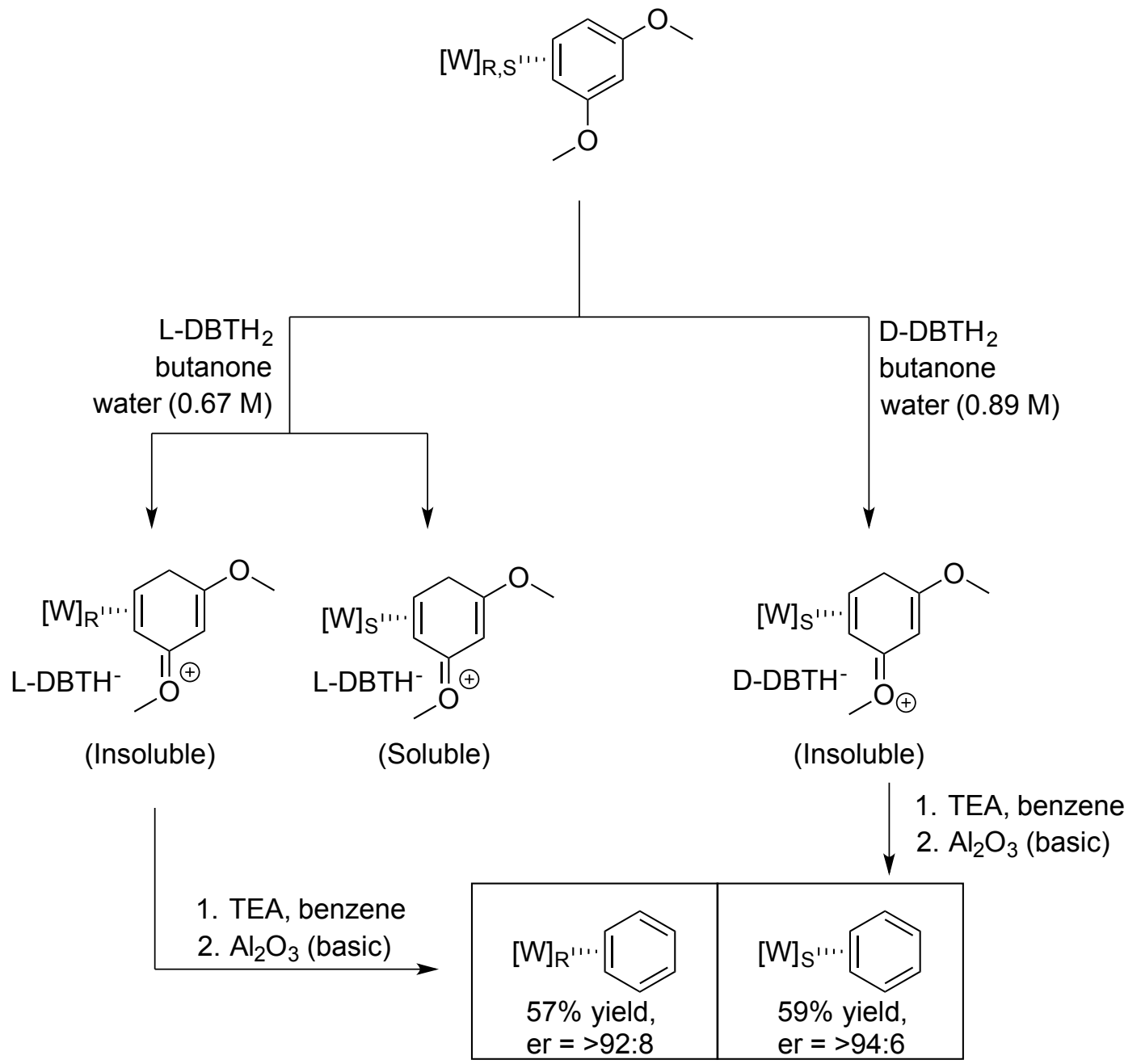

Coordination of a limited number of aromatic molecules has been shown using $\mathrm{P}(\mathrm{OMe})_{3}$ as the ancillary ligand on the tungsten complex, ${ }^{62}$ but broad applicability is only seen with tungsten for the $\mathrm{PMe}_{3}$ complex. The high cost of this one ligand of the complex is a severe limitation to the adoption of this dearomatization methodology for synthetic applications, due to the stoichiometric nature of this chemistry.

In comparison to $\left\{\mathrm{Os}\left(\mathrm{NH}_{3}\right)_{5}\right\}^{+2}$ and $\mathrm{TpRe}(\mathrm{CO})(\mathrm{L})$, [W] has shown a greater propensity to undergo oxidative addition into $\mathrm{C}-\mathrm{H}$ and $\mathrm{N}-\mathrm{H}$ bonds. As such, multiple 7coordinate hydride complexes have been reported during the binding of various ligands 
(i.e., benzene, ${ }^{63} \alpha, \alpha, \alpha$-trifluorotoluene (TFT), ${ }^{63}$ and substituted pyrroles ${ }^{69}$ ) to $\left\{\mathrm{TpW}(\mathrm{NO})\left(\mathrm{PMe}_{3}\right)\right\}$. For instance, in the binding of 2-methylpyrrole, a 7-coordinate hydride complex is made by oxidative addition into the N-H bond. This hydride complex can be converted to an eta- 2 bound, pyrrolium complex via protonation and then subjected to a Michael addition and ring closure to form an indolizidine core (Scheme 1.11).

In addition to the greater propensity for oxidative additions into $\mathrm{C}-\mathrm{H}$ and $\mathrm{N}-\mathrm{H}$ bonds, [W] has also demonstrated an ability to oxidatively add C-F bonds of fluorinated aromatics. ${ }^{70}$ Unlike the hydride complexes described above, the product of an oxidative addition into $\mathrm{C}-\mathrm{F}$ bonds cannot be converted to an eta- 2 bound complex via protonation. For this reason, tungsten is limited in the dearomatization of fluorinated aromatics where the fluorine is bound directly to the arene.

Scheme 1.11. Synthesis of the eta-2 bound pyrrolium complex and subsequent synthesis of an indolizidine core.

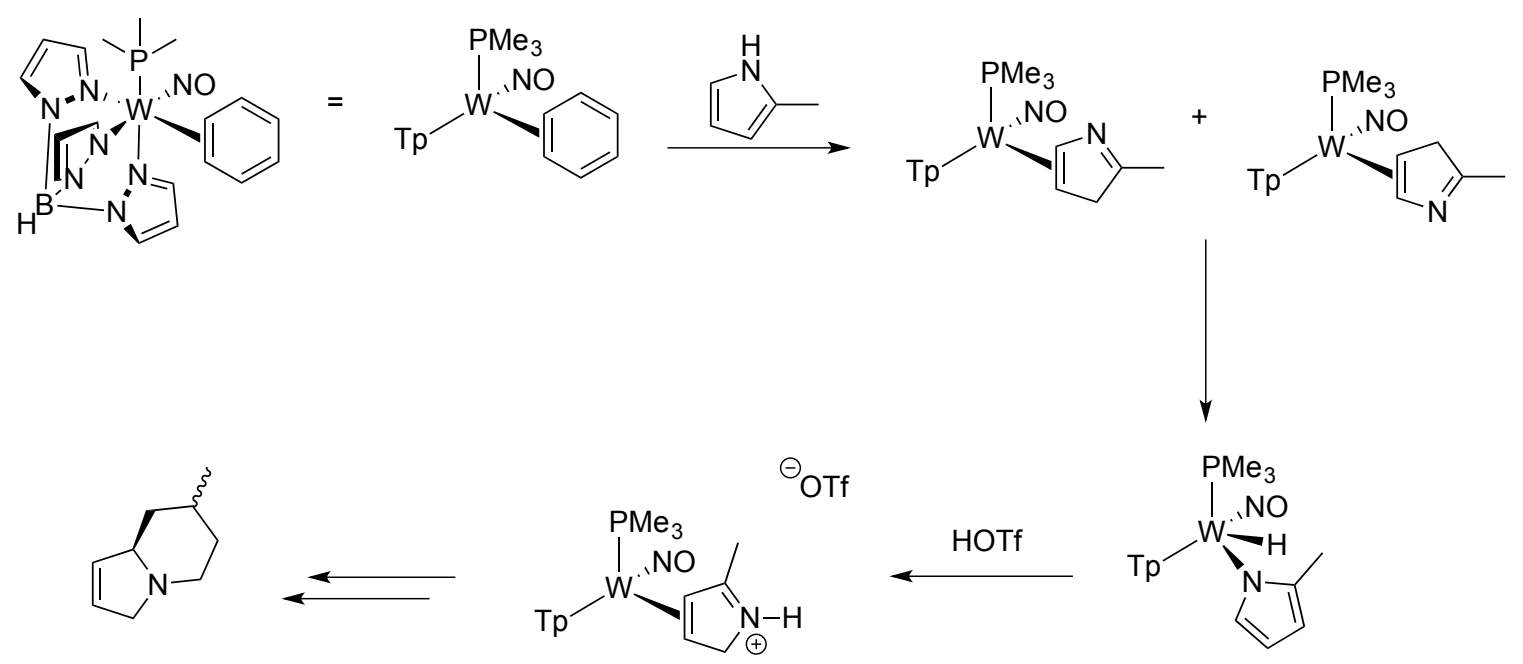


Furthermore, to isolate the modified organics via oxidative decomplexation, harsh oxidants (i.e., ceric ammonium nitrate (CAN) and 2,3-Dichloro-5,6-dicyano-1,4benzoquinone (DDQ)) are often required, which can create unwanted side reactions. To address this issue, further development of complexes of the form $\{\mathrm{TpMo}(\mathrm{NO})(\mathrm{L})\}$ has been explored and will be discussed herein.

\subsection{Developing the $\{\mathrm{TpMo}(\mathrm{NO})(\mathrm{L})\}$ Dearomatization Agent}

The project presented in this dissertation seeks to develop a dearomatization agent that contains all of the advantages of the previous three decades worth of exploration in one metal scaffold. Mainly, we are looking to incorporate the following characteristics: recyclability, scalability, adjustability in the ligand set, and the ability to enantioenrich the metal center. As mentioned previously, $\operatorname{TpRe}_{S}(\mathrm{CO})(\mathrm{MeIm})(\alpha$-pinene) can be oxidized and subsequently reduced to yield $\operatorname{TpRe}_{(\mathrm{S})}(\mathrm{CO})(\mathrm{MeIm})\left(\eta^{2}\right.$-benzene). With a great stability in its plus one oxidation state, we believe $\{\mathrm{TpMo}(\mathrm{NO})(\mathrm{L})\}$ has the potential to follow a similar method for recyclability. With the demonstrated ability to consistently isolate [W]-aromatic complexes on multigram scales, we wish to develop a molybdenum analogue that can form isolable eta-2 bound complexes on the same or greater scale as [W]. Furthermore, we aim to develop a metal center where electronic and steric factors can be tuned by the use of various ancillary ligands (L) to allow for the binding of a broad array of aromatic ligands and their subsequent organic transformation. The ability to enantioenrich the metal center is beyond the scope of this project; however, progress on the project described above is reported herein. 


\subsection{References}

1. Lovering, F.; Bikker, J.; Humblet, C. J. Med. Chem. 2009, 52, (21), 6752.

2. Mander, L. N.; Hook, J. M. Nat. Prod. Reports 1986, 37.

3. Birch, A. J.; Hinde, A. L.; Radom, L. J. Am. Chem. Soc. 1981, 103, 284.

4. Schreiber, S. L. Science 1985, 227, 857.

5. Hudlicky, T. Pure \& Appl. Chem. 1994, 66, 2067.

6. Amurrio, D.; Kahn, K.; Kündig, E. P. J. Org. Chem. 1996, 61, 2258.

7. Barluenga, J.; Trabanco, A. A.; Fløorez, J.; García-Granda, S.; Martín, E. J. Am. Chem. Soc. 1996, 118, 13099.

8. Davies, S. G.; Coote, S. J.; Goodfellow, C. L., Synthetic Applications of Chromium Tricarbonyl Stabilized Benzylic Carbanions. JAI Press, Ltd.: London, 1991; Vol. 2, p 2.

9. Blagg, J. Contemporary Organic Synthesis 1995, 2, (1), 43.

10. Davies, S. G.; McCarthy, T. D., Comprehensive Organometallic Chemistry II. Abel, E. W., Ed. Pergamon Press: Oxford, 1995; Vol. 12, Chapter 9.3.

11. Thompson, R. L.; Lee, S.; Rheingold, A. L.; Cooper, N. J. Organometallics 1991, $10,1657$.

12. Astruc, D. Tetrahedron 1983, 39, (24), 4027

13. Bakhtiar, R.; Jacobson, D. B. J. Am. Soc. Mass Spectrom. 1996, 7, 938.

14. Brooks, B. C.; Gunnoe, T. B.; Harman, W. D. Coord. Chem. Rev. 2000, (206207), 3 .

15. Keane, J. M.; Harman, W. D. Organometallics 2005, 24, 1786.

16. Mocella, C. J.; Delafuente, D. A.; Keane, J. M.; Warner, G. R.; Friedman, L. A.; Sabat, M.; Harman, W. D. Organometallics 2004, 23, (16), 3772.

17. Meiere, S. H.; Keane, J. M.; Gunnoe, T. B.; Sabat, M.; Harman, W. D. J. Am. Chem. Soc. 2003, 125, (8), 2024.

18. Harman, W. D.; Taube, H. J. Am. Chem. Soc. 1987, 109, 1883.

19. Silverthorn, W. E. Adv. Organomet. Chem. 1975, 13, 48. 
20. Browning, J.; Green, M.; Spencer, J. L.; Stone, F. G. J. Chem. Soc., Dalton Trans. 1974, 97.

21. Boese, R.; Stanger, A.; Stellberg, P.; Shazar, A. Angew. Chem. Int. Ed. Engl. 1993, 32, 1475 .

22. Harman, W. D.; Hasegawa, T.; Taube, H. Inorg. Chem. 1991, 113, 453.

23. Chordia, M. D.; Harman, W. D. J. Am. Chem. Soc. 2000, 122, (12), 2725.

24. Harman, W. D.; Taube, H. J. Am. Chem. Soc 1988, 110, 7906.

25. Kopach, M. E.; Harman, W. D. J. Am. Chem. Soc. 1994, 116, 6581.

26. Kopach, M. E.; Harman, W. D.; Hipple, W. G. J. Am. Chem. Soc. 1992, 114, 1737.

27. Winemiller, M. D.; Harman, W. D. J. Org. Chem. 2000, 65, 1249.

28. Gonzalez, J.; Koontz, J. I.; Myers, W. H.; Hodges, L. M.; Sabat, M.; Nilsson, K.

R.; Neely, L. K.; Harman, W. D. J. Am. Chem. Soc. 1995, 117, 3405.

29. Cordone, R.; Taube, H. J. Am. Chem. Soc. 1987, 109, 8101.

30. Spera, M. L.; Harman, W. D. Organometallics 1995, 14, 1559.

31. Chen, H.; Hodges, L. M.; Liu, R.; Stevens, W. C.; Sabat, M.; Harman, W. D. J. Am. Chem. Soc. 1994, 116, 5499.

32. Harman, W. D.; Sekine, M.; Taube, H. J. Am. Chem. Soc. 1988, 110, 5725.

33. Kopach, M. E.; Gonzalez, J.; Harman, W. D. J. Am. Chem. Soc. 1991, 113, 8972.

34. Cordone, R.; Harman, W. D.; Taube, H. J. Am. Chem. Soc. 1989, 111, 5969.

35. Chordia, M. D.; Harman, W. D. J. Am. Chem. Soc. 1998, 120, 5637.

36. Barrera, J.; Orth, S. D.; Harman, W. D. J. Am. Chem. Soc. 1992, 114, 7316.

37. Orth, S. D.; Barrera, J.; Rowe, S. M.; Helberg, L. E.; Harman, W. D. Inorg. Chim. Acta 1998, 270, 337.

38. Sweet, J. R.; Graham, W. A. G. Organometallics 1983, 2, (1), 135.

39. Sweet, J. R.; Graham, W. A. G. J. Am. Chem. Soc. 1983, 105, 305.

40. v.d.Heijden, H.; Orpen, A. G.; Pasman, P. J. Chem. Soc. Chem. Commun. 1985, 1576. 
41. Brooks, B. C.; Chin, R. M.; Harman, W. D. Organometallics 1998, 17, 4716.

42. Heijden, H. v. d.; Orpen, A. G.; Pasman, P. J. Chem. Soc., Chem. Commun. 1985, 1576.

43. Curtis, M. D.; Shiu, K.-B.; Butler, W. M. J. Am. Chem. Soc. 1986, 108, 1550.

44. Curtis, M. D.; Shiu, K. Inorg. Chem. 1985, 24, 1213.

45. Gelabert, R.; Moreno, M.; Lluch, J. M.; Lledos, A. Organometallics 1997, 16, 3805 .

46. Oldham, J., W. J.; Hinkle, A. S.; Heinekey, D. M. J. Am. Chem. Soc. 1997, 119, 11028.

47. Gunnoe, T. B.; Sabat, M.; Harman, W. D. J. Am. Chem. Soc. 1999, 121, 6499.

48. Meiere, S. H.; Brooks, B. C.; Gunnoe, T. B.; Sabat, M.; Harman, W. D.

Organometallics 2001, 20, 1038.

49. Meiere, S. H.; Brooks, B. C.; Gunnoe, T. B.; Carrig, E. H.; Sabat, M.; Harman, W. D. Organometallics 2001, 20, (17), 3661.

50. Harrison, D. P.; Nichols-Nielander, A. C.; Zottig, V. E.; Strausberg, L.; Salomon, R. J.; Trindle, C. O.; Sabat, M.; Gunnoe, T. B.; Iovan, D. A.; Myers, W. H.; Harman, W. D. Organometallics 2011, 30, (9), 2587.

51. Brooks, B. C.; Meiere, S. H.; Friedman, L. A.; Gunnoe, T. B.; Harman, W. D. J. Am. Chem. Soc. 2001, 123, 3541.

52. Ding, F.; Valahovic, M. T.; Keane, J. M.; Anstey, M. R.; Sabat, M.; Trindle, C. O.; Harman, W. D. J. Org. Chem. 2004, 69, (7), 2257.

53. Valahovic, M. T.; Gunnoe, T. B.; Sabat, M.; Harman, W. D. J. Am. Chem. Soc. 2002, 124, 3309 .

54. Meiere, S. H.; Valahovic, M. T.; Harman, W. D. J. Am. Chem. Soc. 2002, 124, (50), 15099.

55. Friedman, L. A.; You, F.; Sabat, M.; Harman, W. D. J. Am. Chem. Soc. 2003, $125,14980$.

56. Keane, J. M.; Ding, F.; Sabat, M.; Harman, W. D. J. Am. Chem. Soc. 2004, 126, 785.

57. Keane, J. M. Synthesis and Nucleophilic Activity of Eta-2 Arene Complexes. Ph. D. Dissertation, University of Virginia, 2003. 
58. McCleverty, J. A.; Seddon, D.; Bailey, N. A.; Joe' Walker, N. W. Journal of the Chemical Society, Dalton Transactions 1976, (10), 898.

59. Liu, W.; You, F.; Mocella, C. J.; Harman, W. D. J. Am. Chem. Soc. 2006, 128, (5), 1426.

60. Trofimenko, S. Inorganic Chemistry 1969, 8, (12), 2675.

61. Graham, P.; Meiere, S. H.; Sabat, M.; Harman, W. D. Organometallics 2003, 22, 4364.

62. Ha, Y.; Dilsky, S.; Graham, P. M.; Liu, W.; Reichart, T.; Sabat, M.; Kenae, J. M.; Harman, W. D. Organometallics 2006, 25, 5184.

63. Welch, K. D.; Harrison, D. P.; Lis, E. C.; Liu, W.; Salomon, R. J.; Harman, W. D.; Myers, W. H. Organometallics 2007, 26, (10), 2791.

64. Salomon, R. J.; Todd, M. A.; Sabat, M.; Myers, W. H.; Harman, W. D. Organometallics 2010, 29, (4), 707.

65. MacLeod, B. L.; Pienkos, J. A.; Myers, J. T.; Sabat, M.; Myers, W. H.; Harman, W. D. Organometallics 2014, 33, (22), 6286.

66. MacLeod, B. L.; Pienkos, J. A.; Wilson, K. B.; Sabat, M.; Myers, W. H.; Harman, W. D. Organometallics 2016, 35, (3), 370.

67. Graham, P. M.; Delafuente, D. A.; Liu, W.; Myers, W. H.; Sabat, M.; Harman, W. D. J. Am. Chem. Soc. 2005, 127, (30), 10568.

68. Lankenau, A. W.; Iovan, D. A.; Pienkos, J. A.; Salomon, R. J.; Wang, S.; Harrison, D. P.; Myers, W. H.; Harman, W. D. J. Am. Chem. Soc. 2015, 137, (10), 3649.

69. Welch, K. D.; Harrison, D. P.; Sabat, M.; Hejazi, E. Z.; Parr, B. T.; Fanelli, M. G.; Gianfrancesco, N. A.; Nagra, D. S.; Myers, W. H.; Harman, W. D. Organometallics 2009, 28, (20), 5960.

70. $\quad$ Liu, W.; Welch, K.; Trindle, C. O.; Sabat, M.; Myers, W. H.; Harman, W. D. Organometallics 2007, 26, (10), 2589. 


\section{Chapter Two}

\section{Synthesis of 2-Substituted 1,2-}

\section{Dihydronaphthalenes and 1,2-}

Dihydroanthracenes Using a Recyclable

Molybdenum Dearomatization Agent 


\subsection{Introduction}

The dearomatization of polycyclic aromatic hydrocarbons (PAHs), such as naphthalene or anthracene, has been explored via organometallic methods, but far less so than for benzenes. ${ }^{1-7}$ In $\eta^{6}$ complexes such as $\mathrm{Cr}(\mathrm{CO})_{3}\left(\eta^{6}-\mathrm{L}\right)$, metal-arene bonds are weaker for PAH ligands (L) than for benzenes, whereas for the corresponding $\eta^{2}$ coordinated complexes, the opposite is true. For example, naphthalene has been reported to form stable dihapto-coordinated complexes with a wide range of metals, including $\mathrm{Ni}^{8}{ }^{8}$ $\mathrm{Ru},{ }^{9} \mathrm{Mo},{ }^{10} \mathrm{~W},{ }^{11} \mathrm{Re},{ }^{5}$ and Os, ${ }^{1}$ with much greater kinetic stability toward arene substitution than their benzene counterparts. ${ }^{12}$ Furthermore, in the case of the third row transition metal systems $\left\{\mathrm{Os}^{\mathrm{II}}\left(\mathrm{NH}_{3}\right)_{5}\right\}^{2+},{ }^{1-3}\left\{\mathrm{TpRe}^{\mathrm{I}}(\mathrm{MeIm})(\mathrm{CO})\right\}, \quad 4, \quad 5, \quad 13$ and $\left\{\mathrm{TpW}\left(\mathrm{PMe}_{3}\right)(\mathrm{NO})\right\},{ }^{14}$ tandem electrophilic-nucleophilic additions have been demonstrated, via an arenium intermediate. Such a synthetic approach has led to novel cis-1,2- or cis-1,4-disubstituted dihydronaphthalenes.

Previous reports ${ }^{15}$ have suggested that the complex TpMo(NO)(MeIm) $\left(\eta^{2}-\right.$ naphthalene), 4, prepared from $\mathrm{Mo}(\mathrm{CO})_{6}$ in four steps (Scheme 2.1), may exhibit similar chemical reactivity to the known tungsten complex $\mathrm{TpW}(\mathrm{NO})\left(\mathrm{PMe}_{3}\right)\left(\eta^{2}\right.$-naphthalene).$^{14}$ Furthermore, $\mathrm{TpMo}(\mathrm{NO})(\mathrm{MeIm})(\mathrm{X})$, the expected product of $\mathrm{TpMo}(\mathrm{NO})(\mathrm{MeIm})(\mathrm{L} \pi)$ reacting with a halogen oxidant $\left(\mathrm{X}_{2}=\mathrm{Br}_{2}\right.$ or $\left.\mathrm{I}_{2}\right)$, exhibits a broader range of electrochemical stability for the $\mathrm{Mo}^{\mathrm{I}}$ oxidation state (e.g., $\sim+0.6 \mathrm{~V}$ to $-1.5 \mathrm{~V}$ ), compared to its $\mathrm{W}^{\mathrm{I}}$ analogue, $\left(\sim+0.3 \mathrm{~V}\right.$ to $-1.4 \mathrm{~V}$ for $\left.\mathrm{TpW}(\mathrm{NO})\left(\mathrm{PMe}_{3}\right)(\mathrm{Br})\right) .{ }^{16}$ For the tungsten system, the potential required to oxidize, and thereby remove the final organic product (typically $>+0.5 \mathrm{~V}$ ), is greater than that required to further oxidize $\mathrm{TpW}(\mathrm{NO})\left(\mathrm{PMe}_{3}\right)$ (solvent) to $\mathrm{W}^{\mathrm{II}}$ (0.1 - $0.3 \mathrm{~V}$ for most common solvents). However, the 
greater stability of the $\mathrm{Mo}^{\mathrm{I}}$ analogues toward oxidation suggested that a decomplexation procedure could be developed that would return an intact $\mathrm{Mo}^{\mathrm{I}}$ complex, which in turn could serve as the direct precursor to the naphthalene complex 4 . This process is similar to that previously reported for rhenium analogues, which uses AgOTf as the oxidant. ${ }^{17}$ Herein, we describe a promising new approach for the dearomatization of PAHs on a practical scale (up to $\sim 800 \mathrm{mg}$ of organic product) with highly efficient recovery of the dearomatization agent.

\subsection{Results}

\subsubsection{Improved Synthesis of TpMo(NO)(MeIm) $(L \pi)(L \pi=$ naphthalene or anthracene)}

Although the syntheses of $\mathbf{4}$ and $\mathrm{TpMo}(\mathrm{NO})(\mathrm{MeIm})\left(\eta^{2}\right.$-anthracene) (5) have been previously reported, several steps in the synthetic sequence have been optimized in order for the envisioned method to be practical (Scheme 2.1). The reflux time for the synthesis of $\mathrm{TpMo}(\mathrm{NO})(\mathrm{CO})(\mathrm{MeIm})$ (2) from the dicarbonyl (1) was reduced from 48 to 1 hour by using dimethylformamide (DMF) as the solvent in place of xylenes. Replacing xylenes with DMF achieves a higher temperature for reflux $\left(154{ }^{\circ} \mathrm{C}\right)$, which increases the rate of the reaction. Also improved was the isolation of $\mathbf{2}$, which can now be accomplished through a precipitation in water rather than the previously reported chromatographic workup. Notably, the syntheses of 2 and $\mathrm{TpMo}(\mathrm{NO})(\mathrm{MeIm})(\mathrm{I})$ (3) were found to be successful in air with no noticeable degradation to yield or purity of the isolated products. Overall, the precursor (3) for the dearomatization products (4 and 5) could be isolated on a multigram scale $(>100 \mathrm{~g})$ with an overall yield of $68 \%$ from $\mathrm{Mo}(\mathrm{CO})_{6}$. In comparison, the previously reported $\mathrm{TpMo}(\mathrm{NO})(\mathrm{MeIm})(\mathrm{Br})$ was made on a 21 gram scale with an overall 47\% yield from $\mathrm{Mo}(\mathrm{CO})_{6}$ with 2 and 3 being made under a nitrogen atmosphere. 
To improve the reductive formation of the arene complexes $\mathbf{4}$ and $\mathbf{5}$, we sought to move away from the use of mercury in these reactions by replacing the sodium-mercury amalgam with sodium dispersion. By using hexanes to dissolve the wax from the sodium dispersion in paraffin, elemental sodium flakes could be obtained. With the use of these flakes, the reduction of $\mathbf{3}$ to make $\mathbf{4}$ was found to be uncontrollably fast. This is believed to be due to the in situ reduction of naphthalene to sodium naphthalenide, which provides a homogeneous, rather than heterogeneous, reducing agent. Sodium naphthalenide then reduces 3 at an uncontrolled rate, yielding reaction completion in less than 5 minutes, followed by decomposition due to over-reductive conditions. This over-reduction was previously not an issue as a sodium mercury amalgam was used. The amalgam is believed to have slowly integrated sodium into the reaction mixture. In a similar manner we found that adding sodium dispersion in paraffin wax directly to the reaction solution delivers the reducing agent at a slower, more controlled rate as the wax dissolves. Using this method, sodium-mercury amalgam was replaced by sodium dispersion and the scale was increased to $5 \mathrm{~g}$ yielding 4 at $34 \%$ yield from 3 . The synthesis of the anthracene analogue, $\mathbf{5}$, was also accomplished under mercury-free conditions. 
Scheme 2.1. Synthesis of dihapto-coordinated naphthalene and anthracene complexes of molybdenum.

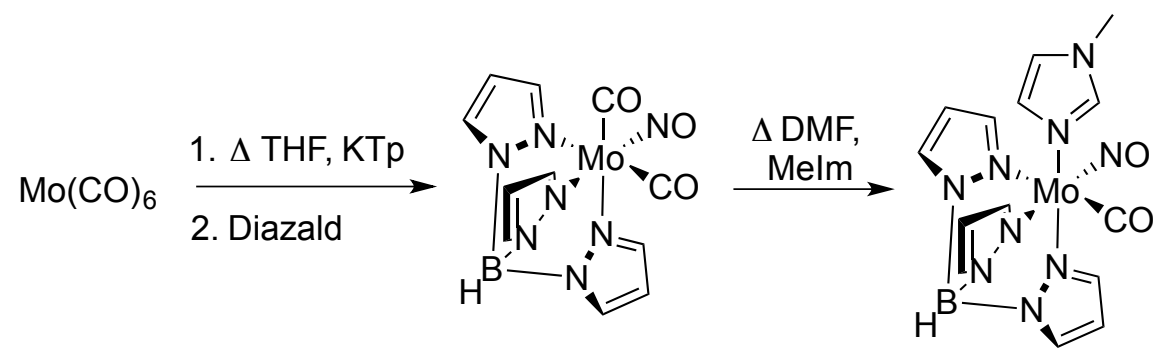<smiles>[Mg][Mg][Mg][Mg]</smiles>

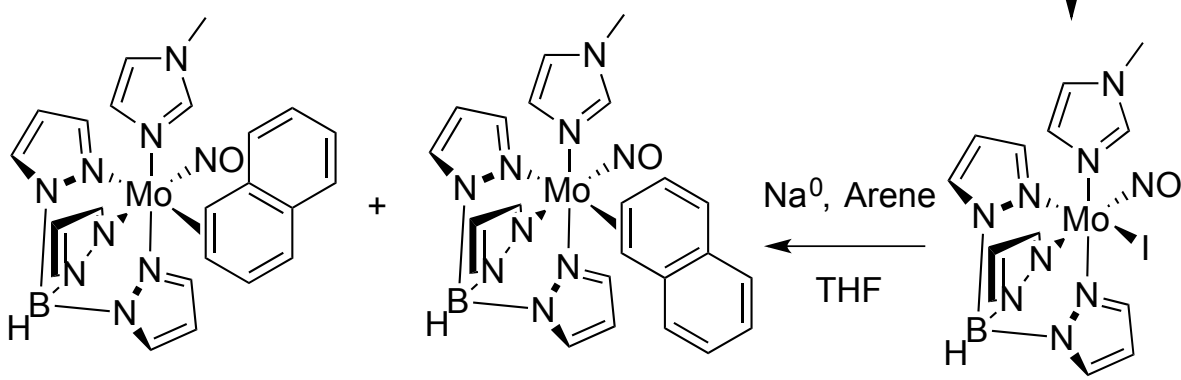
$4 \quad: \quad 1$

$4 p$

4d

3

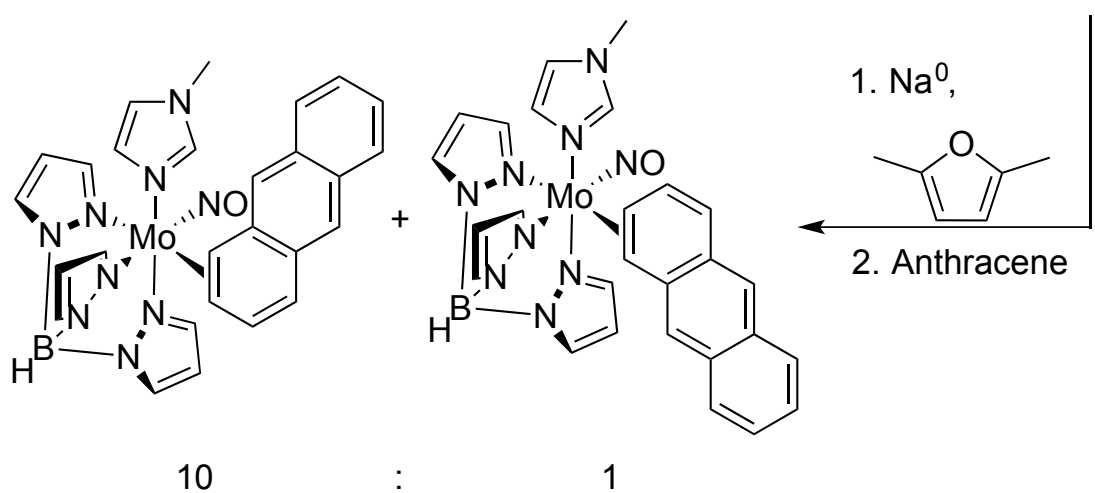

$5 p$

$5 d$

Adding the sodium dispersion in paraffin wax directly into THF increased the reaction time from 5 min with the sodium flakes to $24 \mathrm{~h}$, but provided $\mathbf{5}$ in serviceable yield $(19 \%)$. However, the product was contaminated $(\sim 20 \%)$ by another material, thought to be the binuclear complex $\left[(\mathrm{TpMo}(\mathrm{MeIm})(\mathrm{NO}))_{2}\left(\eta^{2}: \eta^{2}-\mu\right.\right.$-anthracene $\left.)\right]$ based 
on the ${ }^{1} \mathrm{H}$ NMR spectrum contatining resonances at 3.95 and 3.14 ppm with coupling identical to the desired product's proton resonances. This byproduct could be avoided by synthesizing TpMo(NO)(MeIm) $\left(\eta^{2}-2,5\right.$-dimethylfuran) in situ, removing the sodium, and subsequently stirring in the presence of anthracene. Thus, the anthracene derivative $\mathbf{5}$ was cleanly produced on a multigram scale in yields over $50 \%$ from 3 .

\subsubsection{Oxidation of TpMo(NO)(MeIm) $\left(\eta^{2}\right.$-naphthalene) with Iodine}

After improving the synthesis of $\mathbf{4}$, the possibility of recycling the dearomatization agent was investigated. Following a tandem electrophilic-nucleophilic addition reaction on $\mathbf{4}$, it was theorized that an electron could be removed from the metal center in the presence of an oxidant (i.e., a halogen $\left(\mathrm{X}_{2}\right)$ ). After oxidation, the ability for $\mathrm{Mo}^{\mathrm{I}}$ to backbond into this dihydronaphthalene dramatically decreases, as the metal center is substantially less electron-rich, significantly destabilizing the metal ligand bond. Thus the modified organic would be released and a halide could subsequently coordinate to the metal center. This coordination would produce $\mathrm{TpMo}(\mathrm{NO})(\mathrm{MeIm})(\mathrm{X})$, which could then be reduced in the presence of naphthalene to regenerate 4. Although the incompatibility of the reagents used in this sequence prevent a one-pot catalytic cycle, the stepwise isolation of each complex would result in a formal catalytic cycle for the organic modification of aromatics using a molybdenum dearomatization agent (Scheme 2.2). 
Scheme 2.2. Proposed formal catalytic cycle for the dearomatization of naphthalene with $\{\mathrm{TpMo}(\mathrm{NO})(\mathrm{MeIm})\}$.
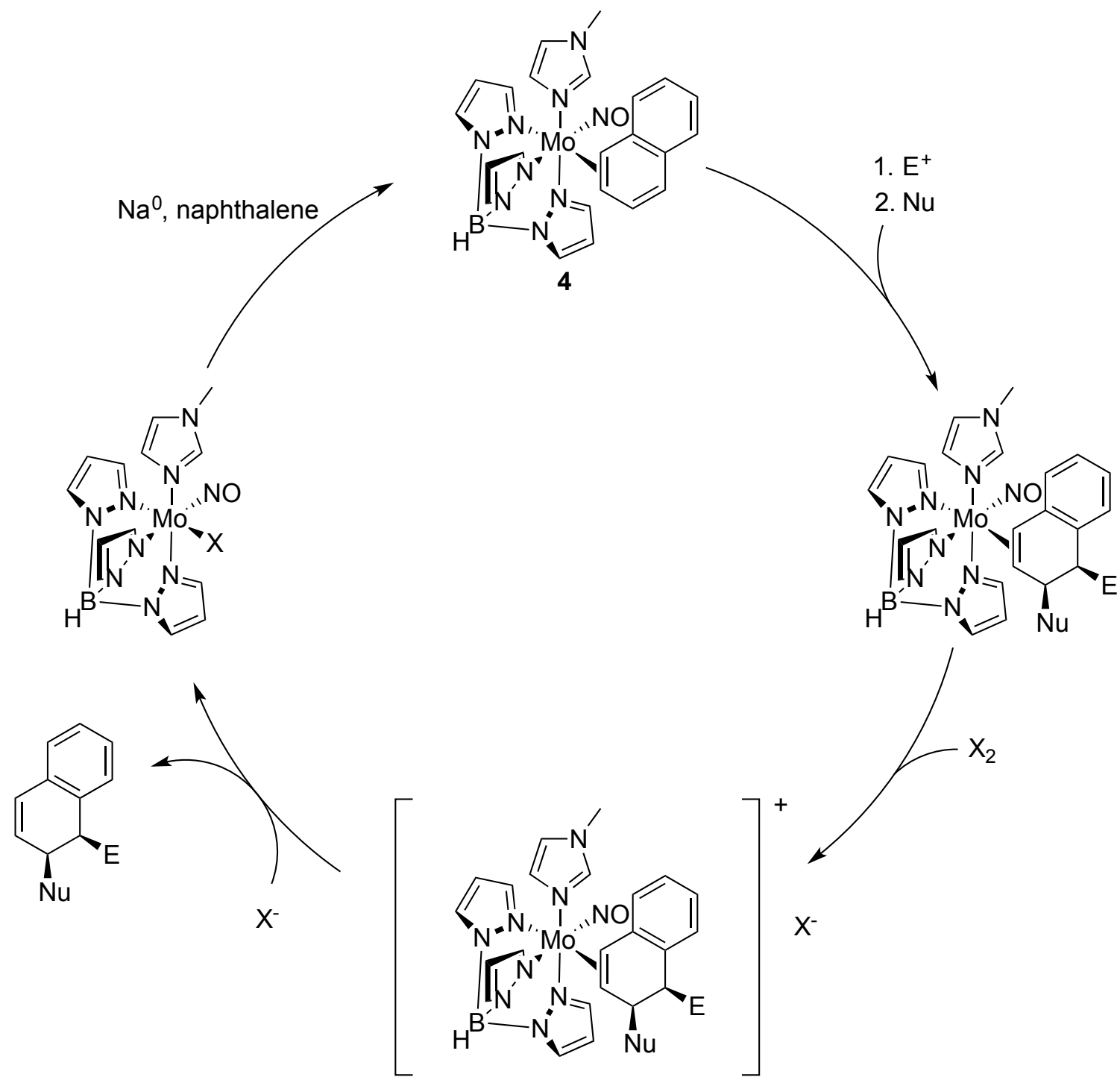

To test the feasibility of this proposed recycling scheme, 4 was subjected to oxidation with iodine in dichloromethane (DCM). In addition to having a mild, but sufficiently strong reduction potential, iodine offers an ease of use, as it is less volatile and toxic than the alternative halogen oxidants. A notable color change from yellow to green was observed upon addition of iodine to a DCM solution of $\mathbf{4}$ that is in agreement 
with the formation of the emerald green product, 3 . This solution was added to hexanes to yield a precipitate that was then isolated on a fritted disc. This solid was confirmed through cyclic voltammetry to be $3(91 \%)$. The filtrate from this precipitation was evaporated in vacuo and analyzed by ${ }^{1} \mathrm{H}$ NMR to confirm that it contained pure naphthalene (70\%) (Scheme 2.3).

Scheme 2.3. Oxidation of 4 with iodine.

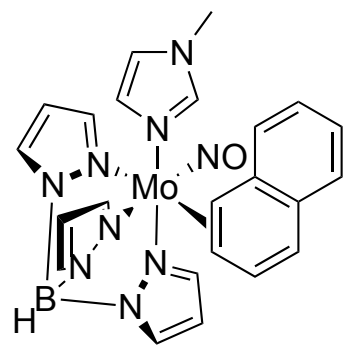

4

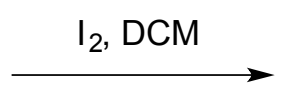

$3(91 \%)$<smiles>c1ccc2ccccc2c1</smiles>

$70 \%$

Although the ability to recover $\mathbf{3}$ and free naphthalene from the oxidation of $\mathbf{4}$ in good yields was promising, the applicability of this method needed to be proven on modified organics (e.g., dihydroarenes). Given that there had been only one example of the organic modification of a molybdenum $\eta^{2}$-arene complex (vide infra), the expansion of organic transformations of PAHs bound in an eta-2 fashion to molybdenum was pursued. 


\subsubsection{Spectroscopic Profile of TpMo(NO)(MeIm) $\left(\eta^{2}\right.$-1,2-dihydroarene)}

For the purposes of this study, we focused on three addition reactions, thought to represent a broader reactivity pattern for the PAH ligands of $\mathbf{4}$ and $\mathbf{5}$ with carbon nucleophiles. The only previous report of ligand-centered reactivity for a molybdenum $\eta^{2}$-arene complex is the protonation of $\mathbf{4}$, followed by the addition of the ketene acetal MTDA (1-methoxy-2-methyl-1-trimethylsiloxy-propene). In addition to optimizing this reaction, the addition of an enolate salt (lithium dimethylmalonate; LiDMM), and an aromatic heterocycle ( $N$-methylpyrrole) were explored as nucleophiles.

While the protonation and addition of MTDA were previously reported for $\mathbf{4},{ }^{15}$ the product complex 6 was never characterized. The NO stretching frequency $\left(1565 \mathrm{~cm}^{-1}\right)$ of 6 is modestly lower than its precursor $4\left(c f . v_{\mathrm{NO}}=1576 \mathrm{~cm}^{-1}\right)$, an observation that suggests that the dihydronaphthalene ligand is a weaker $\pi$-acid than napththalene itself. However, the electrochemical analysis of $\mathrm{TpMo}(\mathrm{NO})(\mathrm{MeIm})\left(\eta^{2}\right.$-1,2-dihydronaphthalene) reveals an anodic wave with $E_{\mathrm{p}, \mathrm{a}}=+0.12 \mathrm{~V}(100 \mathrm{mV} / \mathrm{s})$ indicating its diminished susceptibility to oxidation, relative to $4\left(c f . E_{\mathrm{p}, \mathrm{a}}=-0.26 \mathrm{~V}\right)$. While at first glance, the electrochemical and infrared data seem at odds, the more negative anodic peak for $\mathbf{4}$ is likely a consequence of the decreased kinetic stability of $\left[\mathrm{TpMo}^{\mathrm{I}}(\mathrm{NO})(\mathrm{MeIm})\left(\eta^{2}-\right.\right.$ naphthalene) $]^{+},{ }^{18}$ which is expected to undergo rapid dissociation of the arene ligand. For this type of chemically coupled electrochemical process $\left(\mathrm{E}_{\mathrm{r}} \mathrm{C}_{\mathrm{i}}\right)$, a larger rate constant for the chemical step (i.e., arene dissociation) results in a negative shift for $E_{\mathrm{p}, \mathrm{a}}$. This explanation is more likely than a true shift in the formal $\mathrm{d}^{5} / \mathrm{d}^{6}$ reduction potential in the comparison of $\mathbf{6}$ and $\mathbf{4}$. 


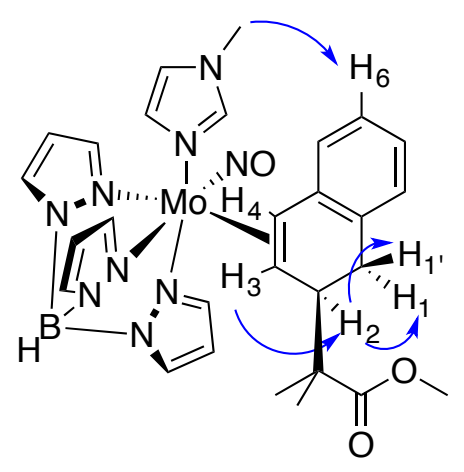

$6 p$ (major)

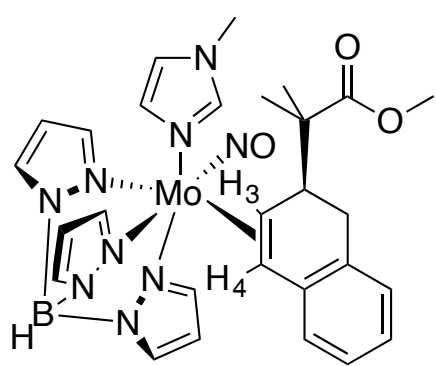

6d (minor)

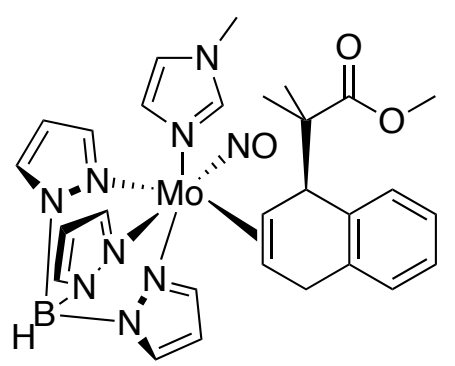

1,4-addition

Figure 2.1. NOESY correlations for $\mathbf{6 p}$ and the proposed assignment of $\mathbf{6 d}$ as its coordination diastereomer, rather than a constitutional isomer resulting from a 1,4addition. $J_{\mathrm{H} 1 \mathrm{~b}-\mathrm{H} 2}<1 \mathrm{~Hz}$.

In addition to resonances for the nine Tp and three MeIm protons, ${ }^{1} \mathrm{H}$ NMR data reveal a diastereotopic methylene group, H1' (doublet of doublets at $3.52 \mathrm{ppm}$ ) and $\mathrm{H} 1$ (doublet at $2.55 \mathrm{ppm}$ ), identified by its large coupling constant $(17.2 \mathrm{~Hz})$, and confirmed by HSQC data. Proton H2 (doublet at $3.23 \mathrm{ppm}$ ) was identified through NOESY and COSY correlations with $\mathrm{H} 1$ and H1'. Proton H3 was identified through its NOESY and COSY correlations to $\mathrm{H} 2$, as well as a COSY correlation to H1. A distinctive doublet of triplets is seen at $2.05 \mathrm{ppm}$ due to $\mathrm{H} 3$ coupling to $\mathrm{H} 4$ (a doublet at $3.13 \mathrm{ppm}$ ), H1', and H2. These data, along with a strong NOESY correlation between Pz3A and H2, confirm that the MTDA addition occurs anti to the metal. This stereochemistry was confirmed by the X-ray crystal structure analysis of $\mathbf{6}$ (Figure 2.2). 


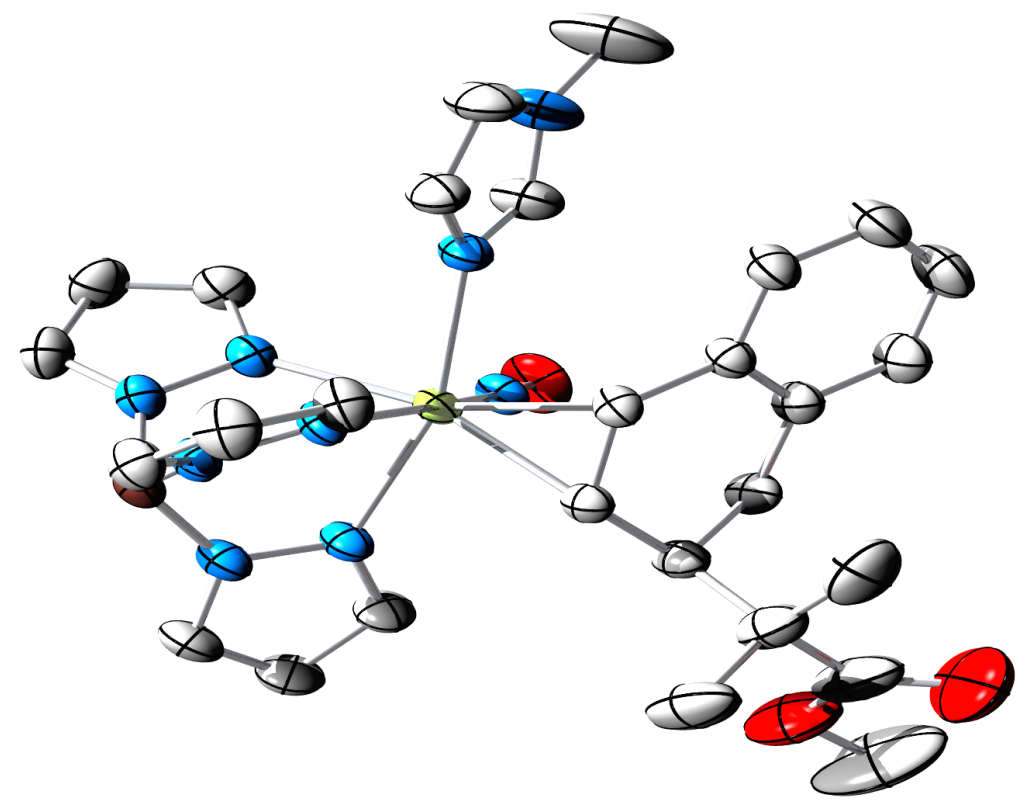

Figure 2.2. Crystal structure of dihydronaphthalene complex 6. Bond lengths $(\AA)$ : MoC(4) 2.232(2); Mo-C(3) 2.242(2); Mo-C(4) 2.32(2); Mo-N(MeIm) 2.2103(17); $\mathrm{Mo}-\mathrm{N}(\mathrm{NO}) 1.7544(17)$.

The axial positioning observed for the $\mathrm{C} 2$ substituent of the dihydronaphthalene ligand is also present in solution according to $\mathrm{COSY}$ and $\mathrm{H} / \mathrm{H}$ coupling data, which indicate an H1-C1-C2-H2 dihedral angle approaching $90^{\circ}$. Identification of the major coordination diastereomer, in which the remaining aromatic portion of the ring is positioned proximal to the MeIm ligand, was determined in solution by the observation of an NOE interaction between $\mathrm{H} 6$ and the methyl of the MeIm ligand.

Signals for an additional complex (6d) are observed in the ${ }^{1} \mathrm{H}$ NMR spectrum of $\mathbf{6}$ at a 25:1 ratio of $\mathbf{6 p : 6 d}$, showing signals and splitting patterns very similar to those of the major species (6p). Most notably, a doublet-of-triplets at $1.84 \mathrm{ppm}$ has the same coupling constants as $\mathrm{H} 3$ of the major isomer. This minor product is thought to be a coordination diastereomer of $\mathbf{6 p}$ in which the unbound aromatic ring is oriented distal to the imidazole (Figure 2.1). While overlapping signals in the NMR spectrum prevent the conclusive 
assignment of the minor species, the oxidation of a sample of $\mathbf{6}$ upon exposure to air generates a single new dihydronaphthalene product, consistent with $\mathbf{6 p}$ and $\mathbf{6 d}$ being coordination diastereomers (see Figure 2.1). Were 6p and 6d constitutional isomers (e.g., 1,2- vs 1,4-addition), then oxidation of $\mathbf{6}$ would have yielded constitutional isomers of $\mathbf{1 2}$ upon oxidative decomplexation.

\subsubsection{Isolation of 1,2-Dihydroarenes Through Air Oxidation}

Similar 1,2-addition reactions for the naphthalene ligand of $\mathbf{4}$ are accomplished through the protonation and subsequent addition of either $N$-methylpyrrole or LiDMM. Further, all three nucleophiles are successfully added to the anthracene ligand of 5 (Table 2.1). Of note, addition products $(\mathbf{C})$ are isolated as racemic mixtures of both enantiomers of the chiral metal center thus yielding the organics $(\mathbf{O})$ as racemic mixtures as well.

Complexes of type $\mathbf{C}$ (Table 2.1) are all capable of being oxidized via exposure to air. Although these organics are obtained in poor yield (average 28\%), their synthesis demonstrates the ability to remove the metal using a mild oxidant that does not react with the sensitive organic products. Of note, when $N$-methylpyrrole is used as the nucleophile, isolation of the organic product reveals two isomers for both naphthalene and anthracene. Using COSY correlations, it was determined that $N$-methylpyrrole adds primarily at the alpha position of the pyrrole, but a small amount $(\sim 10 \%)$ of the beta-substituted analogue is also formed. For the alpha isomer, the ${ }^{1} \mathrm{H}$ NMR spectrum shows triplets at 6.60 and $6.07 \mathrm{ppm}$ as well as a doublet-of-doublets at $5.98 \mathrm{ppm}$. For the beta form, triplets at 6.52 and 6.43 as well as a triplet at 6.04 are observed. The protons on the isolated alkene bond of the naphthalene ring retain their splitting as would be expected if both products were the result of 1,2-additions. The beta addition apparently occurs during the initial addition 
Table 2.1. Tandem electrophilic-nucleophilic addition to 4 and 5.

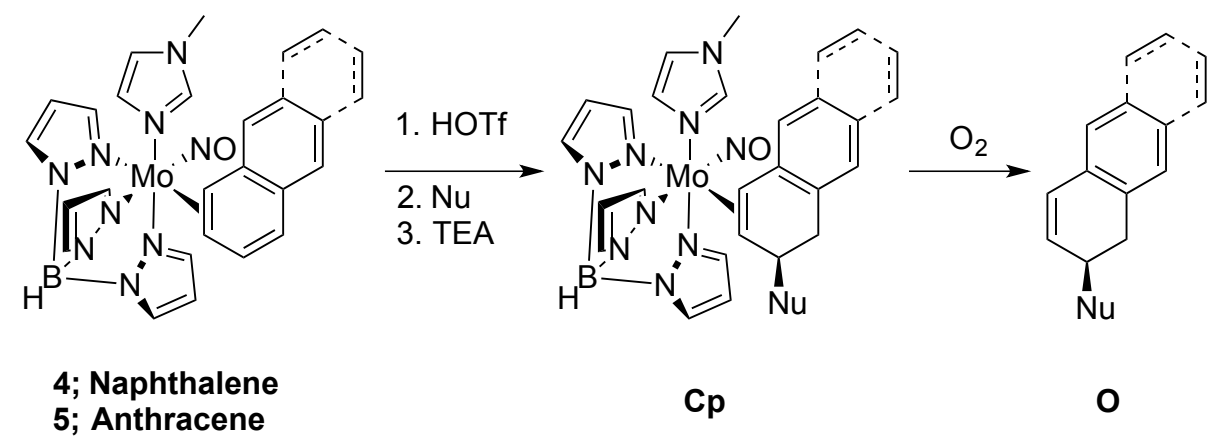

\section{Arene}

Complex

4

5<smiles>COC(=O)C(C)(C)[C@H]1C=Cc2cc3ccccc3cc2C1</smiles>

4<smiles>COC(=O)C(C(=O)OC)[C@H]1C=Cc2cc3ccccc3cc2C1</smiles>

4

5<smiles>Cn1cccc1[C@@H]1C=Cc2cc3ccccc3cc2C1</smiles>

C (\% yield)

6; 61 (5 g scale)

10:1

$7 ; 43$

$25: 1$

$13 ; 22$

8; 66

$8: 1^{\mathrm{a}}$

$14 ; 24$

9; 48

$15: 1^{\mathrm{a}}$

$15 ; 44$

$10 ; 53$

$5: 1$

$16 ; 14$

$11 ; 27$

$8: 1$

$17 ; 39$

a The values reported in this table are more accurate than those published 
process, as NMR spectra for both pyrrole derivatives $\mathbf{1 0}$ and $\mathbf{1 1}$ show $\sim 10 \%$ of an additional molybdenum complex, besides the minor coordination diastereomer (10d, 11d).

\subsubsection{Recyclable Oxidation of TpMo(NO)(MeIm) $\left(\eta^{2}\right.$-1,2-dihydroarenes)}

As discussed above, decomplexation of the organic product for the ester derivative $\mathbf{6}$ can be accomplished using air to oxidize the molybdenum. However, as is the case with $\left\{\mathrm{TpW}(\mathrm{NO})\left(\mathrm{PMe}_{3}\right)(\right.$ alkene $\left.)\right\}$ complexes, a $\mathrm{M}^{\mathrm{I}}$ co-product could not be recovered. ${ }^{19}$ This is illustrated by cyclic voltammetric data, which shows that, upon exposure to air, no electrochemically active molybdenum products are observed over a range of +1.0 to $-1.7 \mathrm{~V}$ (Figure 2.3). A similar result was observed using ceric ammonium nitrate (CAN) as the oxidant (Figure 2.3). However, the ease of oxidation for the metal complexes $\mathbf{6}$ - $\mathbf{1 1}(\sim+0.2 \mathrm{~V})$ suggests that milder oxidants can be used, including iodine.

The $\mathrm{I}_{2} / 2 \mathrm{I}^{-}$standard reduction potential, $+0.53 \mathrm{~V}$, is sufficiently large that it can oxidize the dihydronaphthalene and dihydroanthracene product complexes but is not powerful enough to over-oxidize the metal (the oxidation of $\mathrm{Mo}^{\mathrm{I}}$ to $\mathrm{Mo}^{\mathrm{II}}$ for [TpMo(NO)(MeIm)(I)] occurs with an $E_{\mathrm{p}, \mathrm{a}}=+0.65 \mathrm{~V}$ at $\left.100 \mathrm{mV} / \mathrm{s}\right)$. In contrast to that observed when air or CAN is used to oxidize ester complex 6 (Figure 2.3), a cyclic voltammogram of the reaction mixture resulting from iodine oxidation of $\mathbf{6}$ contains a pseudo-reversible couple near $-1.4 \mathrm{~V}$ and $E_{\mathrm{p}, \mathrm{a}} \sim+0.65 \mathrm{~V}$. This feature is consistent with $\mathrm{TpMo}(\mathrm{NO})(\mathrm{MeIm})(\mathrm{I})$ being generated as the major inorganic product. 


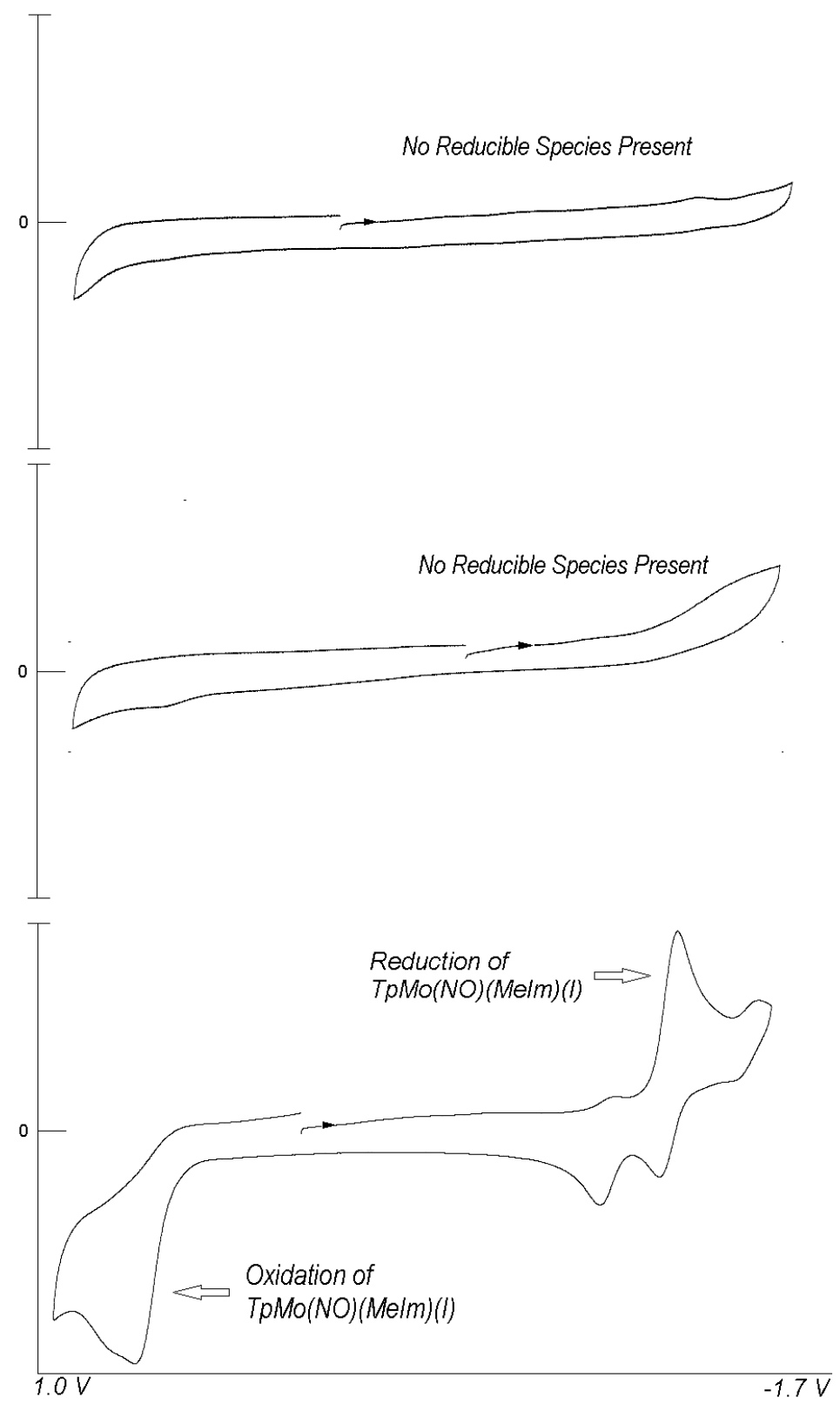

Figure 2.3. Cyclic voltammogram of the reaction mixture following oxidation of 6 and air (top), CAN (middle), or iodine (bottom).

Inspired by the electrochemical data in Figure 2.3, we set out to develop conditions to liberate the organic compounds from complexes $\mathbf{6}$ - $\mathbf{1 1}$ using $\mathrm{I}_{2}$ and then recover 3 by precipitation from DCM:hexanes. Isolated yields for both organic products and 3 are reported in Table 2.2. The average recovery of 3 is $84 \%$ (recovering up to $3 \mathrm{~g}$ 
of 3) and the use of iodine significantly improves yields of the isolated organic products (average 64\%). Of note, $\sim 800 \mathrm{mg}$ of $\mathbf{1 2}$ was isolated from the oxidation of $\mathbf{6}$ with iodine on a $4 \mathrm{~g}$ scale, truly representing the great scale at which $\{\mathrm{TpMo}(\mathrm{NO})(\mathrm{MeIm})\}$ can yield modified organics. With the recovery of $\mathbf{3}$, a formal catalytic cycle is completed for the PAH dearomatization, although incompatibilities of the chemical reagents defeat any possibility of a practical 'one-pot' process.

Table 2.2. Formal catalytic cycle for the generation of dihydronaphthalenes and dihydroanthracenes.

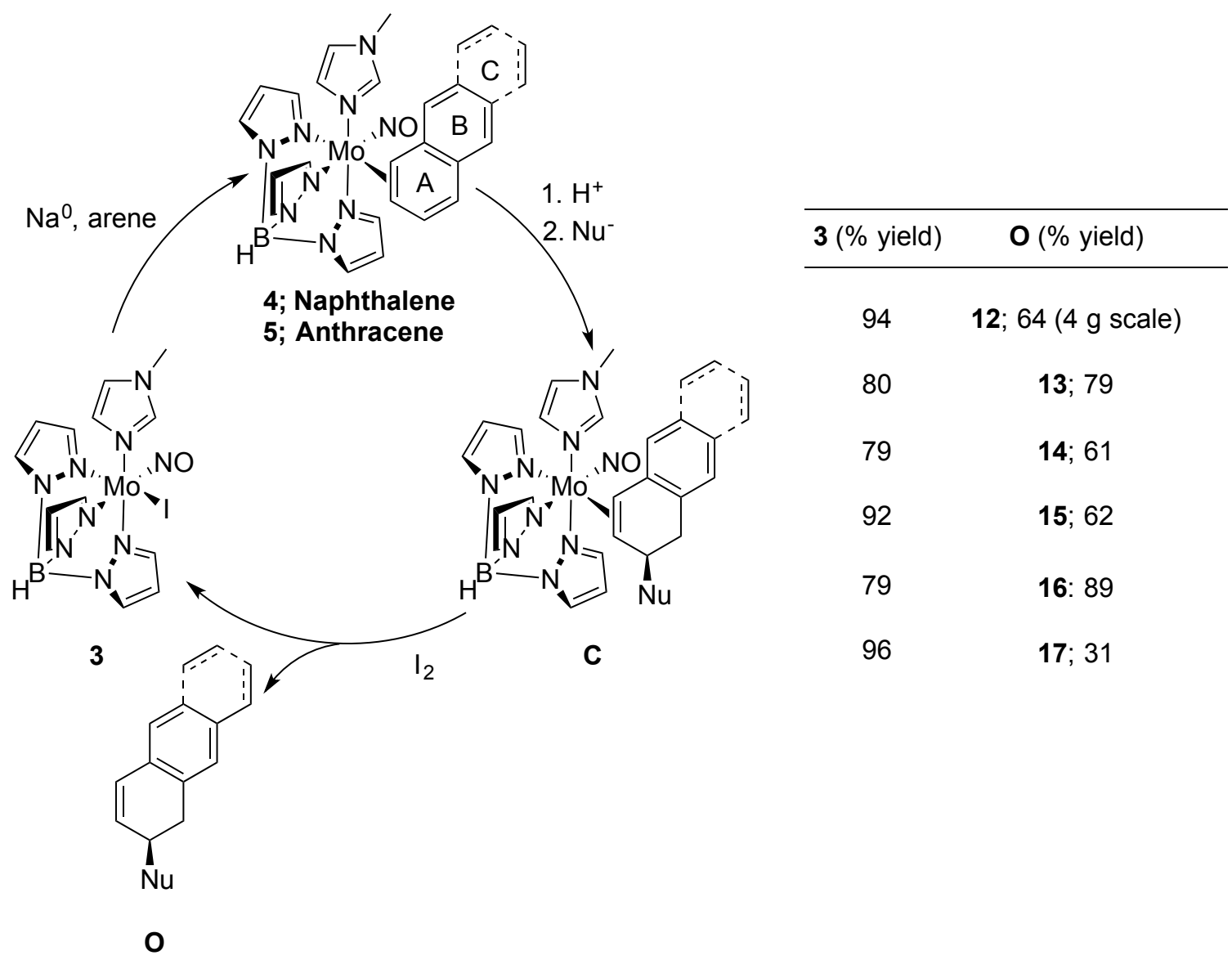




\subsubsection{Chemical Elaboration of Isolated 1,2-Dihydroarenes}

The organic products $12-17$ are themselves susceptible to oxidative dehydrogenation, ${ }^{20}$ especially in the presence of base, but can be stabilized by addition across the isolated alkene bond. To stabilize the products and illustrate the synthetic potential of this dearomatization method, we elected to apply an iodolactonization process to isolated esters $\mathbf{1 2}$ and $\mathbf{1 3}$. Hence, these esters were first hydrolyzed to their carboxylic acids (18 and 19) then cyclized in the presence of iodine to form $\mathbf{2 0}$ and $\mathbf{2 1}$ (Scheme 2.4). Through full 2D analysis, the stereochemistry of 20 and 21 was determined. In particular, the proposed cis ring juncture was confirmed by a strong NOE interaction of the bridgehead protons, a feature that would not be expected in the trans isomer.

Scheme 2.4. Iodolactonization of 12 and 13.

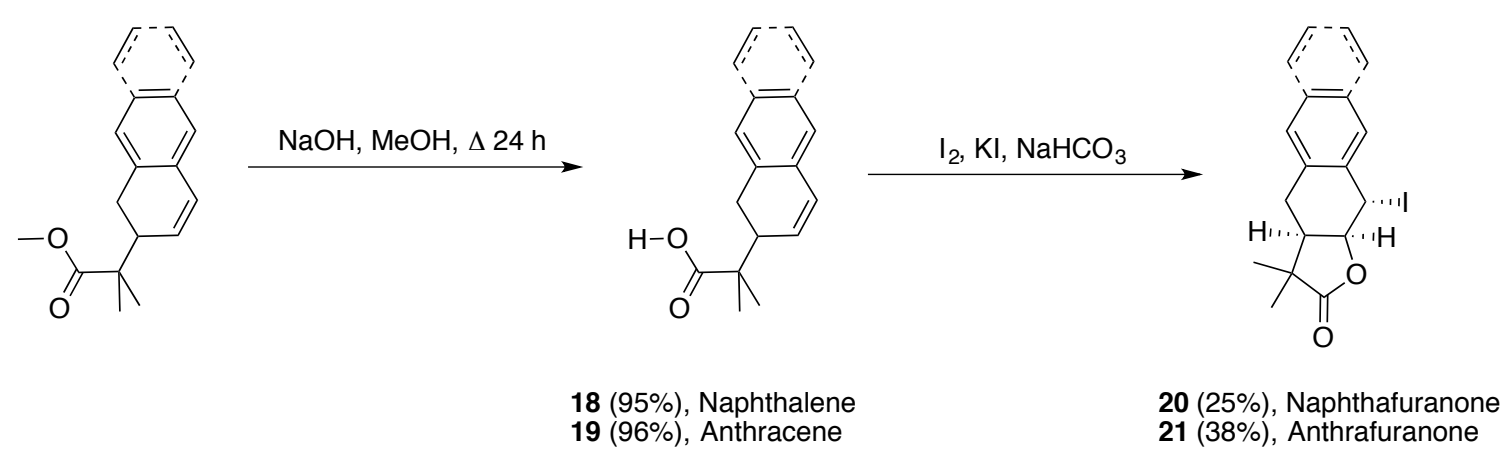

\subsection{Discussion}

Naphthalenes are typically derivatized through Friedel-Crafts acylation ${ }^{21}$ and alkylation $^{22,23}$ to yield substituted naphthalene products. In one report, $\left\{\mathrm{Cr}(\mathrm{CO})_{3}\right\}$ was used to promote the nucleophilic addition of various sulfur-stabilized carbanions to 1methoxynaphthalene. ${ }^{24}$ While subsequent addition of methyl iodide can produce 
acetylated dihydronaphthalenes, the regioselectivity for these reactions is typically poor (Scheme 2.5).

Scheme 2.5. Dearomatization of naphthalene using $\left\{\mathrm{Cr}(\mathrm{CO})_{3}\right\}$.

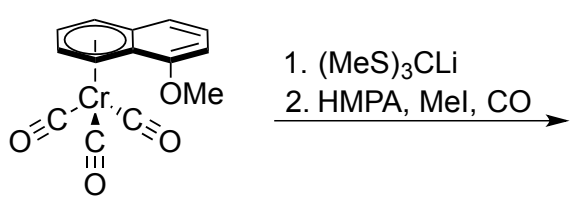<smiles>COc1cccc2c1C=CC(C(C)(C)C)C2C(C)(C)C</smiles>

$86 \%$<smiles>COc1cccc2c1C(C(C)(C)C)C(C(C)(C)C)C=C2</smiles>

$14 \%$

Anthracenes are inherently more reactive at the internal (B) ring than the terminal (A or C) rings due to a greater thermodynamic stability yielded from the retention of two benzene rings (Scheme 2.6). ${ }^{25}$ This trend in reactivity can be seen in the formation of 9,10-dihdyro-9,10-dimethoxyanthracenes as well as in the reductive nitration of anthracene in the presence of hydrochloric acid to form 9,10-dihydroanthracenes. As a result of the relative lack of reactivity at the terminal rings, methods for the preparation of 1,2-dihydroanthracenes directly from anthracene are practically unknown. Aside from minor amounts of cycloaddition and dihydroxylation products formed as part of complex reaction mixtures, reports of addition reactions that involve the terminal rings of anthracene are unknown.

Scheme 2.6. Organic additions to anthracene.

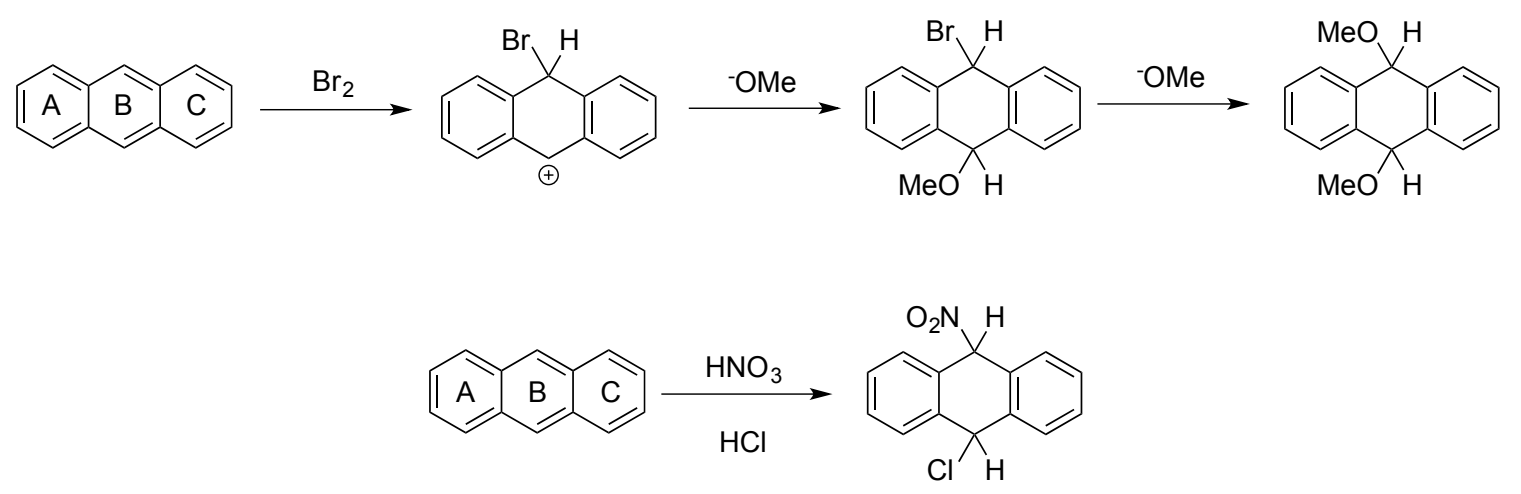


$\{\mathrm{TpMo}(\mathrm{NO})(\mathrm{MeIm})\}$ can be used to regioselectively control tandem electrophilic-nucleophilic additions to naphthalene and anthracene in fair yields, subsequently regenerating the dearomatization precursor 3 through an oxidation with iodine. Furthermore, these 1,2-dihydroarenes have been shown to undergo iodolactonization to yield organics with three new stereogenic centers when compared to their aromatic precursors. This methodology offers a complementary approach to the above-mentioned methods found in the literature for the derivatization of PAHs. These derivatized PAHs represent important classes of compounds of biological interest. For instance, dialin cores are found in many naturally occurring products (i.e., cannabisin C, negundin $\mathrm{B},(+)-$ Phyltetralin, etc.) and pharmaceuticals (i.e., sertraline, tametraline, lometraline, etc.). Lactone $\mathbf{2 0}$ contains the same naphthafuranone core as pygmaeocin $\mathrm{A},{ }^{26}$ used to treat inflammation and malaria, and several arthrinins, ${ }^{27}$ a well-known class of natural products. Furthermore, the anthrafuranone core of $\mathbf{2 1}$ is present in olivinolide, a derivative of the aglycone for the anticancer drug olivomycin. ${ }^{28}$ A similar $1,2,3,4-$ tetrahydroanthracene core is found in toyomycin (chromomycin) (Figure 2.4). ${ }^{29}$ For this reason, compounds $12-\mathbf{1 7}, \mathbf{2 0}$, and 21 have been submitted to Eli Lilly and Company for screening. 


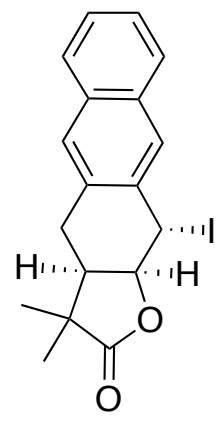

21<smiles>CC1(C)C(=O)O[C@@H]2[C@H]1Cc1ccccc1[C@H]2I</smiles>

20

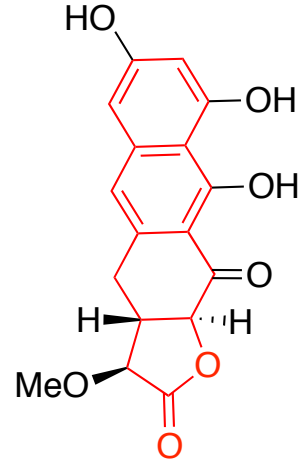

olivinolide

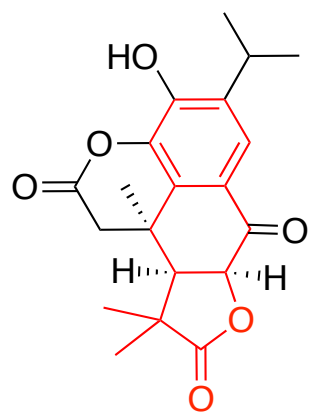

pygmaeocin A

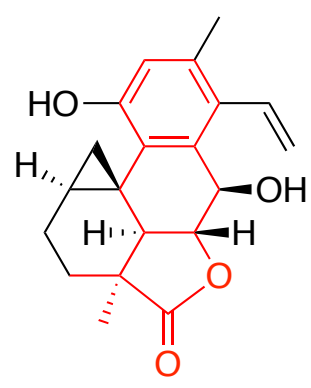

$\operatorname{arthrinin} \mathrm{A}$

Figure 2.4. Naphthafuranones pygmaeocin A and arthrinin A, and the anthrafuranone olivinolide.

Complexes of the form $\mathbf{C}$ (Scheme 2.7) represent the first isolation of a complex from the organic modification of aromatics using $\{\mathrm{TpMo}(\mathrm{NO})(\mathrm{MeIm})\}$. For this reason, explanation of how these reactions are monitored is discussed here to aid future work on these and related systems. The general reaction scheme for the tandem electrophilicnucleophilic addition reactions to form complexes of the form $\mathbf{C}$ is given in Scheme 2.7. First, a heterogeneous mixture of $\mathbf{4}$ or $\mathbf{5}$ suspended in $\mathrm{CH}_{3} \mathrm{CN}$ is cooled at $-30{ }^{\circ} \mathrm{C}$ for 15 min. Next, a $-30{ }^{\circ} \mathrm{C}$ solution of between 3 and 4 equivalents of the electrophile in $\mathrm{CH}_{3} \mathrm{CN}$ is added to the reaction mixture and the resulting solution is left at $-30{ }^{\circ} \mathrm{C}$ for $15 \mathrm{~min}$. Between 7 to 8 equivalents of the nucleophile is then added to the reaction mixture and 
this solution is left at $-30{ }^{\circ} \mathrm{C}$. At this point, the reaction is quenched by the addition of a base and the product $(\mathbf{C})$ can be isolated by chromatography.

Scheme 2.7. Tandem electrophilic-nucleophilic addition reactions to 4 or 5 .

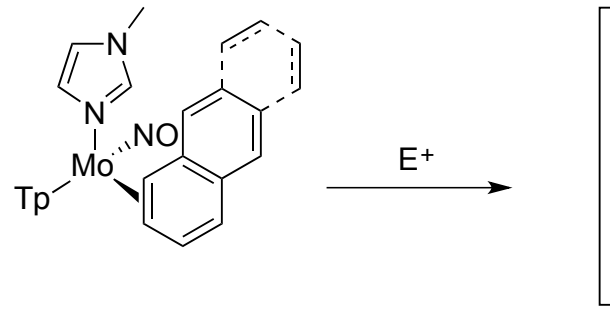

4 or 5

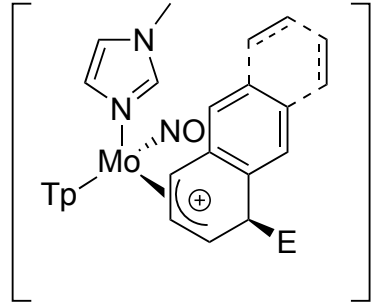

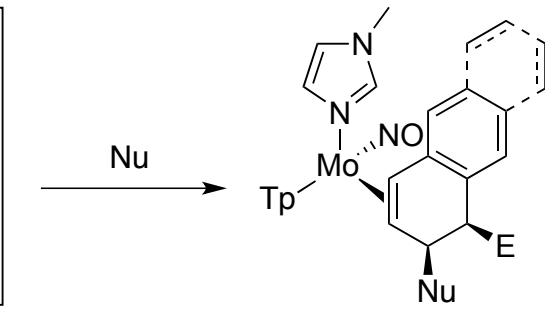

C

Whereas the $\left\{\mathrm{TpW}(\mathrm{NO})\left(\mathrm{PMe}_{3}\right)\right\}$ fragment has a phosphine ligand, the $\{\mathrm{TpMo}(\mathrm{NO})(\mathrm{MeIm})\}$ fragment lacks a convenient handle for the monitoring of the reaction mixture by NMR spectroscopy. Also of difficulty is the sensitivity of the reactant, intermediate, and product complexes, to air and high temperatures $\left(>-20{ }^{\circ} \mathrm{C}\right)$ under the oxidative conditions of the reaction. This sensitivity adds more difficulty to ${ }^{1} \mathrm{H}$ NMR monitoring, in addition to the potentially overwhelming signals yielded from electrophiles or nucleophiles used.

We have found that a useful technique for monitoring the reactions to yield complexes of type $\mathbf{C}$ is cyclic voltammetry (CV). CV allows for the analysis of only the electrochemically active species present in a solution. In this manner we can distinguish metal complexes, utilizing knowledge of the measured potentials of the $\mathrm{Mo}^{0} / \mathrm{Mo}^{1+}$ oxidation obtained from their isolated products, with minimal interference from the organic reagents and solvents also present in the reaction mixture. To monitor these reactions by $\mathrm{CV}$ the following protocol is followed after the addition of both the electrophile and nucleophile at $-30{ }^{\circ} \mathrm{C}$ : an aliquot of the reaction mixture is removed, 
added to base (e.g., TEA), and the resulting solution is added to a CV cell containing room temperature DMA and electrolyte.

The base is added to the aliquot before $\mathrm{CV}$ analysis to quench any unreacted electrophile present in the reaction mixture that could potentially react with the starting material or product of this reaction at the higher temperatures of the $\mathrm{CV}$ experiment. It should be noted that the allylic species from an electrophilic addition to $\mathbf{4}$ or $\mathbf{5}$ is very unstable at temperatures above $-20{ }^{\circ} \mathrm{C}$ (vide infra) and therefore is not observed in this $\mathrm{CV}$ monitoring. Were the allylic species still present in the reaction mixture, it is believed to revert back to starting material via deprotonation (vide infra). Reaction progress in these reactions is indicated by a decrease in the signal for the oxidation of the starting material $\left(E_{\mathrm{p}, \mathrm{a}} \approx-0.26 \mathrm{~V}\right)$ and an increase in a signal at $\sim+0.2 \mathrm{~V}$, which corresponds to the oxidation of the 1,2-dihydroarene complexes (C). For example, when complex $\mathbf{6}$ is reacted with HOTf and MTDA, by monitoring the reaction through the disappearance of the $E_{\mathrm{p}, \mathrm{a}}$ at $-0.26 \mathrm{~V}$ and the appearance of an $E_{\mathrm{p}, \mathrm{a}} \sim+0.12 \mathrm{~V}$, the reaction was determined to be complete 15 minutes after the addition of MTDA to the reaction mixture.

Other key features observed during these addition reactions were color change and homogeneity of the reaction mixture. The reaction starts as a vibrant orange heterogeneous mixture and, upon the addition of acid, changes to a blood red homogeneous solution, indicating the formation of an allyl intermediate (Scheme 2.8, Pathway i). The enhanced basicity of naphthalene when bound to $\{\mathrm{TpMo}(\mathrm{NO})(\mathrm{MeIm})\}$ was compared to its $\{\mathrm{TpRe}(\mathrm{CO})(\mathrm{RIm})\}$ and $[\mathrm{Os}]$ analogues in a previous investigation. ${ }^{30}$ It was found that when $\mathbf{4}$ was added to a solution containing $\operatorname{TpRe}(\mathrm{CO})(\mathrm{BuIm})\left(2,3,4-\eta^{3}\right.$ $(1 H$-naphthalenium $))](\mathrm{OTf})$ no observable proton transfer occurred over $0.5 \mathrm{~h}$ at $-20{ }^{\circ} \mathrm{C}$. 
However, in contrast to the [Os]-naphthalene complex, $\mathbf{4}$ is capable of being protonated by diphenylammonium triflate (DPHAT) (pKa $\sim 6$ in $\mathrm{CH}_{3} \mathrm{CN}$ ). These experiments show that naphthalene is more basic when bound to $\{\mathrm{TpMo}(\mathrm{NO})(\mathrm{MeIm})\}$ than when it's bound to $[\mathrm{Os}]$; however, naphthalene is less basic when bound to $\{\mathrm{TpMo}(\mathrm{NO})(\mathrm{MeIm})\}$ than when it is bound to $\{\mathrm{TpRe}(\mathrm{CO})(\mathrm{BuIm})\}$. Having a greater basicity, $\mathrm{TpMo}(\mathrm{NO})(\mathrm{L})(\mathrm{L} \pi)$ complexes are predicted to have the ability to react with weaker electrophiles than those used with the $[\mathrm{Os}]$ fragment.

Scheme 2.8. Potential pathways from the addition of an electrophile to 4 or 5 .

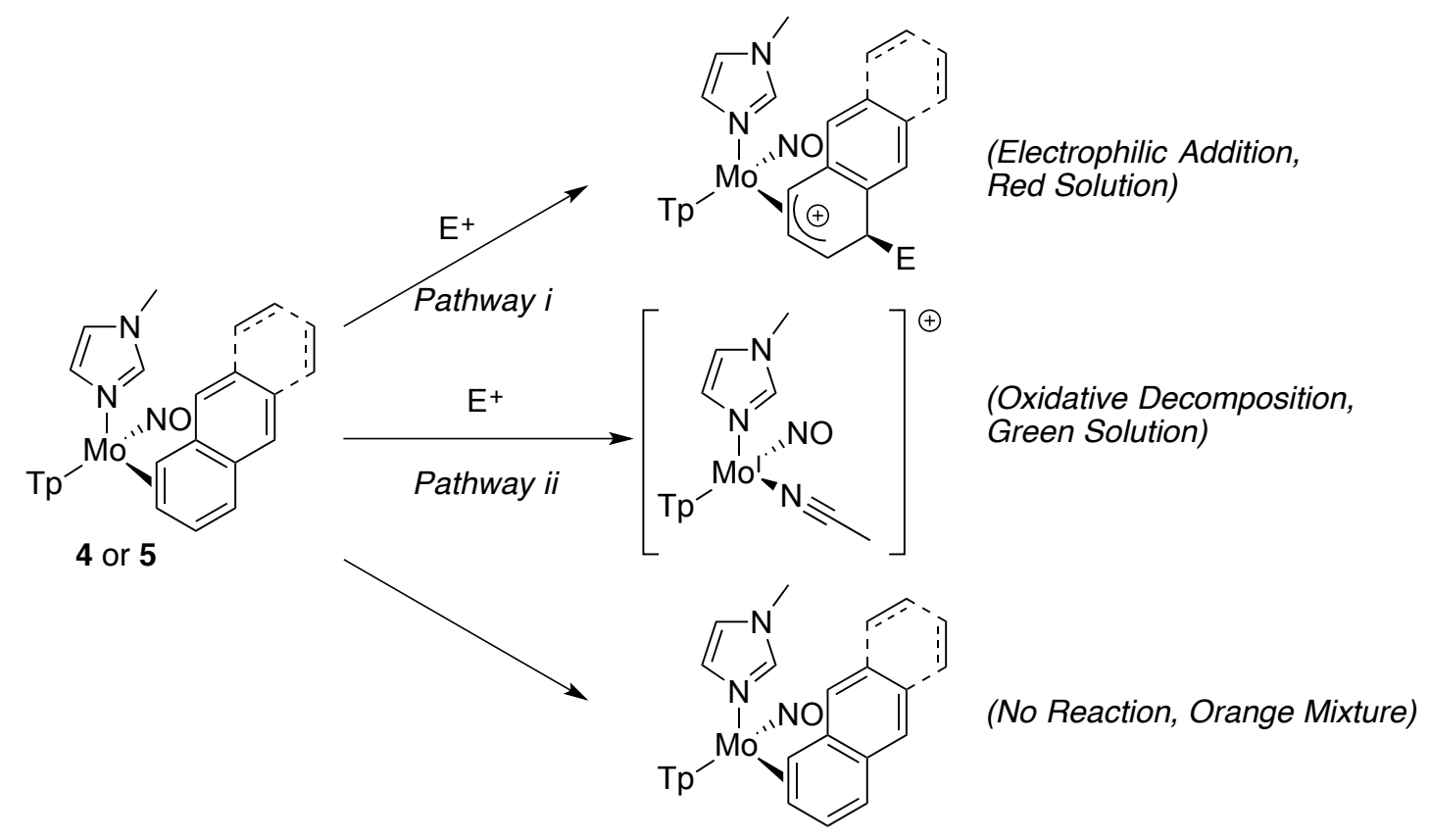

Electrophiles, other than a strong acid $(\mathrm{pKa}<1)$, were attempted (i.e., iminiums, NCS, NBS, selectfluor, acetals, Michael acceptors, etc.); however, either an orange mixture containing a reductive species corresponding to the respective starting material (e.g., 4 or 5) or a green solution containing a strong $E_{\mathrm{p}, \mathrm{c}} \sim-0.9 \mathrm{~V}$ were observed. The observation of starting material indicated 4 and 5's lack of nucleophilicity under these 
conditions (i.e., $-30{ }^{\circ} \mathrm{C}$ ), but raising the temperature appeared to favor the formation of the green solution mentioned above. This green solution contains the cationic acetonitrile complex, $\left[\mathrm{TpMo}^{\mathrm{I}}(\mathrm{NO})(\mathrm{MeIm})\left(\kappa^{1} \text {-acetonitrile }\right)\right]^{1+}$ complex (Scheme 2.8, Pathway ii) resulting from oxidation of the metal complex.

Previous reports have described the ability to exchange furan with acetonitrile to make TpMo(NO)(MeIm)( $\eta^{2}$-acetonitrile) complex in situ. ${ }^{31}$ Although this complex could not be isolated, the addition of benzylbromide yielded a benzonitrilium complex. Similarly, by stirring $\mathrm{TpMo}(\mathrm{NO})(\mathrm{MeIm})\left(\eta^{2}-2,5\right.$-dimethylfuran) complex in acetonitrile, the 2,5-dimethylfuran can be exchanged with $\mathrm{CH}_{3} \mathrm{CN}$ and the purported $\operatorname{TpMo}(\mathrm{NO})(\mathrm{MeIm})\left(\eta^{2}\right.$-acetonitrile) complex is observed in situ with an anodic peak around $+0.2 \mathrm{~V}$. Of note, after the electrochemical oxidation of this complex, a cathodic peak appears at $-0.9 \mathrm{~V}$ that is absent when the oxidation does not occur. This signal is believed to be due to the reduction of the $\left[\operatorname{TpMo}^{\mathrm{I}}(\mathrm{NO})(\mathrm{MeIm})\left(\kappa^{1} \text {-acetonitrile }\right)\right]^{1+}$ cation, formed by a linkage isomerization of the $\eta^{2}$-acetonitrile complex upon oxidation (Scheme 2.9). Under oxidative conditions it is believed that 4 and 5 can be oxidized to release their eta-2 bound aromatic, thereby allowing solvent coordination (e.g., $\mathrm{CH}_{3} \mathrm{CN}$ ). This represents a chemical, rather than electrochemical, process to access the $\operatorname{TpMo}^{\mathrm{I}}(\mathrm{NO})(\mathrm{MeIm})\left(\kappa^{1}\right.$-acetonitrile) complex and explains the observation of the complex in the monitoring of tandem electrophilic-nucleophilic addition reactions to $\mathbf{4}$ and $\mathbf{5}$. 
Scheme 2.9. Exchange of 2,5-dimethylfuran with $\mathrm{CH}_{3} \mathrm{CN}$ to form $\mathrm{TpMo}(\mathrm{NO})(\mathrm{MeIm})\left(\eta^{2}-\right.$ acetonitrile), and the resulting complex observed through CV.

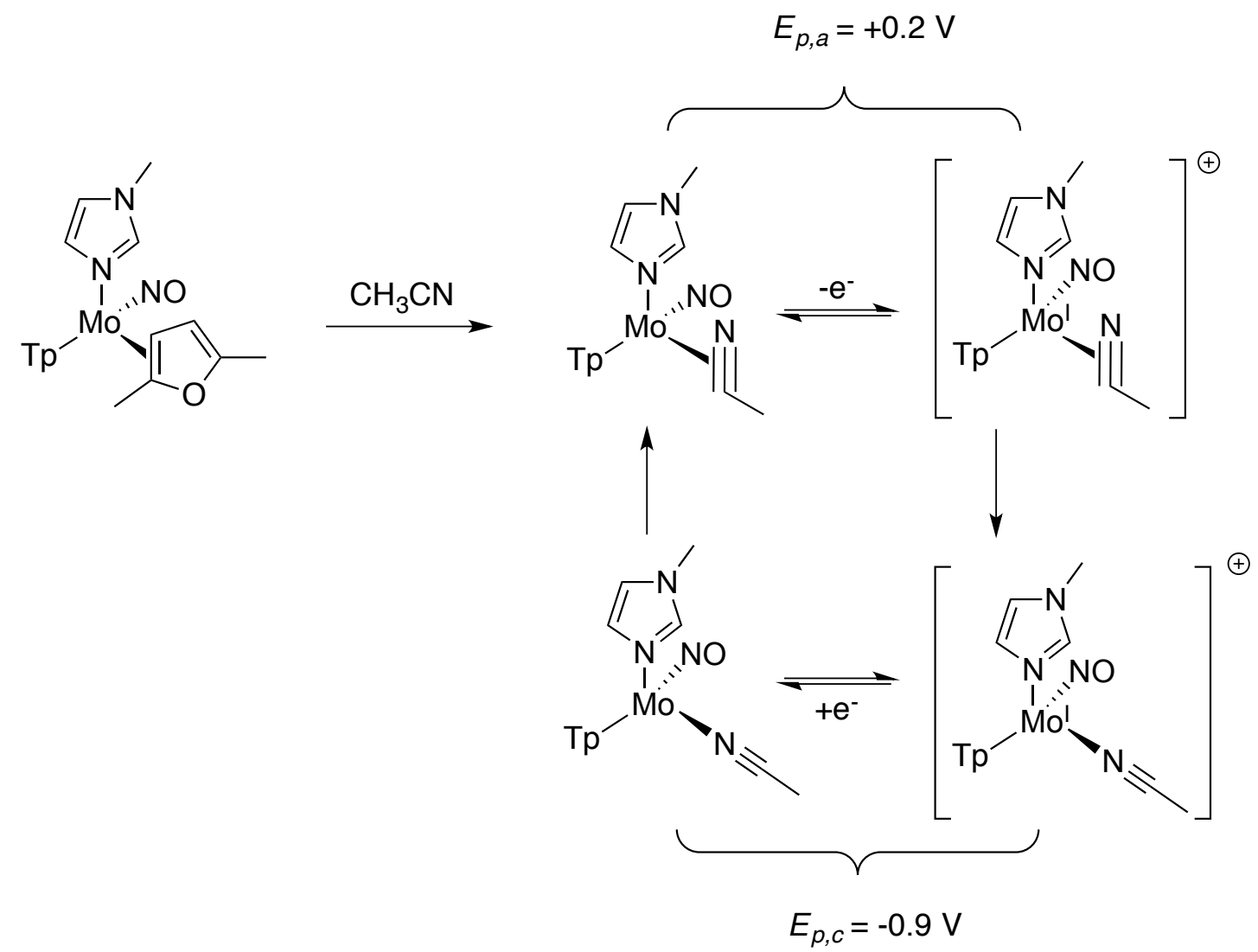

Previous reports also show $\left[\mathrm{TpMo}(\mathrm{NO})(\mathrm{MeIm})\left(\eta^{3}\right.\right.$-naphthalenium) $](\mathrm{OTf})$, with a $\mathrm{t}_{1 / 2}$ of several hours at $-20{ }^{\circ} \mathrm{C}$, to be significantly more unstable than rhenium (I) and osmium (II) analogues. ${ }^{30}$ Evidence to support this instability can be seen in a more downfield shift of the allylic carbons on $\left[\mathrm{TpMo}(\mathrm{NO})(\mathrm{MeIm})\left(2,3,4,-\eta^{3}-\right.\right.$ naphthalenium)](OTf) $(143.4,101.7$, and $88.1 \mathrm{ppm})$ compared to the allylic carbons of $\left[\mathrm{TpRe}(\mathrm{CO})(\mathrm{MeIm})\left(2,3,4,-\eta^{3}\right.\right.$-naphthalenium)](OTf) $(99.6,79.0$, and $73.0 \mathrm{ppm})$, a trend suggesting weaker $\pi$-donation from molybdenum (0). This weaker donation results in a more electrophilic allyl ligand and allows for the addition of weaker nucleophiles (i.e., 
aromatic heterocycles) in comparison to $\{\operatorname{TpRe}(\mathrm{CO})(\mathrm{L})\}$. However, this also implies a greater acidity of the conjugate acid from the protonation of $\mathbf{4}$ and $\mathbf{5}$, relative to the [Os] analogue.

If electrophilic addition to the arene complex is successful (Scheme 2.8, Pathway i), then several possible outcomes can occur upon addition of the nucleophile to the reaction mixture (Scheme 2.10).

Scheme 2.10. Potential pathways after adding a nucleophile to an allylic species of 4 or 5.

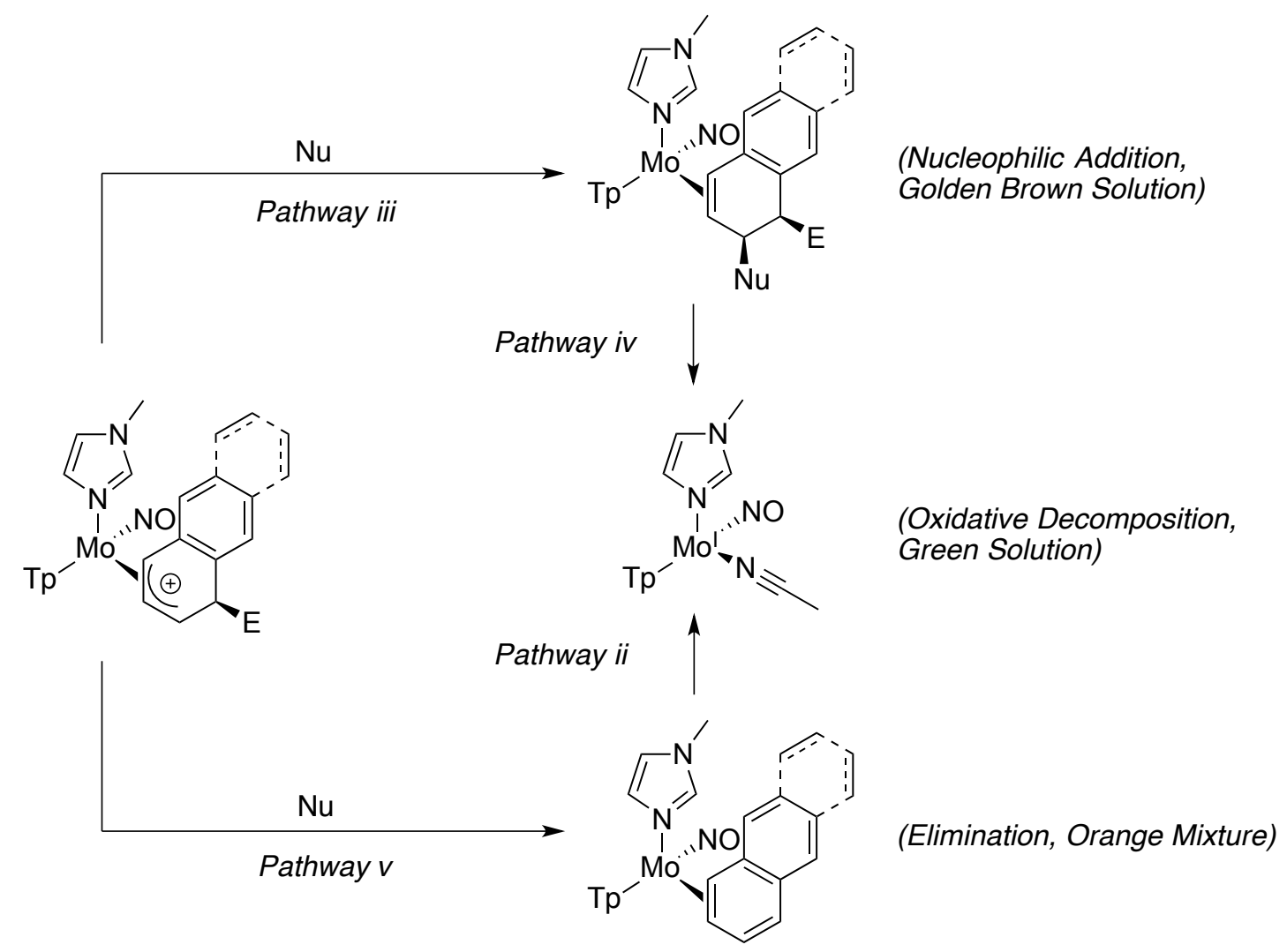

The addition of a nucleophile to this allylic species could yield a 1,2-dihydroarene complex (Scheme 2.10, Pathway iii). Successful formation of 1,2-dihydroarene complexes results in a color change from the blood red of the allyl, to a golden brown 
solution. However, it is also possible that after the successful addition of the nucleophile, the newly formed 1,2-dihydroarene complex is oxidized by the remaining excess of electrophilic reagent in the reaction mixture, generating the $\kappa^{1}$-acetonitrile complex (Scheme 2.10, Pathway iv). Another possibility upon addition of the nucleophilic reagent is that the allylic species formed could revert back to the original arene complex (4 or 5 ) through deprotonation (Scheme 2.10, Pathway v). After deprotonation, oxidative decomposition of $\mathbf{4}$ or $\mathbf{5}$ can occur through Pathway ii described above.

The observation of a single anodic peak around $+0.2 \mathrm{~V}$ with no starting material or $\mathrm{Mo}^{\mathrm{I}}$-acetonitrile complex, gives promise for the success of isolating a clean tandem addition products after workup. Through this method the nucleophiles MTDA, LiDMM, and $N$-methylpyrrole were found to be compatible with HOTf as an electrophile. Additional nucleophiles (i.e., primary amines, thiol salts, triethylsilane as a hydride donor, etc.) were attempted using HOTf as the electrophile; however, none of these produced the desired dihydroarenes under the conditions described here. The above possible pathways make the full analysis of the success or failure of attempted reactions on 4 and 5 difficult. Therefore, although the addition of only one electrophile and three nucleophiles seems limited, it is believed that, given the right conditions, a great breadth of chemical reactivity can be discovered using $\{\mathrm{TpMo}(\mathrm{NO})(\mathrm{MeIm})\}$.

It is also worth addressing the influence of the metal on the stereochemistry of these tandem addition reactions. After protonation of $\mathrm{TpMo}(\mathrm{NO})(\mathrm{MeIm})\left(\eta^{2}-\mathrm{PAH}\right)$, the nucleophiles add to the arenium stereoselectively anti to the metal center. This, in tandem with the stereogenic metal center, gives the potential for forming enantioenriched organic products using this methodology. As of this writing, two issues prevent the further 
development of this chemistry. First, strategies for enantioenrichment or chiral resolution of the $\{\mathrm{TpMo}(\mathrm{NO})(\mathrm{L})\}$ fragment are still being investigated. Therefore, all complexes and all organic compounds are synthesized as racemic mixtures. Second, the relatively low diastereocontrol of the metal-arene coordination (i.e., cdr of $\mathbf{1 0}=5: 1$ ) limits the effectiveness of the stereoselectivity imparted by the metal on the tandem addition chemistry. In contrast to our current understanding of the $\{\mathrm{TpMo}(\mathrm{NO})(\mathrm{L})\}$ system, $\{\mathrm{TpRe}(\mathrm{CO})(\mathrm{L})\}$ and $\left\{\mathrm{TpW}(\mathrm{NO})\left(\mathrm{PMe}_{3}\right)\right\}$ demonstrate potential pathways for controlling these ratios (i.e., sterics or solid state kinetic preference (SICKUS)), and it is our hope that similar strategies may be found for this chemistry. In spite of these limitations, we believe that the $\{\mathrm{TpMo}(\mathrm{NO})(\mathrm{MeIm})\}$ system provides several advantages over these other systems in cost, reactivity, and, most excitingly, recyclability, and it is worth exploring the reactivity of this system. One significant limitation exposed by these tandem addition reactions is the limited scope of the electrophilic additions tolerated by the metal complex. In order to broaden the utility of the $\{\mathrm{TpMo}(\mathrm{NO})(\mathrm{L})\}$ fragment as a dearomatization agent, greater stability under electrophilic conditions is needed. This issue is addressed in the following chapter.

\subsection{Conclusion}

Naphthalene and anthracene form thermally stable $\eta^{2}$-bound complexes with the molybdenum system $\{\mathrm{TpMo}(\mathrm{NO})(\mathrm{MeIm})\}$. Complexation leads to an enhanced reactivity toward protonation to form an arenium cation. This electrophilic species reacts with several common carbon nucleophiles to generate 1,2-dihydronaphthalene and 1,2dihydroanthracene complexes, with the nucleophile adding anti to the metal. Through the use of iodine, a clean oxidative decomplexation is effected, providing the free organic, 
efficiently returning the $\mathrm{Mo}^{\mathrm{I}}$ precursor used to prepare the initial $\eta^{2}$-aromatic complex. Thus, a formal catalytic cycle for the dearomatization of arenes has been demonstrated that is based on a dihapto-coordinated arene complex that does not involve a heavy metal. For the cases where naphthalene and anthracene are converted to tetrahydro- analogues, three new stereogenic centers are created from carbons that were part of the original aromatic rings. 


\subsection{Experimental}

General Methods. NMR spectra were obtained on a 600 or $800 \mathrm{MHz}$ spectrometer. All chemical shifts are reported in ppm, and proton and carbon shifts are referenced to tetramethylsilane (TMS) utilizing residual ${ }^{1} \mathrm{H}$ or ${ }^{13} \mathrm{C}$ signals of the deuterated solvents as an internal standard. Coupling constants $(J)$ are reported in hertz $(\mathrm{Hz})$. Infrared spectra (IR) were recorded as a glaze on a spectrometer fitted with a horizontal attenuated total reflectance (HATR) accessory or on a diamond anvil ATR assembly. Electrochemical experiments were performed under a dinitrogen atmosphere. Cyclic voltammetry data were taken at ambient temperature $\left(\sim 25^{\circ} \mathrm{C}\right)$ at $100 \mathrm{mV} / \mathrm{s}$ in a standard three-electrode cell with a glassy carbon working electrode, $N, N$-dimethylacetamide (DMA) or acetonitrile $\left(\mathrm{CH}_{3} \mathrm{CN}\right)$ solvent (unless otherwise specified), and tetrabutylammonium hexafluorophosphate (TBAH) electrolyte (approximately $0.5 \mathrm{M}$ ). All potentials are reported versus NHE (normal hydrogen electrode) using cobaltocenium hexafluorophosphate $\left(E_{1 / 2}=-0.78 \mathrm{~V}\right)$, ferrocene $\left(E_{1 / 2}=+0.55 \quad \mathrm{~V}\right)$, or decamethylferrocene $\left(E_{1 / 2}=+0.04 \mathrm{~V}\right)$ as an internal standard. The peak-to-peak separation was less than $100 \mathrm{mV}$ for all reversible couples. High-resolution mass spectra were acquired in ESI mode, from samples dissolved in a 3:1 acetonitrile/water solution containing sodium trifluoroacetate (NaTFA). Mass spectra are reported as $\mathrm{M}+$ for monocationic complexes or as $\left[\mathrm{M}+\mathrm{H}^{+}\right]$or $\left[\mathrm{M}+\mathrm{Na}^{+}\right]$for neutral complexes, using $[\mathrm{Na}(\mathrm{NaTFA}) \mathrm{x}]+$ clusters as an internal standard. In all cases, observed isotopic envelopes were consistent with the molecular composition reported. For organic products, the monoisotopic ion is reported; for complexes, the major peaks in the isotopic envelope are reported. Unless otherwise noted, all synthetic reactions were performed in a glovebox 
under a dry nitrogen atmosphere. Deuterated solvents were used as received. Pyrazole $(\mathrm{Pz})$ protons of the (trispyrazolyl) borate (Tp) ligand were uniquely assigned (e.g., "Pz3B") using a combination of two-dimensional NMR data and methylimidazole-proton NOE interactions (see Figure 2.5 below). When unambiguous assignments were not possible, Tp protons were labeled as "Pz3/5 or Pz4". All $J$ values for Pz protons are $2( \pm 0.2) \mathrm{Hz}$. Due to their sensitivity to air, elemental analysis attempts for complexes $\mathbf{6}-12$ were unsuccessful. Column chromatography was employed as the main method of purification for the isolation of complexes $6-12$; however, basic extraction was found to provide equally pure complexes along with comparable yields; this was especially useful in smaller scale $(<300 \mathrm{mg})$ reactions. Compounds $\mathbf{1}, \mathbf{4}$, and $\mathbf{5}$ were previously reported; however, an alternate synthesis avoiding the use of $\mathrm{Na} / \mathrm{Hg}$ amalgam for $\mathbf{4}$ and $\mathbf{5}$ is reported below. ${ }^{15}$

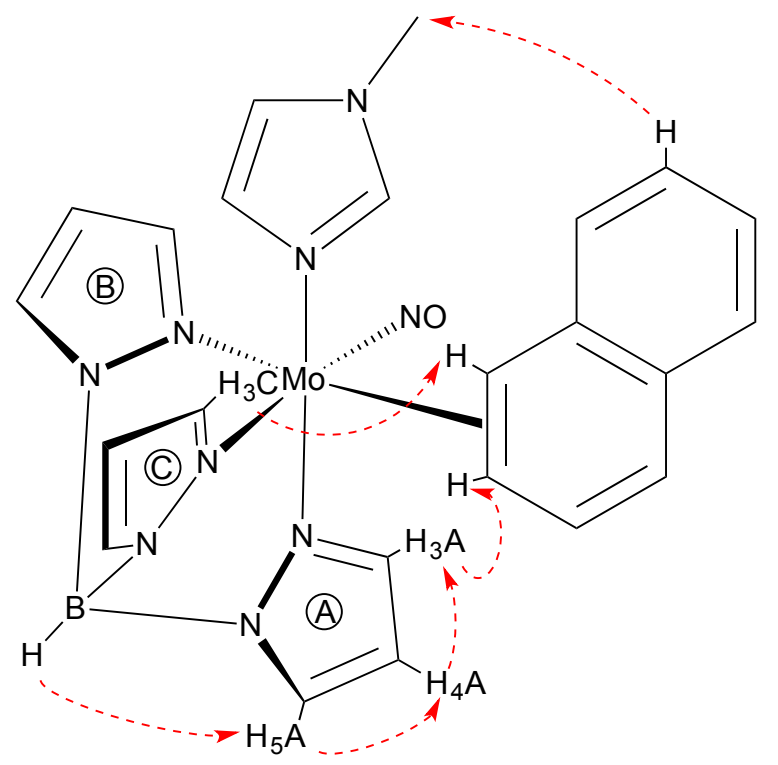

Figure 2.5. Analysis using NOE (red arrows). 


\section{Synthesis of TpMo(NO)(CO)(MeIm) (2).}

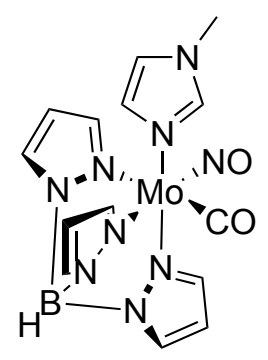

Under an air atmosphere, 1 (80 g, $0.203 \mathrm{~mol}), N, N$-dimethylformamide $(400 \mathrm{~mL})$, and MeIm $(60 \mathrm{~mL}, 0.73 \mathrm{~mol})$ were added to a 3 neck 3 L round bottom flask charged with a stir egg, reflux condenser, thermometer and septum. The resulting orange mixture was heated at reflux $\left(\sim 154{ }^{\circ} \mathrm{C}\right)$ for $1 \mathrm{~h}$ then cooled to $\mathrm{RT}$ for $15 \mathrm{~min}$. The reaction mixture was then added to stirring $\mathrm{H}_{2} \mathrm{O}(1 \mathrm{~L})$ and the resulting green mixture was stirred at RT. After $18 \mathrm{~h}$, the blue precipitate was isolated on a $350 \mathrm{~mL}$ medium porosity fritted disc, washed with $\mathrm{H}_{2} \mathrm{O}(250 \mathrm{~mL}), 20 \% \mathrm{MeOH} \mathrm{Et}_{2} \mathrm{O}(2 \times 250 \mathrm{~mL})$, and $\mathrm{Et}_{2} \mathrm{O}(3 \times 250 \mathrm{~mL})$, and desiccated overnight to yield $2(68.2 \mathrm{~g}, 75 \%)$. CV (DMAc) $E_{\mathrm{p}, \mathrm{a}}=+0.22 \mathrm{~V}$ (NHE). IR: $v(\mathrm{BH})=2480 \mathrm{~cm}^{-1}, v(\mathrm{CO})=1867 \mathrm{~cm}^{-1}, v(\mathrm{NO})=1577 \mathrm{~cm}^{-1} \cdot{ }^{1} \mathrm{H}$ NMR $\left(\mathrm{CDCl}_{3}, \delta\right): 7.73$ (d, Pz3/5), 7.72 (1H, d, Pz3/5), 7.67 (2H, m, Pz3/5), 7.56 (1H, s, MeIm), $7.42(1 \mathrm{H}, \mathrm{d}$, Pz3/5), 7.31 (1H, d, Pz3/5), 6.80 (1H, t, $J=1.4, \mathrm{MeIm}), 6.64(1 \mathrm{H}, \mathrm{t}, J=1.4, \mathrm{MeIm}), 6.19$ (2H, m, Pz4), 6.11 (1H, t, Pz4), 3.68 (3H, s, MeIm).

\section{Synthesis of TpMo(NO)(MeIm)(I) (3).}

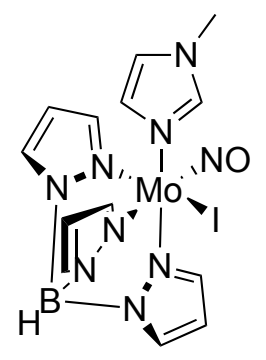


Under an air atmosphere, $2(67 \mathrm{~g}, 0.122 \mathrm{~mol})$ and DCM $(250 \mathrm{~mL})$ were added to a $2 \mathrm{~L}$ Erlenmeyer flask charged with a stir bar and the resulting mixture was stirred at RT until homogeneous. To a $500 \mathrm{~mL}$ Erlenmeyer flask charged with a stir bar were added $\mathrm{I}_{2}$ $(18.83 \mathrm{~g}, 0.074 \mathrm{~mol})$ and $\mathrm{Et}_{2} \mathrm{O}(200 \mathrm{~mL})$, and the mixture was stirred $15 \mathrm{~min}$ at $\mathrm{RT}$ until homogeneous. (CAUTION: Evolution of $\mathrm{CO}$ ) With the hood sash down, the $\mathrm{I}_{2}$ solution was slowly added to the solution of $\mathbf{2}$ and the resulting green solution was left stirring at RT. After $15 \mathrm{~min}, \mathrm{Et}_{2} \mathrm{O}(1.5 \mathrm{~L})$ was added to the reaction mixture creating a green precipitation. The precipitate was isolated on a $600 \mathrm{~mL}$ fine porosity fritted disc, washed with $\mathrm{Et}_{2} \mathrm{O}(4 \times 500 \mathrm{~mL})$, and desiccated overnight to yield 3 (78.1 g, 95\%). CV (DMAc) $E_{\mathrm{p}, \mathrm{a}}=+0.65 \mathrm{~V}(\mathrm{NHE}), \mathrm{E}_{1 / 2}=-1.47 \mathrm{~V}(\mathrm{NHE}) . \mathrm{IR}: v(\mathrm{BH})=2477 \mathrm{~cm}^{-1}, v(\mathrm{NO})=1602 \mathrm{~cm}^{-1}$.

\section{Synthesis of TpMo(MeIm)(NO)(3,4- $\eta^{2}$-naphthalene) (4). ${ }^{15}$}

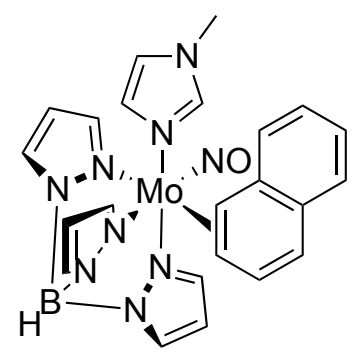

To a $500 \mathrm{~mL}$ round bottom flask with stir bar was added 3 (12.0 g, $23.9 \mathrm{mmol})$, naphthalene (40.0 g, $312 \mathrm{mmol})$, THF $(240 \mathrm{~mL})$ and sodium dispersion $(5.00 \mathrm{~g}, 75.0$ mmol). This was stirred at room temperature for $18 \mathrm{~h}$ at which point the mixture was filtered through a $150 \mathrm{~mL}$ fine porosity fritted disc with $2 \mathrm{~cm}$ of celite. $\mathrm{Et}_{2} \mathrm{O}(600 \mathrm{~mL})$ was added to the filtrate that was then filtered through a $600 \mathrm{~mL}$ fine porosity fritted disc with $4 \mathrm{~cm} \mathrm{SiO} \mathrm{Si}_{2}$ and an orange band was collected with 2:1 $\mathrm{Et}_{2} \mathrm{O}$ :THF $(700 \mathrm{~mL})$. The orange solution was then evaporated in vacuo to $100 \mathrm{~mL}$, pentane was added (900 mL) 
and a yellow precipitate formed. This yellow precipitate was collected on a $60 \mathrm{~mL}$ fine porosity fritted disc, washed with pentane $(3 \times 50 \mathrm{~mL})$, and desiccated $1 \mathrm{~h}$ to yield 4 (4.42 g, $34 \%)$.

\section{Synthesis of TpMo(MeIm)(NO)(3,4- $\eta^{2}$-anthracene) (5). ${ }^{15}$}

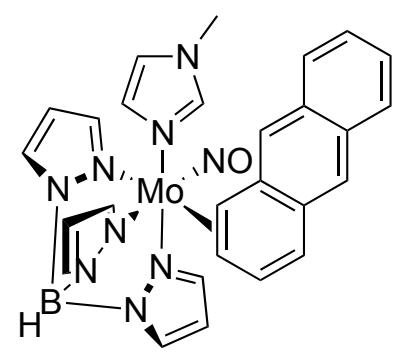

In a $50 \mathrm{~mL}$ round bottom flask with stir bar was added sodium dispersion $(1.00 \mathrm{~g}, 15.0$ $\mathrm{mmol})$ and hexanes $(50 \mathrm{~mL})$. This was stirred at room temperature for $30 \mathrm{~min}$. The organic layer was decanted, hexanes $(50 \mathrm{~mL})$ was added to the flask, and the mixture was stirred at room temperature $1 \mathrm{~h}$. The organic layer was decanted, THF $(50 \mathrm{~mL})$ was added and the organic layer was decanted off. THF $(10 \mathrm{~mL})$ was added to the flask and the mixture was transferred to a $100 \mathrm{~mL}$ round bottom flask charged with stir bar, $3(1.00 \mathrm{~g}$, $2.00 \mathrm{mmol})$, THF $(12 \mathrm{~mL})$, and 2,5-dimethylfuran $(2.00 \mathrm{~mL}, 18.5 \mathrm{mmol})$. This mixture was stirred at room temperature for $2 \mathrm{~h}$ and then filtered through a $15 \mathrm{~mL}$ medium porosity fritted disc with $2 \mathrm{~cm}$ celite and washed with THF $(2 \times 4 \mathrm{~mL})$. This solution was then transferred to a $100 \mathrm{~mL}$ round bottom flask charged with stir bar and anthracene (3.56 g, $20.0 \mathrm{mmol}$ ). This brown mixture was stirred at room temperature $48 \mathrm{~h}$ yielding a red mixture. $\mathrm{Et}_{2} \mathrm{O}(15 \mathrm{~mL})$ was added to the flask and filtered through a $30 \mathrm{~mL}$ medium porosity fritted disc with $2 \mathrm{~cm} \mathrm{SiO}_{2}$. A red filtrate was eluted with 1:1 $\mathrm{Et}_{2} \mathrm{O}: \mathrm{THF}(25 \mathrm{~mL})$ and $\mathrm{Et}_{2} \mathrm{O}(10 \mathrm{~mL})$. This was evaporated to dryness in vacuo. The red solid was then 
washed with $\mathrm{CH}_{3} \mathrm{CN}(20 \mathrm{~mL})$ and the free anthracene was removed on a $30 \mathrm{~mL}$ fine porosity fritted disc. This red solution was then evaporated in vacuo, dissolved in DCM $(6 \mathrm{~mL})$, and precipitated in hexanes $(50 \mathrm{~mL})$. The red solid was then collected on a 30 $\mathrm{mL}$ fine porosity fritted disc, washed with hexanes $(2 \times 10 \mathrm{~mL})$ and desiccated $1 \mathrm{~h}$ to yield $5(520 \mathrm{mg}, 44 \%)$

\section{TpMo(MeIm)(NO)(3,4- $\eta^{2}$-(methyl-2-(1,2-dihydronaphthalen-2-yl)-2-}

methylpropanoate)) (6).

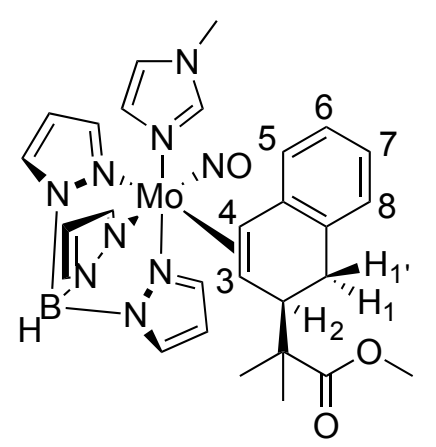

To a $250 \mathrm{~mL}$ round bottom flask was added $4(5.00 \mathrm{~g}, 9.12 \mathrm{mmol})$ and $100 \mathrm{~mL} \mathrm{CH} \mathrm{CHN}_{3}$. This orange mixture was cooled while stirring for $30 \mathrm{~min}$ at $-35^{\circ} \mathrm{C}$. To this orange mixture was added $1 \mathrm{M} \mathrm{HOTf} / \mathrm{CH}_{3} \mathrm{CN}(35 \mathrm{~mL}, 35.0 \mathrm{mmol})$ cooled to $-35^{\circ} \mathrm{C}$. This red solution was left stirring $30 \mathrm{~min}$ at $-35^{\circ} \mathrm{C}$. This red mixture was stirred at room temperature 15 min yielding a red solution that was then cooled to $-35{ }^{\circ} \mathrm{C}$. A $-35^{\circ} \mathrm{C}$ solution of MTDA (10 mL, $49.2 \mathrm{mmol})$ was added and the yellow solution was left standing at $-35^{\circ} \mathrm{C}$ for $15 \mathrm{~min}$. Next, a $-35{ }^{\circ} \mathrm{C}$ solution of triethylamine $(10 \mathrm{~mL}, 71.7$ mmol) was added and the golden solution was warmed to room temperature. The solution was then evaporated in vacuo to dryness, picked up in 1:1 $\mathrm{Et}_{2} \mathrm{O}$ :benzene $(50 \mathrm{~mL})$ and chromatographed through a $300 \mathrm{~mL}$ medium porosity fritted disc with $4 \mathrm{~cm}$ of silica. The 
product was eluted with 1:1 $\mathrm{Et}_{2} \mathrm{O}$ :benzene $(1 \mathrm{~L})$. The yellow filtrate was then evaporated in vacuo, dissolved in DCM $(25 \mathrm{~mL})$ and precipitated in stirring pentane $(250 \mathrm{~mL})$. The precipitate was then collected on a $150 \mathrm{~mL}$ fine porosity fritted disc, washed with pentane (3 x $100 \mathrm{~mL})$, and dried for $1 \mathrm{~h}$ yielding the yellow precipitate $6(3.62 \mathrm{~g}, 61 \%)$.

\section{Basic Extraction Workup of 6}

To a test tube was added $4(300 \mathrm{mg}, 0.546 \mathrm{mmol})$ and $6 \mathrm{~mL} \mathrm{CH}_{3} \mathrm{CN}$. This orange mixture was cooled while stirring for $15 \mathrm{~min}$ at $-35^{\circ} \mathrm{C}$. To this orange mixture was added $1 \mathrm{M}$ HOTf $/ \mathrm{CH}_{3} \mathrm{CN}(2.1 \mathrm{~mL}, 2.10 \mathrm{mmol})$ cooled to $-35^{\circ} \mathrm{C}$. This red solution was left stirring $15 \mathrm{~min}$ at $-35^{\circ} \mathrm{C}$. A $-35^{\circ} \mathrm{C}$ solution of MTDA $(0.6 \mathrm{~mL}, 2.95 \mathrm{mmol})$ was added and the yellow solution was left stirring at $-35{ }^{\circ} \mathrm{C}$ for $20 \mathrm{~min}$. Next, a $-35{ }^{\circ} \mathrm{C}$ solution of triethylamine $(0.6 \mathrm{~mL}, 4.32 \mathrm{mmol})$ was added and the golden solution was warmed to room temperature. The solution was then evaporated in vacuo to dryness, picked up in $\mathrm{CHCl}_{3}(10 \mathrm{~mL})$, and washed with sat. aq. $\mathrm{NaHCO}_{3}(3 \times 10 \mathrm{~mL})$. The organic layer was then dried over $\mathrm{MgSO}_{4}$ and the drying agent was filtered off. The yellow filtrate was then evaporated in vacuo, dissolved in DCM $(1 \mathrm{~mL})$ and precipitated in stirring pentane $(50$ $\mathrm{mL}$ ). The precipitate was then collected on a $15 \mathrm{~mL}$ fine porosity fritted disc, washed with pentane $(3 \times 30 \mathrm{~mL})$, and dried yielding the yellow precipitate $6(218 \mathrm{mg}, 62 \%)$. $\mathrm{CV}(\mathrm{DMAc}) E_{\mathrm{p}, \mathrm{a}}=+0.16 \mathrm{~V}(\mathrm{NHE}) .{ }^{1} \mathrm{H}$ NMR (d -Acetone, $\left.\delta\right): 8.00$ (1H, d, Pz5B), 7.93 (1H, d, Pz5C), 7.92 (1H, d, Pz5A), 7.82 (1H, d, Pz3C), 7.74 (1H, d, Pz3A), 7.16 (1H, d, Pz3B), $7.14(1 \mathrm{H}, \mathrm{t}, J=1.7, \mathrm{MeIm}), 6.98(1 \mathrm{H}, \mathrm{bt}, J=1.5, \mathrm{MeIm}), 6.92(1 \mathrm{H}, \mathrm{d}, J=7.3$, H8), 6.78 (1H, tt, $J=7.3,1.0, \mathrm{H6}), 6.40(1 \mathrm{H}, \mathrm{t}, J=1.4, \mathrm{MeIm}), 6.39$ (1H, t, Pz4A), 6.36 (1H, t, Pz4C), 6.08 (1H, t, Pz4B), 5.88 (1H, dd, $J=7.3,1.3, \mathrm{H} 5), 3.74$ (3H, s, MeIm), 
$3.52\left(1 \mathrm{H}, \mathrm{dd}, J=16.7,8.9, \mathrm{H} 1^{\prime}\right), 3.32(3 \mathrm{H}, \mathrm{s}, \mathrm{OMe}), 3.23(1 \mathrm{H}, \mathrm{d}, J=8.1, \mathrm{H} 2), 3.13(1 \mathrm{H}$, $\mathrm{d}, J=9.5, \mathrm{H} 4), 2.55(1 \mathrm{H}, \mathrm{d}, J=16.7, \mathrm{H} 1), 2.06(1 \mathrm{H}, \mathrm{dt}, J=9.5,1.8, \mathrm{H} 3), 1.11(3 \mathrm{H}, \mathrm{s}$, Me), 0.95 (3H, s, Me). ${ }^{13} \mathrm{C}$ NMR (d $\mathrm{d}^{6}$-Acetone, $\left.\delta\right): 178.9$ (CO), 144.7, 142.7 (Pz5B), 141.8 (Pz3B), 141.7 (Pz3C), 139.1 (MeIm), 137.2 (Pz5C), 137.0 (Pz5A), 135.4 (Pz3A), 133.8 , 129.0, 125.5 (C5), 124.1 (C6), 123.1 (C7), 122.1 (MeIm), 106.5 (Pz4), 106.3 (Pz4), 106.0 (Pz4), 69.0 (C4), 51.2 (C3), 51.0 (OMe), 43.9 (C2), 34.3 (NMe), 30.1 (C1), $23.4(\mathrm{Me})$, 23.0 (Me). HRMS: $\quad \mathrm{C}_{28} \mathrm{H}_{34} \mathrm{~N}_{9} \mathrm{O}_{3} \mathrm{BMo}+\mathrm{Na}^{+}$obsd (\%), calcd (\%), ppm: 670.1867 (46), 670.1843 (52), 3.6; 672.1854 (48), 672.1840 (49), 2.0; 673.1843 (77), 673.1839 (81), 0.5; 674.1854 (83), 674.1835 (87), 2.8; 675.1850 (77), 675.1847 (76), 0.4; 676.1849 (100), 676.1834 (100), 2.2; 677.1870 (36), 677.1862 (38), 1.2; 678.1883 (32), 678.1854 (39), 4.3. Minor Isomer: ${ }^{1} \mathrm{H}$ NMR $\left(\mathrm{d}^{6}\right.$-Acetone, $\left.\delta\right): 8.20(1 \mathrm{H}, \mathrm{d}, \mathrm{Pz} 3 / 5), 7.88(1 \mathrm{H}, \mathrm{d}, \mathrm{Pz} 3 / 5)$, $7.86(1 \mathrm{H}, \mathrm{d}, \mathrm{Pz3} / 5), 6.75$ (2H, m, naphthalene), 6.30 (1H, t, Pz4), 3.57 (1H, dd, $J=18.8$, 8.6, H1a), 2.51(1H, d, $J=18.8, \mathrm{H} 1 \mathrm{~b}), 1.86(1 \mathrm{H}, \mathrm{dt}, J=9.8,1.8, \mathrm{H} 3)$.

\section{TpMo(MeIm)(NO)(3,4- $\eta^{2}$-(methyl-(R)-2-(1,2-dihydroanthracen-2-yl)-2-}

\section{methylpropanoate)) (7).}

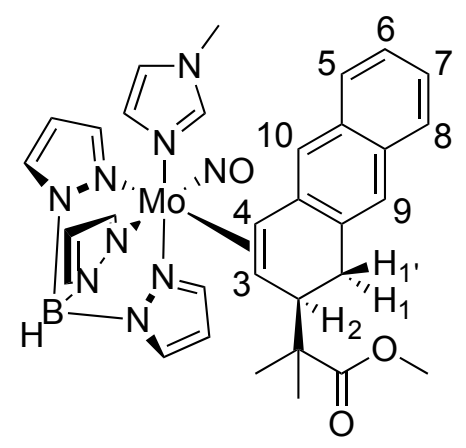

To a test tube was added $5(200 \mathrm{mg}, 0.333 \mathrm{mmol})$ and $4 \mathrm{~mL} \mathrm{CH}_{3} \mathrm{CN}$. This red solution was cooled for $15 \mathrm{~min}$ at $-35^{\circ} \mathrm{C}$. To this solution was added $1 \mathrm{M} \mathrm{HOTf} / \mathrm{CH}_{3} \mathrm{CN}(1.4 \mathrm{~mL}$, 
$1.40 \mathrm{mmol}$ ) cooled to $-35^{\circ} \mathrm{C}$. This dark red solution was left standing at $-35^{\circ} \mathrm{C}$ for 15 min at which point $\mathrm{a}-35^{\circ} \mathrm{C}$ solution of MTDA $(0.4 \mathrm{~mL}, 1.96 \mathrm{mmol})$ was added and left standing at $-35^{\circ} \mathrm{C}$ for $2 \mathrm{~h}$. Next, a $-35^{\circ} \mathrm{C}$ solution of triethylamine $(0.4 \mathrm{~mL}, 2.87 \mathrm{mmol})$ was added and the orange solution was warmed to room temperature. The solution was then evaporated in vacuo to dryness, picked up in 1:1 benzene:THF ( $2 \mathrm{~mL})$, and chromatographed through a $15 \mathrm{~mL}$ medium porosity fritted disc with $2 \mathrm{~cm}$ silica. The product was eluted with 1:1 $\mathrm{Et}_{2} \mathrm{O}$ :benzene $(20 \mathrm{~mL})$. The orange filtrate was then evaporated in vacuo, dissolved in DCM $(1 \mathrm{~mL})$ and precipitated in stirring pentane $(25$ $\mathrm{mL})$. The precipitate was then collected on a $15 \mathrm{~mL}$ fine porosity fritted disc and dried for $15 \mathrm{~min}$ yielding the yellow precipitate 7 (89 $\mathrm{mg}, 41 \%$ ). CV (DMAc) $E_{\mathrm{p}, \mathrm{a}}=+0.18 \mathrm{~V}$ (NHE). IR: $v(\mathrm{BH})=2480 \mathrm{~cm}^{-1}, v(\mathrm{CO})=1720 \mathrm{~cm}^{-1}, v(\mathrm{NO})=1562 \mathrm{~cm}^{-1} .{ }^{1} \mathrm{H}$ NMR $\left(\mathrm{CDCl}_{3}, \delta\right): 8.07$ (1H, d, Pz3C), 7.77 (1H, d, Pz5C), $7.71(1 \mathrm{H}, \mathrm{d}, \mathrm{Pz} 5 \mathrm{~A}), 7.69(1 \mathrm{H}, \mathrm{d}, J=$ 8.0, H8), 7.67 (1H, d, Pz3A), 7.58 (1H, d, Pz3/5B), 7.51 (1H, s, H9), 7.38 (1H, d, J=8.2, H5), 7.23 (1H, m, H6), 7.19 (1H, m, H7), $7.06(1 \mathrm{H}, \mathrm{d}, \mathrm{Pz3} / 5 \mathrm{~B}), 6.79(1 \mathrm{H}, \mathrm{t}, J=1.5$, MeIm), $6.63(1 \mathrm{H}, \mathrm{s}, \mathrm{H} 10), 6.34(1 \mathrm{H}$, buried s, MeIm), $6.33(1 \mathrm{H}, \mathrm{t}, \mathrm{Pz} 4 \mathrm{C}), 6.29(1 \mathrm{H}, \mathrm{t}$, Pz4A), 6.18 (1H, s, MeIm), 6.01(1H, t, Pz4B), 3.70(1H, dd, $J=17.4,7.9, \mathrm{H} 1$ ') 3.35 (3H, s, OMe), 3.34 (3H, s, MeIm), $3.31(1 \mathrm{H}, \mathrm{d}, J=7.1, \mathrm{H} 2), 3.26(1 \mathrm{H}, \mathrm{d}, J=9.3, \mathrm{H} 4), 2.89$ $(1 \mathrm{H}, \mathrm{d}, J=17.4, \mathrm{H} 1), 2.25(1 \mathrm{H}, \mathrm{dt}, J=9.6,1.7, \mathrm{H} 3), 1.18(3 \mathrm{H}, \mathrm{s}, \mathrm{Me}), 1.03(3 \mathrm{H}, \mathrm{s}, \mathrm{Me})$. ${ }^{13} \mathrm{C} \mathrm{NMR}\left(\mathrm{CDCl}_{3}, \delta\right): 179.3$ (CO), 142.9, 142.5, 141.3, 140.8, 138.3, 136.3, 136.1, 134.4, $143.3,132.0,128.8,127.4,125.7,125.4,124.2,123.2,120.6,120.5,105.8,105.6,105.4$, 100.2, 69.6, 66.0, 65.1, 51.3, 50.2, 43.2, 34.4, 22.8. HRMS: $\left[\mathrm{M}+\mathrm{Na}^{+}\right]=$ $\left[\mathrm{C}_{32} \mathrm{H}_{36} \mathrm{~N}_{9} \mathrm{O}_{3} \mathrm{BMo}+\mathrm{Na}^{+}\right]$obsd (\%), calcd (\%), ppm: 720.2013 (47), 720.2000 (51), 1.8; 722.1990 (40), 722.1998 (48), -1.1; 723.2007 (73), 723.1997 (80), 1.4; 724.1985 (83), 
724.1993 (87), -1.1; 725.1990 (77), 725.2005 (77), -2.0; 726.1980 (100), 726.1992 (100), -1.7; 727.1992 (36), 727.2019 (41), -3.8; 728.2007 (34), 728.2012 (40), -0.7. Minor Isomer: ${ }^{1} \mathrm{H}$ NMR $\left(\mathrm{CDCl}_{3}, \delta\right): 8.28(1 \mathrm{H}, \mathrm{d}, \mathrm{Pz} 3 / 5), 7.79(1 \mathrm{H}, \mathrm{d}, \mathrm{Pz} 3 / 5), 7.78(1 \mathrm{H}, \mathrm{d}$, Pz3/5), 6.11 (1H, t, Pz4), 6.03 (2H, t, Pz4), 2.03 (1H, dt, $J=10.0,1.7, \mathrm{H} 3)$.

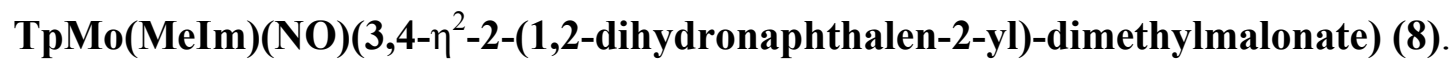

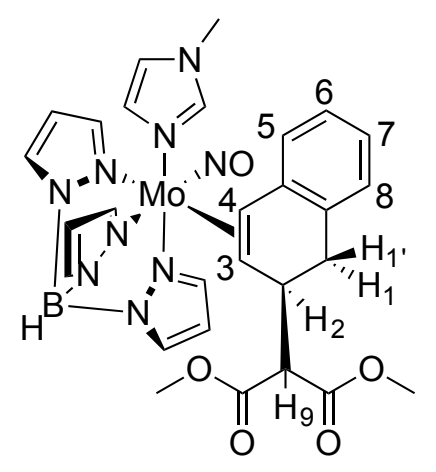

To a 4 dram vial was added $4(200 \mathrm{mg}, 0.364 \mathrm{mmol})$ and $4 \mathrm{~mL} \mathrm{CH}_{3} \mathrm{CN}$. This orange mixture was cooled with stirring for $15 \mathrm{~min}$ at $-35^{\circ} \mathrm{C}$. To this orange mixture was added a $1 \mathrm{~mL}(1 \mathrm{mmol})$ of a $1 \mathrm{M} \mathrm{HOTf} / \mathrm{CH}_{3} \mathrm{CN}$ cooled to $-35^{\circ} \mathrm{C}$. This red solution was left standing $15 \mathrm{~min}$ at $-35^{\circ} \mathrm{C}$. Lithium dimethylmalonate $(270 \mathrm{mg}, 1.94 \mathrm{mmol})$ was added and the yellow solution was left standing at $-35^{\circ} \mathrm{C}$ for $1 \mathrm{~h}$. Next, a $-35^{\circ} \mathrm{C}$ solution of triethylamine $(0.4 \mathrm{~mL}, 2.86 \mathrm{mmol})$ was added and the golden solution was warmed to room temperature. The solution was then evaporated in vacuo to dryness, picked up in benzene $(2 \mathrm{~mL})$ and chromatographed through a $15 \mathrm{~mL}$ medium porosity fritted disc with $2 \mathrm{~cm}$ silica. The product was eluted with 1:1 $\mathrm{Et}_{2} \mathrm{O}$ :benzene $(40 \mathrm{~mL})$. The yellow filtrate was then evaporated in vacuo, dissolved in DCM $(2 \mathrm{~mL})$ and precipitated in stirring pentane $(40 \mathrm{~mL})$. The precipitate was then collected on a $15 \mathrm{~mL}$ medium porosity fritted disc, washed with pentane $(3 \times 10 \mathrm{~mL})$, and dried for 15 min yielding the yellow 
precipitate $8(165 \mathrm{mg}, 66 \%)$. CV (DMAc) $E_{\mathrm{p}, \mathrm{a}}=+0.23 \mathrm{~V}(\mathrm{NHE}) . \mathrm{IR}: v(\mathrm{BH})=2484 \mathrm{~cm}^{-1}$, $v(\mathrm{CO})=1751 \& 1732 \mathrm{~cm}^{-1}, v(\mathrm{NO})=1574 \mathrm{~cm}^{-1} .{ }^{1} \mathrm{H}$ NMR $\left(\mathrm{d}^{6}\right.$-Acetone, $\left.\delta\right): 7.93(2 \mathrm{H}, \mathrm{d}$, Pz3A \& C), 7.92 (1H, d, Pz5C), 7.90 (1H, d, Pz5A), 7.76 (1H, d, Pz3B), 7.72 (1H, d, Pz5B), $7.16(1 \mathrm{H}, \mathrm{t}, J=1.7, \mathrm{MeIm}), 7.04(1 \mathrm{H}, \mathrm{bt}, J=1.8, \mathrm{MeIm}), 6.92(1 \mathrm{H}, \mathrm{d}, J=7.6$, H8), $6.87(1 \mathrm{H}, \mathrm{tt}, J=7.5,1.2, \mathrm{H} 6), 6.78(1 \mathrm{H}, \mathrm{td}, J=7.4,1.3, \mathrm{H} 7), 6.42(1 \mathrm{H}, \mathrm{t}, J=1.3$, MeIm), 6.40 (1H, t, Pz4A), 6.35 (1H, t, Pz4C), 6.09 (1H, t, Pz4B), 5.97 (1H, dd, J = 7.4, 1.4, H5), 3.79 (3H, s, MeIm), 3.61 (3H, s, DMM), 3.56 (1H, dd, $\left.J=16.3,6.0, \mathrm{H} 1^{\prime}\right), 3.46$ (1H, m, H2), 3.42 (1H, s, H9), $3.36(3 \mathrm{H}, \mathrm{s}, \mathrm{DMM}), 3.05(1 \mathrm{H}, \mathrm{d}, J=9.5, \mathrm{H} 4), 2.50(1 \mathrm{H}, \mathrm{d}$, $J=16.3, \mathrm{H} 1), 2.10(1 \mathrm{H}, \mathrm{dt}, J=9.5,2.3, \mathrm{H} 3) .{ }^{13} \mathrm{C} \mathrm{NMR}\left(\mathrm{CDCl}_{3}, \delta\right): 170.1(\mathrm{CO}), 170.0$ (CO), 144.1, 143.3 (Pz5C), 141.9 (MeIm), 141.4 (Pz5B), 139.1 (MeIm), 137.4 (Pz3A \& Pz3C), 136.9 (Pz5A), 135.4 (Pz3B), 131.4, 129.4 (C8), 129.0 (MeIm), 125.8 (C5), 124.9 (C6), 123.2 (C7), 122.2 (MeIm), 106.5 (Pz4A \& Pz4C), 106.1 (Pz4B), 67.3 (2C's C4 and C3), 59.8 (C9), 52.1 (OMe), 51.8 (OMe), 38.2 (C9), 34.8 (NMe), 34.2, 32.8 (C1). HRMS: $\left[\mathrm{M}^{+}\right]=\left[\mathrm{C}_{28} \mathrm{H}_{32} \mathrm{~N}_{9} \mathrm{O}_{5} \mathrm{BMo}^{+}\right]$obsd (\%), calcd (\%), ppm: $677.1682(48), 677.1687$ (52), -0.8; 679.1673 (44), 679.1685 (49), -1.7; 680.1685 (77), 680.1684 (80), 0.2; 681.1668 (86), 681.1679 (87), -1.6; 682.1681 (79), 682.1691 (76), -1.5; 683.1668 (100), $683.1679(100),-1.6 ; 684.1691(38), 684.1706(38),-2.3 ; 685.1672(41), 685.1698(40),-$ 3.9. Minor Isomer ${ }^{1} \mathrm{H}$ NMR ( $\mathrm{d}^{6}$-Acetone, $\left.\delta\right): 8.13(1 \mathrm{H}, \mathrm{d}, \mathrm{Pz3} / 5), 7.85(1 \mathrm{H}, \mathrm{d}, \mathrm{Pz} 3 / 5)$, 7.62 (1H, d, Pz3/5), 6.30 (1H, t, Pz4), 6.06 (1H, t, Pz4), 1.84 (1H, dt, J=9.7, 1.6, H3). 
TpMo(MeIm)(NO)(3,4- $\eta^{2}-(\mathrm{R})-$ dimethyl-2-(1,2-dihydroanthracen-2-yl)malonate) (9).

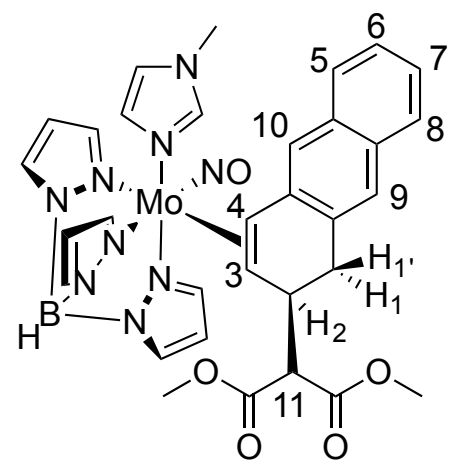

To a test tube was added $5(200 \mathrm{mg}, 0.333 \mathrm{mmol})$ and $4 \mathrm{~mL} \mathrm{CH}_{3} \mathrm{CN}$. This red solution was cooled for $15 \mathrm{~min}$ at $-35^{\circ} \mathrm{C}$. To this solution was added $1 \mathrm{M} \mathrm{HOTf} / \mathrm{CH}_{3} \mathrm{CN}(1.4 \mathrm{~mL}$, $1.40 \mathrm{mmol}$ ) cooled to $-35^{\circ} \mathrm{C}$. This dark red solution was left standing at $-35^{\circ} \mathrm{C}$ for 15 min at which point LiDMM (270 $\mathrm{mg}, 1.97 \mathrm{mmol})$ was added and left standing at $-35^{\circ} \mathrm{C}$ for $15 \mathrm{~min}$. Next, a $-35^{\circ} \mathrm{C}$ solution of triethylamine $(0.4 \mathrm{~mL}, 2.87 \mathrm{mmol})$ was added and the orange solution was warmed to room temperature. The solution was then evaporated in vacuo to dryness and the resulting oil was dissolved in $\mathrm{CHCl}_{3}$. This solution was then washed with $\mathrm{NaHCO}_{3}$ (sat. aq., $10 \mathrm{~mL}$ ), dried over $\mathrm{MgSO}_{4}$, and filtered through a $15 \mathrm{~mL}$ medium porosity fritted disc. The filtrate was then evaporated in vacuo and the resulting oil was dissolved in DCM $(1 \mathrm{~mL})$. This solution was added to stirred pentane $(50 \mathrm{~mL})$ yielding a yellow precipitate that was then isolated on a $15 \mathrm{~mL}$ fine porosity fritted disc. This precipitate was then added to acetone $(2 \mathrm{~mL})$ and the resulting mixture was stirred for $15 \mathrm{~min}$. This precipitate was then isolated on a $15 \mathrm{~mL}$ fine porosity fritted disc, washed with acetone $(2 \times 2 \mathrm{~mL})$, and desiccated for 15 min to yield the yellow solid 9 $(104 \mathrm{mg}, 48 \%) . \mathrm{CV}(\mathrm{DMAc}) E_{\mathrm{p}, \mathrm{a}}=+0.25 \mathrm{~V}(\mathrm{NHE}) . \mathrm{IR}: v(\mathrm{BH})=2453 \mathrm{~cm}^{-1}, v(\mathrm{CO})=$ $1739 \mathrm{~cm}^{-1}, 1720 \mathrm{~cm}^{-1} v(\mathrm{NO})=1562 \mathrm{~cm}^{-1} .{ }^{1} \mathrm{H}$ NMR $\left(\mathrm{CDCl}_{3}, \delta\right): 7.99(1 \mathrm{H}, \mathrm{d}, \mathrm{Pz} 3 \mathrm{~A}), 7.75$ (1H, d, Pz5C), 7.73 (1H, d, Pz5A), 7.69 (1H, d, J=8.1, H8), 7.58 (1H, d, Pz5B), 7.57 
(1H, d, Pz3C), 7.52 (1H, s, H9), 7.43 (1H, d, $J=8.3, \mathrm{H} 5), 7.27$ (1H, t, $J=7.4, \mathrm{H} 6), 7.22$ $(1 \mathrm{H}, \mathrm{t}, J=7.4, \mathrm{H} 7), 7.06$ (1H, t, Pz3B), 6.34 (1H, t, Pz4A), $6.27(1 \mathrm{H}, \mathrm{s}, \mathrm{H} 10), 6.25$ (1H, t, Pz4C), 6.02 (1H, t, Pz4B), 3.75 (1H, m, H1), 3.61 (3H, s, DMM), 3.58 (1H, m, H2), 3.56 (1H, d, $J=9.7, \mathrm{H} 11), 3.45$ (3H, s, DMM), 3.41 (3H, s, MeIm), 3.18 (1H, d, $J=9.4, \mathrm{H} 4)$, $2.85\left(1 \mathrm{H}, \mathrm{d}, J=16.6, \mathrm{H} 1^{\prime}\right), 2.31(1 \mathrm{H}, \mathrm{dt}, J=9.4,2.0, \mathrm{H} 3) .{ }^{13} \mathrm{C} \mathrm{NMR}\left(\mathrm{CDCl}_{3}, \delta\right): 179.3$, 143.3, 142.9, 142.5, 141.3 (Pz3), 140.8, 138.3 (Pz5C), 136.3 (Pz5A), 136.1, 134.4 (Pz5B)), 132.0, 128.8 (C8), 127.4 (C9), 125.7 (C5), 125.4 (C7), 124.2, 123.2 (C10), 120.6, 120.5, 105.8 (Pz4), 105.6 (Pz4), 105.4 (Pz4), 100.2, 69.6 (C4), 66.0 (C3), 65.1, $51.3(\mathrm{OMe}), 50.2$ (OMe), 43.2 (C2), 34.4 (NMe), 22.8 (C1). HRMS: $\left[\mathrm{M}^{+}\right]=$ $\left[\mathrm{C}_{32} \mathrm{H}_{34} \mathrm{~N}_{9} \mathrm{O}_{5} \mathrm{BMo}^{+}\right]$obsd (\%), calcd (\%), ppm: 727.1868 (50), 727.1844 (51), 3.3; 729.1831 (43), 729.1843 (48), -1.6; 730.1827 (75), 730.1841 (80), -1.9; 731.1848 (86), 731.1837 (87), 1.5; 732.1832 (75), 732.1849 (77), -2.3; 733.1844 (100), 733.1837 (100), 1.0; 734.1862 (35), 734.1864 (41), -0.2; 735.1854 (27), 735.1857 (40), -0.4. Minor Isomer: ${ }^{1} \mathrm{H}$ NMR ( $\mathrm{d}^{6}$-Acetone, $\left.\delta\right): 8.24(1 \mathrm{H}, \mathrm{d}, \mathrm{Pz} 3 / 5), 6.33(1 \mathrm{H}, \mathrm{d}, \mathrm{Pz} 4), 6.01(1 \mathrm{H}, \mathrm{t}$, Pz4).

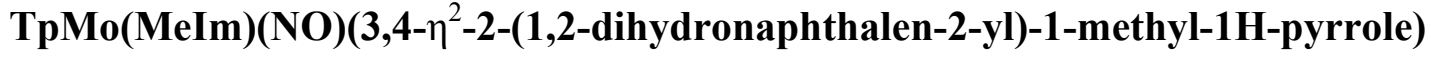

(10).

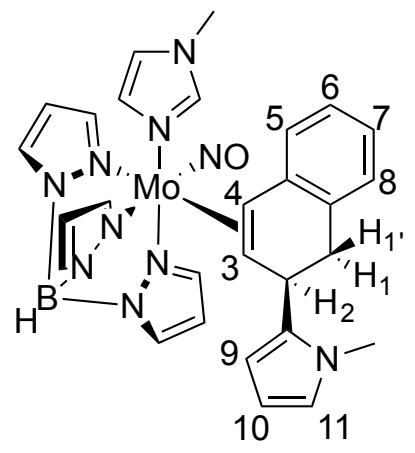


To a $50 \mathrm{~mL}$ round bottom charged with stir bar was added $4(1.00 \mathrm{~g}, 1.82 \mathrm{mmol})$ and 20 $\mathrm{mL} \mathrm{CH} \mathrm{CH}_{3} \mathrm{CN}$. This orange mixture was cooled with stirring for $15 \mathrm{~min}$ at $-35^{\circ} \mathrm{C}$. To this orange mixture was added $1 \mathrm{M} \mathrm{HOTf} / \mathrm{CH}_{3} \mathrm{CN}(9.2 \mathrm{~mL}, 9.20 \mathrm{mmol})$ cooled to $-35^{\circ} \mathrm{C}$. This orange mixture was stirred at room temperature 5 min yielding a red solution that was then cooled to $-35^{\circ} \mathrm{C}$. After 15 min standing at $-35^{\circ} \mathrm{C}, \mathrm{N}$-methylpyrrole $(16 \mathrm{~mL}, 179$ mmol) was added and left standing at $-35{ }^{\circ} \mathrm{C}$ for $18 \mathrm{~h}$. Next, a $-35{ }^{\circ} \mathrm{C}$ solution of triethylamine ( $2 \mathrm{~mL}, 14.3 \mathrm{mmol})$ was added and the golden solution was warmed to room temperature. The solution was then evaporated in vacuo to half volume $(\sim 10 \mathrm{~mL})$ and chromatographed through a $150 \mathrm{~mL}$ coarse porosity fritted disc filled $3 \mathrm{~cm}$ with silica. The product was eluted with 1:1 $\mathrm{Et}_{2} \mathrm{O}$ :benzene $(175 \mathrm{~mL})$. The yellow filtrate was then evaporated in vacuo, dissolved in DCM $(7 \mathrm{~mL})$ and precipitated in stirring pentane $(125$ $\mathrm{mL}$ ). The precipitate was then collected on a $30 \mathrm{~mL}$ fine porosity fritted disc and dried for 15 min yielding the yellow precipitate $10(600 \mathrm{mg}, 53 \%)$. CV (DMAc) $E_{\mathrm{p}, \mathrm{a}}=+0.14$ V (NHE). IR: $v(\mathrm{BH})=2480 \mathrm{~cm}^{-1}, v(\mathrm{NO})=1562 \mathrm{~cm}^{-1} \cdot{ }^{1} \mathrm{H}$ NMR $\left(\mathrm{d}^{6}\right.$-Acetone, $\left.\delta\right): 8.13$ (1H, d, Pz3A), 7.93 (1H, d, Pz5A), 7.91 (1H, d, Pz5B), 7.78 (1H, d, Pz3B), 7.77 (1H, d, Pz5C), 7.19 (1H, d, Pz3C), 7.17 (1H, t, $J=1.5$, MeIm), 7.12 (1H, s, MeIm), 6.93 (1H, d, $J=7.5, \mathrm{H} 8), 6.84(1 \mathrm{H}, \mathrm{t}, J=7.5, \mathrm{H} 6), 6.73(1 \mathrm{H}, \mathrm{dt}, J=7.5,1.3, \mathrm{H} 7), 6.47(1 \mathrm{H}, \mathrm{t}, J=1.4$, MeIm), $6.40(1 \mathrm{H}, \mathrm{t}, \mathrm{Pz} 4 \mathrm{~A}), 6.33(1 \mathrm{H}, \mathrm{t}, \mathrm{Pz} 4 \mathrm{~B}), 6.32(1 \mathrm{H}, \mathrm{t}, J=2.2, \mathrm{H} 11), 6.10(1 \mathrm{H}, \mathrm{t}$, Pz4C), $5.98(1 \mathrm{H}, \mathrm{d}, J=7.2, \mathrm{H} 5), 5.68(1 \mathrm{H}, \mathrm{dd}, J=3.4,1.9, \mathrm{H} 10), 5.64(1 \mathrm{H}, \mathrm{t}, J=2.9$, H9), 4.02 (1H, d, $J=7.4, \mathrm{H} 2), 3.78$ (3H, s, MeIm), 3.75 (1H, dd, $J=15.7,7.0, \mathrm{H1}$ '), 3.4 (3H, s, NMP), $3.11(1 \mathrm{H}, \mathrm{d}, J=9.0, \mathrm{H} 4), 2.74(1 \mathrm{H}, \mathrm{d}, J=15.7, \mathrm{H} 1) 2.34(1 \mathrm{H}, \mathrm{dt}, J=9.0$, 1.9, H3). ${ }^{13} \mathrm{C}$ NMR (d $\mathrm{d}^{6}$-Acetone, $\left.\delta\right): 144.5,143.1,141.9,141.8,141.7,139.2,137.1$, $137.0,135.5,132.8,129.1,128.8,125.9,124.4,123.0,122.2,120.4,106.6,106.5,106.5$, 
106.2, $106.1, \quad 70.3, \quad 66.9, \quad 34.4, \quad 34.3, \quad 33.7, \quad 33.5$. $\quad$ HRMS: $\left[\mathrm{M}+\mathrm{Na}^{+}\right]=$ $\left[\mathrm{C}_{28} \mathrm{H}_{31} \mathrm{~N}_{10} \mathrm{OBMo}+\mathrm{Na}^{+}\right]$obsd (\%), calcd (\%), ppm: 649.1767 (50), 649.1741 (52), 4.1; 651.1740 (47), 651.1738 (48), 0.3; 652.1746 (77), 652.1737 (81), 1.4; 653.1745 (87), 653.1732 (87), 2.0; 654.1745 (75), 654.1744 (76), 0.1; 655.1742 (100), 655.1732 (100), 1.6; 656.1780 (36), 656.1759 (38), 3.2; 657.1766 (37), 657.1751 (39), 2.2. Minor Isomer: ${ }^{1} \mathrm{H}$ NMR $\left(\mathrm{d}^{6}\right.$-Acetone, $\left.\delta\right): 8.01(1 \mathrm{H}, \mathrm{d}, \mathrm{Pz3} / 5), 7.90(1 \mathrm{H}, \mathrm{d}, \mathrm{Pz} 3 / 5), 7.75(1 \mathrm{H}, \mathrm{d}$, Pz3/5), 6.90 (1H, d, naphthalene), $6.38(1 \mathrm{H}, \mathrm{t}, \mathrm{MeIm}), 6.26(1 \mathrm{H}, \mathrm{t}, \mathrm{Pz} 4), 6.25(1 \mathrm{H}, \mathrm{t}$, Pz4), 6.08 (1H, t, Pz4), 3.83 (d, $J=7.2, \mathrm{H} 4), 2.46(1 \mathrm{H}, \mathrm{dt}, J=9.4,2.2, \mathrm{H} 3)$

\section{TpMo(MeIm)(NO)(3,4- $\left.\eta^{2}-2-(1,2-d i h y d r o a n t h r a c e n-2-y l)-1-m e t h y l-1 H-p y r r o l e\right)(11)$.}

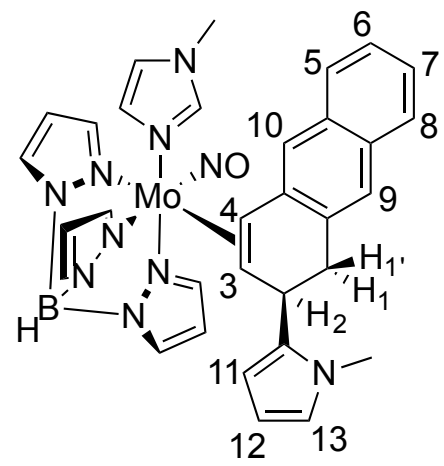

To a test tube was added $5(300 \mathrm{mg}, 0.501 \mathrm{mmol})$ and $6 \mathrm{~mL} \mathrm{CH}_{3} \mathrm{CN}$. This red solution was cooled for $15 \mathrm{~min}$ at $-35^{\circ} \mathrm{C}$. To this solution was added $1 \mathrm{M} \mathrm{HOTf} / \mathrm{MeOH}(2.1 \mathrm{~mL}$, $2.10 \mathrm{mmol}$ ) cooled to $-35^{\circ} \mathrm{C}$. This dark red solution was left standing at $-35^{\circ} \mathrm{C}$ for 15 min at which point $N$-methylpyrrole $(4.8 \mathrm{~mL}, 53.7 \mathrm{mmol})$ was added and left standing at $35^{\circ} \mathrm{C}$ for $18 \mathrm{~h}$. Next, a $-35^{\circ} \mathrm{C}$ solution of triethylamine $(0.6 \mathrm{~mL}, 4.30 \mathrm{mmol})$ was added and the orange solution was warmed to room temperature. The solution was then evaporated in vacuo to dryness, dissolved in THF $(2 \mathrm{~mL})$, and chromatographed through a $60 \mathrm{~mL}$ medium porosity fritted disc $2 \mathrm{~cm}$ full with silica. The product was eluted with 
1:1 $\mathrm{Et}_{2} \mathrm{O}$ :benzene $(50 \mathrm{~mL})$. The orange filtrate was then evaporated in vacuo, dissolved in DCM $(1 \mathrm{~mL})$ and precipitated in stirring pentane $(100 \mathrm{~mL})$. The precipitate was then collected on a $15 \mathrm{~mL}$ fine porosity fritted disc and dried for 15 min yielding the yellow precipitate 11 (85 mg, 27\%). CV (DMAc) $E_{\mathrm{p}, \mathrm{a}}=+0.15 \mathrm{~V}$ (NHE). IR: $v(\mathrm{BH})=2476 \mathrm{~cm}^{-1}$, $v(\mathrm{NO})=1554 \mathrm{~cm}^{-1} .{ }^{1} \mathrm{H}$ NMR ( $\mathrm{d}^{6}$-Acetone, $\left.\delta\right): 8.15(1 \mathrm{H}, \mathrm{d}, \mathrm{Pz3A}), 7.94(2 \mathrm{H}, \mathrm{m}, \mathrm{Pz} 5 \mathrm{~A} \&$ C), $7.85(1 \mathrm{H}, \mathrm{d}, \mathrm{Pz3C}), 7.76(1 \mathrm{H}, \mathrm{d}, \mathrm{Pz3} / 5 \mathrm{~B}), 7.55(1 \mathrm{H}, \mathrm{d}, J=8.3, \mathrm{H} 8), 7.49(1 \mathrm{H}, \mathrm{d}, J=$ 8.1, H5), 7.43 (1H, s, H9), 7.25 (1H, t, $J=7.5, \mathrm{H6}), 7.21$ (1H, t, $J=1.5$, MeIm), 7.19 (1H, m, H7), $7.19(1 \mathrm{H}$, buried d, Pz3/5B), $7.05(1 \mathrm{H}, \mathrm{bt}, \mathrm{MeIm}), 6.51(1 \mathrm{H}, \mathrm{t}, J=1.3$, MeIm), 6.42 (1H, t, Pz4A), 6.35 (1H, t, Pz4C), 6.32 (1H, buried s, H10), 6.33 (1H, buried multiplet, H13), 6.09 (1H, t, Pz4B), 5.70 (1H, dd, $J=3.7,1.8, \mathrm{H} 12), 5.61$ (1H, m, H11), 4.09 (1H, d, $J=6.9, \mathrm{H} 2), 3.92$ (1H, ddd, $J=15.7,7,1.3, \mathrm{H1}$ '), 3.64 (3H, s, MeIm), 3.47 (3H, s, NMP), $3.33(1 \mathrm{H}, \mathrm{d}, J=9.6, \mathrm{H} 4), 3.00(1 \mathrm{H}, \mathrm{d}, J=15.7, \mathrm{H} 1), 2.47$ (1H, dt, $J=9.6$, 2.0, H3). ${ }^{13} \mathrm{C}$ NMR ( $\mathrm{d}^{6}$-Acetone, $\delta$ ): 143.9, 143.3, 142.0 (Pz3/5), 141.7 (Pz3C), 139.0, 137.2 (Pz5A \& Pz5C), 137.1, 135.6 (Pz3/5), 134.1, 133.3, 132.1, 129.1, 127.6 (C8), 126.7 (C5), 126.5 (C9), 124.9 (C6), 123.6 (C7), 122.2 (C10), 121.6, 120.6, 106.6, 106.6, 106.6, 106.3, 106.1, 70.8 (C3), $67.4(\mathrm{C} 4), 34.8$ (C1), 34.5 (NMe), 33.7 (C2), $33.5(\mathrm{NMe})$. HRMS: $\left[\mathrm{M}+\mathrm{Na}^{+}\right]=\left[\mathrm{C}_{32} \mathrm{H}_{33} \mathrm{~N}_{10} \mathrm{OBMo}+\mathrm{Na}^{+}\right]$obsd (\%), calcd (\%), ppm: 699.1895 (52), 699.1898 (51), -0.4; 701.1902 (38), 701.1896 (48), 0.9; 702.1914 (79), 702.1894 (80), 2.8; 703.1904 (88), 703.1890 (88), 2.0; 704.1865 (76), 704.1902 (77), -5.2; 705.1867 (100), 705.1890 (100), -3.2; 706.1896 (36), 706.1916 (41), -2.9; 707.1875 (39), 707.1910 (39), -4.9. 


\section{Methyl-2-(1,2-dihydronaphthalen-2-yl)-2-methylpropanoate (12).}<smiles>COC(=O)C(C)(C)C1C=Cc2ccccc2C1</smiles>

To a $500 \mathrm{~mL}$ Erlenmeyer flask charged with stir bar was added 6 (4.00 g, $6.14 \mathrm{mmol})$ and DCM (100 mL). Next, iodine $(756 \mathrm{mg}, 3.00 \mathrm{mmol})$ dissolved in $\mathrm{Et}_{2} \mathrm{O}(\sim 25 \mathrm{~mL})$ was added to the stirring yellow solution changing instantly to green. This solution was stirred for $5 \mathrm{~min}$ and was then add to $300 \mathrm{~mL}$ stirring pentane. The green precipitate $\mathbf{3}$ was then isolated on a $60 \mathrm{~mL}$ fine porosity fritted disc and washed with pentane $(3 \mathrm{x} 60 \mathrm{~mL})$ and $\mathrm{Et}_{2} \mathrm{O}(3 \times 60 \mathrm{~mL})$. The colorless filtrate was then removed from the glovebox and evaporated in vacuo. This oil was then filtered through a $30 \mathrm{~mL}$ medium porosity fritted disc $2 \mathrm{~cm}$ full with silica along with $200 \mathrm{~mL}$ DCM. The filtrate was then evaporated in vacuo, loaded (6 x $0.6 \mathrm{~mL}$ DCM) onto a silica preparatory plate, and was eluted with $10 \%$ EtOAc:hexanes (HPLC grade, $200 \mathrm{~mL}$ ). A $\mathrm{KMnO}_{4}$ positive band at $\mathrm{R}_{\mathrm{f}}$ 0.50-1.00 was isolated and sonicated in HPLC grade EtOAc $(100 \mathrm{~mL})$ for $20 \mathrm{~min}$. The silica was then filtered off on a $30 \mathrm{~mL}$ fine porosity fritted disc and was washed with DCM (3 x $20 \mathrm{~mL})$. The filtrate was then evaporated in vacuo and dried $1 \mathrm{~h}$ yielding the colorless oil 12 (836 mg, 64\%). This sample matched previous reports. ${ }^{23}$ 


\section{Methyl-2-(1,2-dihydroanthracen-2-yl)-2-methylpropanoate (13).}

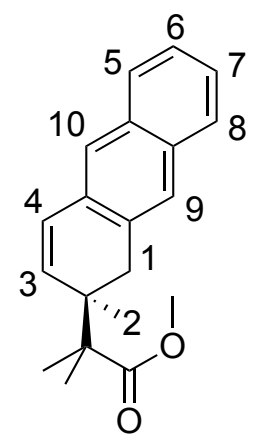

To a $25 \mathrm{~mL}$ filter flask charged with stir bar was added 7 (600 mg, $0.855 \mathrm{mmol})$ and DCM (5 mL). Next, $0.06 \mathrm{M} \mathrm{I}_{2} / \mathrm{Et}_{2} \mathrm{O}(7.83 \mathrm{~mL}, 0.469 \mathrm{mmol})$ was added to the stirring yellow solution changing instantly to green. This solution was stirred for 5 min and was then evaporated in vacuo to $1 \mathrm{~mL}$ and added to stirring hexanes $(40 \mathrm{~mL})$. The green precipitate 3 was then isolated on a $15 \mathrm{~mL}$ medium porosity fritted disc and washed with hexanes $(3 \times 10 \mathrm{~mL})$ and $\mathrm{Et}_{2} \mathrm{O}(10 \mathrm{~mL})$. The colorless filtrate was then removed from the glovebox and evaporated in vacuo. This oil was then loaded ( 3 x $0.3 \mathrm{~mL}$ DCM) onto a silica preparatory plate and was eluted with 10\% EtOAc:hexanes (HPLC grade, $200 \mathrm{~mL}$ ). A $\mathrm{KMnO}_{4}$ positive band at $\mathrm{R}_{\mathrm{f}}$ 0.56-0.68 was scraped off and sonicated in EtOAc (HPLC grade, $25 \mathrm{~mL}$ ) for $10 \mathrm{~min}$. The silica gel was then filtered off on a $30 \mathrm{~mL}$ fine porosity fritted disc and was washed with DCM (4 x $15 \mathrm{~mL})$. The filtrate was then evaporated in vacuo and dried $1 \mathrm{~h}$ yielding the colorless oil $13(162 \mathrm{mg}, 79 \%)$. IR: $v\left(\mathrm{C}-\mathrm{H} \mathrm{sp}{ }^{2}\right)=3051$ $\mathrm{cm}^{-1}, v(\mathrm{CO})=1732 \mathrm{~cm}^{-1} .{ }^{1} \mathrm{H}$ NMR $\left(\mathrm{CDCl}_{3}, \delta\right): 7.74(1 \mathrm{H}, \mathrm{m}, \mathrm{H} 5 / 8), 7.71(1 \mathrm{H}, \mathrm{m}, \mathrm{H} 5 / 8)$, $7.51(1 \mathrm{H}, \mathrm{s}, \mathrm{H} 9), 7.45$ (1H, s, H10), 7.39 (2H, m, H6 \& H9), $6.71(1 \mathrm{H}, \mathrm{dd}, J=9.8,1.6$, H4), $5.95(1 \mathrm{H}, \mathrm{dd}, J=9.8,3.7, \mathrm{H} 3), 3.70\left(3 \mathrm{H}, \mathrm{s}, \mathrm{O}-\mathrm{CH}_{3}\right), 2.97(1 \mathrm{H}, \mathrm{dd}, J=13.3,5.8$, H1'), 2.92 (1H, m, H2), 2.91 (1H, m, H1), 1.24 (3H, s, MMTP), 1.20 (3H, s, MMTP). ${ }^{13} \mathrm{C}$ NMR $\left(\mathrm{CDCl}_{3}, \delta\right): 178.0$ (CO), 133.9, 133.3, 133.0, 132.2, 130.1 (C3), 129.4 (C4), 127.8 
(C5/C8), 127.3 (C5/C8), 125.8, 125.5, 124.3, 52.0 (OMe), 45.9, 41.8 (C2), 30.2 (C1), $22.7(\mathrm{Me}), 22.1(\mathrm{Me})$. LRMS: $\left[\mathrm{M}^{+}\right]=280$.

\section{Dimethyl-2-(1,2-dihydronaphthalen-2-yl)malonate (14).}

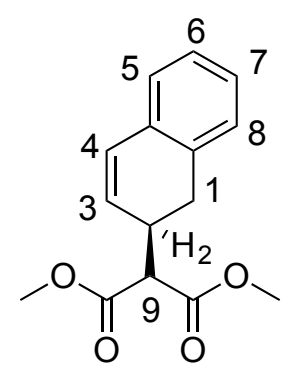

To a $25 \mathrm{~mL}$ filter flask charged with stir bar was added 8 (100 mg, $0.146 \mathrm{mmol})$ and DCM (5 mL). Next, $0.06 \mathrm{M} \mathrm{I}_{2} / \mathrm{Et}_{2} \mathrm{O}(1.16 \mathrm{~mL}, 0.07 \mathrm{mmol})$ was added to the stirring yellow solution changing instantly to green. This solution was stirred for 5 min and was then evaporated in vacuo to $1 \mathrm{~mL}$ and added to stirring pentane $(20 \mathrm{~mL})$. The green precipitate 3 was isolated on a $15 \mathrm{~mL}$ fine porosity fritted disc, washed with pentane $(3 \mathrm{x}$ $10 \mathrm{~mL}$ ). The colorless filtrate was then removed from the glovebox and evaporated in vacuo. This oil was then loaded $(3 \times 0.3 \mathrm{~mL}$ DCM) onto a silica preparatory plate and was eluted with 10\% EtOAc:hexanes (HPLC grade, $200 \mathrm{~mL}$ ). $\mathrm{A} \mathrm{KMnO}_{4}$ positive band at $\mathrm{R}_{\mathrm{f}}$ 0.30-0.40 was scraped off and sonicated in 10\% EtOAc:hexanes (HPLC grade, $25 \mathrm{~mL}$ ) for $10 \mathrm{~min}$. The silica was then filtered off on a $30 \mathrm{~mL}$ fine porosity fritted disc and was washed with HPLC grade hexanes $(3 \times 20 \mathrm{~mL})$. The filtrate was evaporated in vacuo and dried $1 \mathrm{~h}$ yielding the colorless oil $14(22 \mathrm{mg}, 61 \%)$. IR: $(\mathrm{CO})=1736 \mathrm{~cm}^{-1} \cdot{ }^{1} \mathrm{H}$ NMR $\left(\mathrm{CDCl}_{3}, \delta\right): 7.16(2 \mathrm{H}, \mathrm{m}, \mathrm{H} 6$ \& H7), $7.09(1 \mathrm{H}, \mathrm{d}, J=7.2, \mathrm{H} 8), 7.05(1 \mathrm{H}, \mathrm{d}, J=7.2, \mathrm{H} 5)$, $6.53(1 \mathrm{H}, \mathrm{dd}, J=9.9,1.6, \mathrm{H} 4), 5.95(1 \mathrm{H}, \mathrm{dd}, J=9.9,4.6, \mathrm{H} 3), 3.73$ (3H, s, DMM), 3.71 (3H, s, DMM), $3.41(1 \mathrm{H}, \mathrm{d}, J=9.5, \mathrm{H} 9), 3.22(1 \mathrm{H}, \mathrm{m}, \mathrm{H} 2), 2.97(1 \mathrm{H}, \mathrm{dd}, J=15.8,6.9$, 
H1'), $2.73(1 \mathrm{H}, \mathrm{dd}, J=15.8,8.1, \mathrm{H1}) \cdot{ }^{13} \mathrm{C} \mathrm{NMR}\left(\mathrm{CDCl}_{3}, \delta\right): 168.8(\mathrm{CO}), 168.7(\mathrm{CO})$, 133.4, 133.2, 129.3 (C4), 128.6 (C3), 128.2, 127.7, 127.0, 126.3, 54.6 (C9), 52.7 (OMe), $52.6(\mathrm{OMe}), 33.7(\mathrm{C} 2), 31.7(\mathrm{C} 1)$. HRMS: $\left[\mathrm{C}_{15} \mathrm{H}_{16} \mathrm{O}_{4}+\mathrm{Na}^{+}\right]$obsd (\%), calcd (\%), ppm: 283.0933 (100), $283.0941(100),-2.8$.

\section{Dimethyl-2-(1,2-dihydroanthracen-2-yl)malonate (15).}<smiles>COC(=O)C(C(=O)OC)[C@@H]1[CH]c2cc3ccccc3cc2C=C1</smiles>

To a $25 \mathrm{~mL}$ filter flask charged with stir bar was added $9(100 \mathrm{mg}, 0.137 \mathrm{mmol})$ and DCM (5 mL). Next, $0.06 \mathrm{M} \mathrm{I}_{2} / \mathrm{Et}_{2} \mathrm{O}(1.2 \mathrm{~mL}, 0.070 \mathrm{mmol})$ was added to the stirring yellow solution changing instantly to green. This solution was stirred for 5 min and was then evaporated in vacuo to $1 \mathrm{~mL}$ and added to stirring hexanes $(25 \mathrm{~mL})$. The green precipitate 3 was then isolated on a $15 \mathrm{~mL}$ fine porosity fritted disc and washed with $\mathrm{Et}_{2} \mathrm{O}$ $(3 \times 10 \mathrm{~mL})$. The colorless filtrate was then removed from the glovebox and evaporated in vacuo. This oil was then loaded $(3 \times 0.3 \mathrm{~mL}$ DCM) onto a silica preparatory plate and was eluted with 10\% EtOAc:hexanes (HPLC grade, $200 \mathrm{~mL}$ ). A $\mathrm{KMnO}_{4}$ positive band at $\mathrm{R}_{\mathrm{f}}$ 0-0.4 was scraped off and sonicated in EtOAc (HPLC grade, $25 \mathrm{~mL}$ ) for $10 \mathrm{~min}$. The $\mathrm{SiO}_{2}$ gel was then filtered off on a $30 \mathrm{~mL}$ fine porosity fritted disc and was washed with DCM $(3 \times 15 \mathrm{~mL})$. The filtrate was then evaporated in vacuo and dried $1 \mathrm{~h}$ yielding the colorless oil $15(23 \mathrm{mg}, 62 \%)$. IR: $v\left(\mathrm{C}-\mathrm{H} \mathrm{sp}{ }^{2}\right)=3047 \mathrm{~cm}^{-1}, v(\mathrm{CO})=1736 \mathrm{~cm}^{-1} .{ }^{1} \mathrm{H} \mathrm{NMR}$ 
$\left(\mathrm{CDCl}_{3}, \delta\right): 7.75(1 \mathrm{H}, \mathrm{m}, \mathrm{H} 8), 7.72(1 \mathrm{H}, \mathrm{m}, \mathrm{H} 5), 7.49$ (1H, s, H9), 7.40 (2H, m, H6 \& H7), $6.7(1 \mathrm{H}, \mathrm{d}, J=9.2, \mathrm{H} 4), 6.06(1 \mathrm{H}, \mathrm{dd}, J=9.2,4.5, \mathrm{H} 3), 3.75$ (3H, s, DMM), 3.71 (3H, s, DMM), 3.42 (1H, d, $J=9.1, \mathrm{H} 11), 3.30$ (1H, m, H4), 3.13 (1H, dd, $J=15.8,6.0$, H1'), $2.91(1 \mathrm{H}, \mathrm{dd}, J=15.8,6.9, \mathrm{H} 1) .{ }^{13} \mathrm{C} \mathrm{NMR}\left(\mathrm{CDCl}_{3}, \delta\right): 168.9(\mathrm{CO}), 168.8(\mathrm{CO})$, 133.5, 133.2, 132.2, 131.8, 130.0 (C3), 129.7 (C4), 128.0 (C8), 127.5 (C5), 126.7 (C10), 126.2 (C6), 125.9 (C7), 124.9 (C9), 55.0 (C11), 52.9 (OMe), 34.3 (C2), 32.5 (C1. HRMS: $\left[\mathrm{C}_{19} \mathrm{H}_{18} \mathrm{O}_{4}+\mathrm{Na}^{+}\right]$obsd (\%), calcd (\%), ppm: 333.1092 (100), 333.1097 (100), -1.6.

\section{2-(1,2-Dihydronaphthalen-2-yl)-1-methyl-1H-pyrrole (16).}

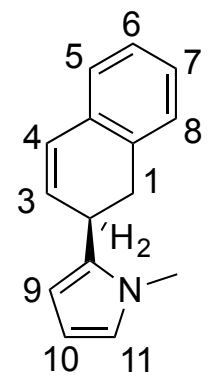

To a $25 \mathrm{~mL}$ filter flask charged with stir bar was added $\mathbf{1 0}(100 \mathrm{mg}, 0.158 \mathrm{mmol})$ and DCM $(5 \mathrm{~mL})$. Next, $0.06 \mathrm{M} \mathrm{I}_{2} / \mathrm{Et}_{2} \mathrm{O}(1.33 \mathrm{~mL}, 0.08 \mathrm{mmol})$ was added to the stirring yellow solution changing instantly to green. This solution was stirred for $5 \mathrm{~min}$ and was then evaporated in vacuo to $1 \mathrm{~mL}$ and added to stirring hexanes $(25 \mathrm{~mL})$. The green precipitate 3 was then isolated on a $15 \mathrm{~mL}$ fine porosity fritted disc and was washed with hexanes $(3 \times 10 \mathrm{~mL})$. The colorless filtrate was then removed from the box and evaporated in vacuo. This oil was then loaded $(3 \times 0.3 \mathrm{~mL}$ DCM) onto a silica preparatory plate and was eluted with 10\% EtOAc:hexanes (HPLC grade, $200 \mathrm{~mL}$ ). A $\mathrm{KMnO}_{4}$ positive band at $\mathrm{R}_{\mathrm{f}}$ 0.5-0.7 was scraped off and sonicated in 10\% EtOAc:hexanes (HPLC grade, $25 \mathrm{~mL}$ ) for $10 \mathrm{~min}$. The silica was then filtered off on a $30 \mathrm{~mL}$ fine 
porosity fritted disc and was washed with $10 \%$ EtOAc:hexanes $(3 \times 15 \mathrm{~mL})$. The filtrate was then evaporated in vacuo and dried $1 \mathrm{~h}$ yielding the colorless oil 16 (29 $\mathrm{mg}, 89 \%)$. IR: 2935, 1724, $1489 \mathrm{~cm}^{-1} .{ }^{1} \mathrm{H}$ NMR $\left(\mathrm{CDCl}_{3}, \delta\right): 7.21$ (4H, m, H11, H6, H7 \& H8), 6.58 $(1 \mathrm{H}, \mathrm{dd}, J=4.9,2.2, \mathrm{H} 4), 6.57(1 \mathrm{H}, \mathrm{t}, J=2.3, \mathrm{H} 11), 6.03(1 \mathrm{H}, \mathrm{dd}, J=9.7, \mathrm{H} 3), 5.87(1 \mathrm{H}$, t, $J=3.3, \mathrm{H} 10), 5.79(1 \mathrm{H}, \mathrm{ddd}, J=3.6,1.9,0.5, \mathrm{H} 9), 3.80(1 \mathrm{H}, \mathrm{m}, \mathrm{H} 2), 3.64(3 \mathrm{H}, \mathrm{s}$, NMe), $3.05\left(1 \mathrm{H}, \mathrm{dd}, J=15.4,6.8, \mathrm{H} 1^{\prime}\right), 2.91(1 \mathrm{H}, \mathrm{dd}, J=15.4,10.9, \mathrm{H} 1) .{ }^{13} \mathrm{C}$ NMR $\left(\mathrm{CDCl}_{3}, \delta\right): 135.2,134.8,133.8,131.3,128.3,128.0,127.6,127.1,126.4,122.2,107.1$, 106.0, 35.1, 34.2, 32.7. LRMS: $\left[\mathrm{M}^{+}\right]=209$.

\section{2-(1,2-Dihydroanthracen-2-yl)-1-methyl-1H-pyrrole (17).}

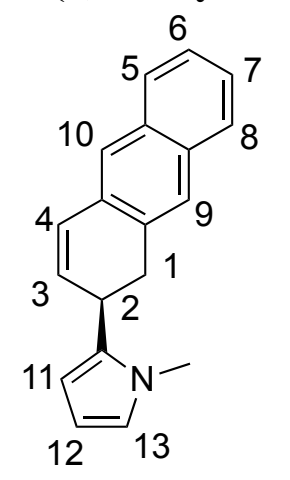

To a $25 \mathrm{~mL}$ filter flask charged with stir bar was added 11 (75 mg, $0.109 \mathrm{mmol})$ and DCM $(5 \mathrm{~mL})$. Next, $0.06 \mathrm{M} \mathrm{I}_{2} / \mathrm{Et}_{2} \mathrm{O}(0.83 \mathrm{~mL}, 0.05 \mathrm{mmol})$ was added to the stirring yellow solution changing instantly to green. This solution was stirred for 5 min and was then evaporated in vacuo to $1 \mathrm{~mL}$ and added to stirring hexanes $(25 \mathrm{~mL})$. The green precipitate 3 was then isolated on a $15 \mathrm{~mL}$ fine porosity fritted disc and was washed with hexanes $(3 \times 10 \mathrm{~mL})$. The colorless filtrate was then removed from the box and evaporated in vacuo. This oil was then loaded $(3 \times 0.3 \mathrm{~mL}$ DCM $)$ onto a silica preparatory plate and was eluted with 10\% EtOAc:hexanes (HPLC grade, $200 \mathrm{~mL}$ ). A band from $\mathrm{R}_{\mathrm{f}}: 0.5-1.0$ was scraped off and sonicated in EtOAc $(25 \mathrm{~mL})$ for $10 \mathrm{~min}$. The 
silica was then filtered off on a $30 \mathrm{~mL}$ fine porosity fritted disc and was washed with EtOAc (HPLC grade, $3 \times 15 \mathrm{~mL}$ ). The filtrate was then evaporated in vacuo and dried $1 \mathrm{~h}$ yielding the colorless oil 17 (11 mg, 31\%). IR: bands at 2924, 3379, and $1697 \mathrm{~cm}^{-1} .{ }^{1} \mathrm{H}$ NMR $\left(\mathrm{CDCl}_{3}, \delta\right): 7.77(1 \mathrm{H}, \mathrm{m}, \mathrm{H} 5 / \mathrm{H} 8), 7.72(1 \mathrm{H}, \mathrm{m}, \mathrm{H} 5 / 8), 7.53(1 \mathrm{H}, \mathrm{s}, \mathrm{H} 9), 7.52(1 \mathrm{H}$, s, H10), 7.40 (2H, m, H6 \& H7), 6.77 (1H, dd, $J=9.6,2.4, \mathrm{H} 4), 6.59$ (1H, t, $J=2.3$, H13), 6.15 (1H, dd, $J=9.6,3.8, \mathrm{H} 3), 6.05$ (1H, t, $J=3.3, \mathrm{H} 12), 5.96(1 \mathrm{H}, \mathrm{m}, \mathrm{H} 11), 3.87$ (1H, m, H2), 3.65 (3H, s, NMP), 3.24 (1H, dd, $J=15.1,6.4, \mathrm{H} 1), 3.15$ (1H, ddd, $J=$ 15.1, 9.9, 1.0, H1). ${ }^{13} \mathrm{C} \mathrm{NMR}\left(\mathrm{CDCl}_{3}, \delta\right): 135.1,133.4,133.3,132.4,132.3,128.5,128.1$, $127.56,126.2,126.1,125.8,127.7,122.3,107.2,106.2,35.7,34.2,32.3$. LRMS: $\left[\mathrm{M}^{+}\right]=$ 259.

\section{2-(1,2-dihydronaphthalen-2-yl)-2-methylpropanoic acid (18)}<smiles>CC(C)(C(=O)O)C1C=Cc2ccccc2C1</smiles>

A solution of 12 (100 mg, $0.43 \mathrm{mmol}), \mathrm{MeOH}(5 \mathrm{~mL})$, and aqueous $\mathrm{NaOH}(1 \mathrm{M}, 4.3 \mathrm{~mL}$, $4.3 \mathrm{mmol}$ ), was added to a $25 \mathrm{~mL}$ round bottom flask charged with a stir pea and reflux condenser. This solution was stirred at reflux for $24 \mathrm{~h}$ at which point the reaction mixture was cooled to room temperature and neutralized $(\mathrm{pH} \sim 7)$ with glacial acetic acid $(\sim 1$ $\mathrm{mL})$. The organic layer was then extracted from this solution with $\mathrm{CHCl}_{3}(15 \mathrm{~mL})$, washed with $\mathrm{H}_{2} \mathrm{O}(30 \mathrm{~mL})$ and brine (sat. aq. $\mathrm{NaCl}, 30 \mathrm{~mL}$ ), and dried over $\mathrm{MgSO}_{4}$. The drying agent was filtered off on a $15 \mathrm{~mL}$ medium porosity fritted disc, washed with $\mathrm{CHCl} 3(3 \times 5 \mathrm{~mL})$ and the filtrate was evaporated in vacuo yielding the colorless oil $\mathbf{1 8}$ 
(89 mg, $96 \%) .{ }^{1} \mathrm{H}$ NMR $\left(\mathrm{CDCl}_{3}, \delta\right): 12.36(1 \mathrm{H}, \mathrm{bs}), 7.16(3 \mathrm{H}, \mathrm{m}), 7.06(1 \mathrm{H}, \mathrm{dd}, J=6.5$, 1.8), $6.59(1 \mathrm{H}, \mathrm{dd}, J=9.9,2.3), 5.96(1 \mathrm{H}, \mathrm{dd}, J=9.7,3.4), 2.96(1 \mathrm{H}, \mathrm{m}), 2.91(1 \mathrm{H}, \mathrm{dd}, J$ $=15.7,6.9), 2.87(1 \mathrm{H}, \mathrm{dd}, J=15.7,11.6), 1.31(3 \mathrm{H}, \mathrm{s}), 1.27(3 \mathrm{H}, \mathrm{s}) .{ }^{13} \mathrm{C} \mathrm{NMR}\left(\mathrm{CDCl}_{3}\right.$, б): $184.3,134.8,133.5,129.2,128.4,127.7,127.3,126.6,125.9,45.5,41.2,29.5,22.4$, 21.8.

\section{2-(1,2-dihydroanthracen-2-yl)-2-methylpropanoic acid (19)}<smiles>CC(C)(C)C(=O)O</smiles>

A solution of 13 (144 mg, $0.51 \mathrm{mmol}), \mathrm{MeOH}(5 \mathrm{~mL})$, and aqueous $\mathrm{NaOH}(1 \mathrm{M}, 4.3 \mathrm{~mL}$, $4.3 \mathrm{mmol}$ ), was added to a $25 \mathrm{~mL}$ round bottom flask charged with a stir pea and reflux condenser. This solution was stirred at reflux for $24 \mathrm{~h}$ at which point the reaction mixture was cooled to room temperature and neutralized $(\mathrm{pH} \sim 7)$ with glacial acetic acid $(\sim 1$ $\mathrm{mL})$. The organic layer was then extracted from this solution with DCM $(20 \mathrm{~mL})$, washed with $\mathrm{H}_{2} \mathrm{O}(30 \mathrm{~mL})$ and brine (sat. aq. $\mathrm{NaCl}, 30 \mathrm{~mL}$ ), and dried over $\mathrm{MgSO}_{4}$. The drying agent was filtered off on a $15 \mathrm{~mL}$ medium porosity fritted disc, washed with DCM $(3 \times 5$ $\mathrm{mL})$ and the filtrate was evaporated in vacuo yielding the colorless oil 19 (130 mg, 95 \%). ${ }^{1} \mathrm{H} \mathrm{NMR}\left(\mathrm{CDCl}_{3}, \delta\right): 11.21(1 \mathrm{H}, \mathrm{bs}), 7.75(1 \mathrm{H}, \mathrm{m}), 7.71(1 \mathrm{H}, \mathrm{m}), 7.53(1 \mathrm{H}, \mathrm{s}), 7.46$ $(1 \mathrm{H}, \mathrm{s}), 7.39(2 \mathrm{H}, \mathrm{m}), 6.74(1 \mathrm{H}, \mathrm{dd}, J=10.1,1.6), 6.01(1 \mathrm{H}, \mathrm{dd}, J=10.1,3.3), 3.06(1 \mathrm{H}$, m), $2.96(2 \mathrm{H}, \mathrm{m}), 1.26(3 \mathrm{H}, \mathrm{s}), 1.22(3 \mathrm{H}, \mathrm{s}) .{ }^{13} \mathrm{C} \mathrm{NMR}\left(\mathrm{CDCl}_{3}, \delta\right): 183.4,133.7,133.3$, 
$132.9,132.1,129.7,129.6,127.8,127.3,125.8(2 \mathrm{C}), 125.5,124.4,45.9,41.5,30.2,22.6$, 21.8

\section{9-Iodo-3,3-dimethyl-3a,4,9,9a-tetrahydronaphtho[2,3-b]furan-2(3H)-one (20).}

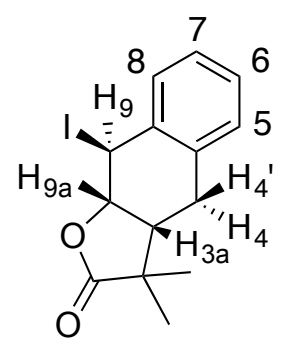

To a 4 dram vial was added $18(90 \mathrm{mg}, 0.416 \mathrm{mmol})$, water $(4 \mathrm{~mL})$, saturated $\mathrm{NaHCO}_{3}$ aq. (1 mL), KI (112 mg, $0.675 \mathrm{mmol}), \mathrm{I}_{2}(79 \mathrm{mg}, 0.313 \mathrm{mmol})$. This was stirred at room temperature for 6 days at which point $30 \mathrm{~mL}$ of DCM were added and the organic layer was evaporated in vacuo. This was loaded (3 x $0.3 \mathrm{~mL} \mathrm{DCM})$ onto a silica preparatory plate and was eluted with 1:4 EtOAc:hexanes (HPLC grade, $200 \mathrm{~mL}$ ). Scraped off a $\mathrm{KMNO}_{4}$ band at Rf : $0.25-0.5$ and sonicated in EtOAc (HPLC grade, $25 \mathrm{~mL}$ ) for 10 min. Filtered off silica on a $15 \mathrm{~mL}$ fine porosity fritted disc, washed with DCM $(3 \times 5$ $\mathrm{mL})$, and evaporated in vacuo to isolate the product $20(35 \mathrm{mg}, 25 \%)$. IR: $v(\mathrm{CO})=1766$ $\mathrm{cm}^{-1} .{ }^{1} \mathrm{H} \mathrm{NMR}\left(\mathrm{CDCl}_{3}, \delta\right): 7.29(1 \mathrm{H}, \mathrm{d}, J=7.5, \mathrm{H} 8), 7.25(1 \mathrm{H}, \mathrm{t}, J=7.5, \mathrm{H} 6), 7.21(1 \mathrm{H}$, $\mathrm{t}, J=7.5, \mathrm{H} 7), 7.13(1 \mathrm{H}, \mathrm{d}, J=7.5, \mathrm{H} 5), 5.55(1 \mathrm{H}, \mathrm{d}, J=2.9, \mathrm{H} 9), 5.36(1 \mathrm{H}, \mathrm{dd}, J=7.6$, 2.9, H9a), 3.06 (1H, dd, $\left.J=16.5,8.5, \mathrm{H} 4^{\prime}\right), 2.89$ (1H, t, $\left.J=7.8, \mathrm{H} 3 \mathrm{a}\right), 2.83(1 \mathrm{H}, \mathrm{d}, J=$ 16.5, H4), $1.36(3 \mathrm{H}, \mathrm{s}, \mathrm{Me}), 1.22(3 \mathrm{H}, \mathrm{s}, \mathrm{Me}) .{ }^{13} \mathrm{C} \mathrm{NMR}\left(\mathrm{CDCl}_{3}, \delta\right): 180.8(\mathrm{CO}), 135.7$, 134.2, 129.7 (C5), 128.8, 128.7, 128.6 (C7), 79.8 (C9a), 43.2 (C3a), 43.1, 28.7 (Me), 27.2 (C4), 24.1 (C9), 20.6 (Me). MP: 102.8-104.3. Anal. Calc'd for $\mathrm{C}_{14} \mathrm{H}_{15} \mathrm{IO}_{2}$ : C, 49.14; $\mathrm{H}$, 4.42. Found: C, 49.15; H, 4.27. 
11-Iodo-3,3-dimethyl-3a,4,11,11a-tetrahydroanthra[2,3-b]furan-2(3H)-one (21).

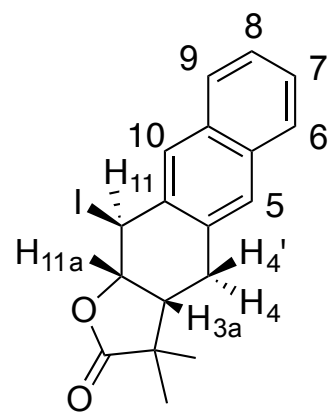

To a 4 dram vial was added $19(120 \mathrm{mg}, 0.451 \mathrm{mmol})$, water $(5 \mathrm{~mL})$, saturated $\mathrm{NaHCO}_{3}$ aq. (2 mL), KI (150 mg, $0.903 \mathrm{mmol}), \mathrm{I}_{2}(100 \mathrm{mg}, 0.397 \mathrm{mmol})$. This was stirred at room temperature for 2 days at which point $30 \mathrm{~mL}$ of DCM were added and the organic layer was evaporated in vacuo. Dissolved in DCM $(15 \mathrm{~mL})$ and $\mathrm{Et}_{2} \mathrm{O}(15 \mathrm{~mL})$, reduced to half volume and cooled in an ice bath for $30 \mathrm{~min}$. Isolated white precipitate on a $2 \mathrm{~mL}$ medium porosity frit, washed with $\mathrm{Et}_{2} \mathrm{O}(3 \times 2 \mathrm{~mL})$ and desiccated to yield product 21 (79 mg, 38\%). IR: $v(\mathrm{CO})=1759 \mathrm{~cm}^{-1} .{ }^{1} \mathrm{H}$ NMR $\left(\mathrm{CDCl}_{3}, \delta\right): 7.83(1 \mathrm{H}, \mathrm{s}, \mathrm{H} 10), 7.78(1 \mathrm{H}$, $\mathrm{d}, J=8.3, \mathrm{H} 6), 7.73(1 \mathrm{H}, \mathrm{d}, J=8.3, \mathrm{H} 9), 7.59(1 \mathrm{H}, \mathrm{s}, \mathrm{H} 5), 7.48(1 \mathrm{H}, \mathrm{t}, J=6.8, \mathrm{H} 7), 7.44$ $(1 \mathrm{H}, \mathrm{t}, J=7.6, \mathrm{H} 8), 5.76(1 \mathrm{H}, \mathrm{d}, J=3.1, \mathrm{H} 11) 5.40(1 \mathrm{H}, \mathrm{dd}, J=7.5 \& 3.1, \mathrm{H} 11 \mathrm{a}), 3.36$ (1H, ddd, $J=15.6,8.4,1.7, \mathrm{H}^{\prime}$ '), 3.02 (1H, d, $\left.J=15.6, \mathrm{H} 4\right), 2.95$ (1H, t, $\left.J=7.5, \mathrm{H} 3 \mathrm{a}\right)$, $1.38(3 \mathrm{H}, \mathrm{s}, \mathrm{Me}), 1.27(3 \mathrm{H}, \mathrm{s}, \mathrm{Me}) .{ }^{13} \mathrm{C} \mathrm{NMR}\left(\mathrm{CDCl}_{3}, \delta\right): 180.7$ (CO), 134.0, 132.8, 132.6, 132.2, 128.2, 128.1, 127.4, 127.3, 127.0, 126.3, 79.6 (H11a), 43.3 (H3a), 43.2, 28.7 (Me), 27.3 (H4), 24.5 (H11), 20.5 (Me). MP: 118.5-121.5. 


\subsection{References}

1. Winemiller, M. D.; Kelsch, B. A.; Sabat, M.; Harman, W. D. Organometallics 1997, 16, (16), 3672.

2. Winemiller, M. D.; Harman, W. D. J. Am. Chem. Soc. 1998, 120, (31), 7835.

3. Winemiller, M. D.; Harman, W. D. J. Org. Chem. 2000, 65, (5), 1249.

4. Valahovic, M. T.; Gunnoe, T. B.; Sabat, M.; Harman, W. D. J. Am. Chem. Soc. 2002, 124, (13), 3309.

5. $\quad$ Ding, F.; Valahovic, M. T.; Keane, J. M.; Anstey, M. R.; Sabat, M.; Trindle, C. O.; Harman, W. D. J. Org. Chem. 2004, 69, (7), 2257.

6. Ding, F.; Valahovic, M. T.; Keane, J. M.; Anstey, M. R.; Sabat, M.; Trindle, C. O.; Harman, W. D. J. Org. Chem. 2004, 69, (7), 2257.

7. Valahovic, M. T.; Gunnoe, T. B.; Sabat, M.; Harman, W. D. J. Am. Chem. Soc. 2002, 124, 3309.

8. Benn, R.; Mynott, R.; Topalovic, I.; Scott, F. Organometallics 1989, 8, 2299.

9. Tagge, C. D.; Bergman, R. G. J. Am. Chem. Soc. 1996, 118, 6908.

10. Mocella, C. J.; Delafuente, D. A.; Keane, J. M.; Warner, G. R.; Friedman, L. A.; Sabat, M.; Harman, W. D. Organometallics 2004, 23, (16), 3772.

11. Pienkos, J. A.; Zottig, V. E.; Iovan, D. A.; Li, M.; Harrison, D. P.; Sabat, M.; Salomon, R. J.; Strausberg, L.; Teran, V. A.; Myers, W. H.; Harman, W. D. Organometallics 2013, 32, (2), 691.

12. Graham, P.; Meiere, S. H.; Sabat, M.; Harman, W. D. Organometallics 2003, 22, 4364.

13. Chordia, M. D.; Smith, P. L.; Meiere, S. H.; Sabat, M.; Harman, W. D. J. Am. Chem. Soc. 2001, 123, (43), 10756.

14. Pienkos, J. A.; Knisely, A. T.; Liebov, B. K.; Teran, V.; Zottig, V. E.; Sabat, M.; Myers, W. H.; Harman, W. D. Organometallics 2013, 33, (1), 267.

15. Meiere, S. H.; Keane, J. M.; Gunnoe, T. B.; Sabat, M.; Harman, W. D. J. Am. Chem. Soc. 2003, 125, (8), 2024.

16. Cyclic voltammogram taken in DMA at $100 \mathrm{mV} / \mathrm{s}$, the positive value represents Epa and the negative value represents a reversible couple from a I/II Epa 
17. Meiere, S. H.; Valahovic, M. T.; Harman, W. D. J. Am. Chem. Soc. 2002, 124, (50), 15099.

18. Bard, A. J.; Faulkner, L. R., Electrochemical Methods Fundamentals and Applications. John Wiley \& Sons: New York, 1980.

19. Electrochemical analysis reveals that

20. Pines, H., Base-Catalyzed Reactions of Hydrocarbons and Related Compounds. Academic Press; Elsevier: New York, 1977.

21. Dowdy, D.; Gore, P. H.; Waters, D. N. J. Chem. Soc., Perkins Trans. 2 1991, (8), 1149.

22. Subrahmanyam, C.; Viswanathan, B.; Varadarajan, T. K. Journal of Molecular Catalysis A: Chemical 2005, 226, (2), 155.

23. Price, C. C.; Ciskowski, J. M. J. Am. Chem. Soc. 1938, 60, (10), 2499.

24. Kuendig, E. P.; Inage, M.; Bernardinelli, G. Organometallics 1991, 10, (8), 2921.

25. Atherton, J. C. C.; Jones, S. Tetrahedron 2003, 59, (46), 9039.

26. Zhou, J.; Xie, G.; Yan, X., Encyclopedia of Traditional Chinese Medicines Molecular Structures, Pharmacological Activities, Natural Sources and Applications: Vol. 4: Isolated Compounds N-S. Springer Science and Business Media: 2011; Vol. 4, p 636.

27. Ebada, S. S.; Schulz, B.; Wray, V.; Totzke, F.; Kubbutat, M. H. G.; Müller, W. E. G.; Hamacher, A.; Kassack, M. U.; Lin, W.; Proksch, P. Bioorganic \& Medicinal Chemistry 2011, 19, (15), 4644.

28. Bakhaeva, G. P.; Berlin, Y. A.; Chuprunova, O. A.; Kolosov, M. N.; Peck, G. Y.; Piotrovich, L. A.; Shemyakin, M. M.; Vasina, I. V. Chemical Communications (London) 1967, (1), 10.

29. Faulkner, D. J. Natural Product Reports 1991, 8, (2), 97.

30. Keane, J. M. Synthesis and Nucleophilic Activity of Eta-2 Arene Complexes. Ph. D. Dissertation, University of Virginia, 2003.

31. Lis, E. C.; Delafuente, D. A.; Lin, Y.; Mocella, C. J.; Todd, M. A.; Liu, W.; Sabat, M.; Myers, W. H.; Harman, W. D., Organometallics 2006, 25 (21), 5051. 


\title{
Chapter 3
}

\section{4-(Dimethylamino)pyridine (DMAP) as an Acid-}

\section{Modulated Donor Ligand for PAH}

\author{
Dearomatization
}




\subsection{Introduction}

In the previous chapter the ability to perform 1,2-addition reactions to naphthalene and anthracene using the $\{\mathrm{TpMo}(\mathrm{NO})(\mathrm{MeIm})\} \quad(\mathrm{Tp}=$ hydridotris(pyrazolyl)borate; MeIm = 1-methylimidazole) system was demonstrated. ${ }^{1,} 2$ This molybdenum dearomatization agent offers the advantages of facile liberation of organic products and recyclability of the $\{\mathrm{TpMo}(\mathrm{NO})(\mathrm{MeIm})\}$ moiety, as well as a substantially lower cost than its heavy metal congeners (e.g., $\left\{\mathrm{Os}\left(\mathrm{NH}_{3}\right)_{5}\right)^{2+}, \quad\{\mathrm{TpRe}(\mathrm{CO})(\mathrm{L})\}$, $\left.\left\{\mathrm{TpW}(\mathrm{NO})\left(\mathrm{PMe}_{3}\right)\right\}\right)$. However, the $\{\mathrm{TpMo}(\mathrm{NO})(\mathrm{MeIm})\}$ fragment is more vulnerable to oxidation by electrophilic reagents such as triflic acid (Scheme 3.1), compared to its Os, Re, and $\mathrm{W}$ analogues. ${ }^{1}$ Previous research with rhenium has shown the ability to significantly influence the reduction potential of the metal by altering the ancillary ligand. We hoped to find an alternative to the MeIm complex that can still bind and activate polycyclic aromatic hydrocarbons (PAHs), but is less susceptible to metal oxidation by electrophilic reagents.

Herein, we report that by the simple replacement of MeIm with 4(dimethylamino)pyridine (DMAP), a significantly greater scope of addition reactions is accessed for both naphthalene and anthracene, while the economic and environmental benefits of the imidazole system are still maintained. The key to this success lies in the ability of the DMAP ligand to accept a proton at the dimethylamino group, and thereby reduce the donation of electron density to the metal from the pyridine ring. $^{3}$ Consequently, the $\mathrm{d}^{5} / \mathrm{d}^{6}$ reduction potential of the metal is temporarily raised, rendering it less susceptible to unintended oxidation by electrophilic reagents (Scheme 3.1). While the protonation of a DMAP ligand has been used previously as a "switch" to modulate 
the chemistry of a transition metal, ${ }^{4}$ the acid normally effects the removal of the DMAP, opening a coordination site on the metal, rather than influencing the redox properties of the metal via the coordinated DMAP.

Scheme 3.1. Arene protonation versus metal oxidation.<smiles></smiles>

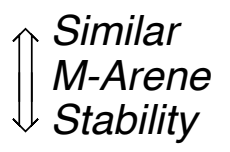<smiles>CCC[18O]c1cccc2ccccc12</smiles><smiles>[Y]C(C)(C)C(=O)Oc1cccc2ccccc12</smiles>
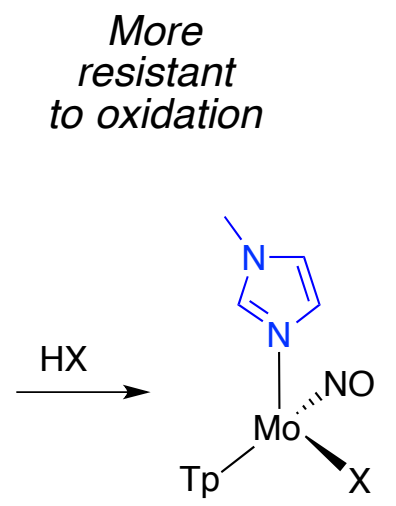

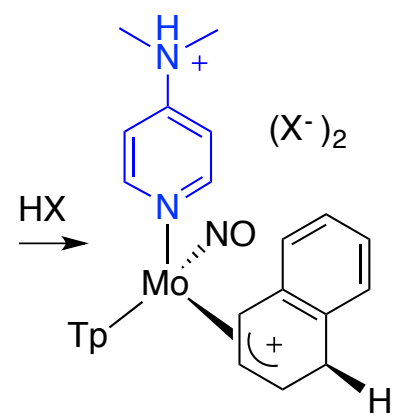

Electrophilic addition

\subsection{Results}

\subsubsection{Synthesis of $T p M o(N O)(D M A P)(L \pi)$}

Similar to the synthesis of $\mathrm{TpMo}(\mathrm{NO})(\mathrm{MeIm})(\mathrm{I}), \mathrm{TpMo}(\mathrm{NO})(\mathrm{DMAP})(\mathrm{I})$ (23) can be synthesized from $\operatorname{Mo}(\mathrm{CO})_{6}$ in air and without chromatography in an overall yield of $66 \%$ on a $170 \mathrm{~g}$ scale (> $80 \%$ per step; Scheme 3.2). Of note, the Mo(I) complex (23) was found to be stable in air for over three months, showing no signs of decomposition. This 
is in dramatic contrast to its tungsten counterpart, $\mathrm{TpW}(\mathrm{NO})\left(\mathrm{PMe}_{3}\right)(\mathrm{Br})$, which decomposes rapidly upon exposure to air. ${ }^{5}$

The precursor to 23, TpMo(NO)(CO)(DMAP) (22), was found to be only slightly less electron-rich in comparison to its MeIm analogue, as judged from an increase in the nitrosyl (from 1577 to $1589 \mathrm{~cm}^{-1}$ ) and carbonyl (from 1867 to $1871 \mathrm{~cm}^{-1}$ ) stretch frequencies as well as a more positive potential for the $\mathrm{Mo}^{0} / \mathrm{Mo}^{\mathrm{I}}$ oxidation observed in a voltammogram (from $E_{\mathrm{p}, \mathrm{a}}=+0.22$ to $0.27 \mathrm{~V}$; NHE, $\left.100 \mathrm{mV} / \mathrm{s}, \mathrm{DMAc}\right){ }^{1}$

In order to compare the ability of $\{\mathrm{TpMo}(\mathrm{NO})(\mathrm{DMAP})\}$ to bind aromatic molecules with its predecessor, $\{\mathrm{TpMo}(\mathrm{NO})(\mathrm{MeIm})\}$, three ligands were investigated: naphthalene (24), anthracene (25) and 2,5-dimethylfuran (26). The naphthalene and anthracene complexes were prepared on 13 -gram scales $(\sim 50 \%$ yield $)$ via the reduction of $\mathbf{2 3}$ in the presence of an excess of the arene. The 2,5-dimethylfuran complex 26 was chosen because of its anticipated role as a precursor to other $\{\mathrm{TpMo}(\mathrm{NO})(\mathrm{DMAP})\}$ complexes, similar to that found for the furan and 2,5-dimethylfuran complexes of $\{\mathrm{TpMo}(\mathrm{NO})(\mathrm{MeIm})\} .{ }^{6,7}$ Reducing 23 with elemental sodium in the presence of 2,5dimethylfuran generates 26, which can be isolated in pure form from MeOH (CAUTION: exothermic production of hydrogen) in 54\% yield (13 g scale), without the need for chromatography.

The dimethylfuran complex $\mathbf{2 6}$ undergoes substitution in acetone forming the complex TpMo(NO)(DMAP) $\left(\eta^{2}\right.$-acetone), with a $k_{o b s}$ of $8.8 \times 10^{-5} \mathrm{~s}^{-1}$ at $22 \pm 1{ }^{\circ} \mathrm{C}$. This is similar to that observed for the MeIm analogue, where $k_{o b s}=8.2 \times 10^{-3} \mathrm{~s}^{-1}\left(22 \pm 1{ }^{\circ} \mathrm{C}\right)$. This represents a free energy of activation of 23.0 and $22.9 \pm 0.1 \mathrm{kcal} / \mathrm{mol}$ for these complexes, respectively, values well below that observed for naphthalene $(26.6 \pm 0.1$ 
$\mathrm{kcal} / \mathrm{mol}$ at $\left.100{ }^{\circ} \mathrm{C} ; \mathrm{L}=\mathrm{MeIm}\right){ }^{7}$ Utilization of this low barrier for substitution of 2,5dimethylfuran is demonstrated by its exchange with $\alpha, \alpha, \alpha$-trifluorotoluene and 2methoxypyridine in Chapters 5 and 6, respectively.

Scheme 3.2. Synthesis of $\eta^{2}$-coordinated naphthalene, anthracene, and 2,5-dimethylfuran complexes.

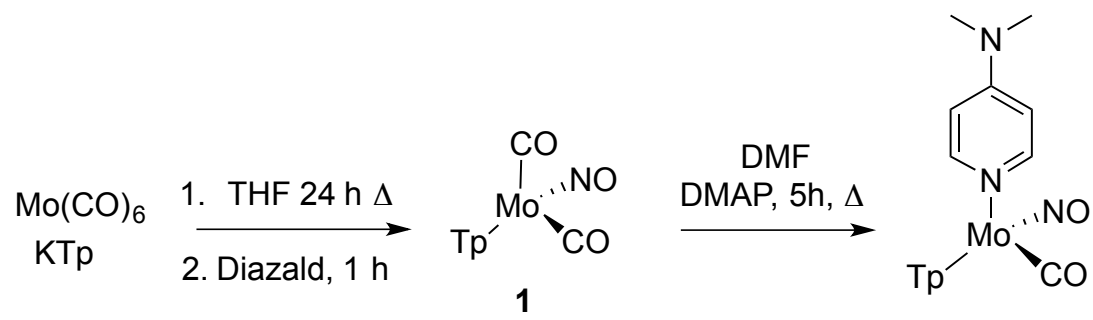

22, $80 \%$

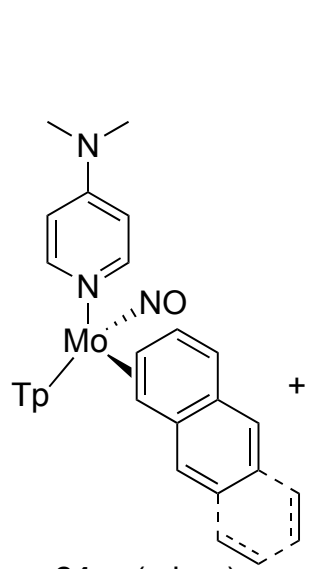

24 ; (minor)

25d; (minor)

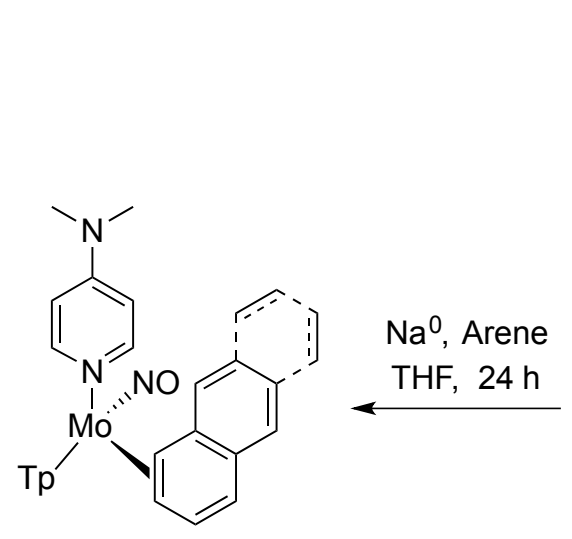

$\mathrm{I}_{2} \mathrm{DCM}$

24p, 50\%; Naphthalene (major)

25 , 47\%; Anthracene (major)

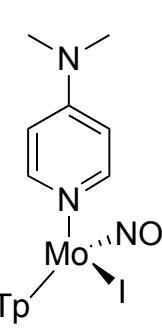

23, $88 \%$

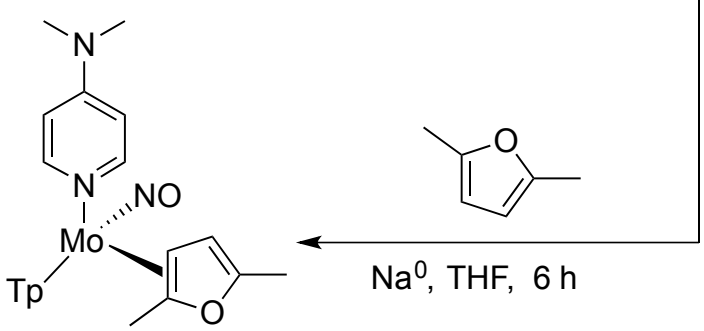

26, $54 \%$ 


\subsubsection{Synthesis of TpMo(NO)(DMAP) $\left(\eta^{2}\right.$-arenium) complexes}

In order to test our central hypothesis that DMAP could be protonated and thereby better prevent metal oxidation than its MeIm forbearer, the naphthalene complex 24 was subjected to HOTf (in $\mathrm{CD}_{3} \mathrm{CN} ;-30{ }^{\circ} \mathrm{C}$ ) and was monitored over time by ${ }^{1} \mathrm{H}$ NMR (25 $\left.{ }^{\circ} \mathrm{C}\right)$. In contrast to that observed for the MeIm complex, with DMAP the formation of a stable allyl complex (24-H) was observed. Key spectroscopic features include diastereotopic methylene protons at 4.78 and $4.75 \mathrm{ppm}$, both doublets with $J=27.6 \mathrm{~Hz}$. This large coupling constant is consistent with a strong $\pi$-acceptor adjacent to the geminal protons. ${ }^{8}$ Another spectroscopic feature for $\mathbf{2 4 - H}$ is a broad singlet at $9.97 \mathrm{ppm}$ that has correlations in COSY and NOESY spectra to two distinct methyl signals near $3.26 \mathrm{ppm}$ (both d with $J=5.0 \mathrm{~Hz}$ ). These data support the hypothesis that both the dimethylamino group and the naphthalene in $\mathbf{2 4 - H}$ are protonated (Scheme 3.3). Although attempts to isolate this dicationic species were unsuccessful, it was found that a triflate salt could be precipitated from the reaction mixture with $\mathrm{Et}_{2} \mathrm{O}$, and the intact allyl complex (27) could be isolated (95\%), observed at room temperature ( ${ }^{1} \mathrm{H}$ NMR), and stored indefinitely at $-30{ }^{\circ} \mathrm{C}$. Of note, the amino group in 27 no longer appears to be protonated, but allylic protons at 7.12, 5.72 and $5.22 \mathrm{ppm}$ and a diastereotopic methylene group (4.77 and $4.74 \mathrm{ppm}$ ) indicate that the allyl-like naphthalenium ligand is intact. In contrast, an allyl complex analogous to $\mathbf{2 7}$ could not be isolated or fully characterized in solution for the MeIm system. ${ }^{1}$

In a similar fashion, the allyl complex of anthracene (28) was isolated, via the formation of the purported dicationic intermediate $\mathbf{2 5 - H}$. The thermal stability of $\mathbf{2 8}$ is similar to that observed for its naphthalene derivative, 27. In both $\mathbf{2 7}$ and $\mathbf{2 8}$, two of the 
three allylic carbon resonances are well upfield of the third (129.1 (C2), 102.1 (C3) and $88.3(\mathrm{C} 4) \mathrm{ppm}$ ), indicating that the allyl is asymmetrically bound to the metal center. This feature has been observed previously for naphthalenium complexes of $\mathrm{W}$ and $\mathrm{Re},{ }^{9}$ as well as a cyclopentadiene-derived allyl complex of $\mathrm{TpMo}(\mathrm{NO})(\mathrm{MeIm}){ }^{7}$ Where available, structural data indicate that this downfield allylic carbon is $\sim 0.2-0.3 \AA$ farther from the metal than the other allylic carbons and is cationic in nature. ${ }^{9}$ We therefore portray allyl complexes as $\eta^{2}$-coordinated allyl cations (Eq 3.1).

Scheme 3.3. Protonation and isolation of allylic naphthalene and anthracene complexes.<smiles></smiles><smiles>c1ccc2ccccc2c1</smiles><smiles>CCCP(=O)(O)N(C)c1ccc(N(C)C)cc1</smiles>

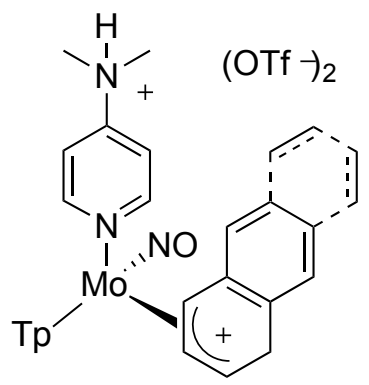

27; 95\%; Naphthalene 28; 96\%; Anthracene 


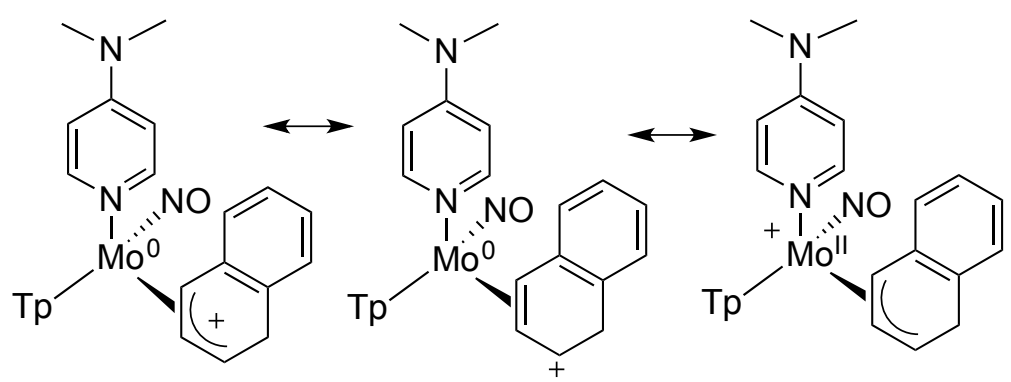

Eq 3.1.

To estimate the impact on the metal from protonating the amino group of DMAP, the model compound $\mathrm{TpMo}(\mathrm{NO})($ pyridine$)\left(\eta^{2}\right.$-naphthalene) (29) was synthesized. ${ }^{10}$ Importantly, there is a minor decrease in the electron density on molybdenum for $\operatorname{TpMo}(\mathrm{NO})(\mathrm{py})\left(\eta^{2}\right.$-naphthalene), as compared to its DMAP analogue, (24), as judged by an increase in nitrosyl stretching frequency $\left(1585 \mathrm{~cm}^{-1}\right.$ vs. $\left.1580 \mathrm{~cm}^{-1}\right)$ and position of the oxidation wave $\left(E_{\mathrm{p}, \mathrm{a}}=-0.07 \mathrm{~V}\right.$ vs. $\left.-0.16 \mathrm{~V} ; 100 \mathrm{mV} / \mathrm{s}\right)$. Furthermore, the ligand exchange in acetone $\left(t_{1 / 2}=29 \mathrm{~h}\right.$ at $\left.22{ }^{\circ} \mathrm{C} \pm 1{ }^{\circ} \mathrm{C}\right)$ leads to a $\Delta \mathrm{G}^{\ddagger}$ for 29 of $24.5 \pm 0.1 \mathrm{kcal} / \mathrm{mol}$, significantly lower than that of $24\left(26.0 \pm 0.1 \mathrm{kcal} / \mathrm{mol} ; \mathrm{T}=22 \pm 1{ }^{\circ} \mathrm{C}\right)$. This difference indicates weaker backbonding into the arene $\pi$-system due to less electron density donated from the ancillary ligand (py $c f$. DMAP). Similar to $\mathbf{2 4}$, the pyridine complex 29 can be protonated to form an allyl species analogous to 27 at $-30{ }^{\circ} \mathrm{C}$; however, upon warming to $25^{\circ} \mathrm{C}$ the complex decomposes. This observation demonstrates the potential dual role that the amino group of DMAP plays in supporting the formation and stability of an allyl complex: in its protonated form it protects the metal from oxidation while the allyl is being formed. Once formed, however, the amino group can be deprotonated to provide more electron density and stabilize the highly electrophilic arenlenium cation (see Scheme 3.3). 


\subsubsection{Protonation and nucleophilic addition to 24 and 25}

Next, we endeavored to determine if the replacement of DMAP for MeIm impacted the ability of the metal to activate the arene toward addition reactions. Previously, ${ }^{1}$ we reported that protonation could be accomplished at $\mathrm{C} 1$ followed by the addition of a carbon nucleophile at $\mathrm{C} 2$, and that the final dihydroarene product $(\mathbf{O})$ could be removed by action of iodine (Scheme 3.4).

Scheme 3.4. Formal catalytic cycle for the generation of dihydronaphthalenes and dihydroanthracenes.
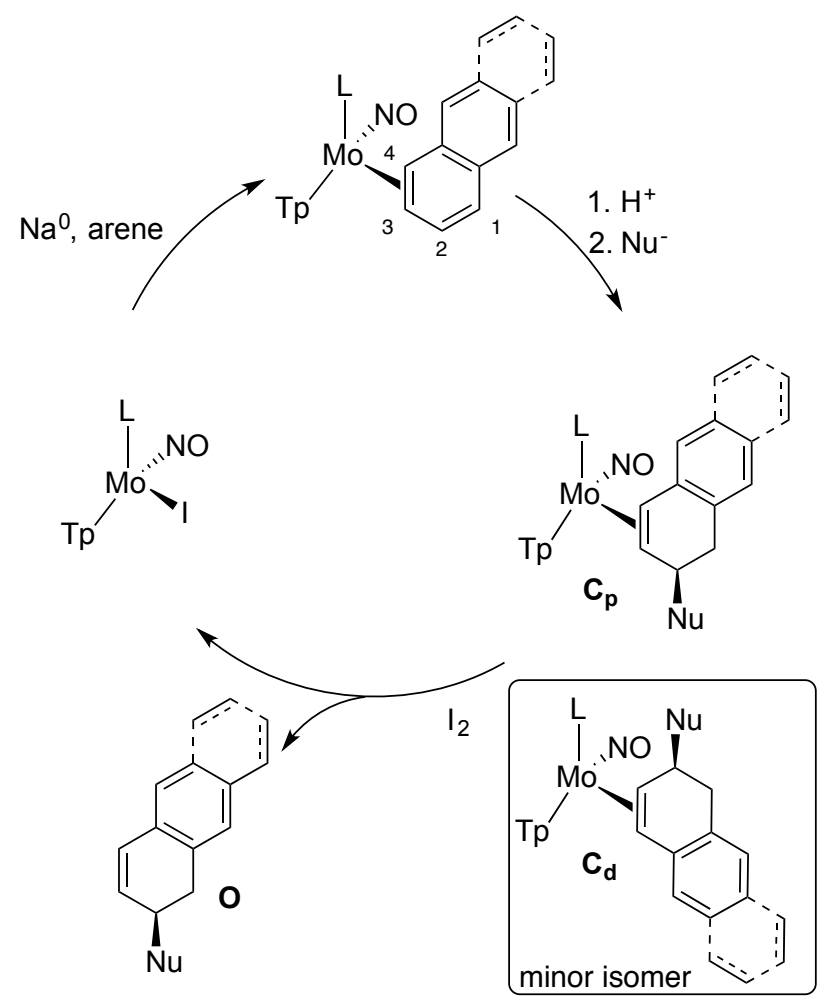

Table 3.1 presents summarized results for conjugate additions to naphthalene (24) and anthracene (25) complexes using triflic acid as the electrophile and three types of carbon nucleophiles - a pyrrole, a silylated ester enolate, and a lithiated enolate - for both 
MeIm and DMAP systems. We found that when MeIm is replaced by DMAP, the yield for isolation of $\mathbf{C}$ (ranging from $41-95 \%$ ) improved by an average of $34 \%$ compared to those yields obtained with the MeIm system (ranging from 27-66\%). Although the yield for organic isolation $\mathbf{O}$ generally decreased directly from $\mathbf{C}$, the improved yield of $\mathbf{C}$ led to an average $30 \%$ increase in overall yield of $\mathbf{O}$ when starting from $\mathbf{2 4}$ or $\mathbf{2 5 .}$

Table 3.1. Isolated Dihydroarenes (O) from Iodine Oxidation of $\mathbf{C}$.

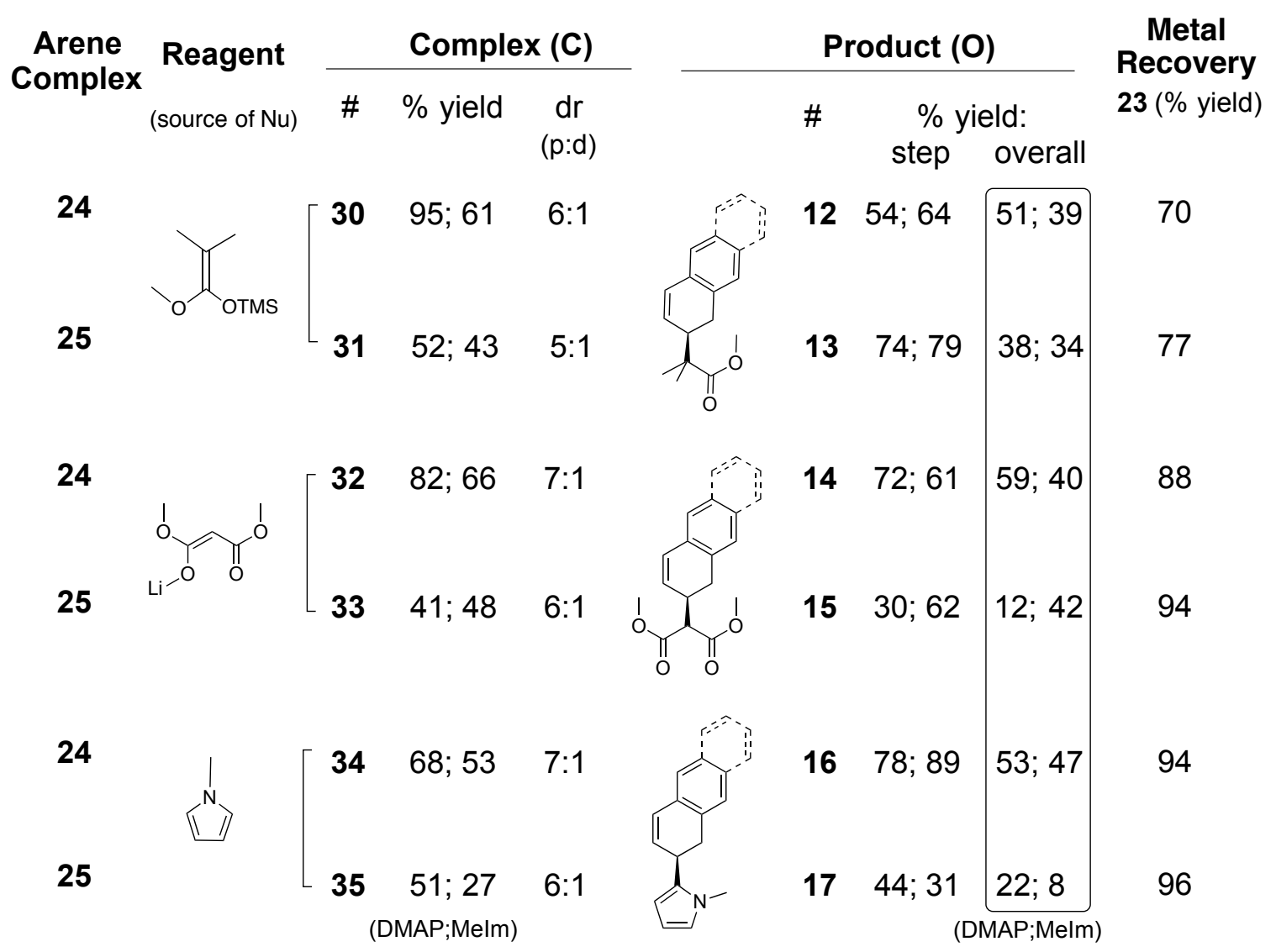

Of note, the product complexes were isolated as a mixture of two coordination diastereomers, differing by which face of the ring is coordinated. These are represented as $\mathbf{C}_{\mathbf{p}}$ (proximal to $\mathrm{L}$ ) and $\mathbf{C}_{\mathbf{d}}$ (distal to $\mathrm{L}$ ) (Scheme 3.4). In all cases observed, the 
dominant isomer $\left(\mathbf{C}_{\mathbf{p}}\right)$ featured the uncoordinated ring(s) oriented proximal to the DMAP. The allyl intermediates (27 and 28) were observed in solution as a 6:1 ratio of diastereomers causing a decrease in the stereoselectivity of $\mathbf{C}_{\mathbf{p}}: \mathbf{C}_{\mathbf{d}}$ from 25:1 to $\sim 5: 1$ compared to the MeIm system. ${ }^{1}$ Even with the clear preference in both DMAP and MeIm systems for the $\mathbf{C}_{\mathbf{p}}$ isomer to dominate, a crystal grown from a solution of the ester product $\mathbf{3 0}$ was found to contain the minor distal stereoisomer $\left(\mathbf{C}_{\mathbf{d}}\right)$. In Figure 3.2, this solid-state structure $\left(\mathbf{3 0}_{\mathbf{d}}\right)$ is compared to the proximal isomer of the MeIm analogue, where the reader will note the enantiomeric relationship of the dihydronaphthalene ligands, despite similar metal configurations.
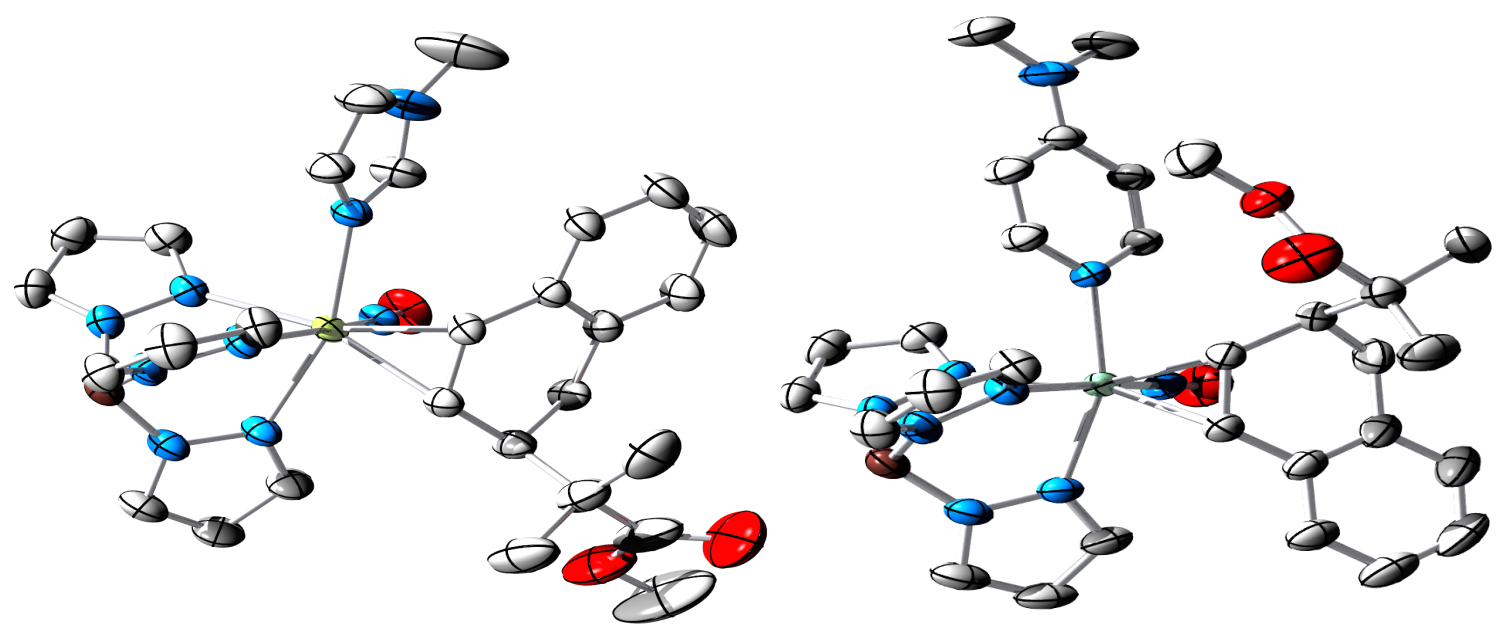

Figure 3.1. Solid-state structures of two dihydronaphthalene complexes 30, using MeIm $(\mathrm{Left})^{11}$ and DMAP (Right). Bond lengths of $30(\AA)$ : Mo-C(4) 2.2492(19); Mo-C(3) 2.2157(19); Mo-N(DMAP) 2.2228(16); Mo-N(NO) 1.7549(17). 


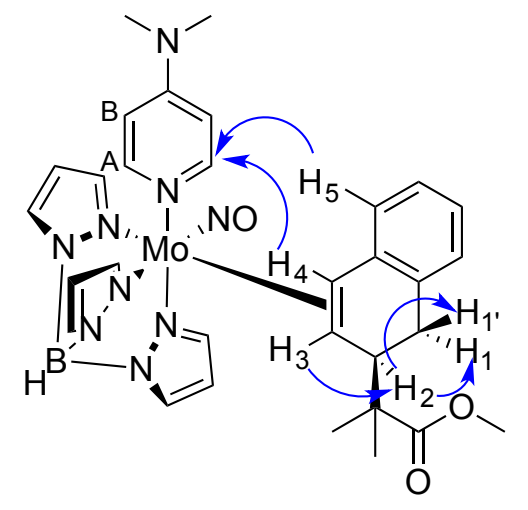

Figure 3.2. NOESY correlations for 30p.

In addition to the nine protons of the Tp ligand observed in the ${ }^{1} \mathrm{H}$ NMR spectra of these complexes, the DMAP ancillary ligand offers the following three sets of protons: a broad singlet integrating for 2 protons around 7.40 ppm (DMAP-A), a multiplet integrating for 2 protons around $6.50 \mathrm{ppm}$ (DMAP-B), and a singlet integrating for 6 protons around $3.00 \mathrm{ppm}$ (N-Me's). Identically to the MeIm analogue, 30p has a diastereotopic methylene group ( $\mathrm{H} 1$ and $\mathrm{H}^{\prime}$ ') that was confirmed by its large coupling constant $(16.6 \mathrm{~Hz})$ and HSQC. Similarly, H2 (3.19 ppm) was identified through COSY and NOESY correlations, leading to the identification of $\mathrm{H} 3$, which is a doublet of triplets at $2.13 \mathrm{ppm}$ identical to that found for the MeIm system. Through COSY correlation, H4 was found to be a doublet at $3.24 \mathrm{ppm}$. Using NOESY, H4 was found to correlate through space with a doublet in the aromatic region (H5) as well as with DMAP-A, confirming the major isomer in the solution phase to orient the bulk of the organic ligand proximal, rather than distal, to the DMAP ligand. This is a different coordination isomer to the one seen in the crystal structure for this complex (vide infra). To further confirm this, H5 was found to strongly correlate via NOE with DMAP-A (Figure 3.2). 


\subsubsection{Nucleophilic additions to $\mathbf{2 7}$ and $\mathbf{2 8}$}

Table 3.2. Synthesis of nitromethyl- and pyrazolyl- substituted dihydroarenes.

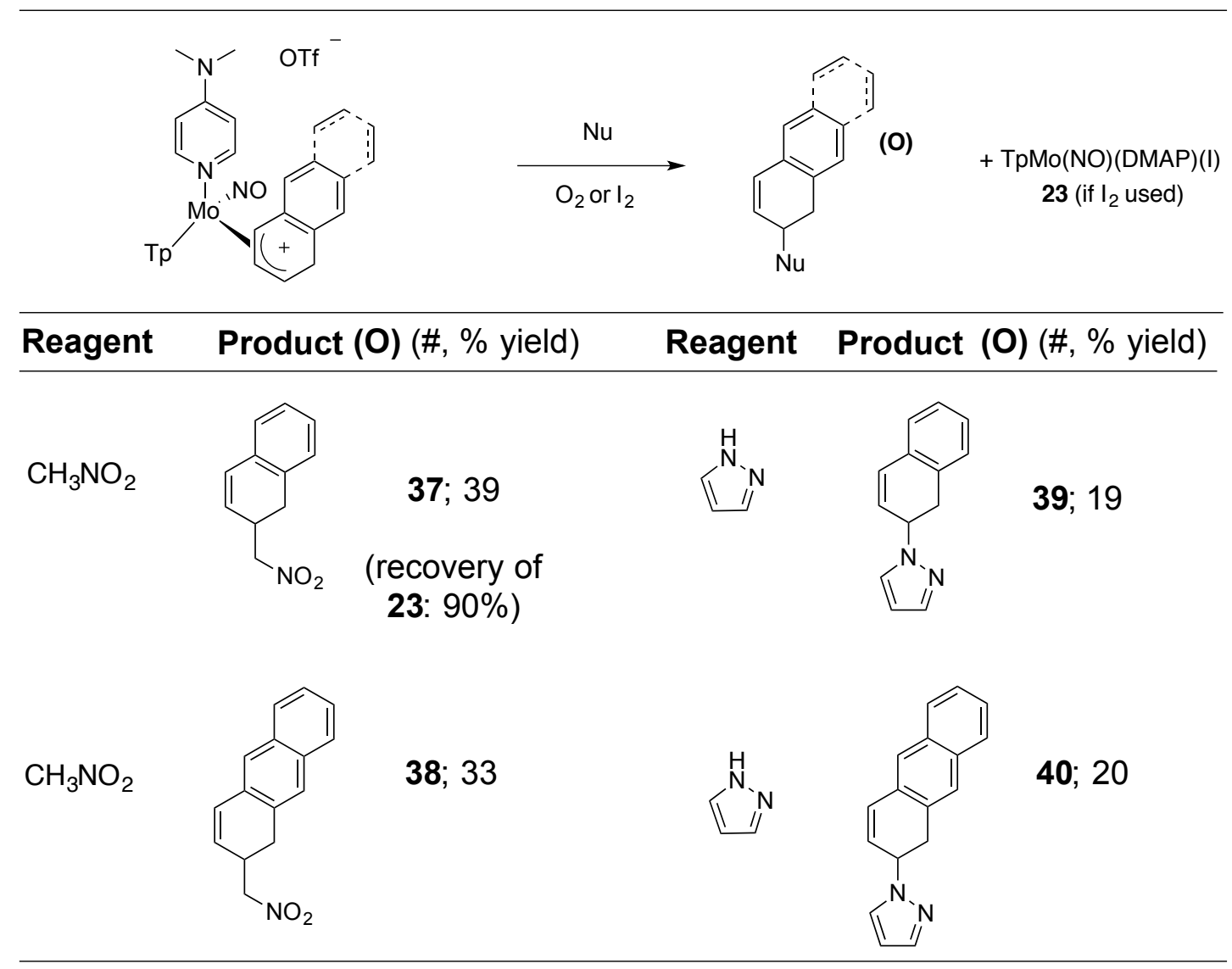

The ability to isolate allyl complexes $\mathbf{2 7}$ and $\mathbf{2 8}$ away from the strong acid required for their preparation presents the opportunity to explore other classes of nucleophiles that would otherwise be compromised by Brønsted acids. Gratifyingly, we found that both nitromethane (Henry reaction) and pyrazole could be added to C2 of either $\mathbf{2 7}$ or $\mathbf{2 8}$. In general, the corresponding complexes proved difficult to isolate, but when the reaction mixture was subjected to air, the desired organic products were obtained in moderate yield. In the case of the nitromethane addition to $\mathbf{2 7}$, the complex (36) could be cleanly isolated (63\%). When $\mathbf{3 6}$ was subsequently treated with iodine the 
precursor $\mathbf{2 3}$ was recovered in $90 \%$ yield. Of the four organic products formed in Table 3.2, only compound $\mathbf{4 0}$ could be obtained from the $\{\mathrm{TpMo}(\mathrm{NO})(\mathrm{MeIm})\}$ system $(12 \%)$.

\subsubsection{Varying the electrophiles added to $\mathbf{2 4}$ and $\mathbf{2 5}$}

The enhanced ability of the DMAP system to resist oxidation by acid also provides the opportunity to expand the range of electrophiles that add to $\mathrm{C} 1$. Attempts were made to add a single equivalent of acid, be it Brønsted or Lewis, to the dimethylamino group to achieve a greater reductive potential at the metal center and subsequently add a greater variety of electrophiles; however, this process was unsuccessful. Reagents explored include halogens, epoxidation agents, and nitrilium salts, all of which led to oxidation of the metal complex. However, when treated with trifluoromethylsilyl triflate (TMSOTf) at $-60{ }^{\circ} \mathrm{C}$, the acetal 2,2-dimethoxypropane reacts with $\mathbf{2 4}$ or $\mathbf{2 5}$ to give an allyl which can be trapped with lithium dimethylmalonate (LiDMM) to form complexes $\mathbf{4 1}$ and $\mathbf{4 2}$, respectively (Scheme 3.5).

Unlike the other arene reactions in this account, the products $\mathbf{4 1}$ and $\mathbf{4 2}$ are the result of 1,4-addition reactions (Scheme 3.5). While the yields are moderate $(\sim 60 \%)$, these complexes are formed with good stereocontrol $(\mathrm{dr}>8: 1$ for $\mathbf{C p}: \mathbf{C d})$, with both additions occurring anti to the metal. Upon treatment with iodine, organics 43 and 44 were obtained in moderate yield and with good recovery of the precursor $\mathbf{2 3}$ (Scheme 3.5). A compound similar to dihydronaphthalene $\mathbf{4 3}$ has been previously obtained on a 40 mg scale from naphthalene, lithium dimethylmalonate, and dimethoxymethane using osmium(II) or rhenium(I) dearomatization agents, ${ }^{11,12}$ but these expensive heavy metals were not recovered. 
Scheme 3.5. Tandem addition of dimethoxypropane, and LiDMM, to 24 and 25.<smiles>CC(C)N1C=CC=c2cc3ccccc3cc2=C1</smiles>

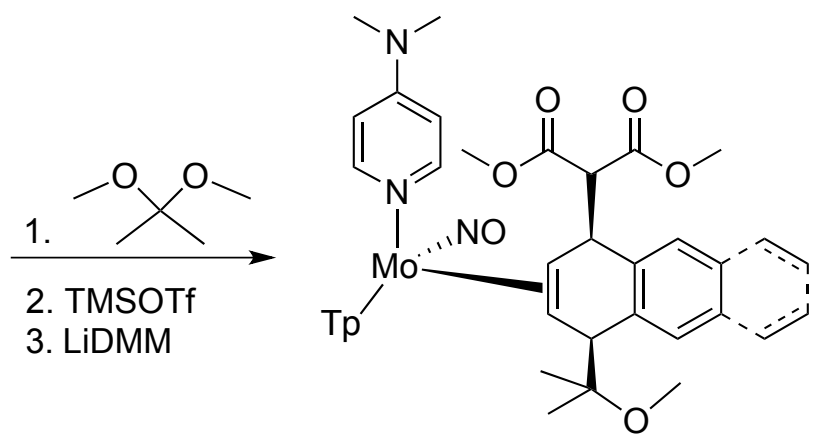

41; $63 \%$; Naphthalene

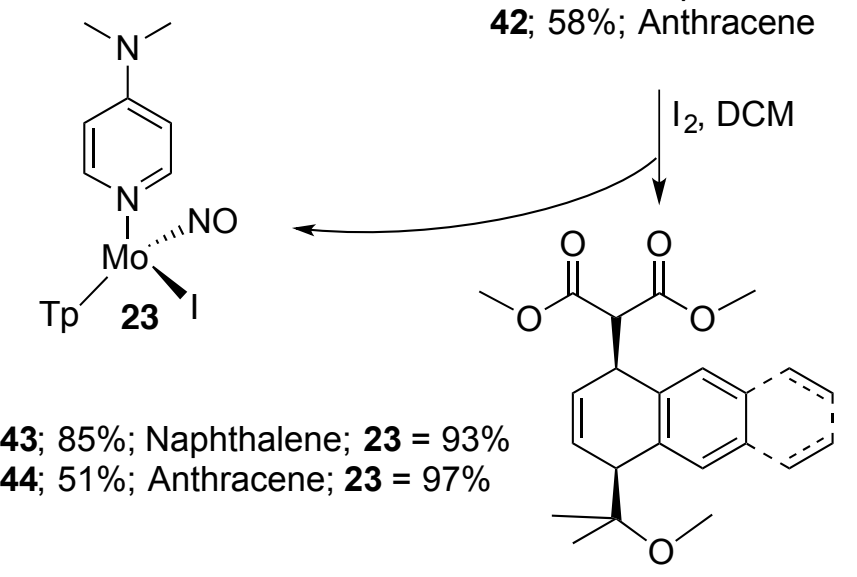

Other 1,4-tandem additions were achieved by using various carbon electrophiles paired with sodium cyanoborohydride $\left(\mathrm{NaCNBH}_{3}\right)$ as the nucleophilic partner (Table 3.3). In addition to acetals (1,1-dimethoxycyclohexane as well as the previously mentioned dimethoxypropane), Michael acceptors were found to successfully add to $\mathrm{C} 1$ of the PAH, as demonstrated by the use of 3-penten-2-one and cyclopentenone (entries 49-52). 
Table 3.3. Addition of various electrophiles to 24 and 25 .
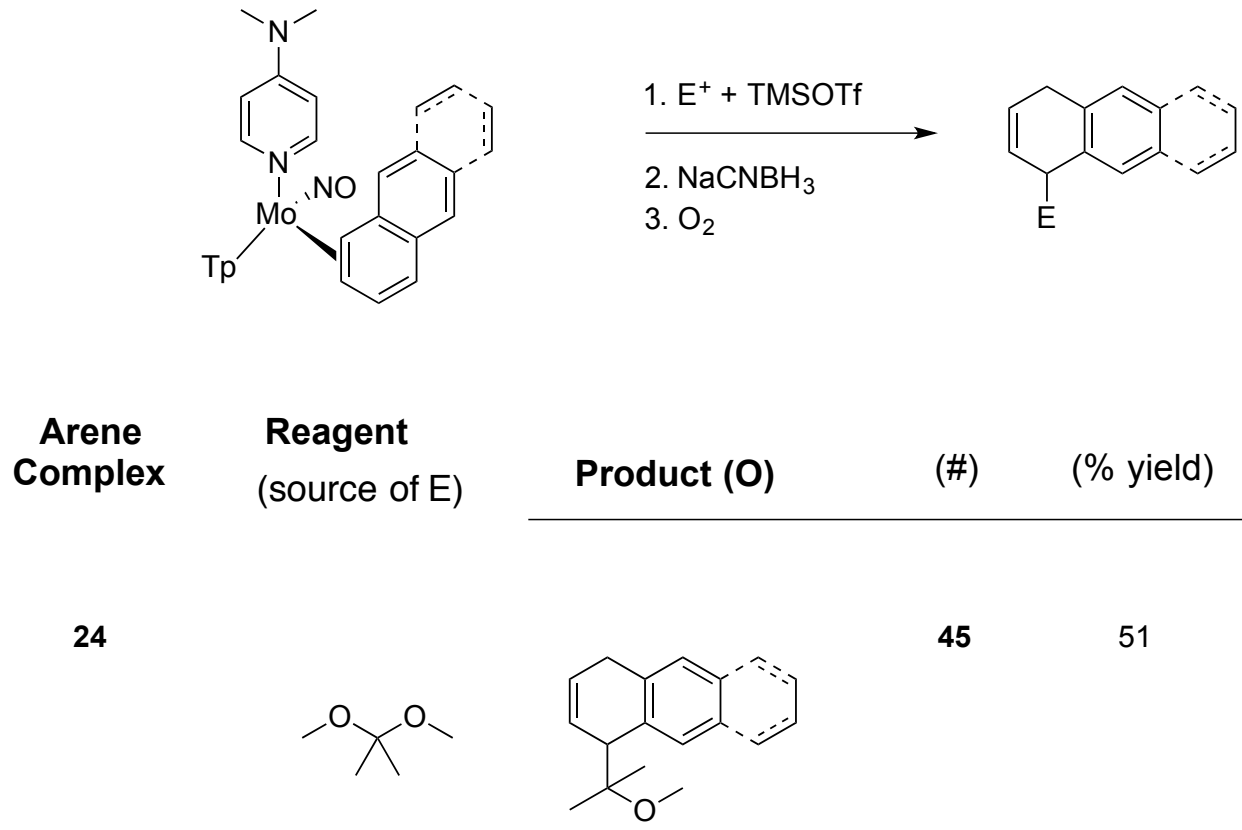

25

46

25

$>20: 1$
24

25<smiles>COC1(OC)CCCCC1</smiles>

24

25<smiles>C/C=C/C(C)=O</smiles><smiles>CC(=O)CC(C)(C)C1C=CCc2cc3ccccc3cc21</smiles>

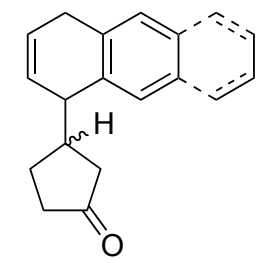

25<smiles>O=C1C=CCC1</smiles>

4<smiles>C1CCCC1</smiles>

5
45

51

$>20: 1$<smiles>COC1(C2C=CCc3cc4ccccc4cc32)CCCCC1</smiles>

48

28

4:1

49

50

38

61

$>20: 1$

$d r=2: 1$

2:1

$d r=2: 1$

and 3.5:1

51

27

$>20: 1$

$d r=10: 1$ 
In general, the addition of enones to the PAH complexes resulted in the formation of 1,4-addition products, isolated as a mixture of two diastereomers that presumably are epimers at the benzylic position. This presumption is based on the ${ }^{1} \mathrm{H}$ NMR observation of identical splitting patterns with small differences in chemical shifts. In one case (50) the dihydroanthracene product is believed to be a mixture of four isomers, as supported by the observation of four individual singlets at $2.15,2.05,2.01$, and $1.97 \mathrm{ppm}$ as well as doublets at $1.03,0.90,0.88$ and $0.73 \mathrm{ppm}$. These isomers are believed to be a mixture of 1,4 and 1,2-addition products, as well as the epimers of these 1,4 and 1,2-addition products. Corresponding alkene peaks for the 4 isomers are overlapping, preventing their unambiguous assignment. Due to the complex mixture, $\mathbf{5 0}$ was not fully characterized; however, combustion analysis revealed this isomeric mixture to be a match for the desired product when solvent was taken into account. To further test the enhanced stability of DMAP in comparison to MeIm, 45 was also prepared using TpMoNO(MeIm) $\left(\eta^{2}\right.$-naphthalene) with a significant $26 \%$ decrease in yield in comparison to 24 .

\subsection{Discussion}

Unlike the $\left\{\mathrm{TpW}(\mathrm{NO})\left(\mathrm{PMe}_{3}\right)\right\}$ fragment, the ancillary ligand (L) of the $\{\mathrm{TpMo}(\mathrm{NO})(\mathrm{L})\}$ fragment can be changed while still retaining the metal center's ability to bind a wide variety of aromatics. In this way, greater control of the metal center's electron donating abilities is obtained. Isolation of the allyls $\mathbf{2 7}$ and $\mathbf{2 8}$ represents a unique ability of DMAP to have a sequential dual role. In its protonated state, DMAP 
protects the metal center from oxidation by reducing electron donation into the metal. In its deprotonated state, DMAP donates electron density into the metal to help stabilize the allylic species. The allylic species $\mathbf{2 7}$ and $\mathbf{2 8}$ can be isolated and observed at room temperature via ${ }^{1} \mathrm{H}$ NMR. As seen in Figure 3.3, the degradation of 27 (noted by the increased formation of free naphthalene) is $\sim 50 \%$ after $30 \mathrm{~min}$; however, after $1.5 \mathrm{~h}$ at room temperature, significant decomposition has occurred. This is noteworthy when considering that with MeIm as the ancillary ligand, observation of $\left[\mathrm{TpMo}(\mathrm{NO})(\mathrm{MeIm})\left(2,3,4-\eta^{3}\right.\right.$-naphthalenium) $](\mathrm{OTf})$ at room temperature has not been achieved.

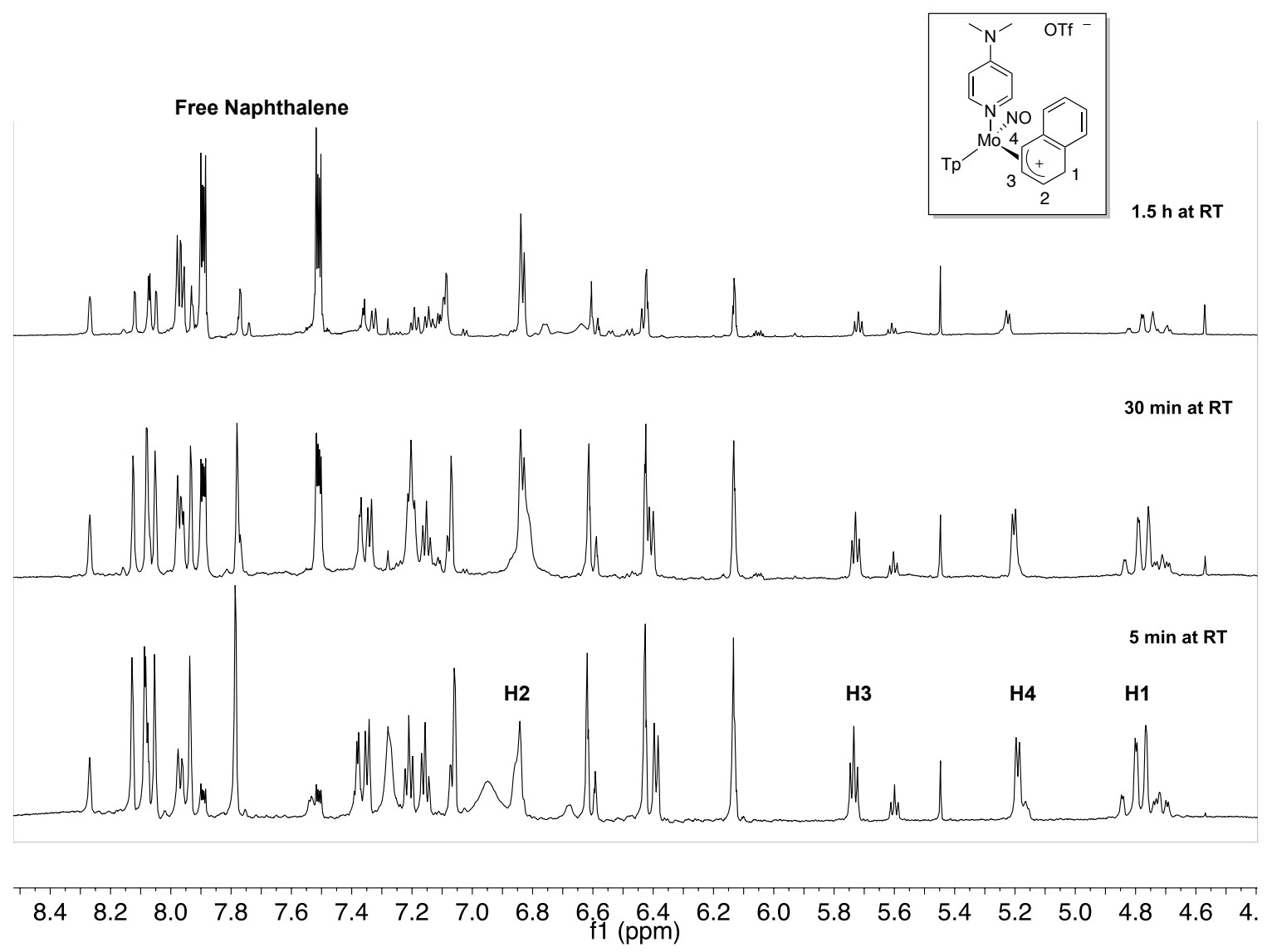

Figure 3.3. Monitoring the decomposition of 27 by ${ }^{1} \mathrm{H}$ NMR over $1.5 \mathrm{~h}$ at $\mathrm{RT}$ in $\mathrm{CD}_{3} \mathrm{CN}$. 
The cationic allyl of 27 significantly withdraws the electron density from the metal center as seen by an increase in the nitrosyl stretching frequency compared to the starting material 24 (from $1580 \mathrm{~cm}^{-1}$ to $1620 \mathrm{~cm}^{-1}$ ). These allylic complexes are asymmetric in their orientation of binding unlike typical complexes binding in an eta-3 fashion. In agreement with this asymmetry, the chemical shifts for the carbons associated with the allyl (27) are as follows: 129.1 (C2), 102.1 (C3) and $88.3(\mathrm{C} 4)$. Of note, C2 is significantly more upfield in $\mathbf{2 7}$ than in the MeIm analogue where C2 is at $143.4 \mathrm{ppm}$.

As proposed in Chapter 2, a greater stability under oxidative conditions has indeed proven to enhance the breadth of chemical reactivity available for $\{\mathrm{TpMo}(\mathrm{NO})(\mathrm{L})\}$. A few points should be made referring back to the potential pathways of the stepwise electrophilic-nucleophilic additions discussed in Chapter 2. The same qualitative observations are seen with the DMAP analogue as with the MeIm analogue (e.g., initially orange mixture, changes to a red or green solution upon the presence of an electrophile, and then changes to a golden brown or green solution upon presence of a nucleophile). As discussed in Chapter 2, the oxidation of starting material can be accomplished by the presence of an electrophile (Scheme 3.6, Pathway ii). For the DMAP analogue (where the electrophile is a Brønsted acid), oxidation is less likely due to a more positive reduction potential upon protonation of the amino group of DMAP. 
Scheme 3.6. Possible pathways after the addition of electrophile $\left(E^{+}\right)$to $\mathbf{2 4}$ or $\mathbf{2 5}$.

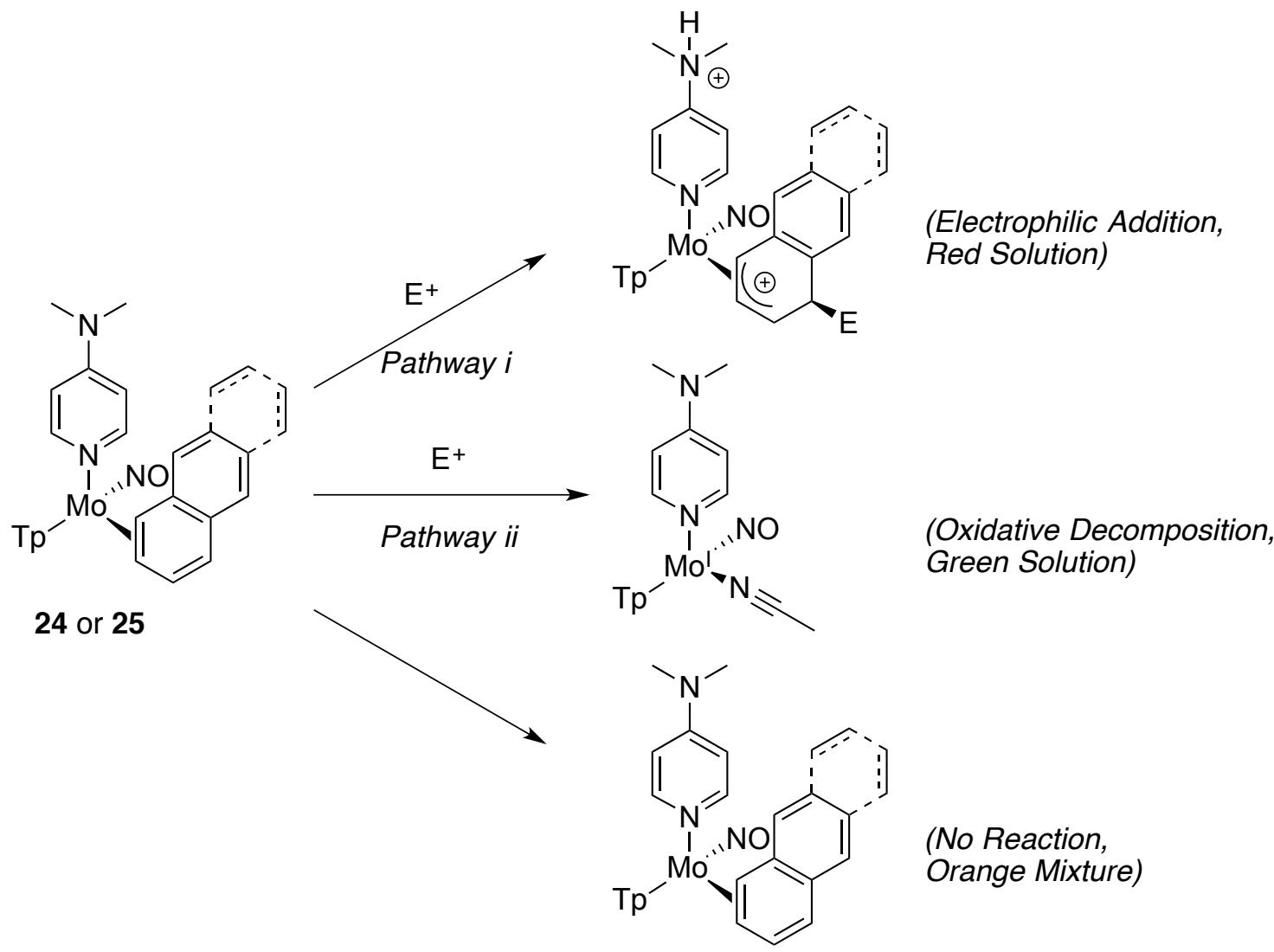

Once the allyl is formed, the DMAP ancillary ligand is believed to offer greater stability in two regards. First, the allyl itself is more stable as can be seen by the ability to isolate $\mathbf{2 7}$ and $\mathbf{2 8}$. This greater stability is believed to be due to a greater donating ability of the DMAP through resonance donation from the amino group. Secondly, the final product of a nucleophilic addition $(\mathbf{C})$ is also more stable to oxidation by excess electrophile present in the reaction mixture, as proposed with MeIm as the ancillary ligand (Scheme 3.7, Pathway iv). 
Scheme 3.7. Oxidative decomposition of $\mathbf{C}$ by excess electrophile, $\mathrm{H}^{+}$in this case.
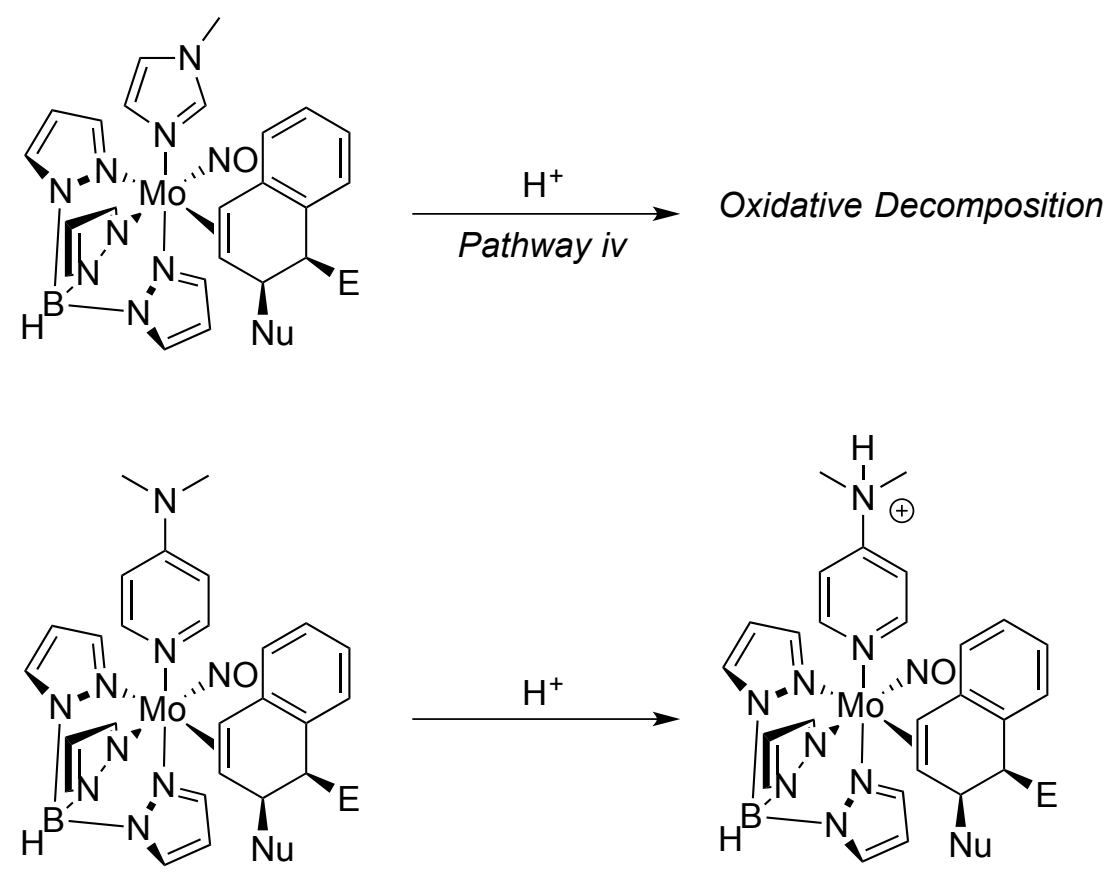

In the case of the DMAP complex, excess acid is believed to preferentially react with the amino group of the DMAP ligand rather than oxidize the metal center. After replicating the previously reported nucleophilic additions observed with the MeIm complex, we hoped to show greater scope in reactivity for the DMAP complex by using a variety of electrophiles.

Initial investigations of electrophile scope began by attempting to add one equivalent of acid (theoretically protonating the amino group) to make the metal center resistant towards oxidation; these attempts proved unsuccessful. However, success has been demonstrated using electrophiles that can be activated with either Brønsted or Lewis acids. For instance, acetals can readily form a more electrophilic, oxonium species in the presence of acid. Similarly, Michael acceptors are enolizable in the presence of an acid, increasing the electophilicity of the beta position. 
That arene complexes of osmium (II) and rhenium (I) have shown success in using both acetals and Michael acceptors for electrophilic-nucleophilic addition reactions, encouraged the use of these electrophiles for the molybdenum system. It was found that by activating acetals and Michael acceptors with HOTf, 24 and 25 could undergo carboncarbon bond formation (Scheme 3.8) at $-60{ }^{\circ} \mathrm{C}$ using proprionitrile $\left(\mathrm{CH}_{3} \mathrm{CH}_{2} \mathrm{CN}\right)$ as the solvent in order to achieve the desired temperature. For these electrophilic additions, the starting complex 24 or 25 is dissolved in $\mathrm{CH}_{3} \mathrm{CH}_{2} \mathrm{CN}$ containing the inactivated electrophile (either an acetal or Michael acceptor). Once the reaction mixture is cooled to $-60{ }^{\circ} \mathrm{C}$, the addition of a $-60{ }^{\circ} \mathrm{C}$ solution of $\mathrm{HOTf}$ in $\mathrm{CH}_{3} \mathrm{CH}_{2} \mathrm{CN}$ yields a dark red solution from the previously orange solution. After $15 \mathrm{~min}$, the nucleophile is added $\left(\mathrm{LiDMM}\right.$ or $\mathrm{NaCNBH}_{3}$ ). After monitoring these reactions by $\mathrm{CV}$ (for more detail on this refer to Chapter 2), the reaction was determined to be complete after 18 hours, as evidenced by the presence of an $E_{\mathrm{p}, \mathrm{a}} \sim+0.2 \mathrm{~V}$. This reaction mixture was then stirred open to air overnight and the organic product yielded from the oxidation of the metal complex was isolated through chromatography. This method yielded the desired organic products, but the organics were only recovered in low yields $(<5 \%)$ due, in part, to the formation of unwanted side products. One side product of these reactions was identified as the product formed from initial protonation, followed by a nucleophilic addition (Scheme 3.8). It should be noted that this yields the organic products 14 and 15 (where the nucleophile $=$ LiDMM), as well as 1,2-dihydronaphthalene and 1,2dihydroanthracene (where the nucleophile $=\mathrm{NaCNBH}_{3}$ ). 
Scheme 3.8. Addition of dimethoxypropane and LiDMM to $\mathbf{2 4}$ using HOTf.<smiles></smiles>

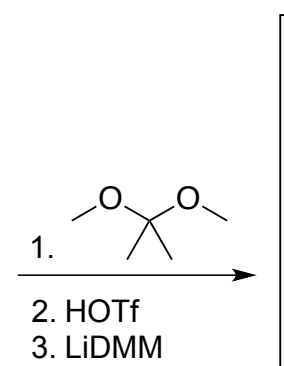

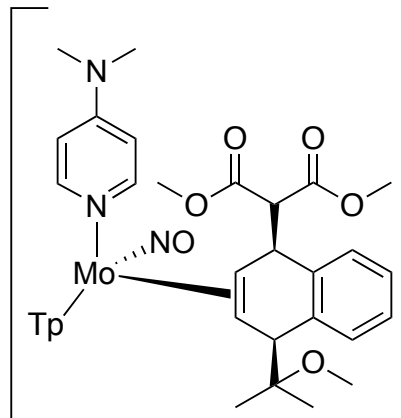<smiles></smiles>

41

32<smiles>COC(=O)C(C(=O)OC)C1c2ccccc2C=C[C@@H]1C(C(=O)OC)C(=O)OC</smiles>

To avoid the undesired protonation side product, TMSOTf was used in place of HOTf to activate the carbon electrophiles. Using the same reaction conditions described above, but replacing HOTf with TMSOTf, complexes 41 and 42 were isolated with minimal signs of complexes 32 or $33(<5 \%)$. Furthermore, in the CV the 1,4dihydroarene complexes (41 and 42) were found to have a smaller positive shift in $\mathrm{Mo}^{0} / \mathrm{Mo}^{\mathrm{I}}$ potential from their aromatic precursors $(\sim 250 \mathrm{mV}$ more positive $)$ in comparison to $\mathbf{3 2}$ and $\mathbf{3 3}$, which are shifted $\sim 350 \mathrm{mV}$ more positive. This less positive 
shift in the anodic wave is in agreement with a decrease in $\pi$-acidity of the resulting dihydroarene of a 1,4-addition. The decrease in $\pi$-acidity is due to the disruption in conjugation with the remaining $\pi$-system.

The preference for regioselectivity in the 1,4-tandem addition reactions is due to the steric interaction between the carbon electrophile added and the incoming nucleophile. To avoid the disfavorable steric repulsion of 1,2-addition of two bulky reagents, the allylic complex can rearrange to provide an electrophilic center without significant steric repulsion (Scheme 3.9). In addition to the difference in their reductive potentials discussed above, the ${ }^{1} \mathrm{H}$ NMR of these 1,4-addition reactions only varies siginifcantly for the "upbound" and "downbound" protons (the protons on the bound alkene proximal and distal to the DMAP ligand, respectively). For 33, the downbound proton $(\mathrm{H} 3)$ is more upfield $(2.32 \mathrm{ppm})$ than the upbound proton $(\mathrm{H} 4,3.35 \mathrm{ppm})$. These alkene protons are more upfield than typically seen for a free alkene, which can be attributed to the large electron density localized on this bond from the metal. Furthermore, anisotropic ring currents from the Tp pyrazole rings gives a greater shielding of protons located between two of the rings of that ligand (Scheme 3.9, quadrant $b$ ). The $\sim 1 \mathrm{ppm}$ difference in chemical shift can also be attributed to a stronger withdrawal of electron density on the $\mathrm{H} 4$ carbon. This was determined by a comparison of 33 with 42, where in $\mathbf{4 2}$ the downbound (H2, $2.51 \mathrm{ppm})$ and the upbound (H3, 2.60 ppm) are much closer in chemical shift. 
Scheme 3.9. Comparison of regiochemistry between 1,2 and 1,4-additions on 25.

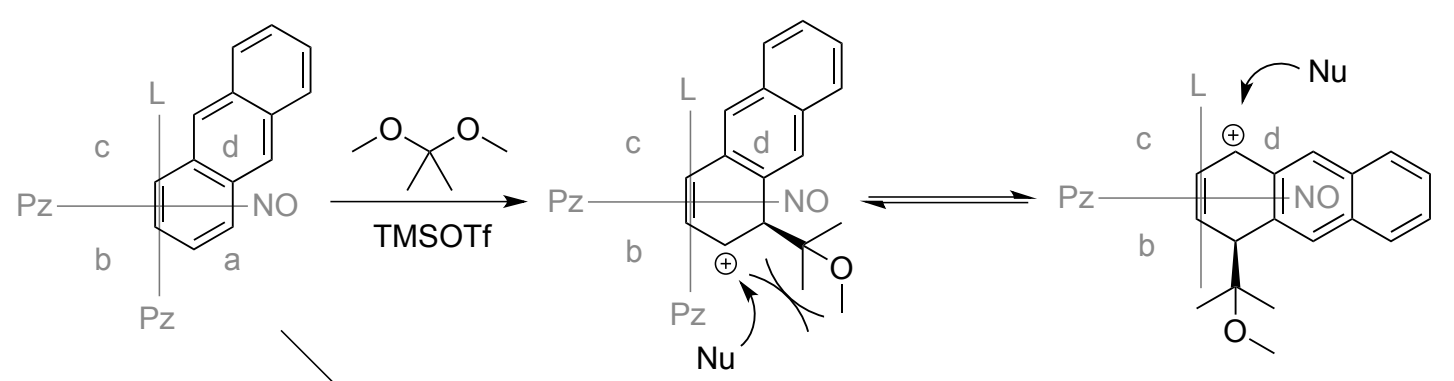

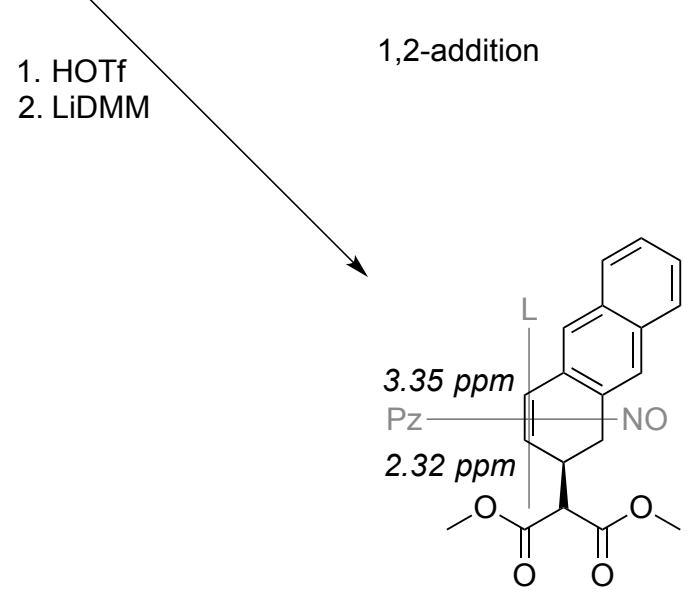

33

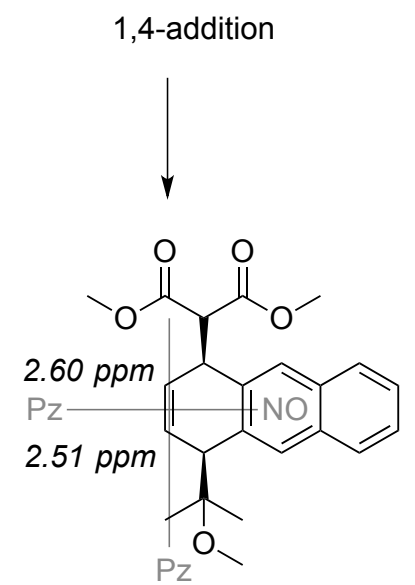

42

Other attempts to add electrophiles activated by TMSOTf with $\mathrm{NaCNBH}_{3}$ as the nucleophile are shown in Table 3.1. These reaction mixtures were analyzed using CV, paying close attention to the subtle difference $(<100 \mathrm{mV})$ between a 1,2 and 1,4addition. After determining the success of these reactions through $\mathrm{CV}$, the reaction mixtures were stirred open to air overnight and the organic products were isolated through chromatography. The products of these 1,2 and 1,4-addition reactions have similar ${ }^{1} \mathrm{H}$ NMR spectra; however, a significant difference is seen in the isolated alkene resonances. In a 1,2-addition reaction, the alkene resonances of the isolated product are typically $\sim 1$ ppm apart and occur between 7.0 and 5.5 ppm. For a 1,4-addition reaction, 
the alkene resonances of the isolated product are typically $\sim 0.2 \mathrm{ppm}$ apart and occur between 6.0 and $5.5 \mathrm{ppm}$. Using this information, the ratio of isomers in a mixture of products yielded from an oxidative decomplexation, can be determined through the integration of the corresponding alkene resonances.

Although oxidation via air does not provide a recyclable metal complex, 1,4dihydroarenes can be isolated as their metal complexes and subjected to iodine oxidation as seen with 41 and $\mathbf{4 2}$. To further demonstrate the recovery of the metal complex precursor in this system, $\mathbf{5 3}$ (the metal-bound complex of the organic 48) was isolated via extraction before exposure to air $(62 \%)$. Oxidation of this complex with iodine generated $48(42 \%)$ and the precursor $(23,98 \%)$ (Scheme 3.10). Encouragingly, the overall yield of 48, via iodine oxidation of $\mathbf{5 3}$, from 25 was $26 \%$, which is comparable to the yield through in situ formation and air oxidation reported in Table 3.3 (28\%). Furthermore, the allyl formed from the addition of dimethoxycyclohexane to 25 (used to make 48 ) was trapped with LiDMM to form the 1,4-tandem addition product 54. This complex was then oxidized with iodine to yield the 1,4-dihydroanthracene 55 in good yield (65\%) with 97\% recovery of $\mathbf{2 3}$ (Scheme 3.10). These observations give the following important implications for future reactions: first, the organics isolated in Table 3.3 can be cleanly isolated as their metal complexes; second, the allyls formed from these new electrophiles can be trapped with a hydride or LiDMM; third, the metal complexes formed from these new electrophilic-nucleophilic additions can be subjected to oxidative decomplexation via iodine, regenerating the metal precursor for these complexes for reuse. 
Scheme 3.10. Addition of dimethoxycyclohexane as an electrophile to $\mathbf{2 5}$, followed by a nucleophilic addition with either $\mathrm{NaCNBH}_{3}$ (bottom) or LiDMM (top).

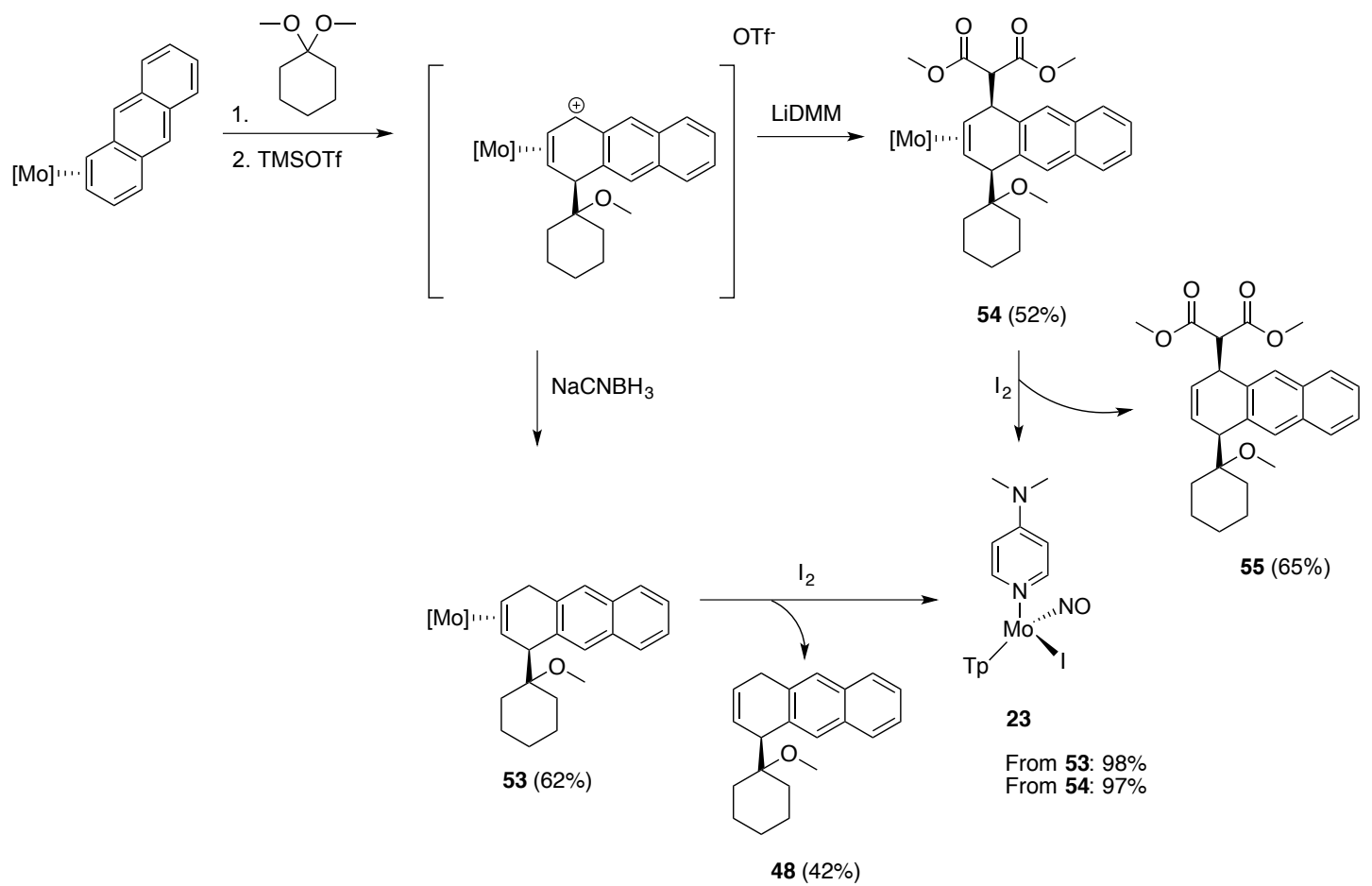

Previous attempts have been made to isolate $\mathbf{4 3}$ and 44 using $\left\{\mathrm{TpW}(\mathrm{NO})\left(\mathrm{PMe}_{3}\right)\right\} \cdot{ }^{13}$ In both cases, the elimination of methanol occurs to yield a transiently stable ylidenium species (Scheme 3.11). For naphthalene, subjecting this intermediate to LiDMM resulted in products that were too unstable to isolate. In the case of anthracene, the ylidinium $\mathbf{5 8}$ reacts with LiDMM at $\mathrm{C} 2$ to form the stable ylidene complex, 59. Treatment of $\mathbf{5 9}$ with ceric ammonium nitrate (CAN) yields trace amounts of an organic compound thought to be the intact ylidine $\mathbf{6 0}$. The full characterization of this compound was not pursued as this ylidine lacks the additional stereocenter found in 43 and 44, again representing a benefit of using molybdenum. 
Scheme 3.11. Formation of the propanylidine dihydroanthracene complex 60.
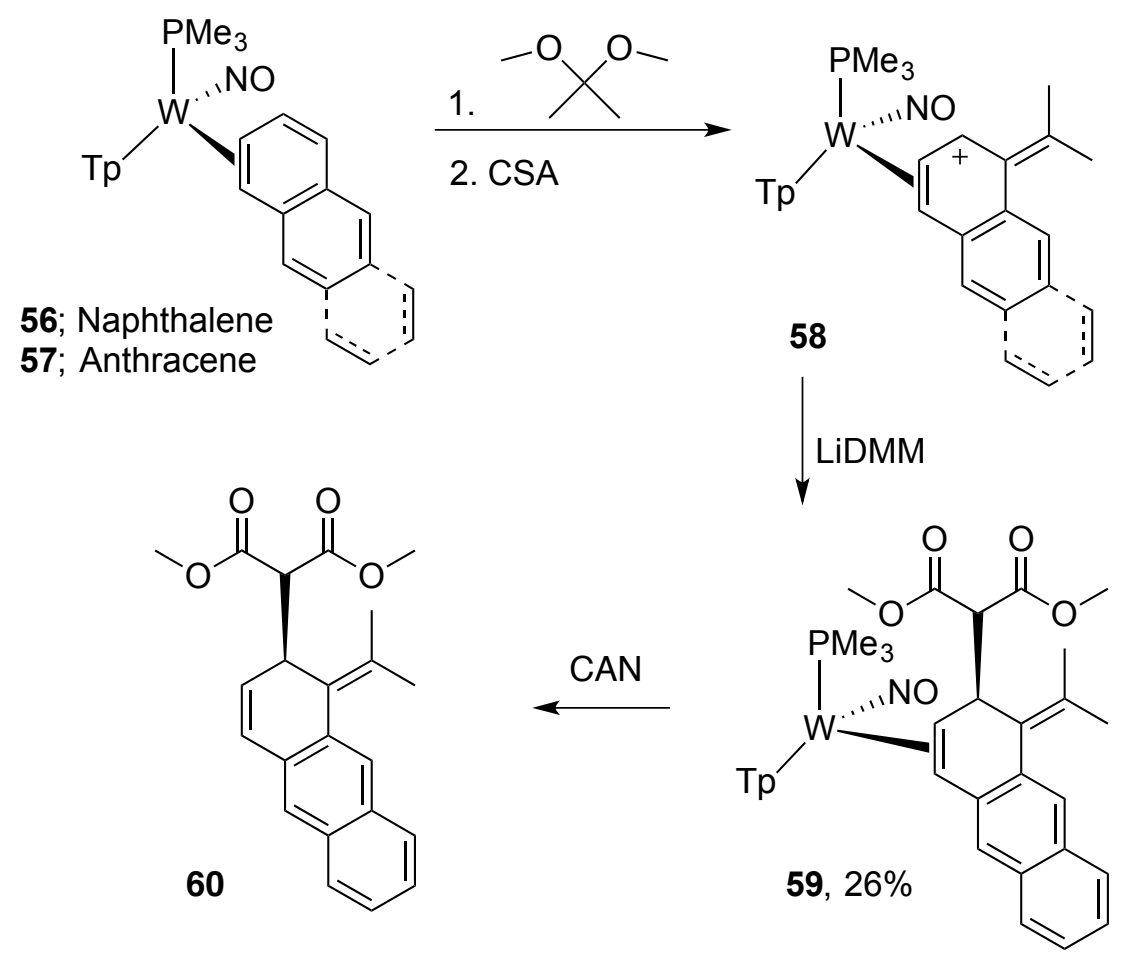

During the initial attempts to vary the electrophile added to $\mathbf{2 4}$, a particularly difficult mixture of products was isolated from the addition of ethyl vinyl ketone (EVK) to 24 using HOTf as the promoter and subsequently trapping the resulting allyl with LiDMM. Subjecting this reaction mixture to an oxidative decomplexation in air and then isolating the resulting products through chromatography gave a mixture initially believed to contain the 1,2 and 1,4-addition products; however, further analysis proved otherwise. Through spectroscopic analysis, the two products of this reaction were identified as the 1,2-tandem addition product, 14, and, surprisingly, the tricyclic hexahydro-phenanthrene, 61, which is the product of a Michael-Michael ring closure (MIMIRC) sequence (Scheme 
3.12). Full analysis and progress on the ongoing investigation of this reaction can be found in Chapter 4.

Scheme 3.12. Electrophilic addition of HOTf and LiDMM to form 14 and the product of a Michael-Michael ring closure, $6 \mathbf{6}$.
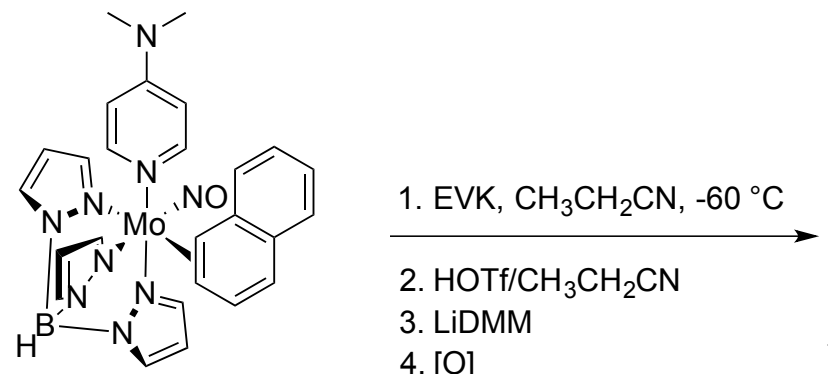

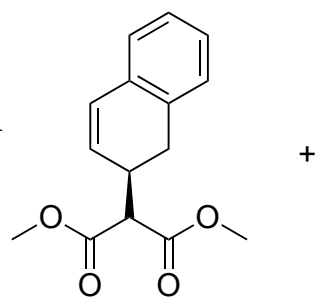

14<smiles>CCC(=O)C1CC(C(=O)CC)C2C=Cc3ccccc3C2C1</smiles>

61

\subsection{Conclusion}

Herein we have described the large-scale synthesis and properties of TpMo(NO)(DMAP)(I) (23), an air-stable precursor for the dearomatization of naphthalene and anthracene. Reduction of $\mathbf{2 3}$ in the presence of the PAH results in a stable $\operatorname{Mo}(0)$ complex in which the arene is dihapto-coordinated and highly activated toward the sequential addition of an electrophile $\left(\mathrm{H}^{+}\right.$or acetal) and nucleophile (enolates, pyrazole, pyrrole). We find that compared to the previously reported $\{\mathrm{TpMo}(\mathrm{NO})(\mathrm{MeIm})\}$ system, the DMAP analog offers significant practical advantages and a wider scope of chemical reactivity. The critical advantage of the DMAP ligand lies in its ability to accept a proton, which renders the metal more resistant to acid oxidation. 


\subsection{Experimental}

\section{General Methods}

NMR spectra were obtained on a 600 or $800 \mathrm{MHz}$ spectrometer. All chemical shifts are reported in ppm, and proton and carbon shifts are referenced to tetramethylsilane (TMS) utilizing residual ${ }^{1} \mathrm{H}$ or ${ }^{13} \mathrm{C}$ signals of the deuterated solvents as an internal standard. Coupling constants $(J)$ are reported in hertz $(\mathrm{Hz})$. Infrared spectra (IR) were recorded as a glaze on a spectrometer fitted with a horizontal attenuated total reflectance (HATR) accessory or on a diamond anvil ATR assembly. Electrochemical experiments were performed under a dinitrogen atmosphere. Cyclic voltammetry data were taken at ambient temperature $\left(\sim 25^{\circ} \mathrm{C}\right)$ at $100 \mathrm{mV} / \mathrm{s}$ in a standard three electrode cell with a glassy-carbon working electrode, $N, N$-dimethylacetamide (DMAc) or acetonitrile $\left(\mathrm{CH}_{3} \mathrm{CN}\right)$ solvent (unless otherwise specified), and tetrabutylammonium hexafluorophosphate (TBAH) electrolyte (approximately $0.5 \mathrm{M}$ ). All potentials are reported versus NHE (normal hydrogen electrode) using cobaltocenium hexafluorophosphate $\left(E_{1 / 2}=-0.78 \mathrm{~V}\right)$, ferrocene $\left(E_{1 / 2}=+0.55 \mathrm{~V}\right)$, or decamethylferrocene $\left(E_{1 / 2}=+0.04 \mathrm{~V}\right)$ as an internal standard. The peak-to-peak separation was less than $100 \mathrm{mV}$ for all reversible couples. High-resolution mass spectra were acquired in ESI mode, from samples dissolved in a 6:1 acetonitrile:water solution containing sodium trifluoroacetate (NaTFA). Mass spectra are reported as $\mathrm{M}+$ for monocationic complexes or as $\mathrm{M}+,\left[\mathrm{M}+\mathrm{H}^{+}\right]$, or $\left[\mathrm{M}+\mathrm{Na}^{+}\right]$for neutral complexes, using $[\mathrm{Na}(\mathrm{NaTFA}) \mathrm{x}]^{+}$clusters as an internal standard. In all cases, observed isotopic envelopes were consistent with the molecular composition reported. For organic products, the monoisotopic ion is reported; for complexes, the major peaks in the isotopic envelope are 
reported. Unless otherwise noted, all synthetic reactions were performed in a glovebox under a dry nitrogen atmosphere. Deuterated solvents were used as received. Pyrazole $(\mathrm{Pz})$ protons of the hydridotris(pyrazolyl)borate $(\mathrm{Tp})$ ligand were uniquely assigned (e.g., "Pz3B") using a combination of two-dimensional NMR data and 4(dimethylamino)pyridine-proton NOE interactions (see Figure 3.4 below). When unambiguous assignments were not possible, Tp protons were labeled as "Pz3/5 or Pz4". BH peaks (around 4-5 ppm) are not identified due to their quadrupole broadening; IR data are used to confirm the presence of a BH group (around $2500 \mathrm{~cm}^{-1}$ ). All $J$ values for Pz protons are $2( \pm 0.2) \mathrm{Hz}$. Due to the sensitivity of complexes 25-35, 36, 41 and 42 to air, elemental analysis attempts were unsuccessful. Elemental analysis of oils 43, 44, 4650 and 52 show residual solvent (DCM) from sample preparation, even after extended time under vacuum. Compounds 1, 24 and 12-17 were previously reported; however, an alternate synthesis of $\mathbf{2 4}$ from $\mathbf{2 3}$ rather than $\mathrm{TpMo}(\mathrm{NO})(\mathrm{Br})_{2}$ is given. ${ }^{1,} 10$

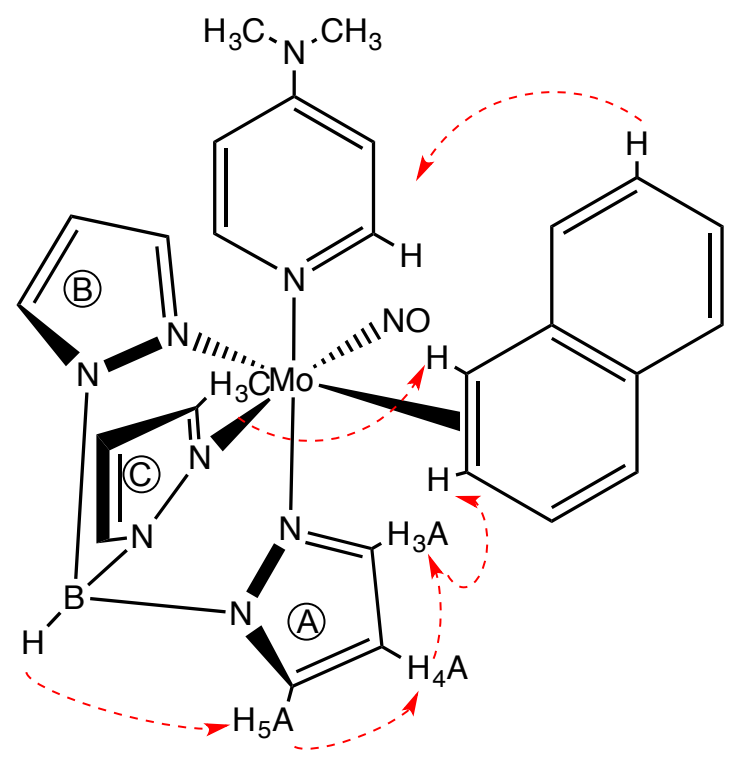

Figure 3.4. Analysis of NOE interactions. 


\section{Synthesis of TpMo(NO)(CO)(DMAP) (22).}

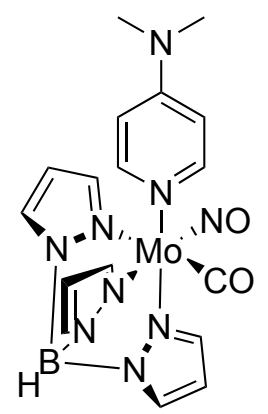

Under an air atmosphere, 1 (166 g, $0.421 \mathrm{~mol}), N, N$-dimethylformamide (1.8 L), and DMAP (165 g, $1.35 \mathrm{~mol})$ were added to a 3 neck 3 L round bottom flask charged with a stir egg, reflux condenser, thermometer and septum. The resulting orange mixture was heated to reflux $\left(\sim 154{ }^{\circ} \mathrm{C}\right)$ for $5 \mathrm{~h}$ then cooled to $\mathrm{RT}$ for $15 \mathrm{~min}$. The reaction mixture was then added to stirring $\mathrm{H}_{2} \mathrm{O}(4 \mathrm{~L})$ and the resulting green mixture was stirred at $\mathrm{RT}$. After $18 \mathrm{~h}$, the green precipitate was isolated on a $3 \mathrm{~L}$ coarse porosity fritted disc, washed with $\mathrm{H}_{2} \mathrm{O}(2 \times 1 \mathrm{~L}), 20 \% \mathrm{MeOH}: \mathrm{Et}_{2} \mathrm{O}(2 \times 1 \mathrm{~L})$, and $\mathrm{Et}_{2} \mathrm{O}(4 \times 1 \mathrm{~L})$, and desiccated overnight to yield $22(164.9 \mathrm{~g}, 80 \%)$. CV (DMAc) $E_{\mathrm{p}, \mathrm{a}}=+0.27 \mathrm{~V}(\mathrm{NHE}) . \mathrm{IR}: v(\mathrm{BH})=$ $2484 \mathrm{~cm}^{-1}, v(\mathrm{CO})=1871 \mathrm{~cm}^{-1}, v(\mathrm{NO})=1589 \mathrm{~cm}^{-1} .{ }^{1} \mathrm{H} \mathrm{NMR}\left(\mathrm{CDCl}_{3}, \delta\right): 7.80(2 \mathrm{H}, \mathrm{m}$, DMAP-A), 7.73 (1H, d, Pz3/5), 7.72 (1H, d, Pz3/5), 7.69 (1H, d, Pz3/5), 7.68 (1H, d, Pz3/5), 7.36 (1H, d, Pz3/5), 7.34 (1H, d, Pz3/5), 6.32 (2H, m, DMAP-B), 6.18 (1H, t, Pz4), 6.17 (1H, t, Pz4), 6.14 (1H, t, Pz4), 2.98 (6H, s, NMe). ${ }^{13} \mathrm{C}$ NMR (d $\mathrm{d}^{6}$-Acetone, $\left.\delta\right)$ : $155.1,152.7,144.8,143.1,142.5,136.7,136.5,136.4,107.8,106.5,106.4,106.3,39.1$. 


\section{Synthesis of TpMo(NO)(DMAP)(I) (23).}

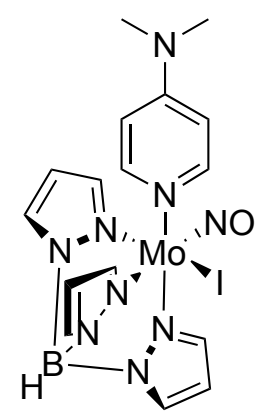

Under an air atmosphere, $22(161 \mathrm{~g}, 0.328 \mathrm{~mol})$ and DCM $(750 \mathrm{~mL})$ were added to a $6 \mathrm{~L}$ Erlenmeyer flask charged with a stir bar and the resulting mixture was stirred at RT until homogeneous. To a 1 L Erlenmeyer flask charged with a stir bar were added $\mathrm{I}_{2}(41.45 \mathrm{~g}$, $0.164 \mathrm{~mol})$ and $\mathrm{Et}_{2} \mathrm{O}(1 \mathrm{~L})$, and the mixture was stirred $15 \mathrm{~min}$ at RT until homogeneous. (CAUTION: Evolution of CO) With the hood sash down, the $I_{2}$ solution was slowly added to the solution of $\mathbf{2 2}$ and the resulting green solution was left stirring at RT. After 15 min, $\mathrm{Et}_{2} \mathrm{O}(4 \mathrm{~L})$ was added to the reaction mixture creating a green precipitation. The precipitate was isolated on a $3 \mathrm{~L}$ coarse porosity fritted disc, washed with $\mathrm{Et}_{2} \mathrm{O}(4 \times 1 \mathrm{~L})$, and desiccated overnight to yield $23\left(170 \mathrm{~g}, 88 \%\right.$ ). CV (DMAc) $E_{\mathrm{p}, \mathrm{a}}=+0.65 \mathrm{~V}(\mathrm{NHE})$, $\mathrm{E}_{1 / 2}=-1.43 \mathrm{~V}(\mathrm{NHE}) . \mathrm{IR}: v(\mathrm{BH})=2477 \mathrm{~cm}^{-1}, v(\mathrm{NO})=1607 \mathrm{~cm}^{-1}$.

\section{Synthesis of TpMo(NO)(DMAP)(3,4- $\eta^{2}$-naphthalene) (24).}

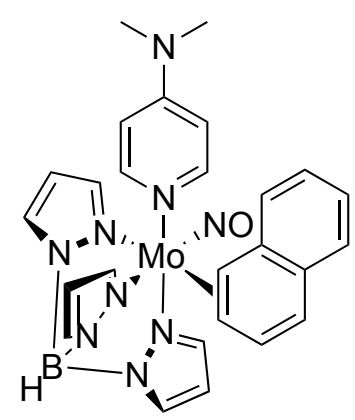

(CAUTION: Pyrophoric!) 23 (24 g, 0.040 mol), THF (500 mL), naphthalene (67 g, 0.508 
mol), and sodium dispersion in wax (30-35\% by weight, $6.8 \mathrm{~g}, 88.6 \mathrm{mmol}$ ) were added to a $1 \mathrm{~L}$ round bottom flask charged with a stir egg and this mixture was stirred at RT. After $24 \mathrm{~h}, \mathrm{Et}_{2} \mathrm{O}(500 \mathrm{~mL})$ was added and the reaction mixture was subsequently loaded onto a $600 \mathrm{~mL}$ medium porosity fritted disc $3 / 4$ full with silica gel. The product was eluted as a yellow band with 1:1 THF: $\mathrm{Et}_{2} \mathrm{O}(400 \mathrm{~mL})$, collected as an orange solution, and then evaporated in vacuo to $\sim 50 \mathrm{~mL}$. This orange solution was added to stirring pentane (900 $\mathrm{mL}$ ) forming an orange precipitate. This precipitate was isolated on a $350 \mathrm{~mL}$ fine porosity fritted disc, washed with pentane $(3 \times 200 \mathrm{~mL})$, and desiccated to yield 24 (11.91 g, 50\% yield).

\section{Synthesis of TpMo(NO)(DMAP)(3,4- $\eta^{2}$-anthracene) (25).}

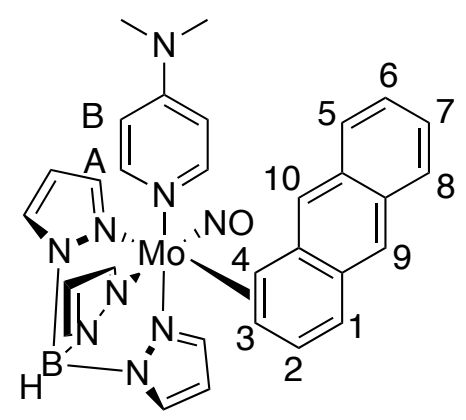

(CAUTION: Pyrophoric!) 23 (24 g, 0.040 mol), THF (500 mL), anthracene (28 g, 0.157 mol), and sodium dispersion in wax (30-35\% by weight, $6.8 \mathrm{~g}, 88.6 \mathrm{mmol}$ ) were added to a $1 \mathrm{~L}$ round bottom flask charged with a stir egg and this mixture was stirred at RT. After $24 \mathrm{~h}, \mathrm{Et}_{2} \mathrm{O}(250 \mathrm{~mL})$ was added and the reaction mixture was subsequently loaded onto a $600 \mathrm{~mL}$ medium porosity fritted disc $3 / 4$ full with silica gel. The product was eluted as a red band with 3:1 THF:Et ${ }_{2} \mathrm{O}(1 \mathrm{~L})$, collected as a red solution, and then evaporated in vacuo. The red solid formed was then suspended in $\mathrm{CH}_{3} \mathrm{CN}(150 \mathrm{~mL})$ and this red mixture was filtered through a $350 \mathrm{~mL}$ fine porosity fritted disc. The orange solid isolated 
was washed with $\mathrm{CH}_{3} \mathrm{CN}(2 \times 150 \mathrm{~mL})$ and the red filtrate was evaporated in vacuo to an oil. This oil was then dissolved in THF $(50 \mathrm{~mL})$ and the product was precipitated in stirring pentane $(800 \mathrm{~mL})$, isolated on a $350 \mathrm{~mL}$ fine porosity fritted disc, washed with pentane ( 3 × $200 \mathrm{~mL})$, and desiccated to yield 25 (11.89 g, 47\% yield). This compound was observed as a 3:1 ratio of diastereomers, the major having theunbound naphthalene ring directed toward the DMAP ligand and the minor having the unbound naphthalene ring directed away from the DMAP ligand. CV (DMAc) $E_{\mathrm{p}, \mathrm{a}}=-0.04 \mathrm{~V}(\mathrm{NHE})$. IR: $v(\mathrm{C}-\mathrm{H}$ $\left.\mathrm{sp}^{2}\right)=2925 \mathrm{~cm}^{-1}, v(\mathrm{BH})=2477 \mathrm{~cm}^{-1}, v(\mathrm{NO})=1585 \mathrm{~cm}^{-1} .{ }^{1} \mathrm{H}$ NMR $\left(\mathrm{d}^{6}\right.$-Acetone, $\left.\delta\right): 8.13$ (1H, d, Pz3A), 8.04 (1H, d, Pz5C), 8.00 (2H, bs, DMAP-A), 7.93 (1H, d, Pz5A), 7.84 (1H, d, Pz5B), 7.81 (1H, d, Pz3C), 7.76 (1H, bd, $J=7.3, \mathrm{H} 8), 7.63$ (1H, s, H9), 7.28 (1H, $\mathrm{d}, J=7.4, \mathrm{H} 5), 7.24$ (2H, m, H6 \& H7), 7.09 (1H, dd, $J=9.1 \& 5.6, \mathrm{H} 2), 6.93(1 \mathrm{H}, \mathrm{d}$, Pz3B), 6.70 (2H, m, DMAP-B), 6.65 (1H, d, J = 9.0, H1), 6.43 (1H, t, Pz4C), 6.37 (1H, t, Pz4A), 6.28 (1H, s, H10), 6.07 (1H, t, Pz4B), 3.79 (1H, d, J=8.0, H4), 3.15 (1H, dd, $J=$ $7.8 \& 5.6, \mathrm{H} 3), 3.06(6 \mathrm{H}, \mathrm{s}, \mathrm{NMe}) .{ }^{13} \mathrm{C}$ NMR (d -Acetone, $\left.\delta\right): 154.9$ (DMAP-C), 150.8 (DMAP-A), 143.6 (Pz3A), 142.6 (Pz3B), 141.8 (Pz3C), 137.6 (Pz5C), 136.8 (Pz5A), 135.9 (Pz5B), 135.7 (C2), 133.0, 132.7, 132.5, 132.1, 127.8 (C8), 126.8 (C5), 124.6 (C6/7), 123.9 (C6/7), 123.2 (C9), 122.9 (C10), 118.7 (C1), 108.4 (DMAP-B), 106.9 (Pz4), 106.3 (Pz4), 106.27 (Pz4), 75.1, 68.9, 39.2 (NMe). HRMS: $\mathrm{C}_{30} \mathrm{H}_{30} \mathrm{~N}_{9} \mathrm{OBMo}+\mathrm{Na}^{+}$ obsd (\%), calcd (\%), ppm: 658.1641 (43), 658.1632 (52), 1.4; $660.1636(40), 660.1630$ (48), 1.0; 661.1614 (78), 661.1628 (81), -2.2; 662.1637 (84), 662.1624 (87), 1.9; 663.1644 (69), 663.1636 (77), 1.2; 664.1643 (100), 664.1624 (100), 2.9; 665.1627 (26), 665.1651 (39), -3.6; 666.1670 (30), 666.1643 (39), 4.0. 


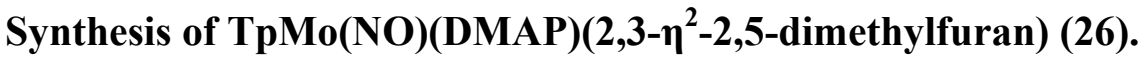

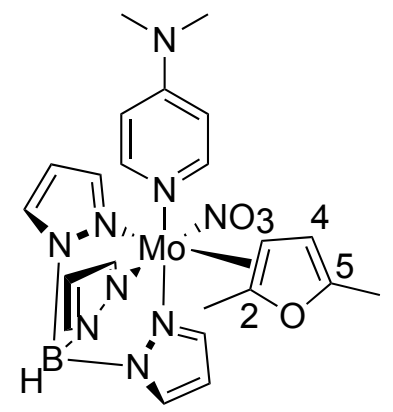

To a $500 \mathrm{~mL}$ round bottom flask charged with a stir egg were added sodium dispersion (30-35\% by weight, $24 \mathrm{~g}$ dispersion, $0.365 \mathrm{~mol})$ and hexanes $(250 \mathrm{~mL})$. The grey mixture formed was stirred for $18 \mathrm{~h}$ at which point the hexanes was decanted off and THF (250 $\mathrm{mL}), 2,5$-dimethlyfuran $(24 \mathrm{~mL}, 0.281 \mathrm{~mol})$ and $23(24 \mathrm{~g}, 0.0408 \mathrm{~mol})$ were added to the remaining solid. The resulting green reaction mixture was stirred for $6 \mathrm{~h}$, transferred to a $1 \mathrm{~L}$ filter flask, and evaporated in vacuo to dryness. (CAUTION: Evolution of hydrogen gas) The resulting black solid was then suspended in $\mathrm{MeOH}(500 \mathrm{~mL})$ forming a dark yellow precipitation. The yellow precipitate was isolated on a $150 \mathrm{~mL}$ medium fritted disc, washed with $\mathrm{MeOH}(3 \times 75 \mathrm{~mL})$ and pentane $(3 \times 75 \mathrm{~mL})$, and desiccated $1 \mathrm{~h}$ to yield 26 (13.03 g, 57\%). CV (DMAc) $E_{\mathrm{p}, \mathrm{a}}=-0.35 \mathrm{~V}(\mathrm{NHE}) . \mathrm{IR}: v(\mathrm{BH})=2494 \mathrm{~cm}^{-1}$, $v(\mathrm{NO})=1620 \mathrm{~cm}^{-1} .{ }^{1} \mathrm{H}$ NMR $\left(\mathrm{d}^{6}\right.$-Acetone, $\left.\delta\right): 8.25$ (1H, d, Pz5A), $7.95(1 \mathrm{H}, \mathrm{d}, \mathrm{Pz} 3 \mathrm{C})$, 7.94 (1H, d, Pz3A), 7.91 (1H, bs, DMAP-A), 7.83 (1H, d, Pz3B), 7.41 (1H, d, Pz5C), $6.95(1 \mathrm{H}, \mathrm{d}, \mathrm{Pz} 5 \mathrm{~B}), 6.65$ (2H, d, $J=7.1$, DMAP-B), $6.38(1 \mathrm{H}, \mathrm{t}, \mathrm{Pz} 4 \mathrm{~A}), 6.30(1 \mathrm{H}, \mathrm{t}$, Pz4C), 6.12 (1H, t, Pz4B), 5.67 (1H, bs, H4), 3.08 (7H, s, H3 \& NMe), 2.13 (3H, s, H5), $0.93(3 \mathrm{H}, \mathrm{s}, \mathrm{H} 2) .{ }^{13} \mathrm{C}$ NMR (d $\mathrm{d}^{6}$-Acetone, d): 155.1, 151.2, 147.7, 142.9, 142.6, 142.5, $137.1,136.3,135.6,119.8,108.4,107.9,106.3,106.3,106.2,69.1,39.2,29.8,21.6,12.9$. 


\section{Synthesis of TpMo(NO)(DMAP)(3,4- $\eta^{2}$-naphthalenium)(OTf) (27).}

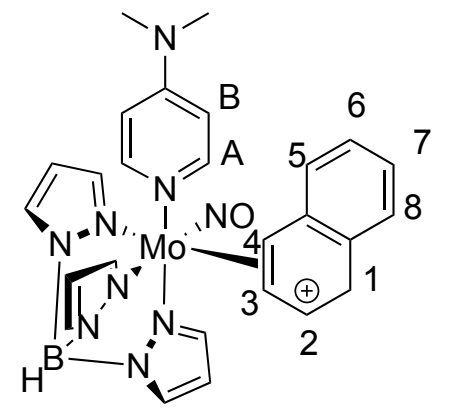

24 (1.0 g, $1.70 \mathrm{mmol})$ and $\mathrm{CH}_{3} \mathrm{CN}(4 \mathrm{~mL})$ were added to a 4-dram vial and the mixture was cooled at $-30{ }^{\circ} \mathrm{C}$ for $15 \mathrm{~min}$. A $-30{ }^{\circ} \mathrm{C}, 1 \mathrm{M}$ solution of $\mathrm{HOTf}$ in $\mathrm{CH}_{3} \mathrm{CN}$ (4.0 mL, 4.0 mmol) was added to the reaction mixture and the resulting red solution was left at $-30{ }^{\circ} \mathrm{C}$ for $15 \mathrm{~min}$. The reaction mixture was evaporated in vacuo to an oil that was redissolved in $\mathrm{DCM}(6 \mathrm{~mL})$. The resulting red solution was added to stirring $\mathrm{Et}_{2} \mathrm{O}(400 \mathrm{~mL})$ forming an orange precipitation. The precipitate was isolated on a $30 \mathrm{~mL}$ fine porosity fritted disc, washed with $\mathrm{Et}_{2} \mathrm{O}(3 \times 30 \mathrm{~mL})$ and desiccated to yield 27 (1.18 g, 95\% yield). CV (DMAc) $E_{\mathrm{p}, \mathrm{a}}=-0.18 \mathrm{~V}(\mathrm{NHE}) . \mathrm{IR}: v(\mathrm{BH})=2500 \mathrm{~cm}^{-1}, v(\mathrm{NO})=1621 \mathrm{~cm}^{-1} .{ }^{1} \mathrm{H} \mathrm{NMR}$ $\left(\mathrm{CD}_{3} \mathrm{CN}, \delta\right): 8.13(1 \mathrm{H}, \mathrm{d}, \mathrm{Pz} 5 \mathrm{C}), 8.09$ (1H, d, Pz3C), 8.05 (1H, d, Pz3A), 7.94 (1H, d, Pz5A), 7.79 (1H, d, Pz3/5B), 7.33 (1H, d, $J=7.6, \mathrm{H} 8), 7.27$ (2H, bs, DMAP-A), 7.20 $(1 \mathrm{H}, \mathrm{t}, J=7.6, \mathrm{H} 7), 7.14(1 \mathrm{H}, \mathrm{t}, J=7.3, \mathrm{H} 6), 7.04$ (1H, d, Pz3/5B), 6.96 (2H, bs, DMAPB), $6.88(1 \mathrm{H}, \mathrm{bd}, J=6.7, \mathrm{H} 2), 6.62(1 \mathrm{H}, \mathrm{t}, \mathrm{Pz} 4 \mathrm{C}), 6.42(1 \mathrm{H}, \mathrm{t}, \mathrm{Pz} 4 \mathrm{~A}), 6.36(1 \mathrm{H}, \mathrm{d}, J=$ 7.6, H5), 6.12 (1H, t, Pz4B), $5.72(1 \mathrm{H}, \mathrm{t}, J=7.3, \mathrm{H} 3), 5.16(1 \mathrm{H}, \mathrm{d}, J=6.3, \mathrm{H} 4), 4.78(1 \mathrm{H}$, $\mathrm{dd}, J=27.6 \& 3.0, \mathrm{H} 1), 4.77(1 \mathrm{H}, \mathrm{d}, J=27.6, \mathrm{H} 1), 3.12(6 \mathrm{H}, \mathrm{s}, \mathrm{NMe}) .{ }^{13} \mathrm{C} \mathrm{NMR}$ (CD $\left.{ }_{3} \mathrm{CN}, \delta\right): 147.5$ (DMAP-C), 143.8 (Pz5C), 143.3 (Pz3C), 140.2, 139.4 (Pz5A), 139.2 (C2), 138.2, 137.2, 136.1 (Pz3/5B), 130.8 (C5), 129.1, 128.7 (C6), 128.3 (C8), 126.9, 122.4, 120.8, 110.3, 108.7 (Pz4C), 107.9 (Pz4A), 107.9 (Pz4B), 102.4, 99.6 (C4), 40.9 (NMe), $33.6(\mathrm{C} 1)$. 


\section{Synthesis of TpMo(NO)(DMAP)(3,4- $\eta^{2}$-anthracenium)(OTf) (28).}

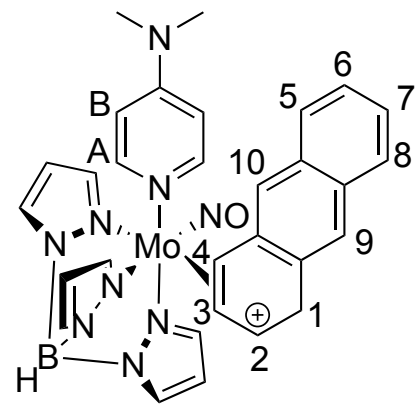

25 (1.0 g, $1.56 \mathrm{mmol})$ and $\mathrm{CH}_{3} \mathrm{CN}(4 \mathrm{~mL})$ were added to a 4-dram vial and the mixture was cooled at $-30{ }^{\circ} \mathrm{C}$ for $15 \mathrm{~min}$. A $-30{ }^{\circ} \mathrm{C}, 1 \mathrm{M}$ solution of $\mathrm{HOTf}$ in $\mathrm{CH}_{3} \mathrm{CN}$ (4.0 mL, 4.0 mmol) was added to the reaction mixture, and the resulting dark red solution was left at $30{ }^{\circ} \mathrm{C}$ for $15 \mathrm{~min}$. The reaction mixture was then evaporated in vacuo to a red oil that was redissolved in DCM $(6 \mathrm{~mL})$ forming a red solution. This solution was added to stirring $\mathrm{Et}_{2} \mathrm{O}(400 \mathrm{~mL})$ forming an orange precipitation. The precipitate was isolated on a $30 \mathrm{~mL}$ fine porosity fritted disc, washed with $\mathrm{Et}_{2} \mathrm{O}(3 \times 30 \mathrm{~mL})$ and desiccated to yield $28(1.14$ g, 93\% yield). CV (DMAc) $E_{\mathrm{p}, \mathrm{a}}=-0.40 \mathrm{~V}(\mathrm{NHE}) . \mathrm{IR}: v\left(\mathrm{C}-\mathrm{H} \mathrm{sp}{ }^{2}\right)=3125 \mathrm{~cm}^{-1}, v(\mathrm{BH})=$ $2515 \mathrm{~cm}^{-1}, v(\mathrm{NO})=1624 \mathrm{~cm}^{-1} .{ }^{1} \mathrm{H} \mathrm{NMR}\left(\mathrm{CD}_{3} \mathrm{CN}, \delta\right): 8.26(1 \mathrm{H}, \mathrm{d}, J=2.2$, DMAP-A $)$, $8.16(1 \mathrm{H}, \mathrm{d}, \mathrm{Pz} 3 \mathrm{C}), 8.08$ (1H, d, Pz3A), 8.08 (1H, s, H10), 8.05 (1H, d, Pz5C), 7.99 (1H, d, $J=2.0$, DMAP-A), $7.94(1 \mathrm{H}, \mathrm{d}, \mathrm{Pz} 5 \mathrm{~A}), 7.82(1 \mathrm{H}, \mathrm{d}, J=8.0, \mathrm{H} 5), 7.77(1 \mathrm{H}, \mathrm{d}$, Pz3/5B), $7.76(1 \mathrm{H}, \mathrm{s}, \mathrm{H} 9), 7.61(1 \mathrm{H}, \mathrm{d}, J=8.1, \mathrm{H} 8), 7.48(1 \mathrm{H}, \mathrm{t}, J=6.8, \mathrm{H} 6), 7.44(1 \mathrm{H}, \mathrm{t}$, $J=7.4, \mathrm{H} 7), 7.07$ (1H, d, Pz3/5B), 6.82 (2H, bs, DMAP-B), 6.65 (1H, m, H2), 6.62 (1H, t, Pz4C), 6.42 (1H, t, Pz4A), 6.10 (1H, t, Pz4B), 5.78 (1H, t, $J=7.1, \mathrm{H} 3), 5.35$ (1H, d, $J=$ 6.6, H4), $4.80(2 \mathrm{H}, 2 \mathrm{~d}$ overlapping, $J=26.5, \mathrm{H} 1), 3.17(6 \mathrm{H}, \mathrm{s}, \mathrm{NMe}) .{ }^{13} \mathrm{C} \mathrm{NMR}\left(\mathrm{CD}_{3} \mathrm{CN}\right.$, d): 147.1 (DMAP-C), 143.2, 142.9, 139.0, 138.8, 136.6 (C2), 134.1, 133.2, 131.9, 131.7, $128.9,128.6,127.9,127.5,127.1,126.9,126.7,126.6,126.5,126.1,122.3,120.2,118.3$, 
108.3 (DMAP-B), 107.5 (Pz4), 107.3 (Pz4), 107.2 (Pz4), 102.6 (C3), 88.8 (C4), 32.9 $(\mathrm{C} 1)$.

\section{Synthesis of TpMo(NO)(pyridine)(n²-naphthalene) (29).}

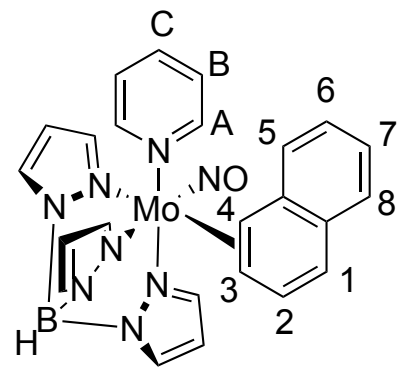

TpMo(NO)(Br) $)_{2}(6.01 \mathrm{~g}, 12.0 \mathrm{mmol})$ and $\mathrm{THF}(125 \mathrm{~mL})$ were added to a $250 \mathrm{~mL}$ round bottom flask charged with a stir bar. Zn dust (1.63 g, $24.9 \mathrm{mmol})$ was added to the stirring reaction mixture and was left stirring. After $10 \mathrm{~min}$, pyridine $(1.21 \mathrm{~mL}, 15.0$ mmol) was added dropwise to the reaction mixture. After stirring for 30 min the solution darkened to a cranberry color, and then changed to dark green after $60 \mathrm{~min}$. $\mathrm{Na}$ dispersion $(30-35 \%, 5.10 \mathrm{~g}, 58.7 \mathrm{mmol})$ was washed with hexanes $(3 \times 30 \mathrm{~mL})$, then THF (25 mL) to produce $\mathrm{Na}^{\circ}$ flakes, which were added to the reaction mixture, followed by naphthalene $(16.46 \mathrm{~g}, 128 \mathrm{mmol})$. The reaction mixture was stirred for $8 \mathrm{~d}$. The dark reaction mixture was loaded onto a $350 \mathrm{~mL}$ medium porosity fritted disc $3 / 4$ full with silica gel and a bright red band was eluted with $\mathrm{Et}_{2} \mathrm{O}(400 \mathrm{~mL})$. The filtrate was evaporated in vacuo, dissolved in DCM $(8 \mathrm{~mL})$, and the product was precipitated in stirring pentane $(200 \mathrm{~mL})$. The precipitate was collected on a $30 \mathrm{~mL}$ fine porosity fritted disc, washed with pentane $(30 \mathrm{~mL})$, and then desiccated yielding 29 (1.60 g, 24\%). CV (DMAc) $E_{\mathrm{p}, \mathrm{a}}=-0.69 \mathrm{~V}(\mathrm{NHE}) . \mathrm{IR}: v\left(\mathrm{C}-\mathrm{H} \mathrm{sp}^{2}\right)=2994 \mathrm{~cm}^{-1}, v(\mathrm{BH})=2485 \mathrm{~cm}^{-1}, v(\mathrm{NO})=$ $1585 \mathrm{~cm}^{-1} .{ }^{1} \mathrm{H}-\mathrm{NMR}\left(\mathrm{d}^{6}\right.$-Acetone, $\left.\delta\right): 8.13(1 \mathrm{H}, \mathrm{d}, \mathrm{Pz} 3 \mathrm{~A}), 8.12(1 \mathrm{H}, \mathrm{bs}, \mathrm{py}-\mathrm{A}), 8.06(1 \mathrm{H}$, d, Pz5C), 7.95 (1H, d, Pz5A), 7.86 (1H, d, Pz3/5B), 7.79 (1H, d, Pz3C), 7.68 (1H, bs, py- 
A), 7.32 (3H, m, py-B \& H8), $7.10(1 \mathrm{H}, \mathrm{dd}, J=8.9 \&$ 5.6, H2), 7.07 (1H, m, py-C), 7.01 $(1 \mathrm{H}, \mathrm{t}, J=7.4, \mathrm{H} 7), 6.84(1 \mathrm{H}, \mathrm{t}, J=7.3, \mathrm{H} 6), 6.79(1 \mathrm{H}, \mathrm{d}, \mathrm{Pz} 3 / 5 \mathrm{~B}), 6.62(1 \mathrm{H}, \mathrm{d}, J=8.9$, H1), 6.44 (1H, t, Pz4C), 6.37 (1H, t, Pz4A), 6.07 (2H, bd \& t, H5 \& Pz4B), 3.88 (1H, d, J $=8.4, \mathrm{H} 4), 3.36(1 \mathrm{H}, \mathrm{dd}, J=8.4 \& 5.6, \mathrm{H} 3) .{ }^{13} \mathrm{C}-\mathrm{NMR}\left(\mathrm{d}^{6}\right.$-Acetone, $\left.\delta\right): 143.3(\mathrm{Pz} 3 / 5)$, 142.3 (Pz3/5), 141.9 (Pz3/5), 137.7 (Pz3/5), 136.9 (Pz5A), 136.2 (Pz3/5), 134.5, 133.8, 132.7, 132.2, 126.6, 125.7 (PyB \& C8), 124.1 (РyC), 123.8 (C6), 119.3 (C1), 107.1 (Pz4C), 106.4 (Pz4A \& Pz4B), 78.1 (C4), 71.7 (C3).

\section{Synthesis of TpMo(NO)(DMAP)(3,4- $\eta^{2}-(2-(2$ '-(2-carbomethoxy)propyl-1,2-} dihydronaphthalene) (30).

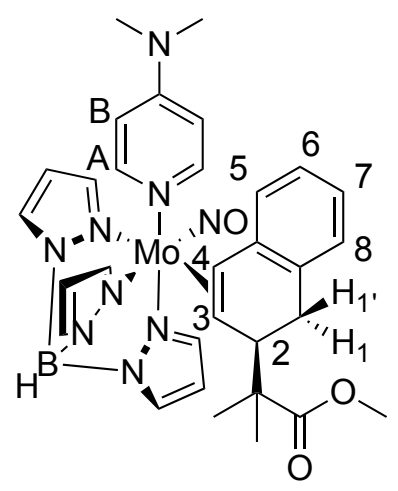

24 (300 $\mathrm{mg}, 0.509 \mathrm{mmol})$ and $\mathrm{CH}_{3} \mathrm{CN}(6 \mathrm{~mL})$ were added to a 4-dram vial and the orange solution was cooled at $-30{ }^{\circ} \mathrm{C}$ for $15 \mathrm{~min}$. A $-30{ }^{\circ} \mathrm{C}$, $1 \mathrm{M}$ solution of $\mathrm{HOTf}$ in $\mathrm{CH}_{3} \mathrm{CN}$ (2.1 $\mathrm{mL}, 2.1 \mathrm{mmol}$ ) was added to the reaction mixture and the resulting red solution was left at $-30{ }^{\circ} \mathrm{C}$. After $15 \mathrm{~min}$, MTDA $(1.0 \mathrm{~mL}, 4.92 \mathrm{mmol})$ was added to this red solution and the resulting mixture was left at $-30^{\circ} \mathrm{C}$. After $18 \mathrm{~h}$, TEA $(0.6 \mathrm{~mL}, 4.3 \mathrm{mmol})$ was added and the resulting brown solution was evaporated in vacuo to a brown oil. This oil was redissolved in DCM (10 mL), washed with $\mathrm{NaHCO}_{3}(10 \mathrm{~mL}$, sat. aq.), and the resulting brown solution was dried over $\mathrm{MgSO}_{4}$. The drying agent was filtered off on a $15 \mathrm{~mL}$ 
coarse porosity fritted disc, washed with DCM $(3 \times 5 \mathrm{~mL})$, and the filtrate was evaporated in vacuo to an oil. This oil was then dissolved in DCM (2 mL) and the product was precipitated in stirring pentane $(50 \mathrm{~mL})$. The precipitate was collected on a $15 \mathrm{~mL}$ medium porosity fritted disc, washed with pentane $(3 \times 15 \mathrm{~mL})$, and desiccated for $15 \mathrm{~min}$, yielding the yellow precipitate $30(333 \mathrm{mg}, 95 \%)$. CV (DMAc) $E_{\mathrm{p}, \mathrm{a}}=+0.20 \mathrm{~V}$ (NHE). IR: $v(\mathrm{BH})=2484 \mathrm{~cm}^{-1}, v(\mathrm{CO})=1724 \mathrm{~cm}^{-1}, v(\mathrm{NO})=1566 \mathrm{~cm}^{-1} .{ }^{1} \mathrm{H}$ NMR $\left(\mathrm{d}^{6}-\right.$

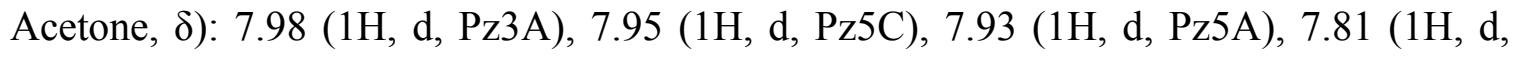
Pz3C), 7.80 (1H, d, Pz5B), 7.39 (2H, bs, DMAP-A), 6.99 (1H, d, Pz3B), 6.95 (1H, d, J= 7.3, H8), 6.78 (1H, t, $J=7.3, \mathrm{H} 7), 6.74(1 \mathrm{H}, \mathrm{t}, J=7.2, \mathrm{H} 6), 6.51$ (2H, m, DMAP-B), 6.41 (1H, t, Pz4A/C), 6.40 (1H, t, Pz4A/C), 6.10 (1H, t, Pz4B), 6.08 (1H, d, J= 7.2, H5), 3.56 $\left(1 \mathrm{H}, \mathrm{dd}, J=16.6 \& 8.8, \mathrm{H} 1^{\prime}\right), 3.31(3 \mathrm{H}, \mathrm{s}, \mathrm{OMe}), 3.24(1 \mathrm{H}, \mathrm{d}, J=7.8, \mathrm{H} 4), 3.19(1 \mathrm{H}, \mathrm{bs}$, H2), $3.06(6 \mathrm{H}, \mathrm{s}, \mathrm{NMe}), 2.62(1 \mathrm{H}, \mathrm{d}, J=16.6, \mathrm{H} 1), 2.19(1 \mathrm{H}, \mathrm{dt}, J=10.1 \& 1.5, \mathrm{H} 3)$, $1.13(3 \mathrm{H}, \mathrm{s}, \mathrm{OMe}), 0.96(3 \mathrm{H}, \mathrm{s}, \mathrm{OMe}) .{ }^{13} \mathrm{C} \mathrm{NMR}\left(\mathrm{d}^{6}\right.$-Acetone, $\left.\delta\right): 178.9$ (CO), 154.9 (DMAP-C), 150.5 (DMAP-A), 144.1, 142.7 (Pz5), 142.7 (Pz5), 142.0 (Pz3B), 137.2, 137.0, 135.7, 135.6, 133.9, 127.9 (C8), 127.2, 127.1, 126.9 (C5), 126.8, 124.6 (C6), 123.3 (C7), 108.2 (DMAP-B), 108.0, 106.9, 106.7 (Pz4A/C), 106.4 (Pz4A/C), 106.2 (Pz4B), 69.5 (OMe), 65.4 (C3), 43.8 (C4), 39.1 (NMe), 29.9 (C1), 23.3 (Me), 23.0. HRMS: $\mathrm{C}_{31} \mathrm{H}_{38} \mathrm{~N}_{9} \mathrm{O}_{3} \mathrm{BMo}+\mathrm{Na}^{+}$obsd (\%), calcd (\%), ppm: 710.2128 (42), 710.2156 (51), -4.0; 712.2114 (43), 712.2154 (48), -5.7; 713.2159 (77), 713.2153 (80), 0.8; 714.2120 (82), 714.2149 (87), -4.1; 715.2155 (67), 715.2161 (77), -0.8; 716.2128 (100), 716.2149 (100), $-2.9 ; 717.2150(34), 717.2176(41),-3.6 ; 718.2139(33), 718.2168(40),-4.1$. 


\section{Synthesis of TpMo(NO)(DMAP)(3,4- $\eta^{2}-(2-(2$ '-(2-carbomethoxy)propyl-1,2-}

\section{dihydroanthracene) (31).}

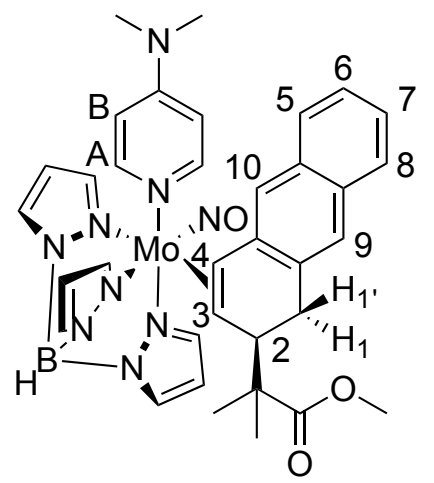

25 (200 $\mathrm{mg}, 0.312 \mathrm{mmol})$ and $\mathrm{CH}_{3} \mathrm{CN}(2 \mathrm{~mL})$ were added to a 4-dram vial and this red solution was left for $15 \mathrm{~min}$ at $-30{ }^{\circ} \mathrm{C}$. A $-30{ }^{\circ} \mathrm{C}, 1 \mathrm{M}$ solution of $\mathrm{HOTf}$ in $\mathrm{CH}_{3} \mathrm{CN}(0.7$ $\mathrm{mL}, 0.7 \mathrm{mmol}$ ) was added to the reaction mixture and the resulting dark red solution was left at $-30{ }^{\circ} \mathrm{C}$. After $15 \mathrm{~min}$, MTDA $(0.4 \mathrm{~mL}, 1.9 \mathrm{mmol})$ was added and the reaction mixture was left at $-30{ }^{\circ} \mathrm{C}$. After $18 \mathrm{~h}$, triethylamine $(0.1 \mathrm{~mL}, 0.717 \mathrm{mmol})$ was added to the reaction mixture and the resulting orange solution was warmed to RT. This solution was then chromatographed through a $15 \mathrm{~mL}$ fine porosity fritted disc $3 / 4$ full with silica gel. The product was eluted with 1:1 $\mathrm{Et}_{2} \mathrm{O}: \mathrm{THF}(50 \mathrm{~mL})$ as a yellow band, collected as a yellow solution, and evaporated in vacuo. The resulting oil was dissolved in DCM (1 $\mathrm{mL})$ and the product was precipitated in stirring hexanes $(50 \mathrm{~mL})$. The precipitate was collected on a $15 \mathrm{~mL}$ fine porosity fritted disc, washed with hexanes $(3 \times 10 \mathrm{~mL})$, and dried for 15 min yielding the yellow solid $31(118 \mathrm{mg}, 52 \%)$ CV (DMAc) $E_{\mathrm{p}, \mathrm{a}}=+0.16 \mathrm{~V}$ (NHE). IR: $v(\mathrm{BH})=2480 \mathrm{~cm}^{-1}, v(\mathrm{CO})=1720 \mathrm{~cm}^{-1}, v(\mathrm{NO})=1570 \mathrm{~cm}^{-1} .{ }^{1} \mathrm{H}-\mathrm{NMR}\left(\mathrm{d}^{6}-\right.$ Acetone, $): 7.99$ (1H, d, Pz5A), 7.96 (1H, d, Pz3A), 7.94 (1H, d, Pz5C), 7.87 (1H, d, Pz3C), 7.80 (1H, d, Pz5B), 7.66 (1H, d, $J=7.9, \mathrm{H} 8), 7.43$ (1H, s, H9), 7.35 (2H, bd, $J=$ 6.6, DMAP-A), 7.24 (2H, m, H5 \& H6), 7.19 (1H, m, H7), 7.05 (1H, d, Pz3B), 6.42 (1H, 
t, Pz4C), 6.41 (2H, bs, DMAP-B), $6.41(1 \mathrm{H}, \mathrm{t}, \mathrm{Pz} 4 \mathrm{~A}), 6.17(1 \mathrm{H}, \mathrm{s}, \mathrm{H} 10), 6.10(1 \mathrm{H}, \mathrm{t}$, Pz4B), $3.68(1 \mathrm{H}, \mathrm{ddd}, J=17.0,8.0, \& 2.0, \mathrm{H} 1), 3.43(1 \mathrm{H}, \mathrm{d}, J=9.7, \mathrm{H} 4), 3.32(3 \mathrm{H}, \mathrm{s}$, OMe), $3.27(1 \mathrm{H}, \mathrm{d}, J=7.7, \mathrm{H} 2), 3.04(6 \mathrm{H}, \mathrm{s}, \mathrm{NMe}), 2.80(1 \mathrm{H}, \mathrm{d}, J=17.0, \mathrm{H} 1$ ') 2.27 $(1 \mathrm{H}, \mathrm{dt}, J=9.5 \& 1.7, \mathrm{H} 3), 1.14(3 \mathrm{H}, \mathrm{s}, \mathrm{Me}), 0.96(3 \mathrm{H}, \mathrm{s}, \mathrm{Me}) .{ }^{13} \mathrm{C}$ NMR $\left(\mathrm{d}^{6}\right.$-Acetone, ס): 179.1 (CO), 155.1 (DMAP-C), 143.4, 143.1 (Pz3), 142.4 (Pz3), 141.8 (Pz5C), 137.6 (Pz5A), 137.2, 135.9, 135.3 (Pz5B), 133.2, 132.4, 127.6 (C8), 126.9 (5/6), 125.6 (C9), 124.9 (C5/6), 123.9 (C7), 123.3 (C10), 109.0, 108.2 (DMAP-B), 107.1 (Pz4), 106.6 (Pz4), 106.4 (Pz4), 70.3 (C4), 65.9 (C3), 51.4 (OMe), 47.9, 43.8 (C2), 39.3 (NMe), 30.6 (C1), $23.5(\mathrm{Me}), 23.1(\mathrm{Me})$. HRMS: $\mathrm{C}_{35} \mathrm{H}_{40} \mathrm{~N}_{9} \mathrm{O}_{3} \mathrm{BMo}^{+}$obsd (\%), calcd (\%), ppm: 737.2397 (26), 737.2416 (50), -2.6; 739.2413 (36), 739.2415 (48), -0.2; 740.2448 (55), 740.2413 (80), 4.8; 741.2416 (85), 741.2409 (88), 0.9; 742.2400 (71), 742.2421 (78), 2.8; 743.2437 (100), 743.2409 (100), 3.8; 744.2445 (21), 744.2435 (43), 1.3; 745.2442 (25), $745.2429(40), 1.7$.

Synthesis of TpMo(NO)(DMAP)(3,4- $\eta^{2}-2-(1,2-d i h y d r o n a p h t h a l e n-2-y l)-$ dimethylmalonate) (32).

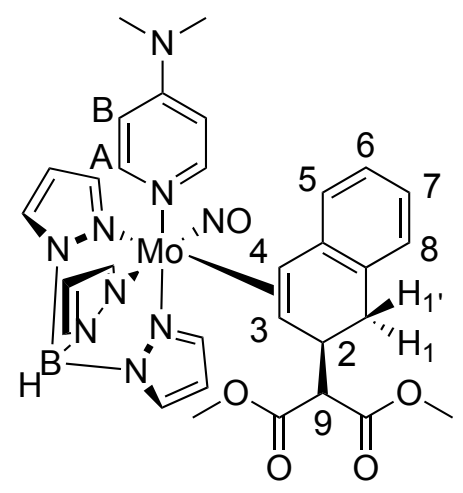

$24(500 \mathrm{mg}, 0.849 \mathrm{mmol})$ and $\mathrm{CH}_{3} \mathrm{CN}(10 \mathrm{~mL})$ were added to a 4-dram vial and this orange mixture was cooled for $15 \mathrm{~min}$ at $-35{ }^{\circ} \mathrm{C}$. A $-35{ }^{\circ} \mathrm{C}, 1 \mathrm{M}$ solution of HOTf in 
$\mathrm{CH}_{3} \mathrm{CN}(3.5 \mathrm{~mL}, 3.5 \mathrm{mmol})$ was added to the reaction mixture and the resulting red solution was left standing at $-35{ }^{\circ} \mathrm{C}$. After $15 \mathrm{~min}$, LiDMM (700 mg, $\left.5.07 \mathrm{mmol}\right)$ was added to the reaction mixture and the resulting red solution was left standing at $-35{ }^{\circ} \mathrm{C}$. After $18 \mathrm{~h}$, a $-35{ }^{\circ} \mathrm{C}$ solution of triethylamine $(1.0 \mathrm{~mL}, 7.17 \mathrm{mmol})$ was added to the reaction mixture and the resulting golden solution was warmed to RT. This solution was evaporated in vacuo to $4 \mathrm{~mL}$ and chromatographed through a $60 \mathrm{~mL}$ medium porosity fritted disc $3 / 4$ full with silica gel. The product was eluted with $1: 1 \mathrm{Et}_{2} \mathrm{O}$ :benzene $(75 \mathrm{~mL})$ as a yellow band, collected as a yellow solution, and evaporated in vacuo. The resulting yellow oil was then dissolved in DCM $(4 \mathrm{~mL})$ and the product was precipitated in stirring hexanes $(100 \mathrm{~mL})$. The precipitate was collected on a $15 \mathrm{~mL}$ medium porosity fritted disc, washed with hexanes $(3 \times 50 \mathrm{~mL})$, and desiccated for 15 min yielding the yellow solid 32 (497 mg, 82\%). CV (DMAc) $E_{\mathrm{p}, \mathrm{a}}=+0.22 \mathrm{~V}(\mathrm{NHE}) . \mathrm{IR}: v(\mathrm{BH})=2484 \mathrm{~cm}^{-1}$, $v(\mathrm{CO})=1751 \& 1732 \mathrm{~cm}^{-1}, v(\mathrm{NO})=1574 \mathrm{~cm}^{-1} .{ }^{1} \mathrm{H}$ NMR $\left(\mathrm{d}^{6}\right.$-Acetone, $\left.\delta\right): 7.95(1 \mathrm{H}, \mathrm{d}$, Pz5C), 7.90 (1H, d, Pz5A), 7.88 (1H, d, Pz3A), 7.69 (1H, d, Pz3C), 7.80 (1H, d, Pz3/5B), 7.38 (2H, bs, DMAP-A), 6.99 (1H, d, Pz3/5B), 6.93 (1H, m, H5), 6.81 (2H, m, H6\&7), 6.50 (2H, m, DMAP-B), 6.39 (1H, t, Pz4A), 6.38 (1H, t, Pz4C), 6.14 (1H, dd, $J=6.4 \&$ 2.8, H8), 6.09 (1H, t, Pz4B), 3.61 (3H, s, OMe), 3.53 (1H, dd, $J=16.0 \& 5.9, \mathrm{H} 1), 3.45$ (1H, m, H2), $3.41(1 \mathrm{H}, \mathrm{s}, \mathrm{H} 9), 3.37(3 \mathrm{H}, \mathrm{s}, \mathrm{OMe}), 3.23(1 \mathrm{H}, \mathrm{d}, J=9.6, \mathrm{H} 4), 3.04(6 \mathrm{H}, \mathrm{s}$, NMe), $2.51\left(1 \mathrm{H}, \mathrm{d}, J=16.0, \mathrm{H} 1^{\prime}\right), 2.16(1 \mathrm{H}, \mathrm{dt}, J=9.5 \& 2.2, \mathrm{H} 3) .{ }^{13} \mathrm{C} \mathrm{NMR}\left(\mathrm{d}^{6}-\right.$ Acetone, $\delta$ ): 170.0 (CO), 169.9 (CO), 154.9 (DMAP-C), 143.5, 142.0, 141.2, 137.5, $136.9,135.8,135.6,131.5,128.4,127.5,127.3,127.1,125.2,123.5,108.1$ (DMAP-B), 106.9 (Pz4), 106.7 (Pz4), 106.5 (Pz4), 106.3, 68.2 (C3), 67.7 (C4), 59.4 (C9), 52.2 (OMe), 52.1 (OMe), 39.1 (NMe), 38.3 (C2), 31.6 (C1). HRMS: $\mathrm{C}_{31} \mathrm{H}_{36} \mathrm{~N}_{9} \mathrm{O}_{5} \mathrm{BMo}^{+}$obsd 
(\%), calcd (\%), ppm: 717.1995 (33), 717.2001 (51), -0.8; 719.1955 (19), 719.1999 (48), 6.1; 720.1998 (82), 720.1997 (80), 0.1; 721.1975 (100), 721.1993 (87), -2.5; 722.2000 (67), 722.2005 (77), -0.7; 723.1968 (83), 723.1993 (100), -3.4; 724.2019 (25), 724.2020 (41), -0.1; $725.2006(22), 725.2013(40),-0.9$.

\section{Synthesis of TpMo(NO)(DMAP)(3,4- $\eta^{2}-2-(1,2-d i h y d r o a n t h r a c e n-2-y l)-$} dimethylmalonate) (33).

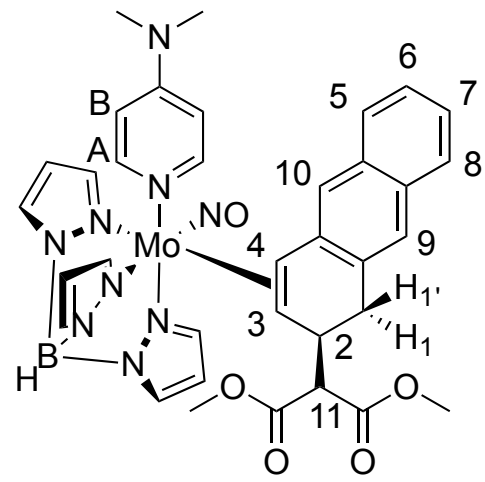

25 (100 $\mathrm{mg}, 0.15 \mathrm{mmol})$ and $\mathrm{CH}_{3} \mathrm{CN}(2 \mathrm{~mL})$ were added to a 4-dram vial and the resulting red solution was cooled at $-30{ }^{\circ} \mathrm{C}$ for $15 \mathrm{~min}$. A $-30{ }^{\circ} \mathrm{C}, 1 \mathrm{M}$ solution of HOTf in $\mathrm{CH}_{3} \mathrm{CN}(0.7 \mathrm{~mL}, 0.7 \mathrm{mmol})$ was added to the reaction mixture giving a dark red solution that was left standing at $-30{ }^{\circ} \mathrm{C}$ for $15 \mathrm{~min}$. LiDMM (138 mg, $\left.1.00 \mathrm{mmol}\right)$ was added to this solution creating an orange solution that was left standing at $-30{ }^{\circ} \mathrm{C}$ for $18 \mathrm{~h}$. Triethylamine $(0.2 \mathrm{~mL}, 1.43 \mathrm{mmol})$ was added to this solution and the reaction mixture was warmed to room temperature. The reaction mixture was extracted with DCM $(6 \mathrm{~mL})$ and $\mathrm{NaHCO}_{3}\left(6 \mathrm{~mL}\right.$, sat. aq.) and the organic layer was dried over $\mathrm{MgSO}_{4}$. The drying agent was removed on a $15 \mathrm{~mL}$ medium porosity fritted disc, washed with DCM $(3 \times 2$ $\mathrm{mL}$ ), and the filtrate was evaporated in vacuo. The oil formed was then dissolved in DCM $(1 \mathrm{~mL})$ and the product was precipitated in stirring pentane $(25 \mathrm{~mL})$, collected on a 
$15 \mathrm{~mL}$ fine porosity fritted disc, washed with pentane $(3 \times 10 \mathrm{~mL})$, and desiccated for 15 min yielding the yellow solid 33 (47 mg, 41\%). CV (DMAc) $E_{\mathrm{p}, \mathrm{a}}=+0.28 \mathrm{~V}$ (NHE). IR: $v\left(\mathrm{C}-\mathrm{H} \mathrm{sp}^{2}\right)=2949 \mathrm{~cm}^{-1}, v(\mathrm{BH})=2485 \mathrm{~cm}^{-1}, v(\mathrm{CO})=1731,1620 \mathrm{~cm}^{-1}, v(\mathrm{NO})=1567 \mathrm{~cm}^{-}$ 1. ${ }^{1} \mathrm{H}$ NMR (d'-Acetone, $\left.\delta\right): 7.99(1 \mathrm{H}, \mathrm{d}, \mathrm{Pz} 5 \mathrm{~A} / \mathrm{C}), 7.92(1 \mathrm{H}, \mathrm{d}, \mathrm{Pz3A}), 7.90(1 \mathrm{H}, \mathrm{d}$, Pz5A/C), 7.82 (1H, d, Pz3/5B), 7.77 (1H, d, Pz3C), $7.70(1 \mathrm{H}, \mathrm{d}, J=8.5, \mathrm{H} 8), 7.46(1 \mathrm{H}, \mathrm{s}$, H9), 7.34 (1H, d, $J=6.7, \mathrm{H} 5), 7.32$ (2H, buried bs, DMAP-A), 7.28 (1H, t, $J=6.6, \mathrm{H} 6$ ), $7.22(1 \mathrm{H}, \mathrm{t}, J=7.6, \mathrm{H} 7), 7.04$ (1H, d, Pz3/5B), 6.41 (4H, m, DMAP-B, Pz4A\&C), 6.29 (1H, s, H10), $6.10(1 \mathrm{H}, \mathrm{t}, \mathrm{Pz} 4 \mathrm{~B}), 3.76(1 \mathrm{H}, \mathrm{dd}, J=16.6 \& 5.9, \mathrm{H} 1), 3.62(3 \mathrm{H}, \mathrm{s}, \mathrm{OMe})$, $3.52(1 \mathrm{H}, \mathrm{m}, \mathrm{H} 2), 3.44(1 \mathrm{H}, \mathrm{d}, J=10.3, \mathrm{H} 11), 3.40(3 \mathrm{H}, \mathrm{s}, \mathrm{OMe}), 3.35(1 \mathrm{H}, \mathrm{d}, J=9.10$, H4), 3.04 (6H, s, NMe), $2.76\left(1 \mathrm{H}, \mathrm{d}, J=16.6, \mathrm{H1}{ }^{\prime}\right), 2.32(1 \mathrm{H}, \mathrm{dt}, J=9.4, \mathrm{H} 3) .{ }^{13} \mathrm{C} \mathrm{NMR}$ (d $\mathrm{d}^{6}$-Acetone, $\left.\delta\right): 170.0$ (CO), 155.0 (DMAP-C), 143.6 (Pz5), 142.5 (Pz3/5), 142.2, 141.4 (Pz3), 137.6 (Pz5), 136.9 (Pz3), 135.8 (Pz3/5), 133.3, 132.5, 132.2, 127.7 (C8), 127.4 (C9), 126.8 (C5), 125.2 (C6), 124.0 (C7), 123.9, 123.6 (H10), 108.1 (DMAP-B), 106.9 (Pz4), 106.7 (Pz4), 106.4 (Pz4), 68.5 (C4), 68.4 (C3), 66.1 (C11), 52.2 (OMe), 51.9 (OMe), $39.3(\mathrm{NMe}), 38.2$ (C2), 32.9 (C1). HRMS: $\mathrm{C}_{35} \mathrm{H}_{38} \mathrm{~N}_{9} \mathrm{O}_{5} \mathrm{BMo}^{+}$obsd (\%), calcd (\%), ppm: 767.2126 (20), 767.2158 (50), -4.1; 769.2081 (10), 769.2157 (48), -9.8; 770.2138 (62), 770.2155 (79), -2.2; 771.2141 (100), 771.2151 (88), -1.3; 772.2177 (72), 772.2163 (78), 1.9; 773.2154 (68), 773.2151 (100), 0.4; 774.2156 (42), 774.2177 (44), 2.7; $775.2039(5), 775.2171(40),-17.0$. 


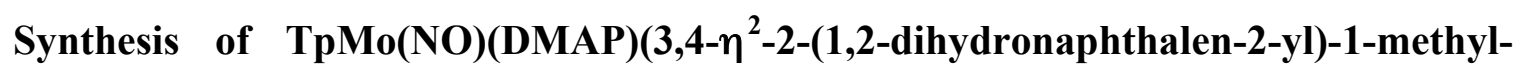
1H-pyrrole) (34).

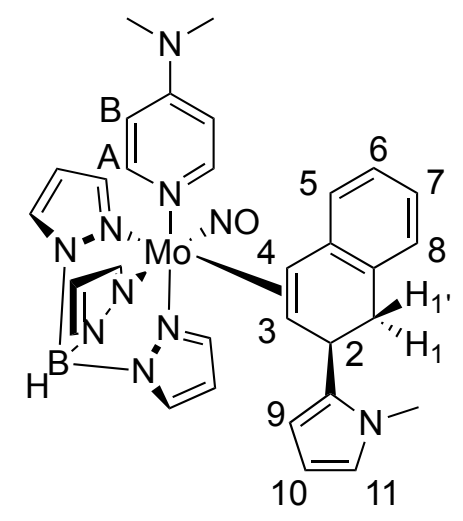

24 (300 mg, $0.509 \mathrm{mmol}$ ) and $\mathrm{CH}_{3} \mathrm{CH}_{2} \mathrm{CN}$ (5 mL) were added to a test tube charged with a stir pea and this orange mixture was cooled with stirring for $15 \mathrm{~min}$ at $-60{ }^{\circ} \mathrm{C}$. A $-60{ }^{\circ} \mathrm{C}$, $1 \mathrm{M}$ solution of HOTf in $\mathrm{CH}_{3} \mathrm{CH}_{2} \mathrm{CN}(2.1 \mathrm{~mL}, 2.1 \mathrm{mmol})$ was added to the reaction mixture and the resulting red solution was left stirring for $15 \mathrm{~min}$ at $-60{ }^{\circ} \mathrm{C} . \mathrm{N}$ methylpyrrole $(1.0 \mathrm{~mL}, 11.3 \mathrm{mmol})$ was added to the reaction mixture and the red solution formed was left stirring at $-60{ }^{\circ} \mathrm{C}$ for $18 \mathrm{~h}$. Next, triethylamine $(0.6 \mathrm{~mL}, 2.1$ mmol) was added to the reaction mixture giving a brown solution that was warmed to RT and evaporated in vacuo. The oil formed was dissolved in DCM (5 mL) and this solution was chromatographed through a $30 \mathrm{~mL}$ medium porosity fritted disc $3 / 4$ full with silica gel. The product was eluted as a yellow band with 1:1 $\mathrm{Et}_{2} \mathrm{O}$ :benzene $(100 \mathrm{~mL})$, collected as a yellow solution, and evaporated in vacuo to an oil. This oil was dissolved in DCM (1 $\mathrm{mL})$ and the product was precipitated in stirring pentane $(50 \mathrm{~mL})$. The precipitate was then collected on a $15 \mathrm{~mL}$ fine porosity fritted disc, washed with pentane $(3 \times 10 \mathrm{~mL})$, and desiccated for 15 min yielding the yellow precipitate 34 (234 mg, 68\%). CV (DMAc) $E_{\mathrm{p}, \mathrm{a}}=+0.18 \mathrm{~V}(\mathrm{NHE}) . \mathrm{IR}: v\left(\mathrm{C}-\mathrm{H} \mathrm{sp}^{2}\right)=2906 \mathrm{~cm}^{-1} v(\mathrm{BH})=2480 \mathrm{~cm}^{-1}, v(\mathrm{NO})=1596 \mathrm{~cm}^{-}$ 1. ${ }^{1} \mathrm{H}-\mathrm{NMR}\left(\mathrm{CDCl}_{3}, \delta\right): 8.12$ (1H, d, Pz3A), 7.99 (1H, d, Pz5C), 7.94 (1H, d, Pz5A), 7.77 
(1H, d, Pz3C), 7.81 (1H, d, Pz5B), 7.47 (2H, bs, DMAP-A), 7.01 (1H, d, Pz3B), 6.94 (1H, buried d, H8), 6.77 (2H, m, H6 \& H7), 6.51 (2H, m, DMAP-B), 6.40 (1H, t, Pz4A), 6.35 (1H, t, Pz4C), 6.31 (1H, dd, $J=2.7 \& 1.9, \mathrm{H} 11), 6.15(1 \mathrm{H}$, buried d, H5), $6.10(1 \mathrm{H}$, t, Pz4B), 5.67 (1H, ddd, $J=3.4,1.9, \&$ 0.4, H10), 5.64 (1H, dd, $J=3.3 \& 2.7, \mathrm{H} 9), 4.01$ $(1 \mathrm{H}, \mathrm{d}, J=7.4, \mathrm{H} 2), 3.75(1 \mathrm{H}, \mathrm{dd}, J=15.5 \& 6.9, \mathrm{H} 1), 3.42(3 \mathrm{H}, \mathrm{s}, \mathrm{NMe}), 3.28(1 \mathrm{H}, \mathrm{d}, J$ $=9.3, \mathrm{H} 4), 3.06(6 \mathrm{H}, \mathrm{s}, \mathrm{NMe}), 2.76\left(1 \mathrm{H}, \mathrm{d}, J=15.5, \mathrm{H1}{ }^{\prime}\right), 2.42(1 \mathrm{H}, \mathrm{dt}, J=9.5 \& 2.1$, H3). ${ }^{13} \mathrm{C}$ NMR $\left(\mathrm{CDCl}_{3}, \delta\right.$ ): 153.6 (DMAP-C), 142.3 (Pz3), 142.2, 141.6 (Pz3), 141.4 (Pz5), 140.6 (Pz5), 135.9 (Pz5), 135.8, 134.5 (Pz3), 132.0, 128.4 (C8), 128.3, 126.7 (C5), 124.0, 122.9 (C6 \& C7), 119.8 (C11), 107.2 (DMAP-B), 106.3 (Pz4A), 105.7 (Pz4C), 105.6 (Pz4B), 105.4 (C9), 105.1 (C10), 70.6, 66.9 (C4), 39.1 (DMAP-NMe), 33.5 (C2), 33.4 (NMe), 32.9. HRMS: $\mathrm{C}_{31} \mathrm{H}_{35} \mathrm{~N}_{10} \mathrm{OBMo}+\mathrm{Na}^{+}$obsd (\%), calcd (\%), ppm: 689.2005 (57), 689.2054 (51), -7.1; 691.2039 (45), 691.2052 (48), -1.9; 692.2043 (87), 692.2051 (80), -1.1; 693.2016 (84), 693.2046 (87), - 4.4; 694.2072 (77), 694.2058 (77), 2.0; 695.2064 (100), 695.2046 (100), 2.6; 696.2089 (27), 696.2073 (41), 2.4; 697.2079 (26), $697.2066(39), 1.9$.

Synthesis of TpMo(NO)(DMAP)(3,4- $\eta^{2}$-2-(1,2-dihydroanthracen-2-yl)-1-methyl-1Hpyrrole) (35).

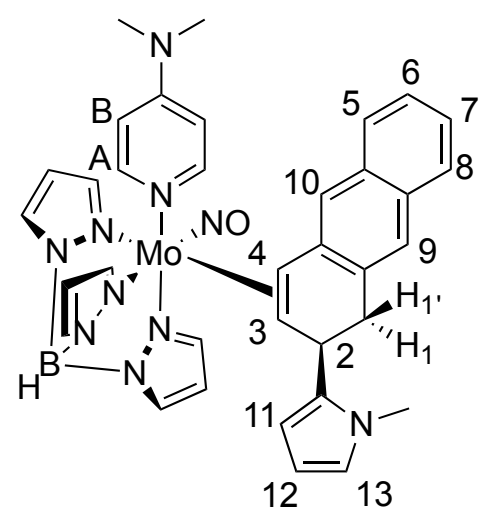


25 (300 mg, $0.468 \mathrm{mmol})$ and $\mathrm{CH}_{3} \mathrm{CN}(6 \mathrm{~mL})$ were added to a 4-dram vial and the resulting red solution was cooled for $15 \mathrm{~min}$ at $-30{ }^{\circ} \mathrm{C}$. A $-30{ }^{\circ} \mathrm{C}, 1 \mathrm{M}$ solution of $\mathrm{HOTf}$ in $\mathrm{CH}_{3} \mathrm{CN}(2.1 \mathrm{~mL}, 2.1 \mathrm{mmol})$ was added to the reaction mixture and the resulting dark red solution was left standing at $-30{ }^{\circ} \mathrm{C}$ for $15 \mathrm{~min} . \mathrm{N}$-methylpyrrole $(4.0 \mathrm{~mL}, 45.0 \mathrm{mmol})$ was added and the reaction mixture was left standing at $-30{ }^{\circ} \mathrm{C}$ for $18 \mathrm{~h}$. Triethylamine (0.6 mL, $4.30 \mathrm{mmol})$ was added to the reaction mixture and the resulting orange solution was warmed to RT then evaporated in vacuo. The oil formed was then dissolved in THF $(1 \mathrm{~mL})$ and the resulting solution was chromatographed through a $30 \mathrm{~mL}$ medium porosity fritted disc $3 / 4$ full with silica gel. The product was eluted as a yellow band with 1:1 $\mathrm{Et}_{2} \mathrm{O}$ :benzene $(50 \mathrm{~mL})$, collected as a yellow solution, and evaporated in vacuo to an oil. This oil was dissolved in DCM $(1 \mathrm{~mL})$ and the product was precipitated in stirring pentane $(50 \mathrm{~mL})$. The precipitate was collected on a $15 \mathrm{~mL}$ fine porosity fritted disc, washed with pentane $(3 \times 10 \mathrm{~mL})$, and dried for 15 min yielding the yellow precipitate 35 $(171 \mathrm{mg}, 51 \%)$. CV (DMAc) $E_{\mathrm{p}, \mathrm{a}}=+0.18 \mathrm{~V}$ (NHE). IR: $v\left(\mathrm{C}-\mathrm{H} \mathrm{sp}^{2}\right)=2916 \mathrm{~cm}^{-1}, v(\mathrm{BH})=$ $2480 \mathrm{~cm}^{-1}, v(\mathrm{NO})=1570 \mathrm{~cm}^{-1} .{ }^{1} \mathrm{H}$ NMR $\left(\mathrm{d}^{6}\right.$-Acetone, $\left.\delta\right): 8.12(1 \mathrm{H}, \mathrm{d}$, Pz3A $), 7.97(1 \mathrm{H}$, d, Pz5C), $7.95(1 \mathrm{H}, \mathrm{d}, \mathrm{Pz5A}), 7.83(1 \mathrm{H}, \mathrm{d}, \mathrm{Pz3C}), 7.81(1 \mathrm{H}, \mathrm{d}, \mathrm{Pz5B}), 7.66(1 \mathrm{H}, \mathrm{d}, J=$ 7.4, H8), 7.45 (2H, bs, DMAP-A), 7.44 (1H, s, H9), 7.30 (1H, d, J = 8.1, H5), $7.25(1 \mathrm{H}$, m, H6), $7.18(1 \mathrm{H}, \mathrm{m}, \mathrm{H} 7), 7.06$ (1H, d, Pz3B), 6.44 (2H, bs, DMAP-B), $6.42(1 \mathrm{H}, \mathrm{t}$, Pz4A), $6.38(1 \mathrm{H}, \mathrm{t}, \mathrm{Pz} 4 \mathrm{C}), 6.32(1 \mathrm{H}, \mathrm{t}, J=2.2, \mathrm{H} 12), 6.31(1 \mathrm{H}, \mathrm{s}, \mathrm{H} 10), 6.11(1 \mathrm{H}, \mathrm{t}$, Pz4B), $5.73(1 \mathrm{H}, \mathrm{m}, \mathrm{H} 11), 5.61(1 \mathrm{H}, \mathrm{m}, \mathrm{H} 13), 4.10(1 \mathrm{H}, \mathrm{d}, J=6.7, \mathrm{H} 2), 3.92(1 \mathrm{H}, \mathrm{dd}, J=$ 15.5 \& 6.9, H1), 3.46 (3H, s, NMe), $3.42(1 \mathrm{H}, \mathrm{d}, J=9.4, \mathrm{H} 4), 3.06$ (6H, s, NMe), 2.99 $\left(1 \mathrm{H}, \mathrm{d}, J=15.5, \mathrm{H} 1^{\prime}\right), 2.57(1 \mathrm{H}, \mathrm{dt}, J=9.7 \& 1.9, \mathrm{H} 3) .{ }^{13} \mathrm{C}$ NMR (d - -Acetone, $\left.\delta\right): 154.0$ (DMAP-C), 143.4 (Pz3/5), 143.2 (Pz3/5), 142.2 (Pz3/5), 141.7 (Pz3/5), 141.6 (Pz3/5), 
141.5 (Pz3/5), 137.3, 135.8, 134.1, 132.2, 129.7, 127.6, 126.8, 126.7, 126.6, 124.9, 123.7, 123.5, 120.6, 120.5 (C13), 108.1 (DMAP-B), 108.0 (C11), 106.9 (Pz4), 106.6 (Pz4), 106.6 (Pz4), 106.3 (C12), 68.1, 39.2 (NMe), 34.7, 33.5, 29.8. HRMS: $\mathrm{C}_{35} \mathrm{H}_{37} \mathrm{~N}_{10} \mathrm{OBMo}{ }^{+}$ obsd (\%), calcd (\%), ppm: 716.2271 (16), $716.2314(50),-5.9 ; 718.2334(23), 718.2312$ (48), 3.1; 719.2303 (69), 719.2310 (80), -1.0; 720.2297 (100), 720.2307 (88), -1.3; 721.2331 (91), 721.2318 (78), 1.8; 722.2318 (95), 722.2306 (100), 1.6; 723.2349 (48), 723.2332 (43), 2.3; 724.2335 (27), $724.2326(40), 1.2$.

Synthesis of TpMo(NO)(DMAP)(3,4- $\eta^{2}-2-(1,2-d i h y d r o n a p h t h a l e n-2-y l)-$ nitromethane) (36).

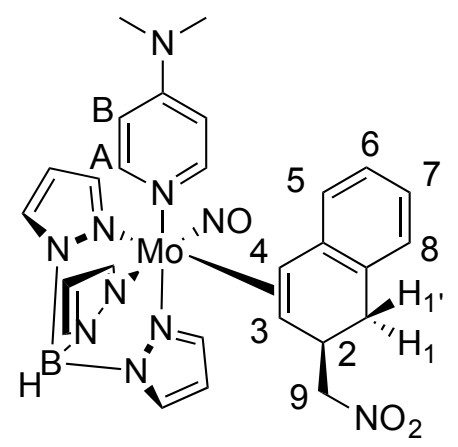

$\mathrm{CH}_{3} \mathrm{CH}_{2} \mathrm{CN}(2 \mathrm{~mL}), \mathrm{MeOH}(2 \mathrm{~mL}), \mathrm{LiOMe}(30 \mathrm{mg}, 0.79 \mathrm{mmol})$, and nitromethane (300 $\mathrm{mg}, 4.90 \mathrm{mmol}$ ) were added to a test tube charged with a stir pea and this solution was cooled at $-60{ }^{\circ} \mathrm{C}$ for $15 \mathrm{~min} .27(250 \mathrm{mg}, 0.338 \mathrm{mmol})$ was added and the resulting brown solution was left stirring at $-60{ }^{\circ} \mathrm{C}$ overnight $(\sim 18 \mathrm{~h})$. This solution was then warmed to RT, extracted with DCM $(6 \mathrm{~mL})$ and $\mathrm{H}_{2} \mathrm{O}(10 \mathrm{~mL})$, and the organic layer was dried over $\mathrm{MgSO}_{4}$ for $15 \mathrm{~min}$. The drying agent was filtered off on a $15 \mathrm{~mL}$ medium porosity fritted disc, washed with DCM (2 x $4 \mathrm{~mL})$ and the yellow filtrate was evaporated in vacuo to an oil. The oil was dissolved in DCM $(1 \mathrm{~mL})$ and the product was precipitated in stirring 
pentane $(50 \mathrm{~mL})$. The precipitate was collected on a $15 \mathrm{~mL}$ fine porosity fritted disc, washed with pentane $(3 \times 10 \mathrm{~mL})$, and desiccated for 15 min yielding the yellow solid 36 (150 mg, 63\%). CV (DMAc) $E_{\mathrm{p}, \mathrm{a}}=+0.25 \mathrm{~V}$ (NHE). IR: $v(\mathrm{BH})=2924 \mathrm{~cm}^{-1}, v(\mathrm{NO})=$ $1574 \& 1543 \mathrm{~cm}^{-1} .{ }^{1} \mathrm{H}$ NMR (d $\mathrm{d}^{6}$-Acetone, $\left.\delta\right): 7.98$ (1H, d, Pz5C), $7.93(1 \mathrm{H}, \mathrm{d}$, Pz5A), 7.89 (1H, d, Pz3A), 7.80 (1H, d, Pz5B), 7.73 (1H, d, Pz3C), 7.39 (2H, bs, DMAP-A), 7.01 (2H, m, H6 \& H7), 7.00 (1H, d, Pz3B), 6.86 (1H, m, H8), 6.53 (2H, m, DMAP-B), $6.41(1 \mathrm{H}, \mathrm{t}, \mathrm{Pz} 4 \mathrm{~A}), 6.39$ (1H, t, Pz4C), $6.20(1 \mathrm{H}, \mathrm{m}, \mathrm{H}), 6.10(1 \mathrm{H}, \mathrm{t}, \mathrm{Pz} 4 \mathrm{~B}), 4.40(1 \mathrm{H}$, dd, $J=10.2 \& 6.6, \mathrm{H} 9), 4.40$ (1H, dd, $J=10.7 \& 8.6, \mathrm{H} 9), 3.56(1 \mathrm{H}, \mathrm{dd}, J=16.1 \& 5.9$, H1), $3.45(1 \mathrm{H}, \mathrm{m}, \mathrm{H} 2), 3.28(1 \mathrm{H}, \mathrm{d}, J=9.5, \mathrm{H} 4), 3.06(6 \mathrm{H}, \mathrm{s}, \mathrm{NMe}), 2.54(1 \mathrm{H}, \mathrm{d}, J=$ 16.1, H1'), $2.13(1 \mathrm{H}, \mathrm{dt}, J=9.5 \& 2.3, \mathrm{H} 3) .{ }^{13} \mathrm{C}$ NMR (d - -Acetone, $\left.\delta\right): 155.1$ (DMAP-C), 150.9 (DMAP-A), 150.7 (Pz5C), 143.2 (Pz3A), 142.1 (Pz3B), 141.5 (Pz3C), 137.1 (Pz5A), 135.8 (Pz5B), 130.6 (C6/7), 129.6 (C6/7), 127.6 (C5), 126.7, 125.5 (C8), 123.7, 108.2 (DMAP-B), 107.0 (Pz4), 106.9 (Pz4), 106.4 (Pz4), 83.1 (C9), 67.0 (C4), 66.5 (C3), 39.1 (NMe), 37.6 (C2), $31.3(\mathrm{C} 1)$. HRMS: $\mathrm{C}_{27} \mathrm{H}_{31} \mathrm{~N}_{10} \mathrm{O}_{3} \mathrm{BMo}+\mathrm{Na}^{+}$obsd (\%), calcd (\%), ppm: 669.1608 (44), 669.1639 (52), -4.6; 671.1629 (41), 671.1636 (49), -1.1; 672.1610 (74), 672.1635 (81), -3.7; 673.1638 (80), 673.1630 (87), 1.1; 674.1628 (79), 674.1643 (76), -2.2; 675.1616 (100), 675.1630 (100), -2.1; 676.1657 (30), 676.1657 (38), -0.1; 677.1651 (29), $677.1650(39), 0.2$.

\section{Synthesis of 2-(nitromethyl)-1,2-dihydronaphthalene (37).}

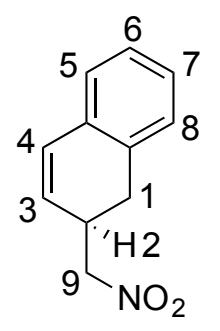


To a $50 \mathrm{~mL}$ filter flask charged with a stir pea were added $\mathbf{3 6}$ (100 mg, $0.123 \mathrm{mmol})$, DCM $(5 \mathrm{~mL})$, and a $0.06 \mathrm{M}$ solution of $\mathrm{I}_{2} / \mathrm{Et}_{2} \mathrm{O}(1 \mathrm{~mL}, 0.06 \mathrm{mmol})$ resulting in a green solution. The solution was stirred at RT for $5 \mathrm{~min}$ and then evaporated in vacuo to an oil. The oil was dissolved in DCM $(1 \mathrm{~mL})$, giving a solution that was then added to stirring pentane $(25 \mathrm{~mL})$, creating a green precipitation. The precipitate was collected on a $15 \mathrm{~mL}$ fine porosity fritted disc, washed with pentane ( $3 \times 10 \mathrm{~mL})$, and desiccated to yield $\mathbf{2 3}$ (65 mg, 90\%). The filtrate was removed from the glovebox and evaporated in vacuo to a brown oil. The oil was dissolved in DCM $(3 \times 0.3 \mathrm{~mL})$ and the resulting solution was dropwise added onto a $250 \mu \mathrm{m}$ silica preparatory plate. The product was eluted with $10 \%$ EtOAc:hexanes $(200 \mathrm{~mL})$, scraped off as a band at $\mathrm{R}_{\mathrm{f}}: 0.51-0.65$ and this silica gel was sonicated in EtOAc $(20 \mathrm{~mL})$ for $15 \mathrm{~min}$. The silica was filtered off on a $15 \mathrm{~mL}$ medium porosity fritted disc and washed with DCM $(3 \times 2 \mathrm{~mL})$. The colorless filtrate was then evaporated in vacuo and desiccated to yield the colorless oil 37 (9 mg, 39\% yield). IR: $v\left(\mathrm{C}-\mathrm{H} \mathrm{sp}{ }^{2}\right)=3020,2916,2850 \mathrm{~cm}^{-1}, v(\mathrm{NO})=1550,1377 \mathrm{~cm}^{-1} .{ }^{1} \mathrm{H} \mathrm{NMR}\left(\mathrm{CDCl}_{3}, \delta\right)$ : $7.20(2 \mathrm{H}, \mathrm{m}, \mathrm{H} 6$ \& H7), 7.14 (1H, d, $J=7.2, \mathrm{H} 5), 7.08(1 \mathrm{H}, \mathrm{d}, J=6.3, \mathrm{H} 8), 6.61(1 \mathrm{H}, \mathrm{d}$, $J=9.5, \mathrm{H} 4), 5.88(1 \mathrm{H}, \mathrm{dd}, J=9.5 \& 5.0, \mathrm{H} 3), 4.35(1 \mathrm{H}, \mathrm{dd}, J=12.2 \& 6.6, \mathrm{H} 9), 4.31$ $(1 \mathrm{H}, \mathrm{dd}, J=12.2 \& 8.8, \mathrm{H} 9), 3.29(1 \mathrm{H}, \mathrm{m}, \mathrm{H} 2), 3.04(1 \mathrm{H}, \mathrm{dd}, J=16.4 \& 6.6, \mathrm{H} 1), 2.74$ $(1 \mathrm{H}, \mathrm{dd}, J=16.4 \& 6.9, \mathrm{H} 1) .{ }^{13} \mathrm{C} \mathrm{NMR}\left(\mathrm{CDCl}_{3}, \delta\right): 132.7,132.4,130.7$ (C4), 128.5 (C5), 128.2 (C6/7), 127.4 (C6/7), 126.7 (C8), 125.8 (C3), 77.5 (C9), 32.9 (C2), 30.9 (C1). LRMS: $\mathrm{C}_{11} \mathrm{H}_{11} \mathrm{NO}_{2}$, mass found: 189.1, mass required: 189.08 . 


\section{Synthesis of 2-(nitromethyl)-1,2-dihydroanthracene (38).}

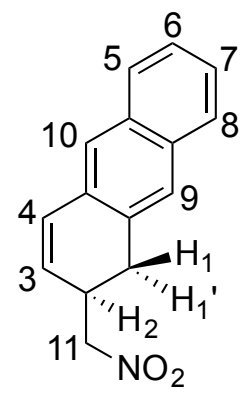

$\mathrm{CH}_{3} \mathrm{CN}(2 \mathrm{~mL}), \mathrm{LiOMe}(15 \mathrm{mg}, 0.39 \mathrm{mmol}), \mathrm{MeOH}(0.5 \mathrm{~mL})$, and nitromethane (120 $\mathrm{mg}, 1.97 \mathrm{mmol}$ ) were added to a 4-dram vial and this solution was cooled at $-30{ }^{\circ} \mathrm{C}$ for $15 \mathrm{~min} .28$ (100 mg, $0.126 \mathrm{mmol})$ was added to this solution and left at $-30{ }^{\circ} \mathrm{C}$ for $18 \mathrm{~h}$. The reaction mixture was removed from the glovebox and left open to air overnight $(\sim 24$ h) resulting in a brown film. The film was dissolved in DCM (3 $\times 0.3 \mathrm{~mL})$ and the resulting solution was dropwise added onto a $250 \mu \mathrm{m}$ silica preparatory plate. The product was eluted with 10\% EtOAc:hexanes $(200 \mathrm{~mL})$, scraped off as a band at $\mathrm{R}_{\mathrm{f}}$ : 0.12 0.43 and this silica gel was sonicated in EtOAc $(20 \mathrm{~mL})$ for $15 \mathrm{~min}$. The silica was filtered off on a $15 \mathrm{~mL}$ fine porosity fritted disc and washed with DCM $(3 \times 2 \mathrm{~mL})$. The filtrate was evaporated in vacuo and desiccated to yield the colorless oil 38 (10 $\mathrm{mg}$, 33\%). IR: $v\left(\mathrm{C}-\mathrm{H} \mathrm{sp}{ }^{2}\right)=2916 \mathrm{~cm}^{-1}, v(\mathrm{NO})=1545 \mathrm{~cm}^{-1} .{ }^{1} \mathrm{H} \mathrm{NMR}\left(\mathrm{CDCl}_{3}, \delta\right): 7.78(1 \mathrm{H}$, m, H5), 7.75 (1H, m, H8), 7.57 (1H, s, H9), 7.53 (1H, s, H10), 7.43 (2H, m, H6 \& H7), $6.79(1 \mathrm{H}, \mathrm{d}, J=9.9, \mathrm{H} 4), 5.99(1 \mathrm{H}, \mathrm{dd}, J=9.9 \& 4.7, \mathrm{H} 3), 4.37(1 \mathrm{H}, \mathrm{dd}, J=13.0 \& 6.3$, H11), 4.34 (1H, dd, $J=13.0 \& 8.6, \mathrm{H} 11), 3.37(1 \mathrm{H}, \mathrm{m}, \mathrm{H} 2), 3.19(1 \mathrm{H}, \mathrm{dd}, J=15.5 \& 6.1$, $\left.\mathrm{H} 1 / \mathrm{H} 1{ }^{\prime}\right), 2.91\left(1 \mathrm{H}, \mathrm{dd}, J=15.5 \& 6.1, \mathrm{H} 1 / \mathrm{H} 1^{\prime}\right) .{ }^{13} \mathrm{C} \mathrm{NMR}\left(\mathrm{CDCl}_{3}, \delta\right): 133.4(\mathrm{C} 10 / 9 \mathrm{a})$, 133.1 (C10/9a), 131.0 (C5a/C8a), 130.9 (C4), 130.7 (C5a/C8a), 127.9 (C10), 127.4 (C9), 127.0 (C5), 126.9 (C6/7), 126.4 (C6/7), 126.0 (C3), 125.4 (C8), 77.7 (C11), 33.4 (C2), $31.5(\mathrm{C} 1)$. 


\section{1-(1,2-dihydronaphthalen-2-yl)-1H-pyrazole (39).}

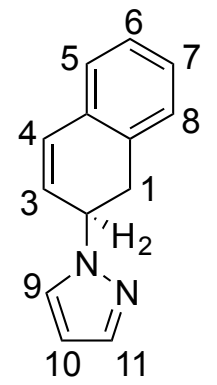

DBU (0.1 mL, $0.068 \mathrm{mmol}), \mathrm{CH}_{3} \mathrm{CH}_{2} \mathrm{CN}(2 \mathrm{~mL})$ and pyrazole (200 mg, $\left.2.93 \mathrm{mmol}\right)$ were added to a test tube and this solution was cooled at $-60{ }^{\circ} \mathrm{C}$ for $15 \mathrm{~min} .27(200 \mathrm{mg}, 0.271$ mmol) was then added to this solution and left at $-60{ }^{\circ} \mathrm{C}$ for $18 \mathrm{~h}$. The reaction mixture was then warmed to RT, extracted with DCM $(6 \mathrm{~mL})$ and $\mathrm{H}_{2} \mathrm{O}(10 \mathrm{~mL})$, and the organic layer was dried over $\mathrm{MgSO}_{4}$. The drying agent was filtered off on a $15 \mathrm{~mL}$ medium porosity fritted disc and washed with DCM $(3 \times 3 \mathrm{~mL})$. The filtrate was evaporated in vacuo to an oil, which was then removed from the glovebox. The oil was dissolved in DCM $(10 \mathrm{~mL})$ and stirred open to air overnight $(18 \mathrm{~h})$ at RT. The brown film formed was dissolved in DCM $(3 \times 0.3 \mathrm{~mL})$ and the resulting solution was dropwise added onto a 250 $\mu \mathrm{m}$ silica preparatory plate. The product was eluted with $10 \%$ EtOAc:hexanes $(200 \mathrm{~mL})$, scraped off as a band at $\mathrm{R}_{\mathrm{f}}: 0.22-0.34$ and this silica gel was sonicated in EtOAc $(20 \mathrm{~mL})$ for $15 \mathrm{~min}$. The silica was filtered off on a $15 \mathrm{~mL}$ fine porosity fritted disc and washed with DCM $(3 \times 2 \mathrm{~mL})$. The filtrate was evaporated in vacuo and desiccated to yield the oil 39 (10 mg, 19\%). IR: $v\left(\mathrm{C}-\mathrm{H} \mathrm{sp}^{2}\right)=3018,2921,2850 \mathrm{~cm}^{-1} .{ }^{1} \mathrm{H}$ NMR $\left(\mathrm{CDCl}_{3}, \delta\right): 7.52$ (1H, d, $J=1.8, \mathrm{H} 11), 7.31(1 \mathrm{H}, \mathrm{d}, J=2.4, \mathrm{H} 9), 7.22(1 \mathrm{H}, \mathrm{t}, J=7.6, \mathrm{H} 7), 7.19(1 \mathrm{H}, \mathrm{td}, J$ $=7.6 \& 1.5, \mathrm{H} 6), 7.16(1 \mathrm{H}, \mathrm{d}, J=7.4, \mathrm{H} 5), 7.11(1 \mathrm{H}, \mathrm{d}, J=7.4, \mathrm{H} 8), 6.76(1 \mathrm{H}, \mathrm{dd}, J=$ $9.6 \& 1.7, \mathrm{H} 4), 6.17(1 \mathrm{H}, \mathrm{t}, J=2.1, \mathrm{H} 10), 6.15(1 \mathrm{H}, \mathrm{dd}, J=9.6 \& 4.4, \mathrm{H} 3), 5.28(1 \mathrm{H}, \mathrm{m}$, H2), $3.33\left(1 \mathrm{H}, \mathrm{dd}, J=16.3 \& 7.4, \mathrm{H} 1 / \mathrm{H} 1^{\prime}\right), 3.28$ (1H, dd, $\left.J=16.3 \& 7.3, \mathrm{H} 1 / \mathrm{H} 1{ }^{\prime}\right) .{ }^{13} \mathrm{C}$ 
NMR (CDCl $3, \delta): 139.3$ (C11), 132.6, 132.2, 131.1 (2C's) (C4), 128.3 (C8), 128.2 (C9), 127.5, 127.2, 126.8, 125.7, $125.6(\mathrm{C} 3), 105.5$ (C10), 56.0 (C2), 35.3 (C1).

\section{Synthesis of 1-(1,2-dihydroanthracen-2-yl)-1H-pyrazole (40).}

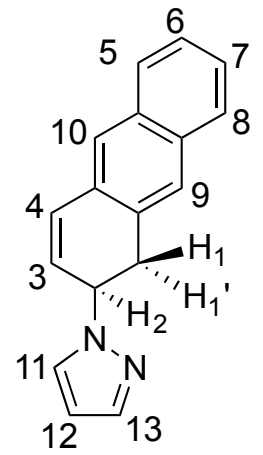

DBU (0.1 mL, $0.068 \mathrm{mmol}), \mathrm{CH}_{3} \mathrm{CH}_{2} \mathrm{CN}(2 \mathrm{~mL})$ and pyrazole (200 mg, $\left.2.93 \mathrm{mmol}\right)$ were added to a test tube and this solution was cooled at $-60{ }^{\circ} \mathrm{C}$ for $15 \mathrm{~min} . \mathbf{2 8}(100 \mathrm{mg}, 0.127$ mmol) was added to this solution and left at $-60{ }^{\circ} \mathrm{C}$ for $18 \mathrm{~h}$. The reaction mixture was warmed to RT, extracted with DCM $(6 \mathrm{~mL})$ and $\mathrm{H}_{2} \mathrm{O}(10 \mathrm{~mL})$, and the organic layer was dried over $\mathrm{MgSO}_{4}$. The drying agent was filtered off on a $15 \mathrm{~mL}$ medium porosity fritted disc and washed with DCM (3 x $4 \mathrm{~mL})$. The filtrate was evaporated in vacuo to an oil that was then removed from the glovebox, dissolved in DCM $(10 \mathrm{~mL})$ and the resulting brown solution was stirred open to air overnight $(18 \mathrm{~h})$. The brown oil formed was dissolved in DCM (3 $\times 0.3 \mathrm{~mL})$ and the resulting solution was dropwise added onto a 250 $\mu \mathrm{m}$ silica preparatory plate. The product was eluted with $10 \%$ EtOAc:hexanes $(200 \mathrm{~mL})$, scraped off as a band at $\mathrm{R}_{\mathrm{f}}: 0.15-0.28$ and this silica gel was sonicated in EtOAc $(20 \mathrm{~mL})$ for $15 \mathrm{~min}$. The silica was filtered off on a $15 \mathrm{~mL}$ fine porosity fritted disc and washed with DCM (3 x $2 \mathrm{~mL})$. The filtrate was evaporated in vacuo and desiccated to yield the oil $40(6 \mathrm{mg}, 20 \%)$. IR: $v\left(\mathrm{C}-\mathrm{H} \mathrm{sp}{ }^{2}\right)=2947 \mathrm{~cm}^{-1} .{ }^{1} \mathrm{H} \mathrm{NMR}\left(\mathrm{CDCl}_{3}, \delta\right): 7.79(1 \mathrm{H}, \mathrm{m}, \mathrm{H} 5)$, 
$7.71(1 \mathrm{H}, \mathrm{m}, \mathrm{H} 8), 7.61(1 \mathrm{H}, \mathrm{s}, \mathrm{H} 10), 7.53(1 \mathrm{H}, \mathrm{s}, \mathrm{H} 9), 7.53(1 \mathrm{H}, \mathrm{d}, J=2.2, \mathrm{H} 13), 7.44$ $(2 \mathrm{H}, \mathrm{m}, \mathrm{H} 6$ \& H7), $7.31(1 \mathrm{H}, \mathrm{d}, J=2.2, \mathrm{H} 11), 6.96$ (1H, d, $J=9.6, \mathrm{H} 4), 6.29(1 \mathrm{H}, \mathrm{dd}, J$ $=9.6 \& 4.4, \mathrm{H} 3), 6.19(1 \mathrm{H}, \mathrm{t}, J=2.2, \mathrm{H} 12), 5.38(1 \mathrm{H}, \mathrm{m}, \mathrm{H} 2), 3.50(1 \mathrm{H}, \mathrm{dd}, J=15.9 \&$ 6.8, H1'), $3.47(1 \mathrm{H}, \mathrm{dd}, J=15.9 \& 6.6, \mathrm{H} 1) .{ }^{13} \mathrm{C} \mathrm{NMR}\left(\mathrm{CDCl}_{3}, \delta\right): 139.5$ (C9), 133.5, 133.0, 131.5 (C4), 130.8, 130.8, 128.0 (C5), 127.6, 127.5 (C11), 127.0 (C8), 126.9 (C3), 126.5 (C9), 126.0 (C6 \& C7), 125.8 (C10), 105.6 (C12), 56.4 (C2), 36.0 (C1). LRMS: $\mathrm{C}_{17} \mathrm{H}_{14} \mathrm{~N}_{2}$, mass found [M+Na $\left.{ }^{+}\right]: 269$, mass required: 246.12 .

\section{Synthesis of TpMo(NO)(DMAP)(2,3- $\eta^{2}$-dimethyl 2-(4-(2-methoxypropan-2-yl)-1,4-} dihydronaphthalen-1-yl)malonate) (41).

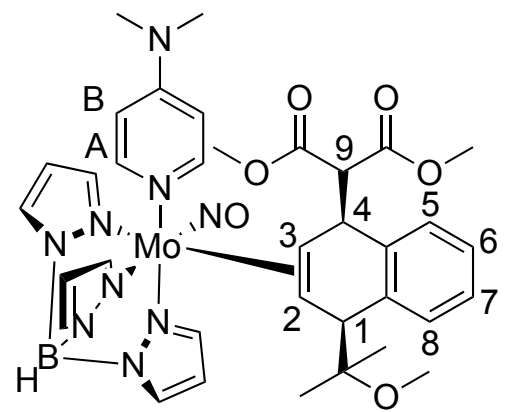

To a test tube charged with a stir pea were added 24 (200 mg, $0.340 \mathrm{mmol}), \mathrm{CH}_{3} \mathrm{CH}_{2} \mathrm{CN}$ (6 $\mathrm{mL})$, and dimethoxypropane $(0.3 \mathrm{~mL}, 2.44 \mathrm{mmol})$. The resulting orange solution was stirred at RT until homogeneous and then cooled at $-60{ }^{\circ} \mathrm{C}$ for $15 \mathrm{~min}$. TMSOTf $(0.1 \mathrm{~mL}$, $0.54 \mathrm{mmol}$ ) was added to the reaction mixture and the resulting red solution was left stirring at $-60{ }^{\circ} \mathrm{C}$ for $15 \mathrm{~min}$. LiDMM (250 $\left.\mathrm{mg}, 1.81 \mathrm{mmol}\right)$ was added to the solution giving a yellow solution that was left stirring at $-60{ }^{\circ} \mathrm{C}$. After $18 \mathrm{~h}$, TEA $(0.2 \mathrm{~mL}, 1.41$ mmol) was added and the reaction mixture was warmed to RT, extracted with DCM (6 $\mathrm{mL})$ and $\mathrm{H}_{2} \mathrm{O}(10 \mathrm{~mL})$, and the organic layer was dried over $\mathrm{MgSO}_{4}$. The drying agent was filtered off on a $15 \mathrm{~mL}$ medium porosity fritted disc, and washed with DCM $(3 \times 2$ 
$\mathrm{mL}$ ), and the filtrate was evaporated in vacuo. The resultant oil was dissolved in DCM (1 $\mathrm{mL})$ and the product was precipitated in stirring pentane $(50 \mathrm{~mL})$. The beige precipitate was isolated on a $15 \mathrm{~mL}$ fine porosity fritted disc, washed with pentane $(3 \times 10 \mathrm{~mL})$, and desiccated to yield 41 (170 mg, 63\% yield). CV (DMAc) $E_{\mathrm{p}, \mathrm{a}}=+0.17 \mathrm{~V}$ (NHE). IR: $v(\mathrm{C}-$ $\left.\mathrm{H} \mathrm{sp}^{2}\right)=2942 \mathrm{~cm}^{-1}, v(\mathrm{BH})=2477 \mathrm{~cm}^{-1}, v(\mathrm{CO})=1730 \mathrm{~cm}^{-1}, v(\mathrm{NO})=1567 \mathrm{~cm}^{-1} \cdot{ }^{1} \mathrm{H}$ NMR (d $\mathrm{d}^{6}$-Acetone, $\left.\delta\right): 7.94$ (1H, d, Pz3A), 7.92 (1H, d, Pz5C), 7.87 (1H, d, Pz5A), 7.80 (2H, bs, DMAP-A), 7.68 (1H, d, Pz5B), 7.58 (1H, d, Pz3C), 7.34 (3H, m, H6, H7, and H8), 7.04 (2H, m, H6 \& H7), 7.02 (1H, d, J = 7.2, H5), 6.88 (1H, d, Pz3B), 6.66 (2H, m, DMAP-B), 6.35 (1H, t, Pz4C), 6.30 (1H, t, Pz4A), 6.00 (1H, t, Pz4B), 4.49 (1H, d, $J=$ 10.7, H9), 4.00 (1H, d, $J=10.7, \mathrm{H} 4), 3.91(1 \mathrm{H}, \mathrm{s}, \mathrm{H} 1), 3.57(3 \mathrm{H}, \mathrm{s}, \mathrm{OMe}), 3.43(3 \mathrm{H}, \mathrm{s}$, OMe), 3.89 (6H, s, NMe), 3.03 (3H, s, OMe), 2.52 (1H, d, $J=10.5, \mathrm{H} 3), 2.45$ (1H, d, $J=$ 10.5, H2), $1.22(3 \mathrm{H}, \mathrm{s}, \mathrm{Me}), 1.21(3 \mathrm{H}, \mathrm{s}, \mathrm{Me}) .{ }^{13} \mathrm{C}$ NMR (d $\mathrm{d}^{6}$-Acetone, $\left.\delta\right): 170.3$ (CO), 170.1 (CO), 154.9 (DMAP-C), 151.2 (DMAP-A), 142.9 (Pz3A), 141.6 (Pz3B), 141.6 (Pz3C), 139.1, 138.5, 137.3 (Pz5A), 137.1 (Pz5C), 135.4 (Pz5B), 133.6 (C5), 129.8, 126.1 (C8), 125.9, 108.4 (DMAP-B), 106.7 (Pz4C), 106.2 (Pz4A), 105.9 (Pz4B), 79.6, 69.4 (C3), 63.6 (C9), 61.7 (C2), 53.9 (C1), 52.2 (OMe), 51.7 (OMe), 48.9 (OMe), 44.7 (C4), $39.1(\mathrm{NMe}), 32.3,25.4(\mathrm{Me}), 24.6(\mathrm{Me})$. 
Synthesis of TpMo(NO)(DMAP)(2,3- $\eta^{2}$-dimethyl 2-(4-(2-methoxypropan-2-yl)-1,4dihydroanthracen-1-yl)malonate) (42).

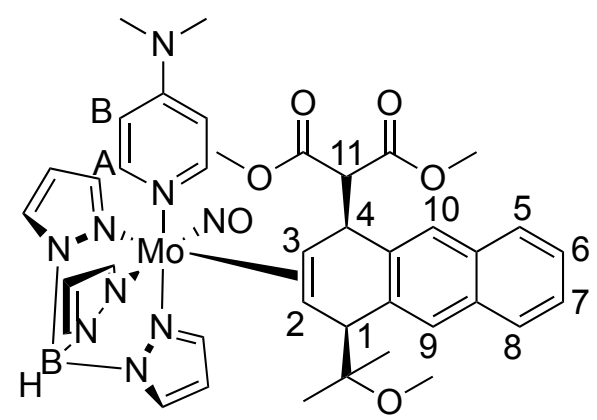

25 (200 mg, $0.313 \mathrm{mmol}), \mathrm{CH}_{3} \mathrm{CH}_{2} \mathrm{CN}$ (6 mL), and dimethoxypropane (0.3 mL, 2.44 mmol) were added to a test tube charged with a stir pea. The reaction mixture was stirred at RT until homogeneous and then cooled at $-60{ }^{\circ} \mathrm{C}$ for $15 \mathrm{~min}$. TMSOTf $(0.1 \mathrm{~mL}, 0.54$ mmol) was added to the reaction mixture and the resulting red solution was left stirring at $-60{ }^{\circ} \mathrm{C}$ for $15 \mathrm{~min}$. LiDMM (150 $\left.\mathrm{mg}, 1.09 \mathrm{mmol}\right)$ was added to the red solution, giving a brown solution that was left stirring at $-60{ }^{\circ} \mathrm{C}$ for $18 \mathrm{~h}$. TEA $(0.2 \mathrm{~mL}, 1.43 \mathrm{mmol})$ was added to the brown solution, which was then warmed to RT, extracted with DCM (6 mL) and $\mathrm{H}_{2} \mathrm{O}(10 \mathrm{~mL})$, and the organic layer was dried over $\mathrm{MgSO}_{4}$. The drying agent was filtered off on a $15 \mathrm{~mL}$ medium porosity fritted disc, washed with DCM (3 x $2 \mathrm{~mL})$, and the filtrate was evaporated in vacuo. The resulting oil was then dissolved in DCM (1 mL) and the product was precipitated in stirring pentane $(50 \mathrm{~mL})$. The beige precipitate was isolated on a $15 \mathrm{~mL}$ fine porosity fritted disc, washed with pentane $(3 \mathrm{x} 10 \mathrm{~mL})$, and desiccated to yield 42 (152 mg, 58\% yield). CV (DMAc) $E_{\mathrm{p}, \mathrm{a}}=+0.07 \mathrm{~V}$ (NHE). IR: $v(\mathrm{C}-$ $\left.\mathrm{H} \mathrm{sp}^{2}\right)=2945 \mathrm{~cm}^{-1}, v(\mathrm{BH})=2475 \mathrm{~cm}^{-1}, v(\mathrm{CO})=1729 \mathrm{~cm}^{-1}, v(\mathrm{NO})=1569 \mathrm{~cm}^{-1} \cdot{ }^{1} \mathrm{H}$ NMR (d $\mathrm{d}^{6}$-Acetone, $\left.\delta\right): 7.96$ (1H, d, Pz3A), 7.93 (1H, d, Pz5C), 7.89 (1H, s, H9), 7.88 (1H, d, Pz5A), 7.84 (2H, bs, DMAP-A), 7.77 (1H, m, H5/H8), 7.73 (1H, m, H5/H8), 7.68 (1H, d, Pz5B), 7.61 (1H, d, Pz3C), 7.59 (1H, s, H10), 7.31 (2H, m, H6 \& H7), 6.85 (1H, 
d, Pz3B), 6.68 (2H, m, DMAP-B), 6.38 (1H, t, Pz4C), $6.32(1 \mathrm{H}, \mathrm{t}, \mathrm{Pz} 4 \mathrm{~A}), 5.97(1 \mathrm{H}, \mathrm{t}$, Pz4B), $4.70(1 \mathrm{H}, \mathrm{d}, J=10.8, \mathrm{H} 11), 4.26(1 \mathrm{H}, \mathrm{d}, J=10.9, \mathrm{H} 4), 4.10(1 \mathrm{H}, \mathrm{s}, \mathrm{H} 1), 3.60$ (3H, s, OMe), $3.39(3 \mathrm{H}, \mathrm{s}, \mathrm{OMe}), 3.09(6 \mathrm{H}, \mathrm{s}, \mathrm{NMe}), 3.07(3 \mathrm{H}, \mathrm{s}, \mathrm{OMe}), 2.60(1 \mathrm{H}, \mathrm{d}, J=$ 10.3, H3), $2.51(1 \mathrm{H}, \mathrm{d}, J=10.3, \mathrm{H} 2), 1.30(3 \mathrm{H}, \mathrm{s}, \mathrm{Me}), 1.25(3 \mathrm{H}, \mathrm{s}, \mathrm{Me}) \cdot{ }^{13} \mathrm{C}$ NMR $\left(\mathrm{d}^{6}-\right.$ Acetone, $\delta$ ): 170.4 (CO), 170.1 (CO), 154.9 (DMAP-C), 151.2 (DMAP-A), 142.9, 141.6 (C5/8), 141.5 (Pz3B), 138.1, 137.9, 137.3 (Pz5C), 137.1 (Pz5A), 135.5 (Pz5B), 133.5, 133.4, 131.7 (C9), 128.1, 127.9 (C5/8), 127.7 (C10), 125.1, 125.0, 108.5 (DMAP-B), 106.7 (Pz4C), 106.3 (Pz4A), 105.9 (Pz4B), 79.9, 68.8 (C3), 63.6 (C11), 61.5 (C7), 54.3 (C1), 52.3 (OMe), $51.8(\mathrm{OMe}), 48.9(\mathrm{OMe}), 44.9(\mathrm{C} 4), 39.1(\mathrm{NMe}), 29.8,25.6(\mathrm{Me})$, $24.6(\mathrm{Me})$.

\section{Synthesis of dimethyl 2-(-4-(2-methoxypropan-2-yl)-1,4-dihydronaphthalen-1-} yl)malonate (43).

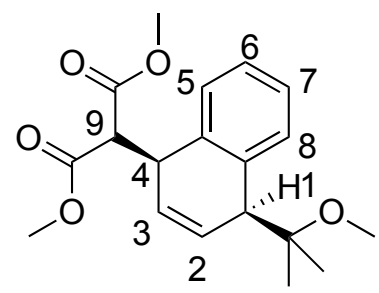

Via Iodine Oxidation

To a $50 \mathrm{~mL}$ filter flask charged with a stir pea were added 41 (100 $\mathrm{mg}, 0.126 \mathrm{mmol})$, DCM $(5 \mathrm{~mL})$, and a $0.06 \mathrm{M}$ solution of $\mathrm{I}_{2} / \mathrm{Et}_{2} \mathrm{O}(1 \mathrm{~mL}, 0.06 \mathrm{mmol})$, resulting in a green solution. This solution was stirred at RT for $5 \mathrm{~min}$ and evaporated in vacuo. The resulting green oil was dissolved in DCM $(1 \mathrm{~mL})$ forming a green solution. This solution was added to stirring hexanes $(50 \mathrm{~mL})$ giving a green precipitation. Collected the green precipitate on a $15 \mathrm{~mL}$ fine porosity fritted disc, washed with hexanes $(3 \times 10 \mathrm{~mL})$, and 
desiccated to yield $\mathbf{2 3}$ (69 $\mathrm{mg}, 93 \%$ ). The filtrate was removed from the glovebox and evaporated in vacuo to an oil. The oil was dissolved in DCM ( $3 \times 0.3 \mathrm{~mL})$ and the resulting solution was dropwise added onto a $250 \mu \mathrm{m}$ silica preparatory plate. The product was eluted with 10\% EtOAc:hexanes $(200 \mathrm{~mL})$, scraped off as a band at $\mathrm{R}_{\mathrm{f}}: 0.42-$ 0.54 and this silica gel was sonicated in EtOAc $(20 \mathrm{~mL})$ for $15 \mathrm{~min}$. The silica was filtered off on a $15 \mathrm{~mL}$ medium porosity fritted disc and washed with DCM (3 x $2 \mathrm{~mL})$. The filtrate was evaporated in vacuo and desiccated to yield the colorless oil 43 (36 mg, $85 \%$ yield).

\section{Via Air Oxidation}

24 (200 mg, $0.339 \mathrm{mmol}), \mathrm{CH}_{3} \mathrm{CH}_{2} \mathrm{CN}$ (6 mL), and dimethoxypropane (0.3 mL, 2.44 mmol) were added to a test tube charged with a stir pea and the orange solution was stirred at RT for $1 \mathrm{~min}$, then at $-60^{\circ} \mathrm{C}$ for $15 \mathrm{~min}$. TMSOTf $(0.1 \mathrm{~mL}, 0.54 \mathrm{mmol})$ was added to the reaction mixture and the resulting red solution was stirred at $-60{ }^{\circ} \mathrm{C}$ for 15 min. LiDMM (250 $\mathrm{mg}, 1.81 \mathrm{mmol})$ was added to the reaction mixture and the resulting brown solution was left at $-60{ }^{\circ} \mathrm{C}$ for $2 \mathrm{~h}$. The reaction mixture was warmed to RT, extracted with DCM $(8 \mathrm{~mL})$ and $\mathrm{H}_{2} \mathrm{O}(8 \mathrm{~mL})$, and the organic layer was dried over $\mathrm{MgSO}_{4}$. The drying agent was filtered off on a $15 \mathrm{~mL}$ medium porosity fritted disc, washed with DCM (3 x $5 \mathrm{~mL})$ and the filtrate was evaporated in vacuo. The resulting brown oil was dissolved in DCM $(1 \mathrm{~mL})$ forming a brown solution. This solution was added to stirring pentane $(50 \mathrm{~mL})$ giving a tan precipitation. The tan precipitate was collected on a $15 \mathrm{~mL}$ fine porosity fritted disc and washed with pentane ( $3 \times 5 \mathrm{~mL})$. The precipitate was removed from the glovebox, dissolved in DCM $(5 \mathrm{~mL})$, and the resulting 
solution was left stirring open to air overnight $(\sim 18 \mathrm{~h})$. The brown oil formed was dissolved in DCM (3 $\times 0.3 \mathrm{~mL})$ and the resulting solution was dropwise added onto a 250 $\mu \mathrm{m}$ silica preparatory plate. The product was eluted with 10\% EtOAc:hexanes $(200 \mathrm{~mL})$, scraped off as a band at $\mathrm{R}_{\mathrm{f}}: 0.15-0.48$ and this silica gel was sonicated in EtOAc $(20 \mathrm{~mL})$ for $15 \mathrm{~min}$. The silica was filtered off on a $15 \mathrm{~mL}$ fine porosity fritted disc and washed with DCM $(3 \times 5 \mathrm{~mL})$. The filtrate was evaporated in vacuo and desiccated for 15 min to yield the colorless oil 43 (49 mg, 43\% yield). IR: $v\left(\mathrm{C}-\mathrm{H} \mathrm{sp}{ }^{2}\right)=2974 \& 2951 \mathrm{~cm}^{-1}, v(\mathrm{CO})$ $=1791,1750, \& 1734 \mathrm{~cm}^{-1} .{ }^{1} \mathrm{H} \operatorname{NMR}\left(\mathrm{CDCl}_{3}, \delta\right): 7.29(1 \mathrm{H}, \mathrm{d}, J=7.9, \mathrm{H} 7), 7.18(1 \mathrm{H}, \mathrm{td}$, $J=7.3 \& 1.8, \mathrm{H} 5 / \mathrm{H} 6), 7.14(1 \mathrm{H}, \mathrm{td}, J=7.8 \& 1.5, \mathrm{H} 5 / \mathrm{H} 6), 7.12(1 \mathrm{H}, \mathrm{dd}, J=7.5 \& 1.8$, H9), 6.09 (2H, m, H2 \& H3), 4.21 (1H, m, H4), 3.77 (1H, d, $J=10.0, \mathrm{H} 8), 3.76$ (3H, s, OMe), 3.64 (3H, s, OMe), 3.55 (1H, m, H1), 3.24 (3H, s, OMe), 1.27 (3H, s, Me), 1.20 $(3 \mathrm{H}, \mathrm{s}, \mathrm{Me}) .{ }^{13} \mathrm{C} \mathrm{NMR}\left(\mathrm{CDCl}_{3}, \delta\right): 169.4(\mathrm{CO}), 168.6(\mathrm{CO}), 136.5,131.1(\mathrm{C} 7), 136.5$, 130.4 (C2 \& C3), 128.4, 128.2, 126.2, 126.1, 77.9, 59.7 (C8), 53.1 (OMe), 52.6 (OMe), 49.4 (OMe), 49.3 (C1), 40.2 (C4), $24.2(\mathrm{Me}), 23.6$ (Me). EA: Calculated for $\mathrm{C}_{19} \mathrm{H}_{24} \mathrm{O}_{5} \cdot 0.08 \mathrm{CH}_{2} \mathrm{Cl}_{2}$ : C, 67.52; H, 7.18. Found: C, 67.98; H, 7.18.

\section{Synthesis of dimethyl 2-(4-(2-methoxypropan-2-yl)-1,4-dihydroanthracen-1-} yl)malonate (44).

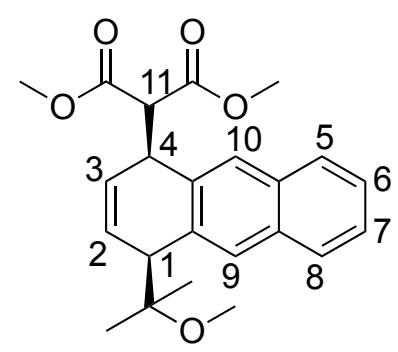

To a $50 \mathrm{~mL}$ filter flask charged with a stir pea were added 42 (100 mg, $0.118 \mathrm{mmol})$, 
$\operatorname{DCM}(5 \mathrm{~mL})$, and a $0.06 \mathrm{M}$ solution of $\mathrm{I}_{2} / \mathrm{Et}_{2} \mathrm{O}(1 \mathrm{~mL}, 0.06 \mathrm{mmol})$. The resulting green solution was stirred at RT for 5 min and then evaporated in vacuo. The resulting green oil was dissolved in DCM (1 mL) forming a green solution. This solution was added to stirring hexanes $(50 \mathrm{~mL})$ creating a green precipitation. The precipitate was collected on a $15 \mathrm{~mL}$ fine porosity fritted disc, washed with hexanes $(3 \times 10 \mathrm{~mL})$, and desiccated to yield 3 (64 mg, 93\%). The filtrate was removed from the glovebox and evaporated in vacuo to an oil. The oil was dissolved in DCM $(3 \times 0.3 \mathrm{~mL})$ and the resulting solution was dropwise added onto a $250 \mu \mathrm{m}$ silica preparatory plate. The product was eluted with 10\% EtOAc:hexanes $(200 \mathrm{~mL})$, scraped off as a band at $\mathrm{R}_{\mathrm{f}}: 0.18-0.42$ and this silica gel was sonicated in EtOAc $(20 \mathrm{~mL})$ for $15 \mathrm{~min}$. The silica was filtered off on a $15 \mathrm{~mL}$ medium porosity fritted disc and washed with DCM $(3 \times 2 \mathrm{~mL})$. The filtrate was evaporated in vacuo and desiccated to yield the colorless oil 44 (22 mg, 49\% yield). IR: $v\left(\mathrm{C}-\mathrm{H} \mathrm{sp}{ }^{2}\right)=3039 \& 2947 \mathrm{~cm}^{-1}, v(\mathrm{CO})=1730 \mathrm{~cm}^{-1} .{ }^{1} \mathrm{H}$ NMR $\left(\mathrm{CDCl}_{3}, \delta\right): 7.77(1 \mathrm{H}, \mathrm{d}, J$ $=7.2, \mathrm{H} 5 / 8), 7.75(1 \mathrm{H}, \mathrm{s}, \mathrm{H} 9), 7.73(1 \mathrm{H}, \mathrm{d}, J=7.2, \mathrm{H} 8), 7.65(1 \mathrm{H}, \mathrm{s}, \mathrm{H} 10), 7.41(2 \mathrm{H}, \mathrm{m}$, H6 \& H7), 6.19 (2H, m, H2 \& H3), $4.41(1 \mathrm{H}, \mathrm{m}, \mathrm{H} 4), 3.99$ (1H, d, $J=10.8, \mathrm{H} 7), 3.80$ (3H, s, OMe), $3.70(1 \mathrm{H}, \mathrm{m}, \mathrm{H} 1), 3.61$ (3H, s, OMe), 3.24 (3H, s, OMe), 1.35 (3H, s, Me), $1.29(3 \mathrm{H}, \mathrm{s}, \mathrm{Me}) .{ }^{13} \mathrm{C} \mathrm{NMR}\left(\mathrm{CDCl}_{3}, \delta\right): 169.5$ (CO), $168.7(\mathrm{CO}), 135.1,134.6,132.0$, 131.8, 130.5 (C2/3), 129.5 (C9), 128.4 (C2/3), 127.6 (C5/8), 127.4 (C5/8), 127.1 (C10), 125.6 (C6 \& 7), 78.1, 59.8 (C7), 52.7 (OMe), 52.5 (OMe), 49.9 (C1), 49.3 (OMe), 40.2 (C4), 24.2 (Me), 23.6 (Me). EA: Calculated for $\mathrm{C}_{23} \mathrm{H}_{26} \mathrm{O}_{5}$ : C, 72.23; H, 6.85. Found: C, $72.02 ; \mathrm{H}, 6.87$. 


\section{Synthesis of 1-(2-methoxypropan-2-yl)-1,4-dihydronaphthalene (45).}

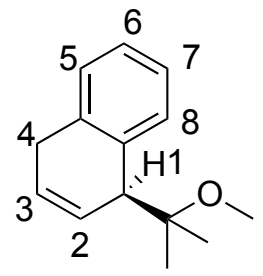

24 (200 mg, $0.339 \mathrm{mmol}), \mathrm{CH}_{3} \mathrm{CH}_{2} \mathrm{CN}$ (6 mL), and dimethoxypropane (0.3 mL, 2.44 mmol) were added to a test tube charged with a stir pea and this orange solution was stirred at $\mathrm{RT}$ for $1 \mathrm{~min}$, then at $-60{ }^{\circ} \mathrm{C}$ for $15 \mathrm{~min}$. TMSOTf $(0.1 \mathrm{~mL}, 0.54 \mathrm{mmol})$ was added to the reaction mixture yielding a red solution that was stirred at $-60{ }^{\circ} \mathrm{C}$ for $15 \mathrm{~min}$. $\mathrm{NaCNBH}_{3}(226 \mathrm{mg}, 3.60 \mathrm{mmol})$ was added to the red solution and the reaction mixture was left at $-60{ }^{\circ} \mathrm{C}$ for $2 \mathrm{~h}$. The yellow solution formed was removed from the glovebox and stirred open to air overnight $(\sim 18 \mathrm{~h})$. Evaporated the resulting brown solution in vacuo to an oil. The oil was dissolved in DCM (3 x $0.3 \mathrm{~mL})$ and the resulting solution was dropwise added onto a $250 \mu \mathrm{m}$ silica preparatory plate. The product was eluted with 10\% EtOAc:hexanes $(200 \mathrm{~mL})$, scraped off as a band at $\mathrm{R}_{\mathrm{f}}:$ 0.53-0.75 and this silica gel was sonicated in EtOAc $(20 \mathrm{~mL})$ for $15 \mathrm{~min}$. The silica was filtered off on a $15 \mathrm{~mL}$ fine porosity fritted disc and washed with DCM $(3 \times 5 \mathrm{~mL})$. The filtrate was evaporated in vacuo and desiccated for $15 \mathrm{~min}$ to yield the colorless oil 45 (35 mg, 51\% yield). IR: v(C$\left.\mathrm{H} \mathrm{sp}^{2}\right)=2978,2827 \mathrm{~cm}^{-1}, v(\mathrm{OMe})=1362 \mathrm{~cm}^{-1} .{ }^{1} \mathrm{H} \mathrm{NMR}\left(\mathrm{CDCl}_{3}, \delta\right): 7.23(1 \mathrm{H}, \mathrm{d}, J=$ 7.5, H8), 7.17 (2H, m, H6 \& H7), $7.13(1 \mathrm{H}, \mathrm{d}, J=7.7, \mathrm{H} 5), 6.13$ (1H, m, H3), 6.00 (1H, m, H2), 3.64 (1H, m, H1), 3.38 (1H, m, H4), 3.31 (3H, s, OMe), 3.30 (1H, m, H4), 1.12 $(3 \mathrm{H}, \mathrm{s}, \mathrm{Me}), 1.03(3 \mathrm{H}, \mathrm{s}, \mathrm{Me}) .{ }^{13} \mathrm{C} \mathrm{NMR}\left(\mathrm{CDCl}_{3}, \delta\right): 136.4,136.1,130.4,128.3,128.0$ $127.1(\mathrm{C} 2), 126.0(\mathrm{C} 3), 125.3,49.5$ (OMe), 47.8 (C1), $31.2(\mathrm{C} 4), 22.5(\mathrm{Me}), 22.3(\mathrm{Me})$. 


\section{Synthesis of 1-(2-methoxypropan-2-yl)-1,4-dihydroanthracene (46).}

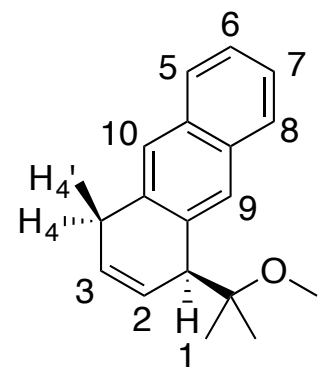

25 (200 mg, $0.312 \mathrm{mmol}), \mathrm{CH}_{3} \mathrm{CH}_{2} \mathrm{CN}$ (6 mL), and dimethoxypropane (0.3 mL, 2.44 mmol) were added to a test tube charged with a stir pea and this red solution was stirred at RT for $1 \mathrm{~min}$, then at $-60{ }^{\circ} \mathrm{C}$ for $15 \mathrm{~min}$. TMSOTf $(0.1 \mathrm{~mL}, 0.54 \mathrm{mmol})$ was added to the reaction mixture and the resulting red solution was left at $-60{ }^{\circ} \mathrm{C}$ for $15 \mathrm{~min}$. $\mathrm{NaCNBH}_{3}(226 \mathrm{mg}, 3.60 \mathrm{mmol})$ was added to this solution and the reaction mixture was left at $-60^{\circ} \mathrm{C}$ for $2 \mathrm{~h}$. The reaction mixture was warmed to RT, extracted with DCM (8 $\mathrm{mL})$ and $\mathrm{H}_{2} \mathrm{O}(8 \mathrm{~mL})$, and the organic layer was dried over $\mathrm{MgSO}_{4}$. The drying agent was filtered off on a $15 \mathrm{~mL}$ medium porosity fritted disc, washed with DCM (3 x $5 \mathrm{~mL})$, and the filtrate was evaporated in vacuo. The brown oil formed was dissolved in DCM (1 mL) creating a brown solution. This solution was added to stirring pentane $(50 \mathrm{~mL})$ forming a tan precipitation. The tan precipitate was collected on a $15 \mathrm{~mL}$ fine porosity fritted disc and washed with pentane $(3 \times 5 \mathrm{~mL})$. The precipitate was removed from the glovebox, dissolved in DCM (5 mL), and stirred open to air overnight $(\sim 18 \mathrm{~h})$. The resulting brown solution was evaporated in vacuo to an oil. The oil was dissolved in DCM (3 x $0.3 \mathrm{~mL})$ and the resulting solution was dropwise added onto a $250 \mu \mathrm{m}$ silica preparatory plate. The product was eluted with 10\% EtOAc:hexanes $(200 \mathrm{~mL})$, scraped off as a band at $\mathrm{R}_{\mathrm{f}}$ : 0.57-0.72 and this silica gel was sonicated in EtOAc $(20 \mathrm{~mL})$ for $15 \mathrm{~min}$. The silica was filtered off on a $15 \mathrm{~mL}$ fine porosity fritted disc, washed with DCM $(3 \times 5 \mathrm{~mL})$. The 
filtrate was evaporated in vacuo and desiccated for $15 \mathrm{~min}$ to yield the colorless oil 46 (20 mg, 25\% yield). IR: $v\left(\mathrm{C}-\mathrm{H} \mathrm{sp}{ }^{2}\right)=2971 \& 2934 \mathrm{~cm}^{-1}, v(\mathrm{OMe})=1074 \mathrm{~cm}^{-1} .{ }^{1} \mathrm{H}$ NMR $\left(\mathrm{CDCl}_{3}, \delta\right): 7.80(1 \mathrm{H}, \mathrm{m}, \mathrm{H} 5), 7.75(1 \mathrm{H}, \mathrm{m}, \mathrm{H} 8), 7.71(1 \mathrm{H}, \mathrm{s}, \mathrm{H} 9), 7.62$ (1H, s, H8), 7.40 (2H, m, H6 \& H7), 6.2 (1H, ddd, $J=9.9,5.5, \& 2.1, \mathrm{H} 3), 6.1(1 \mathrm{H}, \mathrm{ddd}, J=9.9,5.5, \&$ 3.2, H2), $3.82(1 \mathrm{H}, \mathrm{m}, \mathrm{H} 1), 3.58(1 \mathrm{H}, \mathrm{dd}, J=20.6 \& 5.4, \mathrm{H} 4), 3.50(1 \mathrm{H}, \mathrm{d}, J=20.6$,

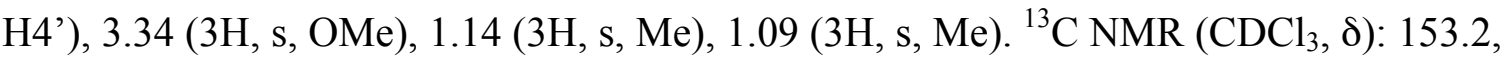
134.8, 132.2, 131.9, 128.8 (C9), 128.4 (C2), 127.8 (C5), 127.3 (C3), 127.0 (C8), 125.9, 125.5 (C6/7), 125.0 (C6/7), 81.0, 49.6 (OMe), 48.5 (C1), 31.3 (C4), 22.6 (Me), 22.3 (Me). EA: Calculated for $\mathrm{C}_{18} \mathrm{H}_{20} \mathrm{O} \cdot 0.5 \mathrm{CH}_{2} \mathrm{Cl}_{2}$ : C, 75.37; H, 7.18. Found: C, 75.12; H, 6.78 .

\section{Synthesis of 1-(1-methoxycyclohexyl)-1,4-dihydronaphthalene (47).}

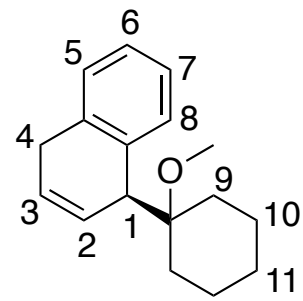

24 (200 mg, $0.339 \mathrm{mmol}), \mathrm{CH}_{3} \mathrm{CH}_{2} \mathrm{CN}(6 \mathrm{~mL})$, and 1,1-dimethoxycyclohexane $(0.3 \mathrm{~mL}$, $1.97 \mathrm{mmol}$ ) were added to a test tube charged with a stir pea and the orange solution was stirred at RT for $1 \mathrm{~min}$, then at $-60{ }^{\circ} \mathrm{C}$ for $15 \mathrm{~min}$. TMSOTf $(0.1 \mathrm{~mL}, 0.54 \mathrm{mmol})$ was added to the reaction mixture and the resulting red solution was stirred at $-60{ }^{\circ} \mathrm{C}$ for 15 min. $\mathrm{NaCNBH}_{3}(226 \mathrm{mg}, 3.60 \mathrm{mmol})$ was added to the solution and the reaction mixture was left at $-60{ }^{\circ} \mathrm{C}$ for $5 \mathrm{~h}$. The reaction mixture was removed from the glovebox and stirred open to air overnight $(\sim 18 \mathrm{~h})$. The resulting brown solution was evaporated in vacuo. The oil formed was then dissolved in DCM $(1 \mathrm{~mL})$ forming a brown solution. 
This solution was added to stirring $\mathrm{Et}_{2} \mathrm{O}(50 \mathrm{~mL})$ forming a brown precipitation. The precipitate was filtered off on a $15 \mathrm{~mL}$ medium porosity fritted disc, washed with $\mathrm{Et}_{2} \mathrm{O}(3$ x $5 \mathrm{~mL}$ ), and the filtrate was evaporated in vacuo. The brown oil formed was dissolved in $\operatorname{DCM}(3 \times 0.3 \mathrm{~mL})$ and the resulting solution was dropwise added onto a $250 \mu \mathrm{m}$ silica preparatory plate. The product was eluted with 10\% EtOAc:hexanes $(200 \mathrm{~mL})$, scraped off as a band at $\mathrm{R}_{\mathrm{f}}$ : $0.72-0.93$ and this silica gel was sonicated in EtOAc $(20 \mathrm{~mL})$ for 15 min. The silica was filtered off on a $15 \mathrm{~mL}$ fine porosity fritted disc and washed with DCM (3 x $5 \mathrm{~mL})$. The filtrate was evaporated in vacuo and desiccated for $15 \mathrm{~min}$ to yield the colorless oil $47(22 \mathrm{mg}, 27 \%$ yield $)$. IR: $v\left(\mathrm{C}-\mathrm{H} \mathrm{sp}{ }^{2}\right)=2925 \& 2853 \mathrm{~cm}^{-1}, v(\mathrm{OMe})=$ $1065 \mathrm{~cm}^{-1} .{ }^{1} \mathrm{H}$ NMR $\left(\mathrm{CDCl}_{3}, \delta\right): 7.17$ (2H, m, H6 \& H7), 7.13 (2H, m, H5 \& H8) 6.15 $(1 \mathrm{H}, \mathrm{ddd}, J=9.9,5.4, \& 2.1, \mathrm{H} 3), 5.95(1 \mathrm{H}, \mathrm{ddd}, J=9.9,5.7, \& 3.3, \mathrm{H} 2), 3.71(1 \mathrm{H}, \mathrm{m}$, H1), 3.39 (3H, s, OMe), 3.38 (1H, d, $J=18.9, \mathrm{H} 4), 3.30$ (1H, dd, $J=18.9 \&$ 5.6, H4), 1.8-0.9 (10H, H9, H10, \& H11). ${ }^{13} \mathrm{C}$ NMR $\left(\mathrm{CDCl}_{3}, \delta\right): 136.8,135.9,129.8(\mathrm{C} 5 / 8), 128.1$ (C5/8), 127.8 (C2), 127.3 (C3), 125.9 (C6/7), 125.4 (C6/7), 81.6, 48.5 (OMe), 45.2 (C1), 31.4 (C4), 30.4 (C9), 30.2 (C10), 25.7 (C11), 21.5. EA: Calculated for $\mathrm{C}_{17} \mathrm{H}_{22} \mathrm{O} \cdot 0.5$ $\mathrm{CH}_{2} \mathrm{Cl}_{2}: \mathrm{C}, 73.80 ; \mathrm{H}, 8.14$. Found: C, 73.95; H, 8.13.

\section{Synthesis of 1-(1-methoxycyclohexyl)-1,4-dihydroanthracene (48).}

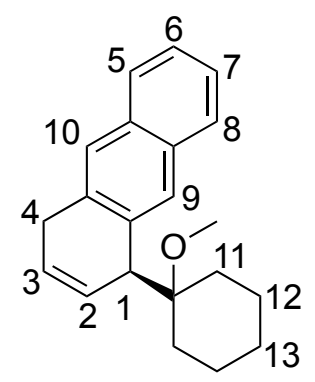


Via Air Oxidation

25 (200 mg, $0.312 \mathrm{mmol}), \mathrm{CH}_{3} \mathrm{CH}_{2} \mathrm{CN}$ (6 mL), and 1,1-dimethoxycyclohexenone (0.3 $\mathrm{mL}, 1.97 \mathrm{mmol}$ ) were added to a test tube charged with a stir pea and this red solution was stirred at RT for $1 \mathrm{~min}$, then at $-60{ }^{\circ} \mathrm{C}$ for $15 \mathrm{~min}$. TMSOTf $(0.1 \mathrm{~mL}, 0.54 \mathrm{mmol})$ was added to the reaction mixture and the resulting red solution was stirred at $-60{ }^{\circ} \mathrm{C}$ for $15 \mathrm{~min} . \mathrm{NaCNBH}_{3}(226 \mathrm{mg}, 3.60 \mathrm{mmol})$ was added to the solution and the reaction mixture was left at $-60^{\circ} \mathrm{C}$ for $1 \mathrm{~h}$. The reaction mixture was warmed to $\mathrm{RT}$, extracted with DCM $(8 \mathrm{~mL})$ and $\mathrm{H}_{2} \mathrm{O}(8 \mathrm{~mL})$, and the organic layer was dried over $\mathrm{MgSO}_{4}$. The drying agent was filtered off on a $15 \mathrm{~mL}$ medium porosity fritted disc, washed with DCM ( $3 \times 5 \mathrm{~mL})$, and the filtrate was evaporated in vacuo. The oil formed was then dissolved in DCM (1 mL) creating a brown solution. This solution was then added to stirring pentane $(50 \mathrm{~mL})$ forming a tan precipitation. The tan precipitate was collected on a 15 $\mathrm{mL}$ fine porosity fritted disc, washed with pentane $(3 \times 5 \mathrm{~mL})$, and removed from the glovebox. The precipitate was dissolved in DCM $(5 \mathrm{~mL})$, and the resulting solution was stirred open to air overnight $(\sim 18 \mathrm{~h})$. The reaction mixture was evaporated in vacuo to an oil. This oil was dissolved in DCM $(3 \times 0.3 \mathrm{~mL})$ and the resulting solution was dropwise added onto a $250 \mu \mathrm{m}$ silica preparatory plate. The product was eluted with $2.5 \%$ EtOAc:hexanes $(200 \mathrm{~mL})$, scraped off as a band at $\mathrm{R}_{\mathrm{f}}$ : $0.33-0.60$ and this silica gel was sonicated in EtOAc $(20 \mathrm{~mL})$ for $15 \mathrm{~min}$. The silica was filtered off on a $15 \mathrm{~mL}$ fine porosity fritted disc and washed with DCM $(3 \times 5 \mathrm{~mL})$. The filtrate was evaporated in vacuo and desiccated for $15 \mathrm{~min}$ to yield the colorless oil 48 (25 mg, 28\% yield). 


\section{Via Iodine Oxidation}

To a $50 \mathrm{~mL}$ filter flask charged with a stir pea were added $\mathbf{5 3}(100 \mathrm{mg}, 0.133 \mathrm{mmol})$, $\operatorname{DCM}(5 \mathrm{~mL})$, and a $0.06 \mathrm{M}$ solution of $\mathrm{I}_{2} / \mathrm{Et}_{2} \mathrm{O}(1.1 \mathrm{~mL}, 0.068 \mathrm{mmol})$. The resulting green solution was stirred at RT for $5 \mathrm{~min}$ and then evaporated in vacuo. The resulting green oil was dissolved in DCM (1 mL) forming a green solution. This solution was added to stirring hexanes $(50 \mathrm{~mL})$ creating a green precipitation. The precipitate was collected on a $15 \mathrm{~mL}$ fine porosity fritted disc, washed with hexanes $(3 \times 10 \mathrm{~mL})$, and desiccated to yield 23 (77 $\mathrm{mg}, 98 \%$ ). The filtrate was removed from the glovebox and evaporated in vacuo to an oil. The oil was dissolved in DCM (3 x $0.3 \mathrm{~mL})$ and the resulting solution was dropwise added onto a $250 \mu \mathrm{m}$ silica preparatory plate. The product was eluted with $10 \%$ EtOAc:hexanes $(200 \mathrm{~mL})$, scraped off as a band at $\mathrm{R}_{\mathrm{f}}: 0.22-$ 0.38 and this silica gel was sonicated in EtOAc $(20 \mathrm{~mL})$ for $15 \mathrm{~min}$. The silica was filtered off on a $15 \mathrm{~mL}$ medium porosity fritted disc and washed with DCM (3 x $2 \mathrm{~mL})$. The filtrate was evaporated in vacuo and desiccated to yield the colorless oil 48 (16.5 mg, $42 \%$ yield $) . \mathrm{IR}: v\left(\mathrm{C}-\mathrm{H} \mathrm{sp}^{2}\right)=2925 \& 2853 \mathrm{~cm}^{-1}, v(\mathrm{OMe})=1060 \mathrm{~cm}^{-1} .{ }^{1} \mathrm{H} \mathrm{NMR}\left(\mathrm{CDCl}_{3}\right.$, ঠ): 7.79 (1H, m, H5/H8), 7.76 (1H, m, H5/H8), 7.62 (1H, s, H10), 7.60 (1H, s, H9), 7.41 (2H, m, H6 \& H7), 6.23 (1H, ddd, $J=9.9,5.6, \&$ 1.9, H3), 6.04 (1H, ddd, $J=9.9,5.6$, 3.3, H2), 3.89 (1H, bs, H1), 3.59 (1H, d, $J=20.4, \mathrm{H} 4), 3.48$ (1H, dd, $J=20.4$ \& 5.6, H4), $3.44(3 \mathrm{H}, \mathrm{s}, \mathrm{OMe}), 1.85(1 \mathrm{H}, \mathrm{d}, J=14.2, \mathrm{H} 11), 1.67(1 \mathrm{H}, \mathrm{d}, J=14.2, \mathrm{H} 11), 1.49(6 \mathrm{H}, \mathrm{m}$, $\mathrm{H} 11$ \& H12), 1.12 (1H, m, H13), 0.92 (1H, m, H13). ${ }^{13} \mathrm{C} \mathrm{NMR}\left(\mathrm{CDCl}_{3}, \delta\right): 135.5,134.8$, 132.2, 131.8, 128.2 (C10), 127.9 (C2), 127.7 (C5/8), 127.5 (C3), 127.0 (C5/8), 126.0 (C9), 125.5 (C6/7), 125.1 (C6/7), 81.8, 48.6 (OMe), 45.8 (C1), 31.5 (C4), 30.4 (C11), 30.3 (C12), 25.7 (C13), 21.5. EA: Calculated for $\mathrm{C}_{21} \mathrm{H}_{24} \mathrm{O} \cdot 0.5 \mathrm{CH}_{2} \mathrm{Cl}_{2}: \mathrm{C}, 77.11 ; \mathrm{H}$, 
7.52. Found: C, 77.27; H, 7.29.

\section{Synthesis of 4-(1,4-dihydronaphthalen-1-yl)pentan-2-one (49).}

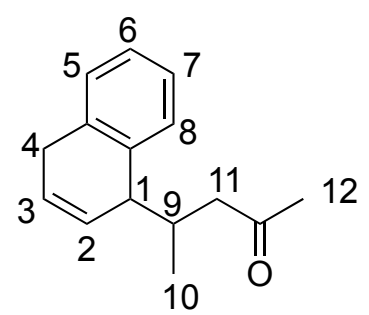

24 (200 mg, $0.339 \mathrm{mmol}), \mathrm{CH}_{3} \mathrm{CH}_{2} \mathrm{CN}$ (6 mL), and 3-penten-2-one (0.2 mL, $\left.2.05 \mathrm{mmol}\right)$ were added to a test tube charged with a stir pea and this orange solution was stirred at RT for $1 \mathrm{~min}$, then at $-60{ }^{\circ} \mathrm{C}$ for $15 \mathrm{~min}$. TMSOTf $(0.1 \mathrm{~mL}, 0.54 \mathrm{mmol})$ was added to the reaction mixture and the resulting red solution was stirred at $-60{ }^{\circ} \mathrm{C}$ for $15 \mathrm{~min}$. $\mathrm{NaCNBH}_{3}(226 \mathrm{mg}, 3.60 \mathrm{mmol})$ was added to the solution and the reaction mixture was left at $-60{ }^{\circ} \mathrm{C}$ for $5 \mathrm{~h}$. The reaction mixture was removed from the glovebox and stirred open to air overnight $(\sim 18 \mathrm{~h})$. The brown solution formed was then evaporated in vacuo to an oil. This oil was dissolved in DCM (1 mL) giving a brown solution. This solution was then added to stirring $\mathrm{Et}_{2} \mathrm{O}(50 \mathrm{~mL})$ forming a brown precipitation. The precipitate was isolated on a $15 \mathrm{~mL}$ medium porosity fritted disc, washed with $\mathrm{Et}_{2} \mathrm{O}(3 \times 5 \mathrm{~mL})$, and the filtrate was evaporated in vacuo. The oil was dissolved in DCM (3 x $0.3 \mathrm{~mL})$ and the resulting solution was dropwise added onto a $250 \mu \mathrm{m}$ silica preparatory plate. The product was eluted with 10\% EtOAc:hexanes $(200 \mathrm{~mL})$, scraped off as a band at $\mathrm{R}_{\mathrm{f}}$ : 0.35 0.44 and this silica gel was sonicated in EtOAc $(20 \mathrm{~mL})$ for $15 \mathrm{~min}$. The silica was filtered off on a $15 \mathrm{~mL}$ fine porosity fritted disc and washed with DCM (3 x $5 \mathrm{~mL})$. The filtrate was evaporated in vacuo and desiccated for 15 min to yield the colorless oil 49 (44 mg, 61\% yield). IR: $v\left(\mathrm{C}-\mathrm{H} \mathrm{sp}{ }^{2}\right)=2960 \mathrm{~cm}^{-1}, v(\mathrm{CO})=1704 \mathrm{~cm}^{-1} .{ }^{1} \mathrm{H} \mathrm{NMR}\left(\mathrm{CDCl}_{3}\right.$, 


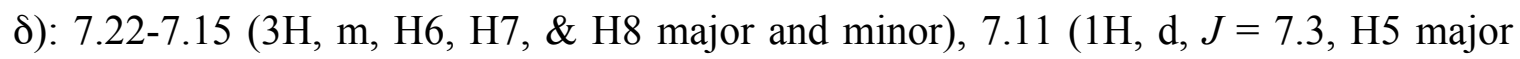
and minor), $6.09(1 \mathrm{H}, \mathrm{m}, \mathrm{H} 2$ minor), $6.06(1 \mathrm{H}, \mathrm{m}, \mathrm{H} 2$ major $), 5.91(1 \mathrm{H}, \mathrm{m}, \mathrm{H} 3), 5.81$ (1H, m, H3), 3.45-3.3 (3H, H1 \& H4 major and minor), 2.61 (1H, dd, $J=16.4 \& 5.7$, H11 minor), 2.52 (2H, m, H9 major and minor), 2.30 (1H, dd, $J=16.4 \& 8.4, \mathrm{H} 11$ minor), 2.16 (3H, s, H12 minor), 2.15 (2H, m, H11 major), 1.96 (3H, s, H12 major), 1.01 $\left(3 \mathrm{H}, \mathrm{d}, J=6.9, \mathrm{H} 10\right.$ major), 0.68 (3H, d, $J=6.8, \mathrm{H} 10$ minor). ${ }^{13} \mathrm{C} \mathrm{NMR}\left(\mathrm{CDCl}_{3}, \delta\right)$ : (major) 209.1 137.2, 135.2, 128.4, 128.3, 126.8, 126.3 126.2, 126.1, 46.2, 44.6, 37.2, 30.4, 17.9; (minor) 208.6, 137.6, 135.3, 128.4, 127.5, 127.4, 126.2, 126.0, 125.9, 48.5, 43.9, 37.1, 30.7, 30.5, 15.3. EA: Calculated for $\mathrm{C}_{15} \mathrm{H}_{18} \mathrm{O} \bullet 0.3 \mathrm{CH}_{2} \mathrm{Cl}_{2}$ : C, 76.64; H, 7.82. Found: C, 76.71; H, 7.58 .

\section{Synthesis of 4-(1,4-dihydroanthracen-1-yl)pentan-2-one (50).}

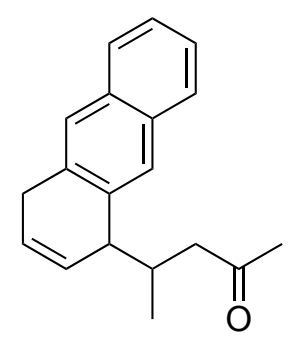

25 (200 mg, $0.312 \mathrm{mmol}), \mathrm{CH}_{3} \mathrm{CH}_{2} \mathrm{CN}(6 \mathrm{~mL})$, and 3-penten-2-one (0.2 mL, $\left.2.0 \mathrm{mmol}\right)$ were added to a test tube charged with a stir pea and the red solution was stirred at RT for $1 \mathrm{~min}$, then at $-60{ }^{\circ} \mathrm{C}$ for $15 \mathrm{~min}$. TMSOTf $(0.1 \mathrm{~mL}, 0.54 \mathrm{mmol})$ was added to the reaction mixture and the resulting red solution was stirred at $-60{ }^{\circ} \mathrm{C}$ for $15 \mathrm{~min}$. $\mathrm{NaCNBH}_{3}(226 \mathrm{mg}, 3.60 \mathrm{mmol})$ was added to the solution and the reaction mixture was left at $-60{ }^{\circ} \mathrm{C}$ for $5 \mathrm{~h}$. The reaction mixture was removed from the glovebox and stirred open to air overnight $(\sim 18 \mathrm{~h})$. The resulting brown solution was evaporated in vacuo to an 
oil. This oil was then dissolved in DCM (1 mL) forming a brown solution. This solution was added to stirring $\mathrm{Et}_{2} \mathrm{O}(50 \mathrm{~mL})$ forming a brown precipitation. The precipitate was collected on a $15 \mathrm{~mL}$ medium porosity fritted disc, washed with $\mathrm{Et}_{2} \mathrm{O}(3 \times 5 \mathrm{~mL})$, and evaporated filtrate in vacuo. The brown oil formed was dissolved in DCM (3 x $0.3 \mathrm{~mL})$ and the resulting solution was dropwise added onto a $250 \mu \mathrm{m}$ silica preparatory plate. The product was eluted with 10\% EtOAc:hexanes $(200 \mathrm{~mL})$, scraped off as a band at $\mathrm{R}_{\mathrm{f}}$ : 0.28-0.56 and this silica gel was sonicated in EtOAc $(20 \mathrm{~mL})$ for $15 \mathrm{~min}$. The silica was filtered off on a $15 \mathrm{~mL}$ fine porosity fritted disc and washed with DCM ( $3 \times 5 \mathrm{~mL})$. The filtrate was evaporated in vacuo and desiccated for $15 \mathrm{~min}$ to yield the colorless oil $\mathbf{5 0}$ (32 mg, 38\% yield). IR: $v\left(\mathrm{C}-\mathrm{H} \mathrm{sp}{ }^{2}\right)=2959 \& 2926 \mathrm{~cm}^{-1}, v(\mathrm{CO})=1703 \mathrm{~cm}^{-1}$. EA: Calculated for $\mathrm{C}_{19} \mathrm{H}_{20} \mathrm{O} \bullet 0.5 \mathrm{CH}_{2} \mathrm{Cl}_{2}$ : C, 76.33; H, 6.90. Found: C, 76.75; H, 6.57.

\section{Synthesis of 3-(1,4-dihydronaphthalen-1-yl)cyclopentan-1-one (51).}

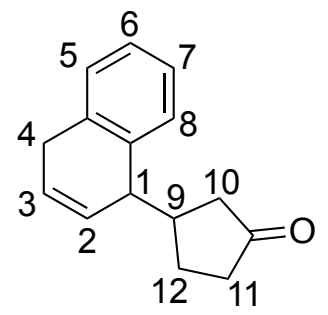

24 (200 mg, $0.339 \mathrm{mmol}), \mathrm{CH}_{3} \mathrm{CH}_{2} \mathrm{CN}$ (6 mL), and cyclopenten-2-one (0.3 mL, 3.59 mmol) were added to a test tube charged with a stir pea and the orange solution was stirred at RT for $1 \mathrm{~min}$, then at $-60^{\circ} \mathrm{C}$ for $15 \mathrm{~min}$. TMSOTf $(0.1 \mathrm{~mL}, 0.54 \mathrm{mmol})$ was added to the reaction mixture and the resulting red solution was stirred at $-60{ }^{\circ} \mathrm{C}$ for 15 min. $\mathrm{NaCNBH}_{3}(226 \mathrm{mg}, 3.59 \mathrm{mmol})$ was added to the solution and the reaction mixture was left at $-60{ }^{\circ} \mathrm{C}$ for $2.5 \mathrm{~h}$. The reaction mixture was removed from the glovebox and stirred open to air overnight ( $\sim 18 \mathrm{~h})$. The brown solution formed was evaporated in vacuo 
to an oil. This oil was dissolved in DCM $(1 \mathrm{~mL})$, creating a brown solution that was then added to stirring $\mathrm{Et}_{2} \mathrm{O}(50 \mathrm{~mL})$, forming a brown precipitation. The precipitate was isolated on a $15 \mathrm{~mL}$ medium porosity fritted disc, washed with $\mathrm{Et}_{2} \mathrm{O}(3 \times 5 \mathrm{~mL})$, and evaporated filtrate in vacuo to an oil. The brown oil was dissolved in DCM ( $3 \times 0.3 \mathrm{~mL})$ and the resulting solution was dropwise loaded onto a $250 \mu \mathrm{m}$ silica preparatory plate. The product was eluted with $10 \%$ EtOAc:hexanes $(200 \mathrm{~mL})$, scraped off as a band at $\mathrm{R}_{\mathrm{f}}$ : $0.25-0.43$, and the silica gel was sonicated in EtOAc $(20 \mathrm{~mL})$ for $15 \mathrm{~min}$. The silica was filtered off on a $15 \mathrm{~mL}$ fine porosity fritted disc and washed with DCM $(3 \times 5 \mathrm{~mL})$. The filtrate was evaporated in vacuo and desiccated for $15 \mathrm{~min}$ to yield the colorless oil $\mathbf{5 1}$ $(20 \mathrm{mg}, 27 \%$ yield $)$. IR: $v\left(\mathrm{C}-\mathrm{H} \mathrm{sp}^{2}\right)=3026 \& 2886 \mathrm{~cm}^{-1}, v(\mathrm{CO})=1737 \mathrm{~cm}^{-1} .{ }^{1} \mathrm{H} \mathrm{NMR}$ $\left(\mathrm{CDCl}_{3}, \delta\right): 7.17(4 \mathrm{H}, \mathrm{m}, \mathrm{H} 5, \mathrm{H} 6, \mathrm{H} 7, \& \mathrm{H} 8), 6.14(1 \mathrm{H}, \mathrm{ddd}, J=10.0,4.7, \& 2.7, \mathrm{H} 3)$, $5.97(1 \mathrm{H}, \mathrm{m}, \mathrm{H} 2), 3.51(1 \mathrm{H}, \mathrm{m}, \mathrm{H} 1), 3.34(2 \mathrm{H}, \mathrm{m}, \mathrm{H} 4), 2.48(1 \mathrm{H}, \mathrm{m}, \mathrm{H} 9), 2.28(1 \mathrm{H}, \mathrm{m}$, H10/H11), 2.09 (3H, m, H10/H11), 1.98 (1H, dd, $J=18.5 \& 11.5, \mathrm{H} 12), 1.74(1 \mathrm{H}, \mathrm{m}$, H12). ${ }^{13} \mathrm{C}$ NMR ( $\left.\mathrm{CDCl}_{3}, \delta\right): 219.2$ (CO), 137.3, 134.9, 128.5, 128.4, 127.8 (C3), 126.9 (C2), 126.3, 126.1, 45.2, 43.2 (C1), 41.7, 38.8, 30.5 (C4), 27.2. LRMS: $\mathrm{C}_{15} \mathrm{H}_{16} \mathrm{O}$, mass found $\left[\mathrm{M}+\mathrm{H}^{+}\right]=213$, mass required: 212.2 .

\section{Synthesis of 3-(1,4-dihydroanthracen-1-yl)cyclopentan-1-one (52).}

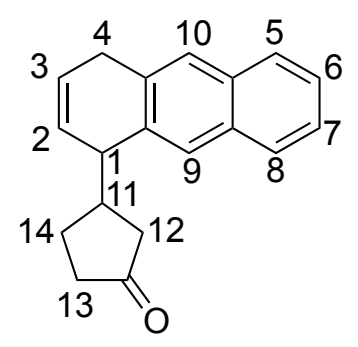

25 (200 mg, $0.312 \mathrm{mmol}), \mathrm{CH}_{3} \mathrm{CH}_{2} \mathrm{CN}(6 \mathrm{~mL})$, and cyclopentenone (0.3 mL, $\left.3.59 \mathrm{mmol}\right)$ 
were added to a test tube charged with a stir pea and the red solution was stirred at RT for $1 \mathrm{~min}$, then at $-60{ }^{\circ} \mathrm{C}$ for $15 \mathrm{~min}$. TMSOTf $(0.1 \mathrm{~mL}, 0.54 \mathrm{mmol})$ was added to the reaction mixture and the resulting red solution was stirred at $-60{ }^{\circ} \mathrm{C}$ for $15 \mathrm{~min}$. $\mathrm{NaCNBH}_{3}$ (226 mg, $3.60 \mathrm{mmol}$ ) was added to the solution and the resulting brown solution was left at $-60^{\circ} \mathrm{C}$ for $2 \mathrm{~h}$. The reaction mixture was warmed to RT, extracted with DCM $(8 \mathrm{~mL})$ and $\mathrm{H}_{2} \mathrm{O}(8 \mathrm{~mL})$, and the organic layer was dried over $\mathrm{MgSO}_{4}$. The drying agent was filtered off on a $15 \mathrm{~mL}$ medium porosity fritted disc, washed with DCM ( $3 \times 5 \mathrm{~mL})$, and the filtrate was evaporated in vacuo. The resulting oil was dissolved in DCM ( $1 \mathrm{~mL})$ forming a brown solution. This solution was added to stirring pentane (50 $\mathrm{mL}$ ) forming a tan precipitation. The tan precipitate was collected on a $15 \mathrm{~mL}$ fine porosity fritted disc and washed with pentane $(3 \times 5 \mathrm{~mL})$. The precipitate was removed from the glovebox, dissolved in DCM $(5 \mathrm{~mL})$, and the resulting brown solution was stirred open to air overnight $(\sim 18 \mathrm{~h})$. The resulting brown solution was evaporated in vacuo to an oil. This oil was dissolved in DCM $(3 \times 0.3 \mathrm{~mL})$ and the resulting solution was dropwise added onto a $250 \mu \mathrm{m}$ silica preparatory plate. The product was eluted with $20 \%$ EtOAc:hexanes $(200 \mathrm{~mL})$, scraped off as a band at $\mathrm{R}_{\mathrm{f}}: 0.38-0.56$ and this silica gel was sonicated in EtOAc $(20 \mathrm{~mL})$ for $15 \mathrm{~min}$. The silica was filtered off on a $15 \mathrm{~mL}$ fine porosity fritted disc and washed with DCM $(3 \times 5 \mathrm{~mL})$. The filtrate was evaporated in vacuo and desiccated for $15 \mathrm{~min}$ to yield the colorless oil $\mathbf{5 2}$ (23 $\mathrm{mg}, 27 \%$ yield). IR: v(C$\left.\mathrm{H} \mathrm{sp}^{2}\right)=3027 \& 2885 \mathrm{~cm}^{-1}, v(\mathrm{CO})=1735 \mathrm{~cm}^{-1} .{ }^{1} \mathrm{H} \mathrm{NMR}\left(\mathrm{CDCl}_{3}, \delta\right): 7.77(2 \mathrm{H}, \mathrm{m}, \mathrm{H} 5 \&$ H8), 7.65 (1H, s, H10), 7.64 (1H, s, H9), 7.42 (2H, m, H6 \& H7), 6.21 (1H, ddd, J = 9.9, 4.3, \& 3.2, H3), $6.08(1 \mathrm{H}, \mathrm{m}, \mathrm{H} 2), 3.68(1 \mathrm{H}, \mathrm{m}, \mathrm{H} 1), 3.55(2 \mathrm{H}, \mathrm{m}, \mathrm{H} 4), 2.52(1 \mathrm{H}, \mathrm{m}$, H11), 2.28 (1H, m, H12/H13), 2.15 (1H, dd, $J=18.2 \&$ 7.2, H12/H13), 2.07 (3H, m, 
H12, H13, \& H14), 1.76 (1H, m, H14). ${ }^{13} \mathrm{C} \mathrm{NMR}\left(\mathrm{CDCl}_{3}, \delta\right): 219.1$ (CO), 136.3, 133.7, 132.3, 132.2, 127.8, 127.6 (C3), 127.4 (C2), 127.2 (C5/8), 126.5 (C10), 125.6 (C6/7), 125.4 (C6/7), 45.4 (C11), 43.9 (C1), 42.1 (C12/13), 38.7 (C12/13), 30.6 (C4), 27.4 (C12/13/14). EA: Calculated for $\mathrm{C}_{19} \mathrm{H}_{18} \mathrm{O} \cdot 0.5 \mathrm{CH}_{2} \mathrm{Cl}_{2}$ : C, 76.84; H, 6.28. Found: C, $78.11 ; \mathrm{H}, 6.36$.

\section{Synthesis of TpMo(NO)(DMAP)(2,3- $\eta^{2}-(S)-1-(1-m e t h o x y c y c l o h e x y l)-1,4-$} dihydroanthracene)) (53).

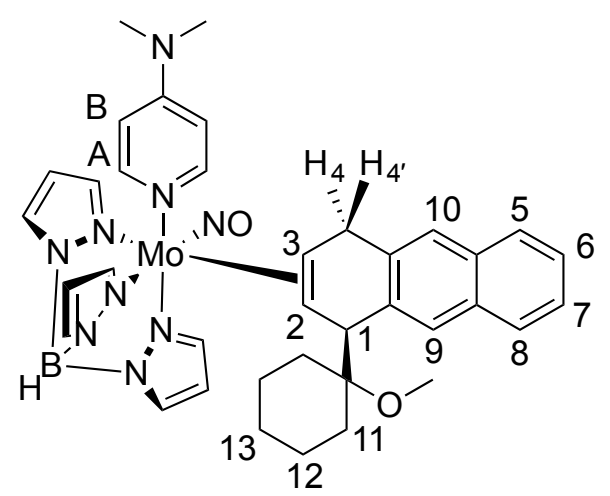

25 (200 mg, $0.313 \mathrm{mmol}), \mathrm{CH}_{3} \mathrm{CH}_{2} \mathrm{CN}(6 \mathrm{~mL})$, and 1,1-dimethoxycyclohexane (0.3 mL, $1.98 \mathrm{mmol}$ ) were added to a test tube charged with a stir pea. The reaction mixture was stirred at RT until homogeneous and then cooled at $-60^{\circ} \mathrm{C}$ for $15 \mathrm{~min}$. TMSOTf $(0.1 \mathrm{~mL}$, $0.54 \mathrm{mmol}$ ) was added to the reaction mixture and the resulting red solution was left stirring at $-60{ }^{\circ} \mathrm{C}$ for $15 \mathrm{~min} . \mathrm{NaCNBH}_{3}(226 \mathrm{mg}, 3.59 \mathrm{mmol})$ was added to the red solution, giving a brown solution that was left stirring at $-60{ }^{\circ} \mathrm{C}$ for $2 \mathrm{~h}$. This brown solution was then warmed to RT and washed with $\mathrm{H}_{2} \mathrm{O}(8 \mathrm{~mL})$. The aqueous layer was back-extracted with DCM $(6 \mathrm{~mL})$, combined with the organic layer, and dried over $\mathrm{MgSO}_{4}$. The drying agent was filtered off on a $15 \mathrm{~mL}$ medium porosity fritted disc, washed with DCM (3 $\times 2 \mathrm{~mL})$, and the filtrate was evaporated in vacuo. The resulting oil 
was then dissolved in DCM $(1 \mathrm{~mL})$ and the product was precipitated in stirring pentane $(50 \mathrm{~mL})$. The beige precipitate was isolated on a $15 \mathrm{~mL}$ fine porosity fritted disc, washed with pentane (3 x $10 \mathrm{~mL})$, and desiccated to yield 53 (146 mg, 62\% yield). CV (DMAc) $E_{\mathrm{p}, \mathrm{a}}=-0.07 \mathrm{~V}(\mathrm{NHE}) . \mathrm{IR}: v\left(\mathrm{C}-\mathrm{H} \mathrm{sp}^{2}\right)=2928 \mathrm{~cm}^{-1}, v(\mathrm{BH})=2471 \mathrm{~cm}^{-1}, v(\mathrm{NO})=1567 \mathrm{~cm}^{-}$ ${ }^{1} .{ }^{1} \mathrm{H}$ NMR (d $\mathrm{d}^{6}$-Acetone, $\left.\delta\right): 7.93$ (2H, m, Pz3A \& Pz5C), 7.90 (2H, buried s, DMAP-A), 7.87 (1H, d, Pz5A), 7.77 (1H, d, $J=7.7$, H5/H8), 7.76 (1H, s, H9), 7.72 (2H, d, Pz3C \& H5/H8), 6.69 (1H, d, Pz5B), 7.58 (1H, s, H10), 7.29 (2H, m, H6 \& H7), 6.88 (1H, d, Pz3B), 6.69 (2H, m, DMAP-B), 6.37 (1H, t, Pz4C), 6.32 (1H, t, Pz4A), 5.98 (1H, t, Pz4B), 4.22 (1H, dd, $\left.J=17.3 \& 3.6, \mathrm{H} 4^{\prime}\right), 4.08$ (1H, s, H1), 3.40 (1H, d, $\left.J=17.3, \mathrm{H} 4\right)$, $3.10(6 \mathrm{H}, \mathrm{N}-\mathrm{Me}), 2.85(3 \mathrm{H}, \mathrm{s}, \mathrm{OMe}), 2.63(1 \mathrm{H}, \mathrm{dd}, J=10.5 \& 3.6, \mathrm{H} 3), 2.50(1 \mathrm{H}, \mathrm{d}, J=$ 10.5, H2), $2.30(1 \mathrm{H}, \mathrm{d}, J=12.8, \mathrm{H} 11), 2.03(1 \mathrm{H}$, buried d, H11), $1.58-1.28(6 \mathrm{H}, \mathrm{m}$, $\mathrm{H} 11, \mathrm{H} 12, \& \mathrm{H} 13), 1.05(1 \mathrm{H}, \mathrm{t}, J=12.8, \mathrm{H} 11), 0.81(1 \mathrm{H}, \mathrm{m}, \mathrm{H} 13) .{ }^{13} \mathrm{C} \mathrm{NMR}\left(\mathrm{d}^{6}-\right.$ Acetone, 8): 154.9 (DMAP-C), 151.2 (DMAP-A), 143.6, 141.6, 141.5, 139.2, 138.6, 137.1, 136.9, 135.5, 133.9, 133.3, 129.9 (C9), 128.1 (C5/8), 127.5 (Pz3C), 126.5 (C10), 124.8 (C6/7), 124.5 (C6/7), 108.5 (DMAP-B), 106.6 (Pz4C), 106.2 (Pz4A), 105.9 (Pz4B), 82.5, 66.8 (C3), 61.7 (C2), 49.7 (C1), 47.5 (OMe), 39.2 (NMe), 35.5 (C4), 32.1 (C11), 31.0, 29.8, 26.7, 22.5, 22.3. 


\section{Synthesis of TpMo(NO)(DMAP)(2,3- $\eta^{2}$-dimethyl 2-((1S,4S)-4-(1-}

methoxycyclohexyl)-1,4-dihydroanthracen-1-yl)malonate)) (54).

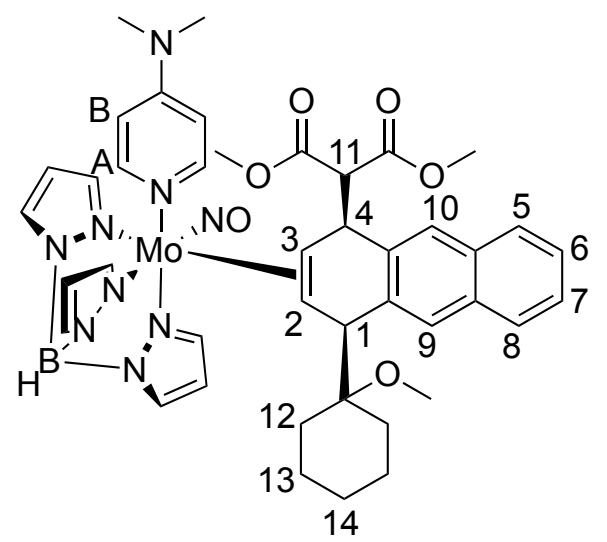

25 (200 mg, $0.313 \mathrm{mmol}), \mathrm{CH}_{3} \mathrm{CH}_{2} \mathrm{CN}$ (6 mL), and 1,1-dimethoxycyclohexane (0.3 mL, $1.98 \mathrm{mmol}$ ) were added to a test tube charged with a stir pea. The reaction mixture was stirred at RT until homogeneous and then cooled at $-60{ }^{\circ} \mathrm{C}$ for $15 \mathrm{~min}$. TMSOTf $(0.1 \mathrm{~mL}$, $0.54 \mathrm{mmol}$ ) was added to the reaction mixture and the resulting red solution was left stirring at $-60{ }^{\circ} \mathrm{C}$ for $15 \mathrm{~min}$. LiDMM (250 $\left.\mathrm{mg}, 1.81 \mathrm{mmol}\right)$ was added to the red solution, giving a brown solution that was left stirring at $-60{ }^{\circ} \mathrm{C}$ for $2 \mathrm{~h}$. This brown solution was then warmed to RT and washed with $\mathrm{H}_{2} \mathrm{O}(8 \mathrm{~mL})$. The aqueous layer was back-extracted with DCM $(6 \mathrm{~mL})$, combined with the organic layer, and dried over $\mathrm{MgSO}_{4}$. The drying agent was filtered off on a $15 \mathrm{~mL}$ medium porosity fritted disc, washed with DCM (3 x $2 \mathrm{~mL})$, and the filtrate was evaporated in vacuo. The resulting oil was then dissolved in DCM $(1 \mathrm{~mL})$ and the product was precipitated in stirring pentane $(50 \mathrm{~mL})$. The beige precipitate was isolated on a $15 \mathrm{~mL}$ fine porosity fritted disc, washed with pentane $(3 \times 10 \mathrm{~mL})$, and desiccated to yield 54 (145 mg, 52\% yield). CV (DMAc) $E_{\mathrm{p}, \mathrm{a}}=+0.7 \mathrm{~V}(\mathrm{NHE}) . \mathrm{IR}: v\left(\mathrm{C}-\mathrm{H} \mathrm{sp}^{2}\right)=2929 \mathrm{~cm}^{-1}, v(\mathrm{BH})=2470 \mathrm{~cm}^{-1}, v(\mathrm{CO})=1730 \mathrm{~cm}^{-}$ ${ }^{1}, v(\mathrm{NO})=1572 \mathrm{~cm}^{-1} .{ }^{1} \mathrm{H}$ NMR $\left(\mathrm{d}^{6}\right.$-Acetone, $\left.\delta\right): 7.96(2 \mathrm{H}, \mathrm{bs}$, DMAP-A $), 7.94(1 \mathrm{H}, \mathrm{d}$, 
Pz5A), 7.89 (1H, d, Pz5C), 7.83 (1H, d, Pz3C), 7.84 (1H, s, H9), 7.78 (1H, m, H5/H8), 7.73 (1H, m, H5/H8), 7.67 (1H, d, Pz5B), 7.63 (1H, s, H10), 7.55 (1H, d, Pz3A), 7.32 (2H, m, H6 \& H7), 6.84 (1H, d, Pz3B), 6.73 (2H, m, DMAP-B), 6.39 (1H, t, Pz4A), 6.33 (1H, t, Pz4C), 5.97 (1H, t, Pz4B), 4.46 (1H, d, $J=11.1, \mathrm{H} 4), 4.34$ (1H, d, $J=11.1, \mathrm{H} 11)$, $4.21(1 \mathrm{H}, \mathrm{s}, \mathrm{H} 1), 3.56(3 \mathrm{H}, \mathrm{s}, \mathrm{OMe}), 3.44(3 \mathrm{H}, \mathrm{s}, \mathrm{OMe}), 3.12(6 \mathrm{H}, \mathrm{s}, \mathrm{NMe}), 2.76(3 \mathrm{H}, \mathrm{s}$, OMe), $2.46(1 \mathrm{H}, \mathrm{d}, J=10.3, \mathrm{H} 3), 2.40(1 \mathrm{H}, \mathrm{d}, J=10.3, \mathrm{H} 2), 2.05$ (3H, buried multiplet, H12), 1.70 (1H, m, H12), 1.60 (1H, m, H13), 1.50 (2H, m, H13 \& H14), 1.35 (2H, m, H13), 1.00 (1H, m, H14). ${ }^{13} \mathrm{C}$ NMR (d $\mathrm{d}^{6}$-Acetone, $\left.\delta\right): 169.9$ (CO), 169.5 (CO), 155.0 (DMAP-C), 151.1 (DMAP-A), 143.3, 141.5 (Pz3A), 141.2 (Pz3B), 138.2, 137.4 (Pz5C), 137.3 (Pz5A), 137.1, 135.6 (Pz5B), 133.5, 133.4, 130.8 (Pz3C), 128.6, 128.1 (C5/8), 127.7 (C5/8), 125.3 (C6), 125.2 (C7), 108.6 (DMAP-B), 106.9 (Pz4C), 106.5 (Pz4B), 105.9 (Pz4A), 80.7, 67.1 (C3), 64.2 (C11), 60.8 (C2), 52.5 (OMe), $51.9(\mathrm{OMe}), 47.7$ (OMe), 44.9 (C4), $39.2(\mathrm{NMe}), 32.1,26.6,22.8$.

\section{Synthesis of dimethyl 2-((1S,4S)-4-(1-methoxycyclohexyl)-1,4-dihydroanthracen-1-} yl)malonate (55).

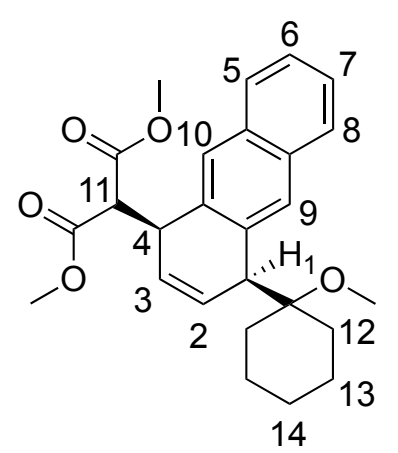

To a $50 \mathrm{~mL}$ filter flask charged with a stir pea were added $\mathbf{5 4}(100 \mathrm{mg}, 0.113 \mathrm{mmol})$, $\operatorname{DCM}(5 \mathrm{~mL})$, and a $0.06 \mathrm{M}$ solution of $\mathrm{I}_{2} / \mathrm{Et}_{2} \mathrm{O}(1 \mathrm{~mL}, 0.06 \mathrm{mmol})$, resulting in a green 
solution. This solution was stirred at RT for 5 min and evaporated in vacuo. The resulting green oil was dissolved in DCM $(1 \mathrm{~mL})$ forming a green solution. This solution was added to stirring hexanes $(50 \mathrm{~mL})$ giving a green precipitation. Collected the green precipitate on a $15 \mathrm{~mL}$ fine porosity fritted disc, washed with hexanes $(3 \times 10 \mathrm{~mL})$, and desiccated to yield 23 (65 mg, 97\%). The filtrate was removed from the glovebox and evaporated in vacuo to an oil. The oil was dissolved in DCM (3 x $0.3 \mathrm{~mL})$ and the resulting solution was dropwise added onto a $250 \mu \mathrm{m}$ silica preparatory plate. The product was eluted with 10\% EtOAc:hexanes $(200 \mathrm{~mL})$, scraped off as a band at $\mathrm{R}_{\mathrm{f}}$ : 0.16 0.34 and this silica gel was sonicated in EtOAc $(20 \mathrm{~mL})$ for $15 \mathrm{~min}$. The silica was filtered off on a $15 \mathrm{~mL}$ medium porosity fritted disc and washed with DCM (3 x $2 \mathrm{~mL})$. The filtrate was evaporated in vacuo and desiccated to yield the colorless oil $\mathbf{5 5}(31 \mathrm{mg}$, $65 \%$ yield $)$. IR: $v\left(\mathrm{C}-\mathrm{H} \mathrm{sp}^{2}\right)=2936 \mathrm{~cm}^{-1}$ and $v(\mathrm{CO})=1735 \mathrm{~cm}^{-1} \cdot{ }^{1} \mathrm{H} \mathrm{NMR}\left(\mathrm{CDCl}_{3}, \delta\right)$ : 7.77 (1H, m, H5/H8), 7.72 (1H, m, H5/H8), 7.66 (1H, s, H10), 7.63 (1H, s, H9), 7.41 (2H, m, H6 \& H7), 6.18 (1H, ddd, $J=10.2,5.2,1.0, \mathrm{H} 3), 6.09$ (1H, ddd, $J=10.2,5.2$, 0.6, H2), 4.43 (1H, ddd, $J=10.8,5.0,2.2, \mathrm{H} 4), 3.94(1 \mathrm{H}, \mathrm{m}, \mathrm{H} 1), 3.84(1 \mathrm{H}, \mathrm{d}, J=10.9$, H11), 3.80 (3H, s, OMe), 3.62 (3H, s, OMe), 3.35 (3H, s, OMe), 1.88 (1H, m, H12), 1.73 (1H, m, H12), 1.60 (6H, m, H12, H13, \& H14), 1.36 (1H, m, H12), 1H, m, H14). ${ }^{13} \mathrm{C}$ NMR $\left(\mathrm{CDCl}_{3}, \delta\right): 169.4$ (OMe), $168.4(\mathrm{OMe}), 134.8,134.2,132.0,131.9,130.1$ (H2), 129.0 (C10), 127.9 (C3), 127.6 (C5/8), 127.4 (C5/8), 127.3 (C9), 125.8 (C6 \& C7), 79.5, 59.8 (C11), 52.7 (OMe), 52.6 (OMe), 48.5 (OMe), 44.8 (C1), 40.0 (C4), 31.9, 31.8, 25.7. 


\subsection{References}

1. Myers, J. T.; Shivokevich, P. J.; Pienkos, J. A.; Sabat, M.; Myers, W. H.; Harman, W. D. Organometallics 2015, 34, (14), 3648.

2. $\quad$ Meiere, S. H.; Keane, J. M.; Gunnoe, T. B.; Sabat, M.; Harman, W. D. J. Am. Chem. Soc. 2003, 125, (8), 2024.

3. Rossi, M. B.; Piro, O. E.; Castellano, E. E.; Alborés, P.; Baraldo, L. M. Inorg. Chem. 2008, 47, (7), 2416.

4. $\quad$ Dunbar, M. A.; Balof, S. L.; Roberts, A. N.; Valente, E. J.; Schanz, H.-J. Organometallics 2011, 30, (2), 199.

5. Graham, P.; Meiere, S. H.; Sabat, M.; Harman, W. D. Organometallics 2003, 22, 4364.

6. Carden, R. G.; Ohane, J. J.; Pike, R. D.; Graham, P. M. Organometallics 2013, $32,(9), 2505$.

7. Mocella, C. J.; Delafuente, D. A.; Keane, J. M.; Warner, G. R.; Friedman, L. A.; Sabat, M.; Harman, W. D. Organometallics 2004, 23, (16), 3772.

8. Cahill, R.; Cookson, R. C.; Crabb, T. A. Tetrahedron 1969, 25, (19), 4681.

9. Harrison, D. P.; Nichols-Nielander, A. C.; Zottig, V. E.; Strausberg, L.; Salomon, R. J.; Trindle, C. O.; Sabat, M.; Gunnoe, T. B.; Iovan, D. A.; Myers, W. H.; Harman, W. D. Organometallics 2011, 30, (9), 2587.

10. Ha, Y.; Dilsky, S.; Graham, P. M.; Liu, W.; Reichart, T.; Sabat, M.; Kenae, J. M.; Harman, W. D. Organometallics 2006, 25, 5184.

11. Winemiller, M. D.; Harman, W. D. J. Am. Chem. Soc. 1998, 120, (31), 7835.

12. Ding, F.; Valahovic, M. T.; Keane, J. M.; Anstey, M. R.; Sabat, M.; Trindle, C. O.; Harman, W. D. J. Org. Chem. 2004, 69, (7), 2257.

13. Strausberg, L. Reactivity and Functionalization of Naphthalene and Anthracene Complexes of $\{\mathrm{TpW}\{\mathrm{NO})(\mathrm{PMe} 3)\}$. Ph. D. Dissertation, University of Virginia, 2008. 


\section{Chapter 4}

Michael-Michael Ring Closures on TpMo(NO)(DMAP)( $\eta^{2}$-Naphthalene) 


\subsection{Introduction}

Methods that form C-C bonds with regio- and stereocontrol make up the backbone of organic synthesis. Classical examples of carbon-carbon bond formation include the Claisen condensation, Mannich and Wittig reactions, Friedel-Crafts acylation and alkylation, the use of Grignard reagents, cross coupling reactions and many more. ${ }^{1}$ Among these well-known syntheses is the Michael reaction which involves the enolate attack on an $\alpha, \beta$-unsaturated carbonyl, yielding a conjugate addition product. ${ }^{2-4}$ Furthermore, the enolate formed immediately after nucleophilic attack onto the Michael acceptor can act as a nucleophile itself and attack a second equivalent of Michael acceptor. That resulting enolate can undergo an intramolecular cyclization to form a new ring system. This Michael-Michael-ring closure (MIMIRC) has been thoroughly explored by Gary Posner and others. ${ }^{5-11}$ Demonstrating the utility of this reaction, Posner reacted the enol silane of tetralone (62) with methyl lithium and subsequently added 2-methyl-2cyclopentenone yielding 64 , an estrone derivative with a steroidal core (Scheme 4.1). ${ }^{11}$ Of note, in this MIMIRC example, three new carbon-carbon bonds and three new stereocenters are formed selectively.

Scheme 4.1. Tandem Michael-Michael-ring closure to yield ( \pm )-9,11-Dehydroestrone.

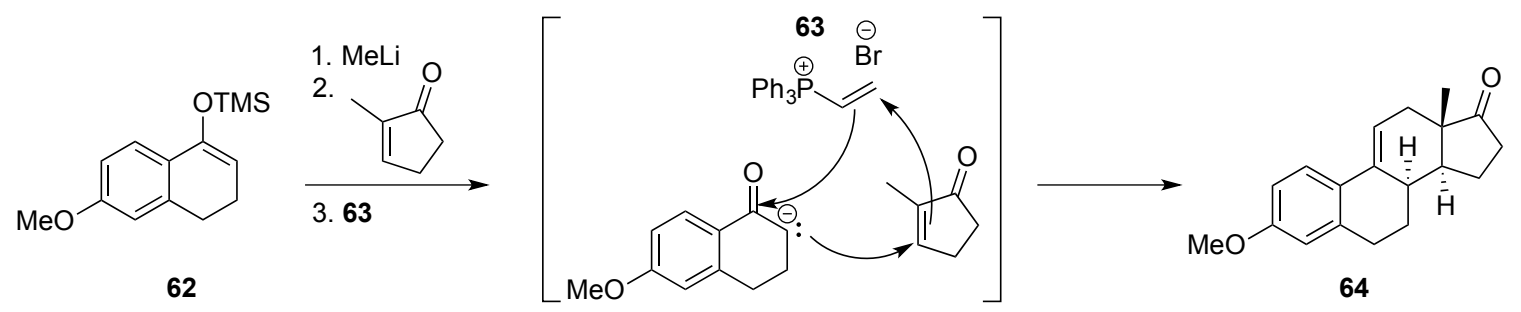


To further expand on the work of Posner, the Harman group has demonstrated MIMIRC reactions with the $\pi$-systems of aromatic molecules bound to a transition metal in an eta-2 fashion. For example, $\left\{\mathrm{Os}\left(\mathrm{NH}_{3}\right)_{5}\left(\eta^{2}-2 \text {-methylfuran }\right)\right\}^{2+}(\mathbf{6 5})$ is capable of undergoing an electrophilic addition at the beta position of the furan with methyl vinyl keton (MVK) activated by boron trifluoride diethyl etherate $\left(\mathrm{BF}_{3} \cdot \mathrm{OEt}_{2}\right)\left(\mathrm{Scheme}_{4} .2\right) .{ }^{12}$ The resulting enolate then reacts with a second equivalent of MVK, which then performs an intramolecular nucleophilic attack to yield a benzofuran skeleton (66). In an additional example, $\mathrm{TpRe}(\mathrm{CO})(\mathrm{MeIm})\left(\eta^{2}\right.$-2-methoxynaphthalene) (67) can undergo an electrophilic addition of methylacrylate, which proceeds through a Michael-Michael-ring closure to yield the final MIMIRC product (68) upon oxidative decomplexation. ${ }^{13}$ Encouraging results from the MIMIRC reaction to form $\mathbf{6 6}$ demonstrate that the metal center induces stereocontrol of the bridgehead formation through the steric preference of the initial electrophilic addition and the final nucleophilic ring closure both to occur anti to the metal. For $\mathbf{6 8}$, the stereocenters set by the metal are lost once the organic is liberated due to an elimination of the methoxy group. It should be noted that Scheme 4.1 shows an example of an $[\mathrm{A}+\mathrm{B}]$ MIMIRC, wherein the second Michael added is different from the initial. In contrast, Scheme 4.2 represents an [A+A] MIMIRC, wherein the two Michael acceptors being added are the same. 
Scheme 4.2. MIMIRC reactions on $\left\{\mathrm{Os}\left(\mathrm{NH}_{3}\right)_{5}\left(\eta^{2}-2-\text { methylfuran }\right)\right\}^{2+}$ and $\operatorname{TpRe}(\mathrm{CO})(\mathrm{MeIm})\left(\eta^{2}-2-m e t h o x y n a p h t h a l e n e\right)$.

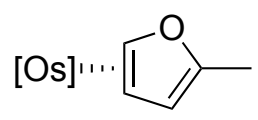

65

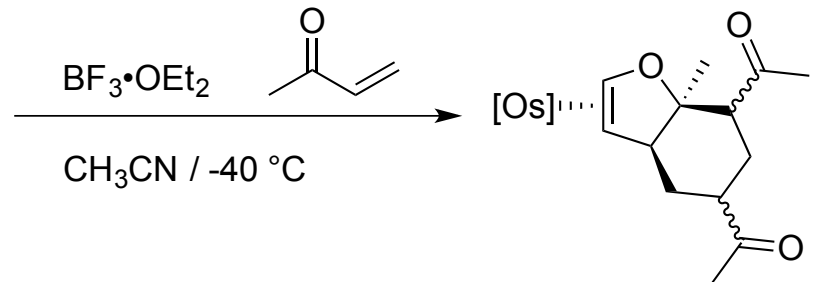

66, $87 \%$

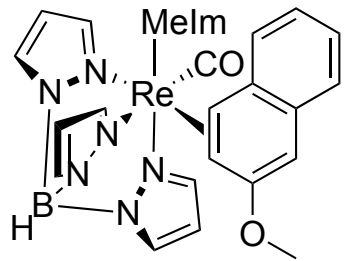

67

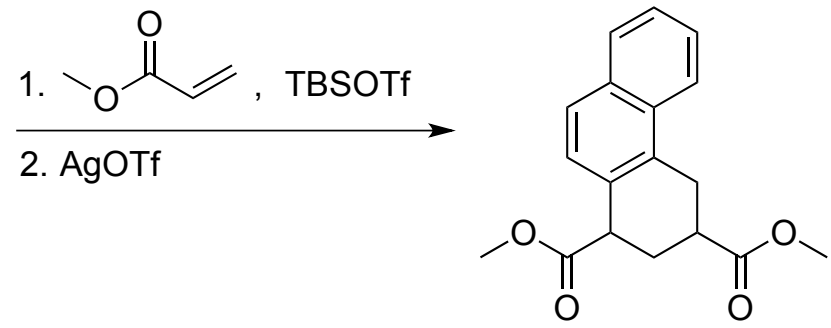

68, $40 \%$

During attempts at carbon-carbon bond formation on $\mathrm{TpMo}(\mathrm{NO})(\mathrm{DMAP})\left(\eta^{2}-\right.$ naphthalene) (24), the electrophilic addition of ethyl vinyl ketone (EVK) followed by the nucleophilic addition of LiDMM yielded the first example of a MIMIRC reaction of an eta-2 bound aromatic molecule performed using molybdenum; however, this was isolated as a mixture of products (vide infra). The following chapter describes the development of a method to perform MIMIRC reactions on 24, determination of the stereoselectivity of these additions, and exploration of further possible MIMIRC reactions, which can be mediated by the $\{\mathrm{TpMo}(\mathrm{NO})(\mathrm{DMAP})\}$ fragment. 


\subsection{Results and Discussion}

During the initial investigation for electrophilic additions of Michael acceptors onto 24, triflic acid (HOTf) was used to promote the reaction by the putative formation of the more electrophilic, protonated species, 69. Under these conditions, 24 is capable of attacking 69 to form an allyl stabilized by the metal center (70). With the intention of adding a carbon nucleophile to $\mathbf{7 0}$, LiDMM was added to the reaction mixture and, upon reaction completion, the resulting solution was exposed to air overnight (Scheme 4.3).

Scheme 4.3. Attempted tandem electrophilic-nucleophilic addition of EVK and LiDMM to 24 .

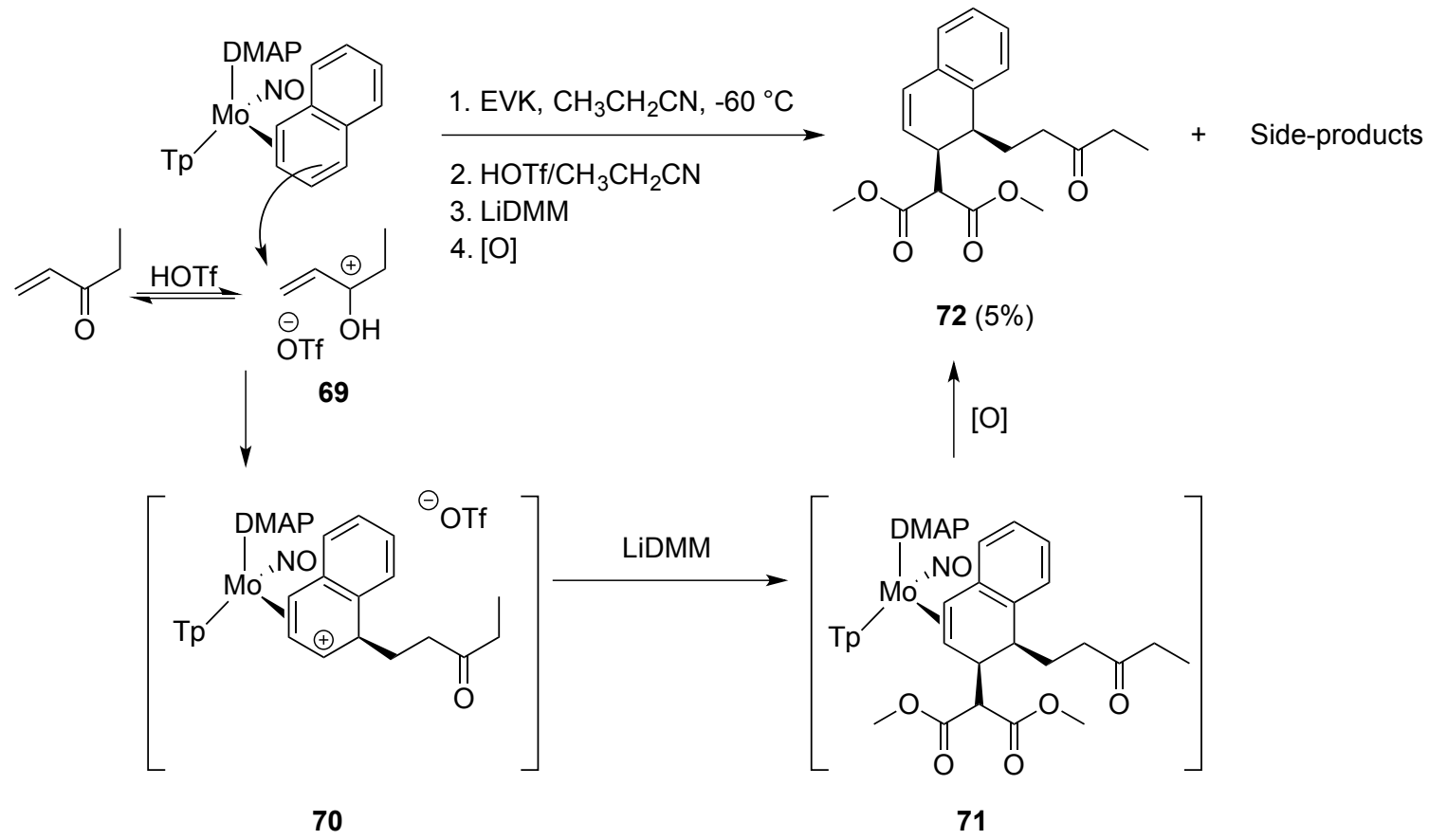

Following oxidative decomplexation to yield $\mathbf{7 2}$, the resulting reaction mixture was chromatographed on a silica gel preparatory plate, which yielded multiple products. 
In contrast to the 1,4-additions seen in Chapter 3, one of the products in this mixture was the 1,2-dihydronaphthalene 72. In the ${ }^{1} \mathrm{H}$ NMR spectrum of $\mathbf{7 2}$, the isolated alkene resonances are at $6.48(\mathrm{dd}, J=10.0$ and $2.7 \mathrm{~Hz}, \mathrm{H} 3)$ and $5.64(\mathrm{~d}, J=10.0 \mathrm{~Hz}, \mathrm{H} 4) \mathrm{ppm}$, which are in agreement with typical chemical shifts of a 1,2-addition product. Two singlets at 3.83 and $3.74 \mathrm{ppm}$, as well as a triplet at $0.98 \mathrm{ppm}$, each integrate for three protons in comparison to $\mathrm{H} 3$ and $\mathrm{H} 4$. In the ${ }^{13} \mathrm{C}$ NMR spectrum of $\mathbf{7 2}$, three carbonyl peaks are seen at 218.9, 168.9, and $168.6 \mathrm{ppm}$ representing the ketone and two ester carbonyls, respectively. Lastly, the three CO stretching frequencies at 1755, 1736 and $1712 \mathrm{~cm}^{-1}$ are observed in the IR spectrum of 72. In agreement with the preference for additions to occur anti to the metal center, a strong NOE interaction is seen between protons $\mathrm{H} 1$ and $\mathrm{H} 2$. Therefore, a cis geometry is assigned for 72. Although this evidence indicates that a successful tandem electrophilic-nucleophilic addition to yield $\mathbf{7 2}$ occurred, the selective synthesis of this product proved inconsistent and low yielding $(<$ $5 \%$ ). Furthermore, a 1,2-addition cannot be assumed as the favored regiochemistry due to the plethora of side-products seen when using HOTf as the acid source.

In the investigation of the side-products isolated from the procedure to yield $\mathbf{7 2}$, six main pathways were considered (Scheme 4.4). Considering the synthesis of $\mathbf{7 2}$, Pathway $\mathrm{i}$ assumes the possibility that two 1,2-additions occurred wherein the cis and trans products could be isolated. Similarly, the cis and trans isomers of a 1,4-addition could have formed (74 and 75), as depicted in Pathway ii. As previously seen in Chapter 3 with the isolation of $\mathbf{1 4}$ during the synthesis of $\mathbf{4 3}$, the likelihood of one of the sideproducts being the product of a protonation and subsequent nucleophilic addition is very high, thus Pathway iii considers 1,2- and 1,4-additon products 14 and 76, respectively. 
The initial addition of EVK to naphthalene is believed to form an enol, which can act as a nucleophile. Pathway iv depicts this enol attacking the allylic naphthalene to form a cyclized 1,2-dihydronaphthalene (77 and $\mathbf{7 8 )}$ ). In a similar mechanism, after the allylic ring rearranges on the metal center, the enol can attack, which would yield a cyclized 1,4dihydronaphthalene (79 and $\mathbf{8 0}$ ) as seen in Pathway v. Lastly, the enol formed can attack a second equivalent of Michael acceptor and the subsequent enol can attack the allylic naphthalene yielding either a 1,2- or 1,4-dihydronaphthalene (61 and 81) as depicted in Pathway vi.

Scheme 4.4. Analysis of mixture resulting from the attempted tandem electrophilicnucleophilic addition of EVK and LiDMM to 24.

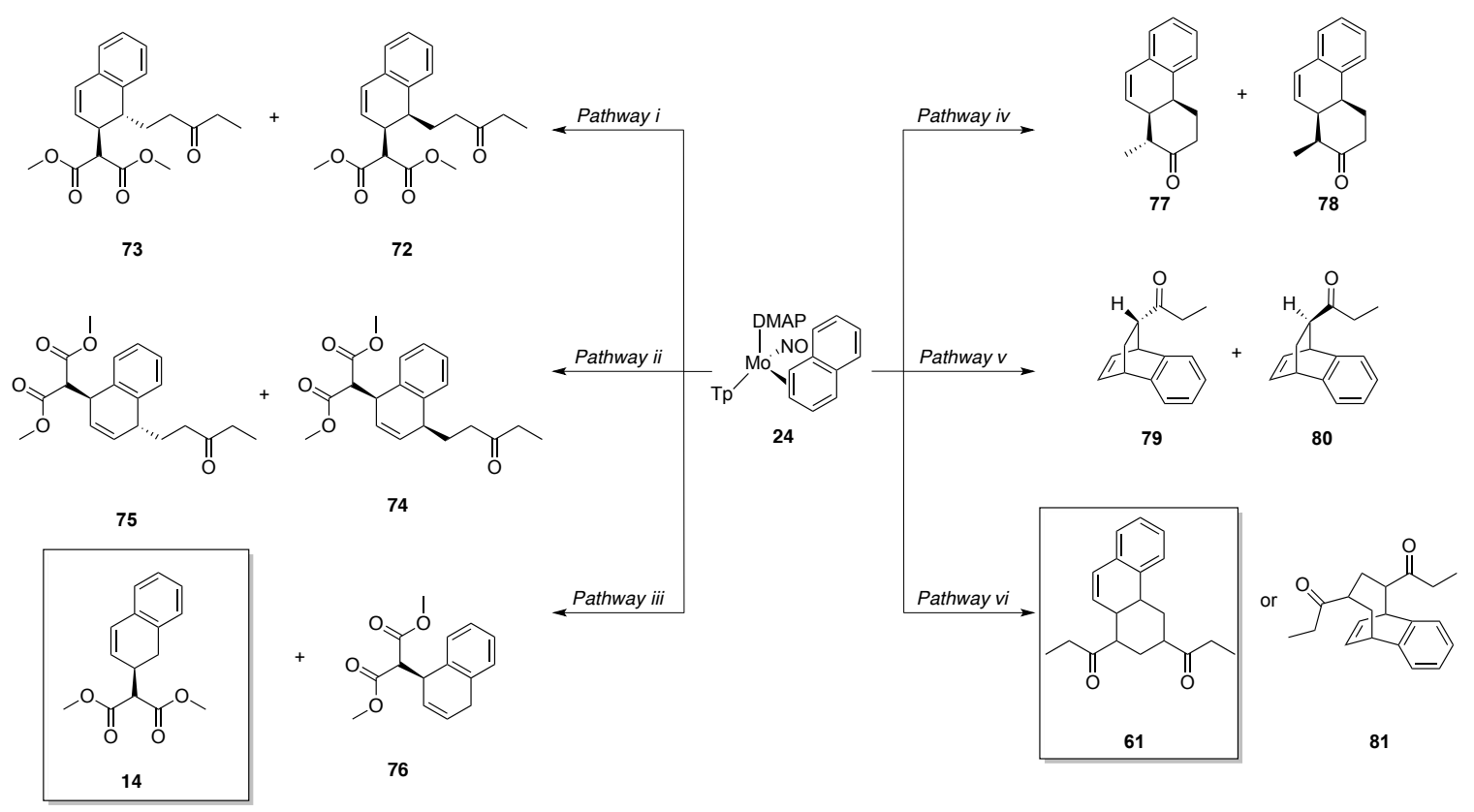

One band collected from the preparatory plate was shown by ${ }^{1} \mathrm{H}$ NMR to have four alkene peaks at $6.52(\mathrm{dd}, J=9.6$ and $1.4 \mathrm{~Hz}), 6.44(\mathrm{~d}, J=9.7 \mathrm{~Hz}), 6.01(\mathrm{dd}, J=9.7$ 
and $6.1 \mathrm{~Hz}$ ), and $5.95(\mathrm{dd}, J=9.6$ and $4.5 \mathrm{~Hz}) \mathrm{ppm}$. This was initially thought to be the two epimers of a 1,2-addition or 1,4-addition on naphthalene (Scheme 4.3, Pathway i or Pathway ii). However, comparing this spectrum with that of the cleanly isolated $\mathbf{7 2}$ shows this not to be the case. Another key feature in disagreement with Pathway i or ii is the fact that four carbonyl carbons $(213.4,213.2,168.8$, and $168.7 \mathrm{ppm})$ are present in the ${ }^{13} \mathrm{C}$ NMR spectrum of this mixture, rather than the expected six carbonyls. Furthermore, the chemical shifts and splitting patterns of the alkene resonances do not match those of a 1,4-addition product, thus Pathway i and Pathway ii were determined not to have contributed to this mixture of products.

Through a comparison with previous results it was found that the alkene peaks at 6.52 and $5.95 \mathrm{ppm}$ were in fact due to the presence of the 1,2-dihydronaphthalene $\mathbf{1 4}$ (See Chapter 2), the product of a tandem electrophilic-nucleophilic addition of HOTf and LiDMM (e.g., Pathway iii). However, the chemical shifts and splitting patterns of the remaining alkene resonances indicate that a 1,4-addition product was not made. Accounting for the presence of $\mathbf{1 4}$, the remaining peaks were compared to the possible products of Pathways iv, v, and vi.

Key ${ }^{1} \mathrm{H}$ NMR features for the remaining product include two peaks at 1.09 and $0.98 \mathrm{ppm}$, both of which are triplets, and each integrating for three protons (relative to the integration of the alkene peaks at 6.44 and 6.01 ). The triplets at 1.09 and 0.98 ppm couple to two separate methylene groups (identified by HSQC) at $2.55(2 \mathrm{H}, \mathrm{m})$ as well as at 2.27 and $2.42(1 \mathrm{H}$ each, m) ppm, respectively. Using HMBC correlations, these methylene groups were assigned as being adjacent to the carbonyl resonances at 213.4 and 213.2 ppm. Pathways $\mathrm{v}$ and vi both yield products with methylene groups adjacent to a 
carbonyl and a methyl group; however, compounds 77 and 78 do not, thus disproving Pathway iv.

There is literature precedent supporting the possibility of the 1,4-cyclizations proposed in Pathway v; ${ }^{13}$ however, were Pathway v taken then both products 79 and 80 would need to be in this mixture to support the presence of the two triplets in the ${ }^{1} \mathrm{H}$ NMR spectrum. As this is not the case (based on the lack of remaining unassigned chemical shifts), Pathway v was ruled out. Following Pathway vi to yield $\mathbf{8 1}$ was unlikely as COSY correlation shows only one of the two alkene peaks $(6.01 \mathrm{ppm})$ to correlate with any other protons in this product. This is a common indicator of a product from a 1,2rather than a 1,4-addition. Thus leading to the investigation of $\mathbf{6 1}$ as the unidentified product. This analysis led to the carbon and proton assignments shown in Figure 4.1.

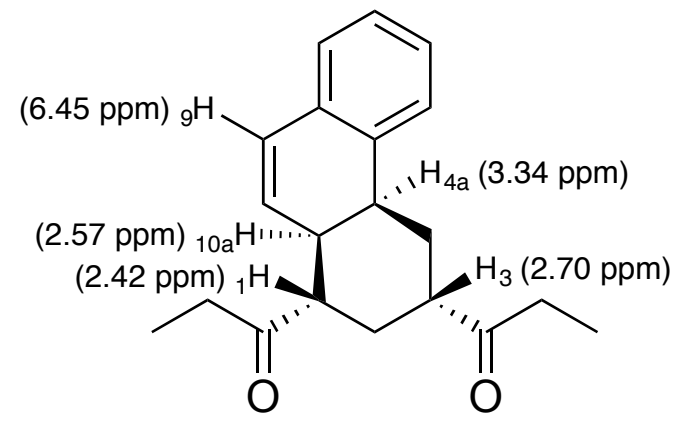

Figure 4.1. Selected proton assignments for $\mathbf{6 1 .}$

Under the reaction conditions to form 72, the following proposed mechanism provides a rationale for the formation of $\mathbf{6 1}$. The initial electrophilic addition of EVK to 24 yields an enol bound allylic species (70), which tautomerizes to the keto form. Upon the addition of LiDMM the ketone is deprotonated yielding a nucleophilic enolate (82), which can then attack another equivalent of EVK yielding the Michael-Michael addition product $(\mathbf{8 3})$. Subsequently, 83 can be deprotonated and the resulting enolate (84) can 
attack the electrophilic allyl, yielding the ring-closed product 61 (Scheme 4.5). This proposed mechanism agrees with the observation that formation of $\mathbf{6 1}$ requires the presence of a base and does not occur simply with HOTf and EVK.

Scheme 4.5. Proposed mechanism for the synthesis of 61.
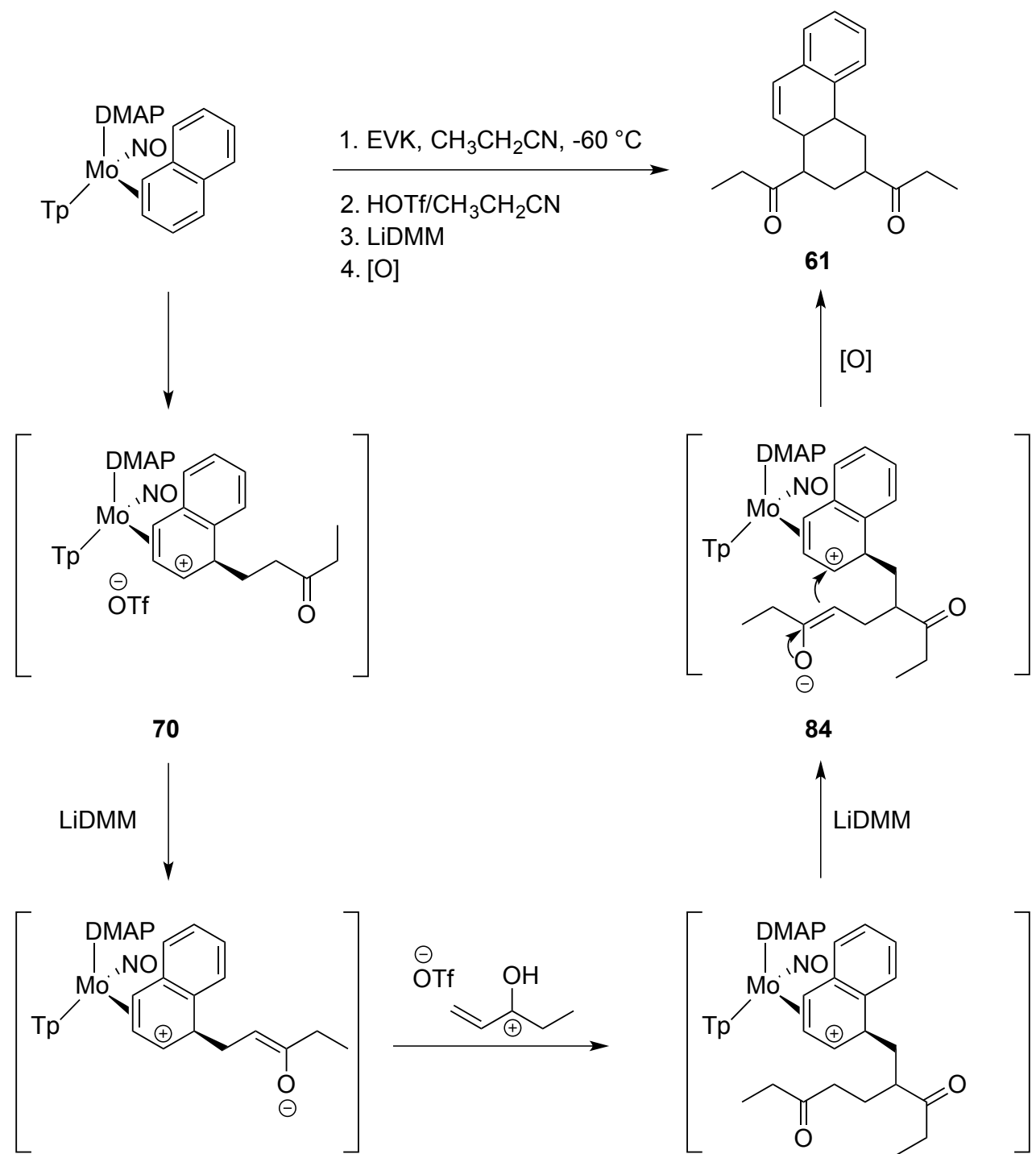

82
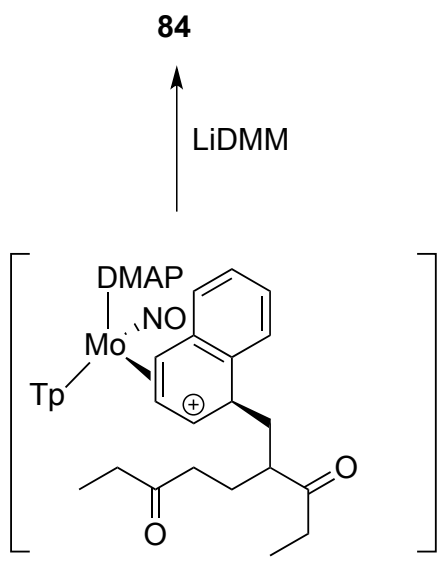

83 
Following determination of the structure of $\mathbf{6 1}$, attempts were made to improve the selectivity, yield, and purity of this MIMIRC reaction product. Optimization of this reaction began by attempting to use LiOMe in place of LiDMM to access 82, while avoiding the nucleophilic addition of LiDMM. With this replacement, an impure metal complex (broad $E_{\mathrm{p}, \mathrm{a}} \sim+220 \mathrm{mV}$ ) was isolated and then subjected to iodine oxidation, This procedure yielded a minute amount of desired product (61) as well as a substitution product $(\mathbf{8 5})$, which was confirmed by literature reports. ${ }^{14}$ This substitution product, $\mathbf{8 5}$, was formed from $\mathbf{7 0}$ undergoing deprotonation at the naphthalenium, rather than addition of a second equivalent of EVK. Attempts to reduce these side-products focused on using Lewis acids to activate the Michael acceptor in place of HOTf. The use of LiOTf or $\mathrm{BF}_{3} \cdot \mathrm{OEt}_{2}$ yielded only free naphthalene upon oxidative decomplexation of their reaction mixtures. However, success was found through the use of alkylsilated triflates.

Trimethylsilyl trifluoromethanesulfonate (TMSOTf) was added to a $-60{ }^{\circ} \mathrm{C}$ solution of 24 and EVK dissolved in $\mathrm{CH}_{3} \mathrm{CH}_{2} \mathrm{CN}$. The resulting solution turned blood red; a change in color consistent with the formation of an allyl. The mixture was left at $60{ }^{\circ} \mathrm{C}$ for 18 hours over which time it gradually turned brown. Subsequently, the resulting brown solution was then stirred exposed to air for $24 \mathrm{~h}$. Chromatographing this solution on a silica gel preparatory plate yielded the desired product $\mathbf{6 1}$, the substitution product $\mathbf{8 5}$, as well as another substitution product 86 (Scheme 4.6). Similar to the synthesis of $\mathbf{8 5}, \mathbf{8 6}$ is the result of the allyl being deprotonated; however, in the synthesis of 86, the enol has attacked another equivalent of EVK before the deprotonation at the naphthalenium occurs. The pure isolation of $\mathbf{8 6}$ has not been accomplished, but key similarities with the ${ }^{1} \mathrm{H}$ NMR spectrum of $\mathbf{8 5}$ allowed for the identification of $\mathbf{8 6}$. For 
instance, $\mathbf{8 6}$ has 7 aromatic peaks (four doublets and three triplets) as well as two triplets at 1.06 and $0.85 \mathrm{ppm}$. The formation of $\mathbf{8 5}$ and $\mathbf{8 6}$ using TMSOTf is believed to occur due to the formation of HOTf when TMSOTf comes into contact with trace amounts of water. This formation of HOTf provides a better electrophile for the enolates $\mathbf{8 2}$ and $\mathbf{8 4}$ to attack, which hinders the desired annulative process and favors the isolation of substitution products $\mathbf{8 5}$ and $\mathbf{8 6}$.

Scheme 4.6. Synthesis of $\mathbf{6 1}$ from 24 as well as common side-products (e.g., 85, 86, and free naphthalene)

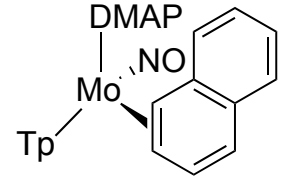

24
1. $\mathrm{EVK}, \mathrm{CH}_{3} \mathrm{CH}_{2} \mathrm{CN}$, Additive

2. Activator

3. Base, [O]<smiles>CCC(=O)C1CC(C(=O)CC)C2C=Cc3ccccc3C2C1</smiles>

61<smiles>CCC(=O)CCC(Cc1cccc2ccccc12)C(=O)CC</smiles>

86

After determining alkylsilylated triflates to be the most efficient activator for these MIMIRC reactions, the reaction was optimized. This optimization was completed using the above description of reaction setup, followed by $\mathrm{CV}$ monitoring of the reaction (as these are products of a 1,2-addition, the anodic wave for their eta-2 bound complexes 
was suspected to be $\sim+200 \mathrm{mV}$; see Chapter 2). After reaction completion was determined, TEA was added and the reaction mixture was stirred while being exposed to air for no less than $18 \mathrm{~h}$. These reaction mixtures were then evaporated in situ and the resulting oil was dissolved in DCM. Adding this brown solution to $\mathrm{Et}_{2} \mathrm{O}(50 \mathrm{~mL})$ induced a precipitation of an undesired inorganic material that was subsequently removed using a $15 \mathrm{~mL}$ fritted disc. The filtrate was then evaporated in vacuo and the resulting crude organic was isolated as a brown oil, dissolved in $\mathrm{CDCl}_{3}$, and observed by ${ }^{1} \mathrm{H}$ NMR.

The success of these reactions was determined through ${ }^{1} \mathrm{H}$ NMR by identifying the relative concentrations of $\mathbf{6 1 , 8 5 , 8 6}$, and free naphthalene. In these crude organic mixtures the following peaks were integrated to compare relative concentrations: product 61, $6.41 \mathrm{ppm}$; product 85, $1.06 \mathrm{ppm}$; product $\mathbf{8 6}, 7.96 \mathrm{ppm}$; and free naphthalene, 7.80 ppm. Using this method of monitoring, the reaction time, temperature, equivalents of TMSOTf, and different alkylsilylated triflates were varied and their results recorded (Table 4.1). Out of the four products described above, free naphthalene was consistently formed in the largest amount. Between $\mathbf{6 1 , 8 5}$, and $\mathbf{8 6}$ it was found that the two major products were $\mathbf{6 1}$ and $\mathbf{8 6}$, with minimal observations of $\mathbf{8 5}$. For this reason, Table 4.1 only compares the relative integrations of $\mathbf{6 1}$ and $\mathbf{8 6}$. The highest yield of the desired MIMIRC was obtained when di-tert-butylpyridine (DTBP) was present (entry 12), which limits the acid source for protonation of $\mathbf{8 4}$ or $\mathbf{8 2}$. This led to a crude organic mixture with only $7 \%$ of $\mathbf{8 6}$ relative to $\mathbf{6 1}$. After a chromatographic workup of the crude organic described in entry 12, 61 was isolated at $21 \%$ from 24 . 
Table 4.1. Summary of experiments performed during the optimization of the synthesis of 61 .

\begin{tabular}{|c|c|c|c|c|c|c|c|}
\hline Entry & EVK (equiv) & \begin{tabular}{|l|} 
Additive (equiv) \\
\end{tabular} & Temperature $\left({ }^{\circ} \mathrm{C}\right)$ & Activator (equiv) & Time (h) & Base & Result \\
\hline 1 & 5.5 & - & -60 & HOTf (2.2) & 18 & TEA & Minute amounts of $\mathbf{6 1}$ and 85 \\
\hline 2 & 10 & - & -60 & LiOTf (2.2) & 18 & TEA & No Reaction \\
\hline 3 & 7 & - & -60 & HOTf (2.2) & 0.33 & LiOMe & Minute amounts of 61 and 85 \\
\hline 4 & 7 & - & -60 & TMSOTf (1.6) & 18 & TEA & 1:1:1 amounts of $\mathbf{6 1 , 8 5}$, and 86 \\
\hline 5 & 7 & - & -60 & $\mathrm{BF}_{3}$-Etherate (2.2) & 18 & TEA & No Reaction \\
\hline 6 & 7 & - & -30 & TMSOTf (1.6) & 18 & TEA & Decomposition \\
\hline 7 & 7 & - & -60 & TMSOTf (1) & 18 & TEA & $86: 61=1.0: 0.7$ \\
\hline 8 & 7 & - & -60 & TMSOTf (1.6) & 1 & TEA & $86: 61=0.7: 1.0$ \\
\hline 9 & 7 & - & -60 & TMSOTf (1.6) & 6 & TEA & $86: 61=0.6: 1.0$ \\
\hline 10 & 7 & - & -60 & TBDMSOTf (1.6) & 18 & TEA & $86: 61=1.0: 1.0$ \\
\hline 11 & 7 & - & -60 & TIPSOTF (1.6) & 18 & TEA & $86: 61=1.0: 0.6$ \\
\hline 12 & 7 & DTBP (0.9) & -60 & TMSOTf (1.6) & 18 & TEA & $86: 61=0.07: 1.0(21 \%$ isolated yield of 61$)$ \\
\hline 13 & 7 & DBU (0.9) & -60 & TMSOTf (1.6) & 18 & TEA & No Reaction \\
\hline
\end{tabular}

Following the conditions described in entry 12, with the exception of the oxidative decomplexation, isolation of $\mathrm{TpMo}(\mathrm{NO})(\mathrm{DMAP})\left(\eta^{2}-\mathbf{6 1}\right)$ was accomplished via chromatography and subsequent precipitation in pentane to yield 87 at $45 \%$ from 24 . In agreement with an eta-2 bound, 1,2-addition to $\mathbf{2 4}$, the anodic wave of $\mathbf{8 7}$ is $+160 \mathrm{mV}$ and the stretching frequency for the nitrosyl is $1567 \mathrm{~cm}^{-1}$. Encouragingly, the ${ }^{1} \mathrm{H}$ NMR of 87 shows there to be one major diastereomer present (dr 8:1), implying stereocontrol not only at the metal center, but also of the newly formed ring. Similar to 61, the HSQC of 87 was used to identify four methylene groups, two of which are part of the ethyl groups attached to the carbonyl, while the other two represent H4 and H2 (Figure 4.3). Furthermore, the up- $(3.31 \mathrm{ppm}, 1 \mathrm{H}, \mathrm{d}, \mathrm{H} 9)$ and down-bound (2.07 ppm, dd, H10) protons were identified by their upfield chemical shift and coupling constant of $9 \mathrm{~Hz}$, which is typical for adjacent alkene coupling. Through COSY correlation to H10, H10a was identified as a multiplet at $2.77 \mathrm{ppm}$. Once H10a was identified, the remaining assignments were made in a similar fashion to that described for $\mathbf{6 1}$ (vide supra). 
With one major diastereomer seen in the isolation of $\mathbf{8 7}$, the assignment of the newly formed stereocenters was pursued. In addition to 2D-NMR analysis, a solid-state analysis was accomplished through x-ray diffraction (Figure 4.2). In this sample, it is shown that both the electrophilic and nucleophilic additions to $\mathbf{2 4}$ occurred anti to the metal center. Furthermore, the ethyl ketone groups are in an equatorial geometry so as to avoid high energy steric interactions found in a 1,3-diaxial orientation. Lastly, from the solid state it was found that the equatorial geometry of the initial Michael addition places H4a close to Pz3A (2.76 $\AA$ ). In Chapter 3 it was demonstrated that the solid-state analysis is not always indicative of the bulk solution; for this reason, the following 2D-NMR analysis is aimed at confirming these stereochemical relationships.

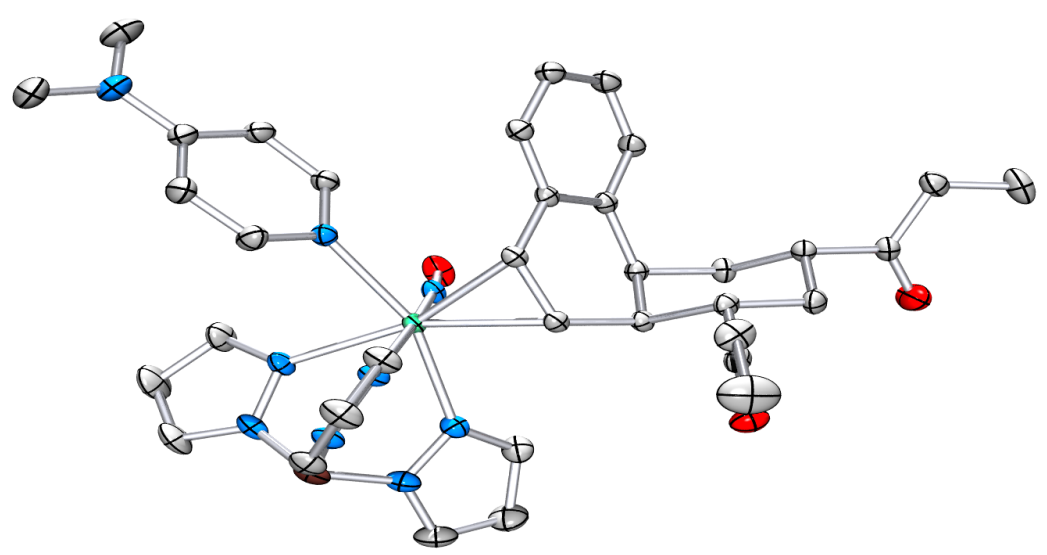

Figure 4.2. Crystal structure of 87. Bond lengths $(\AA)$ : Mo-C(9) 2.2348(15); Mo$\mathrm{C}(10)$ 2.2276(15); Mo-N(DMAP) 2.2233(15); Mo-N(NO) 1.7596(14). 


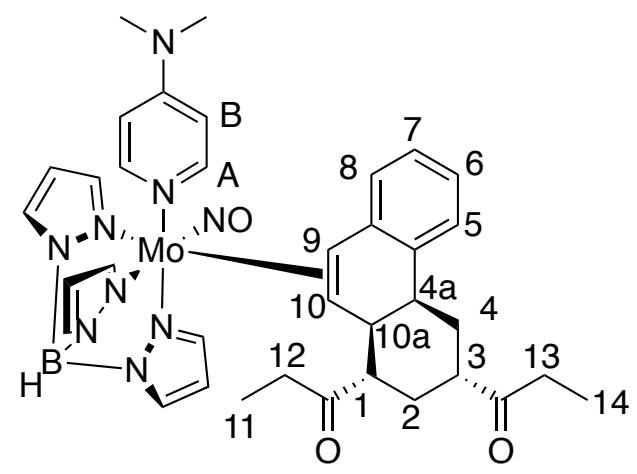

Figure 4.3. Numbering scheme of 87 .

H10a was previously identified, which through COSY correlation led to the assignment of $\mathrm{H} 4 \mathrm{a}$ as a multiplet at $3.73 \mathrm{ppm}$. Furthermore, H10, H4a, and H10a all show strong NOE interaction with Pz3A, which is in agreement with the solid-state depiction of addition occurring anti to the metal center. From this analysis it is assumed that the organic isolated from 87 (61) would also have a cis orientation between H4a and H10a. Dihedral angle analysis to confirm this assumption is not available due the complex splitting pattern of $\mathrm{H} 4 \mathrm{a}$ and $\mathrm{H} 10 \mathrm{a}$; however, NOE interaction between these protons is strong, which is in agreement with a cis orientation.

The $\mathrm{C} 3$ and $\mathrm{C} 1$ carbons of $\mathbf{8 7}$ are enolizable, which indicates the ability to rearrange until a thermodynamically preferred orientation is achieved. In the case of $\mathbf{8 7}$, as well as $\mathbf{6 1}$, the thermodynamic preference is to place both propanonyl groups in an equatorial position rather than a 1,3-diaxial geometry, which would lead to unfavorable steric interaction (Figure 4.4). This is confirmed by a strong NOE interaction seen between H3 and H1 for complex 87 and the organic 61. The combination of enolizable carbons and stereochemical control from the metal center allows for improved stereoselectivity during these MIMIRC reactions. 


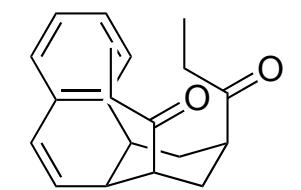

1,3-diaxial geometry (disfavored)

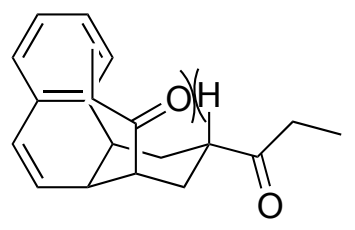

staggered geometry (disfavored)

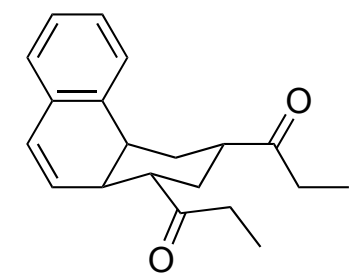

1,3-diequatorial geometry (favored)

Figure 4.4. Analysis of possible steric interaction among different geometries of $\mathbf{6 1}$.

MVK is also capable of undergoing an $[\mathrm{A}+\mathrm{A}] \mathrm{MIMIRC}$ reaction with 24; however, the yields for metal complex isolation (88) are low (10\%). Encouragingly, the spectroscopic assignments made for $\mathbf{8 7}$ are identical to $\mathbf{8 8}$ with the exception of the methylene protons attributed to the ethyl ketone in $\mathbf{8 7}$. Furthermore, the triplets at 1.00 and $0.68 \mathrm{ppm}$ are no longer present; rather, two singlets at 2.19 and $1.89 \mathrm{ppm}$ are seen in the ${ }^{1} \mathrm{H}$ NMR of $\mathbf{8 8}$. This implies similar stereocontrol for $\mathbf{8 8}$ as that seen for $\mathbf{8 7}$, which is expected.

With the isolation of these complexes, the oxidative decomplexation of their hexahydrophenanthrene organic ligands was pursued. Initial attempts at decomplexation involved a one-pot addition/oxidation sequence directly from the parent naphthalene complex, 24. A concern during this initial investigation was the presence of a large amount of free naphthalene seen after oxidation. This was believed to be due to a lack of reaction occurring; therefore, upon stirring the reaction mixture open to air, the unreacted complex 24 simply releases free naphthalene. Alternatively, the presence of naphthalene could result from the retro-addition of the organic addition products (61 and 89) after the oxidation of complexes $\mathbf{8 7}$ and $\mathbf{8 8}$. To discriminate between these two pathways of naphthalene formation, an isolated sample of $\mathbf{8 7}$ was dissolved in DCM and stirred open 
to air overnight. The resulting mixture was observed by ${ }^{1} \mathrm{H}$ NMR to reveal $\mathbf{6 1}$ as the only diamagnetic product of oxidation, demonstrating that the naphthalene seen in early decomplexation reactions was due to the presence of unreacted 24 in the reaction mixture. Following this, the oxidative decomplexation of the organic products directly from $\mathbf{8 7}$ and $\mathbf{8 8}$ was accomplished using iodine (Scheme 4.7) yielding $\mathbf{6 1}$ and $\mathbf{8 9}$ in fair yields with a good recovery of the molybdenum(I) iodo precursor, $\mathbf{2 3}$.

Scheme 4.7. Synthesis of 87 and 88 and their subsequent oxidations to yield 61 and 89.

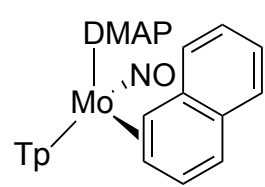

24

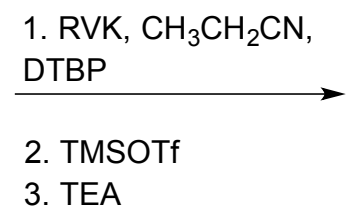

3. TEA

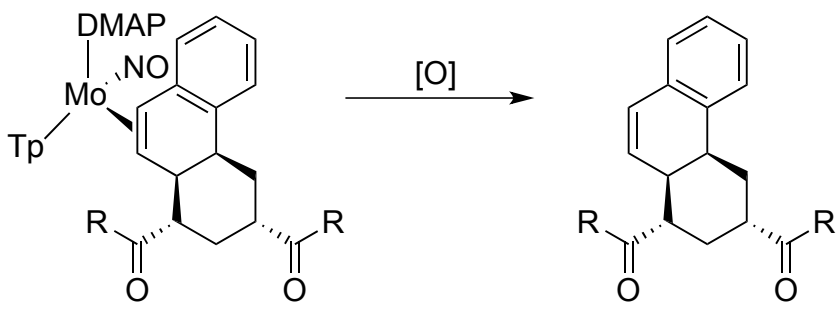

$$
\mathrm{R}=\mathrm{Et}(\mathbf{8 7}) ; 45 \%
$$$$
\mathrm{R}=\mathrm{Me}(\mathbf{8 8}) ; 10 \%
$$

$\mathrm{R}=\mathrm{Et}(61), 46 \%$ via $\mathrm{O}_{2}$;

$26 \%$ via $\mathrm{I}_{2}, \mathbf{2 3}=91 \%$

$\mathrm{R}=\mathrm{Me}(\mathbf{8 9}), 16 \%$

via $I_{2}, 23=85 \%$

To further demonstrate the promise that this method of Michael-Michael ring closure has, $[\mathrm{A}+\mathrm{B}] \mathrm{MIMIRC}$ reactions of $\mathbf{2 4}$ were investigated. The goal of this approach is to limit the amount of reactivity once the first Michael acceptor is added to $\mathbf{2 4}$ to give a single Michael addition product (e.g., the enolate 82) and then introduce another Michael acceptor to continue through the ring closure. This was accomplished by limiting the initial Michael acceptor (e.g., EVK) to one molar equivalent so as to prevent a second addition from occurring. For the reactions described in this section the general reaction scheme is as follows: to a $-60{ }^{\circ} \mathrm{C}$ proprionitrile solution of $\mathbf{2 4}$, Michael acceptor A (1.0 equivalent), and DTBP, TMSOTf is added; after stirring at $-60{ }^{\circ} \mathrm{C}$ for 1 minute, a 
solution of Michael acceptor B (2.5 equivalents) in proprionitrile (1 mL) is added to the reaction mixture, which is then left at $-60{ }^{\circ} \mathrm{C}$ for $18 \mathrm{~h}$.

Using the method described above, success was found when Michael acceptor A was EVK and Michael acceptor B was either MVK or cyclopentenone. The cleanest of these two reactions was the $[\mathrm{EVK}+\mathrm{MVK}]$ additions, which led to the isolation of the metal complex 90; however, a pure isolation of 90 is only achieved at a low yield $(30 \%)$ (Scheme 4.8). This low yield is believed to be due to other side-products from the reaction (vide infra). The ${ }^{1} \mathrm{H}$ NMR of $\mathbf{9 0}$ is identical to $\mathbf{8 7}$ and $\mathbf{8 8}$; however, it contains a singlet and a triplet in the aliphatic region, rather than two singlets or two triplets. Furthermore, a solid-state structure of $\mathbf{9 0}$ was determined through x-ray diffraction as seen in Figure 4.3.

As expected, the solid-state analysis demonstrates a retained 1,3-diequatorial geometry of the alkyl groups. Also seen is a similar cis bridgehead with the additions to naphthalene occurring anti to the metal center. Although the metal center of $\mathbf{9 0}$ can be isolated cleanly at a low yield, more often a higher mass recovery is accomplished with a detriment to purity. For instance, adding iodine to one of these greater mass recovery samples of 90 yields three products, 61, 89 and the desired $[A+B]$ MIMIRC organic, 91, in a 1:1:2 ratio. As expected, the ${ }^{1} \mathrm{H}$ NMR spectrum of 91 shows a great similarity to 61 and 89, with the exception of the methyl groups' splitting and chemical shifts. 


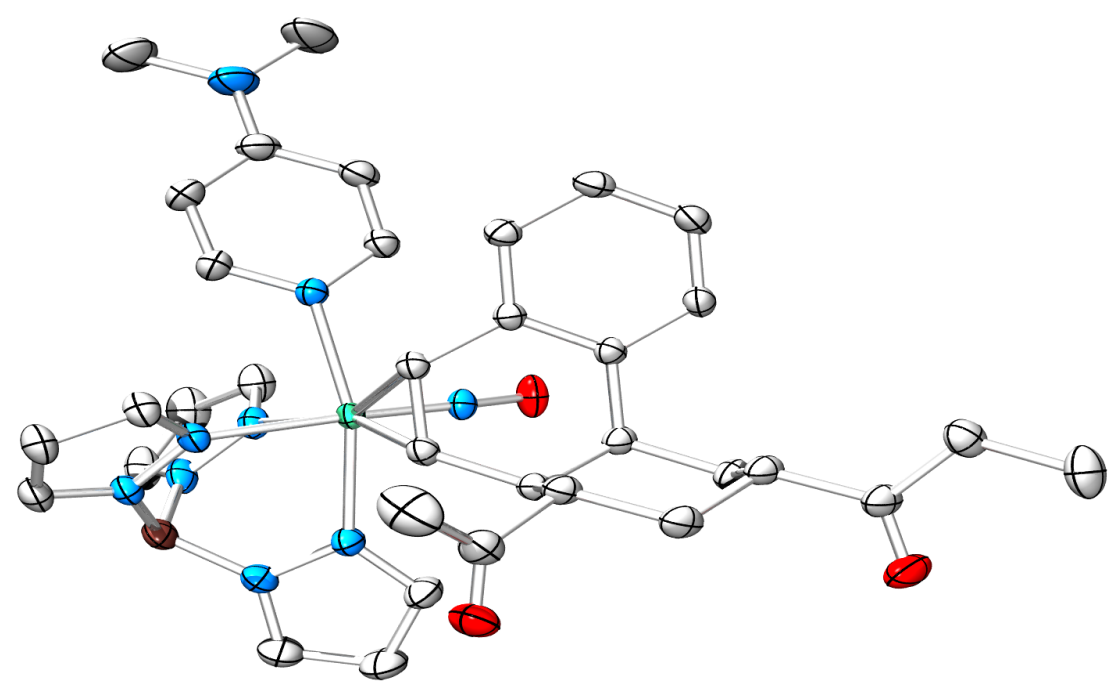

Figure 4.5. Crystal structure of 90. Bond lengths $(\AA)$ : Mo-C(9) 2.2291(19); Mo-C(10) 2.226(2); Mo-N(DMAP) 2.2230(18); Mo-N(NO) 1.7549(17).

Further attempts to increase the yield of $\mathbf{9 0}$ while retaining purity are underway; however, at this point the only yield confidently reported for $\mathbf{9 1}$ is one yielded from an oxidative decomplexation of the reaction mixture, which originates from a pure material, 24. By stirring the reaction mixture to make 90 exposed to air, 91 can be isolated from 24 in a yield of $12 \%$ (Scheme 4.8). In a similar manner, the isolation of $\mathbf{9 2}$, the product of an [EVK + cyclopentenone] MIMIRC addition to 24, has been achieved at 10\% from 24 (Scheme 4.8). 
Scheme 4.8. [A+B] MIMIRC additions to 24 .

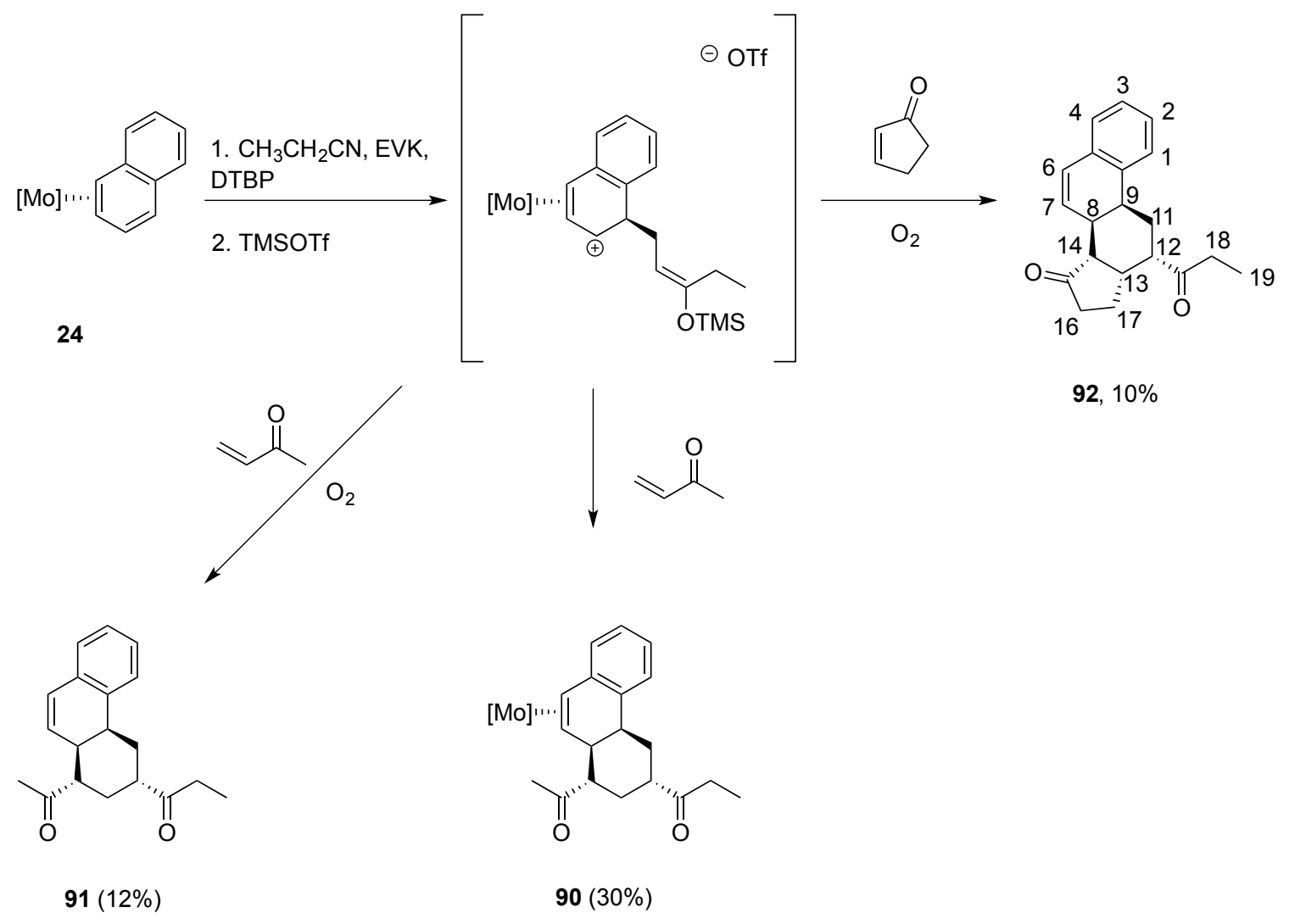

The ${ }^{1} \mathrm{H}$ NMR spectrum of 92 contains one major triplet, indicating one $[\mathrm{A}+\mathrm{B}]$ MIMIRC product, with $<10 \%$ impurity determined to be 86. Although the ${ }^{1} \mathrm{H}$ NMR spectrum of 92 is convoluted between 2.60 and $1.90 \mathrm{ppm}$, it is believed that the full characterization of this compound has been carefully completed including the relative stereochemistry of the five newly created stereocenters (Figure 4.6). Correlations observed in the ${ }^{1} \mathrm{H}-{ }^{13} \mathrm{C}$ HSQC spectrum of 92 show the presence of one primary carbon, four secondary carbons, and five tertiary carbons in the aliphatic region. From a COSY correlation with the alkene peak at 6.15 (dd, H7) ppm, H8 was identified as a multiplet (2.25 ppm). A notably similar peak seen in each of the ${ }^{1} \mathrm{H}$ NMR spectra of $\mathbf{6 1}$, and $\mathbf{8 7}$ - 
90 was a multiplet at $3.26 \mathrm{ppm}$. This peak was determined to represent H9 due to its coupling with H8 as well as with methylene protons at 2.45 and $2.17 \mathrm{ppm}$. These geminal protons show strong NOE interactions with $\mathrm{H} 2$, which confirm their identity as $\mathrm{H} 11$. Furthermore, $\mathrm{H} 11$ couples to a proton at $3.11 \mathrm{ppm}(\mathrm{dt}, J=13.0 \& 4.4 \mathrm{~Hz})$, which is bound to a tertiary carbon identifying this proton as H12. H12 couples to a proton at 2.57 ppm, which is bound to a tertiary carbon, thus 2.57 ppm was identified as corresponding to H13. H13 couples with geminal protons at 1.97 and $1.69 \mathrm{ppm}(\mathrm{H} 17)$ as well as with a proton at $2.06(\mathrm{dd}, J=12.2$ and $6.7 \mathrm{~Hz}) \mathrm{ppm}$ that is bound to a tertiary carbon (H14). As confirmation, it was observed in the NMR spectrum that H15 couples to H8.

Of the protons mentioned above, only two splitting patterns were able to be unambiguously identified - H14 and H12. Due to this lack of coupling information, only a few assumptions can be made about the stereochemical assignments of $\mathbf{9 2}$. Assuming a similar stereoselectivity determined by the metal center, protons $\mathrm{H} 8$ and $\mathrm{H} 9$ will be cis to one another, which is in agreement with their strong NOE interaction. Also assumed is that the enolizable carbons adjacent to $\mathrm{H} 14$ and $\mathrm{H} 12$ will isomerize to a thermodynamically preferred 1,3-diequatorial geometry, which is in agreement with the strong NOE interaction between H12 and H14. Using these assumptions, two models of 92 were built and optimized in Gaussian using a Semi-Empirical method. These two models were based on the two stereoisomers of H13, identified as cis or trans in relation to H12 and H14 (Figure 4.4). From this, the dihedral angles between H12 to H13 and H12 to H11 (labeled cis or trans in relation to H12) were identified and subsequently used to predict coupling constants based on the Karplus equation (Tables 4.2 and 4.3). ${ }^{15}$ 


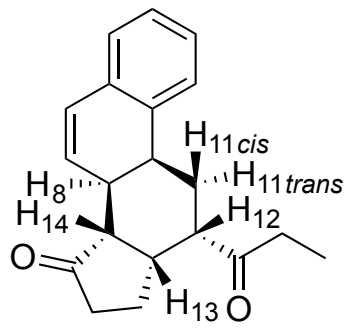

cis

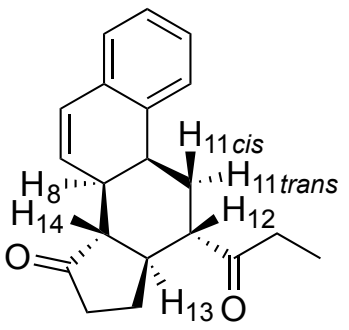

trans

Figure 4.6. Cis and trans orientations of $\mathrm{H} 13$ in 92.

Table 4.2. Predicted coupling constants where H13 is in a cis orientation to H14 and H12.

\begin{tabular}{|l|r|r|r|}
\hline \multicolumn{4}{|c|}{ H13 cis to H14 and H12 } \\
\hline Protons Considered & Dihedral Angle ( $\left.{ }^{\circ}\right)$ & Dihedral Angle (rad) & Coupling Constant (Hz) \\
\hline $\mathrm{H} 14 \mathrm{H} 8$ & 160 & 2.72 & 11.66 \\
\hline $\mathrm{H} 14 \mathrm{H} 13$ & 34 & 0.578 & 7.02 \\
\hline $\mathrm{H} 13 \mathrm{H} 12$ & 46 & 0.782 & 5.03 \\
\hline $\mathrm{H} 12 \mathrm{H} 11$ cis & 62 & 1.054 & 2.44 \\
\hline $\mathrm{H} 12 \mathrm{H} 11$ trans & 177 & 3.009 & 13.76 \\
\hline
\end{tabular}

Table 4.3. Predicted coupling constants where H13 is in a trans orientation to H14 and H12.

\begin{tabular}{|l|r|r|r|}
\hline \multicolumn{5}{|c|}{ H13 trans to H14 and H12 } \\
\hline Protons Considered & Dihedral Angle $\left(^{\circ}\right)$ & Dihedral Angle (rad) & Coupling Constant (Hz) \\
\hline $\mathrm{H} 14 \mathrm{H} 8$ & 179 & 3.043 & 13.86 \\
\hline $\mathrm{H} 14 \mathrm{H} 13$ & 171 & 2.907 & 13.24 \\
\hline $\mathrm{H} 13 \mathrm{H} 12$ & 176 & 2.992 & 13.69 \\
\hline $\mathrm{H} 12 \mathrm{H} 11$ cis & 61 & 1.037 & 2.59 \\
\hline $\mathrm{H} 12 \mathrm{H} 11$ trans & 176 & 2.992 & 13.69 \\
\hline
\end{tabular}

When $\mathrm{H} 13$ is cis to $\mathrm{H} 12$ and H14, the coupling constant between $\mathrm{H} 12$ and $\mathrm{H} 13$ is predicted to be $5.03 \mathrm{~Hz}$, which is similar to the coupling constant predicted between $\mathrm{H} 12$ 
and $\mathrm{H} 11$ cis $(2.44 \mathrm{~Hz})$. The coupling constant between $\mathrm{H} 12$ and H11trans is predicted to be $13.76 \mathrm{~Hz}$. With coupling between $\mathrm{H} 13$ and $\mathrm{H} 11, \mathrm{H} 12$ is expected to resemble a doublet-of-doublet-of-doublets; however, with similar coupling constants between H11 cis and H13, H12 would most likely be observed as a doublet-of-triplets. In contrast, were $\mathrm{H} 13$ in a trans orientation to $\mathrm{H} 12$, a triplet-of-doublets is predicted due to the similarities in coupling constants from $\mathrm{H} 13$ and H11trans $(13.69 \mathrm{~Hz})$. From this prediction, and the identification of the $\mathrm{H} 12$ resonance as a doublet-of-triplets at 3.11 ppm, it was determined that $\mathrm{H} 13$ orients cis to $\mathrm{H} 12$ and H14. To help confirm the accuracy of the predicted values, the only other resolved splitting pattern, H14 (dd, $J=$ 12.2 and $6.7 \mathrm{~Hz}$ ), was also compared. This comparison again shows the cis orientation to be in the best agreement with the predicted values. Future attempts will aim to isolate a crystal structure of $\mathbf{9 2}$ to confirm these stereochemical determinations.

The isolation of 92 demonstrates future potential for the utility of this method. From naphthalene, a steroidal core containing five new stereocenters and three new carbon-carbon bonds can be isolated (Figure 4.5). Although a majority of naturally occurring steroidal cores have a trans bridgehead between rings B and C (Figure 4.5), there have been recent investigations into the effect of inversion of stereochemistry at C8 of steroidal estrogens (93 and 94). ${ }^{16-20}$ These investigations showed 93 and 94 to have an affinity for estrogen receptors while also providing osteoprotective action, a significant benefit for their use in hormone replacement therapy. Although this inversion is not widely studied, the development of a library of $\mathrm{B} / \mathrm{C}$ cis fused ring junctures of steroidal cores could provide more detail into their use. Furthermore, compounds 61, 89, and 91 contain the same hexhydrophenanthrene core as substituted tetralins that have been 
studied for their efficacy as selective estrogen receptor-beta agonists. ${ }^{21}$ These hexahydrophenanthrenes also share a similar core as aromatic abietane diterpenoids (i.e., carnosic acid, ferruginol, and abietic acid). These abietane diterpenoids have been thoroughly studied for their antimicrobial and antibacterial properties. ${ }^{22,23}$

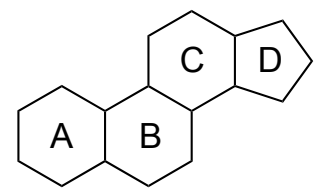

Steroidal Core<smiles>CCC(=O)[C@H]1CC2c3ccccc3C=CC2[C@@H]2C(=O)CC[C@@H]12</smiles>

92

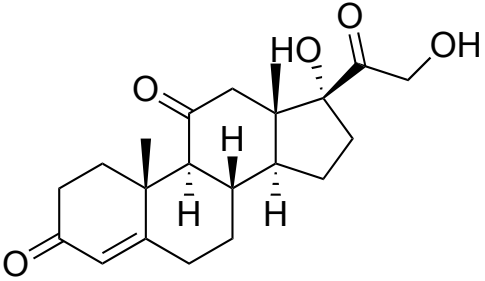

Cortisone<smiles>COc1cc(C)c2c(c1)CC[C@@H]1[C@H]2CC[C@]2(O)[C@@H](C(C)=O)CC[C@H]12</smiles>

93<smiles>C[C@]12CC[C@H]3c4ccc(O)cc4CC[C@H]3[C@@H]1CC[C@@H]2O</smiles>

Estradiol<smiles>COc1cc(C)c2c(c1)CC[C@@H]1C2CC[C@]2(C)C(=O)CC[C@H]12</smiles>

94

Figure 4.7. Examples of steroidal cores found in the literature.

To our knowledge, only one other example of the synthesis of a steroidal core from naphthalene has been shown: the report by Mathias Berndt et al. using samarium diiodide to close a pendant cyclopentanone (Scheme 4.8). Furthermore, Berndt demonstrated the synthetic potential of this methodology by subjecting 96 to additions like catalytic hydrogenation (97), oxidation (98) with $\mathrm{MnO}_{2}$, as well as epoxidation with mCPBA (99). ${ }^{24,25}$ It is presumed that a similar reactivity with 92 would be seen, which would lead to further derivatization. 
Scheme 4.8. Samarium diiodide-induced cyclization of naphthyl-substituted ketone (95).

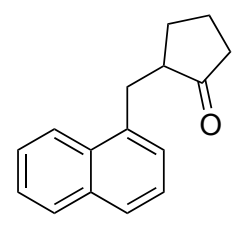

95

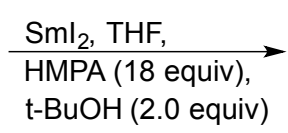

t-BuOH (2.0 equiv)

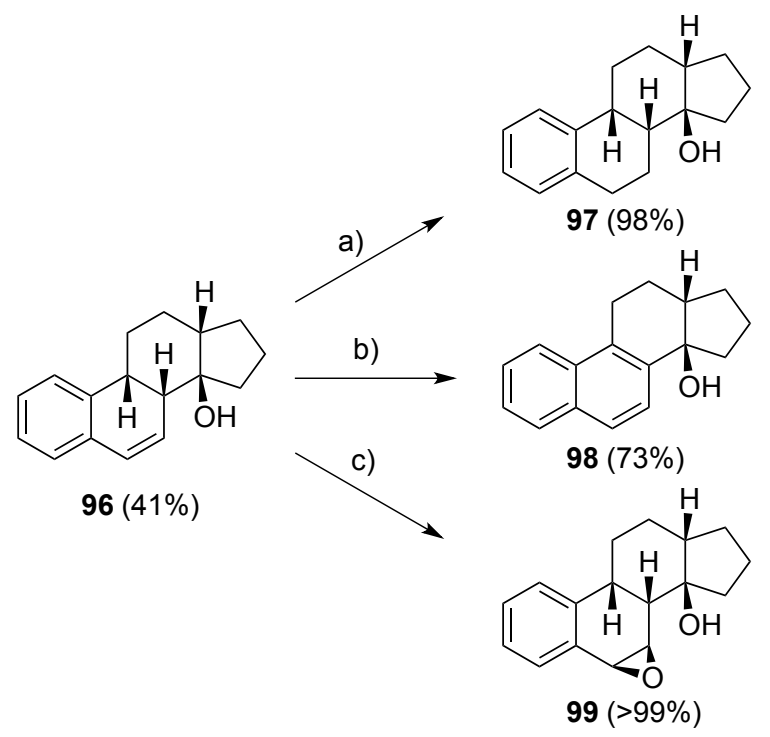

Conditions: (a) Pd/C, $\mathrm{H}_{2}, \mathrm{MeOH}$, rt; (b) $\mathrm{MNO}_{2}$, THF, D; (c) $m$-CPBA, $\mathrm{MeOH}, 0{ }^{\circ} \mathrm{C}$

The optimization of MIMIRC additions to $\mathbf{2 4}$ has provided good insight into the reaction conditions needed for their synthesis. As mentioned previously, a significant amount of free naphthalene is obtained during the isolation of $\mathbf{6 1 , 8 9 , 9 1}$, and 92 from their respective reaction mixtures. CV monitoring of these reactions often shows a $1: 1$ ratio of the desired complex $(\sim+200 \mathrm{mV})$ and $\mathbf{2 4}(\sim-160 \mathrm{mV})$. In circumstances where the ratio is in favor of the desired complex in comparison to $\mathbf{2 4}$, an increase in decomposition is seen $\left(E_{\mathrm{p}, \mathrm{c}} \sim-900 \mathrm{mV}\right)$. The primary cause of this is believed to be due to a competition between the protonation of $\mathbf{2 4}$ and the electrophilic addition of EVK to $\mathbf{2 4}$ (Scheme 4.9). Once 24 is protonated, the allyl 27 (see Chapter 3) remains unreactive until TEA is added to stop the reaction, which deprotonates 27 back to 24 . 
Scheme 4.9. Competitive pathways encountered during the synthesis of $\mathbf{8 7}$.<smiles>Cc1ccc2ccccc2c1</smiles>

24

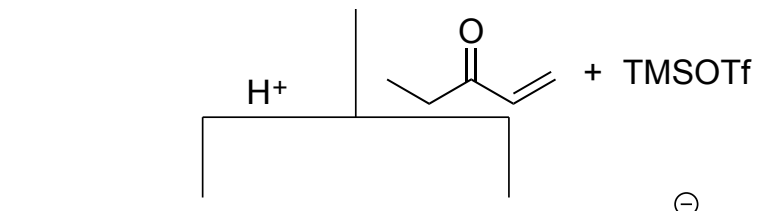

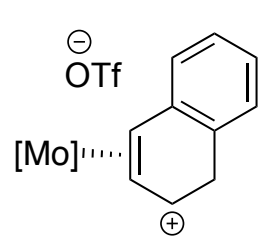

27<smiles>Cc1cccc2ccccc12</smiles>

24

$$
[\mathrm{Mo}]=\{\mathrm{TpMo}(\mathrm{NO})(\mathrm{DMAP})\}
$$

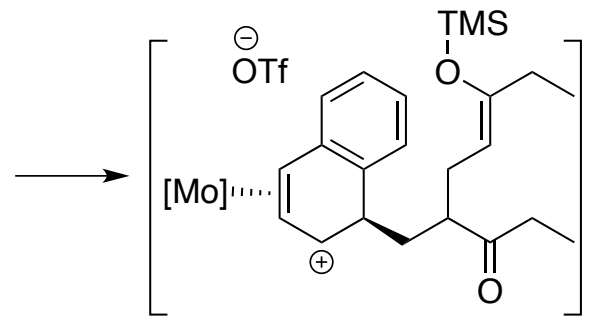<smiles>[3H][In]</smiles><smiles>CCC(=O)[C@H]1C[C@@H]2c3ccccc3C=C[C@@H]2[C@H](C(=O)CC)C1</smiles>

87

The conditions described in entry 12 of Table 4.1 were varied in attempts to increase the reaction progress; however, no further improvements have been made to date. These attempts include increasing the temperature, equivalents of TMSOTf and DTBP, and strength of base present in the reaction mixture. Although these attempts have not improved the reaction progress, a significant increase in decomposition $\left(E_{\mathrm{p}, \mathrm{c}} \sim-900\right.$ $\mathrm{mV}$ ) is seen. Efforts to purify all reagents through distillation and storage over molecular sieves do not appear to prevent the purported protonation of $\mathbf{2 4}$. 
Also desired is a better control during the $[\mathrm{A}+\mathrm{B}]$ MIMIRC additions. For instance, in the $[\mathrm{EVK}+\mathrm{MVK}]$ addition to $\mathbf{2 4}$, limiting the equivalents of EVK did not provide full control as $\mathbf{6 1}$ and $\mathbf{8 9}$ were also obtained in these reactions. While the optimization of this reaction is underway, future attempts to use substituted naphthalenes, vinyl ketones, and cyclopentenones could provide a simple one-pot method for synthesizing various steroidal cores with improved stereocontrol.

\subsection{Conclusion}

With these examples of MIMIRC additions to 24, several key features should be noted. First, from a fully aromatic core (naphthalene), organic products with no less than four new stereocenters and three new carbon-carbon bonds have been isolated and characterized. Second, the isolation of complexes from [A+A] MIMIRC reactions has been demonstrated with EVK and MVK, giving promise for optimization of the additional MIMIRC reactions described above. Finally, the regenerative oxidative decomplexation of these complexes with iodine to liberate the functionalized organic ligands, while also recovering a metal precursor complex, has been accomplished. 


\subsection{Experimental}

\section{General Methods}

NMR spectra were obtained on a 600 or $800 \mathrm{MHz}$ spectrometer. All chemical shifts are reported in ppm, and proton and carbon shifts are referenced to tetramethylsilane (TMS) utilizing residual ${ }^{1} \mathrm{H}$ or ${ }^{13} \mathrm{C}$ signals of the deuterated solvents as an internal standard. Coupling constants $(J)$ are reported in hertz $(\mathrm{Hz})$. Infrared spectra (IR) were recorded as a glaze on a spectrometer fitted with a horizontal attenuated total reflectance (HATR) accessory or on a diamond anvil ATR assembly. Electrochemical experiments were performed under a dinitrogen atmosphere. Cyclic voltammetry data were taken at ambient temperature $\left(\sim 25{ }^{\circ} \mathrm{C}\right)$ at $100 \mathrm{mV} / \mathrm{s}$ in a standard three electrode cell with a glassy-carbon working electrode, N,N-dimethylacetamide (DMAc) or acetonitrile $\left(\mathrm{CH}_{3} \mathrm{CN}\right)$ solvent (unless otherwise specified), and tetrabutylammonium hexafluorophosphate (TBAH) electrolyte (approximately $0.5 \mathrm{M})$. All potentials are reported versus NHE (normal hydrogen electrode) using cobaltocenium hexafluorophosphate $\left(E_{1 / 2}=-0.78 \mathrm{~V}\right)$, ferrocene $\left(E_{1 / 2}=+0.55 \mathrm{~V}\right)$, or decamethylferrocene $\left(E_{1 / 2}=+0.04 \mathrm{~V}\right)$ as an internal standard. The peak-to-peak separation was less than $100 \mathrm{mV}$ for all reversible couples. Unless otherwise noted, all synthetic reactions were performed in a glovebox under a dry nitrogen atmosphere. Deuterated solvents were used as received. Pyrazole $(\mathrm{Pz})$ protons of the hydridotris(pyrazolyl)borate (Tp) ligand were uniquely assigned (e.g., "Pz3B") using a combination of two-dimensional NMR data and 4-(dimethylamino)pyridine-proton NOE interactions (See Figure 3.4). When unambiguous assignments were not possible, Tp protons were labeled as "Pz3/5 or Pz4". BH peaks (around 4-5 ppm) are not identified 
due to their quadrupole broadening; IR data are used to confirm the presence of a $\mathrm{BH}$ group (around $2500 \mathrm{~cm}^{-1}$ ). All $J$ values for Pz protons are $2( \pm 0.2) \mathrm{Hz}$.

\section{Synthesis of $1,1^{\prime}-((1 S, 3 S, 4 \mathrm{a} S, 10 \mathrm{a} S)-1,2,3,4,4 \mathrm{a}, 10 \mathrm{a}-h e x a h y d r o p h e n a n t h r e n e-1,3-$ diyl)bis(propan-1-one) (61).}

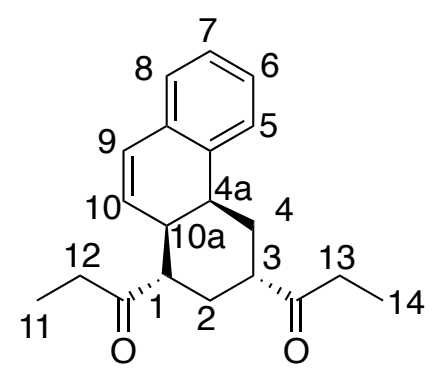

24 (200 mg, $0.34 \mathrm{mmol}), \mathrm{CH}_{3} \mathrm{CH}_{2} \mathrm{CN}$ (6 mL), EVK (0.1 mL, $\left.1.0 \mathrm{mmol}\right)$, and DTBP (54 $\mathrm{mg}, 0.3 \mathrm{mmol}$ ) were added to a test tube. The reaction mixture was then cooled at $-60{ }^{\circ} \mathrm{C}$ for $15 \mathrm{~min}$. TMSOTf $(0.1 \mathrm{~mL}, 0.5 \mathrm{mmol})$ was added to the reaction mixture, and the resulting red solution was left stirring at $-60{ }^{\circ} \mathrm{C}$ overnight $(\sim 18 \mathrm{~h})$. TEA $(0.1 \mathrm{~mL}, 0.74$ mmol) was added to the reaction mixture, which was subsequently removed from the glovebox and stirred open to air overnight $(\sim 18 \mathrm{~h})$. The resulting brown mixture was evaporated in vacuo, and the resulting film was dissolved in DCM (1 mL). This solution was then added stirring $\mathrm{Et}_{2} \mathrm{O}(50 \mathrm{~mL})$, yielding a brown precipitation. The brown precipitate was filtered off on a $15 \mathrm{~mL}$ medium porosity fritted disc and washed with $\mathrm{Et}_{2} \mathrm{O}(3 \times 5 \mathrm{~mL})$. The filtrate was then evaporated in vacuo. This oil was then dissolved in $\operatorname{DCM}(3 \times 0.3 \mathrm{~mL})$ and the resulting solution was dropwise added onto a $250 \mu \mathrm{m}$ silica preparatory plate. The product was eluted with 10\% EtOAc:hexanes $(200 \mathrm{~mL})$, scraped off as a band at $\mathrm{R}_{\mathrm{f}}$ : $0.30-0.40$ and this silica gel was sonicated in EtOAc $(20 \mathrm{~mL})$ for 15 
min. The silica was filtered off on a $15 \mathrm{~mL}$ fine porosity fritted disc and washed with DCM (3 x $5 \mathrm{~mL})$. The filtrate was evaporated in vacuo and desiccated for 15 min to yield the colorless oil $61(23 \mathrm{mg}, 23 \%$ yield $)$. IR: $v\left(\mathrm{C}-\mathrm{H} \mathrm{sp}^{2}\right)=2933 \mathrm{~cm}^{-1}, v(\mathrm{CO})=1702 \mathrm{~cm}^{-1}$. ${ }^{1} \mathrm{H} \mathrm{NMR}\left(\mathrm{CDCl}_{3}, \delta\right): 7.28(1 \mathrm{H}, \mathrm{d}, J=7.3, \mathrm{H} 5), 7.26(1 \mathrm{H}, \mathrm{t}, J=7.0, \mathrm{H} 6 / 7), 7.21(1 \mathrm{H}, \mathrm{t}, J$ $=7.0, \mathrm{H} 6 / 7), 7.10(1 \mathrm{H}, \mathrm{d}, J=7.3, \mathrm{H} 8), 6.45(1 \mathrm{H}, \mathrm{d}, J=9.6, \mathrm{H} 9), 6.01(1 \mathrm{H}, \mathrm{dd}, J=9.6 \&$ 6.20, H10), $3.34(1 \mathrm{H}, \mathrm{bs}, \mathrm{H} 4 \mathrm{a}), 2.70(2 \mathrm{H}, \mathrm{m}, \mathrm{H} 3 \mathrm{\&} \mathrm{H} 4), 2.57(1 \mathrm{H}, \mathrm{m}, \mathrm{H} 10 \mathrm{a}), 2.55(2 \mathrm{H}$, m, H13), 2.42 (2H, m, H1 \& H12), 2.27 (1H, dq, $J=18.0 \&$ 7.3, H12), 1.85 (1H, m, H2), $1.80(1 \mathrm{H}, \mathrm{m}, \mathrm{H} 4), 1.43(1 \mathrm{H}, \mathrm{m}, \mathrm{H} 2), 1.09$ (3H, t, $J=7.4, \mathrm{H} 14), 0.98$ (3H, t, $J=7.2, \mathrm{H} 11)$.

${ }^{13} \mathrm{C} \mathrm{NMR}\left(\mathrm{CDCl}_{3}, \delta\right): 213.4(\mathrm{CO}), 213.2(\mathrm{CO}), 135.4(\mathrm{C} 8 \mathrm{a} / 4 \mathrm{~b}), 134.2(\mathrm{C} 8 \mathrm{a} / 4 \mathrm{~b}), 131.2$ (C10), 128.2 (C9), 127.9 (C6/7), 127.1 (C8), 126.7 (C6/7), 124.8 (C4), 49.3 (C1), 44.4 (C3), 37.2 (C10a), 36.5 (C12), 35.9 (C4a), 34.0 (C13), 30.9 (C2), 27.8 (C4), 7.9 (C11/14), 7.6 (C11/14). EA: Calculated for $\mathrm{C}_{20} \mathrm{H}_{24} \mathrm{O}_{2} \cdot 0.25 \mathrm{CH}_{2} \mathrm{Cl}_{2}: \mathrm{C}, 76.57$; H, 7.77. Found: C, 76.50; H, 7.88. 


\section{Synthesis of dimethyl 2-((1S,2S)-1-(3-oxopentyl)-1,2-dihydronaphthalen-2-} yl)malonate (72).

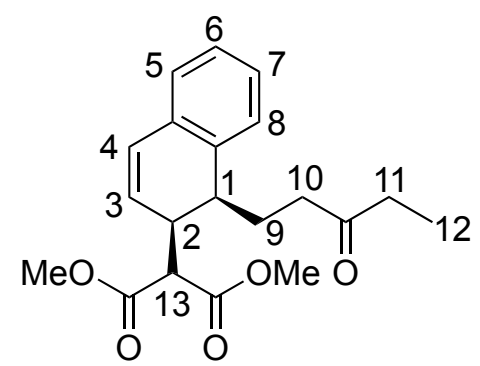

24 (100 mg, $0.17 \mathrm{mmol}), \mathrm{CH}_{3} \mathrm{CH}_{2} \mathrm{CN}(3 \mathrm{~mL})$, and $\mathrm{EVK}(0.1 \mathrm{~mL}, 1.0 \mathrm{mmol})$ were added to a test tube. The reaction mixture was then cooled at $-50{ }^{\circ} \mathrm{C}$ for $15 \min$. A $-50{ }^{\circ} \mathrm{C} 1 \mathrm{M}$ solution of HOTf in $\mathrm{CH}_{3} \mathrm{CH}_{2} \mathrm{CN}(0.4 \mathrm{~mL}, 0.4 \mathrm{mmol})$ was added to the reaction mixture, and the resulting red solution was left stirring at $-50{ }^{\circ} \mathrm{C}$ overnight $(\sim 18 \mathrm{~h})$. LiDMM (248 $\mathrm{mg}, 1.8 \mathrm{mmol}$ ) was added to the reaction mixture, which was then left at $-50{ }^{\circ} \mathrm{C}$ for 30 min. The resulting brown solution was evaporated to an oil that was then dissolved in $\mathrm{CHCl}_{3}(10 \mathrm{~mL})$. This solution was then washed with $\mathrm{NaHCO}_{3}$ (sat. aq., $10 \mathrm{~mL}$ ), dried with $\mathrm{MgSO}_{4}$, and filtered through a $15 \mathrm{~mL}$ medium porosity fritted disc. The filtrate was evaporated in vacuo to an oil that was then dissolved in DCM (1 mL) and added to stirring hexanes $(50 \mathrm{~mL})$. The resulting precipitate was collected on a $15 \mathrm{~mL}$ fine porosity fritted disc, washed with hexanes $(3 \times 15 \mathrm{~mL})$, and taken outside of the glovebox. The precipitate was dissolved in DCM $(10 \mathrm{~mL})$ and this solution was stirred open to air overnight. The resulting oil was dissolved in DCM ( $3 \times 0.3 \mathrm{~mL})$ and this solution was dropwise added onto a $250 \mu \mathrm{m}$ silica preparatory plate. The product was eluted with 10\% EtOAc:hexanes $(200 \mathrm{~mL})$, scraped off as a band at $\mathrm{R}_{\mathrm{f}}:$ 0.3-0.4 and this silica gel was sonicated in EtOAc $(20 \mathrm{~mL})$ for $15 \mathrm{~min}$. The silica was filtered off on a 15 $\mathrm{mL}$ medium porosity fritted disc and washed with DCM $(3 \times 2 \mathrm{~mL})$. The filtrate was 
evaporated in vacuo and desiccated to yield the colorless oil 72 (3 mg, 5\% yield). IR: $v\left(\mathrm{C}-\mathrm{H} \mathrm{sp}^{2}\right)=2974 \mathrm{~cm}^{-1}, v(\mathrm{CO})=1755,1736$, and $1712 \mathrm{~cm}^{-1} .{ }^{1} \mathrm{H}$ NMR $\left(\mathrm{CDCl}_{3}, \delta\right): 7.21$ $(1 \mathrm{H}, \mathrm{t}, J=7.7, \mathrm{H} 6 / 7), 7.16(1 \mathrm{H}, \mathrm{t}, J=7.7, \mathrm{H} 6 / 7), 7.07(1 \mathrm{H}, \mathrm{d}, J=7.4, \mathrm{H} 5), 6.98(1 \mathrm{H}, \mathrm{d}, J$ $=7.2, \mathrm{H} 8), 6.48(1 \mathrm{H}, \mathrm{dd}, J=10.0,2.7, \mathrm{H} 4), 5.64(1 \mathrm{H}, \mathrm{d}, J=8.3, \mathrm{H} 3), 3.85(3 \mathrm{H}, \mathrm{s}, \mathrm{OMe})$, $3.79(1 \mathrm{H}, \mathrm{d}, J=11.6, \mathrm{H} 13), 3.76(3 \mathrm{H}, \mathrm{s}, \mathrm{OMe}), 3.47(1 \mathrm{H}, \mathrm{m}, \mathrm{H} 2), 2.72(1 \mathrm{H}, \mathrm{m}, \mathrm{H} 1), 2.30$ (2H, q, $J=7.9, \mathrm{H} 11), 2.17$ (2H, m, H10), 1.75 (2H, m, H9), 0.98 (1H, t, $J=7.9, \mathrm{H} 12)$. ${ }^{13} \mathrm{C}$ NMR $\left(\mathrm{CDCl}_{3}, \delta\right): 210.9,168.9,168.6,137.5,132.9,128.5,127.8,127.2,126.8$, $126.5,52.8,52.7,52.5,52.3,39.8,39.1,35.9,20.6,7.8$.

Synthesis of TpMo(NO)(DMAP) $\left(\eta^{2}-1,1^{\prime}-((1 S, 3 S, 4 a S, 10 a S)-1,2,3,4,4 a, 10 a-\right.$ hexahydrophenanthrene-1,3-diyl)bis(propan-1-one)) (87).

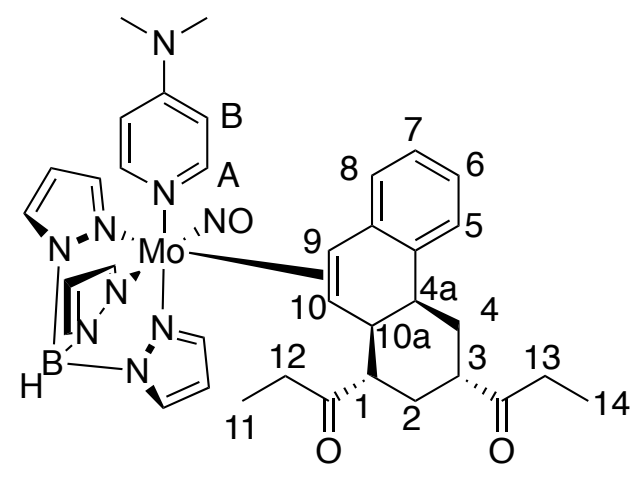

24 (200 mg, $0.34 \mathrm{mmol}), \mathrm{CH}_{3} \mathrm{CH}_{2} \mathrm{CN}$ (6 mL), EVK (84 mg, $1.0 \mathrm{mmol}$ ), and DTBP (54 $\mathrm{mg}, 0.3 \mathrm{mmol}$ ) were added to a test tube. The reaction mixture was then cooled at $-60{ }^{\circ} \mathrm{C}$ for $15 \mathrm{~min}$. TMSOTf $(0.1 \mathrm{~mL}, 0.5 \mathrm{mmol})$ was added to the reaction mixture, and the resulting red solution was left stirring at $-60{ }^{\circ} \mathrm{C}$ overnight $(\sim 18 \mathrm{~h})$. TEA $(0.1 \mathrm{~mL}, 0.74$ mmol) was added to the reaction mixture, which was subsequently warmed to room temperature. This solution was then chromatographed through a $30 \mathrm{~mL}$ fine porosity 
fritted disc $3 / 4$ full with silica gel. The product was eluted with $1: 1 \mathrm{Et}_{2} \mathrm{O}: \mathrm{THF}(50 \mathrm{~mL})$ as a yellow band, collected as a yellow solution, and evaporated in vacuo. The resulting oil was dissolved in DCM $(1 \mathrm{~mL})$ and the product was precipitated in stirring pentane (50 $\mathrm{mL}$ ). The precipitate was collected on a $15 \mathrm{~mL}$ fine porosity fritted disc, washed with pentane $(3 \times 10 \mathrm{~mL})$, and dried for 15 min yielding the yellow solid 87 (115 mg, 45\%). $\mathrm{CV}(\mathrm{DMAc}) E_{\mathrm{p}, \mathrm{a}}=+0.16 \mathrm{~V}(\mathrm{NHE}) . \mathrm{IR}: v\left(\mathrm{C}-\mathrm{H} \mathrm{sp}{ }^{2}\right)=2932 \mathrm{~cm}^{-1}, v(\mathrm{~B}-\mathrm{H})=2480 \mathrm{~cm}^{-1}$, $v(\mathrm{CO})=1703$ and $1620 \mathrm{~cm}^{-1}, v(\mathrm{NO})=1562 \mathrm{~cm}^{-1} .{ }^{1} \mathrm{H}$ NMR $\left(\mathrm{d}^{6}\right.$-Acetone, $\left.\delta\right): 7.93(1 \mathrm{H}, \mathrm{d}$ Pz5A), 7.87 (1H, d, Pz3A), 7.79 (1H, d, Pz5B), 7.69 (1H, d, Pz3C), 7.41 (2H, bs, DMAPA), $7.35(1 \mathrm{H}, \mathrm{d}, J=7.9, \mathrm{H} 5), 6.98(1 \mathrm{H}, \mathrm{d}, \mathrm{Pz3B}), 6.93(1 \mathrm{H}, \mathrm{t}, J=7.5, \mathrm{H} 6), 6.86(1 \mathrm{H}, \mathrm{t}, J$ $=7.5, \mathrm{H} 7), 6.51(2 \mathrm{H}, \mathrm{m}, \mathrm{DMAP}-\mathrm{B}), 6.37$ (1H, t, Pz4A), 6.35 (1H, t, Pz4C), $6.21(1 \mathrm{H}, \mathrm{d}, J$ = 7.2, H8), 6.09 (1H, t, Pz4B), $3.73(1 \mathrm{H}, \mathrm{m}, \mathrm{H} 4 \mathrm{a}), 3.31(1 \mathrm{H}, \mathrm{d}, J=9.0, \mathrm{H} 9), 3.06(6 \mathrm{H}, \mathrm{s}$, NMe), 2.84 (1H, m, H4), 2.77 (1H, m, H10a), 2.65 (3H, m, H3, H1, \& H13/12), 2.55 (1H, m, H13/H12), 2.40 (1H, m, H13/H12), 2.30 (1H, m, H13/12), 2.07 (1H, dd, $J=9.0$ \& 2.6, H10), $1.87(1 \mathrm{H}, \mathrm{m}, \mathrm{H} 4), 1.70(1 \mathrm{H}, \mathrm{m}, \mathrm{H} 2), 1.52(1 \mathrm{H}, \mathrm{q}, J=12.4, \mathrm{H} 2), 1.00(3 \mathrm{H}, \mathrm{t}$, $J=7.3, \mathrm{H} 11 / \mathrm{H} 14), 0.68(3 \mathrm{H}, \mathrm{t}, J=7.2, \mathrm{H} 11 / 14) .{ }^{13} \mathrm{C}$ NMR $\left(\mathrm{d}^{6}\right.$-Acetone, $\left.\delta\right): 215.1(\mathrm{CO})$, 213.8 (CO), 155.1 (DMAP-C), 150.7 (DMAP-A), 144.7 (Pz5), 143.6 (Pz3A), 142.1 (Pz3B), 141.2 (Pz3C), 137.6 (Pz5), 136.9 (Pz5), 135.8, 134.1, 128.1 (C8), 125.6 (C5), 125.1 (C7), 123.9 (C6), 108.3 (2C, DMAP-B), 106.9 (Pz4A/C), 106.8 (Pz4A/C), 106.5 (Pz4B), 70.9 (C10), 68.1 (C9), 55.0, 45.3, 43.1 (C10a), 39.3, 37.5, 35.9 (C4a), 34.3, 32.6 (C2), 31.0 (C4), 8.2 (C11/14), 7.8 (C11/14). 
Synthesis of TpMo(NO)(DMAP) $\left(\eta^{2}-1,1^{\prime}-((1 S, 3 S, 4 a S, 10 a S)-1,2,3,4,4 a, 10 a-\right.$ hexahydrophenanthrene-1,3-diyl)bis(ethan-1-one)) (88).

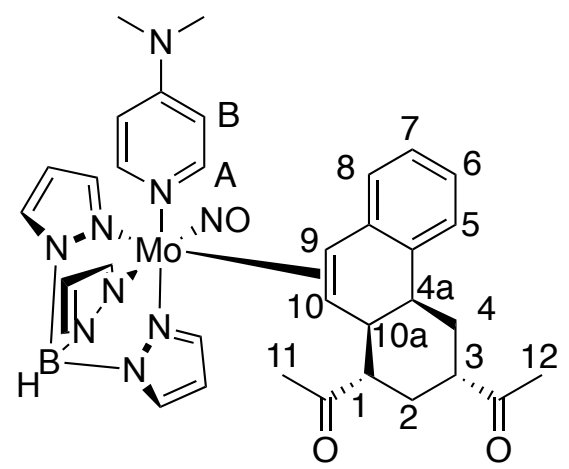

24 (200 mg, $0.34 \mathrm{mmol}), \mathrm{CH}_{3} \mathrm{CH}_{2} \mathrm{CN}$ (6 mL), MVK (0.1 mL, $\left.1.0 \mathrm{mmol}\right)$, and DTBP (54 $\mathrm{mg}, 0.3 \mathrm{mmol}$ ) were added to a test tube. The reaction mixture was then cooled at $-60{ }^{\circ} \mathrm{C}$ for $15 \mathrm{~min}$. TMSOTf $(0.1 \mathrm{~mL}, 0.5 \mathrm{mmol})$ was added to the reaction mixture, and the resulting red solution was left stirring at $-60{ }^{\circ} \mathrm{C}$ overnight $(\sim 18 \mathrm{~h})$. TEA $(0.1 \mathrm{~mL}, 0.74$ mmol) was added to the reaction mixture, which was subsequently warmed to room temperature. This solution was then chromatographed through a $30 \mathrm{~mL}$ fine porosity fritted disc $3 / 4$ full with silica gel. The product was eluted with $1: 1 \mathrm{Et}_{2} \mathrm{O}$ :THF $(50 \mathrm{~mL})$ as a yellow band, collected as a yellow solution, and evaporated in vacuo. The resulting oil was dissolved in DCM $(1 \mathrm{~mL})$ and the product was precipitated in stirring pentane $(50$ $\mathrm{mL}$ ). The precipitate was collected on a $15 \mathrm{~mL}$ fine porosity fritted disc, washed with pentane $(3 \times 10 \mathrm{~mL})$, and dried for $15 \mathrm{~min}$ yielding the yellow solid $\mathbf{8 8}$ (25 mg, 10\%). CV (DMAc) $E_{\mathrm{p}, \mathrm{a}}=+0.17 \mathrm{~V}(\mathrm{NHE}) . \mathrm{IR}: v\left(\mathrm{C}-\mathrm{H} \mathrm{sp}^{2}\right)=2920 \mathrm{~cm}^{-1}, v(\mathrm{~B}-\mathrm{H})=2478 \mathrm{~cm}^{-1}, v(\mathrm{CO})$ $=1698$ and $1619 \mathrm{~cm}^{-1}, v(\mathrm{NO})=1562 \mathrm{~cm}^{-1} \cdot{ }^{1} \mathrm{H}$ NMR $\left(\mathrm{d}^{6}\right.$-Acetone, $\left.\delta\right): 7.94(1 \mathrm{H}, \mathrm{d}$, Pz5A/C), 7.91 (1H, d, Pz3A), 7.88 (1H, d, Pz5A/C), 7.78 (1H, d, Pz5B), 7.72 (1H, d Pz3C), 7.37 (2H, bs, DMAP-A), 7.34 (1H, d, $J=7.5, \mathrm{H} 5), 6.98$ (1H, d, Pz3B), 6.94 (1H, $\mathrm{td}, J=7.5 \& 1.5, \mathrm{H} 6), 6.86(1 \mathrm{H}, \mathrm{t}, J=7.4, \mathrm{H} 7), 6.50(2 \mathrm{H}, \mathrm{m}, \mathrm{DMAP}-\mathrm{B}), 6.36(2 \mathrm{H}, \mathrm{m}$, 
Pz4A\&C), 6.24 (1H, dd, $J=7.6 \&$ 1.0, H8), 6.09 (1H, t, Pz4B), 3.79 (1H, m, H4a), 3.72 $(1 \mathrm{H}, \mathrm{d}, J=9.5, \mathrm{H} 9), 3.05(6 \mathrm{H}, \mathrm{s}, \mathrm{NMe}), 2.87(1 \mathrm{H}, \mathrm{m}, \mathrm{H} 4), 2.79(1 \mathrm{H}, \mathrm{m}, \mathrm{H} 10 \mathrm{a}), 2.59$ (2H, m, H3 \& H1), 2.19 (3H, s, H13), 2.14 (1H, dd, $J=9.5 \& 2.5, \mathrm{H} 10), 1.89$ (3H, s, H12), $1.86(1 \mathrm{H}, \mathrm{m}, \mathrm{H} 4), 1.77(1 \mathrm{H}, \mathrm{m}, \mathrm{H} 2), 1.52(1 \mathrm{H}, \mathrm{q}, J=12.6, \mathrm{H} 2) .{ }^{13} \mathrm{C}$ NMR $\left(\mathrm{d}^{6}\right.$-Acetone, ঠ): 212.5 (CO), 211.0 (CO), 154.9 (DMAP-C), 151.1 (2C, DMAP-A), 144.6 (C4b/9a), 143.5 (Pz3A), 141.9 (Pz3B), 141.3 (Pz3C), 137.5 (Pz5A/C), 136.8 (Pz5A/C), 135.7 (Pz5B), 133.8 (C4b/9a), 128.0 (C8), 125.4 (C5), 124.9 (C7), 123.8 (C6), 108.1 (2C, DMAP-B), 106.9 (Pz4A/C), 106.6 (Pz4A/C), 106.3 (Pz4B), 70.4 (C10), 68.0 (C9), 55.9 (C1/3), 45.9 (C1/3), 42.7 (C10a), 39.1 (2C, DMAP-Me), 35.5 (C4a), 32.2 (C2), 30.7 (C4), 30.2 (C11), 28.1 (C12).

\section{Synthesis of $1,1^{\prime}-((1 S, 3 S, 4 a S, 10 a S)-1,2,3,4,4 a, 10 a-h e x a h y d r o p h e n a n t h r e n e-1,3-$} diyl)bis(ethan-1-one) (89).

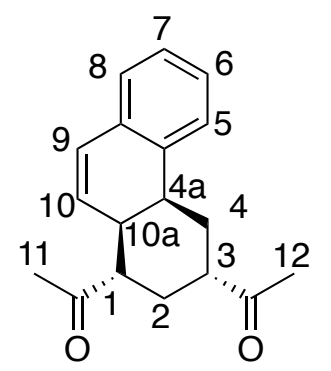

To a $50 \mathrm{~mL}$ filter flask charged with a stir pea were added $24(50 \mathrm{mg}, 0.07 \mathrm{mmol}), \mathrm{DCM}$ $(5 \mathrm{~mL})$, and a $0.06 \mathrm{M}$ solution of $\mathrm{I}_{2} / \mathrm{Et}_{2} \mathrm{O}(0.6 \mathrm{~mL}, 0.035 \mathrm{mmol})$, resulting in a green solution. This solution was stirred at RT for $5 \mathrm{~min}$ and evaporated in vacuo. The resulting green oil was dissolved in DCM (1 mL) forming a green solution. This solution was added to stirring hexanes $(50 \mathrm{~mL})$ giving a green precipitation. The green precipitate was collected on a $15 \mathrm{~mL}$ fine porosity fritted disc, washed with hexanes ( $3 \times 10 \mathrm{~mL})$, and 
desiccated to yield 3 (35 mg, 85\%). The filtrate was removed from the glovebox and evaporated in vacuo to an oil. The oil was dissolved in DCM ( $3 \times 0.3 \mathrm{~mL})$ and the resulting solution was dropwise added onto a $250 \mu \mathrm{m}$ silica preparatory plate. The product was eluted with $10 \%$ EtOAc:hexanes $(200 \mathrm{~mL})$, scraped off as a band at $\mathrm{R}_{\mathrm{f}}$ : 0.27 0.4 and this silica gel was sonicated in EtOAc $(20 \mathrm{~mL})$ for $15 \mathrm{~min}$. The silica was filtered off on a $15 \mathrm{~mL}$ medium porosity fritted disc and washed with DCM $(3 \times 2 \mathrm{~mL})$. The filtrate was evaporated in vacuo and desiccated to yield the colorless oil 89 (3 mg, 16\% yield). IR: $v\left(\mathrm{C}-\mathrm{H} \mathrm{sp}^{2}\right)=2921 \mathrm{~cm}^{-1} v(\mathrm{CO})=1701 \mathrm{~cm}^{-1} .{ }^{1} \mathrm{H} \mathrm{NMR}\left(\mathrm{CDCl}_{3}, \delta\right): 7.28(1 \mathrm{H}, \mathrm{d}$, $J=7.3, \mathrm{H} 5), 7.27(1 \mathrm{H}, \mathrm{m}, \mathrm{H} 6 / 7), 7.22(1 \mathrm{H}, \mathrm{m}, \mathrm{H} 6 / 7), 7.10(1 \mathrm{H}, \mathrm{d}, J=7.3, \mathrm{H} 8), 6.46(1 \mathrm{H}$, d, $J=9.6, \mathrm{H} 9), 1 \mathrm{H}, \mathrm{dd}, J=9.6 \& 6.08, \mathrm{H} 10), 3.35(1 \mathrm{H}, \mathrm{m}, \mathrm{H} 4 \mathrm{a}), 2.73(1 \mathrm{H}, \mathrm{m}, \mathrm{H} 4), 2.70$ (1H, m, H3), $2.54(1 \mathrm{H}, \mathrm{m}, \mathrm{H} 10 \mathrm{a}), 2.45(1 \mathrm{H}, \mathrm{td}, J=12.2 \& 3.5, \mathrm{H} 1), 2.23(3 \mathrm{H}, \mathrm{s}, \mathrm{H} 13)$, 2.07 (3H, s, H12), $1.95(1 \mathrm{H}, \mathrm{m}, \mathrm{H} 2), 1.77(1 \mathrm{H}, \mathrm{m}, \mathrm{H} 4), 1.42(1 \mathrm{H}, \mathrm{q}, J=12.3, \mathrm{H} 2) .{ }^{13} \mathrm{C}$ NMR (CDCl 3 , ঠ): 210.8 (CO), 210.6 (CO), 135.3 (C9a/4b), 134.2 (C9a/4b), 131.2 (C10), 128.2 (C9), 128.0 (C6/7), 127.1 (C8), 126.8 (C6/7), 124.8 (C5), 50.2 (C1), 45.3 (C3), 37.0 (C10a), 35.9 (C4a), 30.5 (C2), 30.2 (C11), 28.2 (C12), 27.7 (C4). 
Synthesis of TpMo(NO)(DMAP) $\left(\eta^{2}-1-(((1 S, 3 S, 4 a S, 10 a S)-1-a c e t y l-1,2,3,4,4 a, 10 a-\right.$ hexahydrophenanthren-3-yl)propan-1-one)) (90).

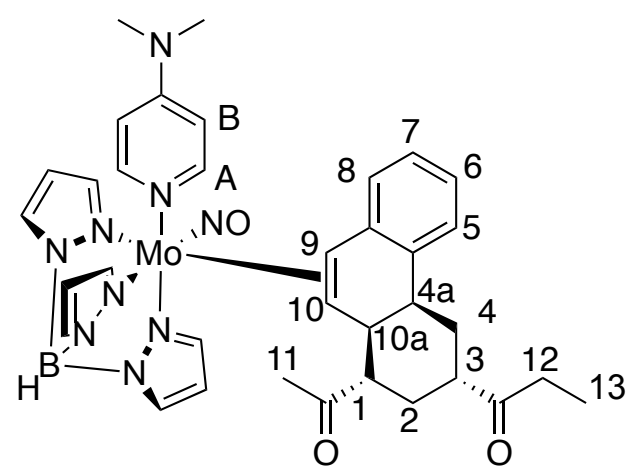

TpMo(NO)(DMAP)(naphthalene) (200 mg, $0.34 \mathrm{mmol}), \mathrm{CH}_{3} \mathrm{CH}_{2} \mathrm{CN}$ (6 mL), EVK (33 mg, $0.4 \mathrm{mmol}$ ), and DTBP (54 $\mathrm{mg}, 0.3 \mathrm{mmol}$ ) were added to a test tube. The reaction mixture was then cooled at $-60^{\circ} \mathrm{C}$ for $15 \mathrm{~min}$. TMSOTf $(0.1 \mathrm{~mL}, 0.5 \mathrm{mmol})$ was added to the reaction mixture, and the resulting red solution was left stirring at $-60{ }^{\circ} \mathrm{C} 1 \mathrm{~min}$. Next, MVK (50 mg, $0.7 \mathrm{mmol})$ was added to the reaction mixture, which was then left stirring at $-60{ }^{\circ} \mathrm{C}$ overnight $(\sim 18 \mathrm{~h})$. TEA $(0.1 \mathrm{~mL}, 0.74 \mathrm{mmol})$ was added to the reaction mixture, which was subsequently warmed to room temperature. This solution was then chromatographed through a $60 \mathrm{~mL}$ medium porosity fritted disc $3 / 4$ full with silica gel. The product was eluted with $\mathrm{Et}_{2} \mathrm{O}(50 \mathrm{~mL})$ as a yellow band, collected as a yellow solution, and evaporated in vacuo. The resulting oil was dissolved in DCM (1 mL) and the product was precipitated in stirring pentane $(50 \mathrm{~mL})$. The precipitate was collected on a $15 \mathrm{~mL}$ fine porosity fritted disc, washed with pentane $(3 \times 10 \mathrm{~mL})$, and dried for $15 \mathrm{~min}$ yielding the yellow solid 26 (77 mg, 30\%). CV (DMAc) $E_{\mathrm{p}, \mathrm{a}}=+0.16 \mathrm{~V}$ (NHE). IR: $v(\mathrm{~B}-$ $\left.\mathrm{H} \mathrm{sp}^{2}\right)=2474 \mathrm{~cm}^{-1} v(\mathrm{CO})=1702 \& 1618 \mathrm{~cm}^{-1}, v(\mathrm{NO})=1561 \mathrm{~cm}^{-1} \cdot{ }^{1} \mathrm{H}$ NMR $\left(\mathrm{d}^{6}-\right.$ Acetone, $): 7.94$ (1H, d, Pz5A/C), 7.91 (1H, d, Pz3A), 7.88 (1H, d, Pz5A/C), 7.78 (1H, d, Pz5B), 7.72 (1H, d, Pz3C), 7.37 (2H, bs, DMAP-A), 7.35 (1H, d, J = 7.4, H5), 7.00 
(1H, d, Pz3B), $6.94(1 \mathrm{H}, \mathrm{t}, J=7.4, \mathrm{H} 6), 6.86(1 \mathrm{H}, \mathrm{t}, J=7.5, \mathrm{H} 7), 6.51$ (2H, m, DMAPB), $6.37(1 \mathrm{H}, \mathrm{t}, \mathrm{Pz} 4 \mathrm{~A} / \mathrm{C}), 6.36(1 \mathrm{H}, \mathrm{t}, \mathrm{Pz} 4 \mathrm{~A} / \mathrm{C}), 6.21(1 \mathrm{H}, \mathrm{d}, J=7.5, \mathrm{H} 8), 6.09(1 \mathrm{H}, \mathrm{t}$, Pz4B), 3.78 (1H, m, H4a), 3.31 (1H, d, $J=9.5, \mathrm{H} 9), 3.05(6 \mathrm{H}, \mathrm{s}, \mathrm{N}-\mathrm{Me}), 2.83(1 \mathrm{H}, \mathrm{m}$, H4), 2.79 (1H, ddd, $J=10.6,5.1,2.4, \mathrm{H} 10 \mathrm{a}), 2.63$ (2H, m, H3 \& H12), 2.56 (2H, m, H1 \& H12), $2.14(1 \mathrm{H}, \mathrm{dd}, J=9.5,2.5, \mathrm{H} 10), 1.88(3 \mathrm{H}, \mathrm{s}, \mathrm{H} 11), 1.74(1 \mathrm{H}, \mathrm{d}, J=12.7, \mathrm{H} 2)$, $1.55(1 \mathrm{H}, \mathrm{q}, J=12.7, \mathrm{H} 2), 1.01(3 \mathrm{H}, \mathrm{t}, J=7.3, \mathrm{H} 13) .{ }^{13} \mathrm{C}$ NMR (d -Acetone, $\left.\delta\right): 213.7$ (CO), 212.5 (CO), 155.11 (DMAP-C), 150.7 (2C, DMAP-A), 144.7 (C4b/9a), 143.6 (Pz3A), 142.1 (Pz3B), 141.4 (Pz3C), 137.6 (Pz5A/C), 137.0 (Pz5A/C), 135.8 (Pz5B), 133.9 (C4b/9a), 128.2 (C8), 125.6 (C5), 125.1 (C7), 123.9 (C6), 108.3 (2C, DMAP-B), 107.0 (Pz4A/C), 106.7 (Pz4A/C), 106.5 (Pz4B), 70.6 (C10), 68.2 (C9), 56.2 (C1), 45.2 (C3), 42.9 (C10a), 39.3 (2C, DMAP-Me), 35.7 (C4a), 34.4 (C12), 32.4 (C2), 31.0 (C4), $8.24(\mathrm{C} 13)$.

\section{Synthesis of 1-((1S,3S,4aS,10aS)-1-acetyl-1,2,3,4,4a,10a-hexahydrophenanthren-3-} yl)propan-1-one (91).

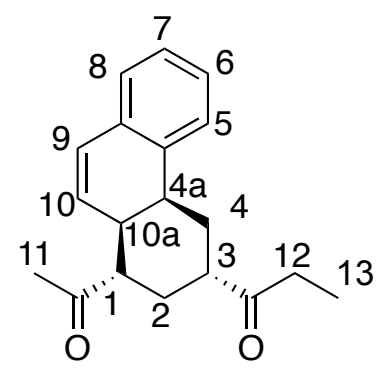

24 (200 mg, $0.34 \mathrm{mmol}), \mathrm{CH}_{3} \mathrm{CH}_{2} \mathrm{CN}$ (6 mL), EVK (33 mg, $0.4 \mathrm{mmol}$ ), and DTBP (54 $\mathrm{mg}, 0.3 \mathrm{mmol}$ ) were added to a test tube. The reaction mixture was then cooled at $-60{ }^{\circ} \mathrm{C}$ for $15 \mathrm{~min}$. TMSOTf $(0.1 \mathrm{~mL}, 0.5 \mathrm{mmol})$ was added to the reaction mixture, and the resulting red solution was left stirring at $-60{ }^{\circ} \mathrm{C} 1 \mathrm{~min}$. Next, MVK (50 mg, $0.7 \mathrm{mmol}$ ) 
was added to the reaction mixture, which was then left stirring at $-60{ }^{\circ} \mathrm{C}$ overnight $(\sim 18$ h). TEA ( $0.1 \mathrm{~mL}, 0.74 \mathrm{mmol})$ was added to the reaction mixture, which was subsequently removed from the glovebox and stirred open to air overnight $(\sim 18 \mathrm{~h})$. The resulting brown mixture was evaporated in vacuo, and the resulting film was dissolved in DCM (1 $\mathrm{mL})$. This solution was then added stirring $\mathrm{Et}_{2} \mathrm{O}(50 \mathrm{~mL})$, yielding a brown precipitation. The brown precipitate was filtered off on a $15 \mathrm{~mL}$ medium porosity fritted disc and washed with $\mathrm{Et}_{2} \mathrm{O}(3 \times 5 \mathrm{~mL})$. The filtrate was then evaporated in vacuo. This oil was then dissolved in DCM $(3 \times 0.3 \mathrm{~mL})$ and the resulting solution was dropwise added onto a $250 \mu \mathrm{m}$ silica preparatory plate. The product was eluted with $10 \%$ EtOAc:hexanes (200 mL), scraped off as a band at $\mathrm{R}_{\mathrm{f}}: 0.32-0.41$ and this silica gel was sonicated in EtOAc $(20 \mathrm{~mL})$ for $15 \mathrm{~min}$. The silica was filtered off on a $15 \mathrm{~mL}$ fine porosity fritted disc and washed with DCM $(3 \times 5 \mathrm{~mL})$. The filtrate was evaporated in vacuo and desiccated for $15 \mathrm{~min}$ to yield the colorless oil 27 (11 mg, 12\% yield). ${ }^{1} \mathrm{H} \mathrm{NMR}\left(\mathrm{CDCl}_{3}\right.$,

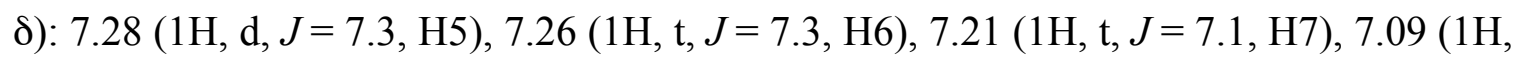
d, $J=7.2, \mathrm{H} 8), 6.44(1 \mathrm{H}, \mathrm{d}, J=9.6, \mathrm{H} 10), 6.08(1 \mathrm{H}, \mathrm{dd}, J=9.6 \& 5.9, \mathrm{H} 9), 3.34$ (1H, bs, H4a), 2.70 (2H, m, H3 \& H4), 2.58 (1H, m, H10a), 2.53 (2H, q, H12), 2.42 (1H, m, H1), $2.06(3 \mathrm{H}, \mathrm{s}, \mathrm{H} 11), 1.92(1 \mathrm{H}, \mathrm{m}, \mathrm{H} 2), 1.78(1 \mathrm{H}, \mathrm{m}, \mathrm{H} 4), 1.43(1 \mathrm{H}, \mathrm{m}, \mathrm{H} 2), 1.09$ (3H, t, $J=$ 7.3, H13). ${ }^{13} \mathrm{C}$ NMR $\left(\mathrm{CDCl}_{3}, \delta\right): 213.2$ (CO), $210.8(\mathrm{CO}), 135.4(\mathrm{C} 4 \mathrm{~b} / 9 \mathrm{a}), 134.2$ (C4b/9a), 131.2 C9), 128.1 (C10), 127.9 (C6/7), 127.1 (C8), 126.1 (C6/7), 124.8 (C5), 50.3 (C1), 44.4 (C3), 36.9 (C10a), 35.9 (C4a), 34.1 (C12), 30.6 (C2), 30.1 (C11), 27.9 (C4), 7.9 (C13). 
Synthesis of $(8 S, 9 S, 12 S, 13 S, 14 S)-12$-propionyl-8,9,11,12,13,14,16,17-octahydro-15Hcyclopenta $[a]$ phenanthren-15-one $(91)$.

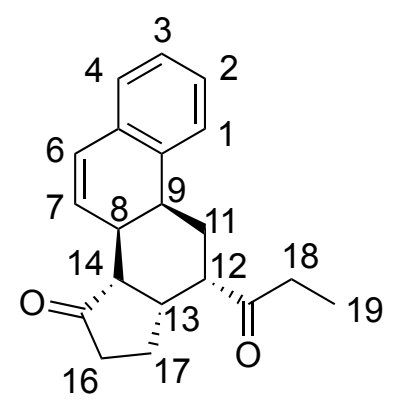

24 (200 mg, $0.34 \mathrm{mmol}), \mathrm{CH}_{3} \mathrm{CH}_{2} \mathrm{CN}$ (6 mL), EVK (33 mg, $\left.0.4 \mathrm{mmol}\right)$, and DTBP (54 $\mathrm{mg}, 0.3 \mathrm{mmol}$ ) were added to a test tube. The reaction mixture was then cooled at $-60{ }^{\circ} \mathrm{C}$ for $15 \mathrm{~min}$. TMSOTf $(0.1 \mathrm{~mL}, 0.5 \mathrm{mmol})$ was added to the reaction mixture, and the resulting red solution was left stirring at $-60^{\circ} \mathrm{C} 1 \mathrm{~min}$. Next, cyclopentenone $(57 \mathrm{mg}, 0.7$ mmol) was added to the reaction mixture, which was then left stirring at $-60^{\circ} \mathrm{C}$ overnight $(\sim 18 \mathrm{~h})$. TEA $(0.1 \mathrm{~mL}, 0.74 \mathrm{mmol})$ was added to the reaction mixture, which was subsequently removed from the glovebox and stirred open to air overnight $(\sim 18 \mathrm{~h})$. The resulting brown mixture was evaporated in vacuo, and the resulting film was dissolved in DCM $(1 \mathrm{~mL})$. This solution was then added stirring $\mathrm{Et}_{2} \mathrm{O}(50 \mathrm{~mL})$, yielding a brown precipitation. The brown precipitate was filtered off on a $15 \mathrm{~mL}$ medium porosity fritted disc and washed with $\mathrm{Et}_{2} \mathrm{O}(3 \times 5 \mathrm{~mL})$. The filtrate was then evaporated in vacuo. This oil was then dissolved in DCM (3 x $0.3 \mathrm{~mL})$ and the resulting solution was dropwise added onto a $250 \mu \mathrm{m}$ silica preparatory plate. The product was eluted with $10 \%$ EtOAc:hexanes (200 mL), scraped off as a band at $\mathrm{R}_{\mathrm{f}}: 0.42-0.50$ and this silica gel was sonicated in EtOAc $(20 \mathrm{~mL})$ for $15 \mathrm{~min}$. The silica was filtered off on a $15 \mathrm{~mL}$ fine porosity fritted disc and washed with DCM (3 x $5 \mathrm{~mL})$. The filtrate was evaporated in vacuo and desiccated for $15 \mathrm{~min}$ to yield the colorless oil $28(10 \mathrm{mg}, 10 \%$ yield $)$. IR: $v\left(\mathrm{C}-\mathrm{H} \mathrm{sp}{ }^{2}\right)=$ 
$2932 \mathrm{~cm}^{-1}, v(\mathrm{CO})=1731$ and $1703 \mathrm{~cm}^{-1} .{ }^{1} \mathrm{H}$ NMR $\left(\mathrm{CDCl}_{3}, \delta\right): 7.22(3 \mathrm{H}, \mathrm{m}, \mathrm{H} 1, \mathrm{H} 2, \&$ H3), $7.11(1 \mathrm{H}, \mathrm{d}, J=7.3, \mathrm{H} 4), 6.58(1 \mathrm{H}, \mathrm{d}, J=9.6, \mathrm{H} 6), 6.15(1 \mathrm{H}, \mathrm{dd}, J=9.6 \& 6.2, \mathrm{H} 7)$, 3.27 (1H, m, H9), 3.11 (1H, dt, $J=13.0$ \& 4.4, H12), 2.58 (2H, m, H13 \& H18), 2.47 (3H, m, H11, H16, H18), 2.25 (1H, m, H8), 2.17 (2H, m, H11 \& H16), 2.06 (1H, dd, $J=$ 12.2 \& 6.7, H14), $1.97(1 \mathrm{H}, \mathrm{m}, \mathrm{H} 17), 1.69(1 \mathrm{H}, \mathrm{m}, \mathrm{H} 17), 1.10(3 \mathrm{H}, \mathrm{t}, J=7.2, \mathrm{H} 19) .{ }^{13} \mathrm{C}$ NMR ( $\left.\mathrm{CDCl}_{3}, \delta\right): 218.5(\mathrm{CO}), 212.5$ (CO), 134.6 (C5/10), 134.3 (C5/10), 130.6 (C7), 128.7 (C6), 127.9 (C2/3), 127.2 (C4), 126.8 (C2/3), 124.9 (C1), 51.1 (C14), 43.8 (C12), 37.9 (C13), 37.1 (C16), 35.2 (C9), 34.5 (C18), 30.8 (C8), 21.2 (C11), 21.0 (C17), 7.9 (C19). 


\subsection{References}

1. Bruice, P. Y., Organic Chemistry. 5th ed.; Pearson/Prentice Hall: Upper Saddle River, NJ, 2007.

2. Boehm, M.; Lorthiois, E.; Meyyappan, M.; Vasella, A. Helvetica Chimica Acta 2003, 86, (11), 3787.

3. Cheng, W.; Wu, D.; Liu, Y. Biomacromolecules 2016, 17, (10), 3115.

4. Zhang, Y.; Wang, W. Catalysis Science \& Technology 2012, 2, (1), 42.

5. $\quad$ Genna, D. T.; Maio, W. A. Tetrahedron 2016, 72, (40), 5956.

6. Posner, G. H. Chem. Rev. 1986, 86, 831.

7. Posner, G. H.; Asirvatham, E. Tetrahedron Letters 1986, 27, (6), 663.

8. $\quad$ Posner, G. H.; Lu, S.-B.; Asirvatham, E. Tetrahedron Letters 1986, 27, (6), 659.

9. Posner, G. H.; Lu, S. B.; Asirvatham, E.; Silversmith, E. F.; Shulman, E. M. J. Am. Chem. Soc. 1986, 108, (3), 511.

10. Posner, G. H.; Mallamo, J. P.; Black, A. Y. Tetrahedron 1981, 37, (23), 3921.

11. Posner, G. H.; Switzer, C. J. Am. Chem. Soc. 1986, 108, (6), 1239.

12. Chen, H.; Liu, R.; Myers, W. H.; Harman, W. D. J. Am. Chem. Soc. 1998, 120, 509.

13. Ding, F.; Valahovic, M. T.; Keane, J. M.; Anstey, M. R.; Sabat, M.; Trindle, C. O.; Harman, W. D. J. Org. Chem. 2004, 69, (7), 2257.

14. Zhang, L.; Xie, X.; Fu, L.; Zhang, Z. J. Org. Chem. 2013, 78, (7), 3434.

15. Karplus, M. J. Chem. Phys. 1959, 30, 11.

16. Belov, V. N.; Dudkin, V. Y.; Urusova, E. A.; Starova, G. L.; Selivanov, S. I.; Nikolaev, S. V.; Eshchenko, N. D.; Morozkina, S. N.; Shavva, A. G. Russian Journal of Bioorganic Chemistry 2007, 33, (3), 293.

17. Dauben, W. G.; Fullerton, D. S. J. Org. Chem. 1971, 36, (22), 3277.

18. Egorov, M. S.; Grinenko, E. V.; Zorina, A. D.; Balykina, L. V.; Selivanov, S. I.; Shavva, A. G. Russ. J. Org. Chem. 2001, 37, (6), 802.

19. Morozkina, S. N.; Abusalimov, S. N.; Selivanov, S. I.; Shavva, A. G. Russ. J. Org. Chem. 2013, 49, (4), 603. 
20. Morozkina, S. N.; Abusalimov, S. N.; Starova, G. L.; Selivanov, S. I.; Shavva, A. G. Russ. J. Org. Chem. 2010, 80, (7), 1324.

21. Dodge, J. A.; Krishnan, V. G.; Lugar, C. W.; Neubauer, B. L.; Norman, B. H.; Pfeifer, L. A.; Richardson, T. I., Substituted benzopyrans as selective estrogen receptorbeta agonists. Google Patents: 2003.

22. Gonzalez, M. A. Nat. Prod. Rep. 2015, 32, (5), 684.

23. González, M. A. Euro. J. Med. Chem. 2014, 87, 834.

24. Aulenta, F.; Berndt, M.; Bruedgam, I.; Hartl, H.; Soergel, S.; Reissig, H.-U. Chem. - Eur. J. 2007, 13, (21), 6047.

25. Berndt, M.; Hlobilová, I.; Reissig, H.-U. Org. Lett. 2004, 6, (6), 957. 


\section{Chapter 5}

Binding and Activation of $\alpha, \alpha, \alpha$-Trifluorotoluene with $\{$ TpMo(NO)(DMAP) $\}$ 


\subsection{Introduction}

Although the $\{\mathrm{TpMo}(\mathrm{NO})(\mathrm{L})\}$ fragment has been shown to bind a wide variety of aromatics in an eta-2 fashion, ${ }^{1-3}$ one limitation that has discouraged the use of this system has been its inability to form a stable and isolable eta- 2 bound benzene complex. This inability can be rationalized by the comparison of free energy of activation $\left(\Delta \mathrm{G}^{\ddagger}\right)$ for the dissociative substitution of ligands eta- 2 bound to $\left\{\mathrm{Os}\left(\mathrm{NH}_{3}\right)_{5}\right\}^{2+4,} 5$ $\{\mathrm{TpRe}(\mathrm{CO})(\mathrm{MeIm})\},{ }^{6}\left\{\mathrm{TpW}(\mathrm{NO})\left(\mathrm{PMe}_{3}\right)\right\},^{7}$ and $\{\mathrm{TpMo}(\mathrm{NO})(\mathrm{DMAP})\}^{8}$ with acetone (Table 5.1). According to published reports, the entropy of activation for benzene displacement is typically small and positive, usually $2-5$ eu. ${ }^{9-12}$ From this assumption, and the previously reported energies of activation in Table 5.1, the enthalpy of activation $\left(\Delta \mathrm{H}^{\ddagger}\right)$ for these complexes can be estimated. The $\Delta \mathrm{H}^{\ddagger}$ provides a more accurate representation of the bond strength between these metals and their eta- 2 bound ligands. Taking the average of the data available ( $3 \mathrm{eu}$ ) we calculated an approximate value for $\Delta \mathrm{H}^{\ddagger}$ of $27.0 \mathrm{kcal} / \mathrm{mol}$ for $\mathrm{TpMo}(\mathrm{NO})(\mathrm{DMAP})\left(\eta^{2}\right.$-naphthalene) (24), which is an upper limit for the approximate bond dissociation energy (i.e., metal-arene bond strength) for a heterolytic cleavage.

From the differences in enthalpies of activation between the metal-benzene and metal-naphthalene complexes reported in Table 5.1 (between $6-8 \mathrm{kcal} / \mathrm{mol}$ ), an enthalpy of activation close to $20 \mathrm{kcal} / \mathrm{mol}$ is predicted for the purported TpMo(NO)(DMAP) $\left(\eta^{2}-\right.$ benzene) complex; as determined by comparison with the enthalpy of activation for the TpMo(NO)(DMAP) $\left(\eta^{2}\right.$-naphthalene) (24) complex $(27.0 \mathrm{kcal} / \mathrm{mol})$. This substantially lower predicted bond strength for $\mathrm{TpMo}(\mathrm{NO})(\mathrm{DMAP})\left(\eta^{2}\right.$-benzene) would account for the inability of its isolation at ambient temperature. From the data in Table 5.1 it can also 
be seen that there would be a predicted gain of three $\mathrm{kcal} / \mathrm{mol}$ of stability achieved by the introduction of a single $\mathrm{CF}_{3}$ group on benzene (entries 3 and 8 ). This three $\mathrm{kcal} / \mathrm{mol}$ increase would give a predicted enthalpy of activation of $23 \mathrm{kcal} / \mathrm{mol}$ for the purported TpMo(NO)(DMAP) $\left(\eta^{2}-\alpha, \alpha, \alpha-\right.$ trifluortoluene (TFT)) (100) complex, which could provide enough stability for its isolation. Thus the synthesis of an eta-2 bound TFT complex was pursued in the hopes that its stability would allow for isolation and characterization.

Table 5.1. Comparison of energies for the exchange of various ligands bound to Os, Re, W, and Mo with acetone.

\begin{tabular}{|c|c|c|c|c|c|c|}
\hline Entry & Compound & $\mathrm{T}(\mathrm{K})$ & $\Delta \mathrm{G}^{\ddagger}(\mathrm{kcal} / \mathrm{mol})$ & $\Delta S^{\ddagger}$ (eu) & $\Delta \mathbf{H}^{\ddagger}(\mathrm{kcal} / \mathrm{mol})$ & Reference \\
\hline 1 & {$\left[\mathrm{Os}\left(\mathrm{NH}_{3}\right)_{5}\left(\eta^{2} \text {-naphthalene }\right)\right]^{2+}$} & 373 & 29.4 & $\sim 3$ & 30.5 & 5 \\
\hline 2 & {$\left[\mathrm{Os}\left(\mathrm{NH}_{3}\right)_{5}\left(\eta^{2} \text {-benzene }\right)\right]^{2+}$} & 298 & 23.9 & $\sim 3$ & 24.8 & 4 \\
\hline 3 & {$\left[\mathrm{Os}\left(\mathrm{NH}_{3}\right)_{5}\left(\eta^{2}-\mathrm{CF}_{3} \mathrm{Ph}\right)\right]^{2+}$} & 298 & 25.6 & $\sim 3$ & 26.5 & 4 \\
\hline 4 & TpRe(CO)(Melm)( $\eta^{2}$-naphthalene) & 373 & 28.5 & $\sim 3$ & 29.7 & 6 \\
\hline 5 & $\operatorname{TpRe}(\mathrm{CO})(\mathrm{Melm})\left(\eta^{2}\right.$-benzene) & 295 & 22.6 & $\sim 3$ & 23.4 & 6 \\
\hline 6 & $\mathrm{TpW}(\mathrm{NO})\left(\mathrm{PMe}_{3}\right)\left(\eta^{2}\right.$-naphthalene $)$ & 351 & 30.4 & $\sim 3$ & 31.4 & 7 \\
\hline 7 & $\mathrm{TpW}(\mathrm{NO})\left(\mathrm{PMe}_{3}\right)\left(\eta^{2}\right.$-benzene $)$ & 295 & 22.3 & $\sim 3$ & 23.2 & 7 \\
\hline 8 & $\mathrm{TpW}(\mathrm{NO})\left(\mathrm{PMe}_{3}\right)\left(\eta^{2}-\mathrm{CF}_{3} \mathrm{Ph}\right)$ & 295 & 25.4 & $\sim 3$ & 26.2 & 7 \\
\hline 9 & TpMo(NO)(DMAP)( $\eta^{2}$-naphthalene) & 298 & 25.4 & $\sim 3$ & 27.0 & 8 \\
\hline
\end{tabular}

\subsection{Results}

As discussed in Chapter 3, the $\mathrm{TpMo}(\mathrm{NO})(\mathrm{DMAP})\left(\eta^{2}-2,5\right.$-dimethylfuran) complex (26) has a half-life $\left(\mathrm{t}_{1 / 2}\right)$ of $130 \mathrm{~min}$ in $\mathrm{d}^{6}$-acetone $\left(25^{\circ} \mathrm{C}\right),{ }^{8}$ which would allow for a substitution of furan for another ligand within a reasonable timeframe. Following this information, 26 was stirred in an excess of neat TFT and observed over time. The reaction mixture changed from a yellow to a red mixture after $8 \mathrm{~h}$ and a shift in the anodic wave from $-350 \mathrm{mV}$ to $-280 \mathrm{mV}(100 \mathrm{mV} / \mathrm{s}$, NHE) was seen. The red precipitate 
from this exchange was then collected, and weighed to determine a yield of $52 \%$. This represents the first substituted benzene eta-2 bound to the $\{\mathrm{TpMo}(\mathrm{NO})(\mathrm{L})\}$ fragment (100) (Scheme 5.1).

Scheme 5.1. Exchange of $\mathbf{2 6}$ with TFT to yield $\mathbf{1 0 0}$.

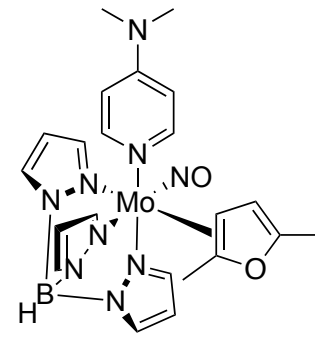

26
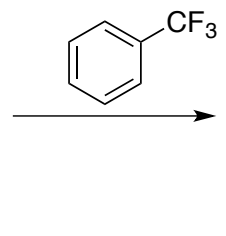

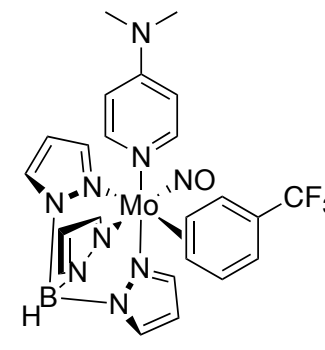

100-A

1

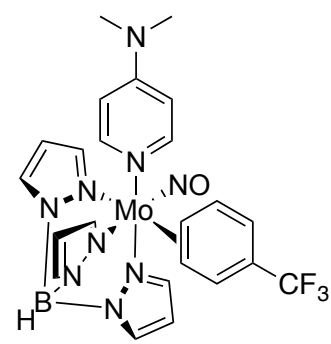

100-B

1

$52 \%$ yield

NMR analysis shows that the TFT ligand is fluxional on the NMR timescale (600 $\mathrm{MHz}$ ) with severely broadened peaks. However, reducing the temperature of a $\mathrm{d}^{6}$-acetone solution of $\mathbf{1 0 0}$ to $-30{ }^{\circ} \mathrm{C}$ allows for full characterization of the complex (Figure 5.1).

Various isomers of $\mathbf{1 0 0}$ were modeled using the Gaussian 03 program suite. Relative energies were determined using a hybrid density functional B3LYP expressed in a hybrid basis. The basis incorporates the Los Alamos pseudopotential LANL2DZ and the associated basis functions for molybdenum and Pople's 6-31G(d) basis for all other atoms. This combination has proved reliable for Os, Mo, Re, and W systems for relative (binding) energies, charge transfer, and preferred structures, especially in similar systems. ${ }^{13}$ These results showed that the two thermodynamically preferred orientations of $\operatorname{TpMo}(\mathrm{NO})(\mathrm{DMAP})\left(\eta^{2}-\mathrm{TFT}\right)$ are those which place the $\mathrm{CF}_{3}$ group in quadrants a and $\mathrm{d}$ (100-A and 100-B, Figure 5.2). 


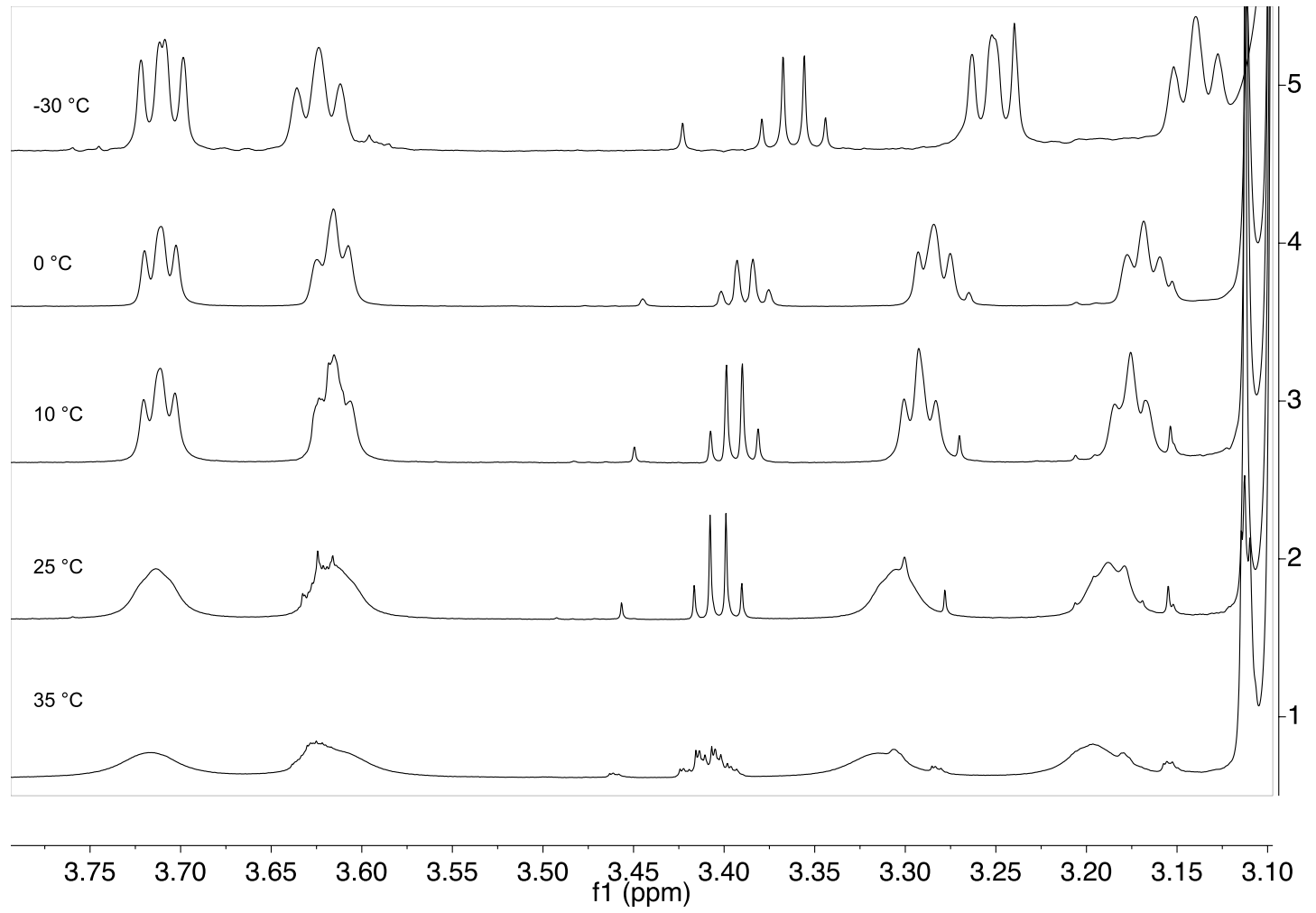

Figure 5.1. ${ }^{1} \mathrm{H}$ NMR spectra of $\mathrm{TpMo}(\mathrm{NO})(\mathrm{DMAP})\left(\eta^{2}-\mathrm{TFT}\right)(\mathbf{1 0 0})$ in $\mathrm{d}^{6}$-Acetone at various temperatures.

Energies for each isomer are expressed relative to that of isomer 100-A, which was determined to be the lowest energy orientation. 100-A and 100-B are related to one another through either an intrafacial or interfacial isomerization with very little difference in their free energies $(0.12 \mathrm{kcal} / \mathrm{mol})$. Spin-saturation experiments have revealed intrafacial isomerization as the dominant dynamic process (vide infra). Through intrafacial isomerization of $\mathbf{1 0 0 - A}$ or $\mathbf{1 0 0 - B}$, orientations in which the $\mathrm{CF}_{3}$ group is proximal (100-C) or distal (100-D) to the DMAP ligand can be obtained with slightly higher free energies relative to $\mathbf{1 0 0 - A}(0.59-1.79 \mathrm{kcal} / \mathrm{mol})$. With a small decrease in thermodynamic stability relative to $\mathbf{1 0 0 - A}$, constitutional isomers $\mathbf{1 0 0 - C}$ and $\mathbf{1 0 0}-\mathbf{D}$ could be observed; however, no empirical evidence of this has been found. Lastly, through an 
intrafacial isomerization of $\mathbf{1 0 0}-\mathbf{C}$ or $\mathbf{1 0 0}-\mathbf{D}$, orientations in which the $\mathrm{CF}_{3}$ group is placed in a sterically disfavored quadrant can be obtained (100-E and 100-F). However, such steric hindrance yields a significant $5 \mathrm{kcal} / \mathrm{mol}$ decrease in the thermodynamic stability of orientations $\mathbf{1 0 0 - E}$ and $\mathbf{1 0 0 - F}$, relative to $\mathbf{1 0 0 - A}$.

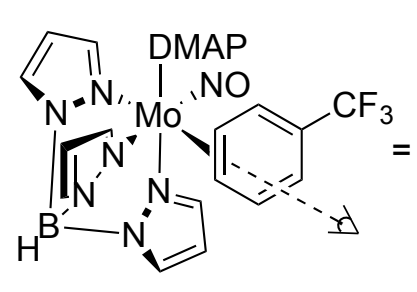

100

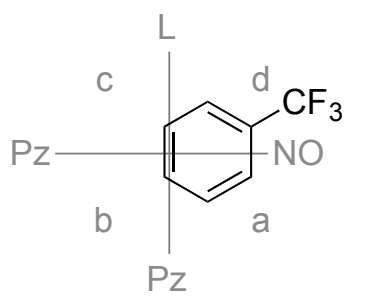

$100-\mathrm{A}(0 \mathrm{kcal} / \mathrm{mol})$

Ring-

Walk

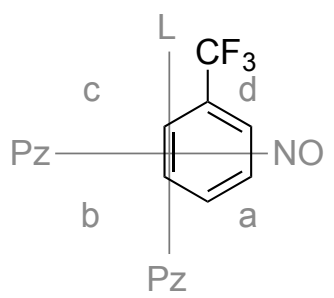

$100-\mathrm{C}(0.59 \mathrm{kcal} / \mathrm{mol})$
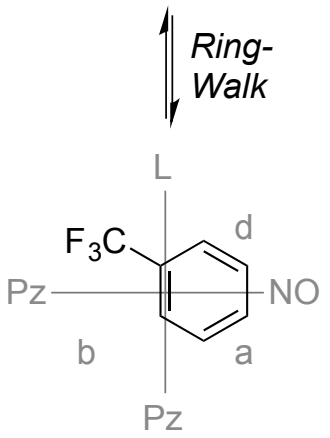

100-E $(5.02 \mathrm{kcal} / \mathrm{mol})$

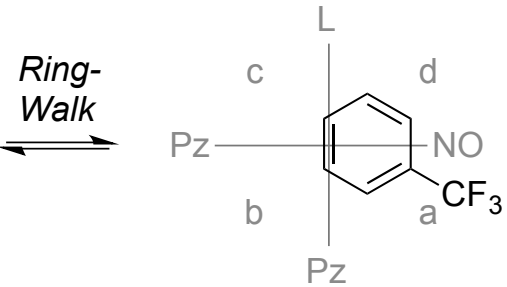

100-B $(0.12 \mathrm{kcal} / \mathrm{mol})$

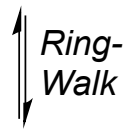

Face-
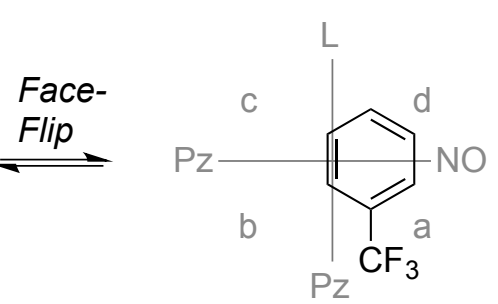

100-D $(1.76 \mathrm{kcal} / \mathrm{mol})$

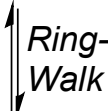

Face-
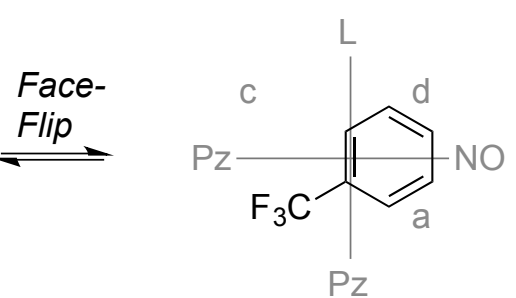

$100-\mathrm{F}(5.00 \mathrm{kcal} / \mathrm{mol})$

Figure 5.2. Coordination diastereomers of $\mathbf{1 0 0}$ and their relative free energies as predicted from DFT calculations.

Analysis of NOESY data of $\mathbf{1 0 0}$ (via spin-saturation exchange) reveal that the dominant dynamic process is an intrafacial (ring-walk) isomerization occurring on the 
seconds time-scale at a temperature of $25^{\circ} \mathrm{C}$ (Figure 5.3). Through COSY analysis, peaks at 3.61, 3.30, and $7.10 \mathrm{ppm}$ were identified as belonging to the same spin-system. Likewise peaks at $3.18,3.71$, and 6.70 ppm were also identified as belonging to the same spin-system. The resonance for the upbound proton of 100-A (H3, $3.61 \mathrm{ppm})$ exchanges with a peak at $6.70 \mathrm{ppm}$, identified as H5 of 100-B (Figure 5.3). Upon intrafacial isomerization, the downbound proton of 100-A $(\mathrm{H} 4,3.30 \mathrm{ppm})$ would be expected to still have significant shielding from the metal center, which agrees with the observed exchange between the resonance for $\mathrm{H} 4$ of 100-A and the peak at $3.71 \mathrm{ppm}(\mathrm{H} 4$ of 100B). Furthermore, the resonance for H5 of 100-A (7.11 ppm), identified through COSY as being adjacent to $\mathrm{H} 4$ of $\mathbf{1 0 0 - A}$, would be expected to shift significantly upfield from the aromatic region. This is in agreement with the observed exchange between $\mathrm{H} 5$ of 100-A and the peak at $3.18 \mathrm{ppm}(\mathrm{H} 3$ of $\mathbf{1 0 0}-\mathbf{B})$.

A ${ }^{1} \mathrm{H}$ NMR experiment of a $\mathrm{d}^{6}$-acetone solution of $\mathbf{1 0 0}$ at $0{ }^{\circ} \mathrm{C}$ revealed the coupling constant between $\mathrm{H} 4$ and $\mathrm{H} 5$ of $\mathbf{1 0 0 B}$ to be $6.2 \mathrm{~Hz}$. The difference in chemical shift between $\mathrm{H} 4$ of $\mathbf{1 0 0 A}$ and $\mathrm{H} 4$ of $\mathbf{1 0 0 B}$ is $341 \mathrm{~Hz}$ at $0{ }^{\circ} \mathrm{C}$. The approximate coalescence temperature $\left(50{ }^{\circ} \mathrm{C}\right)$ between 100A and 100B was obtained by VT-NMR in $\mathrm{d}^{6}$-acetone $\left(0-50{ }^{\circ} \mathrm{C}\right)$. The ${ }^{1} \mathrm{H}$ NMR spectrum of $\mathbf{1 0 0}$ obtained at $50{ }^{\circ} \mathrm{C}$ shows the complex to be very near coalescene and so an upper limit $\left(60{ }^{\circ} \mathrm{C}\right)$ and a lower limit $(40$ $\left.{ }^{\circ} \mathrm{C}\right)$ were used to approximate a window of error $( \pm 0.5 \mathrm{kcal} / \mathrm{mol})$. From this information, the approximate rate of isomerization was determined $\left(k=750 \mathrm{~s}^{-1}\right)$ and with it the free energy of activation of isomerization was approximated to be $14.7 \pm 0.5 \mathrm{kcal} / \mathrm{mol}$ at 50 ${ }^{\circ} \mathrm{C} .{ }^{14}$ This value is similar to that observed for $\left[\mathrm{Os}\left(\mathrm{NH}_{3}\right)_{5}\left(\eta^{2} \text {-benzene }\right)\right]^{2+}\left(\Delta \mathrm{G}^{\ddagger}=12.5\right.$ $\mathrm{kcal} / \mathrm{mol}$ at $\left.10^{\circ} \mathrm{C}\right)$. 


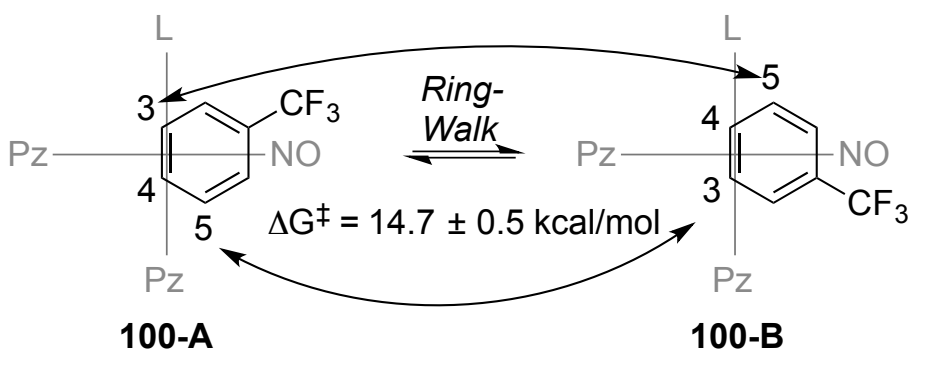

Figure 5.3. Intrafacial isomerization between 100-A and 100-B.

In contrast to that observed in the synthesis of the TFT complex, attempts to replace the furan for benzene have not been successful; however, the additional $\pi$-acidity provided by the presence of a $\mathrm{CF}_{3}$ group causes a stronger $\pi$-backbond donation, thereby stabilizing the eta-2 bond with TFT. The TFT complex (100) is stable at ambient temperature and slowly undergoes substitution in neat acetone with a half-life $\left(\mathrm{t}_{1 / 2}=39\right.$ minutes) that corresponds to a $\Delta \mathrm{G}^{\dot{+}}=22.3 \pm 0.08 \mathrm{kcal} / \mathrm{mol}$ at $25^{\circ} \mathrm{C}$.

Exchange of the furan ligand of $\mathbf{2 6}$ with TFT provided a sufficient method for the isolation of 100 (52\% yield); however, an alternate pathway to this complex was investigated. Although the synthesis of $\mathbf{2 6}$ can be done with good yields, a direct synthesis of 100 from TpMo(NO)(DMAP)(I) (23) was desired. Removing the furan complex intermediate would also eliminate the presence of $\mathbf{2 6}$, commonly seen as an impurity $(\sim 10 \%)$ in the isolation of $\mathbf{1 0 0}$ through the ligand exchange reaction. Initial reduction attempts to yield $\mathbf{1 0 0}$ from $\mathbf{2 3}$ were performed using THF as a cosolvent, with TFT and sodium dispersion in paraffin wax, but an unexplained decomposition was seen. This was believed to be due to the formation of an overly reduced metal complex reacting with the THF co-solvent (vide infra). Success was found by removing the co-solvent. In a similar method for the synthesis of $\mathrm{TpW}(\mathrm{NO})\left(\mathrm{PMe}_{3}\right)\left(\eta^{2}\right.$-benzene) complex, the reduction 
of 23 with elemental sodium in TFT (neat) yielded 100. However, difficulties in achieving consistent reaction outcomes required an investigation into the isolation of various sizes of elemental sodium.

It was found that after washing sodium dispersion in paraffin wax with hexanes, different sizes of elemental sodium could be obtained. For every gram of sodium dispersion in paraffin wax, approximately $30 \mathrm{~mL}$ of hexanes was used for washing. For the reduction of $\mathbf{2 3}(3 \mathrm{~g})$ to $\mathbf{1 0 0}, 1.5 \mathrm{~g}$ of sodium dispersion was added to a $50 \mathrm{~mL}$ round bottom flask charged with a $1 / 2$ inch stir egg. To this flask was added hexanes $(50 \mathrm{~mL})$ and the resulting grey mixture was stirred overnight $(18 \mathrm{~h})$. It was observed that the size of the resulting elemental sodium was a function of the rate of stirring used during the solvation of the wax. For example, in one set of experiments stirring at $1150 \mathrm{rpm}$ resulted in elemental sodium described herein as "flecks" $(\sim 1 / 4 \mathrm{~cm}$ in diameter $)$, at $\sim 700 \mathrm{rpm}$ yields sodium "flakes" $(\sim 1 / 2-1 \mathrm{~cm}$ in diameter $)$, and at $\sim 350 \mathrm{rpm}$ yields sodium "sheets" ( 1 $-2 \mathrm{~cm}$ in diameter).

After decanting the hexanes away from this elemental sodium, $\alpha, \alpha, \alpha-$ trifluorotoluene was added, followed by $\mathbf{2 3}$. The resulting green mixture was then stirred at room temperature and the reaction was monitored by cyclic voltammetry (CV). As this reaction is heterogeneous, due to the insolubility of $\mathbf{2 3}$ and sodium in TFT, the surface area of the sodium has a significant impact on the rate of the reaction (e.g., the greater the surface area, the greater the rate of the reaction). The most important factors in controlling this surface area include rate of stirring during the washing process, as described above, as well as during the reaction itself. For example, in reactions with otherwise identical conditions, the reaction time for the reduction of $\mathbf{2 3}$ to $\mathbf{1 0 0}$ increases 
from $6 \mathrm{~h}$ with sodium flecks, to $24 \mathrm{~h}$ with sodium flakes (Scheme 5.2). Furthermore, with otherwise identical conditions, the reaction time increases from $6 \mathrm{~h}$ with the reaction mixture stirring at $1150 \mathrm{rpm}$, to 3 days with the reaction mixture stirring at $500 \mathrm{rpm}$. Attempts to solubilize $\mathbf{2 3}$ with a co-solvent (i.e., THF, DME or benzene) result in significant decomposition (assumed based on the black color of the reaction mixture and absence of any reducible species in the CV).

Scheme 5.2. Reduction of 23 to $\mathbf{1 0 0}$.

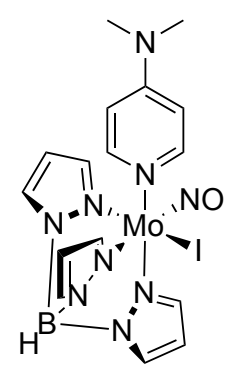

23

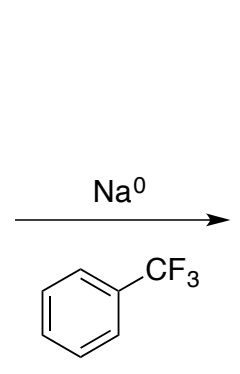

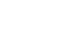

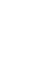

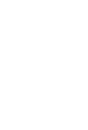

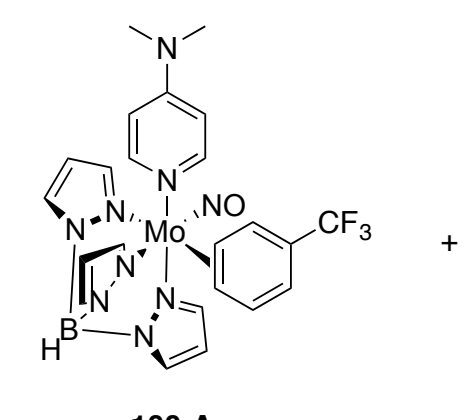

100-A

1

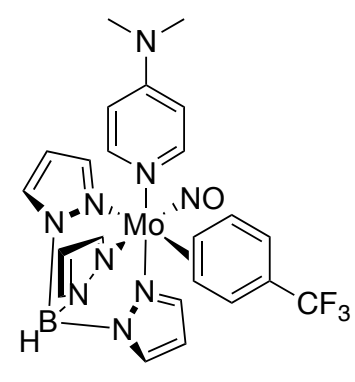

100-B

1

$65 \%$ yield

Following optimization of the reduction of $\mathbf{2 3}$ to $\mathbf{1 0 0}$, the isolation of $\mathbf{1 0 0}$ proved difficult. As $\mathbf{1 0 0}$ is insoluble in TFT, it can be isolated from the reaction mixture through filtration; however, the resulting precipitate also contains elemental sodium. After filtering the reaction mixture, THF was added in an attempt to dissolve $\mathbf{1 0 0}$. The addition of THF to this red solid resulted in a violent, effervescent reaction, which yielded a black solution. For the same reason, attempts to chromatograph $\mathbf{1 0 0}$ away from the sodium proved ineffective. In a similar workup to that of the $\operatorname{TpMo}(\mathrm{NO})(\mathrm{DMAP})\left(\eta^{2}-2,5-\right.$ dimethylfuran) complex, the reaction mixture resulting from the reduction of $\mathbf{2 3}$ in TFT can be added slowly to stirring $\mathrm{MeOH}$ (CAUTION: hydrogen evolution!), which yields a 
red precipitate and the elemental sodium is consumed. The resulting precipitate can then be isolated through filtration and purified with trituration in $\mathrm{Et}_{2} \mathrm{O}$. Utilizing a fast stir rate, sodium flecks, and $\mathrm{MeOH}$ precipitation, the scale for the reduction of $\mathbf{2 3}$ to $\mathbf{1 0 0}$ was increased to $17 \mathrm{~g}$ with a decent yield (65\%) without chromatography.

Having developed a reliable synthesis of the complex, the potential synthetic utility of $\mathbf{1 0 0}$ was then evaluated. Previous studies of third-row metal benzene complexes document a broad range of electrophile-nucleophile tandem addition reactions to the benzene ligand resulting in 1,4-hexadienes such as $\mathbf{1 0 2}$ in Scheme 5.3. ${ }^{15}$

Scheme 5.3. Tandem addition reaction sequence with $\operatorname{TpRe}(\mathrm{CO})(\mathrm{MeIm})\left(\eta^{2}\right.$-toluene $)$ (101).

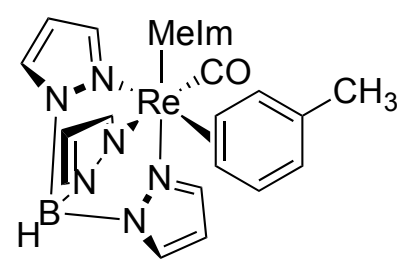

101

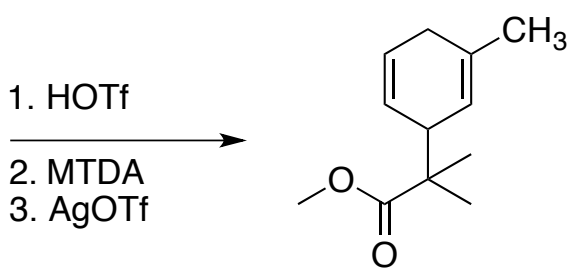

$102(62 \%)$

The two nucleophiles chosen to test the viability of $\mathbf{1 0 0}$ for this type of reaction, $\mathrm{N}$ methylpyrrole and 1-methoxy-2-methyl-1-(trimethylsilyloxy)-1-propene (MTDA), were chosen as representatives of two important classes of carbon-carbon bond forming reactions: Friedel-Crafts alkylation of aromatic heterocycles and addition of protected enolates. 
Scheme 5.4. Tandem electrophilic-nucleophilic additions to $\operatorname{TpMo}(\mathrm{NO})(\mathrm{DMAP})\left(\eta^{2}-\right.$ TFT) (100) complex.<smiles>COC(=O)C(C)(C)[C@@H]1CC(C(C)(C)C)=C[C@H](C)C1</smiles>

100

$100-\mathrm{H}$

$103(50 \%)$
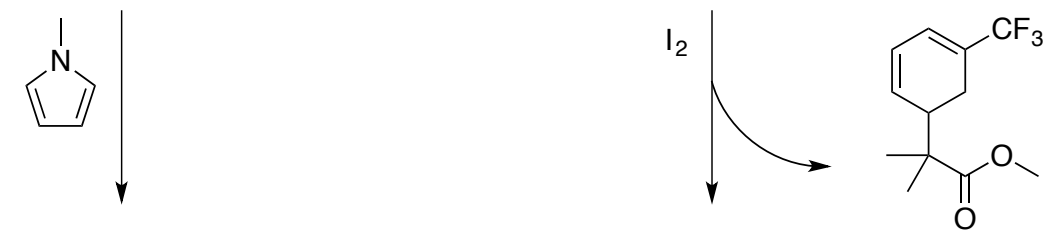

$105(46 \%)$

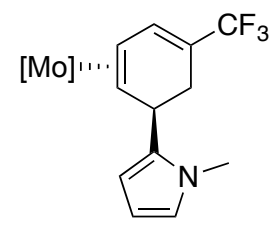

$104(37 \%)$

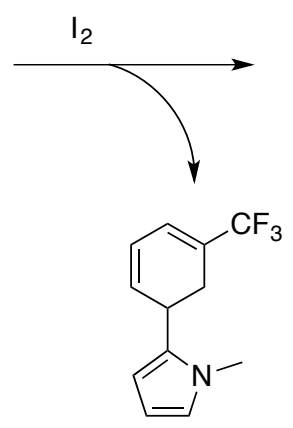

$106(52 \%)$

Attempts to characterize the purported allyl intermediate (100-H) from the protonation of 100 with HOTf have been unsuccessful. However, when a solution of the trifluorotoluene complex $\mathbf{1 0 0}$ is protonated followed by the addition of MTDA at $-60{ }^{\circ} \mathrm{C}$, a new complex is formed, 103 (47\% yield). A similar reaction sequence can be successfully carried out with $N$-methylpyrrole in place of MTDA, to yield 104 (37\%) (Scheme 5.4).

${ }^{1} \mathrm{H}$ NMR, COSY, NOESY, IR and electrochemical data are consistent with $\mathbf{1 0 3}$ and 104 being 1,3-cyclohexadiene complexes; contrast to that observed for electrophilicnucleophilic additions to the benzene or toluene third-row metal complexes described 
above, which yield non-conjugated dienes. In the ${ }^{13} \mathrm{C}$ NMR spectrum of $\mathbf{1 0 3}$ and $\mathbf{1 0 4}$, large fluorine coupling with the carbon nuclei is seen for the $\mathrm{CF}_{3}$ carbon $\left(\mathrm{q},{ }^{1} J_{\mathrm{F}, \mathrm{C}} \sim 260\right.$ $\mathrm{Hz})$, the carbon adjacent to the $\mathrm{CF}_{3}$ group $\left(\mathrm{C} 4, \mathrm{q},{ }^{2} J_{\mathrm{F}, \mathrm{C}} \sim 39 \mathrm{~Hz}\right)$, as well as the allylic carbon $\left(\mathrm{C} 3, \mathrm{q},{ }^{3} J_{\mathrm{F}, \mathrm{C}} \sim 7 \mathrm{~Hz}\right)$. The anodic wave for $\mathbf{1 0 3}$ and $\mathbf{1 0 4}$ is significantly positive of the anodic wave of $\mathbf{1 0 0}(+0.30 \mathrm{~V}$ vs $-0.30 \mathrm{~V})$; however, a better representation of electron density at the metal center can be seen in the nitrosyl stretch frequencies for $\mathbf{1 0 0}$ in comparison to 103 (e.g., 1586 vs $1571 \mathrm{~cm}^{-1}$ ). As the resulting ligand of a 1,2-addition to 100 has a decreased amount of conjugation relative to the its aromatic precursor, the metal center is more electron-rich in the case of $\mathbf{1 0 3}$ and 104, resulting in a lower stretch frequency.

Both protonation and nucleophilic addition were found to be regioselective, and the addition of the nucleophile proved to be stereoselective, with addition occurring anti to the metal. Also, in contrast to the complexes described in Chapters 2 and 3, 103 and 104 are isolated as single coordination diastereomers, even though the aromatic precursor 100 showed virtually no diastereoselectivity. Apparently, the electron-withdrawing nature of the $\mathrm{CF}_{3}$ group directs protonation to the ortho carbon such that the resulting positive charge build-up in the " $\eta^{2}$-allyl" complex intermediate (100-H) is farther from the $\mathrm{CF}_{3}$ group and distal to the DMAP ligand. Nucleophilic addition to the allyl group occurs to the meta carbon adjacent to protonation, resulting in 1,3-diene complexes. After isolation, complexes 103 and 104 can be subjected to oxidative decomplexation with iodine to recover 23 at an average 80\% yield and the organics 105 and 106 at an average 50\% yield. 


\subsection{Discussion}

Selective fluorination has been a significant focus for the development of biologically active small molecules. ${ }^{16-20}$ The presence of fluorine substituents yields greater lipophilicity and bioavailability, thus improving the pharmacokinetic profile for small molecules containing fluorine. Common fluorine containing pharmaceuticals include fluoxetine, bicalutamide, and fipronil (Figure 5.4). Furthermore, fluorinecontaining compounds have demonstrated desirable uses as refrigerants, polymers, dyes, and agrochemicals. ${ }^{21-24}$ The important benefits from the inclusion of fluorine have made the selective synthesis of fluorine containing molecules a well-studied area. Classic synthetic methods for fluorination include using electrophilic (i.e., elemental fluorine, organofluoroxy reagents, and perchloryl fluoride) and nucleophilic (i.e., Olah's reagent, $\mathrm{SF}_{4}$, and $\mathrm{BrF}_{3}$ ) sources of fluorine. ${ }^{18}$ Also of great focus is the transition metal mediated trifluoromethylation of aromatics. ${ }^{25}$

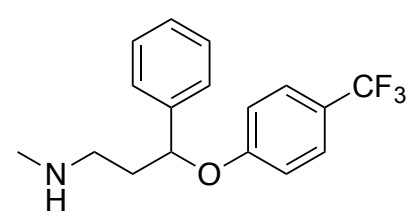

Fluoxetine

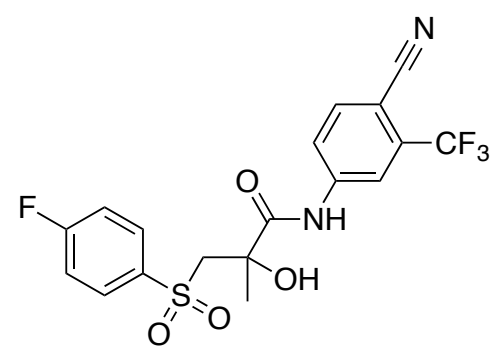

Bicalutamide

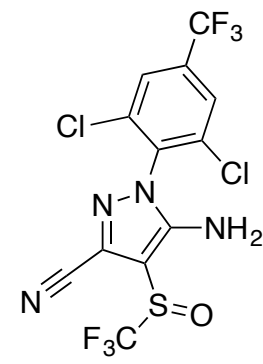

Fipronil

Figure 5.4. Pharmaceuticals containing $\mathrm{CF}_{3}$ groups.

As a complement to these methods, our approach for the synthesis of small molecules containing fluorine utilizes a stable, commercially available starting material (TFT). After binding this aromatic, the $\{\mathrm{TpMo}(\mathrm{NO})(\mathrm{DMAP})\}$ fragment can control the tandem electrophilic-nucleophilic additions to yield 1,3-dienes, 105 and $\mathbf{1 0 6}$, with great 
regio- and stereocontrol. The formation of these two 1,3-dienes represents a simple method for the synthesis of a variety of new organic molecules bearing a $\mathrm{CF}_{3}$ group. Of interest is the potential to perform an iodolactonization on $\mathbf{1 0 5}$ to potentially yield the benzofuranone 107 (Scheme 5.5). Furthermore, the recent advances in Michael-Michael ring closing (MIMIRC) reactions could yield multicyclic organics $(\mathbf{1 0 8})$ with $\mathrm{CF}_{3}$ groups (Scheme 5.5).

Scheme 5.5. Future reactivity for chemical elaboration from $\mathbf{1 0 0 .}$

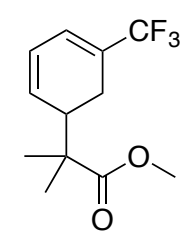

105

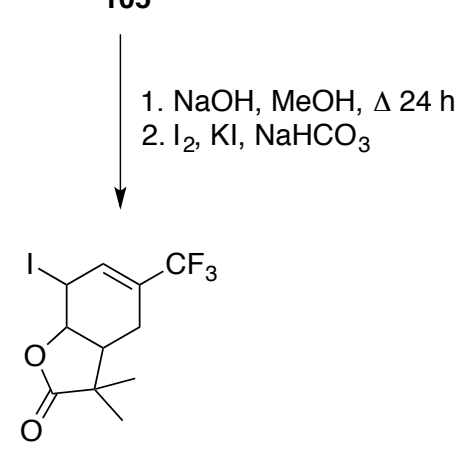

107
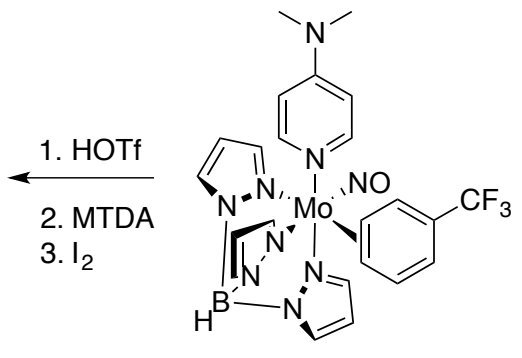

100
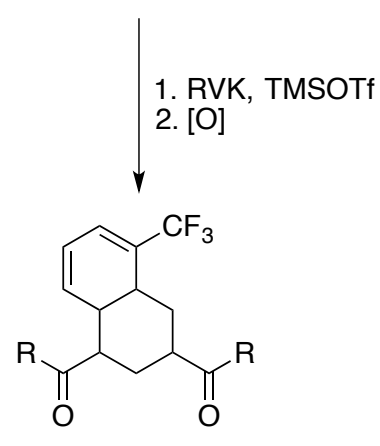

108

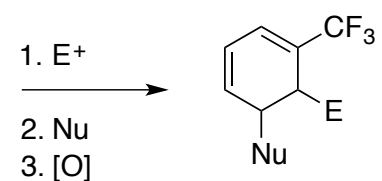

109
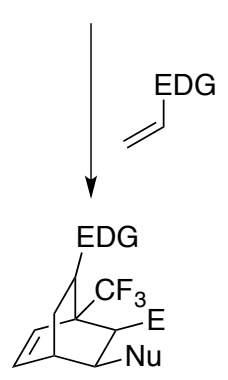

110

With the compatibility of $\mathbf{1 0 0}$ and HOTf, similar methods for chemical elaboration mentioned in Chapters 2-4 are expected to be applicable to the synthesis of various novel organics from 100. Future exploration of this chemistry will focus on the use of additional electrophiles (i.e., acetals, Michael acceptors, etc.) and nucleophiles (i.e., pyrazole, LiDMM, nitromethane, etc.) for tandem addition reactions to make $\mathbf{1 0 9}$. 
Reactivity of these 1,3-dienes (105 and 106) is virtually unknown due to a lack of practical methods for their synthesis. As such, they represent potential candidates for inverse-electron-demand dienes that could be utilized in Diels-Alder reactions (IEDD) (Scheme 5.5). ${ }^{21}$

With the success demonstrated in the isolation of $\mathbf{1 0 0}$, the isolation of other eta-2 bound fluorinated aromatic complexes of $\{\mathrm{TpMo}(\mathrm{NO})(\mathrm{DMAP})\}$ is being pursued. Utilizing the significantly lower $t_{1 / 2}$ for exchange of $\mathbf{1 0 0}$, in comparison to TpMo(NO)(DMAP)( $\eta^{2}$-dimethylfuran) complex, the ability to exchange the TFT ligand of $\mathbf{1 0 0}$ with other $\pi$-ligands has shown to be an invaluable tool in binding previously inaccessible ligands. Another benefit in using TpMo(NO)(DMAP)( $\left.\eta^{2}-\mathrm{TFT}\right)$ in these exchanges is that the $\{\mathrm{TpMo}(\mathrm{NO})(\mathrm{DMAP})\}$ fragment is a weaker $\pi$-base than its thirdrow congeners. Although this is classically referred to as a disadvantage of the $\{\mathrm{TpMo}(\mathrm{NO})(\mathrm{L})\}$ fragment, having a decreased $\pi$-basicitiy decreases the potential for $\mathrm{C}-\mathrm{F}$ oxidative addition, which has shown to be the dominant pathway for the attempted exchange reactions of $\mathrm{TpW}(\mathrm{NO})\left(\mathrm{PMe}_{3}\right)\left(\eta^{2}\right.$-benzene) with many fluorinated aromatics. As such, great progress has been achieved as a separate project aimed at binding a variety of fluorinated aromatics without inserting into the C-F bond (Figure 5.5). ${ }^{26}$ As a future project, these newly discovered complexes will be investigated for their ability to undergo chemical transformation with the hopes of achieving novel, fluorinated organics. Furthermore, the high off-rate of the TFT ligand has been utilized to achieve the binding of free benzene, to yield TpMo(NO)(DMAP) $\left(\eta^{2}\right.$-benzene) (111). 
Figure 5.5. Newly bound fluorinated aromatic complexes of $\{\mathrm{TpMo}(\mathrm{NO})(\mathrm{DMAP})\}{ }^{26}$

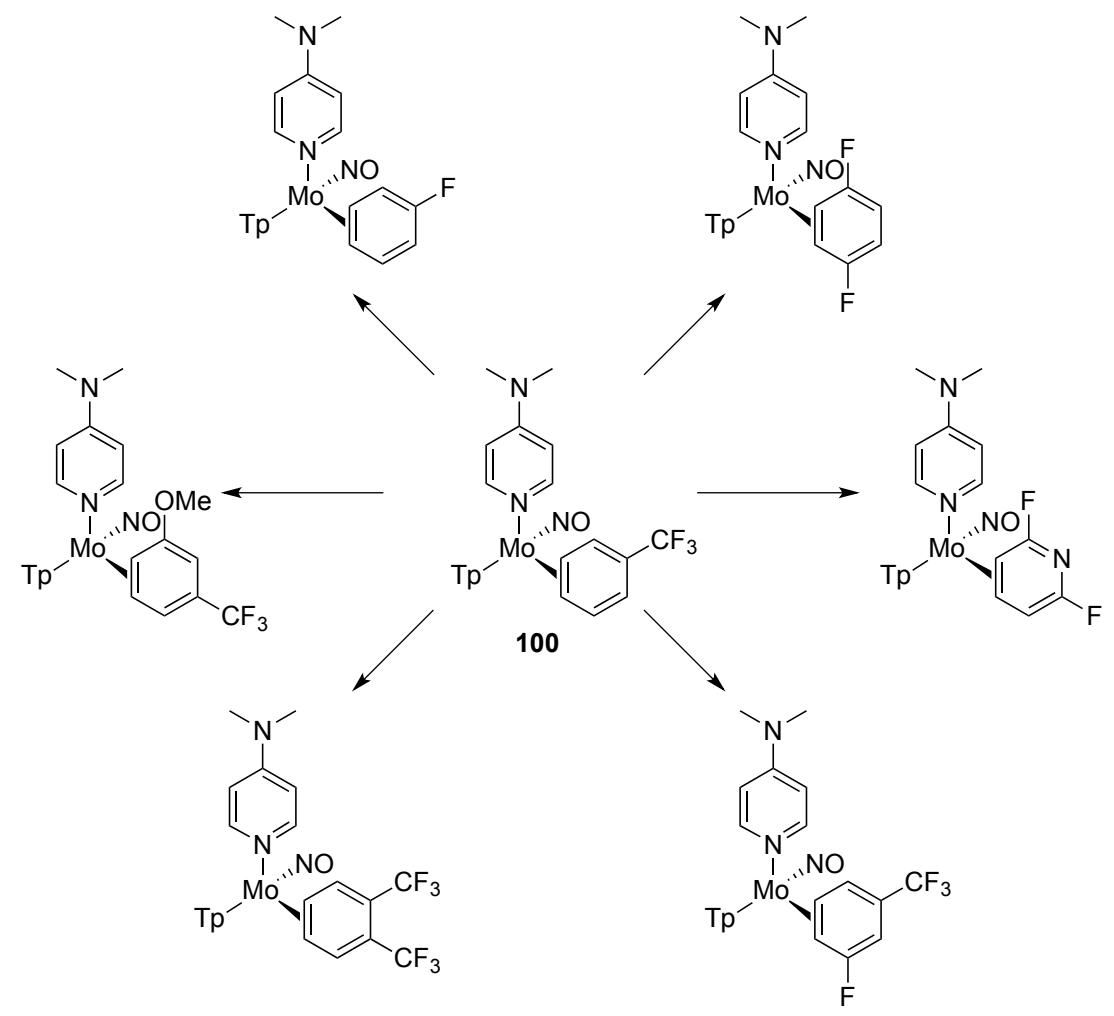

By stirring 100 in a large excess of benzene, 111 can be isolated and characterized by $\mathrm{CV}$ and ${ }^{1} \mathrm{H}$ NMR (Scheme 5.6). ${ }^{27}$ Considering the TFT complex has a $\Delta \mathrm{G}^{\ddagger}=22.3 \pm$ $0.08 \mathrm{kcal} / \mathrm{mol}$, along with the approximated three $\mathrm{kcal} / \mathrm{mol}$ additional stability gained from having a $\mathrm{CF}_{3}$ group on the benzene ring, one would expect this newly reported complex (111) to have a $\Delta \mathrm{G}^{\ddagger} \approx 19 \mathrm{kcal} / \mathrm{mol}$. Monitoring the rate of the exchange of the benzene ligand of $\mathbf{1 1 1}$ for $\mathrm{d}^{6}$-acetone, we determined through the Eyring equation that TpMo(NO)(DMAP) $\left(\eta^{2}\right.$-benzene) has a $\Delta \mathrm{G}^{\ddagger}=19.4 \mathrm{kcal} / \mathrm{mol}$, which is in agreement with the predicted value. This breakthrough in second-row dearomatization is currently being investigated as a separate project with the expectation that the expansive chemical 
libraries developed with the $\left\{\mathrm{TpW}(\mathrm{NO})\left(\mathrm{PMe}_{3}\right)\right\}$ fragment will soon be within reach of the $\{$ TpMo(NO)(DMAP) $\}$ fragment.

\subsection{Conclusion}

To our knowledge, complex $\mathbf{1 0 0}$ is the first example of an eta-2 bound substituted benzene complex, not derived from a third-row metal, that is thermally stable and capable of undergoing electrophilic-nucleophilic additions on the arene. The synthesis of $\mathbf{1 0 0}$ can be done on a $50 \mathrm{~g}$ scale, with fair yields $(65 \%)$, and its air-stable precursor (23) has been produced on a $170 \mathrm{~g}$ scale in $66 \%$ overall yield from $\mathrm{Mo}(\mathrm{CO})_{6}$. Although Os, Re, and W have demonstrated similar reactivity, the low cost, superior yields, ease of handling, and recyclability offered with Mo make 100 a potentially valuable new tool for organic synthesis. 


\subsection{Experimental}

General Methods. NMR spectra were obtained on a 600 or $800 \mathrm{MHz}$ spectrometer. All chemical shifts are reported in ppm, and proton and carbon shifts are referenced to tetramethylsilane (TMS) utilizing residual ${ }^{1} \mathrm{H}$ or ${ }^{13} \mathrm{C}$ signals of the deuterated solvents as an internal standard. Fluorine chemical shifts are referenced to a solution of hexafluorobenzene in $\mathrm{d}^{6}$-Acetone $\left(-164.9 \mathrm{ppm}\right.$ relative to $\left.\mathrm{CFCl}_{3}\right)$ contained in a capillary tube. Coupling constants $(J)$ are reported in hertz $(\mathrm{Hz})$. Infrared spectra (IR) were recorded as a glaze on a spectrometer fitted with a horizontal attenuated total reflectance (HATR) accessory or on a diamond anvil ATR assembly. Electrochemical experiments were performed under a dinitrogen atmosphere. Cyclic voltammetry data were taken at ambient temperature $\left(\sim 25^{\circ} \mathrm{C}\right)$ at $100 \mathrm{mV} / \mathrm{s}$ in a standard three-electrode cell with a glassy carbon working electrode, $\mathrm{N}, \mathrm{N}$-dimethylacetamide (DMA) or acetonitrile $\left(\mathrm{CH}_{3} \mathrm{CN}\right)$ solvent (unless otherwise specified), and tetrabutylammonium hexafluorophosphate (TBAH) electrolyte (approximately $0.5 \mathrm{M}$ ). All potentials are reported versus NHE (normal hydrogen electrode) using cobaltocenium hexafluorophosphate $\left(E_{1 / 2}=-0.78 \mathrm{~V}\right)$, ferrocene $\left(E_{1 / 2}=+0.55 \mathrm{~V}\right)$, or decamethylferrocene $\left(E_{1 / 2}=+0.04 \mathrm{~V}\right)$ as an internal standard. The peak-to-peak separation was less than $100 \mathrm{mV}$ for all reversible couples. Unless otherwise noted, all synthetic reactions were performed in a glovebox under a dry nitrogen atmosphere. Deuterated solvents were used as received. Pyrazole (Pz) protons of the (trispyrazolyl) borate (Tp) ligand were uniquely assigned (e.g., "Pz3B") using a combination of two-dimensional NMR data and (dimethylamino)pyridine-proton NOE interactions (see Figure 3.4). When unambiguous assignments were not possible, Tp protons were labeled as "Pz3/5 or Pz4". All $J$ values for Pz protons are $2( \pm 0.2) \mathrm{Hz}$. 


\section{Synthesis of TpMo(NO)(DMAP)(3,4- $\eta^{2}-\alpha, \alpha, \alpha-$ trifluorotoluenene) (100)}

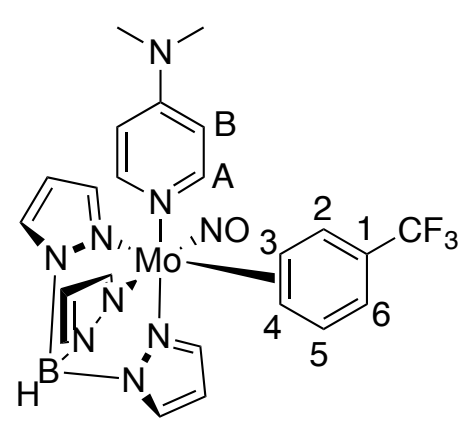

A

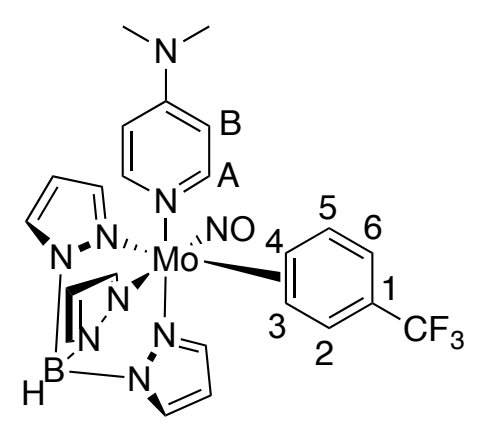

B

To a $250 \mathrm{~mL}$ round bottom flask charged with a stir egg were added sodium dispersion (30-35\% by weight, $12 \mathrm{~g}$ dispersion, $0.183 \mathrm{~mol}$ ) and hexanes $(200 \mathrm{~mL})$. The grey mixture formed was stirred at $1150 \mathrm{RPM}$ for $18 \mathrm{~h}$ at which point the hexanes was decanted off. Then, TFT (75 mL, $0.431 \mathrm{~mol}), \mathrm{Et}_{2} \mathrm{O}(25 \mathrm{~mL})$ and $23(24 \mathrm{~g}, 0.0408 \mathrm{~mol})$ were added to the remaining solid. The resulting green reaction mixture was stirred at 1150 RPM for 24 h. This dark red mixture was then transferred to a $500 \mathrm{~mL}$ Erlenmeyer flask containing $\mathrm{Et}_{2} \mathrm{O}(200 \mathrm{~mL})$ and the resulting mixture was stirred vigorously. While purging the box with nitrogen, half of this mixture was then slowly added to stirring $\mathrm{MeOH}(150 \mathrm{~mL})$, yielding a red precipitate. This precipitate was then collected on a $350 \mathrm{~mL}$ fine porosity fritted disc. The second half of the reaction mixture was then slowly added to a separate stirring solution of $\mathrm{MeOH}(150 \mathrm{~mL})$, yielding a red precipitate. This precipitate was then collected on the same $350 \mathrm{~mL}$ fine porosity fritted disc and was washed with $\mathrm{Et}_{2} \mathrm{O}(4 \times 50$ $\mathrm{mL}$ ). The resulting red precipitate was transferred to a $250 \mathrm{~mL}$ Erlenmeyer flask containing $\mathrm{Et}_{2} \mathrm{O}(100 \mathrm{~mL})$ and this mixture was stirred for $1 \mathrm{~h}$. This precipitate was then collected on a $350 \mathrm{~mL}$ fine porosity fritted disc, washed with $\mathrm{Et}_{2} \mathrm{O}(3 \times 50 \mathrm{~mL})$, and 
dessicated for $2 \mathrm{~h}$ to yield the solid $100(13.10 \mathrm{~g}, 65 \%)$ CV (DMAc) $E_{\mathrm{p}, \mathrm{a}}=-0.28 \mathrm{~V}$ (NHE). IR: $v(\mathrm{BH})=2481 \mathrm{~cm}^{-1}, v(\mathrm{NO})=1586 \mathrm{~cm}^{-1}$. Two coordination diastereomers $\mathbf{A}: \mathbf{B}=1: 1{ }^{1} \mathrm{H}$ NMR $\left(\mathrm{d}^{6}\right.$-Acetone, $\left.\delta,-30{ }^{\circ} \mathrm{C}\right): 8.17(4 \mathrm{H}$, buried broad s, DMAP-A for A and B), $8.11(2 \mathrm{H}$, overlapping doubletsy , Tp3,5), $8.02(1 \mathrm{H}, \mathrm{d}, \mathrm{Tp} 3,5), 8.01(1 \mathrm{H}, \mathrm{d}$, Tp3,5), $7.95(3 \mathrm{H}, \mathrm{m}, \mathrm{Tp} 3,5), 7.84(1 \mathrm{H}, \mathrm{d}, \mathrm{Tp} 3,5), 7.58(1 \mathrm{H}, \mathrm{d}, \mathrm{Tp} 3,5), 7.57(1 \mathrm{H}, \mathrm{d}$, Tp3,5), $7.46(1 \mathrm{H}, \mathrm{d}, J=6.3, \mathrm{H} 2 \mathbf{B}), 7.12(1 \mathrm{H}, \mathrm{dd}, J=9.1 \& 6.2, \mathrm{H} 5 \mathbf{A}), 7.08(1 \mathrm{H}, \mathrm{d}, J=$ 5.8, H2A), $6.90(1 \mathrm{H}, \mathrm{d}, \mathrm{Tp} 3,5), 6.88(1 \mathrm{H}, \mathrm{d}, \mathrm{Tp} 3,5), 6.74(4 \mathrm{H}$, buried broad s, DMAP-B for $\mathbf{A}$ and B $), 6.70(1 \mathrm{H}, \mathrm{dd}, J=9.1 \& 6.2, \mathrm{H} 5 \mathbf{B}), 6.41$ (3H, m, Tp4), $6.37(1 \mathrm{H}, \mathrm{t}, \mathrm{Tp} 4)$, $6.29(1 \mathrm{H}, \mathrm{dd}, J=9.1 \& 1.5, \mathrm{H} 6 \mathbf{A}), 6.25(1 \mathrm{H}, \mathrm{dd}, J=9.1 \& 1.5, \mathrm{H} 6 \mathbf{B}), 6.15(2 \mathrm{H}, \mathrm{m}, \mathrm{Tp} 4)$, $3.71(1 \mathrm{H}, \mathrm{dd}, J=8.5 \& 6.2, \mathrm{H} 4 \mathbf{B}), 3.60(1 \mathrm{H}, \mathrm{t}, J=7.3, \mathrm{H} 3 \mathbf{A}), 3.30(1 \mathrm{H}, \mathrm{dd}, J=8.5 \&$ 6.3, H4A), $3.14(1 \mathrm{H}, \mathrm{t}, J=7.2, \mathrm{H} 3 \mathbf{B}), 3.09(12 \mathrm{H}, \mathrm{s}, \mathrm{NMe}) .{ }^{13} \mathrm{C}$ NMR (d $\mathrm{d}^{6}$-Acetone, $\left.\delta,-30{ }^{\circ} \mathrm{C}\right): 154.7$ (DMAP-C), 150.2 (DMAP-C), 142.4 (Tp3,5), 142.3 (Tp3,5), 142.0 (Tp3,5), 141.5 (2C, Tp3,5), 137.5 (2C, Tp3,5), 136.9 (Tp3,5), 136.8 (Tp3,5), 135.9 (4C, C6A \& C2B, Tp3,5), 135.8 (2C, C1B \& C1A), 135.2 (2C, C2A \& C5B), 127.3 (q, $J=$ 261.0, $\left.\mathrm{CF}_{3}\right), 125.5$ (q, $\left.J=261.0, \mathrm{CF}_{3}\right), 111.7(\mathrm{C} 5 \mathrm{~A}), 111.4$ (C6B), 108.0 (4C, DMAP-B), 106.9 (3C, Tp4), 106.4 (3C, Tp4), 78.7 (C4B), 76.2 (C4A), 75.4 (C3A), 72.9 (C3B), $30.07(\mathrm{NMe}) .{ }^{19} \mathrm{~F}$ NMR (d - Acetone, $\left.\delta\right):-62.41$. 


\section{Synthesis of TpMo(NO)(DMAP)( $\eta^{2}$-methyl 2-methyl-2-(5-(trifluoromethyl)} cyclohexa-2,4-dien-1-yl)propanoate) (103).

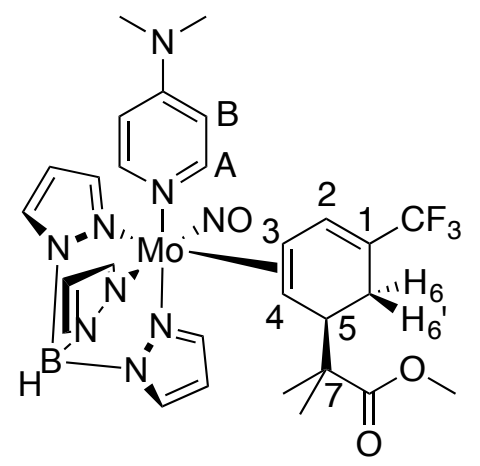

100 (500 mg, $0.80 \mathrm{mmol}), \mathrm{CH}_{3} \mathrm{CH}_{2} \mathrm{CN}(5 \mathrm{~mL})$ and a stir pea, were added to a test tube and this orange mixture was cooled for $15 \mathrm{~min}$ at $-60^{\circ} \mathrm{C}$. A $-60^{\circ} \mathrm{C}, 1 \mathrm{M}$ solution of HOTf in $\mathrm{CH}_{3} \mathrm{CH}_{2} \mathrm{CN}(2.0 \mathrm{~mL}, 2.0 \mathrm{mmol})$ was added to the reaction mixture and the resulting red solution was left standing at $-60^{\circ} \mathrm{C}$. After $15 \mathrm{~min}$, MTDA $(1.0 \mathrm{~mL}, 4.9 \mathrm{mmol})$ was added to the reaction mixture and the resulting red solution was left stirring at $-60{ }^{\circ} \mathrm{C}$. After $18 \mathrm{~h}$, a $-60{ }^{\circ} \mathrm{C}$ solution of triethylamine $(1.0 \mathrm{~mL}, 7.17 \mathrm{mmol})$ was added to the reaction mixture and the resulting brown solution was chromatographed through a $60 \mathrm{~mL}$ medium porosity fritted disc $3 / 4$ full with silica gel. The product was eluted with $1: 1$ $\mathrm{Et}_{2} \mathrm{O}$ :benzene $(100 \mathrm{~mL})$ as a yellow band, collected as a yellow solution, and evaporated in vacuo. The resulting yellow oil was then dissolved in DCM $(1 \mathrm{~mL})$ and the product was precipitated in stirring pentane $(20 \mathrm{~mL})$. The precipitate was collected on a $15 \mathrm{~mL}$ fine porosity fritted disc, washed with pentane $(3 \times 50 \mathrm{~mL})$, and desiccated for $15 \mathrm{~min}$ yielding the light yellow solid $103(299 \mathrm{mg}, 50 \%)$. CV (DMAc) $E_{\mathrm{p}, \mathrm{a}}=+0.32 \mathrm{~V}$ (NHE). IR: $v(\mathrm{BH})=2480 \mathrm{~cm}^{-1}, v(\mathrm{CO})=1721 \mathrm{~cm}^{-1}, v(\mathrm{NO})=1571 \mathrm{~cm}^{-1} . \mathrm{H}$ NMR $\left(\mathrm{d}^{6}\right.$-Acetone,

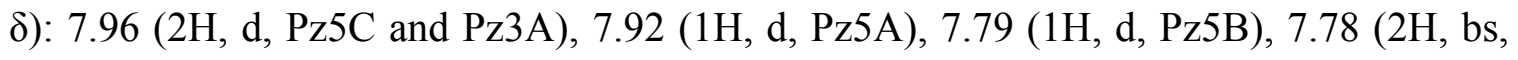
DMAP-A), 7.66 (1H, d, Pz3C), 7.09 (1H, d, Pz3B), 6.62 (3H, m, DMAP-B \& H2), 6.38 
(1H, t, Pz4A), 6.37 (1H, t, Pz4C), 6.13 (1H, t, Pz4B), 3.29 (1H, d, J = 9.8, H5), 3.24 (3H, s, OMe), $3.08(6 \mathrm{H}, \mathrm{s}, \mathrm{N}-\mathrm{Me}), 2.93(1 \mathrm{H}, \mathrm{m}, \mathrm{H} 6), 2.86(1 \mathrm{H}, \mathrm{t}, J=7.9, \mathrm{H} 3), 2.11(1 \mathrm{H}, \mathrm{d}, J=$ 18.6, H6'), 1.89 (1H, dt, $J=9.8 \& 1.8, \mathrm{H} 4), 1.23$ (3H, s, Me), 1.04 (3H, s, Me). ${ }^{13} \mathrm{C}$ NMR (d $\mathrm{d}^{6}$-Acetone, $\left.\mathrm{d}\right): 178.2$ (CO), 155.3 (DMAP-C), 151.1 (DMAP-A), 142.6 (Pz3A), 142.1 (Pz3B), 141.6 (Pz3C), 137.5 (q, $J=6.9, \mathrm{C} 2), 137.4$ (Pz5C), 137.2 (Pz5A), 135.8 (Pz3B), 126.8 (q, $\left.J=264.0, \mathrm{CF}_{3}\right), 115.5$ (1C, q, $\left.J=29.0, \mathrm{C} 1\right), 108.2$ (DMAP-B), 106.9 (Pz4), 106.4 (Pz4), 106.3 (Pz4), 65.3 (C4), 60.8 (C3), 51.2 (OMe), 51.1 (C7), 42.6 (C5), 39.2 (NMe), 22.9 (Me), 22.4 (C6), $22.3(\mathrm{Me}) .{ }^{19} \mathrm{~F}$ NMR (d ${ }^{6}$-Acetone, $\left.\delta\right):-65.38$. EA: Calculated for $\mathrm{C}_{28} \mathrm{H}_{35} \mathrm{BF}_{3} \mathrm{MoN}_{9} \mathrm{O}_{3}: \mathrm{C}, 47.41 ; \mathrm{H}, 4.97 ; \mathrm{N}, 17.77$. Found: $\mathrm{C}, 47.68 ; \mathrm{H}, 5.22$; N, 17.65.

\section{Synthesis of TpMo(NO)(DMAP)( $\eta^{2}$ - 1-methyl-2-(5-(trifluoromethyl)cyclohexa-2,4-}

dien-1-yl)-1 $H$-pyrrole) (104).

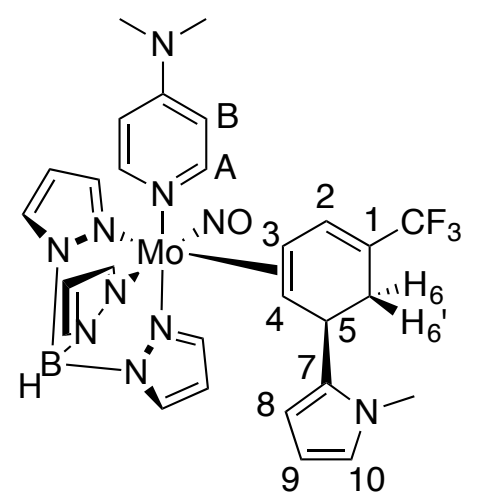

100 (500 mg, $0.80 \mathrm{mmol}), \mathrm{CH}_{3} \mathrm{CH}_{2} \mathrm{CN}(5 \mathrm{~mL}$ ) and a stir pea, were added to a test tube and this orange mixture was cooled for 15 min at $-60{ }^{\circ} \mathrm{C}$. A $-60{ }^{\circ} \mathrm{C}, 1 \mathrm{M}$ solution of HOTf in $\mathrm{CH}_{3} \mathrm{CH}_{2} \mathrm{CN}(2.0 \mathrm{~mL}, 2.0 \mathrm{mmol})$ was added to the reaction mixture and the resulting red solution was left standing at $-60{ }^{\circ} \mathrm{C}$. After $15 \mathrm{~min}, \mathrm{~N}$-methylpyrrole $(1.0 \mathrm{~mL}, 11.0$ mmol) was added to the reaction mixture and the resulting red solution was left stirring at 
$-60{ }^{\circ} \mathrm{C}$. After $18 \mathrm{~h}$, a $-60{ }^{\circ} \mathrm{C}$ solution of triethylamine $(1.0 \mathrm{~mL}, 7.17 \mathrm{mmol})$ was added to the reaction mixture and the resulting brown solution was chromatographed through a 60 $\mathrm{mL}$ medium porosity fritted disc $3 / 4$ full with silica gel. The product was eluted with $\mathrm{Et}_{2} \mathrm{O}$ $(100 \mathrm{~mL})$ as a yellow band, collected as a yellow solution, and evaporated in vacuo. The resulting yellow oil was then dissolved in DCM $(2 \mathrm{~mL})$ and the product was precipitated in stirring pentane $(75 \mathrm{~mL})$. The precipitate was collected on a $15 \mathrm{~mL}$ fine porosity fritted disc, washed with pentane ( $3 \times 10 \mathrm{~mL})$, and desiccated for 15 min yielding the light yellow solid $104(205 \mathrm{mg}, 37 \%)$. CV (DMAc) $E_{\mathrm{p}, \mathrm{a}}=+0.35 \mathrm{~V}(\mathrm{NHE}) . \mathrm{IR}: v(\mathrm{BH})=2479$ $\mathrm{cm}^{-1}, v(\mathrm{NO})=1574 \mathrm{~cm}^{-1} .{ }^{1} \mathrm{H}$ NMR $\left(\mathrm{d}^{6}\right.$-Acetone, $\left.\delta\right): 8.12(1 \mathrm{H}, \mathrm{d}, \mathrm{Pz} 3 \mathrm{~A}), 7.94(1 \mathrm{H}, \mathrm{d}$, Pz3B), 7.93 (1H, d, pz5A), 7.83 (2H, m, DMAP-A), 7.80 (1H, d, Pz5B), $7.61(1 \mathrm{H}, \mathrm{d}$, Pz3C), 7.12 (1H, d, Pz3B), $6.81(1 \mathrm{H}, \mathrm{m}, \mathrm{H} 2), 6.63$ (2H, m, DMAP-B), 6.40 (1H, t, Pz4A), 6.35 (1H, t, $J=2.0, \mathrm{H} 10), 6.33$ (1H, t, Pz4C), 6.14 (1H, t, Pz4B), $6.00(1 \mathrm{H}, \mathrm{m}$, H8), $5.77(1 \mathrm{H}, \mathrm{t}, J=3.0, \mathrm{H} 9), 4.03(1 \mathrm{H}, \mathrm{d}, J=8.2, \mathrm{H} 5), 3.34(3 \mathrm{H}, \mathrm{s}, \mathrm{NMe}), 3.16(1 \mathrm{H}, \mathrm{m}$, H6), 3.08 (6H, s, NMe), 2.85 (1H, t, $J=9.1, \mathrm{H} 3), 2.33$ (1H, t, $J=9.1, \mathrm{H} 6$ '), 2.24 (1H, dt, $J=9.1 \& 1.7, \mathrm{H} 4) .{ }^{13} \mathrm{C}$ NMR ( $\mathrm{d}^{6}$-Acetone, d): 155.3 (DMAP-C), 151.1 (DMAP-A), 142.8 (Pz3A), 142.2 (Pz3C), 141.6 (Pz3B), 141.3 (C7), 137.5 (q, $J=6.1, \mathrm{C} 2), 137.4$ (Pz5C and Pz5A), 135.8 (Pz5B), 126.8 (q, $J=268.0$, CF3), 120.7 (C10), 114.4 (q, $J=$ 30.0, C1), 108.2 (DMAP-B), 106.9 (Pz4C), 106.8 (C9), 106.6 (Pz4A), 106.4 (Pz4B), 105.7 (C8), 71.8 (C4), 58.8 (C3), 39.2 (DMAP Mes), 33.4 (NMe), 32.9 (C5), 27.3 (C6). ${ }^{19}$ F NMR ( $d^{6}$-Acetone, $\left.\delta\right):-65.40$. 


\section{Synthesis of methyl 2-methyl-2-(5-(trifluoromethyl)cyclohexa-2,4-dien-1-} yl)propanoate (105).

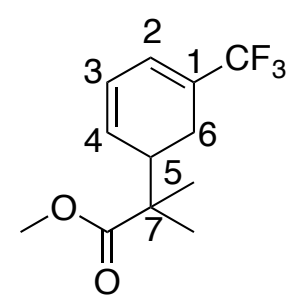

To a $50 \mathrm{~mL}$ filter flask charged with a stir pea were added 103 (100 mg, $0.145 \mathrm{mmol})$, $\mathrm{DCM}(5 \mathrm{~mL})$, and a $0.06 \mathrm{M}$ solution of $\mathrm{I}_{2} / \mathrm{Et}_{2} \mathrm{O}(1.2 \mathrm{~mL}, 0.073 \mathrm{mmol})$ resulting in a green solution. The solution was stirred at room temperature for $5 \mathrm{~min}$ and then evaporated in vacuo to an oil. The oil was dissolved in DCM (1 mL), giving a solution that was then added to stirring pentane $(25 \mathrm{~mL})$, creating a green precipitation. The precipitate was collected on a $15 \mathrm{~mL}$ fine porosity fritted disc, washed with pentane $(3 \times 10 \mathrm{~mL})$, and desiccated to yield 23 (73 $\mathrm{mg}, 89 \%$ ). The filtrate was removed from the glovebox and evaporated in vacuo to a brown oil. The oil was dissolved in DCM (3 x $0.3 \mathrm{~mL})$ and the resulting solution was dropwise added onto a $250 \mu \mathrm{m}$ silica preparatory plate. The product was eluted with 10\% EtOAc:hexanes $(200 \mathrm{~mL})$, scraped off as a band at $\mathrm{R}_{\mathrm{f}}$ : 0.41 0.62 and this silica gel was sonicated in EtOAc $(20 \mathrm{~mL})$ for $15 \mathrm{~min}$. The silica was filtered off on a $15 \mathrm{~mL}$ medium porosity fritted disc and washed with DCM $(3 \times 2 \mathrm{~mL})$. The colorless filtrate was then evaporated in vacuo and desiccated to yield the colorless oil $105\left(16 \mathrm{mg}, 46 \%\right.$ yield). IR: $v\left(\mathrm{C}-\mathrm{H} \mathrm{sp}{ }^{2}\right)=22925 \mathrm{~cm}^{-1}, v(\mathrm{CO})=1731 \mathrm{~cm}^{-1} \cdot{ }^{1} \mathrm{H}$ NMR $\left(\mathrm{d}^{6}\right.$-Acetone, $\left.\delta\right): 6.36(1 \mathrm{H}, \mathrm{m}, \mathrm{H} 2), 6.04(1 \mathrm{H}, \mathrm{m}, \mathrm{H} 3), 5.83(1 \mathrm{H}, \mathrm{dd}, J=9.6$ \& 3.9, H4), $3.69(3 \mathrm{H}, \mathrm{s}, \mathrm{OMe}), 2.86(1 \mathrm{H}, \mathrm{m}, \mathrm{H} 6), 2.32(1 \mathrm{H}, \mathrm{m}, \mathrm{H} 5), 2.21(1 \mathrm{H}, \mathrm{m}, \mathrm{H} 6), 1.19(3 \mathrm{H}, \mathrm{s}$, Me), $1.18(3 \mathrm{H}, \mathrm{s}, \mathrm{Me}) .{ }^{13} \mathrm{C}$ NMR (d $\mathrm{d}^{6}$-Acetone, $\left.\delta\right): 177.4(\mathrm{CO}), 131.4(\mathrm{C} 4), 125.6$ (q, $J=$ 30.9, C1), $124.8(\mathrm{q}, J=6.5, \mathrm{C} 2), 124.0\left(\mathrm{q}, J=270.0, \mathrm{CF}_{3}\right), 123.5(\mathrm{C} 3), 52.2(\mathrm{OMe}), 45.7$ 


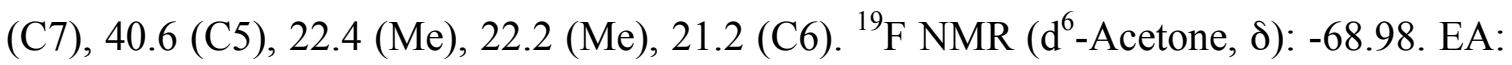

Calculated for $\mathrm{C}_{12} \mathrm{H}_{15} \mathrm{~F}_{3} \mathrm{O}_{2}$ : C, 58.06; H, 6.09. Found: C, 58.61; H, 6.22.

\section{Synthesis of 1-methyl-2-(5-(trifluoromethyl)cyclohexa-2,4-dien-1-yl)-1H-pyrrole} (106).

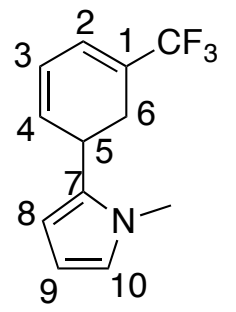

To a $50 \mathrm{~mL}$ filter flask charged with a stir pea were added 104 (100 mg, $0.145 \mathrm{mmol})$, $\operatorname{DCM}(5 \mathrm{~mL})$, and a $0.06 \mathrm{M}$ solution of $\mathrm{I}_{2} / \mathrm{Et}_{2} \mathrm{O}(1.2 \mathrm{~mL}, 0.073 \mathrm{mmol})$ resulting in a green solution. The solution was stirred at room temperature for $5 \mathrm{~min}$ and then evaporated in vacuo to an oil. The oil was dissolved in DCM (1 mL), giving a solution that was then added to stirring hexanes $(25 \mathrm{~mL})$, creating a green precipitation. The precipitate was collected on a $15 \mathrm{~mL}$ fine porosity fritted disc, washed with hexanes $(3 \times 10 \mathrm{~mL})$, and desiccated to yield $\mathbf{2 3}$ (76 $\mathrm{mg}, 89 \%$ ). The filtrate was removed from the glovebox and evaporated in vacuo to a brown oil. The oil was dissolved in DCM $(3 \times 0.3 \mathrm{~mL})$ and the resulting solution was dropwise added onto a $250 \mu \mathrm{m}$ silica preparatory plate. The product was eluted with 10\% EtOAc:hexanes $(200 \mathrm{~mL})$, scraped off as a band at $\mathrm{R}_{\mathrm{f}}$ : 0.50 0.71 and this silica gel was sonicated in EtOAc $(20 \mathrm{~mL})$ for $15 \mathrm{~min}$. The silica was filtered off on a $15 \mathrm{~mL}$ medium porosity fritted disc and washed with DCM $(3 \times 2 \mathrm{~mL})$. The colorless filtrate was then evaporated in vacuo and desiccated to yield the colorless oil 106 (17 mg, 52\% yield). ${ }^{1} \mathrm{H}$ NMR (d ${ }^{6}$-Acetone, $\left.\delta\right): 6.59(1 \mathrm{H}, \mathrm{t}, J=2.3, \mathrm{H} 10), 6.49$ (1H, m, H2), 6.10 (1H, m, H4), $6.08(1 \mathrm{H}, 1, J=3.1, \mathrm{H} 8), 6.07(1 \mathrm{H}, \mathrm{m}, \mathrm{H} 3), 6.00(1 \mathrm{H}, \mathrm{m}$, 
H9), $3.78(1 \mathrm{H}, \mathrm{m}, \mathrm{H} 5), 3.68(3 \mathrm{H}, \mathrm{s}, \mathrm{NMe}), 2.62(1 \mathrm{H}, \mathrm{dd}, J=17.2,8.9, \mathrm{H} 5), 2.49(1 \mathrm{H}, \mathrm{m}$, H6). ${ }^{13} \mathrm{C}$ NMR (d $\mathrm{d}^{6}$-Acetone, $\delta$ ): 133.6 (C7), 133.4 (C4), 125.0 (q, $\left.J=30.5, \mathrm{C} 1\right), 124.9$ (q, $J=6.8, \mathrm{C} 2), 124.0\left(\mathrm{q}, J=272.0, \mathrm{CF}_{3}\right), 122.6(\mathrm{C} 10), 122.4(\mathrm{C} 3), 107.1(\mathrm{C} 8), 106.2(\mathrm{C} 9)$,

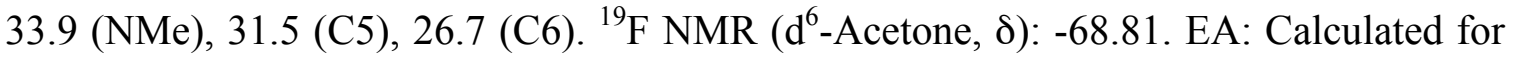
$\mathrm{C}_{12} \mathrm{H}_{12} \mathrm{~F}_{3} \mathrm{~N}$ : C, 63.43; H, 5.32; N, 6.16. Found: C, 63.22; H, 5.36; N, 6.13. 


\subsection{References}

1. Ha, Y.; Dilsky, S.; Graham, P. M.; Liu, W.; Reichart, T.; Sabat, M.; Kenae, J. M.; Harman, W. D. Organometallics 2006, 25, 5184.

2. Meiere, S. H.; Keane, J. M.; Gunnoe, T. B.; Sabat, M.; Harman, W. D. J. Am. Chem. Soc. 2003, 125, (8), 2024.

3. Mocella, C. J.; Delafuente, D. A.; Keane, J. M.; Warner, G. R.; Friedman, L. A.; Sabat, M.; Harman, W. D. Organometallics 2004, 23, (16), 3772.

4. Harman, W. D.; Sekine, M.; Taube, H. J. Am. Chem. Soc. 1988, 110, 5725.

5. Harman, W. D. Chem. Rev. 1997, 97, 1953.

6. Brooks, B. C.; Meiere, S. H.; Friedman, L. A.; Gunnoe, T. B.; Harman, W. D. J. Am. Chem. Soc. 2001, 123, 3541.

7. Welch, K. D.; Harrison, D. P.; Lis, E. C.; Liu, W.; Salomon, R. J.; Harman, W. D.; Myers, W. H. Organometallics 2007, 26, (10), 2791.

8. $\quad$ Myers, J. T.; Dakermanji, S. J.; Chastanet, T. R.; Shivokevich, P. J.; Strausberg, L. J.; Sabat, M.; Myers, W. H.; Harman, W. D. Organometallics 2016.

9. Bengali, A. A. Organometallics 2000, 19, (19), 4000.

10. Bengali, A. A.; Charlton, S. B. Journal of Chemical Education 2000, 77, (10), 1348.

11. Bengali, A. A.; Grunbeck, A. R. Organometallics 2005, 24, (24), 5919.

12. Bengali, A. A.; Leicht, A. Organometallics 2001, 20, (7), 1345.

13. Rassolov, V. A.; Ratner, M. A.; Pople, J. A.; Redfern, P. C.; Curtiss, L. A. J. Comput. Chem 2001, 22, (9), 976.

14. Mann, B. E. Ann. Rep. on NMR Spec. 1982, 12, 263.

15. Ding, F.; Harman, W. D. J. Am. Chem. Soc. 2004, 126, (42), 13752.

16. Bégué, J.-P.; Bonnet-Delpon, D. J. Fluorine Chem. 2006, 127, (8), 992.

17. Isanbor, C.; O’Hagan, D. J. Fluorine Chem. 2006, 127, (3), 303.

18. Kirk, K. L. J. Fluorine Chem. 2006, 127, (8), 1013.

19. Wang, J.; Sánchez-Roselló, M.; Aceña, J. L.; del Pozo, C.; Sorochinsky, A. E.; Fustero, S.; Soloshonok, V. A.; Liu, H. Chem. Rev. 2014, 114, (4), 2432. 
20. Yerien, D. E.; Bonesi, S.; Postigo, A. Organic \& Biomolecular Chemistry 2016, 14, (36), 8398.

21. Nagai, T.; Nasu, Y.; Shimada, T.; Shoda, H.; Koyama, M.; Ando, A.; Miki, T.; Kumadaki, I. J. Fluorine Chem. 1992, 57, (1-3), 245.

22. Braden, R. P. J.; Klauke, E. Pesticide Science 1986, 17, (4), 418.

23. Ameduri, B. Chemical Reviews 2009, 109, (12), 6632.

24. Engel, A., Fluorine-Containing Dyes. In Organofluorine Chemistry: Principles and Commercial Applications, Banks, R. E.; Smart, B. E.; Tatlow, J. C., Eds. Springer US: Boston, MA, 1994; pp 315.

25. Tomashenko, O. A.; Grushin, V. V. Chemical Reviews 2011, 111, (8), 4475.

26. Full credit is given to Jacob Smith for discovery and isolation of these fluorinated aromatics.

27. Full credit is given to Jacob Smith for discovery and characterization of TpMo(NO)(DMAP) $\left(\eta^{2}\right.$-benzene). 


\section{Chapter 6}

Activation of Pyridines Using

$\{$ TpMo(NO)(DMAP)\} 


\subsection{Introduction}

Pyridines are ubiquitous in nature, commercially available, and stable, making them an attractive starting material for the synthesis of alkaloids. Furthermore, dihydropyridines are often used as building blocks for the syntheses of piperidines ${ }^{1}$ (a fully saturated pyridine), which can be found in many pharmaceuticals, ${ }^{2}$ agrochemicals, ${ }^{3}$ and petrochemicals. ${ }^{4}$ The functionalization of pyridine through aromatic substitution (either with electrophiles or nucleophiles) is challenging due to poor chemoselectivity. ${ }^{5}$ Furthermore, in comparison to benzene, pyridine has a lower energy $\pi$-system and thus is less reactive with electrophiles. This decrease in reactivity requires harsher reagents for the functionalization of pyridines. For this reason, the synthesis of dihydro-, tetrahydro-, and perhydropyridines from pyridine is most effective when the nitrogen is functionalized first. ${ }^{6}$ This method has been widely utilized in the syntheses of pyridines and 1,2-dihydropyridines, often with good regio- and stereocontrol (Scheme 6.1).

Scheme 6.1. Traditional synthetic methods for the reduction of pyridines.<smiles>Oc1cccnc1</smiles>

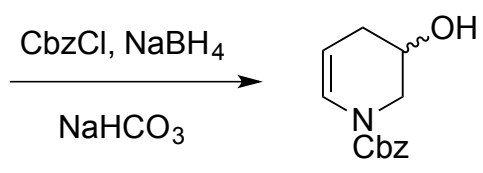

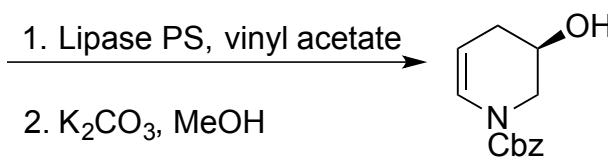<smiles>[X]OC(=O)c1cccnc1</smiles>

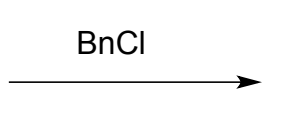<smiles>[X]OC(=O)c1ccc[n+](Cc2ccccc2)c1</smiles><smiles>COC(=O)C1=CN(Cc2ccccc2)CCC1</smiles>

Although previous reports have shown the ability for metals to bind pyridines in an eta-2 fashion, ${ }^{7-13}$ the $\left\{\mathrm{TpW}(\mathrm{NO})\left(\mathrm{PMe}_{3}\right)\right\}$ fragment is the first dearomatization agent reported that can promote electrophilic additions and subsequent derivatization to yield 
dihydropyridines. ${ }^{14-21}$ Initial investigations into the coordination of pyridines by the dearomatization agent $\left\{\mathrm{TpW}(\mathrm{NO})\left(\mathrm{PMe}_{3}\right)\right\}$ found that pyridine preferentially binds to the metal center through the nitrogen in a $\kappa^{1}$-fashion (112, Scheme 6.2).

Scheme 6.2. Coordination and reactivity of pyridines dearomatized through coordination to $\left\{\mathrm{TpW}(\mathrm{NO})\left(\mathrm{PMe}_{3}\right)\right\}$.

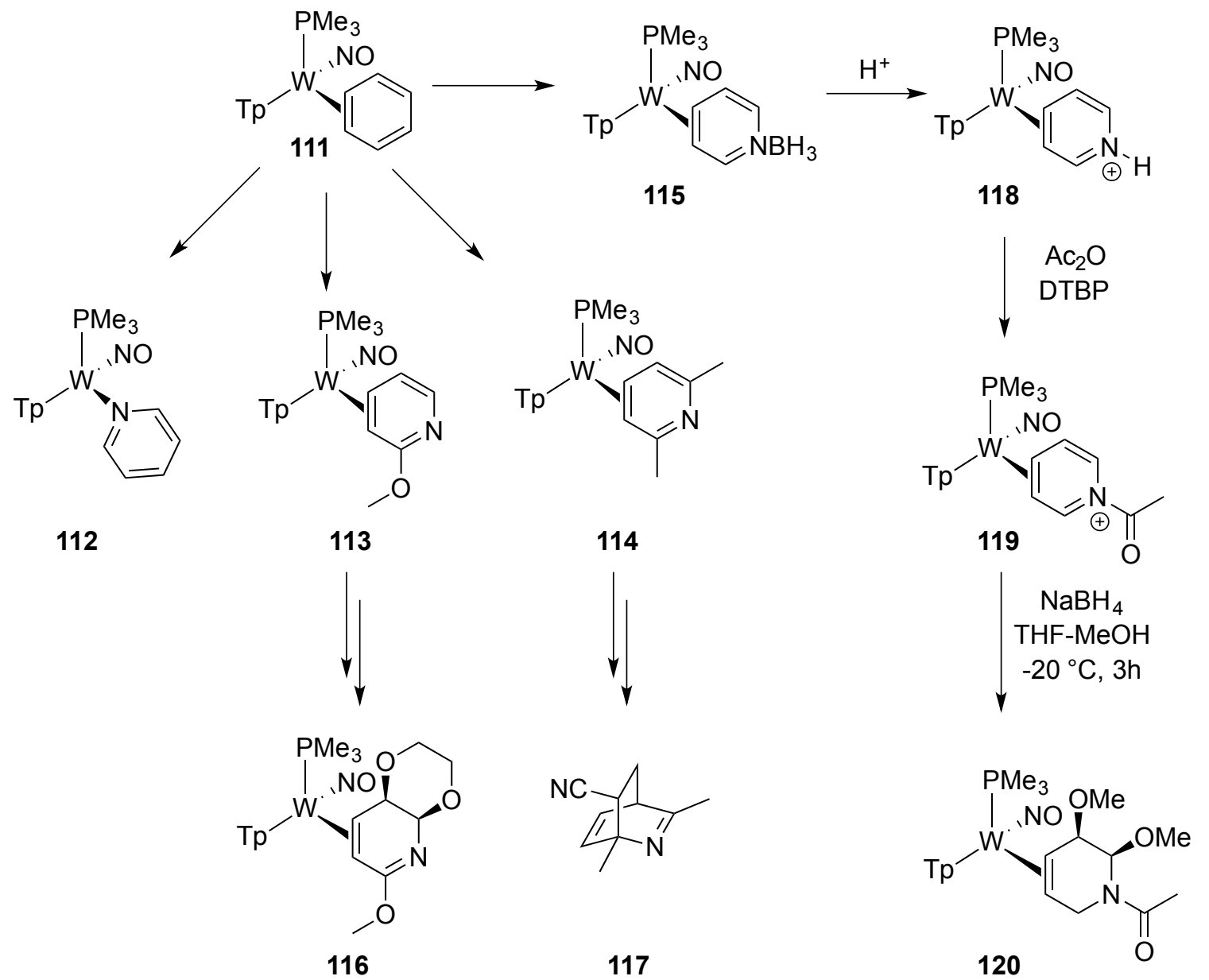

However, by taking advantage of either steric or electronic effects, the isolation of primarily $\eta^{2}$-bound complexes can be accomplished (113 and 114). ${ }^{22}$ Furthermore, using $N$-protected pyridines, the isolation of $\eta^{2}$-pyridine complexes without substituents on the ring carbons can also be achieved, such as the pyridine-borane complex (115) ${ }^{23}$ From 
these $\eta^{2}$-bound pyridine complexes a variety of alkaloid cores have been synthesized with great regio- and stereocontrol by taking advantage of the increased reactivity imparted by coordination to the electron-rich metal (Scheme 6.2). With a goal of replicating the reactivity demonstrated by the dearomatization of pyridines by $\left\{\mathrm{TpW}(\mathrm{NO})\left(\mathrm{PMe}_{3}\right)\right\}$, the coordination of pyridines to the more economically viable $\{\mathrm{TpMo}(\mathrm{NO})(\mathrm{DMAP})\}$ fragment was investigated.

\subsection{Results and Discussion}

As discussed in Chapter 3, the TpMo(NO)(DMAP) $\left(\eta^{2}-2,5\right.$-dimethylfuran) (26) complex can undergo substitution of the furan ligand in the presence of an excess of another viable backbond acceptor. Initial attempts to obtain an eta-2 bound pyridine complex with $\{\mathrm{TpMo}(\mathrm{NO})(\mathrm{DMAP})\}$ focused on ligand exchange reactions with unsubstituted pyridine. These exchanges show similar reactivity to that reported for $\mathrm{TpW}(\mathrm{NO})\left(\mathrm{PMe}_{3}\right)\left(\eta^{2}\right.$-benzene): when $\mathbf{2 6}$ was stirred in neat pyridine, a turquoise reaction mixture was obtained. As with $\left\{\mathrm{TpW}(\mathrm{NO})\left(\mathrm{PMe}_{3}\right)\right\}$, this turquoise color results from a metal-to-ligand charge transfer and indicates coordination through the nitrogen. ${ }^{24}$ Due to the inherent stability of the $\kappa^{1}$-pyridine ligands in these systems, isolation of this complex was not pursued with $\{\mathrm{TpMo}(\mathrm{NO})(\mathrm{DMAP})\}$. Next, we focused on ligand exchanges with nitrogen-protected pyridinium triflates; however, these reactions resulted in oxidative decomposition of the metal center. We then attempted to bind pyridine-borane by stirring 26 in neat pyridine-borane; however, as a dark turquoise reaction mixture was observed, an impurity of free pyridine is believed to have led to the formation of $\kappa^{1}$-pyridine complex. 
Successful isolation of a $\{\mathrm{TpMo}(\mathrm{NO})(\mathrm{DMAP})\}$ complex with an eta-2 bound substituted pyridine was achieved by stirring $\mathbf{2 6}$ in neat 2-methoxypyridine. The initial yellow mixture of $\mathbf{2 6}$ changes to a slightly darker orange heterogeneous mixture after 24 h. The success of the ligand exchange was determined by the observation of a shift in the first anodic wave over the course of the reaction from $-350 \mathrm{mV}$ to $-220 \mathrm{mV}$ as seen by $\mathrm{CV}(100 \mathrm{mV} / \mathrm{s}, \mathrm{NHE})$. The orange precipitate was isolated and triturated in $\mathrm{Et}_{2} \mathrm{O}$ to give the $\mathrm{TpMo}(\mathrm{NO})(\mathrm{DMAP})\left(\eta^{2}-2\right.$-methoxypyridine) complex (120) in yields of $73 \%$ on a $5 \mathrm{~g}$ scale. Similarly, the successful isolation of $\operatorname{TpMo}(\mathrm{NO})(\mathrm{DMAP})\left(\eta^{2}-2,6-\right.$ dimethoxypyridine) (121) can be accomplished by stirring 26 in neat 2,6dimethoxypyridine and filtering the resulting orange mixture to give a yellow precipitate on a $500 \mathrm{mg}$ scale with a yield of $54 \%$ (Scheme 6.3).

From the ${ }^{1} \mathrm{H}$ NMR spectrum of $\mathbf{1 2 1}$ the resonances for $\mathrm{H} 3$ and $\mathrm{H} 4$ for both coordination diastereomers were assigned. For 121A the resonance for $\mathrm{H} 4$ is seen as a doublet-of-doublets at $3.65 \mathrm{ppm}$ and the resonance for $\mathrm{H} 3$ is a doublet at $2.96 \mathrm{ppm}$. For 121B the resonance for $\mathrm{H} 3$ is seen as a doublet at $3.28 \mathrm{ppm}$ and the resonance for $\mathrm{H} 4$ is a doublet-of-doublets at $3.23 \mathrm{ppm}$. Using COSY analysis, the H5 assignments for both diastereomers were determined (6.24 ppm for 121A and $5.85 \mathrm{ppm}$ for 121B) and through NOE interaction with DMAP protons or Pz3A the coordination diastereomers were assigned. In contrast to $\mathrm{TpW}(\mathrm{NO})\left(\mathrm{PMe}_{3}\right)\left(\eta^{2}\right.$-2-methoxypyridine), 121 shows only a slight preference to orient the methoxy towards the ancillary ligand, and so $121 \mathrm{~A}$ and 121B are isolated in a 1:1.4 ratio (Scheme 6.3). 
Scheme 6.3. Exchange of 2,5-dimethylfuran with 2-methoxy- and 2,6-dimethoxypyridine to form $\mathrm{TpMo}(\mathrm{NO})(\mathrm{DMAP})\left(\eta^{2}-2-\right.$ methoxypyridine) and $\mathrm{TpMo}(\mathrm{NO})(\mathrm{DMAP})\left(\eta^{2}-2,6-\right.$ dimethoxypyridine complexes 121 and 122, respectively.

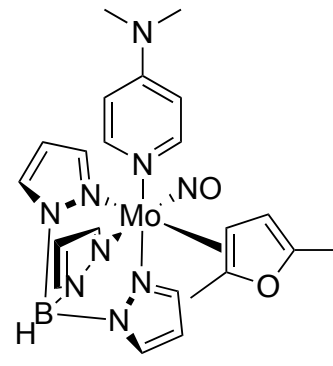

26

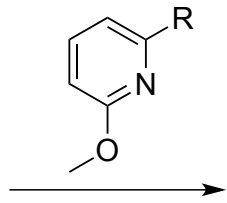

A

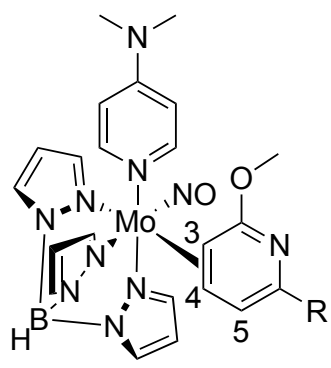

B

121: $R=H, 1: 1.4(A: B)$

122: $R=O M e, 1: 1(A: B)$

The exchange of 2-methoxypyridine with solvent ( $\mathrm{d}^{6}$-acetone) is very slow such that no $\mathrm{TpMo}(\mathrm{NO})(\mathrm{DMAP})\left(\eta^{2}\right.$-acetone) complex is observed over $6 \mathrm{~h}$ sitting in $\mathrm{d}^{6}$ acetone. The lack of exchange with acetone implies that complete dissociation of the ligand does not occur during the isomerization from $121 \mathrm{~A}$ to $121 \mathrm{~B}$, and so it is assumed that 121A and 121B are undergoing intramolecular isomerization. As 121A and 121B are not interconverted through an intrafacial isomerization, it is assumed that an interfacial (face-flip) isomerization is taking place.

Similar to $\mathrm{TpW}(\mathrm{NO})\left(\mathrm{PMe}_{3}\right)\left(\eta^{2}-2,6\right.$-dimethoxypyridine), $\mathbf{1 2 2}$ is isolated as a 1:1 mixture of coordination diastereomers (122A and 122B). The ${ }^{1} \mathrm{H}$ NMR spectrum of 122 has significant line-broadening due to isomerization occurring on the NMR time-scale. From spin-saturation exchange experiments it was found that the broadened peaks identified as the resonances for $\mathrm{H} 3$ and $\mathrm{H} 4$ of $\mathbf{1 2 2 A}$ and $\mathbf{1 2 2 B}$ exchange with peaks in the aromatic region, which led to the conclusion that the primary method of isomerization is 
intrafacial. A ${ }^{1} \mathrm{H}$ NMR experiment of a $\mathrm{CDCl}_{3}$ solution of $\mathbf{1 2 2}$ at $0{ }^{\circ} \mathrm{C}$ revealed the coupling constant between $\mathrm{H} 4$ and $\mathrm{H} 5$ of $\mathbf{1 2 2 A}$ to be $5.9 \mathrm{~Hz}$. The difference in chemical shift between $\mathrm{H} 4$ of $\mathbf{1 2 2} \mathrm{A}$ and $\mathrm{H} 4$ of $\mathbf{1 2 2 B}$ is $189 \mathrm{~Hz}$ at $0{ }^{\circ} \mathrm{C}$. The approximate coalescence temperature $\left(55^{\circ} \mathrm{C}\right)$ between $122 \mathrm{~A}$ and 122B was obtained by VT-NMR in $\mathrm{CDCl}_{3}\left(0-55^{\circ} \mathrm{C}\right)$. The low solubility of $\mathbf{1 2 2}$ limited the available deuterated solvents to $\mathrm{CDCl}_{3}$, which also limited the temperature range available for these experiments. However, the ${ }^{1} \mathrm{H}$ NMR spectrum of $\mathbf{1 2 2}$ obtained at $55^{\circ} \mathrm{C}$ shows the complex to be very near coalescene and so an upper limit $\left(65^{\circ} \mathrm{C}\right)$ and a lower limit $\left(45^{\circ} \mathrm{C}\right)$ were used to approximate a window of error $( \pm 0.5 \mathrm{kcal} / \mathrm{mol})$. From this information, the approximate rate of isomerization was determined $\left(\mathrm{k}=420 \mathrm{~s}^{-1}\right)$ and with it the free energy of activation of isomerization was approximated to be $15.5 \pm 0.5 \mathrm{kcal} / \mathrm{mol}$ at $55{ }^{\circ} \mathrm{C} .{ }^{25}$ Further analysis is needed for the full characterization of 122; however, the conjugate acid of 122 (127) has a significantly higher barrier of isomerization for the pyridinium ligand, making its full characterization possible (vide infra).

The observed coordination of these methoxypyridines to the $\{\mathrm{TpMo}(\mathrm{NO})(\mathrm{DMAP})\}$ fragment mirrors that seen with $\left\{\mathrm{TpW}(\mathrm{NO})\left(\mathrm{PMe}_{3}\right)\right\}$. For the latter, an investigation of binding for a wide range of substituted pyridines determined that the steric presence of a bulky group at the 2 position of pyridine is not sufficient to prevent binding through the nitrogen in the tungsten system, as seen by the $\kappa^{1}$ coordination of both 2-picoline and 2-ethylpyridine. Rather, a combination of sterics and electronic stabilization is needed to favor eta-2 coordination, as seen with 2methoxypyridine. The increased $\pi$-donation from the placement of a methoxy group at 
the ortho position provides delocalization of electron density over the uncoordinated portion of the ring (Figure 6.1).

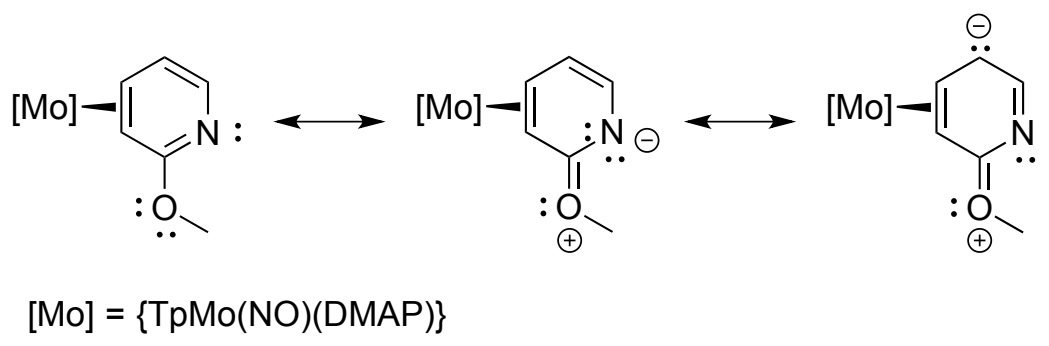

Figure 6.1. Resonance structures showing the delocalization of electron density on 2methoxypyridine via coordinating at the C3-C4 double bond.

Furthermore, this stabilization provides an energetic preference for coordination diastereomers that retain linear conjugation in the uncoordinated portion of the pyridine ligand (e.g., binding across the $\mathrm{C} 3-\mathrm{C} 4$ bond) and disfavors those with cross conjugation (e.g., binding across the C4-C5 bond). With TpW(NO) $\left(\mathrm{PMe}_{3}\right)\left(\eta^{2}-2-\right.$ methoxypyridine) the methoxy group orients proximal to the $\mathrm{PMe}_{3}$ ligand in the major isomer, ${ }^{22}$ whereas the minor isomer has the methoxy group distal $(\mathrm{cdr}=2: 1)$.

The investigation of reactivity of these preliminary pyridine complexes has focused on the organic modification of 2-methoxypyridine. Similar to organic reactions of uncoordinated pyridines, initial electrophilic additions to $\mathbf{1 2 1}$ were found to occur at the nitrogen. For instance, the addition of triflic acid (HOTf) or methyl triflate (MeOTf) to 121 yields the pyridinium complexes 123 and 124 , respectively (Scheme 6.4). 
Scheme 6.4. Electrophilic addition to 121.

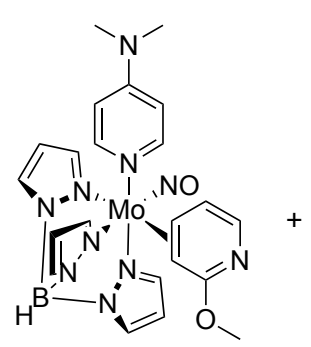

121-A

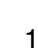

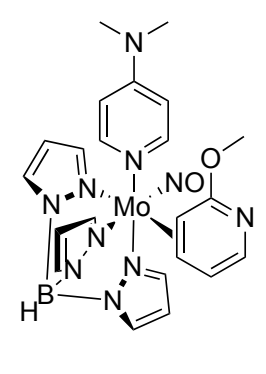

121-B

1.4

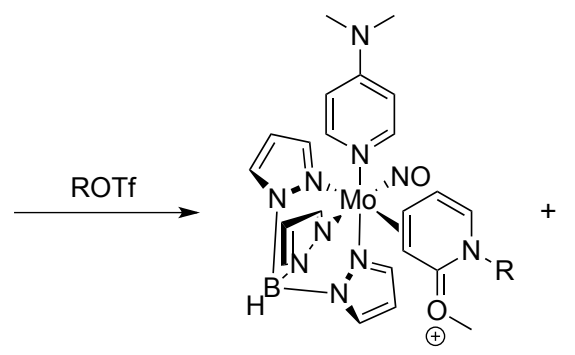

A

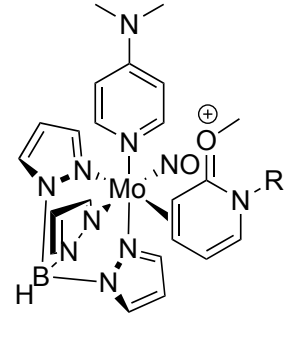

B

In the ${ }^{1} \mathrm{H}$ NMR spectrum of $\mathbf{1 2 3}$, the methoxy groups shift downfield to 3.83 and $3.80 \mathrm{ppm}$ from 3.79 and $3.62 \mathrm{ppm}$, the values seen for 121. Furthermore, the anodic waves (which approximate the formal reduction potentials) shifts from $-0.22 \mathrm{~V}$ to $\sim+0.80$ $\mathrm{V}$ upon electrophilic addition in these reactions, representing a significant decrease in electron density at the metal center. This greater electron withdrawing ability of the cationic ligands is also observed in the nitrosyl stretch frequencies, where $\mathbf{1 2 3}$ and $\mathbf{1 2 4}$ have significantly higher frequencies (1599 and $1596 \mathrm{~cm}^{-1}$, respectively) than $\mathbf{1 2 1}$ (1575 $\left.\mathrm{cm}^{-1}\right)$.

The coordination diastereomer ratio (cdr) for pyridinium complexes 123 (2:1 $A: B)$ and $124(3: 1 \mathrm{~A}: \mathrm{B})$ is higher than $121(1: 1.4 \mathrm{~A}: \mathrm{B})$. Similar to TpW(NO)(PMe $)\left(\eta^{2}-\right.$ 2-methoxypyridine), ${ }^{22}$ the oxonium species $\mathbf{1 2 3}$ and $\mathbf{1 2 4}$ favor the $\mathbf{A}$ isomer wherein the methoxy group is distal to ancillary ligand. To access the thermodynamic ratio of $\mathbf{1 2 3}$, a $\mathrm{CD}_{3} \mathrm{CN}$ solution of $\mathbf{1 2 3}$ was added to a $\mathrm{CD}_{3} \mathrm{CN}$ solution of di-tert-butylpyridine (DTBP) and observed by ${ }^{1} \mathrm{H}$ NMR over $2 \mathrm{~h}$. After $5 \mathrm{~min}$, an ${ }^{1} \mathrm{H}$ NMR spectrum of this solution revealed it to contain $\mathbf{1 2 3}$ at a 4:1 ratio of $\mathbf{A}: \mathbf{B}$. Observing this solution after 2 hours 
yielded no further change in the ratio of $\mathbf{A}: \mathbf{B}$, leading to the conclusion that the thermodynamic ratio of $\mathbf{1 2 3}$ is $4: 1$.

For the methylation of $\mathbf{1 2 1}$, the resulting product, 124, is isolated as a 3:1 ratio of A:B. This observed increase in cdr could be due to the selective precipitation of one diastereomer over the other. Considering the reported $89 \%$ yield, were the remaining theoretical yield $(137 \mathrm{mg})$ assumed to be 124B that was not collected during the precipitation, then the overall ratio would be $2: 1 \mathbf{A}: \mathbf{B}$, which is still higher than the original ratio $(1: 1.4)$. This analysis implies that the rate of methylation is slower than the rate of isomerization for 121. Interestingly, stirring a solution of 123 in $\mathrm{CH}_{3} \mathrm{CN}$ overnight yields an orange precipitate. A ${ }^{1} \mathrm{H}$ NMR spectrum of this solid reveals it to contain a 25:1 ratio of $124 \mathrm{~A}: 124 \mathrm{~B}$, with $34 \%$ of the mass recovered. However, the filtrate from the isolation of this solid contains $\mathbf{1 2 4 A}$ and $\mathbf{1 2 4 B}$ at a 2:1 ratio, which indicates this single diastereomer to be isolated by a selective precipitation, not a thermodynamic equilibration. In addition to 2D-NMR, solid-state analysis of $\mathbf{1 2 4}$ through $\mathrm{x}$-ray diffraction shows an orientation in which the methoxy group is distal to DMAP (Figure 6.2). Furthermore, the C-O bond length $(1.33 \AA)$ indicates partial double bond character in comparison to an alcohol C-O (1.41 $\AA$ ) bond and a ketone C-O (1.21 $\AA$ ) bond. ${ }^{26}$ This double bond character is in agreement with the resonance structures of $\mathbf{1 2 4}$ wherein the positive charge is distributed between an oxonium and an iminium structure. 


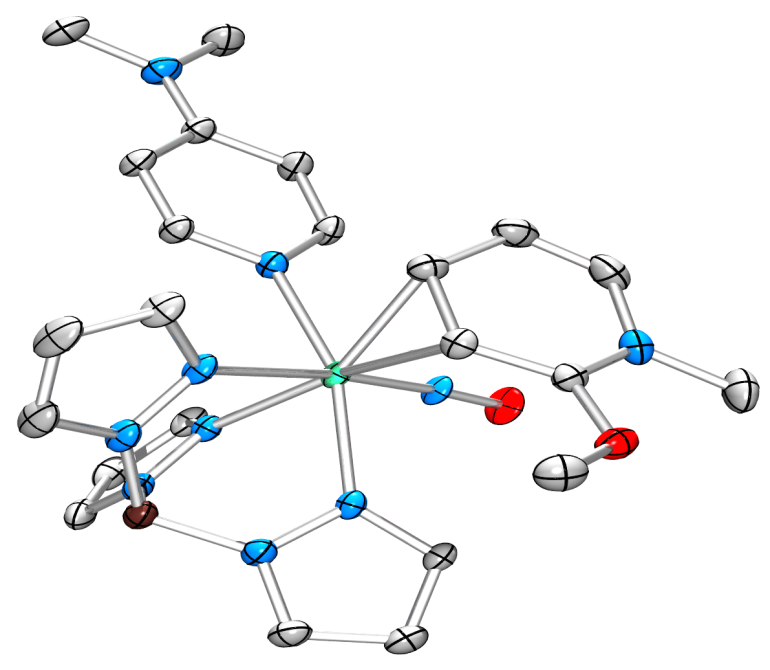

Figure 6.2. Crystal structure of 124A. The triflate anion is omitted for clarity. Bond lengths $(\AA \AA)$ : Mo-C(4) 2.231(4); Mo-C(3) 2.291(4); Mo-N(DMAP) 2.228(3); Mo$\mathrm{N}(\mathrm{NO}) 1.763(3) ; \mathrm{C}(2)-\mathrm{N}(\mathrm{NMe}) 1.335(6) ; \mathrm{C}(2)-\mathrm{O}(\mathrm{OMe}) 1.331(5)$.

After the initial electrophilic additions to form 123 and 124, tandem electrophilicnucleophilic additions were attempted (Scheme 6.5). Adding a $-30{ }^{\circ} \mathrm{C}$ solution of HOTf in $\mathrm{CH}_{3} \mathrm{CN}$ to $\mathbf{1 2 3}$ or $\mathbf{1 2 4}$, followed by a nucleophile (e.g., MTDA, $N$-methylpyrrole, LiDMM, imidazole, or propylamine) results in the isolation of starting material. Attempts to use other electrophiles result in either oxidation of the metal complex, as is the case for halogen electrophiles (e.g., Selectfluor, NBS, and NCS), or no reactivity, as is the case for carbon-based electrophiles (e.g., acetals and Michael acceptors). The lack of electrophilic addition to $\mathbf{1 2 3}$ or $\mathbf{1 2 4}$ is believed to be due to the significant electrondensity withdrawal from the metal center, which reduces the nucleophilicity of the uncoordinated $\pi$-bond (highlighted in red). 
Scheme 6.5. Attempted electrophilic-nucleophilic additions to $\mathbf{1 2 3}$ and $\mathbf{1 2 4}$.

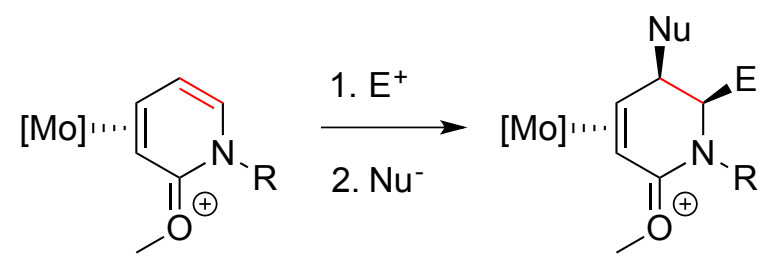

We then endeavored to reduce the oxonium in an attempt to access a neutral dihydropyridine complex. It was found that $\mathrm{NaBH}_{4}$ and $\mathrm{NaCNBH}_{3}$ were too weak to reduce the oxonium. However, adding lithium aluminum hydride (LAH) to a solution of 124 revealed a new reduced species with an $E_{\mathrm{p}, \mathrm{a}}$ at $-0.2 \mathrm{~V}$, which indicates the presence of a more electron-rich ligand than before the reaction. NMR analysis of this new product showed a lack of any methoxy groups; rather, geminal protons at 3.81 and $3.61 \mathrm{ppm}$ were identified using HSQC. This new species was identified as the product of an overreduction of $\mathbf{1 2 4}$ to yield the $N$-methylated dihydropyridine complex, 125 (Scheme 6.6).

Scheme 6.6. Reduction of 124 to yield 125 .

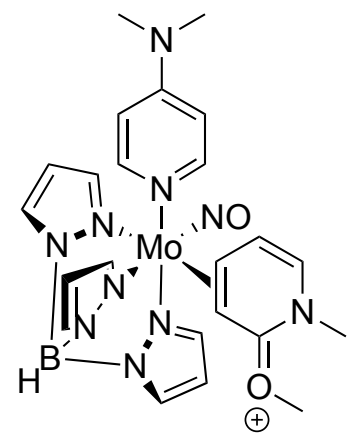

124
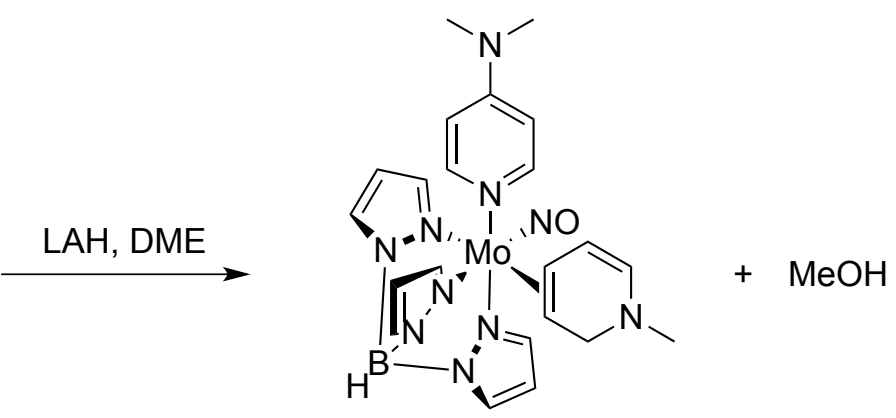

$125(95 \%)$ 
With the intention of adding propylamine as a nucleophile to the oxonium carbon, a solution of propylamine in DCM was added to $\mathbf{1 2 4}$ at room temperature and this mixture was stirred for $18 \mathrm{~h}$. The resulting green solution was then washed with $\mathrm{NaHCO}_{3}$ (aq. sat.), dried with $\mathrm{MgSO}_{4}$, and added to stirring pentane. The resulting precipitate was isolated, washed with pentane, and desiccated to yield a light green solid. The ${ }^{1} \mathrm{H}$ NMR spectrum of this solid lacks the presence of a methoxy resonance, similar to that observed for 125; however, no geminal proton signals are observed, indicating a lack of propylamine addition. Furthermore, resonances for the alkene peaks (H5 and H6) are still seen at 5.33 and $6.07 \mathrm{ppm}$, respectively, but this upfield shift in their resonance (from 6.43 and $6.34 \mathrm{ppm}$ ) would imply a loss of the oxonium. Interestingly, the ${ }^{13} \mathrm{C}$ NMR of this solid still has a carbonyl resonance at $173.6 \mathrm{ppm}$; however, this is more upfield from the carbonyl observed in $\mathbf{1 2 4}$ (175.7 ppm). With this information this new species was identified as the product of a demethylation of the oxonium, yielding 126 (Scheme 6.7).

Scheme 6.7. Oxonium demethylation of $\mathbf{1 2 4}$.

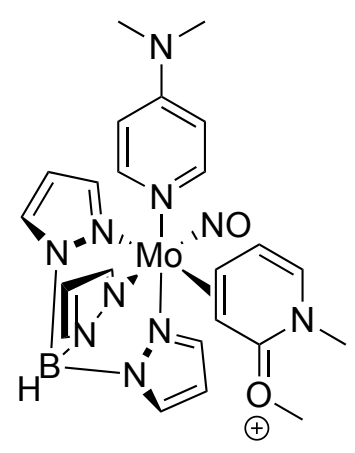

124
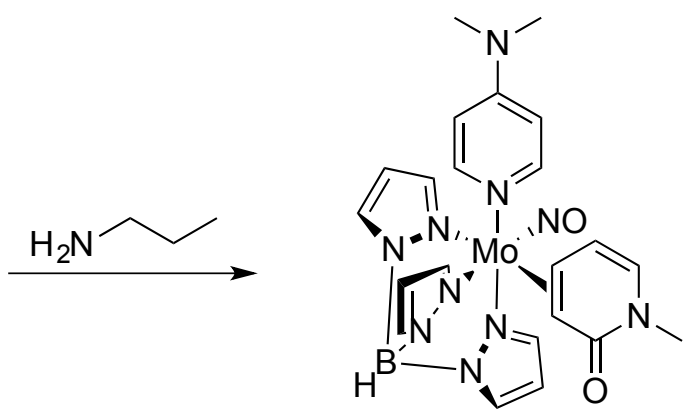

$126(98 \%)$ 
In agreement with this assessment is the negative shift in anodic wave (from $+0.80 \mathrm{~V}$ to $+0.08 \mathrm{~V})$ and a weaker nitrosyl stretch frequency than for $\mathbf{1 2 4}\left(1579 \mathrm{vs} 1596 \mathrm{~cm}^{-1}\right)$. Furthermore, a carbonyl stretch at $1615 \mathrm{~cm}^{-1}$ is in agreement with $\mathbf{1 2 6}$ containing an amide.

Attempts to perform tandem electrophilic-nucleophilic additions (Scheme 6.8) to 125 and $\mathbf{1 2 6}$ have been unsuccessful. Of interest is whether the metal or the nitrogen will control the reactivity. Interestingly, although not unexpected, subjecting a single coordination diastereomer of $\mathbf{1 2 4}$ (isolated by the selective precipitation described above) to the over-reduction conditions to form $\mathbf{1 2 5}$, or the demethylation conditions to yield 126, yields retention of the cdr in the final product.

Scheme 6.8. Attempted tandem electrophilic-nucleophilic additions to $\mathbf{1 2 3}$ and $\mathbf{1 2 4}$.

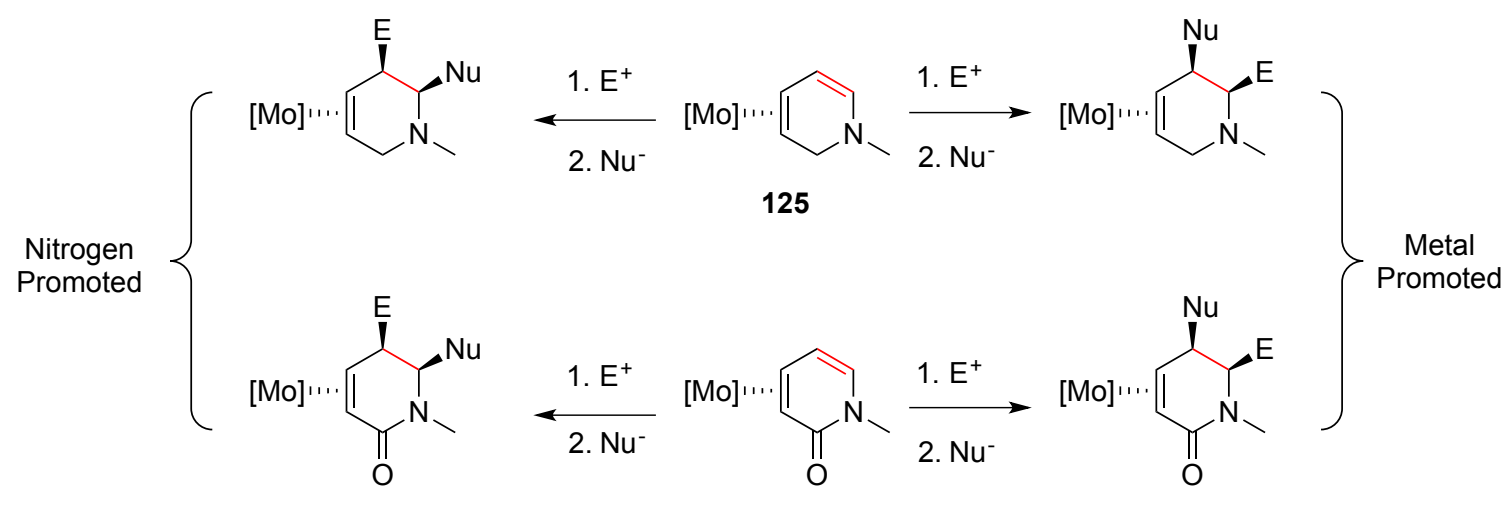

An interesting observation was made in the ${ }^{1} \mathrm{H}$ NMR of $\mathbf{1 2 3}$ and $\mathbf{1 2 4}$. The protons bound to the DMAP ancillary ligand experience a significant broadening. Typically the DMAP-B protons have a splitting pattern resembling a doublet-of-doublets while the 
DMAP-A protons are observed as a broad singlet. A good example of this splitting pattern is seen in the ${ }^{1} \mathrm{H}$ NMR of $\mathbf{1 2 5}$ (Figure 6.3).

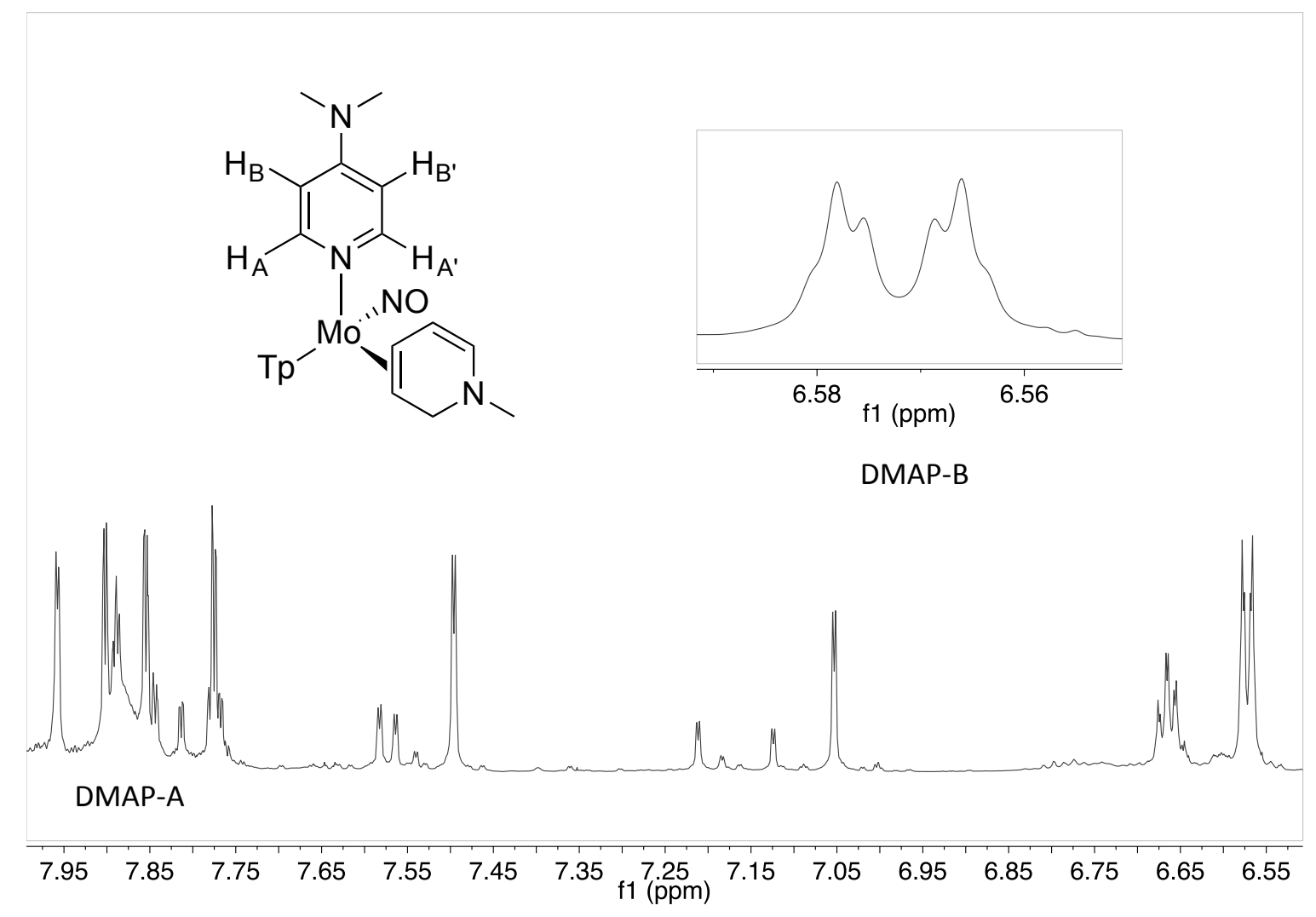

Figure 6.3. ${ }^{1} \mathrm{H}$ NMR of $\mathbf{1 2 5}\left(\mathrm{d}^{6}\right.$-Acetone, $\left.25^{\circ} \mathrm{C}\right)$.

The averaging of these signals is due to the rotation at the DMAP-Mo bond. The DMAP-B protons experience little difference in magnetic environment upon rotation and so their signal averaging will occur at a lower temperature than the DMAP-A protons. The DMAP-A protons, being close to the metal center, experience significantly different magnetic environments upon rotation at the DMAP-Mo bond. For this reason, DMAP-A protons are observed as a broad singlet because the rotational barrier at the DMAP-Mo bond is great enough to slow signal averaging.

As discussed in Chapter 3, the p-orbitals at the nitrogen within the ring of DMAP are expected to donate into the $\mathrm{d} \pi$ symmetric orbitals of molybdenum to stabilize electron 
withdrawal from the metal center. As the eta- 2 bound ligand's $\pi$-acidity increases, so will the donation from DMAP, subsequently increasing the barrier of rotation at the DMAPMo bond. This effect can be seen in the ${ }^{1} \mathrm{H}$ NMR of $\mathbf{1 2 4}$ at room temperature (Figure 6.4). Contrast to the typical doublet-of-doublets seen for the DMAP-B protons, in $\mathbf{1 2 4}$ the DMAP-B protons are observed as a broad singlet while the DMAP-A protons are very near to coalescence. A ${ }^{1} \mathrm{H}$ NMR experiment of a $\mathrm{CD}_{3} \mathrm{CN}$ solution of 124 at $10{ }^{\circ} \mathrm{C}$ revealed the coupling constant between DMAP-A of 124B and DMAP-B of 124B to be 6.8 Hz. The difference in chemical shift between DMAP-A of 124B and DMAP-A' of 124B was determined to be $605 \mathrm{~Hz}$ at $10^{\circ} \mathrm{C}$. The approximate coalescence temperature $\left(50{ }^{\circ} \mathrm{C}\right)$ between DMAP-A and DMAP-A' of 124B was obtained by VT-NMR in $\mathrm{CD}_{3} \mathrm{CN}$ $\left(0-75^{\circ} \mathrm{C}\right)$. From this information, the approximate rate of isomerization was determined $\left(\mathrm{k}=1343 \mathrm{~s}^{-1}\right)$ and with it the free energy of activation of isomerization was approximated to be $14.3 \pm 0.5 \mathrm{kcal} / \mathrm{mol}$ at $50^{\circ} \mathrm{C}$. As expected a similar increase in rotational barrier is seen for the $\left[\mathrm{TpMo}(\mathrm{NO})(\mathrm{DMAP})\left(2,3,4-\eta^{2} \text {-naphthalenium) }\right]^{+}\right.$complex 27 (Chapter 3, Figure 3.3). Measuring the rotational barrier of the DMAP-Mo bond gives insight into the electron density at the metal center. However, considering the need for lower temperature ${ }^{1} \mathrm{H}$ NMR experiments (e.g., $<0{ }^{\circ} \mathrm{C}$ ), other methods for determining relative electron density at the metal center (e.g., CV and IR) remain more advantageous. 


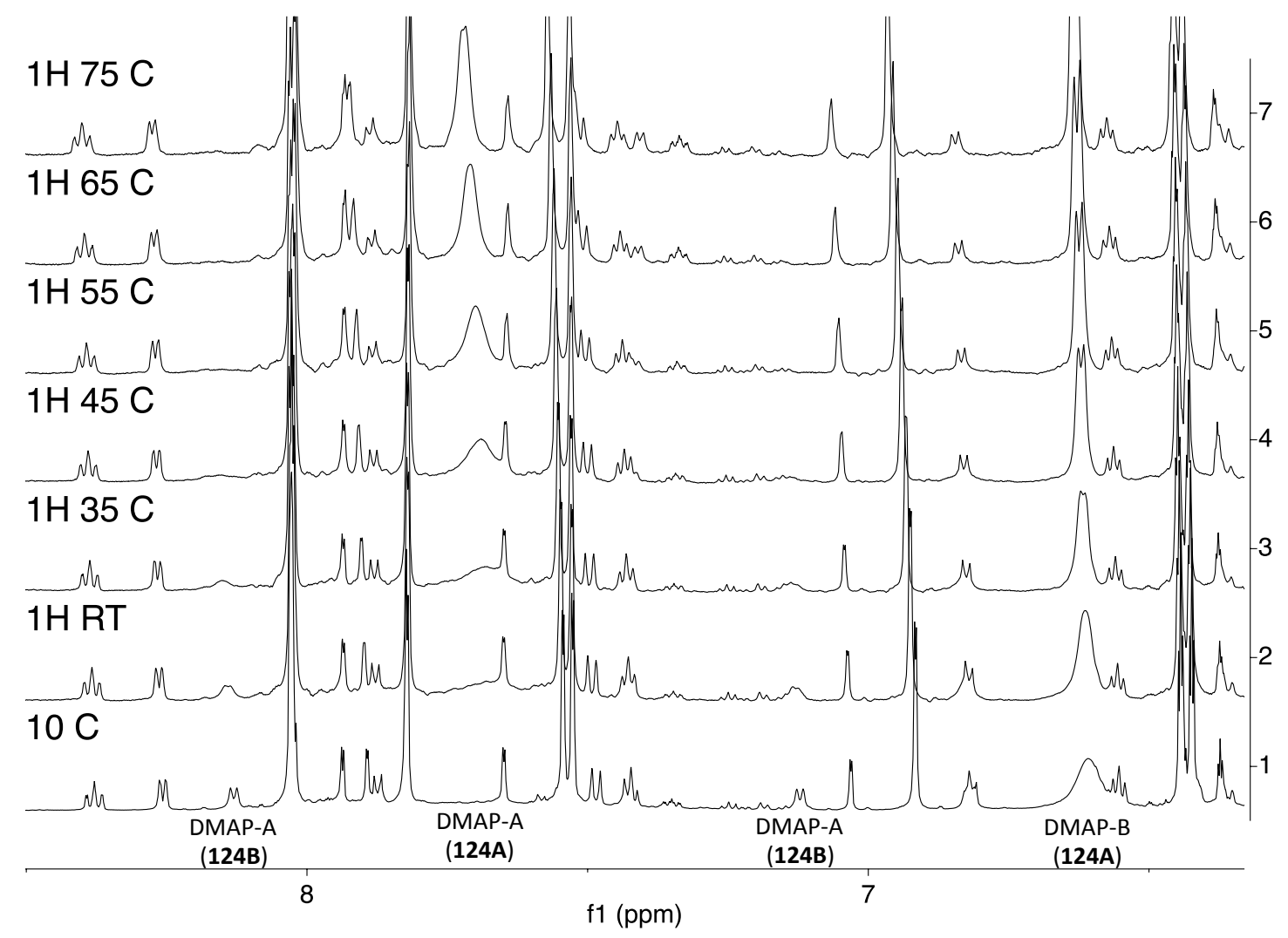

Figure 6.4. ${ }^{1} \mathrm{H}$ NMR spectra of $\mathbf{1 2 4}$ at various temperatures in $\mathrm{CD}_{3} \mathrm{CN}$.

Similar to the protonation of 121, complex 122 can be protonated by HOTf $/ \mathrm{CH}_{3} \mathrm{CN}$ at $-30{ }^{\circ} \mathrm{C}$ and isolated as a precipitate upon addition to $\mathrm{Et}_{2} \mathrm{O}$ (32\% yield) (Scheme 6.9). A ${ }^{1} \mathrm{H}$ NMR of this precipitate shows it to contain two main isomers. The expected protonation of the nitrogen to form 127I is identified by a unique set of $\mathrm{Tp}$ signals and a doublet at $5.35 \mathrm{ppm}$. This doublet represents H5, which, through COSY, was used to identify $\mathrm{H} 4$ as a triplet at $4.11 \mathrm{ppm}$ and $\mathrm{H} 3$ as a doublet at $2.81 \mathrm{ppm}$. These chemical shifts are in agreement with the oxonium orienting distal to the DMAP ancillary ligand. However, 127I is not the major isomer isolated from the protonation of $\mathbf{1 2 2}$. Although the initial protonation of $\mathbf{1 2 2}$ is believed to occur at the nitrogen, $\mathbf{1 2 7 I}$ undergoes a tautomerization to yield $127 \mathrm{~A}$, the product of protonating $122 \mathrm{~A}$ at $\mathrm{C} 5$. A mixture of 127A and 127I is isolated as a 3:1 ratio after precipitation. A key feature used 
to identify $127 \mathrm{~A}$ is the geminal proton signals at 4.04 and $3.43 \mathrm{ppm}$, which have a large coupling constant of $22.5 \mathrm{~Hz}$. One of the protons, $\mathrm{H} 5$, has a strong NOE interaction with the DMAP-A protons at $7.70 \mathrm{ppm}$, and this observation identifies the major isomer to be where the oxonium is distal to the DMAP.

Scheme 6.9. Protonation of 122.

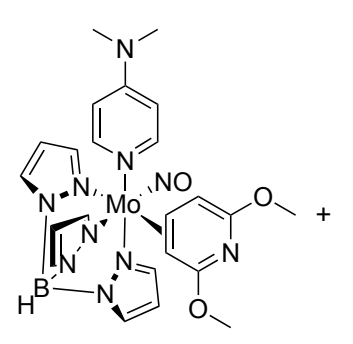

122-A

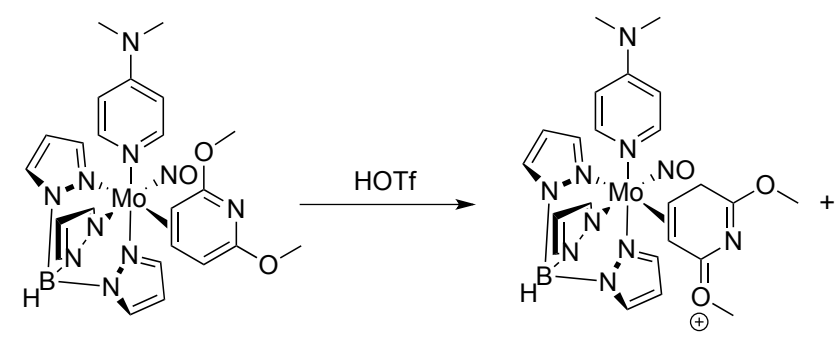

122-B
$127 \mathrm{~A}$

1

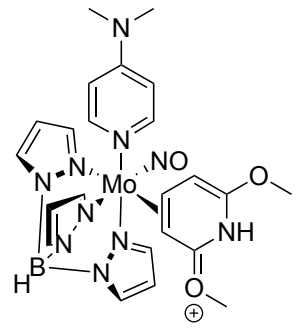

$127 \mathrm{I}$

Further investigation into the dearomatization of pyridines is currently being pursued as a separate project. We have demonstrated the potential to use

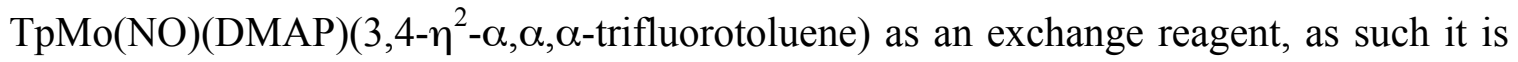
currently being used to investigate the binding and activation of pyridines. Encouragingly, the binding of pyridine-borane has shown to be possible, granted the pyridine-borane is stringently purified before use (Scheme 6.10$).{ }^{36}$ Preliminary results show that this pyridine-borane can be protonated to yield $\mathbf{1 2 9}$, which can subsequently be deprotonated in the presence of methyl vinyl ketone (MVK) to yield $\mathbf{1 3 0}$. The ability to bind pyridine-borane represents a significant breakthrough in the dearomatization of pyridines. The tungsten analogue of the pyridine-borane complex was heavily utilized in the synthesis of a large variety of substituted piperidines. 
Scheme 6.10. Exchange of TFT ligand for pyridine-borane and subsequent reactivity.

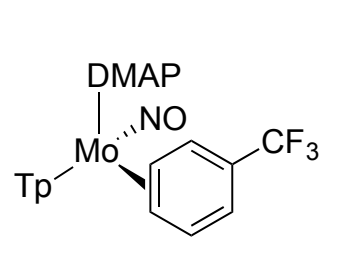

100

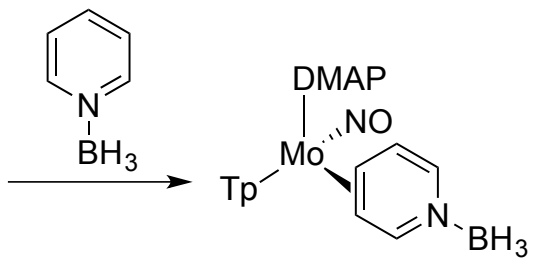

128

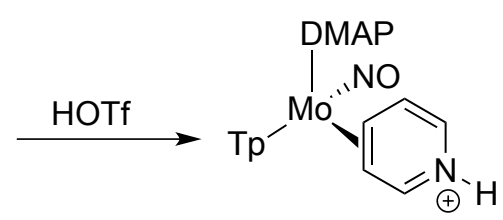

129

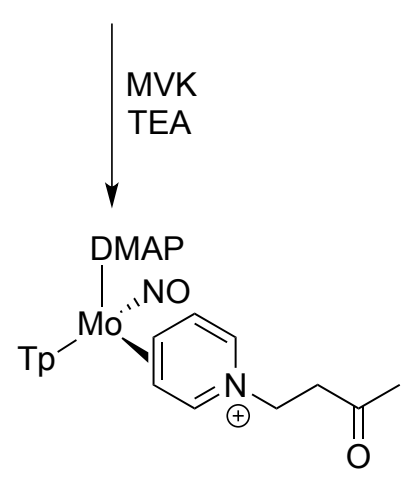

130

It is our belief that similar reactivity patterns will be found using $\{\mathrm{TpMo}(\mathrm{NO})(\mathrm{DMAP})\}$, which will offer a more economically viable alternative for the dearomatization of pyridines than the $\left\{\mathrm{TpW}(\mathrm{NO})\left(\mathrm{PMe}_{3}\right)\right\}$ fragment.

In the Harman Lab, we have demonstrated an ability to selectively bind pyridines to transition metals in an eta-2 fashion. While not the first reported eta-2 complexes of pyridines, the $\mathrm{TpW}(\mathrm{NO})\left(\mathrm{PMe}_{3}\right)\left(\eta^{2}-2,6-\right.$ lutidine $)$ and $\mathrm{TpW}(\mathrm{NO})\left(\mathrm{PMe}_{3}\right)\left(\eta^{2}-2-\right.$ dimethylaminopyridine) complexes were the first to demonstrate metal-promoted reactivity of the coordinated pyridine. These addition reactions subsequently yielded a wide variety of substituted dihydro and tetrahydropyridines. Pyridines themselves belong to a broader class of compounds that are referred to as aromatic, nitrogen-containing heterocycles (ANHs). Examples of ANHs include azacycles (i.e., pyrroles and pyridines), 
diazacycles (i.e., pyrimidines pyrazoles, and imidazoles), and triazacycles (i.e., triazines and triazoles). Similar to their aromatic, hydrocarbon analogues, ANHs often yield substituted aromatics during attempted addition reactions. While there are synthetic methods to dearomatize ANHs through hydrogenation, ${ }^{27}$ a lack of selectivity and functional group compatibility often hinders this approach.

To complement current methods for the synthesis of dearomatized azacycles from their aromatic precursors, the Harman Lab has thoroughly explored the binding and subsequent organic transformations of ANHs with $\left\{\mathrm{Os}\left(\mathrm{NH}_{3}\right)_{5}\right\}^{2+},\{\mathrm{TpRe}(\mathrm{CO})(\mathrm{MeIm})\}$, and $\left\{\mathrm{TpW}(\mathrm{NO})\left(\mathrm{PMe}_{3}\right)\right\} .{ }^{28-33}$ From the synthesis of epibatidine derivatives from pyrroles, ${ }^{30,}{ }^{34}$ to the synthesis of azabicyclo[2.2.2] octadienes from pyridines, ${ }^{35}$ the dearomatization of ANHs has been a prime example of the powerful synthetic ability of these dearomatization agents. In one example, the product of a [4+2] cycloaddition between acrylonitrile and an enantioenriched lutidinium complex yielded an azabicyclic compound with an enantiomeric ratio (er) of 9:1 (ee $=80 \%) .{ }^{35}$ The impressive chemistry developed with these dearomatization agents provides a great incentive for investigating ANH dearomatization with $\{\mathrm{TpMo}(\mathrm{NO})(\mathrm{L})\}$.

The eta- 2 pyridine complexes described above represent the first examples of binding and activating ANHs with $\{\operatorname{TpMo}(\mathrm{NO})(\mathrm{L})\}$. Molybdenum is far from the expansive chemistry demonstrated with its third-row congeners. However, the initial binding and reactivity described in this chapter gives insight into the electronic and steric conditions required to isolate $\mathrm{TpMo}(\mathrm{NO})(\mathrm{L})\left(\eta^{2}-\mathrm{ANH}\right)$ complexes. With a broad horizon ahead, exploring the dearomatization of ANHs with $\{\mathrm{TpMo}(\mathrm{NO})(\mathrm{L})\}$ is sure to further 
the fundamental understanding of the synthesis of alkaloid cores from aromatic precursors.

\subsection{Conclusion}

The binding of 2-methoxy- and 2,6-dimethoxypyridine to $\{\mathrm{TpMo}(\mathrm{NO})(\mathrm{DMAP})\}$ represents the first thermally stable eta- 2 bound pyridine complexes of molybdenum. Similar to that seen with the $\operatorname{TpW}(\mathrm{NO})\left(\mathrm{PMe}_{3}\right)\left(\eta^{2}-2-\right.$ methoxypyridine $)$ complex, initial electrophilic additions to $\mathbf{1 2 1}$ occur at the nitrogen. Subsequently, the syntheses of protonated and methylated pyridinium complexes (e.g., 123 and 124) were accomplished. Attempts to add electrophiles on the remaining $\pi$-bond of 123 and 124 were unsuccessful; however, reduction of the oxonium of $\mathbf{1 2 4}$ was accomplished with LAH to yield $\mathbf{1 2 5}$. Furthermore, the demethylation of the oxonium methyl of $\mathbf{1 2 4}$ was accomplished to yield the pyridone complex 126. Future investigations will focus on the organic transformations of the newly isolated $\mathrm{TpMo}(\mathrm{NO})(\mathrm{DMAP})\left(\eta^{2}\right.$-pyridine-borane $)$ complex. 


\subsection{Experimental}

General Methods. NMR spectra were obtained on a 600 or $800 \mathrm{MHz}$ spectrometer. All chemical shifts are reported in ppm, and proton and carbon shifts are referenced to tetramethylsilane (TMS) utilizing residual ${ }^{1} \mathrm{H}$ or ${ }^{13} \mathrm{C}$ signals of the deuterated solvents as an internal standard. Coupling constants $(J)$ are reported in hertz $(\mathrm{Hz})$. Infrared spectra (IR) were recorded as a glaze on a spectrometer fitted with a horizontal attenuated total reflectance (HATR) accessory or on a diamond anvil ATR assembly. Electrochemical experiments were performed under a dinitrogen atmosphere. Cyclic voltammetry data were taken at ambient temperature $\left(\sim 25^{\circ} \mathrm{C}\right)$ at $100 \mathrm{mV} / \mathrm{s}$ in a standard three-electrode cell with a glassy carbon working electrode, $N, N$-dimethylacetamide (DMA) or acetonitrile $\left(\mathrm{CH}_{3} \mathrm{CN}\right)$ solvent (unless otherwise specified), and tetrabutylammonium hexafluorophosphate (TBAH) electrolyte (approximately $0.5 \mathrm{M}$ ). All potentials are reported versus NHE (normal hydrogen electrode) using cobaltocenium hexafluorophosphate $\left(E_{1 / 2}=-0.78 \mathrm{~V}\right)$, ferrocene $\left(E_{1 / 2}=+0.55 \mathrm{~V}\right)$, or decamethylferrocene $\left(E_{1 / 2}=+0.04 \mathrm{~V}\right)$ as an internal standard. The peak-to-peak separation was less than $100 \mathrm{mV}$ for all reversible couples. Unless otherwise noted, all synthetic reactions were performed in a glovebox under a dry nitrogen atmosphere. Deuterated solvents were used as received. Pyrazole $(\mathrm{Pz})$ protons of the (trispyrazolyl) borate (Tp) ligand were uniquely assigned (e.g., "Pz3B") using a combination of twodimensional NMR data and (dimethylamino)pyridine-proton NOE interactions (see Figure 3.4). When unambiguous assignments were not possible, Tp protons were labeled as “Pz3/5 or Pz4”. All $J$ values for Pz protons are $2( \pm 0.2) \mathrm{Hz}$. 
Synthesis of TpMo(NO)(DMAP)(3,4- $\eta^{2}-2-$ methoxypyridine) (121).

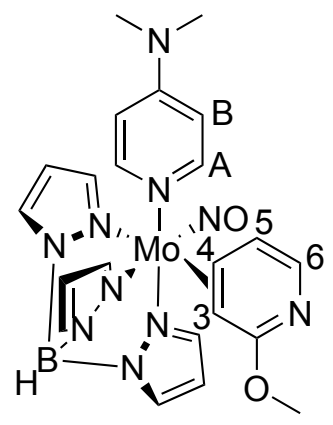

$121 \mathrm{~A}$

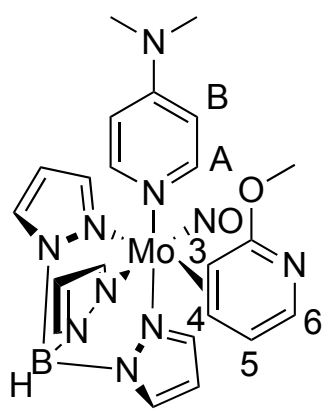

121B

To a $50 \mathrm{~mL}$ round bottom flask charged with a stir pea was added $\mathbf{2 6}(6 \mathrm{~g}, 10.77 \mathrm{mmol})$ and 2-methoxypyridine $(20 \mathrm{~mL})$. This yellow mixture was left stirring at room temperature overnight $(42 \mathrm{~h})$. The resulting orange precipitate was isolated on a $60 \mathrm{~mL}$ fine porosity fritted disc, washed with pentane $(5 \times 60 \mathrm{~mL})$, and desiccated $1 \mathrm{~h}$ to yield 121 (4.48 g, 73\% yield). CV (DMAc) $E_{\mathrm{p}, \mathrm{a}}=-0.22 \mathrm{~V}(\mathrm{NHE}) . \mathrm{IR}: v\left(\mathrm{C}-\mathrm{H} \mathrm{sp}{ }^{2}\right)=2934 \mathrm{~cm}^{-1}$, $v(\mathrm{~B}-\mathrm{H})=2475 \mathrm{~cm}^{-1}, v(\mathrm{NO})=1575 \mathrm{~cm}^{-1} .1: 1.4 \mathbf{A}: \mathbf{B}$ ratio. ${ }^{1} \mathrm{H}$ NMR $\left(\mathrm{d}^{6}-\right.$ Acetone, 600 MHz): $\delta 8.03(1 \mathrm{H}, \mathrm{d}, \mathrm{Pz3} / 5), 8.00(1 \mathrm{H}, \mathrm{d}, \mathrm{Pz3} / 5), 7.92(1 \mathrm{H}, \mathrm{d}, \mathrm{Pz3} / 5), 7.87(1 \mathrm{H}, \mathrm{d}$, Pz3/5), 7.85 (3H, m, Pz3/5), 7.75 (4H, buried bs, DMAP-A 121A and 121B), $7.51(1 \mathrm{H}, \mathrm{d}$, Pz3/5), 7.50 (1H, d, Pz3/5), 6.97 (1H, d, Pz3/5), 6.95 (1H, d, Pz3/5), 6.67 (2H, bd, DMAP-B 121A/121B), 6.61 (2H, bd, DMAP-B 121A/121B), $6.58(1 \mathrm{H}, \mathrm{d}, J=6.4,1 \mathrm{H}$, H6 121B), 6.56 (1H, d, $J=6.4, \mathrm{H} 6$ 121A), 6.37 (2H, m, Pz4), 6.33 (1H, t, Pz4), 6.26 (1H, t, Pz4), 6.24 (1H, m, H5 121B), 6.13 (1H, t, Pz4), 6.12 (1H, t, Pz4), 5.85 (1H, m, H5 121A), 3.79 (3H, s, OMe 121A), $3.65(1 \mathrm{H}, \mathrm{dd}, J=8.8,5.5, \mathrm{H} 3$ 121A), $3.62(3 \mathrm{H}, \mathrm{s}, \mathrm{OMe}$ 121B), $3.28(1 \mathrm{H}, \mathrm{d}, J=8.8, \mathrm{H} 3$ 121B $), 3.23(1 \mathrm{H}, \mathrm{dd}, J=8.8,5.5, \mathrm{H} 4$ 121B $), 3.08(6 \mathrm{H}, \mathrm{s}$, NMe 121A), 3.07 (6H, s, NMe 121B), $2.96\left(1 \mathrm{H}, \mathrm{d}, J=8.8, \mathrm{H} 4\right.$ 121A). Note: $\mathrm{CDCl}_{3}$ used for carbon experiment due to solubility issues in $\mathrm{d}^{6}$-Acetone making ${ }^{13} \mathrm{C} \mathrm{NMR}$ 
experiments too low in concentration. ${ }^{13} \mathrm{C}$ NMR $\left(\mathrm{CDCl}_{3}, 200 \mathrm{MHz}\right): \delta 171.4,170.7$, $153.9,153.8,146.9,144.0,142.2,142.1,141.9,140.2,139.9,138.7,136.4,136.3,136.0$, $135.9,134.7,134.7,128.4,128.0,118.2,117.3,116.8,111.0,107.4,105.9,105.8,105.6$ $105.5, \quad 105.4, \quad 105.2, \quad 78.1, \quad 77.2, \quad 75.4,65.0, \quad 62.4, \quad 52.4, \quad 52.1, \quad 39.3$. HRMS: $\mathrm{C}_{22} \mathrm{H}_{27} \mathrm{~N}_{10} \mathrm{O}_{2} \mathrm{BMo}+\mathrm{H}^{+}$obsd (\%), calcd (\%), ppm: 567.1613 (45), 567.1556 (54), 10.0; 569.1550 (66), 569.1553 (49), -0.5; 570.1533 (88), 570.1552 (81), -3.4; 571.1549 (100), 571.1547 (86), 0.4; 572.1552 (87), 572.1560 (74), -1.3; 573.1544 (92), 573.1546 (100), 0.4; 574.1537 (40), 574.1575 (34), -6.6; 575.1546 (30), 575.1566 (39), -3.4.

\section{Synthesis of TpMo(NO)(DMAP)(3,4- $\eta^{2}$-2,6-dimethoxypyridine) (122).}

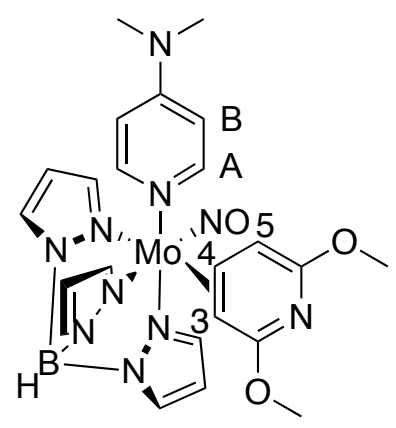

$122 A$

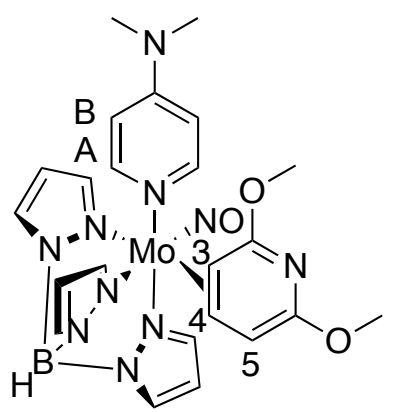

122B

To a $25 \mathrm{~mL}$ round bottom flask charged with a stir pea was added 26 (1.0 g, $1.79 \mathrm{mmol})$ and 2,6-dimethoxypyridine $(3 \mathrm{~mL})$. This mixture was stirred at room temperature for 20 h. The resulting orange precipitate was then collected on a $15 \mathrm{~mL}$ medium porosity fritted disc, washed with pentane ( $3 \times 15 \mathrm{~mL})$, and dried in vacuo to yield $122(580 \mathrm{mg}, 54 \%$ yield). CV (DMAc) $E_{\mathrm{p}, \mathrm{a}}=-0.37 \mathrm{~V}(\mathrm{NHE}) . \mathrm{IR}: v\left(\mathrm{C}-\mathrm{H} \mathrm{sp}{ }^{2}\right)=2935 \mathrm{~cm}^{-1}, v(\mathrm{~B}-\mathrm{H})=2471$ $\mathrm{cm}^{-1}, v(\mathrm{NO})=1600 \mathrm{~cm}^{-1} .{ }^{1} \mathrm{H} \mathrm{NMR}\left(\mathrm{CDCl}_{3}, 600 \mathrm{MHz}, @ 0{ }^{\circ} \mathrm{C}\right): \delta 5.51(1 \mathrm{H}, \mathrm{d}, J=5.0$, H5 122B), $5.12(1 \mathrm{H}, \mathrm{d}, J=5.0, \mathrm{H} 5$ 122A), $3.71(1 \mathrm{H}, \mathrm{dd}, J=8.6,5.0, \mathrm{H} 4$ 122A), 3.50 
$(1 \mathrm{H}, \mathrm{dd}, J=8.6,5.0, \mathrm{H} 4$ 122B $), 3.18(1 \mathrm{H}, \mathrm{d}, J=8.6, \mathrm{H} 3 \mathbf{1 2 2 B}), 3.06(1 \mathrm{H}$, buried under OMe, H3 122A). ${ }^{13} \mathrm{C}$ NMR (201 MHz, $\left.\mathrm{CDCl}_{3}, @ 0{ }^{\circ} \mathrm{C}\right): \delta 172.4,171.6,163.1,154.3$, $153.9,153.8,153.7,150.7,144.0,142.1,142.0,141.1,140.9,140.3,140.0,136.3,136.2$, 135.9, 135.9, 134.7, 134.7, 107.4, 105.9, 105.8, 105.6, 105.4, 105.3, 105.2, 100.8, 88.4, $87.8,80.5,61.6,59.2,55.0,54.8,52.9,52.5,39.3,39.3$.

\section{Synthesis of [TpMo(NO)(DMAP)(3,4- $\eta^{2}$-2-methoxypyridinium) $]^{+}$(OTf) (123).}

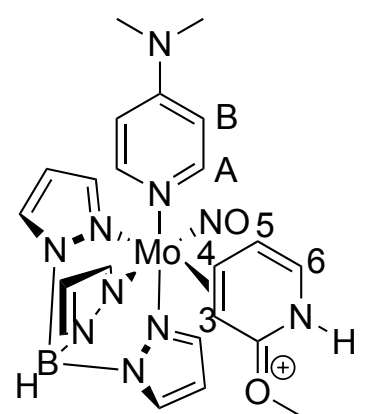

123A

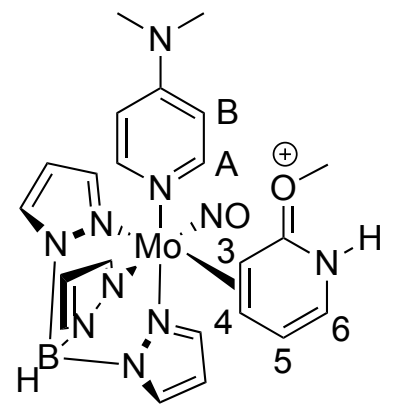

123B

To a 4-dram vial was added $121(1 \mathrm{~g}, 1.7 \mathrm{mmol})$ and $\mathrm{CH}_{3} \mathrm{CN}(10 \mathrm{~mL})$. This mixture was cooled at $-30{ }^{\circ} \mathrm{C}$ for $15 \mathrm{~min}$. Next, a $-30{ }^{\circ} \mathrm{C} 1 \mathrm{M}$ solution of $\mathrm{HOTf} / \mathrm{CH}_{3} \mathrm{CN}(3.6 \mathrm{~mL}, 3.6$ mmol) was added to the reaction mixture and the resulting yellow solution was left at -30 ${ }^{\circ} \mathrm{C}$. After $15 \mathrm{~min}$, this solution was then added slowly to stirring $\mathrm{Et}_{2} \mathrm{O}(400 \mathrm{~mL})$, which yielded a yellow precipitate. This precipitate was collected on a $30 \mathrm{~mL}$ fine porosity fritted disc, washed with $\mathrm{Et}_{2} \mathrm{O}(3 \times 30 \mathrm{~mL})$, and desiccated to yield $123(1.14 \mathrm{~g}, 93 \%$ yield). CV (DMAc) $E_{\mathrm{p}, \mathrm{a}}=+0.87 \mathrm{~V}(\mathrm{NHE})$. IR: $v\left(\mathrm{C}-\mathrm{H} \mathrm{sp}{ }^{2}\right)=3103 \mathrm{~cm}^{-1}, v(\mathrm{~B}-\mathrm{H})=2503$ $\mathrm{cm}^{-1}, v(\mathrm{NO})=1599 \mathrm{~cm}^{-1}$. Product isolated as a 2:1 A:B ratio. ${ }^{1} \mathrm{H}$ NMR $\left(\mathrm{d}^{6}\right.$-Acetone, 600 MHz): $\delta 11.10$ (2H, bs, NH 123A \& 123B), 8.15 (1H, d, Pz5C 123A), 8.14 (1H, d, Pz5C 123B), 8.10 (1H, d, Pz5A 123A), 8.03 (1H, d, Pz5A 123B), 7.94 (2H, d, Pz5B, 123A and 
123B), 7.92 (1H, d, Pz3A/Pz3C, 123B), 7.88 (1H, d, Pz3A/Pz3C 123B), 7.80 (4H, bs, DMAP-A 123A and 123B), 7.77 (1H, d, Pz3C 123A), 7.62 (1H, d, Pz3A 123A), 7.15 (1H, d, Pz3B 123B), 7.03 (1H, d, Pz3B 123A), 6.75 (2H, bs, DMAP-B 123A), 6.64 (1H, t, $J=5.9, \mathrm{H} 5$ 123B $), 6.50(1 \mathrm{H}, \mathrm{t}, \mathrm{Pz} 4 \mathrm{C}$ 123A $), 6.48(1 \mathrm{H}, \mathrm{t}, \mathrm{Pz} 4 \mathrm{C}$ 123B $), 6.45(2 \mathrm{H}, \mathrm{m}$, Pz4A 123A and H6 123B), 6.42 (1H, m, H6 123A), 6.40 (1H, t, Pz4A 123B), 6.31 (1H, t, $J=5.9, \mathrm{H} 5$ 123A $), 6.19$ (1H, t, Pz4B 123B), 6.18 (1H, t, Pz4B 123A), $4.17(1 \mathrm{H}, \mathrm{dd}, J=$ 8.5, 5.4, H4 123A), 3.83 (3H, s, OMe 123B), $3.80(3 \mathrm{H}, \mathrm{s}$, OMe 123A), $3.54(1 \mathrm{H}, \mathrm{d}, J=$ 8.5, H2 123B), 3.47 (1H, dd, $J=8.5,5.4, \mathrm{H} 4$ 123B), 3.14 (6H, s NMe 123A), 3.12 (6H, s NMe 123B), 2.92 (1H, d, $J=8.5, \mathrm{H} 2$ 123A $).{ }^{13} \mathrm{C}$ NMR ( $\mathrm{d}^{6}$-Acetone, $200 \mathrm{mHz}$ ): $\delta 175.9$ (CO), 155.6 (DMAP-C), 150.4 (2C, DMAP-A), 143.8 (Pz3A), 143.2 (Pz3B), 142.0 (Pz3C), 138.5 (2C, Pz5A \& Pz5C), 136.8 (Pz5B), 120.8 (C6), 114.2 (C5), 108.9 (2C, DMAP-B), 107.8 (Pz4A/C), 107.5 (Pz4A/C), 106.9 (Pz4B), 78.2 (C4), 57.9 (OMe), 56.1 (C3), 39.2 (2C, DMAP-Me). HRMS: $\mathrm{C}_{22} \mathrm{H}_{28} \mathrm{~N}_{10} \mathrm{O}_{2} \mathrm{BMo}^{+}$obsd (\%), calcd (\%), ppm: 567.1544 (40), 567.1556 (54), -2.2; 569.1545 (64), 569.1553 (49), -1.4; 570.1541 (96), 570.1552 (81), -2.0; 571.1561 (89), 571.1547 (86), 2.5; 572.1544 (100), 572.1560 (74), 2.7; 573.1549 (77), 573.1546 (100), 0.5; 574.1586 (40), 574.1575 (34), 2.0; 575.1566 (31), $575.1566(39), 0.0$. 


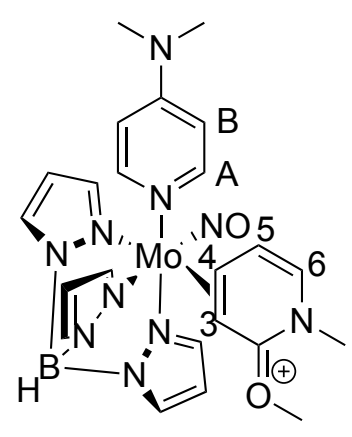

124A

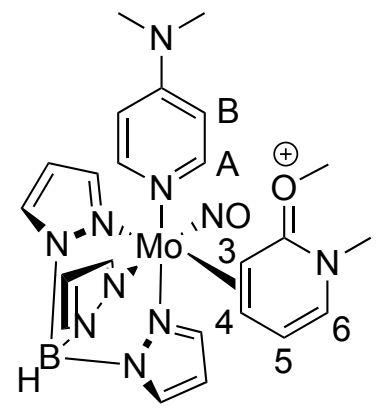

124B

To a 4-dram vial was added 121 (1 g, $1.7 \mathrm{mmol}), \mathrm{CH}_{3} \mathrm{CN}(5 \mathrm{~mL})$, and $\mathrm{MeOTf}(400 \mathrm{mg}$, $2.44 \mathrm{mmol}$ ). This orange mixture was left sitting at room temperature for $10 \mathrm{~min}$, yielding a dark red solution. This solution was added to stirring $\mathrm{Et}_{2} \mathrm{O}(100 \mathrm{~mL})$ to yield a red precipitate, which was then isolated on a $30 \mathrm{~mL}$ fine porosity fritted disc, washed with $\mathrm{Et}_{2} \mathrm{O}(3 \times 30 \mathrm{~mL})$, and desiccated to yield the dark orange product $124(1.72 \mathrm{~g}, 89 \%$ yield). $\mathrm{CV}(\mathrm{DMAc}) E_{\mathrm{p}, \mathrm{a}}=+0.79 \mathrm{~V}(\mathrm{NHE}) . \mathrm{IR}: v\left(\mathrm{C}-\mathrm{H} \mathrm{sp}{ }^{2}\right)=3105 \mathrm{~cm}^{-1}, v(\mathrm{~B}-\mathrm{H})=2484$ $\mathrm{cm}^{-1}, v(\mathrm{NO})=1596 \mathrm{~cm}^{-1}$. Product isolated as a 3:1 A:B ratio. ${ }^{1} \mathrm{H}$ NMR $\left(\mathrm{d}^{6}\right.$-Acetone, 600 MHz): 8.27 (1H, d, $J=7.3$, DMAP-A 124B), $8.16(1 \mathrm{H}, \mathrm{d}, \operatorname{Pz} 5 \mathrm{C}$ 124A), $8.15(1 \mathrm{H}, \mathrm{d}$, Pz5A 124A), 8.13 (1H, d, Pz3/5 124B), 8.20 (1H, d, Pz3/5 124B), 7.96 (1H, d, Pz3/5 124B), 7.94 (1H, d, Pz3/5 124B), 7.93 (1H, d, Pz5B 124A), 7.79 (2H, bs, DMAP-B 124A), 7.74 (1H, d, Pz3C 124A), 7.65 (1H, d, Pz3A 124A), 7.28 (1H, d, $J=7.3$, DMAPA 124B), 7.09 (1H, d, Pz3B 124B), 7.02 (1H, bs, DMAP-B 124B), 7.00 (1H, d, Pz3B 124A), 6.75 (2H, bs, DMAP-B 124A), 6.61 (1H, bs, DMAP-B 124B), $6.51(1 \mathrm{H}, \mathrm{t}, \mathrm{Pz} 4 \mathrm{C}$ 124A), 6.49 (1H, t, Pz4A 124A), 6.48 (1H, t, Pz4 124B), 6.43 (1H, d, $J=7.2, \mathrm{H} 6$ 124A), $6.34(1 \mathrm{H}, \mathrm{t}, J=6.3, \mathrm{H} 5$ 124A), 6.39 (1H, t, Pz4 124B), 6.18 (1H, t, Pz4 124B), 6.17 (1H, 
t, Pz4B 124A), $4.21(1 \mathrm{H}, \mathrm{dd}, J=8.6,6.3, \mathrm{H} 4$ 124A), 3.75 (3H, s, NMe 124A), $3.69(1 \mathrm{H}$, $\mathrm{d}, J=8.6, \mathrm{H} 3$ 124B), $3.54(1 \mathrm{H}, \mathrm{dd}, J=8.6,5.8, \mathrm{H} 4$ 124B), 3.50 (3H, s, OMe 124A), 3.12 $\left(6 \mathrm{H}, \mathrm{s}, \mathrm{NMe}\right.$ 124A), $3.00\left(1 \mathrm{H}, \mathrm{d}, J=8.6, \mathrm{H} 3\right.$ 124A). ${ }^{13} \mathrm{C}$ NMR (d - -Acetone, $\left.200 \mathrm{MHz}\right)$ ठ 124A: 175.7 (OMe), 155.89 (DMAP-C), 150.5 (2C, DMAP-A), 143.9 (Pz3A), 143.2 (Pz3B), 142.1 (Pz3C), 138.9 (Pz5A/5C), 138.6 (Pz5A/5C), 136.9 (Pz5B), 120.9 (C5/C6), 120.8 (C5/C6), 109.1 (2C, DMAP-B), 108.1 (Pz4A/4C), 107.9 (Pz4A/4C), 106.9 (Pz4B), 78.1 (C4), 58.5 (OMe), 55.9 (C3), 39.3 (DMAP-Me), 37.3 (NMe). 124B (signals are too small for unambiguous assignment): 175.6, 155.9, 150.2, 143.0, 142.5, 138.5, 137.8, $136.5,121.5,121.2,108.9,107.7,107.0,106.9,76.7,66.1,58.3,56.4,39.3,29.8,15.6$. HRMS: $\mathrm{C}_{23} \mathrm{H}_{30} \mathrm{~N}_{10} \mathrm{O}_{2} \mathrm{BMo}^{+}$obsd (\%), calcd (\%), ppm: 581.1728 (49), 581.1713 (54), 2.6; 583.1713 (48), 583.1710 (49), 0.6; 584.1723 (75), 584.1709 (81), 2.4; $585.1716(85)$, 585.1704 (86), 2.1; 586.1716 (73), 586.1716 (74), -0.1; 587.1721 (100), 587.1703 (100), 3.0; 588.1724 (32), 588.1731 (34), -1.3; 589.1738 (39), 589.1723 (39), 2.6.

\section{Synthesis of TpMo(NO)(DMAP)(3,4- $\eta^{2}-N$-methyldihydropyridine) (125).}

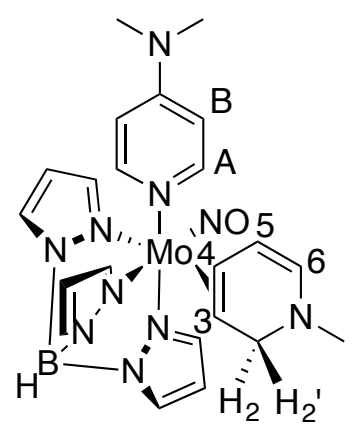

125A

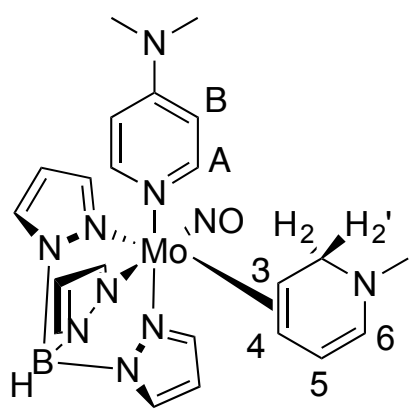

125B 
To a solution of $124(500 \mathrm{mg}, 0.679 \mathrm{mmol})$ and dimethoxyethane $(5 \mathrm{~mL})$ was added lithium aluminum hydride (LAH) $(75 \mathrm{mg}, 1.97 \mathrm{mmol})$. The resulting dark red solution was then stirred at room temperature for $5 \mathrm{~min}$. This solution was then added to stirring $\mathrm{H}_{2} \mathrm{O}(30 \mathrm{~mL})$ in a $50 \mathrm{~mL}$ round bottom flask, yielding a green precipitate. This mixture was let stirring at room temperature for $20 \mathrm{~h}$. The resulting yellow precipitate was isolated on a $30 \mathrm{~mL}$ medium porosity fritted disc, washed with $\mathrm{H}_{2} \mathrm{O}(3 \times 20 \mathrm{~mL})$ and hexanes $(3 \times 20 \mathrm{~mL})$ (Note: do not stir the precipitate with washing solvent, just wash through), and dried in vacuo $1 \mathrm{~h}$. This precipitate was then triturated in stirring hexanes $(20 \mathrm{~mL}$ ) for $20 \mathrm{~h}$ and the resulting precipitate was isolated on a $15 \mathrm{~mL}$ fine porosity fritted disc, washed with hexanes ( $3 \times 10 \mathrm{~mL})$, and desiccated to yield 125 (360 mg, 95\% yield). CV (DMAc) $E_{\mathrm{p}, \mathrm{a}}=-0.23 \mathrm{~V}$ (NHE). IR: $v\left(\mathrm{C}-\mathrm{H} \mathrm{sp}^{2}\right)=2922 \mathrm{~cm}^{-1}, v(\mathrm{~B}-\mathrm{H})=2468$ $\mathrm{cm}^{-1}, v(\mathrm{NO})=1566 \mathrm{~cm}^{-1}$. Product isolated as a 4:1 A:B ratio. Confirmed $\mathbf{A}$ isomer by isolating $\mathbf{1 2 5}$ after selective precipitation of $\mathbf{1 2 4}$, therefore the only peaks assigned to $\mathbf{B}$ are based on clean separation from $\mathbf{A}$ isomer. ${ }^{1} \mathrm{H}$ NMR ( $\mathrm{d}^{6}$-Acetone, $\left.600 \mathrm{MHz}\right)$ : $\delta 7.95$ (1H, d, Pz3A 125A), 7.90 (1H, d, Pz5A/C 125A ), 7.88 (2H, buried bs, DMAP-A 125A), 7.85 (1H, d, Pz5A/C 125A), 7.77 (1H, d, Pz5B 125A), 7.49 (1H, d, Pz3C 125A), 7.04 (1H, d, Pz3B 125A), 6.66 (2H, m, DMAP-B 125B), 6.58 (2H, m, DMAP-B 125A), 6.30 (2H, t, Pz4A \& C 125A), 6.28 (1H, t, Pz4 125B), 6.24 (1H, t, Pz4 125B), 6.12 (1H, t, Pz4 125B), $6.11(1 \mathrm{H}, \mathrm{t}, \mathrm{Pz} 4 \mathrm{~B}$ 125A), 5.50 (1H, d, $J=7.4, \mathrm{H} 6$ 125A $), 5.44(1 \mathrm{H}, \mathrm{d}, J=7.4, \mathrm{H} 6$ 125B), $5.06(1 \mathrm{H}, \mathrm{ddd}, J=7.4,4.6, \& 0.9, \mathrm{H} 5$ 125B $), 4.75$ (1H, ddd, $J=7.4,4.6, \& 0.9$, H5 125A), 3.81 (1H dd, $J=10.9 \& 2.8, \mathrm{H} 2$ 125A), 3.61 (1H, dd, $J=10.9 \& 5.0, \mathrm{H} 2$ ' 125A), 3.05 (6H, s, N-Me 125A), 2.62 (3H, s, N-Me 125A), 2.59 (1H, m, H4 125A), 2.03 $(1 \mathrm{H}, \mathrm{m}, \mathrm{H} 3 \mathbf{1 2 5 A}) .{ }^{13} \mathrm{C}$ NMR of 125A only. ${ }^{13} \mathrm{C}$ NMR (d -Acetone, $\left.200 \mathrm{MHz}\right): \delta 155.0$ 
(DMAP-C), 151.1 (2C, DMAP-A), 143.5 (Pz3A), 142.0 (Pz3B), 141.3 (Pz3C), 137.1 (Pz5A/C), 136.7 (Pz5A/C), 135.5 (Pz5B), 131.6 (C6), 107.9 (DMAP-B), 106.5 (Pz4A/C), 106.2 (Pz4A/C), 106.1 (Pz4B), 103.6 (C5), 63.5 (C4), 61.7 (C3), 53.9 (C2), 43.2 (NMe), 39.1 (DMAP-Me). HRMS: $\mathrm{C}_{22} \mathrm{H}_{29} \mathrm{~N}_{10} \mathrm{OBMo}+\mathrm{H}^{+}$obsd (\%), calcd (\%), ppm: 553.1769 (36), 553.1764 (54), 1.0; 555.1762 (65), 555.1760 (49), $0.3 ; 556.1734$ (77), 556.1760 (82), -4.6; 557.1730 (92), 557.1754 (86), -4.3; 558.1783 (80), 558.1767 (74), 2.9; 559.1740 (100), 559.1754 (100), -2.4; 560.1814 (46), 560.1782 (34), 5.7; 561.1866 (44), $561.1773(39), 16.6$.

Synthesis of TpMo(NO)(DMAP)(3,4- $\eta^{2}-N$-methylpyridin-2(1H)-one) (126).
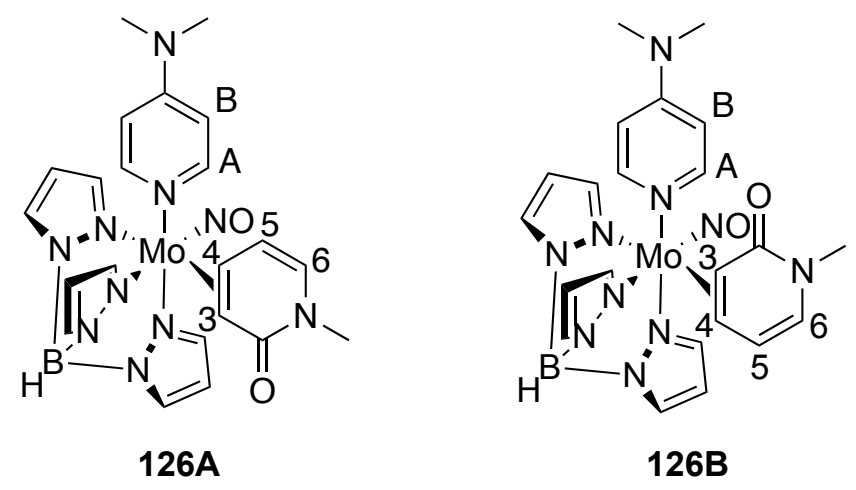

To a 4-dram vial was added $124(1.0 \mathrm{~g}, 0.0013 \mathrm{~mol})$, DCM $(5 \mathrm{~mL})$, and propylamine (1.2 g, $0.020 \mathrm{~mol})$. This solution was stirred overnight at room temp $(20 \mathrm{~h})$. The resulting green solution was washed with $\mathrm{NaHCO}_{3}$ (sat. aq., $10 \mathrm{~mL}$ ) and the aqueous layer was back-extracted with DCM (3 x $5 \mathrm{~mL})$. The organic layers were combined and subsequently dried with $\mathrm{MgSO}$. The $\mathrm{MgSO}_{4}$ was removed on a $15 \mathrm{~mL}$ medium porosity fritted disc, washed with DCM (3 $\times 5 \mathrm{~mL})$, and the resulting filtrate was evaporated in vacuo to an oil. This oil was then dissolved in DCM $(15 \mathrm{~mL})$ and the resulting solution was added to stirring pentane $(200 \mathrm{~mL})$, yielding a green precipitate. This precipitate was 
isolated on a $30 \mathrm{~mL}$ fine porosity fritted disc, washed with pentane $(3 \times 10 \mathrm{~mL})$ and desiccated to yield 126 (728 mg, 98\% yield). CV (DMAc) $E_{\mathrm{p}, \mathrm{a}}=+0.08 \mathrm{~V}$ (NHE). IR: $v\left(\mathrm{C}-\mathrm{H} \mathrm{sp}^{2}\right)=2927 \mathrm{~cm}^{-1}, v(\mathrm{~B}-\mathrm{H})=2481 \mathrm{~cm}^{-1}, v(\mathrm{CO})=1615 \mathrm{~cm}^{-1}, v(\mathrm{NO})=1579 \mathrm{~cm}^{-1} .{ }^{1} \mathrm{H}$ NMR (d $\mathrm{d}^{6}$-Acetone, 600 MHz): $\delta 8.44$ (1H, d, Pz5A 126A), 8.01 (1H, d, Pz3/5 126B), 7.99 (1H, d, Pz3/5 126B), 7.98 (1H, d, Pz5C 126A), 7.91 (1H, d, Pz3/5 126B), $7.84(1 \mathrm{H}$, d, Pz5B 126A), 7.83 (1H, d, Pz3A 126A), 7.78 (2H, bs, DMAP-A 126A), 7.71 (3H, d, Pz3/5 \& DMAP-A 126B), 7.60 (1H, d, Pz3C 126A), 7.51 (1H, d, Pz3/5 126B), 7.03 (1H, d, Pz3B 126A), 7.00 (1H, d, Pz3/5 126B), 6.63 (2H, bd, DMAP-B 126A), 6.51 (2H, bd, DMAP-B 126B), 6.37 (1H, t, Pz4 126B), 6.35 (1H, t, Pz4C 126A), 6.30 (1H, t, Pz4 126B), 6.21 (1H, t, Pz4A 126A), 6.12 (1H, t, Pz4B 126A), 6.11 (1H, t, Pz4 126B), 6.08 $(1 \mathrm{H}, \mathrm{d}, J=7.5, \mathrm{H} 6$ 126B), 6.07 (1H, d, $J=7.5$, H6 126A), 5.73 (1H, t, $J=7.4, \mathrm{H} 5$ 126B), $5.33(1 \mathrm{H}, \mathrm{dd}, J=7.5,5.4, \mathrm{H} 5$ 126A), $3.48(1 \mathrm{H}, \mathrm{dd}, J=8.6,5.4, \mathrm{H} 4 \mathbf{1 2 6} \mathrm{A}), 3.29(3 \mathrm{H}, \mathrm{s}$, NMe 126A), 3.28 (3H, s, NMe 126B), 3.07 (6H, s, NMe 126A), 3.05 (6H, s, NMe 126A), $3.00\left(1 \mathrm{H}, \mathrm{m}, \mathrm{H} 3\right.$ 126B), $2.69(1 \mathrm{H}, \mathrm{d}, J=8.6, \mathrm{H} 3 \mathbf{1 2 6 A}), 1.69(1 \mathrm{H}, \mathrm{m}, \mathrm{H} 4 \mathbf{1 2 6 B}) .{ }^{13} \mathrm{C}$ NMR (d'-Acetone, 200 MHz): $\delta$ 173.6 (CO), 155.2 (DMAP-C), 150.5 (DMAP-A), 146.7 (Pz5A), 142.5 (Pz3B), 141.6 (Pz3C), 137.7 (Pz5C), 136.7 (Pz3A/5B), 135.8 (Pz3A/5B), 123.4 (C6), 109.8 (C5), 108.3 (DMAP-B), 106.9 (Pz4C), 106.4 (Pz4B), 105.7 (Pz4A), 75.3 (C4), 62.8 (C3), 39.1 (DMAP-Me), 34.6 (NMe). 


\section{Synthesis of [TpMo(NO)(DMAP)(3,4- $\left.\left.\eta^{2}-2,6-d i m e t h o x y p y r i d i n i u m\right)\right]^{+}$(OTf) (127).}

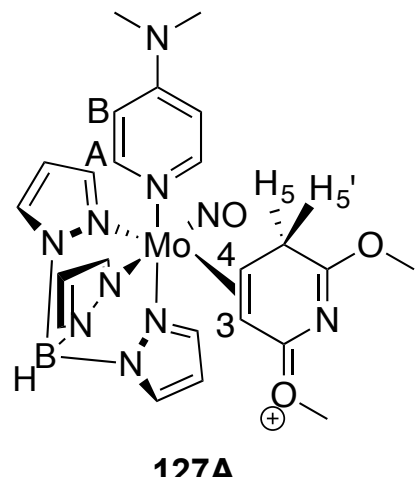

127A

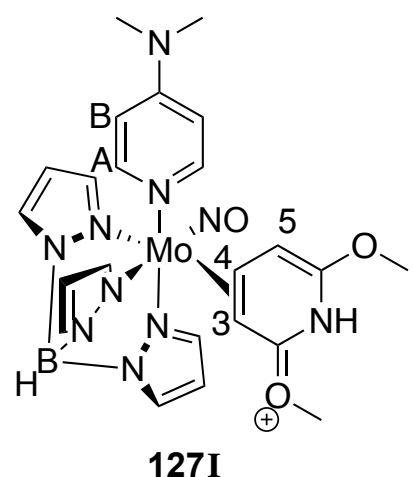

$127 \mathrm{I}$

To a $-30{ }^{\circ} \mathrm{C}$ solution of $122(100 \mathrm{mg}, 0.17 \mathrm{mmol})$ and $\mathrm{CH}_{3} \mathrm{CN}(2 \mathrm{~mL})$ was added a -30 ${ }^{\circ} \mathrm{C}, 1 \mathrm{M}$ solution of $\mathrm{HOTf} / \mathrm{CH}_{3} \mathrm{CN}(0.7 \mathrm{~mL}, 0.7 \mathrm{mmol})$. The resulting orange solution was left at $-30{ }^{\circ} \mathrm{C}$ for $15 \mathrm{~min}$ and was subsequently added to stirring $\mathrm{Et}_{2} \mathrm{O}(50 \mathrm{~mL})$, yielding a bright orange precipitate. This precipitate was isolated on a $15 \mathrm{~mL}$ fine porosity fritted disc, washed with Et2O (3 x $15 \mathrm{~mL}$ ), and dried in vacuo to yield 127 (40 mg, 32\% yield). $\mathrm{CV}(\mathrm{DMAc}) E_{\mathrm{p}, \mathrm{a}}=+0.98 \mathrm{~V}(\mathrm{NHE}) . \mathrm{IR}: v\left(\mathrm{C}-\mathrm{H} \mathrm{sp}{ }^{2}\right)=2954 \mathrm{~cm}^{-1}, v(\mathrm{~B}-\mathrm{H})=2511 \mathrm{~cm}^{-1}$ $v(\mathrm{NO})=1615 \mathrm{~cm}^{-1} .{ }^{1} \mathrm{H}$ NMR $\left(\mathrm{d}^{6}\right.$-Acetone, $\left.600 \mathrm{MHz}\right): \delta 9.93(1 \mathrm{H}, \mathrm{bs}, \mathrm{N}-\mathrm{H}$ 127I $), 8.01$ (1H, d, Pz3/5 127I), 7.99 (1H, d, Pz3/5 127I), 7.98 (1H, d, Pz5A 127A), 7.93 (1H, d, Pz5C 127A), 7.82 (1H, d, Pz5B 127A), 7.74 (1H, d, Pz3C 127A), 7.73 (2H, buried bs, DMAP-A 127A and 127I), 7.58 (1H, d, Pz3/5 127I), 7.57 (1H, d, Pz3/5 127I), 7.35 (1H, d, Pz3A 127A), 7.15 (1H, d, Pz3B 127A), 6.98 (1H, d, Pz3/5 127I), 6.83 (2H, m, DMAPB 127I), 6.66 (2H, bs, DMAP-B 127A), $6.44(1 \mathrm{H}, \mathrm{t}, \mathrm{Pz} 4 \mathrm{C}$ 127A $), 6.41(1 \mathrm{H}, \mathrm{t}, \mathrm{Pz} 4 \mathrm{~A}$ 127A), 6.17 (1H, t, Pz4B 127A), 6.13 (1H, t, Pz4 127I), 5.34 (1H, d, $J=5.8, \mathrm{H} 5$ 127I), $4.22(3 \mathrm{H}, \mathrm{s}, \mathrm{OMe}$ 127A $), 4.20(3 \mathrm{H}, \mathrm{s}, \mathrm{OMe}$ 127A $), 4.11(1 \mathrm{H}, \mathrm{t}, J=6.8, \mathrm{H} 4$ 127I), 4.04 $(1 \mathrm{H}, \mathrm{dd}, J=22.5,8.4, \mathrm{H} 5$ ' 127A), $3.84(3 \mathrm{H}, \mathrm{s}, \mathrm{OMe} \mathbf{1 2 7 I}), 3.73(1 \mathrm{H} \mathrm{t}, J=7.9, \mathrm{H} 4$ 127A), 3.49 (3H, s, OMe 127I), $3.47(1 \mathrm{H}, \mathrm{dt}, J=22.5,1.3, \mathrm{H} 5$ 127A), $3.03(6 \mathrm{H}, \mathrm{s}, \mathrm{NMe}$ 127I), $3.02(6 \mathrm{H}, \mathrm{s}, \mathrm{NMe}$ 127A $), 2.94(1 \mathrm{H}, \mathrm{dd}, J=7.9,1.3, \mathrm{H} 3$ 127A) $2.81(1 \mathrm{H}, \mathrm{d}, J=8.3$, 
H3 127I). ${ }^{13} \mathrm{C}$ NMR (d $\mathrm{d}^{6}$-Acetone, $200 \mathrm{MHz}$ ): 191.9 (CO), 186.7 (CO), 155.6 (DMAP-C), 149.9 (2C, DMAP-A), 144.8 (Pz3A), 143.1 (Pz3B), 141.9 (Pz3C), 138.7 (Pz5A/5C), 138.3 (Pz5A/5C), 137.4 (Pz5B), 109.5 (2C, DMAP-B), 107.9 (Pz4A/4C), 107.6 (Pz4A/4C), 107.3 (Pz4B), 71.3 (C4), 58.8 (OMe), 58.4 (OMe), 57.0 (C3), 39.4 (DMAPMe), 33.4 (C5). HRMS: $\mathrm{C}_{23} \mathrm{H}_{30} \mathrm{~N}_{10} \mathrm{O}_{3} \mathrm{BMo}^{+}$obsd (\%), calcd (\%), ppm: 597.1692 (50), 597.1662 (54), 5.0; 599.1664 (55), 599.1659 (49), 0.8; 600.1662 (88), 600.1658 (81), 0.6; 601.1654 (93), 601.1653 (86), 0.2; 602.1664 (87), 602.1666 (75), -0.3; 603.1664 (100), 603.1652 (100), 1.9; 604.1700 (41), 604.1681 (35), 3.2; 605.1690 (41), 605.1672 (39), 3.0 . 


\subsection{References}

1. $\quad$ Bates, R. W.; Sa-Ei, K. Tetrahedron 2002, 58, (30), 5957.

2. Baumann, M.; Baxendale, I. R. Beilstein J. Org. Chem. 2013, 9, 2265.

3. Katritzky Ar Fau - Katritzky, A. R.; Wang Z Fau - Wang, Z.; Slavov S Fau Slavov, S.; Tsikolia M Fau - Tsikolia, M.; Dobchev D Fau - Dobchev, D.; Akhmedov Ng Fau - Akhmedov, N. G.; Hall Cd Fau - Hall, C. D.; Bernier Ur Fau - Bernier, U. R.; Clark Gg Fau - Clark, G. G.; Linthicum Kj Fau - Linthicum, K. J., (0027-8424 (Print)).

4. Metkar, P. S.; Scialdone, M. A.; Moloy, K. G. Green Chem. 2014, 16, (10), 4575.

5. Bull, J. A.; Mousseau, J. J.; Pelletier, G.; Charette, A. B. Chemical Rev. 2012, $112,(5), 2642$.

6. Buffat, M. G. P. Tetrahedron 2004, 60, (8), 1701.

7. Cordone, R.; Harman, W. D.; Taube, H. J. Am. Chem. Soc. 1989, 111, 2896.

8. Cordone, R.; Taube, H. J. Am. Chem. Soc. 1987, 109, (26), 8101.

9. Meiere, S. H.; Brooks, B. C.; Gunnoe, T. B.; Sabat, M.; Harman, W. D. Organometallics 2001, 20, 1038.

10. Bonanno, J. B.; Veige, A. S.; Wolczanski, P. T.; Lobkovsky, E. B. Inorg. Chim. Act. 2003, 345, 173.

11. Kleckley, T. S.; Bennett, J. L.; Wolczanski, P. T.; Lobkovsky, E. B. J. Am. Chem. Soc. 1997, 119, 247.

12. Neithamer, D. R.; Parkanyi, L.; Mitchell, J. F.; Wolczanski, P. T. J. Am. Chem. Soc. 1988, 110, 4421.

13. Covert, K. J.; Neithamer, D. R.; Zonnevylle, M. C.; LaPointe, R. E.; Schaller, C. P.; Wolczanski, P. T. Inorg. Chem. 1991, 30, (11), 2494.

14. Harrison, D. P.; Harman, W. D. Aldrichimica Acta 2012, 45, 45.

15. Harrison, D. P.; Iovan, D. A.; Myers, W. H.; Sabat, M.; Wang, S.; Zottig, V. E.; Harman, W. D. J. Am. Chem. Soc. 2011, 133, (45), 18378.

16. Harrison, D. P.; Kosturko, G. W.; Ramdeen, V. M.; Nichols-Nielander, A. C.; Payne, S. J.; Sabat, M.; Myers, W. H.; Harman, W. D. Organometallics 2010, 29, (8), 1909.

17. Harrison, D. P.; Sabat, M.; Myers, W. H.; Harman, W. D. J. Am. Chem. Soc. 2010, 132, (48), 17282. 
18. Harrison, D. P.; Zottig, V. E.; Kosturko, G. W.; Welch, K. D.; Sabat, M.; Myers, W. H.; Harman, W. D. Organometallics 2009, 28, (19), 5682.

19. Kosturko, G. W.; Harrison, D. P.; Sabat, M.; Myers, W. H.; Harman, W. D. Organometallics 2009, 28, (2), 387.

20. Lankenau, A. W.; Iovan, D. A.; Pienkos, J. A.; Salomon, R. J.; Wang, S.; Harrison, D. P.; Myers, W. H.; Harman, W. D. J. Am. Chem. Soc. 2015, 137, (10), 3649.

21. Pienkos, J. A.; Zottig, V. E.; Iovan, D. A.; Li, M.; Harrison, D. P.; Sabat, M.; Salomon, R. J.; Strausberg, L.; Teran, V. A.; Myers, W. H.; Harman, W. D. Organometallics 2013, 32, (2), 691.

22. Delafuente, D. A.; Kosturko, G. W.; Graham, P. M.; Harman, W. H.; Myers, W. H.; Surendranath, Y.; Klet, R. C.; Welch, K. D.; Trindle, C. O.; Sabat, M.; Harman, W. D. J. Am. Chem. Soc. 2006, 129, (2), 406.

23. Harrison, D. P.; Welch, K. D.; Nichols-Nielander, A. C.; Sabat, M.; Myers, W. H.; Harman, W. D. J. Am. Chem. Soc. 2008, 130, (50), 16844.

24. Trindle, C.; Harman, W. D. J. Comput. Chem. 2005, 26, 194.

25. Mann, B. E. Ann. Rep. on NMR Spec. 1982, 12, 263.

26. Allen, F. H.; Kennard, O.; Watson, D. G.; Brammer, L.; Orpen, A. G.; Taylor, R. J. Chem. Soc., Perkin Trans. 2 1987, (12), S1.

27. Glorius, F. Organic \& Biomolecular Chemistry 2005, 3, (23), 4171.

28. Welch, K. D.; Smith, P. L.; Keller, A. P.; Myers, W. H.; Sabat, M.; Harman, W. D. Organometallics 2006, 25, (21), 5067.

29. Welch, K. D.; Harrison, D. P.; Sabat, M.; Hejazi, E. Z.; Parr, B. T.; Fanelli, M. G.; Gianfrancesco, N. A.; Nagra, D. S.; Myers, W. H.; Harman, W. D. Organometallics 2009, 28, (20), 5960.

30. Shen, T. Y.; Harman, W. D.; Huang, D. F.; Gonzalez, J. Preparation of 7azabicyclo[2.2.1] heptane and -heptene derivatives as cholinergic receptor ligands. US5817679A, 1998.

31. Myers, W. H.; Welch, K. D.; Graham, P. M.; Keller, A.; Sabat, M.; Trindle, C. O.; Harman, W. D. Organometallics 2005, 24, (22), 5267.

32. Myers, W. H.; Sabat, M.; Harman, W. D. J. Am. Chem. Soc. 1991, 113, 6682.

33. Myers, W. H.; Koontz, J. I.; Harman, W. D. J. Am. Chem. Soc. 1992, 114, 5684.

34. Badio, B.; Garraffo, M.; Plummer, C. V.; Padgett, W. L.; Daly, J. W. Euro. J.

Pharm. 1997, 321, 189. 
35. Graham, P. M.; Delafuente, D. A.; Liu, W.; Myers, W. H.; Sabat, M.; Harman, W. D. J. Am. Chem. Soc. 2005, 127, (30), 10568.

36. Full credit is given to Justin Wilde for the discovery and isolation of the complexes in Scheme 6.10. side 


\section{Concluding Remarks}


The goal of this research on molybdenum dearomatization agents has been to develop a new system that incorporated the following characteristics: recyclability, scalability, and adjustability in the ligand set. In this vein, we have demonstrated the ability to bind a variety of aromatics by a reduction of $\mathrm{TpMo}(\mathrm{NO})(\mathrm{L})(\mathrm{I})($ where $\mathrm{L}=\mathrm{N}$ methylimidazole (MeIm) or $N, N$-dimethylaminopyridine (DMAP)) in the presence of the desired coordinating ligand ( $L \pi)$. This air-stable iodo complex can be synthesized on a large scale $(>150 \mathrm{~g})$ without chromatography. Coordination complexes of certain aromatic molecules can be isolated from the reduction of this iodo precursor in the presence of the desired ligand on scales up to $13 \mathrm{~g}$. Once bound, the dearomatized ligands are activated toward organic transformations, which, after oxidative decomplexation, yield novel small molecules.

Oxidative decomplexation of the modified organic ligand can be accomplished with a mild oxidant (e.g., air) or with iodine to recover the $\mathrm{TpMo}(\mathrm{NO})(\mathrm{L})(\mathrm{I})$ complex. Initial investigations demonstrated the ability to recycle the molybdenum complex and to carry out said process on $\mathbf{6}$ at a $4 \mathrm{~g}$ scale to yield $800 \mathrm{mg}$ of $\mathbf{1 2}$ using the $\{\mathrm{TpMo}(\mathrm{NO})(\mathrm{MeIm})\}$ fragment. Variability in the ligand set was explored by exchanging the MeIm ligand with DMAP. This seemingly more robust $\{$ TpMo(NO)(DMAP)\} fragment has been the focus of our attention over the past four years.

From novel organic reactivity of known arene complexes (Chapters 3 and 4) to newly found aromatics capable of being bound (Chapters 5 and 6), we have demonstrated the true potential for future dearomatization with molybdenum using $\{\mathrm{TpMo}(\mathrm{NO})(\mathrm{L})\}$. The novel organics isolated during the research for this dissertation have been submitted for screening through Eli-Lilly's Open Innovation Drug Discovery Program. Although no 
pharmacological uses have yet been found for the submitted compounds, the larger the chemical library we are able to develop, the better understanding we can obtain of the chemistry of these seemingly simple, yet novel molecules.

Furthermore, this work demonstrates that the conditions developed for the organic transformations of one metal-coordinated aromatic (i.e., naphthalene), assist in the understanding of organic transformations of other aromatics (i.e., TFT and substituted pyridines) in these systems. We have demonstrated with the naphtha- and anthrafuranone cores 20 and 21 that after oxidative decomplexation, the final products after organic transformations are more reactive than their aromatic precursor. Specifically, the double bond that was protected by coordination with the metal can undergo further chemical transformations without the use of transition metals. The formation of two new carboncarbon bonds (41 and 42) to the $\pi$-system of an aromatic while still bound to molybdenum, demonstrates the stereocontrol of not only the nucleophilic addition (as seen in Chapter 2), but also of the initial electrophilic addition. In both additions the incoming electrophile or nucleophile adds anti to the metal center. Similarly, novel hexahydrophenanthrenes $\mathbf{6 1}, \mathbf{8 9}, \mathbf{9 1}$, and $\mathbf{9 2}$, can be synthesized by $[A+A]$ or $[A+B]$ MIMIRC additions to $\mathbf{2 4}$.

In work to widen the scope of the chemistry of these molybdenum systems, the TFT complex (100) has proven to be a more effective exchange reagent than the dimethylfuran complex (26) and its use in accessing a broader array of aromatic complexes (i.e., 1-fluorobenzene, 1-fluoro-3-trifluoromethylbenzene, 1,2difluorobenzene, etc.) has been shown. Additionally, the organic transformations demonstrated with $\mathbf{1 0 0}$ suggest that similar reactivity may be possible with the newly 
isolated fluorinated aromatics, which were incompatible with previous dearomatization systems. In addition to fluorinated arenes, the advent of the pyridine complexes $\mathbf{1 2 0}$ and 121 has laid the groundwork for an unexplored realm of chemistry, dearomatization of ANHs with molybdenum. The recent binding of pyridine-borane to $\{\mathrm{TpMo}(\mathrm{NO})(\mathrm{DMAP})\}$ is a significant advance in this chemistry and will continue to be explored.

A significant challenge for the synthetic utility of the molybdenum systems is the current lack of a process for enantioenrichment of the metal center. Without an ability to resolve the enantiomers of the aromatic complexes, the use of this molybdenum system for the enantioselective synthesis of organics is limited to the use of chiral auxiliaries on the organic molecule or chiral reagents. However, consider the 13 years of in-depth investigation of dearomatization using the $\left\{\mathrm{TpW}(\mathrm{NO})\left(\mathrm{PMe}_{3}\right)\right\}$ fragment before a broadly applicable method for its enantioenrichment was discovered. The lesson from this previous work is that the more we investigate the surprising chemistry of the molybdenum system, the better equipped we will be to solve this problem.

The broader applicability of this research can be divided into two main points. For the organic community, the synthesis of novel small molecules mentioned in this dissertation represents a proof-of-concept for the versatility of this molybdenum scaffold. Although nowhere near an exhaustive search of all electrophiles, nucleophiles, reaction conditions, etc., this dissertation provides a few examples of the wide range of products potentially available through the use of $\{\mathrm{TpMo}(\mathrm{NO})(\mathrm{L})\}$. For the inorganic community, the broader applicability of this dissertation can be seen in the similarities between this second-row dearomatization agent and its third-row congeners. The Harman lab has 
previously demonstrated that by fine-tuning the electronics of the metal center, we can invoke similar reactivity between the third-row metals osmium, rhenium and tungsten. This dissertation shows an ability to continue this impressive control of metal electronics while moving up a row on the periodic table. This approach not only affords a decreased toxicity and cost per mole, but also provides insight into the fundamental nature of the chemistry of transition metals. 


\section{Appendix}




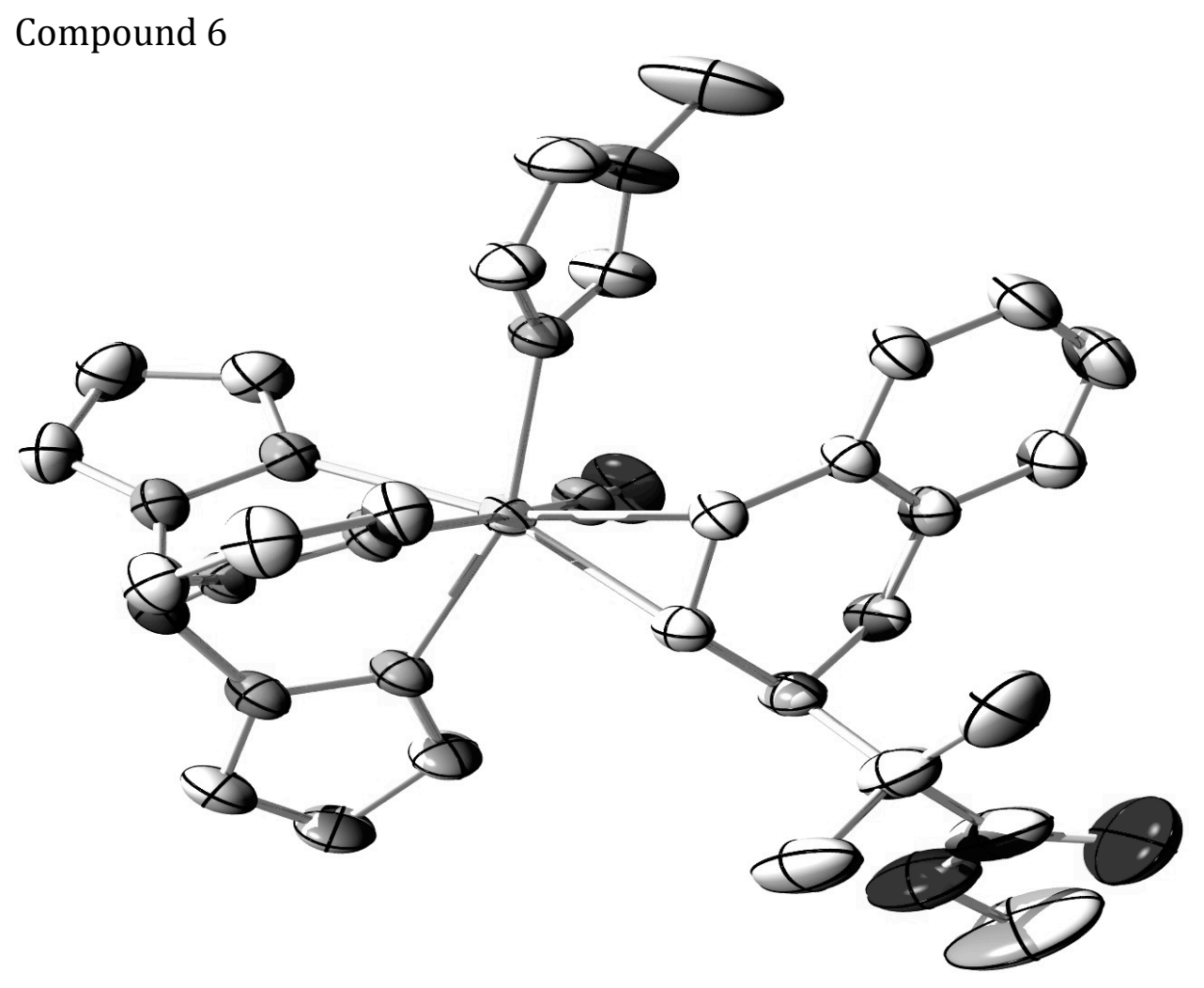


Compound 6

Table 1. Crystal Data for $\mathrm{C}_{32} \mathrm{H}_{42} \mathrm{BN}_{9} \mathrm{O}_{4} \mathrm{Mo}$.

\begin{tabular}{|c|c|}
\hline Empirical formula & C32 H42 B Mo N9 O4 \\
\hline Formula weight & 651.39 \\
\hline Temperature & $233(2) \mathrm{K}$ \\
\hline Wavelength & $0.71073 \AA$ \\
\hline Crystal system & Orthorhombic \\
\hline Space group & Pbca \\
\hline \multirow[t]{3}{*}{ Unit cell dimensions } & $\mathrm{a}=13.4488(9) \AA$ \\
\hline & $\mathrm{b}=21.3636(15) \AA$ \\
\hline & $\mathrm{c}=24.1495(16) \AA$ \\
\hline Volume & $6938.5(8) \AA^{3}$ \\
\hline $\mathrm{Z}$ & 8 \\
\hline Density (calculated) & $1.247 \mathrm{Mg} / \mathrm{m}^{3}$ \\
\hline Absorption coefficient & $0.418 \mathrm{~mm}^{-1}$ \\
\hline $\mathrm{F}(000)$ & 2688 \\
\hline Crystal size & $0.390 \times 0.270 \times 0.08 \mathrm{~mm}^{3}$ \\
\hline Theta range for data collection & 3.286 to $30.168^{\circ}$. \\
\hline Index ranges & $-19<=\mathrm{h}<=19,-30<=\mathrm{k}<=30,-34<=\mathrm{l}<=34$ \\
\hline Reflections collected & 125378 \\
\hline Independent reflections & $10233[\mathrm{R}(\mathrm{int})=0.0359]$ \\
\hline Completeness to theta $=25.242^{\circ}$ & $99.8 \%$ \\
\hline Absorption correction & Empirical \\
\hline Refinement method & Full-matrix least-squares on $\mathrm{F}^{2}$ \\
\hline Data / restraints / parameters & $10233 / 0 / 461$ \\
\hline Goodness-of-fit on $\mathrm{F}^{2}$ & 1.054 \\
\hline Final $R$ indices $[\mathrm{I}>2 \operatorname{sigma}(\mathrm{I})]$ & $\mathrm{R} 1=0.0379, \mathrm{wR} 2=0.0959$ \\
\hline $\mathrm{R}$ indices (all data) & $\mathrm{R} 1=0.0534, \mathrm{wR} 2=0.1072$ \\
\hline Largest diff. peak and hole & 0.697 and -0.450 e. $\AA^{-3}$ \\
\hline
\end{tabular}




\section{Compound 6}

Table 2. Fractional coordinates $\left(\times 10^{4}\right)$ and equivalent isotropic displacement parameters $\left(\AA^{2} \times 10^{3}\right)$ for $\mathrm{C}_{32} \mathrm{H}_{42} \mathrm{BN}_{9} \mathrm{O}_{4} \mathrm{Mo}$. $\mathrm{U}(\mathrm{eq})$ is defined as one third of the trace of the orthogonalized $\mathrm{U}^{\mathrm{ij}}$ tensor.

\begin{tabular}{|c|c|c|c|c|}
\hline & $\mathrm{x}$ & $\mathrm{y}$ & $\mathrm{z}$ & $\mathrm{U}(\mathrm{eq})$ \\
\hline Mo & $3822(1)$ & $7221(1)$ & $6012(1)$ & $31(1)$ \\
\hline $\mathrm{O}(1)$ & $1276(2)$ & $4671(2)$ & $7004(2)$ & $133(1)$ \\
\hline $\mathrm{O}(2)$ & $762(2)$ & $5218(1)$ & $6286(1)$ & $97(1)$ \\
\hline $\mathrm{O}(3)$ & 2071(2) & $7830(1)$ & $6534(1)$ & $67(1)$ \\
\hline $\mathrm{O}(4)$ & $4352(6)$ & $5427(8)$ & $3983(6)$ & $320(6)$ \\
\hline $\mathrm{N}(1)$ & $3022(1)$ & $6985(1)$ & $5240(1)$ & $39(1)$ \\
\hline $\mathrm{N}(2)$ & $3423(1)$ & $7107(1)$ & $4735(1)$ & $41(1)$ \\
\hline $\mathrm{N}(3)$ & $4126(1)$ & $8080(1)$ & $5517(1)$ & $38(1)$ \\
\hline $\mathrm{N}(4)$ & $4384(1)$ & $8060(1)$ & 4974(1) & $40(1)$ \\
\hline $\mathrm{N}(5)$ & $5168(1)$ & $6872(1)$ & $5517(1)$ & $36(1)$ \\
\hline $\mathrm{N}(6)$ & $5235(1)$ & $7023(1)$ & $4969(1)$ & $37(1)$ \\
\hline $\mathrm{N}(7)$ & $4806(1)$ & $7699(1)$ & $6611(1)$ & $40(1)$ \\
\hline $\mathrm{N}(8)$ & $5220(2)$ & $8213(1)$ & $7362(1)$ & $70(1)$ \\
\hline $\mathrm{N}(9)$ & $2778(1)$ & $7554(1)$ & $6333(1)$ & $39(1)$ \\
\hline $\mathrm{C}(1)$ & $2481(2)$ & 5961(1) & $6383(1)$ & $42(1)$ \\
\hline $\mathrm{C}(2)$ & $3493(2)$ & $6216(1)$ & $6209(1)$ & $36(1)$ \\
\hline $\mathrm{C}(3)$ & $4150(1)$ & $6457(1)$ & $6616(1)$ & $34(1)$ \\
\hline $\mathrm{C}(4)$ & $3829(1)$ & $6535(1)$ & $7193(1)$ & $35(1)$ \\
\hline $\mathrm{C}(5)$ & $4513(2)$ & $6680(1)$ & $7610(1)$ & $47(1)$ \\
\hline$C(6)$ & $4223(2)$ & $6734(2)$ & $8160(1)$ & $60(1)$ \\
\hline$C(7)$ & $3246(2)$ & $6635(2)$ & $8299(1)$ & $64(1)$ \\
\hline $\mathrm{C}(8)$ & $2565(2)$ & $6485(1)$ & $7895(1)$ & $52(1)$ \\
\hline $\mathrm{C}(9)$ & $2832(2)$ & $6437(1)$ & $7340(1)$ & $40(1)$ \\
\hline$C(10)$ & $2080(2)$ & $6302(1)$ & $6899(1)$ & $42(1)$ \\
\hline $\mathrm{C}(11)$ & 2493(2) & $5233(1)$ & $6457(1)$ & $59(1)$ \\
\hline$C(12)$ & $1463(3)$ & $5009(2)$ & $6620(2)$ & $86(1)$ \\
\hline$C(13)$ & $3215(3)$ & $5013(2)$ & $6906(2)$ & $82(1)$ \\
\hline $\mathrm{C}(14)$ & $2750(3)$ & $4910(2)$ & $5905(2)$ & $84(1)$ \\
\hline $\mathrm{C}(15)$ & $-236(3)$ & $5011(3)$ & $6487(3)$ & $165(3)$ \\
\hline$C(16)$ & $2749(2)$ & $6977(1)$ & $4339(1)$ & $51(1)$ \\
\hline
\end{tabular}




$\begin{array}{lllll}\mathrm{C}(17) & 1900(2) & 6770(1) & 4581(1) & 55(1) \\ \mathrm{C}(18) & 2096(2) & 6784(1) & 5146(1) & 47(1) \\ \mathrm{C}(19) & 4525(2) & 8643(1) & 4783(1) & 51(1) \\ \mathrm{C}(20) & 4358(2) & 9054(1) & 5206(1) & 58(1) \\ \mathrm{C}(21) & 4112(2) & 8682(1) & 5658(1) & 48(1) \\ \mathrm{C}(22) & 6078(2) & 6784(1) & 4754(1) & 46(1) \\ \mathrm{C}(23) & 6576(2) & 6471(1) & 5163(1) & 53(1) \\ \mathrm{C}(24) & 5981(2) & 6535(1) & 5629(1) & 45(1) \\ \mathrm{C}(25) & 4474(2) & 8017(1) & 7041(1) & 55(1) \\ \mathrm{C}(26) & 6079(2) & 8015(2) & 7127(1) & 66(1) \\ \mathrm{C}(27) & 5809(2) & 7693(1) & 6663(1) & 51(1) \\ \mathrm{C}(28) & 5081(4) & 8541(3) & 7892(2) & 126(2) \\ \mathrm{C}(29 B) & 3894(14) & 5066(10) & 3541(7) & 139(5) \\ \mathrm{C}(29 \mathrm{~A}) & 3730(20) & 5699(13) & 3586(11) & 208(10) \\ \mathrm{C}(30) & 2940(20) & 5256(10) & 3579(7) & 284(11) \\ \mathrm{C}(31) & 2751(7) & 5115(5) & 4142(7) & 205(5) \\ \mathrm{C}(32) & 3667(11) & 5304(4) & 4442(4) & 185(4) \\ \mathrm{B}(1) & 4445(2) & 7422(1) & 4679(1) & 40(1)\end{array}$




\section{Compound 6}

Table 3. Bond Lengths (A) and Angles (deg) for $\mathrm{C}_{32} \mathrm{H}_{42} \mathrm{BN}_{9} \mathrm{O}_{4} \mathrm{Mo}$.

\begin{tabular}{|c|c|}
\hline Mo-N(9) & $1.7544(17)$ \\
\hline Mo-N(1) & $2.2103(17)$ \\
\hline Mo-N(7) & $2.2107(18)$ \\
\hline Mo-N(3) & $2.2291(18)$ \\
\hline Mo-C(3) & $2.232(2)$ \\
\hline $\mathrm{Mo}-\mathrm{C}(2)$ & $2.242(2)$ \\
\hline Mo-N(5) & $2.2937(16)$ \\
\hline $\mathrm{O}(1)-\mathrm{C}(12)$ & $1.201(5)$ \\
\hline $\mathrm{O}(2)-\mathrm{C}(12)$ & $1.319(5)$ \\
\hline $\mathrm{O}(2)-\mathrm{C}(15)$ & $1.495(5)$ \\
\hline $\mathrm{O}(3)-\mathrm{N}(9)$ & $1.220(2)$ \\
\hline $\mathrm{O}(4)-\mathrm{C}(29 \mathrm{~A})$ & $1.40(3)$ \\
\hline $\mathrm{O}(4)-\mathrm{C}(29 \mathrm{~B})$ & $1.45(2)$ \\
\hline $\mathrm{O}(4)-\mathrm{C}(32)$ & $1.466(11)$ \\
\hline $\mathrm{N}(1)-\mathrm{C}(18)$ & $1.337(3)$ \\
\hline $\mathrm{N}(1)-\mathrm{N}(2)$ & $1.360(2)$ \\
\hline $\mathrm{N}(2)-\mathrm{C}(16)$ & $1.346(3)$ \\
\hline $\mathrm{N}(2)-\mathrm{B}(1)$ & $1.537(3)$ \\
\hline $\mathrm{N}(3)-\mathrm{C}(21)$ & $1.331(3)$ \\
\hline $\mathrm{N}(3)-\mathrm{N}(4)$ & $1.358(2)$ \\
\hline N(4)-C(19) & $1.340(3)$ \\
\hline $\mathrm{N}(4)-\mathrm{B}(1)$ & $1.541(3)$ \\
\hline $\mathrm{N}(5)-\mathrm{C}(24)$ & $1.336(3)$ \\
\hline $\mathrm{N}(5)-\mathrm{N}(6)$ & $1.366(2)$ \\
\hline $\mathrm{N}(6)-\mathrm{C}(22)$ & $1.347(3)$ \\
\hline $\mathrm{N}(6)-\mathrm{B}(1)$ & $1.532(3)$ \\
\hline $\mathrm{N}(7)-\mathrm{C}(25)$ & $1.319(3)$ \\
\hline $\mathrm{N}(7)-\mathrm{C}(27)$ & $1.355(3)$ \\
\hline $\mathrm{N}(8)-\mathrm{C}(25)$ & $1.336(3)$ \\
\hline $\mathrm{N}(8)-\mathrm{C}(26)$ & $1.355(4)$ \\
\hline $\mathrm{N}(8)-\mathrm{C}(28)$ & $1.471(4)$ \\
\hline$C(1)-C(2)$ & $1.525(3)$ \\
\hline $\mathrm{C}(1)-\mathrm{C}(10)$ & $1.542(3)$ \\
\hline
\end{tabular}




\begin{tabular}{|c|c|}
\hline$C(1)-C(11)$ & $1.566(4)$ \\
\hline $\mathrm{C}(2)-\mathrm{C}(3)$ & $1.419(3)$ \\
\hline $\mathrm{C}(3)-\mathrm{C}(4)$ & $1.468(3)$ \\
\hline$C(4)-C(5)$ & $1.399(3)$ \\
\hline $\mathrm{C}(4)-\mathrm{C}(9)$ & $1.403(3)$ \\
\hline $\mathrm{C}(5)-\mathrm{C}(6)$ & $1.387(3)$ \\
\hline $\mathrm{C}(6)-\mathrm{C}(7)$ & $1.373(4)$ \\
\hline $\mathrm{C}(7)-\mathrm{C}(8)$ & $1.376(4)$ \\
\hline $\mathrm{C}(8)-\mathrm{C}(9)$ & $1.391(3)$ \\
\hline$C(9)-C(10)$ & $1.497(3)$ \\
\hline$C(11)-C(12)$ & $1.517(4)$ \\
\hline$C(11)-C(13)$ & $1.530(5)$ \\
\hline$C(11)-C(14)$ & $1.541(4)$ \\
\hline$C(16)-C(17)$ & $1.358(4)$ \\
\hline$C(17)-C(18)$ & $1.391(3)$ \\
\hline$C(19)-C(20)$ & $1.365(4)$ \\
\hline$C(20)-C(21)$ & $1.390(4)$ \\
\hline$C(22)-C(23)$ & $1.368(4)$ \\
\hline$C(23)-C(24)$ & $1.388(3)$ \\
\hline$C(26)-C(27)$ & $1.363(4)$ \\
\hline C(29B)-C(30) & $1.34(3)$ \\
\hline$C(29 A)-C(30)$ & $1.42(3)$ \\
\hline $\mathrm{C}(30)-\mathrm{C}(31)$ & $1.416(18)$ \\
\hline$C(31)-C(32)$ & $1.484(11)$ \\
\hline N(9)-Mo-N(1) & $94.30(7)$ \\
\hline N(9)-Mo-N(7) & $90.19(8)$ \\
\hline N(1)-Mo-N(7) & $161.48(7)$ \\
\hline N(9)-Mo-N(3) & $92.83(8)$ \\
\hline N(1)-Mo-N(3) & $79.88(7)$ \\
\hline N(7)-Mo-N(3) & $81.96(7)$ \\
\hline N(9)-Mo-C(3) & $99.58(8)$ \\
\hline N(1)-Mo-C(3) & $118.72(7)$ \\
\hline N(7)-Mo-C(3) & $78.03(7)$ \\
\hline N(3)-Mo-C(3) & $156.40(7)$ \\
\hline N(9)-Mo-C(2) & $97.87(8)$ \\
\hline
\end{tabular}




\begin{tabular}{|c|c|}
\hline $\mathrm{N}(1)-\mathrm{Mo}-\mathrm{C}(2)$ & $82.20(7)$ \\
\hline N(7)-Mo-C(2) & $115.00(7)$ \\
\hline $\mathrm{N}(3)-\mathrm{Mo}-\mathrm{C}(2)$ & $159.74(7)$ \\
\hline $\mathrm{C}(3)-\mathrm{Mo}-\mathrm{C}(2)$ & $36.98(7)$ \\
\hline N(9)-Mo-N(5) & $173.43(8)$ \\
\hline $\mathrm{N}(1)-\mathrm{Mo}-\mathrm{N}(5)$ & $82.57(6)$ \\
\hline $\mathrm{N}(7)-\mathrm{Mo}-\mathrm{N}(5)$ & $91.04(7)$ \\
\hline $\mathrm{N}(3)-\mathrm{Mo}-\mathrm{N}(5)$ & $80.95(6)$ \\
\hline $\mathrm{C}(3)-\mathrm{Mo}-\mathrm{N}(5)$ & $86.99(7)$ \\
\hline $\mathrm{C}(2)-\mathrm{Mo}-\mathrm{N}(5)$ & $87.45(7)$ \\
\hline $\mathrm{C}(12)-\mathrm{O}(2)-\mathrm{C}(15)$ & $110.1(4)$ \\
\hline $\mathrm{C}(29 \mathrm{~A})-\mathrm{O}(4)-\mathrm{C}(29 \mathrm{~B})$ & $57.6(11)$ \\
\hline $\mathrm{C}(29 \mathrm{~A})-\mathrm{O}(4)-\mathrm{C}(32)$ & $102.3(12)$ \\
\hline $\mathrm{C}(29 \mathrm{~B})-\mathrm{O}(4)-\mathrm{C}(32)$ & $101.1(11)$ \\
\hline $\mathrm{C}(18)-\mathrm{N}(1)-\mathrm{N}(2)$ & $106.14(17)$ \\
\hline $\mathrm{C}(18)-\mathrm{N}(1)-\mathrm{Mo}$ & $132.07(16)$ \\
\hline $\mathrm{N}(2)-\mathrm{N}(1)-\mathrm{Mo}$ & $121.38(13)$ \\
\hline $\mathrm{C}(16)-\mathrm{N}(2)-\mathrm{N}(1)$ & $109.35(19)$ \\
\hline $\mathrm{C}(16)-\mathrm{N}(2)-\mathrm{B}(1)$ & $129.04(19)$ \\
\hline $\mathrm{N}(1)-\mathrm{N}(2)-\mathrm{B}(1)$ & $121.18(16)$ \\
\hline $\mathrm{C}(21)-\mathrm{N}(3)-\mathrm{N}(4)$ & $106.35(19)$ \\
\hline $\mathrm{C}(21)-\mathrm{N}(3)-\mathrm{Mo}$ & $130.98(16)$ \\
\hline $\mathrm{N}(4)-\mathrm{N}(3)-\mathrm{Mo}$ & $122.67(14)$ \\
\hline $\mathrm{C}(19)-\mathrm{N}(4)-\mathrm{N}(3)$ & $109.80(19)$ \\
\hline $\mathrm{C}(19)-\mathrm{N}(4)-\mathrm{B}(1)$ & $131.00(19)$ \\
\hline $\mathrm{N}(3)-\mathrm{N}(4)-\mathrm{B}(1)$ & $119.18(18)$ \\
\hline $\mathrm{C}(24)-\mathrm{N}(5)-\mathrm{N}(6)$ & $105.61(16)$ \\
\hline $\mathrm{C}(24)-\mathrm{N}(5)-\mathrm{Mo}$ & $135.64(14)$ \\
\hline $\mathrm{N}(6)-\mathrm{N}(5)-\mathrm{Mo}$ & $118.76(12)$ \\
\hline $\mathrm{C}(22)-\mathrm{N}(6)-\mathrm{N}(5)$ & $109.90(18)$ \\
\hline $\mathrm{C}(22)-\mathrm{N}(6)-\mathrm{B}(1)$ & $128.16(18)$ \\
\hline $\mathrm{N}(5)-\mathrm{N}(6)-\mathrm{B}(1)$ & $121.92(16)$ \\
\hline $\mathrm{C}(25)-\mathrm{N}(7)-\mathrm{C}(27)$ & $105.6(2)$ \\
\hline $\mathrm{C}(25)-\mathrm{N}(7)-\mathrm{Mo}$ & $123.39(16)$ \\
\hline $\mathrm{C}(27)-\mathrm{N}(7)-\mathrm{Mo}$ & $130.73(16)$ \\
\hline $\mathrm{C}(25)-\mathrm{N}(8)-\mathrm{C}(26)$ & $107.3(2)$ \\
\hline
\end{tabular}




\begin{tabular}{|c|c|}
\hline $\mathrm{C}(25)-\mathrm{N}(8)-\mathrm{C}(28)$ & $124.0(3)$ \\
\hline $\mathrm{C}(26)-\mathrm{N}(8)-\mathrm{C}(28)$ & $128.5(3)$ \\
\hline $\mathrm{O}(3)-\mathrm{N}(9)-\mathrm{Mo}$ & $174.72(19)$ \\
\hline$C(2)-C(1)-C(10)$ & $111.49(17)$ \\
\hline$C(2)-C(1)-C(11)$ & $112.21(19)$ \\
\hline$C(10)-C(1)-C(11)$ & $112.3(2)$ \\
\hline $\mathrm{C}(3)-\mathrm{C}(2)-\mathrm{C}(1)$ & $119.65(18)$ \\
\hline $\mathrm{C}(3)-\mathrm{C}(2)-\mathrm{Mo}$ & $71.11(12)$ \\
\hline $\mathrm{C}(1)-\mathrm{C}(2)-\mathrm{Mo}$ & $125.22(15)$ \\
\hline$C(2)-C(3)-C(4)$ & $121.06(17)$ \\
\hline $\mathrm{C}(2)-\mathrm{C}(3)-\mathrm{Mo}$ & $71.91(12)$ \\
\hline $\mathrm{C}(4)-\mathrm{C}(3)-\mathrm{Mo}$ & $118.67(14)$ \\
\hline$C(5)-C(4)-C(9)$ & $118.59(19)$ \\
\hline$C(5)-C(4)-C(3)$ & $121.06(18)$ \\
\hline$C(9)-C(4)-C(3)$ & $120.30(18)$ \\
\hline$C(6)-C(5)-C(4)$ & $121.5(2)$ \\
\hline$C(7)-C(6)-C(5)$ & $119.4(2)$ \\
\hline$C(6)-C(7)-C(8)$ & $119.9(2)$ \\
\hline $\mathrm{C}(7)-\mathrm{C}(8)-\mathrm{C}(9)$ & $121.9(2)$ \\
\hline$C(8)-C(9)-C(4)$ & $118.7(2)$ \\
\hline $\mathrm{C}(8)-\mathrm{C}(9)-\mathrm{C}(10)$ & $121.7(2)$ \\
\hline $\mathrm{C}(4)-\mathrm{C}(9)-\mathrm{C}(10)$ & $119.63(18)$ \\
\hline$C(9)-C(10)-C(1)$ & $115.46(18)$ \\
\hline$C(12)-C(11)-C(13)$ & $107.4(3)$ \\
\hline$C(12)-C(11)-C(14)$ & $106.8(2)$ \\
\hline$C(13)-C(11)-C(14)$ & $109.5(3)$ \\
\hline $\mathrm{C}(12)-\mathrm{C}(11)-\mathrm{C}(1)$ & $109.5(2)$ \\
\hline $\mathrm{C}(13)-\mathrm{C}(11)-\mathrm{C}(1)$ & $113.1(2)$ \\
\hline$C(14)-C(11)-C(1)$ & $110.3(3)$ \\
\hline $\mathrm{O}(1)-\mathrm{C}(12)-\mathrm{O}(2)$ & $121.8(4)$ \\
\hline $\mathrm{O}(1)-\mathrm{C}(12)-\mathrm{C}(11)$ & $125.5(5)$ \\
\hline $\mathrm{O}(2)-\mathrm{C}(12)-\mathrm{C}(11)$ & $112.7(4)$ \\
\hline $\mathrm{N}(2)-\mathrm{C}(16)-\mathrm{C}(17)$ & $109.2(2)$ \\
\hline$C(16)-C(17)-C(18)$ & $104.8(2)$ \\
\hline $\mathrm{N}(1)-\mathrm{C}(18)-\mathrm{C}(17)$ & $110.6(2)$ \\
\hline $\mathrm{N}(4)-\mathrm{C}(19)-\mathrm{C}(20)$ & $108.5(2)$ \\
\hline
\end{tabular}




$\begin{array}{lc}\mathrm{C}(19)-\mathrm{C}(20)-\mathrm{C}(21) & 105.0(2) \\ \mathrm{N}(3)-\mathrm{C}(21)-\mathrm{C}(20) & 110.3(2) \\ \mathrm{N}(6)-\mathrm{C}(22)-\mathrm{C}(23) & 108.5(2) \\ \mathrm{C}(22)-\mathrm{C}(23)-\mathrm{C}(24) & 104.8(2) \\ \mathrm{N}(5)-\mathrm{C}(24)-\mathrm{C}(23) & 111.1(2) \\ \mathrm{N}(7)-\mathrm{C}(25)-\mathrm{N}(8) & 111.4(2) \\ \mathrm{N}(8)-\mathrm{C}(26)-\mathrm{C}(27) & 106.0(2) \\ \mathrm{N}(7)-\mathrm{C}(27)-\mathrm{C}(26) & 109.6(2) \\ \mathrm{C}(30)-\mathrm{C}(29 \mathrm{~B})-\mathrm{O}(4) & 101.1(14) \\ \mathrm{O}(4)-\mathrm{C}(29 \mathrm{~A})-\mathrm{C}(30) & 100(2) \\ \mathrm{C}(31)-\mathrm{C}(30)-\mathrm{C}(29 \mathrm{~A}) & 105.5(17) \\ \mathrm{C}(31)-\mathrm{C}(30)-\mathrm{C}(29 \mathrm{~B}) & 100.0(16) \\ \mathrm{C}(29 \mathrm{~A})-\mathrm{C}(30)-\mathrm{C}(29 \mathrm{~B}) & 59.7(19) \\ \mathrm{C}(30)-\mathrm{C}(31)-\mathrm{C}(32) & 105.0(11) \\ \mathrm{O}(4)-\mathrm{C}(32)-\mathrm{C}(31) & 101.7(8) \\ \mathrm{N}(6)-\mathrm{B}(1)-\mathrm{N}(2) & 109.65(19) \\ \mathrm{N}(6)-\mathrm{B}(1)-\mathrm{N}(4) & 108.56(17) \\ \mathrm{N}(2)-\mathrm{B}(1)-\mathrm{N}(4) & 107.43(18)\end{array}$




\section{Compound 6}

Table 4. Anisotropic Thermal Displacement Parameters $\left(\AA^{2} \times 10^{3}\right)$ for $\mathrm{C}_{32} \mathrm{H}_{42} \mathrm{BN}_{9} \mathrm{O}_{4}$ Mo. The anisotropic displacement factor exponent takes the form: $-2 \pi^{2}\left[h^{2} a^{* 2} U^{11}+\ldots+2 h k a^{*} b^{*} U^{12}\right]$

\begin{tabular}{|c|c|c|c|c|c|c|}
\hline & $\mathrm{U}^{11}$ & $\mathrm{U}^{22}$ & $\mathrm{U}^{33}$ & $\mathrm{U}^{23}$ & $\mathrm{U}^{13}$ & $\mathrm{U}^{12}$ \\
\hline Mo & $25(1)$ & $42(1)$ & $26(1)$ & $-3(1)$ & $-1(1)$ & 2(1) \\
\hline $\mathrm{O}(1)$ & $119(3)$ & $153(3)$ & $128(3)$ & $15(2)$ & $43(2)$ & $-67(2)$ \\
\hline $\mathrm{O}(2)$ & $58(1)$ & $91(2)$ & $142(2)$ & $-26(2)$ & $19(2)$ & $-29(1)$ \\
\hline $\mathrm{O}(3)$ & $51(1)$ & $85(1)$ & $63(1)$ & $-7(1)$ & $14(1)$ & $31(1)$ \\
\hline $\mathrm{O}(4)$ & $159(7)$ & $510(20)$ & $290(13)$ & $1(13)$ & $-7(7)$ & $-6(9)$ \\
\hline $\mathrm{N}(1)$ & $32(1)$ & $52(1)$ & $32(1)$ & $-2(1)$ & $-3(1)$ & $2(1)$ \\
\hline $\mathrm{N}(2)$ & $37(1)$ & $54(1)$ & $31(1)$ & $-4(1)$ & $-5(1)$ & $4(1)$ \\
\hline $\mathrm{N}(3)$ & $38(1)$ & $42(1)$ & $35(1)$ & $1(1)$ & $0(1)$ & $6(1)$ \\
\hline $\mathrm{N}(4)$ & $40(1)$ & $46(1)$ & $35(1)$ & $5(1)$ & 1(1) & $5(1)$ \\
\hline $\mathrm{N}(5)$ & $31(1)$ & $46(1)$ & $30(1)$ & 1(1) & $3(1)$ & $5(1)$ \\
\hline $\mathrm{N}(6)$ & $34(1)$ & $45(1)$ & $31(1)$ & $-2(1)$ & $6(1)$ & $2(1)$ \\
\hline $\mathrm{N}(7)$ & $36(1)$ & $48(1)$ & $35(1)$ & $-2(1)$ & $-5(1)$ & $-3(1)$ \\
\hline $\mathrm{N}(8)$ & $71(2)$ & $79(2)$ & $58(1)$ & $-26(1)$ & $-18(1)$ & $0(1)$ \\
\hline $\mathrm{N}(9)$ & $32(1)$ & $51(1)$ & $33(1)$ & $-5(1)$ & $0(1)$ & $6(1)$ \\
\hline $\mathrm{C}(1)$ & $34(1)$ & $52(1)$ & $40(1)$ & $-8(1)$ & $2(1)$ & $-6(1)$ \\
\hline $\mathrm{C}(2)$ & $33(1)$ & $43(1)$ & $33(1)$ & $-5(1)$ & $4(1)$ & $-1(1)$ \\
\hline$C(3)$ & $25(1)$ & $45(1)$ & $32(1)$ & $1(1)$ & $3(1)$ & $2(1)$ \\
\hline$C(4)$ & $33(1)$ & 41(1) & $32(1)$ & $-2(1)$ & $2(1)$ & $2(1)$ \\
\hline$C(5)$ & $42(1)$ & $63(1)$ & $35(1)$ & $5(1)$ & $-4(1)$ & $0(1)$ \\
\hline$C(6)$ & $67(2)$ & $77(2)$ & $35(1)$ & $-1(1)$ & $-9(1)$ & $0(1)$ \\
\hline$C(7)$ & $74(2)$ & $87(2)$ & $31(1)$ & $-4(1)$ & $9(1)$ & $4(2)$ \\
\hline $\mathrm{C}(8)$ & $50(1)$ & $65(2)$ & $43(1)$ & $-3(1)$ & $15(1)$ & $0(1)$ \\
\hline $\mathrm{C}(9)$ & $38(1)$ & $44(1)$ & $36(1)$ & $-3(1)$ & $7(1)$ & $1(1)$ \\
\hline$C(10)$ & $30(1)$ & $52(1)$ & $45(1)$ & $-5(1)$ & $8(1)$ & $-3(1)$ \\
\hline$C(11)$ & $54(2)$ & $50(1)$ & $72(2)$ & $-15(1)$ & $17(1)$ & $-14(1)$ \\
\hline$C(12)$ & $74(2)$ & $73(2)$ & $113(3)$ & $-39(2)$ & $33(2)$ & $-32(2)$ \\
\hline$C(13)$ & $90(2)$ & $49(2)$ & $107(3)$ & $13(2)$ & $9(2)$ & $1(2)$ \\
\hline$C(14)$ & $77(2)$ & $74(2)$ & 101(3) & $-43(2)$ & $32(2)$ & $-24(2)$ \\
\hline$C(15)$ & $62(2)$ & $130(4)$ & $301(8)$ & $-48(5)$ & $42(4)$ & $-44(3)$ \\
\hline$C(16)$ & $53(1)$ & $64(2)$ & $36(1)$ & $-8(1)$ & $-13(1)$ & $5(1)$ \\
\hline
\end{tabular}




$\begin{array}{lcccccc}\mathrm{C}(17) & 45(1) & 68(2) & 51(1) & -4(1) & -21(1) & -2(1) \\ \mathrm{C}(18) & 33(1) & 61(1) & 47(1) & 0(1) & -10(1) & -1(1) \\ \mathrm{C}(19) & 51(1) & 53(1) & 48(1) & 12(1) & 4(1) & 4(1) \\ \mathrm{C}(20) & 64(2) & 41(1) & 67(2) & 4(1) & 5(1) & 3(1) \\ \mathrm{C}(21) & 51(1) & 43(1) & 50(1) & -4(1) & 2(1) & 8(1) \\ \mathrm{C}(22) & 40(1) & 56(1) & 42(1) & -3(1) & 14(1) & 4(1) \\ \mathrm{C}(23) & 39(1) & 62(2) & 57(1) & 1(1) & 11(1) & 16(1) \\ \mathrm{C}(24) & 37(1) & 55(1) & 43(1) & 5(1) & 4(1) & 11(1) \\ \mathrm{C}(25) & 49(1) & 65(2) & 51(1) & -18(1) & -7(1) & 4(1) \\ \mathrm{C}(26) & 48(1) & 88(2) & 63(2) & -14(2) & -16(1) & -15(1) \\ \mathrm{C}(27) & 37(1) & 70(2) & 45(1) & -4(1) & -2(1) & -11(1) \\ \mathrm{C}(28) & 123(4) & 150(4) & 104(3) & -77(3) & -35(3) & 12(3) \\ \mathrm{C}(29 \mathrm{~B}) & 147(12) & 149(14) & 120(11) & -28(9) & 27(10) & -3(10) \\ \mathrm{C}(29 \mathrm{~A}) & 230(30) & 200(20) & 190(20) & -5(17) & 70(20) & -78(19) \\ \mathrm{C}(30) & 390(30) & 290(20) & 174(13) & 73(12) & -85(16) & -110(20) \\ \mathrm{C}(31) & 135(7) & 135(7) & 345(16) & 17(10) & 13(9) & 3(5) \\ \mathrm{C}(32) & 270(13) & 151(7) & 135(6) & 12(5) & 7(8) & 52(7) \\ \mathrm{B}(1) & 41(1) & 50(1) & 28(1) & -1(1) & 1(1) & 4(1)\end{array}$




\section{Compound 6}

Table 5. Hydrogen Atom Fractional Coordinates and Thermal Displacement Parameters $\left(\AA^{2} \mathrm{x} 10^{3}\right)$ for $\mathrm{C}_{32} \mathrm{H}_{42} \mathrm{BN}_{9} \mathrm{O}_{4} \mathrm{Mo}$.

\begin{tabular}{|c|c|c|c|c|}
\hline & $\mathrm{x}$ & $\mathrm{y}$ & z & $\mathrm{U}(\mathrm{eq})$ \\
\hline $\mathrm{H}(5)$ & 5177 & 6741 & 7518 & 56 \\
\hline $\mathrm{H}(6)$ & 4686 & 6837 & 8431 & 71 \\
\hline $\mathrm{H}(7)$ & 3045 & 6669 & 8666 & 77 \\
\hline $\mathrm{H}(8)$ & 1907 & 6414 & 7996 & 63 \\
\hline $\mathrm{H}(10 \mathrm{~A})$ & 1785 & 6695 & 6782 & 51 \\
\hline $\mathrm{H}(10 \mathrm{~B})$ & 1554 & 6049 & 7060 & 51 \\
\hline $\mathrm{H}(13 \mathrm{~A})$ & 3033 & 5197 & 7254 & 123 \\
\hline $\mathrm{H}(13 \mathrm{~B})$ & 3879 & 5139 & 6810 & 123 \\
\hline $\mathrm{H}(13 \mathrm{C})$ & 3188 & 4566 & 6936 & 123 \\
\hline $\mathrm{H}(14 \mathrm{~A})$ & 2672 & 4466 & 5944 & 126 \\
\hline $\mathrm{H}(14 \mathrm{~B})$ & 3425 & 5004 & 5806 & 126 \\
\hline $\mathrm{H}(14 \mathrm{C})$ & 2312 & 5060 & 5620 & 126 \\
\hline $\mathrm{H}(15 \mathrm{~A})$ & -445 & 4649 & 6282 & 247 \\
\hline $\mathrm{H}(15 \mathrm{~B})$ & -709 & 5342 & 6434 & 247 \\
\hline $\mathrm{H}(15 \mathrm{C})$ & -197 & 4908 & 6873 & 247 \\
\hline $\mathrm{H}(16)$ & 2850 & 7022 & 3960 & 61 \\
\hline $\mathrm{H}(17)$ & 1315 & 6645 & 4407 & 66 \\
\hline $\mathrm{H}(18)$ & 1645 & 6670 & 5421 & 57 \\
\hline H(19) & 4706 & 8749 & 4424 & 61 \\
\hline $\mathrm{H}(20)$ & 4401 & 9488 & 5194 & 69 \\
\hline $\mathrm{H}(21)$ & 3959 & 8833 & 6009 & 58 \\
\hline $\mathrm{H}(22)$ & 6286 & 6825 & 4388 & 55 \\
\hline $\mathrm{H}(23)$ & 7180 & 6262 & 5135 & 63 \\
\hline $\mathrm{H}(24)$ & 6128 & 6365 & 5974 & 54 \\
\hline $\mathrm{H}(25)$ & 3806 & 8095 & 7112 & 66 \\
\hline $\mathrm{H}(26)$ & 6721 & 8084 & 7256 & 79 \\
\hline $\mathrm{H}(27)$ & 6247 & 7499 & 6419 & 61 \\
\hline $\mathrm{H}(28 \mathrm{~A})$ & 4415 & 8702 & 7912 & 189 \\
\hline $\mathrm{H}(28 \mathrm{~B})$ & 5546 & 8881 & 7918 & 189 \\
\hline
\end{tabular}




$\begin{array}{lllll}\mathrm{H}(28 \mathrm{C}) & 5191 & 8255 & 8192 & 189 \\ \mathrm{H}(29 \mathrm{~A}) & 4178 & 5167 & 3183 & 166 \\ \mathrm{H}(29 \mathrm{~B}) & 3954 & 4619 & 3606 & 166 \\ \mathrm{H}(29 \mathrm{C}) & 4049 & 5731 & 3228 & 249 \\ \mathrm{H}(29 \mathrm{D}) & 3496 & 6109 & 3701 & 249 \\ \mathrm{H}(31 \mathrm{~A}) & 2624 & 4672 & 4190 & 246 \\ \mathrm{H}(31 \mathrm{~B}) & 2181 & 5348 & 4276 & 246 \\ \mathrm{H}(32 \mathrm{~A}) & 3557 & 5677 & 4663 & 222 \\ \mathrm{H}(32 \mathrm{~B}) & 3909 & 4970 & 4678 & 222 \\ \mathrm{H}(1) & 2010(20) & 6035(13) & 6088(10) & 48(7) \\ \mathrm{H}(1 \mathrm{~B}) & 4674(16) & 7489(11) & 4252(10) & 36(6) \\ \mathrm{H}(2) & 3768(18) & 6020(13) & 5895(11) & 46(7) \\ \mathrm{H}(3) & 4852(18) & 6384(10) & 6551(9) & 37(6) \\ \mathrm{H}(30 \mathrm{~A}) & 2870(90) & 5530(60) & 3380(40) & 30(9) \\ \mathrm{H}(30 B) & 2980(30) & 4821(15) & 3795(16) & 49(9)\end{array}$


${ }^{1}$ H NMR (d6-Acetone) of Compound 6:

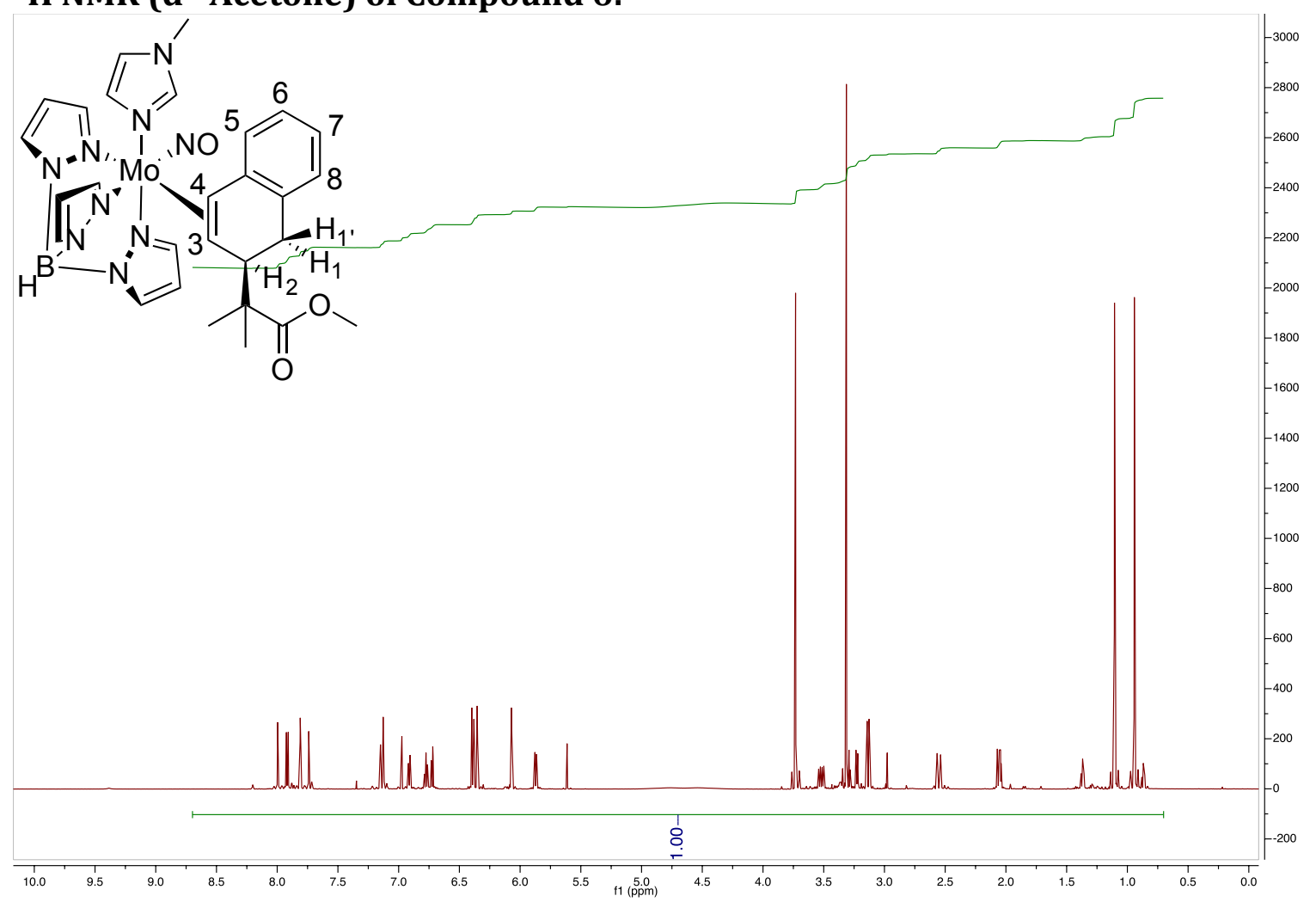

${ }^{13}$ C NMR (d6-Acetone) of Compound 6:

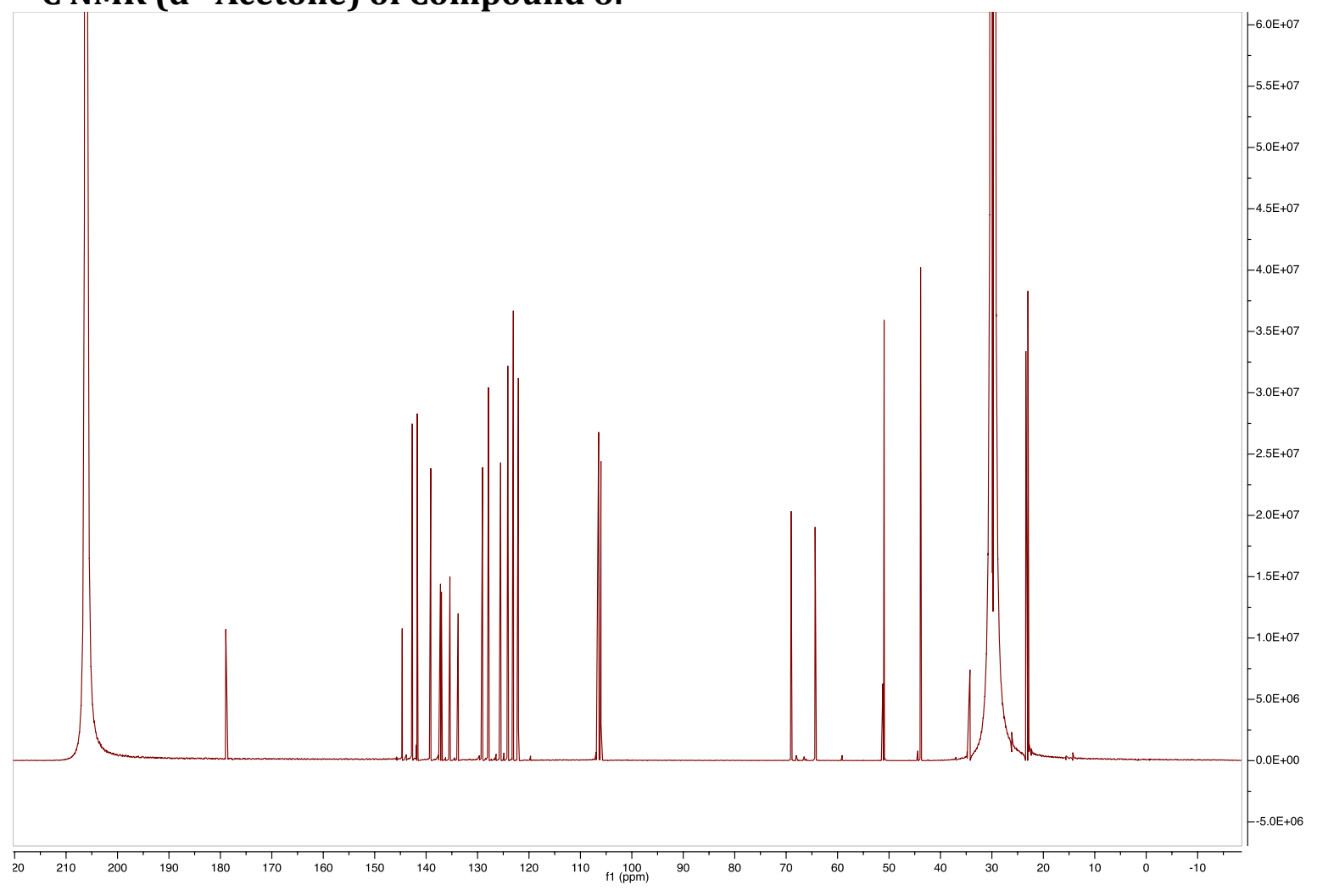


${ }^{1} \mathrm{H}$ NMR $\left(\mathrm{CDCl}_{3}\right)$ of Compound 7:

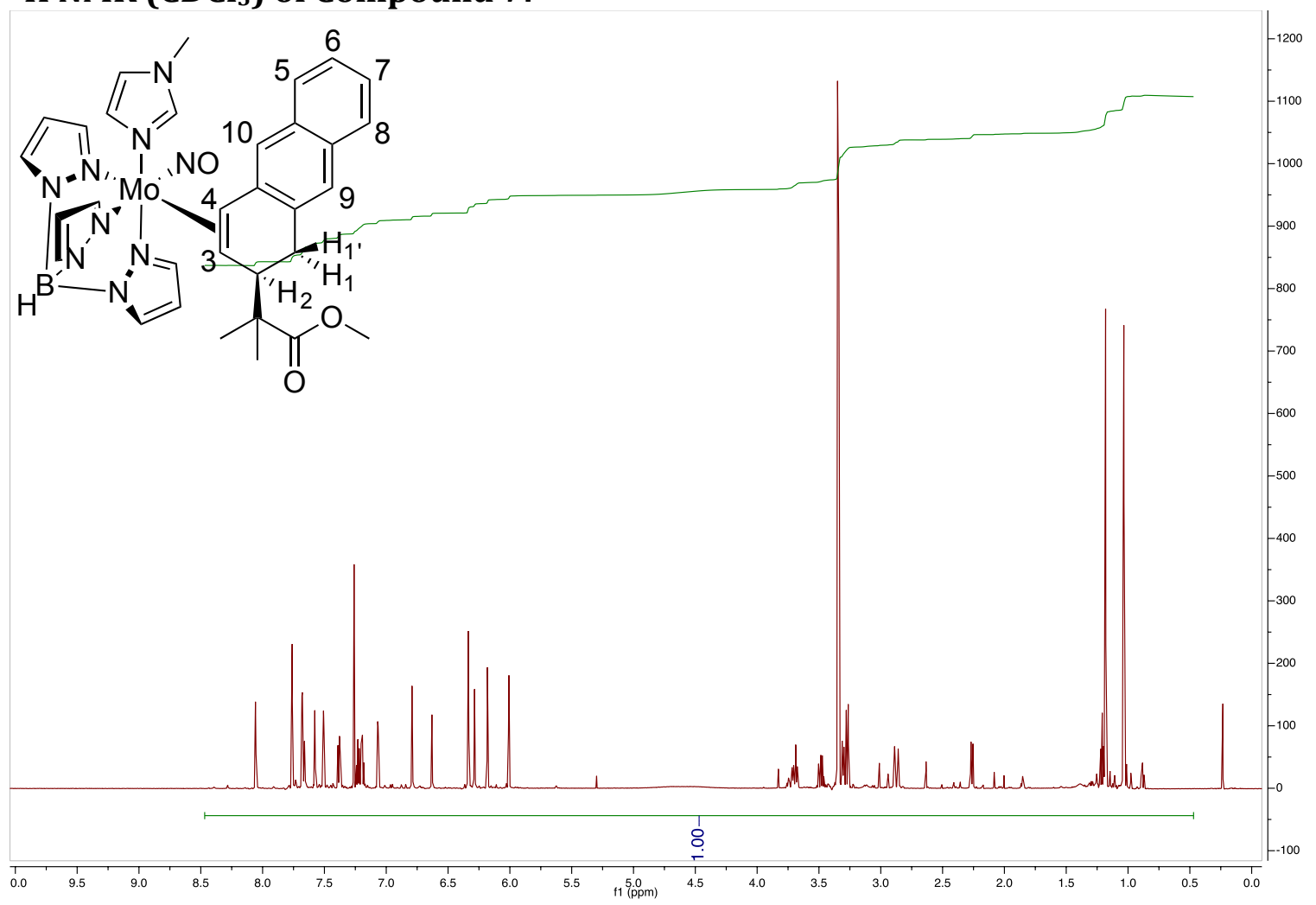

${ }^{13} \mathrm{C}$ NMR $\left(\mathrm{CDCl}_{3}\right)$ of Compound 7:

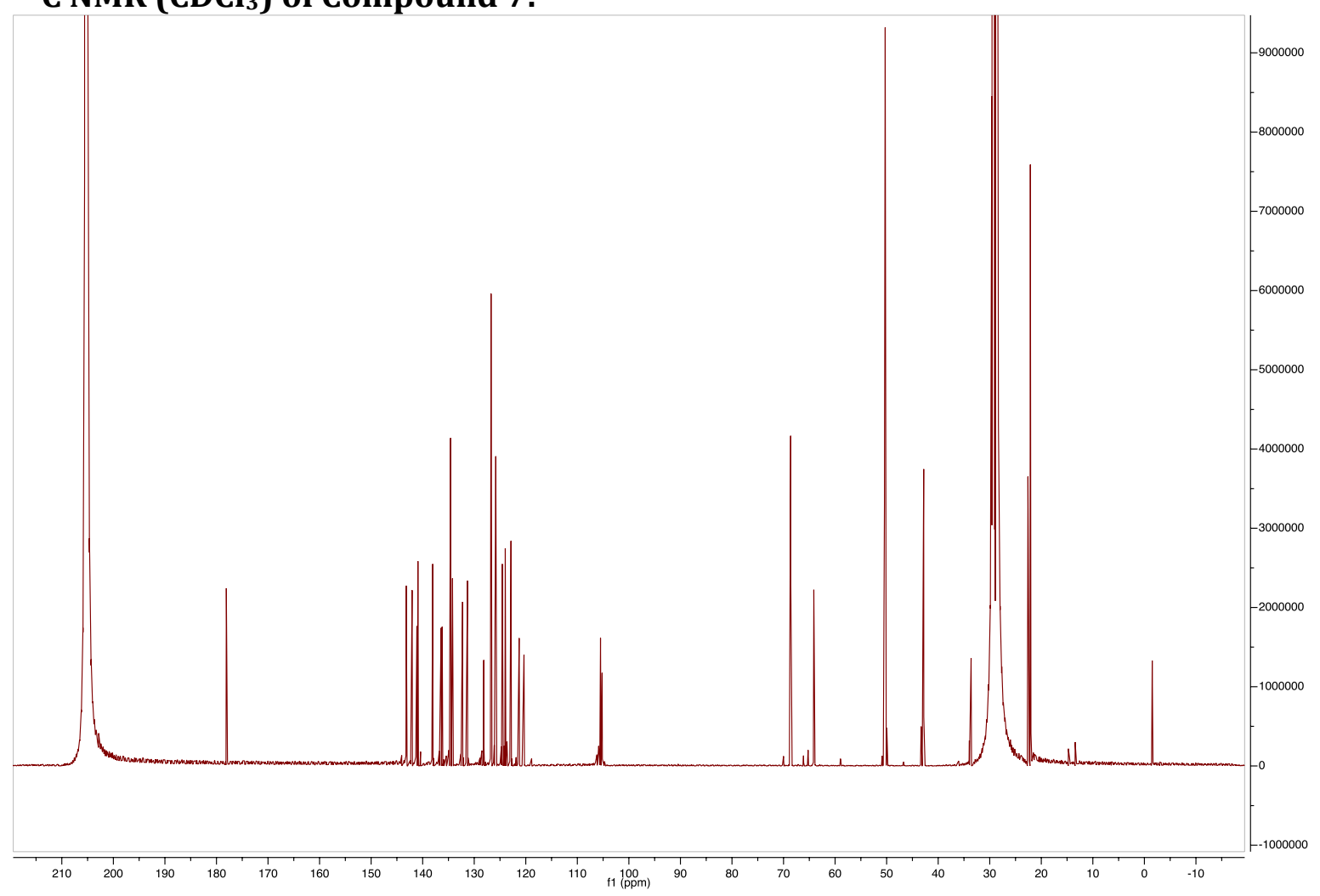


${ }^{1}$ H NMR (d ${ }^{6}$-Acetone) of Compound 8:

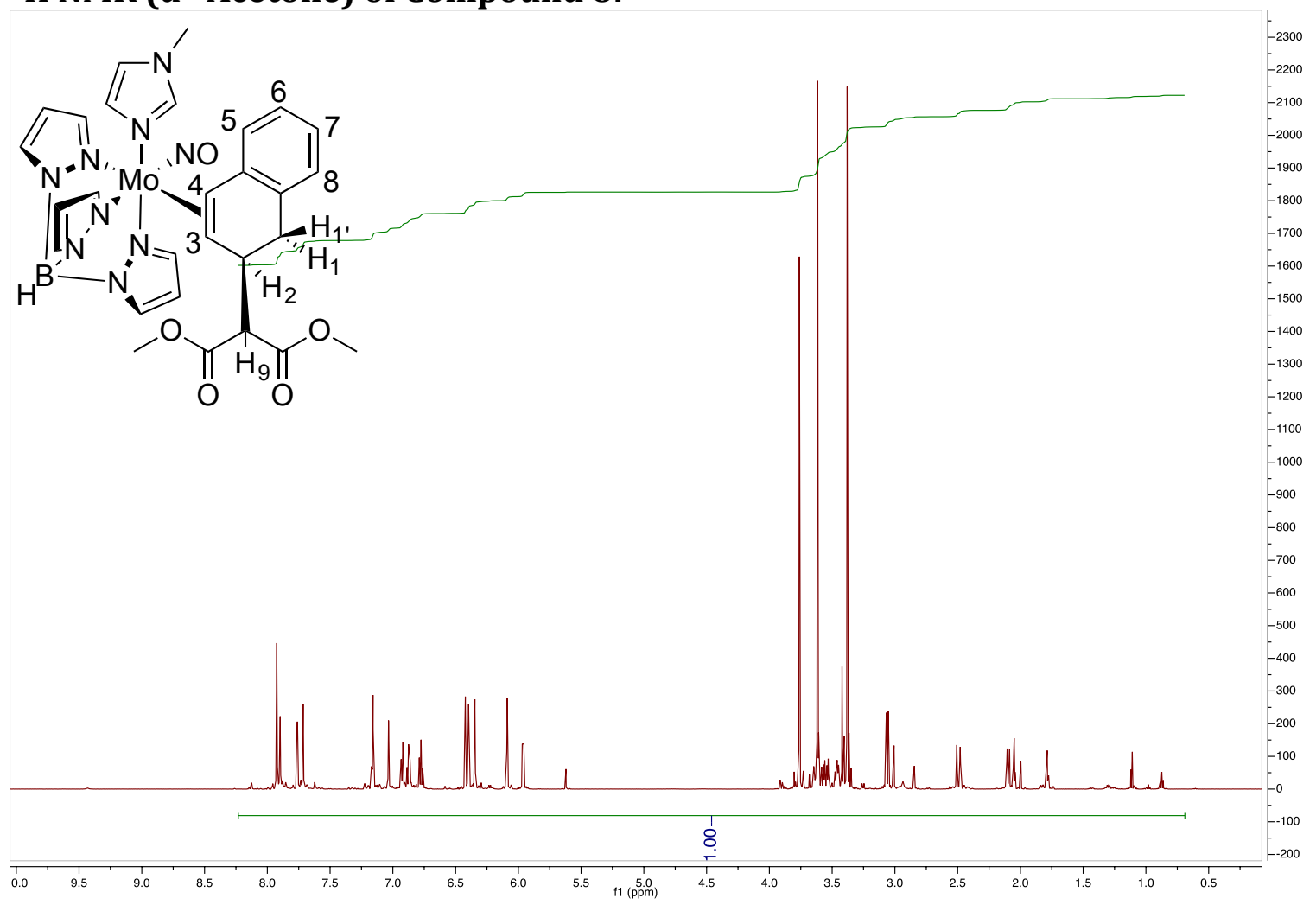

${ }^{13}$ C NMR ( $d^{6}$-Acetone) of Compound 8:

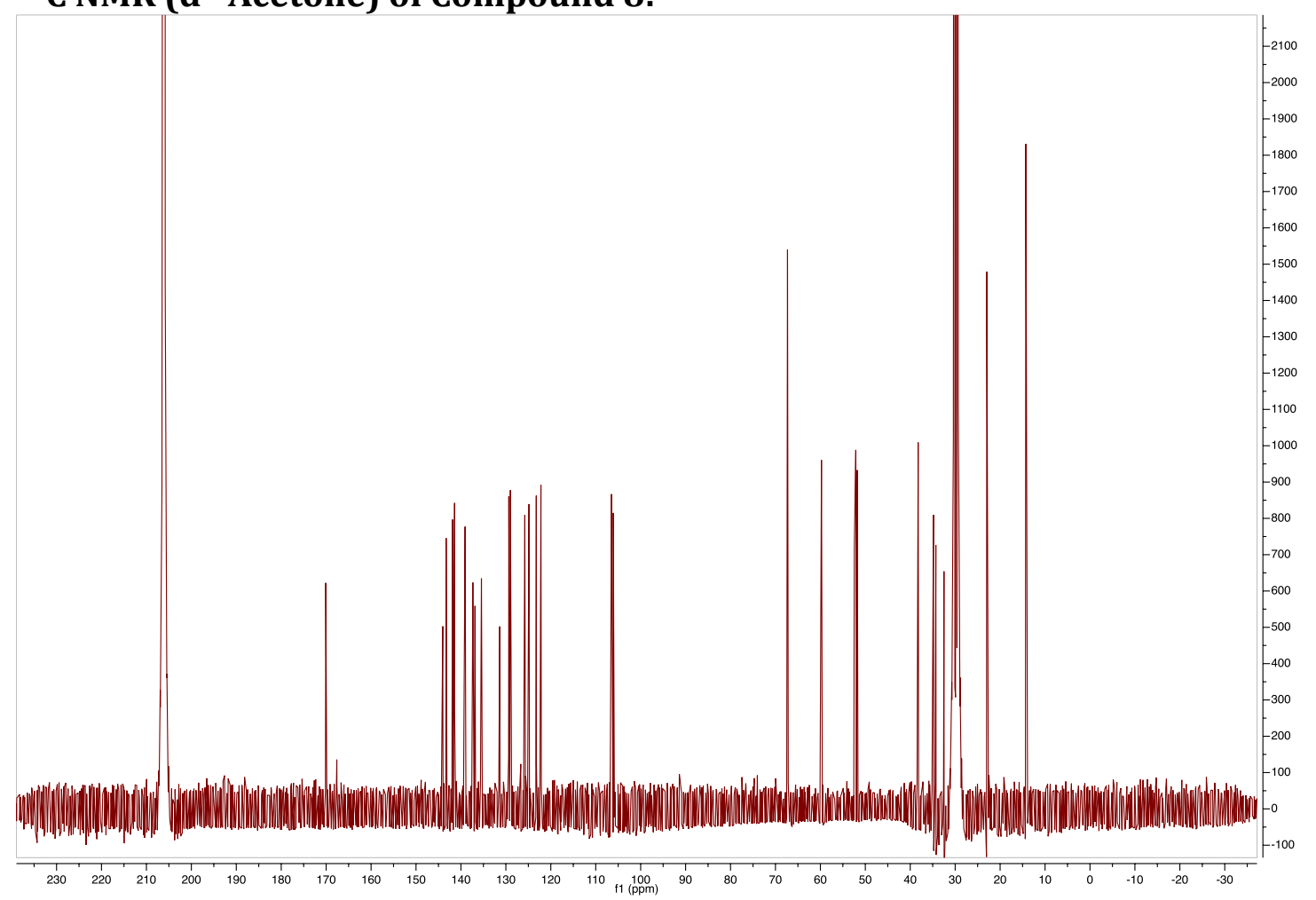


${ }^{1} \mathrm{H}$ NMR $\left(\mathrm{CDCl}_{3}\right)$ of Compound 9:

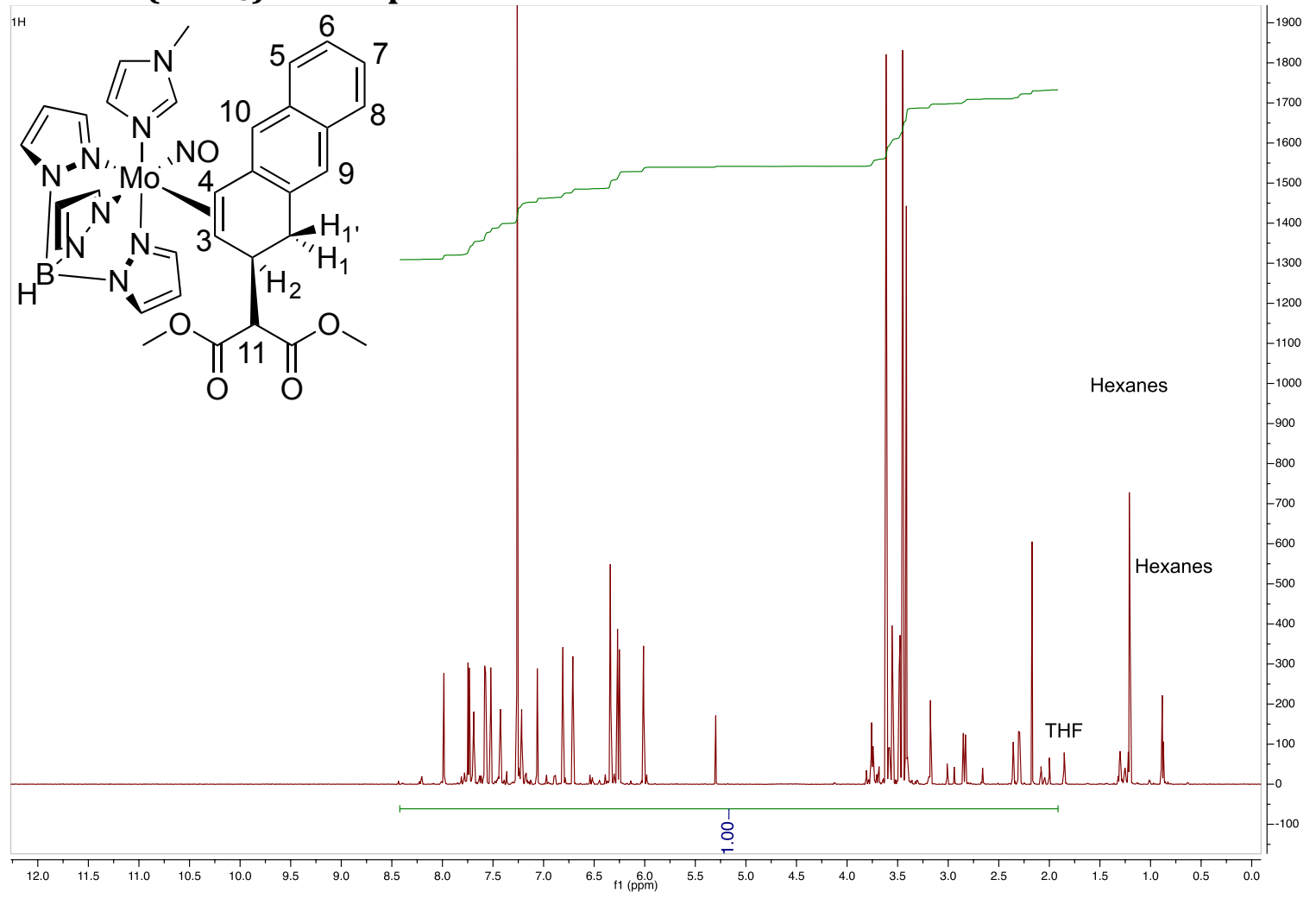

${ }^{13} \mathrm{C} \mathrm{NMR}\left(\mathrm{CDCl}_{3}\right)$ of Compound 9:

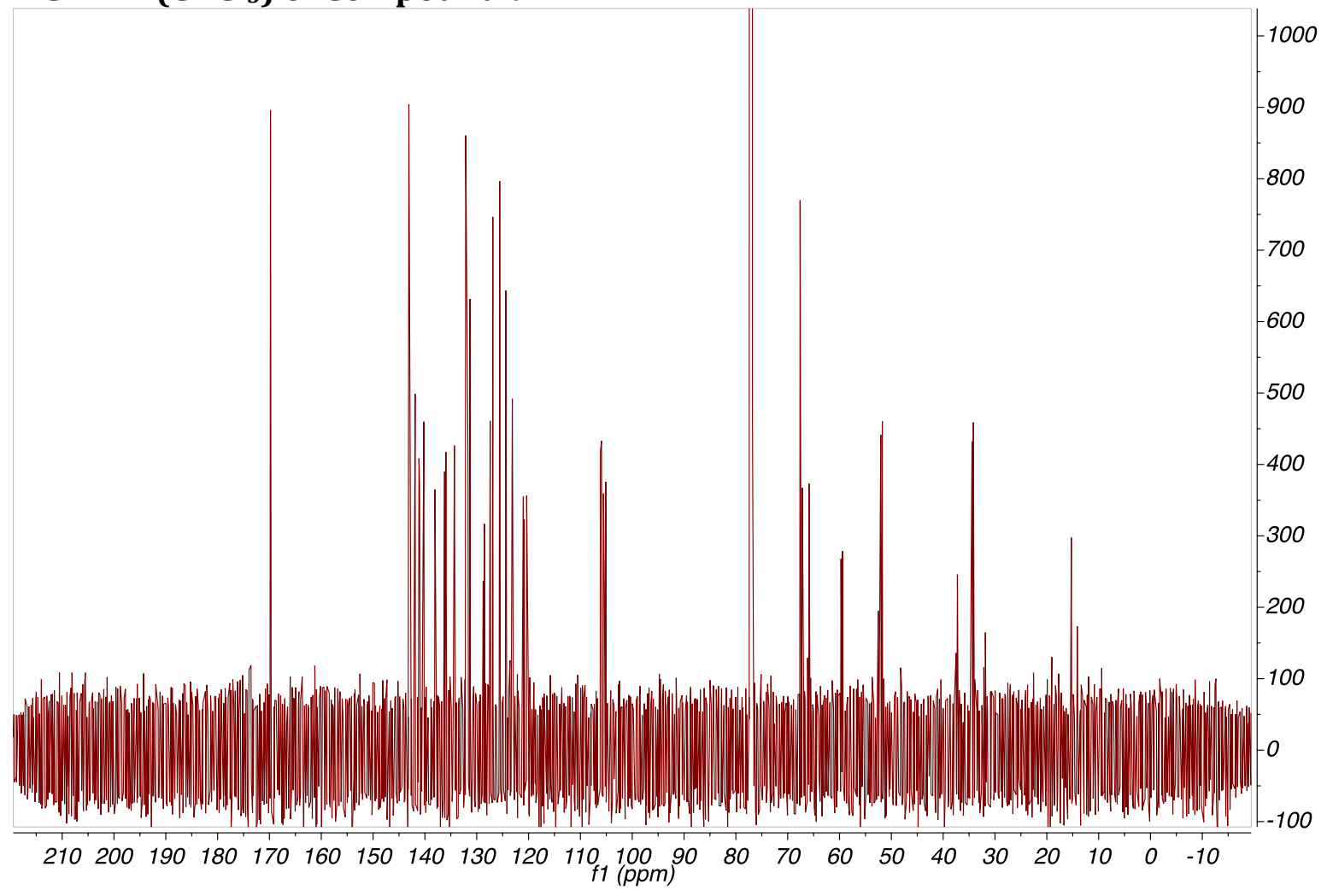


${ }^{1} \mathrm{H}$ NMR (d6-Acetone) of Compound 10:

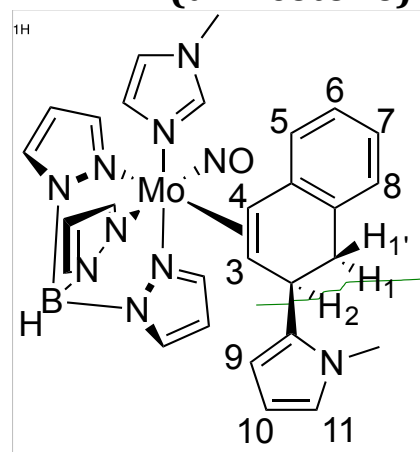

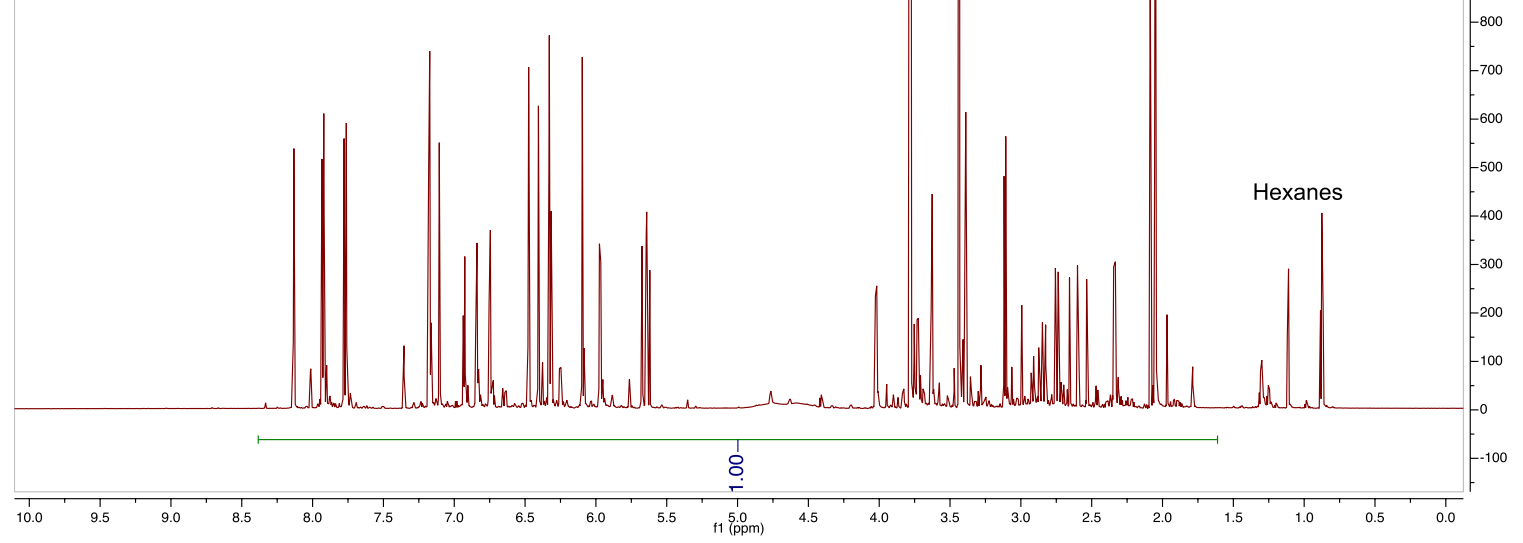

${ }^{13} \mathrm{C}$ NMR (d6-Acetone) of Compound 10:

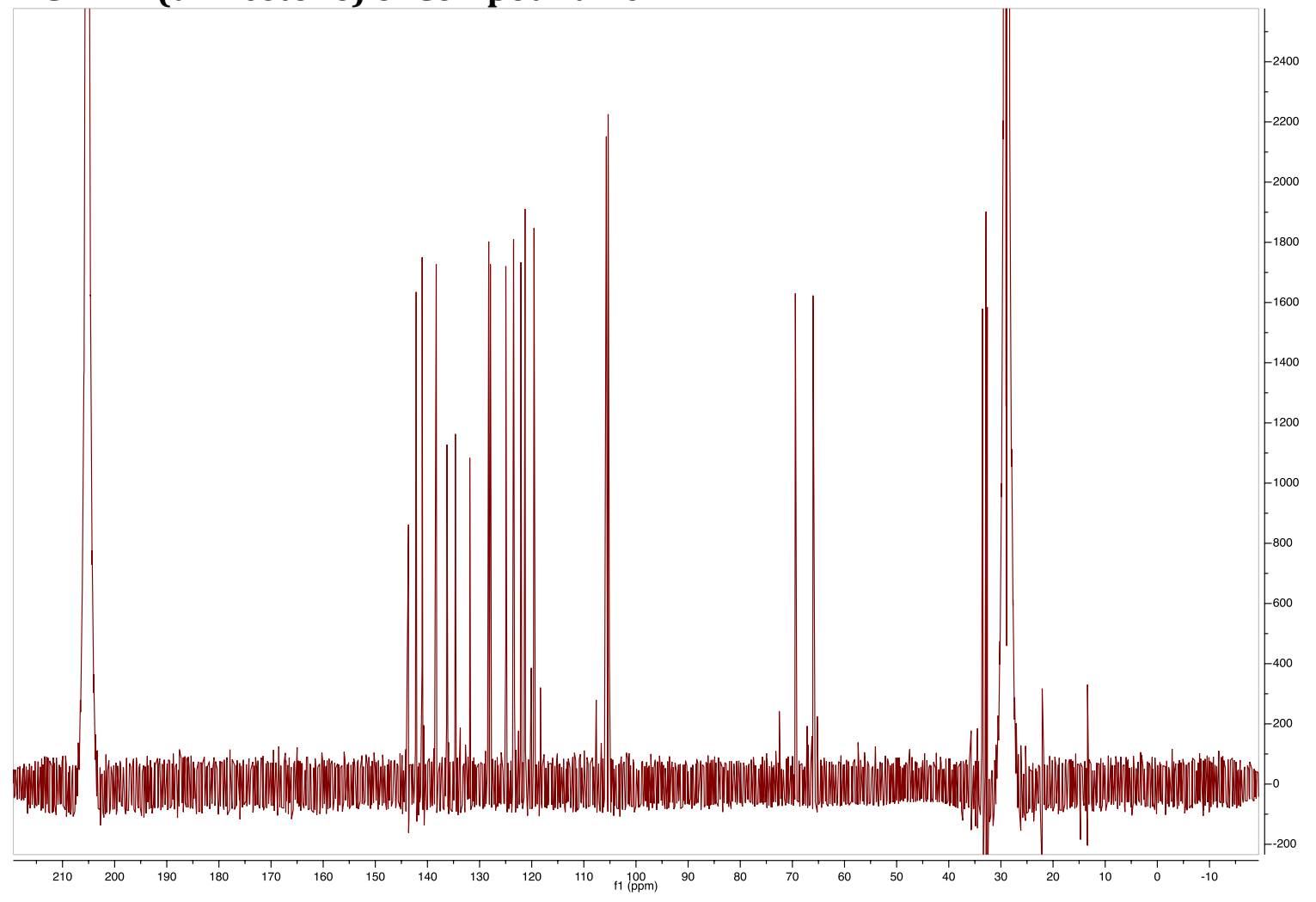



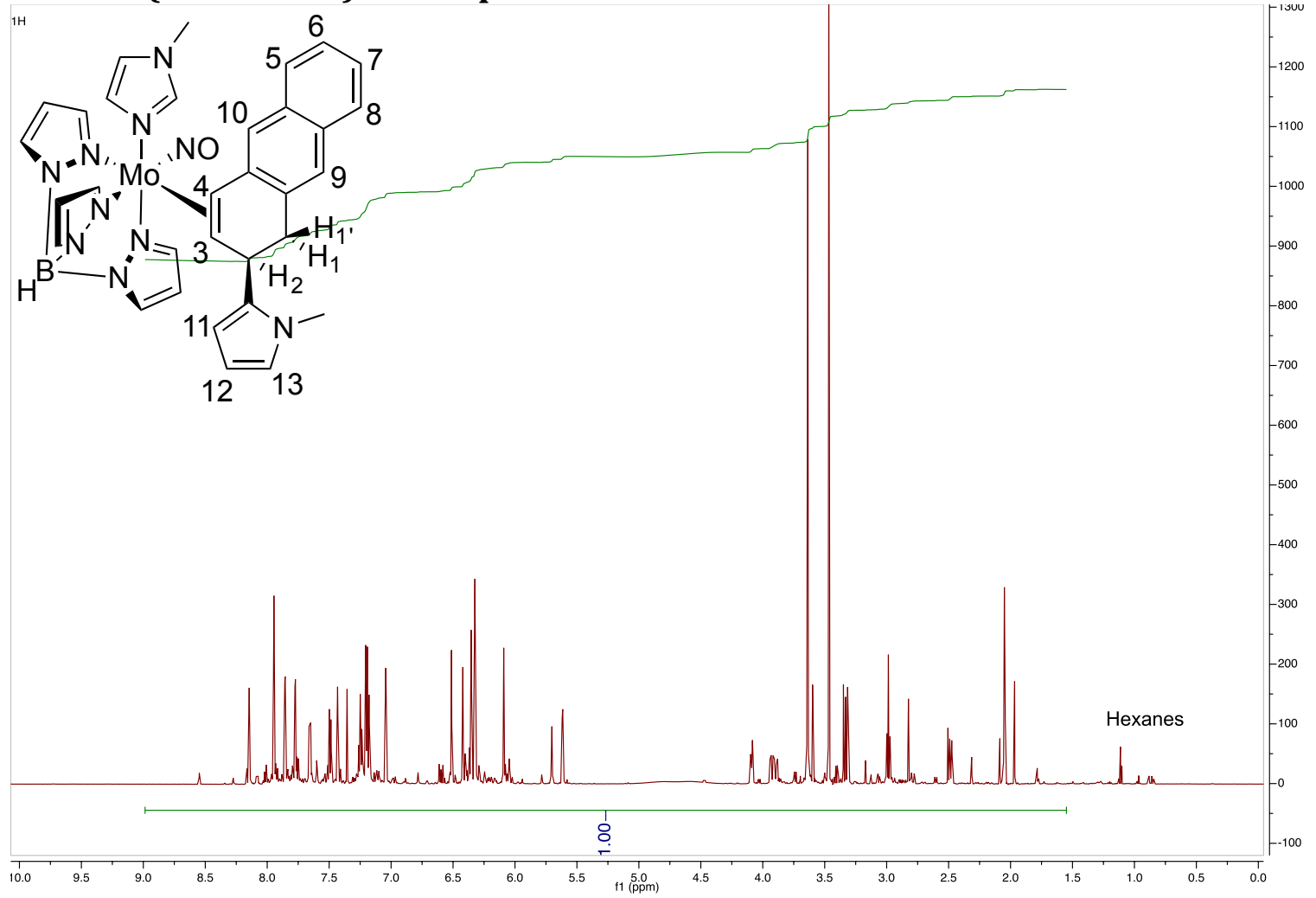

${ }^{13}$ C NMR (d6-Acetone) of Compound 11:

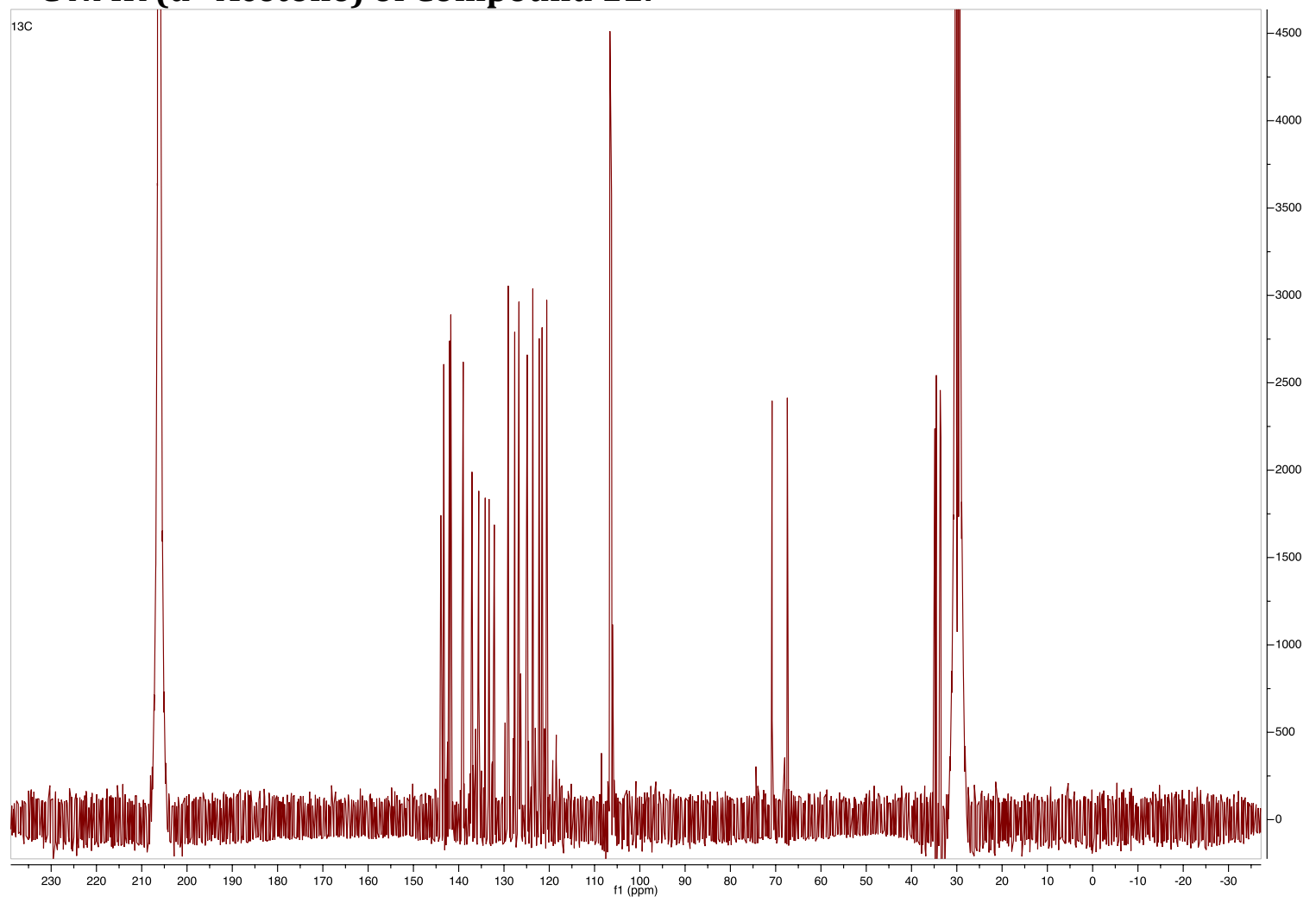


${ }^{1} \mathrm{H}$ NMR $\left(\mathrm{CDCl}_{3}\right)$ of Compound 13:

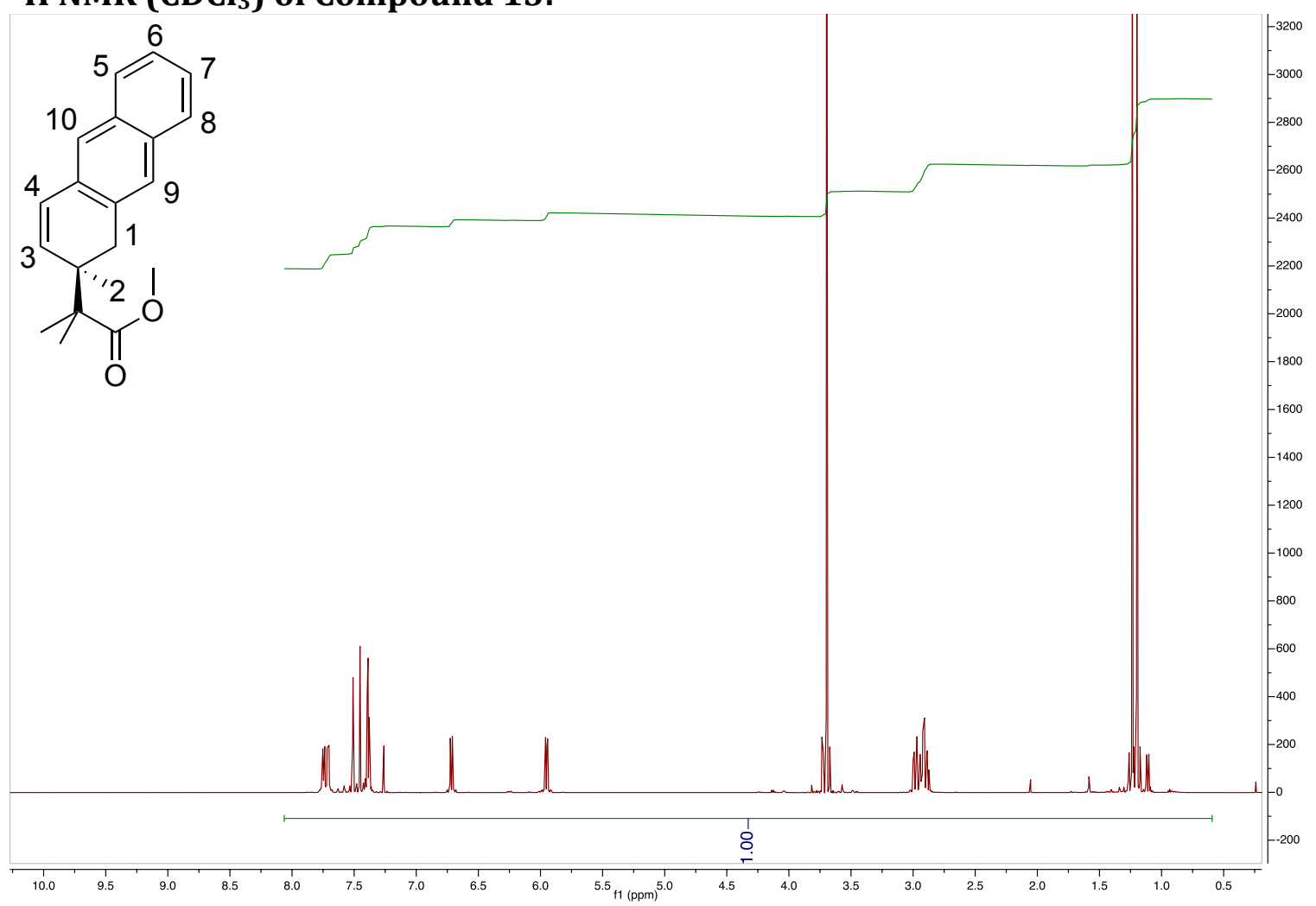

${ }^{13} \mathrm{C} \mathrm{NMR}\left(\mathrm{CDCl}_{3}\right)$ of Compound 13:

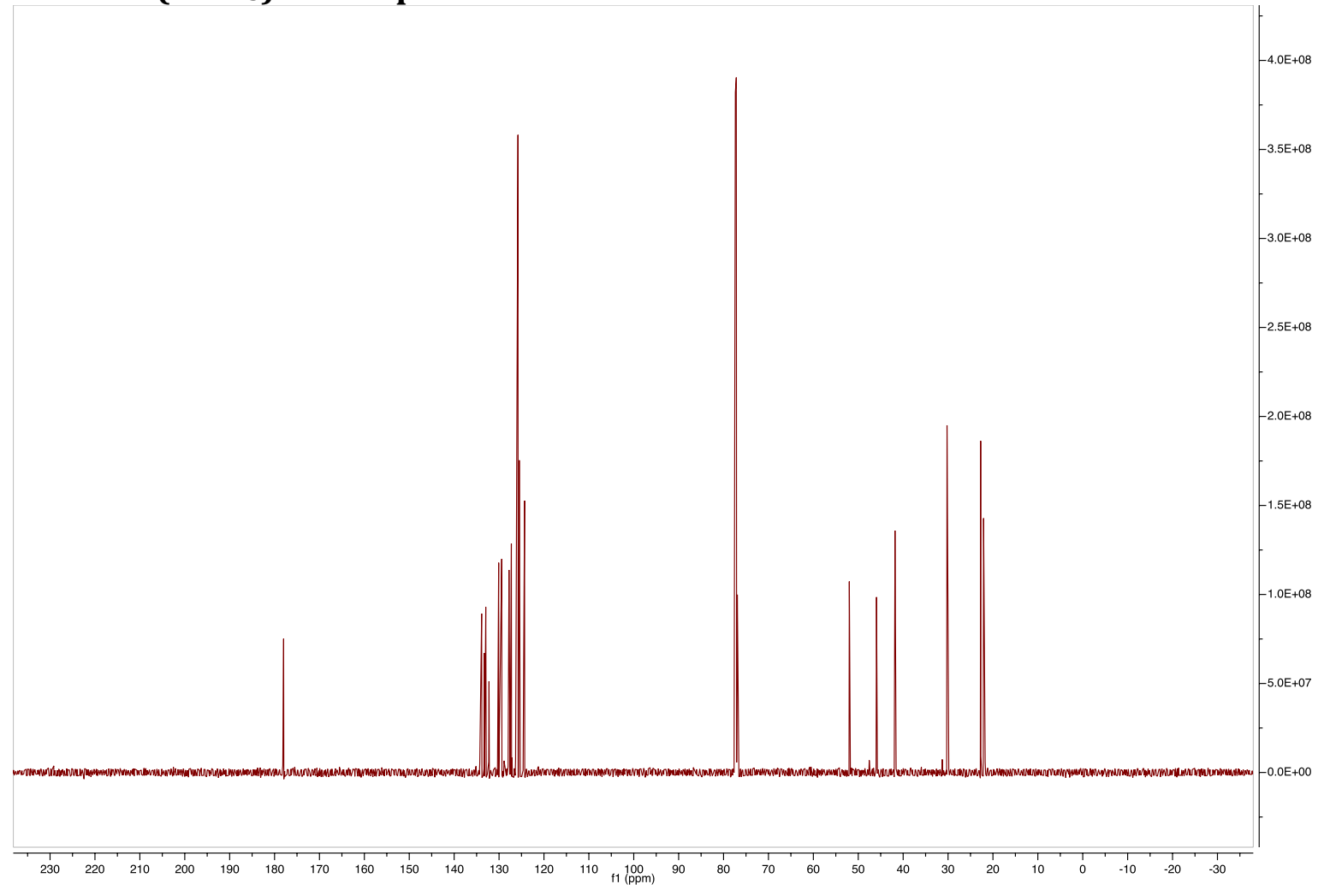


${ }^{1} \mathrm{H}$ NMR $\left(\mathrm{CDCl}_{3}\right)$ of Compound 14:
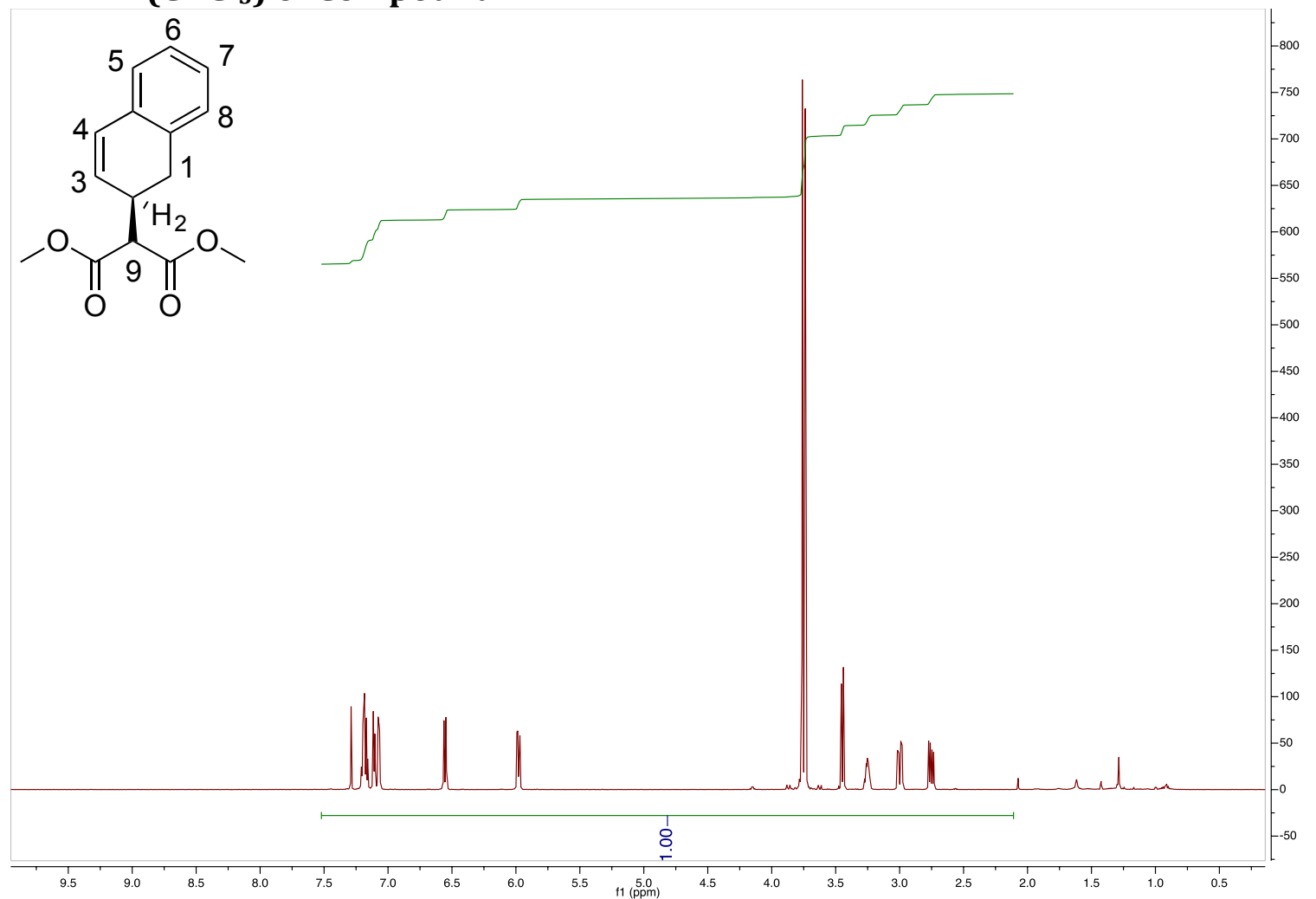

${ }^{13} \mathrm{C} \mathrm{NMR}\left(\mathrm{CDCl}_{3}\right)$ of Compound 14:

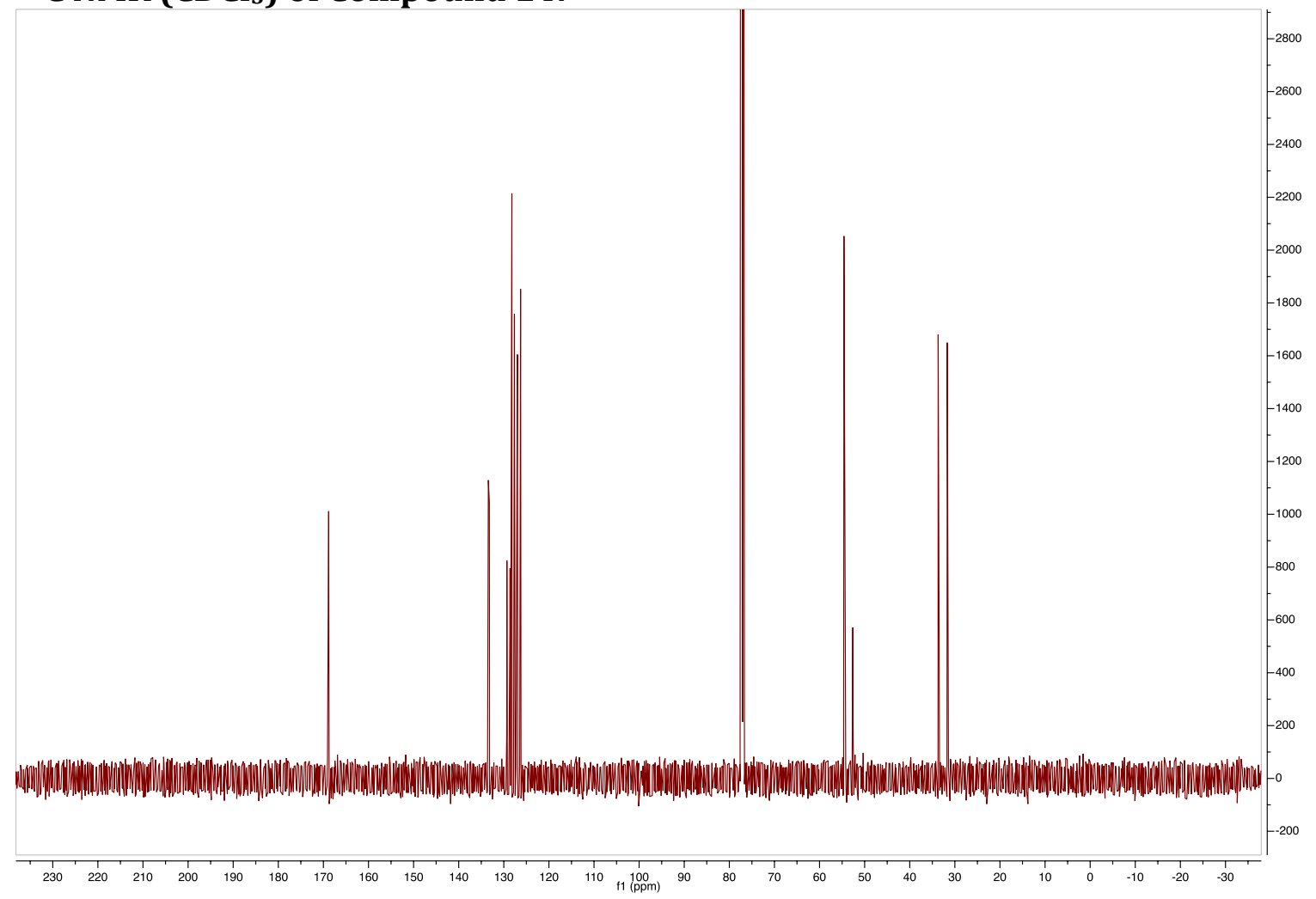


${ }^{1} \mathrm{H}$ NMR $\left(\mathrm{CDCl}_{3}\right)$ of Compound 15:

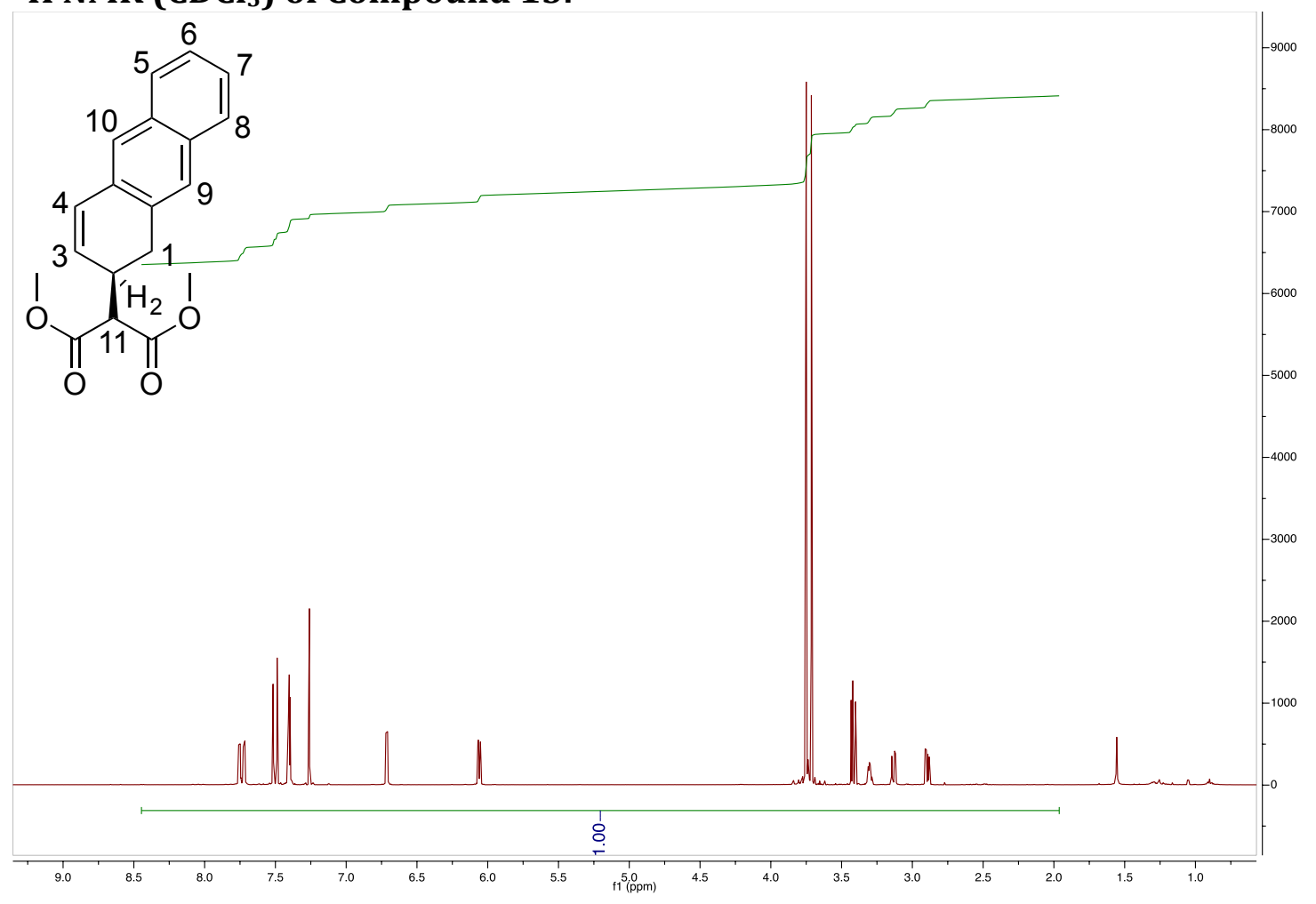

${ }^{13} \mathrm{C} \mathrm{NMR}\left(\mathrm{CDCl}_{3}\right)$ of Compound 15:

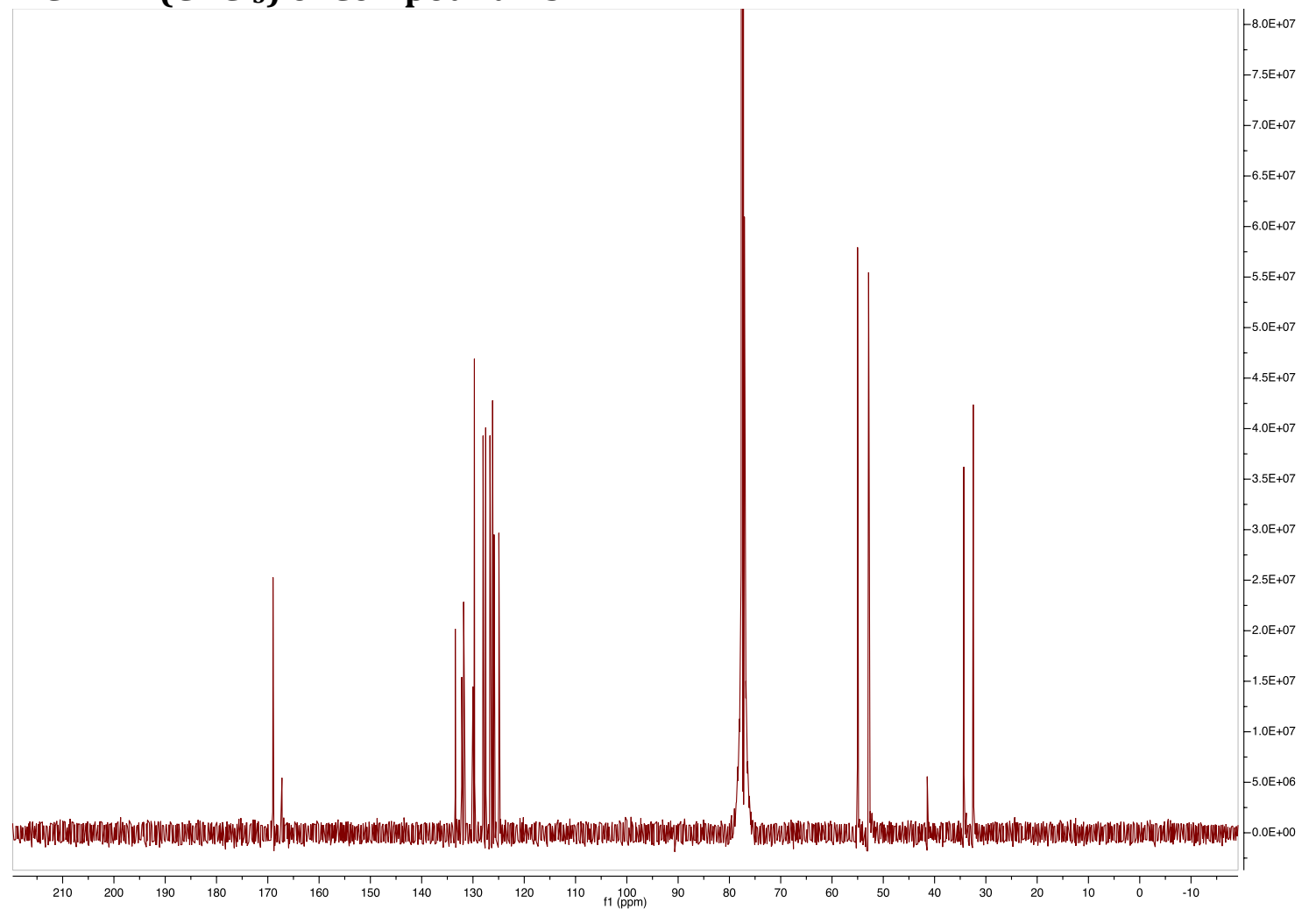


${ }^{1} \mathrm{H}$ NMR $\left(\mathrm{CDCl}_{3}\right)$ of Compound 16:
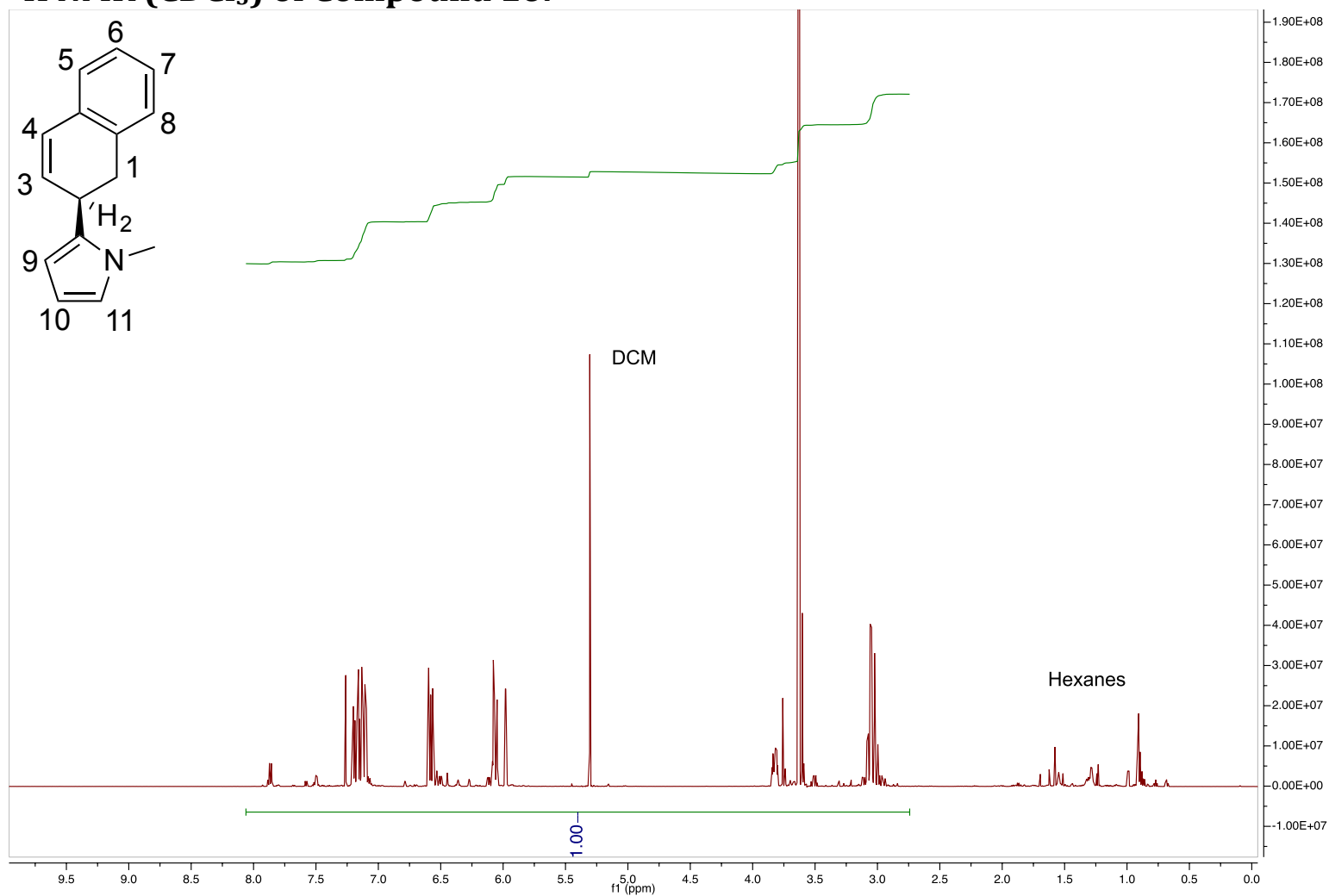

${ }^{13} \mathrm{C} \mathrm{NMR}\left(\mathrm{CDCl}_{3}\right)$ of Compound 16:

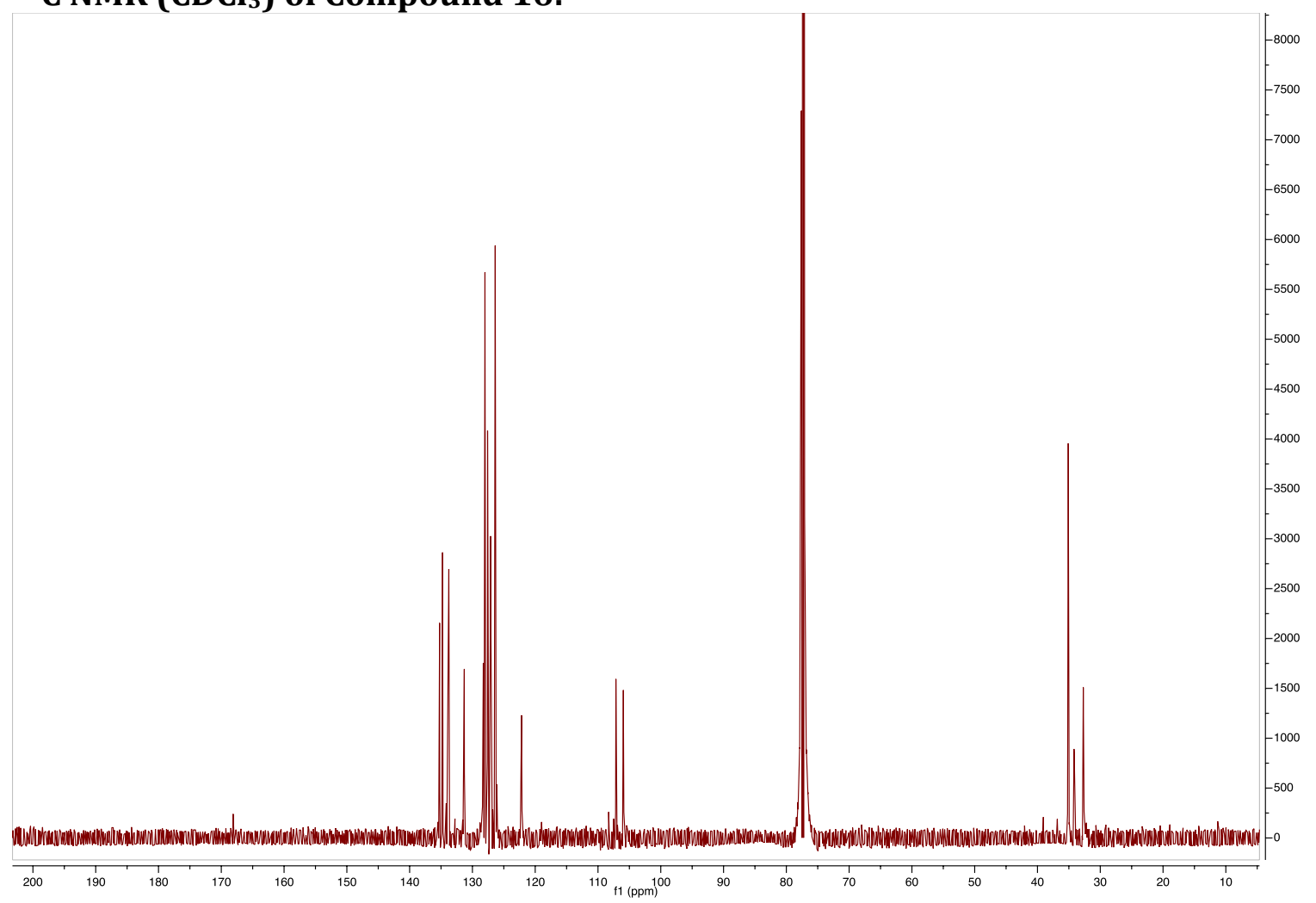




\section{${ }^{1} \mathrm{H}$ NMR $\left(\mathrm{CDCl}_{3}\right)$ of Compound 17:}

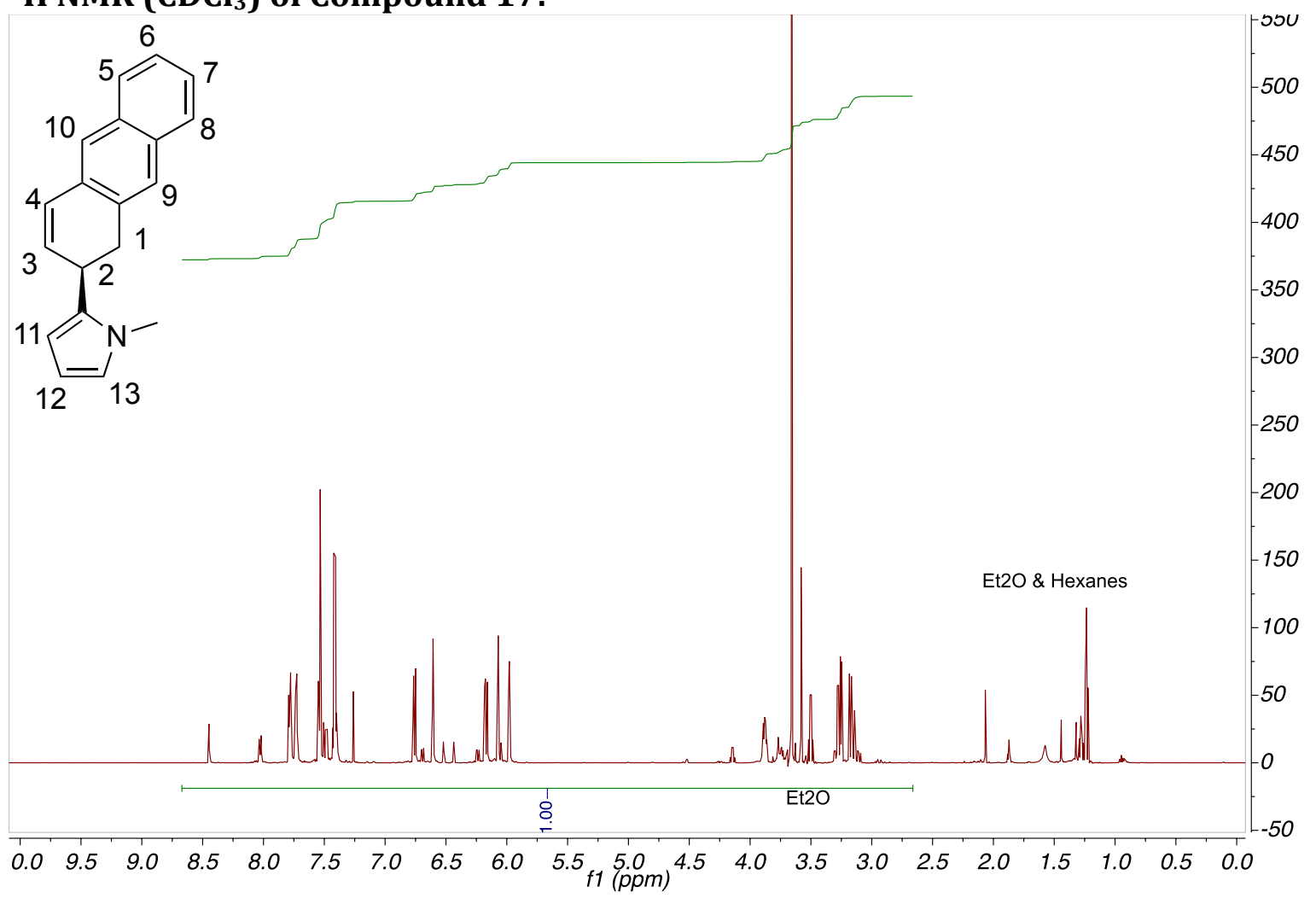

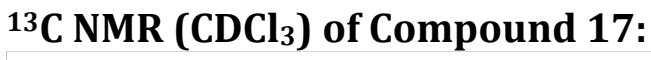

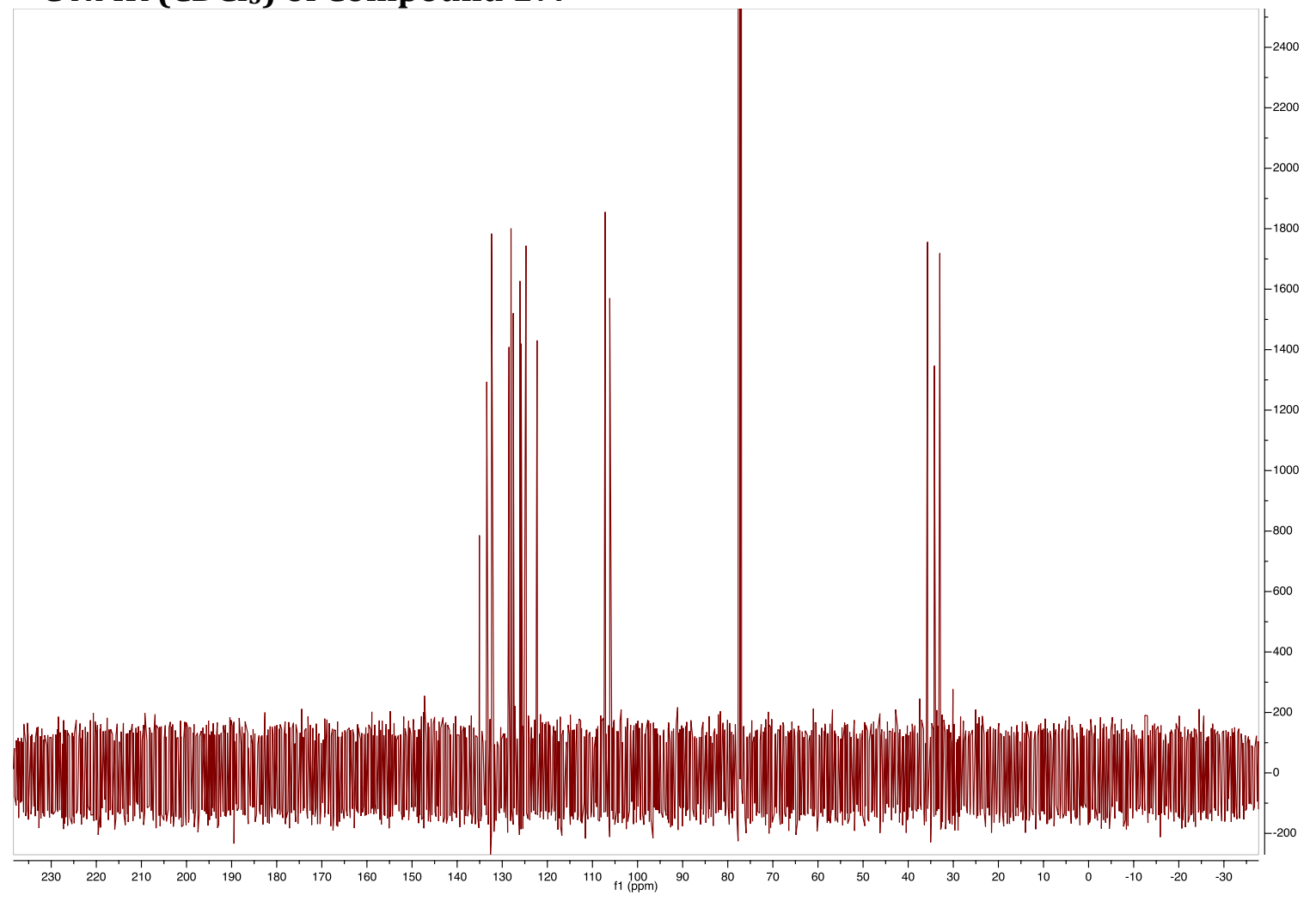


${ }^{1} \mathrm{H}$ NMR $\left(\mathrm{CDCl}_{3}\right)$ of Compound 18:

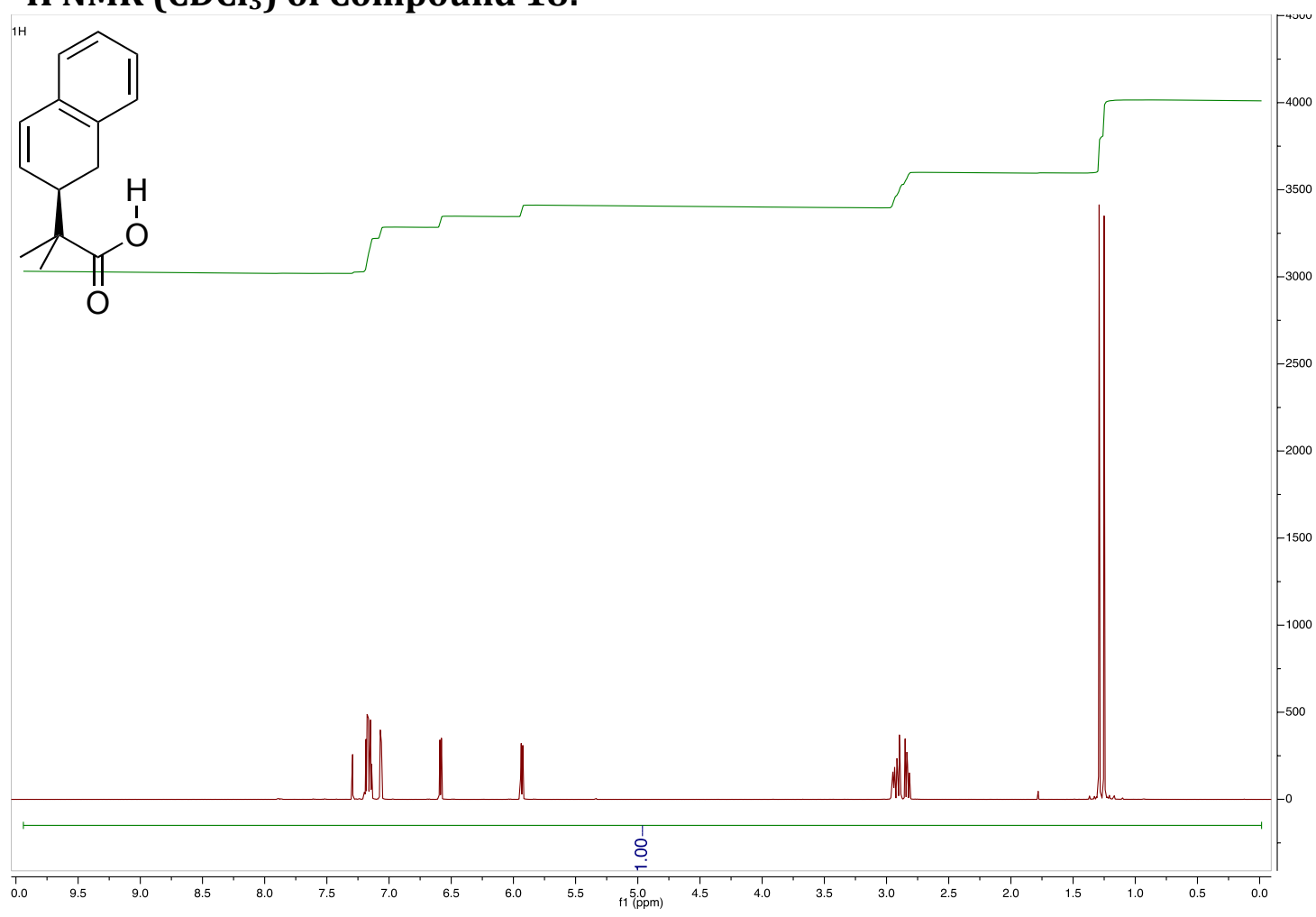

${ }^{13} \mathrm{C} \mathrm{NMR}\left(\mathrm{CDCl}_{3}\right)$ of Compound 18:

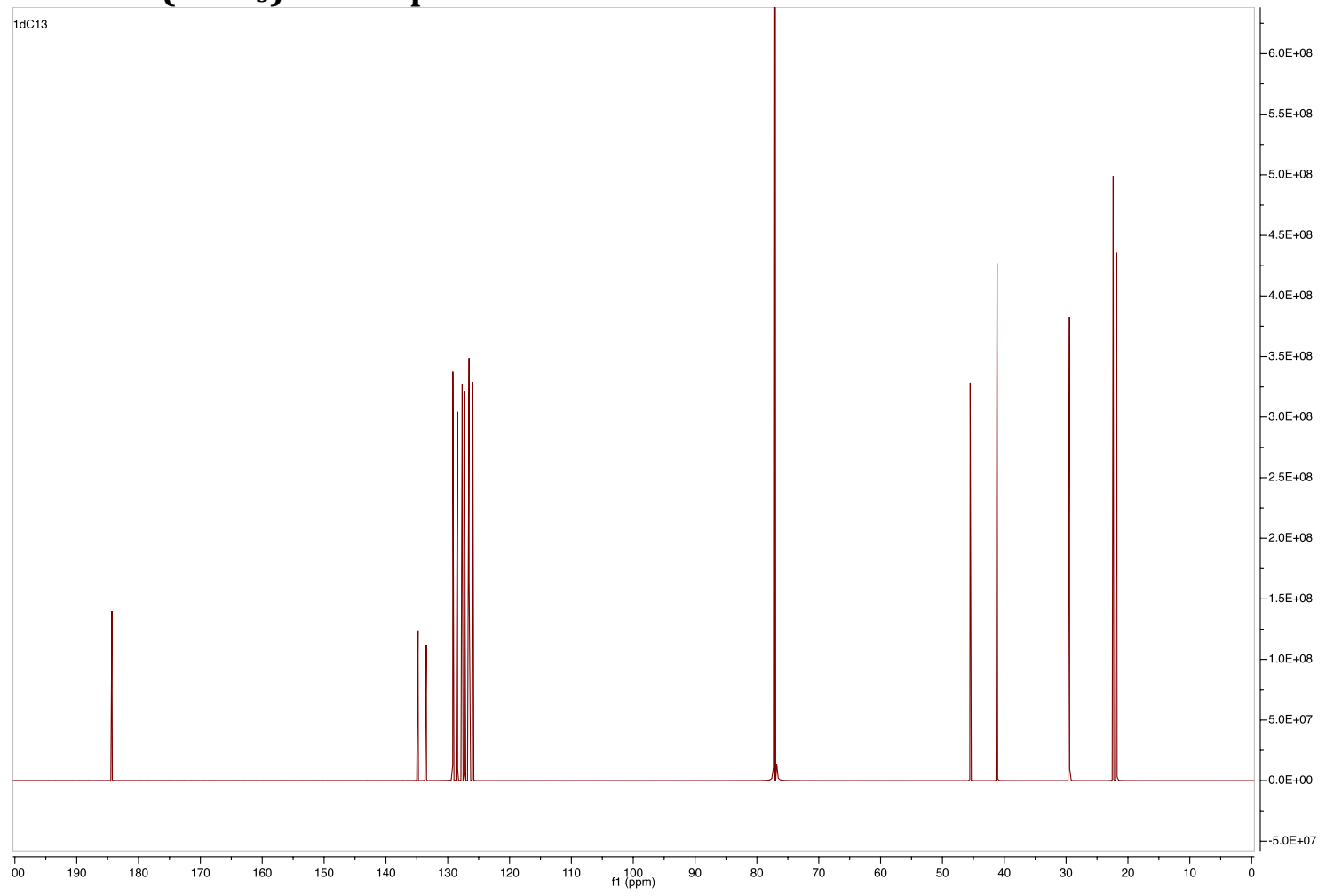


${ }^{1} \mathrm{H}$ NMR $\left(\mathrm{CDCl}_{3}\right)$ of Compound 19:

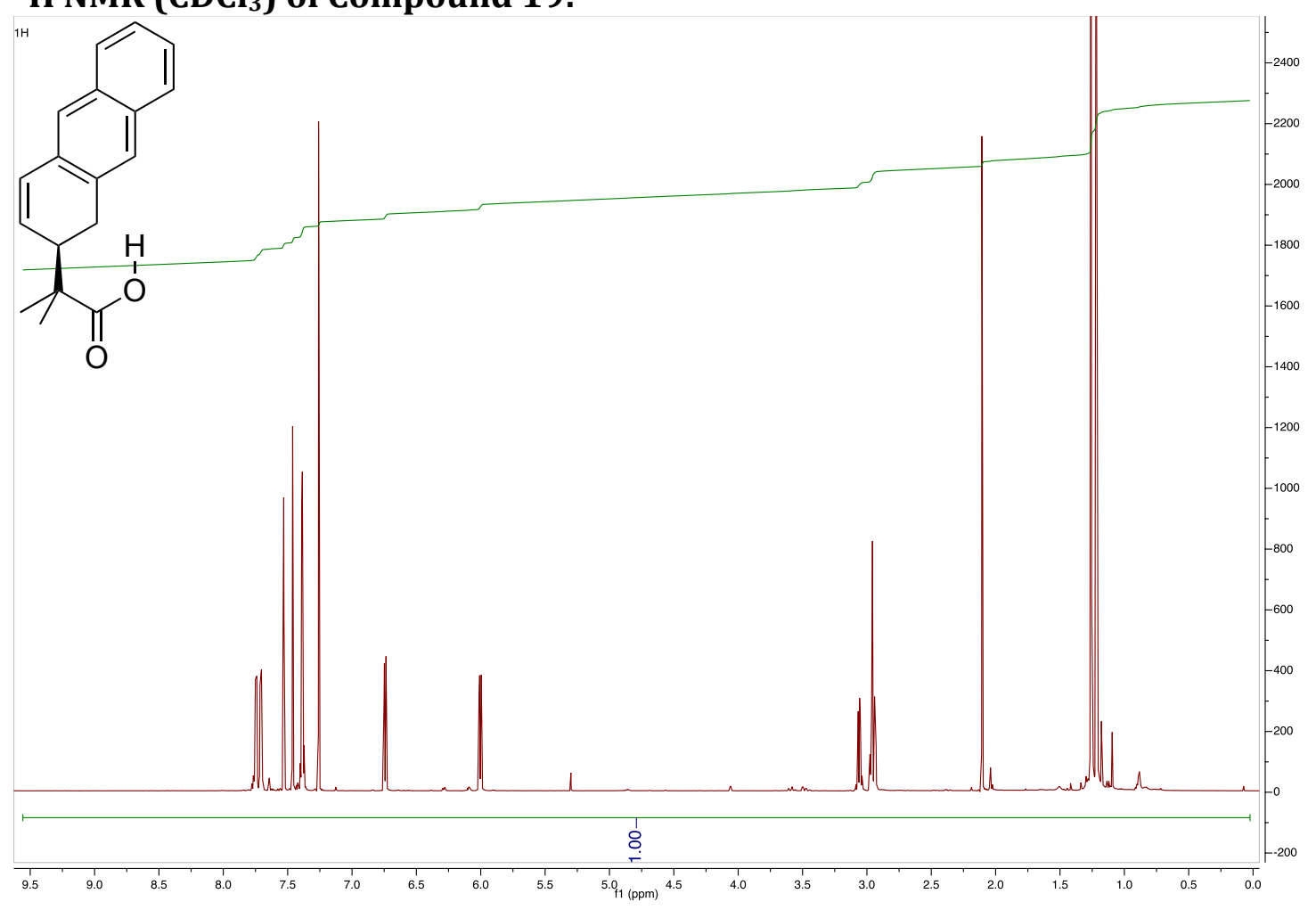

${ }^{13} \mathrm{C}$ NMR $\left(\mathrm{CDCl}_{3}\right)$ of Compound 19:

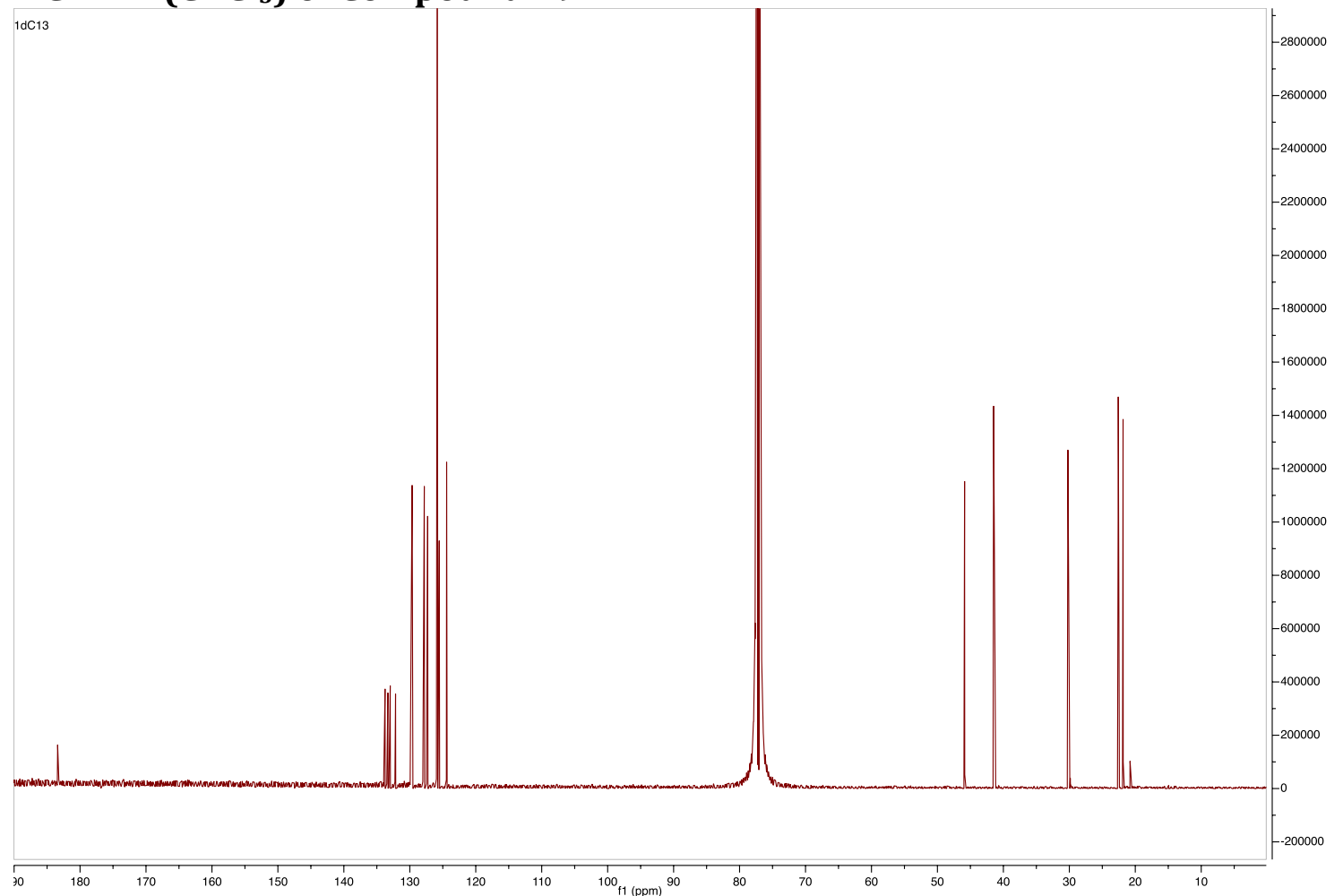




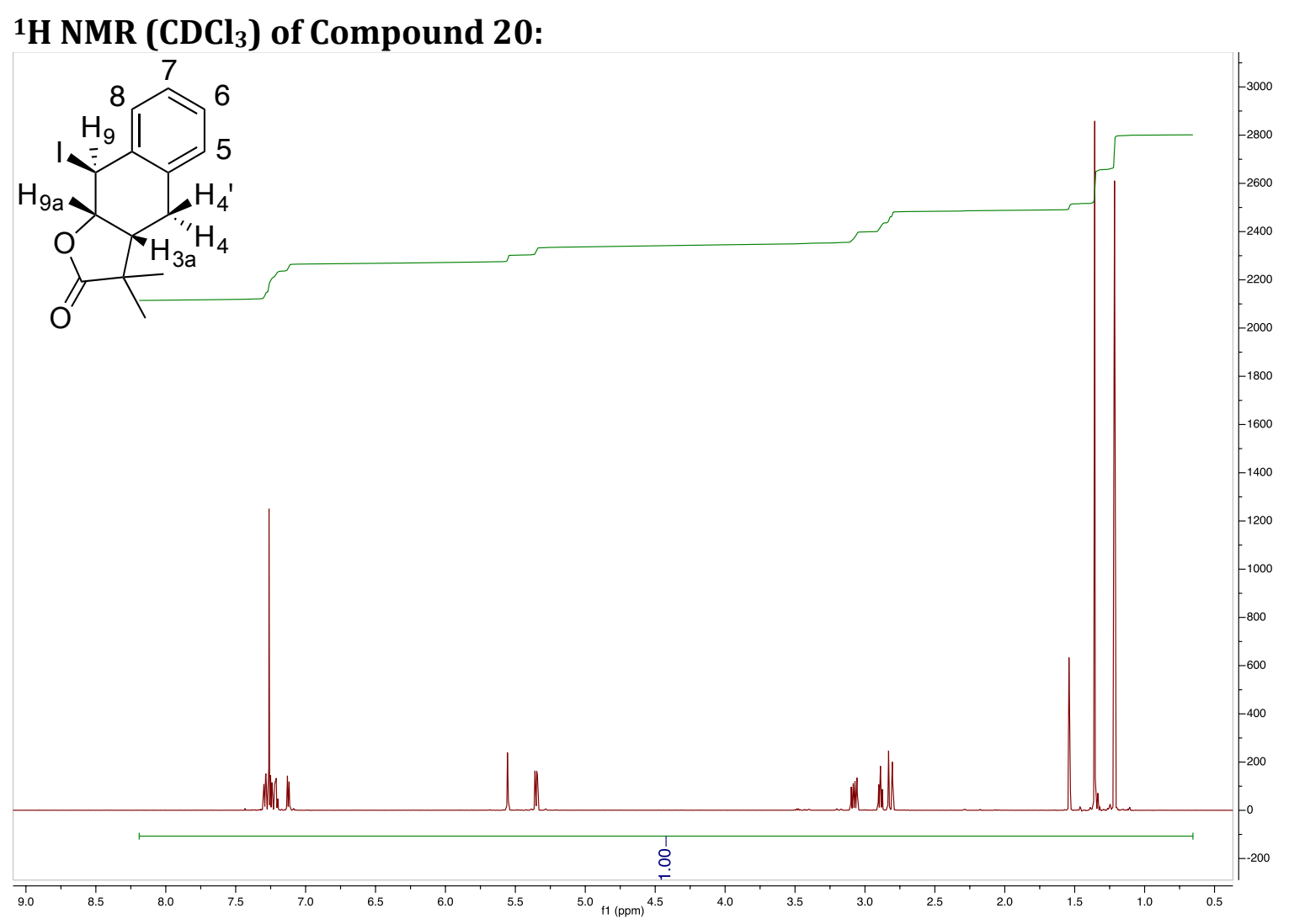

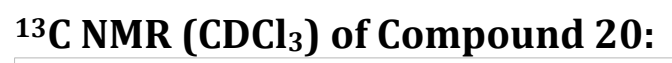

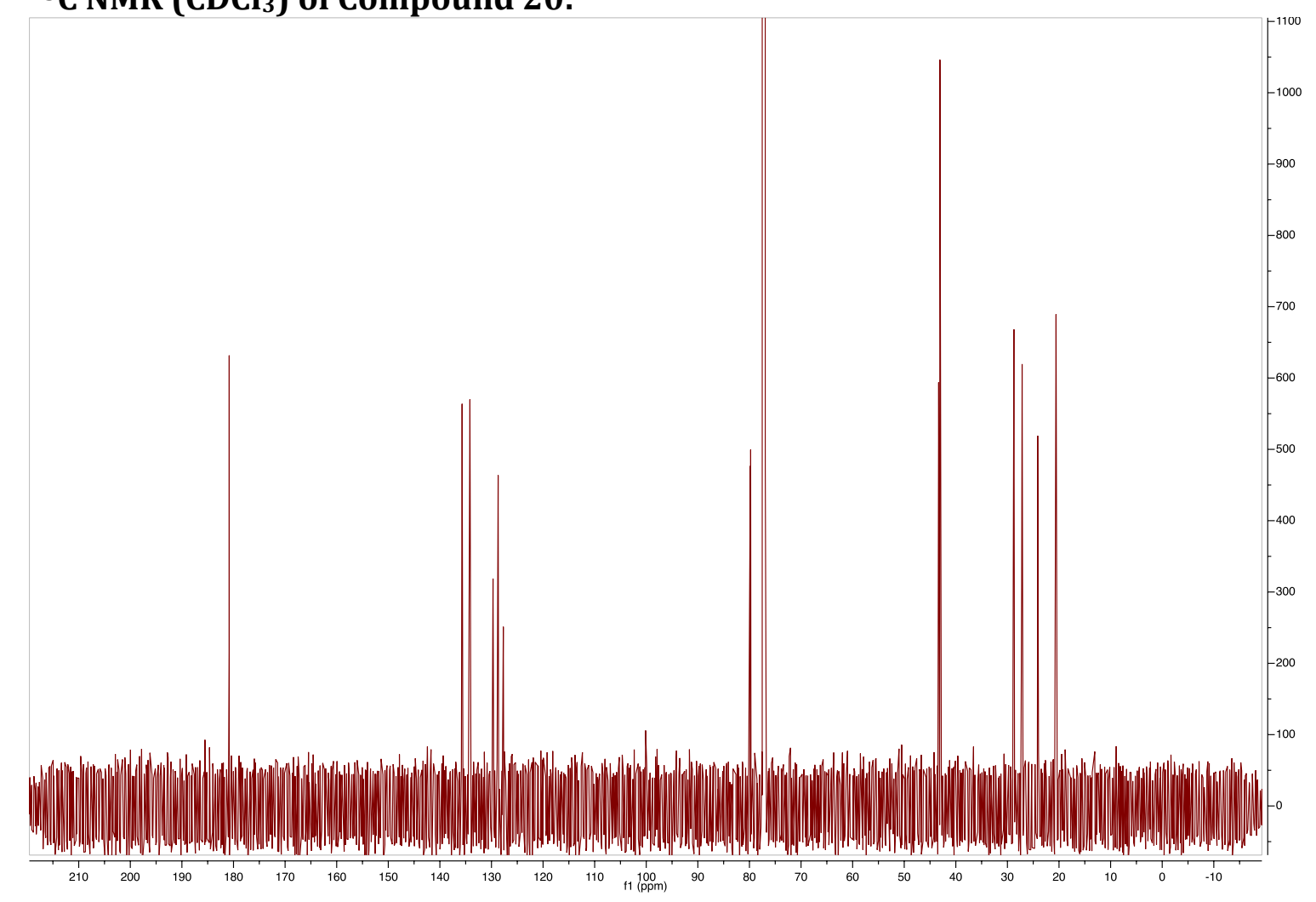


${ }^{1} \mathrm{H}$ NMR $\left(\mathrm{CDCl}_{3}\right)$ of Compound 21:

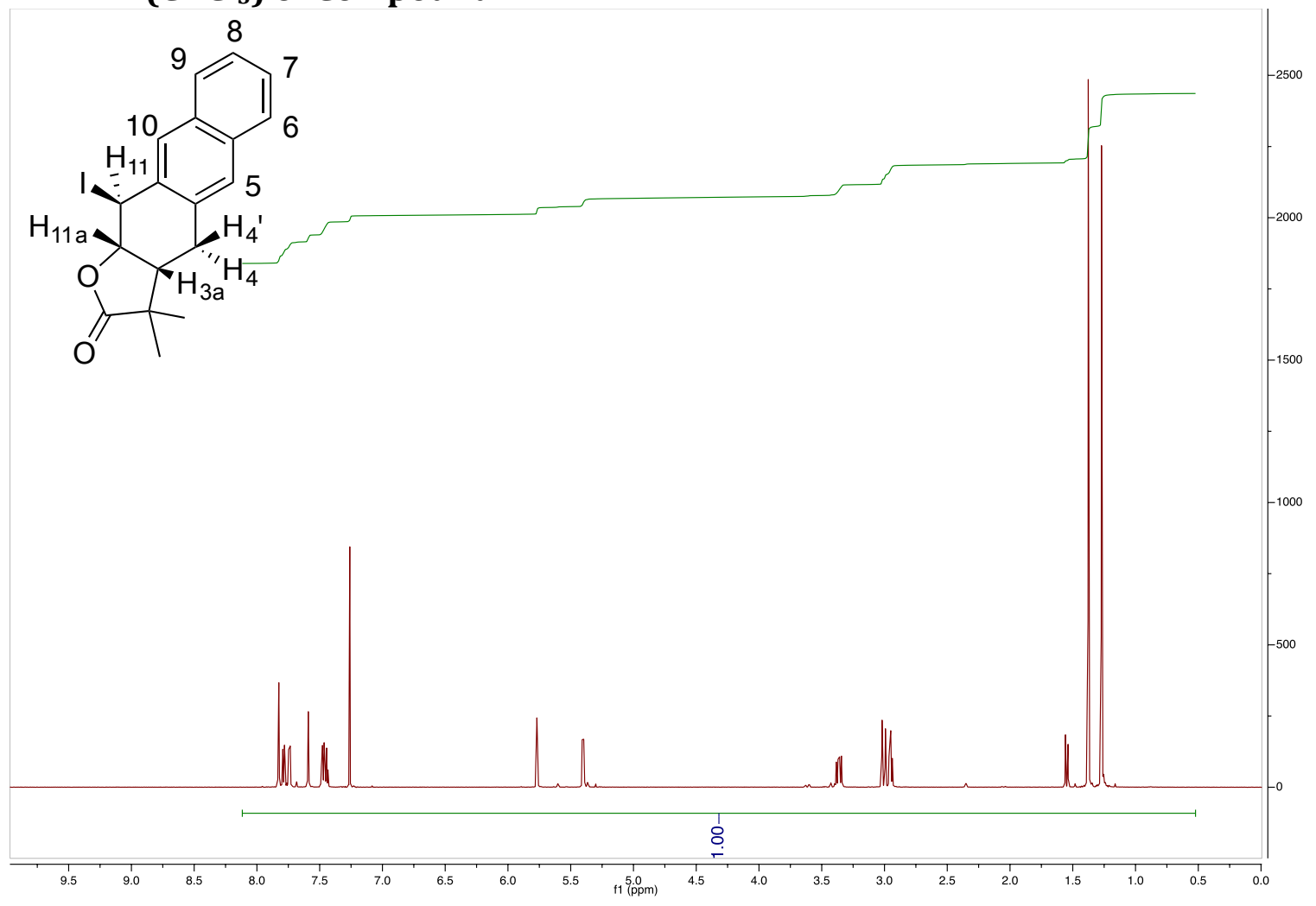

${ }^{13} \mathrm{C} \mathrm{NMR}\left(\mathrm{CDCl}_{3}\right)$ of Compound 21:

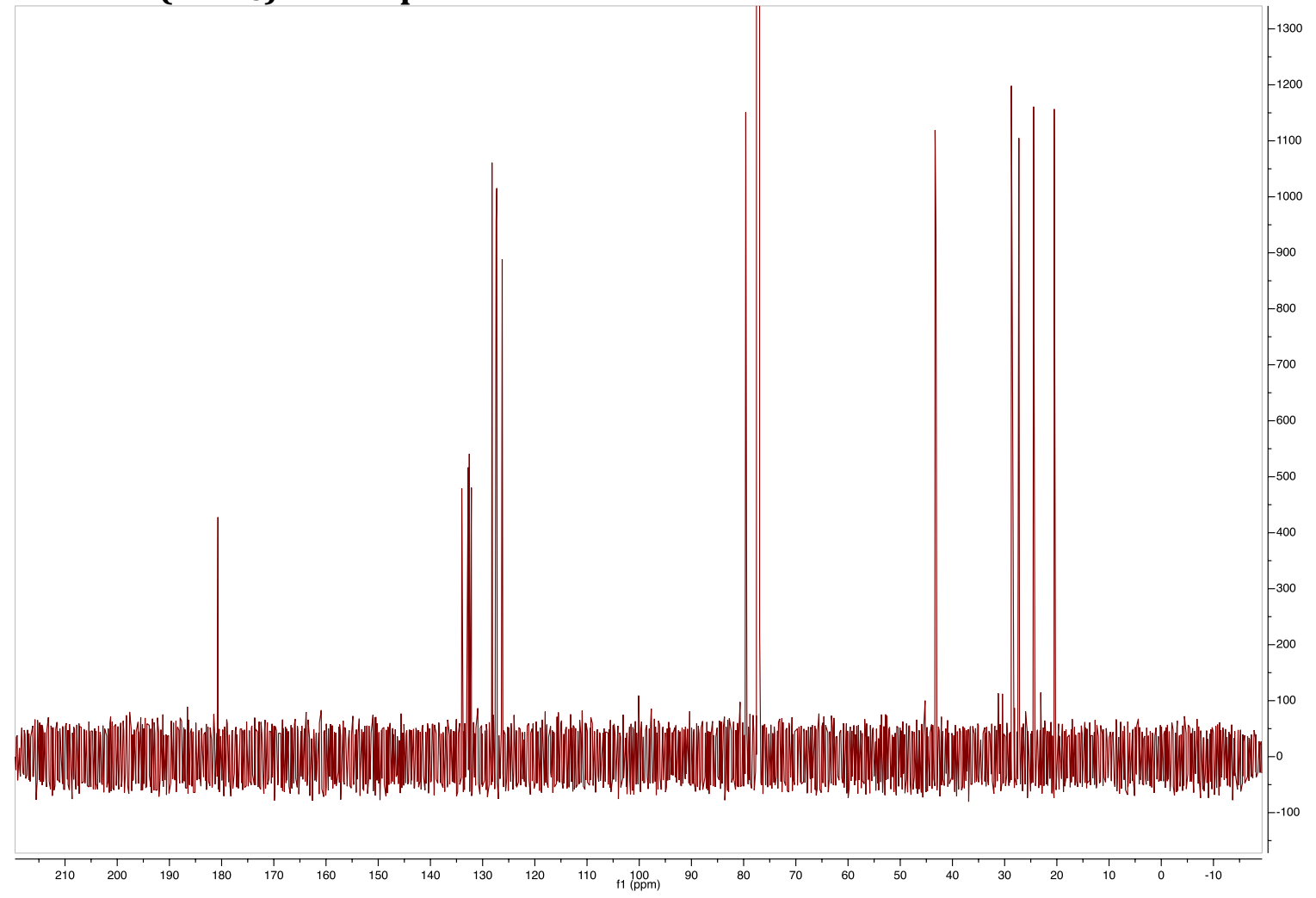


${ }^{1} H_{\text {NMR ( }} \mathrm{d}^{6}$-Acetone) of Compound 22:

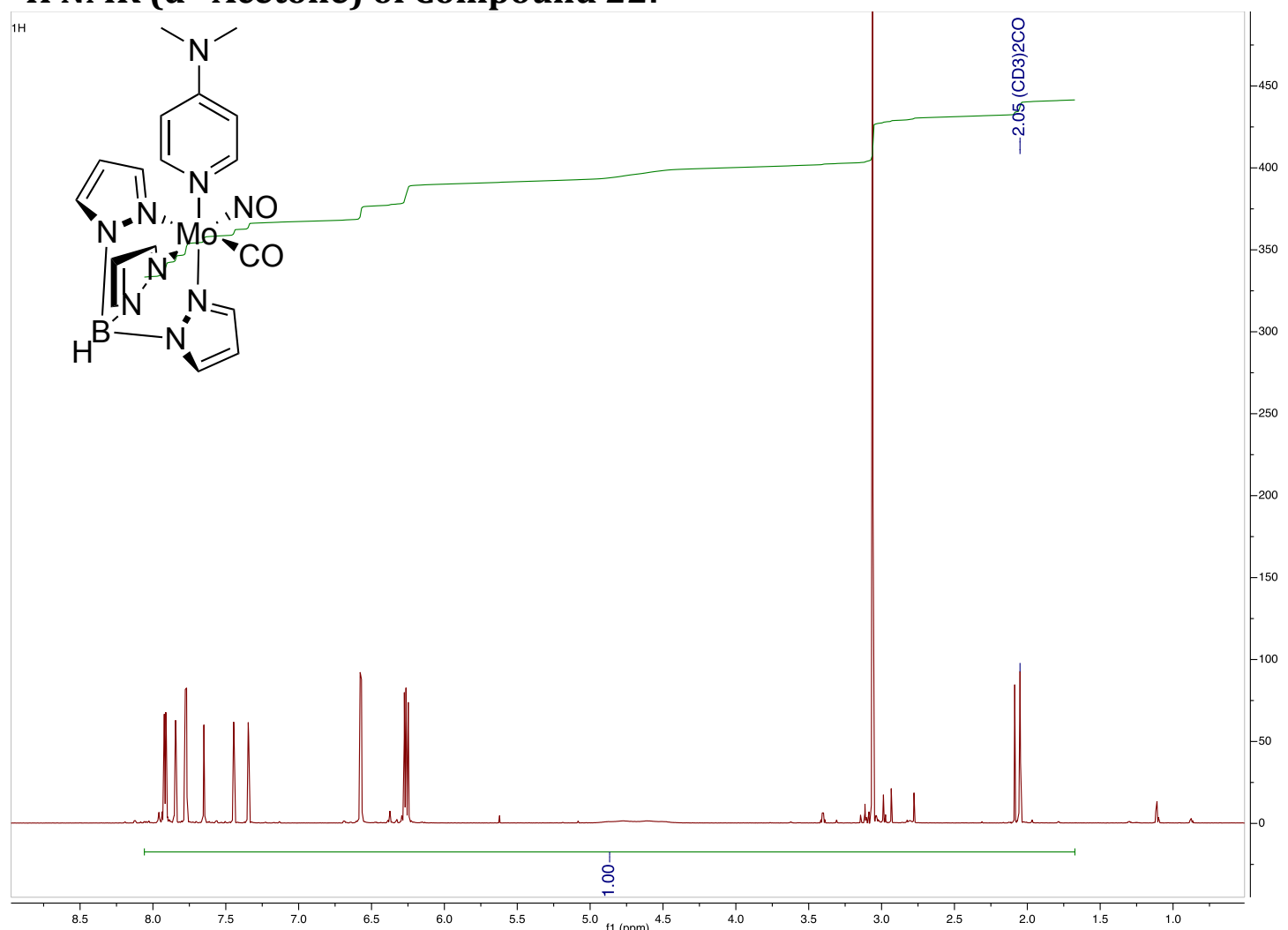

${ }^{13} \mathrm{C}$ NMR (d6-Acetone) of Compound 22:

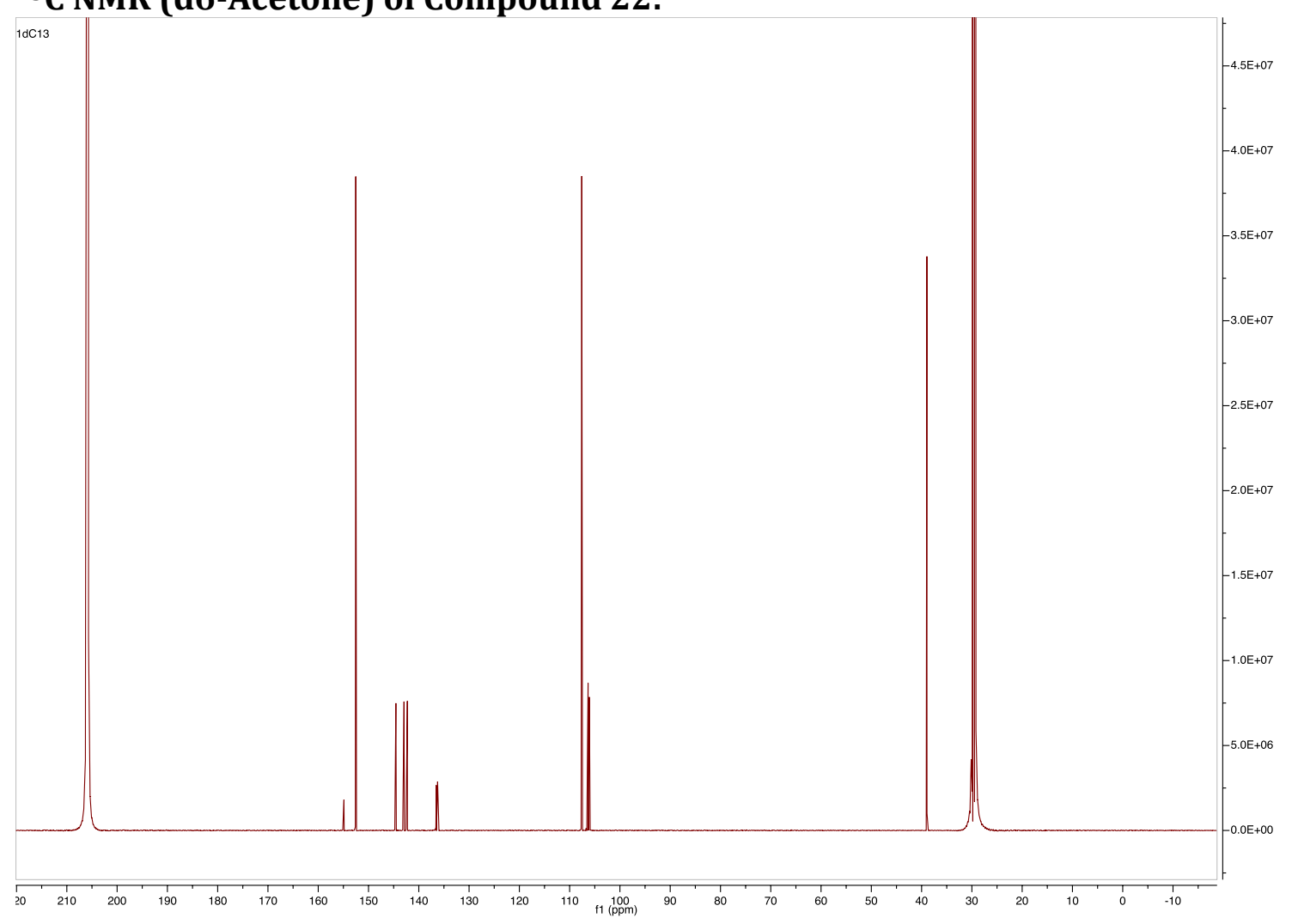


${ }^{1} \mathrm{H}$ NMR ( $\left.\mathrm{CD}_{3} \mathrm{CN}\right)$ of Compound 24-H:

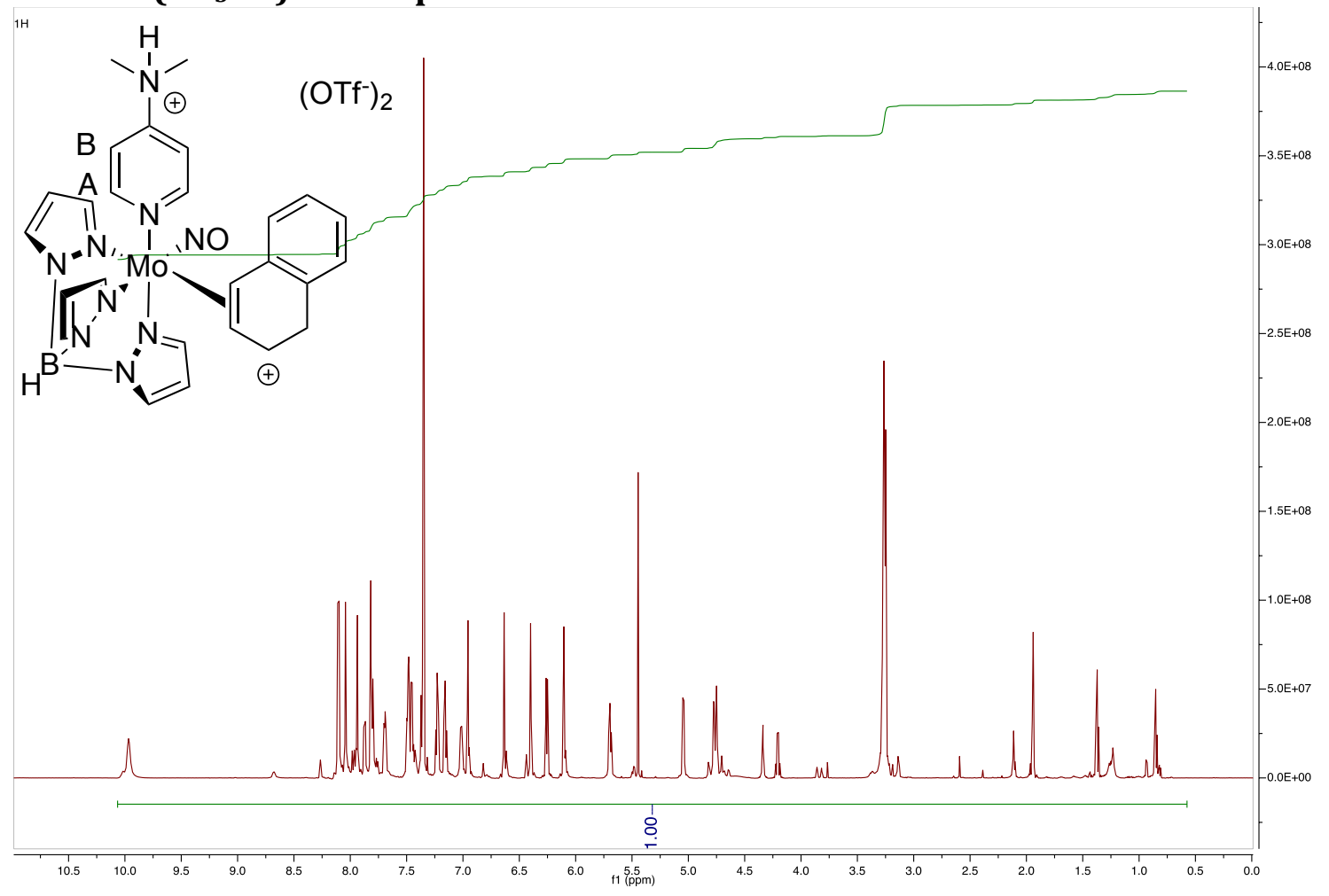


${ }^{1} \mathrm{H}$ NMR ( $d^{6}$-Acetone) of Compound 25:

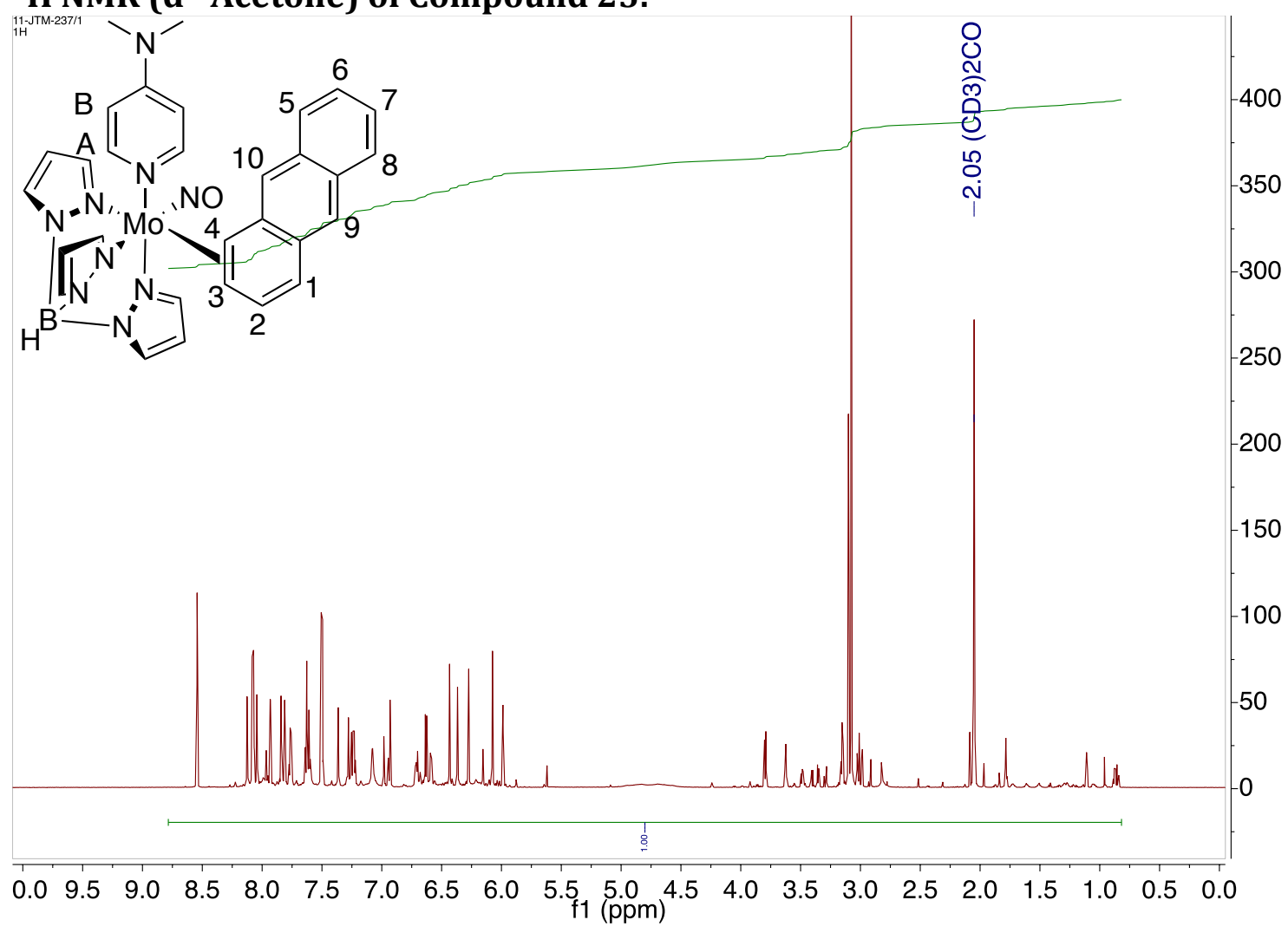

${ }^{13}$ C NMR (d6-Acetone) of Compound 25:

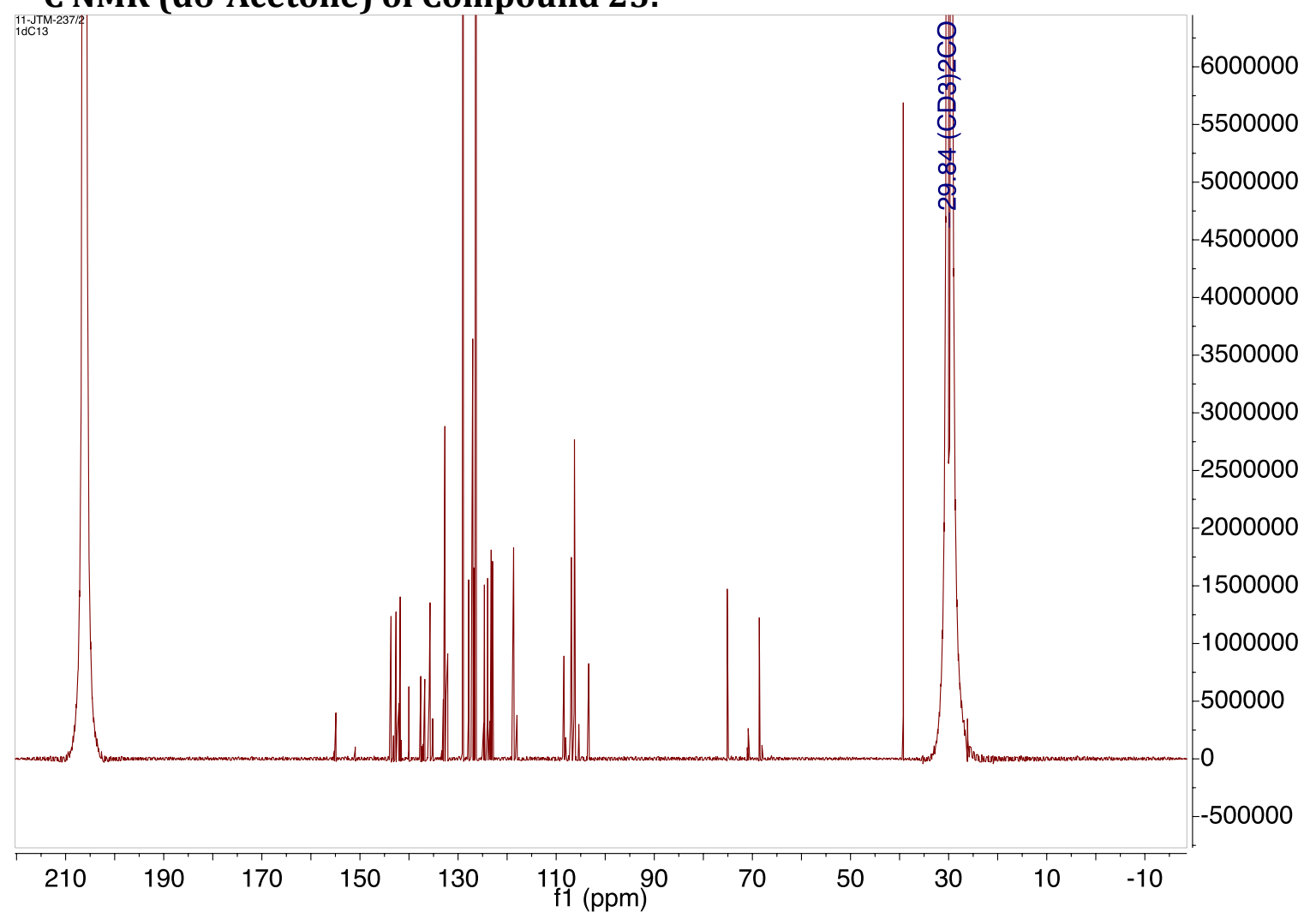


${ }^{1} \mathrm{H}$ NMR (d6-Acetone) of Compound 26:

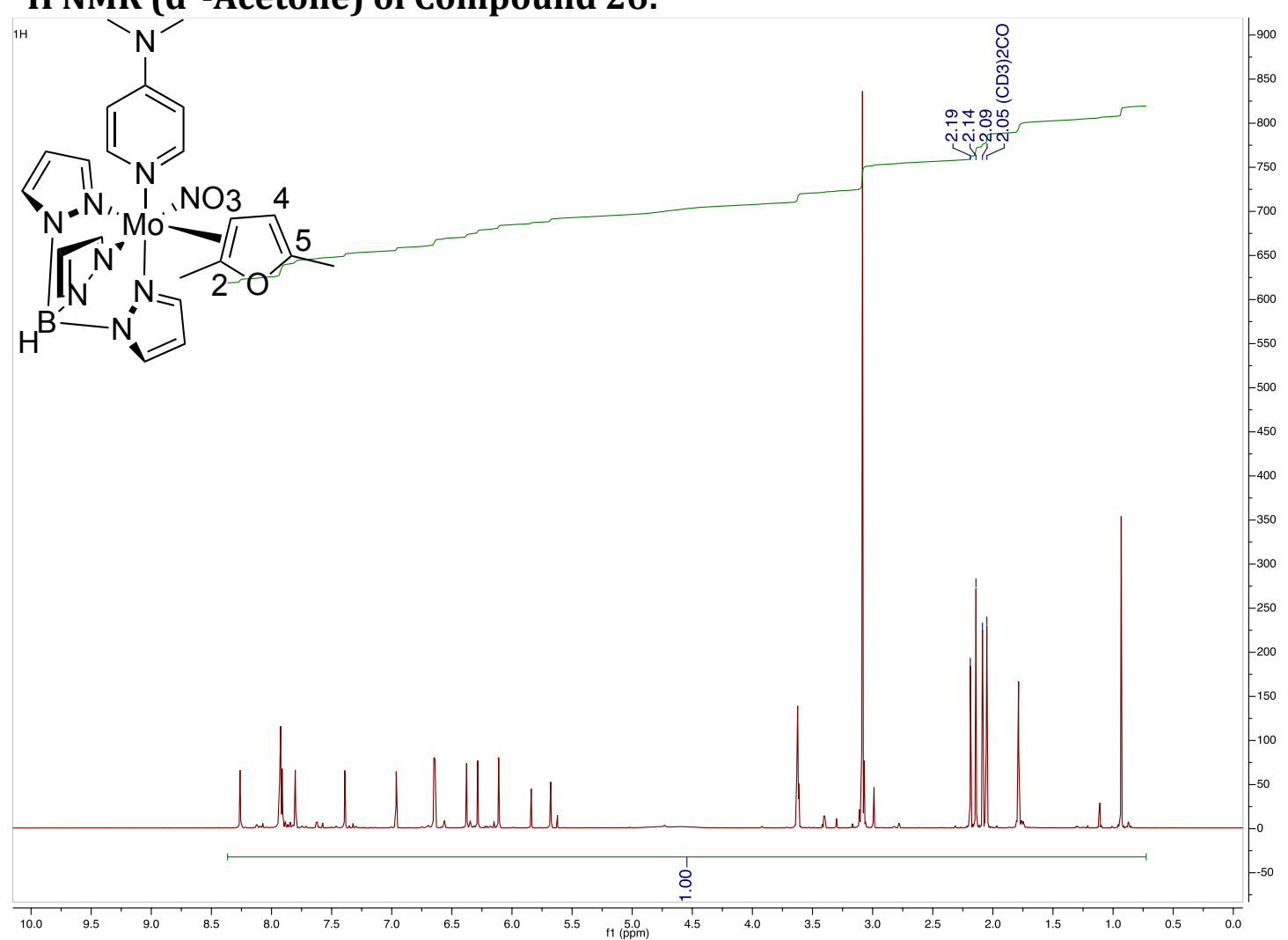

${ }^{13}$ C NMR (d6-Acetone) of Compound 26:

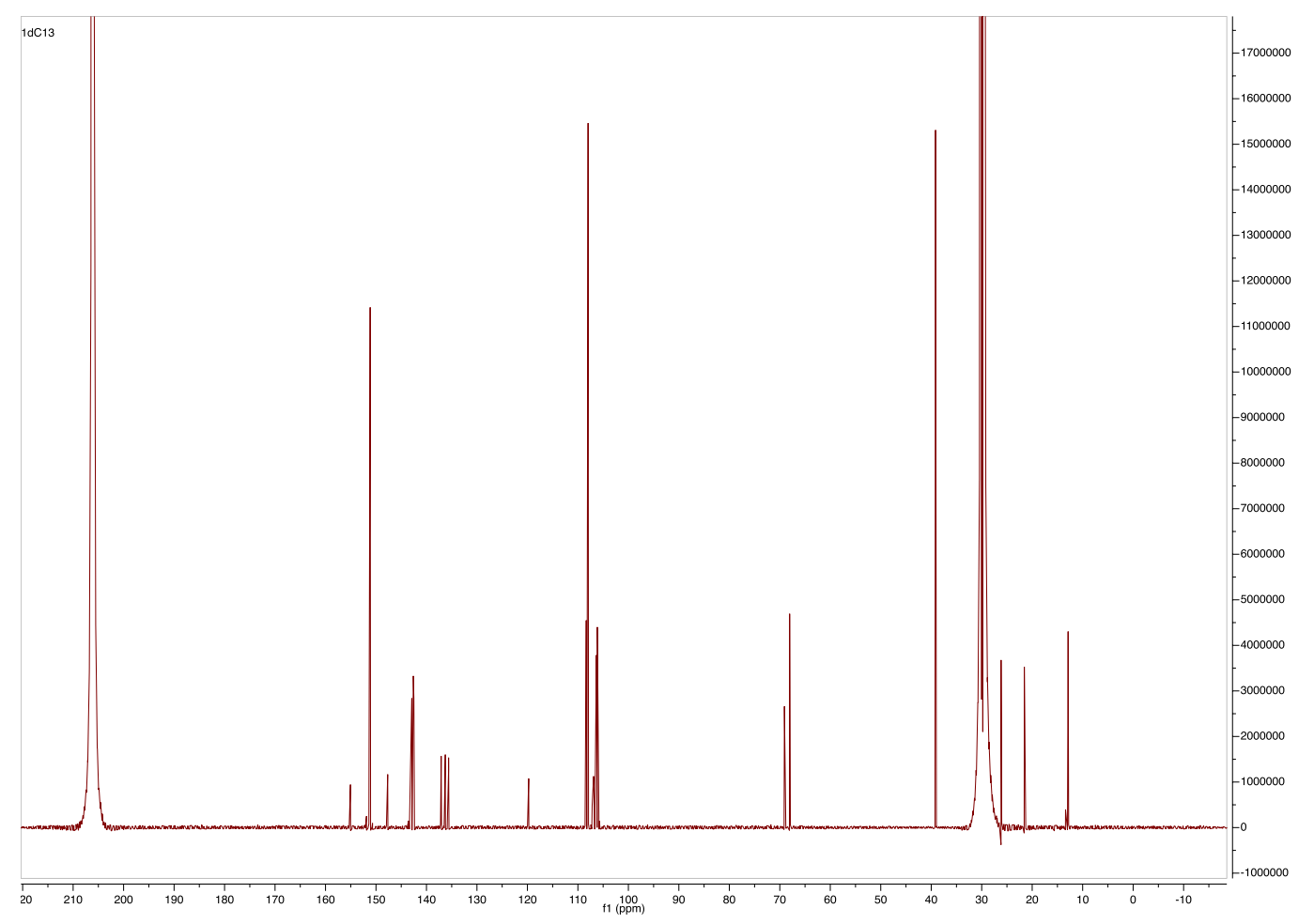


${ }^{1} \mathrm{H}$ NMR ( $\left.\mathrm{CD}_{3} \mathrm{CN}\right)$ of Compound 27:

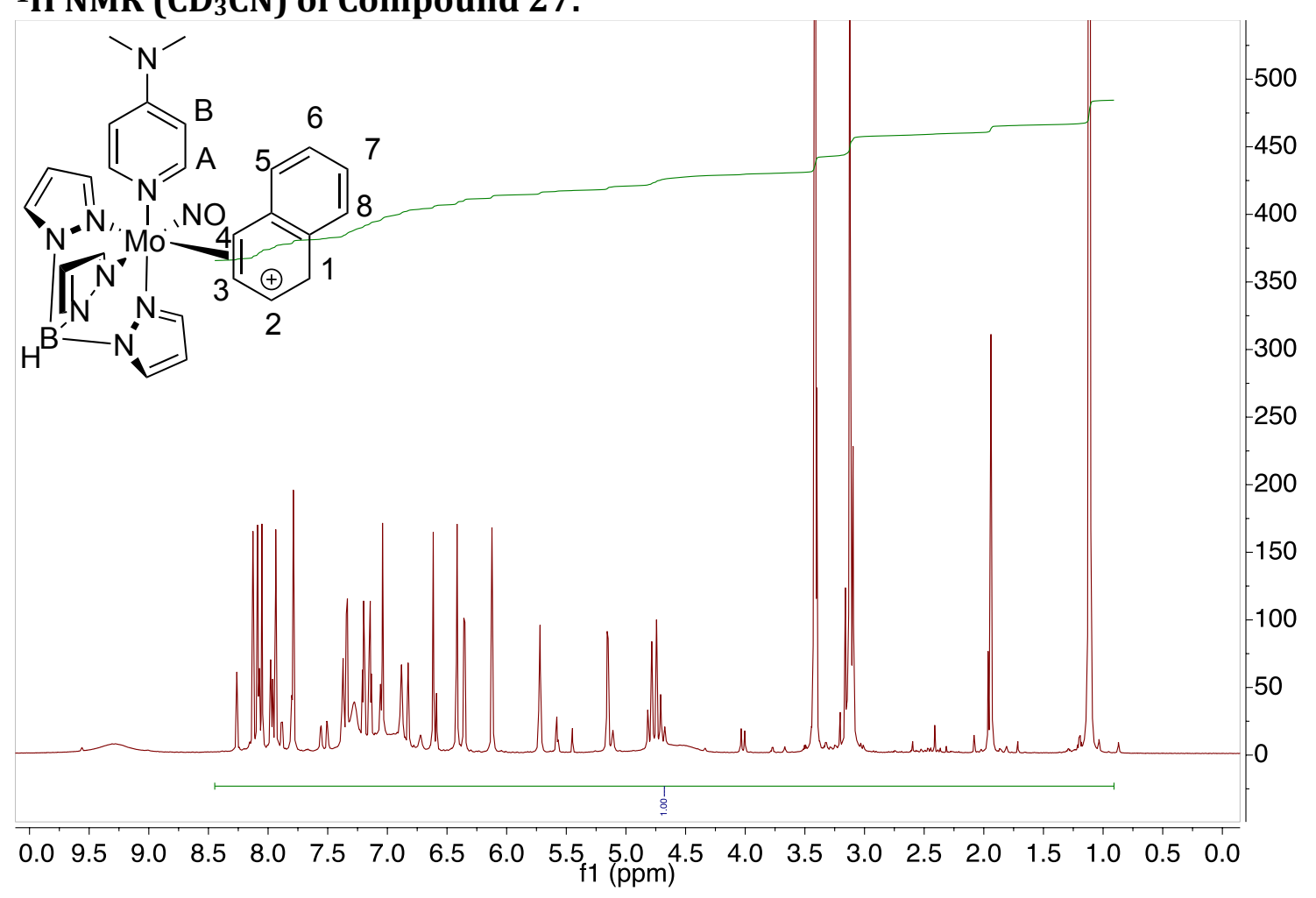

500

450

400

350

300

250

200

150

100

50

${ }^{13} \mathrm{C}$ NMR $\left(\mathrm{CD}_{3} \mathrm{CN}\right)$ of Compound 27:

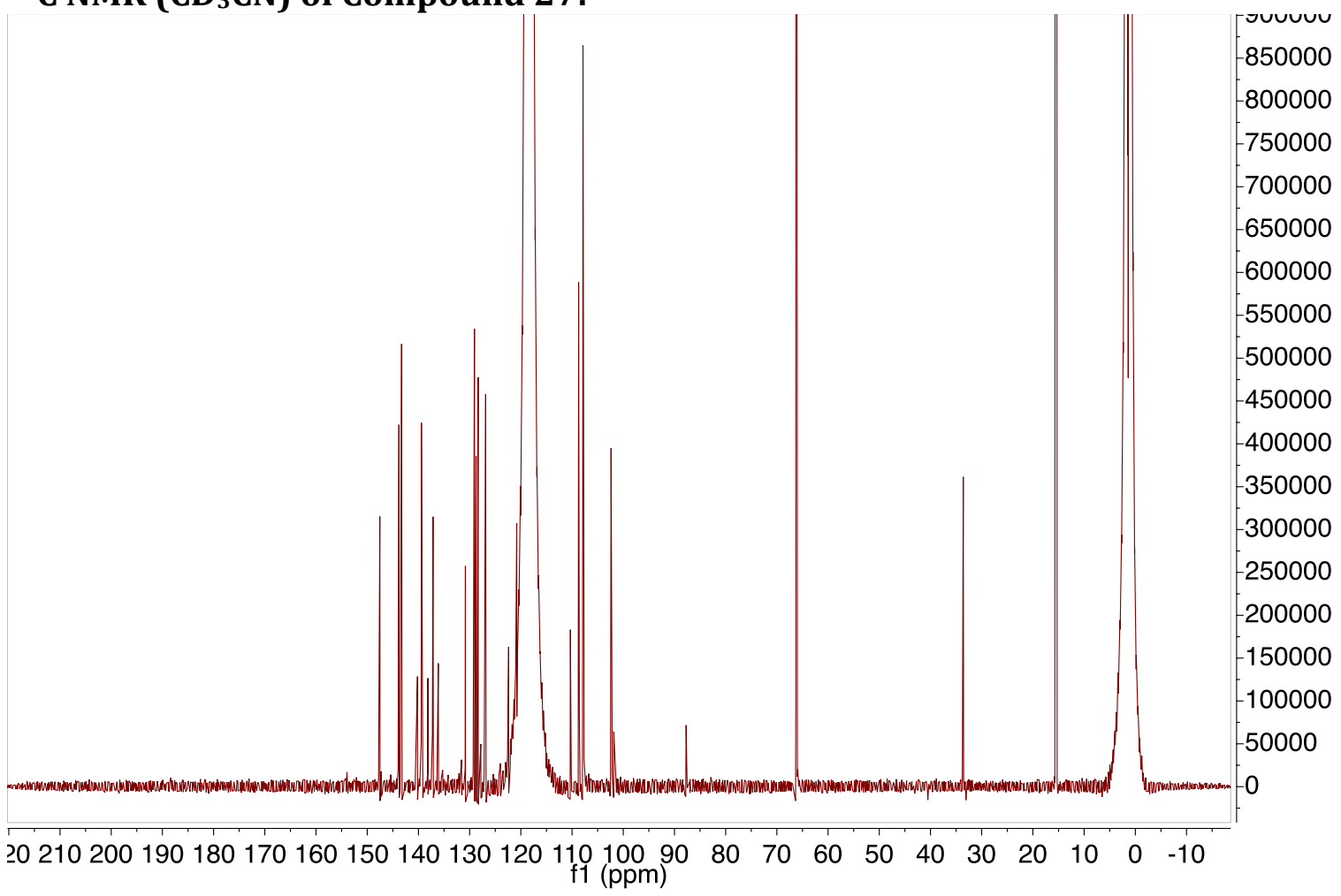


${ }^{1} \mathrm{H}$ NMR $\left(\mathrm{CD}_{3} \mathrm{CN}\right)$ of Compound 28:

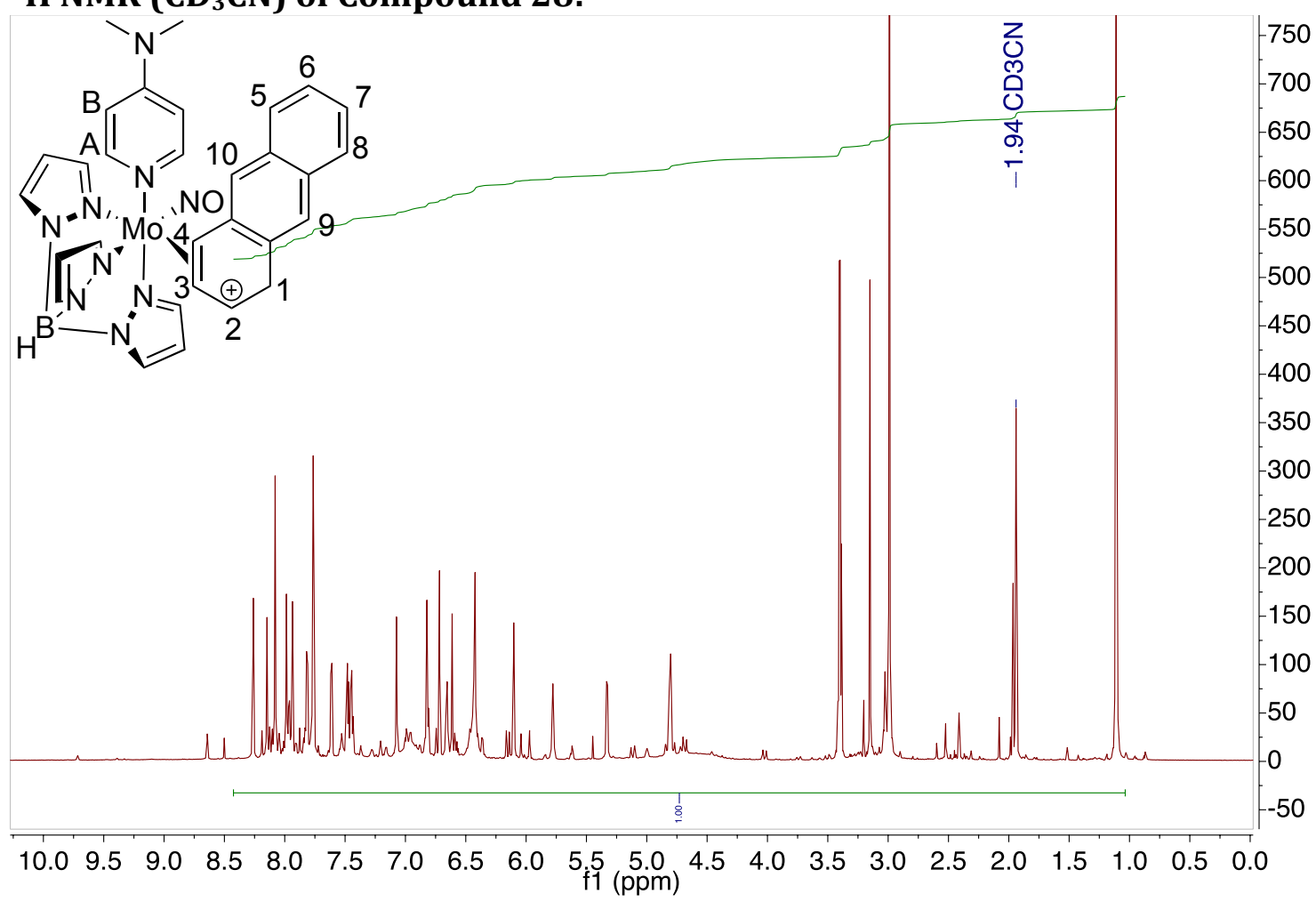

${ }^{13} \mathrm{C}$ NMR $\left(\mathrm{CD}_{3} \mathrm{CN}\right)$ of Compound 28:

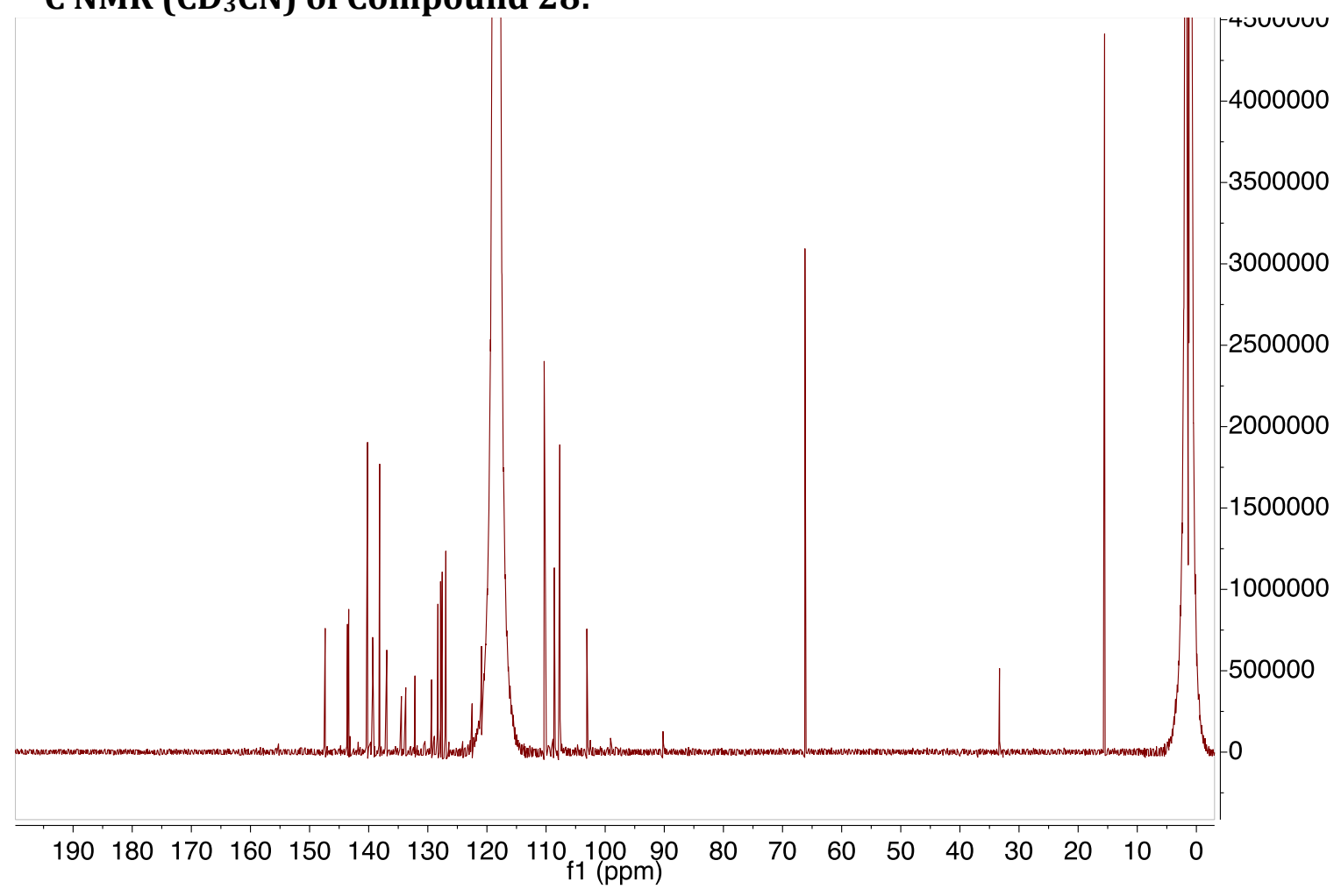


${ }^{1}$ H NMR (d6-Acetone) of Compound 29:

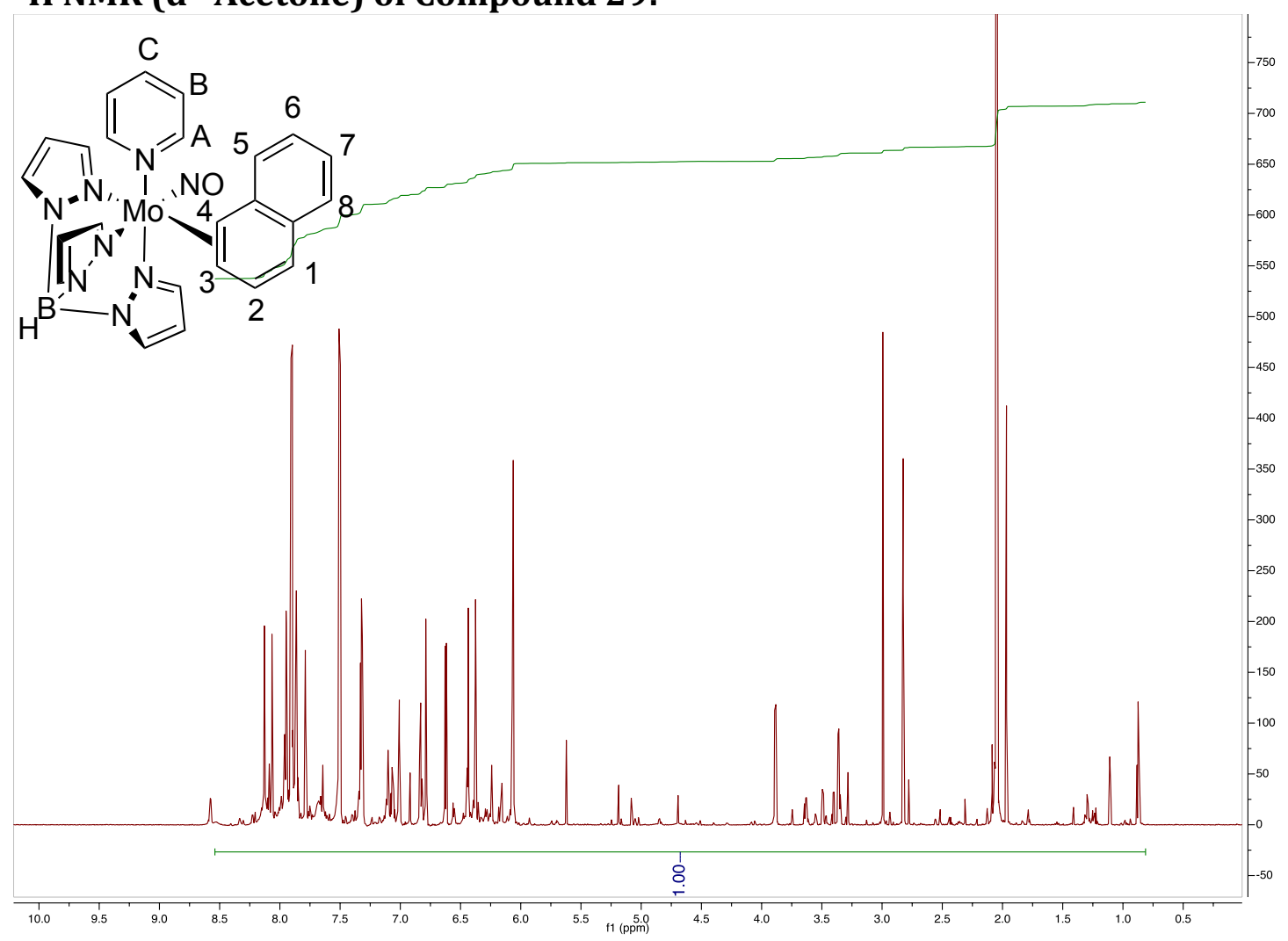

${ }^{13}$ C NMR (d ${ }^{6}$-Acetone) of Compound 29:

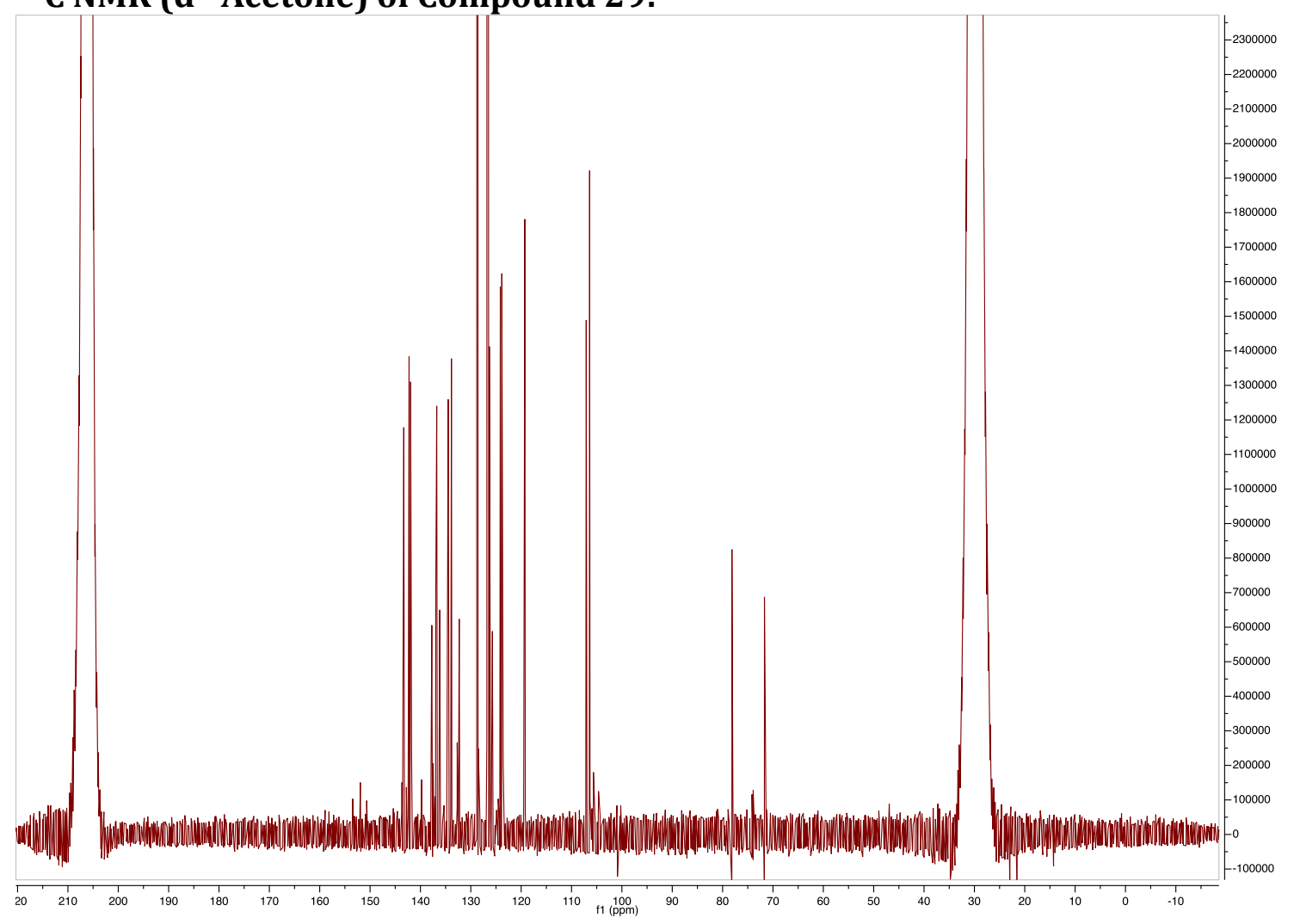


Compound 30

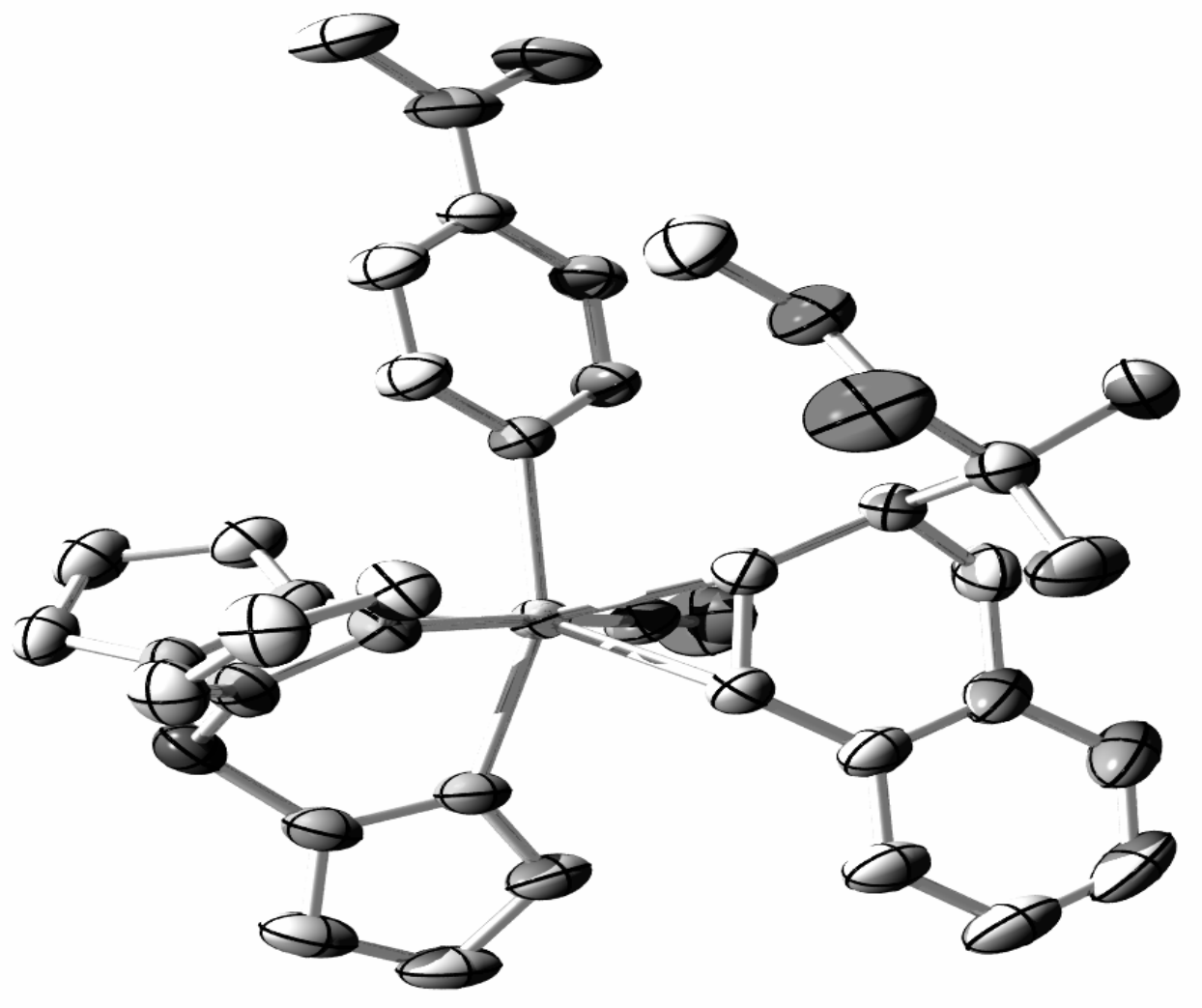




\section{Compound 30}

Table 1. Crystallographic Data for $\mathrm{C}_{31} \mathrm{H}_{38} \mathrm{BN}_{9} \mathrm{O}_{3} \mathrm{Mo}$.

\begin{tabular}{|c|c|}
\hline Empirical formula & $\mathrm{C}_{31} \mathrm{H}_{38} \mathrm{BMoN}_{9} \mathrm{O}_{3}$ \\
\hline Formula weight & 691.45 \\
\hline Temperature & $233(2) \mathrm{K}$ \\
\hline Wavelength & $0.71073 \AA$ \\
\hline Crystal system & Monoclinic \\
\hline Space group & $\mathrm{P} 21_{1} / \mathrm{c}$ \\
\hline \multirow[t]{3}{*}{ Unit cell dimensions } & $a=15.2403(6) \AA$ \\
\hline & $\mathrm{b}=13.9028(6) \AA$ \\
\hline & $\mathrm{c}=16.4382(7) \AA$ \\
\hline Volume & $3249.5(2) \AA^{3}$ \\
\hline $\mathrm{Z}$ & 4 \\
\hline Density (calculated) & $1.413 \mathrm{Mg} / \mathrm{m}^{3}$ \\
\hline Absorption coefficient & $0.451 \mathrm{~mm}^{-1}$ \\
\hline $\mathrm{F}(000)$ & 1432 \\
\hline Crystal size & $0.390 \times 0.380 \times 0.140 \mathrm{~mm}^{3}$ \\
\hline Theta range for data collection & 3.218 to $37.213^{\circ}$. \\
\hline Index ranges & $-25<=\mathrm{h}<=25,-23<=\mathrm{k}<=22,-27<=1<=26$ \\
\hline Reflections collected & 81168 \\
\hline Independent reflections & $16014[\mathrm{R}(\mathrm{int})=0.0292]$ \\
\hline Completeness to theta $=25.242^{\circ}$ & $99.7 \%$ \\
\hline Absorption correction & Empirical \\
\hline Refinement method & Full-matrix least-squares on $\mathrm{F}^{2}$ \\
\hline Data / restraints / parameters & $16014 / 0 / 423$ \\
\hline Goodness-of-fit on $\mathrm{F}^{2}$ & 1.040 \\
\hline Final $R$ indices $[\mathrm{I}>2 \operatorname{sigma}(\mathrm{I})]$ & $\mathrm{R} 1=0.0460, \mathrm{wR} 2=0.1197$ \\
\hline $\mathrm{R}$ indices (all data) & $\mathrm{R} 1=0.0774, \mathrm{wR} 2=0.1359$ \\
\hline Largest diff. peak and hole & 1.511 and -0.764 e. $\AA^{-3}$ \\
\hline
\end{tabular}




\section{Compound 30}

Table 2. Fractional coordinates ( $\mathrm{x} 10^{4}$ ) and equivalent isotropic displacement parameters $\left(\AA^{2} \times 10^{3}\right)$ for $\mathrm{C}_{31} \mathrm{H}_{38} \mathrm{BN}_{9} \mathrm{O}_{3} \mathrm{Mo}$. $\mathrm{U}(\mathrm{eq})$ is defined as one third of the trace of the orthogonalized $\mathrm{Uij}$ tensor.

\begin{tabular}{|c|c|c|c|c|}
\hline & $\mathrm{x}$ & $\mathrm{y}$ & z & $\mathrm{U}(\mathrm{eq})$ \\
\hline Mo & $7344(1)$ & $-716(1)$ & $8006(1)$ & $33(1)$ \\
\hline $\mathrm{O}(1)$ & $7608(2)$ & $3228(2)$ & $8291(2)$ & $92(1)$ \\
\hline $\mathrm{O}(2)$ & $6189(1)$ & $2554(2)$ & 7811(1) & $62(1)$ \\
\hline $\mathrm{O}(3)$ & $6270(2)$ & $-1522(2)$ & $6261(1)$ & $79(1)$ \\
\hline $\mathrm{N}(1)$ & $6036(1)$ & $-74(1)$ & $8072(1)$ & $37(1)$ \\
\hline $\mathrm{N}(2)$ & $3417(2)$ & $752(2)$ & $8160(2)$ & $64(1)$ \\
\hline $\mathrm{N}(3)$ & $8523(1)$ & $-1729(1)$ & $8223(1)$ & $43(1)$ \\
\hline $\mathrm{N}(4)$ & $8947(1)$ & $-2164(1)$ & $9008(1)$ & $48(1)$ \\
\hline $\mathrm{N}(5)$ & 6932(1) & $-1876(1)$ & $8734(1)$ & $41(1)$ \\
\hline $\mathrm{N}(6)$ & $7567(1)$ & $-2290(1)$ & $9454(1)$ & $43(1)$ \\
\hline $\mathrm{N}(7)$ & $8216(1)$ & $-236(1)$ & $9403(1)$ & $40(1)$ \\
\hline $\mathrm{N}(8)$ & $8675(1)$ & $-920(1)$ & $9996(1)$ & $45(1)$ \\
\hline $\mathrm{N}(9)$ & $6721(1)$ & $-1180(1)$ & $6965(1)$ & $44(1)$ \\
\hline $\mathrm{C}(1)$ & $6853(2)$ & $1306(2)$ & $6843(1)$ & $43(1)$ \\
\hline$C(2)$ & $7504(2)$ & 791(1) & $7643(1)$ & $39(1)$ \\
\hline$C(3)$ & $8302(1)$ & $270(2)$ & $7625(1)$ & $41(1)$ \\
\hline$C(4)$ & $8475(2)$ & $155(2)$ & $6815(2)$ & $47(1)$ \\
\hline$C(5)$ & $9337(2)$ & $-199(2)$ & $6817(2)$ & $56(1)$ \\
\hline$C(6)$ & $9514(2)$ & $-301(2)$ & $6058(2)$ & $70(1)$ \\
\hline$C(7)$ & $8842(3)$ & $-3(2)$ & $5277(2)$ & $78(1)$ \\
\hline $\mathrm{C}(8)$ & $8001(2)$ & $374(2)$ & $5267(2)$ & $66(1)$ \\
\hline$C(9)$ & $7803(2)$ & $447(2)$ & $6028(2)$ & $51(1)$ \\
\hline$C(10)$ & $6857(2)$ & $812(2)$ & $5999(2)$ & $55(1)$ \\
\hline $\mathrm{C}(11)$ & $7079(2)$ & 2413(2) & $6892(2)$ & $53(1)$ \\
\hline$C(12)$ & $8060(2)$ & $2629(2)$ & $6897(3)$ & $80(1)$ \\
\hline$C(13)$ & $6341(3)$ & $2965(2)$ & $6147(2)$ & $74(1)$ \\
\hline$C(14)$ & $7010(2)$ & $2783(2)$ & $7740(2)$ & $55(1)$ \\
\hline$C(15)$ & $6080(3)$ & $2803(2)$ & $8613(2)$ & $75(1)$ \\
\hline$C(16)$ & $5243(2)$ & $-39(2)$ & $7357(1)$ & $44(1)$ \\
\hline
\end{tabular}




$\begin{array}{lrrrr}\mathrm{C}(17) & 4384(2) & 235(2) & 7357(2) & 48(1) \\ \mathrm{C}(18) & 4266(2) & 501(2) & 8134(2) & 45(1) \\ \mathrm{C}(19) & 5091(2) & 482(2) & 8876(2) & 53(1) \\ \mathrm{C}(20) & 5931(2) & 195(2) & 8816(1) & 48(1) \\ \mathrm{C}(21) & 2592(2) & 768(3) & 7369(3) & 83(1) \\ \mathrm{C}(22) & 3309(3) & 1075(3) & 8943(3) & 89(1) \\ \mathrm{C}(23) & 9581(2) & -2809(2) & 8944(2) & 62(1) \\ \mathrm{C}(24) & 9574(2) & -2796(2) & 8117(2) & 64(1) \\ \mathrm{C}(25) & 8897(2) & -2116(2) & 7679(2) & 52(1) \\ \mathrm{C}(26) & 7132(2) & -2950(2) & 9779(2) & 53(1) \\ \mathrm{C}(27) & 6202(2) & -2967(2) & 9266(2) & 59(1) \\ \mathrm{C}(28) & 6108(2) & -2290(2) & 8623(2) & 50(1) \\ \mathrm{C}(29) & 9193(2) & -499(2) & 10749(2) & 56(1) \\ \mathrm{C}(30) & 9090(2) & 473(2) & 10660(2) & 61(1) \\ \mathrm{C}(31) & 8472(2) & 609(2) & 9815(2) & 50(1) \\ \mathrm{B}(1) & 8603(2) & -1996(2) & 9767(2) & 48(1)\end{array}$


Compound 30

Table 3. Bond lengths and angles for $\mathrm{C}_{31} \mathrm{H}_{38} \mathrm{BN}_{9} \mathrm{O}_{3} \mathrm{Mo}$.

\begin{tabular}{|c|c|}
\hline Mo-N(9) & $1.7549(17)$ \\
\hline Mo-N(3) & $2.2079(17)$ \\
\hline Mo-C(2) & $2.2157(19)$ \\
\hline Mo-N(1) & $2.2228(16)$ \\
\hline Mo-N(5) & $2.2287(17)$ \\
\hline Mo-C(3) & $2.2492(19)$ \\
\hline Mo-N(7) & $2.2992(17)$ \\
\hline $\mathrm{O}(1)-\mathrm{C}(14)$ & $1.199(4)$ \\
\hline $\mathrm{O}(2)-\mathrm{C}(14)$ & $1.337(3)$ \\
\hline $\mathrm{O}(2)-\mathrm{C}(15)$ & $1.428(3)$ \\
\hline $\mathrm{O}(3)-\mathrm{N}(9)$ & $1.210(2)$ \\
\hline $\mathrm{N}(1)-\mathrm{C}(20)$ & $1.343(3)$ \\
\hline $\mathrm{N}(1)-\mathrm{C}(16)$ & $1.350(3)$ \\
\hline $\mathrm{N}(2)-\mathrm{C}(18)$ & $1.355(3)$ \\
\hline $\mathrm{N}(2)-\mathrm{C}(22)$ & $1.428(4)$ \\
\hline $\mathrm{N}(2)-\mathrm{C}(21)$ & $1.447(4)$ \\
\hline $\mathrm{N}(3)-\mathrm{C}(25)$ & $1.336(3)$ \\
\hline $\mathrm{N}(3)-\mathrm{N}(4)$ & $1.359(3)$ \\
\hline $\mathrm{N}(4)-\mathrm{C}(23)$ & $1.349(3)$ \\
\hline $\mathrm{N}(4)-\mathrm{B}(1)$ & $1.536(3)$ \\
\hline $\mathrm{N}(5)-\mathrm{C}(28)$ & $1.333(3)$ \\
\hline $\mathrm{N}(5)-\mathrm{N}(6)$ & $1.358(2)$ \\
\hline $\mathrm{N}(6)-\mathrm{C}(26)$ & $1.350(3)$ \\
\hline $\mathrm{N}(6)-\mathrm{B}(1)$ & $1.529(3)$ \\
\hline $\mathrm{N}(7)-\mathrm{C}(31)$ & $1.341(3)$ \\
\hline $\mathrm{N}(7)-\mathrm{N}(8)$ & $1.361(2)$ \\
\hline $\mathrm{N}(8)-\mathrm{C}(29)$ & $1.341(3)$ \\
\hline $\mathrm{N}(8)-\mathrm{B}(1)$ & $1.538(3)$ \\
\hline$C(1)-C(2)$ & $1.512(3)$ \\
\hline$C(1)-C(10)$ & $1.550(3)$ \\
\hline$C(1)-C(11)$ & $1.572(3)$ \\
\hline$C(2)-C(3)$ & $1.425(3)$ \\
\hline $\mathrm{C}(3)-\mathrm{C}(4)$ & $1.456(3)$ \\
\hline
\end{tabular}




\begin{tabular}{|c|c|}
\hline $\mathrm{C}(4)-\mathrm{C}(9)$ & $1.391(4)$ \\
\hline$C(4)-C(5)$ & $1.403(3)$ \\
\hline$C(5)-C(6)$ & $1.375(4)$ \\
\hline$C(6)-C(7)$ & $1.387(5)$ \\
\hline $\mathrm{C}(7)-\mathrm{C}(8)$ & $1.379(5)$ \\
\hline $\mathrm{C}(8)-\mathrm{C}(9)$ & $1.392(3)$ \\
\hline $\mathrm{C}(9)-\mathrm{C}(10)$ & $1.513(4)$ \\
\hline $\mathrm{C}(11)-\mathrm{C}(12)$ & $1.523(4)$ \\
\hline $\mathrm{C}(11)-\mathrm{C}(13)$ & $1.537(4)$ \\
\hline$C(11)-C(14)$ & $1.525(4)$ \\
\hline$C(16)-C(17)$ & $1.363(3)$ \\
\hline $\mathrm{C}(17)-\mathrm{C}(18)$ & $1.403(3)$ \\
\hline$C(18)-C(19)$ & $1.402(4)$ \\
\hline$C(19)-C(20)$ & $1.377(3)$ \\
\hline$C(23)-C(24)$ & $1.356(4)$ \\
\hline$C(24)-C(25)$ & $1.394(4)$ \\
\hline$C(26)-C(27)$ & $1.363(4)$ \\
\hline $\mathrm{C}(27)-\mathrm{C}(28)$ & $1.384(4)$ \\
\hline$C(29)-C(30)$ & $1.362(4)$ \\
\hline$C(30)-C(31)$ & $1.383(3)$ \\
\hline N(9)-Mo-N(3) & $92.65(8)$ \\
\hline N(9)-Mo-C(2) & $99.09(8)$ \\
\hline N(3)-Mo-C(2) & $119.01(7)$ \\
\hline N(9)-Mo-N(1) & $90.86(7)$ \\
\hline N(3)-Mo-N(1) & $160.08(6)$ \\
\hline C(2)-Mo-N(1) & $79.67(7)$ \\
\hline N(9)-Mo-N(5) & $95.71(8)$ \\
\hline N(3)-Mo-N(5) & $79.74(6)$ \\
\hline C(2)-Mo-N(5) & $155.27(7)$ \\
\hline N(1)-Mo-N(5) & $80.41(6)$ \\
\hline N(9)-Mo-C(3) & $97.48(8)$ \\
\hline N(3)-Mo-C(3) & $82.08(7)$ \\
\hline C(2)-Mo-C(3) & $37.21(7)$ \\
\hline N(1)-Mo-C(3) & $116.88(7)$ \\
\hline N(5)-Mo-C(3) & $157.96(7)$ \\
\hline
\end{tabular}




\begin{tabular}{|c|c|}
\hline N(9)-Mo-N(7) & $175.13(8)$ \\
\hline N(3)-Mo-N(7) & $83.11(7)$ \\
\hline C(2)-Mo-N(7) & $85.10(7)$ \\
\hline N(1)-Mo-N(7) & $92.33(6)$ \\
\hline $\mathrm{N}(5)-\mathrm{Mo}-\mathrm{N}(7)$ & $81.22(6)$ \\
\hline $\mathrm{C}(3)-\mathrm{Mo}-\mathrm{N}(7)$ & $84.34(7)$ \\
\hline $\mathrm{C}(14)-\mathrm{O}(2)-\mathrm{C}(15)$ & $116.4(2)$ \\
\hline $\mathrm{C}(20)-\mathrm{N}(1)-\mathrm{C}(16)$ & $114.86(17)$ \\
\hline $\mathrm{C}(20)-\mathrm{N}(1)-\mathrm{Mo}$ & $124.02(14)$ \\
\hline $\mathrm{C}(16)-\mathrm{N}(1)-\mathrm{Mo}$ & $120.64(13)$ \\
\hline $\mathrm{C}(18)-\mathrm{N}(2)-\mathrm{C}(22)$ & $122.0(3)$ \\
\hline $\mathrm{C}(18)-\mathrm{N}(2)-\mathrm{C}(21)$ & $120.4(3)$ \\
\hline $\mathrm{C}(22)-\mathrm{N}(2)-\mathrm{C}(21)$ & $117.5(3)$ \\
\hline $\mathrm{C}(25)-\mathrm{N}(3)-\mathrm{N}(4)$ & $106.53(18)$ \\
\hline $\mathrm{C}(25)-\mathrm{N}(3)-\mathrm{Mo}$ & $131.88(17)$ \\
\hline $\mathrm{N}(4)-\mathrm{N}(3)-\mathrm{Mo}$ & $121.25(13)$ \\
\hline $\mathrm{C}(23)-\mathrm{N}(4)-\mathrm{N}(3)$ & $109.3(2)$ \\
\hline $\mathrm{C}(23)-\mathrm{N}(4)-\mathrm{B}(1)$ & $128.9(2)$ \\
\hline $\mathrm{N}(3)-\mathrm{N}(4)-\mathrm{B}(1)$ & $121.22(17)$ \\
\hline $\mathrm{C}(28)-\mathrm{N}(5)-\mathrm{N}(6)$ & $106.06(17)$ \\
\hline $\mathrm{C}(28)-\mathrm{N}(5)-\mathrm{Mo}$ & $132.28(16)$ \\
\hline $\mathrm{N}(6)-\mathrm{N}(5)-\mathrm{Mo}$ & $121.64(12)$ \\
\hline $\mathrm{C}(26)-\mathrm{N}(6)-\mathrm{N}(5)$ & $109.61(18)$ \\
\hline $\mathrm{C}(26)-\mathrm{N}(6)-\mathrm{B}(1)$ & $130.0(2)$ \\
\hline $\mathrm{N}(5)-\mathrm{N}(6)-\mathrm{B}(1)$ & $120.36(16)$ \\
\hline $\mathrm{C}(31)-\mathrm{N}(7)-\mathrm{N}(8)$ & $105.63(17)$ \\
\hline $\mathrm{C}(31)-\mathrm{N}(7)-\mathrm{Mo}$ & $135.79(15)$ \\
\hline $\mathrm{N}(8)-\mathrm{N}(7)-\mathrm{Mo}$ & $118.36(13)$ \\
\hline $\mathrm{C}(29)-\mathrm{N}(8)-\mathrm{N}(7)$ & $109.65(19)$ \\
\hline $\mathrm{C}(29)-\mathrm{N}(8)-\mathrm{B}(1)$ & $128.1(2)$ \\
\hline $\mathrm{N}(7)-\mathrm{N}(8)-\mathrm{B}(1)$ & $122.16(18)$ \\
\hline $\mathrm{O}(3)-\mathrm{N}(9)-\mathrm{Mo}$ & $177.4(2)$ \\
\hline $\mathrm{C}(2)-\mathrm{C}(1)-\mathrm{C}(10)$ & $110.81(18)$ \\
\hline $\mathrm{C}(2)-\mathrm{C}(1)-\mathrm{C}(11)$ & $110.86(19)$ \\
\hline $\mathrm{C}(10)-\mathrm{C}(1)-\mathrm{C}(11)$ & $114.09(18)$ \\
\hline$C(3)-C(2)-C(1)$ & $121.41(18)$ \\
\hline
\end{tabular}




\begin{tabular}{|c|c|}
\hline $\mathrm{C}(3)-\mathrm{C}(2)-\mathrm{Mo}$ & $72.67(11)$ \\
\hline $\mathrm{C}(1)-\mathrm{C}(2)-\mathrm{Mo}$ & $125.53(15)$ \\
\hline$C(2)-C(3)-C(4)$ & $120.73(19)$ \\
\hline $\mathrm{C}(2)-\mathrm{C}(3)-\mathrm{Mo}$ & $70.12(11)$ \\
\hline $\mathrm{C}(4)-\mathrm{C}(3)-\mathrm{Mo}$ & $122.49(15)$ \\
\hline $\mathrm{C}(9)-\mathrm{C}(4)-\mathrm{C}(5)$ & $118.6(2)$ \\
\hline $\mathrm{C}(9)-\mathrm{C}(4)-\mathrm{C}(3)$ & $120.3(2)$ \\
\hline$C(5)-C(4)-C(3)$ & $121.0(2)$ \\
\hline$C(6)-C(5)-C(4)$ & $121.6(3)$ \\
\hline$C(7)-C(6)-C(5)$ & $119.3(3)$ \\
\hline $\mathrm{C}(6)-\mathrm{C}(7)-\mathrm{C}(8)$ & $119.8(3)$ \\
\hline $\mathrm{C}(7)-\mathrm{C}(8)-\mathrm{C}(9)$ & $121.2(3)$ \\
\hline $\mathrm{C}(4)-\mathrm{C}(9)-\mathrm{C}(8)$ & $119.3(3)$ \\
\hline$C(4)-C(9)-C(10)$ & $120.08(19)$ \\
\hline $\mathrm{C}(8)-\mathrm{C}(9)-\mathrm{C}(10)$ & $120.6(3)$ \\
\hline$C(9)-C(10)-C(1)$ & $115.5(2)$ \\
\hline$C(12)-C(11)-C(13)$ & $110.0(2)$ \\
\hline$C(12)-C(11)-C(14)$ & $108.6(3)$ \\
\hline$C(13)-C(11)-C(14)$ & $106.8(2)$ \\
\hline $\mathrm{C}(12)-\mathrm{C}(11)-\mathrm{C}(1)$ & $113.0(2)$ \\
\hline $\mathrm{C}(13)-\mathrm{C}(11)-\mathrm{C}(1)$ & $111.3(2)$ \\
\hline $\mathrm{C}(14)-\mathrm{C}(11)-\mathrm{C}(1)$ & $106.93(18)$ \\
\hline $\mathrm{O}(1)-\mathrm{C}(14)-\mathrm{O}(2)$ & $122.5(3)$ \\
\hline $\mathrm{O}(1)-\mathrm{C}(14)-\mathrm{C}(11)$ & $125.5(3)$ \\
\hline $\mathrm{O}(2)-\mathrm{C}(14)-\mathrm{C}(11)$ & $111.9(2)$ \\
\hline $\mathrm{C}(17)-\mathrm{C}(16)-\mathrm{N}(1)$ & $124.62(19)$ \\
\hline$C(16)-C(17)-C(18)$ & $120.9(2)$ \\
\hline $\mathrm{N}(2)-\mathrm{C}(18)-\mathrm{C}(17)$ & $122.2(2)$ \\
\hline $\mathrm{N}(2)-\mathrm{C}(18)-\mathrm{C}(19)$ & $123.1(2)$ \\
\hline$C(17)-C(18)-C(19)$ & $114.67(19)$ \\
\hline $\mathrm{C}(20)-\mathrm{C}(19)-\mathrm{C}(18)$ & $120.6(2)$ \\
\hline $\mathrm{N}(1)-\mathrm{C}(20)-\mathrm{C}(19)$ & $124.4(2)$ \\
\hline $\mathrm{C}(24)-\mathrm{C}(23)-\mathrm{N}(4)$ & $108.8(2)$ \\
\hline$C(23)-C(24)-C(25)$ & $105.3(2)$ \\
\hline $\mathrm{N}(3)-\mathrm{C}(25)-\mathrm{C}(24)$ & $110.0(2)$ \\
\hline $\mathrm{N}(6)-\mathrm{C}(26)-\mathrm{C}(27)$ & $108.4(2)$ \\
\hline
\end{tabular}




$\begin{array}{ll}\mathrm{C}(26)-\mathrm{C}(27)-\mathrm{C}(28) & 105.0(2) \\ \mathrm{N}(5)-\mathrm{C}(28)-\mathrm{C}(27) & 110.9(2) \\ \mathrm{N}(8)-\mathrm{C}(29)-\mathrm{C}(30) & 109.1(2) \\ \mathrm{C}(29)-\mathrm{C}(30)-\mathrm{C}(31) & 104.7(2) \\ \mathrm{N}(7)-\mathrm{C}(31)-\mathrm{C}(30) & 110.9(2) \\ \mathrm{N}(6)-\mathrm{B}(1)-\mathrm{N}(4) & 107.46(19) \\ \mathrm{N}(6)-\mathrm{B}(1)-\mathrm{N}(8) & 108.43(18) \\ \mathrm{N}(4)-\mathrm{B}(1)-\mathrm{N}(8) & 109.33(19)\end{array}$


Compound 30

Table 4. Anisotropic thermal displacement parameters $\left(\AA^{2} \times 10^{3}\right)$ for $\mathrm{C}_{31} \mathrm{H}_{38} \mathrm{BN}_{9} \mathrm{O}_{3} \mathrm{Mo}$.

The anisotropic displacement factor exponent takes the form: $-2 p^{2}\left[h^{2} a^{* 2} U^{11}+\ldots+2\right.$ $\mathrm{h} \mathrm{k} \mathrm{a*} \mathrm{b}^{*} \mathrm{U}^{12}$ ]

\begin{tabular}{|c|c|c|c|c|c|c|}
\hline & $\mathrm{U}^{11}$ & $\mathrm{U}^{22}$ & $\mathrm{U}^{33}$ & $\mathrm{U}^{23}$ & $\mathrm{U}^{13}$ & $\mathrm{U}^{12}$ \\
\hline Mo & $33(1)$ & $35(1)$ & $35(1)$ & $0(1)$ & $16(1)$ & 2(1) \\
\hline $\mathrm{O}(1)$ & $86(2)$ & $94(2)$ & $99(2)$ & $-34(2)$ & $35(1)$ & $-26(1)$ \\
\hline $\mathrm{O}(2)$ & $66(1)$ & $70(1)$ & $60(1)$ & $-4(1)$ & $33(1)$ & 1(1) \\
\hline $\mathrm{O}(3)$ & $91(2)$ & $90(2)$ & $52(1)$ & $-33(1)$ & $20(1)$ & $-8(1)$ \\
\hline $\mathrm{N}(1)$ & $37(1)$ & $41(1)$ & $38(1)$ & 1(1) & $18(1)$ & $4(1)$ \\
\hline $\mathrm{N}(2)$ & $52(1)$ & $64(1)$ & $87(2)$ & $-3(1)$ & $39(1)$ & $15(1)$ \\
\hline $\mathrm{N}(3)$ & $39(1)$ & $37(1)$ & $59(1)$ & 1(1) & $25(1)$ & $3(1)$ \\
\hline $\mathrm{N}(4)$ & $40(1)$ & $40(1)$ & $66(1)$ & $7(1)$ & $20(1)$ & $8(1)$ \\
\hline $\mathrm{N}(5)$ & $41(1)$ & $41(1)$ & $46(1)$ & $2(1)$ & $20(1)$ & $-2(1)$ \\
\hline $\mathrm{N}(6)$ & $47(1)$ & $38(1)$ & $46(1)$ & $4(1)$ & $20(1)$ & $-1(1)$ \\
\hline $\mathrm{N}(7)$ & $40(1)$ & $38(1)$ & $40(1)$ & 1(1) & 11(1) & 1(1) \\
\hline $\mathrm{N}(8)$ & $40(1)$ & $47(1)$ & $42(1)$ & $4(1)$ & $7(1)$ & $-1(1)$ \\
\hline $\mathrm{N}(9)$ & $47(1)$ & $48(1)$ & $41(1)$ & $-9(1)$ & $22(1)$ & 1(1) \\
\hline $\mathrm{C}(1)$ & $45(1)$ & $42(1)$ & $46(1)$ & $7(1)$ & $22(1)$ & $5(1)$ \\
\hline$C(2)$ & $44(1)$ & $37(1)$ & $39(1)$ & $2(1)$ & $18(1)$ & 1(1) \\
\hline$C(3)$ & $38(1)$ & $42(1)$ & $46(1)$ & $5(1)$ & $19(1)$ & $0(1)$ \\
\hline $\mathrm{C}(4)$ & $52(1)$ & $40(1)$ & $61(1)$ & $0(1)$ & $35(1)$ & $-6(1)$ \\
\hline$C(5)$ & $54(1)$ & $47(1)$ & $82(2)$ & $-3(1)$ & 41(1) & $-7(1)$ \\
\hline$C(6)$ & $73(2)$ & $64(2)$ & $98(2)$ & $-14(2)$ & $62(2)$ & $-17(1)$ \\
\hline$C(7)$ & $114(3)$ & $68(2)$ & $88(2)$ & $-17(2)$ & $79(2)$ & $-22(2)$ \\
\hline $\mathrm{C}(8)$ & $95(2)$ & $61(2)$ & $58(1)$ & $-1(1)$ & $45(2)$ & $-7(2)$ \\
\hline $\mathrm{C}(9)$ & $67(1)$ & $45(1)$ & $51(1)$ & $-2(1)$ & $34(1)$ & $-3(1)$ \\
\hline$C(10)$ & $63(1)$ & $60(1)$ & $40(1)$ & $6(1)$ & $18(1)$ & $3(1)$ \\
\hline$C(11)$ & $61(1)$ & $43(1)$ & $66(1)$ & $10(1)$ & $35(1)$ & $6(1)$ \\
\hline$C(12)$ & $82(2)$ & $53(2)$ & $131(3)$ & $5(2)$ & $71(2)$ & $-7(1)$ \\
\hline$C(13)$ & $99(2)$ & $63(2)$ & $71(2)$ & $26(1)$ & $45(2)$ & $28(2)$ \\
\hline$C(14)$ & $62(1)$ & $43(1)$ & $64(1)$ & $2(1)$ & $26(1)$ & $4(1)$ \\
\hline$C(15)$ & $100(2)$ & $72(2)$ & $68(2)$ & $-1(1)$ & $48(2)$ & $9(2)$ \\
\hline$C(16)$ & $42(1)$ & $54(1)$ & $39(1)$ & 1(1) & $17(1)$ & $7(1)$ \\
\hline
\end{tabular}




$\begin{array}{lllllll}\mathrm{C}(17) & 40(1) & 55(1) & 49(1) & 2(1) & 16(1) & 9(1) \\ \mathrm{C}(18) & 44(1) & 37(1) & 64(1) & 2(1) & 30(1) & 7(1) \\ \mathrm{C}(19) & 55(1) & 62(1) & 50(1) & -8(1) & 29(1) & 5(1) \\ \mathrm{C}(20) & 46(1) & 60(1) & 41(1) & -5(1) & 19(1) & 3(1) \\ \mathrm{C}(21) & 49(2) & 92(2) & 108(3) & 10(2) & 30(2) & 27(2) \\ \mathrm{C}(22) & 85(2) & 100(3) & 107(3) & -4(2) & 67(2) & 23(2) \\ \mathrm{C}(23) & 44(1) & 40(1) & 99(2) & 6(1) & 24(1) & 10(1) \\ \mathrm{C}(24) & 52(1) & 42(1) & 111(2) & -6(1) & 46(2) & 5(1) \\ \mathrm{C}(25) & 51(1) & 40(1) & 78(2) & -6(1) & 40(1) & -3(1) \\ \mathrm{C}(26) & 72(2) & 40(1) & 56(1) & 6(1) & 33(1) & -5(1) \\ \mathrm{C}(27) & 67(2) & 53(1) & 68(2) & 0(1) & 37(1) & -16(1) \\ \mathrm{C}(28) & 46(1) & 51(1) & 58(1) & -2(1) & 23(1) & -10(1) \\ \mathrm{C}(29) & 49(1) & 63(1) & 44(1) & 3(1) & 3(1) & -7(1) \\ \mathrm{C}(30) & 65(2) & 62(1) & 46(1) & -10(1) & 8(1) & -13(1) \\ \mathrm{C}(31) & 56(1) & 42(1) & 45(1) & -5(1) & 11(1) & -2(1) \\ \mathrm{B}(1) & 44(1) & 42(1) & 52(1) & 9(1) & 12(1) & 5(1)\end{array}$


Compound 30

Table 5. Fractional coordinates ( $\times 10^{4}$ ) and isotropic thermal displacement parameters $\left(\AA^{2} \times 10^{3}\right)$ for hydrogen atoms of $\mathrm{C}_{31} \mathrm{H}_{38} \mathrm{BN}_{9} \mathrm{O}_{3} \mathrm{Mo}$.

\begin{tabular}{|c|c|c|c|c|}
\hline & $\mathrm{x}$ & $\mathrm{y}$ & $\mathrm{z}$ & $\mathrm{U}(\mathrm{eq})$ \\
\hline $\mathrm{H}(1)$ & 6208 & 1240 & 6848 & 52 \\
\hline $\mathrm{H}(5)$ & 9806 & -370 & 7349 & 68 \\
\hline $\mathrm{H}(6)$ & 10084 & -570 & 6069 & 84 \\
\hline $\mathrm{H}(7)$ & 8959 & -58 & 4755 & 94 \\
\hline $\mathrm{H}(8)$ & 7554 & 586 & 4737 & 80 \\
\hline $\mathrm{H}(10 \mathrm{~A})$ & 6418 & 269 & 5868 & 66 \\
\hline $\mathrm{H}(10 \mathrm{~B})$ & 6618 & 1271 & 5517 & 66 \\
\hline $\mathrm{H}(12 \mathrm{~A})$ & 8158 & 3319 & 6917 & 120 \\
\hline $\mathrm{H}(12 \mathrm{~B})$ & 8123 & 2370 & 6373 & 120 \\
\hline $\mathrm{H}(12 \mathrm{C})$ & 8525 & 2334 & 7405 & 120 \\
\hline $\mathrm{H}(13 \mathrm{~A})$ & 6431 & 3651 & 6252 & 111 \\
\hline $\mathrm{H}(13 \mathrm{~B})$ & 5716 & 2786 & 6119 & 111 \\
\hline $\mathrm{H}(13 \mathrm{C})$ & 6412 & 2807 & 5599 & 111 \\
\hline $\mathrm{H}(15 \mathrm{~A})$ & 6513 & 2426 & 9084 & 113 \\
\hline $\mathrm{H}(15 \mathrm{~B})$ & 5440 & 2671 & 8569 & 113 \\
\hline $\mathrm{H}(15 \mathrm{C})$ & 6215 & 3482 & 8730 & 113 \\
\hline $\mathrm{H}(16)$ & 5286 & -216 & 6820 & 53 \\
\hline $\mathrm{H}(17)$ & 3865 & 245 & 6830 & 58 \\
\hline $\mathrm{H}(19)$ & 5071 & 666 & 9419 & 63 \\
\hline $\mathrm{H}(20)$ & 6466 & 187 & 9331 & 57 \\
\hline $\mathrm{H}(21 \mathrm{~A})$ & 2664 & 1263 & 6981 & 124 \\
\hline $\mathrm{H}(21 \mathrm{~B})$ & 2040 & 904 & 7512 & 124 \\
\hline $\mathrm{H}(21 \mathrm{C})$ & 2519 & 147 & 7082 & 124 \\
\hline $\mathrm{H}(22 \mathrm{~A})$ & 3366 & 532 & 9329 & 133 \\
\hline $\mathrm{H}(22 \mathrm{~B})$ & 2695 & 1369 & 8803 & 133 \\
\hline $\mathrm{H}(22 \mathrm{C})$ & 3794 & 1543 & 9229 & 133 \\
\hline $\mathrm{H}(23)$ & 9964 & -3203 & 9398 & 74 \\
\hline $\mathrm{H}(24)$ & 9947 & -3167 & 7888 & 76 \\
\hline $\mathrm{H}(25)$ & 8729 & -1954 & 7087 & 62 \\
\hline
\end{tabular}




$\begin{array}{llccc}\mathrm{H}(26) & 7421 & -3333 & 10275 & 64 \\ \mathrm{H}(27) & 5728 & -3354 & 9335 & 71 \\ \mathrm{H}(28) & 5540 & -2143 & 8169 & 60 \\ \mathrm{H}(29) & 9568 & -822 & 11258 & 67 \\ \mathrm{H}(30) & 947 & 11080 & 73 \\ \mathrm{H}(31) & 9375 & 1213 & 9562 & 59 \\ \mathrm{H}(2) & 8260 & 1193(19) & 8185(16) & 43(6) \\ \mathrm{H}(3) & 7669(18) & 270(20) & 8150(20) & 65(8) \\ \mathrm{H}(1 \mathrm{~B}) & 8870(20) & -2417(18) & 10331(16) & 42(6)\end{array}$


${ }^{1} \mathrm{H}$ NMR ( $d^{6}$-Acetone) of Compound 30:

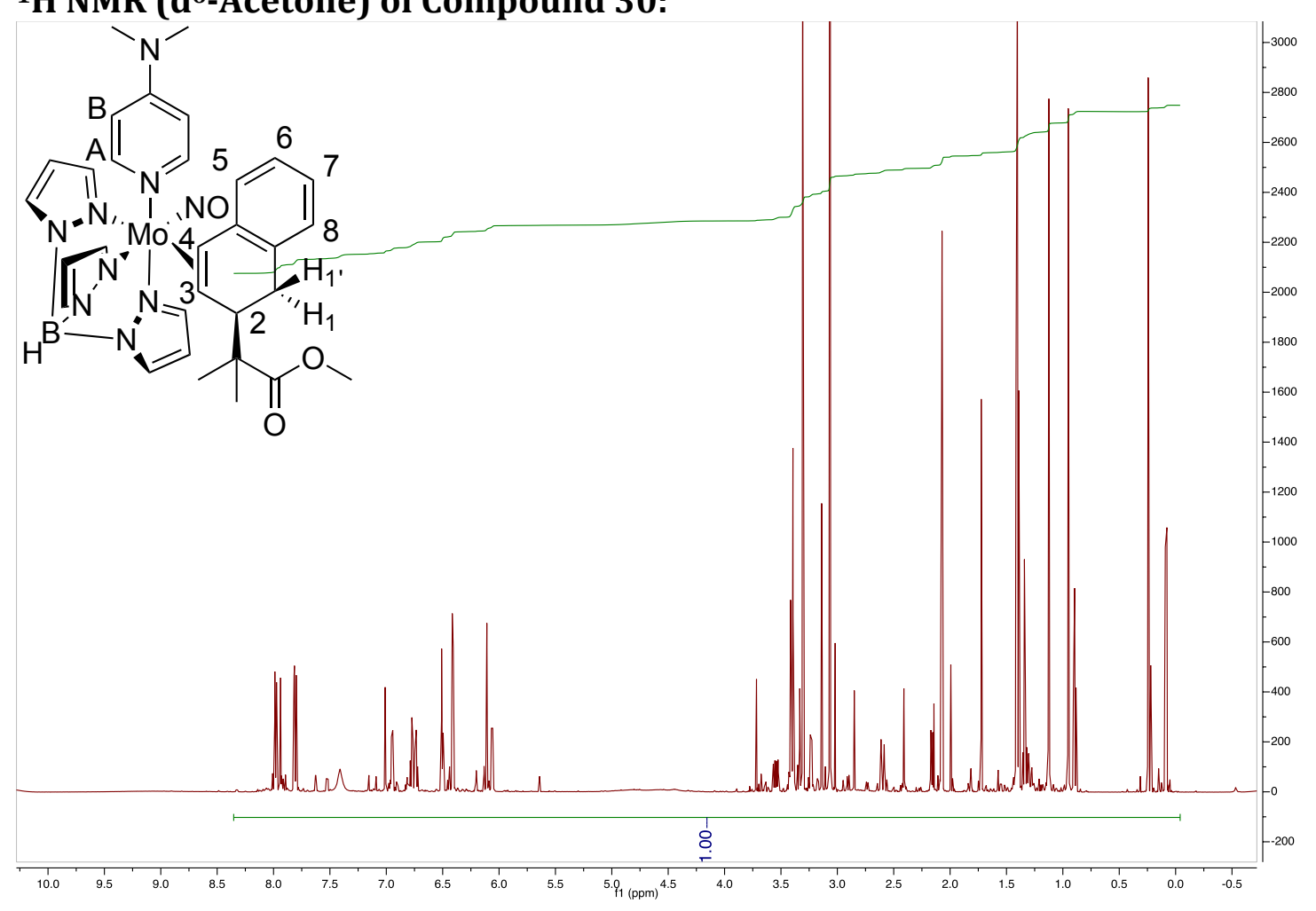

${ }^{13}$ C NMR (d6-Acetone) of Compound 30:

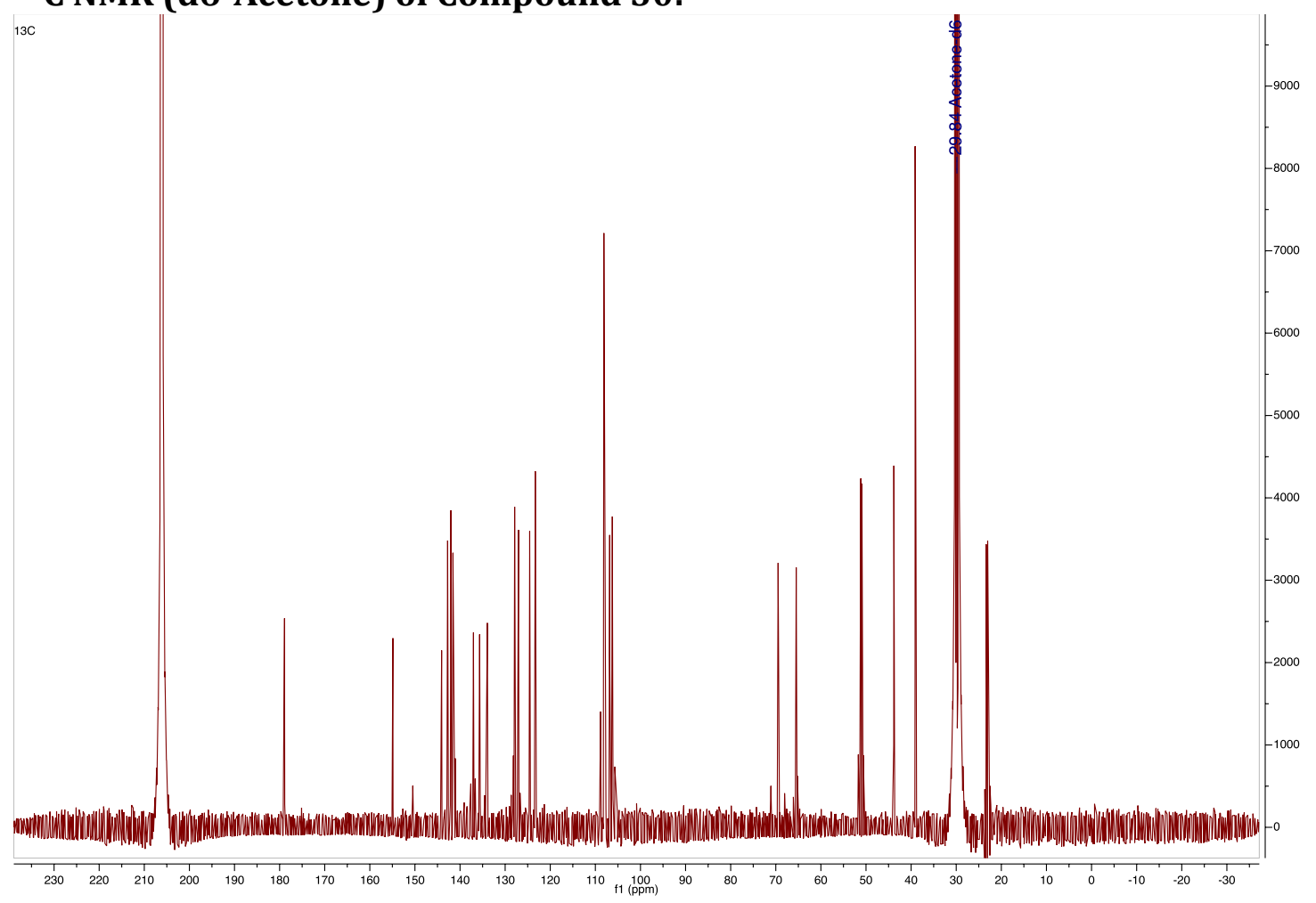


${ }^{1} \mathrm{H}$ NMR (d6-Acetone) of Compound 31:

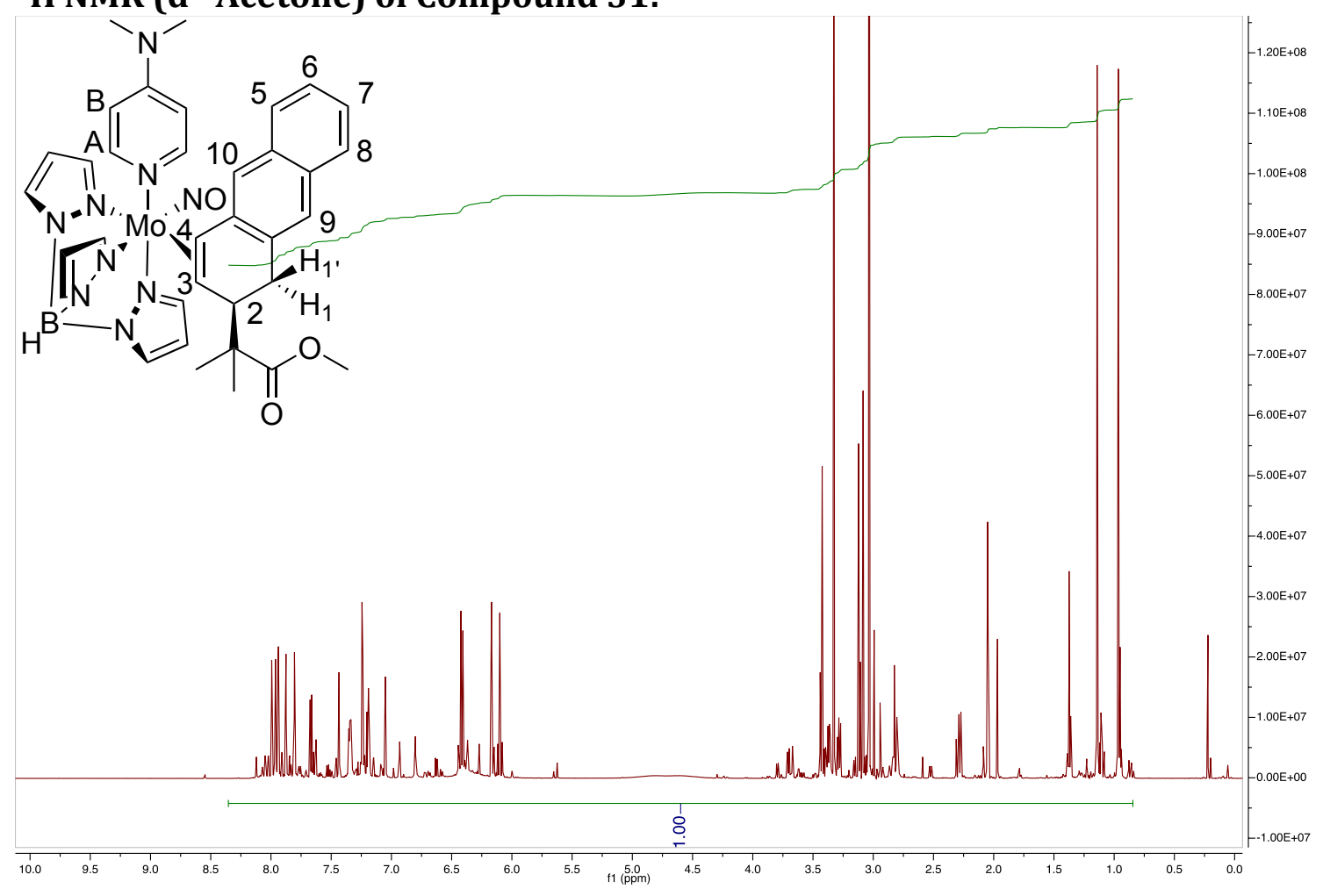

${ }^{13}$ C NMR (d6-Acetone) of Compound 31:

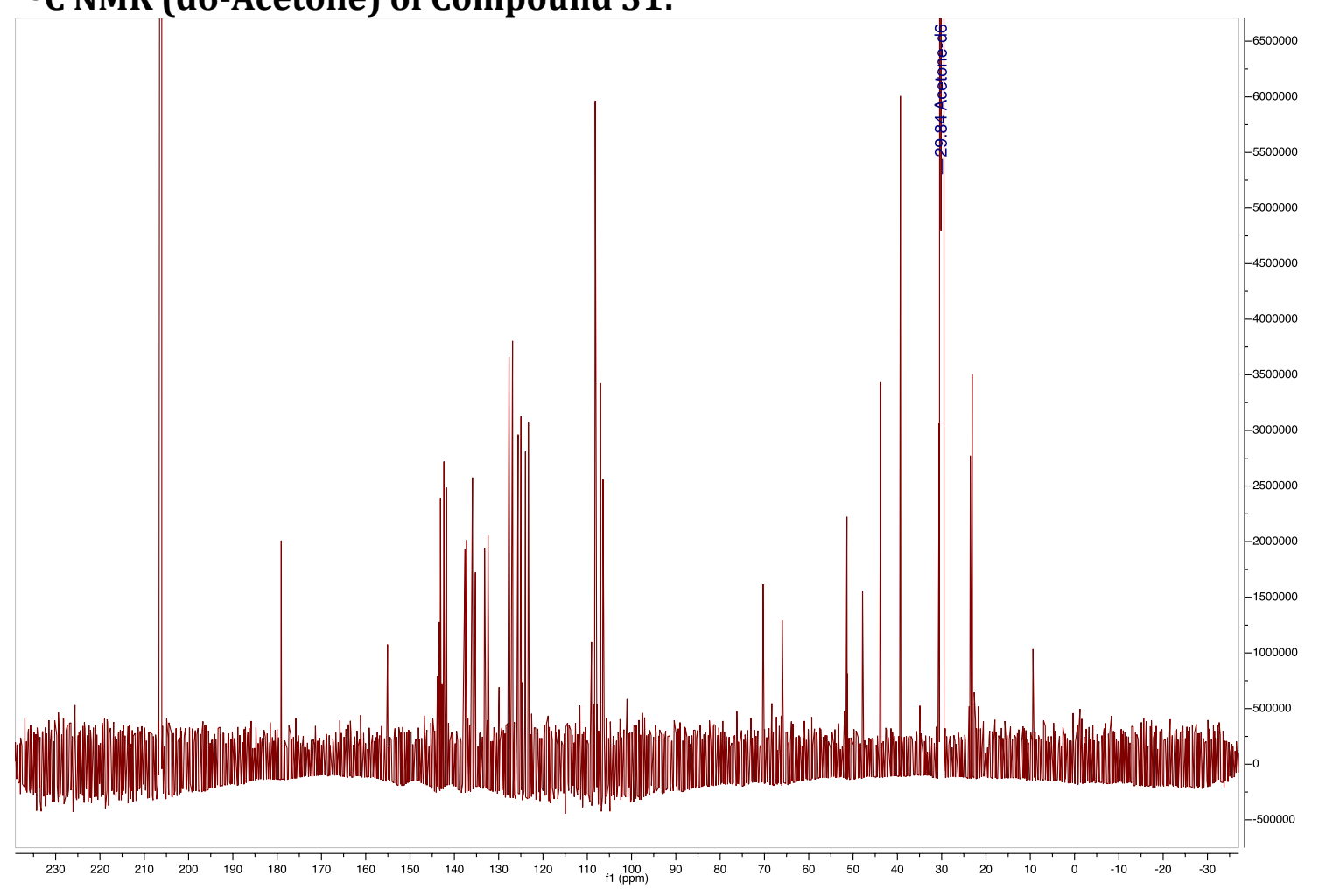


${ }^{1}$ H NMR ( $d^{6}$-Acetone) of Compound 32:

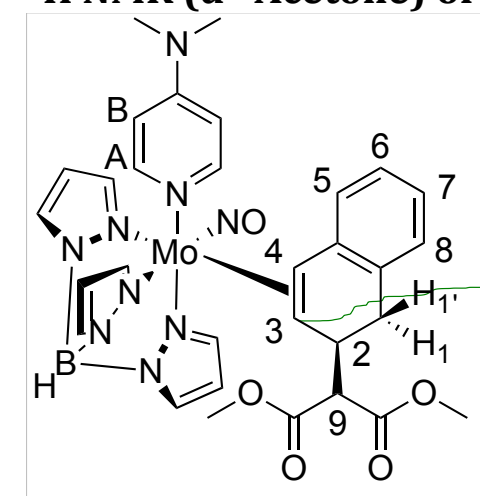

\section{Compound 32:}

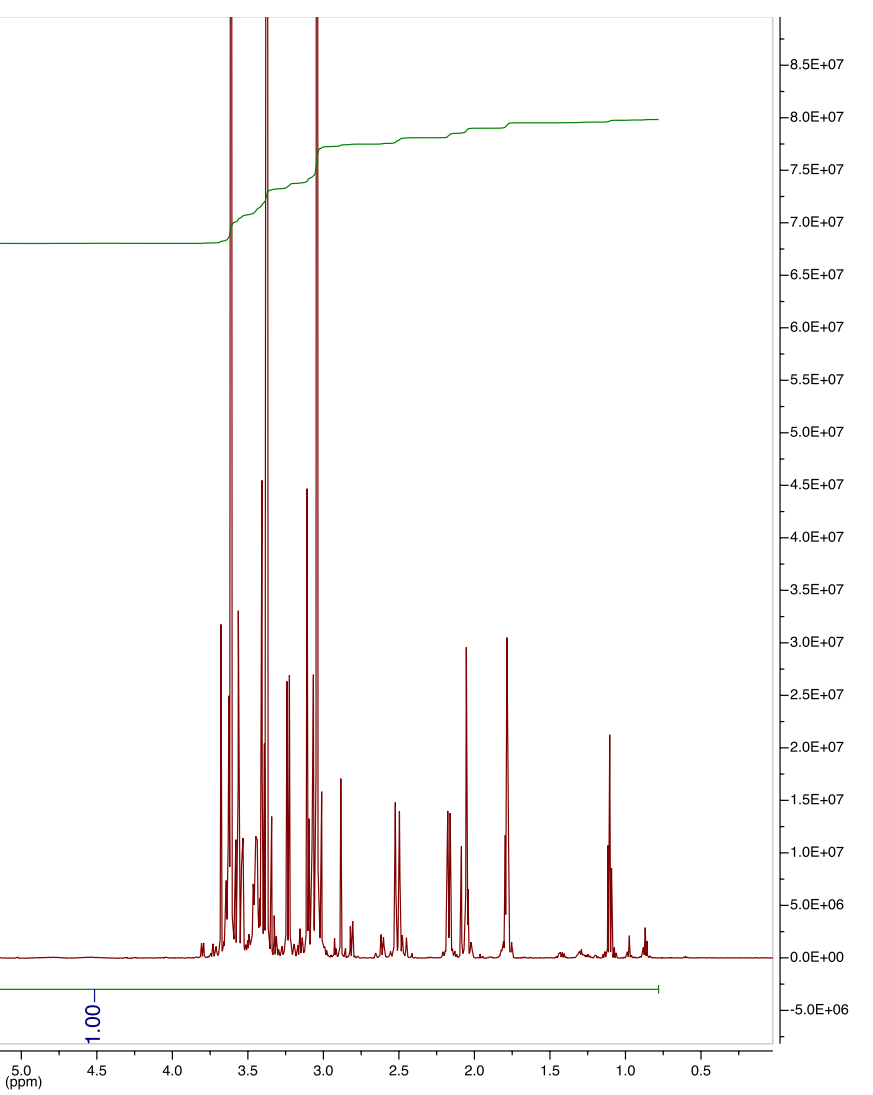

\section{${ }^{13}$ C-NMR (d6-Acetone) of Compound 32:}

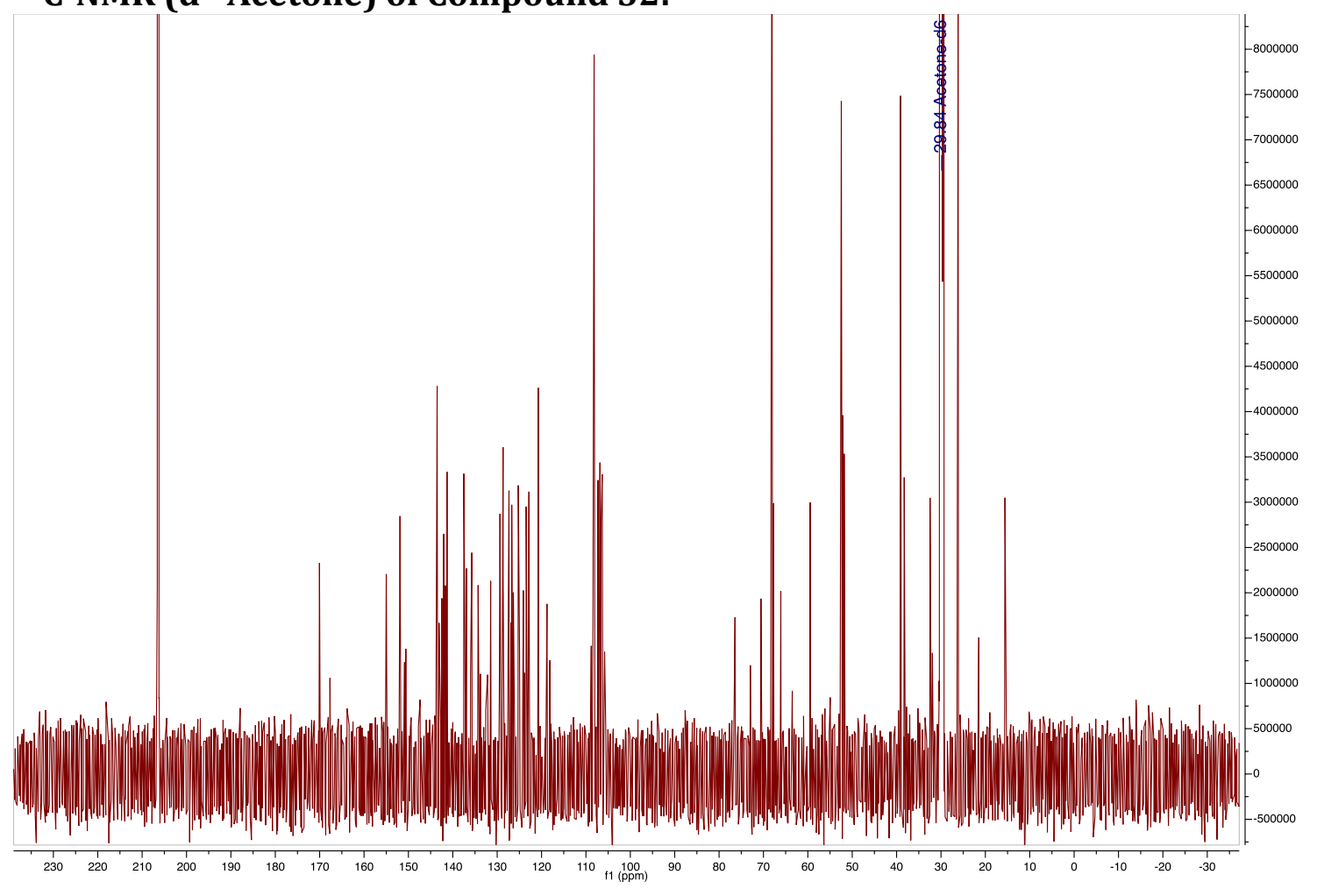


${ }^{1}$ H NMR (d6-Acetone) of Compound 33:

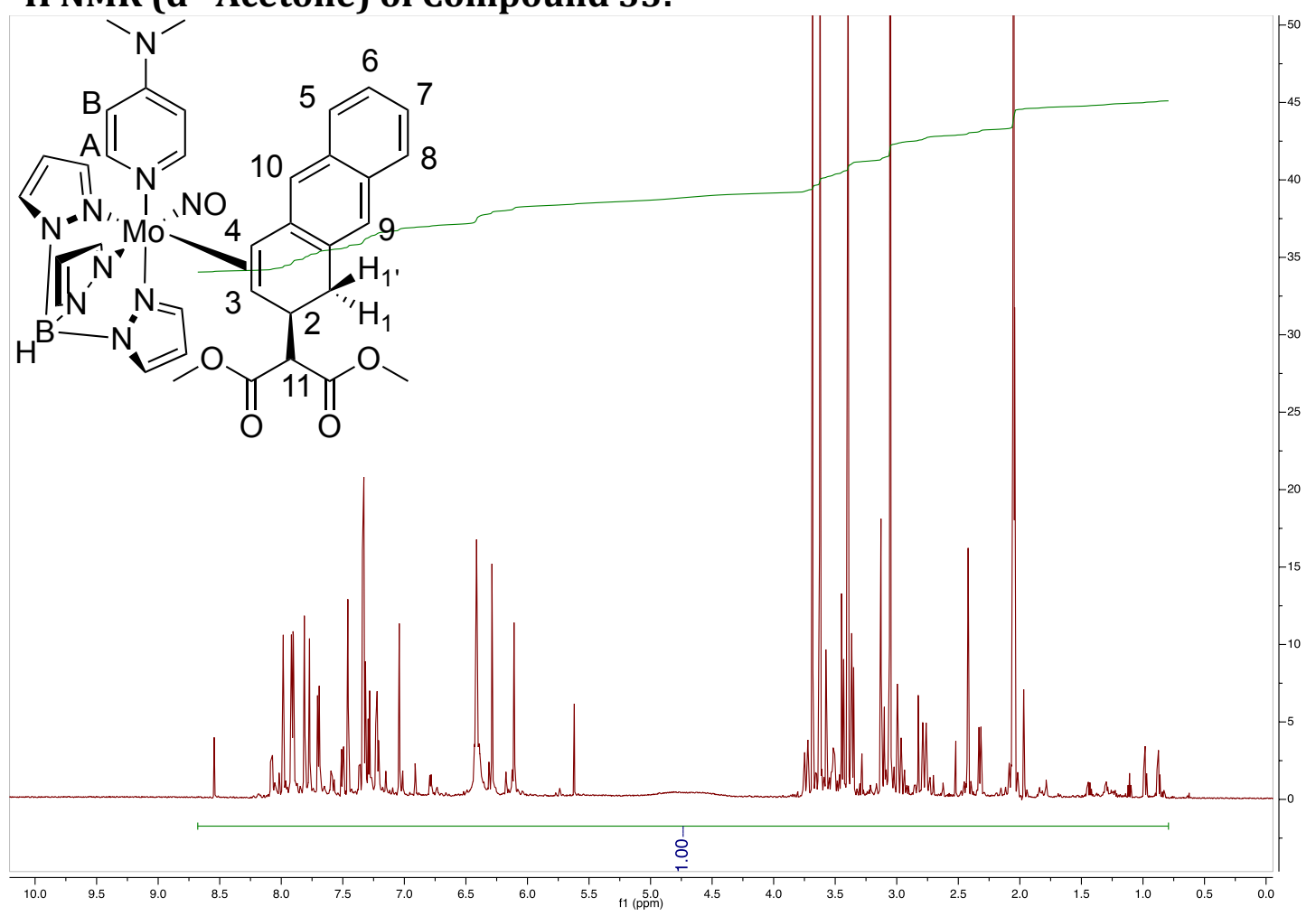

${ }^{13}$ C NMR (d ${ }^{6}$-Acetone) of Compound 33:

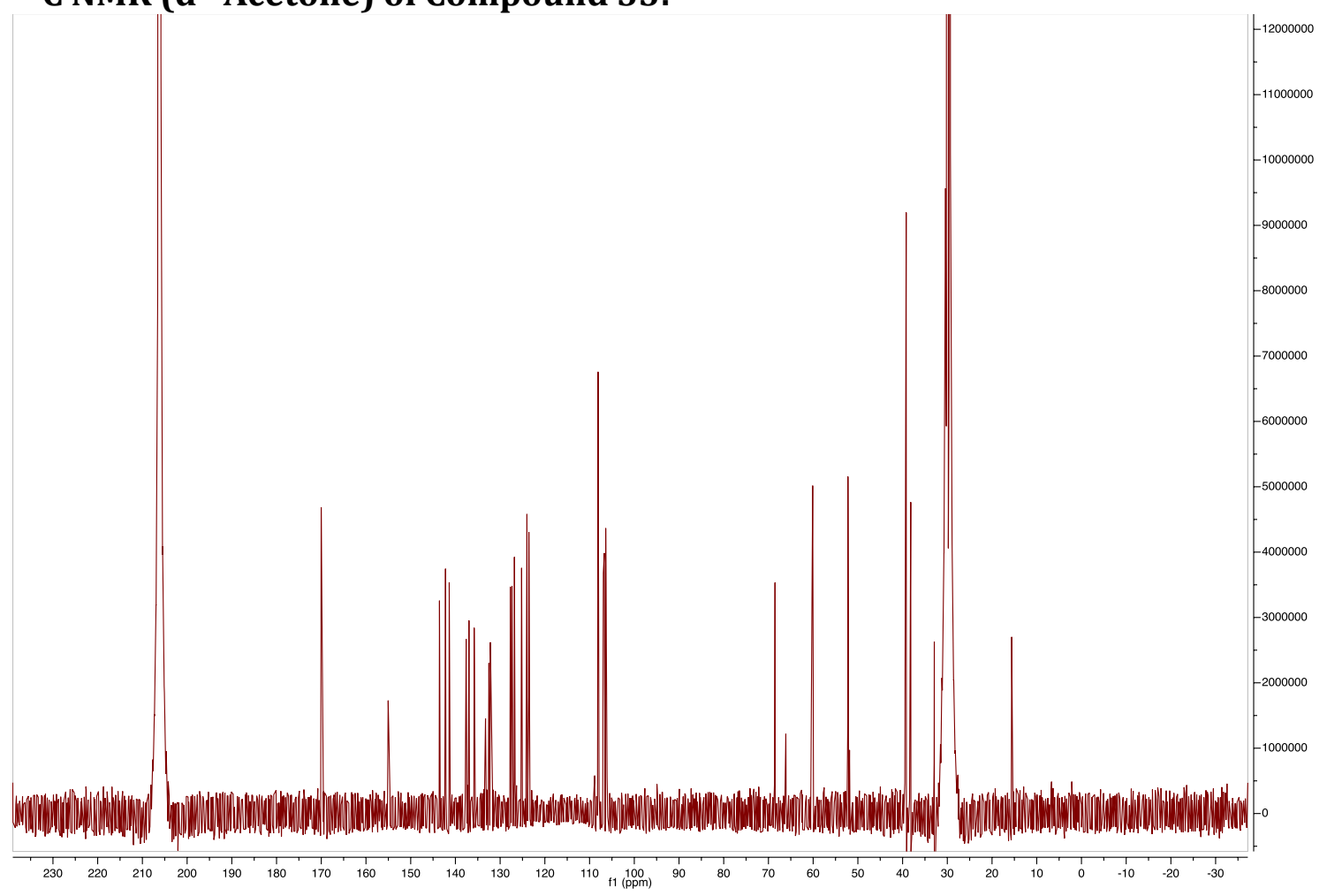


${ }^{1} \mathrm{H}$ NMR ( $\left.\mathrm{CDCl}_{3}\right)$ of Compound 34:

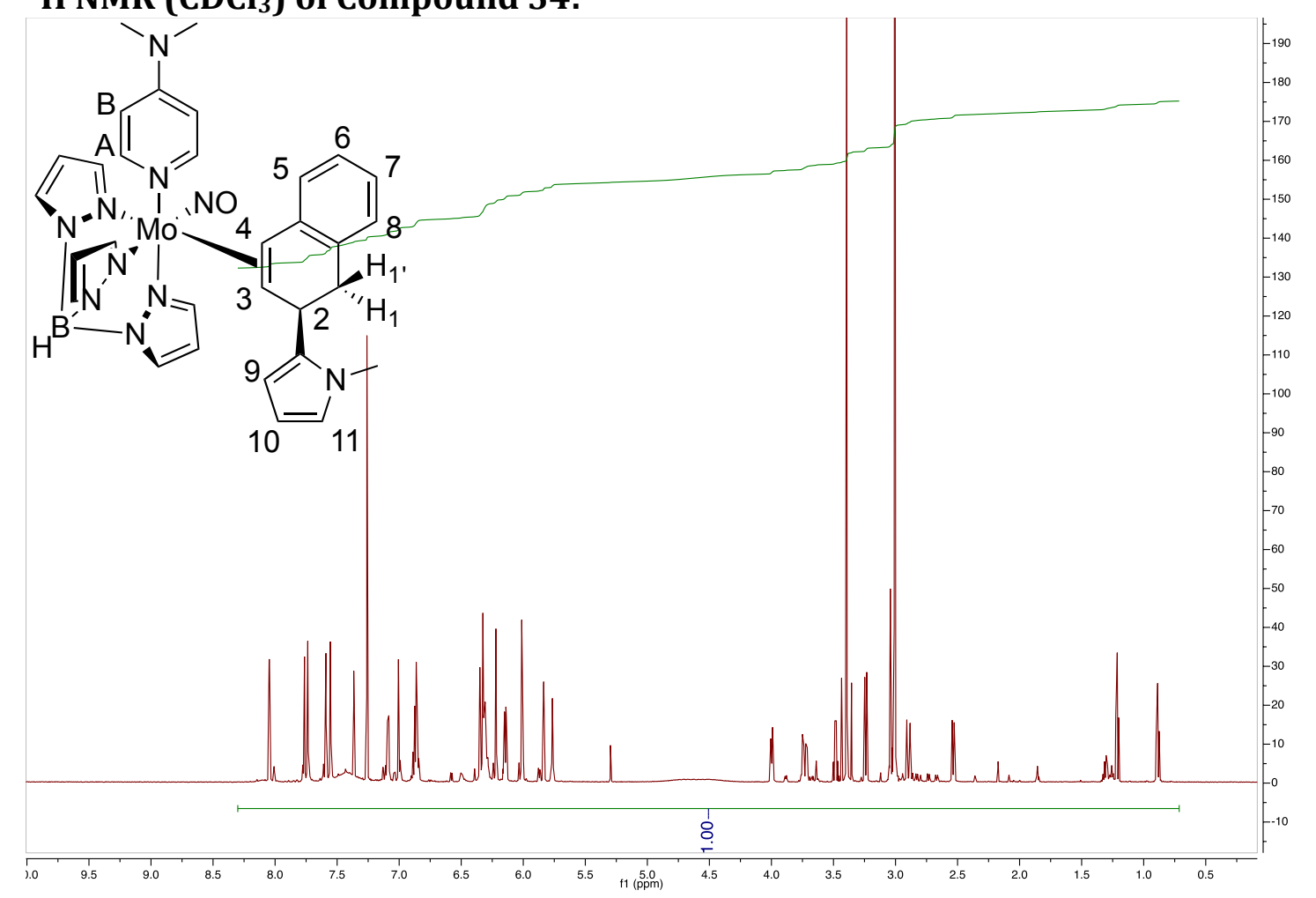

${ }^{13} \mathrm{C} \mathrm{NMR}\left(\mathrm{CDCl}_{3}\right)$ of Compound 34:

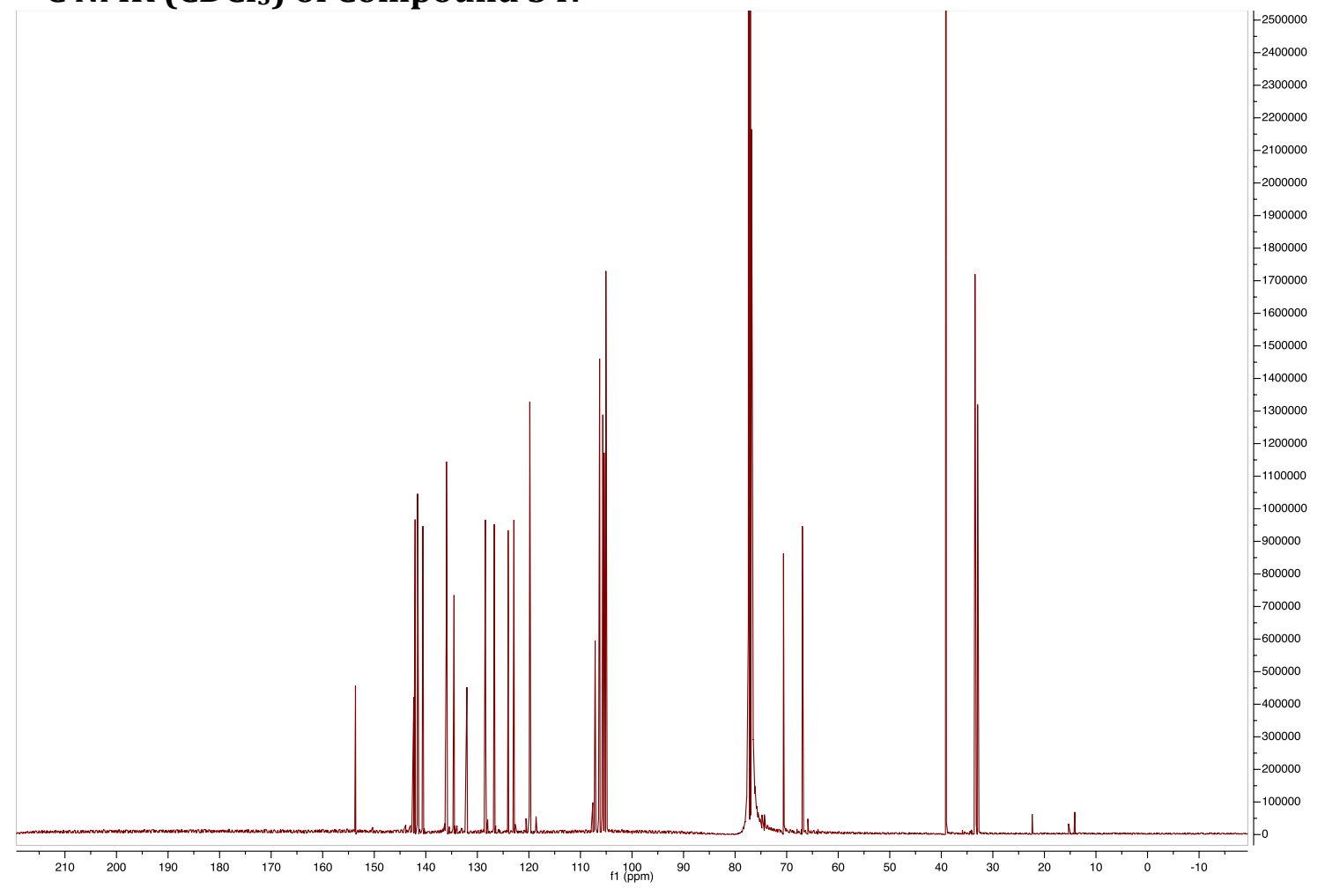


${ }^{1} H_{\text {NMR }}\left(d^{6}\right.$-Acetone) of Compound 35:

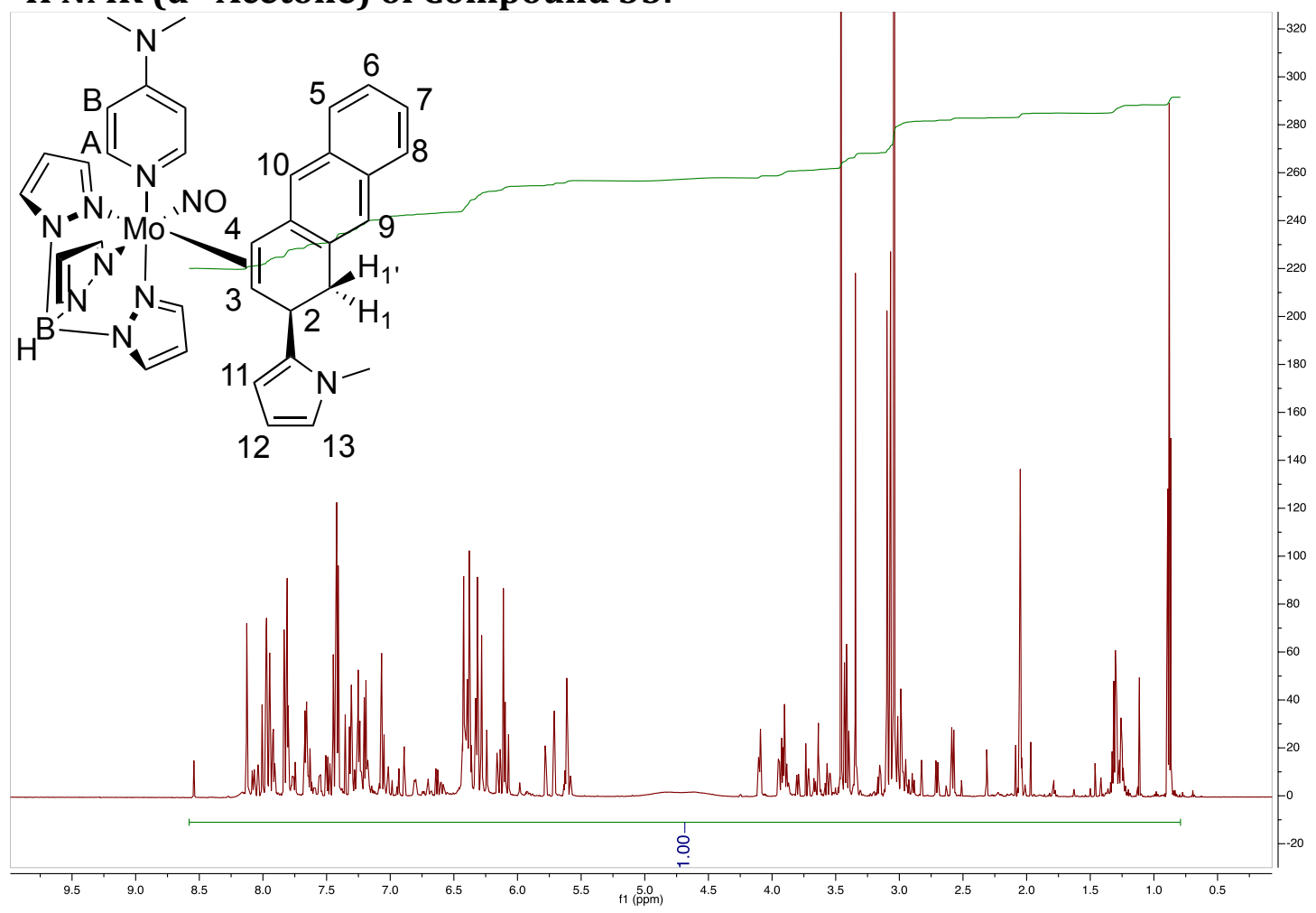

${ }^{13}$ C NMR (d ${ }^{6}$-Acetone) of Compound 35:

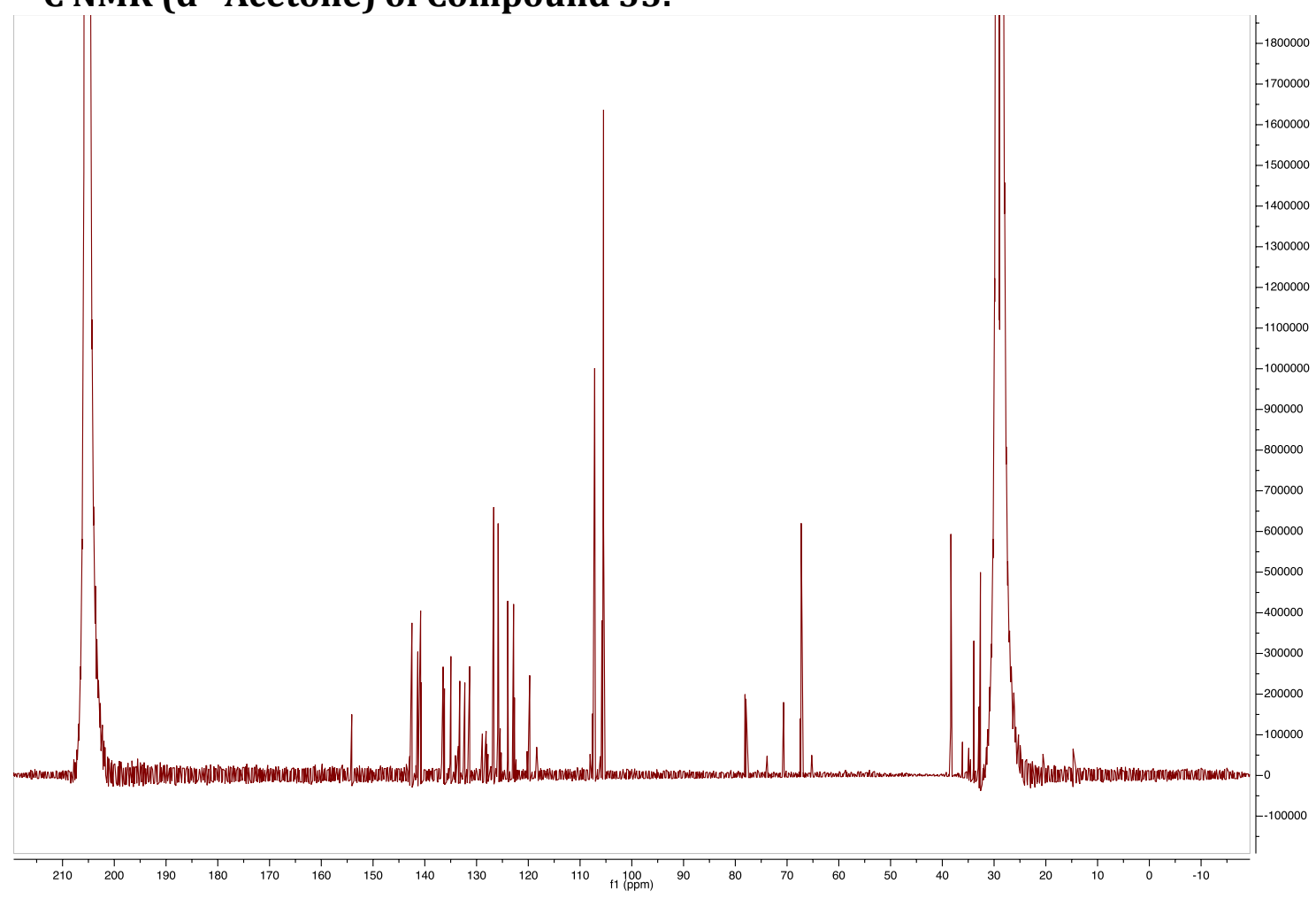




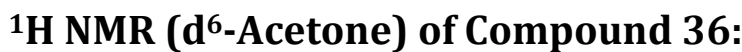

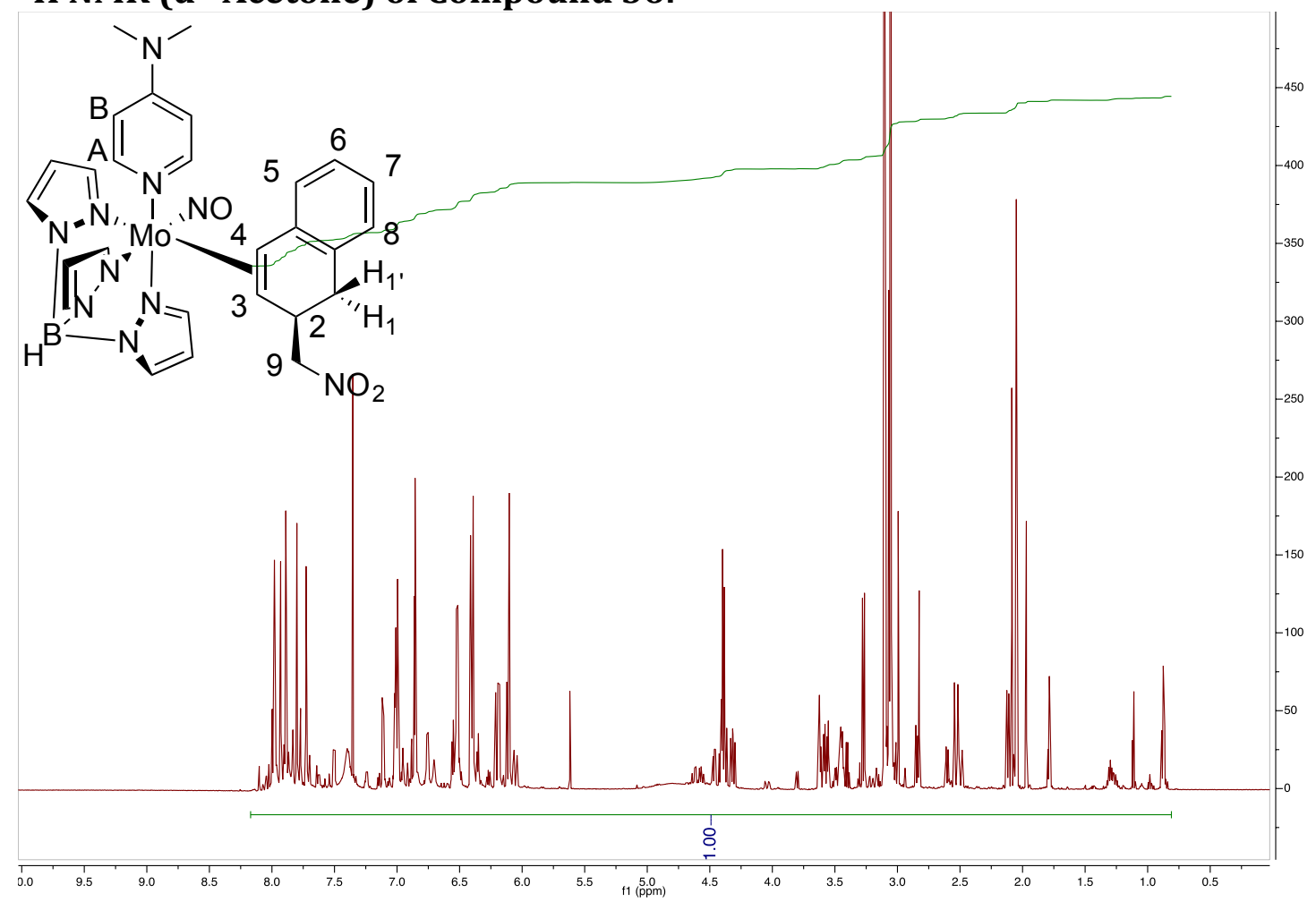

${ }^{13}$ C NMR (d6-Acetone) of Compound 36:

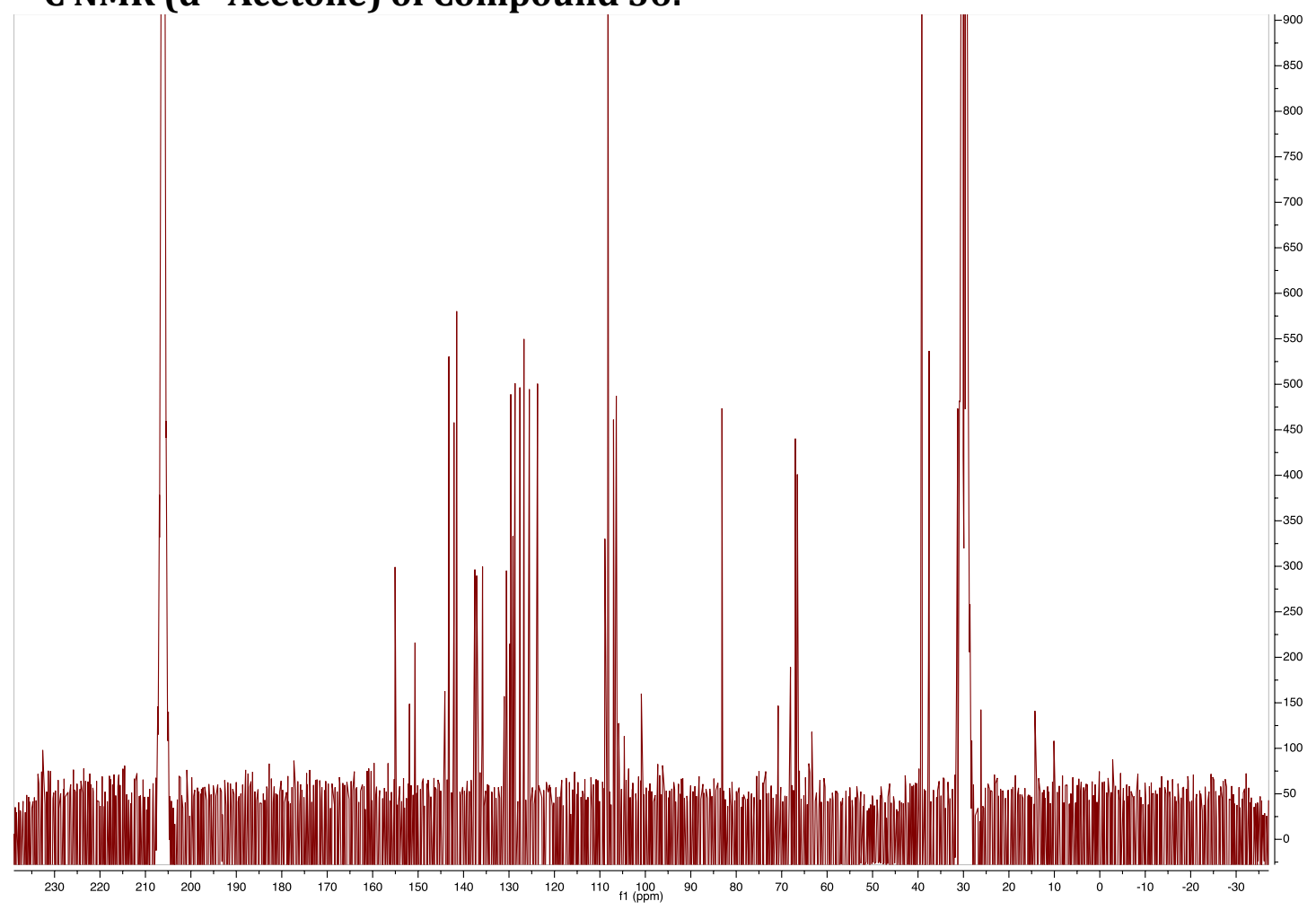




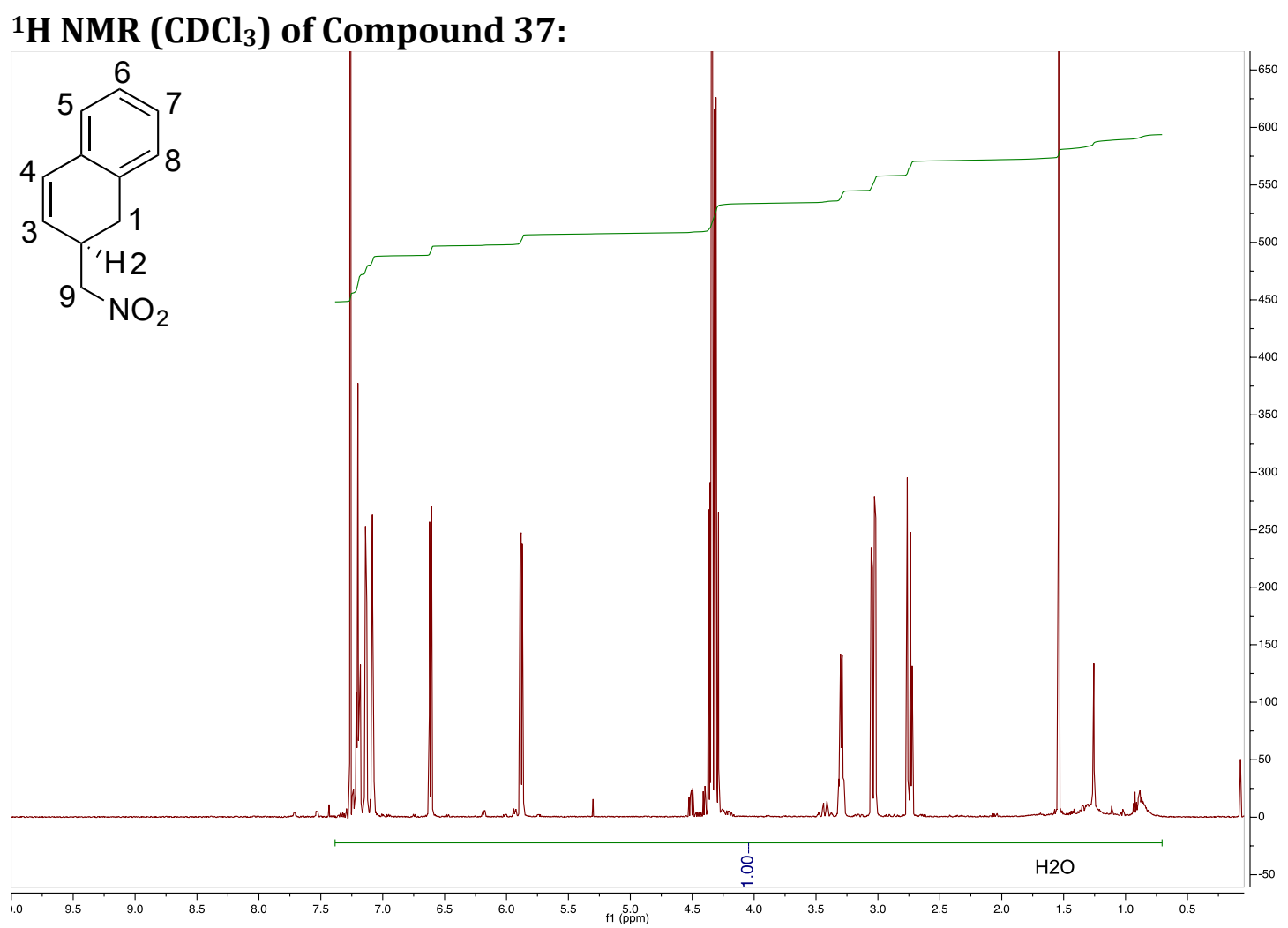

${ }^{13} \mathrm{C} \mathrm{NMR}\left(\mathrm{CDCl}_{3}\right)$ of Compound 37:
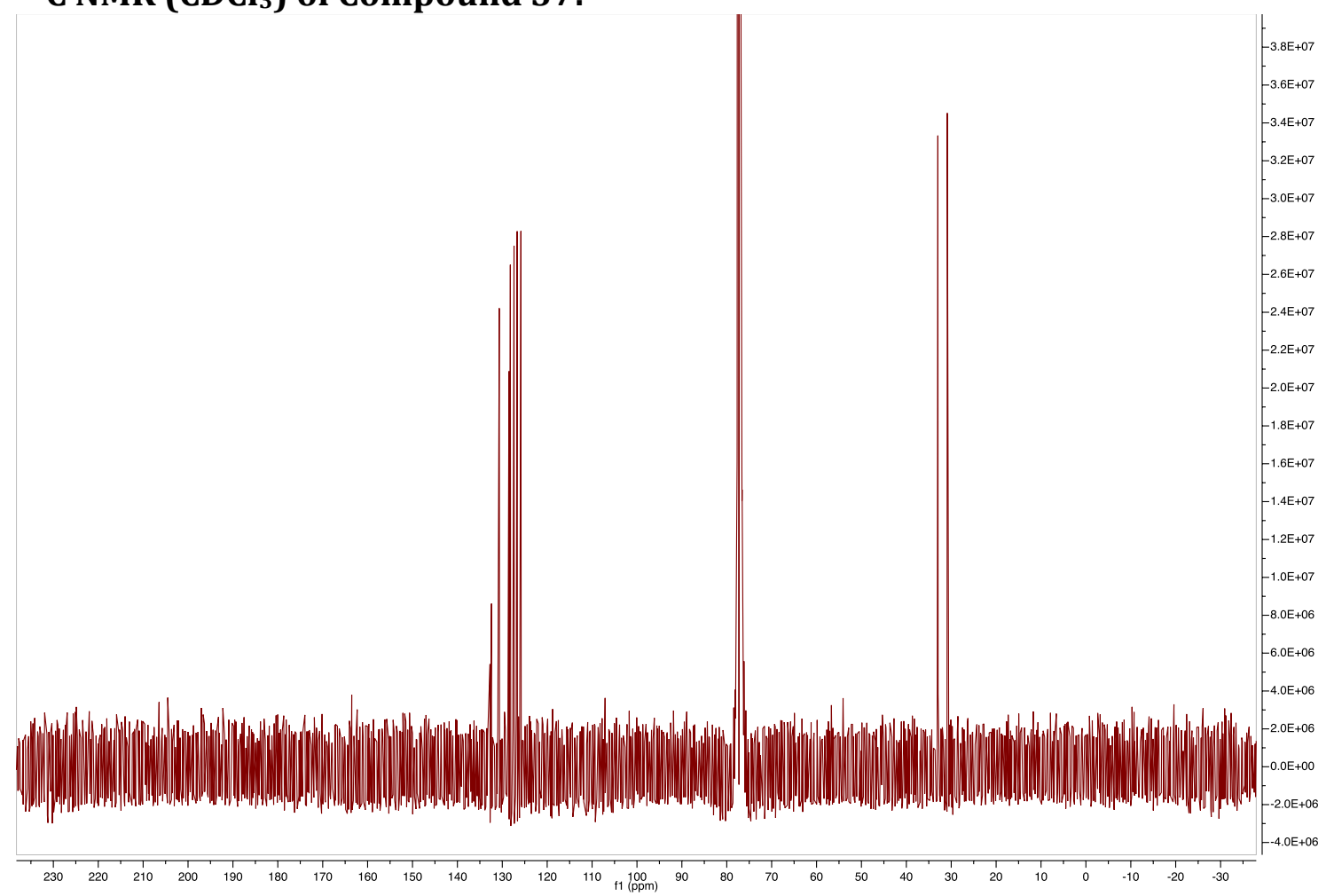


\section{${ }^{1} \mathrm{H}$ NMR $\left(\mathrm{CDCl}_{3}\right)$ of Compound 38:}

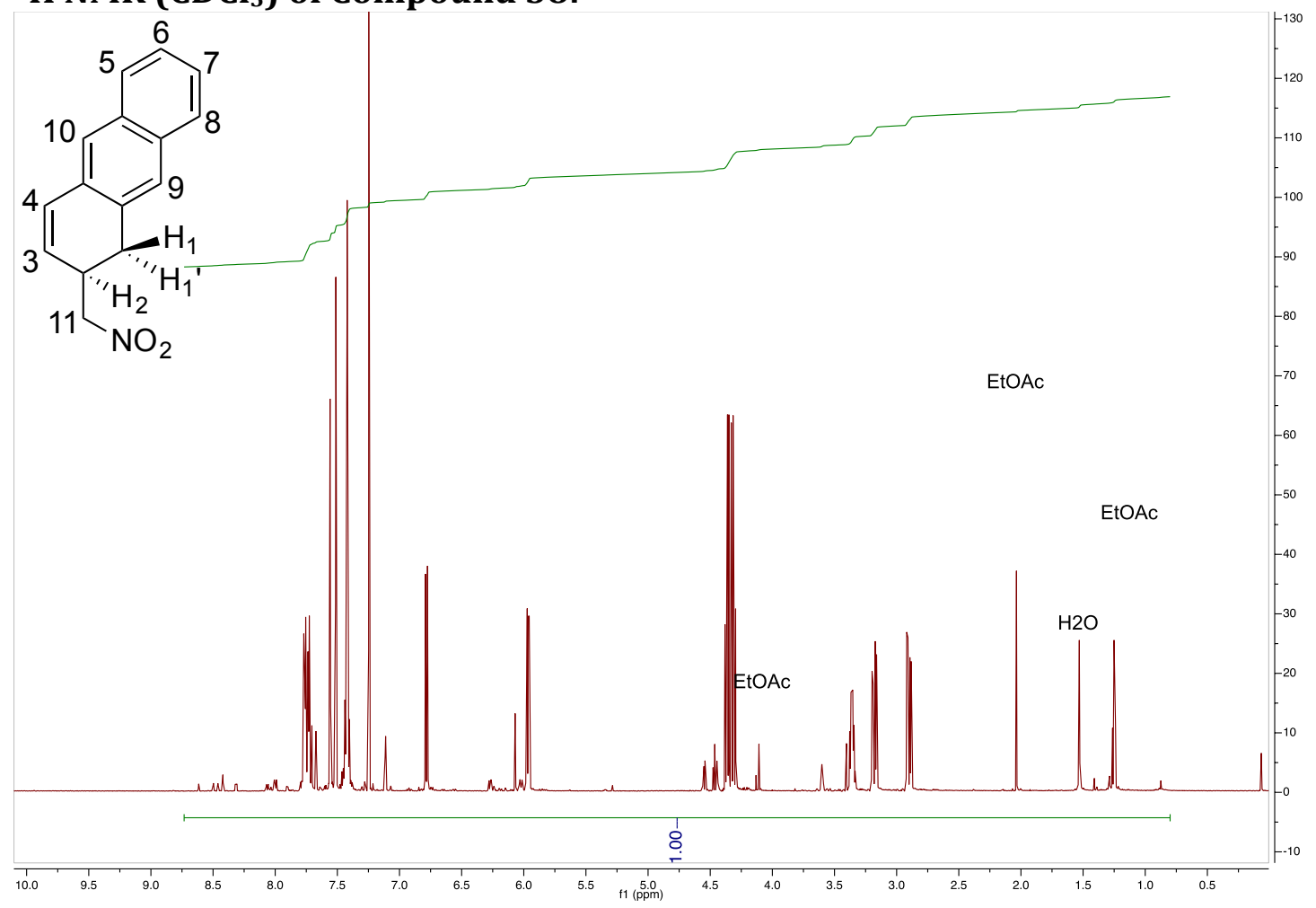

${ }^{13} \mathrm{C} \mathrm{NMR}\left(\mathrm{CDCl}_{3}\right)$ of Compound 38 :

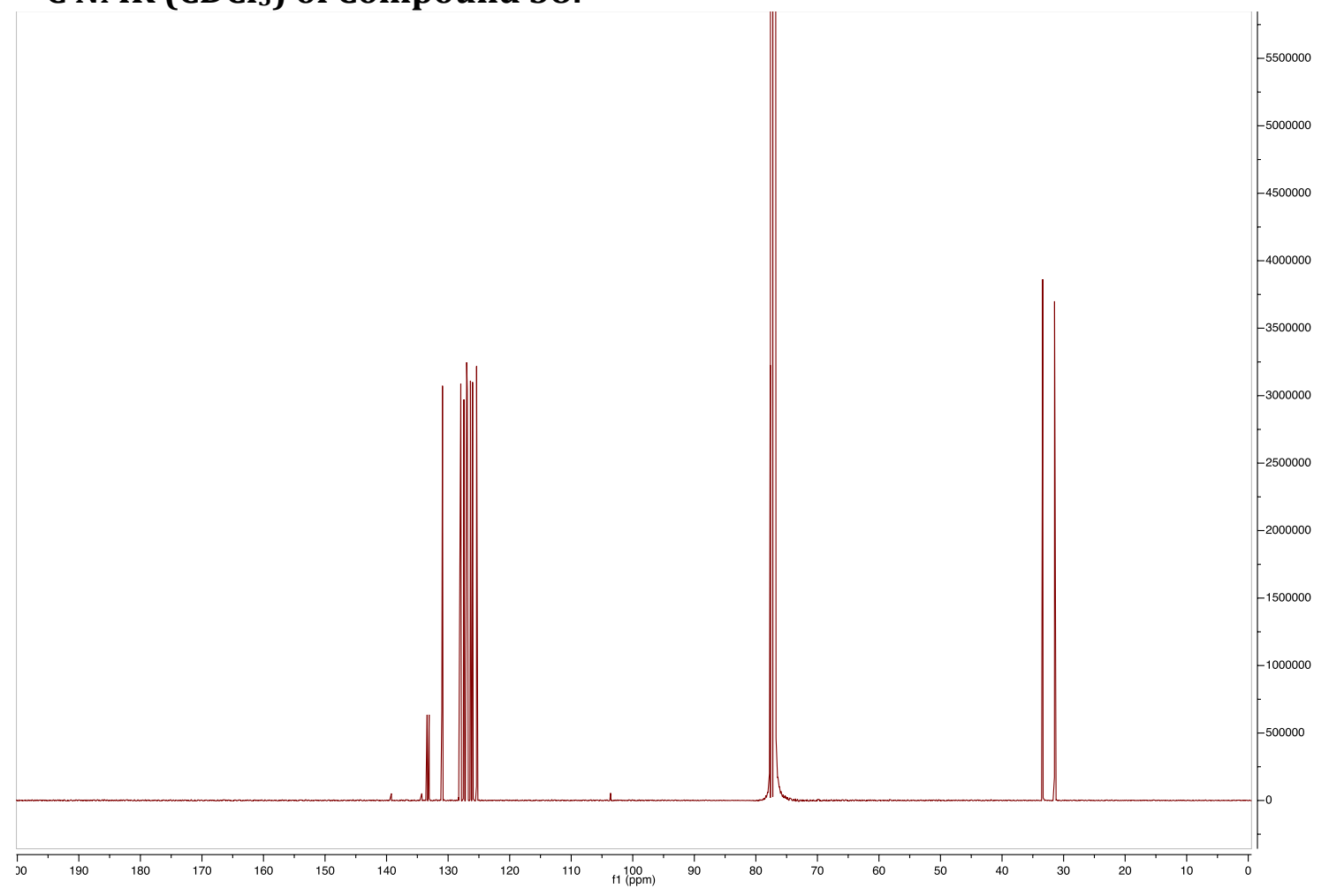




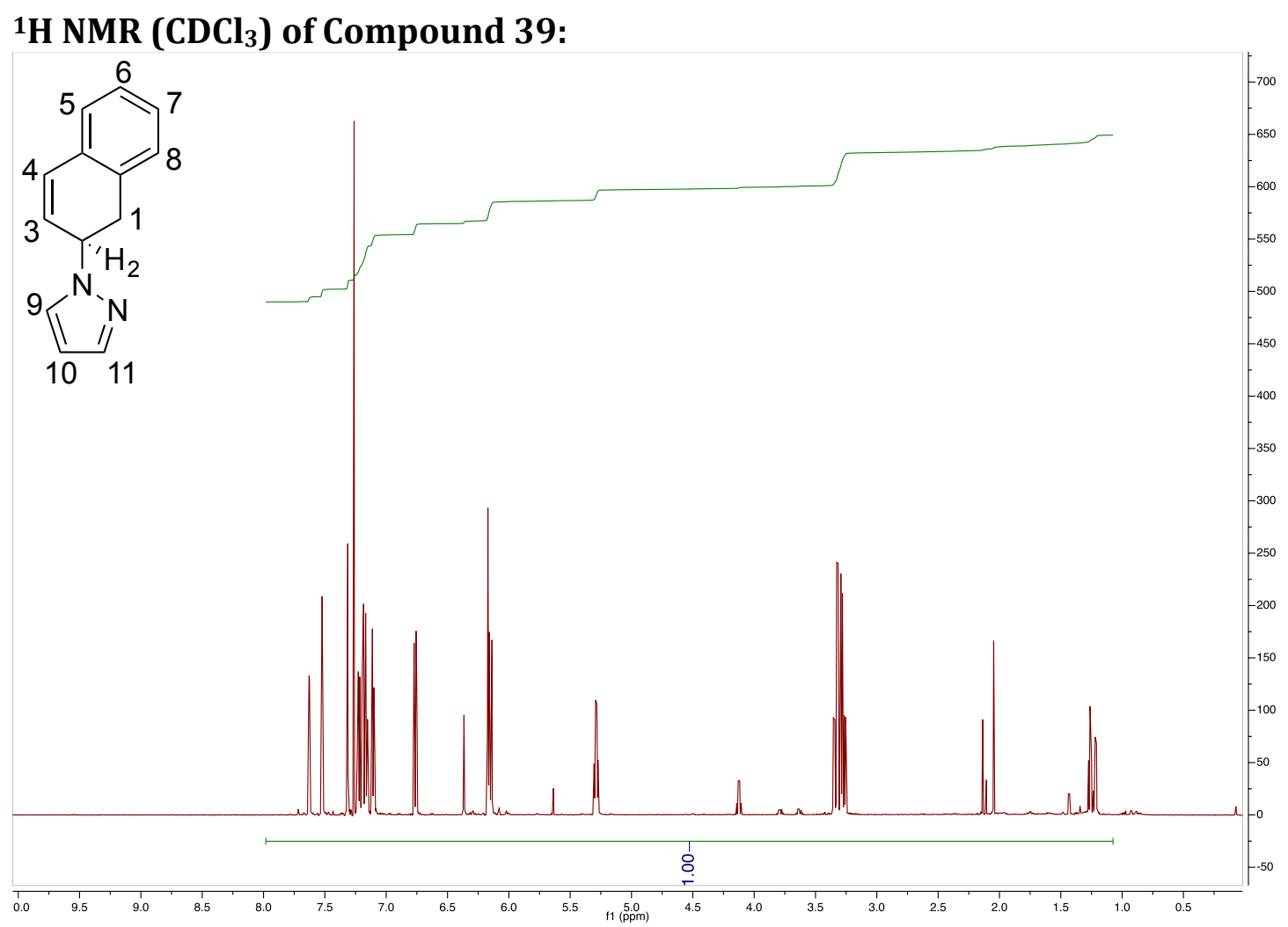

${ }^{13} \mathrm{C} \mathrm{NMR}\left(\mathrm{CDCl}_{3}\right)$ of Compound 39:

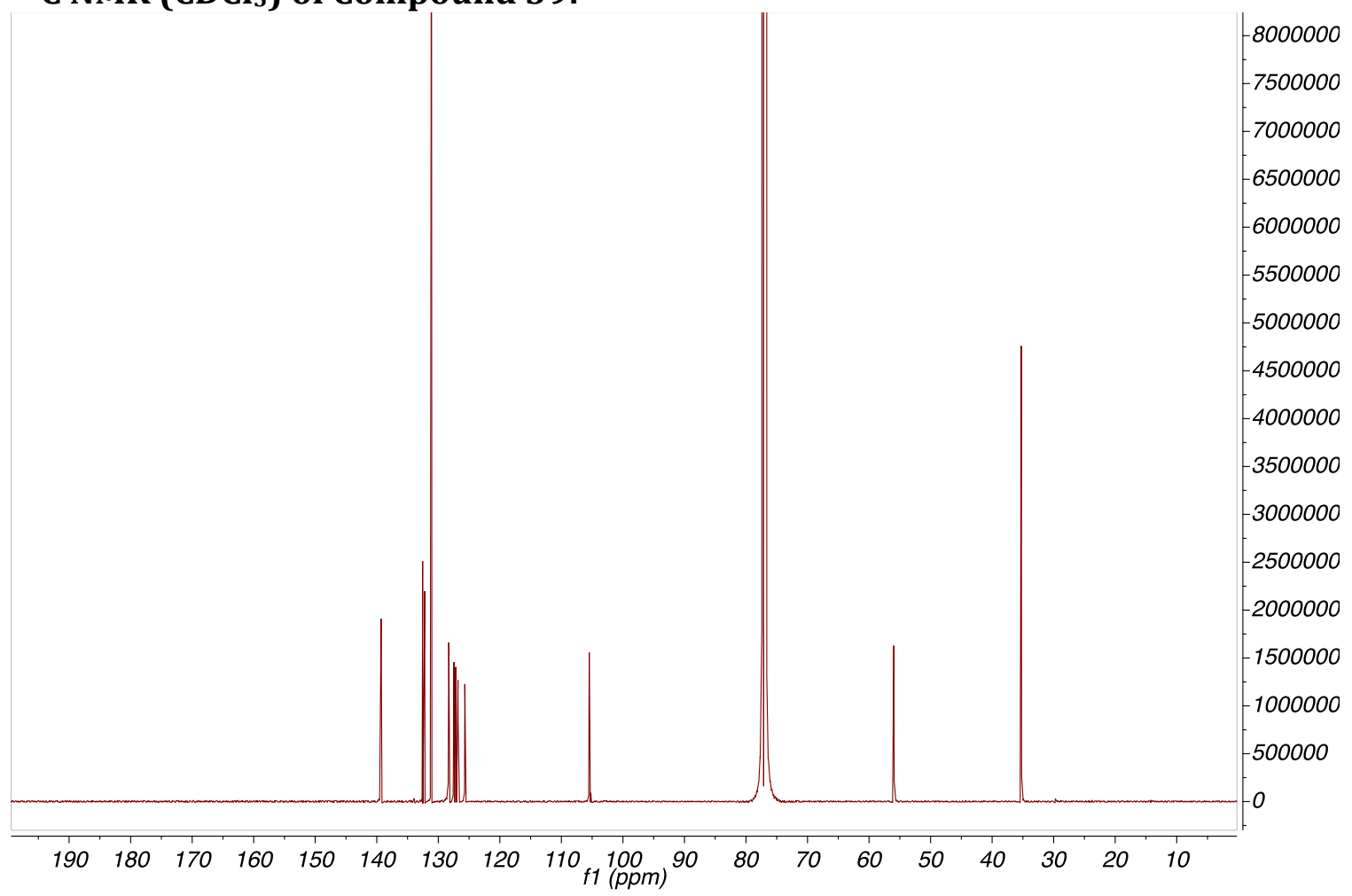




\section{${ }^{1} \mathrm{H}$ NMR $\left(\mathrm{CDCl}_{3}\right)$ of Compound 40:}

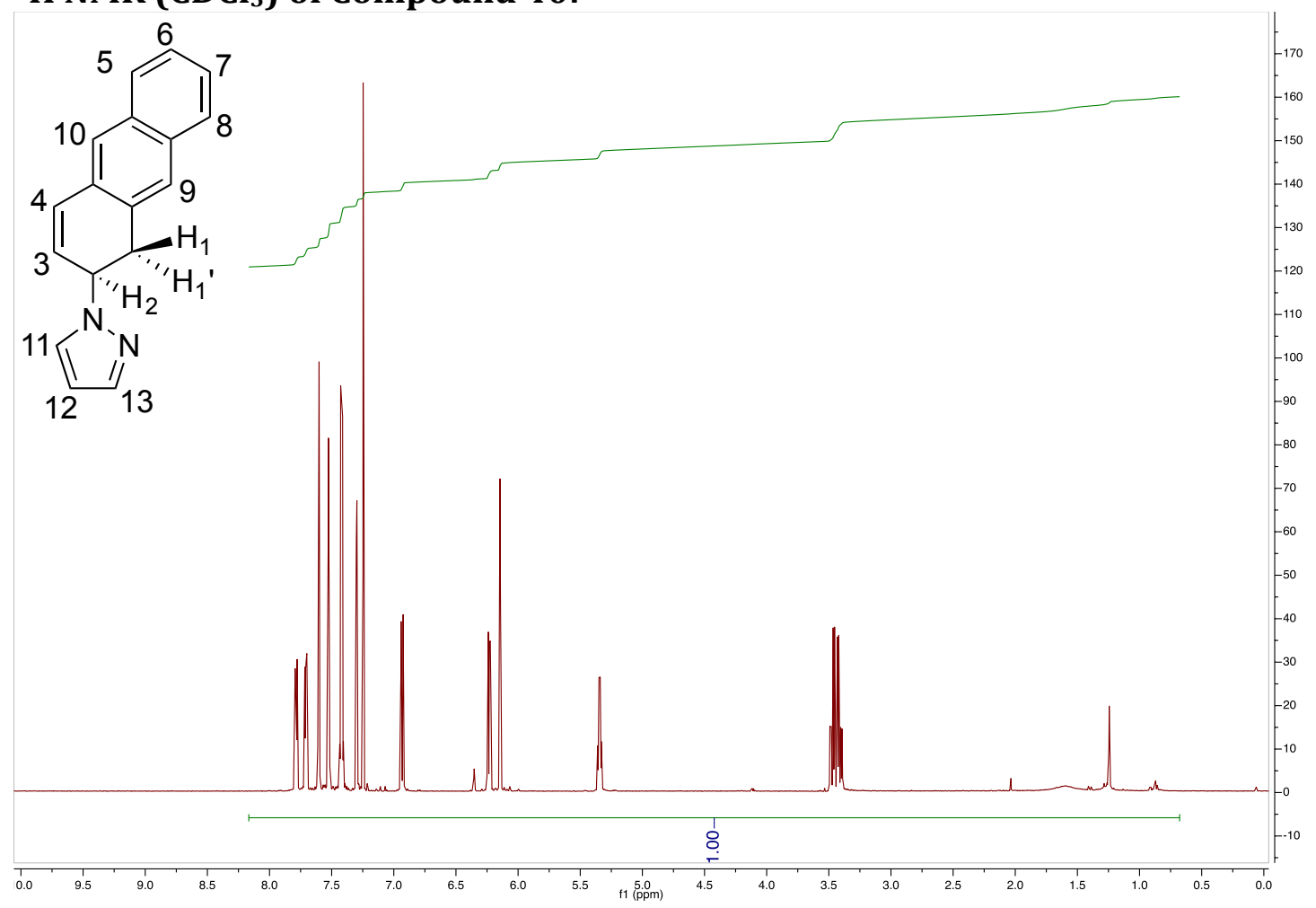

${ }^{13} \mathrm{C} \mathrm{NMR}\left(\mathrm{CDCl}_{3}\right)$ of Compound 40:

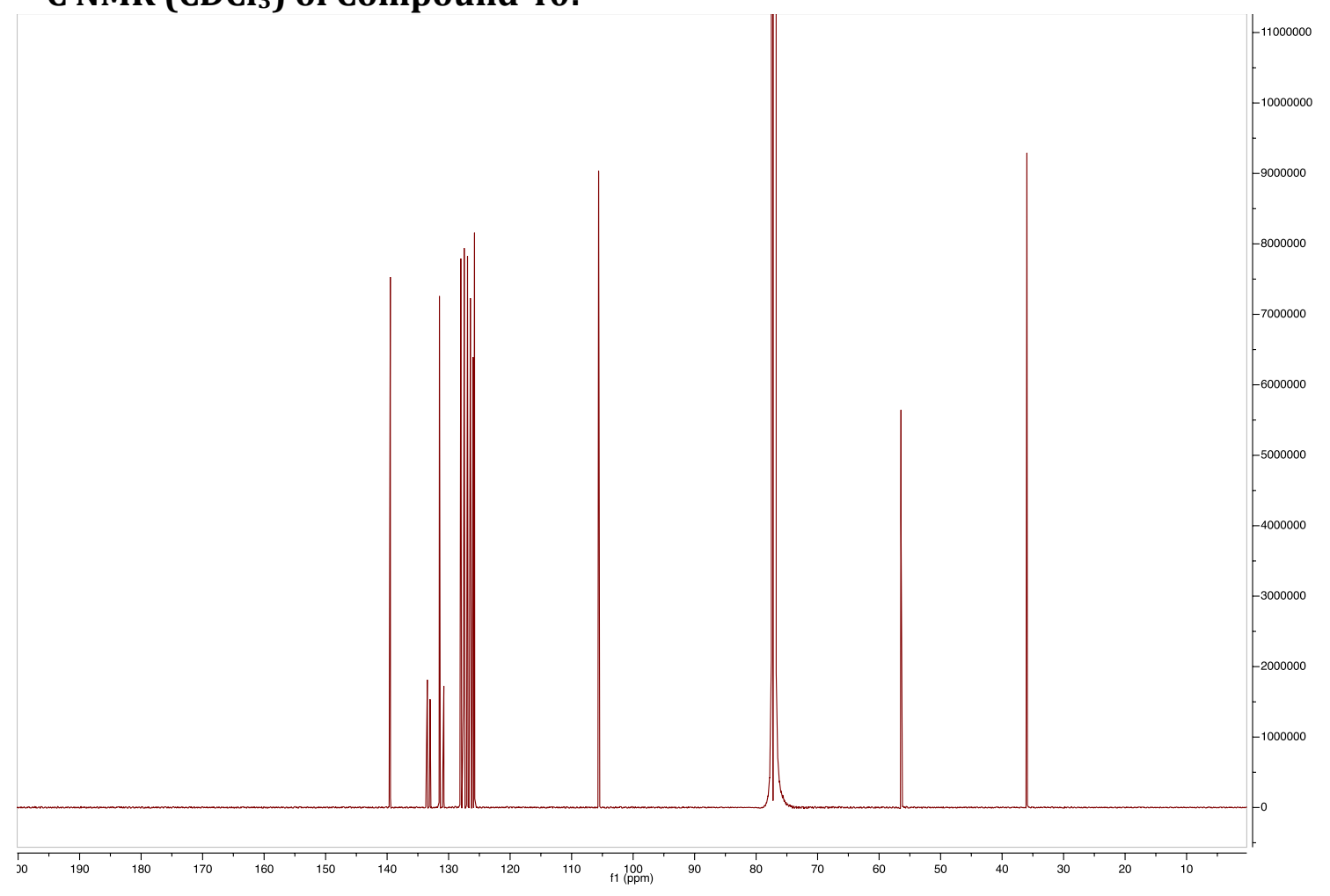


${ }^{1}$ H NMR (d6-Acetone) of Compound 41:

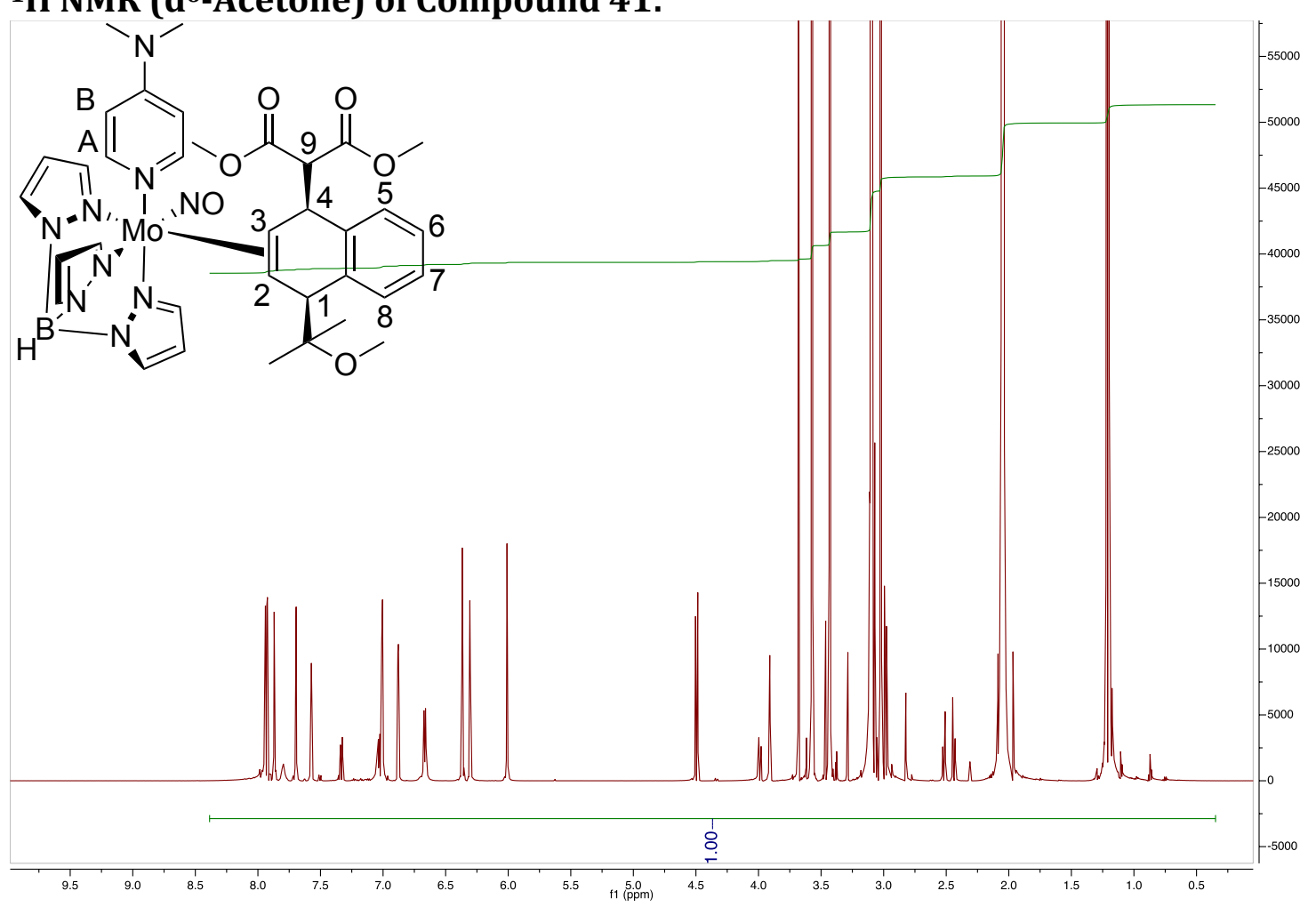

${ }^{13}$ C NMR (d6-Acetone) of Compound 41:

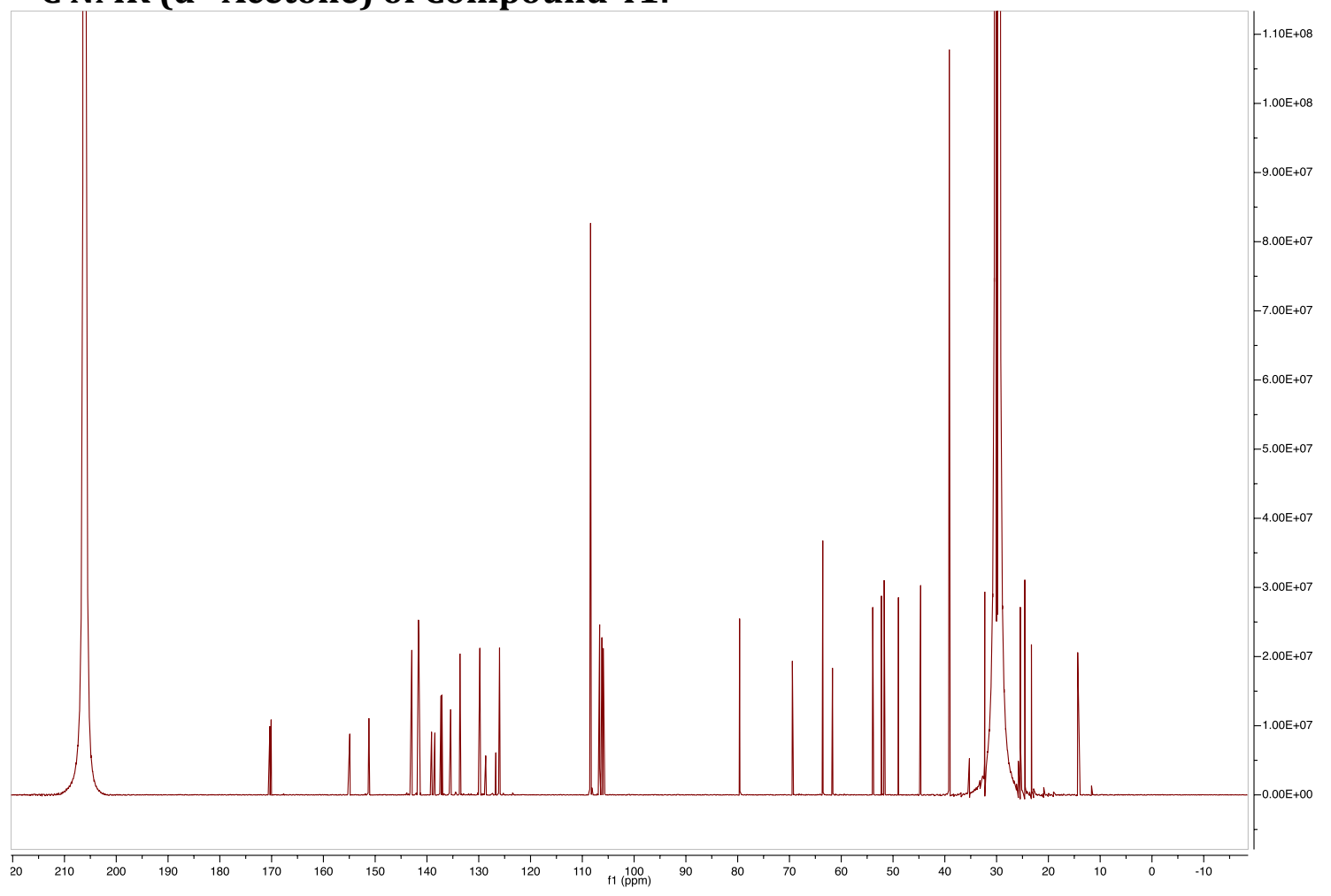


${ }^{1} \mathrm{H}$ NMR (d ${ }^{6}$-Acetone) of Compound 42:

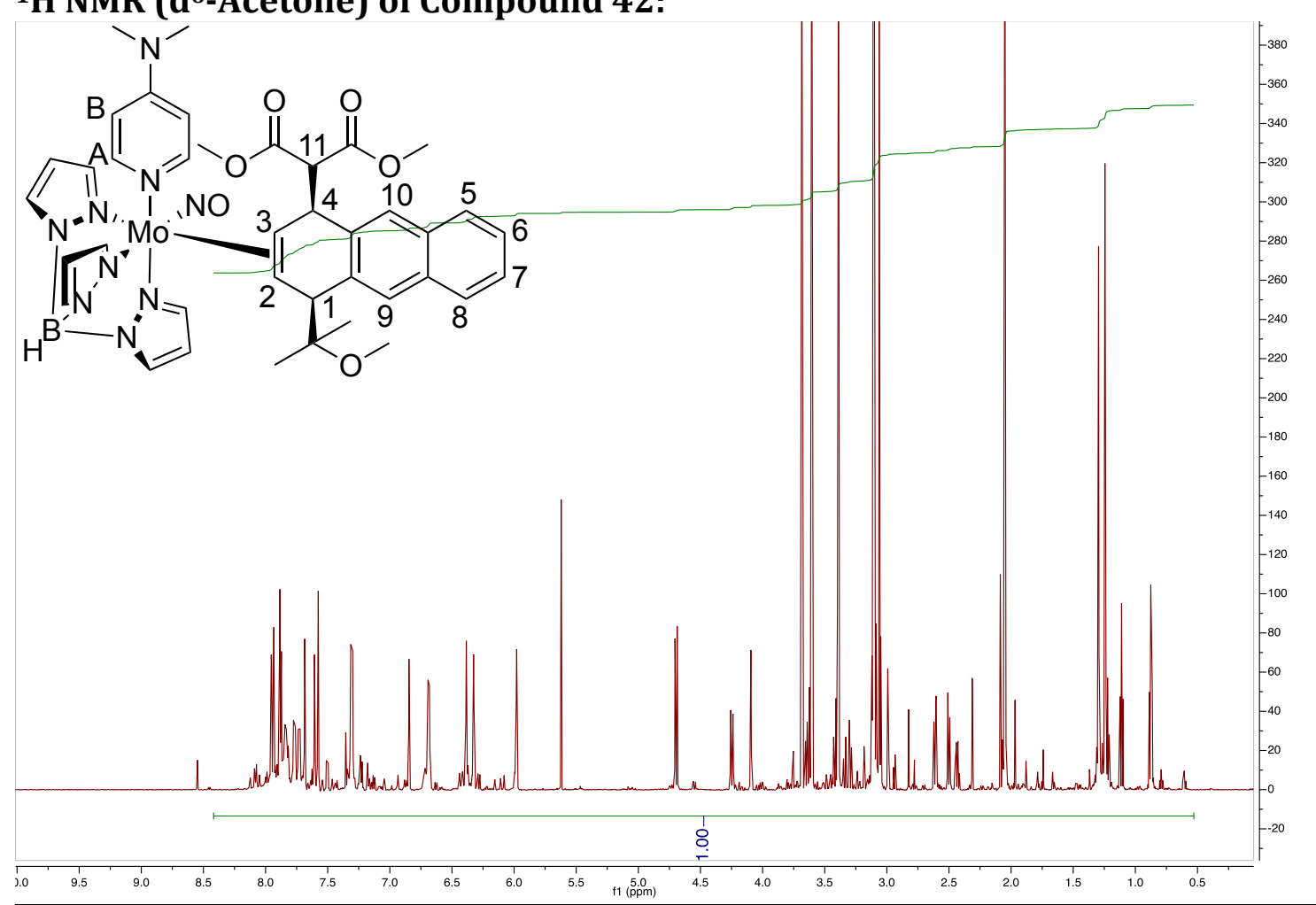

${ }^{13}$ C NMR (d6-Acetone) of Compound 42: ${ }_{11013}$

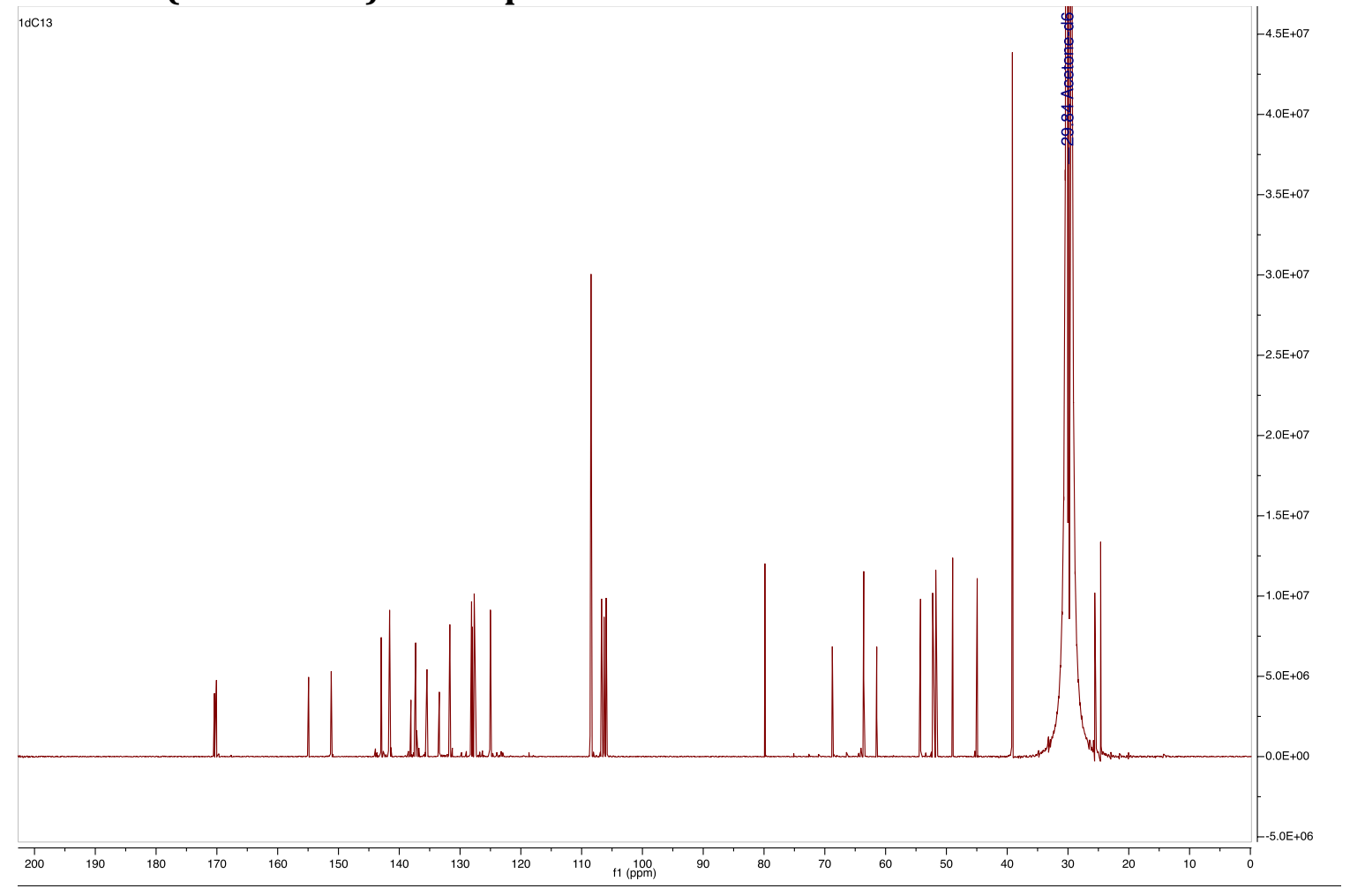


${ }^{1} \mathrm{H}$ NMR $\left(\mathrm{CDCl}_{3}\right)$ of Compound 43:

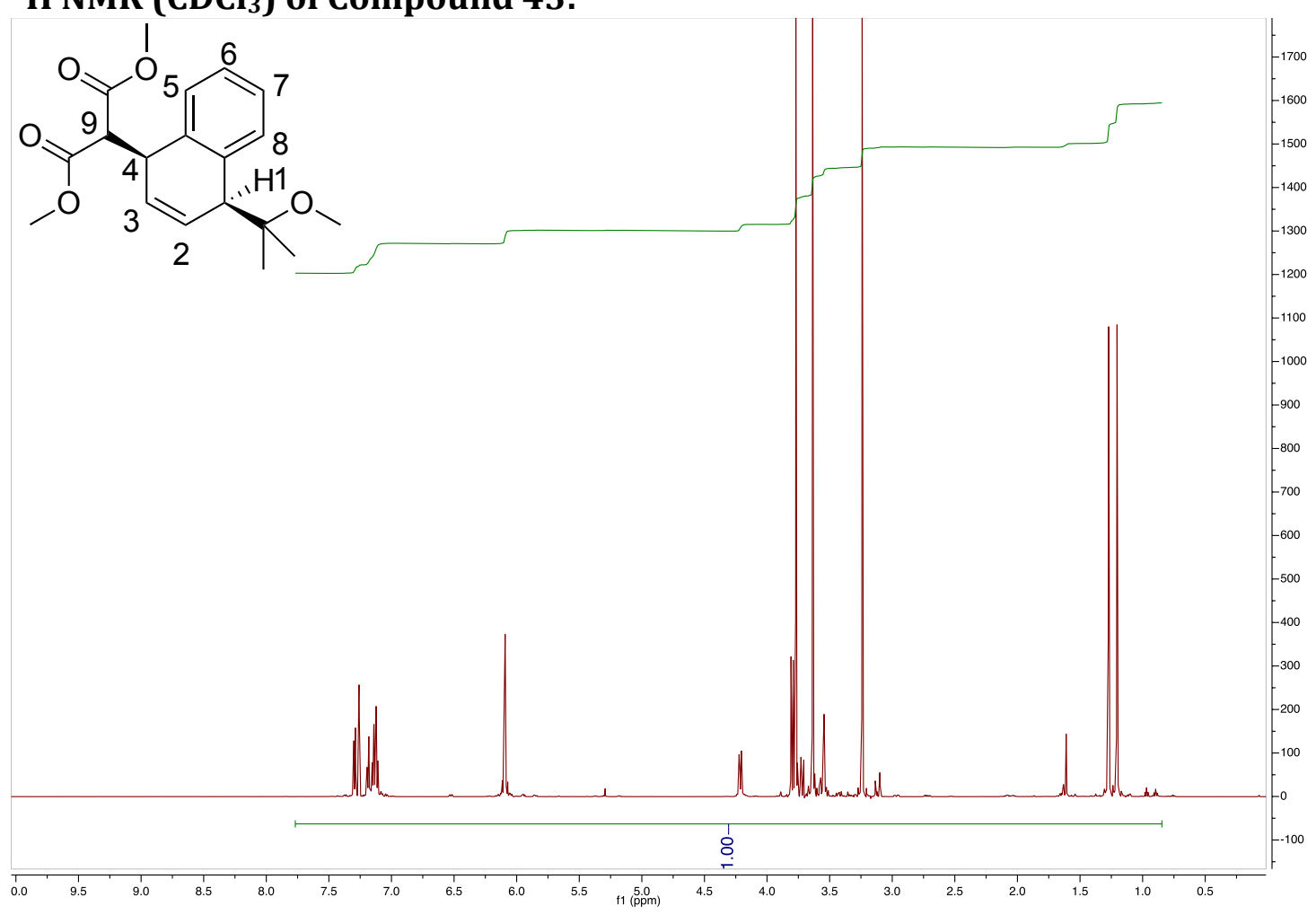

${ }^{13} \mathrm{C} \mathrm{NMR}\left(\mathrm{CDCl}_{3}\right)$ of Compound 43:

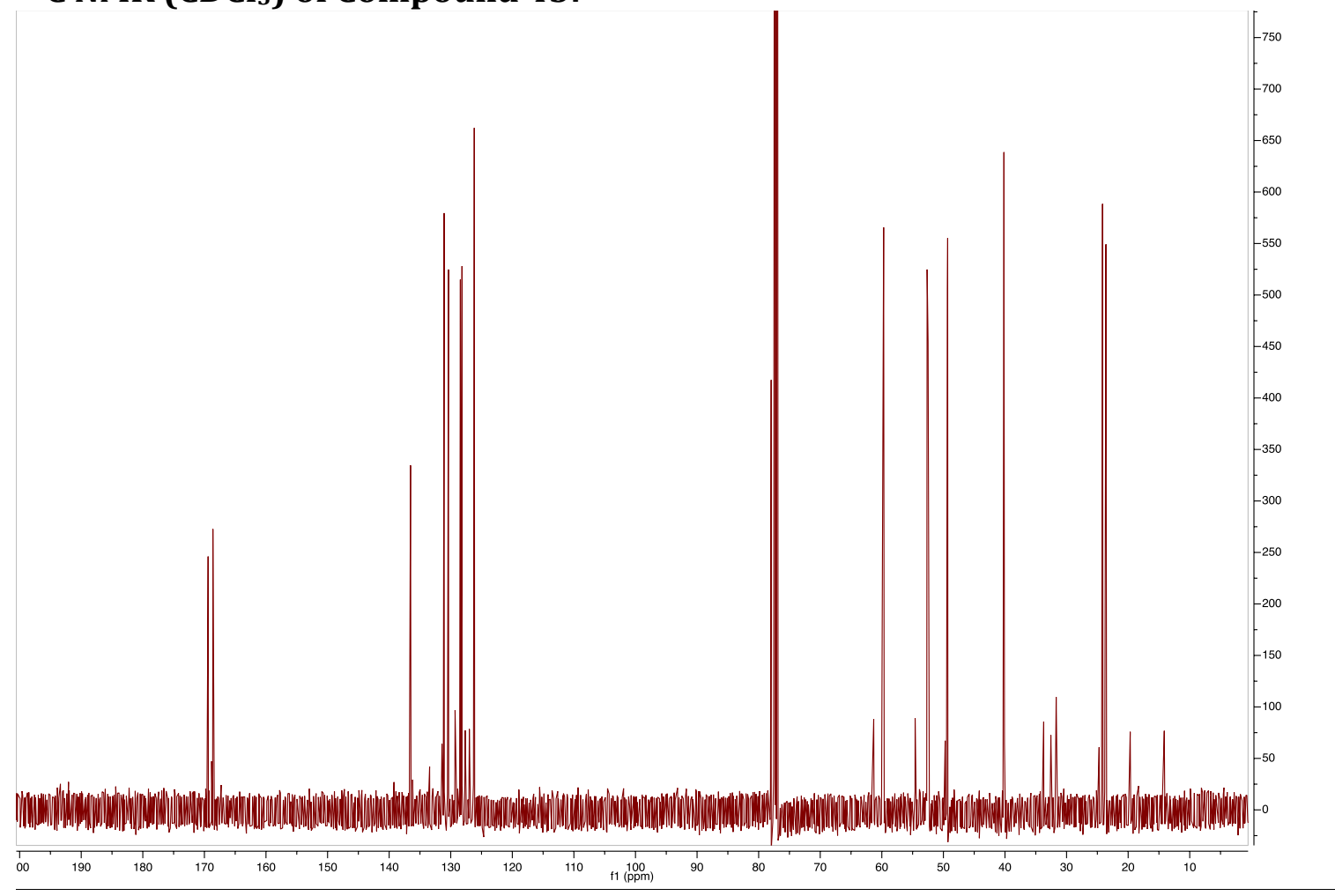




\section{${ }^{1} \mathrm{H}$ NMR $\left(\mathrm{CDCl}_{3}\right)$ of Compound 44:}

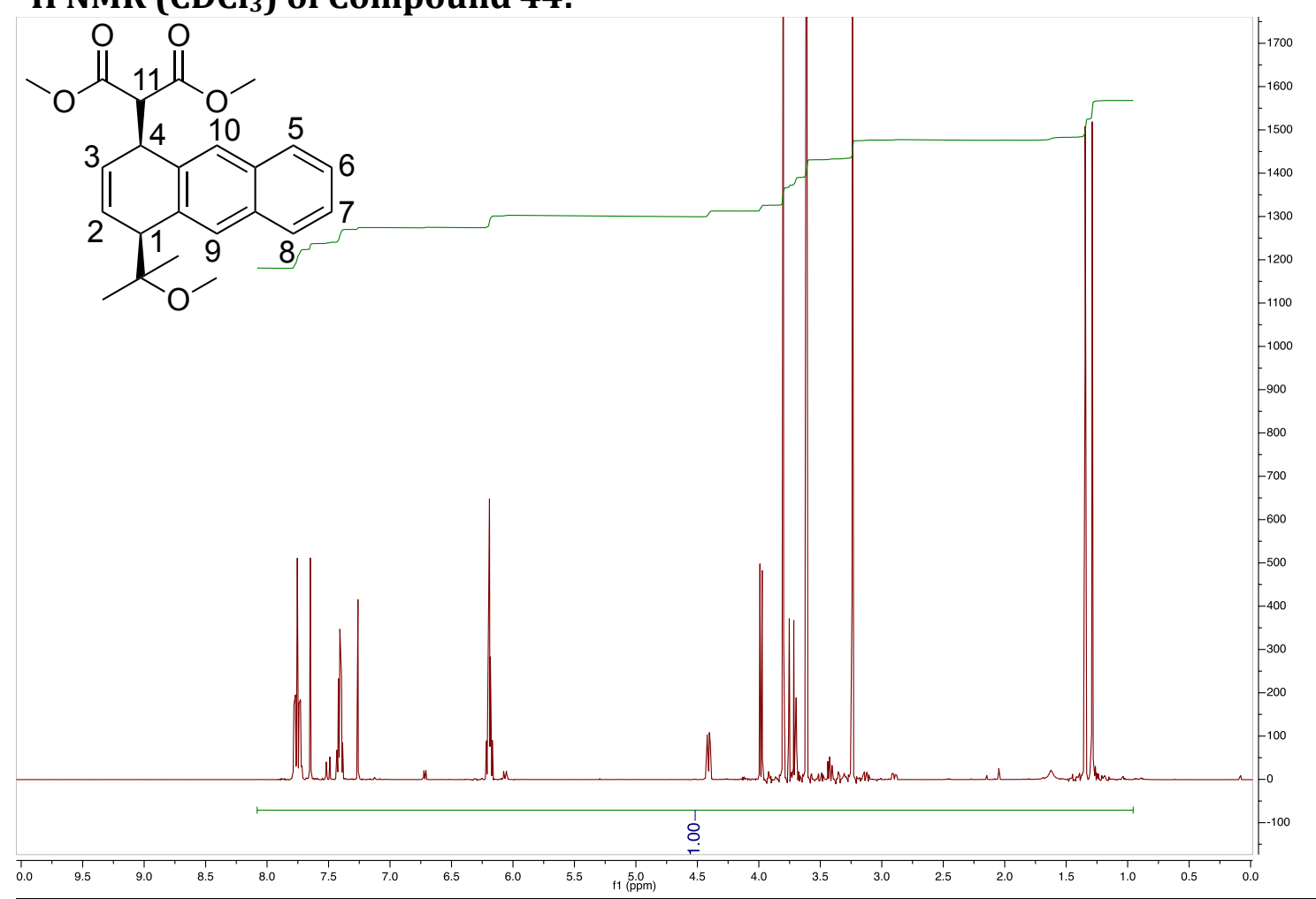

${ }^{13} \mathrm{C} \mathrm{NMR}\left(\mathrm{CDCl}_{3}\right)$ of Compound 44:

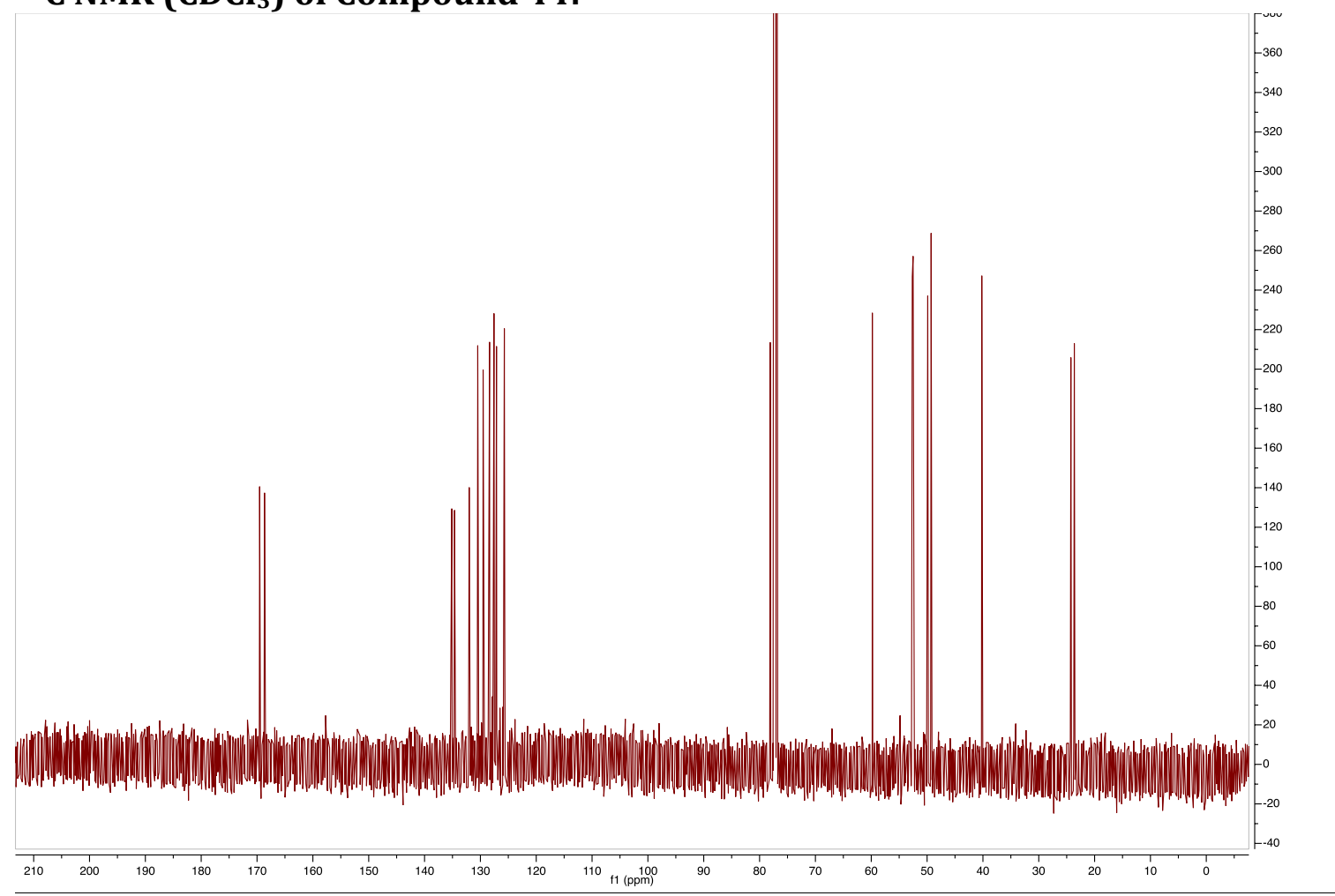




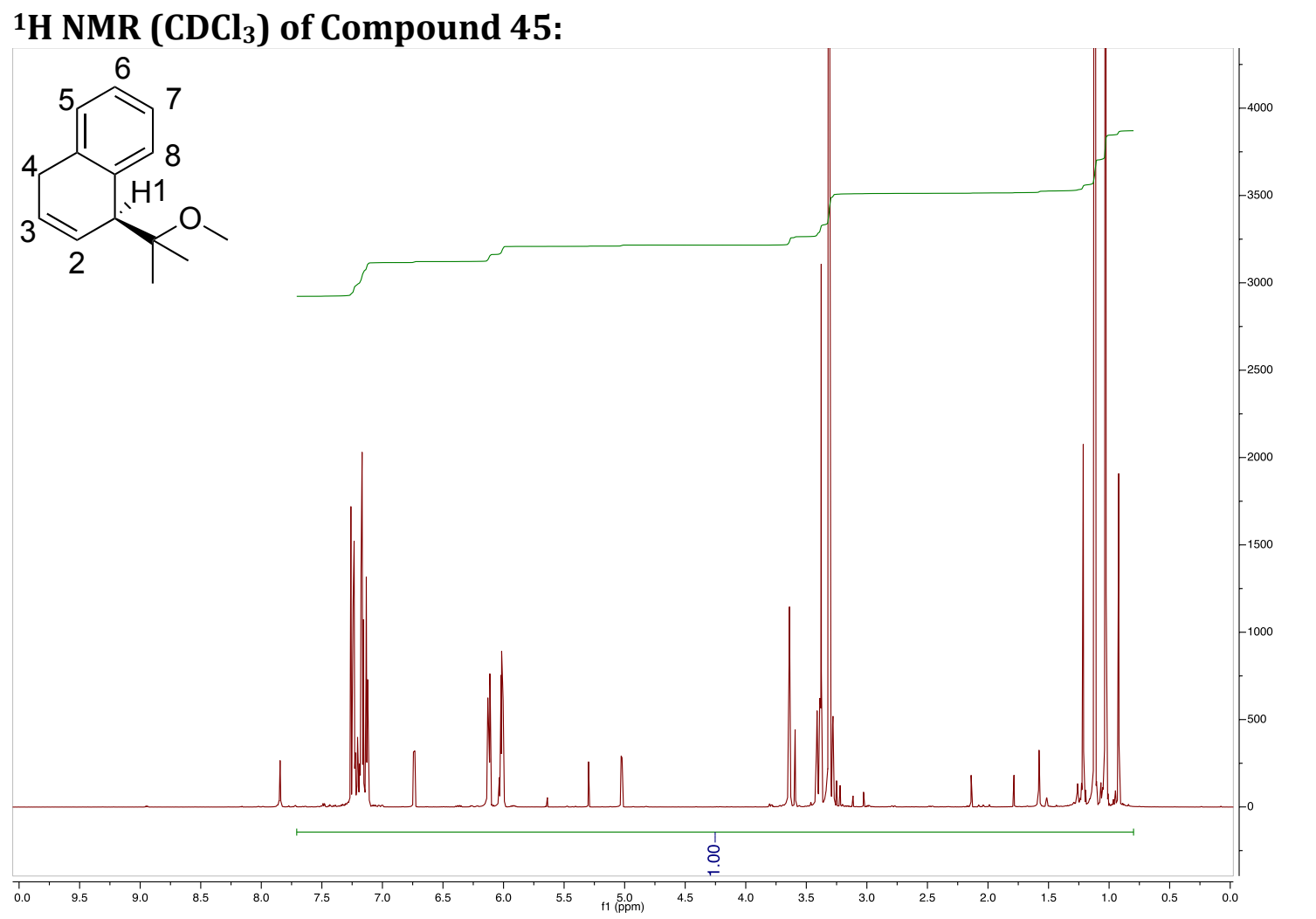

${ }^{13} \mathrm{C} \mathrm{NMR}\left(\mathrm{CDCl}_{3}\right)$ of Compound 45:

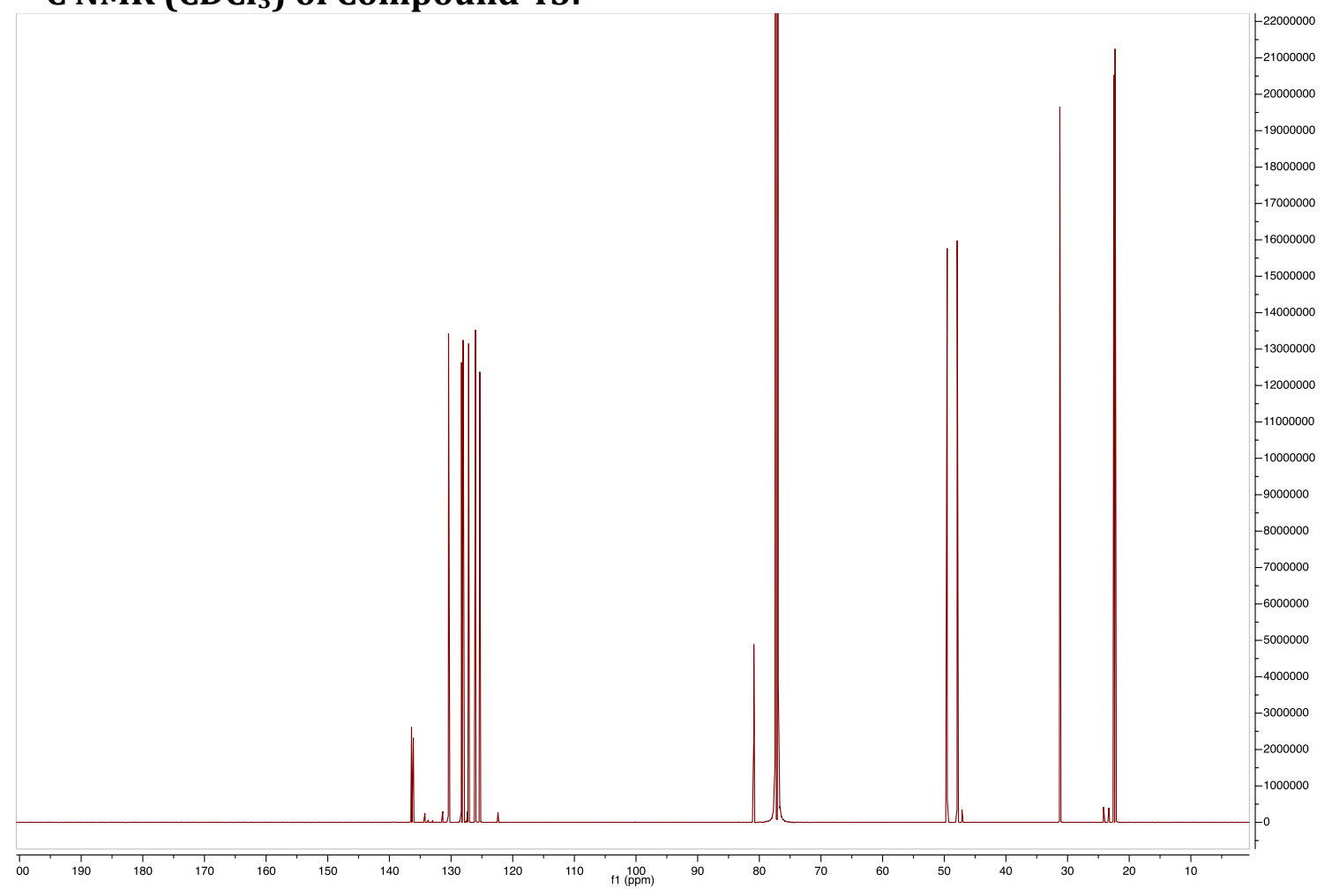




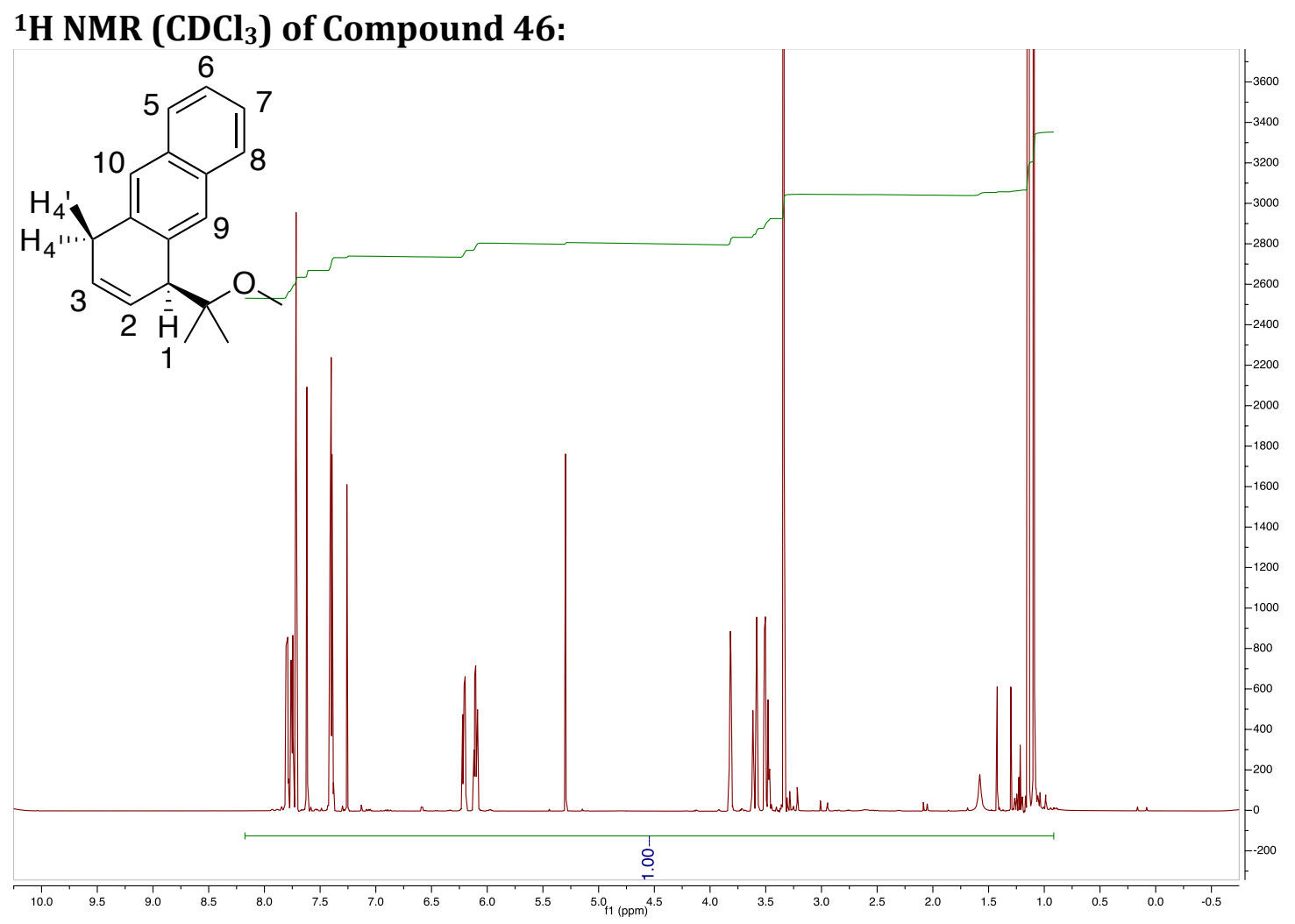

${ }^{13} \mathrm{C} \mathrm{NMR}\left(\mathrm{CDCl}_{3}\right)$ of Compound 46:

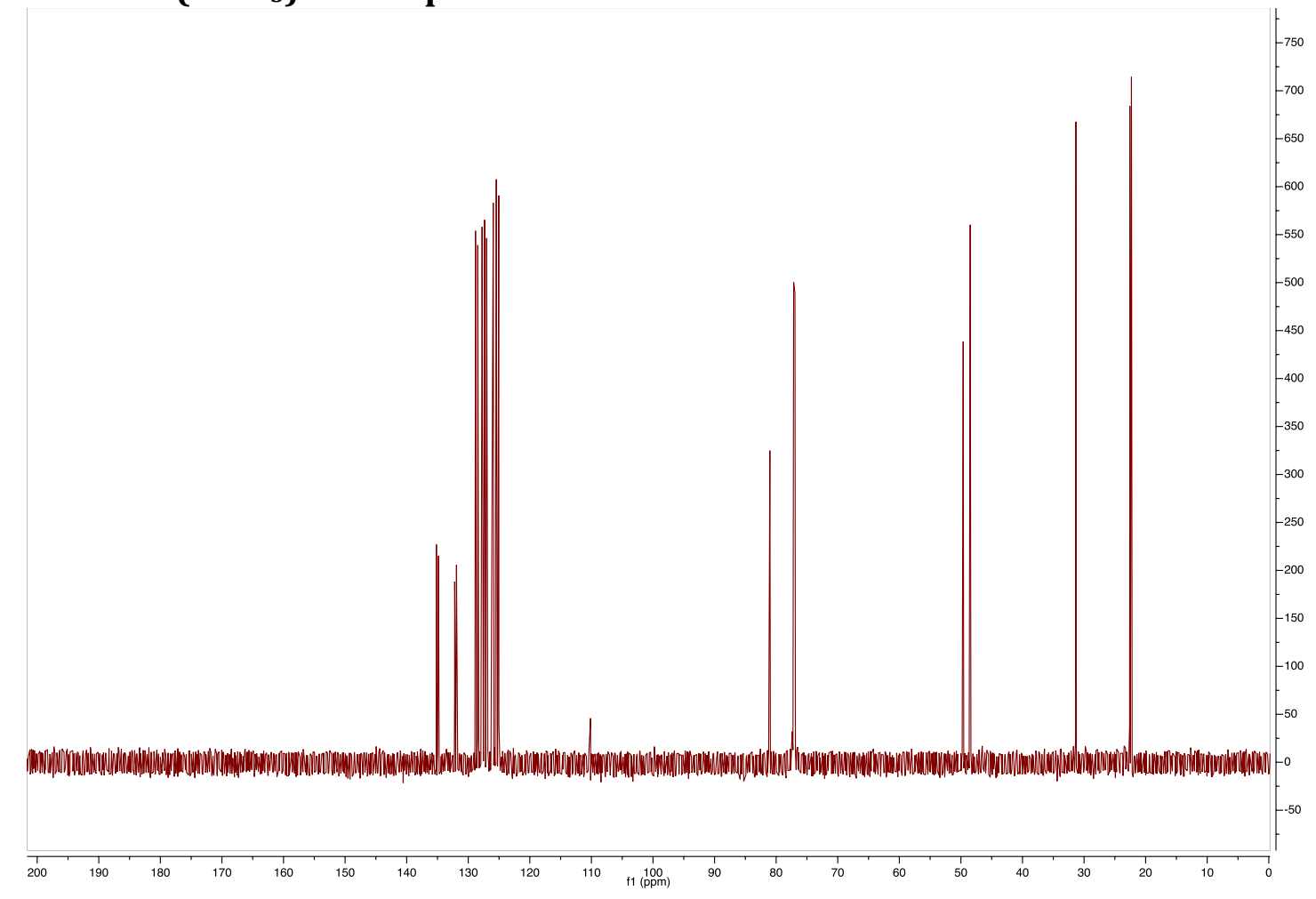




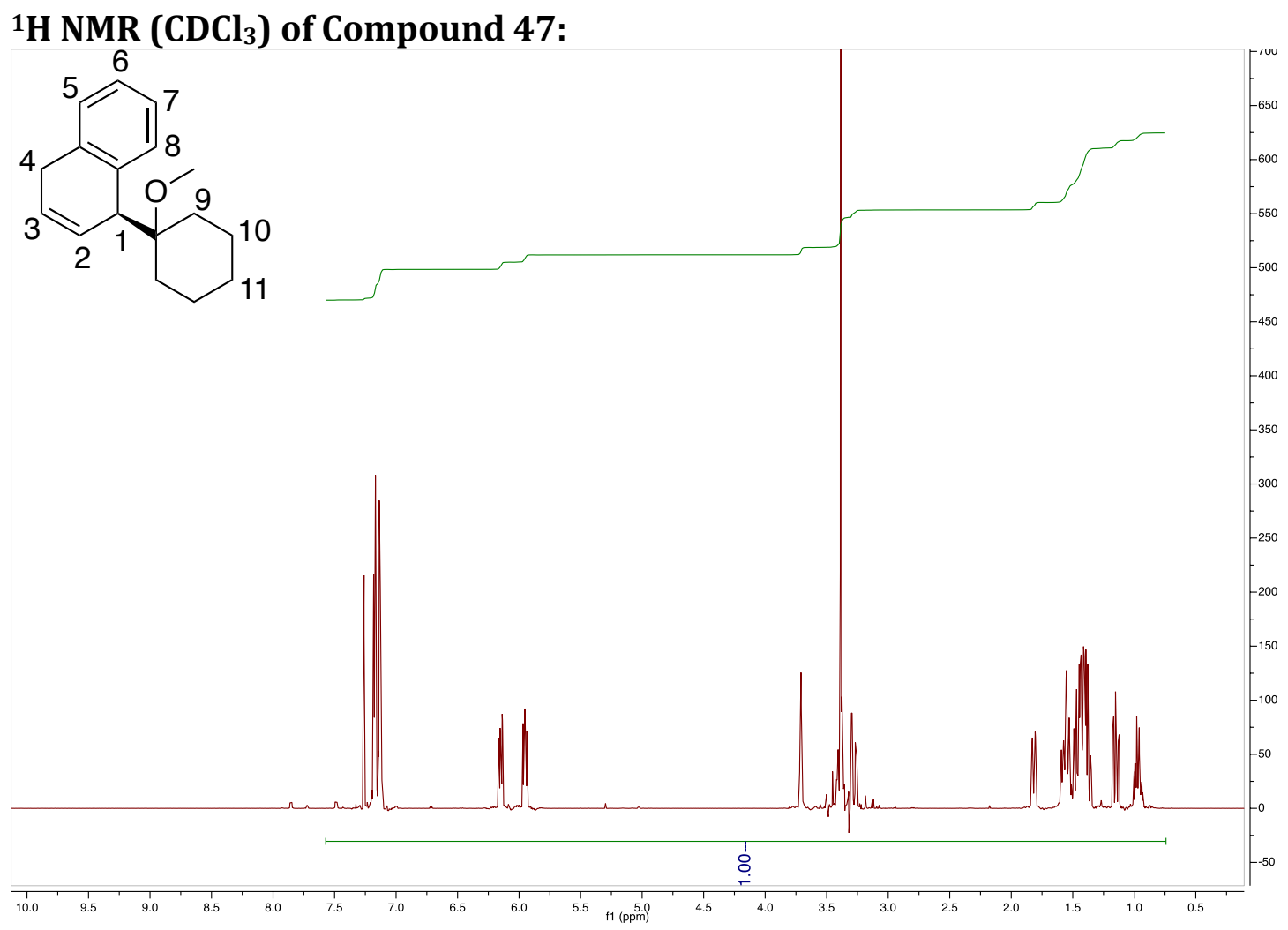

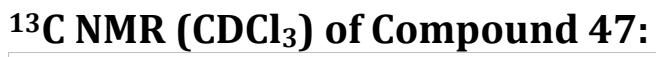

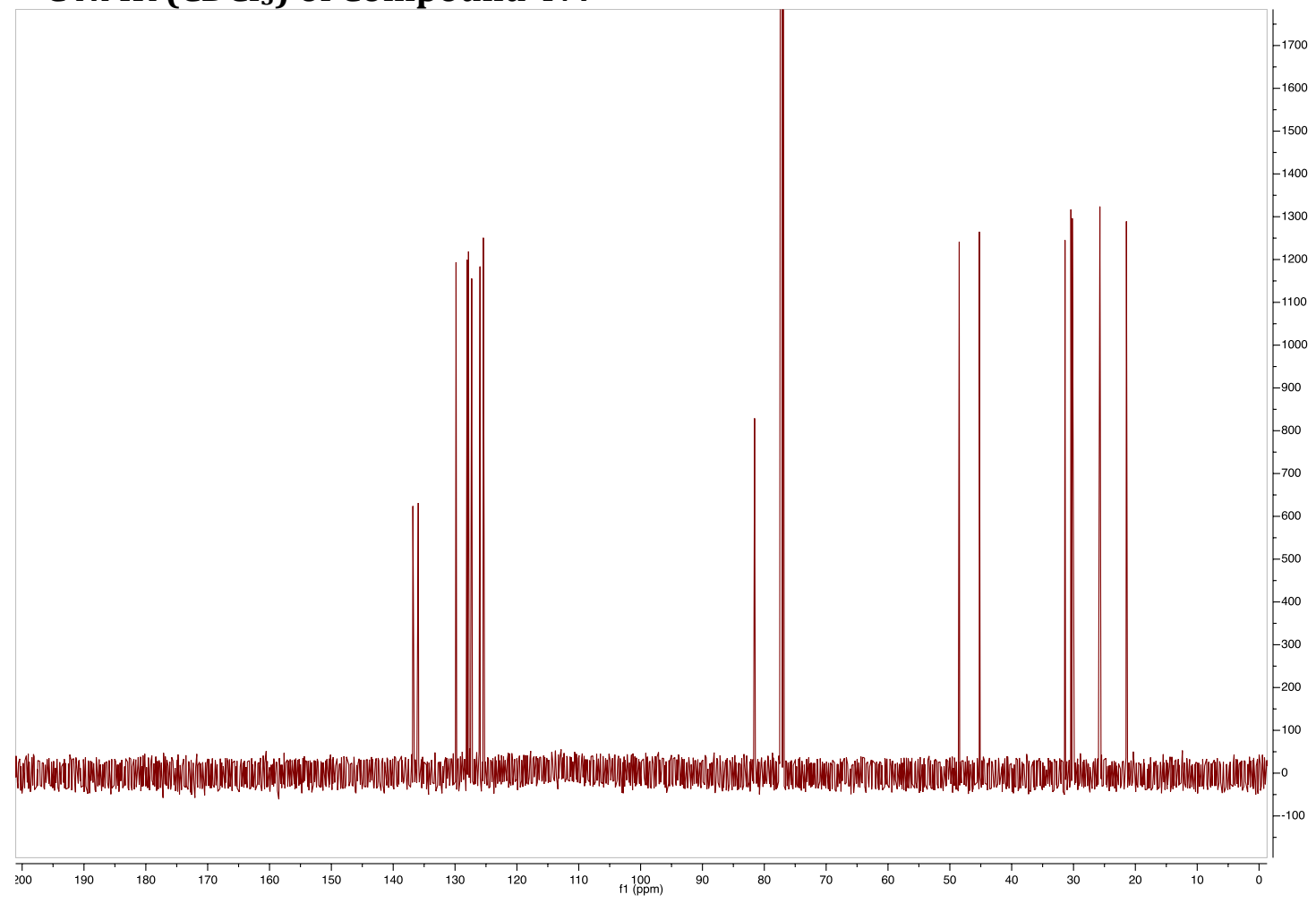


${ }^{1} \mathrm{H}$ NMR $\left(\mathrm{CDCl}_{3}\right)$ of Compound 48:

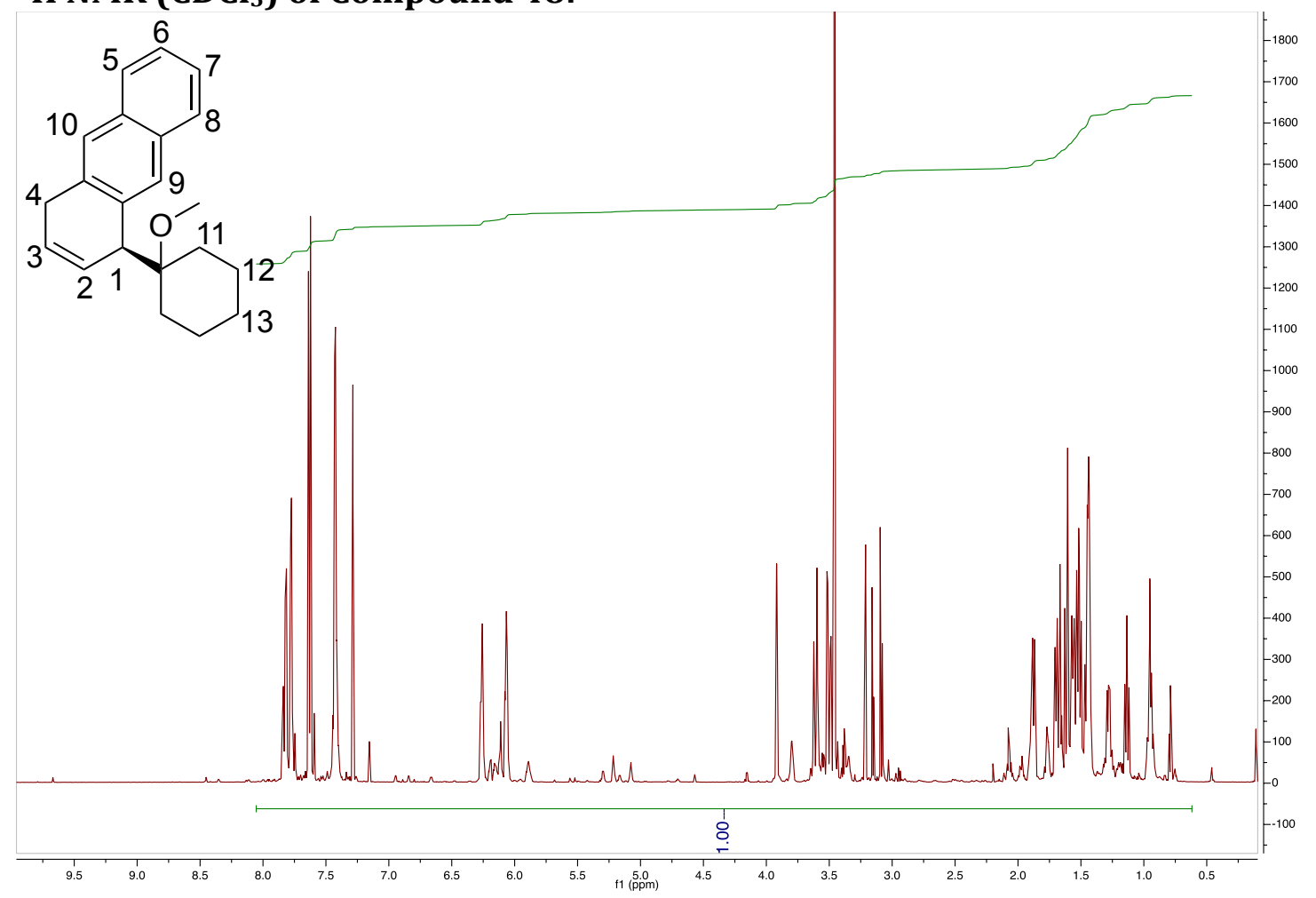

${ }^{13} \mathrm{C} \mathrm{NMR}\left(\mathrm{CDCl}_{3}\right)$ of Compound 48:

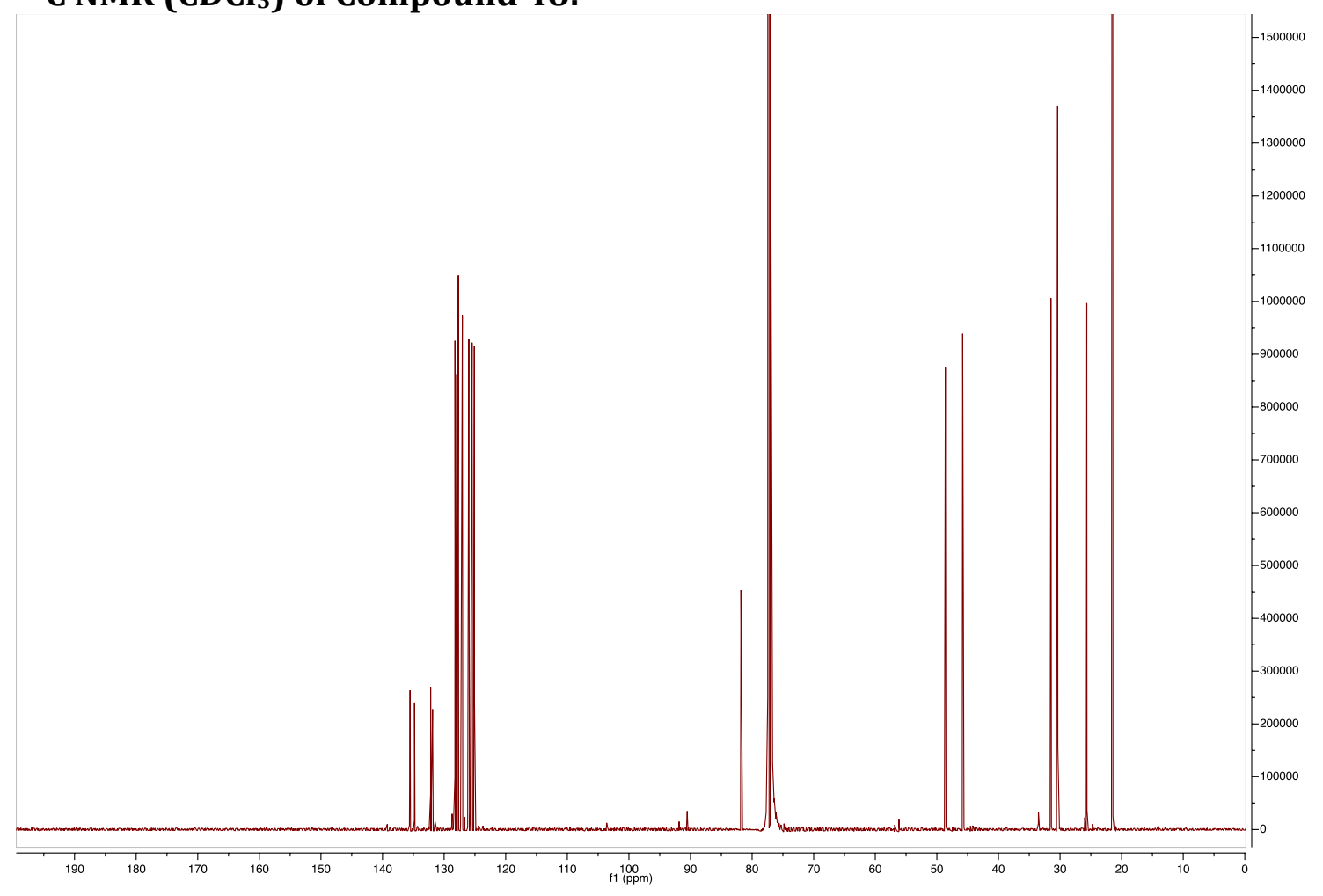


H NMR $\left(\mathrm{CDCl}_{3}\right)$ of Compound 49:

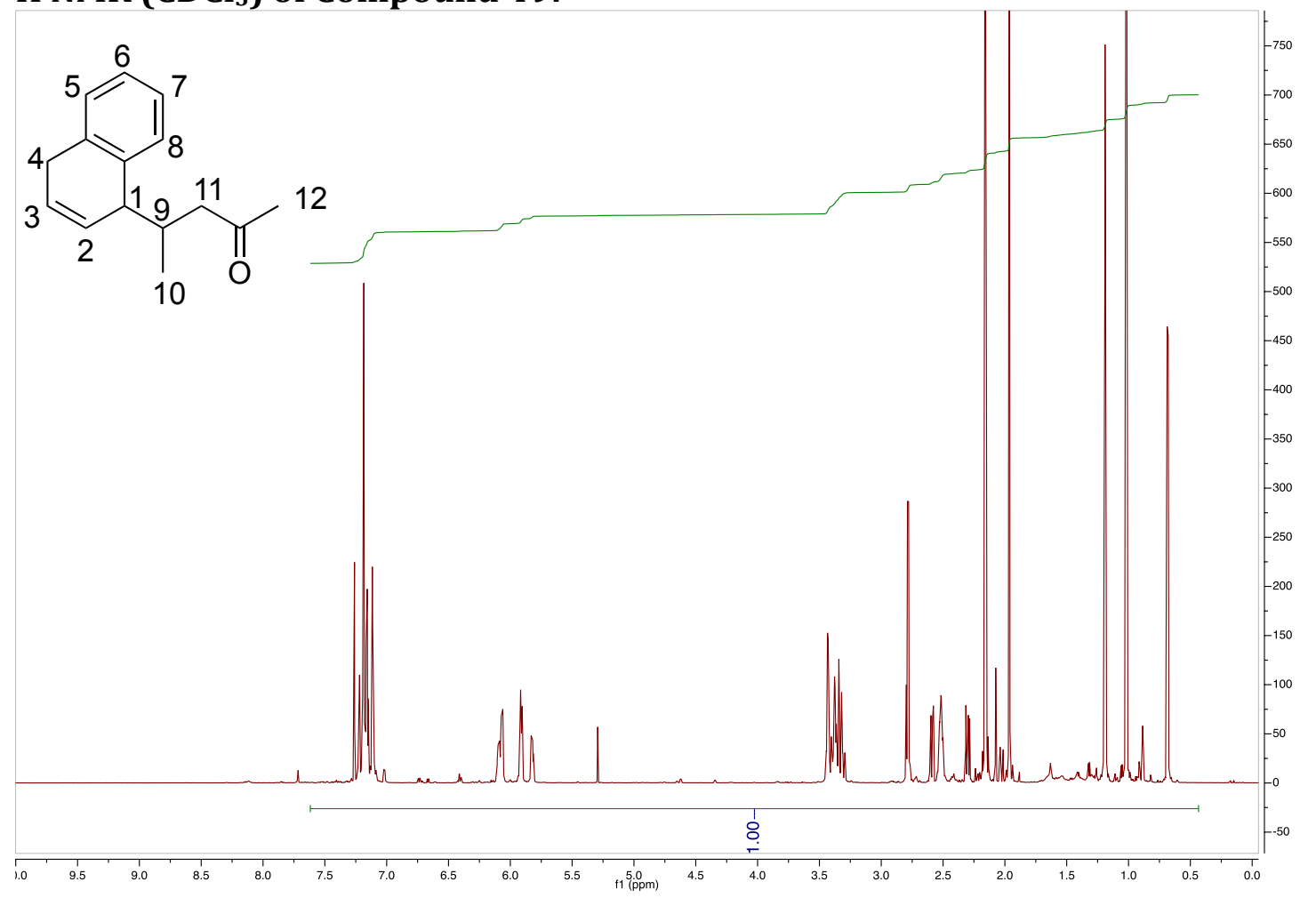

${ }^{13} \mathrm{C} \mathrm{NMR}\left(\mathrm{CDCl}_{3}\right)$ of Compound 49:

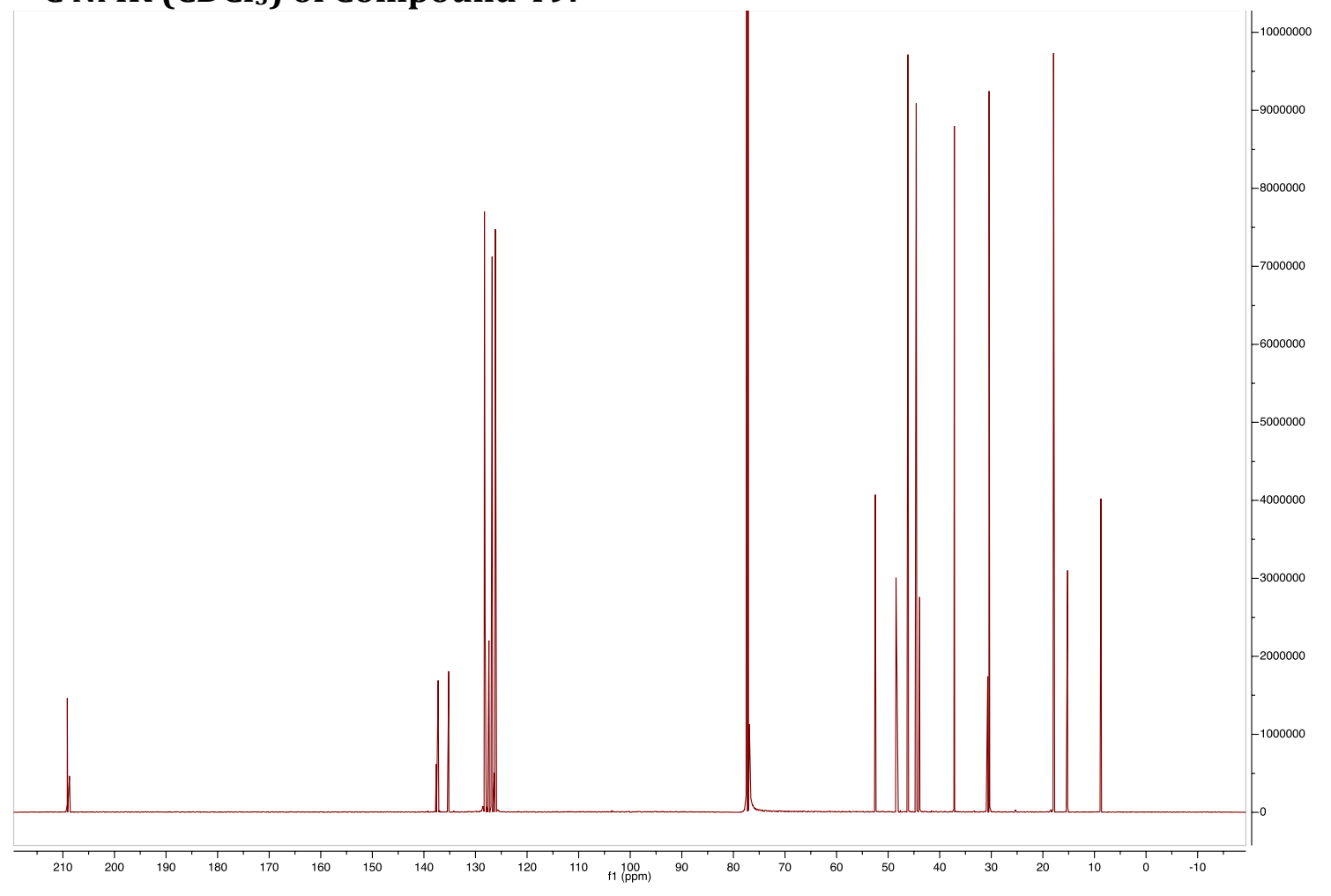


${ }^{1} \mathrm{H}$ NMR $\left(\mathrm{CDCl}_{3}\right)$ of Compound 50:

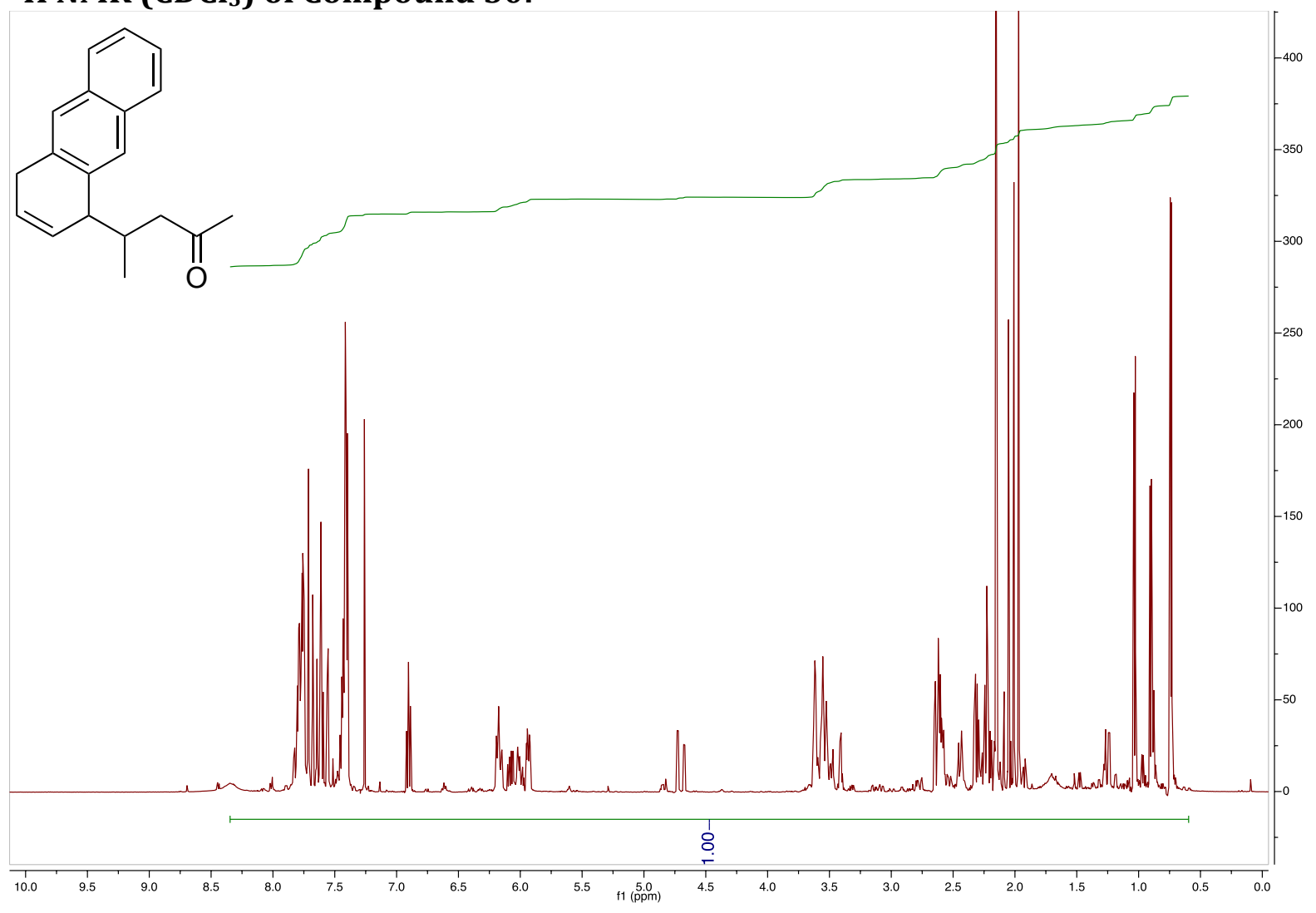


${ }^{1} \mathrm{H}$ NMR $\left(\mathrm{CDCl}_{3}\right)$ of Compound 51:

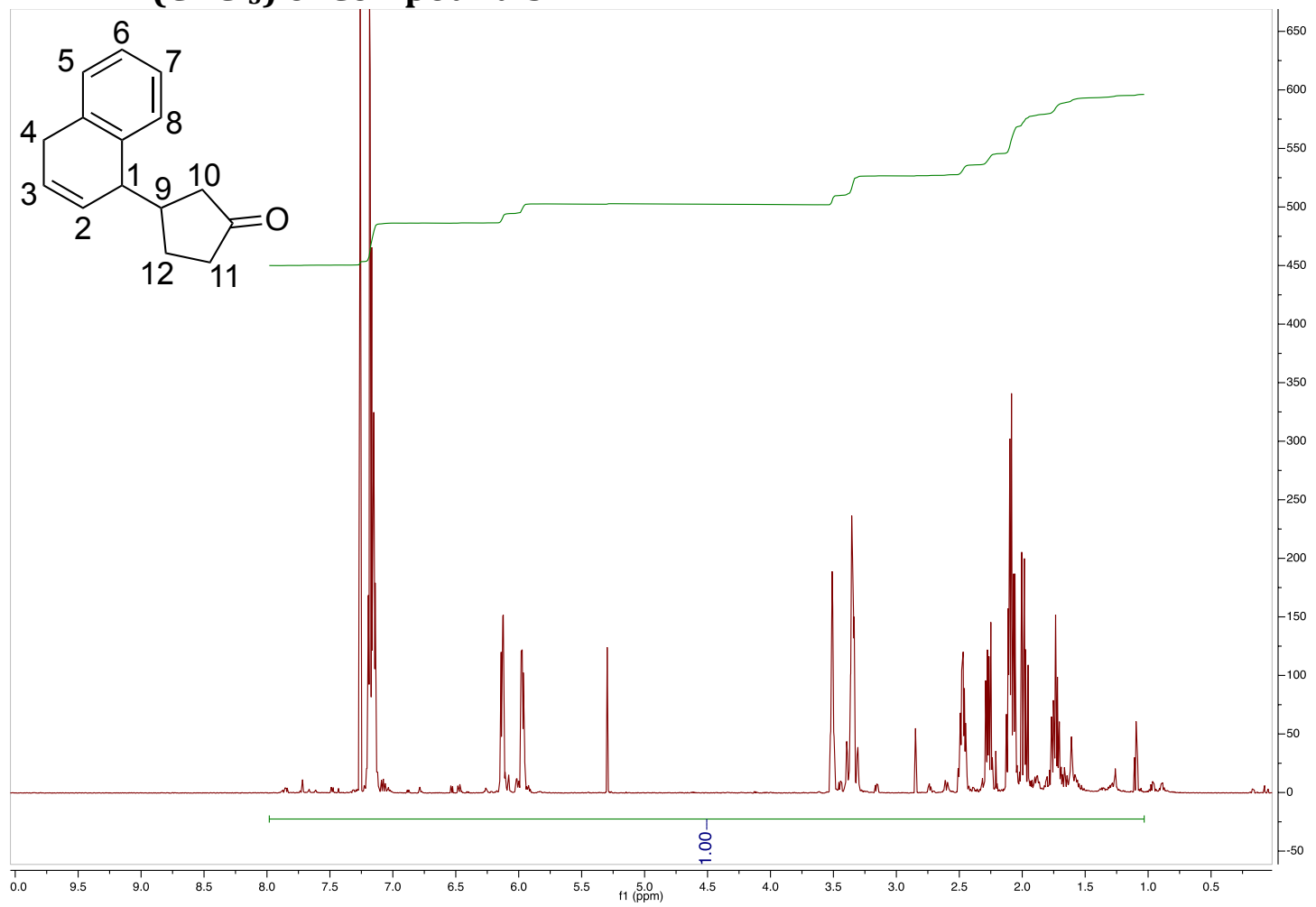

${ }^{13} \mathrm{C} \mathrm{NMR}\left(\mathrm{CDCl}_{3}\right)$ of Compound 51:

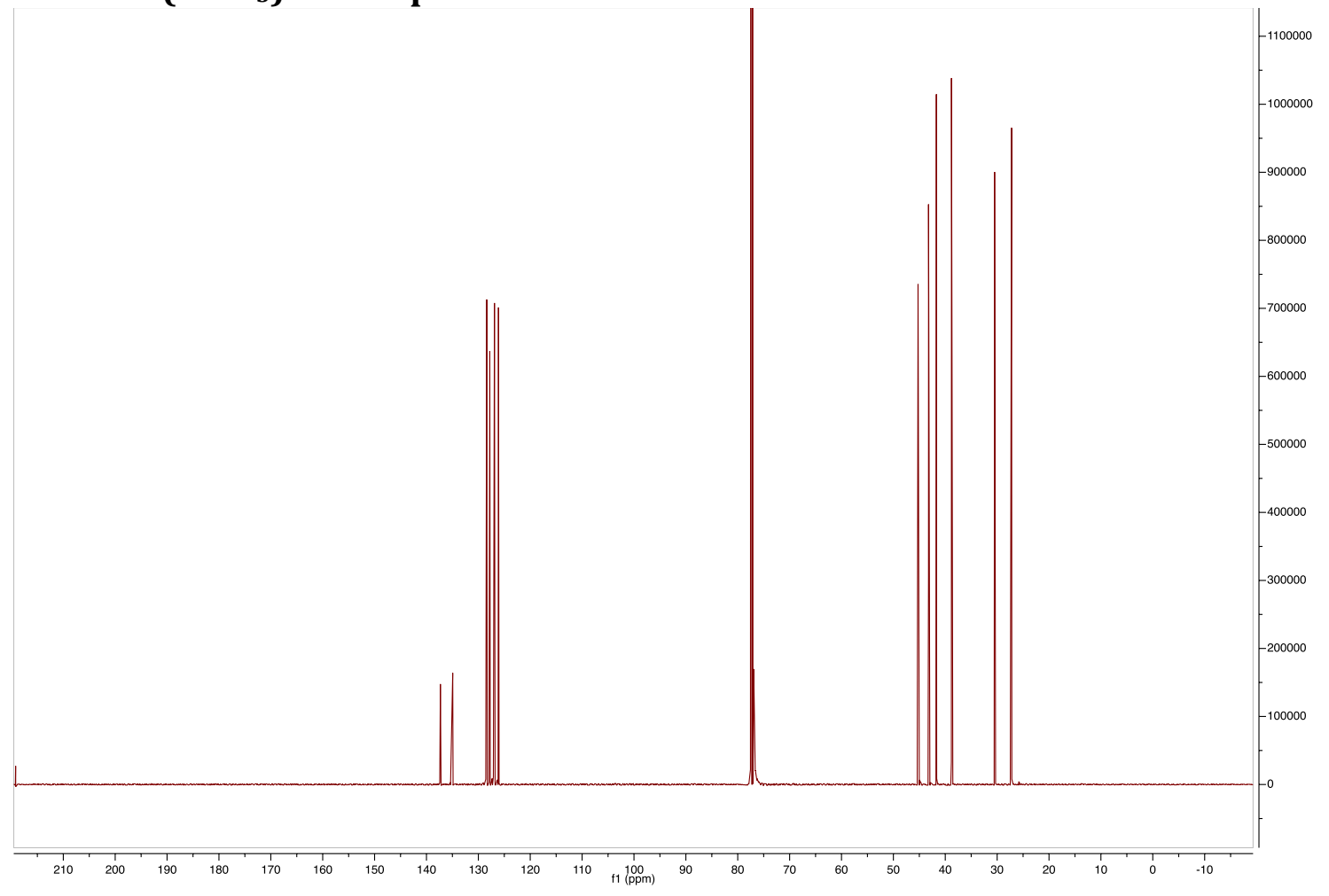


${ }^{1} \mathrm{H}$ NMR $\left(\mathrm{CDCl}_{3}\right)$ of Compound 52 :

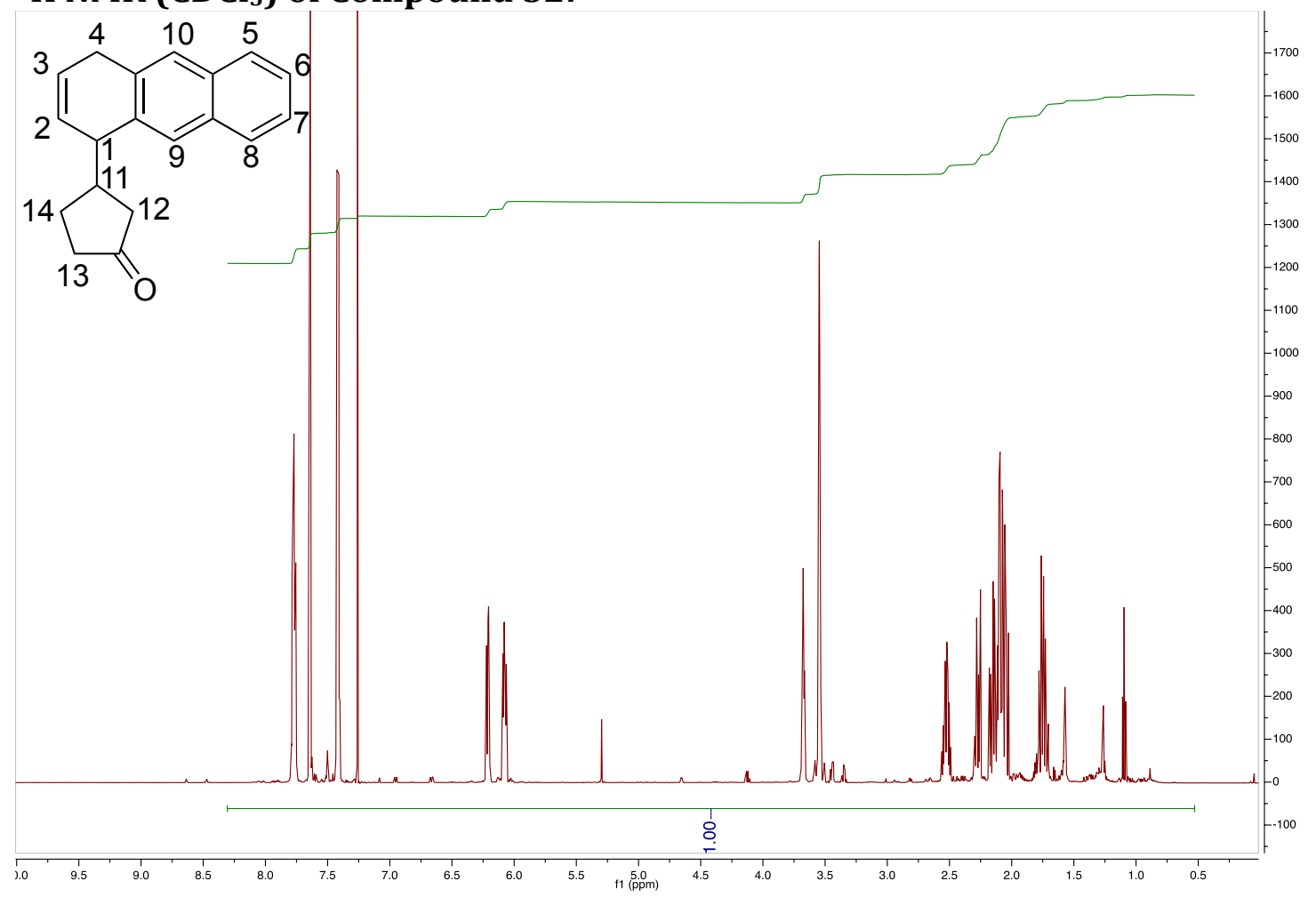

${ }^{13} \mathrm{C}$ NMR $\left(\mathrm{CDCl}_{3}\right)$ of Compound 52 :

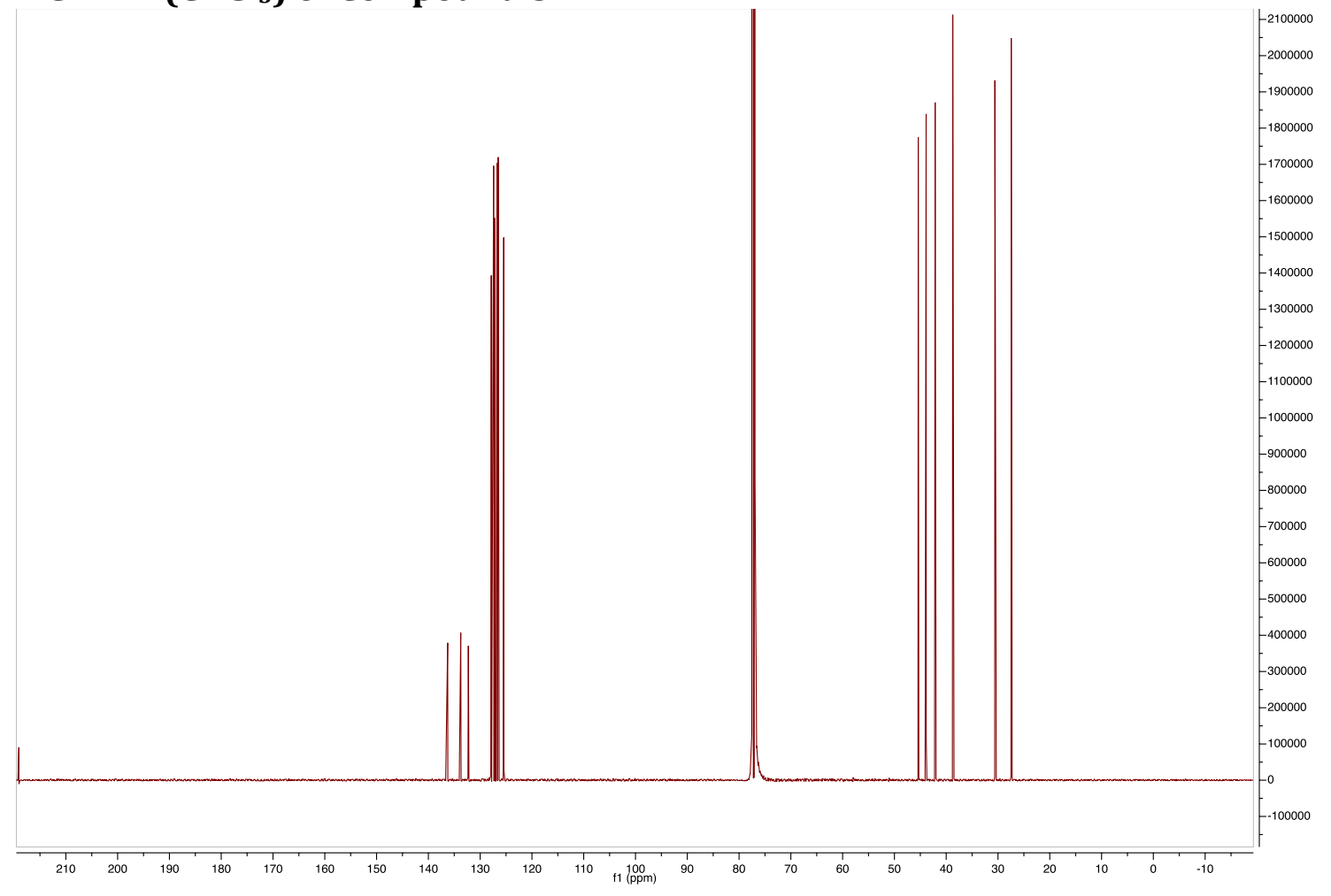


${ }^{1} H_{\text {NMR (d }}{ }^{6}$-Acetone) of Compound 53:

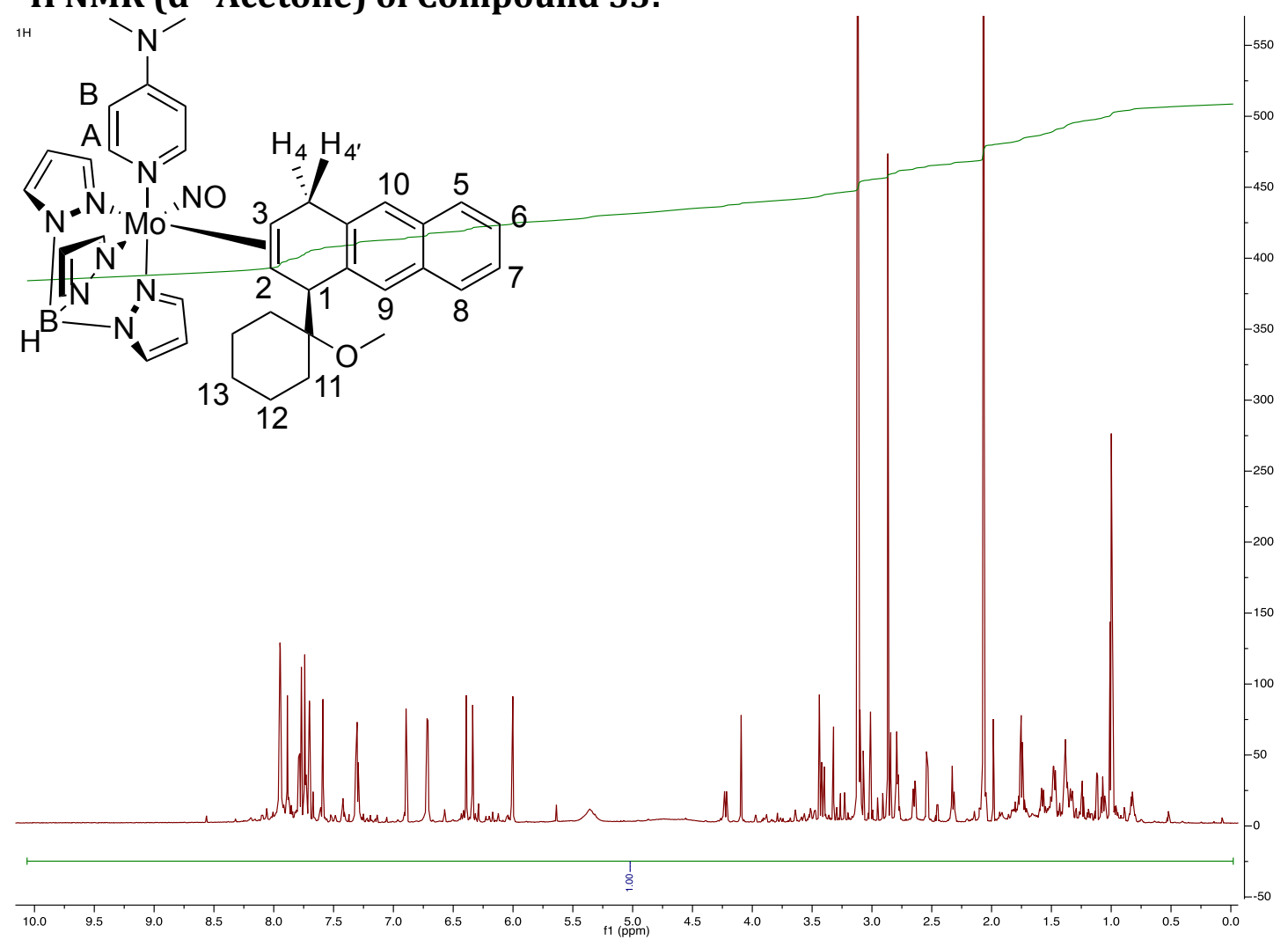

${ }^{13}$ C NMR (d6-Acetone) of Compound 53:

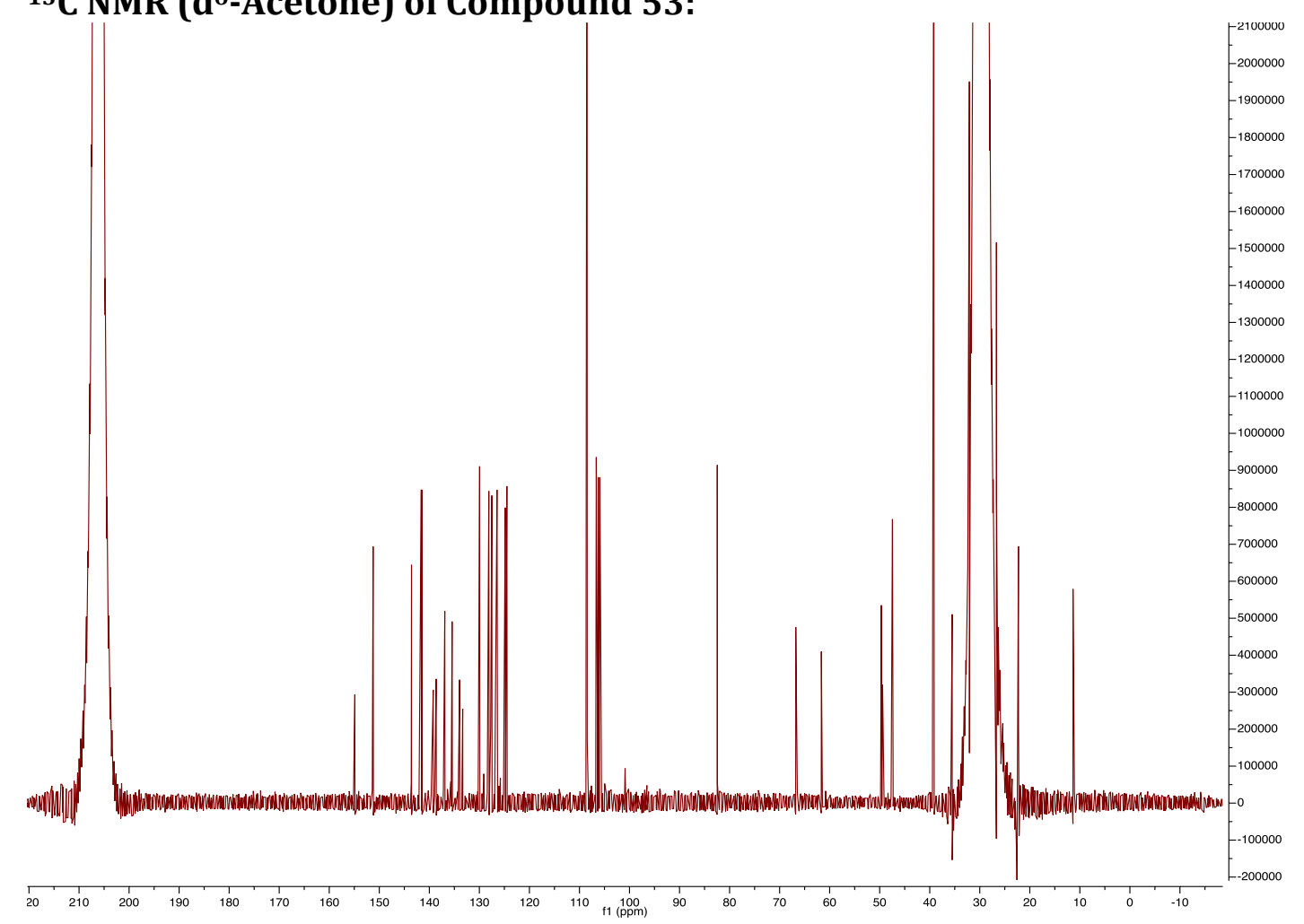


${ }^{1} \mathrm{H}$ NMR ( $d^{6}$-Acetone) of Compound 54:

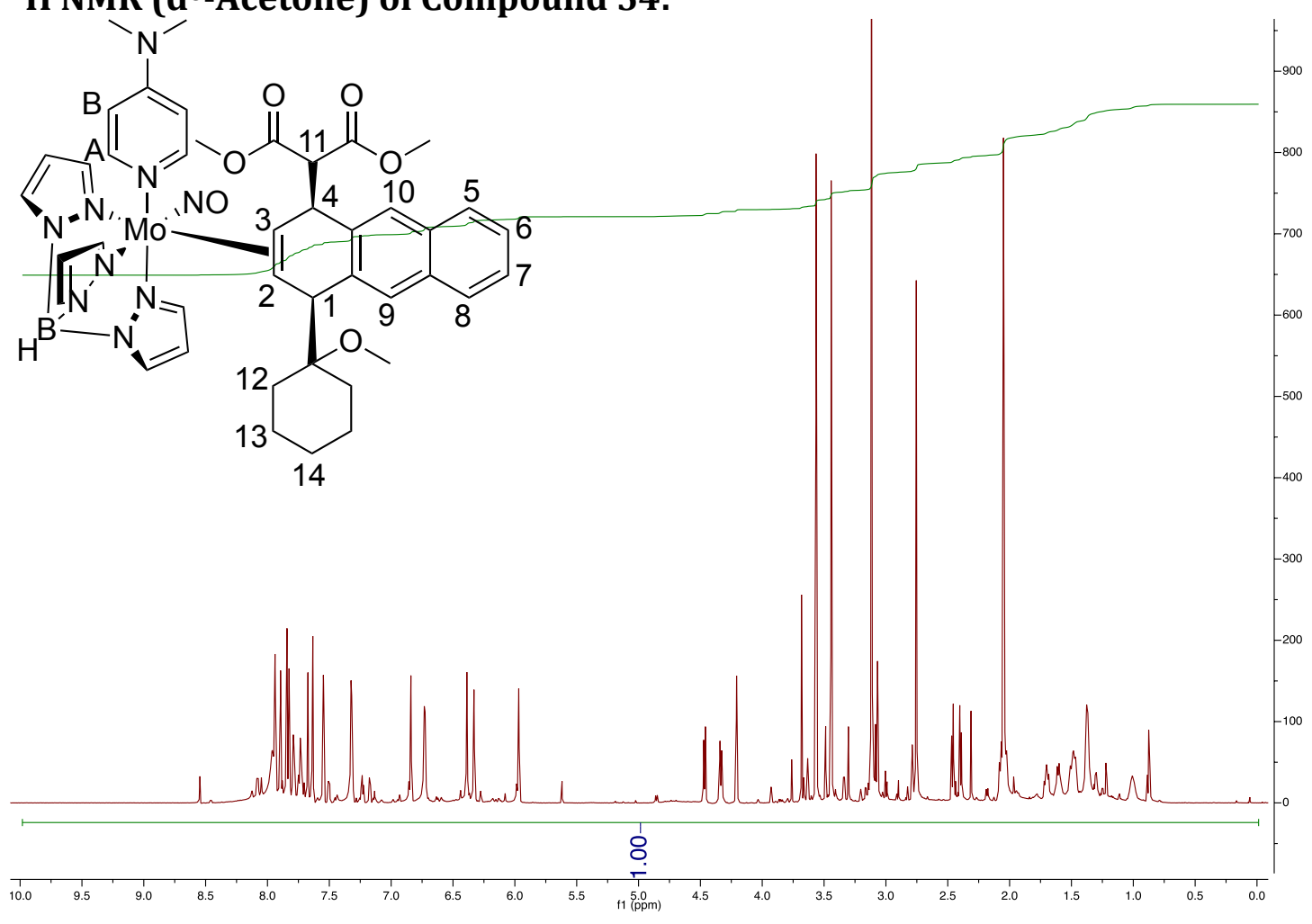

${ }^{13}$ C NMR (d6-Acetone) of Compound 54:

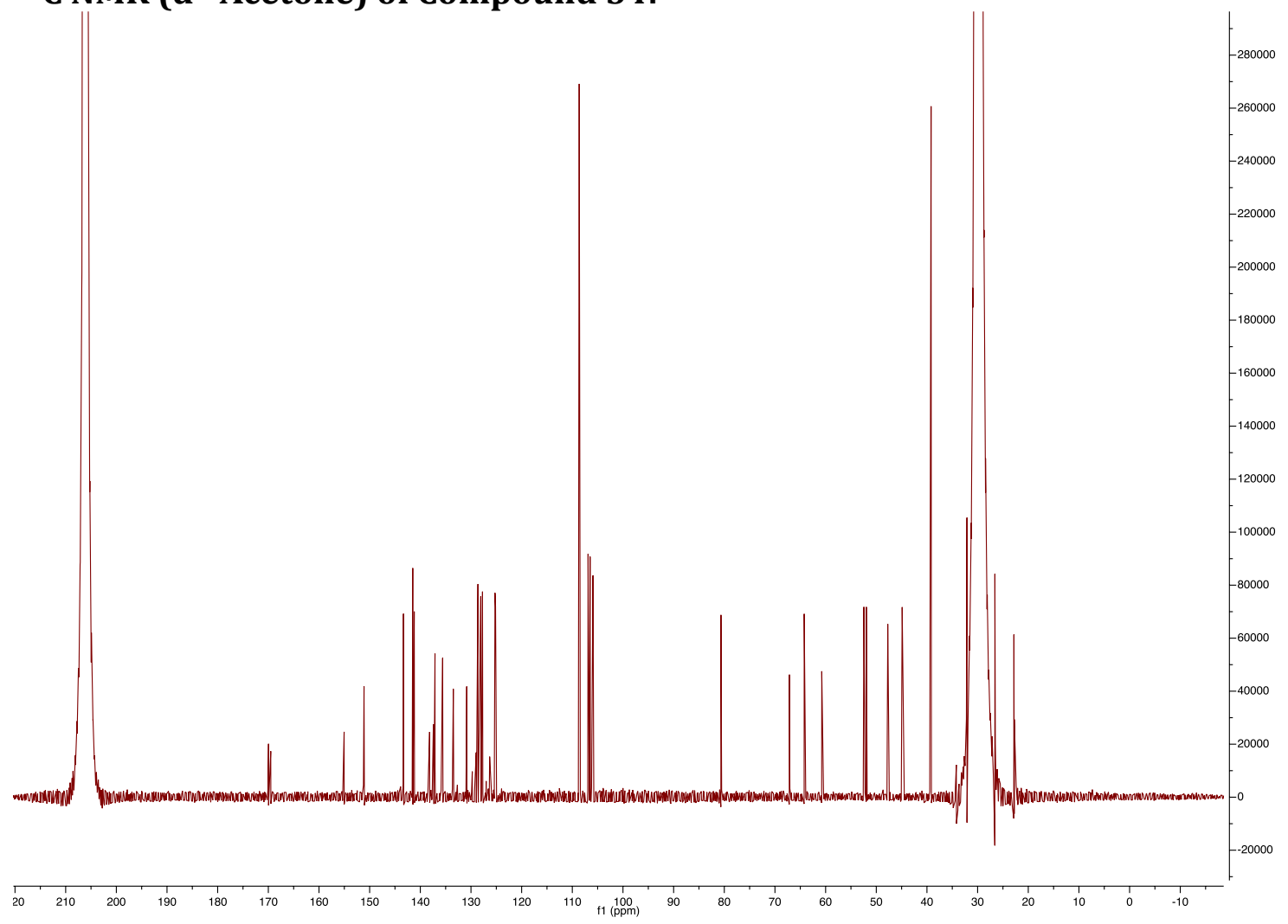


${ }^{1} \mathrm{H}$ NMR ( $\left.\mathrm{CDCl}_{3}\right)$ of Compound 55:

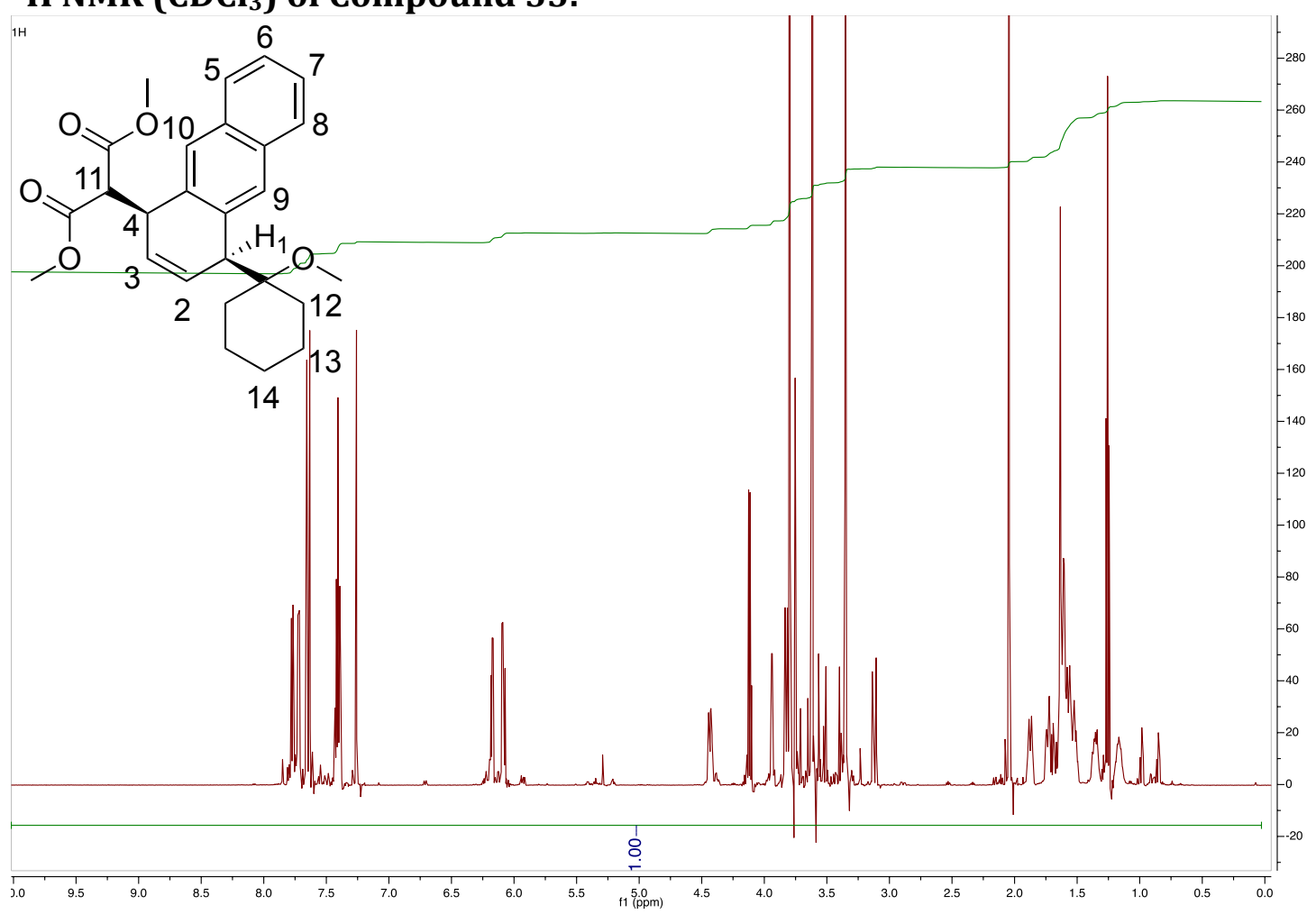

${ }^{13} \mathrm{C} \mathrm{NMR}\left(\mathrm{CDCl}_{3}\right)$ of Compound 55:

${ }_{13 \mathrm{C}}$

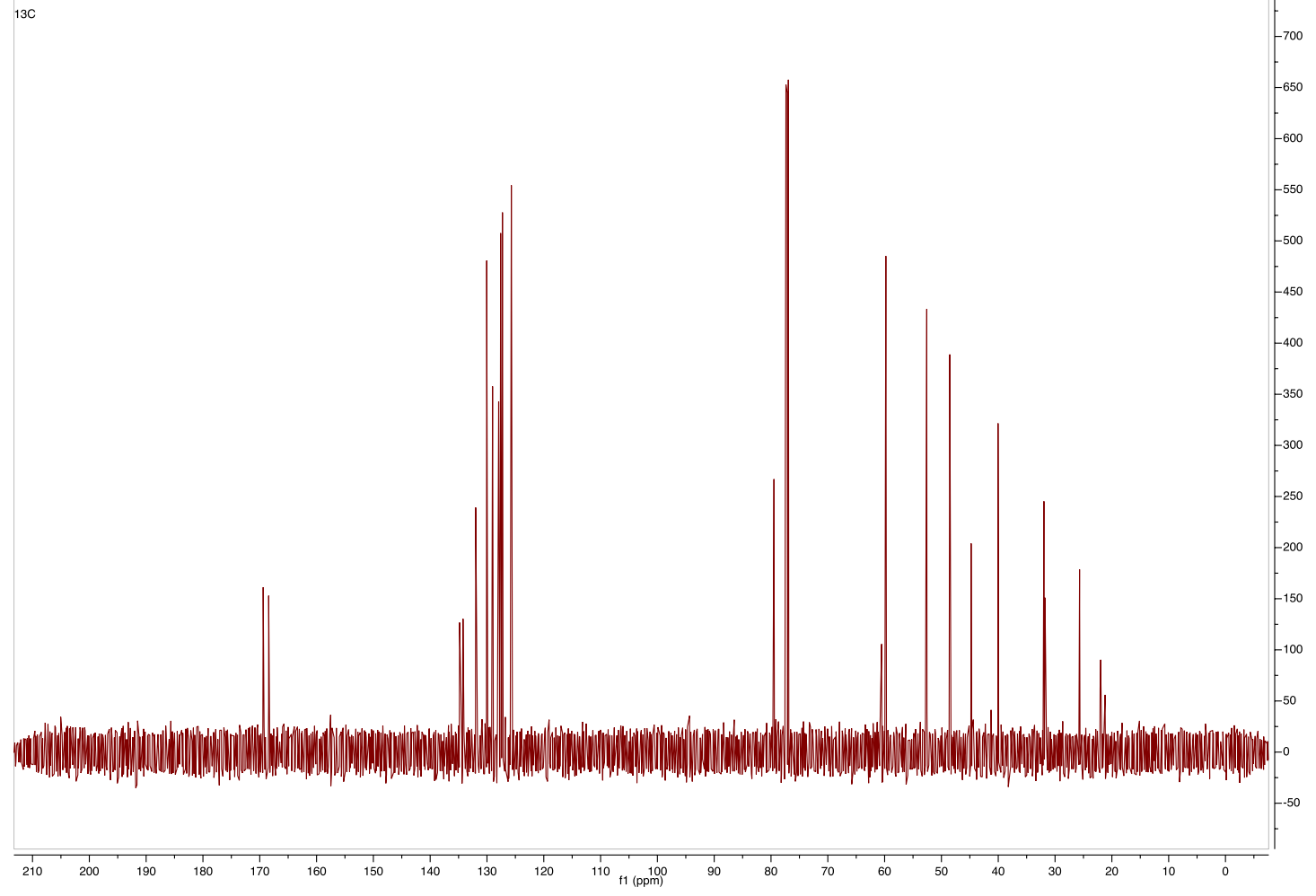


${ }^{1} \mathrm{H}$ NMR ( $\left.\mathrm{CDCl}_{3}\right)$ of Compound 61 :

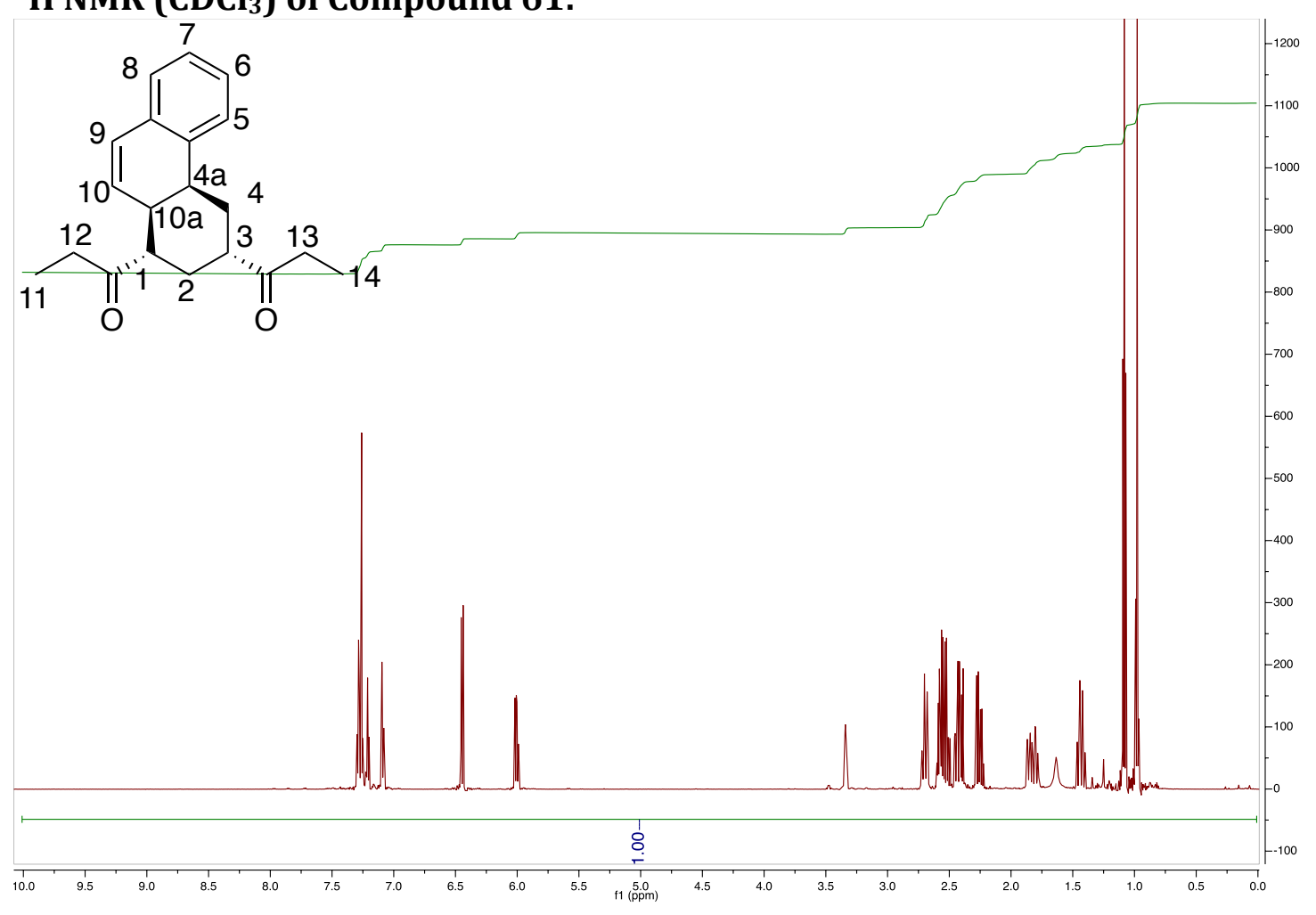

${ }^{13} \mathrm{C} \mathrm{NMR}\left(\mathrm{CDCl}_{3}\right)$ of Compound 61:

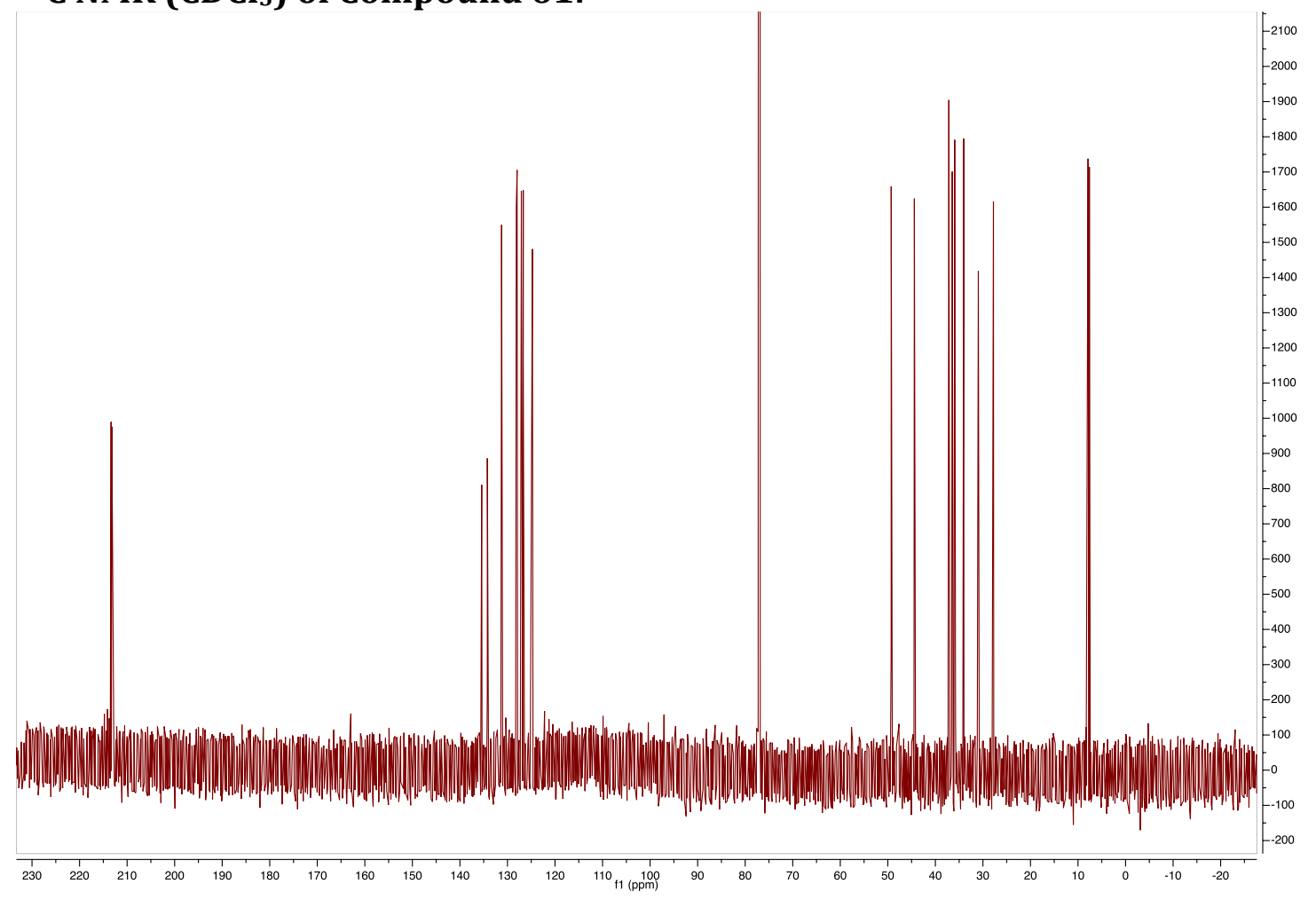


HSQC $\left(\mathrm{CDCl}_{3}\right)$ of Compound 61:

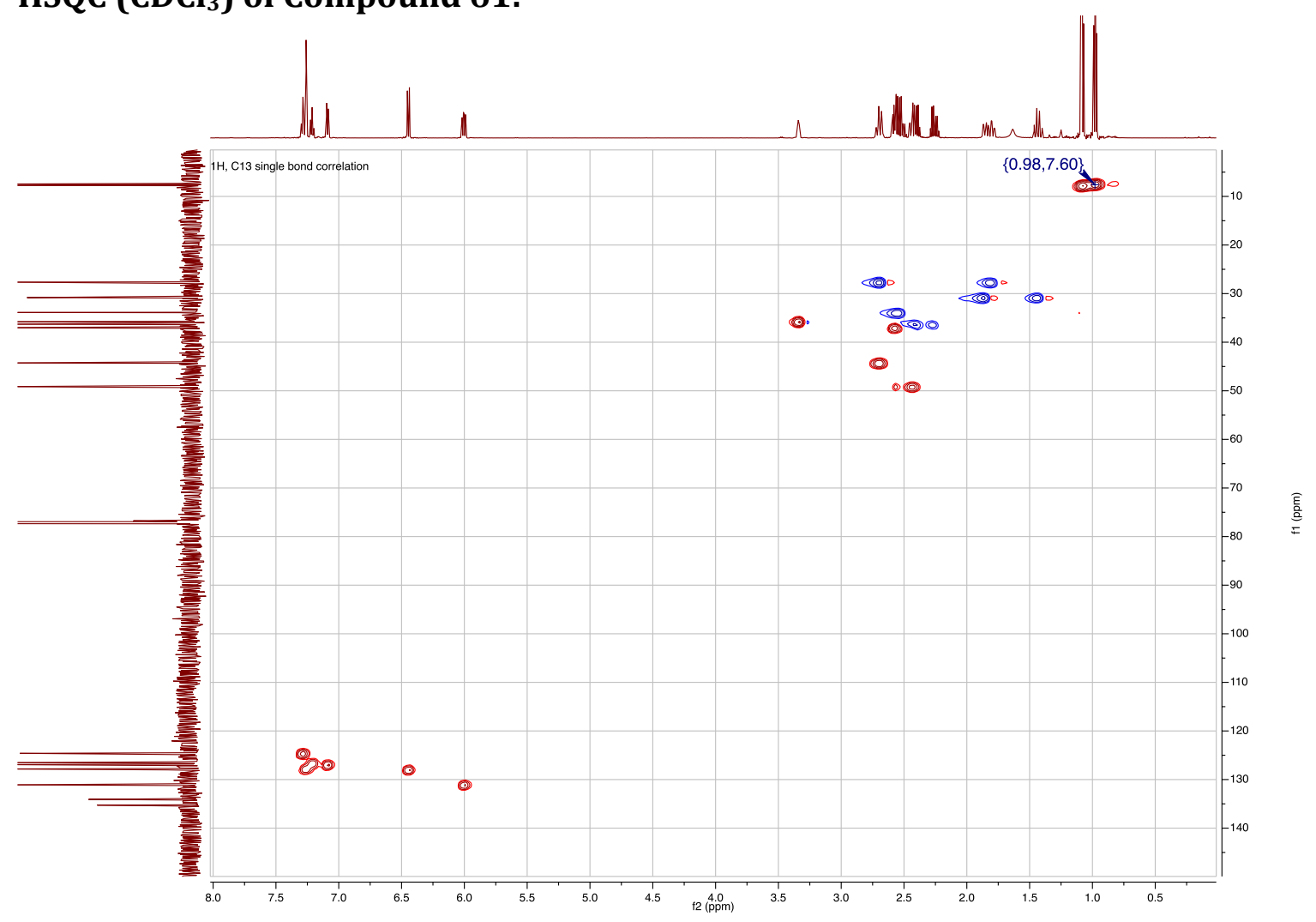

NOESY $\left(\mathrm{CDCl}_{3}\right)$ of Compound 61:

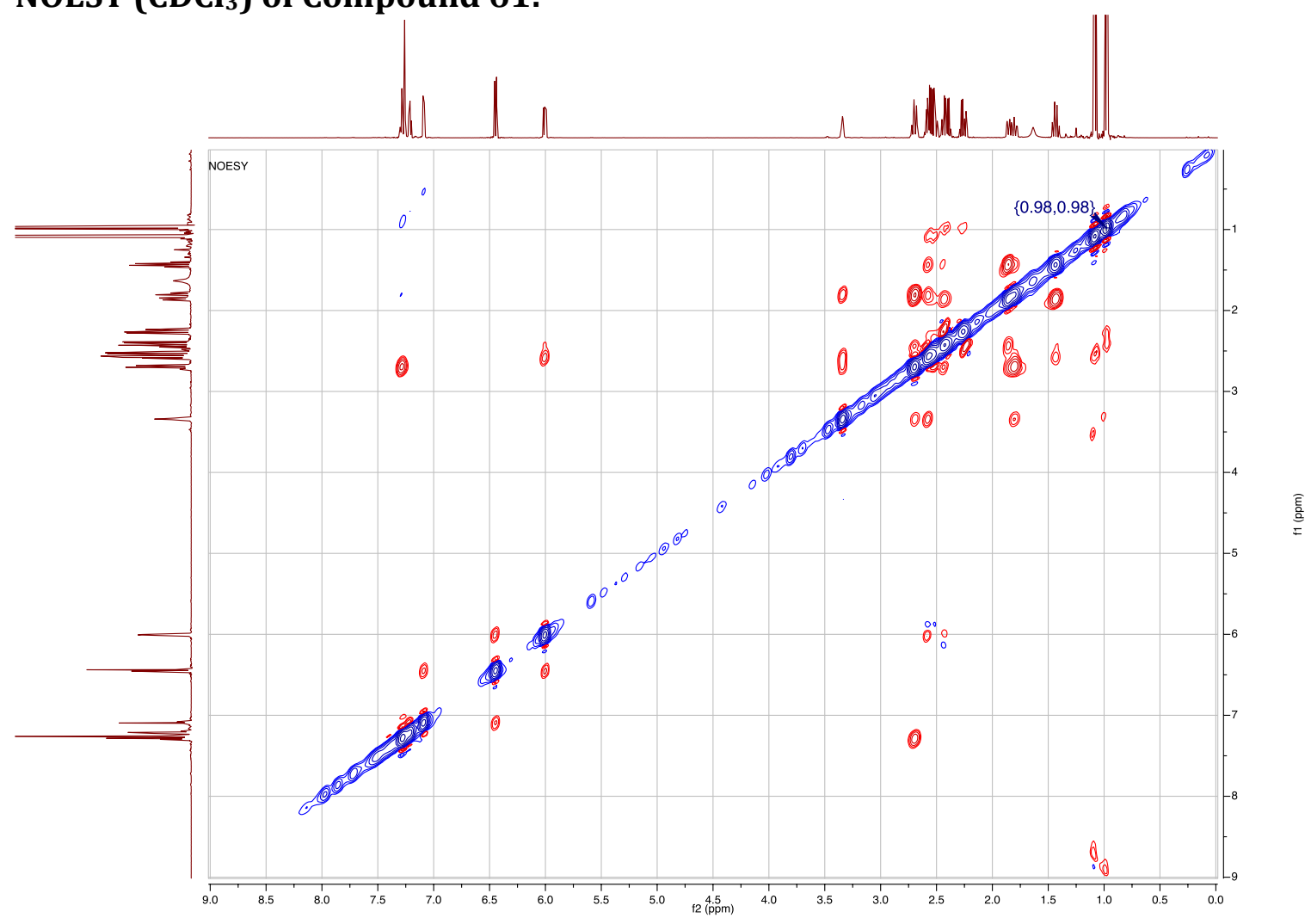


$\operatorname{COSY}\left(\mathrm{CDCl}_{3}\right)$ of Compound 61:

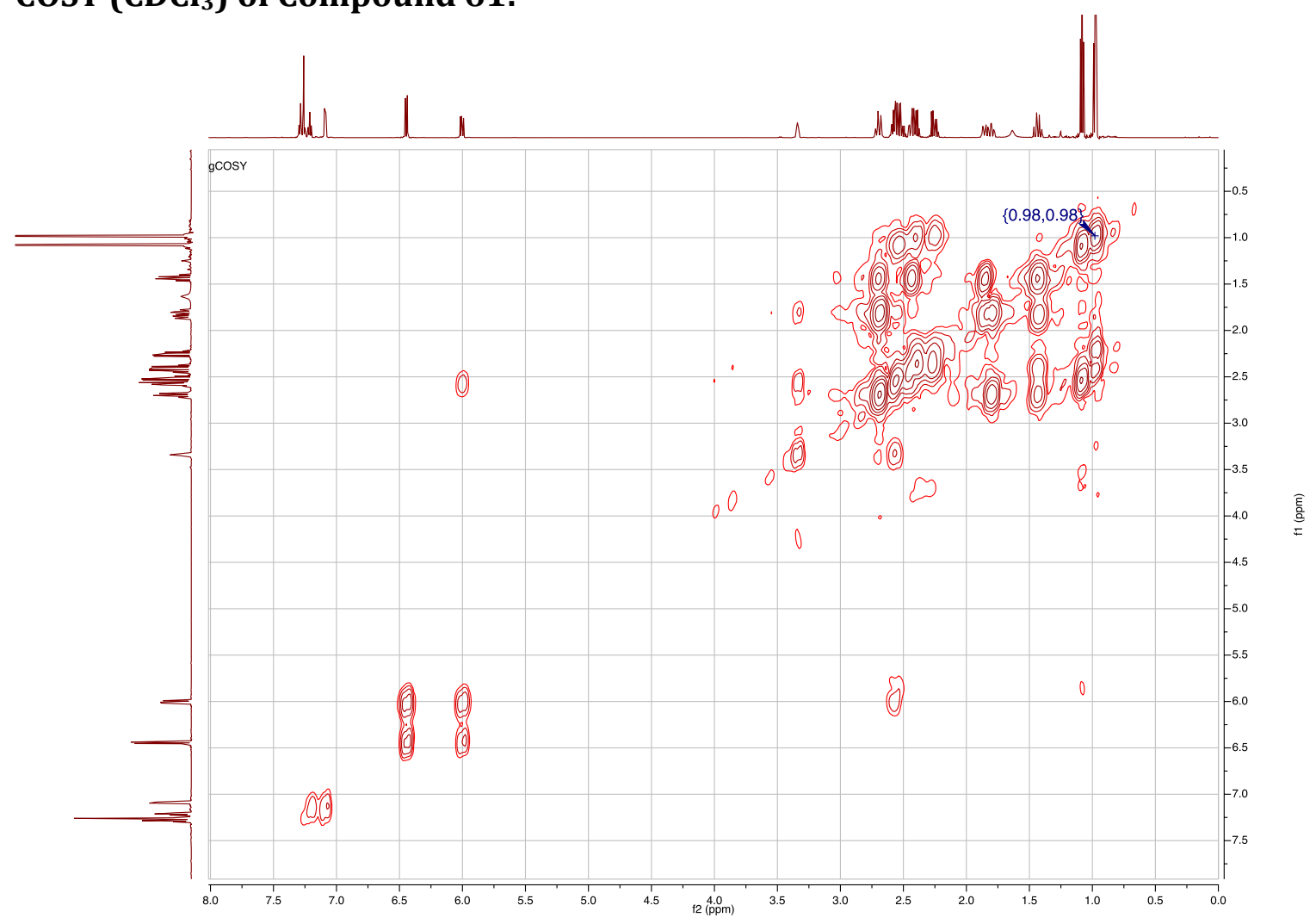




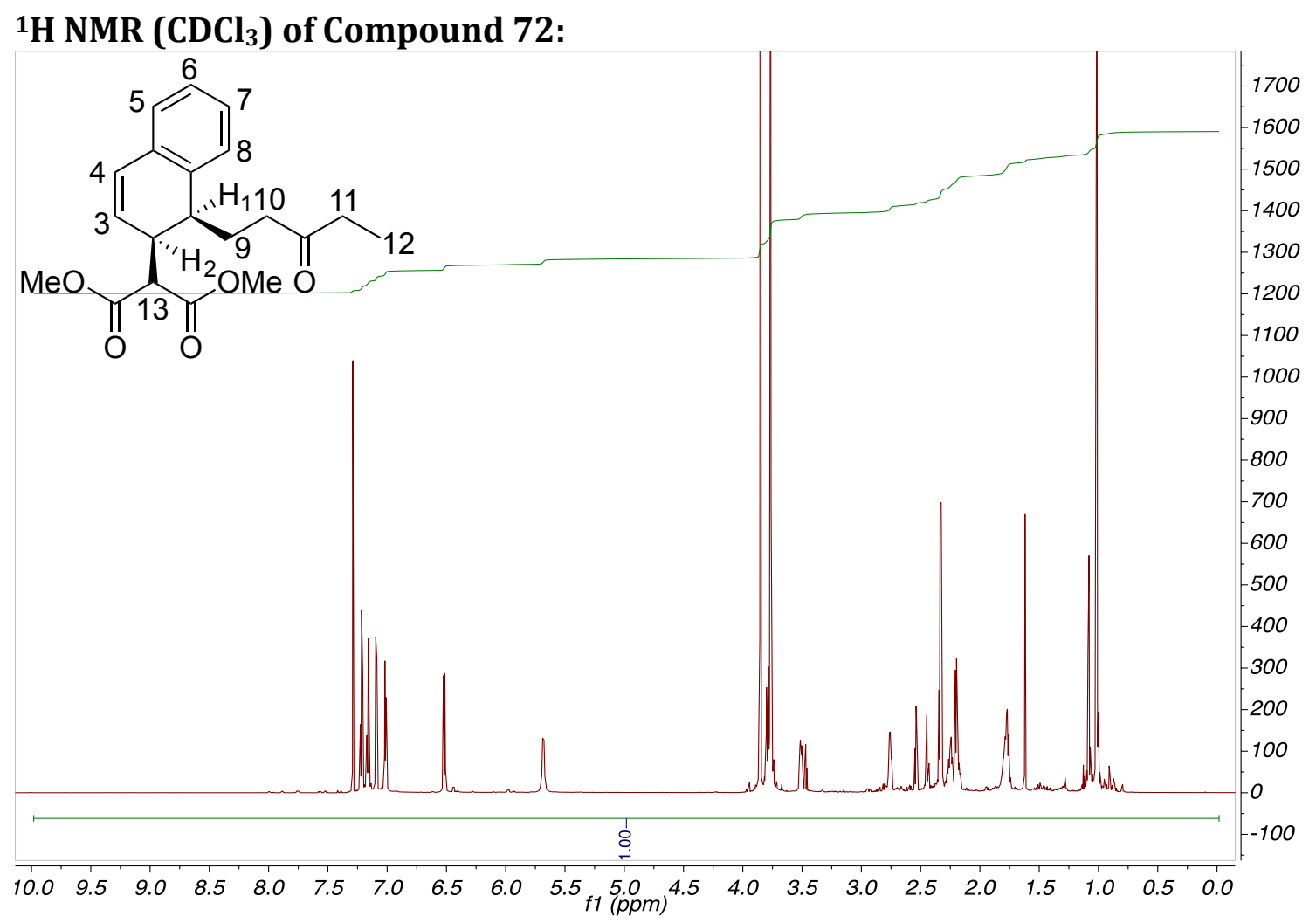

\section{${ }^{13} \mathrm{C}$ NMR $\left(\mathrm{CDCl}_{3}\right)$ of Compound 72:}

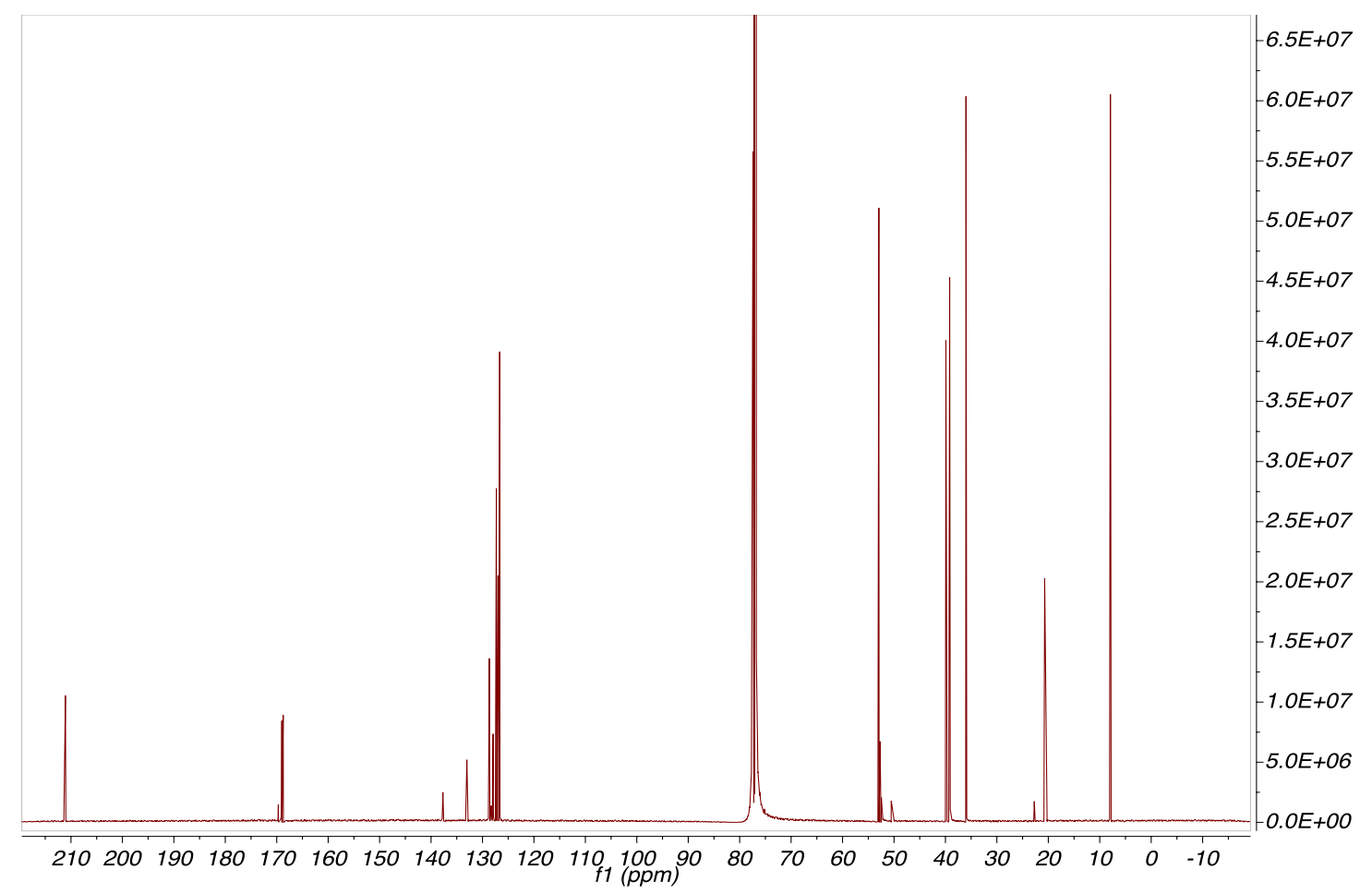




\section{Compound 87}

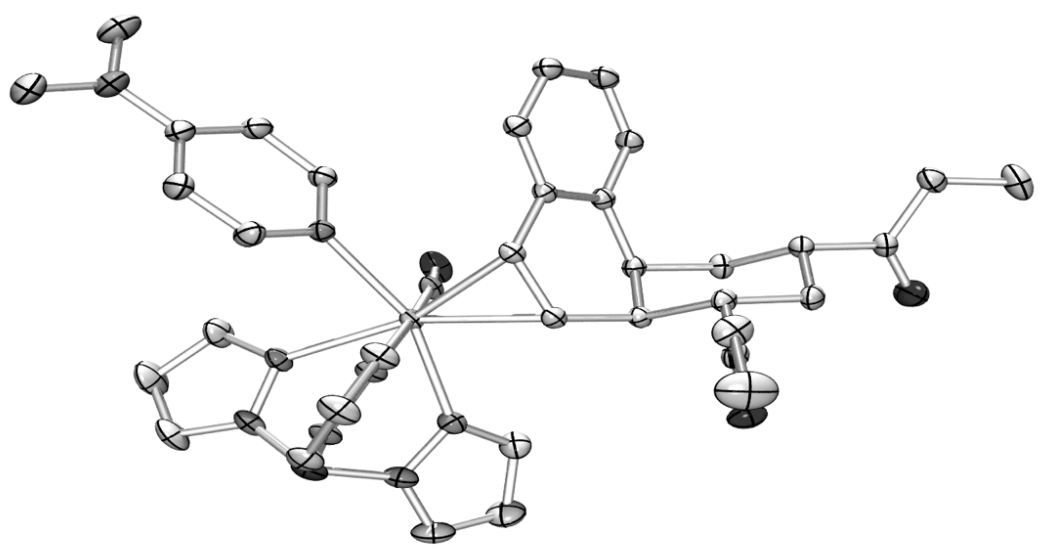




\section{Compound 87}

Table 1. Crystal data and structure refinement for $\mathrm{C}_{42} \mathrm{H}_{56} \mathrm{BMoN}_{9} \mathrm{O}_{4.50}$

Crystal data

Chemical formula

$M_{\mathrm{r}}$

Crystal system, space group

Temperature (K)

$a, b, c(\AA)$

$\beta\left({ }^{\circ}\right)$

$V\left(\AA^{3}\right)$

$Z$

Radiation type

$\mu\left(\mathrm{mm}^{-1}\right)$

Crystal size (mm)

Data collection

Diffractometer

Absorption correction

$T_{\min }, T_{\max }$

No. of measured, independent and observed $[I>2 \sigma(I)]$ reflections

$R_{\text {int }}$

$(\sin \theta / \lambda)_{\max }\left(\AA^{-1}\right)$

Refinement

$R\left[F^{2}>2 \sigma\left(F^{2}\right)\right], w R\left(F^{2}\right), S$

No. of reflections

No. of parameters

$\mathrm{H}$-atom treatment

$\left.\Delta\rangle_{\max }, \Delta\right\rangle_{\min }\left(\mathrm{e} \AA^{-3}\right)$

Compound 87
$\mathrm{C}_{42} \mathrm{H}_{56} \mathrm{BMoN}_{9} \mathrm{O}_{4.50}$

865.70

Monoclinic, $P 2_{1} / n$

153

10.0104 (11), 19.628 (2), 23.526 (3)

$101.949(2)$

$4522.2(9)$

4

Mo $K \alpha$

0.34

$0.44 \times 0.38 \times 0.36$

Bruker APEX-II CCD

Empirical (using intensity measurements) Bruker SADABS

$0.678,0.747$

111328, 21907, 19447

0.026

0.851

$0.051,0.143,1.04$

21907

549

$\mathrm{H}$ atoms treated by a mixture of independent and constrained refinement $1.04,-1.98$ 
Table 2. Fractional atomic coordinates and isotropic or equivalent isotropic displacement parameters $\left(\AA^{2}\right)$ for $\mathrm{C}_{42} \mathrm{H}_{56} \mathrm{BMoN}_{9} \mathrm{O}_{4.5}$

\begin{tabular}{|c|c|c|c|c|}
\hline & $x$ & $y$ & $z$ & $U_{\text {iso }} * / U_{\text {eq }}$ \\
\hline Mo & $0.84250(2)$ & $0.65147(2)$ & $0.32291(2)$ & $0.01664(4)$ \\
\hline $\mathrm{O} 1$ & $0.7640(3)$ & $0.62989(10)$ & $0.65262(8)$ & $0.0474(5)$ \\
\hline $\mathrm{O} 2$ & $0.51219(16)$ & $0.76134(8)$ & $0.45716(7)$ & $0.0317(3)$ \\
\hline $\mathrm{O} 3$ & $1.08043(14)$ & $0.58696(9)$ & $0.40100(7)$ & $0.0316(3)$ \\
\hline $\mathrm{O} 4$ & $0.2012(4)$ & $0.88504(17)$ & $0.53952(14)$ & $0.0889(10)$ \\
\hline O5 & $0.1355(12)$ & $0.0534(10)$ & $0.3473(4)$ & $0.216(9)$ \\
\hline N1 & $0.83314(15)$ & $0.56303(8)$ & $0.26317(6)$ & $0.0212(2)$ \\
\hline $\mathrm{N} 2$ & $0.9071(3)$ & $0.41238(11)$ & $0.14647(10)$ & $0.0419(5)$ \\
\hline N3 & $0.98066(15)$ & $0.68633(8)$ & $0.26574(7)$ & $0.0233(3)$ \\
\hline N4 & $0.97560(16)$ & $0.75017(9)$ & $0.24344(7)$ & $0.0265(3)$ \\
\hline N5 & $0.88812(15)$ & $0.75500(8)$ & $0.35838(7)$ & $0.0227(2)$ \\
\hline N6 & $0.89011(18)$ & $0.80927(8)$ & $0.32225(8)$ & $0.0268(3)$ \\
\hline N7 & $0.68590(14)$ & $0.70855(7)$ & $0.25596(7)$ & $0.0210(2)$ \\
\hline N8 & $0.72334(16)$ & $0.76805(8)$ & $0.23349(7)$ & $0.0231(3)$ \\
\hline N9 & $0.97943(14)$ & $0.61333(8)$ & $0.37146(6)$ & $0.0197(2)$ \\
\hline $\mathrm{C} 1$ & $0.67464(15)$ & $0.59264(8)$ & $0.34973(7)$ & $0.0182(2)$ \\
\hline H1 & 0.5898 & 0.5889 & 0.3188 & $0.022^{*}$ \\
\hline $\mathrm{C} 2$ & $0.68963(15)$ & $0.65663(8)$ & $0.37943(7)$ & $0.0184(2)$ \\
\hline $\mathrm{H} 2$ & 0.6132 & 0.6884 & 0.3631 & $0.022 *$ \\
\hline $\mathrm{C} 3$ & $0.71699(15)$ & $0.65534(8)$ & $0.44561(7)$ & $0.0184(2)$ \\
\hline H3 & 0.7552 & 0.7006 & 0.4603 & $0.022 *$ \\
\hline $\mathrm{C} 4$ & $0.57961(16)$ & $0.64485(9)$ & $0.46529(8)$ & $0.0208(3)$ \\
\hline H4 & 0.5378 & 0.6012 & 0.4484 & $0.025^{*}$ \\
\hline $\mathrm{C} 5$ & 0.59909 (19) & $0.64112(10)$ & $0.53190(8)$ & $0.0250(3)$ \\
\hline $\mathrm{H} 5 \mathrm{~A}$ & 0.6331 & 0.6855 & 0.5491 & $0.030^{*}$ \\
\hline $\mathrm{H} 5 \mathrm{~B}$ & 0.5102 & 0.6318 & 0.5425 & $0.030^{*}$ \\
\hline C6 & 0.70110 (19) & $0.58478(9)$ & $0.55639(7)$ & $0.0231(3)$ \\
\hline H6 & 0.6639 & 0.5401 & 0.5398 & $0.028^{*}$ \\
\hline $\mathrm{C} 7$ & $0.83756(18)$ & $0.59807(10)$ & $0.53816(7)$ & $0.0229(3)$ \\
\hline H7A & 0.9033 & 0.5618 & 0.5542 & $0.027 *$ \\
\hline H7B & 0.8755 & 0.6421 & 0.5546 & $0.027^{*}$ \\
\hline $\mathrm{C} 8$ & $0.82003(16)$ & $0.59989(8)$ & $0.47157(7)$ & $0.0191(2)$ \\
\hline H8 & 0.9102 & 0.6134 & 0.4630 & $0.023^{*}$ \\
\hline C9 & $0.78180(16)$ & $0.53177(8)$ & $0.44195(7)$ & $0.0188(2)$ \\
\hline $\mathrm{C} 10$ & 0.81479 (19) & $0.47026(9)$ & $0.47148(8)$ & $0.0238(3)$ \\
\hline
\end{tabular}




\begin{tabular}{|c|c|c|c|c|}
\hline H10 & 0.8644 & 0.4712 & 0.5105 & $0.029 *$ \\
\hline $\mathrm{C} 11$ & $0.7773(2)$ & $0.40791(10)$ & $0.44542(9)$ & $0.0279(3)$ \\
\hline H11 & 0.8008 & 0.3669 & 0.4665 & $0.033^{*}$ \\
\hline $\mathrm{C} 12$ & $0.7049(2)$ & $0.40571(9)$ & $0.38805(9)$ & $0.0266(3)$ \\
\hline H12 & 0.6767 & 0.3633 & 0.3701 & $0.032 *$ \\
\hline $\mathrm{C} 13$ & $0.67442(18)$ & $0.46618(9)$ & $0.35732(8)$ & $0.0222(3)$ \\
\hline H13 & 0.6275 & 0.4644 & 0.3179 & $0.027^{*}$ \\
\hline $\mathrm{C} 14$ & $0.71126(15)$ & $0.52967(8)$ & $0.38317(7)$ & $0.0179(2)$ \\
\hline $\mathrm{C} 15$ & $0.7202(2)$ & $0.58162(10)$ & $0.62227(8)$ & $0.0258(3)$ \\
\hline $\mathrm{C} 16$ & $0.6814(2)$ & $0.51600(11)$ & $0.64723(9)$ & $0.0294(3)$ \\
\hline H16A & 0.7419 & 0.4795 & 0.6381 & $0.035^{*}$ \\
\hline H16B & 0.5868 & 0.5043 & 0.6277 & $0.035^{*}$ \\
\hline $\mathrm{C} 17$ & $0.6899(3)$ & $0.51693(16)$ & $0.71239(10)$ & $0.0413(5)$ \\
\hline H17A & 0.7846 & 0.5249 & 0.7324 & $0.062 *$ \\
\hline H17B & 0.6587 & 0.4731 & 0.7247 & $0.062 *$ \\
\hline $\mathrm{H} 17 \mathrm{C}$ & 0.6318 & 0.5535 & 0.7221 & $0.062 *$ \\
\hline $\mathrm{C} 18$ & $0.48229(17)$ & $0.70278(10)$ & $0.44298(8)$ & $0.0235(3)$ \\
\hline C19 & $0.3486(2)$ & $0.68405(13)$ & $0.40388(11)$ & $0.0350(4)$ \\
\hline H19A & 0.3004 & 0.6515 & 0.4248 & $0.042 *$ \\
\hline H19B & 0.3678 & 0.6603 & 0.3693 & $0.042 *$ \\
\hline $\mathrm{C} 20$ & $0.2542(3)$ & $0.74337(18)$ & $0.38356(17)$ & $0.0572(8)$ \\
\hline $\mathrm{H} 20 \mathrm{~A}$ & 0.2406 & 0.7698 & 0.4173 & $0.086^{*}$ \\
\hline $\mathrm{H} 20 \mathrm{~B}$ & 0.1660 & 0.7262 & 0.3623 & $0.086^{*}$ \\
\hline $\mathrm{H} 20 \mathrm{C}$ & 0.2948 & 0.7726 & 0.3579 & $0.086^{*}$ \\
\hline $\mathrm{C} 21$ & 0.89953 (19) & $0.50447(9)$ & $0.28137(8)$ & $0.0243(3)$ \\
\hline $\mathrm{H} 21$ & 0.9305 & 0.4979 & 0.3220 & $0.029^{*}$ \\
\hline $\mathrm{C} 22$ & $0.9249(2)$ & $0.45375(10)$ & $0.24462(9)$ & $0.0287(3)$ \\
\hline $\mathrm{H} 22$ & 0.9726 & 0.4139 & 0.2602 & $0.034 *$ \\
\hline $\mathrm{C} 23$ & $0.8805(2)$ & $0.46073(10)$ & $0.18393(9)$ & 0.0300 \\
\hline $\mathrm{C} 24$ & 0.8075 & $0.52061(11)$ & $0.16505(9)$ & $0.0328(4)$ \\
\hline $\mathrm{H} 24$ & 0.7719 & 0.5278 & 0.1248 & $0.039 *$ \\
\hline $\mathrm{C} 25$ & $0.7880(2)$ & $0.56877(10)$ & $0.20513(8)$ & $0.0282(3)$ \\
\hline H25 & 0.7393 & 0.6089 & 0.1909 & $0.034 *$ \\
\hline $\mathrm{C} 26$ & $0.9782(4)$ & $0.35031(14)$ & $0.16825(16)$ & $0.0562(8)$ \\
\hline $\mathrm{H} 26 \mathrm{~A}$ & 1.0657 & 0.3618 & 0.1937 & $0.084 *$ \\
\hline H26B & 0.9944 & 0.3229 & 0.1355 & $0.084 *$ \\
\hline $\mathrm{H} 26 \mathrm{C}$ & 0.9224 & 0.3243 & 0.1902 & $0.084 *$ \\
\hline $\mathrm{C} 27$ & $0.8654(4)$ & $0.42189(16)$ & $0.08404(12)$ & $0.0522(7)$ \\
\hline H27A & 0.7664 & 0.4287 & 0.0737 & $0.078^{*}$ \\
\hline
\end{tabular}




\begin{tabular}{|c|c|c|c|c|}
\hline $\mathrm{H} 27 \mathrm{~B}$ & 0.8902 & 0.3815 & 0.0640 & $0.078^{*}$ \\
\hline $\mathrm{H} 27 \mathrm{C}$ & 0.9117 & 0.4619 & 0.0723 & $0.078^{*}$ \\
\hline $\mathrm{C} 28$ & $1.0785(2)$ & $0.75867(14)$ & $0.21485(11)$ & $0.0382(5)$ \\
\hline $\mathrm{H} 28$ & 1.0972 & 0.7990 & 0.1956 & $0.046^{*}$ \\
\hline $\mathrm{C} 29$ & $1.1515(3)$ & $0.69892(16)$ & $0.21834(13)$ & $0.0447(6)$ \\
\hline $\mathrm{H} 29$ & 1.2292 & 0.6897 & 0.2023 & $0.054 *$ \\
\hline $\mathrm{C} 30$ & $1.0867(2)$ & $0.65482(12)$ & $0.25052(11)$ & $0.0333(4)$ \\
\hline $\mathrm{H} 30$ & 1.1138 & 0.6092 & 0.2602 & $0.040^{*}$ \\
\hline C31 & $0.9353(2)$ & $0.86481(11)$ & $0.35385(11)$ & $0.0362(4)$ \\
\hline H31 & 0.9458 & 0.9089 & 0.3387 & $0.043^{*}$ \\
\hline $\mathrm{C} 32$ & $0.9640(3)$ & $0.84749(13)$ & $0.41174(11)$ & $0.0391(5)$ \\
\hline H32 & 0.9976 & 0.8762 & 0.4440 & $0.047 *$ \\
\hline $\mathrm{C} 33$ & $0.9328(2)$ & $0.77820(11)$ & $0.41236(9)$ & $0.0295(4)$ \\
\hline H33 & 0.9420 & 0.7512 & 0.4465 & $0.035^{*}$ \\
\hline C34 & $0.6168(2)$ & $0.79340(10)$ & $0.19456(9)$ & $0.0284(3)$ \\
\hline H34 & 0.6174 & 0.8344 & 0.1731 & $0.034 *$ \\
\hline $\mathrm{C} 35$ & $0.5071(2)$ & $0.75019(12)$ & $0.19094(10)$ & $0.0323(4)$ \\
\hline H35 & 0.4186 & 0.7549 & 0.1671 & $0.039^{*}$ \\
\hline $\mathrm{C} 36$ & $0.55511(19)$ & $0.69800(10)$ & $0.23004(9)$ & $0.0272(3)$ \\
\hline H36 & 0.5022 & 0.6600 & 0.2373 & $0.033^{*}$ \\
\hline C37 & $0.1838(9)$ & $0.8166(4)$ & $0.5613(2)$ & 0.138 \\
\hline $\mathrm{H} 37 \mathrm{~A}$ & 0.1292 & 0.7880 & 0.5303 & $0.166^{*}$ \\
\hline H37B & 0.1369 & 0.8185 & 0.5944 & $0.166^{*}$ \\
\hline C38 & $0.3268(8)$ & $0.7879(3)$ & $0.5805(3)$ & $0.110(2)$ \\
\hline $\mathrm{H} 38 \mathrm{~A}$ & 0.3446 & 0.7761 & 0.6223 & $0.132 *$ \\
\hline $\mathrm{H} 38 \mathrm{~B}$ & 0.3376 & 0.7463 & 0.5581 & $0.132 *$ \\
\hline C39 & $0.4194(8)$ & $0.8397(3)$ & $0.5700(3)$ & $0.113(2)$ \\
\hline H39A & 0.4961 & 0.8201 & 0.5547 & $0.136^{*}$ \\
\hline H39B & 0.4564 & 0.8660 & 0.6057 & $0.136^{*}$ \\
\hline $\mathrm{C} 40$ & $0.3327(8)$ & $0.8817(3)$ & $0.5267(2)$ & $0.0982(18)$ \\
\hline $\mathrm{H} 40 \mathrm{~A}$ & 0.3717 & 0.9281 & 0.5270 & $0.118^{*}$ \\
\hline H40B & 0.3270 & 0.8619 & 0.4876 & $0.118^{*}$ \\
\hline $\mathrm{C} 42$ & $0.2807(16)$ & $0.0292(10)$ & $0.4234(5)$ & $0.147(7)$ \\
\hline $\mathrm{H} 42 \mathrm{~A}$ & 0.3577 & 0.0618 & 0.4304 & $0.176^{*}$ \\
\hline $\mathrm{H} 42 \mathrm{~B}$ & 0.3057 & -0.0113 & 0.4485 & $0.176^{*}$ \\
\hline $\mathrm{C} 43$ & $0.1550(17)$ & $0.0611(14)$ & $0.4339(5)$ & $0.216(13)$ \\
\hline $\mathrm{H} 43 \mathrm{~A}$ & 0.1005 & 0.0288 & 0.4521 & $0.259^{*}$ \\
\hline H43B & 0.1752 & 0.1022 & 0.4586 & $0.259^{*}$ \\
\hline $\mathrm{C} 41$ & $0.2501(15)$ & $0.0132(10)$ & $0.3727(5)$ & $0.150(7)$ \\
\hline
\end{tabular}




$\begin{array}{lllll}\text { H41A } & 0.2267 & -0.0359 & 0.3689 & 0.180^{*} \\ \text { H41B } & 0.3271 & 0.0219 & 0.3532 & 0.180^{*} \\ \text { C44 } & 0.097(2) & 0.0757(12) & 0.3840(7) & 0.220(13) \\ \text { H44A } & -0.0003 & 0.0624 & 0.3793 & 0.264^{*} \\ \text { H44B } & 0.0991 & 0.1259 & 0.3801 & 0.264^{*} \\ \text { B1 } & 0.8662(2) & 0.79986(11) & 0.25592(10) & 0.0269(4) \\ \text { H1B } & 0.880(3) & 0.8484(14) & 0.2359(12) & 0.027(7)^{*}\end{array}$




\section{Compound 87}

Table 3. Atomic displacement parameters $\left(\AA^{2}\right)$ for $\mathrm{C}_{42} \mathrm{H}_{56} \mathrm{BMoN}_{9} \mathrm{O}_{4.5}$

\begin{tabular}{|c|c|c|c|c|c|c|}
\hline & $U^{11}$ & $U^{22}$ & $U^{33}$ & $U^{12}$ & $U^{13}$ & $U^{23}$ \\
\hline Mo & $0.01410(5)$ & $0.01836(6)$ & $0.01768(6)$ & $0.00136(4)$ & $0.00384(4)$ & $0.00340(4)$ \\
\hline $\mathrm{O} 1$ & $0.0788(15)$ & $0.0388(9)$ & $0.0266(7)$ & $-0.0176(9)$ & $0.0158(8)$ & $-0.0092(6)$ \\
\hline $\mathrm{O} 2$ & $0.0281(7)$ & $0.0267(6)$ & $0.0390(8)$ & $0.0040(5)$ & $0.0039(6)$ & $-0.0035(6)$ \\
\hline $\mathrm{O} 3$ & $0.0170(5)$ & $0.0467(8)$ & $0.0309(7)$ & $0.0097(5)$ & 0.0040 & $0.0157(6)$ \\
\hline $\mathrm{O} 4$ & 0.118 & $0.074(2)$ & $0.0615(17)$ & $0.0042(19)$ & $-0.0121(18)$ & $-0.0102(15)$ \\
\hline O5 & $0.157(9)$ & $0.42(2)$ & $0.064(5)$ & $0.186(13)$ & $-0.002(5)$ & $-0.004(9)$ \\
\hline N1 & $0.0217(6)$ & $0.0215(6)$ & $0.0215(6)$ & $0.0022(5)$ & $0.0069(5)$ & $0.0021(5)$ \\
\hline $\mathrm{N} 2$ & $0.0600(14)$ & $0.0300(9)$ & $0.0410(10)$ & $-0.0009(9)$ & $0.0227(10)$ & $-0.0099(8)$ \\
\hline N3 & $0.0189(6)$ & $0.0265(6)$ & $0.0258(6)$ & $0.0002(5)$ & $0.0078(5)$ & $0.0054(5)$ \\
\hline N4 & $0.0226(6)$ & $0.0303(7)$ & $0.0266(7)$ & $-0.0045(5)$ & $0.0055(5)$ & $0.0091(6)$ \\
\hline N5 & $0.0190(6)$ & $0.0248(6)$ & $0.0241(6)$ & $-0.0026(5)$ & $0.0037(5)$ & $0.0007(5)$ \\
\hline N6 & $0.0267(7)$ & $0.0217(6)$ & $0.0303(7)$ & $-0.0035(5)$ & $0.0019(6)$ & $0.0020(5)$ \\
\hline N7 & $0.0171(5)$ & $0.0211(6)$ & $0.0240(6)$ & $0.0003(4)$ & 0.0021 & $0.0046(5)$ \\
\hline N8 & $0.0220(6)$ & $0.0204(6)$ & $0.0257(6)$ & $0.0011(5)$ & $0.0024(5)$ & $0.0068(5)$ \\
\hline N9 & $0.0137(5)$ & $0.0258(6)$ & $0.0203(6)$ & 0.0024 & 0.0051 & $0.0057(5)$ \\
\hline $\mathrm{C} 1$ & $0.0142(5)$ & $0.0203(6)$ & $0.0200(6)$ & $0.0001(4)$ & $0.0030(5)$ & $0.0026(5)$ \\
\hline $\mathrm{C} 2$ & $0.0148(5)$ & $0.0199(6)$ & $0.0210(6)$ & 0.0023 & $0.0048(5)$ & $0.0024(5)$ \\
\hline $\mathrm{C} 3$ & $0.0153(5)$ & $0.0208(6)$ & $0.0199(6)$ & $0.0004(5)$ & $0.0058(5)$ & $0.0004(5)$ \\
\hline $\mathrm{C} 4$ & $0.0158(6)$ & $0.0238(7)$ & $0.0242(7)$ & $-0.0004(5)$ & $0.0077(5)$ & $-0.0018(5)$ \\
\hline C5 & $0.0233(7)$ & $0.0294(8)$ & $0.0252(7)$ & $0.0006(6)$ & $0.0118(6)$ & $-0.0012(6)$ \\
\hline C6 & $0.0258(7)$ & $0.0242(7)$ & $0.0211(7)$ & $-0.0022(6)$ & $0.0092(6)$ & $-0.0018(5)$ \\
\hline $\mathrm{C} 7$ & $0.0211(7)$ & $0.0292(7)$ & $0.0186(6)$ & $-0.0001(6)$ & $0.0049(5)$ & $0.0000(5)$ \\
\hline $\mathrm{C} 8$ & $0.0160(6)$ & $0.0233(6)$ & $0.0186(6)$ & $0.0015(5)$ & $0.0048(5)$ & $0.0006(5)$ \\
\hline C9 & $0.0165(6)$ & $0.0216(6)$ & $0.0193(6)$ & $0.0029(5)$ & $0.0056(5)$ & $0.0019(5)$ \\
\hline $\mathrm{C} 10$ & $0.0252(7)$ & $0.0250(7)$ & $0.0214(7)$ & $0.0065(6)$ & $0.0059(6)$ & $0.0054(5)$ \\
\hline $\mathrm{C} 11$ & $0.0322(9)$ & $0.0231(7)$ & $0.0304(8)$ & $0.0059(6)$ & $0.0112(7)$ & $0.0062(6)$ \\
\hline $\mathrm{C} 12$ & 0.0288 & $0.0215(7)$ & $0.0317(8)$ & $0.0005(6)$ & $0.0116(7)$ & $0.0004(6)$ \\
\hline $\mathrm{C} 13$ & $0.0209(7)$ & $0.0227(7)$ & $0.0237(7)$ & $-0.0017(5)$ & $0.0062(5)$ & $-0.0002(5)$ \\
\hline $\mathrm{C} 14$ & $0.0140(5)$ & $0.0209(6)$ & $0.0194(6)$ & $-0.0001(4)$ & 0.0049 & $0.0023(5)$ \\
\hline $\mathrm{C} 15$ & $0.0295(8)$ & $0.0283(8)$ & $0.0219(7)$ & $-0.0011(6)$ & $0.0104(6)$ & $-0.0003(6)$ \\
\hline $\mathrm{C} 16$ & $0.0267(8)$ & $0.0315(9)$ & $0.0323(9)$ & $0.0008(7)$ & $0.0114(7)$ & $0.0056(7)$ \\
\hline $\mathrm{C} 17$ & $0.0322(10)$ & $0.0614(15)$ & $0.0321(10)$ & $0.0004(10)$ & $0.0107(8)$ & $0.0158(10)$ \\
\hline $\mathrm{C} 18$ & $0.0164(6)$ & $0.0288(7)$ & $0.0266(7)$ & $0.0020(5)$ & $0.0077(5)$ & $-0.0021(6)$ \\
\hline C19 & $0.0175(7)$ & $0.0421(11)$ & $0.0437(11)$ & $0.0002(7)$ & $0.0020(7)$ & $-0.0052(9)$ \\
\hline
\end{tabular}




$\begin{array}{lllllll}\text { C20 } & 0.0219(10) & 0.0576(17) & 0.083(2) & 0.0055(10) & -0.0091(12) & 0.0039(16) \\ \text { C21 } & 0.0257(7) & 0.0216(7) & 0.0272(8) & 0.0020(6) & 0.0094(6) & 0.0027(6) \\ \text { C22 } & 0.0329(9) & 0.0204(7) & 0.0350(9) & 0.0016(6) & 0.0124(7) & 0.0002(6) \\ \text { C23 } & 0.0364(10) & 0.0241(7) & 0.0333(9) & -0.0052(7) & 0.0160(8) & -0.0052(7) \\ \text { C24 } & 0.0430(11) & 0.0311(9) & 0.0250(8) & -0.0011(8) & 0.0090(8) & -0.0027(7) \\ \text { C25 } & 0.0337(9) & 0.0270(8) & 0.0231(7) & 0.0041(7) & 0.0039(6) & -0.0003(6) \\ \text { C26 } & 0.079(2) & 0.0353(12) & 0.0596(18) & 0.0094(13) & 0.0275(17) & -0.0137(12) \\ \text { C27 } & 0.079(2) & 0.0437(14) & 0.0398(13) & -0.0067(14) & 0.0259(14) & -0.0135(11) \\ \text { C28 } & 0.0301(9) & 0.0484(12) & 0.0392(11) & -0.0081(9) & 0.0145(8) & 0.0143(9) \\ \text { C29 } & 0.0301(10) & 0.0586(15) & 0.0524(14) & -0.0003(10) & 0.0252(10) & 0.0121(12) \\ \text { C30 } & 0.0248(8) & 0.0394(10) & 0.0405(11) & 0.0047(7) & 0.0175(8) & 0.0077(8) \\ \text { C31 } & 0.0362(10) & 0.0235(8) & 0.0460(12) & -0.0061(7) & 0.0016(9) & -0.0039(8) \\ \text { C32 } & 0.0410(12) & 0.0364(11) & 0.0381(11) & -0.0104(9) & 0.0039(9) & -0.0117(9) \\ \text { C33 } & 0.0256(8) & 0.0365(9) & 0.0255(8) & -0.0078(7) & 0.0031(6) & -0.0040(7) \\ \text { C34 } & 0.0276(8) & 0.0262(8) & 0.0297(8) & 0.0050(6) & 0.0016(6) & 0.0091(6) \\ \text { C35 } & 0.0213(7) & 0.0368(10) & 0.0350(9) & 0.0021(7) & -0.0030(7) & 0.0130(8) \\ \text { C36 } & 0.0194(7) & 0.0295(8) & 0.0304(8) & -0.0008(6) & -0.0003(6) & 0.0083(7) \\ \text { C37 } & 0.167(7) & 0.172(7) & 0.059(3) & -0.090(6) & -0.019(3) & 0.014(4) \\ \text { C38 } & 0.147(6) & 0.077(3) & 0.098(4) & 0.003(4) & 0.008(4) & 0.019(3) \\ \text { C39 } & 0.152(6) & 0.101(4) & 0.088(4) & 0.032(4) & 0.028(4) & -0.027(3) \\ \text { C40 } & 0.159(6) & 0.062(3) & 0.080(3) & 0.004(3) & 0.038(4) & -0.011(2) \\ \text { C42 } & 0.126(11) & 0.236(19) & 0.075(7) & 0.087(12) & 0.016(7) & 0.032(10) \\ \text { C43 } & 0.142(13) & 0.43(4) & 0.063(7) & 0.163(19) & 0.001(7) & -0.033(13) \\ \text { C41 } & 0.123(10) & 0.242(19) & 0.069(6) & 0.093(12) & -0.013(6) & -0.049(9) \\ \text { C44 } & 0.25(2) & 0.30(3) & 0.084(9) & 0.22(2) & -0.026(12) & -0.022(13) \\ \text { B1 } & 0.0263(9) & 0.0226(8) & 0.0296(9) & -0.0053(7) & 0.0009(7) & 0.0076(7)\end{array}$




\section{Compound 87}

Table 4. Geometric parameters $\left(\AA,{ }^{\circ}\right)$ for $\mathrm{C}_{42} \mathrm{H}_{56} \mathrm{BMoN}_{9} \mathrm{O}_{4.5}$.

\begin{tabular}{|c|c|c|c|}
\hline $\mathrm{Mo}-\mathrm{N} 9$ & $1.7596(14)$ & $\mathrm{C} 16-\mathrm{H} 16 \mathrm{~A}$ & 0.9900 \\
\hline Mo-N5 & $2.2082(16)$ & $\mathrm{C} 16-\mathrm{H} 16 \mathrm{~B}$ & 0.9900 \\
\hline $\mathrm{Mo}-\mathrm{N} 1$ & $2.2233(15)$ & $\mathrm{C} 17-\mathrm{H} 17 \mathrm{~A}$ & 0.9800 \\
\hline $\mathrm{Mo}-\mathrm{N} 3$ & $2.2271(15)$ & C17-H17B & 0.9800 \\
\hline $\mathrm{Mo}-\mathrm{C} 2$ & $2.2276(15)$ & $\mathrm{C} 17-\mathrm{H} 17 \mathrm{C}$ & 0.9800 \\
\hline $\mathrm{Mo}-\mathrm{C} 1$ & $2.2348(15)$ & $\mathrm{C} 18-\mathrm{C} 19$ & $1.504(3)$ \\
\hline $\mathrm{Mo}-\mathrm{N} 7$ & $2.2740(14)$ & $\mathrm{C} 19-\mathrm{C} 20$ & $1.513(4)$ \\
\hline $\mathrm{O} 1-\mathrm{C} 15$ & $1.212(3)$ & $\mathrm{C} 19-\mathrm{H} 19 \mathrm{~A}$ & 0.9900 \\
\hline $\mathrm{O} 2-\mathrm{C} 18$ & $1.217(2)$ & $\mathrm{C} 19-\mathrm{H} 19 \mathrm{~B}$ & 0.9900 \\
\hline $\mathrm{O} 3-\mathrm{N} 9$ & $1.2174(18)$ & $\mathrm{C} 20-\mathrm{H} 20 \mathrm{~A}$ & 0.9800 \\
\hline $\mathrm{O} 4-\mathrm{C} 40$ & $1.411(7)$ & $\mathrm{C} 20-\mathrm{H} 20 \mathrm{~B}$ & 0.9800 \\
\hline $\mathrm{O} 4-\mathrm{C} 37$ & $1.460(8)$ & $\mathrm{C} 20-\mathrm{H} 20 \mathrm{C}$ & 0.9800 \\
\hline $\mathrm{O} 5-\mathrm{C} 44$ & $1.107(16)$ & $\mathrm{C} 21-\mathrm{C} 22$ & $1.376(3)$ \\
\hline $\mathrm{O} 5-\mathrm{C} 41$ & $1.419(15)$ & $\mathrm{C} 21-\mathrm{H} 21$ & 0.9500 \\
\hline $\mathrm{N} 1-\mathrm{C} 25$ & $1.351(2)$ & $\mathrm{C} 22-\mathrm{C} 23$ & $1.412(3)$ \\
\hline $\mathrm{N} 1-\mathrm{C} 21$ & $1.352(2)$ & $\mathrm{C} 22-\mathrm{H} 22$ & 0.9500 \\
\hline $\mathrm{N} 2-\mathrm{C} 23$ & $1.359(3)$ & $\mathrm{C} 23-\mathrm{C} 24$ & $1.406(3)$ \\
\hline $\mathrm{N} 2-\mathrm{C} 26$ & $1.450(4)$ & $\mathrm{C} 24-\mathrm{C} 25$ & $1.377(3)$ \\
\hline $\mathrm{N} 2-\mathrm{C} 27$ & $1.453(4)$ & $\mathrm{C} 24-\mathrm{H} 24$ & 0.9500 \\
\hline $\mathrm{N} 3-\mathrm{C} 30$ & $1.340(2)$ & $\mathrm{C} 25-\mathrm{H} 25$ & 0.9500 \\
\hline $\mathrm{N} 3-\mathrm{N} 4$ & $1.355(2)$ & $\mathrm{C} 26-\mathrm{H} 26 \mathrm{~A}$ & 0.9800 \\
\hline $\mathrm{N} 4-\mathrm{C} 28$ & $1.352(3)$ & $\mathrm{C} 26-\mathrm{H} 26 \mathrm{~B}$ & 0.9800 \\
\hline N4-B1 & $1.540(3)$ & $\mathrm{C} 26-\mathrm{H} 26 \mathrm{C}$ & 0.9800 \\
\hline $\mathrm{N} 5-\mathrm{C} 33$ & $1.336(2)$ & $\mathrm{C} 27-\mathrm{H} 27 \mathrm{~A}$ & 0.9800 \\
\hline N5-N6 & $1.366(2)$ & $\mathrm{C} 27-\mathrm{H} 27 \mathrm{~B}$ & 0.9800 \\
\hline N6-C31 & $1.344(3)$ & $\mathrm{C} 27-\mathrm{H} 27 \mathrm{C}$ & 0.9800 \\
\hline N6-B1 & $1.540(3)$ & $\mathrm{C} 28-\mathrm{C} 29$ & $1.375(4)$ \\
\hline $\mathrm{N} 7-\mathrm{C} 36$ & $1.341(2)$ & $\mathrm{C} 28-\mathrm{H} 28$ & 0.9500 \\
\hline N7-N8 & $1.366(2)$ & $\mathrm{C} 29-\mathrm{C} 30$ & $1.395(3)$ \\
\hline $\mathrm{N} 8-\mathrm{C} 34$ & $1.349(2)$ & $\mathrm{C} 29-\mathrm{H} 29$ & 0.9500 \\
\hline N8-B1 & $1.550(3)$ & $\mathrm{C} 30-\mathrm{H} 30$ & 0.9500 \\
\hline $\mathrm{C} 1-\mathrm{C} 2$ & $1.430(2)$ & $\mathrm{C} 31-\mathrm{C} 32$ & $1.375(4)$ \\
\hline $\mathrm{C} 1-\mathrm{C} 14$ & $1.470(2)$ & C $31-\mathrm{H} 31$ & 0.9500 \\
\hline $\mathrm{C} 1-\mathrm{H} 1$ & 1.0000 & $\mathrm{C} 32-\mathrm{C} 33$ & $1.396(3)$ \\
\hline $\mathrm{C} 2-\mathrm{C} 3$ & $1.524(2)$ & $\mathrm{C} 32-\mathrm{H} 32$ & 0.9500 \\
\hline
\end{tabular}




\begin{tabular}{|c|c|c|c|}
\hline $\mathrm{C} 2-\mathrm{H} 2$ & 1.0000 & $\mathrm{C} 33-\mathrm{H} 33$ & 0.9500 \\
\hline $\mathrm{C} 3-\mathrm{C} 8$ & $1.536(2)$ & $\mathrm{C} 34-\mathrm{C} 35$ & $1.376(3)$ \\
\hline $\mathrm{C} 3-\mathrm{C} 4$ & $1.553(2)$ & $\mathrm{C} 34-\mathrm{H} 34$ & 0.9500 \\
\hline $\mathrm{C} 3-\mathrm{H} 3$ & 1.0000 & $\mathrm{C} 35-\mathrm{C} 36$ & $1.394(3)$ \\
\hline $\mathrm{C} 4-\mathrm{C} 18$ & $1.518(2)$ & $\mathrm{C} 35-\mathrm{H} 35$ & 0.9500 \\
\hline $\mathrm{C} 4-\mathrm{C} 5$ & $1.540(3)$ & $\mathrm{C} 36-\mathrm{H} 36$ & 0.9500 \\
\hline $\mathrm{C} 4-\mathrm{H} 4$ & 1.0000 & $\mathrm{C} 37-\mathrm{C} 38$ & $1.517(11)$ \\
\hline $\mathrm{C} 5-\mathrm{C} 6$ & $1.534(3)$ & C37-H37A & 0.9900 \\
\hline $\mathrm{C} 5-\mathrm{H} 5 \mathrm{~A}$ & 0.9900 & C37-H37B & 0.9900 \\
\hline $\mathrm{C} 5-\mathrm{H} 5 \mathrm{~B}$ & 0.9900 & $\mathrm{C} 38-\mathrm{C} 39$ & $1.432(9)$ \\
\hline $\mathrm{C} 6-\mathrm{C} 15$ & $1.523(2)$ & $\mathrm{C} 38-\mathrm{H} 38 \mathrm{~A}$ & 0.9900 \\
\hline $\mathrm{C} 6-\mathrm{C} 7$ & $1.536(2)$ & $\mathrm{C} 38-\mathrm{H} 38 \mathrm{~B}$ & 0.9900 \\
\hline C6-H6 & 1.0000 & $\mathrm{C} 39-\mathrm{C} 40$ & $1.450(9)$ \\
\hline $\mathrm{C} 7-\mathrm{C} 8$ & $1.540(2)$ & $\mathrm{C} 39-\mathrm{H} 39 \mathrm{~A}$ & 0.9900 \\
\hline $\mathrm{C} 7-\mathrm{H} 7 \mathrm{~A}$ & 0.9900 & С $39-\mathrm{H} 39 \mathrm{~B}$ & 0.9900 \\
\hline $\mathrm{C} 7-\mathrm{H} 7 \mathrm{~B}$ & 0.9900 & $\mathrm{C} 40-\mathrm{H} 40 \mathrm{~A}$ & 0.9900 \\
\hline $\mathrm{C} 8-\mathrm{C} 9$ & $1.520(2)$ & $\mathrm{C} 40-\mathrm{H} 40 \mathrm{~B}$ & 0.9900 \\
\hline $\mathrm{C} 8-\mathrm{H} 8$ & 1.0000 & $\mathrm{C} 42-\mathrm{C} 41$ & $1.211(16)$ \\
\hline $\mathrm{C} 9-\mathrm{C} 10$ & $1.398(2)$ & $\mathrm{C} 42-\mathrm{C} 43$ & $1.471(18)$ \\
\hline $\mathrm{C} 9-\mathrm{C} 14$ & $1.417(2)$ & $\mathrm{C} 42-\mathrm{H} 42 \mathrm{~A}$ & 0.9900 \\
\hline $\mathrm{C} 10-\mathrm{C} 11$ & $1.385(3)$ & $\mathrm{C} 42-\mathrm{H} 42 \mathrm{~B}$ & 0.9900 \\
\hline $\mathrm{C} 10-\mathrm{H} 10$ & 0.9500 & $\mathrm{C} 43-\mathrm{C} 44$ & $1.232(17)$ \\
\hline $\mathrm{C} 11-\mathrm{C} 12$ & $1.394(3)$ & $\mathrm{C} 43-\mathrm{H} 43 \mathrm{~A}$ & 0.9900 \\
\hline $\mathrm{C} 11-\mathrm{H} 11$ & 0.9500 & $\mathrm{C} 43-\mathrm{H} 43 \mathrm{~B}$ & 0.9900 \\
\hline $\mathrm{C} 12-\mathrm{C} 13$ & $1.390(3)$ & $\mathrm{C} 41-\mathrm{H} 41 \mathrm{~A}$ & 0.9900 \\
\hline $\mathrm{C} 12-\mathrm{H} 12$ & 0.9500 & $\mathrm{C} 41-\mathrm{H} 41 \mathrm{~B}$ & 0.9900 \\
\hline $\mathrm{C} 13-\mathrm{C} 14$ & $1.402(2)$ & $\mathrm{C} 44-\mathrm{H} 44 \mathrm{~A}$ & 0.9900 \\
\hline $\mathrm{C} 13-\mathrm{H} 13$ & 0.9500 & $\mathrm{C} 44-\mathrm{H} 44 \mathrm{~B}$ & 0.9900 \\
\hline $\mathrm{C} 15-\mathrm{C} 16$ & $1.500(3)$ & $\mathrm{B} 1-\mathrm{H} 1 \mathrm{~B}$ & $1.08(3)$ \\
\hline $\mathrm{C} 16-\mathrm{C} 17$ & $1.518(3)$ & & \\
\hline $\mathrm{N} 9-\mathrm{Mo}-\mathrm{N} 5$ & $94.24(6)$ & $\mathrm{C} 16-\mathrm{C} 17-\mathrm{H} 17 \mathrm{C}$ & 109.5 \\
\hline $\mathrm{N} 9-\mathrm{Mo}-\mathrm{N} 1$ & $90.19(6)$ & $\mathrm{H} 17 \mathrm{~A}-\mathrm{C} 17-\mathrm{H} 17 \mathrm{C}$ & 109.5 \\
\hline $\mathrm{N} 5-\mathrm{Mo}-\mathrm{N} 1$ & $159.35(6)$ & $\mathrm{H} 17 \mathrm{~B}-\mathrm{C} 17-\mathrm{H} 17 \mathrm{C}$ & 109.5 \\
\hline $\mathrm{N} 9-\mathrm{Mo}-\mathrm{N} 3$ & $91.43(6)$ & $\mathrm{O} 2-\mathrm{C} 18-\mathrm{C} 19$ & $122.39(18)$ \\
\hline $\mathrm{N} 5-\mathrm{Mo}-\mathrm{N} 3$ & $80.78(6)$ & $\mathrm{O} 2-\mathrm{C} 18-\mathrm{C} 4$ & $120.58(16)$ \\
\hline $\mathrm{N} 1-\mathrm{Mo}-\mathrm{N} 3$ & $78.94(6)$ & $\mathrm{C} 19-\mathrm{C} 18-\mathrm{C} 4$ & $117.03(17)$ \\
\hline $\mathrm{N} 9-\mathrm{Mo}-\mathrm{C} 2$ & $99.84(6)$ & $\mathrm{C} 18-\mathrm{C} 19-\mathrm{C} 20$ & $115.1(2)$ \\
\hline $\mathrm{N} 5-\mathrm{Mo}-\mathrm{C} 2$ & $81.17(6)$ & $\mathrm{C} 18-\mathrm{C} 19-\mathrm{H} 19 \mathrm{~A}$ & 108.5 \\
\hline
\end{tabular}




\begin{tabular}{|c|c|c|c|}
\hline $\mathrm{N} 1-\mathrm{Mo}-\mathrm{C} 2$ & $117.94(6)$ & $\mathrm{C} 20-\mathrm{C} 19-\mathrm{H} 19 \mathrm{~A}$ & 108.5 \\
\hline $\mathrm{N} 3-\mathrm{Mo}-\mathrm{C} 2$ & $159.35(6)$ & $\mathrm{C} 18-\mathrm{C} 19-\mathrm{H} 19 \mathrm{~B}$ & 108.5 \\
\hline $\mathrm{N} 9-\mathrm{Mo}-\mathrm{C} 1$ & $97.30(6)$ & $\mathrm{C} 20-\mathrm{C} 19-\mathrm{H} 19 \mathrm{~B}$ & 108.5 \\
\hline $\mathrm{N} 5-\mathrm{Mo}-\mathrm{C} 1$ & $118.53(6)$ & $\mathrm{H} 19 \mathrm{~A}-\mathrm{C} 19-\mathrm{H} 19 \mathrm{~B}$ & 107.5 \\
\hline $\mathrm{N} 1-\mathrm{Mo}-\mathrm{C} 1$ & $80.75(6)$ & $\mathrm{C} 19-\mathrm{C} 20-\mathrm{H} 20 \mathrm{~A}$ & 109.5 \\
\hline $\mathrm{N} 3-\mathrm{Mo}-\mathrm{C} 1$ & $157.89(6)$ & $\mathrm{C} 19-\mathrm{C} 20-\mathrm{H} 20 \mathrm{~B}$ & 109.5 \\
\hline $\mathrm{C} 2-\mathrm{Mo}-\mathrm{C} 1$ & $37.38(6)$ & $\mathrm{H} 20 \mathrm{~A}-\mathrm{C} 20-\mathrm{H} 20 \mathrm{~B}$ & 109.5 \\
\hline $\mathrm{N} 9-\mathrm{Mo}-\mathrm{N} 7$ & $172.74(6)$ & $\mathrm{C} 19-\mathrm{C} 20-\mathrm{H} 20 \mathrm{C}$ & 109.5 \\
\hline $\mathrm{N} 5-\mathrm{Mo}-\mathrm{N} 7$ & $82.38(6)$ & $\mathrm{H} 20 \mathrm{~A}-\mathrm{C} 20-\mathrm{H} 20 \mathrm{C}$ & 109.5 \\
\hline $\mathrm{N} 1-\mathrm{Mo}-\mathrm{N} 7$ & $90.80(6)$ & $\mathrm{H} 20 \mathrm{~B}-\mathrm{C} 20-\mathrm{H} 20 \mathrm{C}$ & 109.5 \\
\hline $\mathrm{N} 3-\mathrm{Mo}-\mathrm{N} 7$ & $81.70(5)$ & $\mathrm{N} 1-\mathrm{C} 21-\mathrm{C} 22$ & $123.95(18)$ \\
\hline $\mathrm{C} 2-\mathrm{Mo}-\mathrm{N} 7$ & $86.03(6)$ & $\mathrm{N} 1-\mathrm{C} 21-\mathrm{H} 21$ & 118.0 \\
\hline $\mathrm{C} 1-\mathrm{Mo}-\mathrm{N} 7$ & $89.96(5)$ & $\mathrm{C} 22-\mathrm{C} 21-\mathrm{H} 21$ & 118.0 \\
\hline $\mathrm{C} 40-\mathrm{O} 4-\mathrm{C} 37$ & $102.5(5)$ & $\mathrm{C} 21-\mathrm{C} 22-\mathrm{C} 23$ & $120.31(18)$ \\
\hline $\mathrm{C} 44-\mathrm{O} 5-\mathrm{C} 41$ & $105.9(11)$ & $\mathrm{C} 21-\mathrm{C} 22-\mathrm{H} 22$ & 119.8 \\
\hline $\mathrm{C} 25-\mathrm{N} 1-\mathrm{C} 21$ & $115.51(16)$ & $\mathrm{C} 23-\mathrm{C} 22-\mathrm{H} 22$ & 119.8 \\
\hline $\mathrm{C} 25-\mathrm{N} 1-\mathrm{Mo}$ & $122.36(12)$ & $\mathrm{N} 2-\mathrm{C} 23-\mathrm{C} 24$ & $122.5(2)$ \\
\hline $\mathrm{C} 21-\mathrm{N} 1-\mathrm{Mo}$ & $120.65(12)$ & $\mathrm{N} 2-\mathrm{C} 23-\mathrm{C} 22$ & $121.7(2)$ \\
\hline $\mathrm{C} 23-\mathrm{N} 2-\mathrm{C} 26$ & $120.4(2)$ & $\mathrm{C} 24-\mathrm{C} 23-\mathrm{C} 22$ & $115.78(18)$ \\
\hline $\mathrm{C} 23-\mathrm{N} 2-\mathrm{C} 27$ & $120.8(2)$ & $\mathrm{C} 25-\mathrm{C} 24-\mathrm{C} 23$ & $119.70(19)$ \\
\hline $\mathrm{C} 26-\mathrm{N} 2-\mathrm{C} 27$ & $118.8(2)$ & $\mathrm{C} 25-\mathrm{C} 24-\mathrm{H} 24$ & 120.2 \\
\hline $\mathrm{C} 30-\mathrm{N} 3-\mathrm{N} 4$ & $106.94(15)$ & $\mathrm{C} 23-\mathrm{C} 24-\mathrm{H} 24$ & 120.2 \\
\hline $\mathrm{C} 30-\mathrm{N} 3-\mathrm{Mo}$ & $130.17(13)$ & $\mathrm{N} 1-\mathrm{C} 25-\mathrm{C} 24$ & $124.70(19)$ \\
\hline $\mathrm{N} 4-\mathrm{N} 3-\mathrm{Mo}$ & $122.66(12)$ & $\mathrm{N} 1-\mathrm{C} 25-\mathrm{H} 25$ & 117.6 \\
\hline $\mathrm{C} 28-\mathrm{N} 4-\mathrm{N} 3$ & $109.60(18)$ & $\mathrm{C} 24-\mathrm{C} 25-\mathrm{H} 25$ & 117.6 \\
\hline $\mathrm{C} 28-\mathrm{N} 4-\mathrm{B} 1$ & $131.45(18)$ & $\mathrm{N} 2-\mathrm{C} 26-\mathrm{H} 26 \mathrm{~A}$ & 109.5 \\
\hline $\mathrm{N} 3-\mathrm{N} 4-\mathrm{B} 1$ & $118.88(14)$ & $\mathrm{N} 2-\mathrm{C} 26-\mathrm{H} 26 \mathrm{~B}$ & 109.5 \\
\hline $\mathrm{C} 33-\mathrm{N} 5-\mathrm{N} 6$ & $106.30(16)$ & $\mathrm{H} 26 \mathrm{~A}-\mathrm{C} 26-\mathrm{H} 26 \mathrm{~B}$ & 109.5 \\
\hline C33-N5-Mo & $132.47(14)$ & $\mathrm{N} 2-\mathrm{C} 26-\mathrm{H} 26 \mathrm{C}$ & 109.5 \\
\hline $\mathrm{N} 6-\mathrm{N} 5-\mathrm{Mo}$ & $120.73(12)$ & $\mathrm{H} 26 \mathrm{~A}-\mathrm{C} 26-\mathrm{H} 26 \mathrm{C}$ & 109.5 \\
\hline $\mathrm{C} 31-\mathrm{N} 6-\mathrm{N} 5$ & $109.57(18)$ & $\mathrm{H} 26 \mathrm{~B}-\mathrm{C} 26-\mathrm{H} 26 \mathrm{C}$ & 109.5 \\
\hline $\mathrm{C} 31-\mathrm{N} 6-\mathrm{B} 1$ & $128.43(18)$ & $\mathrm{N} 2-\mathrm{C} 27-\mathrm{H} 27 \mathrm{~A}$ & 109.5 \\
\hline $\mathrm{N} 5-\mathrm{N} 6-\mathrm{B} 1$ & $121.20(15)$ & $\mathrm{N} 2-\mathrm{C} 27-\mathrm{H} 27 \mathrm{~B}$ & 109.5 \\
\hline $\mathrm{C} 36-\mathrm{N} 7-\mathrm{N} 8$ & $106.04(14)$ & $\mathrm{H} 27 \mathrm{~A}-\mathrm{C} 27-\mathrm{H} 27 \mathrm{~B}$ & 109.5 \\
\hline $\mathrm{C} 36-\mathrm{N} 7-\mathrm{Mo}$ & $134.99(12)$ & $\mathrm{N} 2-\mathrm{C} 27-\mathrm{H} 27 \mathrm{C}$ & 109.5 \\
\hline $\mathrm{N} 8-\mathrm{N} 7-\mathrm{Mo}$ & $118.96(11)$ & $\mathrm{H} 27 \mathrm{~A}-\mathrm{C} 27-\mathrm{H} 27 \mathrm{C}$ & 109.5 \\
\hline $\mathrm{C} 34-\mathrm{N} 8-\mathrm{N} 7$ & $109.66(15)$ & $\mathrm{H} 27 \mathrm{~B}-\mathrm{C} 27-\mathrm{H} 27 \mathrm{C}$ & 109.5 \\
\hline $\mathrm{C} 34-\mathrm{N} 8-\mathrm{B} 1$ & $128.82(15)$ & $\mathrm{N} 4-\mathrm{C} 28-\mathrm{C} 29$ & $108.36(19)$ \\
\hline $\mathrm{N} 7-\mathrm{N} 8-\mathrm{B} 1$ & $121.38(14)$ & $\mathrm{N} 4-\mathrm{C} 28-\mathrm{H} 28$ & 125.8 \\
\hline
\end{tabular}




\begin{tabular}{|c|c|c|c|}
\hline $\mathrm{O} 3-\mathrm{N} 9-\mathrm{Mo}$ & $174.14(13)$ & $\mathrm{C} 29-\mathrm{C} 28-\mathrm{H} 28$ & 125.8 \\
\hline $\mathrm{C} 2-\mathrm{C} 1-\mathrm{C} 14$ & $119.18(14)$ & $\mathrm{C} 28-\mathrm{C} 29-\mathrm{C} 30$ & $105.13(19)$ \\
\hline $\mathrm{C} 2-\mathrm{C} 1-\mathrm{Mo}$ & $71.04(9)$ & $\mathrm{C} 28-\mathrm{C} 29-\mathrm{H} 29$ & 127.4 \\
\hline $\mathrm{C} 14-\mathrm{C} 1-\mathrm{Mo}$ & $117.82(10)$ & $\mathrm{C} 30-\mathrm{C} 29-\mathrm{H} 29$ & 127.4 \\
\hline $\mathrm{C} 2-\mathrm{C} 1-\mathrm{H} 1$ & 114.1 & $\mathrm{~N} 3-\mathrm{C} 30-\mathrm{C} 29$ & $110.0(2)$ \\
\hline $\mathrm{C} 14-\mathrm{C} 1-\mathrm{H} 1$ & 114.1 & $\mathrm{~N} 3-\mathrm{C} 30-\mathrm{H} 30$ & 125.0 \\
\hline $\mathrm{Mo}-\mathrm{C} 1-\mathrm{H} 1$ & 114.1 & $\mathrm{C} 29-\mathrm{C} 30-\mathrm{H} 30$ & 125.0 \\
\hline $\mathrm{C} 1-\mathrm{C} 2-\mathrm{C} 3$ & $117.58(13)$ & $\mathrm{N} 6-\mathrm{C} 31-\mathrm{C} 32$ & $108.9(2)$ \\
\hline $\mathrm{C} 1-\mathrm{C} 2-\mathrm{Mo}$ & $71.58(9)$ & $\mathrm{N} 6-\mathrm{C} 31-\mathrm{H} 31$ & 125.5 \\
\hline $\mathrm{C} 3-\mathrm{C} 2-\mathrm{Mo}$ & $127.49(10)$ & $\mathrm{C} 32-\mathrm{C} 31-\mathrm{H} 31$ & 125.5 \\
\hline $\mathrm{C} 1-\mathrm{C} 2-\mathrm{H} 2$ & 111.4 & $\mathrm{C} 31-\mathrm{C} 32-\mathrm{C} 33$ & 104.47 (19) \\
\hline $\mathrm{C} 3-\mathrm{C} 2-\mathrm{H} 2$ & 111.4 & $\mathrm{C} 31-\mathrm{C} 32-\mathrm{H} 32$ & 127.8 \\
\hline $\mathrm{Mo}-\mathrm{C} 2-\mathrm{H} 2$ & 111.4 & $\mathrm{C} 33-\mathrm{C} 32-\mathrm{H} 32$ & 127.8 \\
\hline $\mathrm{C} 2-\mathrm{C} 3-\mathrm{C} 8$ & $112.43(13)$ & $\mathrm{N} 5-\mathrm{C} 33-\mathrm{C} 32$ & $110.7(2)$ \\
\hline $\mathrm{C} 2-\mathrm{C} 3-\mathrm{C} 4$ & $108.90(13)$ & $\mathrm{N} 5-\mathrm{C} 33-\mathrm{H} 33$ & 124.6 \\
\hline $\mathrm{C} 8-\mathrm{C} 3-\mathrm{C} 4$ & $110.52(13)$ & $\mathrm{C} 32-\mathrm{C} 33-\mathrm{H} 33$ & 124.6 \\
\hline $\mathrm{C} 2-\mathrm{C} 3-\mathrm{H} 3$ & 108.3 & $\mathrm{~N} 8-\mathrm{C} 34-\mathrm{C} 35$ & $108.85(16)$ \\
\hline $\mathrm{C} 8-\mathrm{C} 3-\mathrm{H} 3$ & 108.3 & $\mathrm{~N} 8-\mathrm{C} 34-\mathrm{H} 34$ & 125.6 \\
\hline $\mathrm{C} 4-\mathrm{C} 3-\mathrm{H} 3$ & 108.3 & $\mathrm{C} 35-\mathrm{C} 34-\mathrm{H} 34$ & 125.6 \\
\hline $\mathrm{C} 18-\mathrm{C} 4-\mathrm{C} 5$ & $108.92(14)$ & $\mathrm{C} 34-\mathrm{C} 35-\mathrm{C} 36$ & $104.52(16)$ \\
\hline $\mathrm{C} 18-\mathrm{C} 4-\mathrm{C} 3$ & $110.00(14)$ & $\mathrm{C} 34-\mathrm{C} 35-\mathrm{H} 35$ & 127.7 \\
\hline $\mathrm{C} 5-\mathrm{C} 4-\mathrm{C} 3$ & $112.10(14)$ & $\mathrm{C} 36-\mathrm{C} 35-\mathrm{H} 35$ & 127.7 \\
\hline $\mathrm{C} 18-\mathrm{C} 4-\mathrm{H} 4$ & 108.6 & $\mathrm{~N} 7-\mathrm{C} 36-\mathrm{C} 35$ & $110.93(17)$ \\
\hline $\mathrm{C} 5-\mathrm{C} 4-\mathrm{H} 4$ & 108.6 & $\mathrm{~N} 7-\mathrm{C} 36-\mathrm{H} 36$ & 124.5 \\
\hline $\mathrm{C} 3-\mathrm{C} 4-\mathrm{H} 4$ & 108.6 & $\mathrm{C} 35-\mathrm{C} 36-\mathrm{H} 36$ & 124.5 \\
\hline $\mathrm{C} 6-\mathrm{C} 5-\mathrm{C} 4$ & $110.52(14)$ & $\mathrm{O} 4-\mathrm{C} 37-\mathrm{C} 38$ & $105.8(5)$ \\
\hline $\mathrm{C} 6-\mathrm{C} 5-\mathrm{H} 5 \mathrm{~A}$ & 109.5 & $\mathrm{O} 4-\mathrm{C} 37-\mathrm{H} 37 \mathrm{~A}$ & 110.6 \\
\hline $\mathrm{C} 4-\mathrm{C} 5-\mathrm{H} 5 \mathrm{~A}$ & 109.5 & $\mathrm{C} 38-\mathrm{C} 37-\mathrm{H} 37 \mathrm{~A}$ & 110.6 \\
\hline $\mathrm{C} 6-\mathrm{C} 5-\mathrm{H} 5 \mathrm{~B}$ & 109.5 & $\mathrm{O} 4-\mathrm{C} 37-\mathrm{H} 37 \mathrm{~B}$ & 110.6 \\
\hline $\mathrm{C} 4-\mathrm{C} 5-\mathrm{H} 5 \mathrm{~B}$ & 109.5 & $\mathrm{C} 38-\mathrm{C} 37-\mathrm{H} 37 \mathrm{~B}$ & 110.6 \\
\hline $\mathrm{H} 5 \mathrm{~A}-\mathrm{C} 5-\mathrm{H} 5 \mathrm{~B}$ & 108.1 & $\mathrm{H} 37 \mathrm{~A}-\mathrm{C} 37-\mathrm{H} 37 \mathrm{~B}$ & 108.7 \\
\hline $\mathrm{C} 15-\mathrm{C} 6-\mathrm{C} 5$ & $110.18(14)$ & $\mathrm{C} 39-\mathrm{C} 38-\mathrm{C} 37$ & $106.9(5)$ \\
\hline $\mathrm{C} 15-\mathrm{C} 6-\mathrm{C} 7$ & $111.07(15)$ & $\mathrm{C} 39-\mathrm{C} 38-\mathrm{H} 38 \mathrm{~A}$ & 110.3 \\
\hline $\mathrm{C} 5-\mathrm{C} 6-\mathrm{C} 7$ & $109.50(14)$ & $\mathrm{C} 37-\mathrm{C} 38-\mathrm{H} 38 \mathrm{~A}$ & 110.4 \\
\hline $\mathrm{C} 15-\mathrm{C} 6-\mathrm{H} 6$ & 108.7 & $\mathrm{C} 39-\mathrm{C} 38-\mathrm{H} 38 \mathrm{~B}$ & 110.3 \\
\hline $\mathrm{C} 5-\mathrm{C} 6-\mathrm{H} 6$ & 108.7 & $\mathrm{C} 37-\mathrm{C} 38-\mathrm{H} 38 \mathrm{~B}$ & 110.3 \\
\hline $\mathrm{C} 7-\mathrm{C} 6-\mathrm{H} 6$ & 108.7 & $\mathrm{H} 38 \mathrm{~A}-\mathrm{C} 38-\mathrm{H} 38 \mathrm{~B}$ & 108.6 \\
\hline $\mathrm{C} 6-\mathrm{C} 7-\mathrm{C} 8$ & $111.55(14)$ & $\mathrm{C} 38-\mathrm{C} 39-\mathrm{C} 40$ & $102.1(6)$ \\
\hline $\mathrm{C} 6-\mathrm{C} 7-\mathrm{H} 7 \mathrm{~A}$ & 109.3 & $\mathrm{C} 38-\mathrm{C} 39-\mathrm{H} 39 \mathrm{~A}$ & 111.4 \\
\hline
\end{tabular}




\begin{tabular}{|c|c|c|c|}
\hline $\mathrm{C} 8-\mathrm{C} 7-\mathrm{H} 7 \mathrm{~A}$ & 109.3 & $\mathrm{C} 40-\mathrm{C} 39-\mathrm{H} 39 \mathrm{~A}$ & 111.4 \\
\hline $\mathrm{C} 6-\mathrm{C} 7-\mathrm{H} 7 \mathrm{~B}$ & 109.3 & $\mathrm{C} 38-\mathrm{C} 39-\mathrm{H} 39 \mathrm{~B}$ & 111.3 \\
\hline $\mathrm{C} 8-\mathrm{C} 7-\mathrm{H} 7 \mathrm{~B}$ & 109.3 & $\mathrm{C} 40-\mathrm{C} 39-\mathrm{H} 39 \mathrm{~B}$ & 111.3 \\
\hline $\mathrm{H} 7 \mathrm{~A}-\mathrm{C} 7-\mathrm{H} 7 \mathrm{~B}$ & 108.0 & $\mathrm{H} 39 \mathrm{~A}-\mathrm{C} 39-\mathrm{H} 39 \mathrm{~B}$ & 109.2 \\
\hline $\mathrm{C} 9-\mathrm{C} 8-\mathrm{C} 3$ & $110.88(13)$ & $\mathrm{O} 4-\mathrm{C} 40-\mathrm{C} 39$ & $109.1(5)$ \\
\hline $\mathrm{C} 9-\mathrm{C} 8-\mathrm{C} 7$ & $114.26(14)$ & $\mathrm{O} 4-\mathrm{C} 40-\mathrm{H} 40 \mathrm{~A}$ & 109.9 \\
\hline $\mathrm{C} 3-\mathrm{C} 8-\mathrm{C} 7$ & $110.27(13)$ & $\mathrm{C} 39-\mathrm{C} 40-\mathrm{H} 40 \mathrm{~A}$ & 109.9 \\
\hline $\mathrm{C} 9-\mathrm{C} 8-\mathrm{H} 8$ & 107.0 & $\mathrm{O} 4-\mathrm{C} 40-\mathrm{H} 40 \mathrm{~B}$ & 109.9 \\
\hline $\mathrm{C} 3-\mathrm{C} 8-\mathrm{H} 8$ & 107.0 & $\mathrm{C} 39-\mathrm{C} 40-\mathrm{H} 40 \mathrm{~B}$ & 109.9 \\
\hline $\mathrm{C} 7-\mathrm{C} 8-\mathrm{H} 8$ & 107.0 & $\mathrm{H} 40 \mathrm{~A}-\mathrm{C} 40-\mathrm{H} 40 \mathrm{~B}$ & 108.3 \\
\hline $\mathrm{C} 10-\mathrm{C} 9-\mathrm{C} 14$ & $118.59(15)$ & $\mathrm{C} 41-\mathrm{C} 42-\mathrm{C} 43$ & $103.0(12)$ \\
\hline $\mathrm{C} 10-\mathrm{C} 9-\mathrm{C} 8$ & $121.39(15)$ & $\mathrm{C} 41-\mathrm{C} 42-\mathrm{H} 42 \mathrm{~A}$ & 111.2 \\
\hline $\mathrm{C} 14-\mathrm{C} 9-\mathrm{C} 8$ & $120.02(14)$ & $\mathrm{C} 43-\mathrm{C} 42-\mathrm{H} 42 \mathrm{~A}$ & 111.1 \\
\hline $\mathrm{C} 11-\mathrm{C} 10-\mathrm{C} 9$ & $121.91(17)$ & $\mathrm{C} 41-\mathrm{C} 42-\mathrm{H} 42 \mathrm{~B}$ & 111.1 \\
\hline $\mathrm{C} 11-\mathrm{C} 10-\mathrm{H} 10$ & 119.0 & $\mathrm{C} 43-\mathrm{C} 42-\mathrm{H} 42 \mathrm{~B}$ & 111.3 \\
\hline $\mathrm{C} 9-\mathrm{C} 10-\mathrm{H} 10$ & 119.0 & $\mathrm{H} 42 \mathrm{~A}-\mathrm{C} 42-\mathrm{H} 42 \mathrm{~B}$ & 109.1 \\
\hline $\mathrm{C} 10-\mathrm{C} 11-\mathrm{C} 12$ & $119.64(17)$ & $\mathrm{C} 44-\mathrm{C} 43-\mathrm{C} 42$ & $101.0(13)$ \\
\hline $\mathrm{C} 10-\mathrm{C} 11-\mathrm{H} 11$ & 120.2 & $\mathrm{C} 44-\mathrm{C} 43-\mathrm{H} 43 \mathrm{~A}$ & 111.9 \\
\hline $\mathrm{C} 12-\mathrm{C} 11-\mathrm{H} 11$ & 120.2 & $\mathrm{C} 42-\mathrm{C} 43-\mathrm{H} 43 \mathrm{~A}$ & 111.4 \\
\hline $\mathrm{C} 13-\mathrm{C} 12-\mathrm{C} 11$ & $119.42(17)$ & $\mathrm{C} 44-\mathrm{C} 43-\mathrm{H} 43 \mathrm{~B}$ & 111.5 \\
\hline $\mathrm{C} 13-\mathrm{C} 12-\mathrm{H} 12$ & 120.3 & $\mathrm{C} 42-\mathrm{C} 43-\mathrm{H} 43 \mathrm{~B}$ & 111.5 \\
\hline $\mathrm{C} 11-\mathrm{C} 12-\mathrm{H} 12$ & 120.3 & $\mathrm{H} 43 \mathrm{~A}-\mathrm{C} 43-\mathrm{H} 43 \mathrm{~B}$ & 109.3 \\
\hline $\mathrm{C} 12-\mathrm{C} 13-\mathrm{C} 14$ & $121.57(17)$ & $\mathrm{C} 42-\mathrm{C} 41-\mathrm{O} 5$ & $106.7(12)$ \\
\hline $\mathrm{C} 12-\mathrm{C} 13-\mathrm{H} 13$ & 119.2 & $\mathrm{C} 42-\mathrm{C} 41-\mathrm{H} 41 \mathrm{~A}$ & 110.5 \\
\hline $\mathrm{C} 14-\mathrm{C} 13-\mathrm{H} 13$ & 119.2 & $\mathrm{O} 5-\mathrm{C} 41-\mathrm{H} 41 \mathrm{~A}$ & 110.1 \\
\hline $\mathrm{C} 13-\mathrm{C} 14-\mathrm{C} 9$ & $118.83(15)$ & $\mathrm{C} 42-\mathrm{C} 41-\mathrm{H} 41 \mathrm{~B}$ & 110.4 \\
\hline $\mathrm{C} 13-\mathrm{C} 14-\mathrm{C} 1$ & $120.07(14)$ & $\mathrm{O} 5-\mathrm{C} 41-\mathrm{H} 41 \mathrm{~B}$ & 110.5 \\
\hline $\mathrm{C} 9-\mathrm{C} 14-\mathrm{C} 1$ & $121.09(14)$ & $\mathrm{H} 41 \mathrm{~A}-\mathrm{C} 41-\mathrm{H} 41 \mathrm{~B}$ & 108.6 \\
\hline $\mathrm{O} 1-\mathrm{C} 15-\mathrm{C} 16$ & $122.14(18)$ & $\mathrm{O} 5-\mathrm{C} 44-\mathrm{C} 43$ & $118.6(14)$ \\
\hline $\mathrm{O} 1-\mathrm{C} 15-\mathrm{C} 6$ & $121.43(18)$ & $\mathrm{O} 5-\mathrm{C} 44-\mathrm{H} 44 \mathrm{~A}$ & 107.0 \\
\hline $\mathrm{C} 16-\mathrm{C} 15-\mathrm{C} 6$ & $116.43(17)$ & $\mathrm{C} 43-\mathrm{C} 44-\mathrm{H} 44 \mathrm{~A}$ & 107.3 \\
\hline $\mathrm{C} 15-\mathrm{C} 16-\mathrm{C} 17$ & $114.9(2)$ & $\mathrm{O} 5-\mathrm{C} 44-\mathrm{H} 44 \mathrm{~B}$ & 108.5 \\
\hline $\mathrm{C} 15-\mathrm{C} 16-\mathrm{H} 16 \mathrm{~A}$ & 108.6 & $\mathrm{C} 43-\mathrm{C} 44-\mathrm{H} 44 \mathrm{~B}$ & 107.8 \\
\hline $\mathrm{C} 17-\mathrm{C} 16-\mathrm{H} 16 \mathrm{~A}$ & 108.6 & $\mathrm{H} 44 \mathrm{~A}-\mathrm{C} 44-\mathrm{H} 44 \mathrm{~B}$ & 107.1 \\
\hline $\mathrm{C} 15-\mathrm{C} 16-\mathrm{H} 16 \mathrm{~B}$ & 108.6 & $\mathrm{~N} 6-\mathrm{B} 1-\mathrm{N} 4$ & $107.56(16)$ \\
\hline $\mathrm{C} 17-\mathrm{C} 16-\mathrm{H} 16 \mathrm{~B}$ & 108.6 & $\mathrm{~N} 6-\mathrm{B} 1-\mathrm{N} 8$ & $109.41(16)$ \\
\hline $\mathrm{H} 16 \mathrm{~A}-\mathrm{C} 16-\mathrm{H} 16 \mathrm{~B}$ & 107.5 & $\mathrm{~N} 4-\mathrm{B} 1-\mathrm{N} 8$ & $108.81(16)$ \\
\hline $\mathrm{C} 16-\mathrm{C} 17-\mathrm{H} 17 \mathrm{~A}$ & 109.5 & $\mathrm{~N} 6-\mathrm{B} 1-\mathrm{H} 1 \mathrm{~B}$ & $109.1(15)$ \\
\hline $\mathrm{C} 16-\mathrm{C} 17-\mathrm{H} 17 \mathrm{~B}$ & 109.5 & $\mathrm{~N} 4-\mathrm{B} 1-\mathrm{H} 1 \mathrm{~B}$ & $108.4(15)$ \\
\hline
\end{tabular}


H17A-C17-H17B 109.5

N8-B1-H1B

$113.4(16)$ 
${ }^{1}$ H NMR ( $d^{6}$-Acetone) of Compound 87:
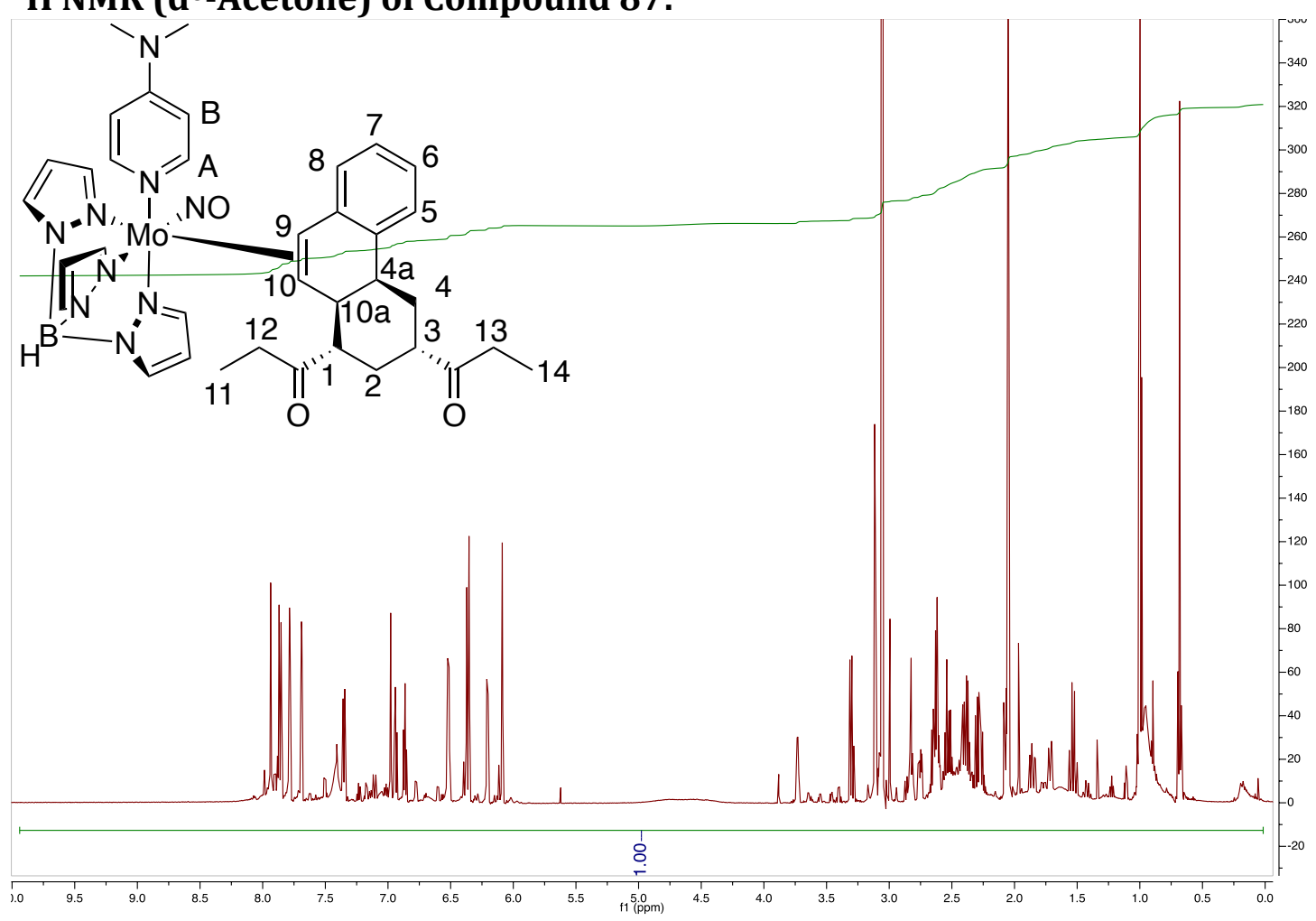

${ }^{13}$ C NMR (d6-Acetone) of Compound 87:

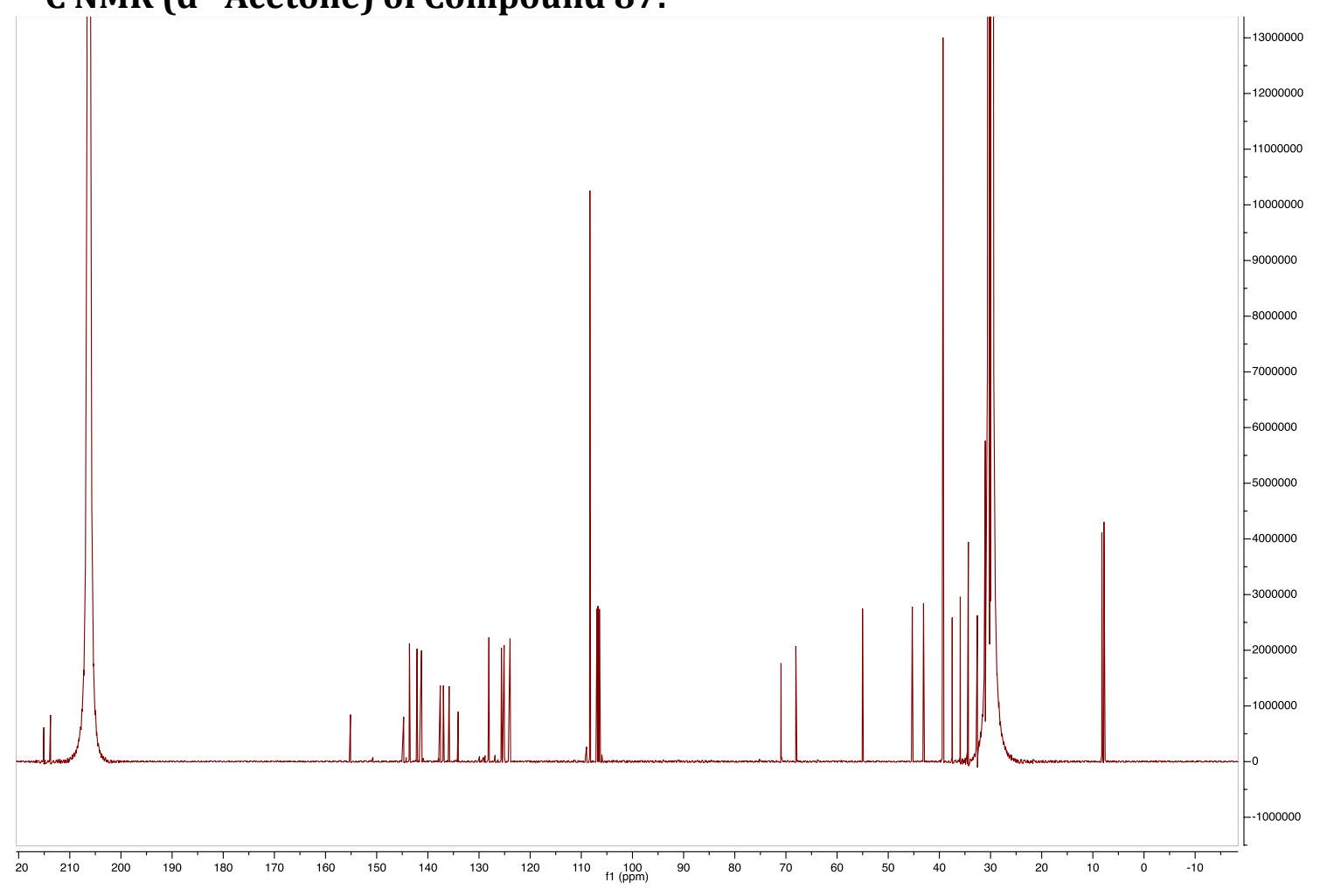


${ }^{1} \mathrm{H}$ NMR ( $d^{6}$-Acetone) of Compound 88:

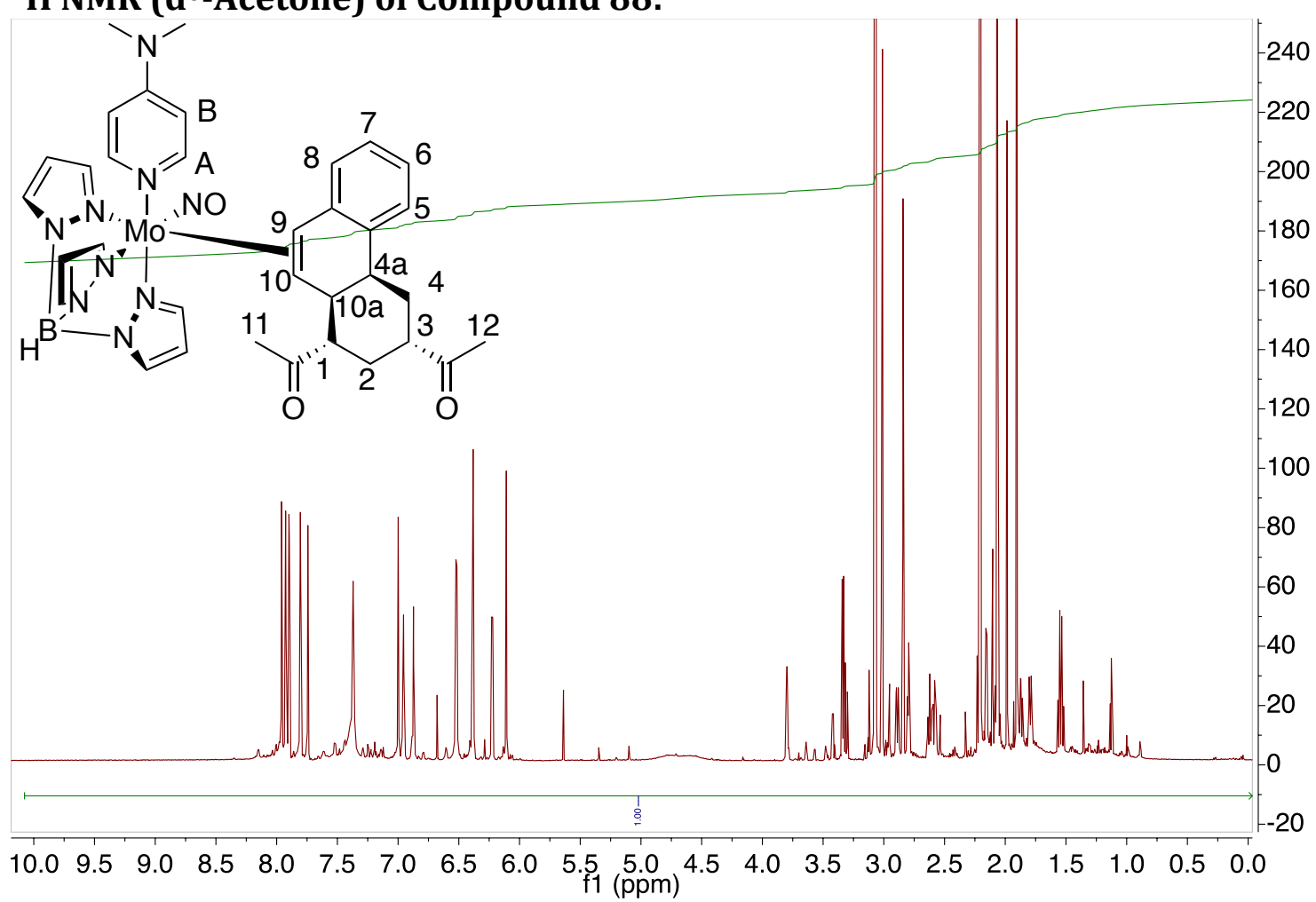

${ }^{13}$ C NMR (d6-Acetone) of Compound 88:

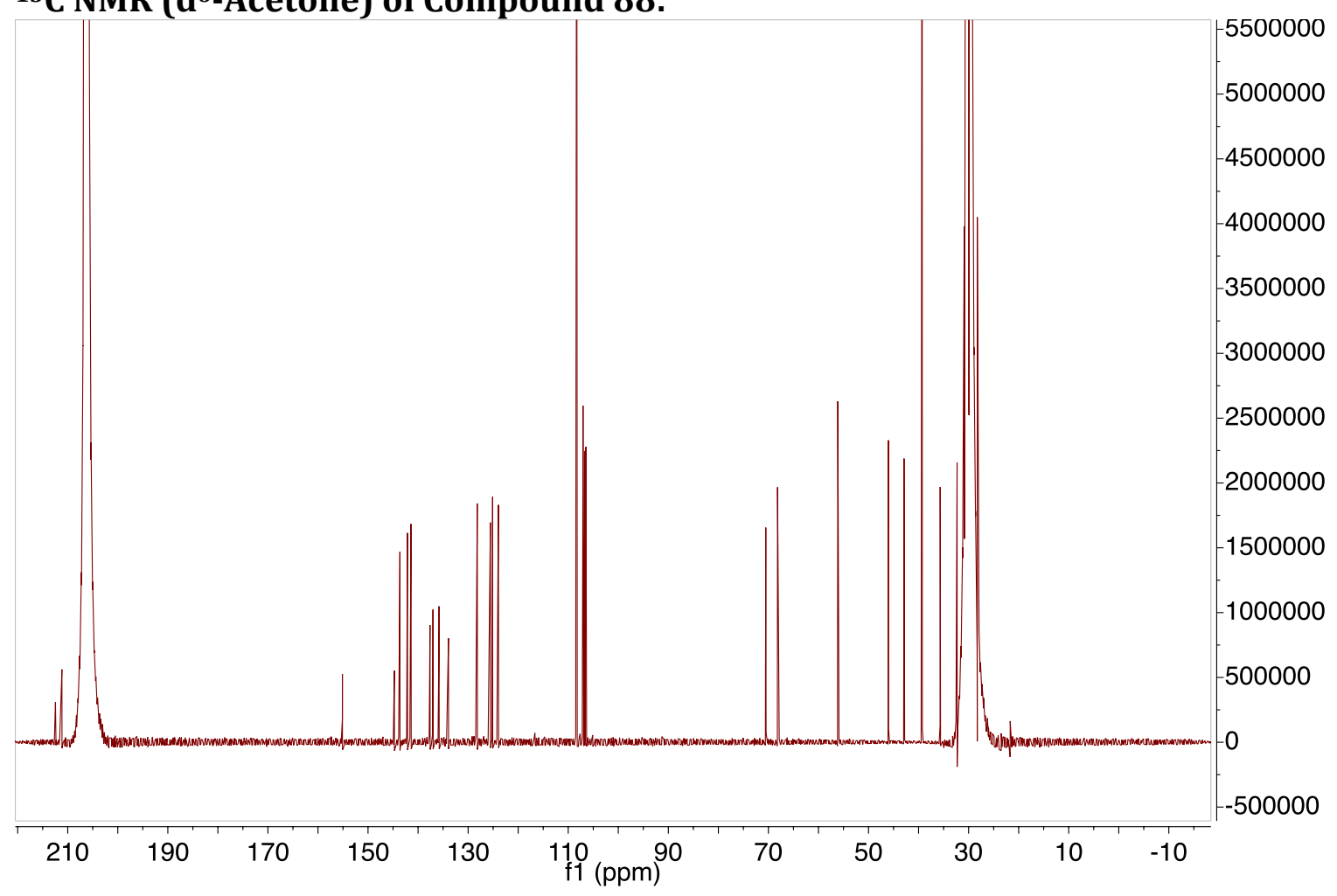




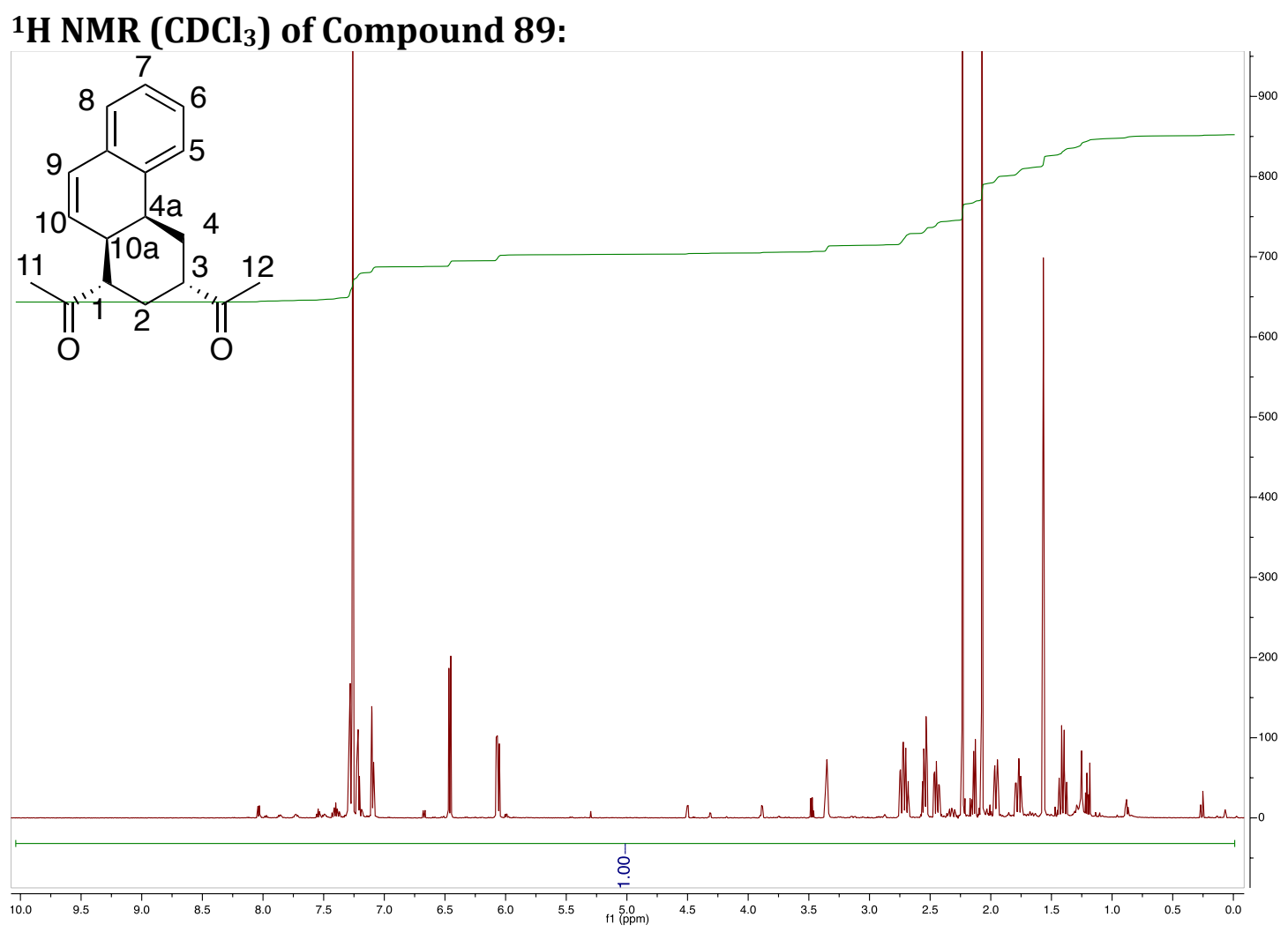

${ }^{13} \mathrm{C} \mathrm{NMR}\left(\mathrm{CDCl}_{3}\right)$ of Compound 89:

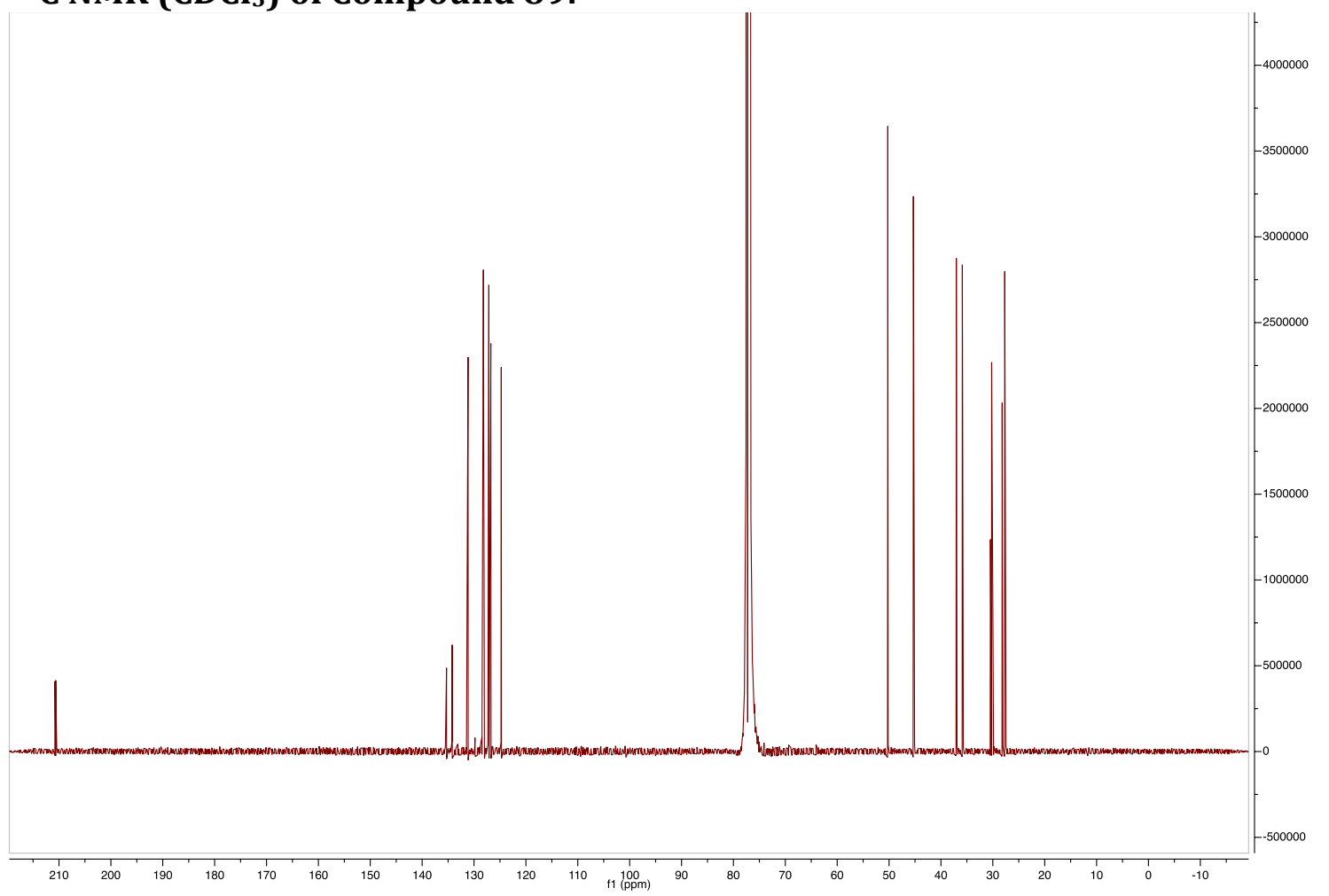


Compound 90

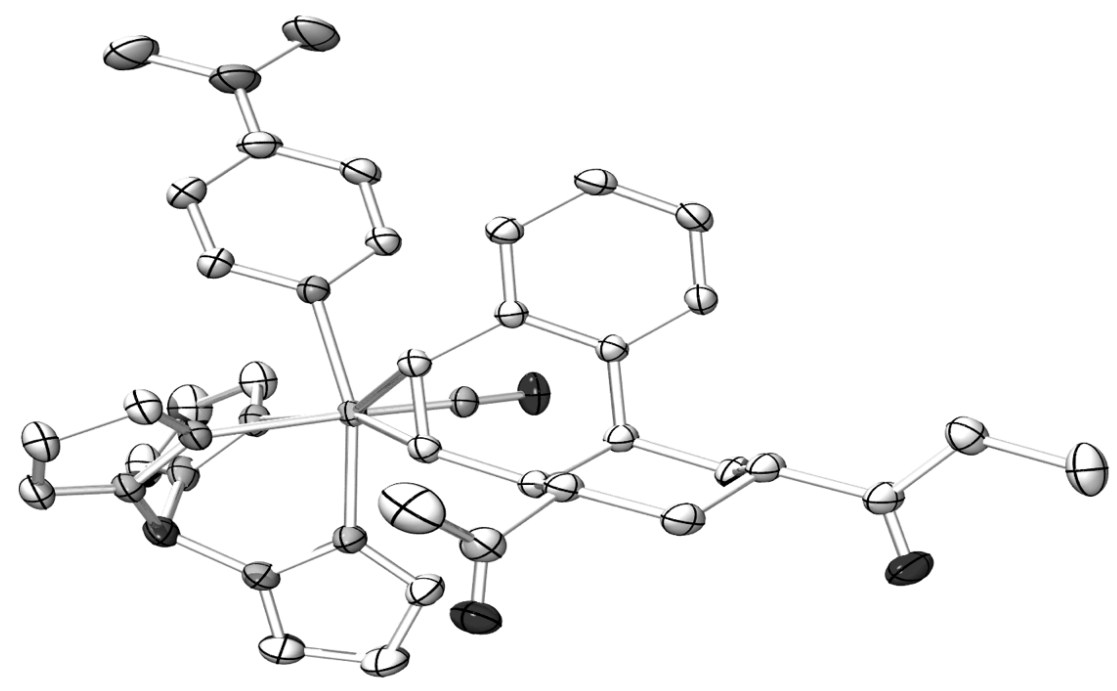




\section{Compound 90}

Table 1. Crystal data and structure refinement for C31H54BN9O4.5Mo.

Empirical formula

Formula weight

Temperature

Wavelength

Crystal system

Space group

Unit cell dimensions

Volume

$\mathrm{Z}$

Density (calculated)

Absorption coefficient

$\mathrm{F}(000)$

Crystal size

Theta range for data collection

Index ranges

Reflections collected

Independent reflections

Completeness to theta $=25.242^{\circ}$

Absorption correction

Refinement method

Data / restraints / parameters

Goodness-of-fit on $\mathrm{F}^{2}$

Final R indices [I $>2 \operatorname{sigma}(\mathrm{I})]$

$\mathrm{R}$ indices (all data)

Largest diff. peak and hole

\section{C41 H54 B Mo N9 O4.50}

851.68

153(2) K

$0.71073 \AA$

Monoclinic

$\mathrm{P} 2 / \mathrm{c}$

$\mathrm{a}=9.7852(18) \AA$

$\mathrm{b}=19.692(4) \AA$

$\beta=99.408(3)^{\circ}$.

$\mathrm{c}=23.463(4) \AA$

4460.3(14) $\AA^{3}$

4

$1.268 \mathrm{Mg} / \mathrm{m}^{3}$

$0.344 \mathrm{~mm}^{-1}$

1784

$0.350 \times 0.230 \times 0.220 \mathrm{~mm}^{3}$

3.136 to $37.212^{\circ}$.

$-16<=\mathrm{h}<=15,-33<=\mathrm{k}<=32,-39<=1<=39$

111241

$21393[\mathrm{R}(\mathrm{int})=0.0351]$

$99.7 \%$

empirical

Full-matrix least-squares on $\mathrm{F}^{2}$

21393 / 0 / 540

1.062

$\mathrm{R} 1=0.0561, \mathrm{wR} 2=0.1433$

$\mathrm{R} 1=0.0865, \mathrm{wR} 2=0.1728$

2.246 and -0.961 e. $\AA^{-3}$ 


\section{Compound 90}

Table 2. Atomic coordinates $\left(\mathrm{x} 10^{4}\right)$ and equivalent isotropic displacement parameters $\left(\AA^{2} \times 10^{3}\right)$ for $\mathrm{C} 31 \mathrm{H} 54 \mathrm{BN} 9 \mathrm{O} 4.5 \mathrm{Mo}$. $\mathrm{U}(\mathrm{eq})$ is defined as one third of the trace of the orthogonalized $\mathrm{U}^{\mathrm{ij}}$ tensor.

\begin{tabular}{|c|c|c|c|c|}
\hline & $\mathrm{x}$ & $\mathrm{y}$ & $\mathrm{z}$ & $\mathrm{U}(\mathrm{eq})$ \\
\hline Mo & $4672(1)$ & $6576(1)$ & $3249(1)$ & $23(1)$ \\
\hline $\mathrm{O}(1)$ & $9282(2)$ & $7725(1)$ & $4533(1)$ & $48(1)$ \\
\hline $\mathrm{O}(2)$ & $8932(3)$ & $6293(1)$ & $6460(1)$ & $55(1)$ \\
\hline $\mathrm{O}(3)$ & $3103(2)$ & $5937(1)$ & $4089(1)$ & $40(1)$ \\
\hline $\mathrm{O}(4)$ & $3329(4)$ & $8805(2)$ & $5463(1)$ & $85(1)$ \\
\hline $\mathrm{O}(5)$ & $2010(10)$ & $4359(8)$ & $8424(4)$ & $180(6)$ \\
\hline $\mathrm{N}(1)$ & $4196(2)$ & 5691(1) & $2663(1)$ & $27(1)$ \\
\hline $\mathrm{N}(2)$ & $2385(3)$ & $4163(1)$ & $1532(1)$ & $57(1)$ \\
\hline $\mathrm{N}(3)$ & $5595(2)$ & $7148(1)$ & $2560(1)$ & $28(1)$ \\
\hline $\mathrm{N}(4)$ & $5022(2)$ & $7754(1)$ & $2360(1)$ & $30(1)$ \\
\hline $\mathrm{N}(5)$ & $2719(2)$ & 6931(1) & $2708(1)$ & $31(1)$ \\
\hline $\mathrm{N}(6)$ & $2569(2)$ & $7571(1)$ & 2491(1) & $34(1)$ \\
\hline $\mathrm{N}(7)$ & $4530(2)$ & $7598(1)$ & $3613(1)$ & $32(1)$ \\
\hline $\mathrm{N}(8)$ & $4187(3)$ & $8148(1)$ & $3264(1)$ & $38(1)$ \\
\hline $\mathrm{N}(9)$ & $3801(2)$ & 6194(1) & $3763(1)$ & $26(1)$ \\
\hline $\mathrm{C}(1)$ & $6788(2)$ & $6635(1)$ & $3768(1)$ & $25(1)$ \\
\hline $\mathrm{C}(2)$ & $6652(2)$ & $6002(1)$ & $3466(1)$ & $25(1)$ \\
\hline$C(3)$ & $6646(2)$ & $5367(1)$ & $3795(1)$ & $23(1)$ \\
\hline $\mathrm{C}(4)$ & $6748(2)$ & $4742(1)$ & $3520(1)$ & $27(1)$ \\
\hline $\mathrm{C}(5)$ & $6811(2)$ & $4133(1)$ & $3825(1)$ & $30(1)$ \\
\hline$C(6)$ & $6717(2)$ & $4142(1)$ & $4409(1)$ & $32(1)$ \\
\hline$C(7)$ & $6583(2)$ & $4756(1)$ & $4684(1)$ & $28(1)$ \\
\hline $\mathrm{C}(8)$ & $6568(2)$ & $5375(1)$ & $4391(1)$ & $24(1)$ \\
\hline $\mathrm{C}(9)$ & $6458(2)$ & $6046(1)$ & $4699(1)$ & $25(1)$ \\
\hline$C(10)$ & $6982(2)$ & $6015(1)$ & $5354(1)$ & $30(1)$ \\
\hline$C(11)$ & $8547(2)$ & $5896(1)$ & $5484(1)$ & $30(1)$ \\
\hline$C(12)$ & $9286(2)$ & $6484(1)$ & $5231(1)$ & $33(1)$ \\
\hline $\mathrm{C}(13)$ & $8787(2)$ & $6548(1)$ & $4576(1)$ & $28(1)$ \\
\hline $\mathrm{C}(14)$ & $7188(2)$ & $6616(1)$ & $4423(1)$ & $25(1)$ \\
\hline$C(15)$ & $9496(3)$ & $7159(1)$ & $4362(1)$ & $37(1)$ \\
\hline
\end{tabular}




\begin{tabular}{|c|c|c|c|c|}
\hline$C(16)$ & $10470(4)$ & $7039(2)$ & $3948(2)$ & $63(1)$ \\
\hline$C(17)$ & $9039(2)$ & $5828(1)$ & $6131(1)$ & $33(1)$ \\
\hline$C(18)$ & $9667(2)$ & $5158(1)$ & $6334(1)$ & $37(1)$ \\
\hline$C(19)$ & $10179(3)$ & $5115(2)$ & $6979(1)$ & $50(1)$ \\
\hline$C(20)$ & $4120(3)$ & $5739(1)$ & $2085(1)$ & $35(1)$ \\
\hline$C(21)$ & $3568(3)$ & $5248(1)$ & $1696(1)$ & $41(1)$ \\
\hline$C(22)$ & $3009(3)$ & $4651(1)$ & 1895(1) & $40(1)$ \\
\hline$C(23)$ & $3127(3)$ & $4590(1)$ & $2500(1)$ & $37(1)$ \\
\hline$C(24)$ & $3709(2)$ & $5105(1)$ & $2854(1)$ & $31(1)$ \\
\hline$C(25)$ & $2297(5)$ & $4236(2)$ & $908(2)$ & $72(1)$ \\
\hline$C(26)$ & $1827(6)$ & $3557(2)$ & $1756(2)$ & $82(2)$ \\
\hline$C(27)$ & $5712(3)$ & $8000(1)$ & 1954(1) & $36(1)$ \\
\hline$C(28)$ & $6759(3)$ & $7561(1)$ & $1883(1)$ & $38(1)$ \\
\hline$C(29)$ & $6646(2)$ & $7037(1)$ & $2271(1)$ & $33(1)$ \\
\hline$C(30)$ & 1261(3) & $7653(2)$ & $2206(1)$ & $46(1)$ \\
\hline $\mathrm{C}(31)$ & $564(3)$ & $7051(2)$ & $2234(1)$ & $53(1)$ \\
\hline$C(32)$ & $1509(2)$ & $6611(1)$ & $2552(1)$ & $38(1)$ \\
\hline$C(33)$ & $4020(4)$ & $8694(1)$ & $3593(1)$ & $52(1)$ \\
\hline$C(34)$ & $4253(4)$ & $8508(2)$ & $4162(1)$ & $57(1)$ \\
\hline$C(35)$ & $4555(3)$ & $7812(1)$ & $4154(1)$ & $42(1)$ \\
\hline$C(36)$ & $1875(7)$ & $8777(3)$ & $5339(2)$ & $88(2)$ \\
\hline$C(37)$ & $1347(8)$ & $8334(3)$ & $5745(3)$ & $102(2)$ \\
\hline$C(38)$ & $2426(10)$ & $7824(4)$ & $5824(5)$ & $155(4)$ \\
\hline$C(39)$ & 3744(9) & $8125(5)$ & $5685(3)$ & $136(3)$ \\
\hline$C(40)$ & $1080(12)$ & $4790(7)$ & $8575(5)$ & $94(3)$ \\
\hline$C(41)$ & $1466(11)$ & $4904(7)$ & $9163(5)$ & $106(4)$ \\
\hline$C(42)$ & $2737(15)$ & $4555(8)$ & $9334(4)$ & $116(5)$ \\
\hline$C(43)$ & $2906(18)$ & $4204(11)$ & $8870(7)$ & $170(9)$ \\
\hline $\mathrm{B}(1)$ & $3798(3)$ & $8066(1)$ & $2607(1)$ & $36(1)$ \\
\hline
\end{tabular}




\section{Compound 90}

Table 3. Bond lengths $[\AA]$ and angles $\left[^{\circ}\right]$ for C31H54BN9O4.5Mo.

\begin{tabular}{|c|c|}
\hline Mo-N(9) & $1.7549(17)$ \\
\hline Mo-N(7) & $2.2003(19)$ \\
\hline Mo-N(1) & $2.2230(18)$ \\
\hline Mo-C(1) & $2.226(2)$ \\
\hline Mo-N(5) & $2.2271(18)$ \\
\hline Mo-C(2) & $2.2291(19)$ \\
\hline Mo-N(3) & $2.2756(17)$ \\
\hline $\mathrm{O}(1)-\mathrm{C}(15)$ & $1.213(3)$ \\
\hline $\mathrm{O}(2)-\mathrm{C}(17)$ & $1.212(3)$ \\
\hline $\mathrm{O}(3)-\mathrm{N}(9)$ & $1.217(2)$ \\
\hline $\mathrm{O}(4)-\mathrm{C}(36)$ & $1.406(7)$ \\
\hline $\mathrm{O}(4)-\mathrm{C}(39)$ & $1.471(8)$ \\
\hline $\mathrm{O}(5)-\mathrm{C}(43)$ & $1.287(16)$ \\
\hline $\mathrm{O}(5)-\mathrm{C}(40)$ & $1.333(15)$ \\
\hline $\mathrm{N}(1)-\mathrm{C}(20)$ & $1.350(3)$ \\
\hline $\mathrm{N}(1)-\mathrm{C}(24)$ & $1.351(3)$ \\
\hline $\mathrm{N}(2)-\mathrm{C}(22)$ & $1.361(3)$ \\
\hline $\mathrm{N}(2)-\mathrm{C}(26)$ & $1.448(5)$ \\
\hline$N(2)-C(25)$ & $1.459(5)$ \\
\hline $\mathrm{N}(3)-\mathrm{C}(29)$ & $1.338(3)$ \\
\hline $\mathrm{N}(3)-\mathrm{N}(4)$ & $1.367(2)$ \\
\hline$N(4)-C(27)$ & $1.345(3)$ \\
\hline $\mathrm{N}(4)-\mathrm{B}(1)$ & $1.542(3)$ \\
\hline$N(5)-C(32)$ & $1.338(3)$ \\
\hline $\mathrm{N}(5)-\mathrm{N}(6)$ & $1.359(3)$ \\
\hline $\mathrm{N}(6)-\mathrm{C}(30)$ & $1.354(3)$ \\
\hline $\mathrm{N}(6)-\mathrm{B}(1)$ & $1.538(4)$ \\
\hline $\mathrm{N}(7)-\mathrm{C}(35)$ & $1.333(3)$ \\
\hline $\mathrm{N}(7)-\mathrm{N}(8)$ & $1.367(3)$ \\
\hline $\mathrm{N}(8)-\mathrm{C}(33)$ & $1.347(3)$ \\
\hline $\mathrm{N}(8)-\mathrm{B}(1)$ & $1.535(3)$ \\
\hline$C(1)-C(2)$ & $1.428(3)$ \\
\hline $\mathrm{C}(1)-\mathrm{C}(14)$ & $1.524(3)$ \\
\hline
\end{tabular}




\begin{tabular}{|c|c|}
\hline $\mathrm{C}(1)-\mathrm{H}(1)$ & 1.0000 \\
\hline $\mathrm{C}(2)-\mathrm{C}(3)$ & $1.471(3)$ \\
\hline $\mathrm{C}(2)-\mathrm{H}(2)$ & 1.0000 \\
\hline $\mathrm{C}(3)-\mathrm{C}(4)$ & $1.400(3)$ \\
\hline $\mathrm{C}(3)-\mathrm{C}(8)$ & $1.413(3)$ \\
\hline $\mathrm{C}(4)-\mathrm{C}(5)$ & $1.394(3)$ \\
\hline $\mathrm{C}(4)-\mathrm{H}(4)$ & 0.9500 \\
\hline $\mathrm{C}(5)-\mathrm{C}(6)$ & $1.387(3)$ \\
\hline $\mathrm{C}(5)-\mathrm{H}(5)$ & 0.9500 \\
\hline $\mathrm{C}(6)-\mathrm{C}(7)$ & $1.386(3)$ \\
\hline $\mathrm{C}(6)-\mathrm{H}(6)$ & 0.9500 \\
\hline $\mathrm{C}(7)-\mathrm{C}(8)$ & $1.398(3)$ \\
\hline $\mathrm{C}(7)-\mathrm{H}(7)$ & 0.9500 \\
\hline $\mathrm{C}(8)-\mathrm{C}(9)$ & $1.519(3)$ \\
\hline$C(9)-C(14)$ & $1.531(3)$ \\
\hline$C(9)-C(10)$ & $1.540(3)$ \\
\hline $\mathrm{C}(9)-\mathrm{H}(9)$ & 1.0000 \\
\hline$C(10)-C(11)$ & $1.530(3)$ \\
\hline $\mathrm{C}(10)-\mathrm{H}(10 \mathrm{~A})$ & 0.9900 \\
\hline $\mathrm{C}(10)-\mathrm{H}(10 \mathrm{~B})$ & 0.9900 \\
\hline$C(11)-C(17)$ & $1.522(3)$ \\
\hline$C(11)-C(12)$ & $1.535(3)$ \\
\hline $\mathrm{C}(11)-\mathrm{H}(11)$ & 1.0000 \\
\hline$C(12)-C(13)$ & $1.539(3)$ \\
\hline $\mathrm{C}(12)-\mathrm{H}(12 \mathrm{~A})$ & 0.9900 \\
\hline $\mathrm{C}(12)-\mathrm{H}(12 \mathrm{~B})$ & 0.9900 \\
\hline$C(13)-C(15)$ & $1.515(3)$ \\
\hline$C(13)-C(14)$ & $1.553(3)$ \\
\hline $\mathrm{C}(13)-\mathrm{H}(13)$ & 1.0000 \\
\hline $\mathrm{C}(14)-\mathrm{H}(14)$ & 1.0000 \\
\hline$C(15)-C(16)$ & $1.488(4)$ \\
\hline $\mathrm{C}(16)-\mathrm{H}(16 \mathrm{~A})$ & 0.9800 \\
\hline $\mathrm{C}(16)-\mathrm{H}(16 \mathrm{~B})$ & 0.9800 \\
\hline $\mathrm{C}(16)-\mathrm{H}(16 \mathrm{C})$ & 0.9800 \\
\hline $\mathrm{C}(17)-\mathrm{C}(18)$ & $1.500(3)$ \\
\hline$C(18)-C(19)$ & $1.517(4)$ \\
\hline
\end{tabular}




\begin{tabular}{|c|c|}
\hline $\mathrm{C}(18)-\mathrm{H}(18 \mathrm{~A})$ & 0.9900 \\
\hline $\mathrm{C}(18)-\mathrm{H}(18 \mathrm{~B})$ & 0.9900 \\
\hline C(19)-H(19A) & 0.9800 \\
\hline C(19)-H(19B) & 0.9800 \\
\hline C(19)-H(19C) & 0.9800 \\
\hline$C(20)-C(21)$ & $1.377(3)$ \\
\hline $\mathrm{C}(20)-\mathrm{H}(20)$ & 0.9500 \\
\hline$C(21)-C(22)$ & $1.408(4)$ \\
\hline $\mathrm{C}(21)-\mathrm{H}(21)$ & 0.9500 \\
\hline$C(22)-C(23)$ & $1.410(4)$ \\
\hline$C(23)-C(24)$ & $1.375(3)$ \\
\hline $\mathrm{C}(23)-\mathrm{H}(23)$ & 0.9500 \\
\hline $\mathrm{C}(24)-\mathrm{H}(24)$ & 0.9500 \\
\hline $\mathrm{C}(25)-\mathrm{H}(25 \mathrm{~A})$ & 0.9800 \\
\hline $\mathrm{C}(25)-\mathrm{H}(25 \mathrm{~B})$ & 0.9800 \\
\hline $\mathrm{C}(25)-\mathrm{H}(25 \mathrm{C})$ & 0.9800 \\
\hline $\mathrm{C}(26)-\mathrm{H}(26 \mathrm{~A})$ & 0.9800 \\
\hline $\mathrm{C}(26)-\mathrm{H}(26 \mathrm{~B})$ & 0.9800 \\
\hline $\mathrm{C}(26)-\mathrm{H}(26 \mathrm{C})$ & 0.9800 \\
\hline$C(27)-C(28)$ & $1.371(4)$ \\
\hline $\mathrm{C}(27)-\mathrm{H}(27)$ & 0.9500 \\
\hline$C(28)-C(29)$ & $1.394(3)$ \\
\hline $\mathrm{C}(28)-\mathrm{H}(28)$ & 0.9500 \\
\hline $\mathrm{C}(29)-\mathrm{H}(29)$ & 0.9500 \\
\hline $\mathrm{C}(30)-\mathrm{C}(31)$ & $1.375(5)$ \\
\hline $\mathrm{C}(30)-\mathrm{H}(30)$ & 0.9500 \\
\hline $\mathrm{C}(31)-\mathrm{C}(32)$ & 1.391(4) \\
\hline $\mathrm{C}(31)-\mathrm{H}(31)$ & 0.9500 \\
\hline $\mathrm{C}(32)-\mathrm{H}(32)$ & 0.9500 \\
\hline$C(33)-C(34)$ & $1.368(4)$ \\
\hline $\mathrm{C}(33)-\mathrm{H}(33)$ & 0.9500 \\
\hline$C(34)-C(35)$ & $1.402(4)$ \\
\hline C(34)-H(34) & 0.9500 \\
\hline $\mathrm{C}(35)-\mathrm{H}(35)$ & 0.9500 \\
\hline$C(36)-C(37)$ & $1.449(8)$ \\
\hline $\mathrm{C}(36)-\mathrm{H}(36 \mathrm{~A})$ & 0.9900 \\
\hline
\end{tabular}




\begin{tabular}{|c|c|}
\hline $\mathrm{C}(36)-\mathrm{H}(36 \mathrm{~B})$ & 0.9900 \\
\hline$C(37)-C(38)$ & $1.447(10)$ \\
\hline $\mathrm{C}(37)-\mathrm{H}(37 \mathrm{~A})$ & 0.9900 \\
\hline $\mathrm{C}(37)-\mathrm{H}(37 \mathrm{~B})$ & 0.9900 \\
\hline$C(38)-C(39)$ & $1.503(12)$ \\
\hline $\mathrm{C}(38)-\mathrm{H}(38 \mathrm{~A})$ & 0.9900 \\
\hline $\mathrm{C}(38)-\mathrm{H}(38 \mathrm{~B})$ & 0.9900 \\
\hline $\mathrm{C}(39)-\mathrm{H}(39 \mathrm{~A})$ & 0.9900 \\
\hline $\mathrm{C}(39)-\mathrm{H}(39 \mathrm{~B})$ & 0.9900 \\
\hline$C(40)-C(41)$ & $1.387(15)$ \\
\hline $\mathrm{C}(40)-\mathrm{H}(40 \mathrm{~A})$ & 0.9900 \\
\hline $\mathrm{C}(40)-\mathrm{H}(40 \mathrm{~B})$ & 0.9900 \\
\hline$C(41)-C(42)$ & $1.420(16)$ \\
\hline $\mathrm{C}(41)-\mathrm{H}(41 \mathrm{~A})$ & 0.9900 \\
\hline $\mathrm{C}(41)-\mathrm{H}(41 \mathrm{~B})$ & 0.9900 \\
\hline$C(42)-C(43)$ & $1.322(16)$ \\
\hline $\mathrm{C}(42)-\mathrm{H}(42 \mathrm{~A})$ & 0.9900 \\
\hline $\mathrm{C}(42)-\mathrm{H}(42 \mathrm{~B})$ & 0.9900 \\
\hline $\mathrm{C}(43)-\mathrm{H}(43 \mathrm{~A})$ & 0.9900 \\
\hline $\mathrm{C}(43)-\mathrm{H}(43 \mathrm{~B})$ & 0.9900 \\
\hline $\mathrm{B}(1)-\mathrm{H}(1 \mathrm{~B})$ & $1.09(3)$ \\
\hline N(9)-Mo-N(7) & $93.28(8)$ \\
\hline N(9)-Mo-N(1) & $90.70(8)$ \\
\hline $\mathrm{N}(7)-\mathrm{Mo}-\mathrm{N}(1)$ & $159.72(7)$ \\
\hline N(9)-Mo-C(1) & $99.18(7)$ \\
\hline N(7)-Mo-C(1) & $81.52(7)$ \\
\hline $\mathrm{N}(1)-\mathrm{Mo}-\mathrm{C}(1)$ & $117.45(7)$ \\
\hline N(9)-Mo-N(5) & $93.34(7)$ \\
\hline N(7)-Mo-N(5) & $80.33(7)$ \\
\hline $\mathrm{N}(1)-\mathrm{Mo}-\mathrm{N}(5)$ & $79.59(7)$ \\
\hline $\mathrm{C}(1)-\mathrm{Mo}-\mathrm{N}(5)$ & $158.46(7)$ \\
\hline N(9)-Mo-C(2) & $97.35(7)$ \\
\hline N(7)-Mo-C(2) & $118.90(7)$ \\
\hline N(1)-Mo-C(2) & $80.19(7)$ \\
\hline $\mathrm{C}(1)-\mathrm{Mo}-\mathrm{C}(2)$ & $37.40(7)$ \\
\hline
\end{tabular}




\begin{tabular}{|c|c|}
\hline N(5)-Mo-C(2) & $157.20(7)$ \\
\hline N(9)-Mo-N(3) & $173.67(7)$ \\
\hline N(7)-Mo-N(3) & $83.13(7)$ \\
\hline N(1)-Mo-N(3) & $90.92(7)$ \\
\hline C(1)-Mo-N(3) & $85.48(7)$ \\
\hline N(5)-Mo-N(3) & $80.94(7)$ \\
\hline C(2)-Mo-N(3) & $88.95(7)$ \\
\hline $\mathrm{C}(36)-\mathrm{O}(4)-\mathrm{C}(39)$ & $104.4(5)$ \\
\hline $\mathrm{C}(43)-\mathrm{O}(5)-\mathrm{C}(40)$ & $109.9(9)$ \\
\hline $\mathrm{C}(20)-\mathrm{N}(1)-\mathrm{C}(24)$ & $115.38(19)$ \\
\hline $\mathrm{C}(20)-\mathrm{N}(1)-\mathrm{Mo}$ & $122.57(15)$ \\
\hline $\mathrm{C}(24)-\mathrm{N}(1)-\mathrm{Mo}$ & $120.90(14)$ \\
\hline $\mathrm{C}(22)-\mathrm{N}(2)-\mathrm{C}(26)$ & $120.8(3)$ \\
\hline $\mathrm{C}(22)-\mathrm{N}(2)-\mathrm{C}(25)$ & $120.4(3)$ \\
\hline $\mathrm{C}(26)-\mathrm{N}(2)-\mathrm{C}(25)$ & $118.8(3)$ \\
\hline $\mathrm{C}(29)-\mathrm{N}(3)-\mathrm{N}(4)$ & $105.97(17)$ \\
\hline $\mathrm{C}(29)-\mathrm{N}(3)-\mathrm{Mo}$ & $134.84(14)$ \\
\hline $\mathrm{N}(4)-\mathrm{N}(3)-\mathrm{Mo}$ & $119.19(13)$ \\
\hline $\mathrm{C}(27)-\mathrm{N}(4)-\mathrm{N}(3)$ & $109.47(19)$ \\
\hline $\mathrm{C}(27)-\mathrm{N}(4)-\mathrm{B}(1)$ & $129.25(19)$ \\
\hline $\mathrm{N}(3)-\mathrm{N}(4)-\mathrm{B}(1)$ & $121.26(17)$ \\
\hline $\mathrm{C}(32)-\mathrm{N}(5)-\mathrm{N}(6)$ & $107.34(18)$ \\
\hline $\mathrm{C}(32)-\mathrm{N}(5)-\mathrm{Mo}$ & $130.30(16)$ \\
\hline $\mathrm{N}(6)-\mathrm{N}(5)-\mathrm{Mo}$ & $122.30(15)$ \\
\hline $\mathrm{C}(30)-\mathrm{N}(6)-\mathrm{N}(5)$ & $109.3(2)$ \\
\hline $\mathrm{C}(30)-\mathrm{N}(6)-\mathrm{B}(1)$ & $131.4(2)$ \\
\hline $\mathrm{N}(5)-\mathrm{N}(6)-\mathrm{B}(1)$ & $119.29(18)$ \\
\hline $\mathrm{C}(35)-\mathrm{N}(7)-\mathrm{N}(8)$ & $106.57(19)$ \\
\hline $\mathrm{C}(35)-\mathrm{N}(7)-\mathrm{Mo}$ & $131.75(16)$ \\
\hline $\mathrm{N}(8)-\mathrm{N}(7)-\mathrm{Mo}$ & $121.15(14)$ \\
\hline $\mathrm{C}(33)-\mathrm{N}(8)-\mathrm{N}(7)$ & $109.2(2)$ \\
\hline $\mathrm{C}(33)-\mathrm{N}(8)-\mathrm{B}(1)$ & $128.7(2)$ \\
\hline $\mathrm{N}(7)-\mathrm{N}(8)-\mathrm{B}(1)$ & $121.01(18)$ \\
\hline $\mathrm{O}(3)-\mathrm{N}(9)-\mathrm{Mo}$ & $174.95(15)$ \\
\hline $\mathrm{C}(2)-\mathrm{C}(1)-\mathrm{C}(14)$ & 117.91(16) \\
\hline $\mathrm{C}(2)-\mathrm{C}(1)-\mathrm{Mo}$ & $71.41(11)$ \\
\hline
\end{tabular}




\begin{tabular}{|c|c|}
\hline $\mathrm{C}(14)-\mathrm{C}(1)-\mathrm{Mo}$ & $127.83(14)$ \\
\hline $\mathrm{C}(2)-\mathrm{C}(1)-\mathrm{H}(1)$ & 111.2 \\
\hline $\mathrm{C}(14)-\mathrm{C}(1)-\mathrm{H}(1)$ & 111.2 \\
\hline Mo-C(1)-H(1) & 111.2 \\
\hline$C(1)-C(2)-C(3)$ & $119.26(16)$ \\
\hline $\mathrm{C}(1)-\mathrm{C}(2)-\mathrm{Mo}$ & $71.18(11)$ \\
\hline $\mathrm{C}(3)-\mathrm{C}(2)-\mathrm{Mo}$ & $118.22(13)$ \\
\hline $\mathrm{C}(1)-\mathrm{C}(2)-\mathrm{H}(2)$ & 113.9 \\
\hline $\mathrm{C}(3)-\mathrm{C}(2)-\mathrm{H}(2)$ & 113.9 \\
\hline Mo-C(2)-H(2) & 113.9 \\
\hline $\mathrm{C}(4)-\mathrm{C}(3)-\mathrm{C}(8)$ & $119.04(17)$ \\
\hline$C(4)-C(3)-C(2)$ & $119.87(17)$ \\
\hline $\mathrm{C}(8)-\mathrm{C}(3)-\mathrm{C}(2)$ & $121.08(17)$ \\
\hline$C(5)-C(4)-C(3)$ & $121.31(19)$ \\
\hline $\mathrm{C}(5)-\mathrm{C}(4)-\mathrm{H}(4)$ & 119.3 \\
\hline $\mathrm{C}(3)-\mathrm{C}(4)-\mathrm{H}(4)$ & 119.3 \\
\hline$C(6)-C(5)-C(4)$ & $119.43(19)$ \\
\hline $\mathrm{C}(6)-\mathrm{C}(5)-\mathrm{H}(5)$ & 120.3 \\
\hline $\mathrm{C}(4)-\mathrm{C}(5)-\mathrm{H}(5)$ & 120.3 \\
\hline$C(7)-C(6)-C(5)$ & $119.85(19)$ \\
\hline $\mathrm{C}(7)-\mathrm{C}(6)-\mathrm{H}(6)$ & 120.1 \\
\hline $\mathrm{C}(5)-\mathrm{C}(6)-\mathrm{H}(6)$ & 120.1 \\
\hline$C(6)-C(7)-C(8)$ & $121.72(19)$ \\
\hline $\mathrm{C}(6)-\mathrm{C}(7)-\mathrm{H}(7)$ & 119.1 \\
\hline $\mathrm{C}(8)-\mathrm{C}(7)-\mathrm{H}(7)$ & 119.1 \\
\hline$C(7)-C(8)-C(3)$ & $118.60(17)$ \\
\hline $\mathrm{C}(7)-\mathrm{C}(8)-\mathrm{C}(9)$ & $121.41(17)$ \\
\hline $\mathrm{C}(3)-\mathrm{C}(8)-\mathrm{C}(9)$ & $119.99(16)$ \\
\hline C(8)-C(9)-C(14) & $111.29(16)$ \\
\hline$C(8)-C(9)-C(10)$ & $113.78(16)$ \\
\hline$C(14)-C(9)-C(10)$ & $110.38(16)$ \\
\hline C(8)-C(9)-H(9) & 107.0 \\
\hline $\mathrm{C}(14)-\mathrm{C}(9)-\mathrm{H}(9)$ & 107.0 \\
\hline $\mathrm{C}(10)-\mathrm{C}(9)-\mathrm{H}(9)$ & 107.0 \\
\hline $\mathrm{C}(11)-\mathrm{C}(10)-\mathrm{C}(9)$ & $111.28(17)$ \\
\hline $\mathrm{C}(11)-\mathrm{C}(10)-\mathrm{H}(10 \mathrm{~A})$ & 109.4 \\
\hline
\end{tabular}




\begin{tabular}{|c|c|}
\hline $\mathrm{C}(9)-\mathrm{C}(10)-\mathrm{H}(10 \mathrm{~A})$ & 109.4 \\
\hline $\mathrm{C}(11)-\mathrm{C}(10)-\mathrm{H}(10 \mathrm{~B})$ & 109.4 \\
\hline $\mathrm{C}(9)-\mathrm{C}(10)-\mathrm{H}(10 \mathrm{~B})$ & 109.4 \\
\hline $\mathrm{H}(10 \mathrm{~A})-\mathrm{C}(10)-\mathrm{H}(10 \mathrm{~B})$ & 108.0 \\
\hline$C(17)-C(11)-C(10)$ & $110.76(19)$ \\
\hline$C(17)-C(11)-C(12)$ & $111.03(18)$ \\
\hline$C(10)-C(11)-C(12)$ & $109.06(17)$ \\
\hline $\mathrm{C}(17)-\mathrm{C}(11)-\mathrm{H}(11)$ & 108.6 \\
\hline $\mathrm{C}(10)-\mathrm{C}(11)-\mathrm{H}(11)$ & 108.6 \\
\hline $\mathrm{C}(12)-\mathrm{C}(11)-\mathrm{H}(11)$ & 108.6 \\
\hline$C(11)-C(12)-C(13)$ & $110.76(17)$ \\
\hline $\mathrm{C}(11)-\mathrm{C}(12)-\mathrm{H}(12 \mathrm{~A})$ & 109.5 \\
\hline $\mathrm{C}(13)-\mathrm{C}(12)-\mathrm{H}(12 \mathrm{~A})$ & 109.5 \\
\hline $\mathrm{C}(11)-\mathrm{C}(12)-\mathrm{H}(12 \mathrm{~B})$ & 109.5 \\
\hline $\mathrm{C}(13)-\mathrm{C}(12)-\mathrm{H}(12 \mathrm{~B})$ & 109.5 \\
\hline $\mathrm{H}(12 \mathrm{~A})-\mathrm{C}(12)-\mathrm{H}(12 \mathrm{~B})$ & 108.1 \\
\hline$C(15)-C(13)-C(12)$ & $107.97(17)$ \\
\hline$C(15)-C(13)-C(14)$ & $110.64(17)$ \\
\hline$C(12)-C(13)-C(14)$ & $112.40(18)$ \\
\hline $\mathrm{C}(15)-\mathrm{C}(13)-\mathrm{H}(13)$ & 108.6 \\
\hline $\mathrm{C}(12)-\mathrm{C}(13)-\mathrm{H}(13)$ & 108.6 \\
\hline $\mathrm{C}(14)-\mathrm{C}(13)-\mathrm{H}(13)$ & 108.6 \\
\hline $\mathrm{C}(1)-\mathrm{C}(14)-\mathrm{C}(9)$ & $112.50(15)$ \\
\hline$C(1)-C(14)-C(13)$ & $108.60(17)$ \\
\hline$C(9)-C(14)-C(13)$ & $111.16(15)$ \\
\hline $\mathrm{C}(1)-\mathrm{C}(14)-\mathrm{H}(14)$ & 108.1 \\
\hline $\mathrm{C}(9)-\mathrm{C}(14)-\mathrm{H}(14)$ & 108.1 \\
\hline $\mathrm{C}(13)-\mathrm{C}(14)-\mathrm{H}(14)$ & 108.1 \\
\hline $\mathrm{O}(1)-\mathrm{C}(15)-\mathrm{C}(16)$ & $121.7(3)$ \\
\hline $\mathrm{O}(1)-\mathrm{C}(15)-\mathrm{C}(13)$ & $120.5(2)$ \\
\hline $\mathrm{C}(16)-\mathrm{C}(15)-\mathrm{C}(13)$ & $117.8(2)$ \\
\hline$C(15)-C(16)-H(16 A)$ & 109.5 \\
\hline $\mathrm{C}(15)-\mathrm{C}(16)-\mathrm{H}(16 \mathrm{~B})$ & 109.5 \\
\hline $\mathrm{H}(16 \mathrm{~A})-\mathrm{C}(16)-\mathrm{H}(16 \mathrm{~B})$ & 109.5 \\
\hline $\mathrm{C}(15)-\mathrm{C}(16)-\mathrm{H}(16 \mathrm{C})$ & 109.5 \\
\hline $\mathrm{H}(16 \mathrm{~A})-\mathrm{C}(16)-\mathrm{H}(16 \mathrm{C})$ & 109.5 \\
\hline
\end{tabular}




\begin{tabular}{|c|c|}
\hline $\mathrm{H}(16 \mathrm{~B})-\mathrm{C}(16)-\mathrm{H}(16 \mathrm{C})$ & 109.5 \\
\hline $\mathrm{O}(2)-\mathrm{C}(17)-\mathrm{C}(18)$ & $122.3(2)$ \\
\hline $\mathrm{O}(2)-\mathrm{C}(17)-\mathrm{C}(11)$ & $121.6(2)$ \\
\hline$C(18)-C(17)-C(11)$ & $116.11(19)$ \\
\hline$C(17)-C(18)-C(19)$ & $114.7(2)$ \\
\hline $\mathrm{C}(17)-\mathrm{C}(18)-\mathrm{H}(18 \mathrm{~A})$ & 108.6 \\
\hline $\mathrm{C}(19)-\mathrm{C}(18)-\mathrm{H}(18 \mathrm{~A})$ & 108.6 \\
\hline $\mathrm{C}(17)-\mathrm{C}(18)-\mathrm{H}(18 \mathrm{~B})$ & 108.6 \\
\hline $\mathrm{C}(19)-\mathrm{C}(18)-\mathrm{H}(18 \mathrm{~B})$ & 108.6 \\
\hline $\mathrm{H}(18 \mathrm{~A})-\mathrm{C}(18)-\mathrm{H}(18 \mathrm{~B})$ & 107.6 \\
\hline $\mathrm{C}(18)-\mathrm{C}(19)-\mathrm{H}(19 \mathrm{~A})$ & 109.5 \\
\hline $\mathrm{C}(18)-\mathrm{C}(19)-\mathrm{H}(19 \mathrm{~B})$ & 109.5 \\
\hline $\mathrm{H}(19 \mathrm{~A})-\mathrm{C}(19)-\mathrm{H}(19 \mathrm{~B})$ & 109.5 \\
\hline $\mathrm{C}(18)-\mathrm{C}(19)-\mathrm{H}(19 \mathrm{C})$ & 109.5 \\
\hline $\mathrm{H}(19 \mathrm{~A})-\mathrm{C}(19)-\mathrm{H}(19 \mathrm{C})$ & 109.5 \\
\hline $\mathrm{H}(19 \mathrm{~B})-\mathrm{C}(19)-\mathrm{H}(19 \mathrm{C})$ & 109.5 \\
\hline $\mathrm{N}(1)-\mathrm{C}(20)-\mathrm{C}(21)$ & $124.6(2)$ \\
\hline $\mathrm{N}(1)-\mathrm{C}(20)-\mathrm{H}(20)$ & 117.7 \\
\hline $\mathrm{C}(21)-\mathrm{C}(20)-\mathrm{H}(20)$ & 117.7 \\
\hline$C(20)-C(21)-C(22)$ & $119.9(2)$ \\
\hline $\mathrm{C}(20)-\mathrm{C}(21)-\mathrm{H}(21)$ & 120.1 \\
\hline $\mathrm{C}(22)-\mathrm{C}(21)-\mathrm{H}(21)$ & 120.1 \\
\hline $\mathrm{N}(2)-\mathrm{C}(22)-\mathrm{C}(21)$ & $122.7(3)$ \\
\hline $\mathrm{N}(2)-\mathrm{C}(22)-\mathrm{C}(23)$ & $121.6(3)$ \\
\hline $\mathrm{C}(21)-\mathrm{C}(22)-\mathrm{C}(23)$ & $115.7(2)$ \\
\hline$C(24)-C(23)-C(22)$ & $120.2(2)$ \\
\hline $\mathrm{C}(24)-\mathrm{C}(23)-\mathrm{H}(23)$ & 119.9 \\
\hline $\mathrm{C}(22)-\mathrm{C}(23)-\mathrm{H}(23)$ & 119.9 \\
\hline $\mathrm{N}(1)-\mathrm{C}(24)-\mathrm{C}(23)$ & $124.3(2)$ \\
\hline $\mathrm{N}(1)-\mathrm{C}(24)-\mathrm{H}(24)$ & 117.9 \\
\hline $\mathrm{C}(23)-\mathrm{C}(24)-\mathrm{H}(24)$ & 117.9 \\
\hline $\mathrm{N}(2)-\mathrm{C}(25)-\mathrm{H}(25 \mathrm{~A})$ & 109.5 \\
\hline $\mathrm{N}(2)-\mathrm{C}(25)-\mathrm{H}(25 \mathrm{~B})$ & 109.5 \\
\hline $\mathrm{H}(25 \mathrm{~A})-\mathrm{C}(25)-\mathrm{H}(25 \mathrm{~B})$ & 109.5 \\
\hline $\mathrm{N}(2)-\mathrm{C}(25)-\mathrm{H}(25 \mathrm{C})$ & 109.5 \\
\hline $\mathrm{H}(25 \mathrm{~A})-\mathrm{C}(25)-\mathrm{H}(25 \mathrm{C})$ & 109.5 \\
\hline
\end{tabular}




\begin{tabular}{|c|c|}
\hline $\mathrm{H}(25 \mathrm{~B})-\mathrm{C}(25)-\mathrm{H}(25 \mathrm{C})$ & 109.5 \\
\hline $\mathrm{N}(2)-\mathrm{C}(26)-\mathrm{H}(26 \mathrm{~A})$ & 109.5 \\
\hline $\mathrm{N}(2)-\mathrm{C}(26)-\mathrm{H}(26 \mathrm{~B})$ & 109.5 \\
\hline $\mathrm{H}(26 \mathrm{~A})-\mathrm{C}(26)-\mathrm{H}(26 \mathrm{~B})$ & 109.5 \\
\hline $\mathrm{N}(2)-\mathrm{C}(26)-\mathrm{H}(26 \mathrm{C})$ & 109.5 \\
\hline $\mathrm{H}(26 \mathrm{~A})-\mathrm{C}(26)-\mathrm{H}(26 \mathrm{C})$ & 109.5 \\
\hline $\mathrm{H}(26 \mathrm{~B})-\mathrm{C}(26)-\mathrm{H}(26 \mathrm{C})$ & 109.5 \\
\hline $\mathrm{N}(4)-\mathrm{C}(27)-\mathrm{C}(28)$ & 109.2(2) \\
\hline $\mathrm{N}(4)-\mathrm{C}(27)-\mathrm{H}(27)$ & 125.4 \\
\hline $\mathrm{C}(28)-\mathrm{C}(27)-\mathrm{H}(27)$ & 125.4 \\
\hline $\mathrm{C}(27)-\mathrm{C}(28)-\mathrm{C}(29)$ & $104.4(2)$ \\
\hline $\mathrm{C}(27)-\mathrm{C}(28)-\mathrm{H}(28)$ & 127.8 \\
\hline $\mathrm{C}(29)-\mathrm{C}(28)-\mathrm{H}(28)$ & 127.8 \\
\hline $\mathrm{N}(3)-\mathrm{C}(29)-\mathrm{C}(28)$ & $111.0(2)$ \\
\hline $\mathrm{N}(3)-\mathrm{C}(29)-\mathrm{H}(29)$ & 124.5 \\
\hline $\mathrm{C}(28)-\mathrm{C}(29)-\mathrm{H}(29)$ & 124.5 \\
\hline $\mathrm{N}(6)-\mathrm{C}(30)-\mathrm{C}(31)$ & $108.1(2)$ \\
\hline $\mathrm{N}(6)-\mathrm{C}(30)-\mathrm{H}(30)$ & 126.0 \\
\hline $\mathrm{C}(31)-\mathrm{C}(30)-\mathrm{H}(30)$ & 126.0 \\
\hline $\mathrm{C}(30)-\mathrm{C}(31)-\mathrm{C}(32)$ & $105.7(2)$ \\
\hline $\mathrm{C}(30)-\mathrm{C}(31)-\mathrm{H}(31)$ & 127.1 \\
\hline $\mathrm{C}(32)-\mathrm{C}(31)-\mathrm{H}(31)$ & 127.1 \\
\hline $\mathrm{N}(5)-\mathrm{C}(32)-\mathrm{C}(31)$ & $109.5(2)$ \\
\hline $\mathrm{N}(5)-\mathrm{C}(32)-\mathrm{H}(32)$ & 125.2 \\
\hline $\mathrm{C}(31)-\mathrm{C}(32)-\mathrm{H}(32)$ & 125.2 \\
\hline $\mathrm{N}(8)-\mathrm{C}(33)-\mathrm{C}(34)$ & 109.2(2) \\
\hline $\mathrm{N}(8)-\mathrm{C}(33)-\mathrm{H}(33)$ & 125.4 \\
\hline $\mathrm{C}(34)-\mathrm{C}(33)-\mathrm{H}(33)$ & 125.4 \\
\hline $\mathrm{C}(33)-\mathrm{C}(34)-\mathrm{C}(35)$ & $104.5(2)$ \\
\hline $\mathrm{C}(33)-\mathrm{C}(34)-\mathrm{H}(34)$ & 127.7 \\
\hline $\mathrm{C}(35)-\mathrm{C}(34)-\mathrm{H}(34)$ & 127.7 \\
\hline $\mathrm{N}(7)-\mathrm{C}(35)-\mathrm{C}(34)$ & $110.5(2)$ \\
\hline $\mathrm{N}(7)-\mathrm{C}(35)-\mathrm{H}(35)$ & 124.8 \\
\hline $\mathrm{C}(34)-\mathrm{C}(35)-\mathrm{H}(35)$ & 124.8 \\
\hline $\mathrm{O}(4)-\mathrm{C}(36)-\mathrm{C}(37)$ & $110.1(5)$ \\
\hline $\mathrm{O}(4)-\mathrm{C}(36)-\mathrm{H}(36 \mathrm{~A})$ & 109.6 \\
\hline
\end{tabular}




\begin{tabular}{ll}
$\mathrm{C}(37)-\mathrm{C}(36)-\mathrm{H}(36 \mathrm{~A})$ & 109.6 \\
$\mathrm{O}(4)-\mathrm{C}(36)-\mathrm{H}(36 \mathrm{~B})$ & 109.6 \\
$\mathrm{C}(37)-\mathrm{C}(36)-\mathrm{H}(36 \mathrm{~B})$ & 109.6 \\
$\mathrm{H}(36 \mathrm{~A})-\mathrm{C}(36)-\mathrm{H}(36 \mathrm{~B})$ & 108.2 \\
$\mathrm{C}(38)-\mathrm{C}(37)-\mathrm{C}(36)$ & $99.8(6)$ \\
$\mathrm{C}(38)-\mathrm{C}(37)-\mathrm{H}(37 \mathrm{~A})$ & 111.8 \\
$\mathrm{C}(36)-\mathrm{C}(37)-\mathrm{H}(37 \mathrm{~A})$ & 111.8 \\
$\mathrm{C}(38)-\mathrm{C}(37)-\mathrm{H}(37 \mathrm{~B})$ & 111.8 \\
$\mathrm{C}(36)-\mathrm{C}(37)-\mathrm{H}(37 \mathrm{~B})$ & 111.8 \\
$\mathrm{H}(37 \mathrm{~A})-\mathrm{C}(37)-\mathrm{H}(37 \mathrm{~B})$ & 109.5 \\
$\mathrm{C}(37)-\mathrm{C}(38)-\mathrm{C}(39)$ & $109.5(6)$ \\
$\mathrm{C}(37)-\mathrm{C}(38)-\mathrm{H}(38 \mathrm{~A})$ & 109.8 \\
$\mathrm{C}(39)-\mathrm{C}(38)-\mathrm{H}(38 \mathrm{~A})$ & 109.8 \\
$\mathrm{C}(37)-\mathrm{C}(38)-\mathrm{H}(38 \mathrm{~B})$ & 109.8 \\
$\mathrm{C}(39)-\mathrm{C}(38)-\mathrm{H}(38 \mathrm{~B})$ & 109.8 \\
$\mathrm{H}(38 \mathrm{~A})-\mathrm{C}(38)-\mathrm{H}(38 \mathrm{~B})$ & 108.2 \\
$\mathrm{O}(4)-\mathrm{C}(39)-\mathrm{C}(38)$ & $103.8(5)$ \\
$\mathrm{O}(4)-\mathrm{C}(39)-\mathrm{H}(39 \mathrm{~A})$ & 111.0 \\
$\mathrm{C}(38)-\mathrm{C}(39)-\mathrm{H}(39 \mathrm{~A})$ & 111.0 \\
$\mathrm{O}(4)-\mathrm{C}(39)-\mathrm{H}(39 \mathrm{~B})$ & 111.0 \\
$\mathrm{C}(38)-\mathrm{C}(39)-\mathrm{H}(39 \mathrm{~B})$ & 111.0 \\
$\mathrm{H}(39 \mathrm{~A})-\mathrm{C}(39)-\mathrm{H}(39 \mathrm{~B})$ & 109.0 \\
$\mathrm{O}(5)-\mathrm{C}(40)-\mathrm{C}(41)$ & $106.2(9)$ \\
$\mathrm{O}(5)-\mathrm{C}(40)-\mathrm{H}(40 \mathrm{~A})$ & 110.5 \\
$\mathrm{C}(41)-\mathrm{C}(40)-\mathrm{H}(40 \mathrm{~A})$ & 110.5 \\
$\mathrm{O}(5)-\mathrm{C}(40)-\mathrm{H}(40 \mathrm{~B})$ & 110.5 \\
$\mathrm{C}(41)-\mathrm{C}(40)-\mathrm{H}(40 \mathrm{~B})$ & 110.5 \\
$\mathrm{H}(40 \mathrm{~A})-\mathrm{C}(40)-\mathrm{H}(40 \mathrm{~B})$ & 108.7 \\
$\mathrm{C}(40)-\mathrm{C}(41)-\mathrm{C}(42)$ & $106.7(9)$ \\
$\mathrm{C}(40)-\mathrm{C}(41)-\mathrm{H}(41 \mathrm{~A})$ & 110.4 \\
$\mathrm{C}(42)-\mathrm{C}(41)-\mathrm{H}(41 \mathrm{~A})$ & 110.4 \\
$\mathrm{C}(40)-\mathrm{C}(41)-\mathrm{H}(41 \mathrm{~B})$ & 110.4 \\
$\mathrm{C}(42)-\mathrm{C}(41)-\mathrm{H}(41 \mathrm{~B})$ & 110.4 \\
$\mathrm{C}(42)-\mathrm{C}(41)-\mathrm{H}(41 \mathrm{~B})$ & 108.6 \\
& $104.0(10)$ \\
& 111.0 \\
& \\
$\mathrm{C}(42 \mathrm{~A})$ & \\
\hline
\end{tabular}




$\begin{array}{ll}\mathrm{C}(41)-\mathrm{C}(42)-\mathrm{H}(42 \mathrm{~A}) & 111.0 \\ \mathrm{C}(43)-\mathrm{C}(42)-\mathrm{H}(42 \mathrm{~B}) & 111.0 \\ \mathrm{C}(41)-\mathrm{C}(42)-\mathrm{H}(42 \mathrm{~B}) & 111.0 \\ \mathrm{H}(42 \mathrm{~A})-\mathrm{C}(42)-\mathrm{H}(42 \mathrm{~B}) & 109.0 \\ \mathrm{O}(5)-\mathrm{C}(43)-\mathrm{C}(42) & 112.6(11) \\ \mathrm{O}(5)-\mathrm{C}(43)-\mathrm{H}(43 \mathrm{~A}) & 109.1 \\ \mathrm{C}(42)-\mathrm{C}(43)-\mathrm{H}(43 \mathrm{~A}) & 109.1 \\ \mathrm{O}(5)-\mathrm{C}(43)-\mathrm{H}(43 \mathrm{~B}) & 109.1 \\ \mathrm{C}(42)-\mathrm{C}(43)-\mathrm{H}(43 \mathrm{~B}) & 109.1 \\ \mathrm{H}(43 \mathrm{~A})-\mathrm{C}(43)-\mathrm{H}(43 \mathrm{~B}) & 107.8 \\ \mathrm{~N}(8)-\mathrm{B}(1)-\mathrm{N}(6) & 107.6(2) \\ \mathrm{N}(8)-\mathrm{B}(1)-\mathrm{N}(4) & 109.9(2) \\ \mathrm{N}(6)-\mathrm{B}(1)-\mathrm{N}(4) & 108.3(2) \\ \mathrm{N}(8)-\mathrm{B}(1)-\mathrm{H}(1 \mathrm{~B}) & 107.5(19) \\ \mathrm{N}(6)-\mathrm{B}(1)-\mathrm{H}(1 \mathrm{~B}) & 109.9(19) \\ \mathrm{N}(4)-\mathrm{B}(1)-\mathrm{H}(1 \mathrm{~B}) & 113.6(19)\end{array}$

Symmetry transformations used to generate equivalent atoms: 


\section{Compound 90}

Table 4. Anisotropic displacement parameters $\left(\AA^{2} \times 10^{3}\right)$ for C31H54BN9O4.5Mo. The anisotropic displacement factor exponent takes the form: $-2 \pi^{2}\left[h^{2} a^{* 2} U^{11}+\ldots+2 h k a^{*} b^{*} U^{12}\right]$

\begin{tabular}{|c|c|c|c|c|c|c|}
\hline & $\mathrm{U}^{11}$ & $\mathrm{U}^{22}$ & $\mathrm{U}^{33}$ & $\mathrm{U}^{23}$ & $\mathrm{U}^{13}$ & $\mathrm{U}^{12}$ \\
\hline Mo & $25(1)$ & $24(1)$ & $20(1)$ & $4(1)$ & $1(1)$ & $3(1)$ \\
\hline $\mathrm{O}(1)$ & $60(1)$ & $31(1)$ & $51(1)$ & $1(1)$ & $-1(1)$ & $-8(1)$ \\
\hline $\mathrm{O}(2)$ & $76(2)$ & $47(1)$ & $33(1)$ & $-12(1)$ & $-14(1)$ & $17(1)$ \\
\hline $\mathrm{O}(3)$ & $30(1)$ & $58(1)$ & $32(1)$ & $17(1)$ & $5(1)$ & $-2(1)$ \\
\hline $\mathrm{O}(4)$ & $111(3)$ & $89(2)$ & $59(2)$ & $-18(2)$ & $26(2)$ & $-18(2)$ \\
\hline $\mathrm{O}(5)$ & $103(6)$ & $361(18)$ & $68(5)$ & $-86(8)$ & $-12(4)$ & $98(9)$ \\
\hline $\mathrm{N}(1)$ & $28(1)$ & $27(1)$ & $23(1)$ & $2(1)$ & $0(1)$ & $0(1)$ \\
\hline $\mathrm{N}(2)$ & $82(2)$ & $35(1)$ & $46(1)$ & $-10(1)$ & $-15(1)$ & $-2(1)$ \\
\hline $\mathrm{N}(3)$ & $30(1)$ & $26(1)$ & $27(1)$ & $6(1)$ & $5(1)$ & $2(1)$ \\
\hline $\mathrm{N}(4)$ & $37(1)$ & $26(1)$ & $27(1)$ & $7(1)$ & $5(1)$ & $4(1)$ \\
\hline $\mathrm{N}(5)$ & $30(1)$ & $35(1)$ & $25(1)$ & $7(1)$ & $2(1)$ & $7(1)$ \\
\hline $\mathrm{N}(6)$ & $36(1)$ & $38(1)$ & $28(1)$ & $12(1)$ & $4(1)$ & $14(1)$ \\
\hline $\mathrm{N}(7)$ & $42(1)$ & $29(1)$ & $25(1)$ & $2(1)$ & $2(1)$ & $9(1)$ \\
\hline $\mathrm{N}(8)$ & $54(1)$ & $26(1)$ & $33(1)$ & $3(1)$ & $6(1)$ & $12(1)$ \\
\hline $\mathrm{N}(9)$ & $25(1)$ & $32(1)$ & $22(1)$ & $6(1)$ & $1(1)$ & $3(1)$ \\
\hline $\mathrm{C}(1)$ & $25(1)$ & $25(1)$ & $25(1)$ & $4(1)$ & $1(1)$ & $-1(1)$ \\
\hline$C(2)$ & $26(1)$ & $25(1)$ & $23(1)$ & $3(1)$ & $3(1)$ & $2(1)$ \\
\hline$C(3)$ & $21(1)$ & $25(1)$ & $22(1)$ & $2(1)$ & $1(1)$ & $2(1)$ \\
\hline$C(4)$ & $28(1)$ & $26(1)$ & $27(1)$ & $-1(1)$ & $3(1)$ & $2(1)$ \\
\hline$C(5)$ & $30(1)$ & $23(1)$ & $36(1)$ & $-2(1)$ & $1(1)$ & $2(1)$ \\
\hline$C(6)$ & $34(1)$ & $25(1)$ & $34(1)$ & $6(1)$ & $-2(1)$ & $-2(1)$ \\
\hline$C(7)$ & $32(1)$ & $29(1)$ & $24(1)$ & $6(1)$ & $1(1)$ & $-1(1)$ \\
\hline $\mathrm{C}(8)$ & $23(1)$ & $24(1)$ & $22(1)$ & $2(1)$ & $-1(1)$ & $0(1)$ \\
\hline$C(9)$ & $24(1)$ & $27(1)$ & $23(1)$ & $0(1)$ & $-1(1)$ & $2(1)$ \\
\hline$C(10)$ & $30(1)$ & $34(1)$ & $22(1)$ & $-2(1)$ & $-2(1)$ & $4(1)$ \\
\hline $\mathrm{C}(11)$ & $30(1)$ & $30(1)$ & $26(1)$ & $-3(1)$ & $-5(1)$ & $6(1)$ \\
\hline$C(12)$ & $29(1)$ & $33(1)$ & $34(1)$ & $-2(1)$ & $-8(1)$ & 1(1) \\
\hline$C(13)$ & $26(1)$ & $26(1)$ & $29(1)$ & $-1(1)$ & $-2(1)$ & 1(1) \\
\hline$C(14)$ & $25(1)$ & $24(1)$ & $25(1)$ & $0(1)$ & $-3(1)$ & $3(1)$ \\
\hline$C(15)$ & $31(1)$ & $37(1)$ & $40(1)$ & $2(1)$ & $-4(1)$ & $-3(1)$ \\
\hline
\end{tabular}




$\begin{array}{lcccccc}\mathrm{C}(16) & 49(2) & 61(2) & 87(3) & 9(2) & 31(2) & 3(2) \\ \mathrm{C}(17) & 31(1) & 35(1) & 29(1) & -2(1) & -7(1) & 5(1) \\ \mathrm{C}(18) & 29(1) & 37(1) & 41(1) & 7(1) & -5(1) & 3(1) \\ \mathrm{C}(19) & 35(1) & 69(2) & 44(1) & 23(1) & 1(1) & 8(1) \\ \mathrm{C}(20) & 45(1) & 35(1) & 25(1) & 0(1) & 5(1) & -3(1) \\ \mathrm{C}(21) & 53(2) & 40(1) & 27(1) & -4(1) & 2(1) & -1(1) \\ \mathrm{C}(22) & 48(1) & 29(1) & 38(1) & -6(1) & -5(1) & 4(1) \\ \mathrm{C}(23) & 44(1) & 25(1) & 40(1) & 2(1) & -2(1) & 1(1) \\ \mathrm{C}(24) & 36(1) & 28(1) & 29(1) & 3(1) & 0(1) & 1(1) \\ \mathrm{C}(25) & 105(3) & 56(2) & 45(2) & -18(1) & -17(2) & 1(2) \\ \mathrm{C}(26) & 131(4) & 35(1) & 65(2) & -4(1) & -30(2) & -22(2) \\ \mathrm{C}(27) & 42(1) & 33(1) & 33(1) & 11(1) & 5(1) & -1(1) \\ \mathrm{C}(28) & 37(1) & 42(1) & 37(1) & 14(1) & 10(1) & 1(1) \\ \mathrm{C}(29) & 32(1) & 34(1) & 34(1) & 8(1) & 9(1) & 4(1) \\ \mathrm{C}(30) & 35(1) & 62(2) & 39(1) & 19(1) & 2(1) & 19(1) \\ \mathrm{C}(31) & 28(1) & 76(2) & 52(2) & 17(2) & -4(1) & 11(1) \\ \mathrm{C}(32) & 26(1) & 50(1) & 38(1) & 11(1) & 1(1) & 3(1) \\ \mathrm{C}(33) & 79(2) & 31(1) & 46(1) & -2(1) & 12(1) & 16(1) \\ \mathrm{C}(34) & 90(3) & 40(1) & 40(1) & -10(1) & 9(2) & 20(2) \\ \mathrm{C}(35) & 57(2) & 41(1) & 27(1) & -2(1) & 5(1) & 16(1) \\ \mathrm{C}(36) & 124(5) & 66(3) & 68(3) & -2(2) & -2(3) & -9(3) \\ \mathrm{C}(37) & 122(5) & 95(4) & 86(4) & -3(3) & 6(3) & -25(4) \\ \mathrm{C}(38) & 157(8) & 82(4) & 239(11) & 47(6) & 69(7) & 16(5) \\ \mathrm{C}(39) & 156(7) & 181(8) & 70(3) & -13(4) & 12(4) & 88(6) \\ \mathrm{C}(40) & 80(6) & 117(9) & 81(6) & 24(6) & -2(5) & -17(6) \\ \mathrm{C}(41) & 80(6) & 121(9) & 124(9) & -80(8) & 35(6) & -15(6) \\ \mathrm{C}(42) & 123(10) & 159(12) & 55(5) & -22(6) & -17(6) & 33(9) \\ \mathrm{C}(43) & 138(13) & 260(20) & 109(10) & -45(12) & 15(9) & 122(14) \\ \mathrm{B}(1) & 47(1) & 28(1) & 34(1) & 8(1) & 7(1) & 13(1)\end{array}$




\section{Compound 90}

Table 5. Hydrogen coordinates ( x 10 $0^{4}$ ) and isotropic displacement parameters $\left(\AA^{2} \times 10^{3}\right)$ for $\mathrm{C} 31 \mathrm{H} 54 \mathrm{BN} 9 \mathrm{O} 4.5 \mathrm{Mo}$.

\begin{tabular}{|c|c|c|c|c|}
\hline & $\mathrm{x}$ & $\mathrm{y}$ & z & $\mathrm{U}(\mathrm{eq})$ \\
\hline $\mathrm{H}(1)$ & 7387 & 6958 & 3591 & 30 \\
\hline $\mathrm{H}(2)$ & 7198 & 5976 & 3141 & 30 \\
\hline $\mathrm{H}(4)$ & 6774 & 4734 & 3117 & 33 \\
\hline $\mathrm{H}(5)$ & 6918 & 3715 & 3635 & 36 \\
\hline $\mathrm{H}(6)$ & 6745 & 3729 & 4619 & 38 \\
\hline $\mathrm{H}(7)$ & 6499 & 4755 & 5081 & 34 \\
\hline $\mathrm{H}(9)$ & 5452 & 6166 & 4647 & 31 \\
\hline $\mathrm{H}(10 \mathrm{~A})$ & 6758 & 6447 & 5534 & 35 \\
\hline $\mathrm{H}(10 \mathrm{~B})$ & 6502 & 5644 & 5526 & 35 \\
\hline $\mathrm{H}(11)$ & 8763 & 5465 & 5292 & 36 \\
\hline $\mathrm{H}(12 \mathrm{~A})$ & 10298 & 6405 & 5306 & 40 \\
\hline $\mathrm{H}(12 \mathrm{~B})$ & 9097 & 6914 & 5424 & 40 \\
\hline $\mathrm{H}(13)$ & 9080 & 6133 & 4383 & 34 \\
\hline $\mathrm{H}(14)$ & 6914 & 7059 & 4580 & 30 \\
\hline $\mathrm{H}(16 \mathrm{~A})$ & 10926 & 7466 & 3876 & 95 \\
\hline $\mathrm{H}(16 \mathrm{~B})$ & 9955 & 6865 & 3583 & 95 \\
\hline $\mathrm{H}(16 \mathrm{C})$ & 11169 & 6705 & 4110 & 95 \\
\hline $\mathrm{H}(18 \mathrm{~A})$ & 10454 & 5065 & 6129 & 44 \\
\hline $\mathrm{H}(18 \mathrm{~B})$ & 8968 & 4798 & 6225 & 44 \\
\hline $\mathrm{H}(19 \mathrm{~A})$ & 10839 & 5483 & 7097 & 75 \\
\hline $\mathrm{H}(19 \mathrm{~B})$ & 10636 & 4677 & 7071 & 75 \\
\hline $\mathrm{H}(19 \mathrm{C})$ & 9392 & 5157 & 7187 & 75 \\
\hline $\mathrm{H}(20)$ & 4471 & 6139 & 1935 & 42 \\
\hline $\mathrm{H}(21)$ & 3565 & 5312 & 1294 & 49 \\
\hline $\mathrm{H}(23)$ & 2802 & 4191 & 2663 & 45 \\
\hline $\mathrm{H}(24)$ & 3774 & 5046 & 3259 & 38 \\
\hline $\mathrm{H}(25 \mathrm{~A})$ & 1744 & 4638 & 777 & 108 \\
\hline $\mathrm{H}(25 \mathrm{~B})$ & 1857 & 3831 & 715 & 108 \\
\hline $\mathrm{H}(25 \mathrm{C})$ & 3230 & 4287 & 813 & 108 \\
\hline
\end{tabular}




\begin{tabular}{|c|c|c|c|c|}
\hline $\mathrm{H}(26 \mathrm{~A})$ & 2589 & 3260 & 1926 & 123 \\
\hline $\mathrm{H}(26 \mathrm{~B})$ & 1238 & 3317 & 1442 & 123 \\
\hline $\mathrm{H}(26 \mathrm{C})$ & 1276 & 3683 & 2053 & 123 \\
\hline $\mathrm{H}(27)$ & 5506 & 8414 & 1748 & 43 \\
\hline $\mathrm{H}(28)$ & 7413 & 7604 & 1627 & 46 \\
\hline $\mathrm{H}(29)$ & 7236 & 6651 & 2324 & 40 \\
\hline $\mathrm{H}(30)$ & 890 & 8055 & 2018 & 55 \\
\hline $\mathrm{H}(31)$ & -370 & 6955 & 2071 & 64 \\
\hline $\mathrm{H}(32)$ & 1324 & 6154 & 2644 & 46 \\
\hline $\mathrm{H}(33)$ & 3779 & 9137 & 3451 & 62 \\
\hline $\mathrm{H}(34)$ & 4218 & 8787 & 4489 & 69 \\
\hline $\mathrm{H}(35)$ & 4751 & 7532 & 4487 & 50 \\
\hline $\mathrm{H}(36 \mathrm{~A})$ & 1583 & 8606 & 4941 & 106 \\
\hline $\mathrm{H}(36 \mathrm{~B})$ & 1488 & 9239 & 5362 & 106 \\
\hline $\mathrm{H}(37 \mathrm{~A})$ & 1274 & 8571 & 6111 & 123 \\
\hline $\mathrm{H}(37 \mathrm{~B})$ & 436 & 8139 & 5580 & 123 \\
\hline $\mathrm{H}(38 \mathrm{~A})$ & 2143 & 7433 & 5567 & 186 \\
\hline $\mathrm{H}(38 \mathrm{~B})$ & 2575 & 7661 & 6228 & 186 \\
\hline $\mathrm{H}(39 \mathrm{~A})$ & 4460 & 8153 & 6035 & 164 \\
\hline $\mathrm{H}(39 \mathrm{~B})$ & 4109 & 7851 & 5389 & 164 \\
\hline $\mathrm{H}(40 \mathrm{~A})$ & 139 & 4591 & 8493 & 113 \\
\hline $\mathrm{H}(40 \mathrm{~B})$ & 1083 & 5221 & 8358 & 113 \\
\hline $\mathrm{H}(41 \mathrm{~A})$ & 749 & 4728 & 9377 & 127 \\
\hline $\mathrm{H}(41 \mathrm{~B})$ & 1587 & 5396 & 9242 & 127 \\
\hline $\mathrm{H}(42 \mathrm{~A})$ & 3510 & 4879 & 9443 & 139 \\
\hline $\mathrm{H}(42 \mathrm{~B})$ & 2682 & 4250 & 9665 & 139 \\
\hline $\mathrm{H}(43 \mathrm{~A})$ & 3848 & 4288 & 8784 & 204 \\
\hline $\mathrm{H}(43 \mathrm{~B})$ & 2828 & 3713 & 8952 & 204 \\
\hline $\mathrm{H}(1 \mathrm{~B})$ & $3490(40)$ & $8562(17)$ & $2428(15)$ & $46(9$ \\
\hline
\end{tabular}


${ }^{1} \mathrm{H}$ NMR (d6-Acetone) of Compound 90:

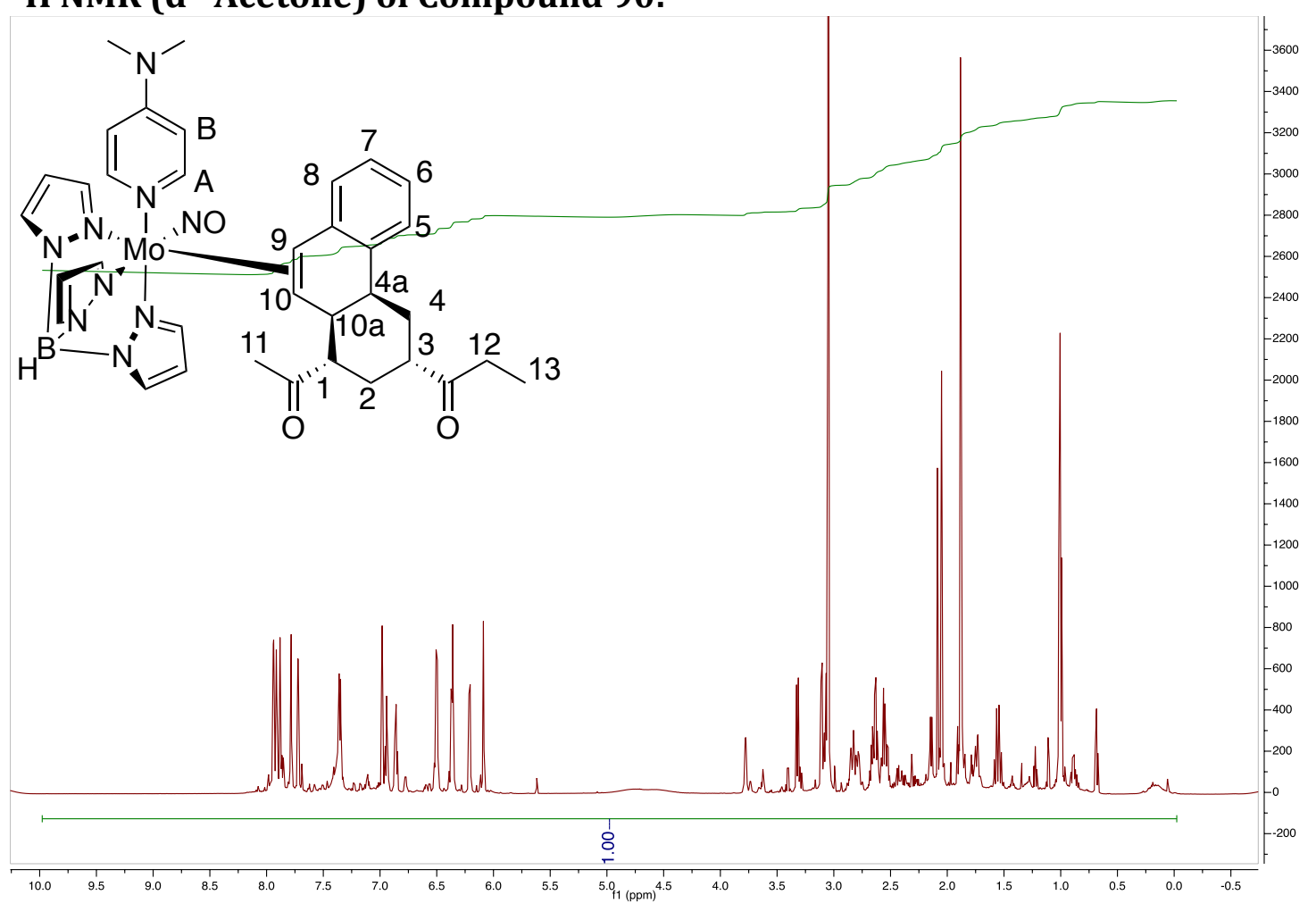

${ }^{13} \mathrm{C}$ NMR ( $\mathrm{d}^{6}$-Acetone) of Compound 90:

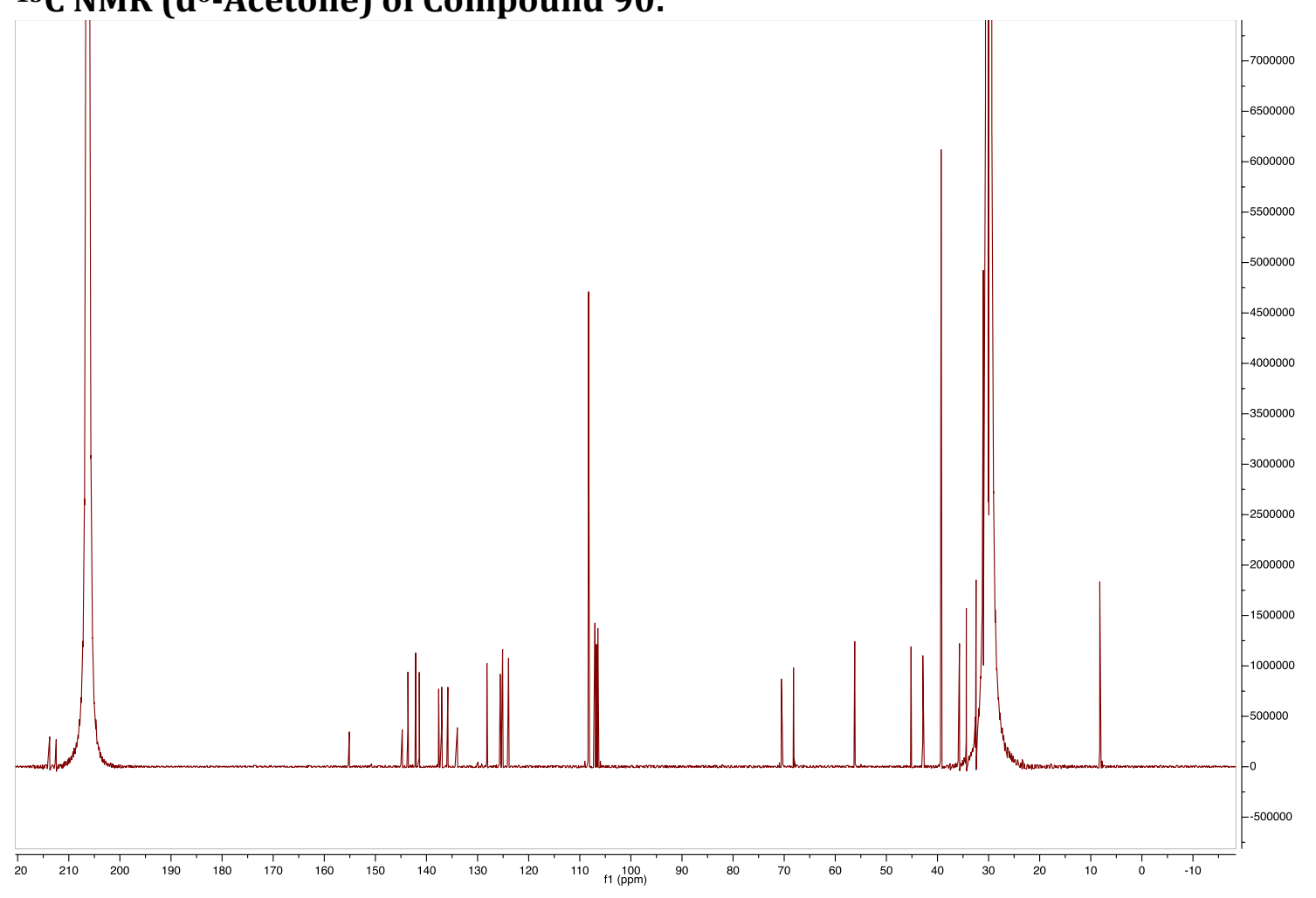


${ }^{1} \mathrm{H}$ NMR $\left(\mathrm{CDCl}_{3}\right)$ of Compound 91:

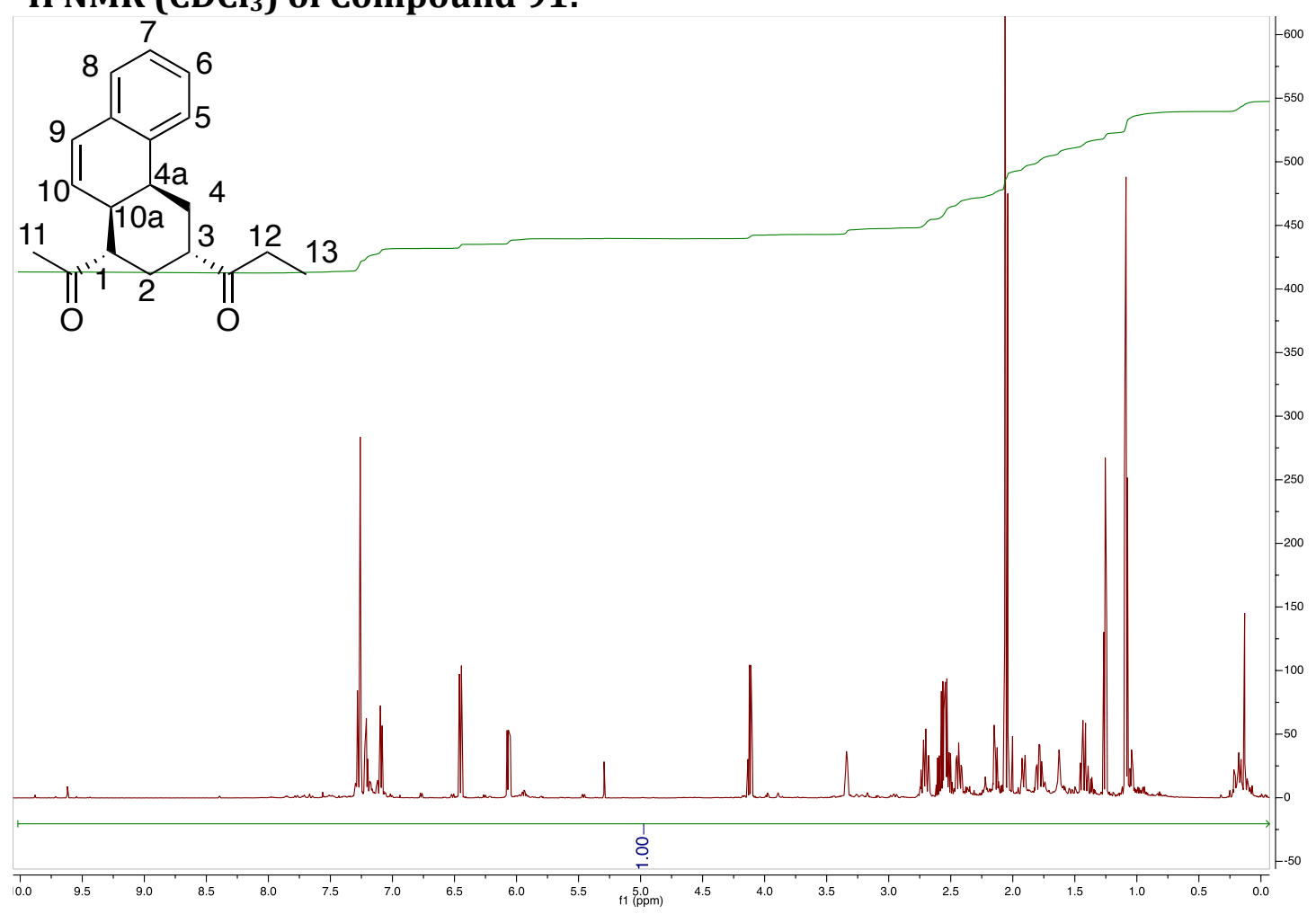

${ }^{13} \mathrm{C} \mathrm{NMR}\left(\mathrm{CDCl}_{3}\right)$ of Compound 91:

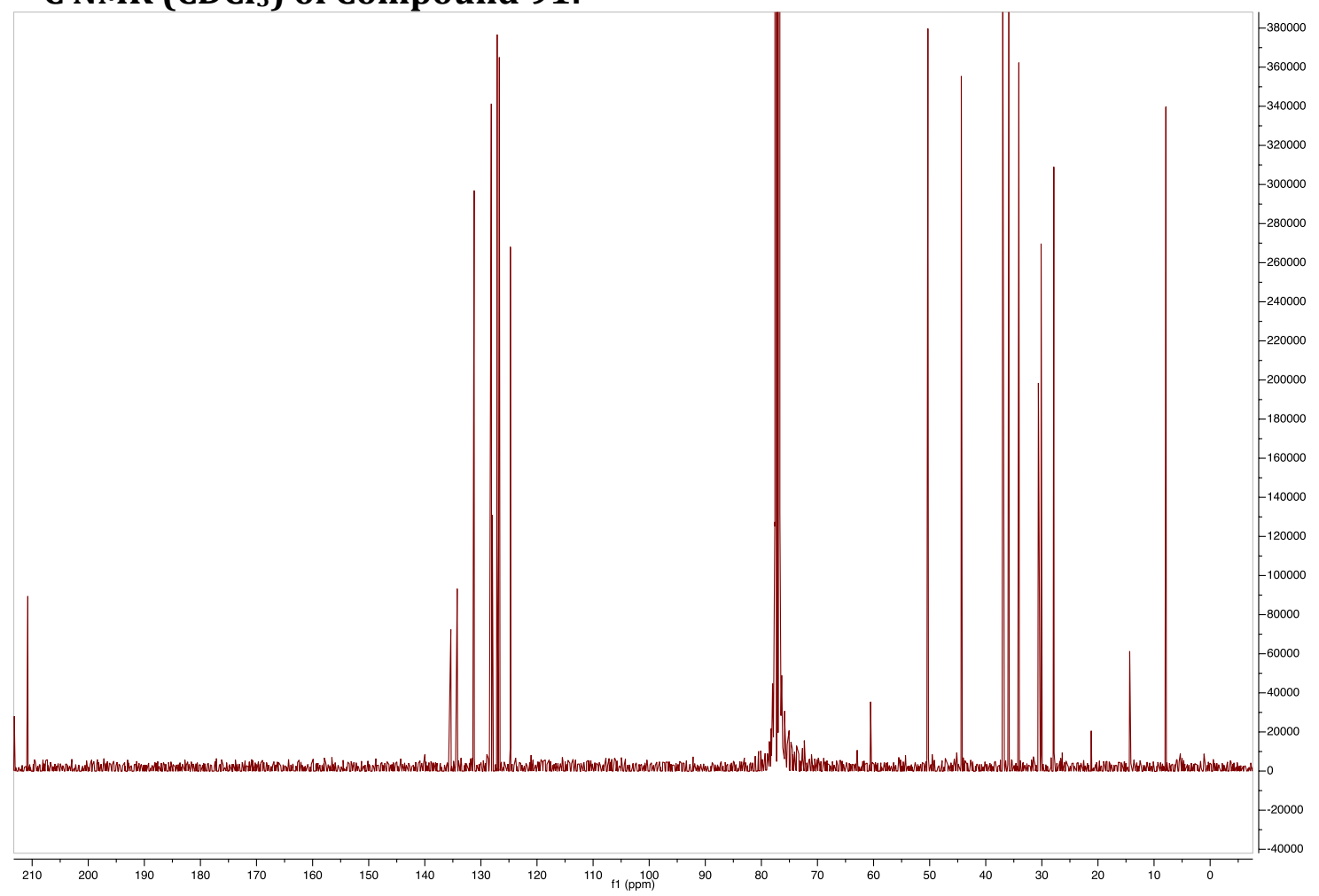


${ }^{1} \mathrm{H}$ NMR $\left(\mathrm{CDCl}_{3}\right)$ of Compound 92:

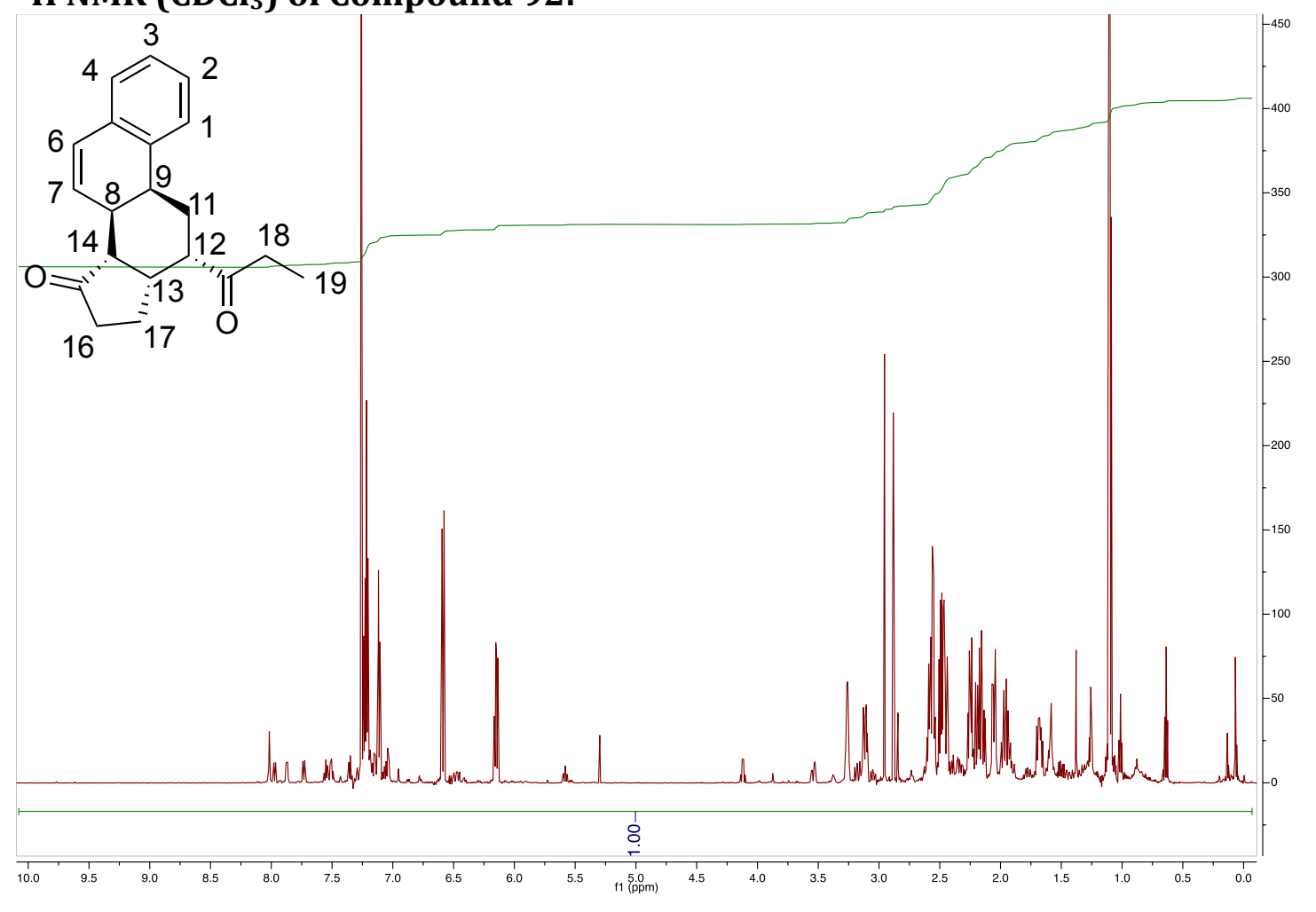

${ }^{13} \mathrm{C} \mathrm{NMR}\left(\mathrm{CDCl}_{3}\right)$ of Compound 92:

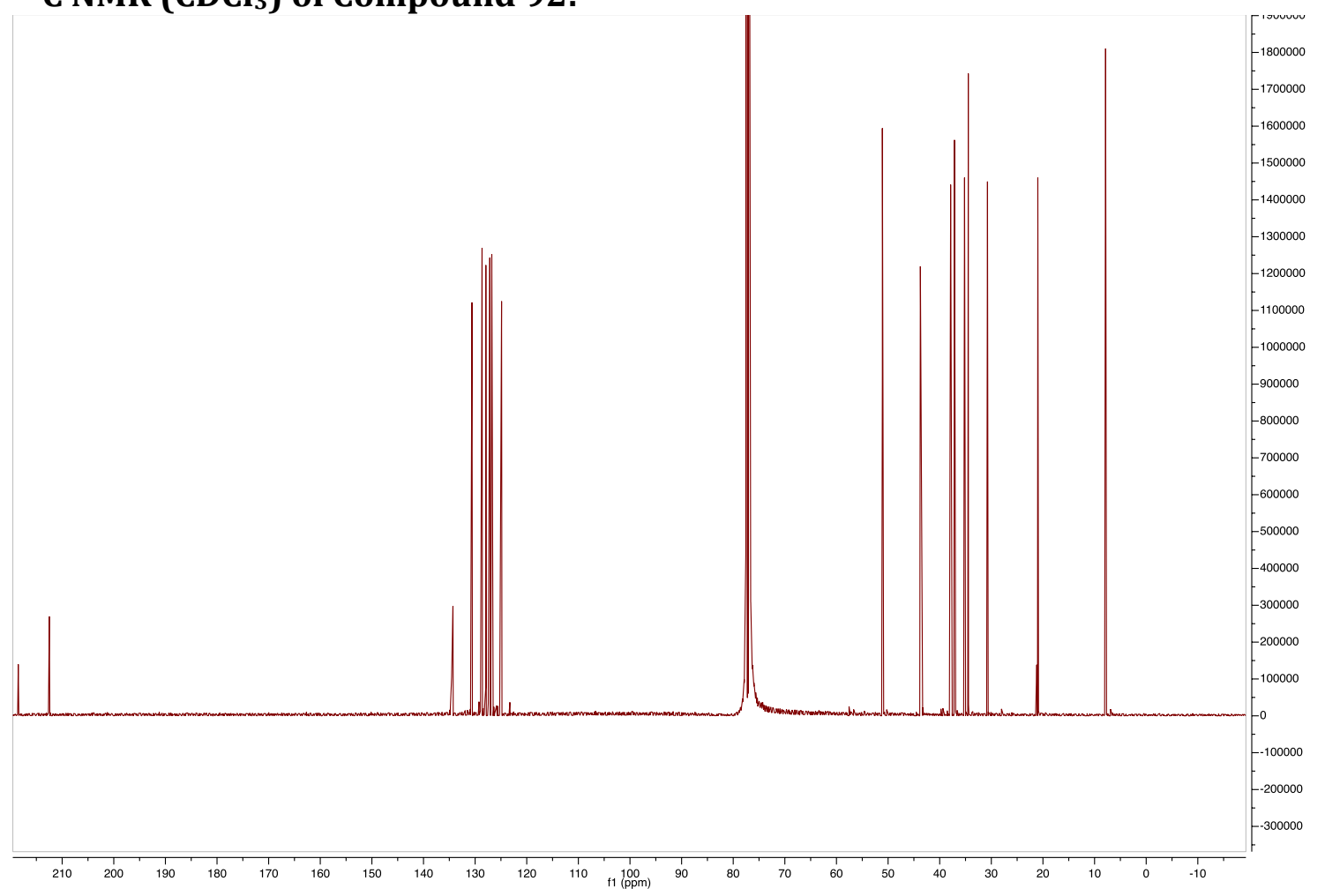


HSQC $\left(\mathrm{CDCl}_{3}\right)$ of Compound 92:

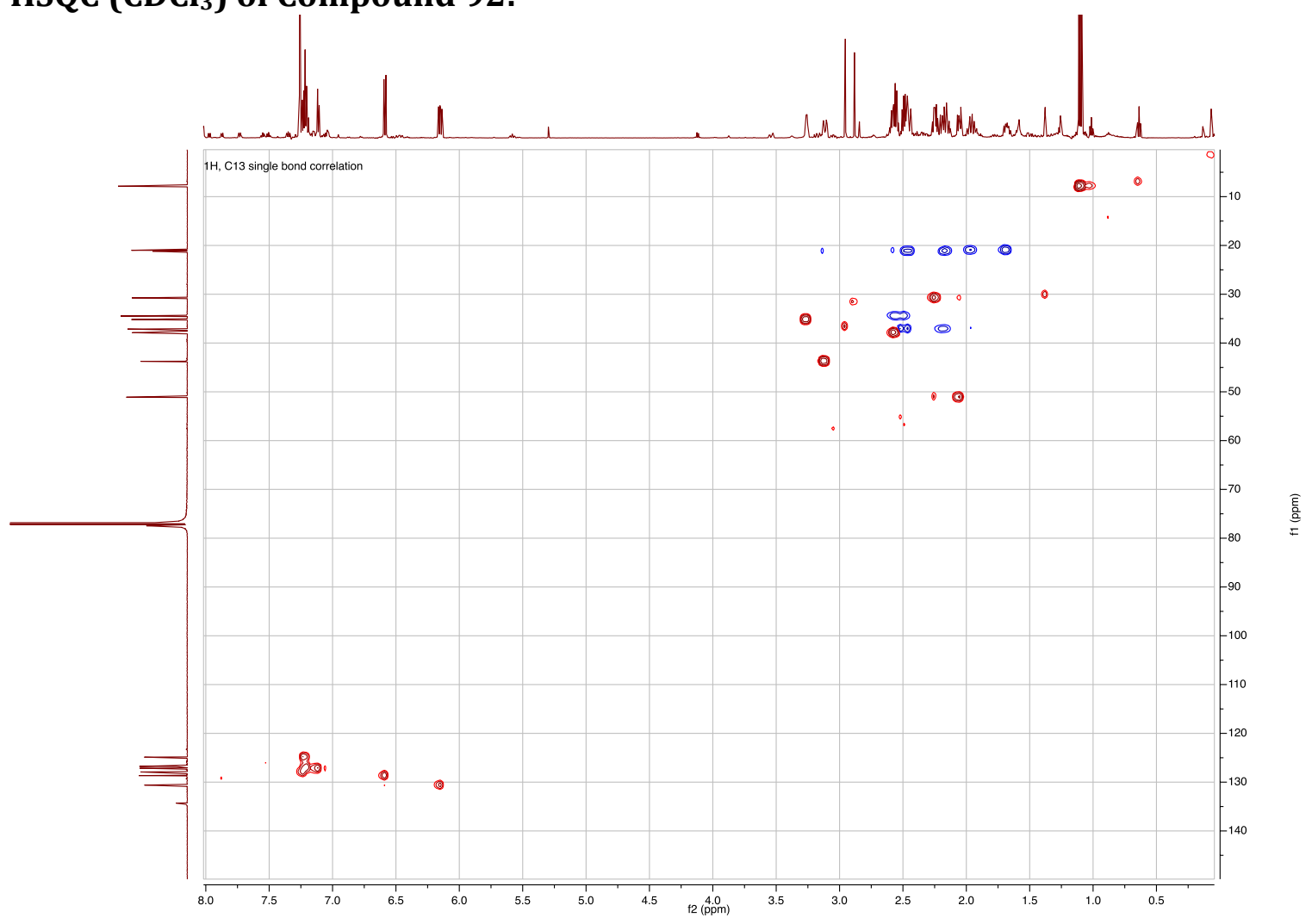

$\operatorname{COSY}\left(\mathrm{CDCl}_{3}\right)$ of Compound 92:

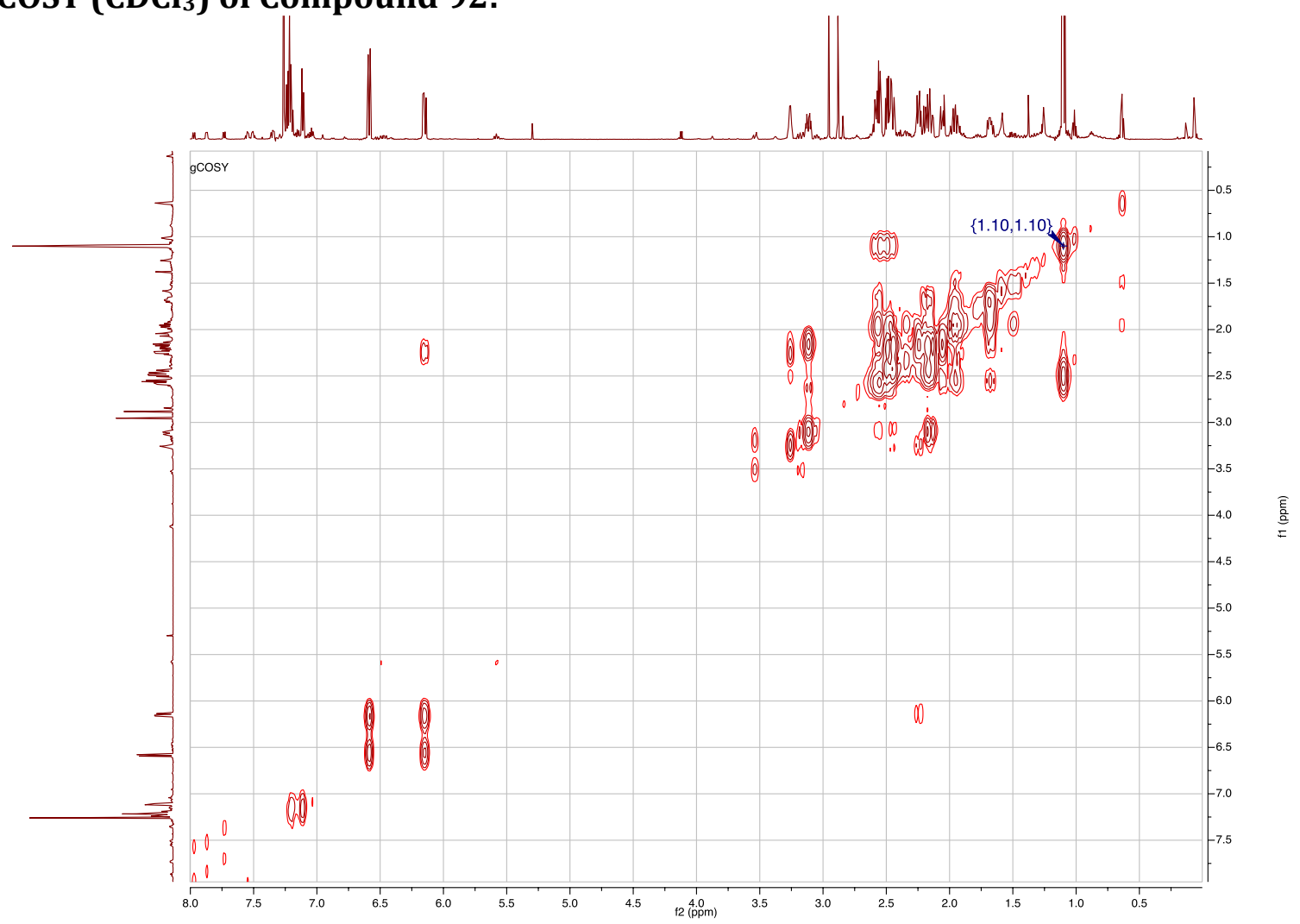


NOESY $\left(\mathrm{CDCl}_{3}\right)$ of Compound 92:

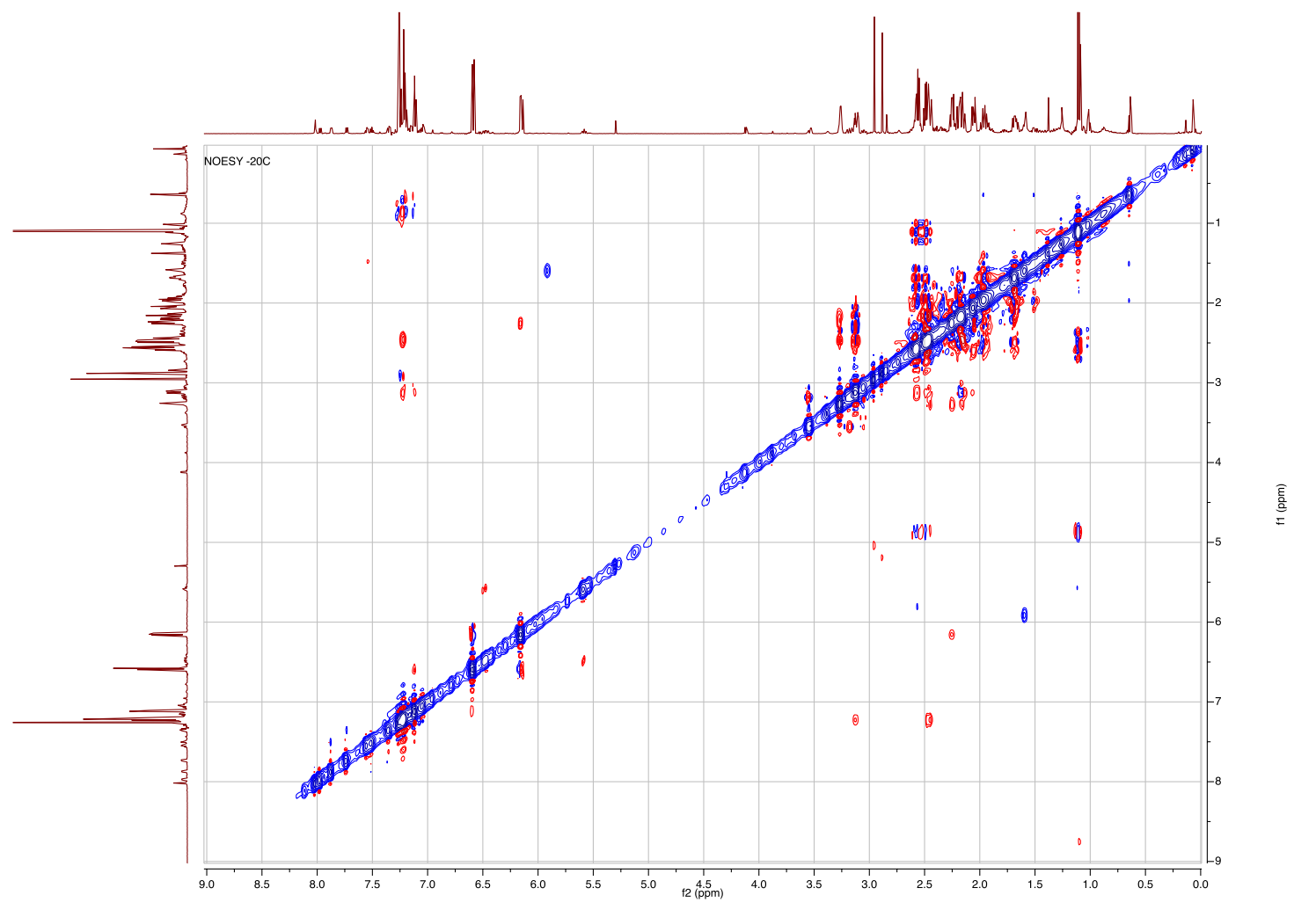




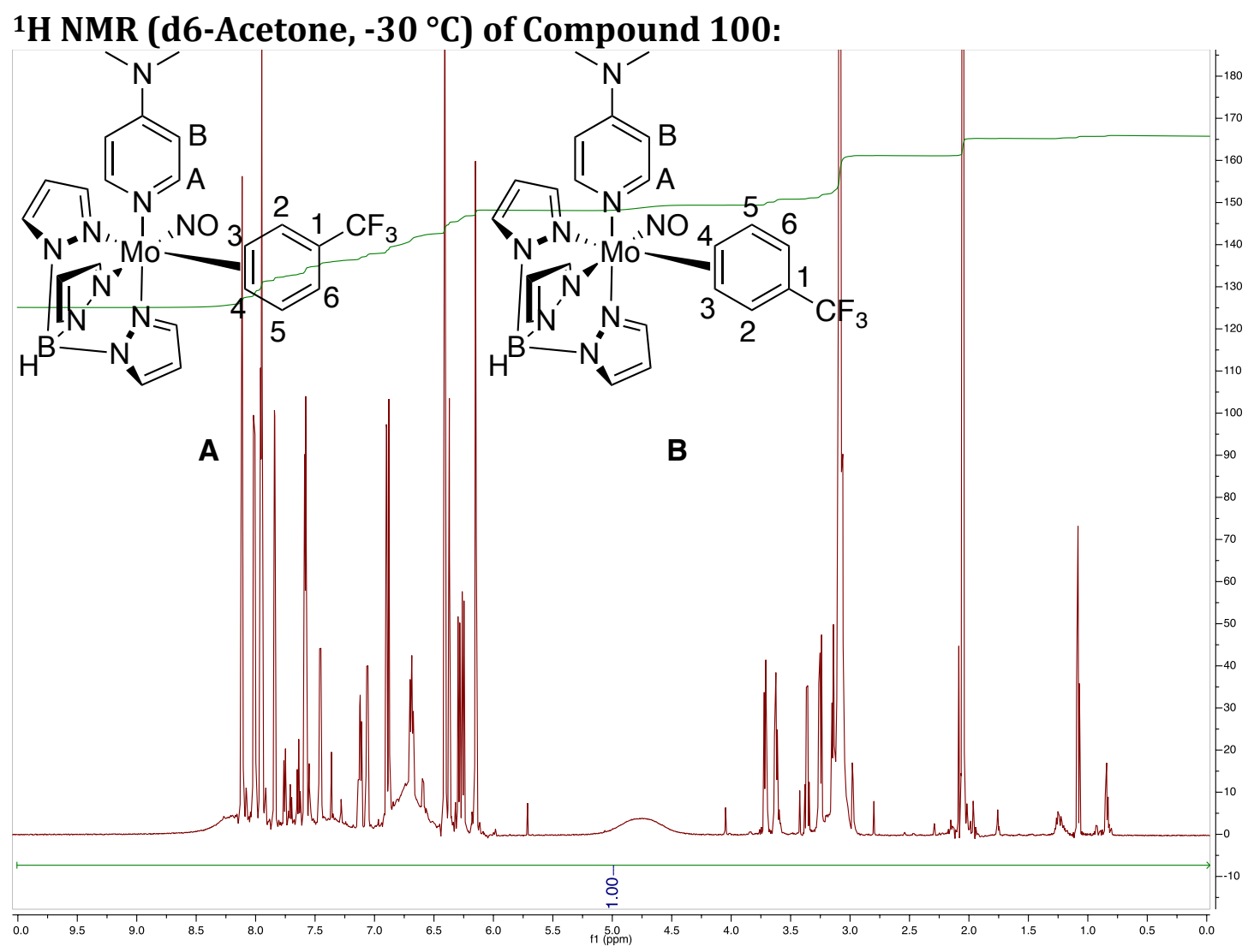

${ }^{13} \mathrm{C}$ NMR (d6-Acetone, $-30^{\circ} \mathrm{C}$ ) of Compound 100:

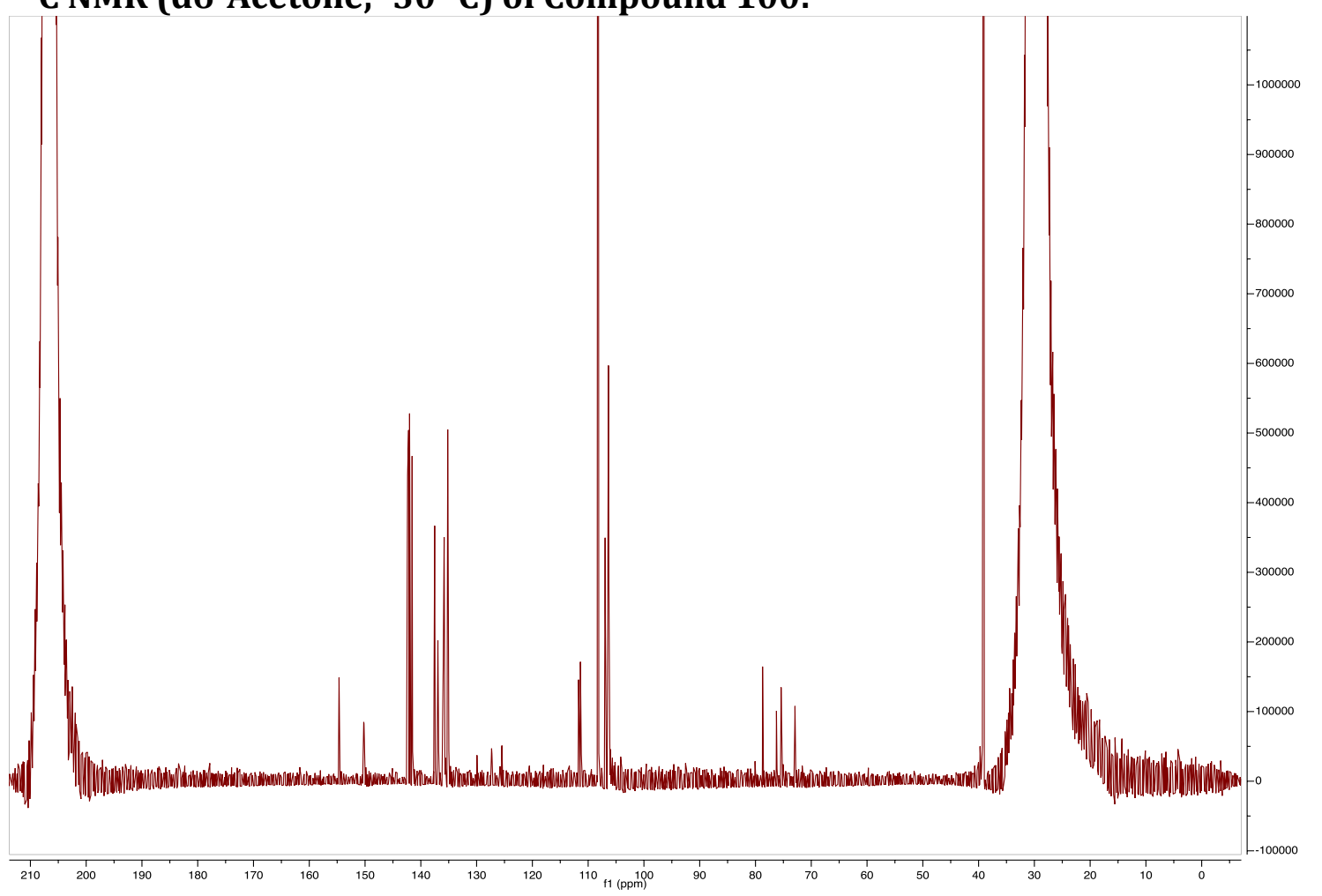


19F NMR (d6-Acetone) of Compound 100:

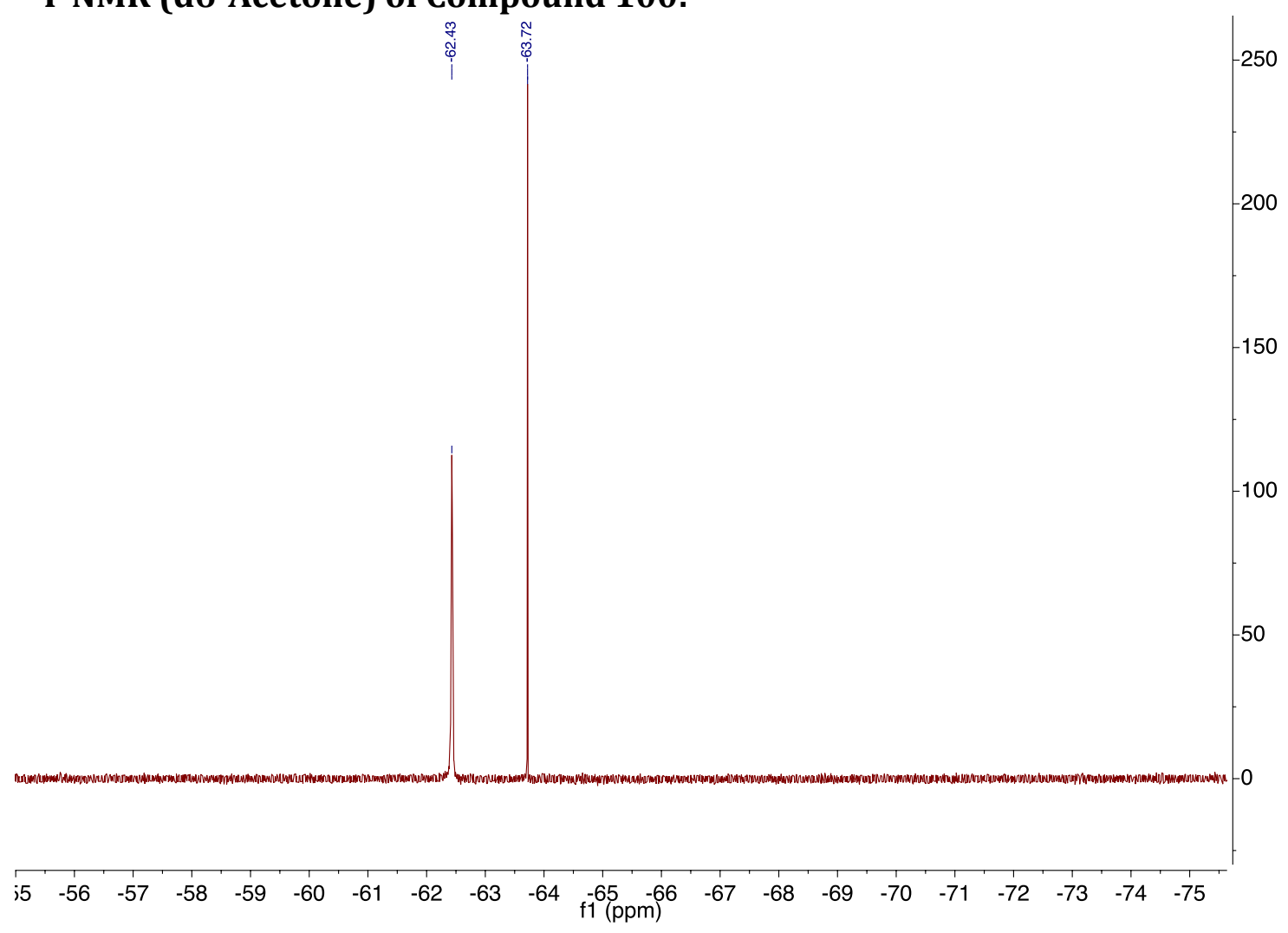

NOESY ( $d^{6}$-Acetone, $-30^{\circ} \mathrm{C}$ ) of Compound 100:

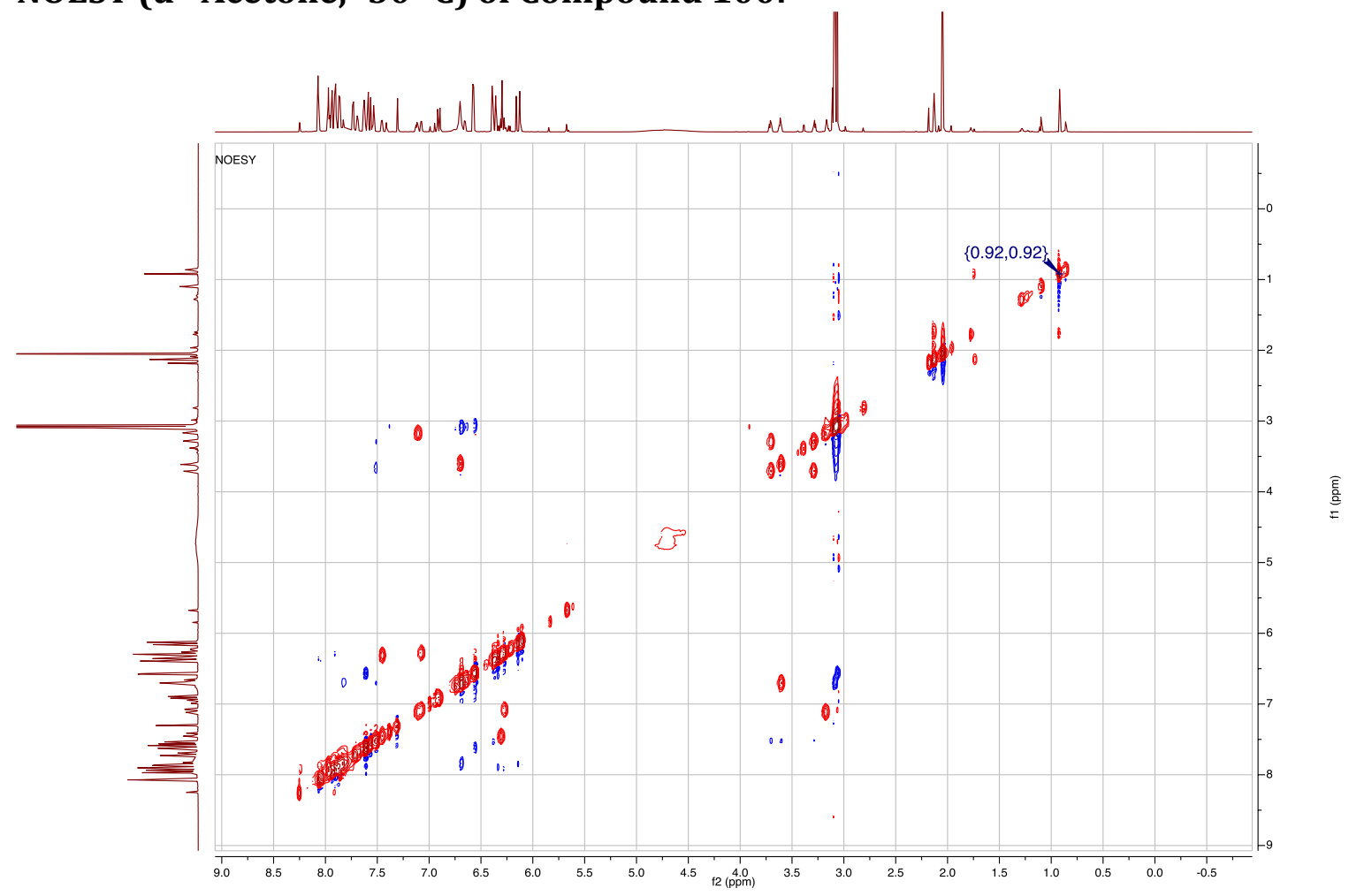




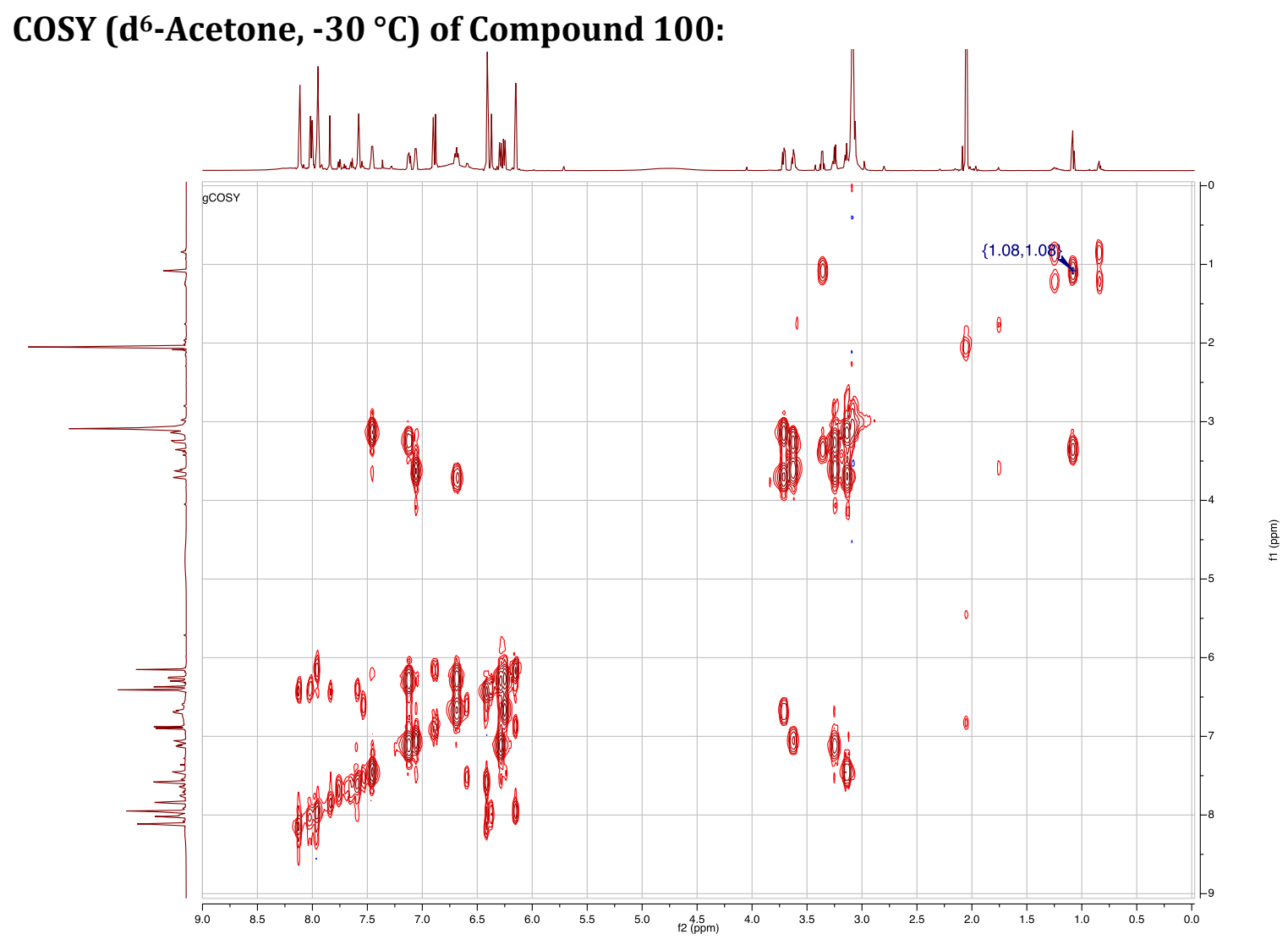


${ }^{1} \mathrm{H}$ NMR (d6-Acetone) of Compound 103:

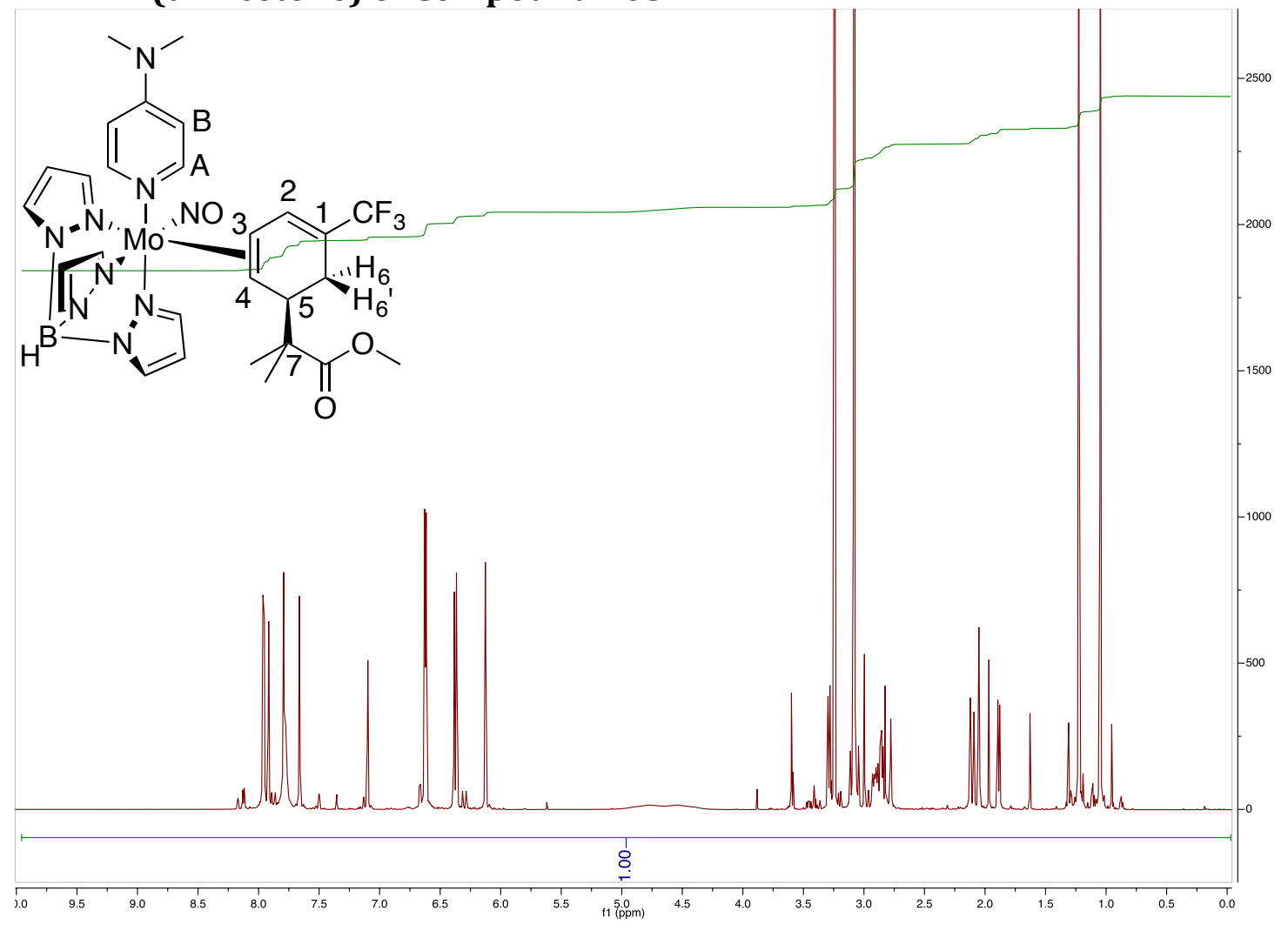

${ }^{13}$ C NMR (d6-Acetone) of Compound 103:

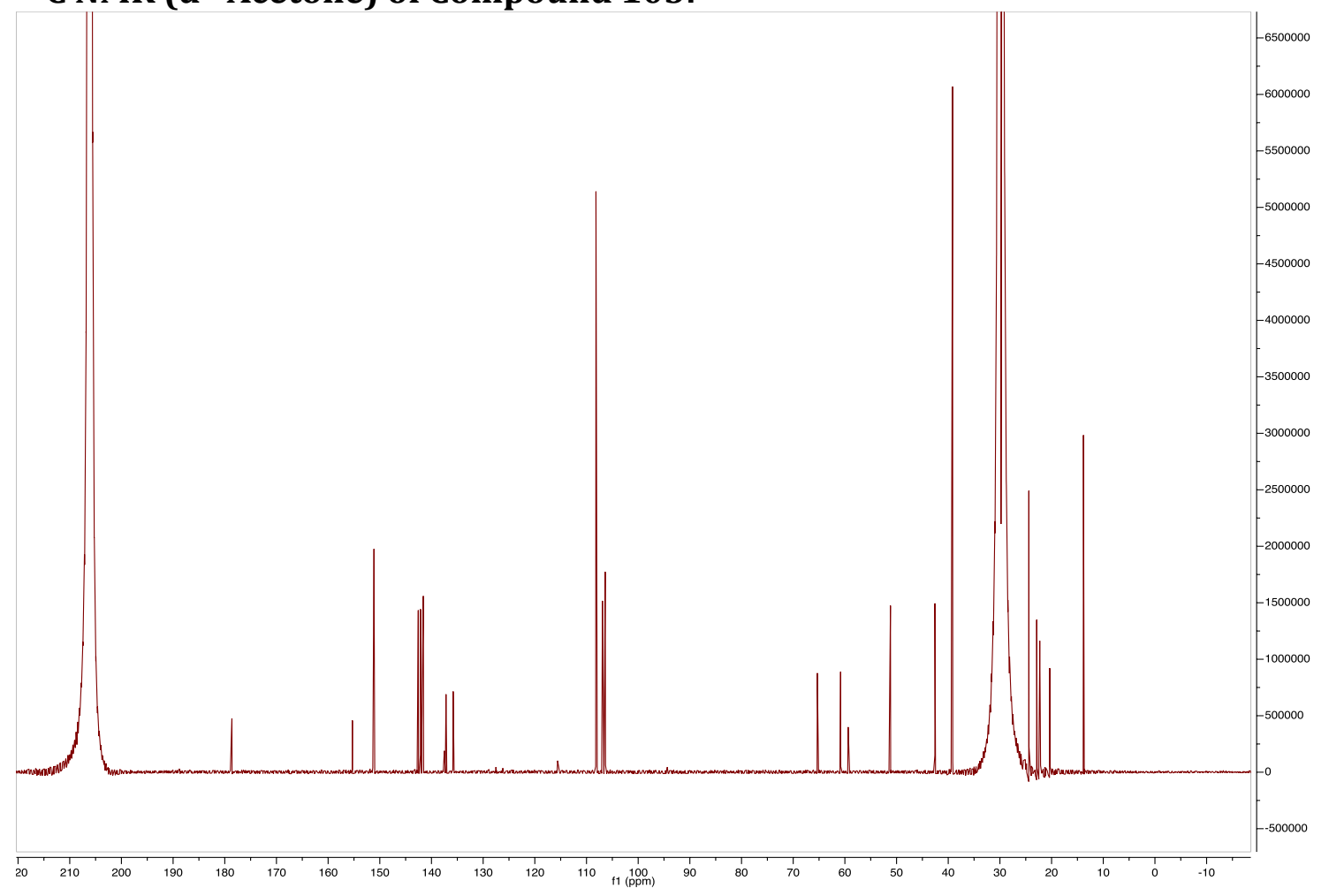


${ }^{19}$ F NMR (d6-Acetone) of Compound 103:

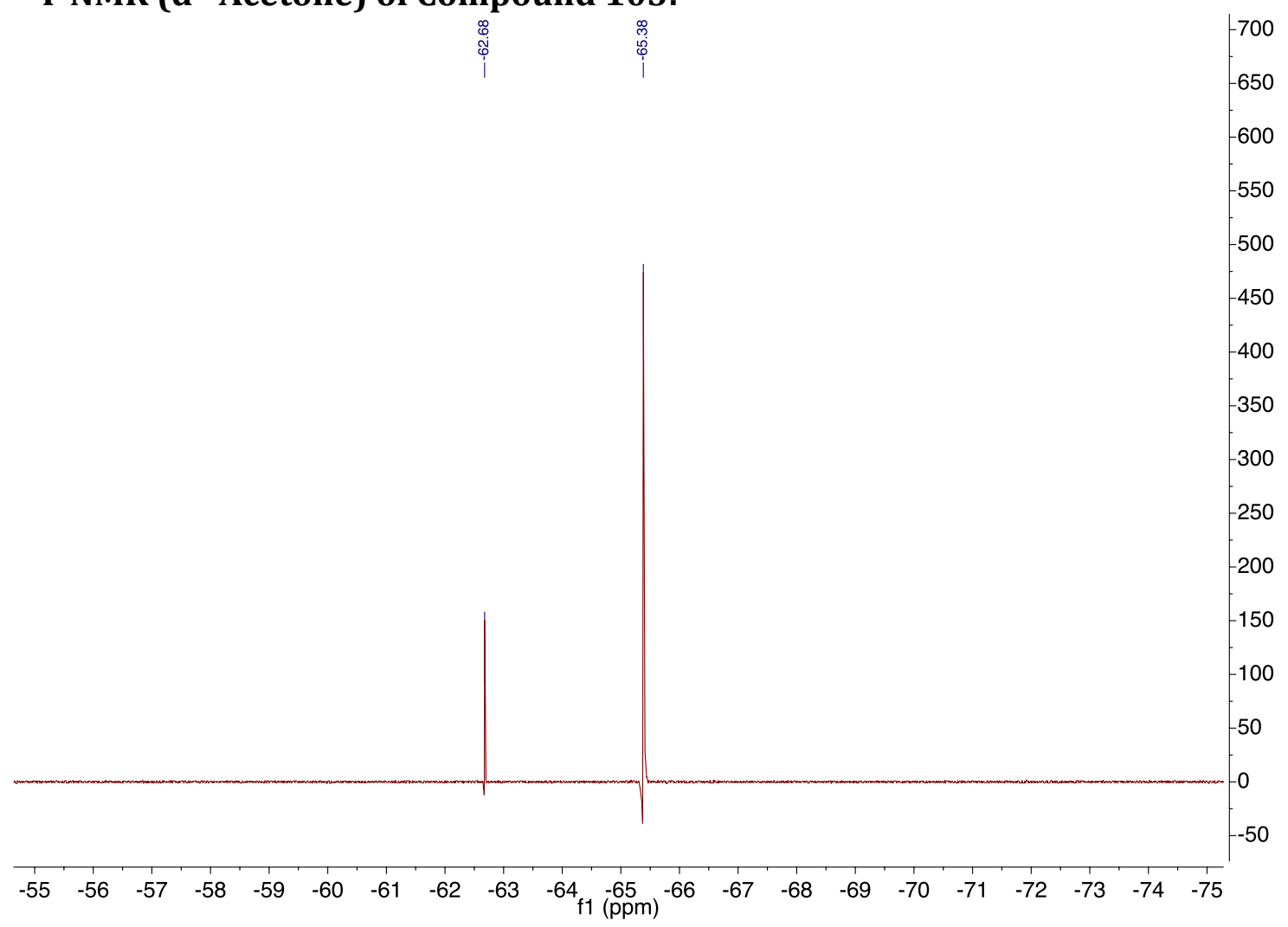


${ }^{1}$ H NMR (d6-Acetone) of Compound 104:

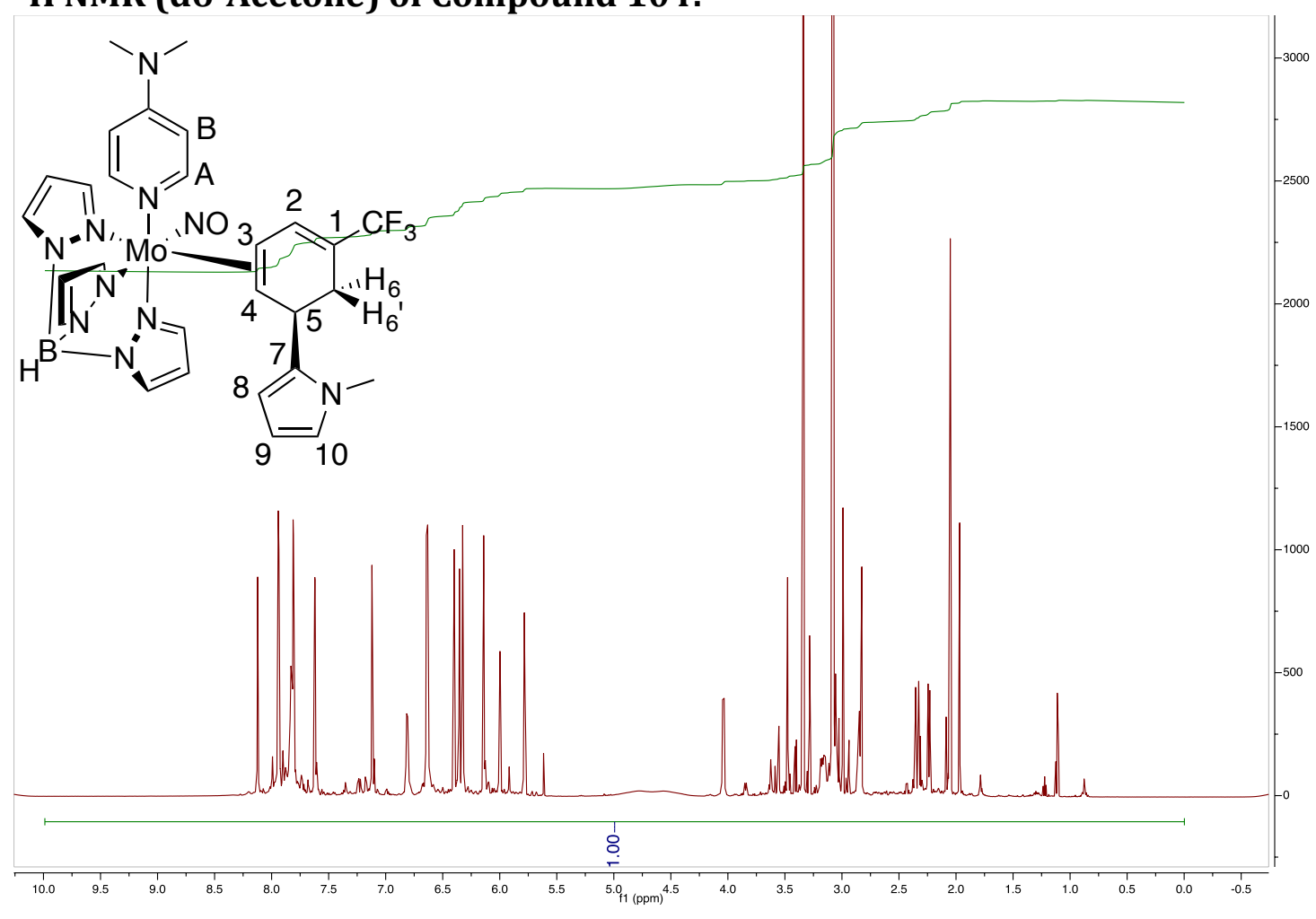

${ }^{13}$ C NMR (d6-Acetone) of Compound 104:

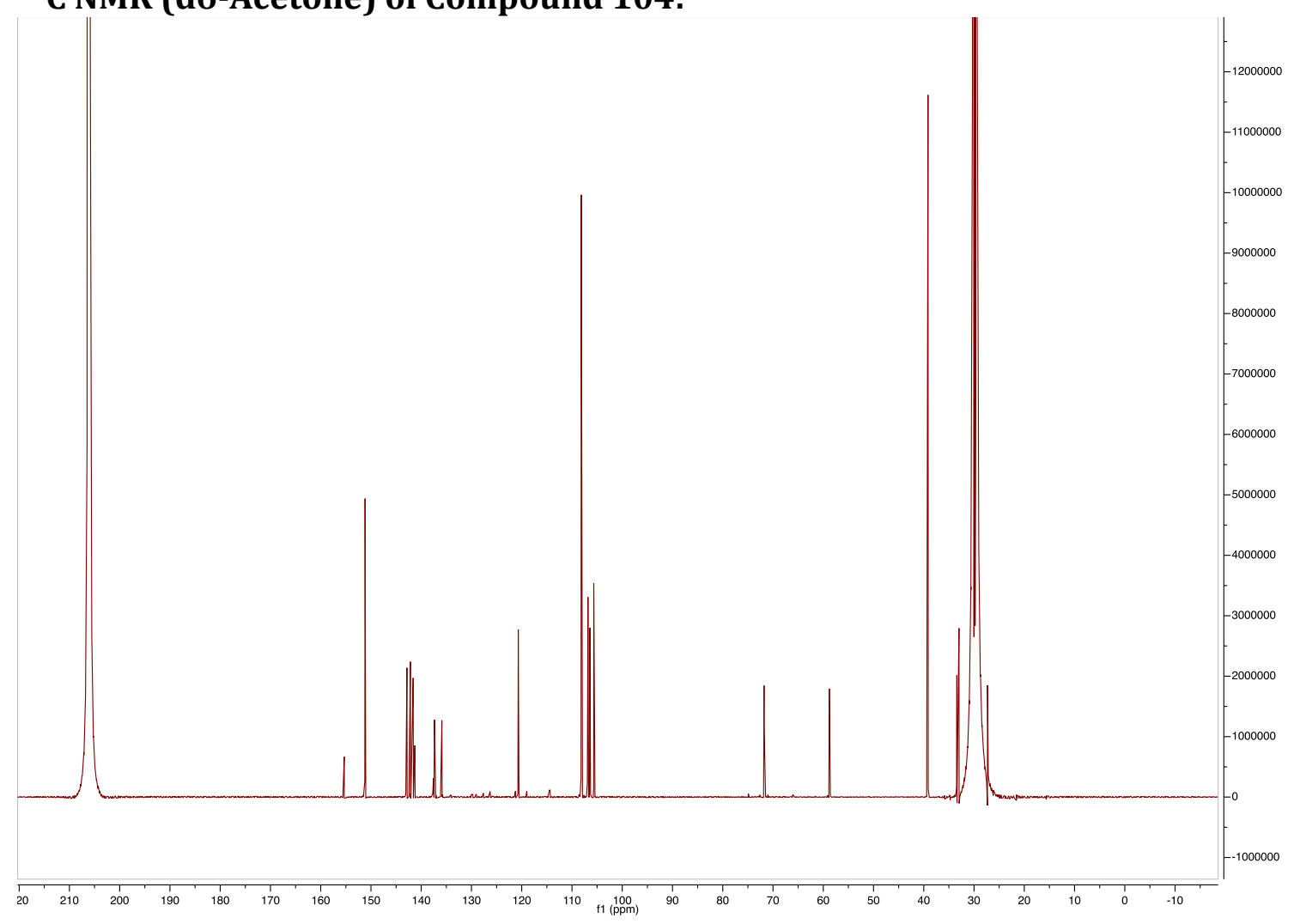


${ }^{19}$ F NMR (d6-Acetone) of Compound 104:

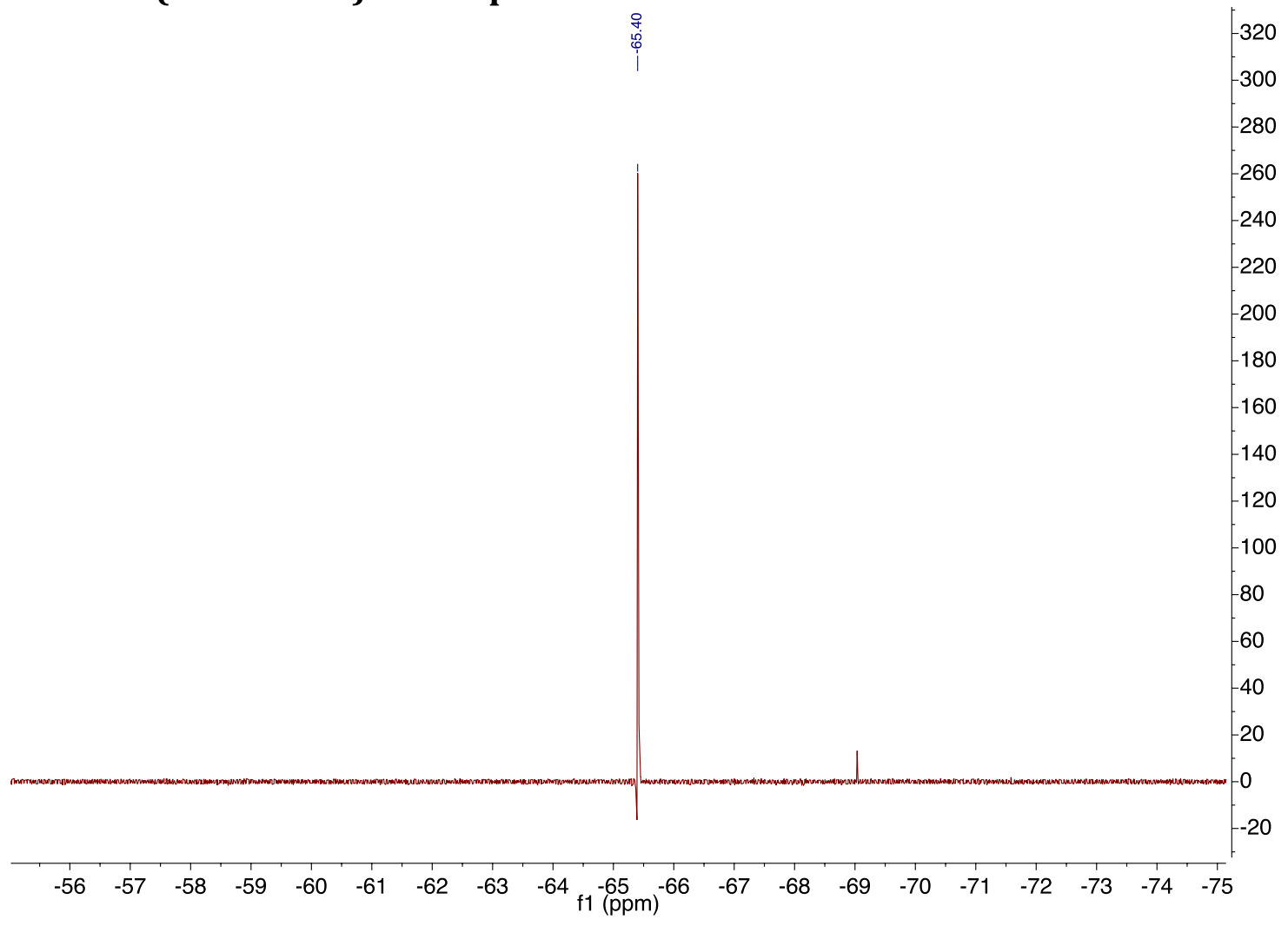




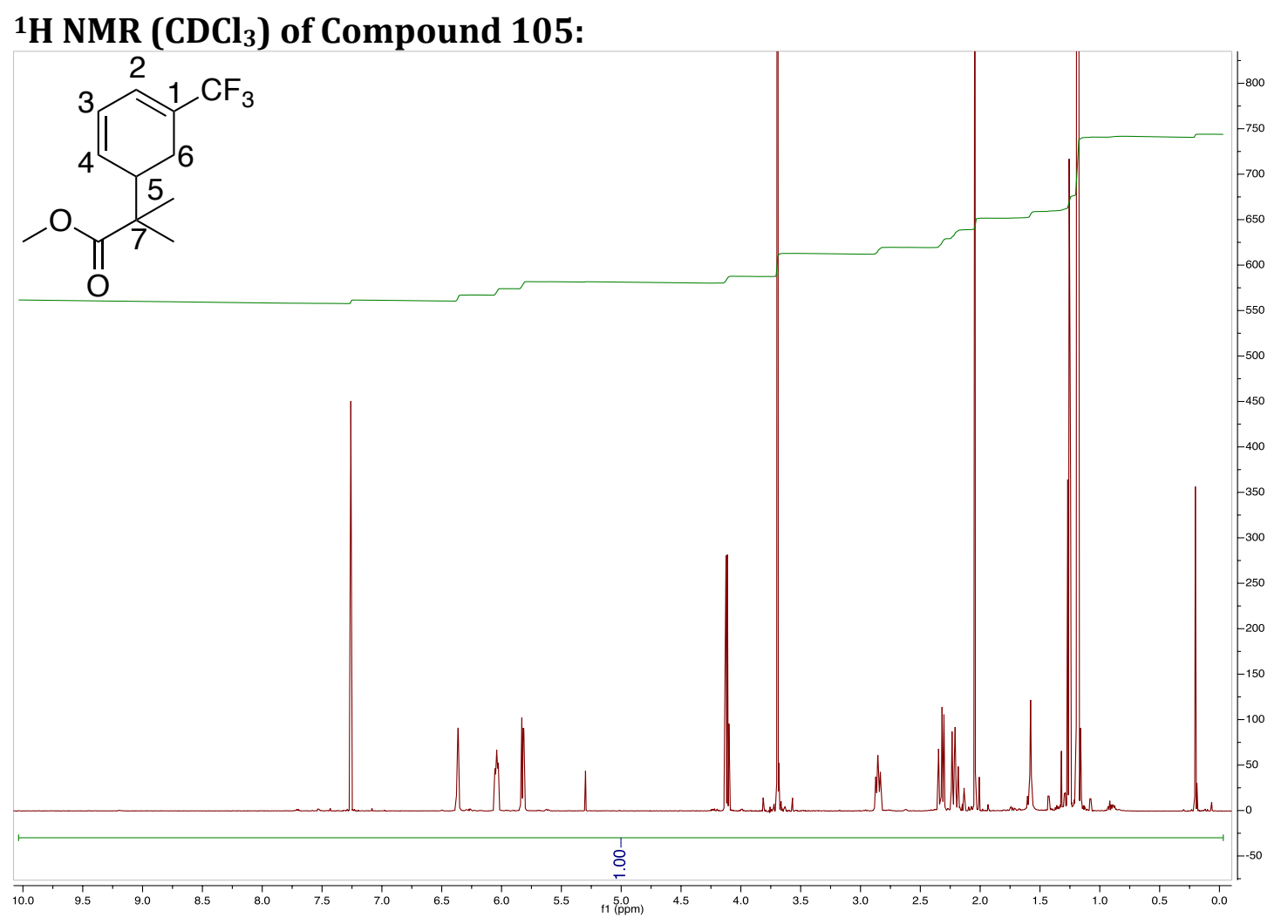

${ }^{13} \mathrm{C} \mathrm{NMR}\left(\mathrm{CDCl}_{3}\right)$ of Compound 105:

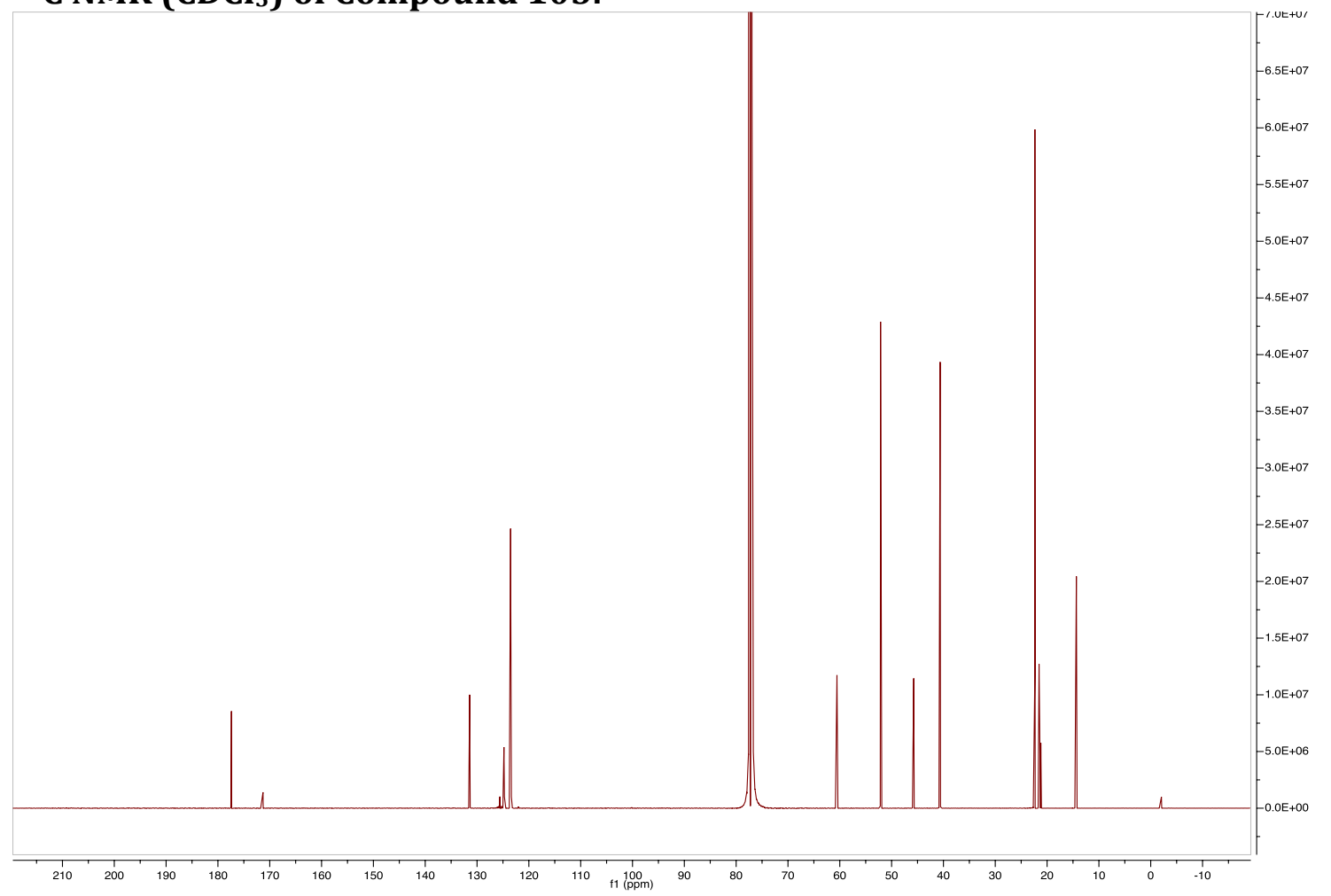


${ }^{19}$ F NMR (d6-Acetone) of Compound 105:

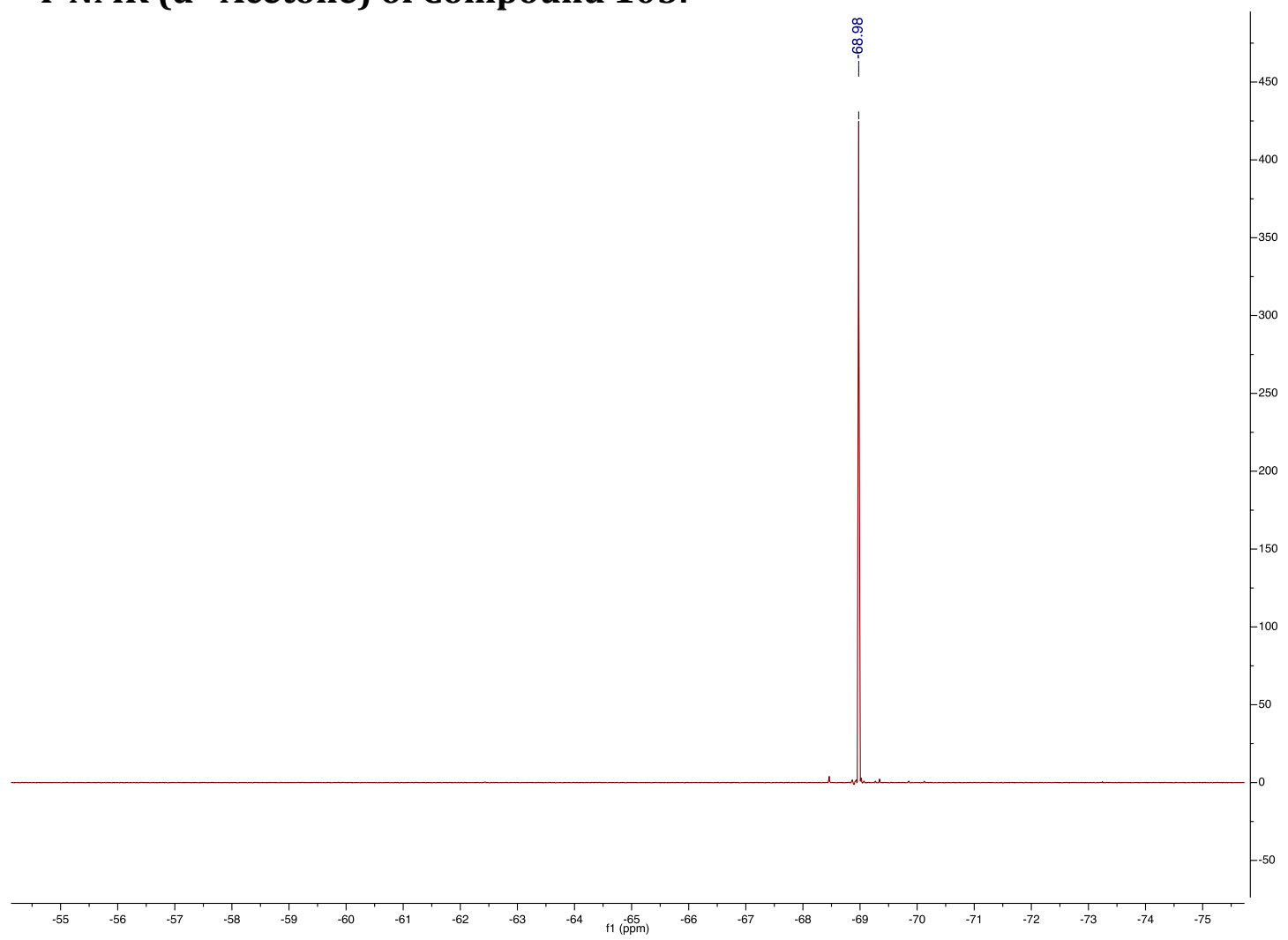


${ }^{1} \mathrm{H}$ NMR $\left(\mathrm{CDCl}_{3}\right)$ of Compound 106:

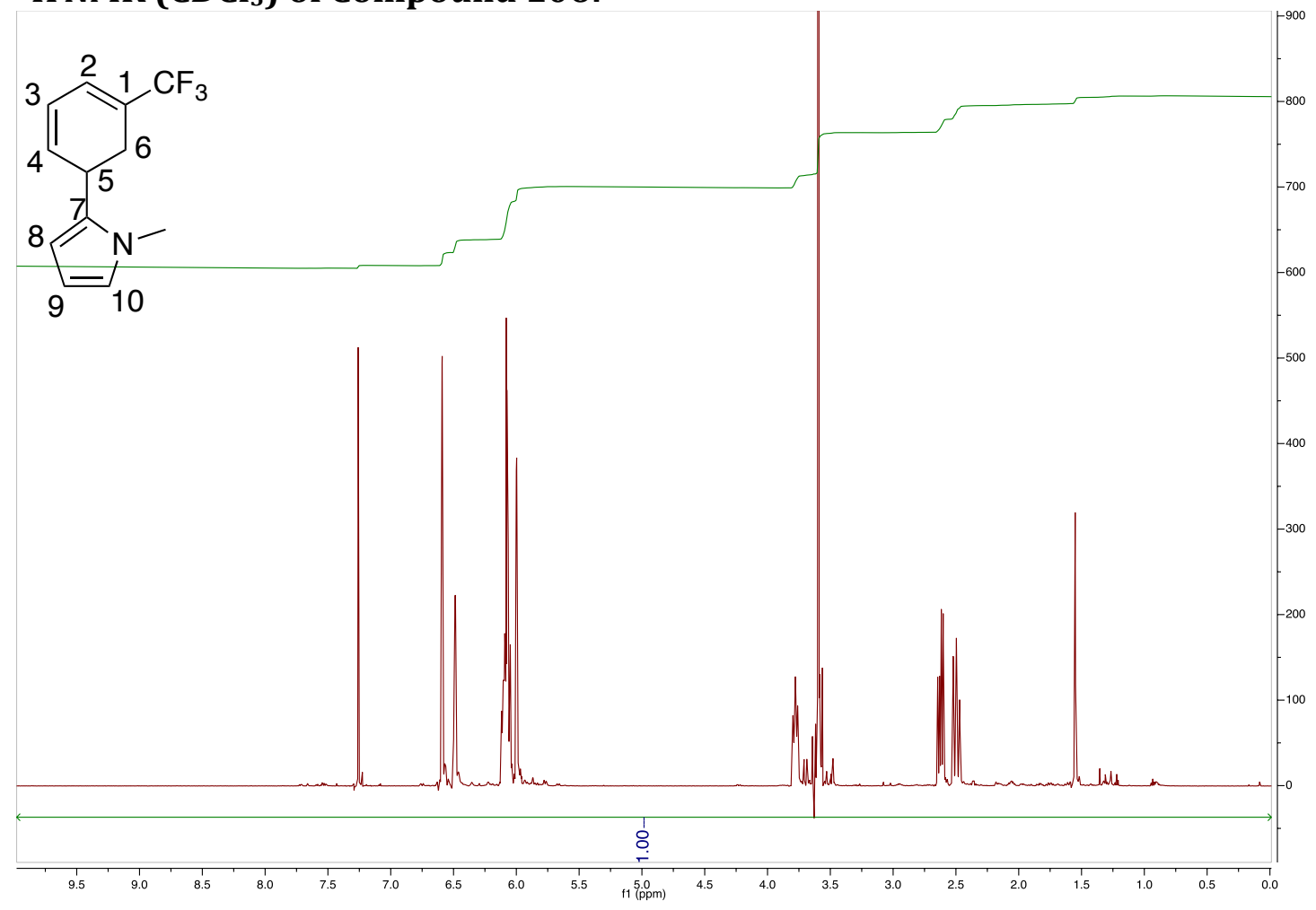

${ }^{13} \mathrm{C} \mathrm{NMR}\left(\mathrm{CDCl}_{3}\right)$ of Compound 106:

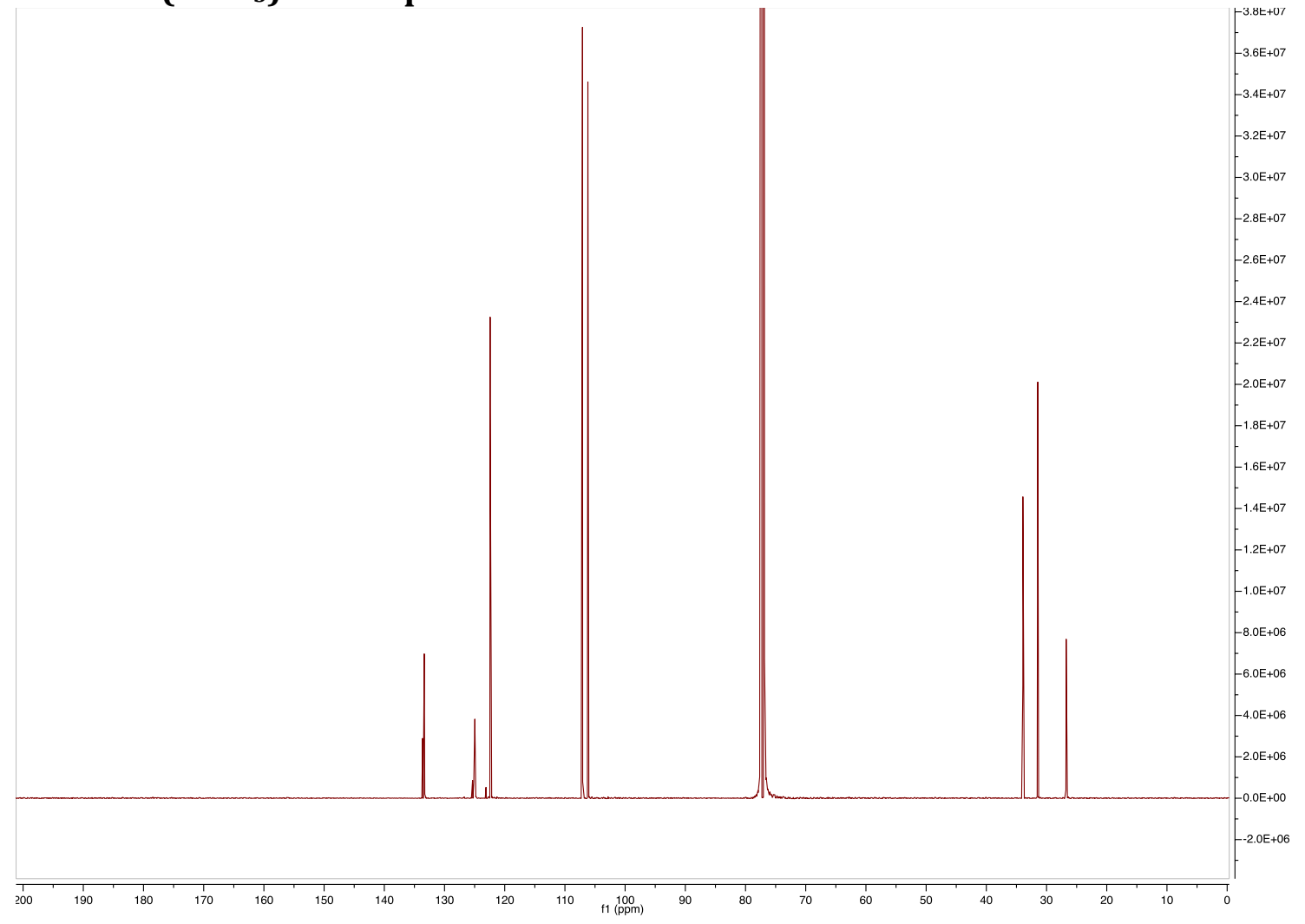


${ }^{19}$ F NMR (d6-Acetone) of Compound 106:

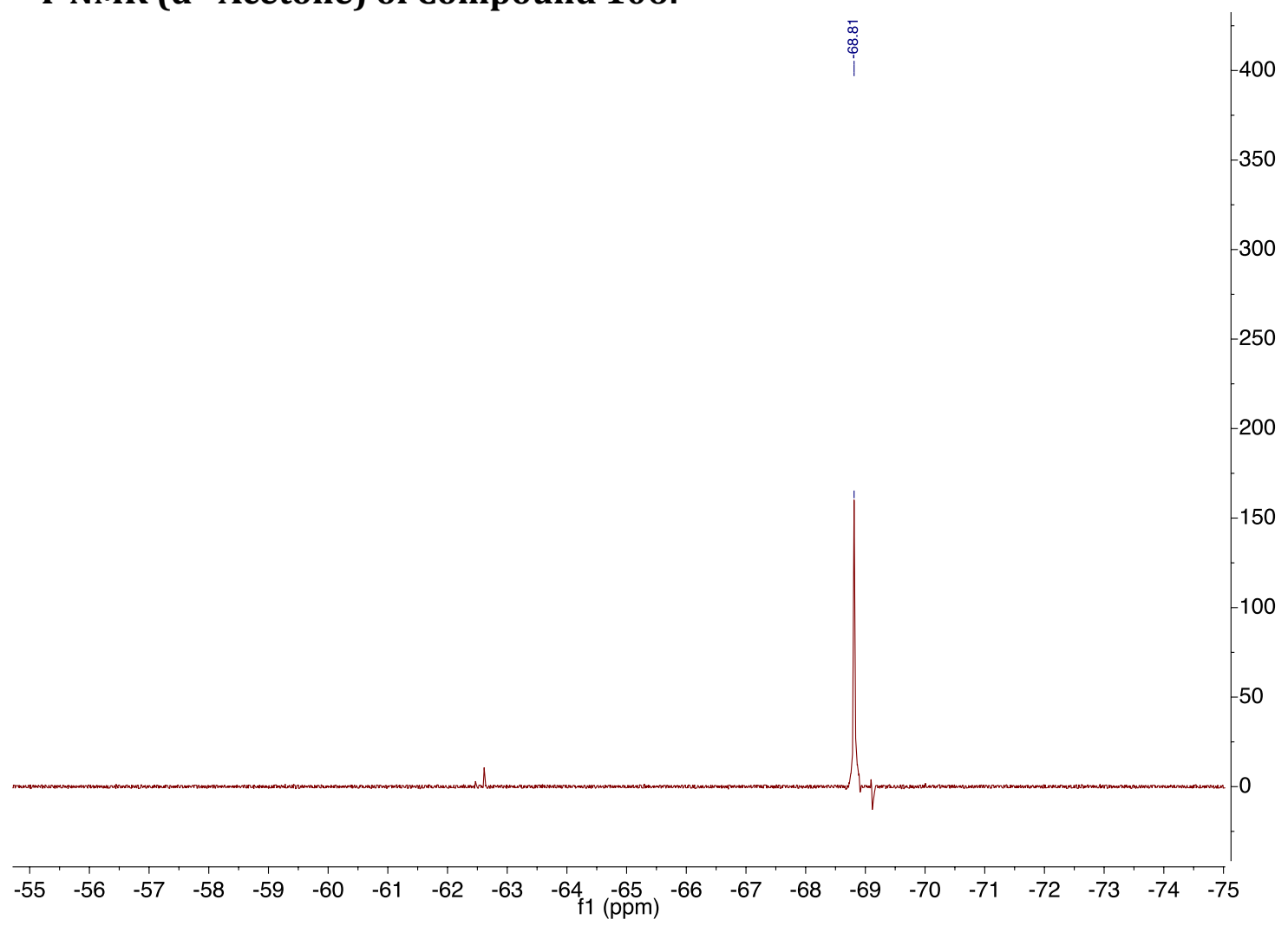


${ }^{1} \mathrm{H}$ NMR (d6-Acetone) of Compound 121:

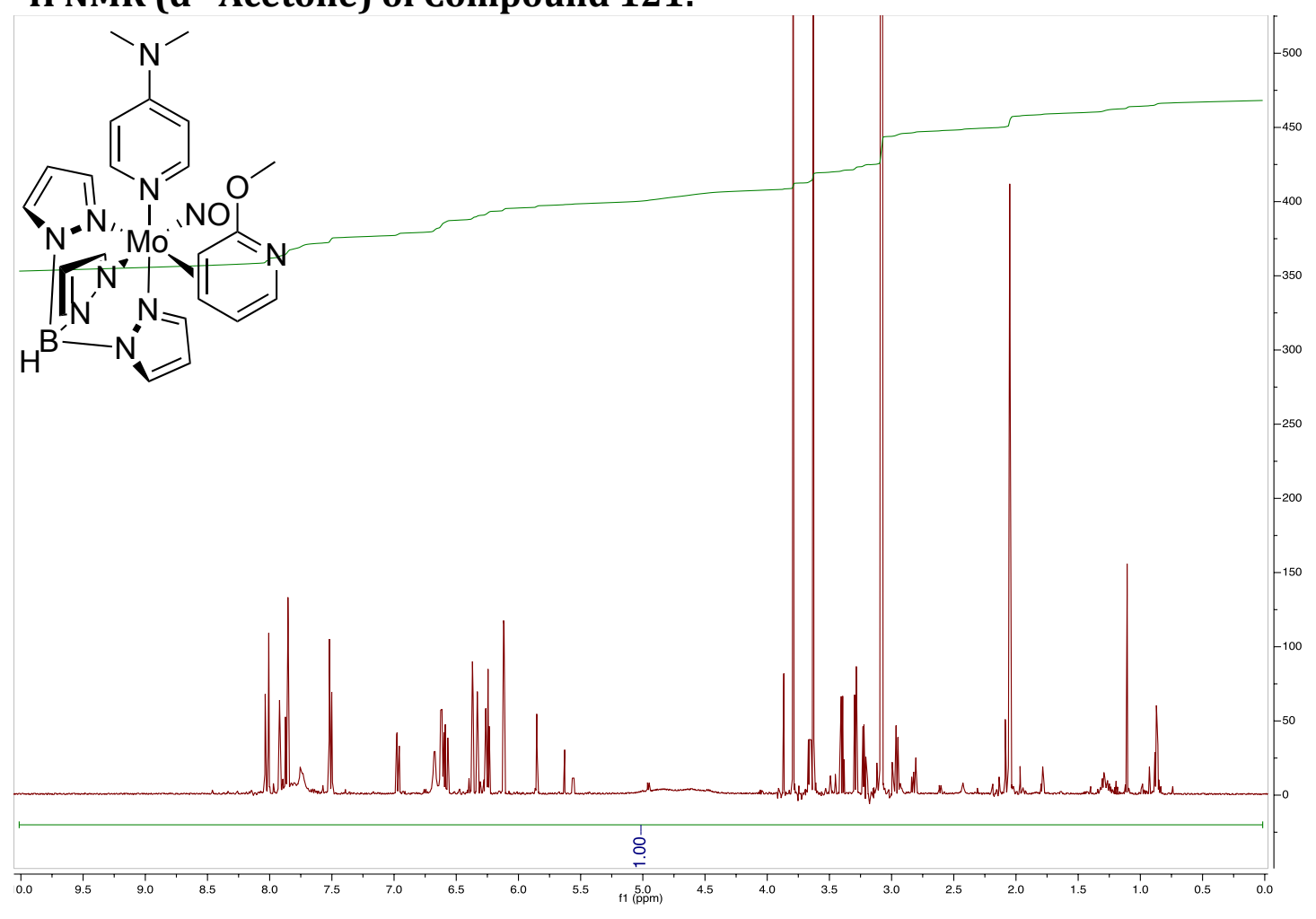

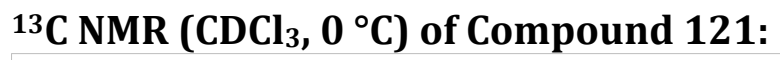

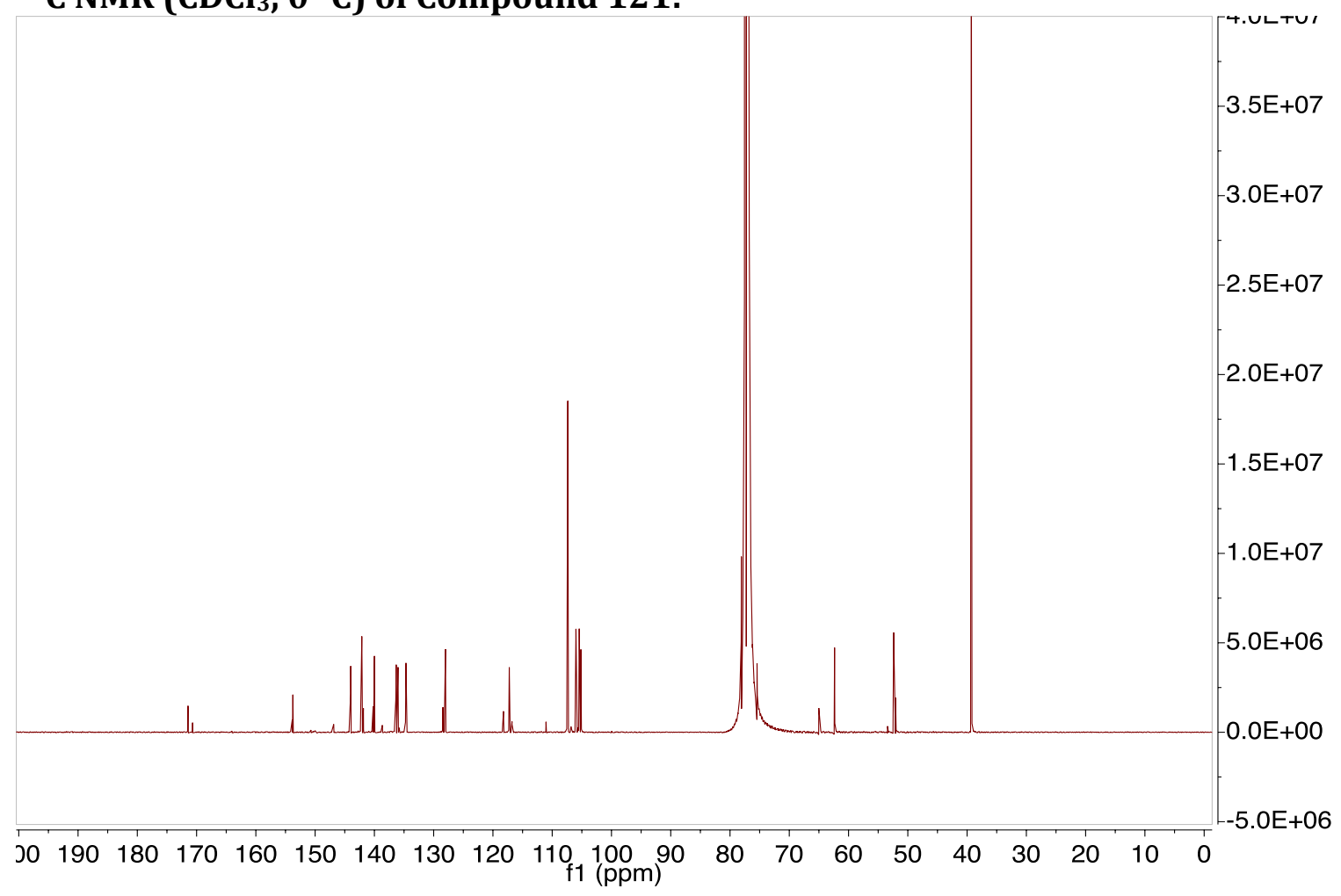




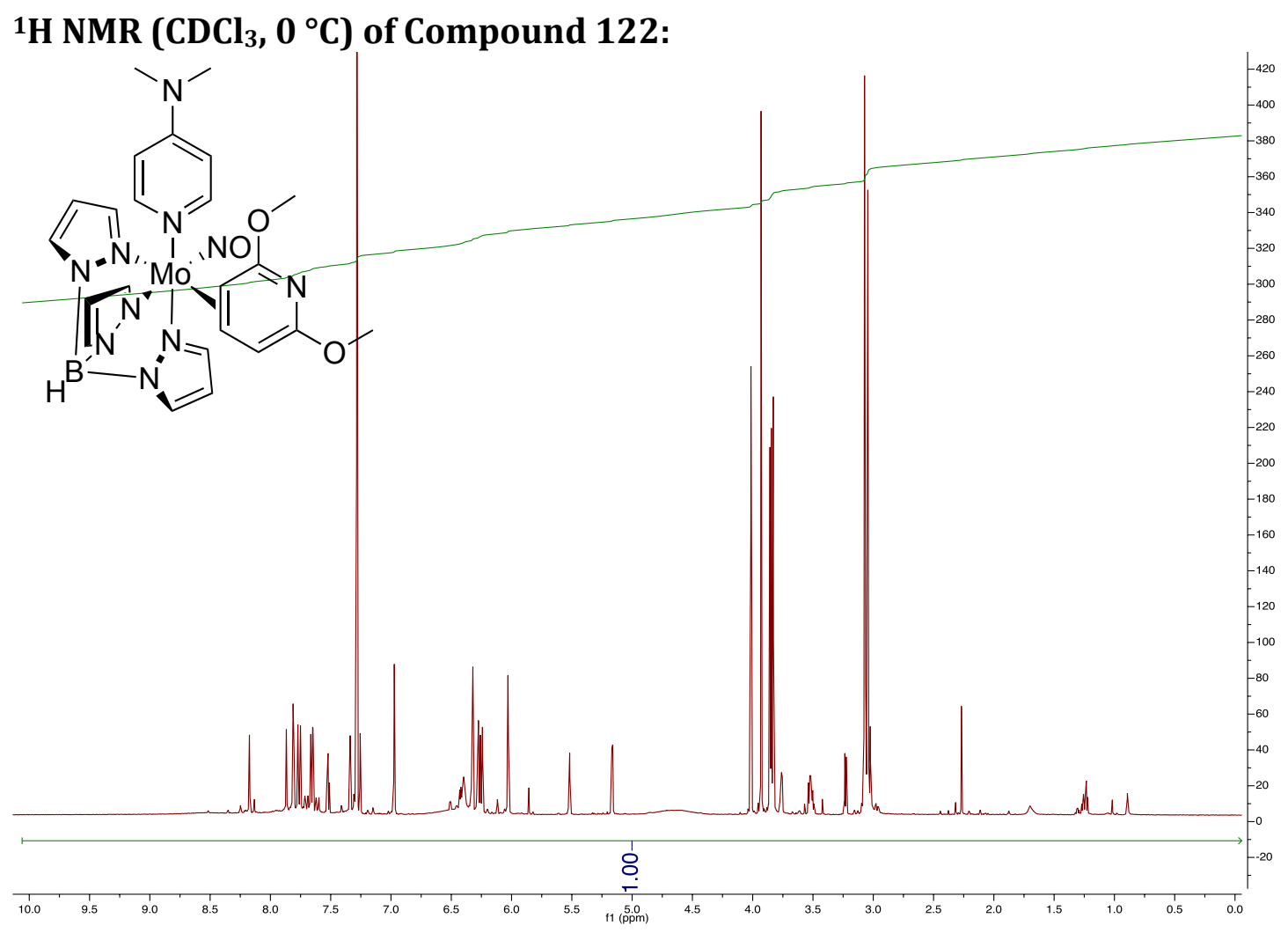

${ }^{13} \mathrm{C} \mathrm{NMR}\left(\mathrm{CDCl}_{3}, \mathrm{O}^{\circ} \mathrm{C}\right)$ of Compound 122 :

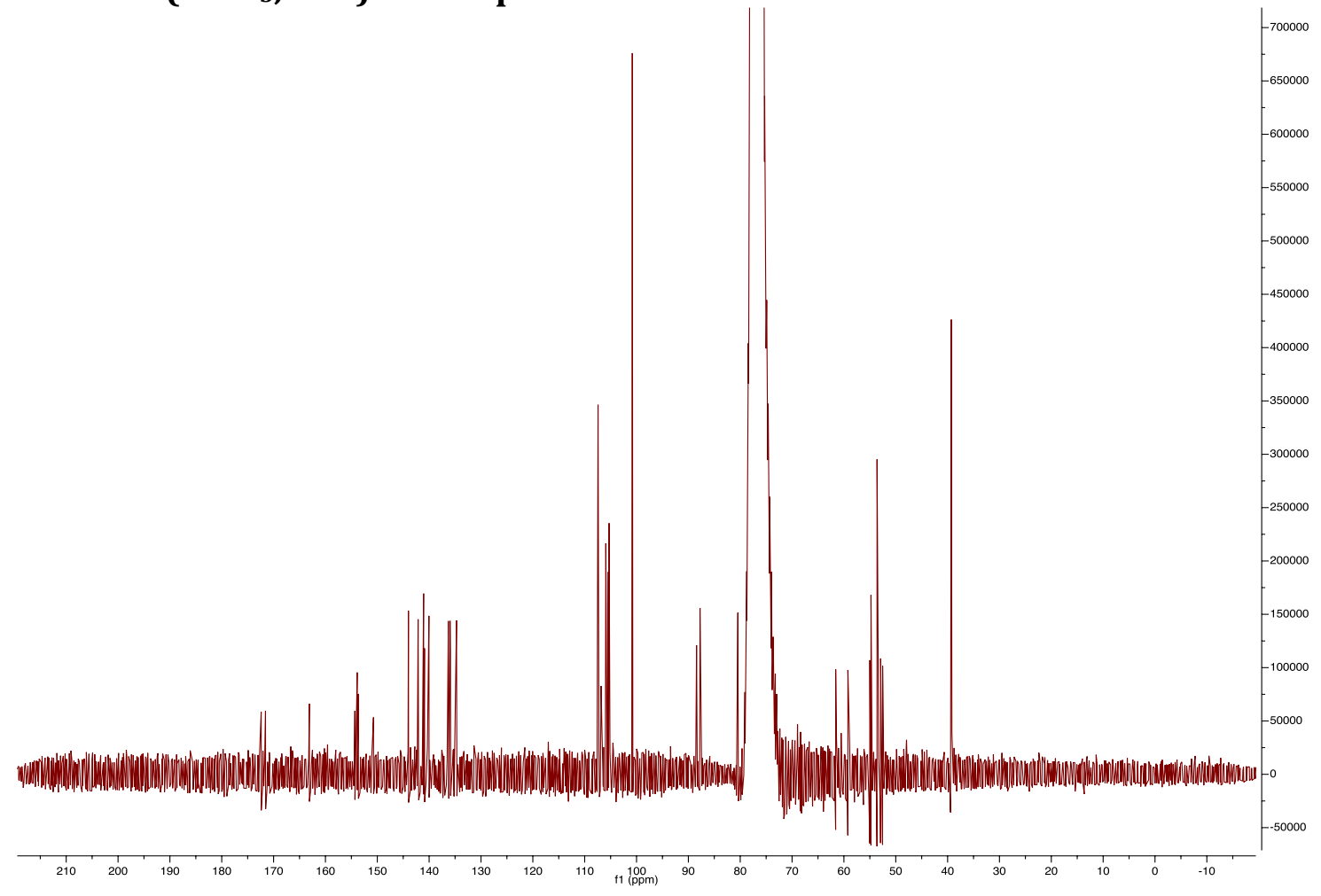


NOESY $\left(\mathrm{CDCl}_{3}, \mathrm{O}^{\circ} \mathrm{C}\right)$ of Compound 122:

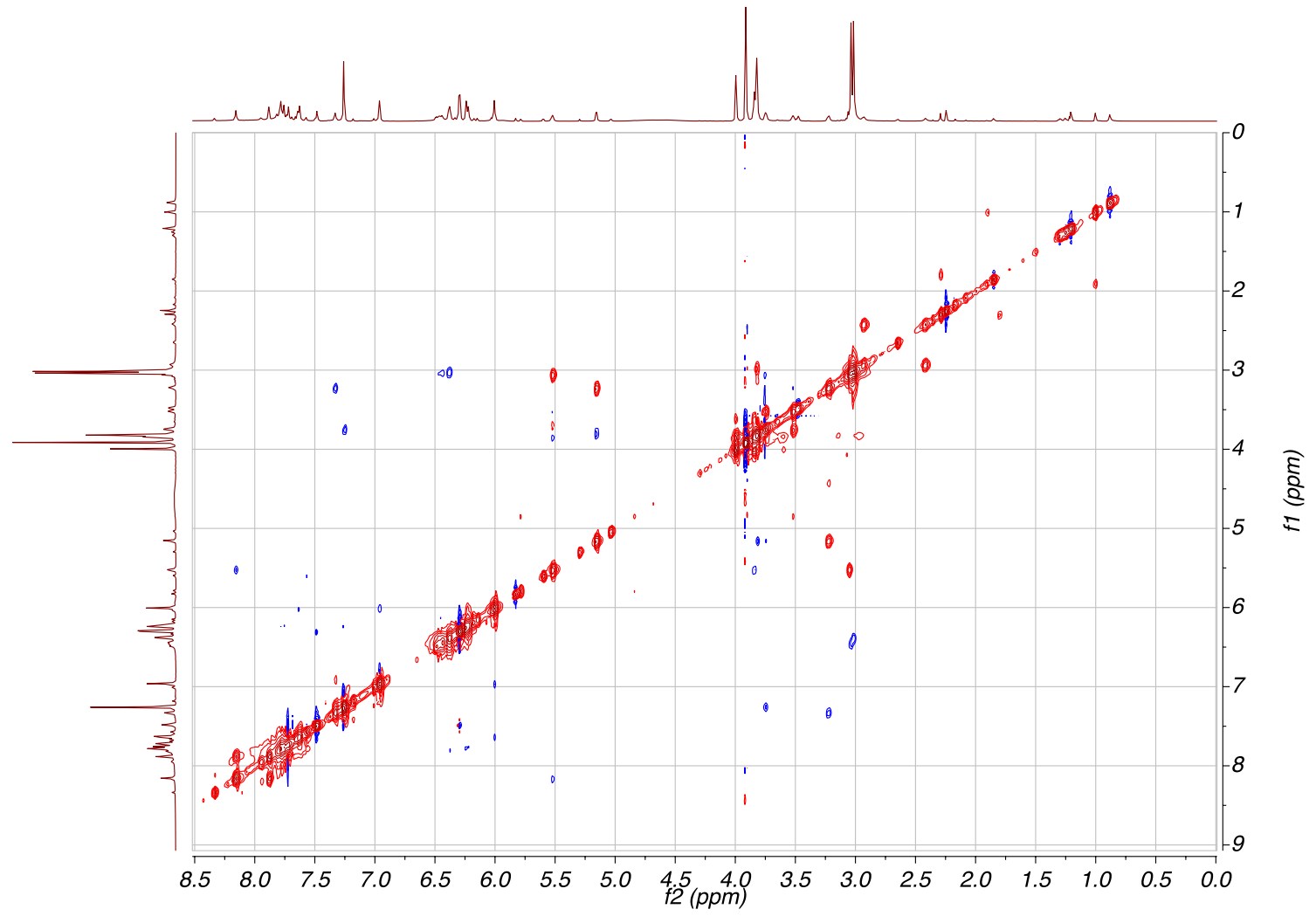


${ }^{1}$ H NMR (d6-Acetone) of Compound 123:

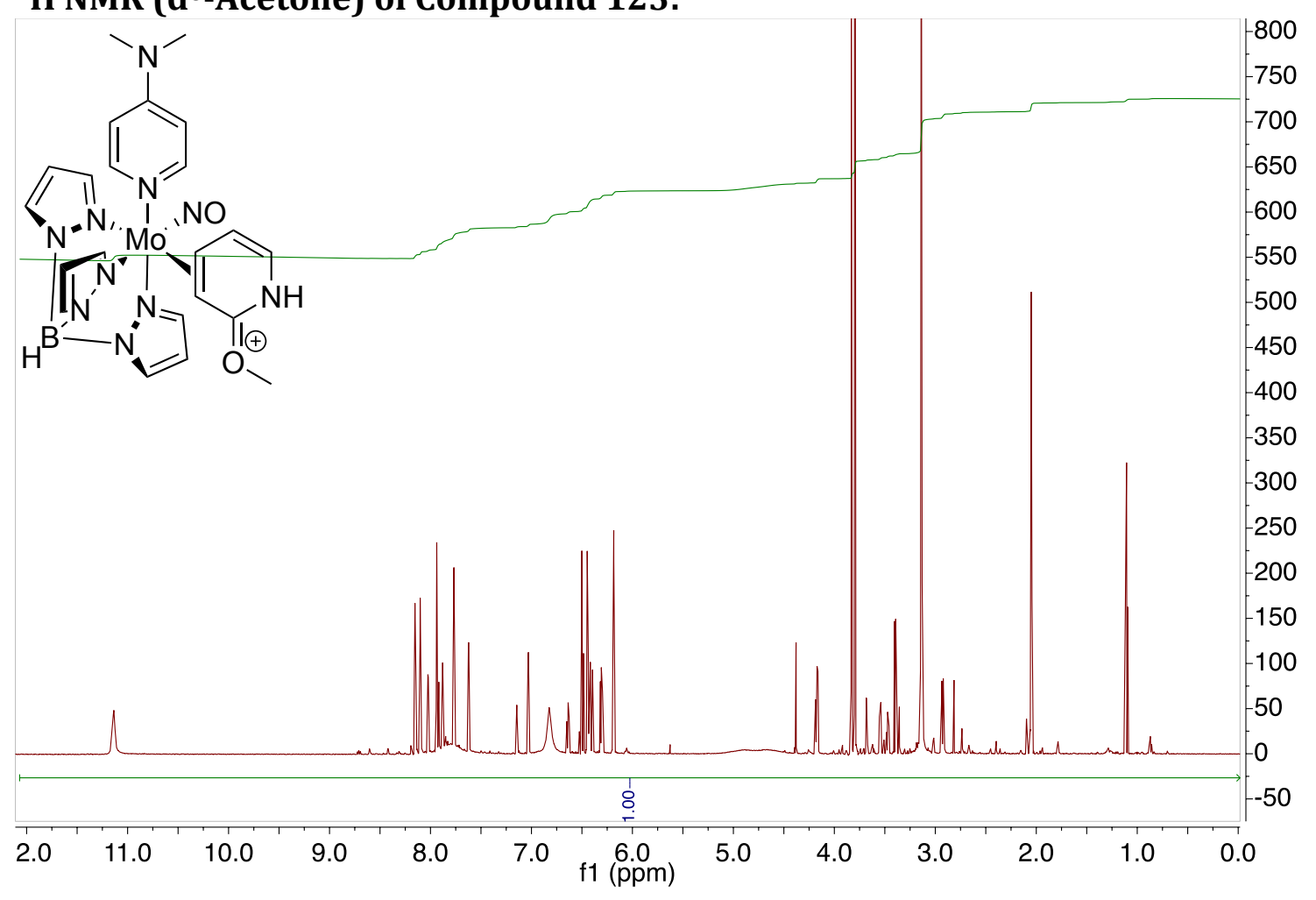

${ }^{1}$ H NMR (d6-Acetone) of Compound 123A Selective Precipitation:

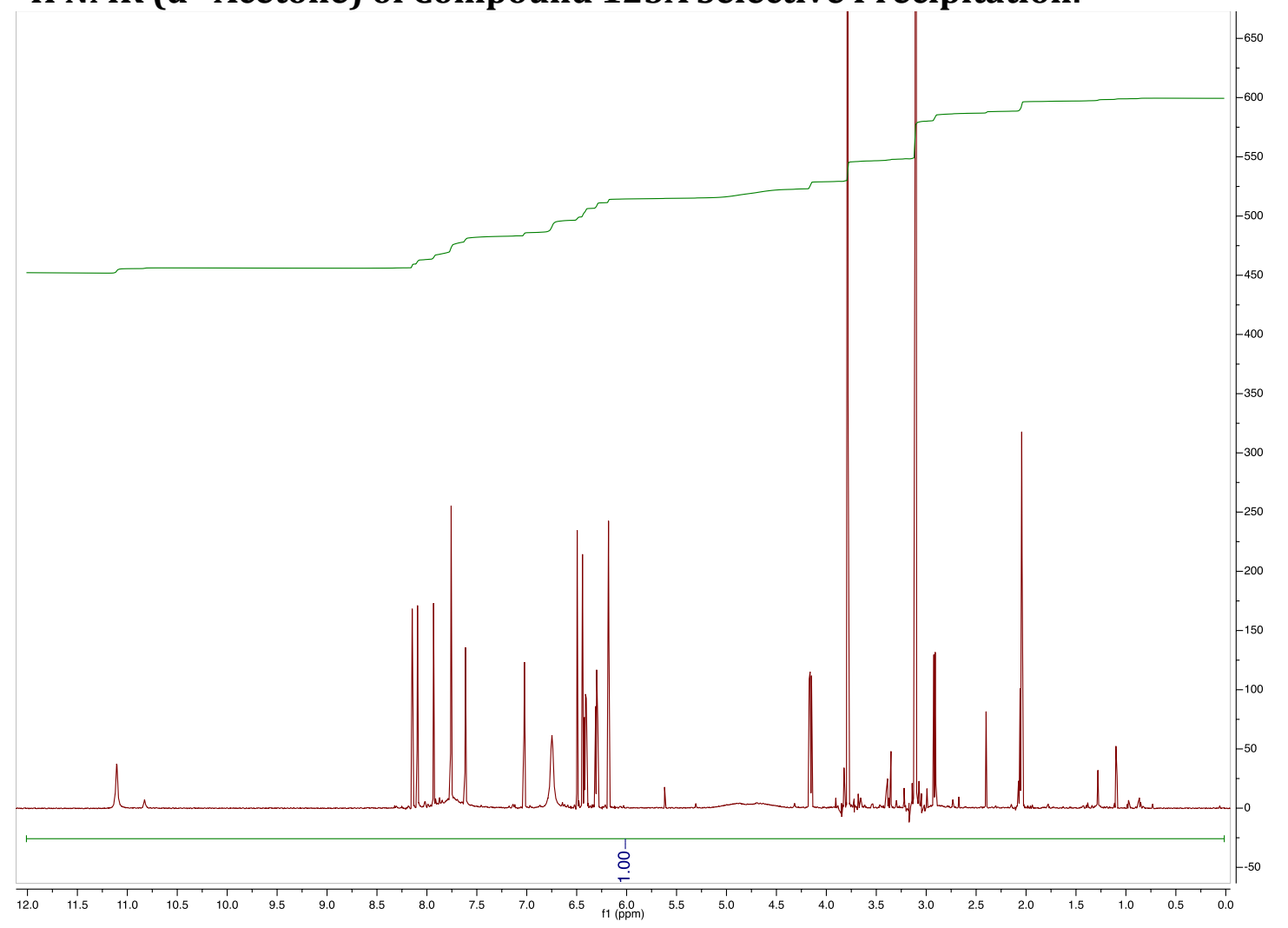


${ }^{13} \mathrm{C}$ NMR (d6-Acetone) of Compound 123:

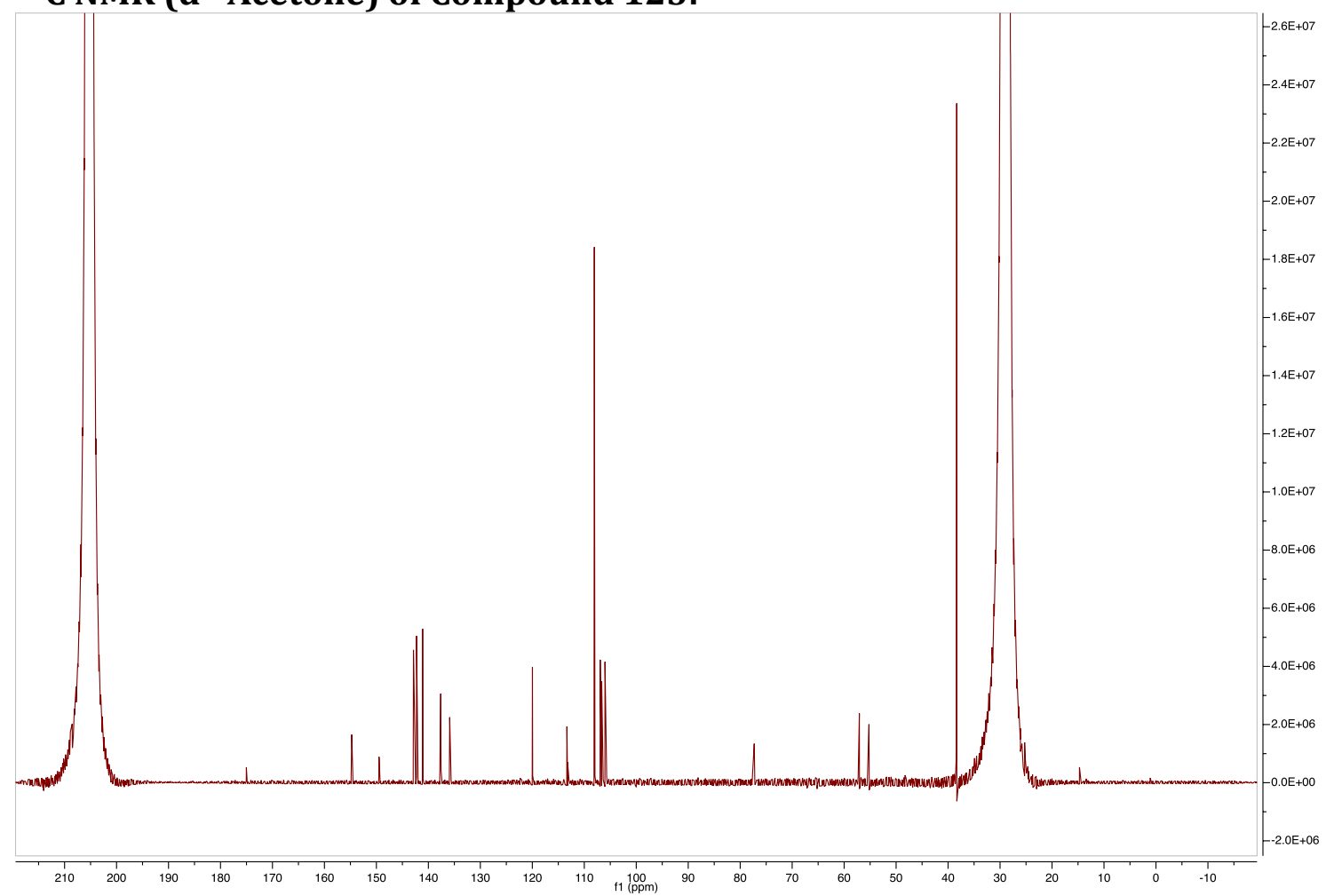


414

Compound 124

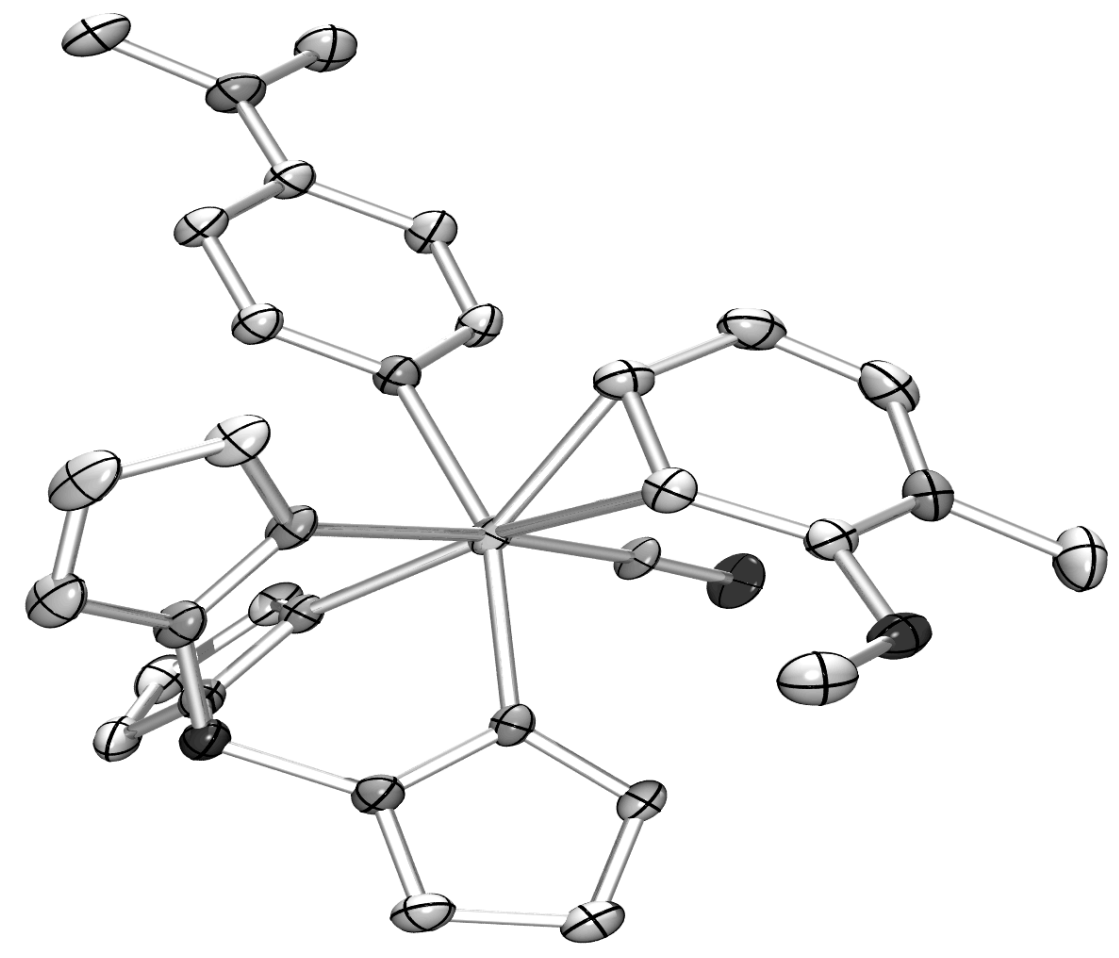




\section{Compound 124}

Table 1. Crystal data and structure refinement for $\mathrm{C}_{24} \mathrm{H}_{30} \mathrm{BF}_{3} \mathrm{MoN}_{10} \mathrm{O}_{5} \mathrm{~S}$.

Empirical formula

Formula weight

Temperature

Wavelength

Crystal system

Space group

Unit cell dimensions

Volume

Z

Density (calculated)

Absorption coefficient

$\mathrm{F}(000)$

Crystal size

Theta range for data collection

Index ranges

Reflections collected

Independent reflections

Completeness to theta $=25.242^{\circ}$

Absorption correction

Refinement method

Data / restraints / parameters

Goodness-of-fit on $\mathrm{F}^{2}$

Final $\mathrm{R}$ indices $[\mathrm{I}>2 \operatorname{sigma}(\mathrm{I})]$

$\mathrm{R}$ indices (all data)

Largest diff. peak and hole
C24 H30 B F3 Mo N10 O5 S

734.39

153(2) K

$0.71073 \AA$

Triclinic

P-1

$$
\begin{array}{ll}
\mathrm{a}=9.612(3) \AA & \alpha=78.584(6)^{\circ} . \\
\mathrm{b}=10.704(3) \AA & \beta=87.543(5)^{\circ} . \\
\mathrm{c}=15.335(4) \AA & \gamma=77.379(6)^{\circ} .
\end{array}
$$

2

$1.616 \mathrm{Mg} / \mathrm{m}^{3}$

$0.576 \mathrm{~mm}^{-1}$

748

$0.410 \times 0.150 \times 0.130 \mathrm{~mm}^{3}$

3.474 to $37.245^{\circ}$.

$-15<=\mathrm{h}<=16,-15<=\mathrm{k}<=18,-25<=\mathrm{l}<=25$

26372

$12051[\mathrm{R}(\mathrm{int})=0.0505]$

$95.4 \%$

Empirical

Full-matrix least-squares on $\mathrm{F}^{2}$

$12051 / 0 / 414$

1.105

$\mathrm{R} 1=0.0879, \mathrm{wR} 2=0.2261$

$\mathrm{R} 1=0.1082, \mathrm{wR} 2=0.2461$

3.728 and -3.495 e. $\AA^{-3}$ 
Compound 124

Table 2. Atomic coordinates ( $\times 10^{4}$ ) and equivalent isotropic displacement parameters $\left(\AA^{2} \times 10^{3}\right)$ for $\mathrm{C}_{24} \mathrm{H}_{30} \mathrm{BF}_{3} \mathrm{MoN}_{10} \mathrm{O}_{5} \mathrm{~S}$. U(eq) is defined as one third of the trace of the orthogonalized $\mathrm{Uij}^{\mathrm{ij}}$ tensor.

\begin{tabular}{|c|c|c|c|c|}
\hline & $\mathrm{x}$ & $\mathrm{y}$ & $\mathrm{z}$ & $\mathrm{U}(\mathrm{eq})$ \\
\hline Mo & $2497(1)$ & $7598(1)$ & $6989(1)$ & $18(1)$ \\
\hline $\mathrm{S}(1)$ & $7003(1)$ & $2858(2)$ & $8477(1)$ & $35(1)$ \\
\hline $\mathrm{F}(1)$ & $8675(5)$ & $1456(5)$ & $7466(3)$ & $64(1)$ \\
\hline $\mathrm{F}(2)$ & $9216(6)$ & $3285(7)$ & $7499(5)$ & $91(2)$ \\
\hline $\mathrm{F}(3)$ & $9707(5)$ & $1685(7)$ & $8611(4)$ & $87(2)$ \\
\hline $\mathrm{O}(1)$ & $6763(3)$ & $7535(4)$ & $6949(2)$ & $30(1)$ \\
\hline $\mathrm{O}(2)$ & $2625(4)$ & $10344(3)$ & $6995(3)$ & $34(1)$ \\
\hline $\mathrm{O}(3)$ & $6125(5)$ & $3356(7)$ & $7704(4)$ & $68(2)$ \\
\hline $\mathrm{O}(4)$ & $6732(7)$ & $1679(6)$ & $9023(4)$ & $65(2)$ \\
\hline $\mathrm{O}(5)$ & $7226(7)$ & $3825(6)$ & $8950(4)$ & $63(2)$ \\
\hline $\mathrm{N}(1)$ & $5451(4)$ & $8563(4)$ & $7936(3)$ & $28(1)$ \\
\hline $\mathrm{N}(2)$ & $744(3)$ & $7508(4)$ & $7983(2)$ & $22(1)$ \\
\hline $\mathrm{N}(3)$ & $-2546(4)$ & $7356(5)$ & $9873(2)$ & $29(1)$ \\
\hline $\mathrm{N}(4)$ & $3628(3)$ & $7739(3)$ & $5706(2)$ & $19(1)$ \\
\hline $\mathrm{N}(5)$ & $3278(3)$ & $7187(4)$ & $5043(2)$ & $23(1)$ \\
\hline $\mathrm{N}(6)$ & $653(3)$ & 8091(3) & $6089(2)$ & 19(1) \\
\hline $\mathrm{N}(7)$ & $670(3)$ & $7588(4)$ & $5343(2)$ & $24(1)$ \\
\hline $\mathrm{N}(8)$ & $2536(4)$ & $5575(4)$ & $6781(2)$ & $22(1)$ \\
\hline $\mathrm{N}(9)$ & $2217(4)$ & $5402(4)$ & $5957(2)$ & $24(1)$ \\
\hline $\mathrm{N}(10)$ & $2533(3)$ & $9227(3)$ & $7022(2)$ & $20(1)$ \\
\hline $\mathrm{C}(1)$ & $5642(4)$ & $7585(5)$ & $7491(2)$ & $23(1)$ \\
\hline $\mathrm{C}(2)$ & $4717(4)$ & $6709(4)$ & $7593(3)$ & $22(1)$ \\
\hline $\mathrm{C}(3)$ & $3631(4)$ & $6791(5)$ & $8285(3)$ & $26(1)$ \\
\hline $\mathrm{C}(4)$ & $3655(5)$ & $7750(6)$ & $8848(3)$ & $33(1)$ \\
\hline$C(5)$ & $4480(5)$ & $8605(6)$ & $8647(3)$ & $34(1)$ \\
\hline$C(6)$ & $35(4)$ & $6529(4)$ & $8139(3)$ & $24(1)$ \\
\hline$C(7)$ & $-1022(4)$ & $6415(5)$ & $8766(3)$ & $25(1)$ \\
\hline $\mathrm{C}(8)$ & $-1452(4)$ & $7399(5)$ & $9283(3)$ & $25(1)$ \\
\hline $\mathrm{C}(9)$ & $-718(4)$ & $8431(5)$ & $9127(3)$ & $28(1)$ \\
\hline
\end{tabular}




\begin{tabular}{lrrrr}
$\mathrm{C}(10)$ & $343(4)$ & $8441(5)$ & $8488(3)$ & $25(1)$ \\
$\mathrm{C}(11)$ & $-3174(5)$ & $6220(6)$ & $10065(3)$ & $35(1)$ \\
$\mathrm{C}(12)$ & $-2958(5)$ & $8362(7)$ & $10415(3)$ & $37(1)$ \\
$\mathrm{C}(13)$ & $4628(4)$ & $8407(4)$ & $5372(2)$ & $21(1)$ \\
$\mathrm{C}(14)$ & $4932(4)$ & $8276(5)$ & $4493(3)$ & $27(1)$ \\
$\mathrm{C}(15)$ & $4060(5)$ & $7485(5)$ & $4315(3)$ & $27(1)$ \\
$\mathrm{C}(16)$ & $-511(5)$ & $8171(6)$ & $4856(3)$ & $31(1)$ \\
$\mathrm{C}(17)$ & $-1347(4)$ & $9084(5)$ & $5297(3)$ & $31(1)$ \\
$\mathrm{C}(18)$ & $-564(4)$ & $9004(5)$ & $6067(3)$ & $24(1)$ \\
$\mathrm{C}(19)$ & $2222(5)$ & $4139(5)$ & $5991(3)$ & $33(1)$ \\
$\mathrm{C}(20)$ & $2534(6)$ & $3453(5)$ & $6842(4)$ & $37(1)$ \\
$\mathrm{C}(21)$ & $2735(5)$ & $4389(4)$ & $7308(3)$ & $29(1)$ \\
$\mathrm{C}(22)$ & $6294(6)$ & $9591(6)$ & $7718(4)$ & $42(1)$ \\
$\mathrm{C}(23)$ & $7255(5)$ & $6359(6)$ & $6585(3)$ & $36(1)$ \\
$\mathrm{C}(24)$ & $8747(6)$ & $2287(8)$ & $7988(5)$ & $48(1)$ \\
$\mathrm{B}(1)$ & $1976(5)$ & $6539(5)$ & $5151(3)$ & $24(1)$ \\
& & & & \\
\hline
\end{tabular}


Compound 124

Table 3. Bond lengths $[\AA]$ and angles $\left[{ }^{\circ}\right]$ for $\mathrm{C}_{24} \mathrm{H}_{30} \mathrm{BF}_{3} \mathrm{MoN}_{10} \mathrm{O}_{5} \mathrm{~S}$.

\begin{tabular}{|c|c|}
\hline Mo-N(10) & $1.763(3)$ \\
\hline Mo-N(6) & $2.200(3)$ \\
\hline Mo-N(4) & $2.201(3)$ \\
\hline Mo-N(2) & $2.228(3)$ \\
\hline Mo-C(3) & $2.231(4)$ \\
\hline Mo-N(8) & $2.242(4)$ \\
\hline Mo-C(2) & $2.291(4)$ \\
\hline $\mathrm{S}(1)-\mathrm{O}(3)$ & $1.428(5)$ \\
\hline $\mathrm{S}(1)-\mathrm{O}(5)$ & $1.432(5)$ \\
\hline $\mathrm{S}(1)-\mathrm{O}(4)$ & $1.438(6)$ \\
\hline $\mathrm{S}(1)-\mathrm{C}(24)$ & $1.836(6)$ \\
\hline $\mathrm{F}(1)-\mathrm{C}(24)$ & $1.322(9)$ \\
\hline $\mathrm{F}(2)-\mathrm{C}(24)$ & $1.331(9)$ \\
\hline $\mathrm{F}(3)-\mathrm{C}(24)$ & $1.323(8)$ \\
\hline $\mathrm{O}(1)-\mathrm{C}(1)$ & $1.331(5)$ \\
\hline $\mathrm{O}(1)-\mathrm{C}(23)$ & $1.454(7)$ \\
\hline $\mathrm{O}(2)-\mathrm{N}(10)$ & $1.210(5)$ \\
\hline $\mathrm{N}(1)-\mathrm{C}(1)$ & $1.335(6)$ \\
\hline $\mathrm{N}(1)-\mathrm{C}(5)$ & $1.407(6)$ \\
\hline $\mathrm{N}(1)-\mathrm{C}(22)$ & $1.484(7)$ \\
\hline $\mathrm{N}(2)-\mathrm{C}(6)$ & $1.348(6)$ \\
\hline $\mathrm{N}(2)-\mathrm{C}(10)$ & $1.363(5)$ \\
\hline $\mathrm{N}(3)-\mathrm{C}(8)$ & $1.360(5)$ \\
\hline $\mathrm{N}(3)-\mathrm{C}(11)$ & $1.447(7)$ \\
\hline $\mathrm{N}(3)-\mathrm{C}(12)$ & $1.465(7)$ \\
\hline $\mathrm{N}(4)-\mathrm{C}(13)$ & $1.345(5)$ \\
\hline $\mathrm{N}(4)-\mathrm{N}(5)$ & $1.361(5)$ \\
\hline $\mathrm{N}(5)-\mathrm{C}(15)$ & $1.343(5)$ \\
\hline $\mathrm{N}(5)-\mathrm{B}(1)$ & $1.546(6)$ \\
\hline $\mathrm{N}(6)-\mathrm{C}(18)$ & $1.348(5)$ \\
\hline $\mathrm{N}(6)-\mathrm{N}(7)$ & $1.356(5)$ \\
\hline$N(7)-C(16)$ & $1.347(5)$ \\
\hline $\mathrm{N}(7)-\mathrm{B}(1)$ & $1.551(6)$ \\
\hline
\end{tabular}




$\begin{array}{ll}\mathrm{N}(8)-\mathrm{C}(21) & 1.342(6) \\ \mathrm{N}(8)-\mathrm{N}(9) & 1.370(5) \\ \mathrm{N}(9)-\mathrm{C}(19) & 1.342(6) \\ \mathrm{N}(9)-\mathrm{B}(1) & 1.539(7) \\ \mathrm{C}(1)-\mathrm{C}(2) & 1.409(6) \\ \mathrm{C}(2)-\mathrm{C}(3) & 1.458(5) \\ \mathrm{C}(2)-\mathrm{H}(2) & 1.0000 \\ \mathrm{C}(3)-\mathrm{C}(4) & 1.471(7) \\ \mathrm{C}(3)-\mathrm{H}(3) & 1.0000 \\ \mathrm{C}(4)-\mathrm{C}(5) & 1.321(8) \\ \mathrm{C}(4)-\mathrm{H}(4) & 0.9500 \\ \mathrm{C}(5)-\mathrm{H}(5) & 0.9500 \\ \mathrm{C}(6)-\mathrm{C}(7) & 1.378(5) \\ \mathrm{C}(6)-\mathrm{H}(6) & 0.9500 \\ \mathrm{C}(7)-\mathrm{C}(8) & 1.422(6) \\ \mathrm{C}(7)-\mathrm{H}(7) & 0.9500 \\ \mathrm{C}(8)-\mathrm{C}(9) & 1.413(7) \\ \mathrm{C}(9)-\mathrm{C}(10) & 1.384(5) \\ \mathrm{C}(9)-\mathrm{H}(9) & 0.9500 \\ \mathrm{C}(10)-\mathrm{H}(10) & 0.9500 \\ \mathrm{C}(11)-\mathrm{H}(11 \mathrm{~A}) & 0.9800 \\ \mathrm{C}(11)-\mathrm{H}(11 \mathrm{~B}) & 0.9800 \\ \mathrm{C}(11)-\mathrm{H}(11 \mathrm{C}) & 0.9800 \\ \mathrm{C}(12)-\mathrm{H}(12 \mathrm{~A}) & 0.9800 \\ \mathrm{C}(12)-\mathrm{H}(12 \mathrm{~B}) & 0.9800 \\ \mathrm{C}(12)-\mathrm{H}(12 \mathrm{C}) & 0.9800 \\ \mathrm{C}(13)-\mathrm{C}(14) & 1.395(5) \\ \mathrm{C}(13)-\mathrm{H}(13) & 0.9500 \\ \mathrm{C}(14)-\mathrm{C}(15) & 1.384(7) \\ \mathrm{C}(14)-\mathrm{H}(14) & 0.9500 \\ \mathrm{C}(15)-\mathrm{H}(15) & 0.9500 \\ \mathrm{C}(16)-\mathrm{C}(17) & 1.389(8) \\ \mathrm{C}(16)-\mathrm{H}(16) & 0.9500 \\ \mathrm{C}(17)-\mathrm{C}(18) & 1.405(6) \\ \mathrm{C}(17)-\mathrm{H}(17) & 0.9500 \\ \mathrm{C}(18)-\mathrm{H}(18) & 0.9500 \\ & \end{array}$




\begin{tabular}{|c|c|}
\hline$C(19)-C(20)$ & $1.374(8)$ \\
\hline C(19)-H(19) & 0.9500 \\
\hline $\mathrm{C}(20)-\mathrm{C}(21)$ & $1.389(7)$ \\
\hline $\mathrm{C}(20)-\mathrm{H}(20)$ & 0.9500 \\
\hline $\mathrm{C}(21)-\mathrm{H}(21)$ & 0.9500 \\
\hline $\mathrm{C}(22)-\mathrm{H}(22 \mathrm{~A})$ & 0.9800 \\
\hline $\mathrm{C}(22)-\mathrm{H}(22 \mathrm{~B})$ & 0.9800 \\
\hline $\mathrm{C}(22)-\mathrm{H}(22 \mathrm{C})$ & 0.9800 \\
\hline $\mathrm{C}(23)-\mathrm{H}(23 \mathrm{~A})$ & 0.9800 \\
\hline $\mathrm{C}(23)-\mathrm{H}(23 \mathrm{~B})$ & 0.9800 \\
\hline $\mathrm{C}(23)-\mathrm{H}(23 \mathrm{C})$ & 0.9800 \\
\hline $\mathrm{B}(1)-\mathrm{H}(1 \mathrm{~B})$ & $1.04(6)$ \\
\hline N(10)-Mo-N(6) & $95.32(14)$ \\
\hline N(10)-Mo-N(4) & $90.89(14)$ \\
\hline N(6)-Mo-N(4) & $80.80(11)$ \\
\hline N(10)-Mo-N(2) & $93.91(14)$ \\
\hline N(6)-Mo-N(2) & $80.05(12)$ \\
\hline N(4)-Mo-N(2) & $160.60(12)$ \\
\hline N(10)-Mo-C(3) & $94.05(17)$ \\
\hline N(6)-Mo-C(3) & $154.85(13)$ \\
\hline N(4)-Mo-C(3) & $122.36(13)$ \\
\hline N(2)-Mo-C(3) & $76.06(13)$ \\
\hline N(10)-Mo-N(8) & $173.26(14)$ \\
\hline N(6)-Mo-N(8) & $82.36(13)$ \\
\hline N(4)-Mo-N(8) & $82.50(13)$ \\
\hline N(2)-Mo-N(8) & $91.92(13)$ \\
\hline C(3)-Mo-N(8) & $90.63(16)$ \\
\hline N(10)-Mo-C(2) & $95.93(15)$ \\
\hline N(6)-Mo-C(2) & $161.81(13)$ \\
\hline N(4)-Mo-C(2) & $84.77(13)$ \\
\hline N(2)-Mo-C(2) & $113.33(13)$ \\
\hline $\mathrm{C}(3)-\mathrm{Mo}-\mathrm{C}(2)$ & $37.59(14)$ \\
\hline N(8)-Mo-C(2) & $84.84(14)$ \\
\hline $\mathrm{O}(3)-\mathrm{S}(1)-\mathrm{O}(5)$ & $114.8(4)$ \\
\hline $\mathrm{O}(3)-\mathrm{S}(1)-\mathrm{O}(4)$ & $115.2(4)$ \\
\hline
\end{tabular}




$\begin{array}{ll}\mathrm{O}(5)-\mathrm{S}(1)-\mathrm{O}(4) & 115.4(3) \\ \mathrm{O}(3)-\mathrm{S}(1)-\mathrm{C}(24) & 101.8(3) \\ \mathrm{O}(5)-\mathrm{S}(1)-\mathrm{C}(24) & 104.6(4) \\ \mathrm{O}(4)-\mathrm{S}(1)-\mathrm{C}(24) & 102.4(4) \\ \mathrm{C}(1)-\mathrm{O}(1)-\mathrm{C}(23) & 118.0(4) \\ \mathrm{C}(1)-\mathrm{N}(1)-\mathrm{C}(5) & 120.2(4) \\ \mathrm{C}(1)-\mathrm{N}(1)-\mathrm{C}(22) & 121.1(4) \\ \mathrm{C}(5)-\mathrm{N}(1)-\mathrm{C}(22) & 118.7(5) \\ \mathrm{C}(6)-\mathrm{N}(2)-\mathrm{C}(10) & 115.8(3) \\ \mathrm{C}(6)-\mathrm{N}(2)-\mathrm{Mo} & 122.9(3) \\ \mathrm{C}(10)-\mathrm{N}(2)-\mathrm{Mo} & 121.4(3) \\ \mathrm{C}(8)-\mathrm{N}(3)-\mathrm{C}(11) & 120.0(4) \\ \mathrm{C}(8)-\mathrm{N}(3)-\mathrm{C}(12) & 120.4(4) \\ \mathrm{C}(11)-\mathrm{N}(3)-\mathrm{C}(12) & 118.9(4) \\ \mathrm{C}(13)-\mathrm{N}(4)-\mathrm{N}(5) & 106.5(3) \\ \mathrm{C}(13)-\mathrm{N}(4)-\mathrm{Mo} & 131.5(3) \\ \mathrm{N}(5)-\mathrm{N}(4)-\mathrm{Mo} & 121.7(2) \\ \mathrm{C}(15)-\mathrm{N}(5)-\mathrm{N}(4) & 110.0(3) \\ \mathrm{C}(15)-\mathrm{N}(5)-\mathrm{B}(1) & 129.4(3) \\ \mathrm{N}(4)-\mathrm{N}(5)-\mathrm{B}(1) & 119.8(3) \\ \mathrm{C}(18)-\mathrm{N}(6)-\mathrm{N}(7) & 106.7(3) \\ \mathrm{C}(18)-\mathrm{N}(6)-\mathrm{Mo} & 130.5(3) \\ \mathrm{N}(7)-\mathrm{N}(6)-\mathrm{Mo} & 122.2(2) \\ \mathrm{C}(16)-\mathrm{N}(7)-\mathrm{N}(6) & 110.1(4) \\ \mathrm{C}(16)-\mathrm{N}(7)-\mathrm{B}(1) & 130.4(4) \\ \mathrm{N}(6)-\mathrm{N}(7)-\mathrm{B}(1) & 119.4(3) \\ \mathrm{C}(21)-\mathrm{N}(8)-\mathrm{N}(9) & 105.7(4) \\ \mathrm{C}(21)-\mathrm{N}(8)-\mathrm{Mo} & 135.1(3) \\ \mathrm{N}(9)-\mathrm{N}(8)-\mathrm{Mo} & 119.1(3) \\ \mathrm{C}(19)-\mathrm{N}(9)-\mathrm{N}(8) & 109.4(4) \\ \mathrm{C}(19)-\mathrm{N}(9)-\mathrm{B}(1) & 129.0(4) \\ \mathrm{N}(8)-\mathrm{N}(9)-\mathrm{B}(1) & 121.6(4) \\ \mathrm{O}(2)-\mathrm{N}(10)-\mathrm{Mo} & 175.4(3) \\ \mathrm{O}(1)-\mathrm{C}(1)-\mathrm{N}(1) & 113.7(4) \\ \mathrm{O}(1)-\mathrm{C}(1)-\mathrm{C}(2) & 124.3(4) \\ \mathrm{N}(1)-\mathrm{C}(1)-\mathrm{C}(2) & 122.0(4)\end{array}$




$\begin{array}{ll}\mathrm{C}(1)-\mathrm{C}(2)-\mathrm{C}(3) & 118.1(4) \\ \mathrm{C}(1)-\mathrm{C}(2)-\mathrm{Mo} & 114.4(3) \\ \mathrm{C}(3)-\mathrm{C}(2)-\mathrm{Mo} & 69.0(2) \\ \mathrm{C}(1)-\mathrm{C}(2)-\mathrm{H}(2) & 115.7 \\ \mathrm{C}(3)-\mathrm{C}(2)-\mathrm{H}(2) & 115.7 \\ \mathrm{Mo}-\mathrm{C}(2)-\mathrm{H}(2) & 115.7 \\ \mathrm{C}(2)-\mathrm{C}(3)-\mathrm{C}(4) & 116.1(4) \\ \mathrm{C}(2)-\mathrm{C}(3)-\mathrm{Mo} & 73.4(2) \\ \mathrm{C}(4)-\mathrm{C}(3)-\mathrm{Mo} & 115.1(3) \\ \mathrm{C}(2)-\mathrm{C}(3)-\mathrm{H}(3) & 115.2 \\ \mathrm{C}(4)-\mathrm{C}(3)-\mathrm{H}(3) & 115.2 \\ \mathrm{Mo}-\mathrm{C}(3)-\mathrm{H}(3) & 115.2 \\ \mathrm{C}(5)-\mathrm{C}(4)-\mathrm{C}(3) & 120.8(4) \\ \mathrm{C}(5)-\mathrm{C}(4)-\mathrm{H}(4) & 119.6 \\ \mathrm{C}(3)-\mathrm{C}(4)-\mathrm{H}(4) & 119.6 \\ \mathrm{C}(4)-\mathrm{C}(5)-\mathrm{N}(1) & 121.5(5) \\ \mathrm{C}(4)-\mathrm{C}(5)-\mathrm{H}(5) & 119.3 \\ \mathrm{~N}(1)-\mathrm{C}(5)-\mathrm{H}(5) & 119.3 \\ \mathrm{~N}(2)-\mathrm{C}(6)-\mathrm{C}(7) & 124.7(4) \\ \mathrm{N}(2)-\mathrm{C}(6)-\mathrm{H}(6) & 117.6 \\ \mathrm{C}(7)-\mathrm{C}(6)-\mathrm{H}(6) & 117.6 \\ \mathrm{C}(6)-\mathrm{C}(7)-\mathrm{C}(8) & 119.4(4) \\ \mathrm{C}(6)-\mathrm{C}(7)-\mathrm{H}(7) & 120.3 \\ \mathrm{C}(8)-\mathrm{C}(7)-\mathrm{H}(7) & 120.3 \\ \mathrm{~N}(3)-\mathrm{C}(8)-\mathrm{C}(9) & 122.5(4) \\ \mathrm{N}(3)-\mathrm{C}(8)-\mathrm{C}(7) & 121.0(4) \\ \mathrm{C}(9)-\mathrm{C}(8)-\mathrm{C}(7) & 116.5(3) \\ \mathrm{C}(10)-\mathrm{C}(9)-\mathrm{C}(8) & 119.4(4) \\ \mathrm{C}(10)-\mathrm{C}(9)-\mathrm{H}(9) & 120.3 \\ \mathrm{C}(8)-\mathrm{C}(9)-\mathrm{H}(9) & 120.3 \\ \mathrm{~N}(2)-\mathrm{C}(10)-\mathrm{C}(9) & 124.2(4) \\ \mathrm{N}(2)-\mathrm{C}(10)-\mathrm{H}(10) & 117.9 \\ \mathrm{C}(9)-\mathrm{C}(10)-\mathrm{H}(10) & 117.9 \\ \mathrm{~N}(3)-\mathrm{C}(11)-\mathrm{H}(11 \mathrm{~A}) & 109.5 \\ \mathrm{~N}(3)-\mathrm{C}(11)-\mathrm{H}(11 \mathrm{~B}) & 109.5 \\ \mathrm{H}(11 \mathrm{~A})-\mathrm{C}(11)-\mathrm{H}(11 \mathrm{~B}) & 109.5 \\ & \end{array}$




$\begin{array}{ll}\mathrm{N}(3)-\mathrm{C}(11)-\mathrm{H}(11 \mathrm{C}) & 109.5 \\ \mathrm{H}(11 \mathrm{~A})-\mathrm{C}(11)-\mathrm{H}(11 \mathrm{C}) & 109.5 \\ \mathrm{H}(11 \mathrm{~B})-\mathrm{C}(11)-\mathrm{H}(11 \mathrm{C}) & 109.5 \\ \mathrm{~N}(3)-\mathrm{C}(12)-\mathrm{H}(12 \mathrm{~A}) & 109.5 \\ \mathrm{~N}(3)-\mathrm{C}(12)-\mathrm{H}(12 \mathrm{~B}) & 109.5 \\ \mathrm{H}(12 \mathrm{~A})-\mathrm{C}(12)-\mathrm{H}(12 \mathrm{~B}) & 109.5 \\ \mathrm{~N}(3)-\mathrm{C}(12)-\mathrm{H}(12 \mathrm{C}) & 109.5 \\ \mathrm{H}(12 \mathrm{~A})-\mathrm{C}(12)-\mathrm{H}(12 \mathrm{C}) & 109.5 \\ \mathrm{H}(12 \mathrm{~B})-\mathrm{C}(12)-\mathrm{H}(12 \mathrm{C}) & 109.5 \\ \mathrm{~N}(4)-\mathrm{C}(13)-\mathrm{C}(14) & 110.2(4) \\ \mathrm{N}(4)-\mathrm{C}(13)-\mathrm{H}(13) & 124.9 \\ \mathrm{C}(14)-\mathrm{C}(13)-\mathrm{H}(13) & 124.9 \\ \mathrm{C}(15)-\mathrm{C}(14)-\mathrm{C}(13) & 104.8(3) \\ \mathrm{C}(15)-\mathrm{C}(14)-\mathrm{H}(14) & 127.6 \\ \mathrm{C}(13)-\mathrm{C}(14)-\mathrm{H}(14) & 127.6 \\ \mathrm{~N}(5)-\mathrm{C}(15)-\mathrm{C}(14) & 108.5(4) \\ \mathrm{N}(5)-\mathrm{C}(15)-\mathrm{H}(15) & 125.7 \\ \mathrm{C}(14)-\mathrm{C}(15)-\mathrm{H}(15) & 125.7 \\ \mathrm{~N}(7)-\mathrm{C}(16)-\mathrm{C}(17) & 108.7(4) \\ \mathrm{N}(7)-\mathrm{C}(16)-\mathrm{H}(16) & 125.7 \\ \mathrm{C}(17)-\mathrm{C}(16)-\mathrm{H}(16) & 125.7 \\ \mathrm{C}(16)-\mathrm{C}(17)-\mathrm{C}(18) & 104.3(4) \\ \mathrm{C}(16)-\mathrm{C}(17)-\mathrm{H}(17) & 127.8 \\ \mathrm{C}(18)-\mathrm{C}(17)-\mathrm{H}(17) & 127.8 \\ \mathrm{~N}(6)-\mathrm{C}(18)-\mathrm{C}(17) & 110.2(4) \\ \mathrm{N}(6)-\mathrm{C}(18)-\mathrm{H}(18) & 124.9 \\ \mathrm{C}(17)-\mathrm{C}(18)-\mathrm{H}(18) & 124.9 \\ \mathrm{~N}(9)-\mathrm{C}(19)-\mathrm{C}(20) & 109.5(4) \\ \mathrm{N}(9)-\mathrm{C}(19)-\mathrm{H}(19) & 125.2 \\ \mathrm{C}(20)-\mathrm{C}(19)-\mathrm{H}(19) & 125.2 \\ \mathrm{C}(19)-\mathrm{C}(20)-\mathrm{C}(21) & 104.1(5) \\ \mathrm{C}(19)-\mathrm{C}(20)-\mathrm{H}(20) & 128.0 \\ \mathrm{C}(21)-\mathrm{C}(20)-\mathrm{H}(20) & 128.0 \\ \mathrm{C}(20)-\mathrm{C}(21)-\mathrm{H}(21) & 124.4 \\ & \end{array}$




$\begin{array}{ll}\mathrm{N}(1)-\mathrm{C}(22)-\mathrm{H}(22 \mathrm{~A}) & 109.5 \\ \mathrm{~N}(1)-\mathrm{C}(22)-\mathrm{H}(22 \mathrm{~B}) & 109.5 \\ \mathrm{H}(22 \mathrm{~A})-\mathrm{C}(22)-\mathrm{H}(22 \mathrm{~B}) & 109.5 \\ \mathrm{~N}(1)-\mathrm{C}(22)-\mathrm{H}(22 \mathrm{C}) & 109.5 \\ \mathrm{H}(22 \mathrm{~A})-\mathrm{C}(22)-\mathrm{H}(22 \mathrm{C}) & 109.5 \\ \mathrm{H}(22 \mathrm{~B})-\mathrm{C}(22)-\mathrm{H}(22 \mathrm{C}) & 109.5 \\ \mathrm{O}(1)-\mathrm{C}(23)-\mathrm{H}(23 \mathrm{~A}) & 109.5 \\ \mathrm{O}(1)-\mathrm{C}(23)-\mathrm{H}(23 \mathrm{~B}) & 109.5 \\ \mathrm{H}(23 \mathrm{~A})-\mathrm{C}(23)-\mathrm{H}(23 \mathrm{~B}) & 109.5 \\ \mathrm{O}(1)-\mathrm{C}(23)-\mathrm{H}(23 \mathrm{C}) & 109.5 \\ \mathrm{H}(23 \mathrm{~A})-\mathrm{C}(23)-\mathrm{H}(23 \mathrm{C}) & 109.5 \\ \mathrm{H}(23 \mathrm{~B})-\mathrm{C}(23)-\mathrm{H}(23 \mathrm{C}) & 109.5 \\ \mathrm{~F}(1)-\mathrm{C}(24)-\mathrm{F}(3) & 107.5(6) \\ \mathrm{F}(1)-\mathrm{C}(24)-\mathrm{F}(2) & 107.7(6) \\ \mathrm{F}(3)-\mathrm{C}(24)-\mathrm{F}(2) & 108.2(6) \\ \mathrm{F}(1)-\mathrm{C}(24)-\mathrm{S}(1) & 111.4(5) \\ \mathrm{F}(3)-\mathrm{C}(24)-\mathrm{S}(1) & 111.3(5) \\ \mathrm{F}(2)-\mathrm{C}(24)-\mathrm{S}(1) & 110.6(5) \\ \mathrm{N}(9)-\mathrm{B}(1)-\mathrm{N}(5) & 109.2(3) \\ \mathrm{N}(9)-\mathrm{B}(1)-\mathrm{N}(7) & 108.7(3) \\ \mathrm{N}(5)-\mathrm{B}(1)-\mathrm{N}(7) & 106.9(4) \\ \mathrm{N}(9)-\mathrm{B}(1)-\mathrm{H}(1 \mathrm{~B}) & 112(4) \\ \mathrm{N}(5)-\mathrm{B}(1)-\mathrm{H}(1 \mathrm{~B}) & 113(3) \\ \mathrm{N}(7)-\mathrm{B}(1)-\mathrm{H}(1 \mathrm{~B}) & 107(3)\end{array}$


Compound 124

Table 4. Anisotropic displacement parameters $\left(\AA^{2} \times 10^{3}\right)$ for $\mathrm{C}_{24} \mathrm{H}_{30} \mathrm{BF}_{3} \mathrm{MoN}_{10} \mathrm{O}_{5} \mathrm{~S}$.

The anisotropic displacement factor exponent takes the form: $-2 \pi^{2}\left[h^{2} a^{* 2} U^{11}+\ldots+2\right.$ $\mathrm{h} \mathrm{k} \mathrm{a*} \mathrm{b}^{*} \mathrm{U}^{12}$ ]

\begin{tabular}{|c|c|c|c|c|c|c|}
\hline & U11 & $\mathrm{U}^{22}$ & $\mathrm{U}^{33}$ & $\mathrm{U}^{23}$ & $\mathrm{U}^{13}$ & $\mathrm{U}^{12}$ \\
\hline Mo & $19(1)$ & $11(1)$ & $25(1)$ & $-2(1)$ & $1(1)$ & $-4(1)$ \\
\hline $\mathrm{S}(1)$ & $36(1)$ & $31(1)$ & $41(1)$ & $-16(1)$ & $6(1)$ & $-8(1)$ \\
\hline $\mathrm{F}(1)$ & $70(3)$ & $55(3)$ & $65(2)$ & $-25(2)$ & $16(2)$ & $1(2)$ \\
\hline $\mathrm{F}(2)$ & $63(3)$ & $69(4)$ & $133(5)$ & $3(4)$ & $44(3)$ & $-22(3)$ \\
\hline $\mathrm{F}(3)$ & $51(3)$ & $82(5)$ & $121(5)$ & $-15(4)$ & $-34(3)$ & $7(3)$ \\
\hline $\mathrm{O}(1)$ & $23(1)$ & $34(2)$ & $32(1)$ & $-4(1)$ & $4(1)$ & $-7(1)$ \\
\hline $\mathrm{O}(2)$ & $44(2)$ & $9(1)$ & $50(2)$ & $-5(1)$ & $0(1)$ & $-9(1)$ \\
\hline $\mathrm{O}(3)$ & $46(2)$ & $77(5)$ & $76(3)$ & $-31(3)$ & $-17(2)$ & $15(3)$ \\
\hline $\mathrm{O}(4)$ & $94(4)$ & $53(3)$ & $62(3)$ & $-25(3)$ & $36(3)$ & $-40(3)$ \\
\hline $\mathrm{O}(5)$ & $92(4)$ & $45(3)$ & $67(3)$ & $-31(3)$ & $10(3)$ & $-27(3)$ \\
\hline $\mathrm{N}(1)$ & $26(1)$ & $26(2)$ & $34(2)$ & $-9(2)$ & $-5(1)$ & $-6(1)$ \\
\hline $\mathrm{N}(2)$ & $21(1)$ & $20(2)$ & $25(1)$ & $-7(1)$ & $3(1)$ & $-5(1)$ \\
\hline $\mathrm{N}(3)$ & $27(1)$ & $31(2)$ & $30(2)$ & $-7(1)$ & $9(1)$ & $-6(1)$ \\
\hline $\mathrm{N}(4)$ & $17(1)$ & $14(1)$ & $26(1)$ & $-4(1)$ & $0(1)$ & $-4(1)$ \\
\hline $\mathrm{N}(5)$ & $24(1)$ & $21(2)$ & $25(1)$ & $-7(1)$ & $5(1)$ & $-7(1)$ \\
\hline $\mathrm{N}(6)$ & $17(1)$ & $14(1)$ & $26(1)$ & $-3(1)$ & $2(1)$ & $-3(1)$ \\
\hline $\mathrm{N}(7)$ & $22(1)$ & $22(2)$ & $26(1)$ & $0(1)$ & $0(1)$ & $-10(1)$ \\
\hline $\mathrm{N}(8)$ & $24(1)$ & $11(1)$ & $31(1)$ & $-3(1)$ & $4(1)$ & $-5(1)$ \\
\hline $\mathrm{N}(9)$ & $28(1)$ & $15(2)$ & $33(2)$ & $-9(1)$ & $6(1)$ & $-6(1)$ \\
\hline $\mathrm{N}(10)$ & $22(1)$ & $8(1)$ & $31(1)$ & $-3(1)$ & $3(1)$ & $-5(1)$ \\
\hline $\mathrm{C}(1)$ & $21(1)$ & $24(2)$ & $24(1)$ & $-3(1)$ & $-2(1)$ & $-3(1)$ \\
\hline $\mathrm{C}(2)$ & $24(1)$ & $15(2)$ & $28(2)$ & $-5(1)$ & $1(1)$ & $-2(1)$ \\
\hline$C(3)$ & $24(2)$ & $28(2)$ & $25(1)$ & $-3(1)$ & $2(1)$ & $-4(2)$ \\
\hline$C(4)$ & $28(2)$ & $44(3)$ & $25(2)$ & $-8(2)$ & $2(1)$ & $-4(2)$ \\
\hline$C(5)$ & $33(2)$ & $40(3)$ & $32(2)$ & $-17(2)$ & $-6(2)$ & $-2(2)$ \\
\hline$C(6)$ & $26(2)$ & $19(2)$ & $27(2)$ & $-7(1)$ & $5(1)$ & $-6(1)$ \\
\hline$C(7)$ & $25(2)$ & $19(2)$ & $30(2)$ & $-4(1)$ & $7(1)$ & $-7(1)$ \\
\hline$C(8)$ & $22(1)$ & $26(2)$ & $26(1)$ & $-5(1)$ & $4(1)$ & $-7(1)$ \\
\hline $\mathrm{C}(9)$ & $26(2)$ & $26(2)$ & $33(2)$ & $-12(2)$ & $8(1)$ & $-8(2)$ \\
\hline
\end{tabular}




$\begin{array}{lllllll}\mathrm{C}(10) & 23(1) & 21(2) & 32(2) & -8(2) & 4(1) & -8(1) \\ \mathrm{C}(11) & 33(2) & 32(3) & 38(2) & -1(2) & 12(2) & -9(2) \\ \mathrm{C}(12) & 35(2) & 44(3) & 38(2) & -16(2) & 13(2) & -12(2) \\ \mathrm{C}(13) & 18(1) & 17(2) & 27(1) & -2(1) & 2(1) & -5(1) \\ \mathrm{C}(14) & 24(2) & 27(2) & 29(2) & -1(2) & 6(1) & -6(2) \\ \mathrm{C}(15) & 30(2) & 26(2) & 25(2) & -6(2) & 9(1) & -7(2) \\ \mathrm{C}(16) & 27(2) & 34(3) & 32(2) & 2(2) & -7(1) & -16(2) \\ \mathrm{C}(17) & 19(1) & 28(2) & 42(2) & 6(2) & -6(1) & -5(2) \\ \mathrm{C}(18) & 16(1) & 18(2) & 35(2) & 2(1) & 2(1) & -1(1) \\ \mathrm{C}(19) & 40(2) & 20(2) & 44(2) & -16(2) & 11(2) & -11(2) \\ \mathrm{C}(20) & 46(3) & 14(2) & 54(3) & -11(2) & 17(2) & -10(2) \\ \mathrm{C}(21) & 32(2) & 11(2) & 40(2) & -3(2) & 6(2) & -2(2) \\ \mathrm{C}(22) & 45(3) & 31(3) & 56(3) & -7(2) & -14(2) & -17(2) \\ \mathrm{C}(23) & 28(2) & 36(3) & 38(2) & -7(2) & 4(2) & 2(2) \\ \mathrm{C}(24) & 36(2) & 41(4) & 63(4) & -3(3) & 3(2) & -6(2) \\ \mathrm{B}(1) & 25(2) & 22(2) & 27(2) & -7(2) & 5(1) & -9(2)\end{array}$


Compound 124

Table 5. Hydrogen coordinates ( x $\left.10^{4}\right)$ and isotropic displacement parameters $\left(\AA^{2} \times 10\right.$ 3 ) for $\mathrm{C}_{24} \mathrm{H}_{30} \mathrm{BF}_{3} \mathrm{MoN}_{10} \mathrm{O}_{5} \mathrm{~S}$.

\begin{tabular}{|c|c|c|c|c|}
\hline & $\mathrm{x}$ & $\mathrm{y}$ & $\mathrm{z}$ & $\mathrm{U}(\mathrm{eq})$ \\
\hline $\mathrm{H}(2)$ & 5155 & 5810 & 7496 & 27 \\
\hline $\mathrm{H}(3)$ & 3444 & 5935 & 8606 & 31 \\
\hline $\mathrm{H}(4)$ & 3069 & 7755 & 9363 & 40 \\
\hline $\mathrm{H}(5)$ & 4415 & 9262 & 8991 & 41 \\
\hline $\mathrm{H}(6)$ & 283 & 5871 & 7791 & 28 \\
\hline $\mathrm{H}(7)$ & -1460 & 5685 & 8852 & 30 \\
\hline $\mathrm{H}(9)$ & -951 & 9111 & 9457 & 33 \\
\hline $\mathrm{H}(10)$ & 822 & 9144 & 8395 & 30 \\
\hline $\mathrm{H}(11 \mathrm{~A})$ & -3605 & 6118 & 9522 & 53 \\
\hline $\mathrm{H}(11 \mathrm{~B})$ & -3910 & 6330 & 10523 & 53 \\
\hline $\mathrm{H}(11 \mathrm{C})$ & -2434 & 5442 & 10279 & 53 \\
\hline $\mathrm{H}(12 \mathrm{~A})$ & -2300 & 8171 & 10919 & 56 \\
\hline $\mathrm{H}(12 \mathrm{~B})$ & -3930 & 8370 & 10639 & 56 \\
\hline $\mathrm{H}(12 \mathrm{C})$ & -2920 & 9217 & 10052 & 56 \\
\hline $\mathrm{H}(13)$ & 5064 & 8897 & 5689 & 25 \\
\hline $\mathrm{H}(14)$ & 5593 & 8647 & 4103 & 33 \\
\hline $\mathrm{H}(15)$ & 4020 & 7201 & 3769 & 33 \\
\hline $\mathrm{H}(16)$ & -735 & 7989 & 4303 & 37 \\
\hline $\mathrm{H}(17)$ & -2244 & 9637 & 5118 & 38 \\
\hline $\mathrm{H}(18)$ & -852 & 9519 & 6508 & 29 \\
\hline $\mathrm{H}(19)$ & 2038 & 3774 & 5503 & 39 \\
\hline $\mathrm{H}(20)$ & 2598 & 2548 & 7061 & 45 \\
\hline $\mathrm{H}(21)$ & 2981 & 4212 & 7920 & 35 \\
\hline $\mathrm{H}(22 \mathrm{~A})$ & 7312 & 9189 & 7785 & 64 \\
\hline $\mathrm{H}(22 \mathrm{~B})$ & 6028 & 10207 & 8123 & 64 \\
\hline $\mathrm{H}(22 \mathrm{C})$ & 6098 & 10057 & 7104 & 64 \\
\hline $\mathrm{H}(23 \mathrm{~A})$ & 7407 & 5595 & 7070 & 53 \\
\hline $\mathrm{H}(23 \mathrm{~B})$ & 8154 & 6403 & 6265 & 53 \\
\hline $\mathrm{H}(23 \mathrm{C})$ & 6537 & 6285 & 6174 & 53 \\
\hline $\mathrm{H}(1 \mathrm{~B})$ & $1750(60)$ & $6240(70)$ & $4580(40)$ & $23(13)$ \\
\hline
\end{tabular}


${ }^{1} H_{\text {NMR }}\left(d^{6}\right.$-Acetone) of Compound 124:

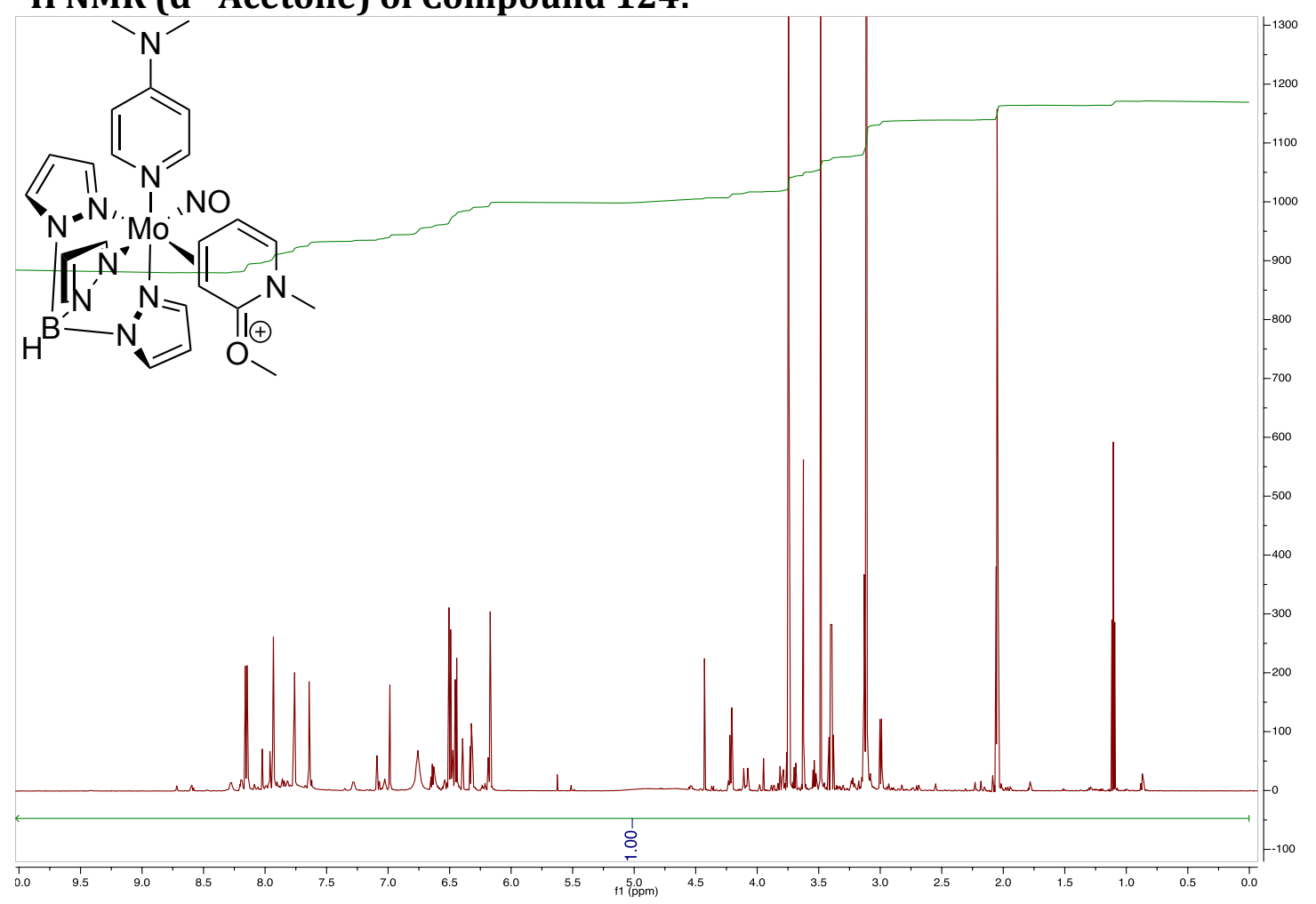

${ }^{1} \mathrm{H}$ NMR (d6-Acetone) of Compound 124A from Selective Precipitation:

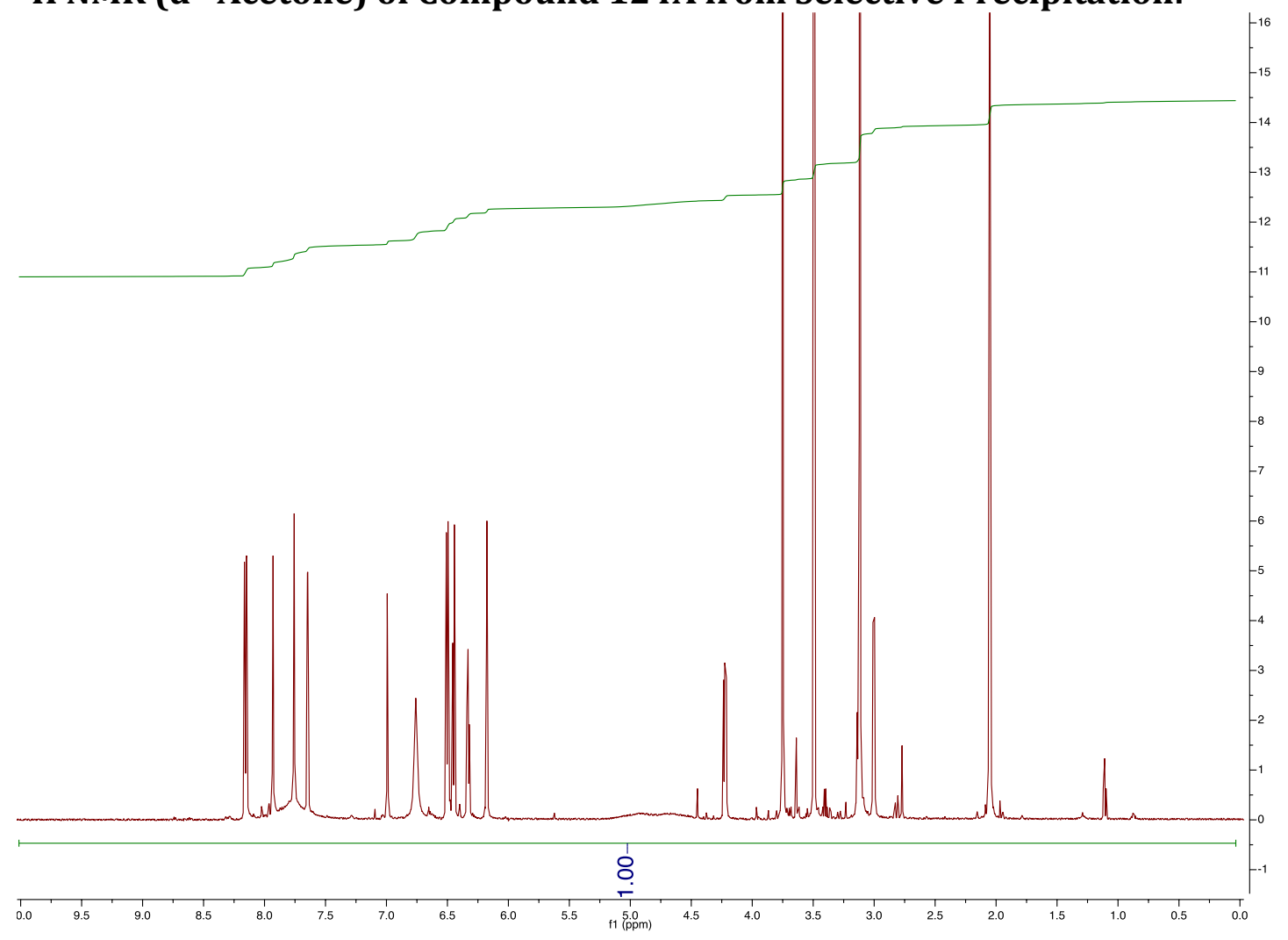


${ }^{13}$ C NMR (d6-Acetone) of Compound 124:

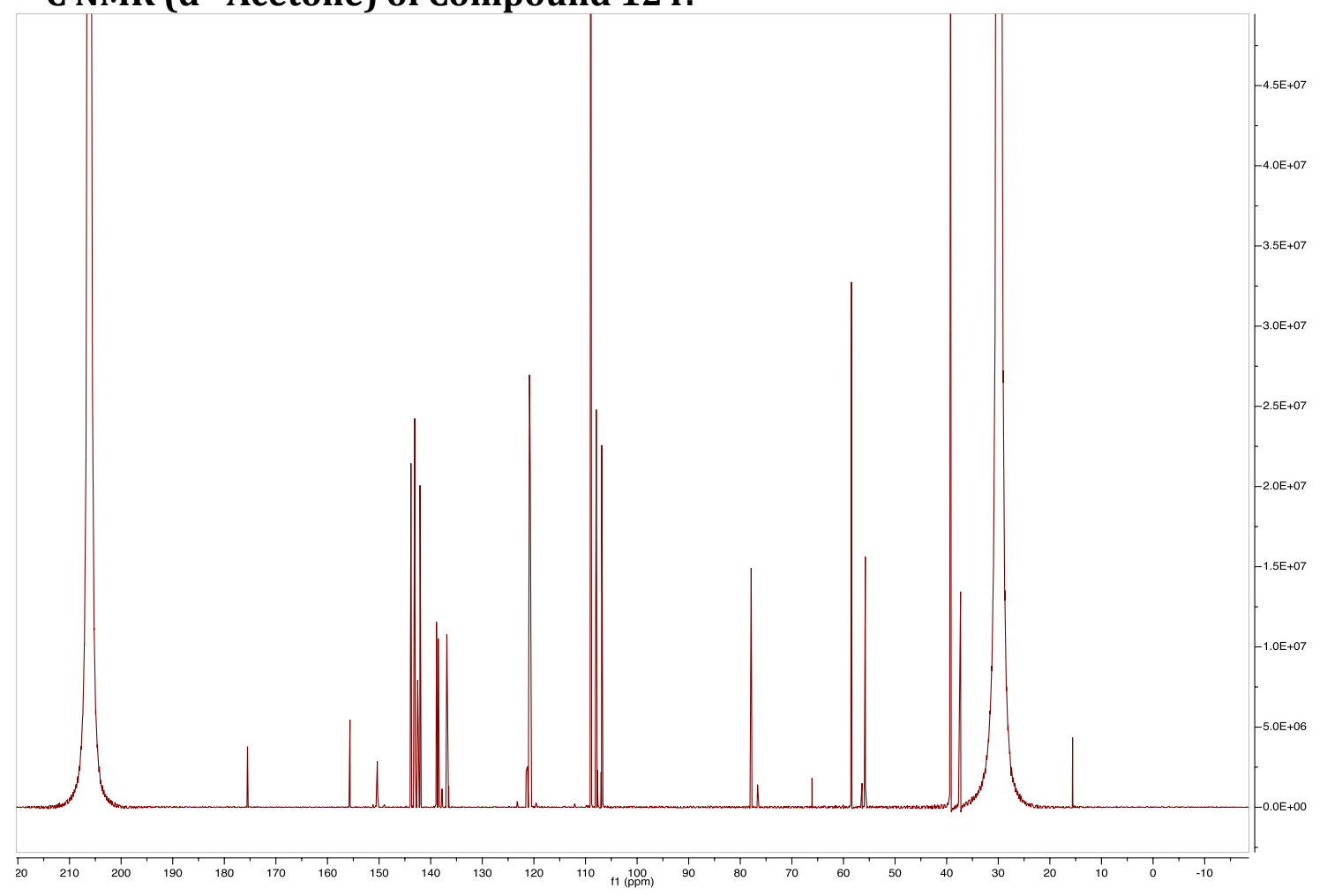


${ }^{1}$ H NMR (d6-Acetone) of Compound 125:

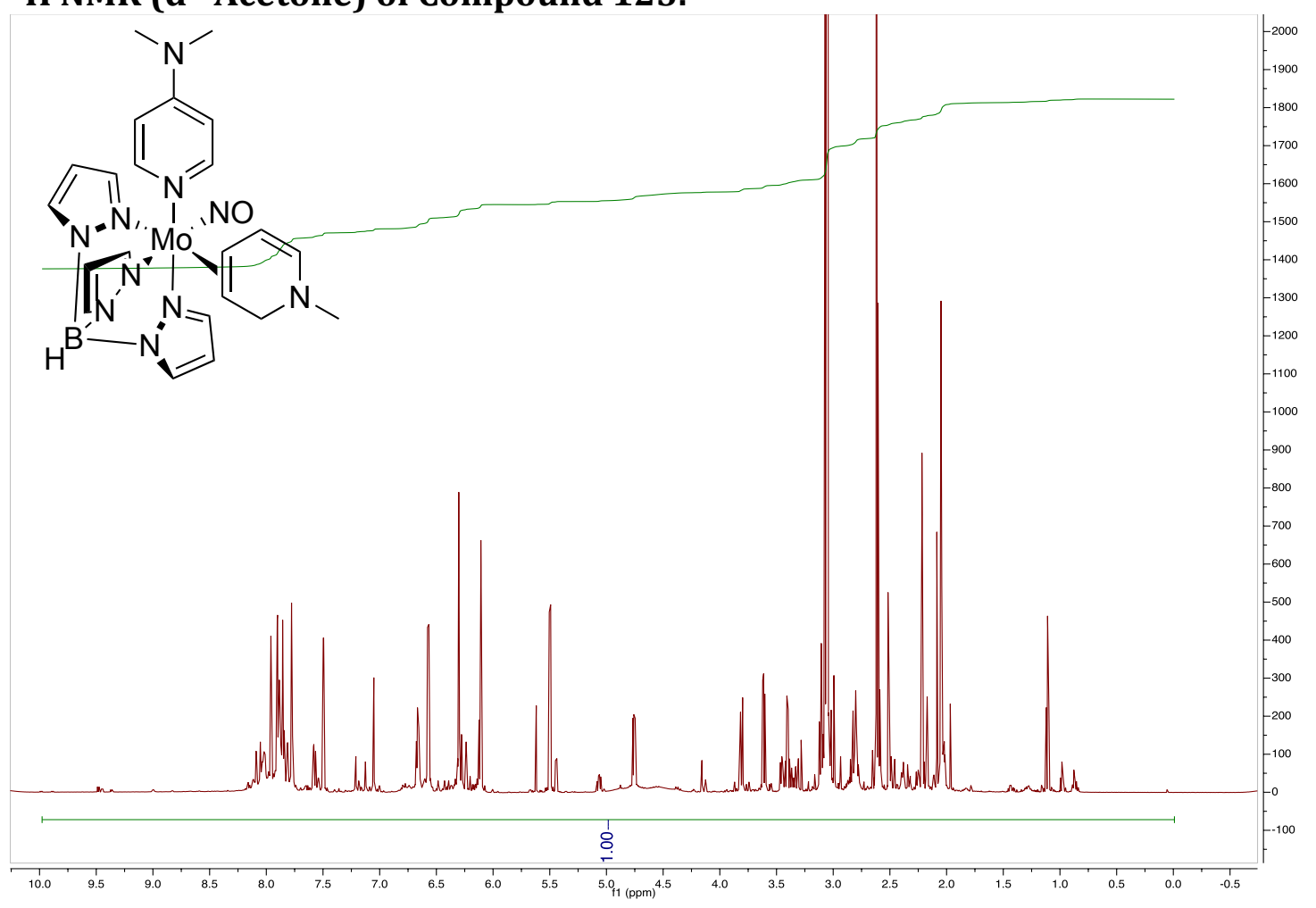

${ }^{1}$ H NMR (d6-Acetone) of Compound 125A From Selective Precipitation:

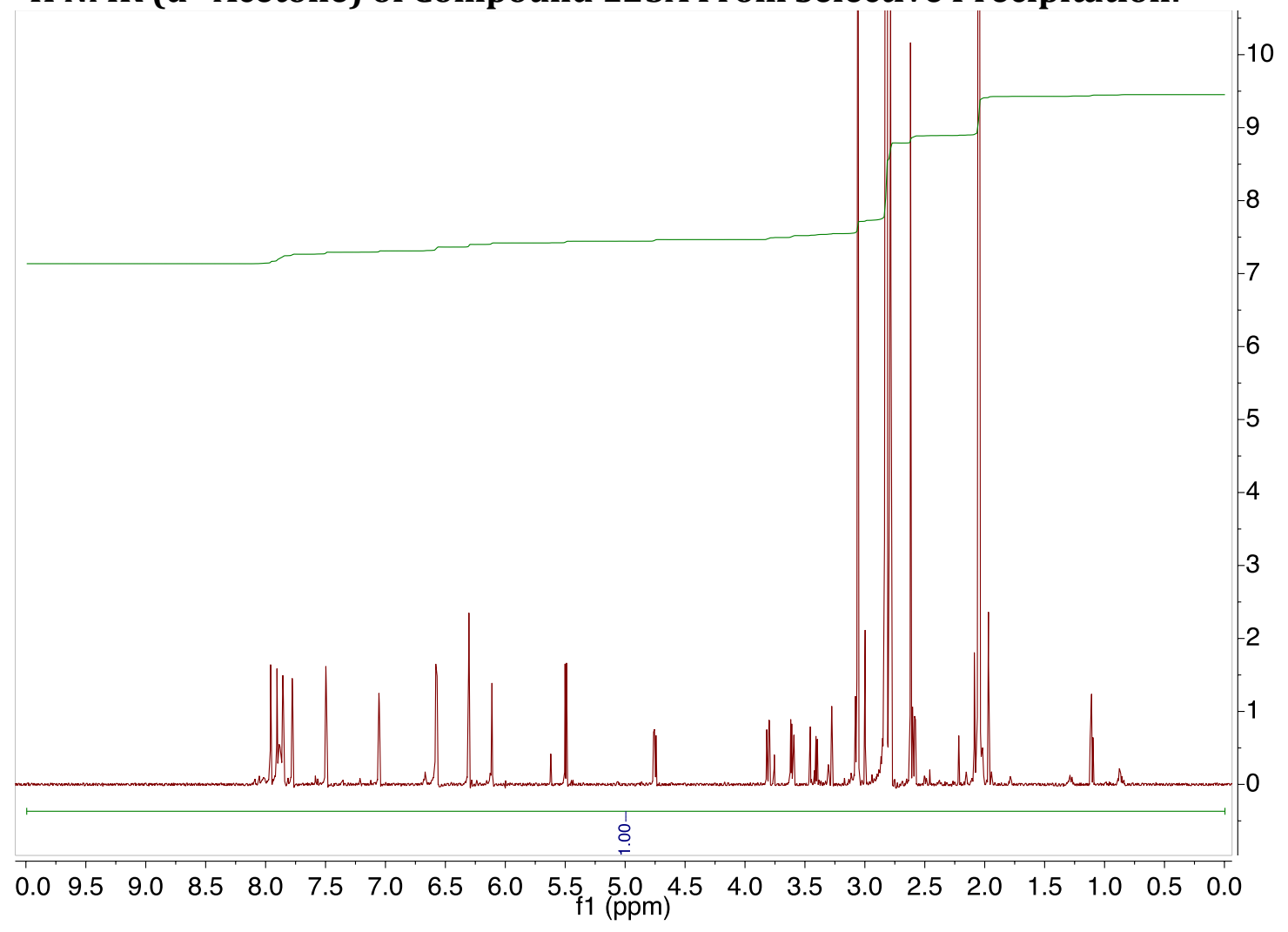


${ }^{13}$ C NMR (d6-Acetone) of Compound 125:

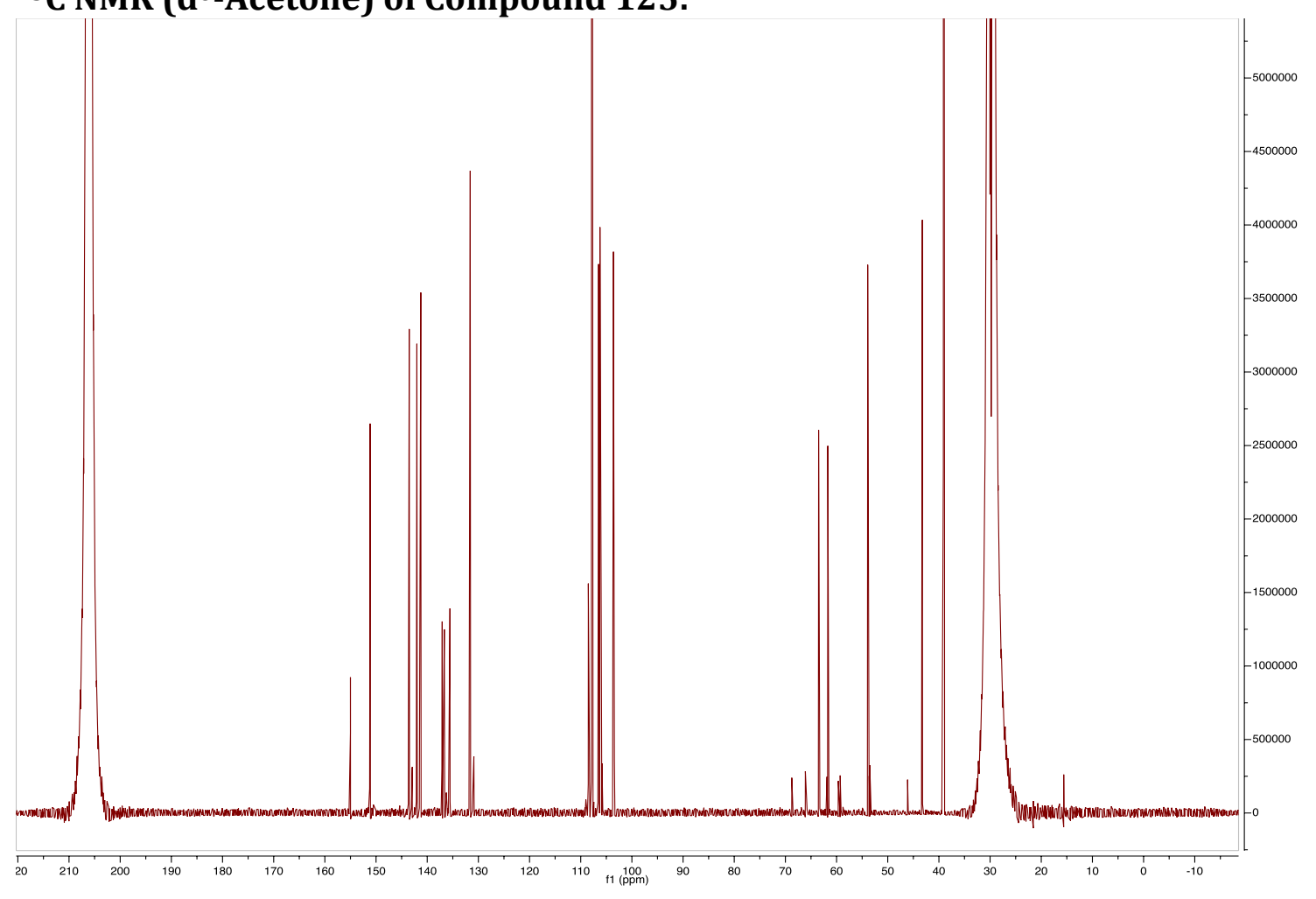


${ }^{1} \mathrm{H}$ NMR (d6-Acetone) of Compound 126:

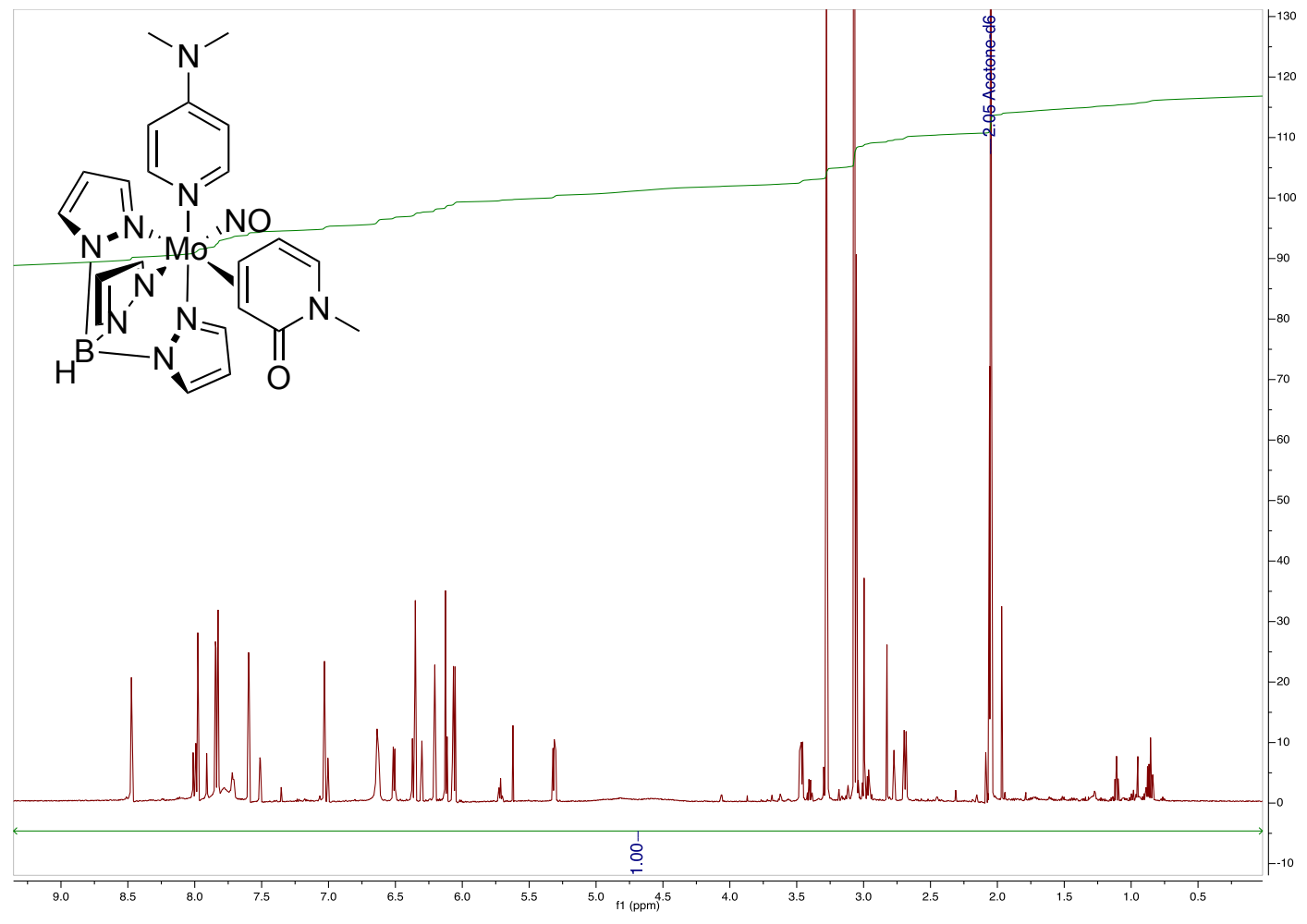

${ }^{1}$ H NMR (d6-Acetone) of Compound 126A:

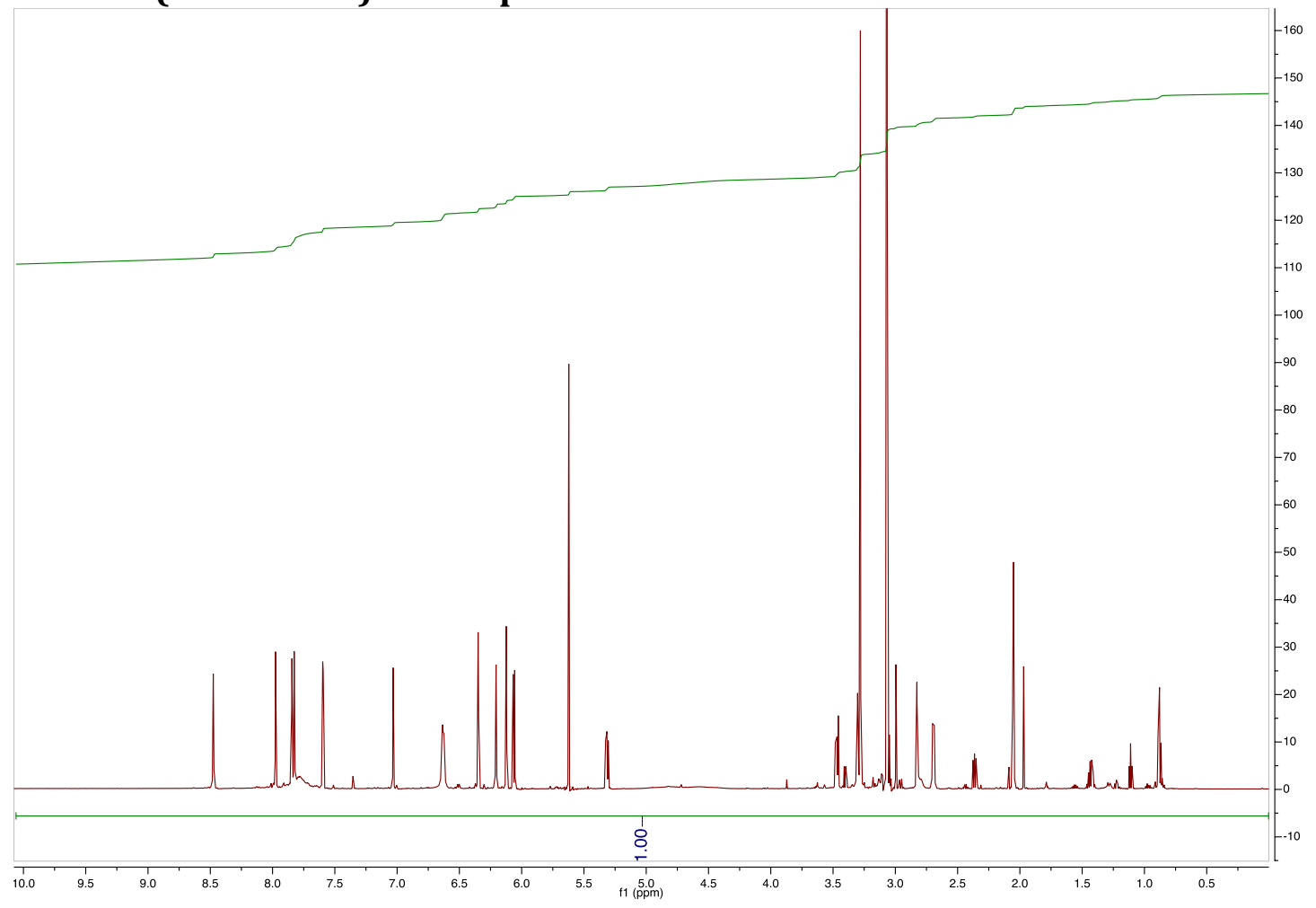


${ }^{13}$ C NMR (d6-Acetone) of Compound 126:

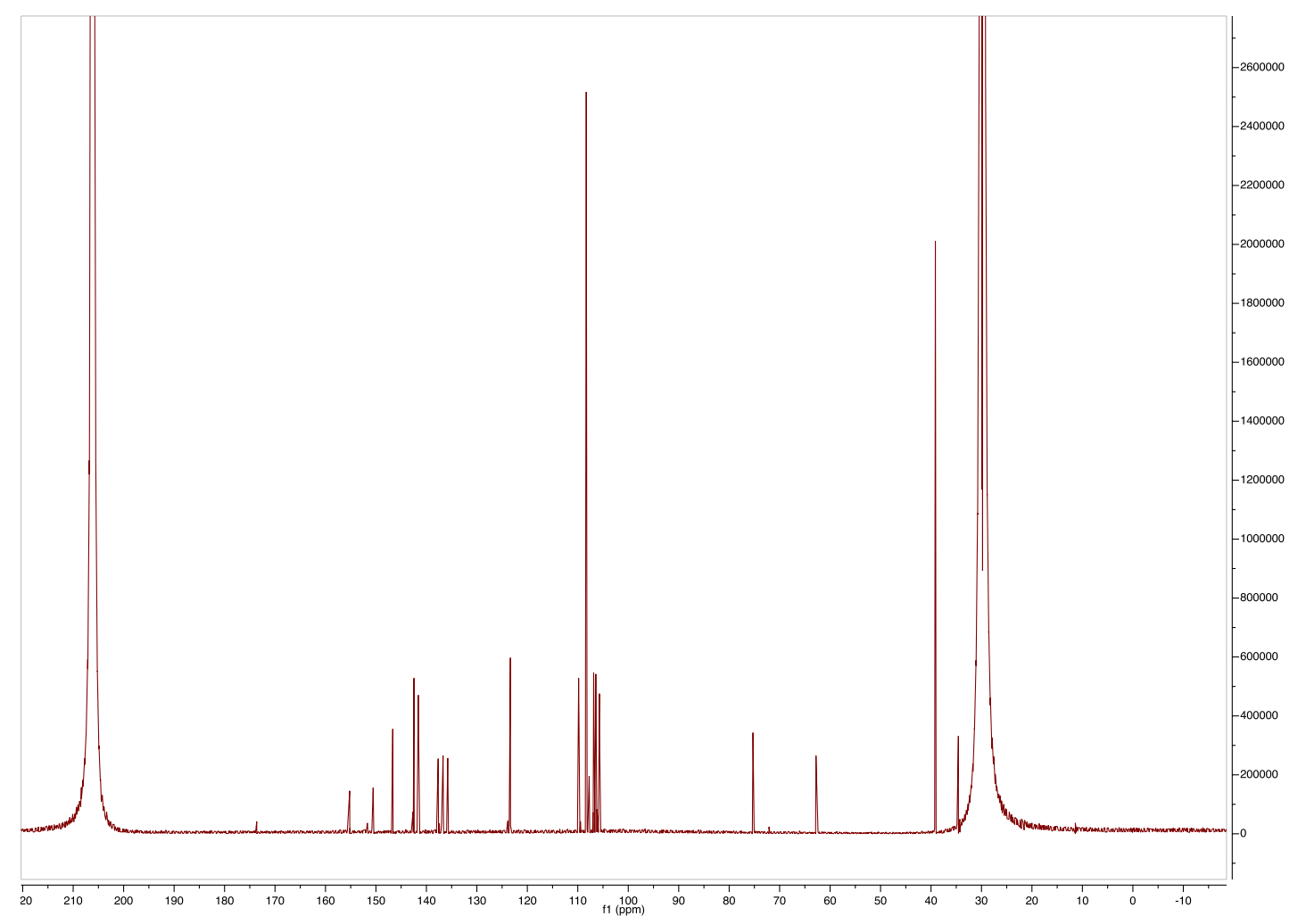


${ }^{1}$ H NMR (d6-Acetone) of Compound 127:
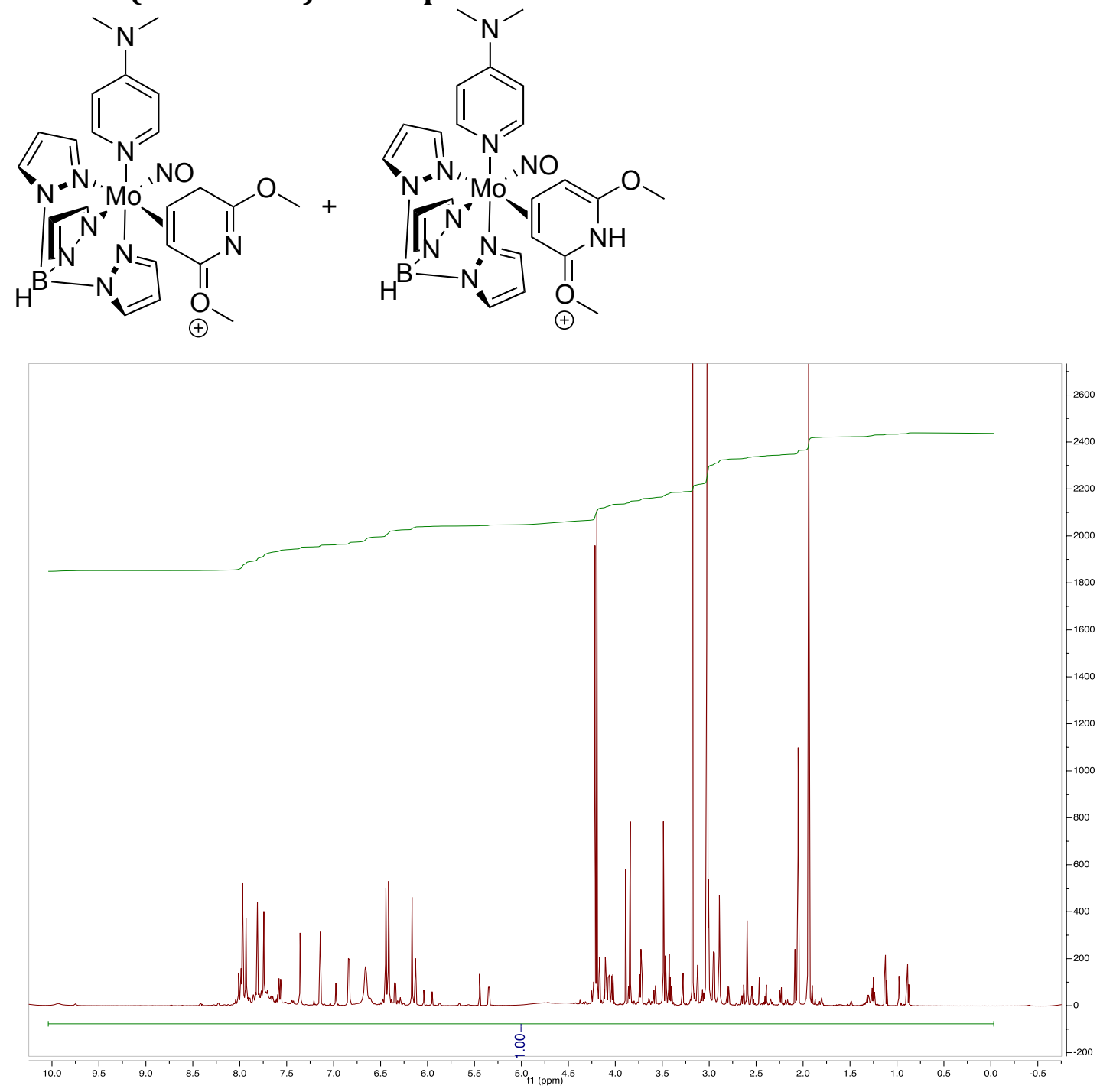
${ }^{13}$ C NMR (d6-Acetone) of Compound 127:

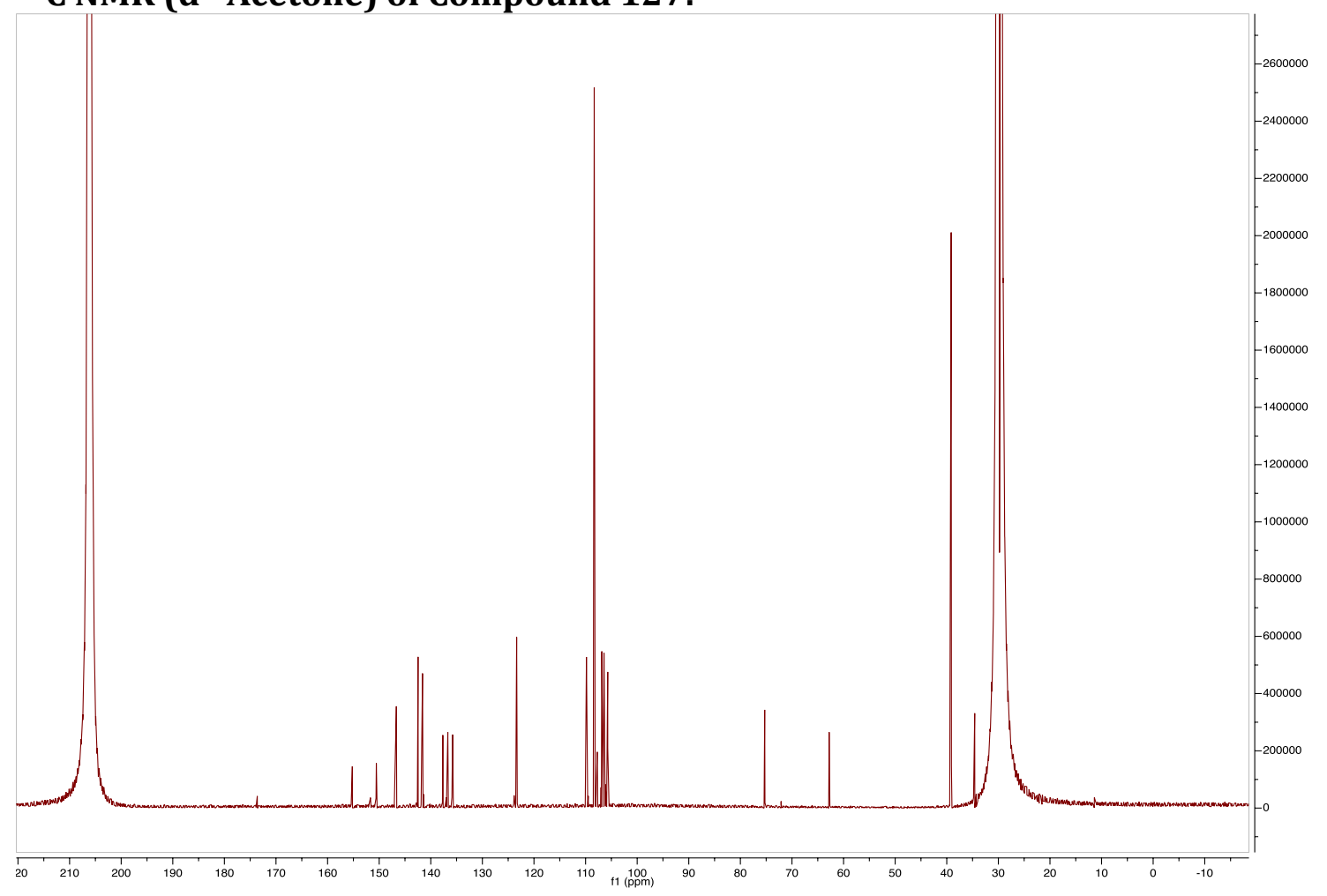



